


\section{MIXED WASTE MANAGEMENT FACILITY GROUNDWATER MONITORING REPORT (U)}

FOURTH QUARTER 1994 AND 1994 SUMMARY

\section{Publication Date: March 1995}

Authorized Derivative Classifier and Reviewing Official:

UNCLASSIFIED

Does Not Contain Unclassified Controlled Nuclear Information

Westinghouse Savannah River Company Savannah River Site Aiken, SC 29808

DISTRIBUTION OF THIS DOCUMENT IS UNLIRMTED M 


\section{DISCLAIMER}

This report was prepared as an account of work sponsored by an agency of the United States Government. Neither the United States Government nor any agency thereof, nor any of their employees, make any warranty, express or implied, or assumes any legal liability or responsibility for the accuracy, completeness, or usefulness of any information, apparatus, product, or process disclosed, or represents that its use would not infringe privately owned rights. Reference herein to any specific commercial product, process, or service by trade name; trademark, manufacturer, or otherwise does not necessarily constitute or imply its endorsement, recommendation, or favoring by the United States Government or any agency thereof. The views and opinions of authors expressed herein do not necessarily state or reflect those of the United States Government or any agency thereof. 


\section{DISCLAIMER}

Portions of this document may be illegible in electronic image products. Images are produced from the best available original document. 


\section{MIXED WASTE MANAGEMENT FACILITY GROUNDWATER MONITORING REPORT (U)}

FOURTH QUARTER 1994

AND 1994 SUMMARY

Publication Date: March 1995

Key Words

BGO wells

FSS wells

tetrachloroethylene

trichloroethylene

tritium

Westinghouse Savannah River Company

Savannah River Site

Aiken, SC 29808 
Unclassified

THIS PAGE LEFT BLANK INTENTIONALLY. 


\section{Abstract}

During fourth quarter 1994, ten constituents exceeded final Primary Drinking Water Standards (PDWS) in groundwater samples from downgradient monitoring wells at the Mixed Waste Management Facility, the Old Burial Ground, the E-Area Vaults, the proposed Hazardous Wastel Mixed Waste Disposal Vaults, and the F-Area Sewage Sludge Application Site. No constituent exceeded final PDWS in samples from the upgradient monitoring wells.

As in previous quarters, tritium and trichloroethylene were the most widespread elevated constituents. Benzene, chloroethene, copper, 1,1-dichloroethylene, dichloromethane, lead, mercury, and tetrachloroethylene also exceeded final Primary Drinking Water Standards in one or more wells. Elevated constituents were found in numerous Aquifer Zone $\| \mathrm{B}_{2}$ (Water Table) and Aquifer Zone IIB (Barnwell/McBean) wells. Elevated constituents were found in three Aquifer Unit IIA (Congaree) wells.

The groundwater flow directions and rates in the three hydrostratigraphic units were similar to those of previous quarters. 
WSRC-TR-94-0610

Unclassified

THIS PAGE LEFT BLANK INTENTIONALLY. 


\section{Contents}

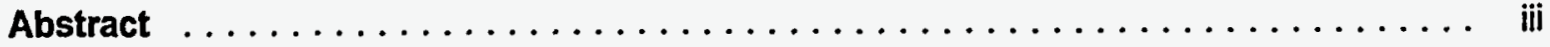

List of Figures $\ldots \ldots \ldots \ldots \ldots \ldots \ldots \ldots \ldots \ldots \ldots \ldots \ldots \ldots \ldots \ldots \ldots$

List of Tables $\ldots \ldots \ldots \ldots \ldots \ldots \ldots \ldots \ldots \ldots \ldots \ldots \ldots \ldots \ldots \ldots \ldots \ldots$

Executive Summary $\ldots \ldots \ldots \ldots \ldots \ldots \ldots \ldots \ldots \ldots \ldots \ldots \ldots \ldots \ldots$

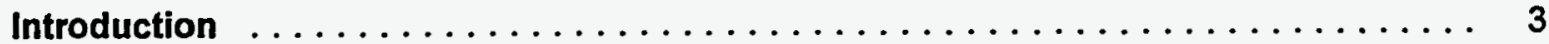

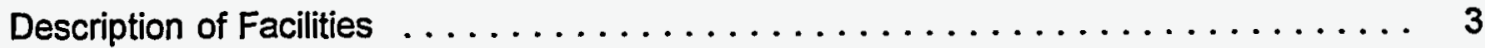

Hydrostratigraphic Units . ......................... 4

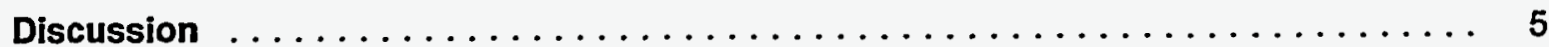

Groundwater Monitoring Data ....................... 5

Integrity of the Monitoring Well Network $\ldots \ldots \ldots \ldots \ldots \ldots \ldots \ldots \ldots$

Analytical Results Exceeding Standards ..................... 8

Trichloroethylene and Tritium Time-Trend Data . . . . . . . . . . . . . 10

Water Levels . . . . . . . . . . . . . . . . . . . . . . . . . . . 11

Groundwater Flow Rates and Directions . . . . . . . . . . . . . . . . 12

Upgradient Versus Downgradient Results $\ldots \ldots \ldots \ldots \ldots \ldots \ldots \ldots \ldots \ldots \ldots$

Quality Control Results $\ldots \ldots \ldots \ldots \ldots \ldots \ldots \ldots \ldots \ldots \ldots \ldots \ldots \ldots$

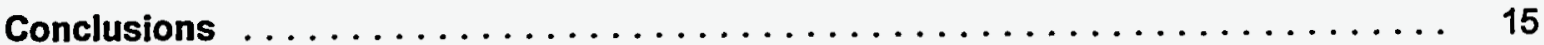

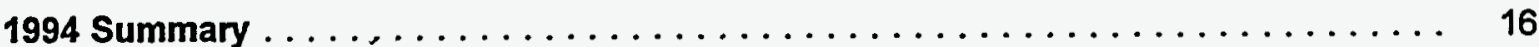

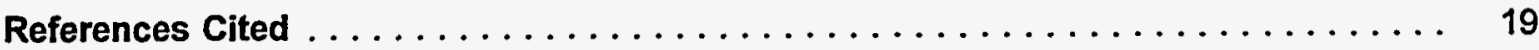

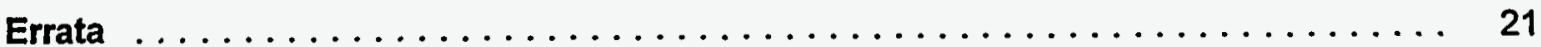

Appendix A-Final Primary Drinking Water Standards $\ldots \ldots \ldots \ldots \ldots \ldots$ A-1

Appendix B-Flagging Criteria $\ldots \ldots \ldots \ldots \ldots \ldots \ldots \ldots \ldots \ldots \ldots \ldots \ldots \ldots \ldots$

Appendix C-Figures $\ldots \ldots \ldots \ldots \ldots \ldots \ldots \ldots \ldots \ldots \ldots \ldots \ldots \ldots \ldots$

Appendix $\mathrm{D}-$ Groundwater Monitoring Results Tables $\ldots \ldots \ldots \ldots \ldots \ldots \ldots$

Appendix E-Data Quality/Usability Assessment $\ldots \ldots \ldots \ldots \ldots \ldots \ldots \ldots$

Appendix F-Time Series Plots $\ldots \ldots \ldots \ldots \ldots \ldots \ldots \ldots \ldots \ldots \ldots \ldots \ldots \ldots$

Appendix G-Hydrographs $\ldots \ldots \ldots \ldots \ldots \ldots \ldots \ldots \ldots \ldots \ldots \ldots \ldots \ldots \ldots$ 


\section{List of Figures}

Page

1. Location of the Mixed Waste Management Facility at the Savannah River Site . . . C C-3

2. Location of the Groundwater Monitoring Wells at the Mixed Waste Management Facility, the Old Burial Ground, the E-Area Vaults, the Hazardous Waste/Mixed Waste Disposal Vaults (the Burial Ground Complex), and the F-Area Sewage Sludge Application Site $\ldots \ldots \ldots \ldots \ldots \ldots \ldots \ldots \ldots \ldots \ldots \ldots \ldots \ldots \ldots$

3. Hydrostratigraphic Nomenclature $\ldots \ldots \ldots \ldots \ldots \ldots \ldots \ldots \ldots \ldots \ldots \ldots \ldots$

4. Regional Correlation of Hydrostratigraphic and Lithostratigraphic Nomenclature .... C-6

5. Location of Aquifer Zone $\mathrm{IIB}_{2}$ (Water Table) Wells at the Burial Ground Complex . . . C-7

6. Location of Aquifer Zone IIB ${ }_{1}$ (Barnwell/McBean) Wells at the Burial Ground Complex ...........................

7. Location of Aquifer Unit IIA (Congaree) Wells at the Burial Ground Complex . . . . . . C-9

8. Lead Concentrations in Aquifer Zone $\|_{2}$ (Water Table) at the Burial Ground Complex, Fourth Quarter $1994 \ldots \ldots \ldots \ldots \ldots \ldots \ldots \ldots \ldots \ldots$ C-10

9. Lead Concentrations in Aquifer Zone $\| B_{1}$ (Barnwell/McBean) at the Burial Ground Complex, Fourth Quarter $1994 \ldots \ldots \ldots \ldots \ldots \ldots \ldots \ldots \ldots \ldots \ldots$ C-11

10. Lead Concentrations in Aquifer Unit IIA (Congaree) at the Burial Ground Complex, Fourth Quarter $1994 \ldots \ldots \ldots \ldots \ldots \ldots \ldots \ldots \ldots \ldots \ldots$. . . . . . . . . . . . . .

11. $\mathrm{pH}$ Values in Aquifer Zone $\|_{\mathrm{B}}$ (Water Table) at the Burial Ground Complex, Fourth Quarter 1994

12. $\mathrm{pH}$ Values in Aquifer Zone $\| \mathrm{B}_{1}$ (Barnwell/McBean) at the Burial Ground Complex, Fourth Quarter 1994

13. pH Values in Aquifer Unit IIA (Congaree) at the Burial Ground Complex, Fourth Quarter 1994

14. Specific Conductance in Aquifer Zone $\| B_{2}$ (Water Table) at the Burial Ground Complex, Fourth Quarter 1994

15. Specific Conductance in Aquifer Zone $\|_{1} B_{1}$ (Barnwell/McBean) at the Burial Ground Complex, Fourth Quarter 1994

16. Specific Conductance in Aquifer Unit IIA (Congaree) at the Burial Ground Complex, Fourth Quarter 1994 
17. Trichloroethylene Concentrations in Aquifer Zone $\|_{2}$ (Water Table) at the Burial Ground Complex, Fourth Quarter 1994

18. Trichloroethylene Concentrations in Aquifer Zone IIB Burial Ground Complex, Fourth Quarter 1994 . .

19. . Trichloroethylene Concentrations in Aquifer Unit IIA (Congaree) at the Burial Ground Complex, Fourth Quarter 1994 . . . . . . . . . . . . . . . . . . . . . . . . . . . . .

20. Tritium Activities in Aquifer Zone IIB $_{2}$ (Water Table) at the Burial Ground Complex, Fourth Quarter 1994

21. Tritium Activities in Aquifer Zone $\mathrm{IIB}_{1}$ (Barnwell/McBean) at the Burial Ground Complex, Fourth Quarter 1994

22. Tritium Activities in Aquifer Unit IIA (Congaree) at the Burial Ground Complex, Fourth Quarter 1994

23. Piezometric Surface Map of Aquifer Zone $\| l_{2}$ (Water Table) at the Burial Ground Complex

24. Potentiometric Surface Map of Aquifer Zone $\| 1_{1}$ (Barnwell/McBean) at the Burial Ground Complex.

25. Potentiometric Surface Map of Aquifer Unit IIA (Congaree) at the Burial Ground Complex 


\section{List of Tables}

In-Text

Page

1. 1994 Groundwater Monitoring Well Installations at the Burial Ground Complex . . . . 7

2. Wells in the Purge Water Containment Program $\ldots \ldots \ldots \ldots \ldots \ldots \ldots$

3. Average Water Elevations ( $\mathrm{ft} \mathrm{ms}$ ) in the Hydrostratigraphic Units beneath the MWMF

4. Estimated Horizontal Groundwater Flow Rates (ft/year) in the Hydrostratigraphic Units beneath the MWMF

\section{Appendix D}

D-1. Maximum Levels of Constituents Exceeding the Final Primary Drinking Water Standards

D-2. Maximum Levels of Constituents Exceeding Other Flag 2 Criteria

D-11

D-3. Groundwater Monitoring Results for Individual Wells

D-15 


\section{Executive Summary}

Currently, 125 wells monitor groundwater quality in the uppermost aquifer beneath the Mixed Waste Management Facility (MWMF) at the Savannah River Site as required by the South Carolina Hazardous Waste Management Regulations and settlement agreements 87-52-SW and 9151-SW. Samples from the wells are analyzed for selected heavy metals, herbicides/pesticides, indicator parameters, radionuclides, volatile organic compounds, and other constituents. As part of the Burial Ground Complex field investigation plan, 23 additional groundwater monitoring wells were installed in the past year. Nine will be sampled beginning first quarter 1995 with the remaining wells to be sampled later in 1995.

During fourth quarter 1994, approximately 60 percent of the downgradient wells at the MWMF contained one or more constituents that exceeded final Primary Drinking Water Standards (PDWS). These elevated constituents included benzene, chloroethene, copper, 1,1-dichloroethylene, dichloromethane, lead, mercury, tetrachloroethylene, trichloroethylene, and tritium. Consistent with historical trends, elevated constituent levels were found primarily in Aquifer Zone $\|_{2}$ (Water Table) and Aquifer Zone IIB (Barnwell/McBean). Three wells in Aquifer Unit IIA (Congaree) contained elevated constituents.

As in previous quarters, tritium and trichloroethylene were the most widespread elevated constituents during fourth quarter 1994. Sixty-three $(50 \%)$ of the 125 monitoring wells contained elevated tritium activities. Trichloroethylene concentrations exceeded the final PDWS in $22(18 \%)$ wells. Chloroethene, 1,1-dichloroethylene, dichloromethane, lead, mercury, tetrachloroethylene, trichloroethylene, and tritium, elevated in one or more wells during fourth quarter 1994, also occurred in elevated levels during third quarters 1994. These constituents generally were elevated in the same wells during both quarters. Gross alpha, which was elevated in only one well during second and third quarters 1994, was not elevated during fourth quarter. Dichloromethane was elevated in four wells for the first time in several quarters.

Groundwater flow directions and rates in Aquifer Zone $\| B_{2}$ (Water Table), Aquifer Zone $\|_{1}$ (Barnwell/McBean), and Aquifer Unit IIA (Congaree) were similar to those of previous quarters. 
WSRC-TR-94-0610

Unclassified

THIS PAGE LEFT BLANK INTENTIONALLY. 


\section{Introduction}

\section{Description of Facilities}

The Mixed Waste Management Facility (MWMF) is part of the Burial Ground Complex, which also includes the Old Burial Ground, the Low-Level Radioactive Waste Disposal Facility (LLRWDF), the E-Area Vaults, and the proposed Hazardous Waste/Mixed Waste Disposal Vaults (HWMWDV). The Burial Ground Complex is located in the central part of the Savannah River Site (SRS) (Figures 1 and 2, Appendix C) within the General Separations Area. The following description outlines important events in the history of the MWMF:

- In 1972, the MWMF began receiving various radioactive and nonradioactive wastes (Heffner and Exploration Resources, 1991).

- A closure plan for the MWMF was filed November 23, 1985, with the South Carolina Department of Health and Environmental Control (SCDHEC) (Jaegge et al., 1987). The closure plan included placing a low permeability cap and final vegetative cover over the facility.

- The MWMF became inactive in 1986 (WSRC, 1993b).

- A consent decree between the U.S. Department of Energy and the Natural Resources Defense Council et al. (Civil Action 1:85-2583-6, U.S. District Court, District of South Carolina, Aiken Division), signed May 26, 1988, and effective June 1,1988, identified the MWMF as subject to the requirements of Subtitle $C$ of the Resource Conservation and Recovery Act (RCRA).

- SCDHEC approved an interim status closure plan for the MWMF in December 1990 and accepted the closure certification in April 1991 in accordance with South Carolina Hazardous Waste Management Regulations (SCHWMR), Subpart G (SCDHEC, 1993).

- A RCRA Part B post-closure care permit application renewal was submitted to SCDHEC in November 1992 (WSRC, 1992a). This permit application included the 58-acre MWMF and the 13-acre Solvent Rag Portions of the LLRWDF as agreed upon by SRS and SCDHEC in settlement agreements 87-52-SW (May 10, 1991) and 91-51-SW (August 26, 1991).

- A closure plan for the Solvent Rag Portions was submitted to SCDHEC in August 1993 (WSRC, 1993b).

- A revised RCRA Part B post-closure care permit application renewal, containing a groundwater corrective-action plan, was submitted to SCDHEC on November 30, 1993 (WSRC, 1993c).

- Beginning first quarter 1994, wells FSS 1D, 2D, 3D, and 4D, located at the F-Area Sewage Sludge Application Site, were added to the MWMF monitoring well network. As a conditional requirement for the closure of the F-Area Sewage Sludge Application Site, the FSS monitoring wells are to be monitored for radionuclides related to the Burial Ground Complex. 
- Revision 1 of the field investigation plan (FIP) for the Burial Ground Complex (WSRC, 1993a), submitted to SCDHEC in September 1993, was approved in full on October 6, 1994. The FIP addresses data uncertainties that were identified during the preparation of the MWMF RCRA Part B post-closure care permit application renewal.

- Phase 1 was divided into the following subphases: $1 \mathrm{~A}$-installation of 12 groundwater monitoring wells, 1B-exploratory work in the southwest corner of the Burial Ground Complex using direct-push technology and exploratory borings, $1 \mathrm{C}$-exploratory work in the northeast corner of the Burial Ground Complex using direct-push technology and exploratory borings, and 1D-soil gas survey. Subphases $1 \mathrm{~B}$ and $1 \mathrm{C}$ determined the horizontal and vertical extent of groundwater contamination away from the Burrial Ground Complex. All activities of Phase 1 of the FIP field work have been completed.

- In January 1995, a field investigation preliminary data report (WSRC, 1995) was submitted to provide the data obtained during Phases $1 \mathrm{~A}, 1 \mathrm{~B}$, and $1 \mathrm{D}$. Phase $1 \mathrm{C}$ data was not completed by submittal time; therefore, it was not included in the report.

- Phase 2 of the FIP field work has been divided into the following subphases: 2A-installation of 11 groundwater monitoring wells, 2B-soil sampling in and around the Burial Ground Complex to determine the source of contamination, $2 \mathrm{C}$-exploratory work around the Burial Ground Complex using direct-push technology and exploratory borings, 2D-vadose sampling in and around the Burial Ground Complex to determine hydraulic conductivity of the vadose zone, and 2E-Fourmile Branch ecological sampling.

Currently, the Environmental Protection Department/Environmental Monitoring Section (EPD/ EMS) conducts quarterly sampling of the 125 wells monitoring the groundwater beneath the MWMF as part of the SRS Groundwater Monitoring Program. The Environmental Restoration Department provides a quarterly report to SCDHEC describing the monitoring results to meet the requirements of SCHWMR (SCDHEC, 1993).

\section{Hydrostratigraphic Units}

Historically, groundwater quality assessment reports for the MWMF have used the lithostratigraphic nomenclature Water Table, Barnwell, McBean, and Congaree to identify hydrologic units. However, an interim alphanumeric system developed by Aadland and Bledsoe (1990) (Figure 3, Appendix $C$ ) defines the aquifer and aquitard units at SRS using hydrostratigraphic designations. Figure 4 (Appendix $C$ ) shows a correlation of these designations. This report uses both nomenclatures. The November 1992 MWMF RCRA Part B post-closure care permit application renewal includes an in-depth explanation of this nomenclature and a detailed description of the geologic and hydrogeologic systems at the Burial Ground Complex (WSRC, 1992a).

The MWMF well network monitors three distinct hydrostratigraphic units in the uppermost aquifer beneath the facility: Aquifer Zone $\mathrm{IIB}_{2}$ (Water Table), which is underlain by Confining Zone $\left\|\mathrm{B}_{1}-\right\|_{\mathrm{B}}$ (Tan Clay); the semi-confined Aquifer Zone IIB $\mathrm{B}_{1}$ (Barnwell/McBean); and the semi-confined Aquifer Unit IIA (Congaree), which is separated from the overlying Aquifer Zone $\|_{1}$ by Confining Unit IIA-IIB (Green Clay). The boundary between the uppermost aquifer and the principal confining unit is the uppermost confining bed of Confining System I-II (Ellenton Formation), which lies approximately $300 \mathrm{ft}$ below the surface of the Burial Ground Complex. 


\section{Discussion .}

\section{Groundwater Monitoring Data}

The sampling procedure (WSRC, 1992b) requires evacuation of a minimum of two well volumes and stabilization of $\mathrm{pH}$, specific conductance, and turbidity prior to sample collection. Stability is established when a minimum of three successive measurements, taken within a given time period, are within a specified tolerance range. If a well pumps dry before two well volumes are purged or before stabilization is achieved, it must be revisited within 24 hours for the data to be considered from a single sampling event. On the second visit within 24 hours, samples are taken without purging or stability measurements; thus, these samples may not be representative of groundwater quality.

All of the wells in the BGO, BGX, FSS, and HMD series and in cluster HSB 85 have single-speed centrifugal downhole pumps.

During fourth quarter 1994, groundwater samples from the MWMF were analyzed for selected heavy metals, herbicides/pesticides, indicator parameters, radionuclides, and other constituents. This report describes the results that equaled or exceeded the following standards:

- Safe Drinking Water Act final Primary Drinking Water Standards (PDWS) or drinking water screening levels, as established by the U.S. Environmental Protection Agency (EPA) (Appendix A)

- the South Carolina final PDWS for lead (Appendix A)

- SRS flagging criteria based on PDWS, Secondary Drinking Water Standards, or method detection limits (Appendix B)

For simplicity, results that equaled or exceeded standards are described as exceeding standards, above standards, or as elevated.

Both field and laboratory $\mathrm{pH}$ results are provided in this report. The field measurements are considered more reflective of actual groundwater conditions; however, laboratory $\mathrm{pH}$ measurements are required by current regulations. Because shipping time to the analytical laboratory for $\mathrm{pH}$ analyses exceeds both the SCDHEC holding time (the time between sample collection and analysis) of 15 minutes and the prescribed analytical method holding time, laboratory $\mathrm{pH}$ measurements always exceed the holding time.

The final PDWS for individual analytes provided in Appendix A may not always match the SRS flagging criteria provided in Appendix B. The final PDWS are used as guidelines in this compliance report to meet regulatory requirements; the flagging criteria are used by EPD/EMS to identify relative levels of constituents in the groundwater and as guides for scheduling groundwater sampling. 


\section{Integrity of the Monitoring Well Network}

The current groundwater monitoring well network at the MWMF (Figure 2, Appendix C) is composed of the following:

- 55 Aquifer Zone $\mathrm{IIB}_{2}$ (Water Table) wells (Figure 5, Appendix C):

BGO 1D, 2D, 3D, 4D, 5D, 6D, 7D, 8D, 9D, 10DR, 11D, 12D, 14DR, 15D, 16D, 17DR, 18D, 19D, 20D, 21D, 22DR, 23D, 24D, 26D, 27D, 28D, 29D, 30D, 31D, 32D, 33D, 34D, 35D, 36D, 37D, 38D, 39D, 40D, 44D, 45D, 46D, 47D, 48D, 49D, 50D; BGX 1D, 9D, 10D, 11D, 12D;

FSS 1D, 2D, 3D, 4D; HSB 85C

- 46 Aquifer Zone IIB, (Barnwell/McBean) wells (Figure 6, Appendix C):

BGO 5C, 6B, 6C, 8C, 10B, 10C, 12CR, 13DR, 14CR, 16B, 27C, 29C, 30C, 31C, 33C, 35C, $37 \mathrm{C}, 42 \mathrm{C}, 43 \mathrm{CR}, 43 \mathrm{D}, 44 \mathrm{~B}, 44 \mathrm{C}, 45 \mathrm{~B}, 45 \mathrm{C}, 46 \mathrm{~B}, 46 \mathrm{C}, 47 \mathrm{C}, 48 \mathrm{C}, 49 \mathrm{C}, 50 \mathrm{C}$; BGX 1C, 2B, 2D, 3D, 4C, 4D, 5D, 6D, 7D, 8DR, 12C; HMD 1D, 2D, 3D, 4D; HSB 85B

- 24 Aquifer Unit IIA (Congaree) wells (Figure 7, Appendix C):

BGO 6A, 8AR, 9AA, 10AA, 10AR, 12AR, 14AR, 16AR, 18A, 25A, 26A, 29A, 41A, 43A, 43AA, 44A, 44AA, 45A, 47A, 49A, 50A; BGX 1A, 4A; HSB 85A

SRS has a program in place to rehabilitate and replace wells that do not produce representative samples from the units being monitored. During 1994, 23 groundwater monitoring wells were or are being installed in and around the Burial Ground Complex as part of Phases 1A and 2A of the Burial Ground Complex Field Investigation Plan (WSRC, 1993a) (Table 1). Well clusters BGO $21,51,52$, and 53 were installed between the Old Burial Ground and the MWMF/LLRWDF to monitor the effect of the groundwater divide on contaminant migration horizontally and vertically between the facilities. Well clusters BGO 3 and 39 were installed upgradient of the Burial Ground Complex to monitor background groundwater quality.

A complete record of well installations, replacements, and abandonments at the MWMF is found in the EPD/EMS well inventory (EPD/EMS, 1994).

The designated background wells for Aquifer Zone $\| \mathrm{B}_{2}$ (Water Table) are $\mathrm{BGO} 1 \mathrm{D}$ and $2 \mathrm{D}$ and HSB 85C; HSB 85B is the background well for Aquifer Zone IIB ${ }_{1}$ (Barnwell/McBean); and HSB $85 A$ is the background well for Aquifer Unit IIA (Congaree).

Table D-3 lists the number of well volumes purged from each of the BGO, BGX, FSS, and HMD series wells and the HSB 85 well cluster during fourth quarter 1994 and provides statements that describe unusual sampling events. Unusual sampling events (with applicable sampling codes in parentheses) occurred as follows.

- Wells that went dry during purging $(X)$ :

BGo 1D, 3D, 4D, 5C, 5D, 6B, 6D, 9AA, 10B, 10C, 10DR, 12CR, 12D, 13DR, 14CR, 16B, 16D, 17DR, 20D, 21D, 22DR, 24D, 25A, 26D, 27D, 28D, 29A, 29C, 29D, 30C, 30D, 31C, 31D, 32D, 33D, 34D, 35D, 36D, 37D, 38D, 39D, 42C, 43CR, 44B, 44C, 45B, 45C, 46C, 49D, 50C; BGX 1C, 1D, 2B, 2D, 10D, 11D, 12D; FSS 1D, 2D, 3D, 4D; HMD 1D, 4D; HSB 85B 
Table 1. 1994 Groundwater Monitoring Well Installations at the Burial Ground Complex

\begin{tabular}{|c|c|c|c|}
\hline Well & Screen Zone & Status & First Sampled \\
\hline BGO $3 A$ & L. Congaree (IIA) & completed & $1 Q 95$ \\
\hline BGO 3C & Barnwell (IIB $\left.B_{1}\right)$ & completed & $1 Q 95$ \\
\hline BGO 20A & U. Congaree (IIA) & installed $^{a}$ & \\
\hline BGO 20AA & L. Congaree (IIA) & installed $^{a}$ & \\
\hline $\mathrm{BGO} 20 \mathrm{~B}$ & McBean (IIB $\left.{ }_{1}\right)$ & installed $^{a}$ & \\
\hline $\mathrm{BGO} 20 \mathrm{C}$ & Barnwell (llB $\left.B_{1}\right)$ & installed $^{a}$ & \\
\hline BGO 39A & L. Congaree (IIA) & completed & $1 Q 95$ \\
\hline BGO $39 \mathrm{C}$ & Barnwell (IIB $\left.{ }_{1}\right)$ & completed & $1 Q 95$ \\
\hline BGO 51A & L. Congaree (IIA) & completed & $1 Q 95$ \\
\hline BGO 51AA & U. Congaree (IIA) & completed & $1 Q 95$ \\
\hline BGO 51B & McBean $\left(\|_{1} B_{1}\right)$ & completed & $1 Q 95$ \\
\hline BGO 51C & Barnwell (IIB ${ }_{1}$ ) & completed & $1 Q 95$ \\
\hline BGO 51D & Water Table $\left(\mathrm{IIB}_{2}\right)$ & completed & $1 Q 95$ \\
\hline BGO 52A & L. Congaree (IIA) & installed $^{a}$ & \\
\hline BGO 52AA & U. Congaree (IIA) & installed $^{a}$ & \\
\hline BGO 52B & McBean $\left(\mathrm{IIB}_{1}\right)$ & installed $^{a}$ & \\
\hline BGO 52C & Barnwell (IIB $\left.B_{q}\right)$ & installed $^{a}$ & \\
\hline BGO 52D & Water Table $\left(\mathrm{IIB}_{2}\right)$ & installed $^{a}$ & \\
\hline BGO 53A & L. Congaree (IIA) & completed & $2 Q 95$ \\
\hline BGO 53AA & U. Congaree (IIA) & completed & $2 Q 95$ \\
\hline BGO 53B & McBean $\left(\mathrm{IIB}_{1}\right)$ & completed & $2 Q 95$ \\
\hline BGO 53C & Barnwell (IIB ${ }_{1}$ ) & completed & $2 Q 95$ \\
\hline BGO 53D & Water Table (IIB ${ }_{2}$ ) & completed & $2 Q 95$ \\
\hline
\end{tabular}

a not ready for sampling.

- Wells that went dry during sampling (l):

BGO 40D

- Wells that could not be sampled because they had mechanical problems $(P)$ :

BGO $46 B$ and $47 \mathrm{C}$ 
- Wells for which volumes purged was estimated because their flowmeters were not working properly:

\section{BGO 4D}

- Wells that had no water in the standpipe during sampling (S):

BGO 49A

- Wells that could not be sampled because they are in the Purge Water Containment (PWC) Program:

The PWC Program was instituted at SRS in 1991 to contain and dispose of purged water that exceeds action levels. SRS presently is establishing a revised set of action levels. Currently, MWMF does not have a method for containing and disposing of purged water; thus, these wells have only water-level measurements taken during sampling (Table 2).

Table 2. Wells in the Purge Water Containment Program

\begin{tabular}{|llll|}
\hline Well & Last Sampled & $\begin{array}{l}\text { Constituent } \\
\text { Exceeding Limit }\end{array}$ & $\begin{array}{l}\text { Level at } \\
\text { Last Sample }\end{array}$ \\
\hline BGO 26A & $3 Q 91$ & $\mathrm{pH}$ & $11.6 \mathrm{pH}$ units \\
BGO 37C & $1 Q 91$ & trichloroethylene & $690 \mu \mathrm{g} / \mathrm{L}$ \\
BGO 41A & $2 Q 93$ & $\mathrm{pH}$ & $12.3 \mathrm{pH}$ units \\
BGO 43A & $2 Q 93$ & $\mathrm{pH}$ & $12.6 \mathrm{pH}$ units \\
BGX 1A & $1 \mathrm{QH}$ & $\mathrm{pH}$ & $12.4 \mathrm{pH}$ units \\
\hline
\end{tabular}

a Field measurement.

\section{Analytical Results Exceeding Standards}

Results for analytes that exceeded the final PDWS during fourth quarter 1994 are summarized in Table D-1 and described below. In the text description, the maximum level for each constituent is indicated in parentheses following the well in which it was detected.

Aquifer Zone $\mathrm{IIB}_{2}$ (Water Table): 41 of the 55 wells scheduled for sampling contained elevated constituents during fourth quarter 1994.

- Tritium was elevated in 36 wells: BGo 3D, 5D, 6D, 7D, 8D, 9D, 10DR, 11D, 15D, 16D, 19D, 20D, 21D, 23D, 27D, 28D (maximum activity at 2.9E+05 pCi/mL), 30D, 31D, 32D, 33D, 34D, 35D, 36D, 37D, 38D, 39D, 44D, 45D, 46D, 47D, 48D, 49D, and 50D; BGX 1D; and FSS $2 \mathrm{D}$ and $3 \mathrm{D}$.

- Trichloroethylene was elevated in 12 wells: BGO 6D, 7D, 12D, 14DR, 15D, 16D, 26D, 28D (maximum concentration at $244 \mu \mathrm{g} / \mathrm{L}$ ), 30D, 46D, 47D, and 48D.

- Tetrachloroethylene was elevated in 5 wells: BGO 6D, 15D, 32D, 46D, and 48D (maximum concentration at $53 \mu \mathrm{g} / \mathrm{L})$. 
- Lead was elevated in 4 wells: BGO 4D and 35D; BGX 10D; and FSS 3D (maximum concentration at $1,740 \mu \mathrm{g} / \mathrm{L})$.

- Chloroethene (vinyl chloride) was elevated in 3 wells: BGO 28D (maximum concentration at $51 \mu \mathrm{g} / \mathrm{L}), 30 \mathrm{D}$, and 46D.

- Copper was elevated in well FSS 3D at 1,810 $\mu \mathrm{g} / \mathrm{L}$.

- 1,1-Dichloroethylene was elevated in well BGO 30D at $7.5 \mu \mathrm{g} / \mathrm{L}$.

- Dichloromethane was elevated in well BGO 14DR at $5.8 \mu \mathrm{g} / \mathrm{L}$.

Aquifer Zone $\|_{1}$ (Barnwell/McBean): 29 of the 46 wells scheduled for sampling contained elevated constituents during fourth quarter 1994.

- Tritium was elevated in 27 wells: BGO 5C, 6B, 6C, 13DR, 14CR, 27C, 29C, 30C, 31C, $33 \mathrm{C}$ (maximum activity at $1.1 \mathrm{E}+04 \mathrm{pCi} / \mathrm{mL}$ ), 35C, 44B, 44C, 45C, 46C, 48C, 49C, and 50C; BGX 1C, 2D, 3D, 4D, 5D, 7D, and 8DR; and HMD 1D and 3D.

- Trichloroethylene was elevated in 10 wells: BGO $12 \mathrm{CR}$ (maximum concentration at $68 \mu \mathrm{g} / \mathrm{L}), 14 \mathrm{CR}, 27 \mathrm{C}, 30 \mathrm{C}, 33 \mathrm{C}, 35 \mathrm{C}, 42 \mathrm{C}, 46 \mathrm{C}$, and 50C and BGX $2 \mathrm{D}$.

- Lead was elevated in well BGO 13DR at $71 \mu \mathrm{g} / \mathrm{L}$.

- Mercury was elevated in 2 wells: BGO 33C (maximum concentration at $4.1 \mu \mathrm{g} / \mathrm{L}$ ) and 45C.

Aquifer Unit IIA (Congaree): 3 of the 24 wells scheduled for sampling contained elevated constituents during fourth quarter 1994.

- Benzene was elevated in 2 wells: BGO 10AR (maximum concentration at $8.3 \mu \mathrm{g} / \mathrm{L}$ ) and 14AR.

- Dichloromethane was elevated in 3 wells: BGO 10AR (maximum concentration at $19 \mu \mathrm{g} / \mathrm{L}), 12 \mathrm{AR}$, and 14AR.

Results for analytes that exceeded other SRS flagging criteria during fourth quarter 1994 are summarized in Table D-2.

Table D-3 shows the results for all of the constituents and indicates the analytical laboratories that conducted the analyses, the dilution factors used in the analyses, and the analyses that received modifiers (which help identify laboratory accuracy and precision) or that exceeded the EPA-approved holding times during fourth quarter 1994. Constituent results in Table D-3 that appear to equal the final PDWS but are not marked in the ST column (exceeded final PDWS or screening level) are below the final PDWS in the database. Database results, the results that are compared to the final PDWS, are entered with more significant digits than the results given in this report. Apparent discrepancies are the result of the rounding of reported results.

In addition to the results tables, Appendix $D$ provides definitions of the abbreviations and the modifiers used in the results tables as well as descriptions of holding times, data rounding, and data qualification practices. Appendix $E$ provides a general assessment of the quality and usability of the data. 
Isoconcentration maps of lead, trichloroethylene, and tritium in Aquifer Zone IIB ${ }_{2}$ (Water Table),

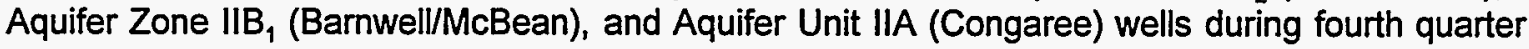
1994 appear in Figures 8 through 10 and 17 through 22 (Appendix C). Postings of pH and specific conductance results appear in Figures 11 through 16.

\section{Trichloroethylene and Tritium Time-Trend Data}

Time series plots from first quarter 1990 through fourth quarter 1994 for wells containing trichloroethylene and tritium are presented in Appendix $F$. A statistical analysis was performed as part of the 1992 MWMF RCRA Part B post-closure care permit application renewal. Trichloroethylene and tritium were identified among the constituents of concern. Statements concerning relative levels of these constituents are provided below.

Trichloroethylene concentrations have exhibited the following trends since first quarter 1992:

- Trichloroethylene concentrations in wells BGo 6A, 6C, 8AR, 8C, 27D, 29D, 31D, 33D, $44 \mathrm{~A}, 44 \mathrm{AA}, 44 \mathrm{~B}, 44 \mathrm{D}, 47 \mathrm{~A}$, and $50 \mathrm{~A}$ have been consistently below or near detection limits.

- Trichloroethylene concentrations in wells BGO 8D, 12AR, 13DR, 14AR, 29A, 29C, 31C, $32 \mathrm{D}, 40 \mathrm{D}, 44 \mathrm{C}, 46 \mathrm{~B}, 47 \mathrm{C}$, and $48 \mathrm{C}$ and $\mathrm{BGX} 2 \mathrm{~B}$ have been consistently near or below the final PDWS.

- Wells BGo 6D, 7D, 12CR, 12D, 14CR, 14DR, 15D, 16D, 27C, 28D, 30C, 30D, 33C, 42C, 46C, 46D, 47D, 48D, and 50D and BGX 2D have exhibited concentrations of trichloroethylene that consistently exceeded the final PDWS.

- Well BGO 28D, screened in Aquifer Zone $\|_{1} B_{2}$ (Water Table), has consistently exhibited the highest trichloroethylene concentrations, usually ranging from approximately $160 \mu \mathrm{g} / \mathrm{L}$ to $340 \mu \mathrm{g} / \mathrm{L}$.

- Other wells have exhibited somewhat erratic activities, ranging from relatively high above the final PDWS to below the final PDWS (i.e., wells BGO 50C and BGX 3D). Data are insufficient to determine trends for other wells. However, trichloroethylene concentrations in wells BGO 6B, 16AR, and 16B have not exceeded the final PDWS since sampling began several quarters ago.

Tritium activities have exhibited the following trends since first quarter 1992:

- Tritium activities in wells BGo 6A, 8AR, 10AR, 12AR, 12CR, 18A, 25A, 43AA, 44A, 44AA, 45A, 47A, 49A, and 50A and BGX 4A and 12C have been consistently near or below detection limits.

- Tritium activities have not exceeded the final PDWS in upgradient wells BGO $1 D$ and HSB $85 \mathrm{~A}, 85 \mathrm{~B}$, and $85 \mathrm{C}$.

- Tritium activities in wells BGO 2D, 5D, 8D, 12D, 14AR, 18D, 20D, 24D, 26D, 29A, 29D, 33D, 35C, 40D, 42C, 43CR, 43D, and 45B; BGX 2B, 4C, 6D, 9D, 10D, 11D, and 12D; FSS 1D and 4D; and HMD 2D, 3D, and 4D have been consistently near or below the final PDWS. 
- Wells BGO 3D, 5C, 6C, 6D, 7D, 11D, 14CR, 15D, 16D, 19D, 21D, 23D, 27C, 27D, 28D, 29C, 30C, 30D, 31C, 31D, 32D, 33C, 34D, 35D, 36D, 37D, 38D, 39D, 44B, 44C, 44D, 45C, 45D, 46C, 46D, 47C, 47D, 48C, 48D, 49C, 49D, 50C, and 50D; BGX 1C, 1D, 2D, $3 \mathrm{D}$, and $8 \mathrm{DR}$; and FSS $2 \mathrm{D}$ and $3 \mathrm{D}$ have exhibited tritium levels that have consistently exceeded the final PDWS.

- Well BGO 28D, located in Aquifer Zone $\mathrm{IIB}_{2}$ (Water Table) at the west edge of the Old Burial Ground, has consistently exhibited the highest tritium activities, exceeding $1.0 \mathrm{E}+05 \mathrm{pCi} / \mathrm{mL}$ during most quarters.

- Some wells have exhibited erratic activities, ranging from relatively high above the final PDWS to below the final PDWS (e.g., wells BGO 9D, 10B, 10C, 13DR, and 46B and $B G X 4 D, 5 D$, and 7D). Data are still insufficient to determine trends for other wells. However, tritium activities in wells BGO 9AA, 10AA, 16AR, and 17DR have been consistently below the final PDWS since sampling began several quarters ago.

Tritium activities in wells BGO 12D, 14AR, 26D, 29A, 34D, 35C, 36D, 37D, 38D, and 39D and FSS 4D appear to have been anomalously high during fourth quarter 1991 as compared to preceding and more recent quarters.

Time series plots of $\mathrm{pH}$ for selected wells also are provided in Appendix F.

\section{Water Levels}

Hydrographs for selected wells and well clusters at the MWMF are provided in Appendix G. Average water elevations for all of the wells in each of the three hydrostratigraphic units beneath the MWMF for the past four quarters are shown in Table 3.

Table 3. Average Water Elevations (ft $\mathrm{ms} /$ ) in the Hydrostratigraphic Units beneath the MWMF

\begin{tabular}{|lllll|}
\hline Unit & 1094 & $2 \mathrm{Q} 94$ & $3 Q 94$ & $4 Q 94$ \\
\hline Aquifer Zone $\|_{1} \mathrm{~B}_{2}$ (Water Table) & $230.70^{\mathrm{a}}$ & 230.48 & 230.34 & 230.38 \\
Aquifer Zone $\| \mathrm{B}_{1}$ (BarnwellMcBean) & 218.15 & 218.79 & 218.46 & 218.12 \\
Aquifer Unit IIA (Congaree) & 159.45 & 159.85 & 158.67 & 163.52 \\
\hline
\end{tabular}

average includes water elevations for wells FSS 1D, 2D, and 3D for the first time.

During fourth quarter 1994, the water level in Aquifer Zone $\| \mathrm{B}_{2}$ (Water Table) increased an average of $0.04 \mathrm{ft}$ compared to third quarter 1994, the water level in Aquifer Zone IIB, (Barnwell/ McBean) decreased an average of $0.34 \mathrm{ft}$, and the water level in Aquifer Unit IIA (Congaree) increased an average of $4.85 \mathrm{ft}$.

A consistent vertical head relationship exists among the hydrostratigraphic units monitored at the MWMF. Flow potential is downward from Aquifer Zone $\|_{1} B_{2}$ (Water Table) to Aquifer Zone IIB (Barnwell/McBean) and downward from Aquifer Zone IIB, (Barnwell/McBean) to Aquifer Unit IIA (Congaree). This relationship, which was first noted in 1988, exists at all BGO well clusters. 


\section{Groundwater Flow Rates and Directions}

Three hydrostratigraphic units lie beneath the Burial Ground Complex. Groundwater flow di-

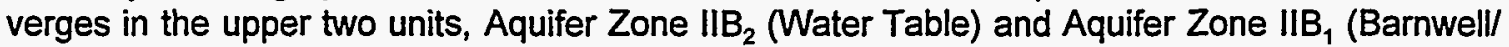
McBean), with flows toward Fourmile Branch to the southwest and Upper Three Runs Creek to the north (using universal transverse Mercator coordinates [UTM]). Groundwater in the lowermost unit, Aquifer Unit IIA (Congaree), flows to the northwest toward Upper Three Runs Creek. Fourmile Branch incises Aquifer Zones $\|_{2} B_{2}$ (Water Table) and IIB ${ }_{1}$ (Barnwell/McBean) but not Aquifer Zone IIA (Congaree), creating a discharge for the upper two units only. Upper Three Runs Creek incises Aquifer Zone $I I B_{2}$ (Water Table), Aquifer Zone IIB, (Barnwell/McBean), and Aquifer Unit IIA (Congaree), creating a discharge for all three units.

Because the topography slopes downward near Upper Three Runs Creek, groundwater conditions change in this area. As indicated in Figure 23, Aquifer Zone $\|_{2} B_{2}$ (Water Table) has a northern limit of saturation. North of this limit, the water table dips below Confining Zone IIB $_{1}-\| l_{2}$ (Tan Clay), and Aquifer Zone IIB, (Barnwell/McBean) becomes unconfined (Figure 24).

Horizontal flow rate calculations provide estimates of the transport rate of constituents originating from the MWMF. Flow rates in Aquifer Zone $\|_{1} B_{2}$ (Water Table) and Aquifer Zone IIB, (Barnwell/ McBean) are calculated along two flow paths (designated flow paths $A$ and $B$ ) to characterize the divergent groundwater flows toward Upper Three Runs Creek and Fourmile Branch. The flow rate for Aquifer Unit IIA (Congaree) is calculated along a single flow path because flow directions within this unit are generally more uniform than in the overlying units. Flow rates are estimated using the following equation:

$$
\text { Flow }(\mathrm{ft} / \text { day })=\frac{\text { Hydraulic Conductivity }(\mathrm{ft} / \text { day) }}{\text { Porosity (unitless) }} \times \frac{d h(\mathrm{ft})}{d l(\mathrm{ft})}
$$

Hydraulic conductivity constants of $3.03 \mathrm{ft} /$ day, $1.5 \mathrm{ft} /$ day, and $45 \mathrm{ft} /$ day are used for Aquifer Zone IIB ${ }_{2}$ (Water Table), Aquifer Zone IIB ${ }_{1}$ (Barnwell/McBean), and Aquifer Unit IIA (Congaree), respectively (WSRC, 1992b). An effective porosity value of 20 percent is used for the two upper units, and an effective porosity value of 25 percent is used for Aquifer Unit IIA (Congaree) (WSRC, 1992b). The value $d h$ is the difference in head, and $d l$ is the length of the flow path.

Flow path lengths are calculated to the nearest $50 \mathrm{ft}$. Flow rate per day is calculated to two significant figures, then multiplied by 365 and rounded to two significant figures for the flow rate per year. Flow rate estimates vary depending on the vertical gradient between wells, the size of the area under consideration, the number of data points, and the length and location of the flow path. Because these are based on inferred or estimated parameters, flow rate estimates should be considered accurate to an order of magnitude only. Horizontal flow rate estimates for the three hydrostratigraphic units during the past four quarters are provided in Table 4.

Table 4. Estimated Horizontal Groundwater Flow Rates (ft'year) in the Hydrostratigraphic Units beneath the MWMF

\begin{tabular}{|lllll|}
\hline Unit & 1094 & $2 Q 94$ & $3 Q 94$ & $4 Q 94$ \\
\hline Aquifer Zone IIB ${ }_{2}$ (Water Table) & $20-31$ & $18-28$ & $19-35$ & $20-47$ \\
Aquifer Zone IIB (Barnwell/McBean) & $8.8-16$ & $8.8-15$ & $5.1-19$ & $7.3-19$ \\
Aquifer Unit IIA (Congaree) & 150 & 220 & 230 & 250 \\
\hline
\end{tabular}


Using UTM coordinates, the fourth quarter 1994 flow directions in Aquifer Zone IIB $_{2}$ (Water Table) were to the west to southwest (flow path $A$ ) and north (flow path $B$ ). Flow directions in Zone $\| \mathrm{B}_{1}$ (Barnwell/McBean) were to the west (flow path $\mathrm{A}$ ) and north (flow path $\mathrm{B}$ ), and the flow direction in Aquifer Unit IIA (Congaree) was to the northwest (Figures 23, 24, and 25, Appendix C).

The flow rate estimates for groundwater in Aquifer Zone $\mathrm{IBB}_{2}$ (Water Table) beneath the MWMF during fourth quarter 1994 are as follows (Figure 23, Appendix C):

- Flow path A (toward Fourmile Branch)

$$
\frac{3.03}{0.20} \times \frac{8}{2,150} \approx 0.056 \mathrm{ft} \text { day }
$$

$0.056 \mathrm{ft} /$ day $\times 365$ days $\approx 20 \mathrm{ft} /$ year

- Flow path B (toward Upper Three Runs Creek)

$$
\begin{aligned}
& \frac{3.03}{0.20} \times \frac{10}{1,200} \approx 0.13 \mathrm{ft} / \text { day } \\
& 0.13 \mathrm{ft} \text { day } \times 365 \text { days } \approx 47 \mathrm{ft} / \text { year }
\end{aligned}
$$

The flow rate estimates for groundwater in Aquifer Zone IIB, (Barnwell/McBean) beneath the MWMF are as follows (Figure 24, Appendix C):

- Flow path A (toward Fourmile Branch)

$$
\frac{1.5}{0.20} \times \frac{6}{2,200} \approx 0.02 \mathrm{ft} / \mathrm{day}
$$

$0.02 \mathrm{ft} /$ day $\times 365$ days $\approx 7.3 \mathrm{ft} /$ year

- Flow path B (toward Upper Three Runs Creek)

$$
\begin{aligned}
& \frac{1.5}{0.20} \times \frac{12}{1,750} \approx 0.051 \mathrm{ft} / \text { day } \\
& 0.051 \mathrm{ft} / \text { day } \times 365 \text { days } \approx 19 \mathrm{ft} / \text { year }
\end{aligned}
$$

The flow rate estimate for groundwater in Aquifer Unit IIA (Congaree) beneath the MWMF is as follows (Figure 25, Appendix C):

$$
\frac{45}{0.25} \times \frac{5}{1,300} \approx 0.69 \mathrm{ft} / \mathrm{day}
$$

$0.69 \mathrm{ft} /$ day $\times 365$ days $\approx 250 \mathrm{ft} /$ year 


\section{Upgradient Versus Downgradient Results}

- Wells BGO 1D and 2D and HSB 85C are upgradient wells in Aquifer Zone IIB $_{2}$ (Water Table); wells HSB 85A and 85B are upgradient wells in Aquifer Unit IIA (Congaree) and Aquifer Zone $\mathrm{IIB}_{1}$ (Barnwell/McBean), respectively. The remaining wells in these hydrostratigraphic units monitor downgradient water quality.

No constituents in excess of the final PDWS were detected in any upgradient wells during fourth quarter 1994. Downgradient wells in the three hydrostratigraphic units contained elevated levels of benzene, chloroethene, copper, 1,1-dichloroethylene, dichloromethane, lead, mercury, tetrachloroethylene, trichloroethylene, and tritium.

\section{Quality Control Results}

Wells BGO 8C, 33C, and 44D and BGX 4D were selected to receive blind replicate analyses during fourth quarter 1994. Blind replicate analyses, representing approximately 5 percent of the quarter's total groundwater samples, are performed by the analytical laboratories each quarter for wells selected by EPD/EMS as part of the EPD/EMS quality assurance program (see Appendix E). The results of the analyses are used for both intralaboratory and interlaboratory comparisons. As a part of intralaboratory quality assurance procedures, certain analyses were duplicated by the laboratory. The results of duplicate and replicate analyses are reported in Table D-3.

The Savannah River Site's Groundwater Monitoring Program, Fourth Quarter 1994 (U) (EPD/EMS, 1995, in preparation) provides a full evaluation of data quality control and an explanation of analytical results for the quarter. 


\section{Conclusions}

The groundwater at the MWMF contains elevated levels of heavy metals, radionuclides, volatile organic compounds, and other constituents resulting from 40 years of operations at the Burial Ground Complex. During fourth quarter 1994, benzene, chloroethene, copper, 1,1-dichloroethylene, dichloromethane, lead, mercury, tetrachloroethylene, trichloroethylene, and tritium exceeded their final PDWS in one or more wells at the MWMF. Except for benzene and copper, all of these constituents also occurred in elevated levels during third quarter 1994. Gross alpha, elevated third quarter 1994, did not exceed the final PDWS in any well during fourth quarter 1994. Elevated lead, which occurred in only one well during second quarter but increased to exceed the final PDWS in 8 wells during third quarter, exceeded the final PDWS in five wells during fourth quarter.

As in previous quarters, tritium and trichloroethylene were the most widespread elevated constituents. Sixty-three $(50 \%)$ of the 125 monitoring wells contained elevated tritium activities, with the maximum activity (2.9E $+05 \mathrm{pCi} / \mathrm{mL}$ ) occurring in well BGO 28D in Aquifer Zone IIB $_{2}$ (Water Table). Trichloroethylene concentrations exceeded the final PDWS in $22(18 \%)$ of the wells, with the maximum concentration of $244 \mu \mathrm{g} / \mathrm{L}$ found in well BGO $28 \mathrm{D}$ in Aquifer Zone $\|_{1} B_{2}$ (Water Table).

Elevated constituent levels were found primarily in Aquifer Zone IIB ${ }_{2}$ (Water Table) and Aquifer Zone IIB, (Barnwell/McBean), consistent with historical data. Past quarters' results for Aquifer Unit IIA (Congaree) have indicated that vertical pathways into this deeper water-bearing unit exist. A current groundwater flow model for the General Separations Area indicates that the vertical component beneath this area is important (GeoTrans, Inc., 1992). During fourth quarter 1994, benzene was elevated in wells BGO 10AR and 14AR and dichloromethane was elevated in wells BGO 10AR, 12AR, 14AR in Aquifer Unit IIA (Congaree).

No constituents in excess of the final PDWS were detected in any upgradient wells during fourth quarter 1994. Downgradient wells contained elevated levels of constituents. Generally, elevated levels of constituents found in downgradient wells but not in upgradient wells at a waste management unit are considered products of the waste management unit.

Groundwater in Aquifer Zone $\|_{2}$ (Water Table) and Aquifer Zone IIB (Barnwell/McBean) diverges beneath the Burial Ground Complex and discharges toward Upper Three Runs Creek or toward Fourmile Branch. During fourth quarter 1994, flow in these units was to the north toward Upper Three Runs Creek or to the west or southwest toward Fourmile Branch (UTM coordinates). The groundwater flow direction in Aquifer Unit IIA (Congaree) was consistently northwest toward Upper Three Runs Creek.

Groundwater flow rate estimates were in the same order of magnitude as previous quarters. Estimates ranged from 20 to $47 \mathrm{ft} / y e a r$ in Aquifer Zone $I I B_{2}$ (Water Table) and from 7.3 to $19 \mathrm{ft} / \mathrm{year}$

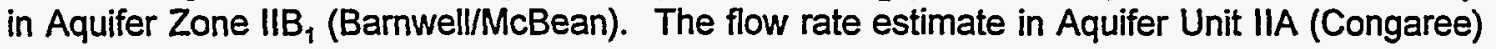
was $250 \mathrm{ft} /$ year. 


\section{Summary}

Results for analytes that exceeded the final PDWS during 1994 are summarized below. The maximum level for each constituent is indicated in parentheses following the well in which it was detected.

Aquifer Zone $\|_{2}$ (Water Table): 48 of the 55 wells contained elevated constituents during 1994.

- Tritium was elevated in 42 wells: BGO 2D, 3D, 5D, 6D, 7D, 8D, 9D, 10DR, 11D, 12D, 15D, 16D, 19D, 20D, 21D, 22DR, 23D, 27D, 28D (maximum activity at 2.9E+05 pCi/mL during fourth quarter), 29D, 30D, 31D, 32D, 33D, 34D, 35D, 36D, 37D, 38D, 39D, 44D, 45D, 46D, 47D, 48D, 49D, and 50D; BGX 1D, 11D, and 12D; and FSS 2D and 3D.

- Trichloroethylene was elevated in 14 wells: BGO 6D, 7D, 12D, 14DR, 15D, 16D, 26D, 28D (maximum concentration at $244 \mu \mathrm{g} / \mathrm{L}$ during fourth quarter), 30D, 32D, 46D, 47D, 48D, and 50D.

- Lead was elevated in 8 wells: BGO 4D, 5D, 26D, 35D, and 40D; BGX 10D; and FSS 1D and $3 D$ (maximum concentration at $1,740 \mu \mathrm{g} / \mathrm{L}$ during fourth quarter). The elevated levels in wells BGO 5D and 40D appeared in improperly preserved or unpreserved samples during third quarter. Elevated lead did not recur in these wells during 1994.

- Tetrachloroethylene was elevated in 6 wells: BGO 6D, 7D, 15D, 32D, 46D, and 48D (maximum concentration at $74 \mu \mathrm{g} / \mathrm{L}$ during third quarter).

- Chloroethene was elevated in 3 wells: BGO 28D (maximum concentration at $140 \mu \mathrm{g} / \mathrm{L}$ during first quarter), 30D, and 46D.

- Copper was elevated in 2 wells: FSS 1D and 3D (maximum concentration at 1,810 $\mu \mathrm{g} / \mathrm{L}$ during fourth quarter).

- 1,1-Dichloroethylene was elevated in 2 wells: BGO 6D and 30D (maximum concentration at $19 \mu \mathrm{g} / \mathrm{L}$ during first quarter). The elevated level in well BGO 6D appeared in an improperly preserved or unpreserved sample during third quarter. Elevated 1,1-dichloroethylene did not recur in this well during 1994.

- Dichloromethane was elevated in 2 wells: BGO 9D (maximum concentration at $8.8 \mu \mathrm{g} / \mathrm{L}$ during third quarter) and 14DR. The elevated level in well BGO 9D appeared in an improperly preserved or unpreserved sample during third quarter. Elevated dichloromethane did not recur in this well during 1994.

- Gross alpha was elevated in well BGO 32D (maximum activity at 2.1E+01 pCi/L during third quarter). 
Aquifer Zone IIB (Barnwell/McBean): 31 of the 46 wells contained elevated constituents during 1994.

- Tritium was elevated in 29 wells: BGO 5C, 6B, 6C, 10B, 10C, 13DR, 14CR, 27C, 29C, $30 \mathrm{C}, 31 \mathrm{C}, 33 \mathrm{C}$ (maximum activity at $1.1 \mathrm{E}+04 \mathrm{pCi} / \mathrm{mL}$ during fourth quarter), $35 \mathrm{C}, 44 \mathrm{~B}$, 44C, 45C, 46C, 48C, 49C, and 50C; BGX 1C, 2D, 3D, 4D, 5D, 7D, and 8DR; and HMD 1D and 3D.

- Trichloroethylene was elevated in 11 wells: BGO 12CR (maximum concentration at $85 \mu \mathrm{g} / \mathrm{L}$ during third quarter), $14 \mathrm{CR}, 27 \mathrm{C}, 30 \mathrm{C}, 33 \mathrm{C}, 35 \mathrm{C}, 42 \mathrm{C}, 46 \mathrm{C}$, and $50 \mathrm{C}$ and BGX 2D and 3D.

- Lead was elevated in 3 wells: BGO 13DR (maximum concentration at $71 \mu \mathrm{g} / \mathrm{L}$ during fourth quarter) and 44B and HMD 1D. The elevated level in well HMD 1D appeared in an improperly preserved or unpreserved sample during third quarter. Elevated lead did not recur in this well during 1994.

- Mercury was elevated in 2 wells: BGO $33 \mathrm{C}$ (maximum concentration at $4.1 \mu \mathrm{g} / \mathrm{L}$ during fourth quarter) and 45C.

- Nonvolatile beta was elevated in well BGX 5D (maximum concentration at 1.5E+02 pCi/L during first quarter).

- Tetrachloroethylene was elevated in well BGO 33C (maximum concentration at $5.2 \mu \mathrm{g} / \mathrm{L}$ during second quarter).

Aquifer Unit IIA (Congaree): 5 of the 24 wells contained elevated constituents during 1994.

- Dichloromethane was elevated in 4 wells: BGO 10AA, 10AR (maximum concentration at $19 \mu \mathrm{g} / \mathrm{L}$ during fourth quarter), 12AR, and 14AR. The elevated level in well BGO 10AA appeared in an improperly preserved or unpreserved sample during third quarter. Elevated dichloromethane did not recur in this well during 1994.

- Benzene was elevated in 2 wells: BGO 10AR (maximum concentration at $8.3 \mu \mathrm{g} / \mathrm{L}$ during fourth quarter) and 14AR.

- Nonvolatile beta was elevated in well BGO 9AA (maximum activity at $7.8 \mathrm{E}+01 \mathrm{pCi} / \mathrm{L}$ during first quarter).

- Tritium was elevated in well BGO 10AA (maximum activity at 2.3E+01 pCi/mL during third quarter).

As in 1993, tritium and trichloroethylene exceeded their final PDWS more frequently than did other constituents. Also, elevated constituents were found primarily in Aquifer Zone ${ } \mathrm{B}_{2}$ (Water Table) and Aquifer Zone IIB ${ }_{1}$ (Barnwell/McBean); however, elevated constituents were found in five wells in Aquifer Unit IIA (Congaree).

As part of the Burial Ground Complex field investigation plan, 23 additional groundwater monitoring wells were installed in the past year. Nine will be sampled beginning first quarter 1995 with the remaining wells to be sampled later in 1995. 
Groundwater flow directions beneath the MWMF during 1994 were generally similar to the directions during 1993. Using UTM coordinates, the flow directions in each hydrostratigraphic unit during 1994 were as follows: Aquifer Zone $\|_{1} B_{2}$ (Water Table) to the north, southwest, or west-southwest; Aquifer Zone IIB, (Barnwell/McBean) to the north, northwest, southwest, westsouthwest, or west; and Aquifer Unit IIA (Congaree) to the northwest. Flow rates during 1994 were in the same order of magnitude as flow rates in 1993.

The average water level in Aquifer Zone $\|_{2}$ (Water Table) decreased $0.75 \mathrm{ft}$ during $1994 \mathrm{com}$ -

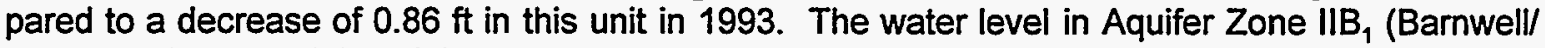
McBean) decreased $1.02 \mathrm{ft}$ in 1994 compared to a decrease of $0.11 \mathrm{ft}$ in 1993. In Aquifer Unit IIA (Congaree), the water level rose $3.23 \mathrm{ft}$ during 1994 compared to an increase of $0.64 \mathrm{ft}$ during 1993. 


\section{References Cited}

Aadland, R. K., and H. W. Bledsoe, 1990. Classification of Hydrostratigraphic Units at the Savannah River Site, South Carolina, WSRC-RP-90-987. Westinghouse Savannah River Company, Savannah River Site, Aiken, SC.

EPD/EMS (Environmental Protection Department/Environmental Monitoring Section), 1994. Environmental Protection Department's Well Inventory (through the second quarter of 1994), ESH-EMS-940518. Westinghouse Savannah River Company, Savannah River Site, Aiken, SC.

EPD/EMS (Environmental Protection Department/Environmental Monitoring Section), 1995. The Savannah River Site's Groundwater Monitoring Program, Fourth Quarter 1994 (U), ESHEMS-940517, in preparation. Westinghouse Savannah River Company, Savannah River Site, Aiken, SC.

GeoTrans, Inc., 1992. Groundwater Flow Model for the General Separations Area, Savannah River Site. Prepared for Westinghouse Savannah River Company, Environmental Restoration Department, Savannah River Site, Aiken, SC.

Heffner, J. D., and Exploration Resources, Inc., 1991. Technical Summary of Groundwater Quality Protection Program at the Savannah River Site (1952-1986), Volume I-Site Geohydrology and Waste Sites, DPSP-88-1002. Westinghouse Savannah River Company, Aiken, SC.

Jaegge, W. J., N. L. Kolb, B. B. Looney, I. W. Marine, O. A. Towler, and J. R. Cook, 1987. Environmental Information Document: Radioactive Waste Burial Grounds, DPST-85-694. Savannah River Laboratory, E. I. du Pont de Nemours \& Company, Aiken, SC.

SCDHEC (South Carolina Department of Health and Environmental Control), 1993. South Carolina Hazardous Waste Management Regulations; R.61-79.124, .260 through .266, .268, and .270. Columbia, SC.

WSRC (Westinghouse Savannah River Company), 1992a. 1992 RCRA Part B Permit Renewal Application. Mixed Waste Management Facility (MWMF) Post-Closure, Volume VII, Revision 0, November 1992, WSRC-IM-91-53. Savannah River Site, Aiken, SC.

WSRC (Westinghouse Savannah River Company), 1992b. Sampling Groundwater Monitoring Wells, Hydrogeologic Data Collection Procedures and Specifications, Manual 3Q5, Rev. 1, Chapter 15. Savannah River Site, Aiken, SC.

WSRC (Westinghouse Savannah River Company), 1993a. A Field Investigation Plan for the Burial Ground Complex, September 1993, WSRC-RP-93-848. Savannah River Site, Aiken, SC.

WSRC (Westinghouse Savannah River Company), 1993b. Mixed Waste Management Facility Closure Plan (LLRWDF), Volume II, August 1993, Q-CLP-E-00001. Savannah River Site, Aiken, SC. 
WSRC (Westinghouse Savannah River Company), 1993c. 1992 RCRA Part B Permit Application, Mixed Waste Management Facility Postclosure, Volume VII, Revision 2, November 1993, WSRC-IM-91-53. Savannah River Site, Aiken, SC.

WSRC (Westinghouse Savannah River Company), 1995. Burial Ground Complex Field Investigation Preliminary Data Report, No. 1, January 1995, WSRC-RP-94-1286. Savannah River Site, Aiken, SC. 


\section{Errata}

In tables with four quarters of data, some values for earlier quarters may differ from values for those same quarters presented in earlier reports because some reanalyses may have been performed by the laboratories after the reports were printed.

Fourth Quarter 1993 and 1993 Summary:

- Page 17: The changes in average water levels during 1993 should be as follows: Aquifer Zone IIB ${ }_{2}$ (Water Table) decreased $0.86 \mathrm{ft}$, Aquifer Zone IIB, (Barnwell/McBean) decreased $0.11 \mathrm{ft}$, and Aquifer Unit IIA (Congaree) increased $0.64 \mathrm{ft}$. The values reported in the Fourth Quarter 1993 report were derived using only three quarters of data. The revised figures measure the difference in water levels from fourth quarter 1992 to fourth quarter 1993.

First Quarter 1994:

- No errata have been reported.

Second Quarter 1994:

- No errata have been reported.

Third Quarter 1994:

- Hydrograph for well BGO 19D: The hydrograph appears different from previous quarters because the top of casing was corrected from 287.2 to $290.1 \mathrm{ft} \mathrm{msl}$ and the screen zone elevation was corrected from 213.9-193.9 ft msl to 216.8-196.8 $\mathrm{ft} \mathrm{msl}$. 
WSRC-TR-94-0610

Unclassified

THIS PAGE LEFT BLANK INTENTIONALLY. 
WSRC-TR-94-0610

Unclassified

\section{Appendix A}

Final Primary Drinking, Water Standards 
WSRC-TR-94-0610

Unclassified

THIS PAGE LEFT BLANK INTENTIONALLY. 
Final Primary Drinking Water Standards

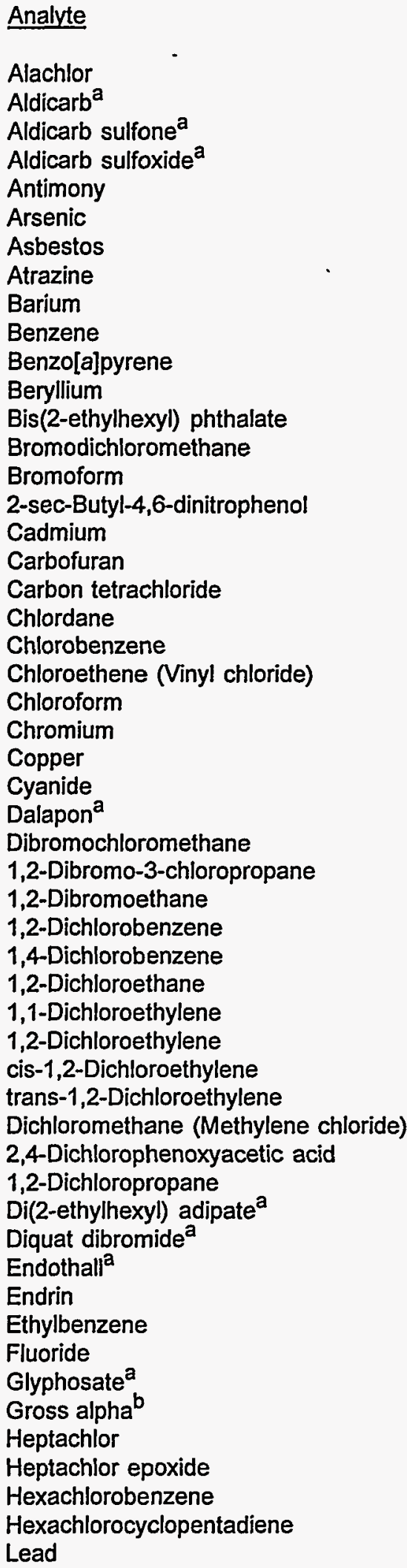

\begin{tabular}{|c|c|c|c|}
\hline Unit & Level & Status & Source \\
\hline$\mu g / L$ & 2 & Final & EPA, 1993 \\
\hline$\mu g / L$ & 3 & Final & EPA, 1993 \\
\hline$\mu \mathrm{g} / \mathrm{L}$ & 2 & Final & EPA, 1993 \\
\hline$\mu g / L$ & 4 & Final & EPA, 1993 \\
\hline$\mu g / L$ & 6 & Final & EPA, 1993 \\
\hline$\mu \mathrm{g} / \mathrm{L}$ & 50 & Final & EPA, 1993 \\
\hline Fibers/L & $7,000,000$ & Final & EPA, 1993 \\
\hline$\mu \mathrm{g} / \mathrm{L}$ & 3 & Final & EPA, 1993 \\
\hline$\mu g / L$ & 2,000 & Final & EPA, 1993 \\
\hline$\mu \mathrm{g} / \mathrm{L}$ & 5 & Final & EPA, 1993 \\
\hline$\mu \mathrm{g} / \mathrm{L}$ & 0.2 & Final & EPA, 1993 \\
\hline$\mu \mathrm{g} / \mathrm{L}$ & 4 & Final & EPA, 1993 \\
\hline$\mu g / L$ & 6 & Final & EPA, 1993 \\
\hline$\mu \mathrm{g} / \mathrm{L}$ & 100 & Final & EPA, 1993 \\
\hline$\mu g / L$ & 100 & Final & EPA, 1993 \\
\hline$\mu \mathrm{g} / \mathrm{L}$ & 7 & Final & EPA, 1993 \\
\hline$\mu \mathrm{g} / \mathrm{L}$ & 5 & Final & EPA, 1993 \\
\hline$\mu \mathrm{g} / \mathrm{L}$ & 40 & Final & EPA, 1993 \\
\hline$\mu g / L$ & 5 & Final & EPA, 1993 \\
\hline$\mu \mathrm{g} / \mathrm{L}$ & 2 & Final & EPA, 1993 \\
\hline$\mu \mathrm{g} / \mathrm{L}$ & 100 & Final & EPA, 1993 \\
\hline$\mu \mathrm{g} / \mathrm{L}$ & 2 & Final & EPA, 1993 \\
\hline$\mu g / L$ & 100 & Final & EPA, 1993 \\
\hline$\mu \mathrm{g} / \mathrm{L}$ & 100 & Final & EPA, 1993 \\
\hline$\mu \mathrm{g} / \mathrm{L}$ & 1,300 & Final & EPA, 1993 \\
\hline$\mu \mathrm{g} / \mathrm{L}$ & 200 & Final & EPA, 1993 \\
\hline$\mu \mathrm{g} / \mathrm{L}$ & 200 & Final & EPA, 1993 \\
\hline$\mu \mathrm{g} / \mathrm{L}$ & 100 & Final & EPA, 1993 \\
\hline$\mu \mathrm{g} / \mathrm{L}$ & 0.2 & Final & EPA, 1993 \\
\hline$\mu \mathrm{g} / \mathrm{L}$ & 0.05 & Final & EPA, 1993 \\
\hline$\mu \mathrm{g} / \mathrm{L}$ & 600 & Final & EPA, 1993 \\
\hline$\mu g / L$ & 75 & Final & EPA, 1993 \\
\hline$\mu \mathrm{g} / \mathrm{L}$ & 5 & Final & EPA, 1993 \\
\hline$\mu \mathrm{g} / \mathrm{L}$ & 7 & Final & EPA, 1993 \\
\hline$\mu \mathrm{g} / \mathrm{L}$ & 50 & Final & EPA, 1993 \\
\hline$\mu \mathrm{g} / \mathrm{L}$ & 70 & Final & EPA, 1993 \\
\hline$\mu \mathrm{g} / \mathrm{L}$ & 100 & Final & EPA, 1993 \\
\hline$\mu \mathrm{g} / \mathrm{L}$ & 5 & Final & EPA, 1993 \\
\hline$\mu \mathrm{g} / \mathrm{L}$ & 70 & Final & EPA, 1993 \\
\hline$\mu \mathrm{g} / \mathrm{L}$ & 5 & Final & EPA, 1993 \\
\hline$\mu \mathrm{g} / \mathrm{L}$ & 400 & Final & EPA, 1993 \\
\hline$\mu g / L$ & 20 & Final & EPA, 1993 \\
\hline$\mu \mathrm{g} / \mathrm{L}$ & 100 & Final & EPA, 1993 \\
\hline$\mu \mathrm{g} / \mathrm{L}$ & 2 & Final & EPA, 1993 \\
\hline$\mu \mathrm{g} / \mathrm{L}$ & 700 & Final & EPA, 1993 \\
\hline$\mu \mathrm{g} / \mathrm{L}$ & 4,000 & Final & EPA, 1993 \\
\hline$\mu \mathrm{g} / \mathrm{L}$ & 700 & Final & EPA, 1993 \\
\hline $\mathrm{pCi} / \mathrm{L}$ & $1.5 \mathrm{E}+01$ & Final & EPA, 1993 \\
\hline$\mu \mathrm{g} / \mathrm{L}$ & 0.4 & Final & EPA, 1993 \\
\hline$\mu \mathrm{g} / \mathrm{L}$ & 0.2 & Final & EPA, 1993 \\
\hline$\mu \mathrm{g} / \mathrm{L}$ & 1 & Final & EPA, 1993 \\
\hline$\mu \mathrm{g} / \mathrm{L}$ & 50 & Final & EPA, 1993 \\
\hline & 50 & Final & SCDHEC, \\
\hline
\end{tabular}




\begin{tabular}{|c|c|c|c|c|}
\hline Analyte & $\underline{\text { Unit }}$ & Level & Status & Source \\
\hline Lindane & $\mu \mathrm{g} / \mathrm{L}$ & 0.2 & Final & EPA, 1993 \\
\hline Mercury & $\mu g / L$ & 2 & Final & EPA, 1993 \\
\hline Methoxychlor & $\mu \mathrm{g} / \mathrm{L}$ & 40 & Final & EPA, 1993 \\
\hline Nickel & $\mu g / L$ & 100 & Final & EPA, 1993 \\
\hline Nitrate as nitrogen & $\mu g / L$ & 10,000 & Final & EPA, 1993 \\
\hline Nitrate-nitrite as nitrogen & $\mu \mathrm{g} / \mathrm{L}$ & 10,000 & Final & EPA, 1993 \\
\hline Nitrite as nitrogen & $\mu g / L$ & 1,000 & Final & EPA, 1993 \\
\hline Nonvolatile beta & pCill & $5 E+01$ & Interim Final & EPA, 1977 \\
\hline Oxamyl ${ }^{a}$ & $\mu \mathrm{g} / \mathrm{L}$ & 200 & Final & EPA, 1993 \\
\hline PCB 1016 & $\mu g / L$ & 0.5 & Final & EPA, 1993 \\
\hline PCB 1221 & $\mu g / L$ & 0.5 & Final & EPA, 1993 \\
\hline PCB 1232 & $\mu g / L$ & 0.5 & Final & EPA, 1993 \\
\hline РCB 1242 & $\mu g / L$ & 0.5 & Final & EPA, 1993 \\
\hline РCB 1248 & $\mu g / L$ & 0.5 & Final & EPA, 1993 \\
\hline PCB 1254 & $\mu \mathrm{g} / \mathrm{L}$ & 0.5 & Final & EPA, 1993 \\
\hline РCB 1260 & $\mu g / L$ & 0.5 & Final & EPA, 1993 \\
\hline PCB 1262 & $\mu g / L$ & 0.5 & Final & EPA, 1993 \\
\hline Pentachlorophenol & $\mu g / L$ & 1 & Final & EPA, 1993 \\
\hline Piclorama & $\mu \mathrm{g} / \mathrm{L}$ & 500 & Final & EPA, 1993 \\
\hline Selenium & $\mu \mathrm{g} / \mathrm{L}$ & 50 & Final & EPA, 1993 \\
\hline Simazine ${ }^{a}$ & $\mu g / L$ & 4 & Final & EPA, 1993 \\
\hline Strontium-89/90 & $\mathrm{pCi} / \mathrm{L}$ & $8 \mathrm{E}+00$ & Final & EPA, 1993 \\
\hline Strontium-90 & $\mathrm{pCi} / \mathrm{L}$ & $8 \mathrm{E}+00$ & Final & EPA, 1993 \\
\hline Styrene & $\mu \mathrm{g} / \mathrm{L}$ & 100 & Final & EPA, 1993 \\
\hline $2,3,7,8-T C D D$ & $\mu \mathrm{g} / \mathrm{L}$ & 0.00003 & Final & EPA, 1993 \\
\hline Tetrachloroethylene & $\mu \mathrm{g} / \mathrm{L}$ & 5 & Final & EPA, 1993 \\
\hline Thallium & $\mu g / L$ & 2 & Final & EPA, 1993 \\
\hline Toluene & $\mu g / L$ & 1,000 & Final & EPA, 1993 \\
\hline Toxaphene & $\mu \mathrm{g} / \mathrm{L}$ & 3 & Final & EPA, 1993 \\
\hline 2,4,5-TP (Silvex) & $\mu g / L$ & 50 & Final & EPA, 1993 \\
\hline 1,2,4-Trichlorobenzene & $\mu \mathrm{g} / \mathrm{L}$ & 70 & Final & EPA, 1993 \\
\hline 1,1,1-Trichloroethane & $\mu g / L$ & 200 & Final & EPA, 1993 \\
\hline 1,1,2-Trichloroethane & $\mu g / L$ & 5 & Final & EPA, 1993 \\
\hline Trichloroethylene & $\mu \mathrm{g} / \mathrm{L}$ & 5 & Final & EPA, 1993 \\
\hline Tritium & $\mathrm{pCi} / \mathrm{mL}$ & $2 E+01$ & Final & EPA, 1993 \\
\hline Xylenes & $\mu g / L$ & 10,000 & Final & EPA, 1993 \\
\hline
\end{tabular}

Note: Final PDWS were assigned to alachlor, aldicarb, aldicarb sulfone, aldicarb sulfoxide, atrazine, carbofuran, dalapon, di(2-ethylhexyl) adipate, diquat dibromide, endothall, glyphosate, oxamyl, picloram, and simazine in the SRS Groundwater Monitoring Program for the first time beginning first quarter 1994.

a At present, EMS does not perform this analysis because the constituent is not in the current contract.

b The standard given is for gross alpha including radium-226 but excluding radon and uranium.

c For double radionuclide analyses where each separate radionuclide has its own standard, the more stringent standard is used. 


\section{References}

EPA (U.S. Environmental Protection Agency), 1977. National Interim Primary Drinking Water Regulations, EPA-570/9-76-003. Washington, DC.

EPA (U.S. Environmental Protection Agency), 1993. National Primary Drinking Water Regulations, Code of Federal Regulations, Title 40, Part 141, pp. 592-732. Washington, DC.

SCDHEC (South Carolina Department of Health and Environmental Control), 1981. State Primary Drinking Water Regulations, R.61-58.5. Columbia, SC. 
WSRC-TR-94-0610

Unclassified

THIS PAGE LEFT BLANK INTENTIONALLY. 


\section{Appendix B}

\section{Flagging Criteria}


WSRC-TR-94-0610

Unclassified

THIS PAGE LEFT BLANK INTENTIONALLY. 


\section{Flagging Criteria}

The Savannah River Site Environmental Protection Department/Environmental Monitoring Section (EPD/EMS) flagging criteria are as follows:

- Flag 2 criteria for constituents equal the Safe Drinking Water Act (SDWA) final Primary Drinking Water Standards (PDWS), the SDWA proposed PDWS, or the SDWA Secondary Drinking Water Standards (SDWS). If a constituent does not have a drinking water standard, the Flag 2 criterion equals 10 times the method detection limit (MDL) calculated as the 90 th percentile detection limit obtained recently by one of the primary analytical laboratories.

- Flag 1 criteria for constituents equal one-half of the final PDWS, one-half the proposed PDWS, or one-half the SDWS. If a constituent does not have a drinking water standard, the Flag 1 criterion equals 5 times the MDL calculated as the 90th percentile detection limit obtained recently by one of the primary analytical laboratories.

- Flag 0 criteria are assigned to constituent levels below Flag 1 criteria, constituent levels below the sample detection limits, or constituents having no flagging criteria.

The following parameters are exceptions to the flagging rules:

- EPD/EMS sets flagging criteria for $\mathrm{pH}$ and specific conductance. No flags are set for alkalinity, calcium, carbonate, magnesium, potassium, silica, sodium, total dissolved solids, total phosphates (as P), and total phosphorus. Analyses for these parameters are conducted as part of the biennial comprehensive analyses or by special request.

- Aesthetic parameters such as color, corrosivity, Eh, odor, surfactants, and turbidity are not assigned flagging criteria but are analyzed by special request.

- Common laboratory contaminants and cleaners such as dichloromethane (methylene chloride), ketones, phthalates, and toluene are not assigned flagging criteria unless they have primary drinking water standards. These constituents are analyzed by special request.

Analyte
Acenaphthene
Acenaphthylene
Acetone
Acetonitrile (Methyl cyanide)
Acetophenone
2-Acetylaminofluorene
Acrolein
Acrylonitrile
Actinium-228
Alachlor
Aldicarb
Aldicarb sulfone ${ }^{\mathrm{b}}$
Aldicarb sulfoxide
Aldrin
Alkalinity (as $\mathrm{CaCO}_{3}$ )
Allyl chloride
Aluminum
Aluminum, dissolved
Aluminum, total recoverable

\begin{tabular}{|c|c|c|}
\hline Unit & Flag 1 & Flag 2 \\
\hline$\mu \mathrm{g} / \mathrm{L}$ & 50 & 100 \\
\hline$\mu \mathrm{g} / \mathrm{L}$ & 50 & 100 \\
\hline$\mu g / L$ & 500 & 1,000 \\
\hline$\mu g / L$ & 500 & 1,000 \\
\hline$\mu g / L$ & 50 & 100 \\
\hline$\mu \mathrm{g} / \mathrm{L}$ & 50 & 100 \\
\hline$\mu g / L$ & 100 & 200 \\
\hline$\mu g / L$ & 100 & 200 \\
\hline pCi/L & $1.64 \mathrm{E}+03$ & $3.27 E+03$ \\
\hline$\mu \mathrm{g} / \mathrm{L}$ & 1 & 2 \\
\hline$\mu \mathrm{g} / \mathrm{L}$ & 1.5 & 3 \\
\hline$\mu \mathrm{g} / \mathrm{L}$ & 1 & 2 \\
\hline$\mu g / L$ & 2 & 4 \\
\hline$\mu \mathrm{g} / \mathrm{L}$ & 0.25 & 0.5 \\
\hline & No flag & No flag \\
\hline mo & 250 & 500 \\
\hline$\mu \mathrm{g} /$ & 25 & 50 \\
\hline$\mu \mathrm{g} / \mathrm{L}$ & 25 & 50 \\
\hline & 25 & 50 \\
\hline
\end{tabular}

$\underline{\text { Source }}^{\mathrm{a}}$

EPA Method 8270

EPA Method 8270

EPA Method 8240

EPA Method 8240

EPA Method 8270

EPA Method 8270

EPA Method 8240

EPA Method 8240

Proposed PDWS (EPA, 1991)

Final PDWS (EPA, 1993a)

Final PDWS (EPA, 1993a)

Final PDWS (EPA, 1993a)

Final PDWS (EPA, 1993a)

EPA Method 8080

Set by EPD/EMS

EPA Method 8240

SDWS (EPA, 1993b)

SDWS (EPA, 1993b)

SDWS (EPA, 1993b) 
Analyte

Americium-241
Americium-243
4-Aminobiphenyl
Ammonia
Ammonia nitrogen
Aniline
Anthracene
Antimony
Antimony, dissolved
Antimony, total recoverable
Antimony-125
Aramite
Arsenic
Arsenic, dissolved
Arsenic, total recoverable
Asbestos
Atrazine
Azobenzene
Barium
Barium, dissolved
Barium, total recoverable
Barium-140
Benzene
alpha-Benzene hexachloride
beta-Benzene hexachloride
delta-Benzene hexachloride
Benzidine
Benzo[a]anthracene
Benzo[b]fluoranthene
Benzo[k]fluoranthene
Benzoic acid
Benzo[g,h,ijperylene
Benzo[a]pyrene
1,4-Benzoquinone
Benzyl alcohol
Beryllium
Beryllium, dissolved
Beryllium, total recoverable
Beryllium-7
Bis(2-chloroethoxy) methane
Bis(2-chloroethyl) ether
Bis(2-chloroisopropyl) ether
Bis(chloromethyl) ether
Bis(2-ethylhexyl) phthalate
Bismuth-214
Boron
Boron, dissolved
Boron, total recoverable
Bromide
Bromodichloromethane
Bromoform
Bromomethane (Methyl bromide)
4-Bromophenyl phenyl ether
Butylbenzyl phthalate
2-sec-Butyl-4,6-dinitrophenol

\begin{tabular}{|c|c|c|c|}
\hline Unit & Flag 1 & Flag 2 & Source \\
\hline $\mathrm{pCi} / \mathrm{L}$ & 3.17E+00 & $6.34 E+00$ & Proposed PDWS (EPA, 1991) \\
\hline $\mathrm{pCill}$ & $3.19 \mathrm{E}+00$ & $6.37 E+00$ & Proposed PDWS (EPA, 1991) \\
\hline$\mu g / L$ & 50 & 100 & EPA Method 8270 \\
\hline$\mu \mathrm{g} / \mathrm{L}$ & 500 & 1,000 & APHA Method 417B \\
\hline$\mu g / L$ & 500 & 1,000 & EPA Method 350.1 \\
\hline$\mu \mathrm{g} / \mathrm{L}$ & 50 & 100 & EPA Method 8270 \\
\hline$\mu g / L$ & 50 & 100 & EPA Method 8270 \\
\hline$\mu \mathrm{g} / \mathrm{L}$ & 3 & 6 & Final PDWS (EPA, 1993a) \\
\hline$\mu \mathrm{g} / \mathrm{L}$ & 3 & 6 & Final PDWS (EPA, 1993a) \\
\hline$\mu \mathrm{g} / \mathrm{L}$ & 3 & 6 & Final PDWS (EPA, 1993a) \\
\hline $\mathrm{pCi} / \mathrm{L}$ & $1.5 E+02$ & $3 E+02$ & Interim Final PDWS (EPA, 1977) \\
\hline$\mu \mathrm{g} / \mathrm{L}$ & 50 & 100 & EPA Method 8270 \\
\hline$\mu \mathrm{g} / \mathrm{L}$ & 25 & 50 & Final PDWS (EPA, 1993a) \\
\hline$\mu \mathrm{g} / \mathrm{L}$ & 25 & 50 & Final PDWS (EPA, 1993a) \\
\hline$\mu g / L$ & 25 & 50 & Final PDWS (EPA, 1993a) \\
\hline Fibers/L & $3,500,000$ & $7,000,000$ & Final PDWS (EPA, 1993a) \\
\hline$\mu \mathrm{g} / \mathrm{L}$ & 1.5 & 3 & Final PDWS (EPA, 1993a) \\
\hline$\mu \mathrm{g} / \mathrm{L}$ & 50 & 100 & EPA Method 625 \\
\hline$\mu g / L$ & 1,000 & 2,000 & Final PDWS (EPA, 1993a) \\
\hline$\mu \mathrm{g} / \mathrm{L}$ & 1,000 & 2,000 & Final PDWS (EPA, 1993a) \\
\hline$\mu g / L$ & 1,000 & 2,000 & Final PDWS (EPA, 1993a) \\
\hline $\mathrm{pCi} / \mathrm{L}$ & $4.5 \mathrm{E}+01$ & $9 \mathrm{E}+01$ & Interim Final PDWS (EPA, 1977) \\
\hline$\mu \mathrm{g} / \mathrm{L}$ & 2.5 & 5 & Final PDWS (EPA, 1993a) \\
\hline$\mu g / L$ & 0.25 & 0.5 & EPA Method 8080 \\
\hline$\mu g / L$ & 0.25 & 0.5 & EPA Method 8080 \\
\hline$\mu \mathrm{g} / \mathrm{L}$ & 0.25 & 0.5 & EPA Method 8080 \\
\hline$\mu \mathrm{g} / \mathrm{L}$ & 250 & 500 & EPA Method 8270 \\
\hline$\mu g / L$ & 0.05 & 0.1 & Proposed PDWS (EPA, 1990) \\
\hline$\mu \mathrm{g} / \mathrm{L}$ & 0.1 & 0.2 & Proposed PDWS (EPA, 1990) \\
\hline$\mu \mathrm{g} / \mathrm{L}$ & 0.1 & 0.2 & Proposed PDWS (EPA, 1990) \\
\hline$\mu g / L$ & 250 & 500 & EPA Method 8270 \\
\hline$\mu \mathrm{g} / \mathrm{L}$ & 50 & 100 & EPA Method 8270 \\
\hline$\mu g / L$ & 0.1 & 0.2 & Final PDWS (EPA, 1993a) \\
\hline$\mu \mathrm{g} / \mathrm{L}$ & 50 & 100 & EPA Method 8270 \\
\hline$\mu \mathrm{g} / \mathrm{L}$ & 50 & 100 & EPA Method 8270 \\
\hline$\mu \mathrm{g} / \mathrm{L}$ & 2 & 4 & Final PDWS (EPA, 1993a) \\
\hline$\mu \mathrm{g} / \mathrm{L}$ & 2 & 4 & Final PDWS (EPA, 1993a) \\
\hline$\mu \mathrm{g} / \mathrm{L}$ & 2 & 4 & Final PDWS (EPA, 1993a) \\
\hline $\mathrm{pCi} / \mathrm{L}$ & $3 E+03$ & $6 E+03$ & Interim Final PDWS (EPA, 1977) \\
\hline$\mu \mathrm{g} / \mathrm{L}$ & 50 & 100 & EPA Method 8270 \\
\hline$\mu \mathrm{g} / \mathrm{L}$ & 50 & 100 & EPA Method 8270 \\
\hline$\mu \mathrm{g} / \mathrm{L}$ & 50 & 100 & EPA Method 8270 \\
\hline$\mu g / L$ & 50 & 100 & EPA Method 8270 \\
\hline$\mu \mathrm{g} / \mathrm{L}$ & 3 & 6 & Final PDWS (EPA, 1993a) \\
\hline $\mathrm{pCi} / \mathrm{L}$ & $9.4 \mathrm{E}+03$ & $1.89 \mathrm{E}+04$ & Proposed PDWS (EPA, 1991) \\
\hline$\mu \mathrm{g} / \mathrm{L}$ & 150 & 300 & EPA Method $6010^{\circ}$ \\
\hline$\mu \mathrm{g} / \mathrm{L}$ & 150 & 300 & EPA Method 6010 \\
\hline$\mu \mathrm{g} / \mathrm{L}$ & 150 & 300 & EPA Method 6010 \\
\hline$\mu \mathrm{g} / \mathrm{L}$ & 5,000 & 10,000 & EPA Method 300.0 \\
\hline$\mu \mathrm{g} / \mathrm{L}$ & 50 & 100 & Final PDWS (EPA, 1993a) \\
\hline$\mu \mathrm{g} / \mathrm{L}$ & 50 & 100 & Final PDWS (EPA, 1993a) \\
\hline$\mu \mathrm{g} / \mathrm{L}$ & 5 & 10 & EPA Method 8240 \\
\hline$\mu \mathrm{g} / \mathrm{L}$ & 50 & 100 & EPA Method 8270 \\
\hline & & No flag & Set by EPD/EMS \\
\hline$\mu$ & 3.5 & 7 & Final PDWS (EPA, 1993a) \\
\hline
\end{tabular}




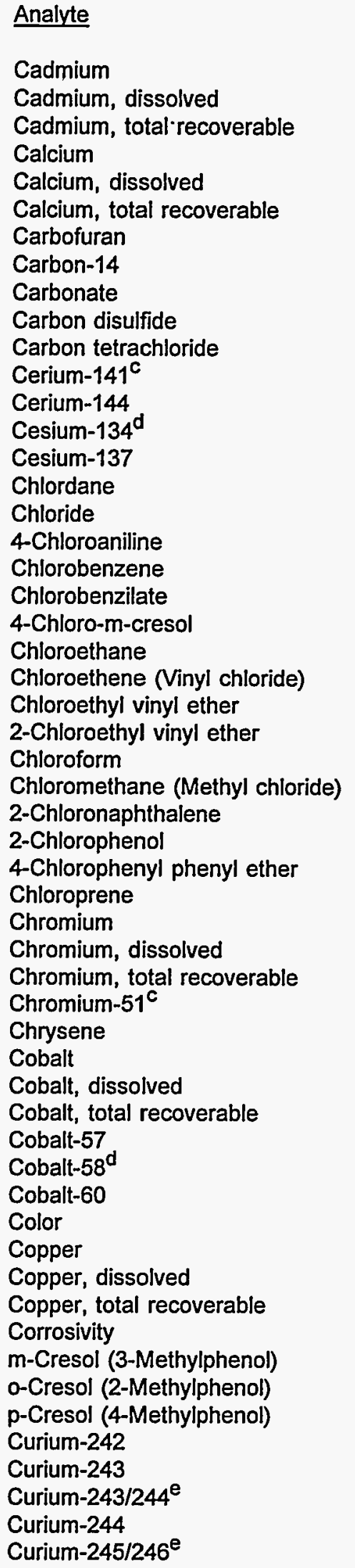

\begin{tabular}{|c|c|c|c|}
\hline Unit & Flag 1 & Flag 2 & Source \\
\hline$\mu \mathrm{g} / \mathrm{L}$ & 2.5 & 5 & Final PDWS (EPA, 1993a) \\
\hline$\mu g / L$ & 2.5 & 5 & Final PDWS (EPA, 1993a) \\
\hline$\mu g / L$ & 2.5 & 5 & Final PDWS (EPA, 1993a) \\
\hline & No flag & No flag & Set by EPD/EMS \\
\hline & No flag & No flag & Set by EPD/EMS \\
\hline & No flag & No flag & Set by EPD/EMS \\
\hline$\mu \mathrm{g} / \mathrm{L}$ & & 40 & Final PDWS (EPA, 1993a) \\
\hline pCill & $1 E+03$ & $2 E+03$ & Interim Final PDWS (EPA, 1977) \\
\hline & No flag & No flag & Set by EPD/EMS \\
\hline$\mu g / L$ & 5 & 10 & EPA Method 8240 \\
\hline$\mu \mathrm{g} / \mathrm{L}$ & 2.5 & 5 & Final PDWS (EPA, 1993a) \\
\hline pCill & $1.5 E+02$ & $3 E+02$ & Interim Final PDWS (EPA, 1977) \\
\hline $\mathrm{pCi} / \mathrm{L}$ & $1.31 E+02$ & $2.61 E+02$ & Proposed PDWS (EPA, 1991) \\
\hline pCi/L & 4.07E+01 & $8.13 E+01$ & Proposed PDWS (EPA, 1991) \\
\hline pCill & $1 E+02$ & $2 \mathrm{E}+02$ & Interim Final PDWS (EPA, 1977) \\
\hline$\mu g / L$ & 1 & 2 & Final PDWS (EPA, 1993a) \\
\hline$\mu g / L$ & 125,000 & 250,000 & SDWS (EPA, 1993b) \\
\hline$\mu \mathrm{g} / \mathrm{L}$ & 50 & 100 & EPA Method 8270 \\
\hline$\mu g / L$ & 50 & 100 & Final PDWS (EPA, 1993a) \\
\hline$\mu g / L$ & 50 & 100 & EPA Method 8270 \\
\hline$\mu g / L$ & 50 & 100 & EPA Method 8270 \\
\hline$\mu \mathrm{g} / \mathrm{L}$ & 5 & 10 & EPA Method 8240 \\
\hline$\mu g / L$ & 1 & 2 & Final PDWS (EPA, 1993a) \\
\hline$\mu \mathrm{g} / \mathrm{L}$ & 5 & 10 & EPA Method 8240 \\
\hline$\mu g / L$ & 5 & 10 & EPA Method 8240 \\
\hline$\mu g / L$ & 50 & 100 & Final PDWS (EPA, 1993a) \\
\hline$\mu \mathrm{g} / \mathrm{L}$ & 5 & 10 & EPA Method 8240 \\
\hline$\mu g / L$ & 50 & 100 & EPA Method 8240 \\
\hline$\mu \mathrm{g} / \mathrm{L}$ & 50 & 100 & EPA Method 8270 \\
\hline$\mu g / L$ & 50 & 100 & EPA Method 8270 \\
\hline$\mu g / L$ & 1,000 & 2,000 & EPA Method 8240 \\
\hline$\mu g / L$ & 50 & 100 & Final PDWS (EPA, 1993a) \\
\hline$\mu \mathrm{g} / \mathrm{L}$ & 50 & 100 & Final PDWS (EPA, 1993a) \\
\hline$\mu \mathrm{g} / \mathrm{L}$ & 50 & 100 & Final PDWS (EPA, 1993a) \\
\hline $\mathrm{pCi} / \mathrm{L}$ & $3 E+03$ & $6 \mathrm{E}+03$ & Interim Final PDWS (EPA, 1977) \\
\hline$\mu g / L$ & 0.1 & 0.2 & Proposed PDWS (EPA, 1990) \\
\hline$\mu \mathrm{g} / \mathrm{L}$ & 20 & 40 & EPA Method 6010 \\
\hline$\mu \mathrm{g} / \mathrm{L}$ & 20 & 40 & EPA Method 6010 \\
\hline$\mu \mathrm{g} / \mathrm{L}$ & 20 & 40 & EPA Method 6010 \\
\hline pCi/L & $5 E+02$ & $1 E+03$ & Interim Final PDWS (EPA, 1977) \\
\hline $\mathrm{pCi} / \mathrm{L}$ & $4.5 E+03$ & $9 E+03$ & Interim Final PDWS (EPA, 1977) \\
\hline pCi/l & $\begin{array}{l}5 E+01 \\
\text { No flag }\end{array}$ & $\begin{array}{l}1 E+02 \\
\text { No flag }\end{array}$ & $\begin{array}{l}\text { Interim Final PDWS (EPA, 1977) } \\
\text { Set by EPD/EMS }\end{array}$ \\
\hline$\mu g /$ & 500 & 1,000 & Final PDWS (SCDHEC, 1981) \\
\hline$\mu \mathrm{g} / \mathrm{L}$ & 500 & 1,000 & Final PDWS (SCDHEC, 1981) \\
\hline$\mu \mathrm{g} / \mathrm{L}$ & $\begin{array}{l}500 \\
\text { No flag }\end{array}$ & $\begin{array}{l}1,000 \\
\text { No flag }\end{array}$ & $\begin{array}{l}\text { Final PDWS (SCDHEC, 1981) } \\
\text { Set by EPD/EMS }\end{array}$ \\
\hline$\mu \mathrm{g} / \mathrm{L}$ & $\begin{array}{l}\text { No llag } \\
50\end{array}$ & $\begin{array}{l}\text { No flag } \\
100\end{array}$ & EPA Method 8270 \\
\hline$\mu \mathrm{g} / \mathrm{L}$ & 50 & 100 & EPA Method 8270 \\
\hline$\mu g / L$ & 50 & 100 & EPA Method 8270 \\
\hline $\mathrm{pCi} / \mathrm{L}$ & $6.65 E+01$ & 1.33E+02 & Proposed PDWS (EPA, 1991) \\
\hline $\mathrm{pCi} / \mathrm{L}$ & $4.15 E+00$ & 8.3E+00 & Proposed PDWS (EPA, 1991) \\
\hline pCi/L & $4.15 E+00$ & $8.3 E+00$ & Proposed PDWS (EPA, 1991) \\
\hline $\mathrm{pCi}$ & $4.92 \mathrm{E}+00$ & $9.84 E+00$ & Proposed PDWS (EPA, 1991) \\
\hline $\mathrm{pCi} / \mathrm{L}$ & $3.12 E+00$ & $6.23 E+00$ & Proposed PDWS (EPA, 1991) \\
\hline
\end{tabular}




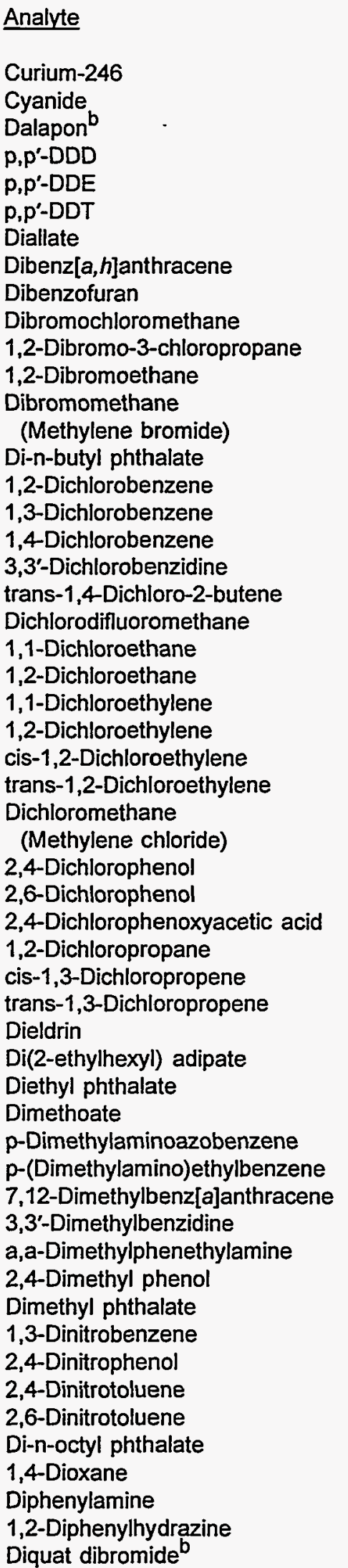

\begin{tabular}{|c|c|c|c|}
\hline Unit & Flag 1 & Flag 2 & Source \\
\hline $\mathrm{pCi} / \mathrm{L}$ & $3.14 E+00$ & $6.27 E+00$ & Proposed PDWS (EPA, 1991) \\
\hline$\mu \mathrm{g} / \mathrm{L}$ & 100 & 200 & Final PDWS (EPA, 1993a) \\
\hline$\mu g / L$ & 100 & 200 & Final PDWS (EPA, 1993a) \\
\hline$\mu \mathrm{g} / \mathrm{L}$ & 0.5 & 1 & EPA Method 8080 \\
\hline$\mu g / L$ & 0.5 & 1 & EPA Method 8080 \\
\hline$\mu \mathrm{g} / \mathrm{L}$ & 0.5 & 1 & EPA Method 8080 \\
\hline$\mu g / L$ & 50 & 100 & EPA Method 8270 \\
\hline$\mu \mathrm{g} / \mathrm{L}$ & 0.15 & 0.3 & Proposed PDWS (EPA, 1990) \\
\hline$\mu g / L$ & 50 & 100 & EPA Method 8270 \\
\hline$\mu g / L$ & 50 & 100 & Final PDWS (EPA, 1993a) \\
\hline$\mu \mathrm{g} / \mathrm{L}$ & 0.1 & 0.2 & Final PDWS (EPA, 1993a) \\
\hline$\mu \mathrm{g} / \mathrm{L}$ & 0.025 & 0.05 & Final PDWS (EPA, 1993a) \\
\hline$\mu g / L$ & 5 & 10 & EPA Method 8240 \\
\hline & No flag & No flag & Set by EPD/EMS \\
\hline$\mu g / L$ & 300 & 600 & Final PDWS (EPA, 1993a) \\
\hline$\mu g / L$ & 50 & 100 & EPA Method 8270 \\
\hline$\mu g / L$ & 37.5 & 75 & Final PDWS (EPA, 1993a) \\
\hline$\mu \mathrm{g} / \mathrm{L}$ & 50 & 100 & EPA Method 8270 \\
\hline$\mu \mathrm{g} / \mathrm{L}$ & 150 & 300 & EPA Method 8240 \\
\hline$\mu g / L$ & 5 & 10 & EPA Method 8240 \\
\hline$\mu g / L$ & 5 & 10 & EPA Method 8240 \\
\hline$\mu g / L$ & 2.5 & 5 & Final PDWS (EPA, 1993a) \\
\hline$\mu g / L$ & 3.5 & 7 & Final PDWS (EPA, 1993a) \\
\hline$\mu g / L$ & 25 & 50 & Final PDWS (EPA, 1993a) \\
\hline$\mu g / L$ & 35 & 70 & Final PDWS (EPA, 1993a) \\
\hline$\mu g / L$ & 50 & 100 & Final PDWS (EPA, 1993a) \\
\hline$\mu \mathrm{g} / \mathrm{L}$ & 2.5 & 5 & Final PDWS (EPA, 1993a) \\
\hline$\mu g / L$ & 50 & 100 & EPA Method 8270 \\
\hline$\mu g / L$ & 50 & 100 & EPA Method 8270 \\
\hline$\mu g / L$ & 35 & 70 & Final PDWS (EPA, 1993a) \\
\hline$\mu g / L$ & 2.5 & 5 & Final PDWS (EPA, 1993a) \\
\hline$\mu g / L$ & 5 & 10 & EPA Method 8240 \\
\hline$\mu g / L$ & 5 & 10 & EPA Method 8240 \\
\hline$\mu \mathrm{g} / \mathrm{L}$ & 2.5 & 5 & EPA Method 8080 \\
\hline$\mu g / L$ & 200 & 400 & Final PDWS (EPA, 1993a) \\
\hline & No flag & No flag & Set by EPD/EMS \\
\hline$\mu \mathrm{g} / \mathrm{L}$ & 50 & 100 & EPA Method 8270 \\
\hline$\mu g / L$ & 50 & 100 & EPA Method 8270 \\
\hline$\mu g / L$ & 50 & 100 & EPA Method 8270 \\
\hline$\mu g / L$ & 50 & 100 & EPA Method 8270 \\
\hline$\mu g / L$ & 50 & 100 & EPA Method 8270 \\
\hline$\mu g / L$ & 50 & 100 & EPA Method 8270 \\
\hline$\mu \mathrm{g} / \mathrm{L}$ & 50 & 100 & EPA Method 8270 \\
\hline & No flag & No flag & Set by EPD/EMS \\
\hline$\mu g / L$ & 50 & 100 & EPA Method 8270 \\
\hline$\mu \mathrm{g} / \mathrm{L}$ & 250 & 500 & EPA Method 8270 \\
\hline$\mu \mathrm{g} / \mathrm{L}$ & 50 & 100 & EPA Method 8270 \\
\hline$\mu g / L$ & 50 & 100 & EPA Method 8270 \\
\hline & No flag & No flag & Set by EPD/EMS \\
\hline$\mu g / L$ & 50 & 100 & EPA Method 8270 \\
\hline$\mu \mathrm{g} / \mathrm{L}$ & 50 & 100 & EPA Method 8270 \\
\hline$\mu g / L$ & 50 & 100 & EPA Method 8270 \\
\hline$\mu \mathrm{g} / \mathrm{L}$ & 10 & 20 & Final PDWS (EPA, 1993a) \\
\hline
\end{tabular}


Analyte

Dissolved organic carbon

Disulfoton

Eh

Endosulfan I

Endosulfan II

Endosulfan sulfate

Endothall ${ }^{b}$

Endrin

Endrin aldehyde

Endrin ketone

Ethylbenzene

Ethyl methacrylate

Ethyl methanesulfonate

Europium-152

Europium-154

Europium-155

Famphur

Fluoranthene

Fluorene

Fluoride

Glyphosate ${ }^{b}$

Gross alpha

Heptachlor

Heptachlor epoxide

Heptachlorodibenzo-p-dioxin isomers

1,2,3,4,6,7,8-HPCDD

Heptachlorodibenzo-p-furan isomers

$1,2,3,4,6,7,8$-HPCDF

Hexachlorobenzene

Hexachlorobutadiene

Hexachlorocyclopentadiene

Hexachlorodibenzo-p-dioxin isomers

$1,2,3,4,7,8-H X C D D$

Hexachlorodibenzo-p-furan isomers

$1,2,3,4,7,8-H X C D F$

Hexachloroethane

Hexachlorophene

Hexachloropropene

2-Hexanone

Indeno[1,2,3-c, d]pyrene

lodine

lodine-129

lodine-131 $\mathrm{c}$

lodomethane (Methyl iodide)

Iron

Iron, dissolved

Iron, total recoverable

Iron- $55^{\mathrm{C}}$

Iron-59c

Isobutyl alcohol

Isodrin

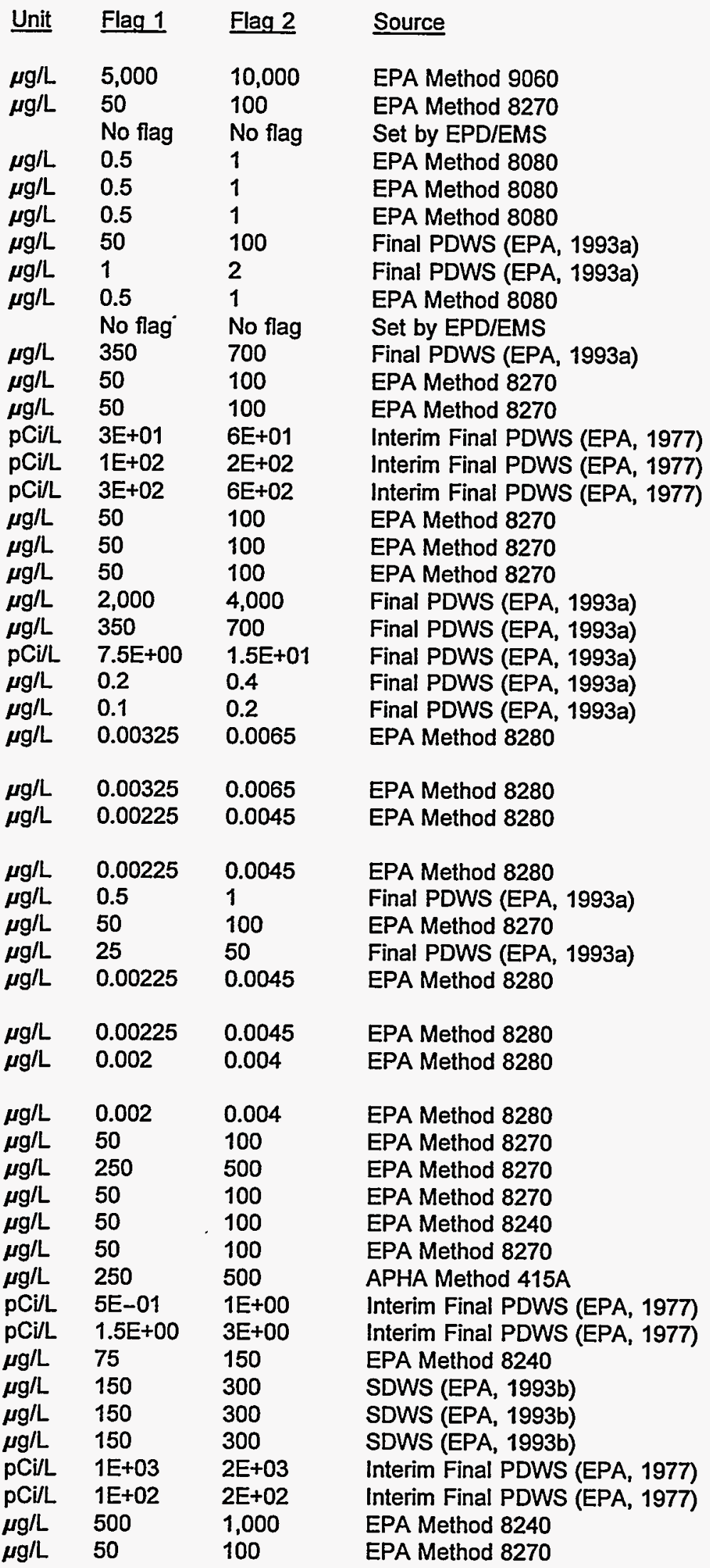




\begin{tabular}{|c|c|c|c|c|}
\hline Analyte & Unit & Flag 1 & Flag 2 & Source \\
\hline Isophorone & $\mu g / L$ & 50 & 100 & EPA Method 8270 \\
\hline Isosafrole & $\mu \mathrm{g} / \mathrm{L}$ & 50 & 100 & EPA Method 8270 \\
\hline Kepone & $\mu \mathrm{g} / \mathrm{L}$ & 50 & 100 & EPA Method 8270 \\
\hline Lanthanum- $140^{\mathrm{C}}$ & $\mathrm{pCi} / \mathrm{L}$ & $3 E+01$ & $6 \mathrm{E}+01$ & Interim Final PDWS (EPA, 1977) \\
\hline Lead & $\mu g / L$ & 25 & 50 & Final PDWS (SCDHEC, 1981) \\
\hline Lead, dissolved & $\mu \mathrm{g} / \mathrm{L}$ & 25 & 50 & Final PDWS (SCDHEC, 1981) \\
\hline Lead, total recoverable & $\mu \mathrm{g} / \mathrm{L}$ & 25 & 50 & Final PDWS (SCDHEC, 1981) \\
\hline Lead-212 & $\mathrm{pCi} / \mathrm{L}$ & $6.2 \mathrm{E}+01$ & $1.23 E+02$ & Proposed PDWS (EPA, 1991) \\
\hline Lindane & $\mu g / L$ & 0.1 & 0.2 & Final PDWS (EPA, 1993a) \\
\hline Lithium & $\mu \mathrm{g} / \mathrm{L}$ & 25 & 50 & EPA Method 6010 \\
\hline Lithium, dissolved & $\mu g / L$ & 25 & 50 & EPA Method 6010 \\
\hline Lithium, total recoverable & $\mu \mathrm{g} / \mathrm{L}$ & 25 & 50 & EPA Method 6010 \\
\hline Magnesium & & No flag & No flag & Set by EPD/EMS \\
\hline Magnesium, dissolved & & No flag & No flag & Set by EPD/EMS \\
\hline Magnesium, total recoverable & & No flag & No flag & Set by EPD/EMS \\
\hline Manganese & $\mu g / L$ & 25 & 50 & SDWS (EPA, 1993b) \\
\hline Manganese, dissolved & $\mu \mathrm{g} / \mathrm{L}$ & 25 & 50 & SDWS (EPA, 1993b) \\
\hline Manganese, total recoverable & $\mu \mathrm{g} / \mathrm{L}$ & 25 & 50 & SDWS (EPA, 1993b) \\
\hline Manganese-54 & $\mathrm{pCi} / \mathrm{L}$ & $1.5 E+02$ & $3 E+02$ & Interim Final PDWS (EPA, 1977) \\
\hline Mercury & $\mu \mathrm{g} / \mathrm{L}$ & 1 & 2 & Final PDWS (EPA, 1993a) \\
\hline Mercury, dissolved & $\mu \mathrm{g} / \mathrm{L}$ & 1 & 2 & Final PDWS (EPA, 1993a) \\
\hline Mercury, total recoverable & $\mu \mathrm{g} / \mathrm{L}$ & 1 & 2 & Final PDWS (EPA, 1993a) \\
\hline Methacrylonitrile & $\mu \mathrm{g} / \mathrm{L}$ & 250 & 500 & EPA Method 8240 \\
\hline Methapyrilene & $\mu \mathrm{g} / \mathrm{L}$ & 50 & 100 & EPA Method 8270 \\
\hline Methoxychlor & $\mu \mathrm{g} / \mathrm{L}$ & 20 & 40 & Final PDWS (EPA, 1993a) \\
\hline 3-Methylcholanthrene & $\mu \mathrm{g} / \mathrm{L}$ & 50 & 100 & EPA Method 8270 \\
\hline 2-Methyl-4,6-dinitrophenol & $\mu \mathrm{g} / \mathrm{L}$ & 250 & 500 & EPA Method 8270 \\
\hline Methyl ethyl ketone & & No flag & No flag & Set by EPD/EMS \\
\hline Methyl isobutyl ketone & & No flag & No flag & Set by EPD/EMS \\
\hline Methyl methacrylate & $\mu g / L$ & 50 & 100 & EPA Method 8270 \\
\hline Methyl methanesulfonate & $\mu \mathrm{g} / \mathrm{L}$ & 50 & 100 & EPA Method 8270 \\
\hline 2-Methyinaphthalene & $\mu \mathrm{g} / \mathrm{L}$ & 50 & 100 & EPA Method 8270 \\
\hline Molybdenum & $\mu \mathrm{g} / \mathrm{L}$ & 250 & 500 & EPA Method 6010 \\
\hline Molybdenum, dissolved & $\mu g / L$ & 250 & 500 & EPA Method 6010 \\
\hline Molybdenum, total recoverable & $\mu g / L$ & 250 & 500 & EPA Method 6010 \\
\hline Naphthalene & $\mu g / L$ & 50 & 100 & EPA Method 8270 \\
\hline 1,4-Naphthoquinone & $\mu \mathrm{g} / \mathrm{L}$ & 50 & 100 & EPA Method 8270 \\
\hline 1-Naphthylamine & $\mu g / L$ & 50 & 100 & EPA Method 8270 \\
\hline 2-Naphthylamine & $\mu g / L$ & 50 & 100 & EPA Method 8270 \\
\hline Neptunium-237 & $\mathrm{pCi} / \mathrm{L}$ & $3.53 E+00$ & $7.06 \mathrm{E}+00$ & Proposed PDWS (EPA, 1991) \\
\hline Nickel & $\mu g / L$ & 50 & 100 & Final PDWS (EPA, 1993a) \\
\hline Nickel, dissolved & $\mu g / L$ & 50 & 100 & Final PDWS (EPA, 1993a) \\
\hline Nickel, total recoverable & $\mu g / L$ & 50 & 100 & Final PDWS (EPA, 1993a) \\
\hline Nickel-59 & $\mathrm{pCi} / \mathrm{L}$ & $1.5 \mathrm{E}+02$ & $3 E+02$ & Interim Final PDWS (EPA, 1977) \\
\hline Nickel-63 ${ }^{c}$ & $\mathrm{pCi} / \mathrm{L}$ & 2.5E+01 & $5 E+01$ & Interim Final PDWS (EPA, 1977) \\
\hline Niobium-95 ${ }^{\mathrm{c}}$ & $\mathrm{pCi} / \mathrm{L}$ & $1.5 E+02$ & 3.E+02 & Interim Final PDWS (EPA, 1977) \\
\hline Nitrate as nitrogen & $\mu \mathrm{g} / \mathrm{L}$ & 5,000 & 10,000 & Final PDWS (EPA, 1993a) \\
\hline Nitrate-nitrite as nitrogen & $\mu g / L$ & 5,000 & 10,000 & Final PDWS (EPA, 1993a) \\
\hline Nitrite as nitrogen & $\mu g / L$ & 500 & 1,000 & Final PDWS (EPA, 1993a) \\
\hline m-Nitroaniline & $\mu g / L$ & 50 & 100 & EPA Method 8270 \\
\hline o-Nitroaniline & $\mu g / L$ & 50 & 100 & EPA Method 8270 \\
\hline p-Nitroaniline & $\mu \mathrm{g} / \mathrm{L}$ & 50 & 100 & EPA Method 8270 \\
\hline Nitrobenzene & $\mu \mathrm{g} / \mathrm{L}$ & 50 & 100 & EPA Method 8270 \\
\hline Nitrogen by Kjeldahl method & $\mu g / L$ & 500 & 1,000 & EPA Method 351.2 \\
\hline 2-Nitrophenol & $\mu g / L$ & 50 & 100 & EPA Method 8270 \\
\hline
\end{tabular}


Analyte

4-Nitrophenol

4-Nitroquinoline-1-oxide

N-Nitrosodi-n-butylamine

$\mathrm{N}$-Nitrosodiethylamine

$\mathrm{N}$-Nitrosodimethylamine

$\mathrm{N}$-Nitrosodiphenylamine

N-Nitrosodipropylamine

$\mathrm{N}$-Nitrosomethylethylamine

$\mathrm{N}$-Nitrosomorpholine

$\mathrm{N}$-Nitrosopiperidine

$\mathrm{N}$-Nitrosopyrrolidine

5-Nitro-o-toluidine

Nonvolatile beta

Octachlorodibenzo-p-dioxin

isomers

Octachlorodibenzo-p-furan

isomers

Odor

Oil \& Grease

Oxamyl ${ }^{b}$

Parathion

Parathion methyl

PCB 1016

PCB 1221

PCB 1232

PCB 1242

PCB 1248

PCB 1254

PCB 1260

PCB 1262

Pentachlorobenzene

Pentachlorodibenzo-p-dioxin isomers

1,2,3,7,8-PCDD

Pentachlorodibenzo-p-furan

isomers

1,2,3,7,8-PCDF

Pentachloroethane

Pentachloronitrobenzene

Pentachlorophenol

$\mathrm{pH}$

$\mathrm{pH}$

Phenacetin

Phenanthrene

Phenol

Phenols

p-Phenylenediamine

Phorate

Picloram ${ }^{b}$

2-Picoline

Pluțonium-238

Plutonium-239

Plutonium-239/240 ${ }^{\mathrm{e}}$

Plutonium-240

Plutonium-241 ${ }^{\mathrm{c}}$

\begin{tabular}{|c|c|c|c|}
\hline Unit & Flag 1 & Flag 2 & $\underline{\text { Source }}$ \\
\hline$g / L$ & 50 & 100 & EPA Method 8270 \\
\hline$g / L$ & 50 & 100 & EPA Method 8270 \\
\hline$g / L$ & 50 & 100 & EPA Method 8270 \\
\hline$g / L$ & 50 & 100 & EPA Method 8270 \\
\hline$g / L$ & 50 & 100 & EPA Method 8270 \\
\hline $\mathrm{g} / \mathrm{L}$ & 50 & 100 & EPA Method 8270 \\
\hline$g / L$ & 50 & 100 & EPA Method 8270 \\
\hline$g / L$ & 50 & 100 & EPA Method 8270 \\
\hline$g / L$ & 50 & 100 & EPA Method 8270 \\
\hline$g / L$ & 50 & 100 & EPA Method 8270 \\
\hline$\mu g / L$ & 50 & 100 & EPA Method 8270 \\
\hline$g / L$ & 50 & 100 & EPA Method 8270 \\
\hline $\mathrm{Ci} / \mathrm{L}$ & $2.5 \mathrm{E}+01$ & $5 \mathrm{E}+01$ & Interim Final PDWS (EPA, 1977) \\
\hline /L & 0.005 & 0.01 & EPA Method 8280 \\
\hline //L & 0.005 & 0.01 & EPA Method 8280 \\
\hline & No flag & No flag & Set by EPD/EMS \\
\hline /L & 5,000 & 10,000 & EPA Method 413.1 \\
\hline$g / L$ & 100 & 200 & Final PDWS (EPA, 1993a) \\
\hline$g / L$ & 0.25 & 0.5 & EPA Method 8080 \\
\hline$g / L$ & 0.25 & 0.5 & EPA Method 8080 \\
\hline $\mathrm{g} / \mathrm{L}$ & 0.25 & 0.5 & Final PDWS (EPA, 1993a) \\
\hline$g / L$ & 0.25 & 0.5 & Final PDWS (EPA, 1993a) \\
\hline$g / L$ & 0.25 & 0.5 & Final PDWS (EPA, 1993a) \\
\hline $3 / L$ & 0.25 & 0.5 & Final PDWS (EPA, 1993a) \\
\hline & 0.25 & 0.5 & Final PDWS (EPA, 1993a) \\
\hline 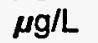 & 0.25 & 0.5 & Final PDWS (EPA, 1993a) \\
\hline 3/L & 0.25 & 0.5 & Final PDWS (EPA, 1993a) \\
\hline$g / L$ & 0.25 & 0.5 & Final PDWS (EPA, 1993a) \\
\hline & 50 & 100 & EPA Method 8270 \\
\hline - & 0.00275 & 0.0055 & EPA Method 8280 \\
\hline & 0.00275 & 0.0055 & EPA Method 8280 \\
\hline & 0.00275 & 0.0055 & EPA Method 8280 \\
\hline$\frac{g}{\mathrm{~L}} \mathrm{~L}$ & 0.00275 & 0.0055 & EPA Method 8280 \\
\hline 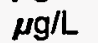 & 50 & 100 & EPA Method 8270 \\
\hline 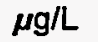 & 50 & 100 & EPA Method 8270 \\
\hline$g / L$ & 0.5 & 1 & Final PDWS (EPA, 1993a) \\
\hline $\mathrm{pH}$ & 8 & 10 & Set by EPD/EMS \\
\hline $\mathrm{pH}$ & 4 & 3 & Set by EPD/EMS \\
\hline$\mu \mathrm{g} / \mathrm{L}$ & 50 & 100 & EPA Method 8270 \\
\hline$g / L$ & 50 & 100 & EPA Method 8270 \\
\hline gl & 50 & 100 & EPA Method 8270 \\
\hline 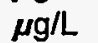 & 25 & 50 & EPA Method 420.1 \\
\hline & 50 & 100 & EPA Method 8270 \\
\hline & 0.5 & 1 & EPA Method 8080 \\
\hline & 250 & 500 & Final PDWS (EPA, 1993a) \\
\hline $3 / \mathrm{L}$ & 50 & 100 & EPA Method 8270 \\
\hline i/L & $3.51 E+00$ & $7.02 E+00$ & Proposed PDWS (EPA, 1991) \\
\hline & $3.11 E+01$ & $6.21 E+01$ & Proposed PDWS (EPA, 1991) \\
\hline & $3.11 E+01$ & $6.21 E+01$ & Proposed PDWS (EPA, 1991) \\
\hline Cil & $3.11 E+01$ & $6.22 E+01$ & Proposed PDWS (EPA, 1991) \\
\hline p & $3.13 E+01$ & $6.26 \mathrm{E}+01$ & Proposed PDWS (EPA, 1991) \\
\hline
\end{tabular}


Analyte

Plutonium-242 ${ }^{\mathrm{C}}$

Potassium

Potassium, dissolved

Potassium, total recoverable

Potassium-40

Promethium-144

Promethium-146

Promethium-147

Pronamid

Propionitrile

Pyrene

Pyridine

Radium (alpha-emitting) ${ }^{f}$

Radium-226

Radium-228

Radon-222

Ruthenium-103 ${ }^{\mathrm{C}}$

Ruthenium-106

Safrole

Selenium

Selenium, dissolved

Selenium, total recoverable

Silica

Silica, dissolved

Silica, total recoverable

Silver

Silver, dissolved

Silver, total recoverable

Simazine ${ }^{b}$

Sodium

Sodium, dissolved

Sodium, total recoverable

Sodium-22

Specific conductance

Strontium-89

Strontium-89/90 ${ }^{\mathrm{e}}$

Strontium-90

Styrene

Sulfate

Sulfide

Sulfotepp

Surfactants

2,3,7,8-TCDD

2,3,7,8-TCDF

Technetium-99

1,2,4,5-Tetrachlorobenzene

Tetrachlorodibenzo-p-dioxin isomers

Tetrachlorodibenzo-p-furan isomers

1,1,1,2-Tetrachloroethane

1,1,2,2-Tetrachloroethane

Tetrachloroethylene

2,3,4,6-Tetrachlorophenol

Thallium

\begin{tabular}{|c|c|c|c|}
\hline Unit & Flag 1 & Flag 2 & $\underline{\text { Source }}$ \\
\hline Ci/L & $3.27 E+01$ & $6.54 \mathrm{E}+01$ & Proposed PDWS (EPA, 1991) \\
\hline & No flag & No flag & Set by EPD/EMS \\
\hline & No flag & No flag & Set by EPD/EMS \\
\hline & No flag & No flag & Set by EPD/EMS \\
\hline Cill & $1.5 E+02$ & $3 E+02$ & Proposed PDWS (EPA, 1986) \\
\hline $\mathrm{Ci} / \mathrm{L}$ & $5 E+01$ & $1 E+02$ & EPA Method 901.1 \\
\hline $\mathrm{pCi} / \mathrm{L}$ & $5 E+01$ & $1 \mathrm{E}+02$ & EPA Method 901.1 \\
\hline Ci/L & $2.62 E+03$ & $5.24 E+03$ & Proposed PDWS (EPA, 1991) \\
\hline$g / L$ & 50 & 100 & EPA Method 8270 \\
\hline$\mu \mathrm{g} / \mathrm{L}$ & 1,000 & 2,000 & EPA Method 8240 \\
\hline$\mu \mathrm{g} / \mathrm{L}$ & 50 & 100 & EPA Method 8270 \\
\hline$\mu \mathrm{g} / \mathrm{L}$ & 50 & 100 & EPA Method 8270 \\
\hline $\mathrm{pCi} / \mathrm{L}$ & $1 E+01$ & $2 E+01$ & Proposed PDWS (EPA, 1991) \\
\hline $\mathrm{pCi} / \mathrm{L}$ & $1 E+01$ & $2 E+01$ & Proposed PDWS (EPA, 1991) \\
\hline $\mathrm{pCi} / \mathrm{L}$ & $1 E+01$ & $2 \mathrm{E}+01$ & Proposed PDWS (EPA, 1991) \\
\hline $\mathrm{pCi} / \mathrm{L}$ & $1.5 \mathrm{E}+02$ & $3 E+02$ & Proposed PDWS (EPA, 1991) \\
\hline $\mathrm{pCi} / \mathrm{L}$ & $1 \mathrm{E}+02$ & $2 E+02$ & Interim Final PDWS (EPA, 1977) \\
\hline $\mathrm{pCi} / \mathrm{L}$ & $1.5 \mathrm{E}+01$ & $3 E+01$ & Interim Final PDWS (EPA, 1977) \\
\hline$\mu g / L$ & 50 & 100 & EPA Method 8270 \\
\hline$\mu \mathrm{g} / \mathrm{L}$ & 25 & 50 & Final PDWS (EPA, 1993a) \\
\hline$\mu \mathrm{g} / \mathrm{L}$ & 25 & 50 & Final PDWS (EPA, 1993a) \\
\hline$\mu \mathrm{g} / \mathrm{L}$ & 25 & 50 & Final PDWS (EPA, 1993a) \\
\hline & No flag & No flag & Set by EPD/EMS \\
\hline & No flag & No flag & Set by EPD/EMS \\
\hline & No flag & No flag & Set by EPD/EMS \\
\hline & 50 & 100 & SDWS (EPA, 1993b) \\
\hline$/ \mathrm{L}$ & 50 & 100 & SDWS (EPA, 1993b) \\
\hline /L & 50 & 100 & SDWS (EPA, 1993b) \\
\hline$\mu \mathrm{g} / \mathrm{L}$ & 2 & 4 & Final PDWS (EPA, 1993a) \\
\hline & No flag & No flag & Set by EPD/EMS \\
\hline & No flag & No flag & Set by EPD/EMS \\
\hline & No flag & No flag & Set by EPD/EMS \\
\hline $\mathrm{i} / \mathrm{L}$ & $2.33 E+02$ & $4.66 E+02$ & Proposed PDWS (EPA, 1991) \\
\hline$\mu \mathrm{S} / \mathrm{cm}$ & 250 & 500 & Set by EPD/EMS \\
\hline $\mathrm{pCi} / \mathrm{L}$ & $1 \mathrm{E}+01$ & $2 E+01$ & Interim Final PDWS (EPA, 1977) \\
\hline $\mathrm{pCi} / \mathrm{L}$ & $4 \mathrm{E}+00$ & $8 \mathrm{E}+00$ & Final PDWS (EPA, 1993a) \\
\hline $\mathrm{pCi} / \mathrm{L}$ & $4 \mathrm{E}+00$ & $8 \mathrm{E}+00$ & Final PDWS (EPA, 1993a) \\
\hline & & 100 & Final PDWS (EPA, 1993a) \\
\hline$\mu \mathrm{g} / \mathrm{L}$ & 200,000 & 400,000 & Proposed PDWS (EPA, 1990) \\
\hline$\mu \mathrm{s}$ & 5,000 & 10,000 & EPA Method 9030 \\
\hline & 50 & 100 & EPA Method 8270 \\
\hline & No flag & No flag & Set by EPD/EMS \\
\hline & 0.000015 & 0.00003 & Final PDWS (EPA, 1993a) \\
\hline & 0.002 & 0.004 & EPA Method 8280 \\
\hline pCill & $4.5 E+02$ & $9 E+02$ & Interim Final PDWS (EPA, 1977) \\
\hline$\mu \mathrm{g} / \mathrm{L}$ & & 100 & EPA Method 8270 \\
\hline & 0.00225 & 0.0045 & EPA Method 8280 \\
\hline & 0.002 & 0.004 & EPA Method 8280 \\
\hline$\mu \mathrm{g} / \mathrm{l}$ & 5 & 10 & EPA Method 8240 \\
\hline$\mu \mathrm{g} /$ & 5 & 10 & EPA Method 8240 \\
\hline & 2.5 & 5 & Final PDWS (EPA, 1993a) \\
\hline & 50 & 100 & EPA Method 8270 \\
\hline & 1 & 2 & Final PDWS (EPA, 1993a) \\
\hline
\end{tabular}


Analyte

Thallium, dissolved

Thallium, total recoverable

Thionazin

Thorium-228

Thorium-230

Thorium-232

Thorium-234

Tin

Tin, dissolved

Tin, total recoverable

Tin-113

Toluene

o-Toluidine

Total carbon

Total coliform

Total dissolved solids

Total hydrocarbons

Total inorganic carbon

Total organic carbon

Total organic halogens

Total organic nitrogen

Total petroleum hydrocarbons

Total phosphates (as P)

Total phosphorus

Toxaphene

2,4,5-TP (Silvex)

Tributyl phosphate

1,2,4-Trichlorobenzene

1,1,1-Trichloroethane

1,1,2-Trichloroethane

Trichloroethylene

Trichlorofluoromethane

2,4,5-Trichlorophenol

2,4,6-Trichlorophenol

2,4,5-Trichlorophenoxyacetic acid

1,2,3-Trichloropropane

$0,0,0$-Triethyl phosphorothioate

1,3,5-Trinitrobenzene

Tritium

Turbidity ${ }^{g}$

Uranium

Uranium, dissolved

Uranium, total recoverable

Uranium alpha activity

Uranium-233/234

Uranium-234

Uranium-235

Uranium-238

Vanadium

Vanadium, dissolved

Vanadium, total recoverable

Vinyl acetate

\begin{tabular}{|c|c|c|}
\hline Unit & Flag 1 & Flag 2 \\
\hline$\mu g / L$ & 1 & 2 \\
\hline$\mu \mathrm{g} / \mathrm{L}$ & 1 & 2 \\
\hline$\mu g / L$ & 50 & 100 \\
\hline $\mathrm{pCi} / \mathrm{L}$ & $6.25 E+01$ & $1.25 E+02$ \\
\hline pCill & $3.96 E \div 01$ & $7.92 E+01$ \\
\hline $\mathrm{pCi} / \mathrm{L}$ & $4.4 E+01$ & 8.8E+01 \\
\hline $\mathrm{pCi} / \mathrm{L}$ & $2 E+02$ & $4.01 E+02$ \\
\hline$\mu \mathrm{g} / \mathrm{L}$ & 10 & 20 \\
\hline$\mu g / L$ & 10 & 20 \\
\hline$\mu \mathrm{g} / \mathrm{L}$ & 10 & 20 \\
\hline $\mathrm{pCi} / \mathrm{L}$ & $1.5 E+02$ & $3 E+02$ \\
\hline$\mu \mathrm{g} / \mathrm{L}$ & 500 & 1,000 \\
\hline$\mu g / L$ & 50 & 100 \\
\hline$\mu \mathrm{g} / \mathrm{L}$ & $\begin{array}{l}5,000 \\
0\end{array}$ & $\begin{array}{l}10,000 \\
0\end{array}$ \\
\hline & No flag & No flag \\
\hline$\mu g / L$ & 5,000 & 10,000 \\
\hline$\mu g / L$ & 5,000 & 10,000 \\
\hline$\mu g / L$ & 5,000 & 10,000 \\
\hline$\mu \mathrm{g} / \mathrm{L}$ & 25 & 50 \\
\hline$\mu g / L$ & 500 & 1,000 \\
\hline$\mu g / L$ & 5,000 & 10,000 \\
\hline & No flag & No flag \\
\hline & No flag & No flag \\
\hline$\mu g / L$ & 1.5 & 3 \\
\hline$\mu \mathrm{g} / \mathrm{L}$ & 25 & 50 \\
\hline$\mu g / L$ & 50 & 100 \\
\hline$\mu \mathrm{g} / \mathrm{L}$ & 35 & 70 \\
\hline$\mu \mathrm{g} / \mathrm{L}$ & 100 & 200 \\
\hline$\mu g / L$ & 2.5 & 5 \\
\hline$\mu g / L$ & 2.5 & 5 \\
\hline$\mu g / L$ & 5 & 10 \\
\hline$\mu \mathrm{g} / \mathrm{L}$ & 50 & 100 \\
\hline$\mu \mathrm{g} / \mathrm{L}$ & 50 & 100 \\
\hline$\mu g / L$ & 2.5 & 5 \\
\hline$\mu \mathrm{g} / \mathrm{L}$ & 5 & 10 \\
\hline$\mu \mathrm{g} / \mathrm{L}$ & 50 & 100 \\
\hline$\mu \mathrm{g} / \mathrm{L}$ & 50 & 100 \\
\hline $\mathrm{pCi} / \mathrm{mL}$ & $1 E+01$ & $2 E+01$ \\
\hline & No flag & No flag \\
\hline$\mu g / L$ & 10 & 20 \\
\hline$\mu \mathrm{g} / \mathrm{L}$ & 10 & 20 \\
\hline$\mu g / L$ & 10 & 20 \\
\hline $\mathrm{pCi} / \mathrm{L}$ & $1.5 E+01$ & $3 E+01$ \\
\hline $\mathrm{pCi} / \mathrm{L}$ & $6.9 E+00$ & $1.38 E \div 01$ \\
\hline $\mathrm{pCi} / L$ & $6.95 E+00$ & 1.39E +01 \\
\hline $\mathrm{pCi} / \mathrm{L}$ & $7.25 E+00$ & $1.45 E+01$ \\
\hline $\mathrm{pCi} / \mathrm{L}$ & 7.3E+00 & $1.46 E+01$ \\
\hline$\mu g / L$ & 40 & 80 \\
\hline$\mu g / L$ & 40 & 80 \\
\hline$\mu g / L$ & 40 & 80 \\
\hline$\mu \mathrm{g} / \mathrm{L}$ & 5 & 10 \\
\hline
\end{tabular}

Source

Final PDWS (EPA, 1993a)

Final PDWS (EPA, 1993a)

EPA Method 8270

Proposed PDWS (EPA, 1991)

Proposed PDWS (EPA, 1991)

Proposed PDWS (EPA, 1991)

Proposed PDWS (EPA, 1991)

EPA Method 282.2

EPA Method 282.2

EPA Method 282.2

Interim Final PDWS (EPA, 1977)

Final PDWS (EPA, 1993a)

EPA Method 8270

EPA Method 9060

Final PDWS (EPA, 1993a)

Set by EPD/EMS

EPA Method 418.1

EPA Method 9060

EPA Method 9060

EPA Method 9020

APHA Method 420

EPA Method 418.1

Set by EPD/EMS

Set by EPD/EMS

Final PDWS (EPA, 1993a)

Final PDWS (EPA, 1993a)

EPA Method 8270

Final PDWS (EPA, 1993a)

Final PDWS (EPA, 1993a)

Final PDWS (EPA, 1993a)

Final PDWS (EPA, 1993a)

EPA Method 8240

EPA Method 8270

EPA Method 8270

EPA Method 8150

EPA Method 8240

EPA Method 8270

EPA Method 8270

Final PDWS (EPA, 1993a)

Set by EPD/EMS

Proposed PDWS (EPA, 1991)

Proposed PDWS (EPA, 1991)

Proposed PDWS (EPA, 1991)

Proposed PDWS (EPA, 1991)

Proposed PDWS (EPA, 1991)

Proposed PDWS (EPA, 1991)

Proposed PDWS (EPA, 1991)

Proposed PDWS (EPA, 1991)

EPA Method 6010

EPA Method 6010

EPA Method 6010

EPA Method 8240 


\begin{tabular}{|c|c|c|c|c|}
\hline Analyte & Unit & Flag 1 & Flag 2 & Source \\
\hline Xylenes & $\mu g / L$ & 5,000 & 10,000 & Final PDWS (EPA, 1993a) \\
\hline Yttrium-88 & $\mathrm{pCi} / \mathrm{L}$ & $5 E \div 01$ & $1 E+02$ & EPA Method 901.1 \\
\hline Zinc & $\mu \mathrm{g} / \mathrm{L}$ & 2,500 & 5,000 & SDWS (EPA, 1993b) \\
\hline Zinc, dissolved & $\mu g / L$ & 2,500 & 5,000 & SDWS (EPA, 1993b) \\
\hline Zinc, total recoverable & $\mu \mathrm{g} / \mathrm{L}$ & 2,500 & 5,000 & SDWS (EPA, 1993b) \\
\hline Zinc-65 & $\mathrm{pCi} / \mathrm{L}$ & $1.5 E+02$ & $3 E+02$ & Interim Final PDWS (EPA, 1977) \\
\hline Zirconium-95 & $\mathrm{pCi} / \mathrm{L}$ & $1 E+02$ & $2 E+02$ & Interim Final PDWS (EPA, 1977) \\
\hline Zirconium/Niobium-95 ${ }^{c}$ & $\mathrm{pCi} / \mathrm{L}$ & $1 E+02$ & $2 E \div 02$ & Interim Final PDWS (EPA, 1977) \\
\hline
\end{tabular}

a References for methods are in Appendix E; references for dated sources are at the end of this appendix.

b EMS is currently unable to perform this analysis.

c EMS discontinued monitoring this radionuclide because it is inappropriate for the SRS Groundwater Monitoring Program.

d EPD/EMS set this flagging criterion using the 1991 proposed PDWS because the final PDWS in 1977 may have been in error.

e For double radionuclide analyses where each separate radionuclide has its own standard, the more

stringent standard is used.

f The applied standard is for radium-226.

9 The primary maximum contaminant level range for turbidity is $1-5$ NTU, which is inappropriate for the SRS Groundwater Monitoring Program.

\section{References Cited}

EPA (U.S. Environmental Protection Agency), 1977. National Interim Primary Drinking Water Regulations, EPA-570/9-76-003. Washington, DC.

EPA (U.S. Environmental Protection Agency), 1986. Water Pollution Control; National Primary Drinking Water Regulations, Radionuclides (Proposed). Federal Register, September 30, 1986, pp. 34835-34862. Washington, DC.

EPA (U.S. Environmental Protection Agency), 1990. National Primary and Secondary Drinking Water Regulations; Synthetic Organic Chemicals and Inorganic Chemicals (Proposed Rule). Federal Register, July 25, 1990, pp. 30369-30448. Washington, DC.

EPA (U.S. Environmental Protection Agency), 1991. National Primary Drinking Water Regulations; Radionuclides; Proposed Rule. Federal Register, July 18, 1991, pp. 33052-33127. Washington, DC.

EPA (U.S. Environmental Protection Agency), 1993a. National Primary Drinking Water Regulations. Code of Federal Regulations, Title 40, Part 141, pp. 592-732. Washington, DC.

EPA (U.S. Environmental Protection Agency), 1993b. National Secondary Drinking Water Regulations. Code of Federal Regulations, Title 40, Part 143, pp. 774-777. Washington, DC.

SCDHEC (South Carolina Department of Health and Environmental Control), 1981. State Primary Drinking Water Regulations, R.61-58.5. Columbia, SC. 


\section{Appendix C}

Figures 
WSRC-TR-94-0610

Unclassified

THIS PAGE LEFT BLANK INTENTIONALLY. 


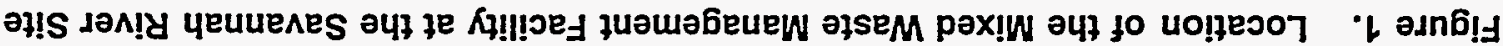

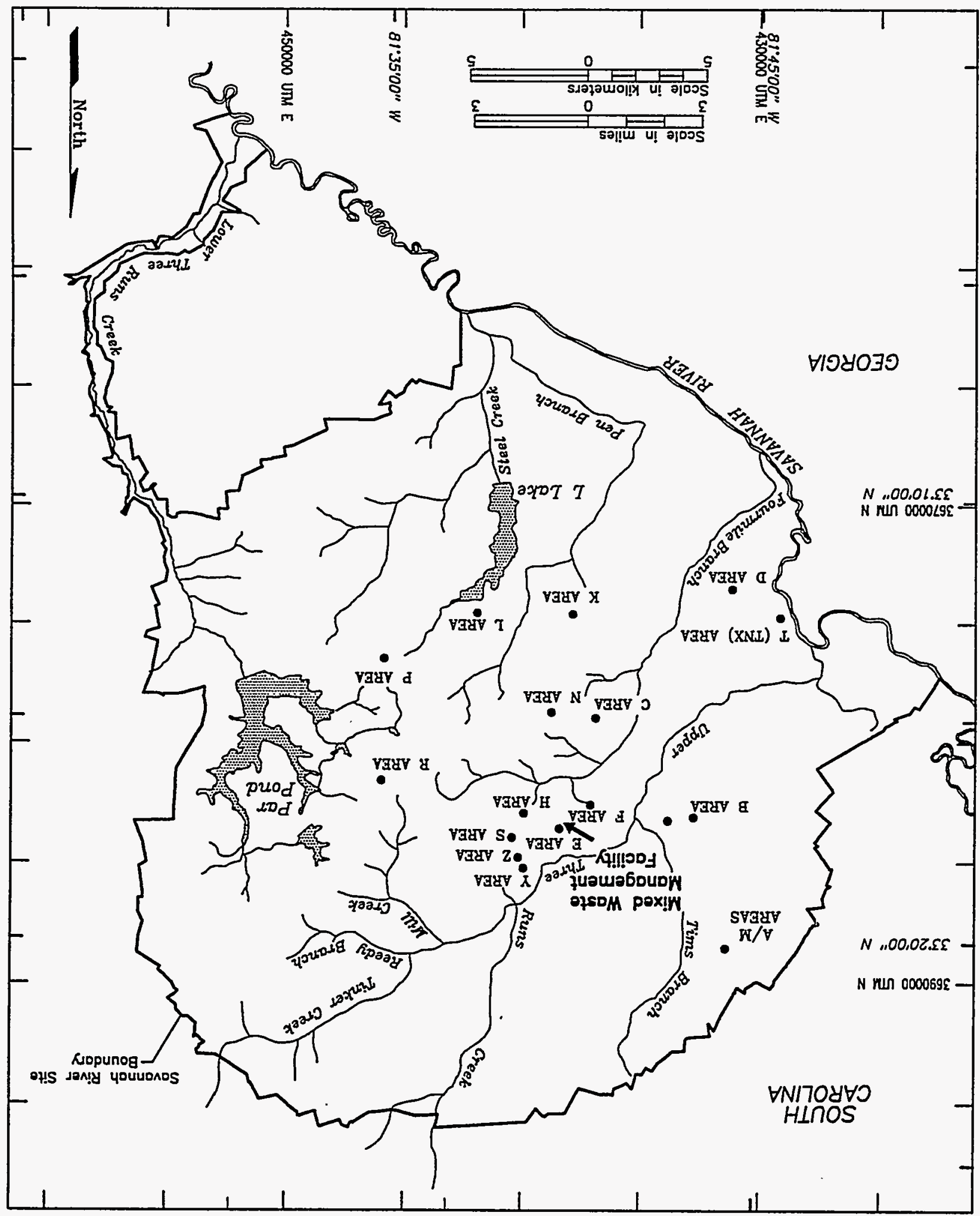




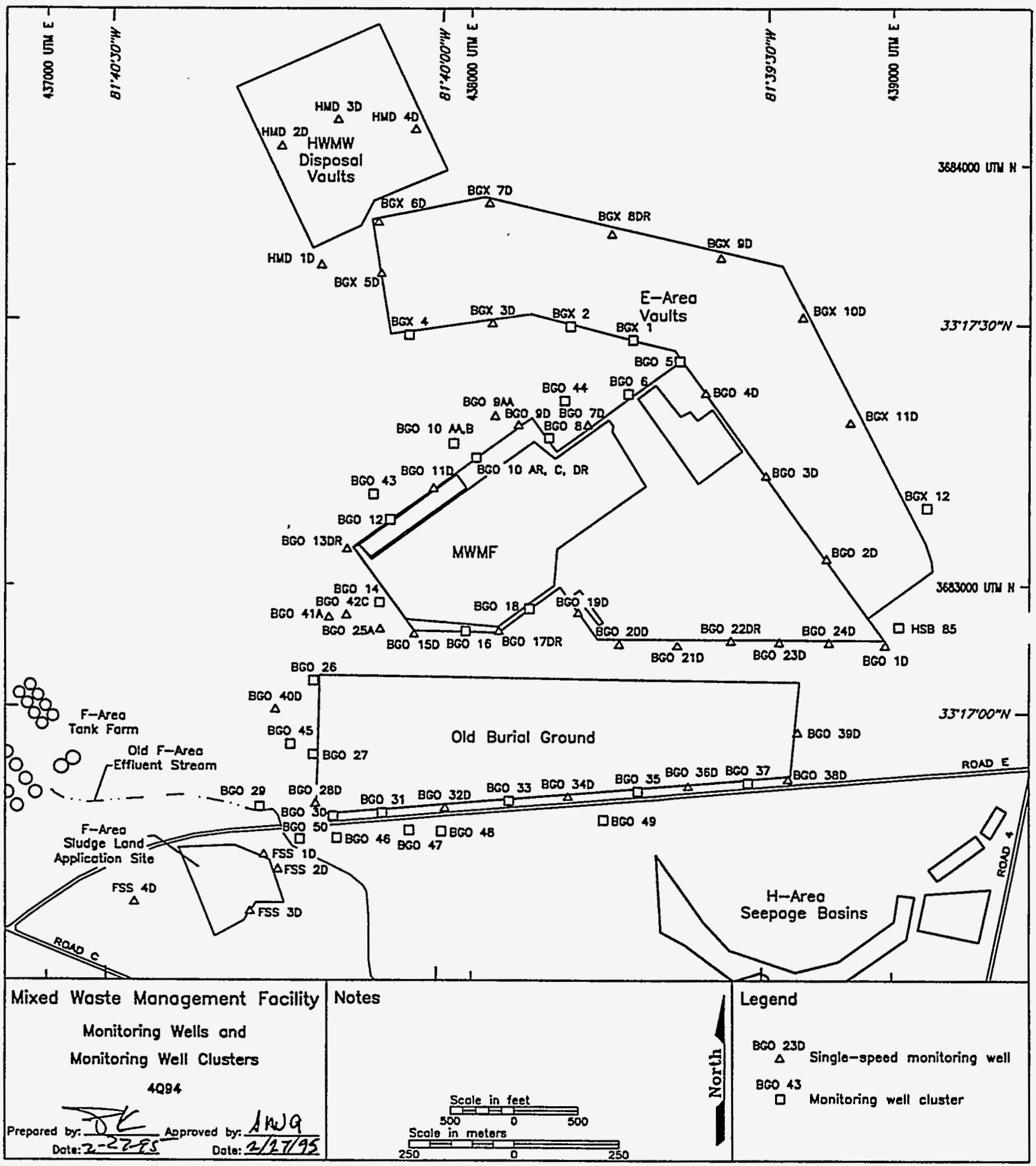

Figure 2. Location of the Groundwater Monitoring Wells at the Mixed Waste Management Facility, the Old Burial Ground, the E-Area Vaults, the Hazardous Waste/Mixed Waste Disposal Vaults (the Burial Ground Complex), and the F-Area Sewage Sludge Application Site 


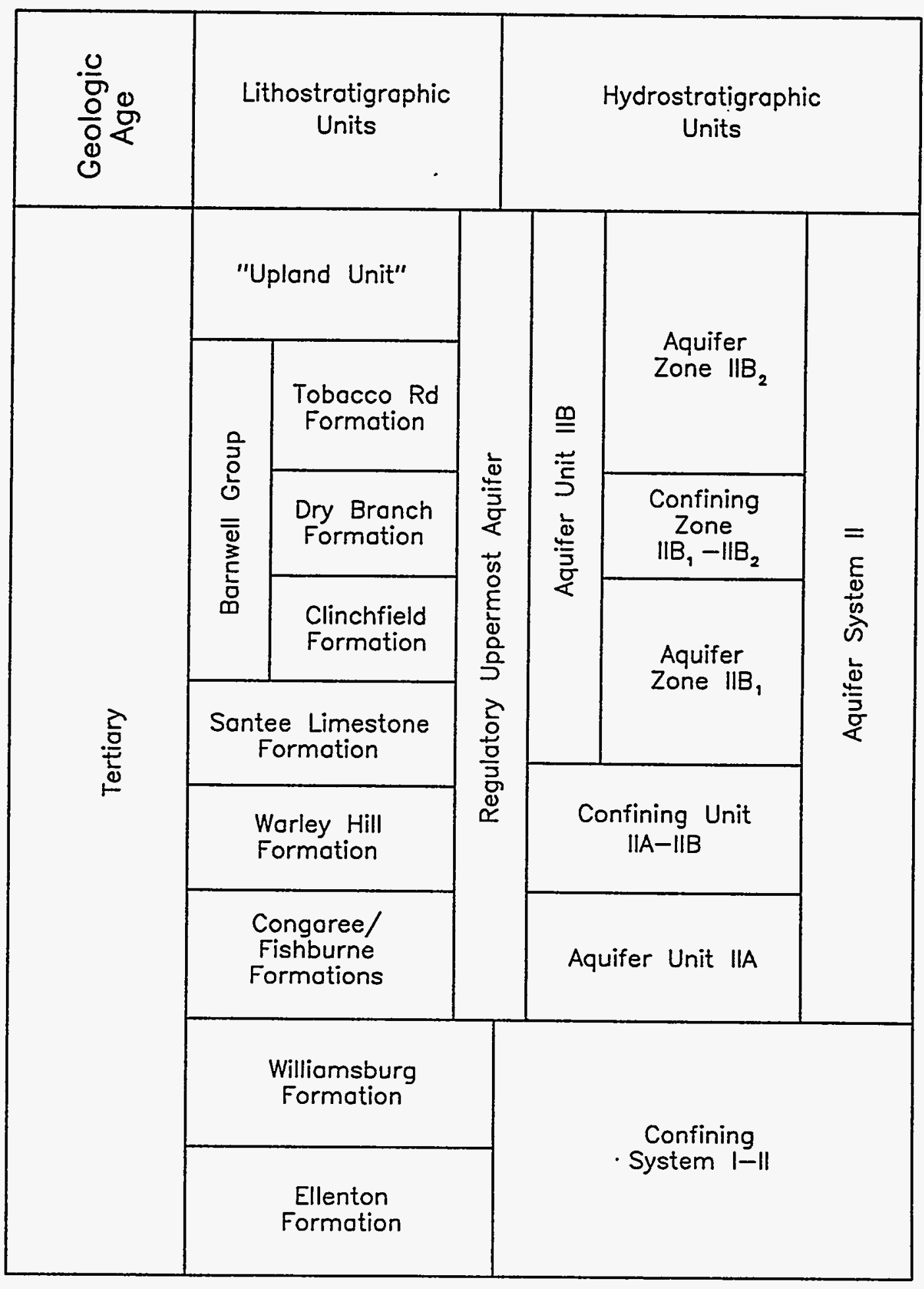

Figure 3. Hydrostratigraphic Nomenclature 


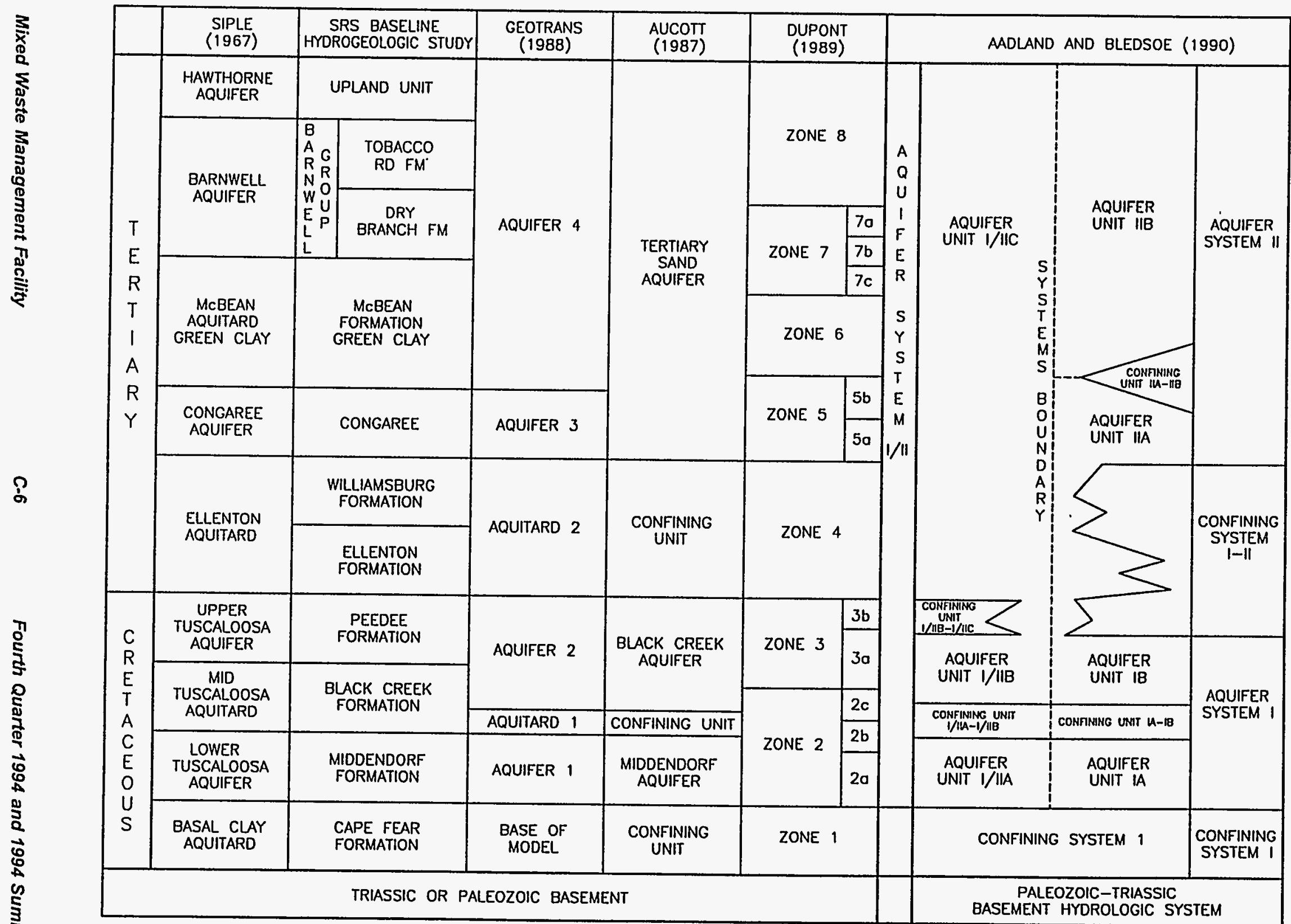

Figure 4. Regional Correlation of Hydrostratigraphic and Lithostratigraphic Nomenclature 


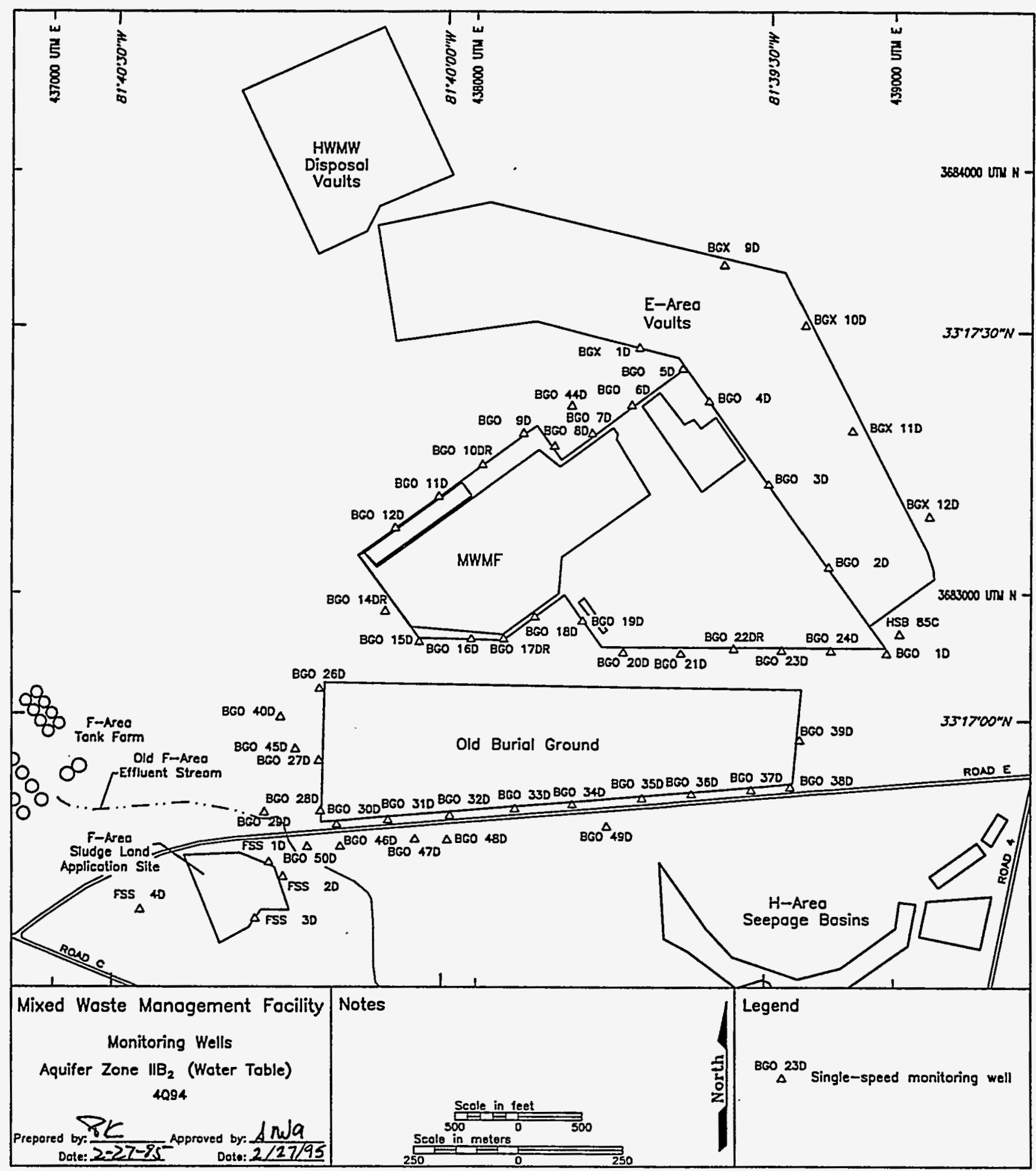

Figure 5. Location of Aquifer Zone $\|_{2} B_{2}$ (Water Table) Wells at the Burial Ground Complex 


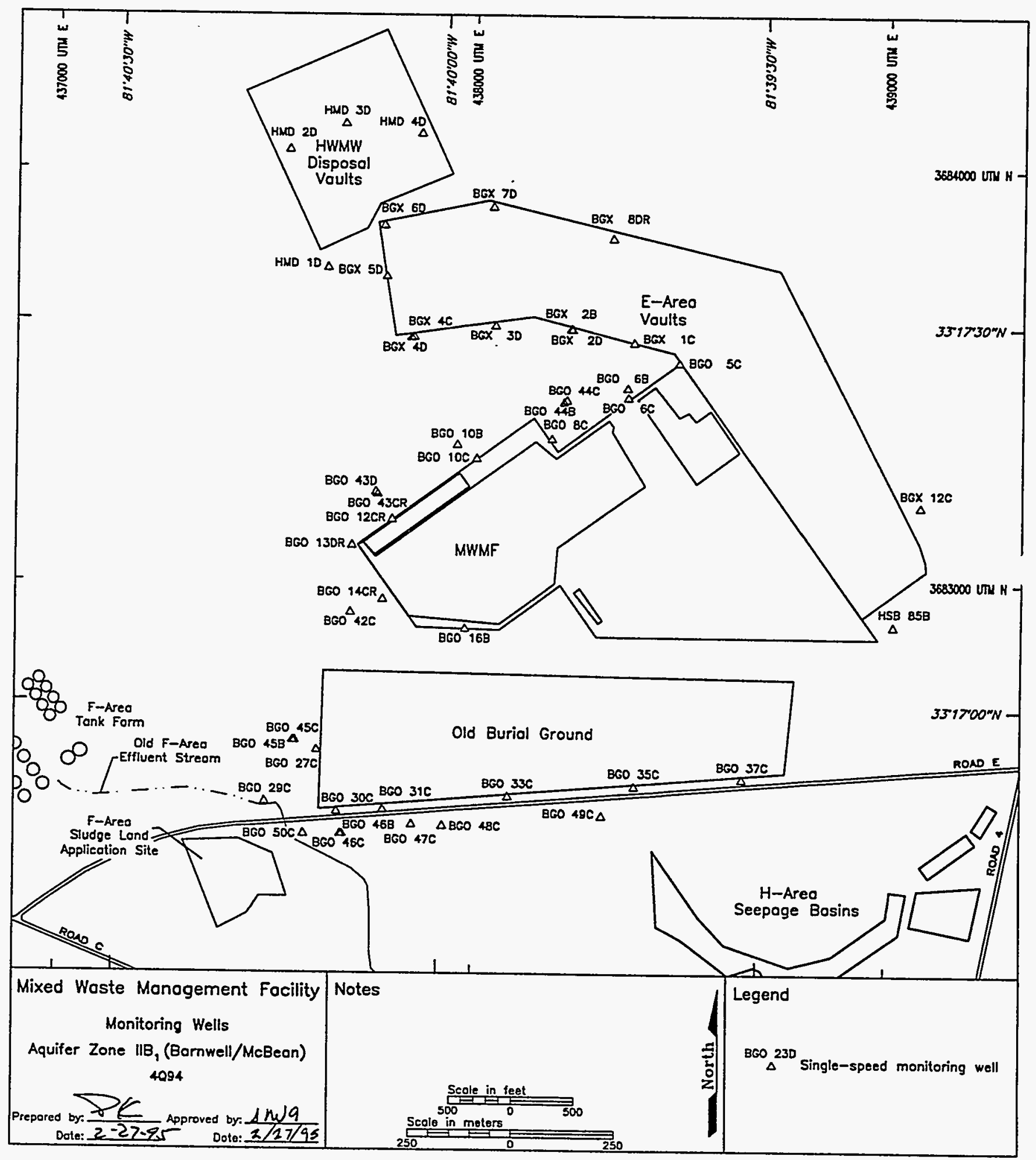

Figure 6. Location of Aquifer Zone IIB, (Barnwell/McBean) Wells at the Burial Ground Complex 


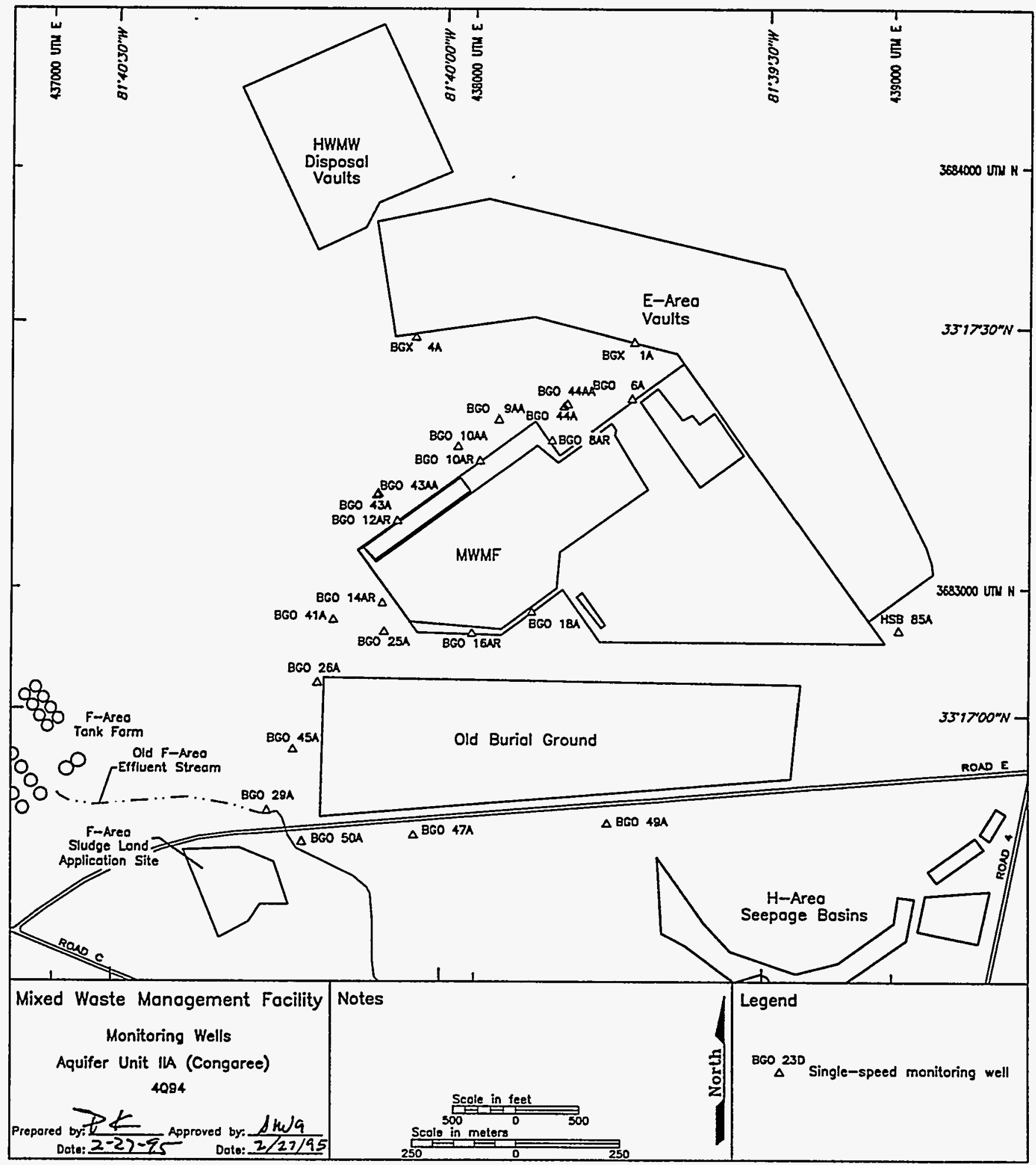

Figure 7. Location of Aquifer Unit IIA (Congaree) Wells at the Burial Ground Complex 


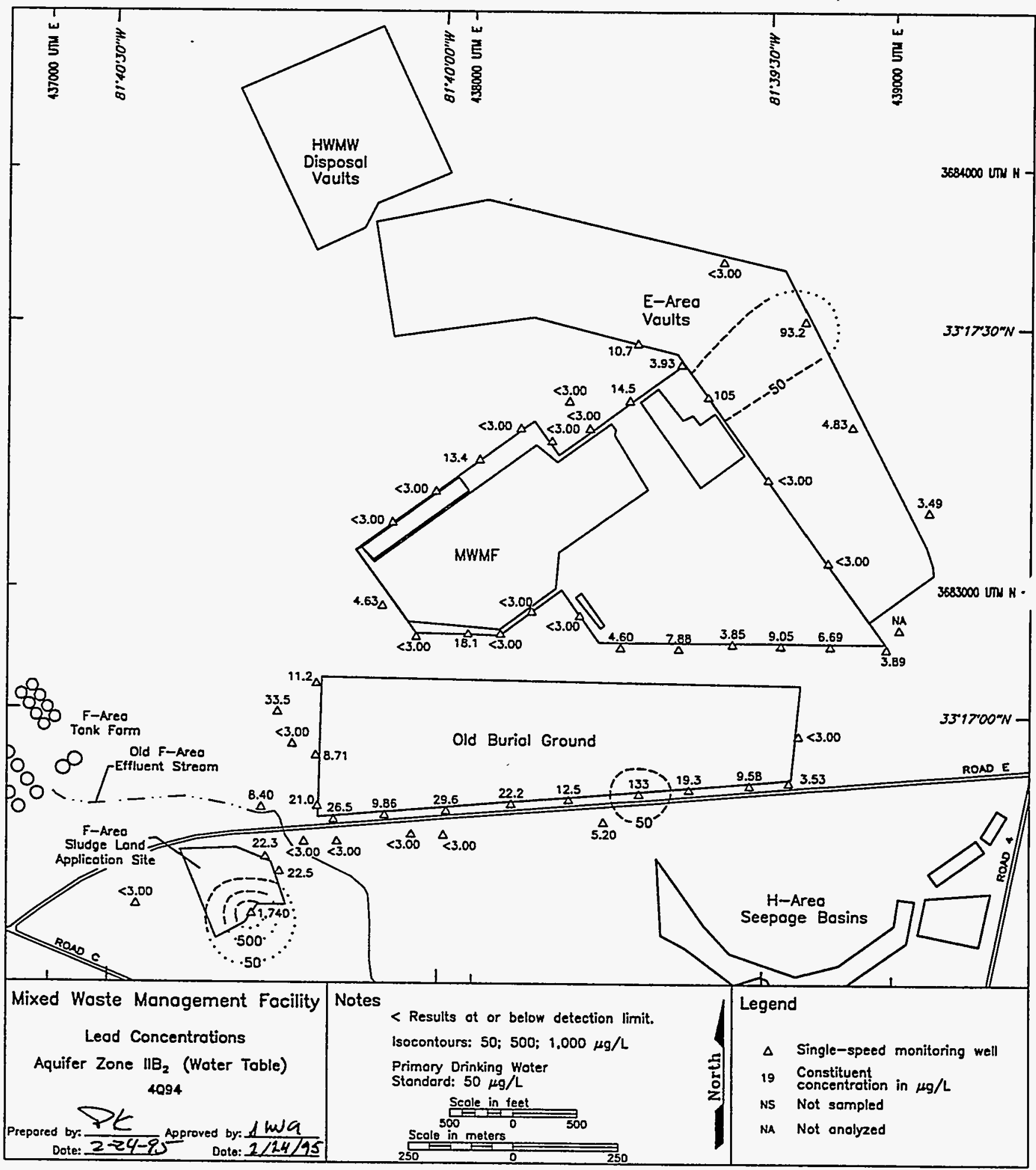

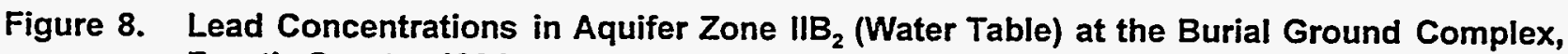
Fourth Quarter 1994 


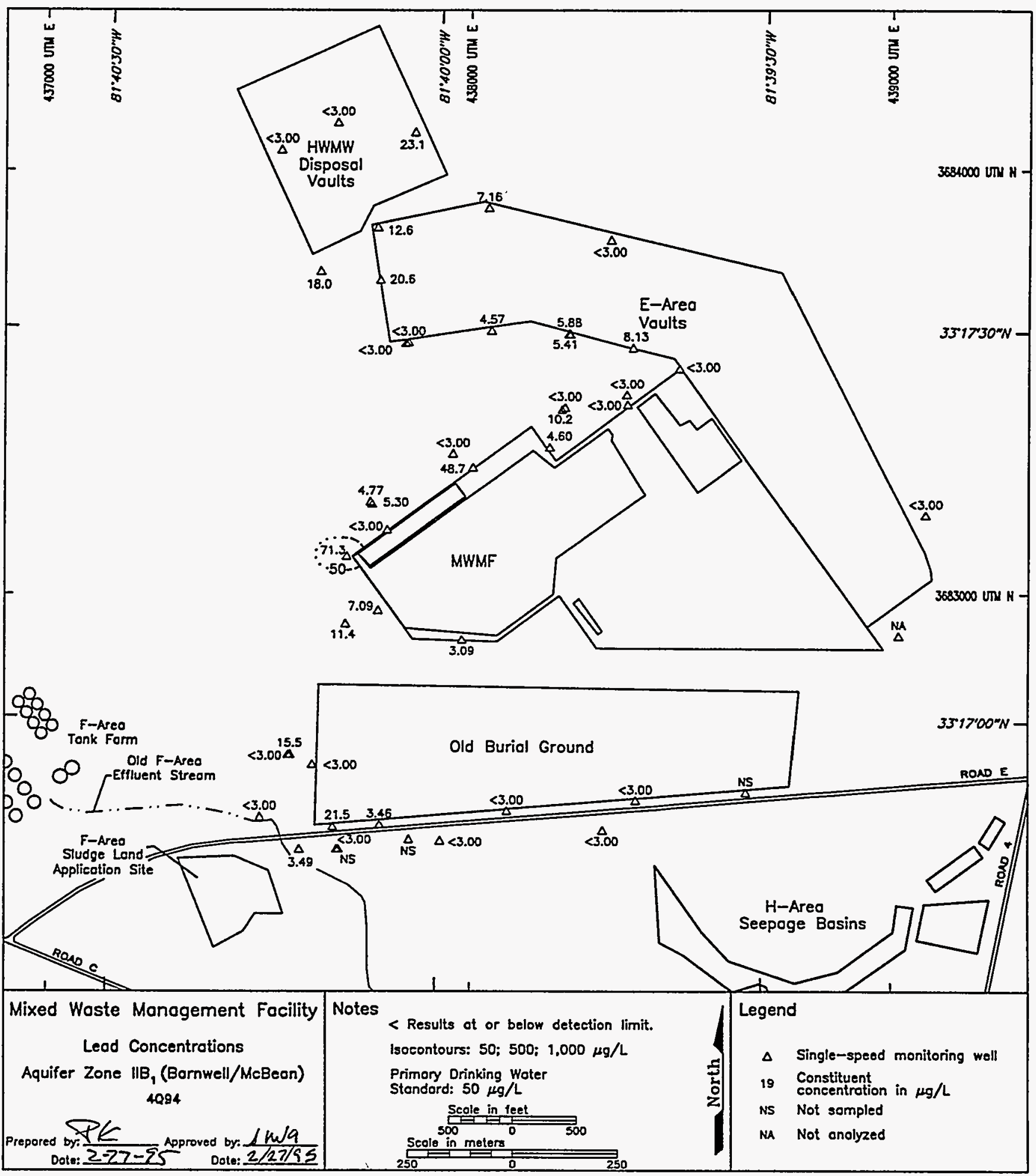

Figure 9. Lead Concentrations in Aquifer Zone $\|_{1} B_{1}$ (Barnwell/McBean) at the Burial Ground Complex, Fourth Quarter 1994 


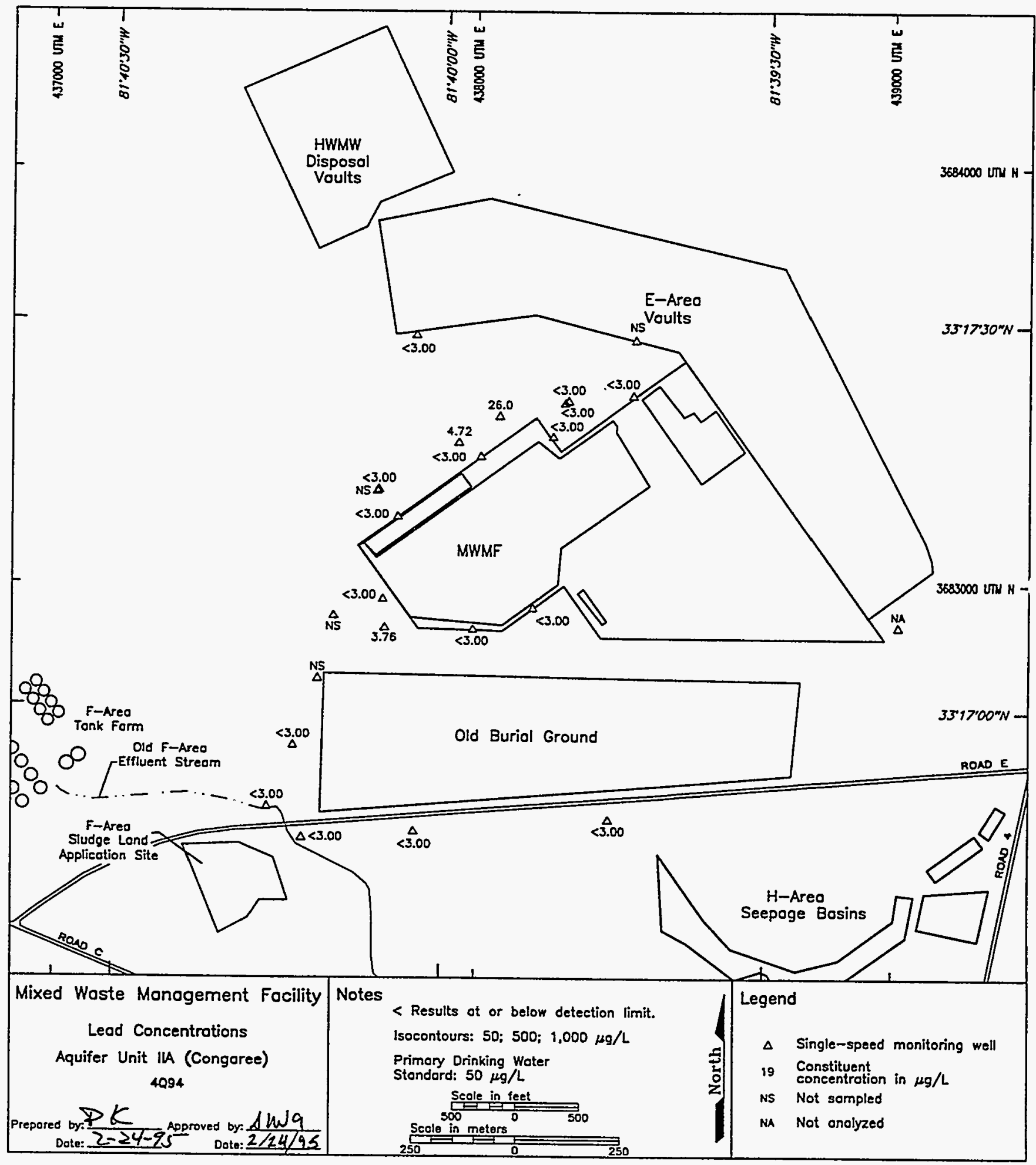

Figure 10. Lead Concentrations in Aquifer Unit IIA (Congaree) at the Burial Ground Complex, Fourth Quarter 1994 


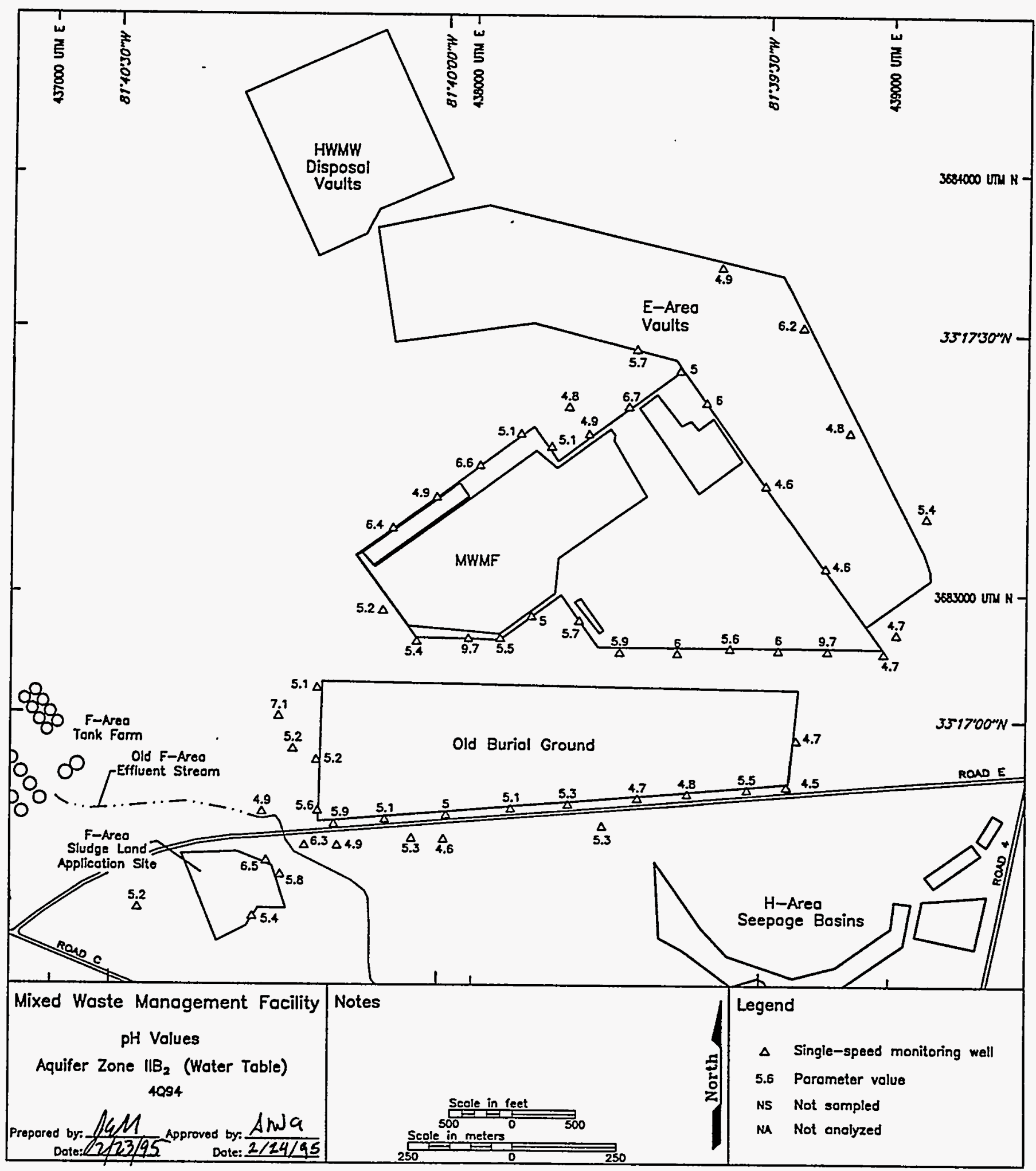

Figure 11. $\mathrm{pH}$ Values in Aquifer Zone $\mathrm{IB}_{2}$ (Water Table) at the Burial Ground Complex, Fourth Quarter 1994 


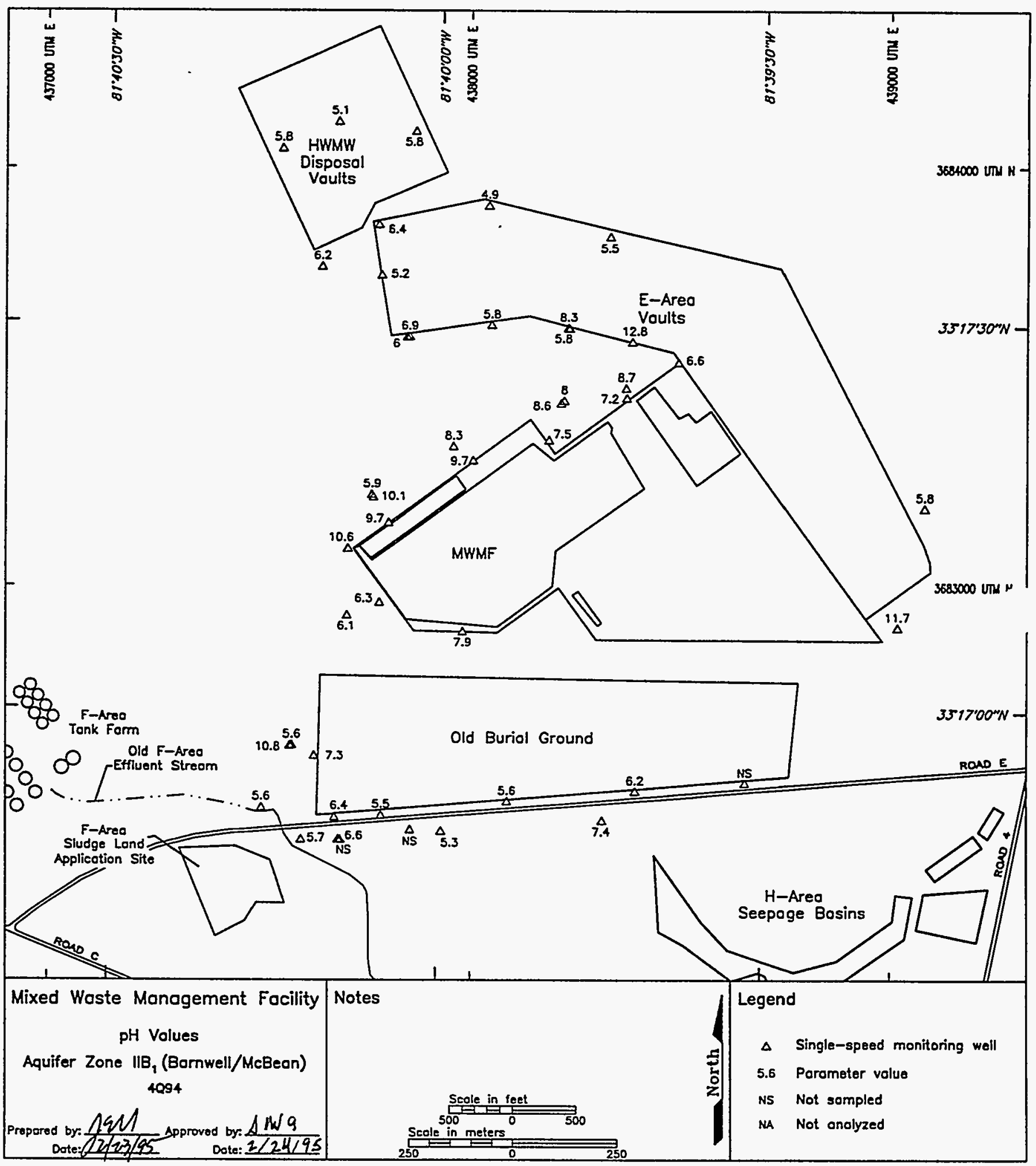

Figure 12. $\mathrm{pH}$ Values in Aquifer Zone $\|_{1} \mathrm{~B}_{1}$ (Barnwell/McBean) at the Burial Ground Complex, Fourth Quarter 1994 


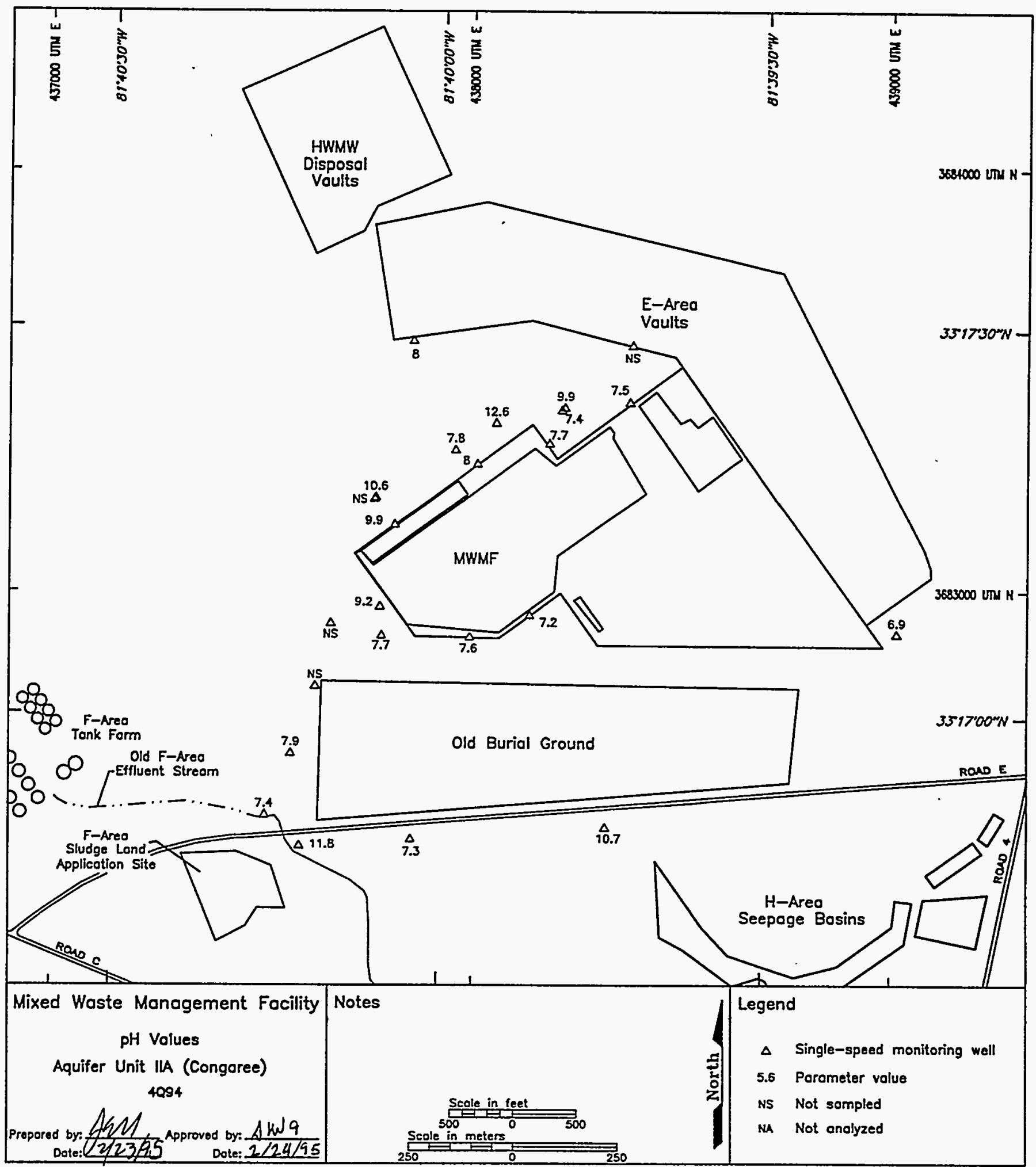

Figure 13. $\mathrm{pH}$ Values in Aquifer Unit IIA (Congaree) at the Burial Ground Complex, Fourth Quarter 1994 


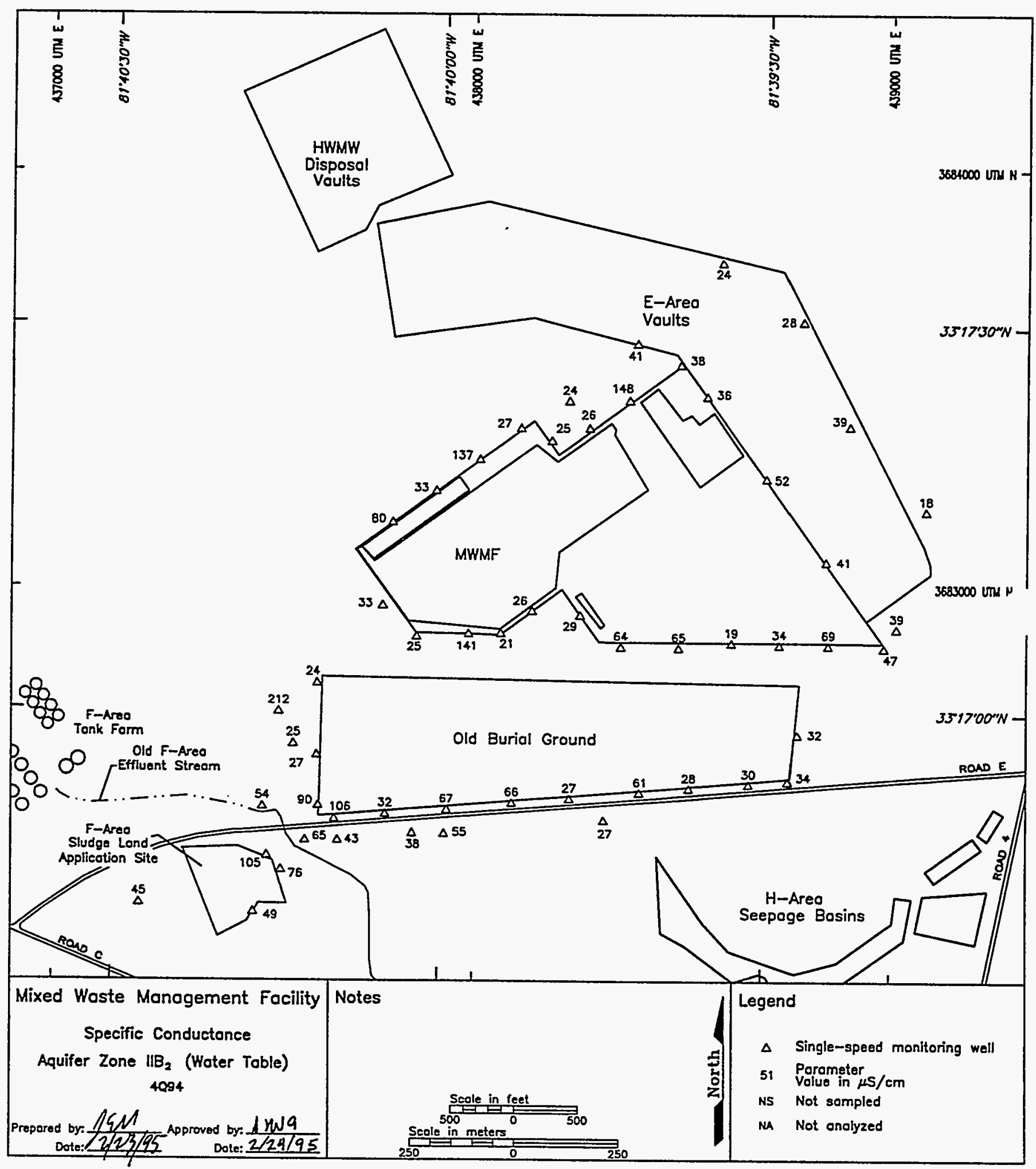

Figure 14. Specific Conductance in Aquifer Zone IIB $_{2}$ (Water Table) at the Burial Ground Complex, Fourth Quarter 1994 


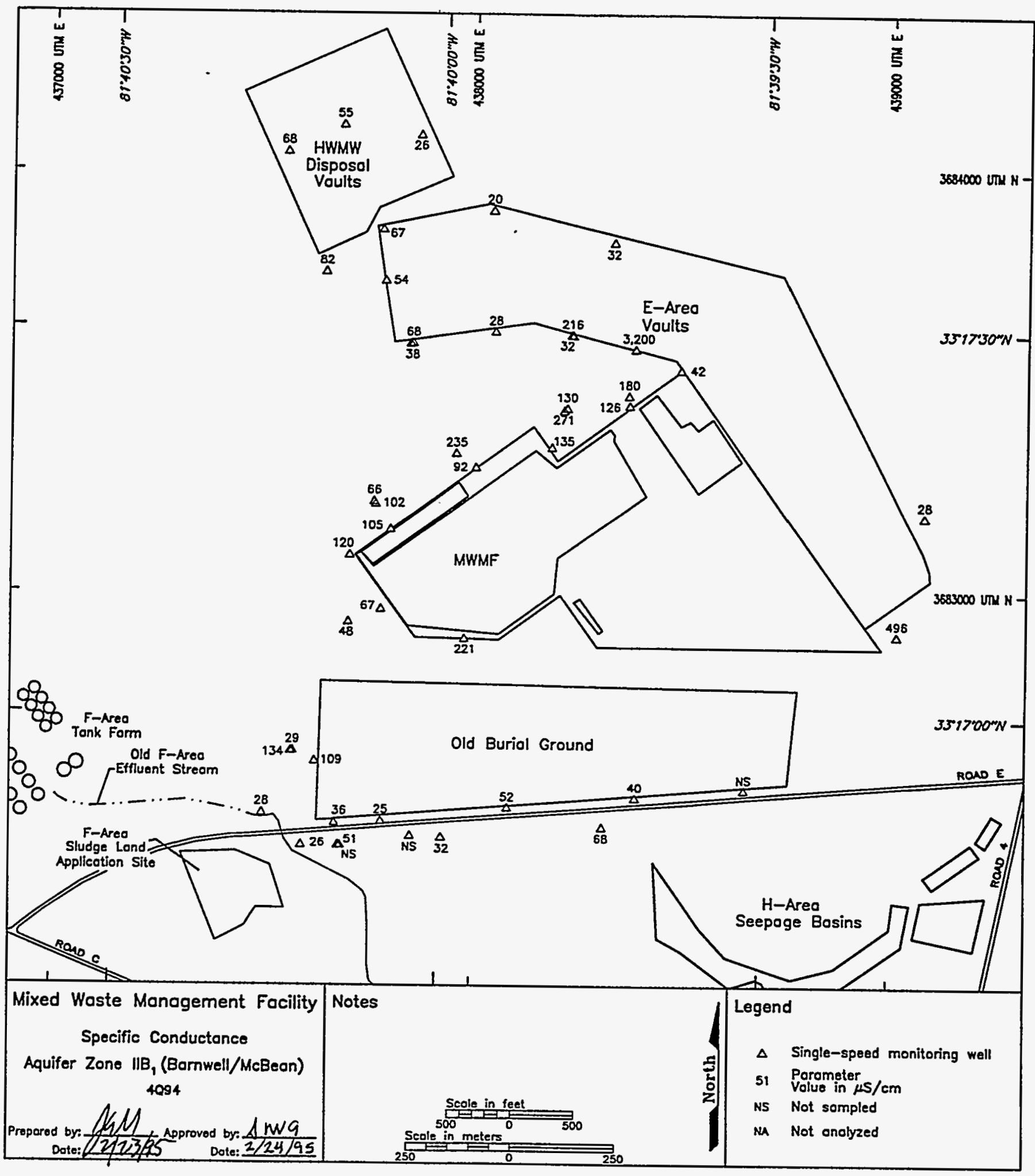

Figure 15. Specific Conductance in Aquifer Zone $\|_{1} \mathrm{~B}_{1}$ (Barnwell/McBean) at the Burial Ground Complex, Fourth Quarter 1994 


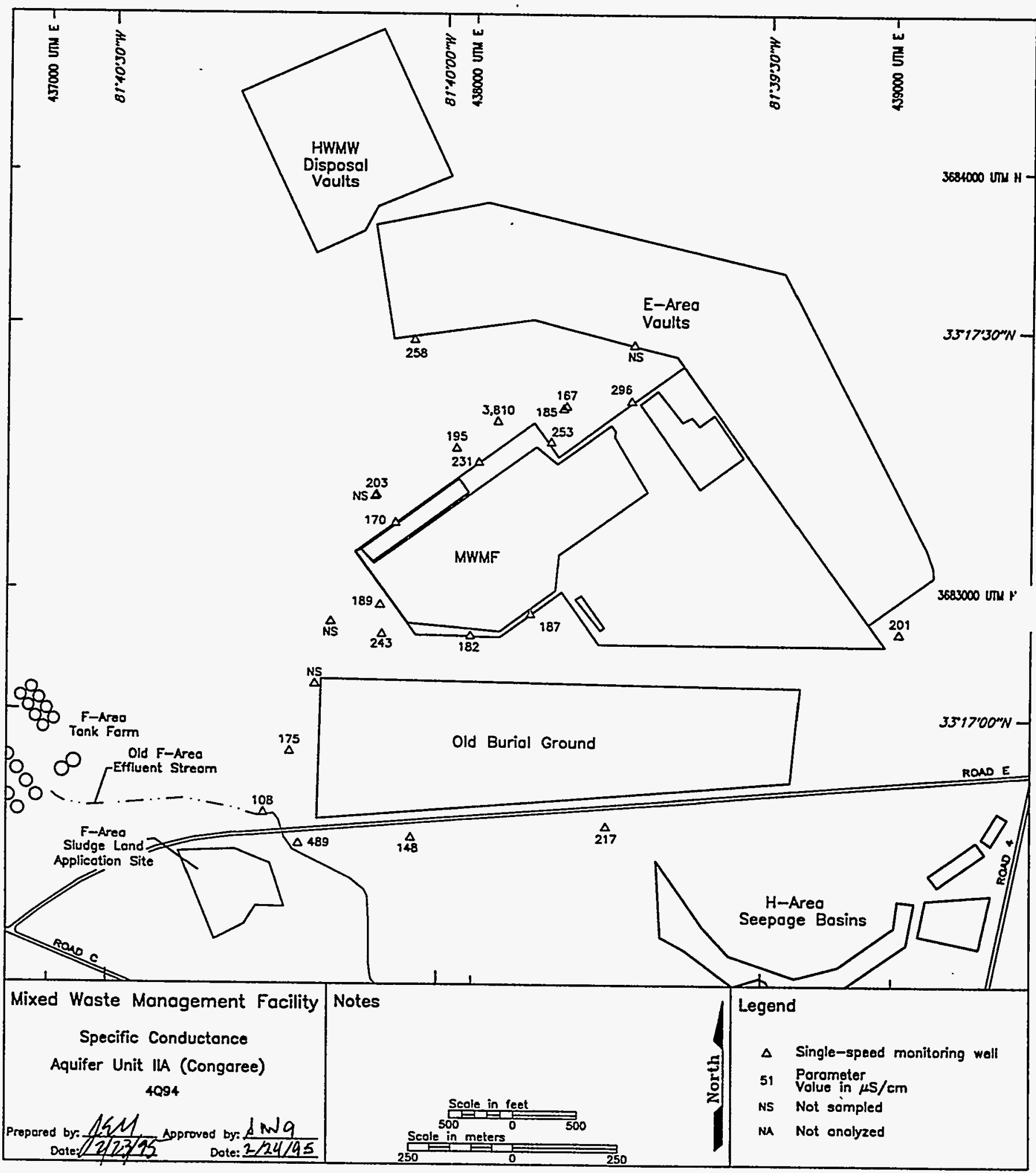

Figure 16. Specific Conductance in Aquifer Unit IIA (Congaree) at the Burial Ground Complex, Fourth Quarter 1994 


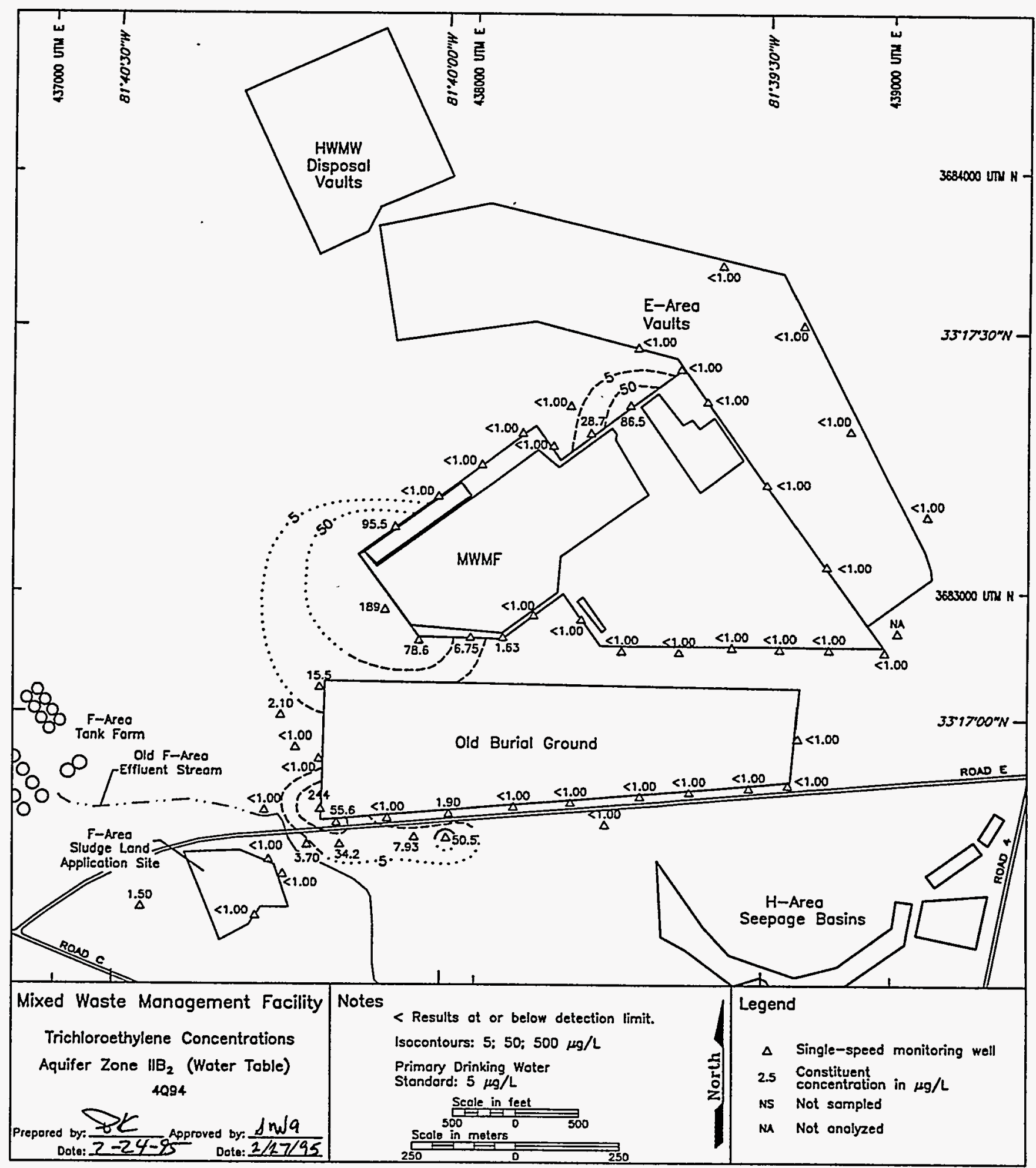

Figure 17. Trichloroethylene Concentrations in Aquifer Zone $\| B_{2}$ (Water Table) at the Burial Ground Complex, Fourth Quarter 1994 


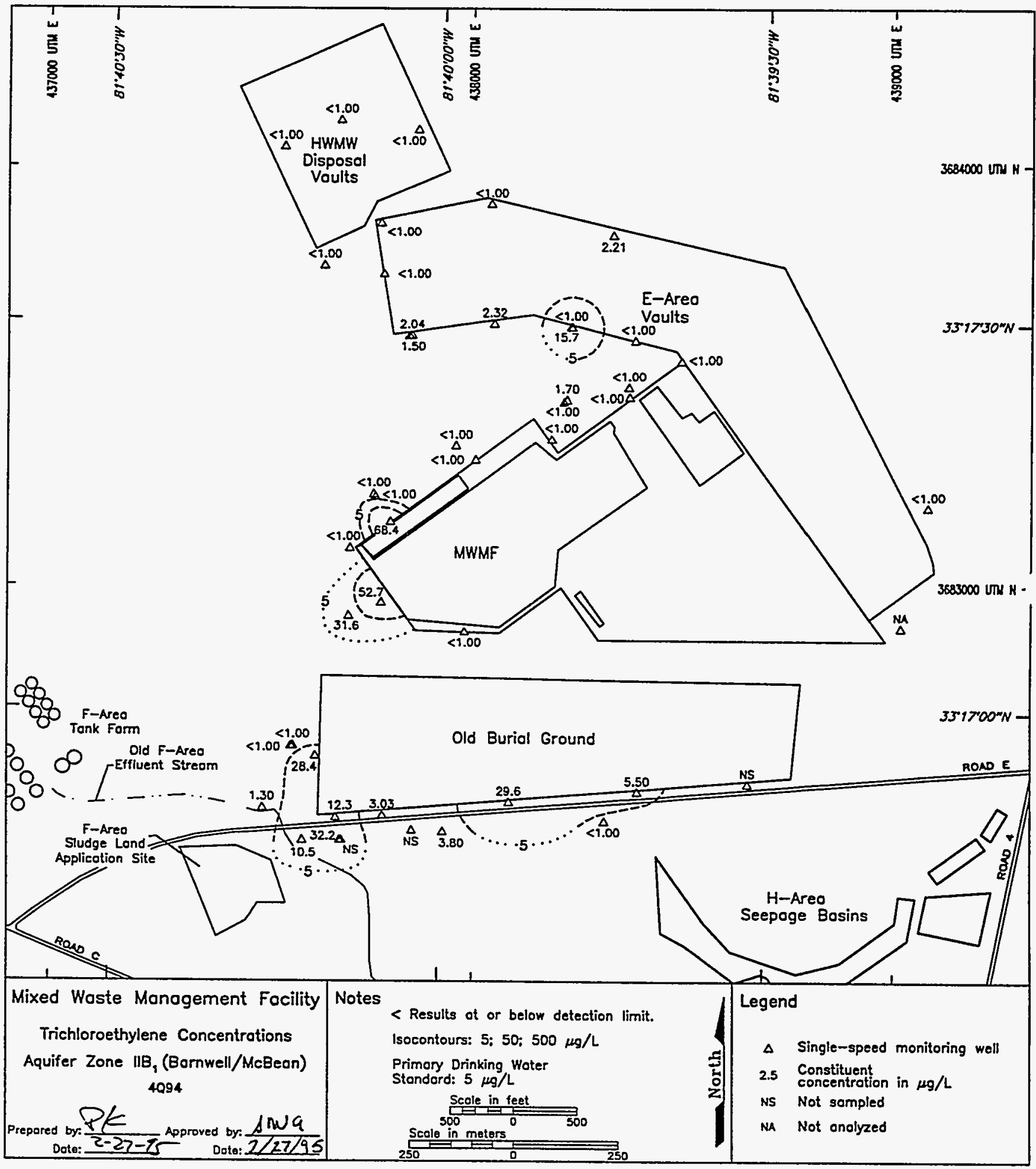

Figure 18. Trichloroethylene Concentrations in Aquifer Zone $I I B_{1}$ (Barnwell/McBean) at the Burial Ground Complex, Fourth Quarter 1994 


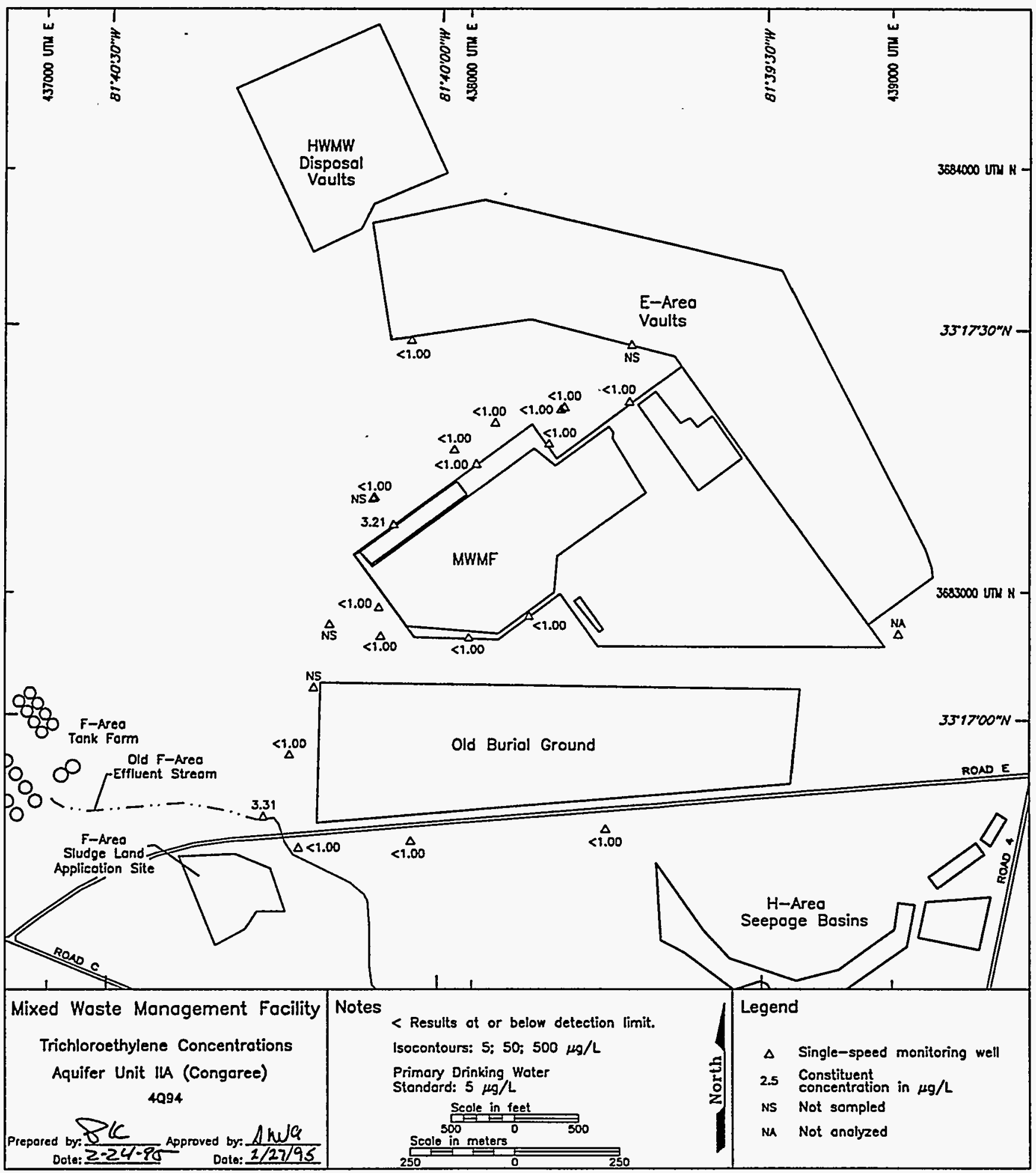

Figure 19. Trichloroethylene Concentrations in Aquifer Unit IIA (Congaree) at the Burial Ground Complex, Fourth Quarter 1994 


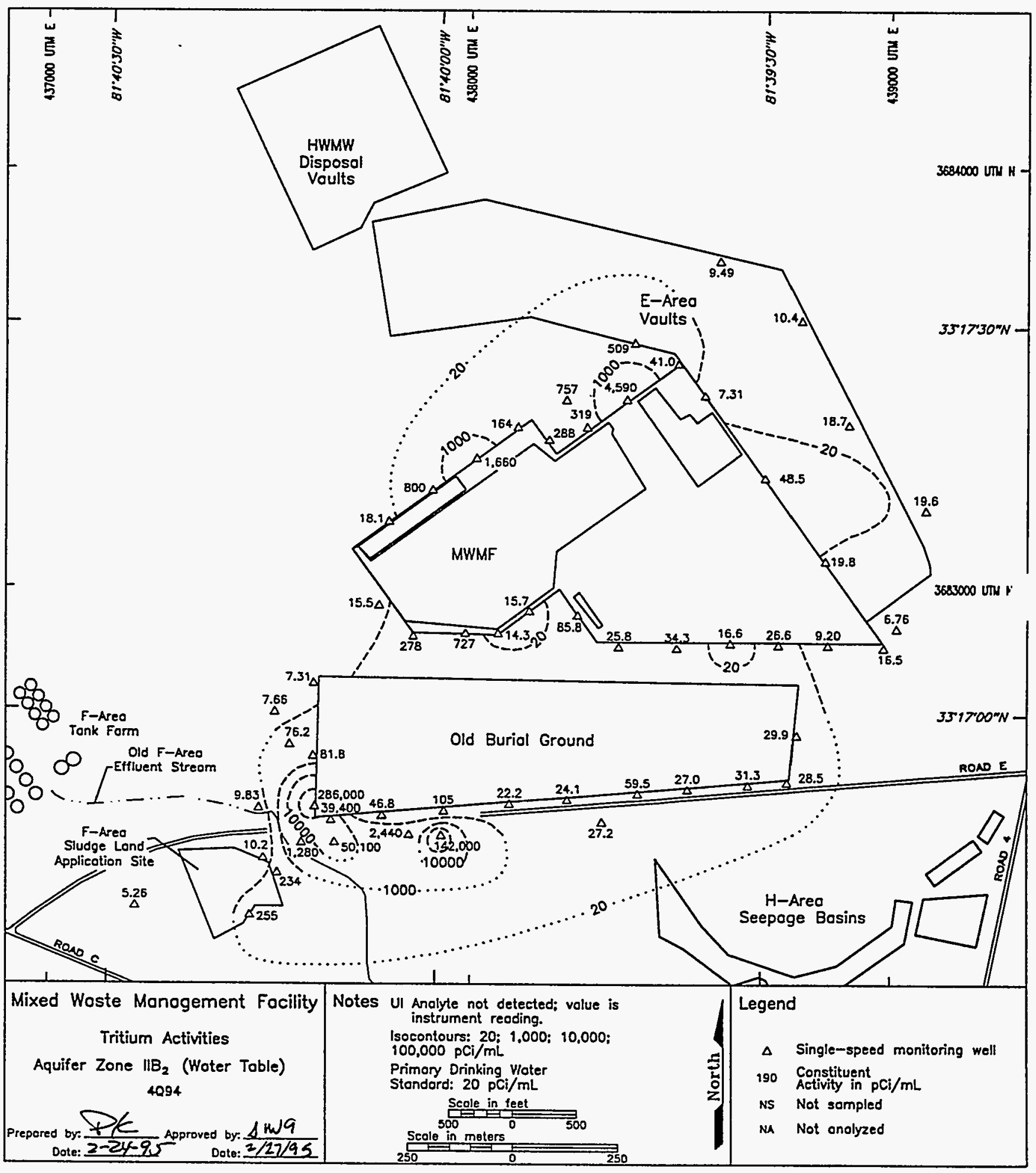

Figure 20. Tritium Activities in Aquifer Zone $\|_{2} B_{2}$ (Water Table) at the Burial Ground Complex, Fourth Quarter 1994 


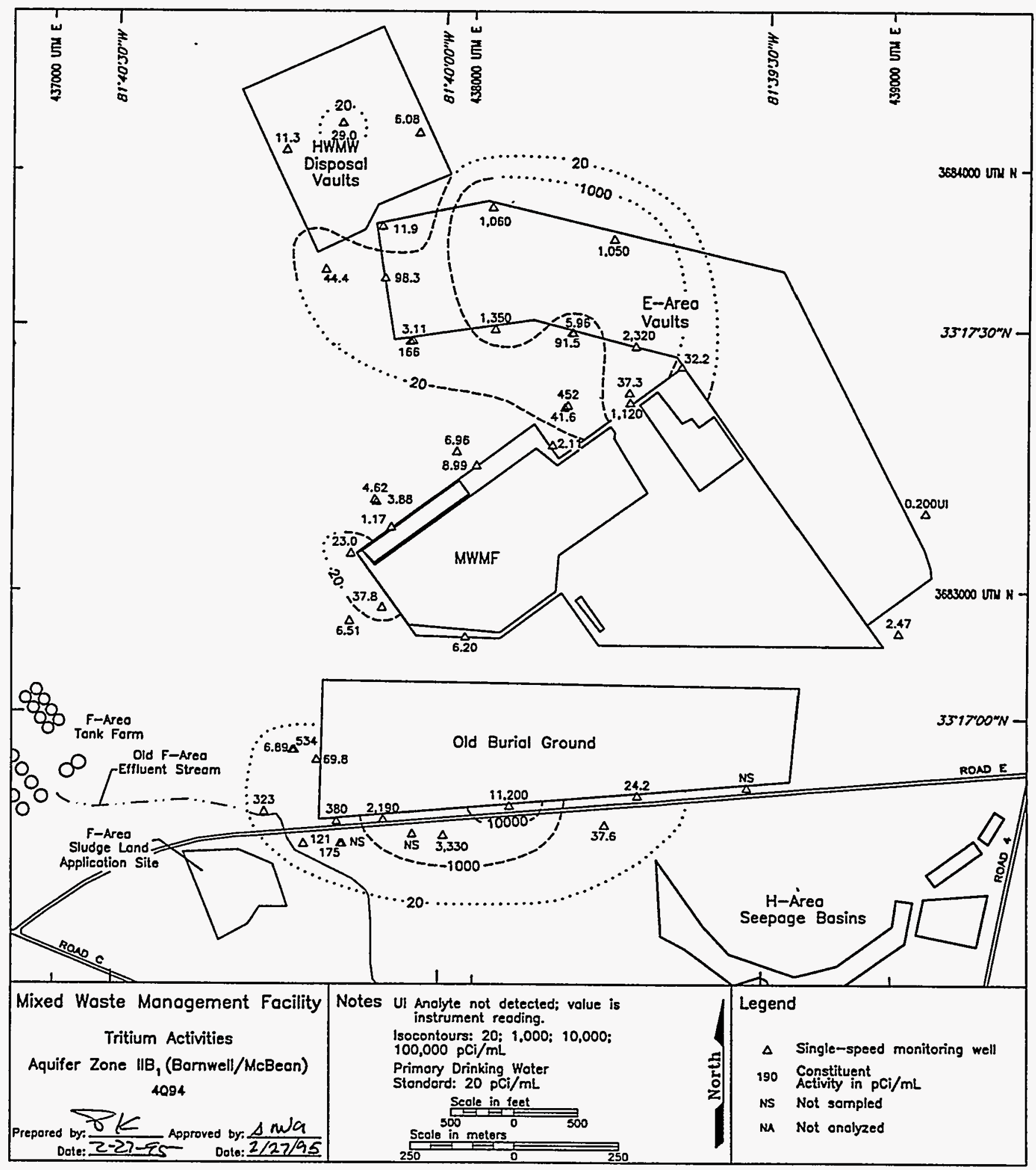

Figure 21. Tritium Activities in Aquifer Zone $\|_{1} B_{1}$ (Barnwell/McBean) at the Burial Ground Complex, Fourth Quarter 1994 


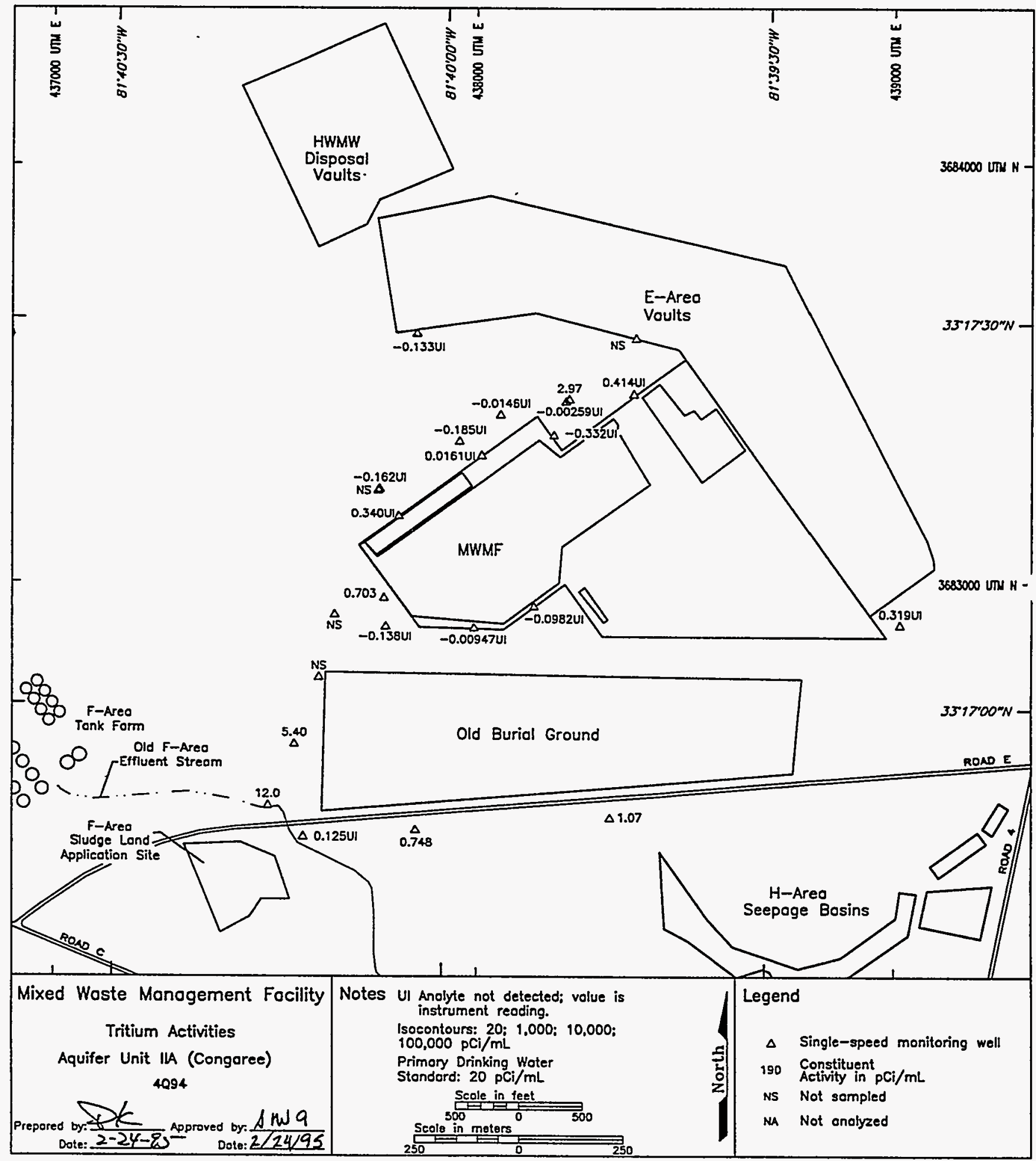

Figure 22. Tritium Activities in Aquifer Unit IIA (Congaree) at the Burial Ground Complex, Fourth Quarter 1994 


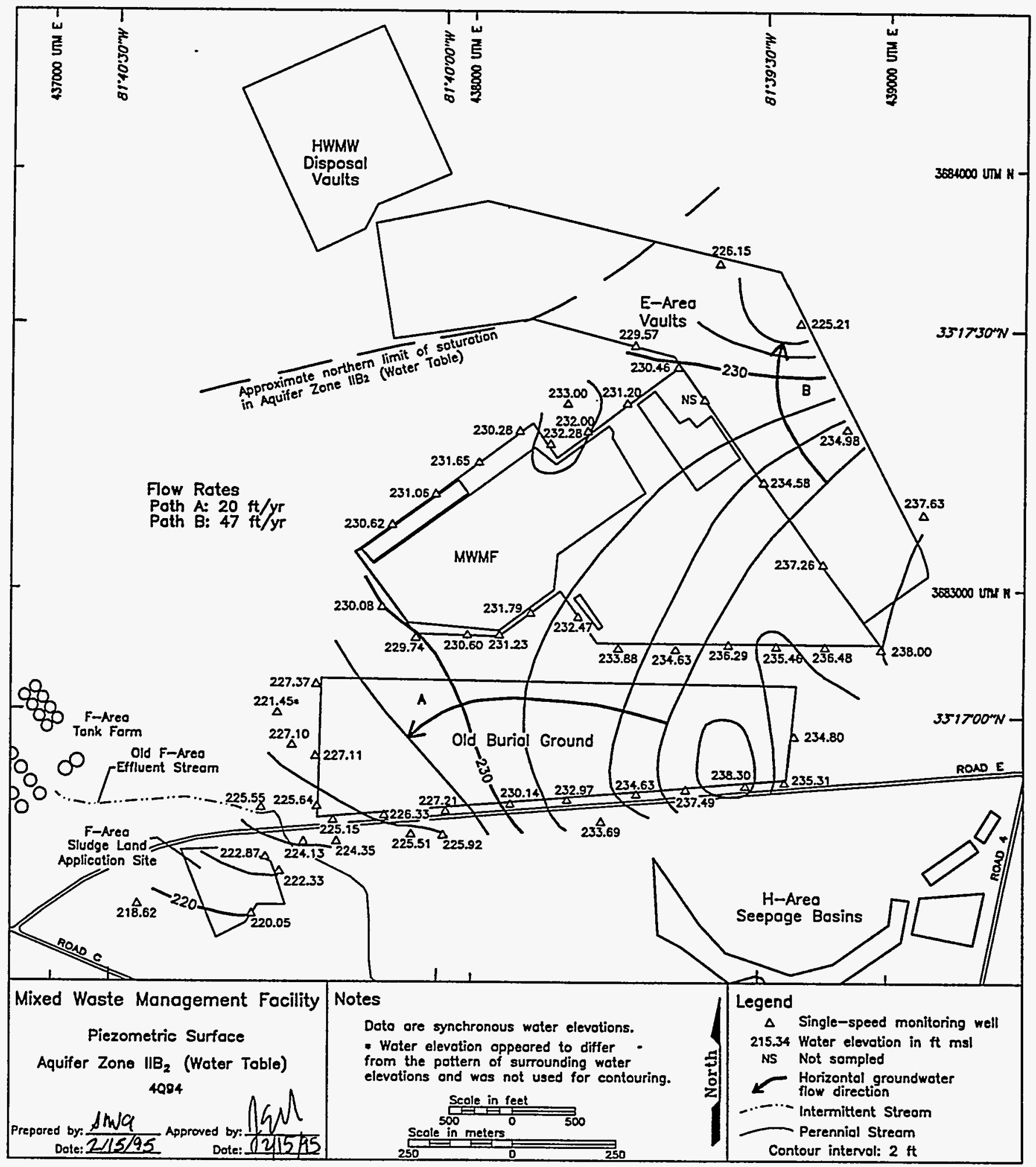

Figure 23. Piezometric Surface Map of Aquifer Zone $\mathrm{IIB}_{2}$ (Water Table) at the Burial Ground Complex 


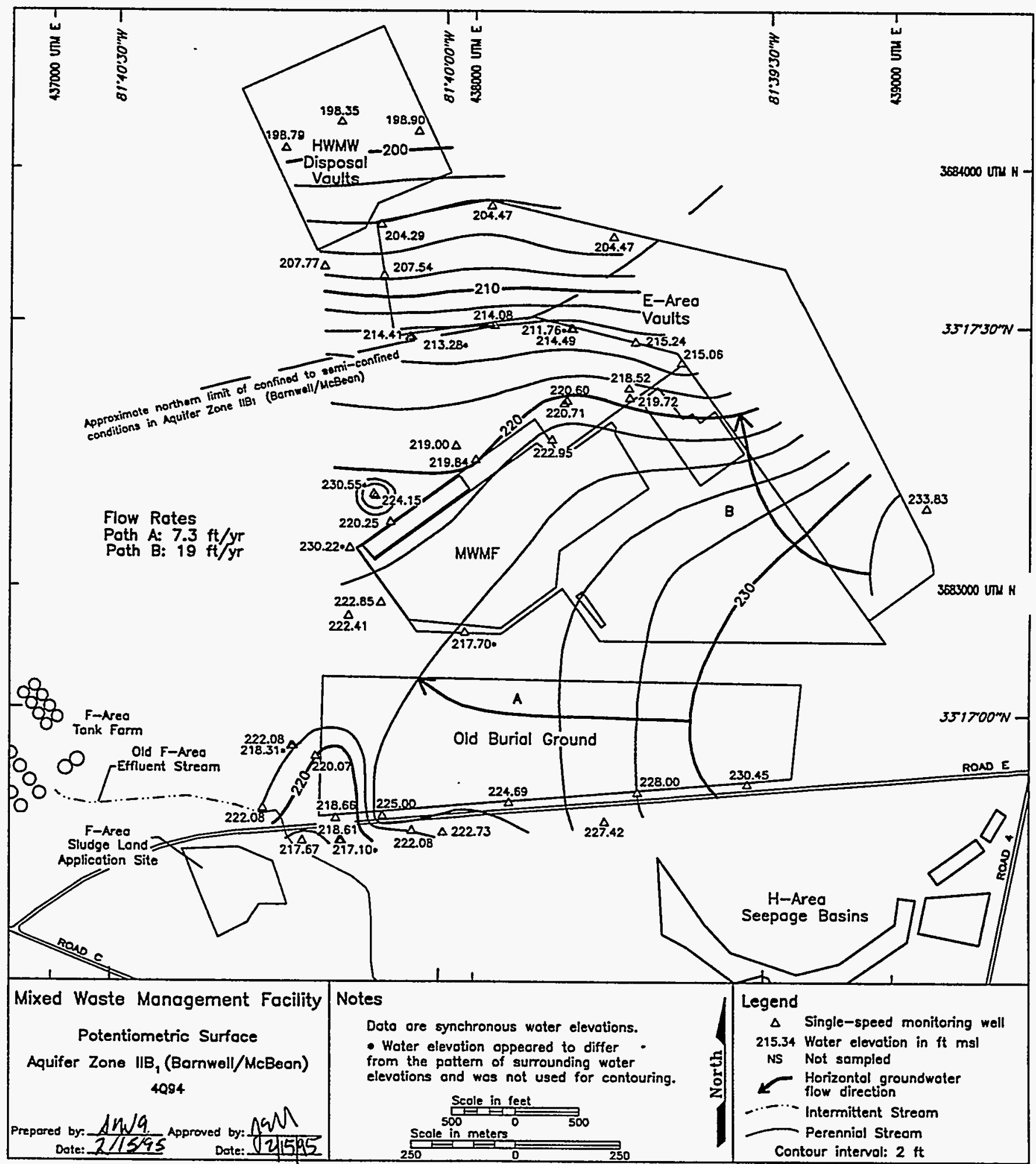

Figure 24. Potentiometric Surface Map of Aquifer Zone IIB, (Barnwell/McBean) at the Burial Ground Complex 


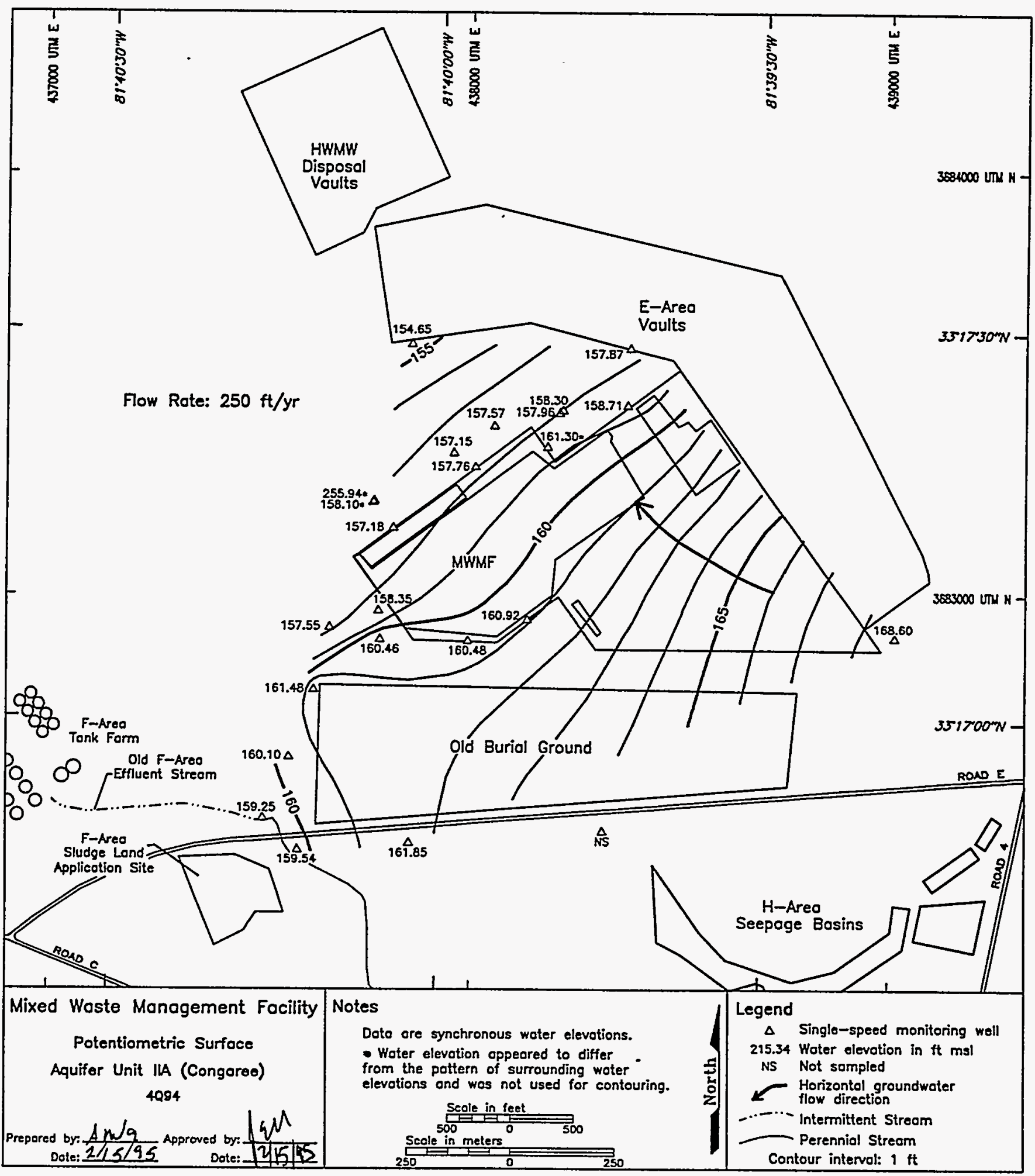

Figure 25. Potentiometric Surface Map of Aquifer Unit IIA (Congaree) at the Burial Ground Complex 


\section{References}

Aadland, R. K., and H. W. Bledsoe, 1990. Classification of Hydrostratigraphic Units at the Savannah River Site, South Carolina, WSRC-RP-90-987. Westinghouse Savannah River Company, Savannah River Site, Aiken, SC.

Aucott, W. R., M. E. Davis, and G. K. Speiran, 1987. Geohydrologic Framework of the Coastal Plain Aquifers of South Carolina, U.S. Geological Survey Water-Resources Investigations Report 85-4271, Columbia, SC.

Du Pont (E. I. du Pont de Nemours \& Company), 1989. Final Safety Analysis Report of the Defense Waste Processing Facility, DPSTSA-200-10. Savannah River Plant, Aiken, SC.

GeoTrans, Inc., 1988. A Numerical Model of the Hydrogeological System Underlying the Savannah River Plant, Final report submitted to Savannah River Laboratory. GeoTrans, Inc., Sterling, VA.

Siple, G. E., 1967. Geology and Ground Water of the Savannah River Plant and Vicinity, South Carolina. Geological Survey Water-Supply Paper 1841, Reston, VA. 
WSRC-TR-94-0610

Unclassified

\section{Appendix D}

\section{Groundwater Monitoring Results Tables}


WSRC-TR-94-0610

Unclassified

THIS PAGE LEFT BLANK INTENTIONALLY. 


\section{Key to Reading the Tables}

The following abbreviations may appear in the data tables:

\section{Constituents}

1,2,3,4,6,7,8-HPCDD
1,2,3,4,6,7,8-HPCDF
$1,2,3,4,7,8-H X C D D$
$1,2,3,4,7,8-H X C D F$
Lindane
PCB
$1,2,3,7,8-P C D D$
$1,2,3,7,8-P C D F$
Sp. conductance
TCDD
TCDF

Laboratories

CN
EM
GE and GP
SC
SP
TM
WA and WS

\section{Sampling Codes}

B

C

D

E

I

$L$

$P$

$\mathrm{S}$

\section{Sampling Methods}

B

$P$

$S$

V 1,2,3,4,6,7,8-heptachlorodibenzo-p-dioxin

$1,2,3,4,6,7,8$-heptachlorodibenzo-p-furan

$1,2,3,4,7,8$-hexachlorodibenzo-p-dioxin

$1,2,3,4,7,8$-hexachlorodibenzo-p-furan

gamma-benzene hexachloride

polychlorinated biphenyl

1,2,3,7,8-pentachlorodibenzo-p-dioxin

1,2,3,7,8-pentachlorodibenzo-p-furan

specific conductance

tetrachlorodibenzo-p-dioxin

tetrachlorodibenzo-p-furan

Clemson Technical Center, Inc.

Environmental Protection Department/Environmental

Monitoring Section (EPD/EMS) Laboratory

General Engineering Laboratories

Savannah River Technology Center

Spencer Testing Services, Inc.

TMA/Eberline

Roy F. Weston, Inc.

blank sample was collected

well was pumping continuously

well was dry

equipment blank was collected

well went dry during sampling; insufficient water to collect

all samples

well went dry before sampling began; only depth to water can be determined

inaccessibility or mechanical failure prevented sample

collection and field analysis of the water

no water in standpipe; for water-level events only

well went dry during purging; samples collected after well recovered

sample collected using an open-bucket bailer

sample collected using a bladder pump

sample collected using a single-speed centrifugal

downhole pump

sample collected using a variable-speed pump 
Units

E

$\mathrm{mg} / \mathrm{L}$

msl

MSL

NTU

$\mathrm{pCi} / \mathrm{L}$

$\mathrm{pCi} / \mathrm{mL}$

$\mathrm{pH}$

$\mu \mathrm{g} / \mathrm{L}$

$\mu \mathrm{S} / \mathrm{cm}$ exponential notation (e.g., $1.1 \mathrm{E}-09=1.1 \times 10^{-9}=$

0.0000000011 )

milligrams per liter

mean sea level

million structures per liter

nephelometric turbidity unit

picocuries per liter

picocuries per milliliter

$\mathrm{pH}$ unit

micrograms per liter

microsiemens per centimeter

Other

CS

DF

H

Mod

PDWS

PVC

ST

carbon steel

dilution factor column in data tables

holding time column in data tables

modifier column in data tables

primary drinking water standard

polyvinyl chloride

exceeded standard column in data tables

TOC

top of casing

\section{Holding Times}

Standard analytical methods include a limit, called holding time, on the maximum elapsed time between sample collection and extraction or analysis by the laboratory. In the data tables, a large bullet $(\cdot)$ in the $H$ (holding time) column indicates that holding time was exceeded. Analyses performed beyond holding times may not yield valid results.

The South Carolina Department of Health and Environmental Control allows only 15 minutes to elapse between sampling and analysis for $\mathrm{pH}$. Thus, only field $\mathrm{pH}$ measurements can meet the holding time criterion; laboratory $\mathrm{pH}$ analyses always will exceed it.

The laboratory procedure used for the determination of specific conductance allows one day to elapse between sampling and analysis. Thus, laboratory specific conductance measurements may exceed the holding time criterion.

\section{Data Rounding}

Constituent results in analytical results tables that appear to equal the final PDWS but are not marked in the ST (exceeded the final PDWS or screening level) column are below the final PDWS in the database. Values stored in the database contain more significant digits than the reported results. Apparent discrepancies in the tables are due to the rounding of reported results. 


\section{Data Qualification}

The contract laboratories continually assess their own accuracy and precision according to U.S. Environmental. Protection Agency (EPA) guidelines. They submit sample- or batch-specific quality assurance/quality control information either at the same time as analytical results or in a quarterly summary. Properly defined and used result modifiers (also referred to as qualifiers) can be a key component in assessing data usability. Result modifiers designed by the EPD/EMS and provided to the primary laboratories are defined below. These modifiers appear in the data tables under the column Mod. The lettered modifiers are based on EPA's STORET codes.

\section{Result Modifiers}

Data are not qualified. Numbers should be interpreted exactly as reported.

The value in the result field is the instrument reading, not the sample quantification limit. Always used with the result qualifier $U$.

Value is estimated because quantitation in the sample or in associated quality control samples did not meet specifications.

Value is off-scale high. The actual value is not known but is known to be greater than the value shown.

Presence of the analyte is verified but not quantified.

Result was rejected because performance requirements in the sample analysis or associated quality control analyses were not met.

Analyte was not detected; if present, it was below the criteria for detection.

Material analyzed for but not detected. Analytical result reported is less than the sample quantitation limit.

Analyte was detected in an associated method blank

Result was obtained from an unpreserved or improperly preserved sample. Data may not be accurate.

Result may be an underestimation of the true value due to analytical bias.

Result may be an overestimation of the true value due to analytical bias.

The associated result may be of poor precision (high variability) due to analytical bias.

Result is associated with $Q A$ results indicating matrix interference.

The associated result is from a reanalysis performed out of holding time due to problems with an earlier analysis. 
Table D-1. Maximum Levels of Constituents Exceeding the Final Primary Drinking Water Standards

Aquifer Zone IIB ${ }_{2}$ (Water Table)

\begin{tabular}{|c|c|c|c|c|c|c|c|}
\hline Well & Constituent & Unit & 1094 & $\underline{2 Q 94}$ & 3Q94 & 4Q94 & Mod \\
\hline BGO 2D & Tritium & $\mathrm{pCi} / \mathrm{mL}$ & $2.1 E+.01$ & $2.1 E+01$ & $2.2 E+01$ & $-{ }^{a}$ & \\
\hline BGO 3D & Tritium & $\mathrm{pCi} / \mathrm{mL}$ & $3.7 E+01$ & $4.5 \mathrm{E}+01$ & $4.5 E+01$ & $4.9 E+01$ & \\
\hline BGO 4D & Lead & $\mu \mathrm{g} / \mathrm{L}$ & $N A^{b}$ & NA & NA & 105 & \\
\hline BGO 5D & $\begin{array}{l}\text { Lead } \\
\text { Tritium }\end{array}$ & $\begin{array}{l}\mu \mathrm{g} / \mathrm{L} \\
\mathrm{pCi} / \mathrm{mL}\end{array}$ & $\overline{2.4 E+01}$ & - & $\begin{array}{l}132 \\
3.0 E+01\end{array}$ & $\overline{4.1 E+01}$ & $J$ \\
\hline$B G O 6 D$ & $\begin{array}{l}\text { 1,1-Dichloroethylene } \\
\text { Tetrachloroethylene } \\
\text { Trichloroethylene } \\
\text { Tritium }\end{array}$ & $\begin{array}{l}\mu \mathrm{g} / \mathrm{L} \\
\mu \mathrm{g} / \mathrm{L} \\
\mu \mathrm{g} / \mathrm{L} \\
\mathrm{pCi} / \mathrm{mL}\end{array}$ & $\begin{array}{l}- \\
- \\
23 \\
1.0 E+03\end{array}$ & $\begin{array}{l}- \\
- \\
35 \\
1.9 E+03\end{array}$ & $\begin{array}{l}7.9 \\
6.6 \\
75 \\
3.8 E+03\end{array}$ & $\begin{array}{l}-\overline{7.4} \\
87 \\
4.6 E+03\end{array}$ & \\
\hline BGO 7D & $\begin{array}{l}\text { Tetrachloroethylene } \\
\text { Trichloroethylene } \\
\text { Tritium }\end{array}$ & $\begin{array}{l}\mu \mathrm{g} / \mathrm{L} \\
\mu \mathrm{g} / \mathrm{L} \\
\mathrm{pCi} / \mathrm{mL}\end{array}$ & $\begin{array}{l}13 \\
175 \\
6.5 E+02\end{array}$ & $\begin{array}{l}9.8 \\
153 \\
5.7 E+02\end{array}$ & $\begin{array}{l}11 \\
193 \\
9.0 E+02\end{array}$ & $\begin{array}{l}- \\
29 \\
3.2 E+02\end{array}$ & \\
\hline$B G O 8 D$ & Tritium & $\mathrm{pCi} / \mathrm{mL}$ & - & - & - & $2.9 E+02$ & \\
\hline BGO 9D & $\begin{array}{l}\text { Dichloromethane } \\
\text { Tritium }\end{array}$ & $\begin{array}{l}\mu \mathrm{g} / \mathrm{L} \\
\mathrm{pCi} / \mathrm{mL}\end{array}$ & $\overline{2.4 E+02}$ & $\overline{1.6 E}+02$ & $\begin{array}{l}8.8 \\
2.5 E+02\end{array}$ & $\overline{1.6 E}+02$ & \\
\hline BGO 10DR & Tritium & $\mathrm{pCi} / \mathrm{mL}$ & $1.0 E+03$ & $2.2 E+03$ & $2.7 E+03$ & $1.7 E+03$ & \\
\hline BGO 11D & Tritium & $\mathrm{pCi} / \mathrm{mL}$ & $1.7 E+03$ & $1.5 E+03$ & $1.0 E+03$ & $8.0 E+02$ & \\
\hline BGO 12D & $\begin{array}{l}\text { Trichloroethylene } \\
\text { Tritium }\end{array}$ & $\begin{array}{l}\mu \mathrm{g} / \mathrm{L} \\
\mathrm{pCi} / \mathrm{mL}\end{array}$ & $\begin{array}{l}127 \\
2.9 E+01\end{array}$ & $\begin{array}{l}83 \\
2.2 E+01\end{array}$ & $\begin{array}{l}100 \\
-\end{array}$ & $\begin{array}{l}96 \\
-\end{array}$ & \\
\hline BGO 14DR & $\begin{array}{l}\text { Dichloromethane } \\
\text { Trichloroethylene }\end{array}$ & $\begin{array}{l}\mu \mathrm{g} / \mathrm{L} \\
\mu \mathrm{g} / \mathrm{L}\end{array}$ & $\overline{61}$ & $\overline{162}$ & $\overline{131}$ & $\begin{array}{l}5.8 \\
189\end{array}$ & \\
\hline BGO 15D & $\begin{array}{l}\text { Tetrachloroethylene } \\
\text { Trichloroethylene } \\
\text { Tritium }\end{array}$ & $\begin{array}{l}\mu \mathrm{g} / \mathrm{L} \\
\mu \mathrm{g} / \mathrm{L} \\
\mathrm{pCi} / \mathrm{mL}\end{array}$ & $\begin{array}{l}- \\
67 \\
2.7 E+02\end{array}$ & $\begin{array}{l}5.8 \\
69 \\
2.6 E+02\end{array}$ & $\begin{array}{l}5.5 \\
70 \\
3.1 E+02\end{array}$ & $\begin{array}{l}6.7 \\
79 \\
2.8 E+02\end{array}$ & \\
\hline BGO 16D & $\begin{array}{l}\text { Trichloroethylene } \\
\text { Tritium }\end{array}$ & $\begin{array}{l}\mu \mathrm{g} / \mathrm{L} \\
\mathrm{pCi} / \mathrm{mL}\end{array}$ & $\begin{array}{l}22 \\
6.3 E+02\end{array}$ & $\begin{array}{l}8.6 \\
6.9 E+02\end{array}$ & $\begin{array}{l}5.0 \\
6.3 E+02\end{array}$ & $\begin{array}{l}6.8 \\
7.3 E+02\end{array}$ & \\
\hline BGO 19D & Tritium & $\mathrm{pCi} / \mathrm{mL}$ & $5.6 \mathrm{E}+01$ & $7.5 \mathrm{E}+01$ & $6.7 E+01$ & $8.6 E+01$ & \\
\hline BGO 20D & Tritium & $\mathrm{pCi} / \mathrm{mL}$ & $2.3 E+01$ & $2.2 E+01$ & $2.7 E+01$ & $2.6 E+01$ & \\
\hline BGO 210 & Tritium & $\mathrm{pCi} / \mathrm{mL}$ & $3.0 E+01$ & $3.2 E+01$ & $3.8 E+01$ & $3.4 E+01$ & \\
\hline BGO 22DR & Tritium & $\mathrm{pCi} / \mathrm{mL}$ & $4.2 E+01$ & $3.7 E+01$ & 2.7E+01 & - & \\
\hline 23D & Tritium & $\mathrm{pCi} / \mathrm{mL}$ & $2.0 E+01$ & $2.1 E+01$ & $2.5 E+01$ & $2.7 E+01$ & \\
\hline
\end{tabular}


Aquifer Zone $\mathrm{IIB}_{2}$ (Water Table)

\begin{tabular}{|c|c|c|c|c|c|c|c|}
\hline Well & Constifuent & Unit & 1094 & $\underline{2 Q 94}$ & $\underline{3094}$ & $\underline{4 Q 94}$ & Mod \\
\hline BGO 26D & $\begin{array}{l}\text { Lead } \\
\text { Trichloroethylene }\end{array}$ & $\begin{array}{l}\mu \mathrm{g} / \mathrm{L} \\
\mu \mathrm{g} / \mathrm{L}\end{array}$ & - & $\begin{array}{l}- \\
-\end{array}$ & $\begin{array}{l}59 \\
12\end{array}$ & $\overline{16}$ & \\
\hline BGO 27D & Tritium & $\mathrm{pCi} / \mathrm{mL}$ & $2.5 E+01$ & $3.1 E+01$ & $6.6 \mathrm{E}+01$ & $8.2 E+01$ & \\
\hline BGO 28D & $\begin{array}{l}\text { Chloroethene } \\
\text { Trichloroethylene } \\
\text { Tritium }\end{array}$ & $\begin{array}{l}\mu \mathrm{g} / \mathrm{L} \\
\mu \mathrm{g} / \mathrm{L} \\
\mathrm{pCi} / \mathrm{mL}\end{array}$ & $\begin{array}{l}140 \\
190 \\
1.2 E+05\end{array}$ & $\begin{array}{l}96 \\
208 \\
1.6 E+05\end{array}$ & $\begin{array}{l}85 \\
163 \\
2.0 E+05\end{array}$ & $\begin{array}{l}51 \\
244 \\
2.9 E+05\end{array}$ & \\
\hline BGO 29D & Tritium & $\mathrm{pCi} / \mathrm{mL}$ & - & $4.2 E+01$ & - & - & \\
\hline BGO 30D & $\begin{array}{l}\text { Chloroethene } \\
\text { 1,1-Dichloroethylene } \\
\text { Trichloroethylene } \\
\text { Tritium }\end{array}$ & $\begin{array}{l}\mu \mathrm{g} / \mathrm{L} \\
\mu \mathrm{g} / \mathrm{L} \\
\mu \mathrm{g} / \mathrm{L} \\
\mathrm{pCi} / \mathrm{mL}\end{array}$ & $\begin{array}{l}7.8 \\
19 \\
81 \\
4.5 E+04\end{array}$ & $\begin{array}{l}5.5 \\
13 \\
60 \\
3.9 E+04\end{array}$ & $\begin{array}{l}14 \\
8.1 \\
79 \\
3.8 \mathrm{E}+04\end{array}$ & $\begin{array}{l}9.0 \\
7.5 \\
56 \\
3.9 E+04\end{array}$ & \\
\hline BGO 31D & Tritium & $\mathrm{pCi} / \mathrm{mL}$ & $3.0 E+01$ & - & $7.9 E+01$ & 4.7E+01 & \\
\hline BGO 32D & $\begin{array}{l}\text { Gross alpha } \\
\text { Tetrachloroethylene } \\
\text { Trichloroethylene } \\
\text { Tritium }\end{array}$ & $\begin{array}{l}\mathrm{pCi} / \mathrm{L} \\
\mu \mathrm{g} / \mathrm{L} \\
\mu \mathrm{g} / \mathrm{L} \\
\mathrm{pCi} / \mathrm{mL}\end{array}$ & $\begin{array}{l}\overline{5.3} \\
5.1 \\
6.2 E+02\end{array}$ & $\begin{array}{l}1.5 E+01 \\
6.9 \\
5.9 \\
1.1 E+03\end{array}$ & $\begin{array}{l}2.1 E+01 \\
8.0 \\
\overline{6} .7 E+02\end{array}$ & $\begin{array}{l}\overline{7.6} \\
\overline{1.1 E+02}\end{array}$ & \\
\hline BGO 33D & Tritium & $\mathrm{pCi} / \mathrm{mL}$ & $2.1 E+01$ & $2.3 E+01$ & 2.3E+01 & $2.2 E \div 01$ & \\
\hline BGO 34D & Tritium & $\mathrm{pCi} / \mathrm{mL}$ & $2.3 E+01$ & $2.5 E+01$ & $2.5 E \div 01$ & 2.4E+01 & \\
\hline BGO 35D & $\begin{array}{l}\text { Lead } \\
\text { Tritium }\end{array}$ & $\begin{array}{l}\mu \mathrm{g} / \mathrm{L} \\
\mathrm{pCi} / \mathrm{mL}\end{array}$ & $\overline{1.0 E+02}$ & $\overline{9.3 E}+01$ & $\overline{9.3 E}+01$ & $\begin{array}{l}133 \\
6.0 E+01\end{array}$ & \\
\hline BGO 36D & Tritium & $\mathrm{pCi} / \mathrm{mL}$ & $2.5 E+01$ & $2.8 E+01$ & $2.9 \mathrm{E}+01$ & 2.7E+01 & \\
\hline BGO 37D & Tritium & $\mathrm{pCi} / \mathrm{mL}$ & $3.0 E+01$ & $3.5 E+01$ & $3.3 E+01$ & 3.1E+01 & \\
\hline BGO 38D & Tritium & $\mathrm{pCi} / \mathrm{mL}$ & $2.6 \mathrm{E}+01$ & $3.0 E+01$ & $3.0 E+01$ & $2.9 E+01$ & \\
\hline BGO 39D & Tritium & $\mathrm{pCi} / \mathrm{mL}$ & $2.9 E+01$ & $3.1 E+01$ & $3.1 E+01$ & $3.0 E+01$ & \\
\hline BGO 4OD & Lead & $\mu \mathrm{g} / \mathrm{L}$ & - & - & 56 & - & \\
\hline BGO 44D & Tritium & $\mathrm{pCi} / \mathrm{mL}$ & $3.8 E+02$ & $3.7 E \div 02$ & $6.3 E+02$ & $7.6 \mathrm{E}+02$ & \\
\hline BGO 45D & Tritium & $\mathrm{pCi} / \mathrm{mL}$ & $4.3 E+02$ & $5.0 E+02$ & $2.2 E+02$ & $7.6 \mathrm{E}+01$ & \\
\hline BGO 46D & $\begin{array}{l}\text { Chloroethene } \\
\text { Tetrachloroethylene } \\
\text { Trichloroethylene } \\
\text { Tritium }\end{array}$ & $\begin{array}{l}\mu \mathrm{g} / \mathrm{L} \\
\mu \mathrm{g} / \mathrm{L} \\
\mu \mathrm{g} / \mathrm{L} \\
\mathrm{pCi} / \mathrm{mL}\end{array}$ & $\begin{array}{l}- \\
6.0 \\
48 \\
3.4 E+04\end{array}$ & $\begin{array}{l}3.6 \\
8.3 \\
44 \\
3.9 E+04\end{array}$ & $\begin{array}{l}2.3 \\
8.2 \\
37 \\
4.5 E+0.4\end{array}$ & $\begin{array}{l}4.1 \\
9.4 \\
34 \\
5.0 E+04\end{array}$ & $J$ \\
\hline BGO 47D & $\begin{array}{l}\text { Trichloroethylene } \\
\text { Tritium }\end{array}$ & $\begin{array}{l}\mu \mathrm{g} / \mathrm{L} \\
\mathrm{pCi} / \mathrm{mL}\end{array}$ & $\begin{array}{l}13 \\
1.7 E+03\end{array}$ & $\begin{array}{l}19 \\
2.7 E+03\end{array}$ & $\begin{array}{l}17 \\
4.8 E+03\end{array}$ & $\begin{array}{l}7.9 \\
2.4 E+03\end{array}$ & \\
\hline
\end{tabular}


Aquifer Zone $\mathrm{IIB}_{2}$ (Water Table)

\begin{tabular}{|c|c|c|c|c|c|c|}
\hline Well & Constituent & $\underline{\text { Unit }}$ & $1 \mathrm{Q94}$ & $\underline{2 Q 94}$ & $\underline{3 Q 94}$ & 4Q94 \\
\hline BGO 48D & $\begin{array}{l}\text { Tetrachloroethylene } \\
\text { Trichloroethylene } \\
\text { Tritium }\end{array}$ & $\begin{array}{l}\mu \mathrm{g} / \mathrm{L} \\
\mu \mathrm{g} / \mathrm{L} \\
\mathrm{pCi} / \mathrm{mL}\end{array}$ & $\begin{array}{l}68 \\
36 \\
7.6 E+04\end{array}$ & $\begin{array}{l}68 \\
41 \\
1.1 E+05\end{array}$ & $\begin{array}{l}74 \\
39 \\
1.5 E+05\end{array}$ & $\begin{array}{l}53 \\
51 \\
1.4 \mathrm{E}+05\end{array}$ \\
\hline BGO 49D & Tritium & $\mathrm{pCi} / \mathrm{mL}$ & 2.1E+01 & $2.4 E+01$ & 2.7E+01 & 2.7E+01 \\
\hline BGO 50D & $\begin{array}{l}\text { Trichloroethylene } \\
\text { Tritium }\end{array}$ & $\stackrel{\mu \mathrm{g} / \mathrm{L}}{\mathrm{pCi} / \mathrm{mL}}$ & $\begin{array}{l}12 \\
4.0 E+03\end{array}$ & $\begin{array}{l}19 \\
6.1 E+03\end{array}$ & $\begin{array}{l}8.8 \\
3.5 E+03\end{array}$ & $\overline{1.3 E}+03$ \\
\hline BGX 1D & Tritium & $\mathrm{pCi} / \mathrm{mL}$ & $3.0 E+02$ & $3.5 E+02$ & 4.7E+02 & $5.1 E+02$ \\
\hline$B G X 10 D$ & Lead & $\mu g / L$ & - & - & 61 & 93 \\
\hline$B G \times 11 D$ & Tritium & $\mathrm{pCi} / \mathrm{mL}$ & - & $2.1 E+01$ & $2.0 E+01$ & - \\
\hline$B G X 12 D$ & Tritium & $\mathrm{pCi} / \mathrm{mL}$ & - & $2.0 \mathrm{E}+01$ & - & - \\
\hline FSS 1D & $\begin{array}{l}\text { Copper } \\
\text { Lead }\end{array}$ & $\begin{array}{l}\mu g / L \\
\mu g / L\end{array}$ & $\begin{array}{l}1,400 \\
119\end{array}$ & - & $\overline{53}$ & - \\
\hline FSS 2D & Tritium & $\mathrm{pCi} / \mathrm{mL}$ & $1.8 \mathrm{E}+02$ & $2.1 E+02$ & $2.3 E+02$ & $2.3 E+02$ \\
\hline FSS 3D & $\begin{array}{l}\text { Copper } \\
\text { Lead } \\
\text { Tritium }\end{array}$ & $\begin{array}{l}\mu \mathrm{g} / \mathrm{L} \\
\mu \mathrm{g} / \mathrm{L} \\
\mathrm{pCi} / \mathrm{mL}\end{array}$ & $\begin{array}{l}\overline{-} \\
\overline{9.7 E}+01\end{array}$ & $\begin{array}{l}- \\
956 \\
2.0 \mathrm{E}+02\end{array}$ & $\begin{array}{l}- \\
492 \\
2.5 E+02\end{array}$ & $\begin{array}{l}1,810 \\
1,740 \\
2.6 \mathrm{E}+02\end{array}$ \\
\hline
\end{tabular}

Aquifer Zone IIB, (BarnwelI/McBean)

\begin{tabular}{|c|c|c|c|c|c|c|}
\hline Well & Constituent & $\underline{\text { Unit }}$ & 1094 & $2 Q 94$ & $\underline{3094}$ & 4Q94 \\
\hline BGO 5C & Tritium & $\mathrm{pCi} / \mathrm{mL}$ & $2.9 E+01$ & $2.9 E+01$ & $3.1 E+01$ & $3.2 E+01$ \\
\hline BGO 6B & Tritium & $\mathrm{pCi} / \mathrm{mL}$ & $2.1 E+01$ & $2.4 \mathrm{E}+01$ & $4.4 E+01$ & $3.7 E+01$ \\
\hline BGO $6 \mathrm{C}$ & Tritium & $\mathrm{pCi} / \mathrm{mL}$ & $8.9 E+02$ & $9.8 E+02$ & $1.3 E+03$ & $1.1 \mathrm{E}+03$ \\
\hline BGO 10B & Tritium & $\mathrm{pCi} / \mathrm{mL}$ & $1.1 E+02$ & - & $2.4 E+01$ & - \\
\hline BGO 10C & Tritium & $\mathrm{pCi} / \mathrm{mL}$ & $1.1 E+02$ & - & - & - \\
\hline BGO 12CR & Trichloroethylene & $\mu g / L$ & 79 & 82 & 85 & 68 \\
\hline BGO 13DR & $\begin{array}{l}\text { Lead } \\
\text { Tritium }\end{array}$ & $\begin{array}{l}\mu \mathrm{g} / \mathrm{L} \\
\mathrm{pCi} / \mathrm{mL}\end{array}$ & - & - & $\begin{array}{l}50 \\
2.1 E+01\end{array}$ & $\begin{array}{l}71 \\
2.3 E+01\end{array}$ \\
\hline BGO 14CR & $\begin{array}{l}\text { Trichloroethylene } \\
\text { Tritium }\end{array}$ & $\begin{array}{l}\mu \mathrm{g} / \mathrm{L} \\
\mathrm{pCi} / \mathrm{mL}\end{array}$ & $\begin{array}{l}56 \\
3.0 E+01\end{array}$ & $\begin{array}{l}52 \\
4.5 E+01\end{array}$ & $\begin{array}{l}67 \\
4.7 E+01\end{array}$ & $\begin{array}{l}53 \\
3.8 E+01\end{array}$ \\
\hline BGO $27 \mathrm{C}$ & Trichloroethylene & $\mu g / L$ & 22 & 26 & 23 & 28 \\
\hline
\end{tabular}


Aquifer Zone IIB 1 (Barnwell/McBean)

\begin{tabular}{|c|c|c|c|c|c|c|}
\hline Well & Constituent & $\underline{\text { Unit }}$ & 1Q94 & $\underline{2 Q 94}$ & 3Q94 & 4Q94 \\
\hline & Tritium & $\mathrm{pCi} / \mathrm{mL}$ & $6.6 \mathrm{E}+01$ & $7.4 \mathrm{E}+01$ & $7.8 \mathrm{E}+01$ & $7.0 \mathrm{E}+01$ \\
\hline BGO 29C & Tritium & $\mathrm{pCi} / \mathrm{mL}$ & $1.9 \mathrm{E}+02$ & - & 3.1E+02 & $3.2 E+02$ \\
\hline BGO 30C & $\begin{array}{l}\text { Trichloroethylene } \\
\text { Tritium }\end{array}$ & $\underset{\mathrm{pCi} / \mathrm{mL}}{\mu \mathrm{L} / \mathrm{L}}$ & $\begin{array}{l}11 \\
6.2 E+02\end{array}$ & $\begin{array}{l}9.9 \\
5.0 \mathrm{E}+02\end{array}$ & $\begin{array}{l}8.9 \\
4.5 E+02\end{array}$ & $\begin{array}{l}12 \\
3.8 \mathrm{E}+02\end{array}$ \\
\hline BGO $31 \mathrm{C}$ & Tritium & $\mathrm{pCi} / \mathrm{mL}$ & $3.1 E+03$ & $2.8 \mathrm{E}+03$ & $2.5 E+03$ & $2.2 E+03$ \\
\hline BGO 33C & $\begin{array}{l}\text { Mercury } \\
\text { Tetrachloroethylene } \\
\text { Trichloroethylene } \\
\text { Tritium }\end{array}$ & $\begin{array}{l}\mu \mathrm{g} / \mathrm{L} \\
\mu \mathrm{g} / \mathrm{L} \\
\mu \mathrm{g} / \mathrm{L} \\
\mathrm{pCi} / \mathrm{mL}\end{array}$ & $\begin{array}{l}2.8 \\
5.1 \\
26 \\
8.2 E+03\end{array}$ & $\begin{array}{l}- \\
5.2 \\
28 \\
8.8 E+03\end{array}$ & $\begin{array}{l}3.4 \\
- \\
27 \\
1.0 E+04\end{array}$ & $\begin{array}{l}4.1 \\
- \\
30 \\
1.1 \mathrm{E}+04\end{array}$ \\
\hline BGO 35C & $\begin{array}{l}\text { Trichloroethylene } \\
\text { Tritium }\end{array}$ & $\begin{array}{l}\mu \mathrm{g} / \mathrm{L} \\
\mathrm{pCi} / \mathrm{mL}\end{array}$ & - & $\begin{array}{l}5.1 \\
2.7 E+01\end{array}$ & $\overline{2.5 E}+01$ & $\begin{array}{l}5.5 \\
2.4 E+01\end{array}$ \\
\hline BGO 42C & Trichloroethylene & $\mu \mathrm{g} / \mathrm{L}$ & 36 & 28 & 34 & 32 \\
\hline BGO 44B & $\begin{array}{l}\text { Lead } \\
\text { Tritium }\end{array}$ & $\underset{\mathrm{pCi} / \mathrm{mL}}{\mu \mathrm{g} / \mathrm{L}}$ & $\begin{array}{l}55 \\
1.1 E+02\end{array}$ & $\overline{6.6 E}+01$ & $\overline{5.6 E}+01$ & $\overline{4.2 E}+01$ \\
\hline BGO 44C & Tritium & $\mathrm{pCi} / \mathrm{mL}$ & $3.2 E+02$ & $3.2 E+02$ & 4.7E+02 & $4.5 E+02$ \\
\hline BGO 45C & $\begin{array}{l}\text { Mercury } \\
\text { Tritium }\end{array}$ & $\begin{array}{l}\mu \mathrm{g} / \mathrm{L} \\
\mathrm{pCi} / \mathrm{mL}\end{array}$ & $\overline{7.4 E+02}$ & $\overline{7.3 E}+02$ & $\overline{5.6 E}+02$ & $\begin{array}{l}2.1 \\
5.3 E+02\end{array}$ \\
\hline BGO 46C & $\begin{array}{l}\text { Trichloroethylene } \\
\text { Tritium }\end{array}$ & $\begin{array}{l}\mu \mathrm{g} / \mathrm{L} \\
\mathrm{pCi} / \mathrm{mL}\end{array}$ & $\begin{array}{l}24 \\
9.2 E+01\end{array}$ & $\begin{array}{l}25 \\
9.8 E+01\end{array}$ & $\begin{array}{l}27 \\
1.7 E+02\end{array}$ & $\begin{array}{l}32 \\
1.8 E+02\end{array}$ \\
\hline BGO 48C & Tritium & $\mathrm{pCi} / \mathrm{mL}$ & - & $3.6 E+03$ & $3.7 \mathrm{E}+03$ & $3.3 E+03$ \\
\hline BGO 49C & Tritium & $\mathrm{pCi} / \mathrm{mL}$ & $2.5 \mathrm{E}+01$ & 3.1E+01 & $3.2 E+01$ & $3.8 E+01$ \\
\hline BGO 50C & $\begin{array}{l}\text { Trichloroethylene } \\
\text { Tritium }\end{array}$ & $\begin{array}{l}\mu \mathrm{g} / \mathrm{L} \\
\mathrm{pCi} / \mathrm{mL}\end{array}$ & $\overline{9.9 E}+01$ & $\begin{array}{l}11 \\
1.0 E+02\end{array}$ & $\begin{array}{l}7.5 \\
1.2 E+02\end{array}$ & $\begin{array}{l}11 \\
1.2 E+02\end{array}$ \\
\hline BGX $1 \mathrm{C}$ & Tritium & $\mathrm{pCi} / \mathrm{mL}$ & $1.5 \mathrm{E}+03$ & $1.6 \mathrm{E}+03$ & $2.1 E+03$ & 2.3E+03 \\
\hline$B G X 2 D$ & $\begin{array}{l}\text { Trichloroethylene } \\
\text { Tritium }\end{array}$ & $\begin{array}{l}\mu \mathrm{g} / \mathrm{L} \\
\mathrm{pCi} / \mathrm{mL}\end{array}$ & $\begin{array}{l}13 \\
8.4 E+01\end{array}$ & $\begin{array}{l}15 \\
9.7 E+01\end{array}$ & $\begin{array}{l}17 \\
9.4 E+01\end{array}$ & $\begin{array}{l}16 \\
9.2 E+01\end{array}$ \\
\hline BGX 3D & $\begin{array}{l}\text { Trichloroethylene } \\
\text { Tritium }\end{array}$ & $\begin{array}{l}\mu \mathrm{g} / \mathrm{L} \\
\mathrm{pCi} / \mathrm{mL}\end{array}$ & $\begin{array}{l}36 \\
1.3 E+03\end{array}$ & $\overline{1.4 E}+03$ & $\overline{1.4 E}+03$ & $\overrightarrow{1.4 E+03}$ \\
\hline$B G X \quad 4 D$ & Tritium & $\mathrm{pCi} / \mathrm{mL}$ & - & - & - & $1.7 E+02$ \\
\hline BGX 5D & $\begin{array}{l}\text { Nonvolatile beta } \\
\text { Tritium }\end{array}$ & $\begin{array}{l}\mathrm{pCi} / \mathrm{L} \\
\mathrm{pCi} / \mathrm{mL}\end{array}$ & $\begin{array}{l}1.5 \mathrm{E}+02 \\
7.1 \mathrm{E}+01\end{array}$ & $\overline{7} .7 E+01$ & $\overline{8} .9 \mathrm{E}+01$ & $\overline{9} .8 \mathrm{E}+01$ \\
\hline$B G X 7 D$ & Tritium & $\mathrm{pCi} / \mathrm{mL}$ & 1.1E+03 & $9.0 E+02$ & $1.2 E+03$ & 1.1E+03 \\
\hline
\end{tabular}


Aquifer Zone IIB, (Barnwell/McBean)

\begin{tabular}{|c|c|c|c|c|c|c|}
\hline Well & Constituent & Unit & $1 \mathrm{Q94}$ & 2Q94 & 3Q94 & 4Q94 \\
\hline BGX 8DR & Tritium & $\mathrm{pCi} / \mathrm{mL}$ & $9.3 E+02$ & $1.0 \mathrm{E}+03$ & 1.1E+03 & 1.1E+03 \\
\hline HMD 1D & $\begin{array}{l}\text { Lead } \\
\text { Tritium }\end{array}$ & $\begin{array}{l}\mu \mathrm{g} / \mathrm{L} \\
\mathrm{pCi} / \mathrm{mL}\end{array}$ & $\overline{3.7 E}+01$ & $\overline{3} .3 E+01$ & $\begin{array}{l}63 \\
3.3 E+01\end{array}$ & $\overline{4.4 E+01}$ \\
\hline AMD 3D & Tritium & $\mathrm{pCi} / \mathrm{mL}$ & - & - & - & $2.9 E+01$ \\
\hline
\end{tabular}

Aquifer Unit IIA (Congaree)

\begin{tabular}{|c|c|c|c|c|c|c|c|}
\hline Well & Constituent & $\underline{\text { Unit }}$ & 1094 & $\underline{2 Q 94}$ & 3Q94 & 4Q994 & Mod \\
\hline BGO 9AA & Nonvolatile beta & $\mathrm{pCi} / \mathrm{L}$ & $7.8 \mathrm{E}+01$ & - & - & - & \\
\hline BGO 10AA & $\begin{array}{l}\text { Dichloromethane } \\
\text { Tritium }\end{array}$ & $\begin{array}{l}\mu \mathrm{g} / \mathrm{L} \\
\mathrm{pCi} / \mathrm{mL}\end{array}$ & - & - & $\begin{array}{l}6.8 \\
2.3 E+01\end{array}$ & - & UI \\
\hline BGO 10AR & $\begin{array}{l}\text { Benzene } \\
\text { Dichloromethane }\end{array}$ & $\begin{array}{l}\mu g / L \\
\mu g / L\end{array}$ & - & - & - & $\begin{array}{l}8.3 \\
19\end{array}$ & \\
\hline BGO 12AR & Dichloromethane & $\mu g / L$ & - & - & - & 10 & \\
\hline BGO 14AR & $\begin{array}{l}\text { Benzene } \\
\text { Dichloromethane }\end{array}$ & $\begin{array}{l}\mu \mathrm{g} / \mathrm{L} \\
\mu \mathrm{g} / \mathrm{L}\end{array}$ & - & - & - & $\begin{array}{l}7.6 \\
17\end{array}$ & \\
\hline
\end{tabular}

Notes: The groundwater samples are unfiltered. Thus, the results for metals are for total recoverable metals. The modifier column applies to fourth quarter 1994 results only.

a $-=$ analyzed but not above final PDWS.

b $N A=$ not analyzed or result was rejected because performance requirements in the sample analysis or associated quality control analyses were not met. 
Table D-2. Maximum Levels of Constituents Exceeding Other Flag 2 Criteria

$$
\text { Aquifer Zone } \mathrm{IIB}_{2} \text { (Water Table) }
$$

\begin{tabular}{|c|c|c|c|c|}
\hline Well & Constituènt & $\underline{\text { Unit }}$ & $\underline{4 Q 94}$ & Mod \\
\hline BGO 1D & Aluminum & $\mu \mathrm{g} / \mathrm{L}$ & 126 & \\
\hline$B G O 2 D$ & Aluminum & $\mu \mathrm{g} / \mathrm{L}$ & 75 & \\
\hline BGO 3D & Aluminum & $\mu \mathrm{g} / \mathrm{L}$ & 214 & \\
\hline BGO 4D & $\begin{array}{l}\text { Aluminum } \\
\text { Iron }\end{array}$ & $\begin{array}{l}\mu \mathrm{g} / \mathrm{L} \\
\mu \mathrm{g} / \mathrm{L}\end{array}$ & $\begin{array}{l}3,710 \\
1,160\end{array}$ & \\
\hline BGO 5D & Aluminum & $\mu \mathrm{g} / \mathrm{L}$ & 61 & \\
\hline BGO $6 D$ & $\begin{array}{l}\text { Aluminum } \\
\text { Total organic halogens }\end{array}$ & $\begin{array}{l}\mu g / L \\
\mu g / L\end{array}$ & $\begin{array}{l}684 \\
64\end{array}$ & \\
\hline BGO 12D & Total organic halogens & $\mu \mathrm{g} / \mathrm{L}$ & 53 & \\
\hline BGO 14DR & Total organic halogens & $\mu \mathrm{g} / \mathrm{L}$ & 99 & \\
\hline BGO 15D & Total organic halogens & $\mu \mathrm{g} / \mathrm{L}$ & 66 & \\
\hline BGO 16D & $\begin{array}{l}\text { Aluminum } \\
\text { Iron }\end{array}$ & $\begin{array}{l}\mu \mathrm{g} / \mathrm{L} \\
\mu \mathrm{g} / \mathrm{L}\end{array}$ & $\begin{array}{l}531 \\
303\end{array}$ & \\
\hline BGO 17DR & Aluminum & $\mu g / L$ & 120 & \\
\hline BGO 19D & Aluminum & $\mu g / L$ & 57 & \\
\hline BGO 24D & Aluminum & $\mu g / L$ & 387 & \\
\hline BGO 26D & $\begin{array}{l}\text { Aluminum } \\
\text { Total organic halogens }\end{array}$ & $\begin{array}{l}\mu \mathrm{g} / \mathrm{L} \\
\mu \mathrm{g} / \mathrm{L}\end{array}$ & $\begin{array}{l}179 \\
58\end{array}$ & \\
\hline BGO 27D & Aluminum & $\mu g / L$ & 113 & \\
\hline BGO 28D & $\begin{array}{l}\text { Aluminum } \\
\text { 1,1-Dichloroethane } \\
\text { Iron } \\
\text { Manganese } \\
\text { Total organic halogens }\end{array}$ & $\begin{array}{l}\mu g / L \\
\mu g / L \\
\mu g / L \\
\mu g / L \\
\mu g / L\end{array}$ & $\begin{array}{l}1,370 \\
18 \\
778 \\
75 \\
428\end{array}$ & $J$ \\
\hline BGO 29D & $\begin{array}{l}\text { Aluminum } \\
\text { Manganese }\end{array}$ & $\begin{array}{l}\mu g / L \\
\mu g / L\end{array}$ & $\begin{array}{l}669 \\
72\end{array}$ & \\
\hline BGO 30D & $\begin{array}{l}\text { Aluminum } \\
1,1 \text {-Dichloroethane } \\
\text { Manganese } \\
\text { Total organic halogens }\end{array}$ & $\begin{array}{l}\mu g / L \\
\mu g / L \\
\mu g / L \\
\mu g / L\end{array}$ & $\begin{array}{l}686 \\
38 \\
98 \\
561\end{array}$ & \\
\hline BGO 31D & Aluminum & $\mu \mathrm{g} / \mathrm{L}$ & 132 & \\
\hline
\end{tabular}


Aquifer Zone $\mathrm{IIB}_{2}$ (Water Table)

\begin{tabular}{|c|c|c|c|c|}
\hline Well & Constituent & $\underline{\text { Unit }}$ & $\underline{4 Q 94}$ & Mod \\
\hline BGO 32D & $\begin{array}{l}\text { Aluminum } \\
\text { Iron }\end{array}$ & $\underset{\mu g / L}{\mu g / L}$ & $\begin{array}{l}826 \\
650\end{array}$ & \\
\hline BGO 33D & $\begin{array}{l}\text { Aluminum } \\
\text { Iron }\end{array}$ & $\begin{array}{l}\mu g / L \\
\mu g / L\end{array}$ & $\begin{array}{l}322 \\
357\end{array}$ & \\
\hline BGO 34D & Aluminum & $\mu \mathrm{g} / \mathrm{L}$ & 261 & \\
\hline BGO 35D & $\begin{array}{l}\text { Aluminum } \\
\text { Iron }\end{array}$ & $\begin{array}{l}\mu g / L \\
\mu g / L\end{array}$ & $\begin{array}{l}530 \\
373\end{array}$ & \\
\hline BGO 36D & Aluminum & $\mu \mathrm{g} / \mathrm{L}$ & 950 & \\
\hline BGO 37D & Aluminum & $\mu \mathrm{g} / \mathrm{L}$ & 146 & \\
\hline BGO 38D & Aluminum & $\mu g / L$ & 280 & \\
\hline BGO 39D & Aluminum & $\mu g / L$ & 146 & \\
\hline BGO 40D & $\begin{array}{l}\text { Aluminum } \\
\text { Iron } \\
\text { Tin }\end{array}$ & $\begin{array}{l}\mu g / L \\
\mu g / L \\
\mu g / L\end{array}$ & $\begin{array}{l}289 \\
396 \\
40\end{array}$ & \\
\hline BGO 44D & Aluminum & $\mu \mathrm{g} / \mathrm{L}$ & 63 & \\
\hline BGO 46D & $\begin{array}{l}\text { 1,1-Dichloroethane } \\
\text { Total organic halogens } \\
\text { Uranium-233/234 } \\
\text { Uranium-238 }\end{array}$ & $\begin{array}{l}\mu \mathrm{g} / \mathrm{L} \\
\mu \mathrm{g} / \mathrm{L} \\
\mathrm{pCi} / \mathrm{L} \\
\mathrm{pCi} / \mathrm{L}\end{array}$ & $\begin{array}{l}16 \\
161 \\
1.1 E+02 \\
2.3 E+02\end{array}$ & $\mathrm{~J}$ \\
\hline BGO $48 D$ & $\begin{array}{l}\text { Aluminum } \\
\text { Total organic halogens }\end{array}$ & $\begin{array}{l}\mu \mathrm{g} / \mathrm{L} \\
\mu \mathrm{g} / \mathrm{L}\end{array}$ & $\begin{array}{l}119 \\
113\end{array}$ & \\
\hline BGO 49D & Aluminum & $\mu \mathrm{g} / \mathrm{L}$ & 89 & \\
\hline BGO 50D & Aluminum & $\mu g / L$ & 92 & \\
\hline $\mathrm{BGX} 1 \mathrm{D}$ & Aluminum & $\mu g / L$ & 229 & \\
\hline$B G \times 10 D$ & $\begin{array}{l}\text { Aluminum } \\
\text { Iron } \\
\text { Manganese }\end{array}$ & $\begin{array}{l}\mu g / L \\
\mu g / L \\
\mu g / L\end{array}$ & $\begin{array}{l}445 \\
435 \\
109\end{array}$ & \\
\hline$B G \times 11 D$ & Aluminum & $\mu \mathrm{g} / \mathrm{L}$ & 312 & \\
\hline$B G X 12 D$ & Aluminum & $\mu g / L$ & 118 & \\
\hline FSS 10 & Aluminum & $\mu g / L$ & 993 & \\
\hline FSS 2D & $\begin{array}{l}\text { Aluminum } \\
\text { Manganese }\end{array}$ & $\begin{array}{l}\mu \mathrm{g} / \mathrm{L} \\
\mu \mathrm{g} / \mathrm{L}\end{array}$ & $\begin{array}{l}426 \\
61\end{array}$ & \\
\hline
\end{tabular}


Aquifer Zone IIB ${ }_{2}$ (Water Table)

\begin{tabular}{|c|c|c|c|c|}
\hline Well & Constituent & Unit & 4Q94 & Mod \\
\hline FSS 3D & $\begin{array}{l}\text { Aluminum } \\
\text { Iron } \\
\text { Manganese }\end{array}$ & $\begin{array}{l}\mu g / L \\
\mu g / L \\
\mu g / L\end{array}$ & $\begin{array}{l}8,110 \\
668 \\
600\end{array}$ & \\
\hline FSS 4D & Aluminum & $\mu g / L$ & 147 & \\
\hline \multicolumn{5}{|c|}{ Aquifer Zone IIB (Barnwell/McBean) } \\
\hline Well & Constituent & Unit & $\underline{4 Q 94}$ & Mod \\
\hline BGO $5 \mathrm{C}$ & $\begin{array}{l}\text { Aluminum } \\
\text { Manganese }\end{array}$ & $\begin{array}{l}\mu g / L \\
\mu g / L\end{array}$ & $\begin{array}{l}697 \\
51\end{array}$ & \\
\hline BGO $8 \mathrm{C}$ & Aluminum & $\mu \mathrm{g} / \mathrm{L}$ & 332 & \\
\hline BGO 10B & Aluminum & $\mu \mathrm{g} / \mathrm{L}$ & 54 & \\
\hline BGO 10C & $\begin{array}{l}\text { Aluminum } \\
\text { Iron } \\
\text { Manganese }\end{array}$ & $\begin{array}{l}\mu \mathrm{g} / \mathrm{L} \\
\mu \mathrm{g} / \mathrm{L} \\
\mu \mathrm{g} / \mathrm{L}\end{array}$ & $\begin{array}{l}394 \\
2,420 \\
114\end{array}$ & \\
\hline BGO 12CR & Aluminum & $\mu \mathrm{g} / \mathrm{L}$ & 215 & \\
\hline BGO 13DR & $\begin{array}{l}\text { Aluminum } \\
\text { Iron } \\
\text { Manganese }\end{array}$ & $\begin{array}{l}\mu g / L \\
\mu g / L \\
\mu g / L\end{array}$ & $\begin{array}{l}95 \\
467 \\
108\end{array}$ & \\
\hline BGO 14CR & $\begin{array}{l}\text { Aluminum } \\
\text { Iron }\end{array}$ & $\underset{\mu \mathrm{g} / \mathrm{L}}{\mu}$ & $\begin{array}{l}231 \\
454\end{array}$ & \\
\hline BGO 16B & Aluminum & $\mu \mathrm{g} / \mathrm{L}$ & 78 & \\
\hline $\mathrm{BGO} 30 \mathrm{C}$ & Aluminum & $\mu g / L$ & 360 & \\
\hline BGO 31C & Aluminum & $\mu g / L$ & 141 & \\
\hline BGO 33C & $\begin{array}{l}\text { Aluminum } \\
\text { Total organic halogens }\end{array}$ & $\underset{\mu g / L}{\mu g / L}$ & $\begin{array}{l}94 \\
192\end{array}$ & \\
\hline $\mathrm{BGO} 42 \mathrm{C}$ & Aluminum & $\mu g / L$ & 55 & \\
\hline BGO 43CR & Aluminum & $\mu \mathrm{g} / \mathrm{L}$ & 111 & \\
\hline BGO 44B & $\begin{array}{l}\text { Aluminum } \\
\text { Manganese }\end{array}$ & $\begin{array}{l}\mu \mathrm{g} / \mathrm{L} \\
\mu \mathrm{g} / \mathrm{L}\end{array}$ & $\begin{array}{l}81 \\
74\end{array}$ & \\
\hline BGO 44C & $\begin{array}{l}\text { Aluminum } \\
\text { Manganese }\end{array}$ & $\begin{array}{l}\mu g / L \\
\mu g / L\end{array}$ & $\begin{array}{l}182 \\
72\end{array}$ & \\
\hline BGO 45B & $\begin{array}{l}\text { Aluminum } \\
\mathrm{pH}\end{array}$ & $\begin{array}{l}\mu \mathrm{g} / \mathrm{L} \\
\mathrm{pH}\end{array}$ & $\begin{array}{l}89 \\
11\end{array}$ & J \\
\hline
\end{tabular}


Aquifer Zone IIB 1 (Barnwell/McBean)

\begin{tabular}{|c|c|c|c|c|}
\hline Well & Constituent & Unit & 4Q94 & Mod \\
\hline $\mathrm{BGX} 1 \mathrm{C}$ & $\begin{array}{l}\text { Aluminum } \\
\mathrm{pH} \\
\text { Specific conductance }\end{array}$ & $\begin{array}{l}\mu \mathrm{g} / \mathrm{L} \\
\mathrm{pH} \\
\mu \mathrm{S} / \mathrm{cm}\end{array}$ & $\begin{array}{l}783 \\
12 \\
1,930\end{array}$ & $J$ \\
\hline$B G \times 2 B$ & Aluminum & $\mu g / L$ & 72 & \\
\hline$B G X 2 D$ & Aluminum & $\mu g / L$ & 51 & \\
\hline$B G X \quad 4 D$ & Aluminum & $\mu g / L$ & 61 & \\
\hline BGX 5D & $\begin{array}{l}\text { Aluminum } \\
\text { Manganese }\end{array}$ & $\begin{array}{l}\mu g / L \\
\mu g / L\end{array}$ & $\begin{array}{l}114 \\
312\end{array}$ & \\
\hline HMD 1D & $\begin{array}{l}\text { Aluminum } \\
\text { Iron }\end{array}$ & $\begin{array}{l}\mu \mathrm{g} / \mathrm{L} \\
\mu \mathrm{g} / \mathrm{L}\end{array}$ & $\begin{array}{l}746 \\
758\end{array}$ & \\
\hline HMD 4D & Aluminum & $\mu g / L$ & 246 & \\
\hline \multicolumn{5}{|c|}{ Aquifer Unit IIA (Congaree) } \\
\hline Well & Constituent & $\underline{\text { Unit }}$ & 4Q94 & Mod \\
\hline BGO 9AA & $\begin{array}{l}\text { Aluminum } \\
\text { pH } \\
\text { Specific conductance } \\
\text { Tin }\end{array}$ & $\begin{array}{l}\mu \mathrm{g} / \mathrm{L} \\
\mathrm{pH} \\
\mu \mathrm{S} / \mathrm{cm} \\
\mu \mathrm{g} / \mathrm{L}\end{array}$ & $\begin{array}{l}925 \\
12 \\
1,580 \\
188\end{array}$ & $\mathrm{~J}$ \\
\hline BGO 12AR & Aluminum & $\mu g / L$ & 223 & \\
\hline BGO 14AR & Aluminum & $\mu \mathrm{g} / \mathrm{L}$ & 118 & \\
\hline BGO 29A & Aluminum & $\mu \mathrm{g} / \mathrm{L}$ & 270 & \\
\hline BGO 43AA & $\begin{array}{l}\text { Aluminum } \\
\mathrm{pH}\end{array}$ & $\begin{array}{l}\mu \mathrm{g} / \mathrm{L} \\
\mathrm{pH}\end{array}$ & $\begin{array}{l}62 \\
10\end{array}$ & $J$ \\
\hline BGO 44AA & Aluminum & $\mu \mathrm{g} / \mathrm{L}$ & 137 & \\
\hline BGO 47A & Total organic halogens & $\mu g / L$ & 112 & \\
\hline BGO 49A & $\begin{array}{l}\text { Aluminum } \\
\mathrm{pH}\end{array}$ & $\underset{\mathrm{pH}}{\mu \mathrm{g} / \mathrm{L}}$ & $\begin{array}{l}549 \\
11\end{array}$ & $\mathbf{J}$ \\
\hline BGO 50A & $\begin{array}{l}\text { Aluminum } \\
\text { pH } \\
\text { Specific conductance }\end{array}$ & $\begin{array}{l}\mu \mathrm{g} / \mathrm{L} \\
\mathrm{pH} \\
\mu \mathrm{S} / \mathrm{cm}\end{array}$ & $\begin{array}{l}409 \\
12 \\
512\end{array}$ & $J$ \\
\hline
\end{tabular}

Notes: These results do not include field data. The groundwater samples are unfiltered. Thus, the results for metals are for total recoverable metals. Flags are established by EPD/EMS and are based on final PDWS, Secondary Drinking Water Standards, or method detection limits (see Appendix B). 
Table D-3. Groundwater Monitoring Results for Individual Wells

WELL BGO 1D

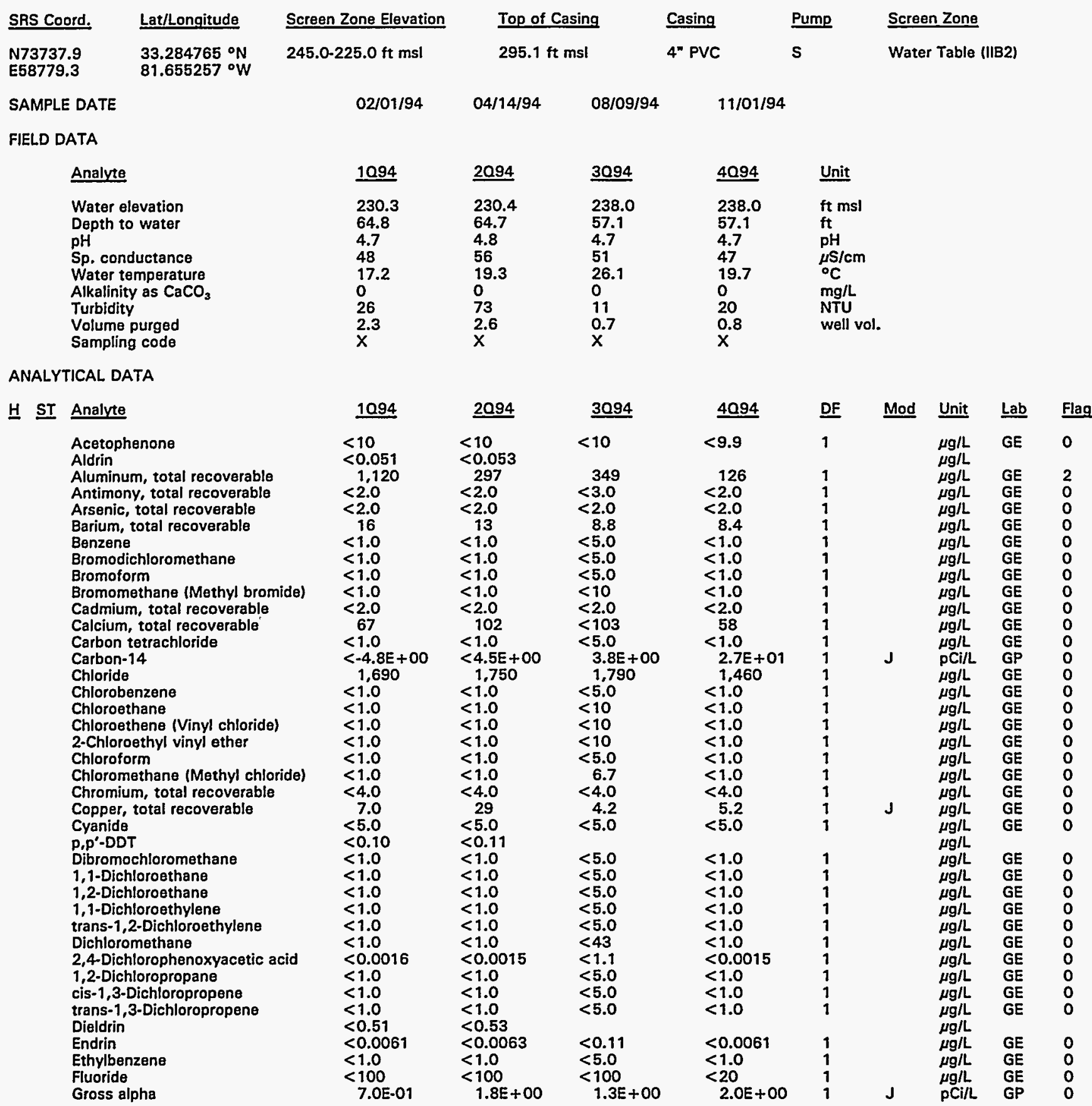

Note: Flagging, dilution factors, modifiers, and laboratories are for fourth quarter 1994 data only. See Appendix B for flagging criteria. - = exceeded holding time for fourth quarter 1994.

- = exceeded screening level or final PDWS for fourth quarter 1994. 


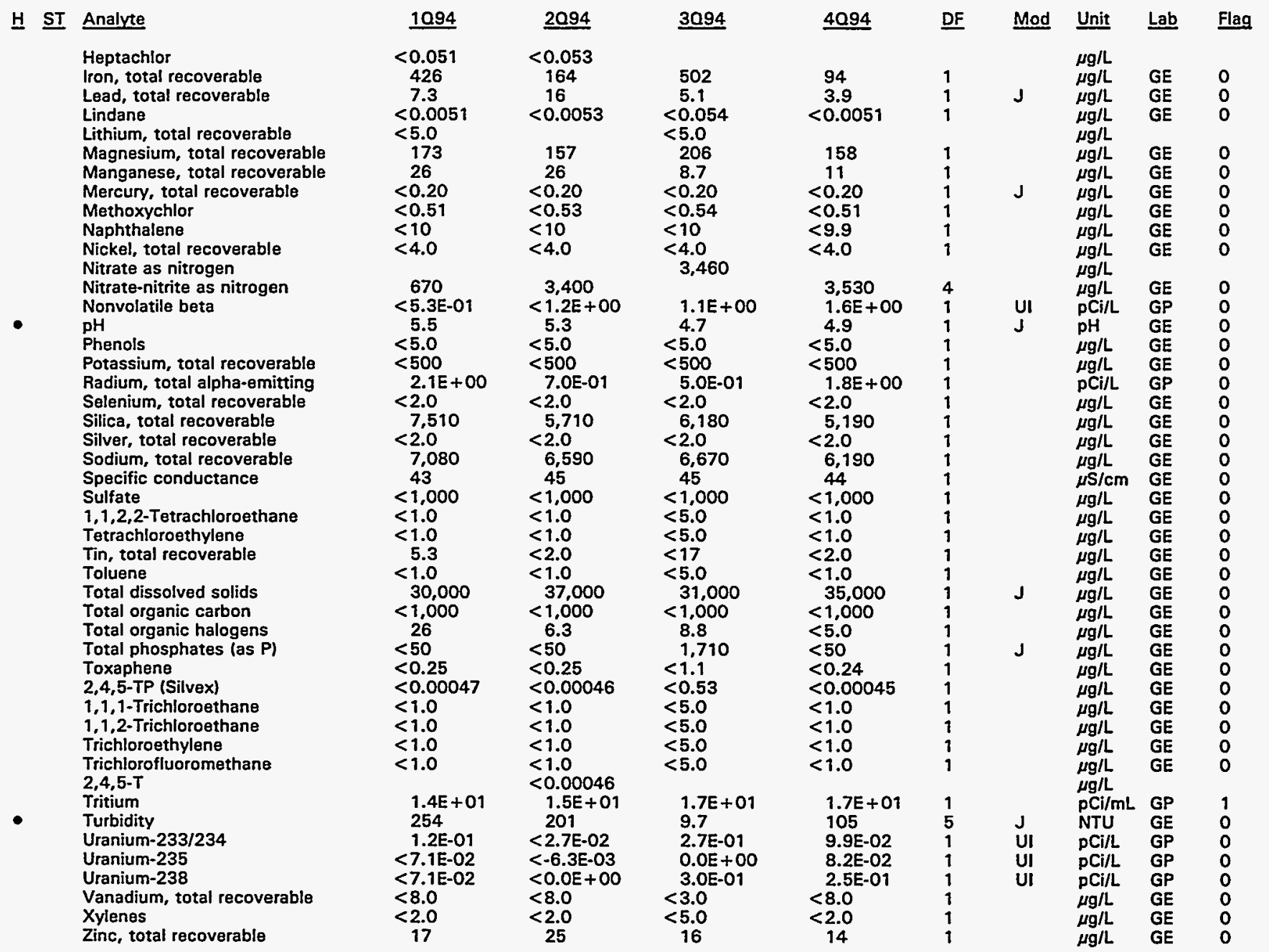

Note: Flagging, dilution factors, modifiers, and laboratories are for fourth quarter 1994 data only. See Appendix B for flagging criteria. - = exceeded holding time for fourth quarter 1994.

- = exceeded screening level or final PDWS for fourth quarter 1994. 
WELL BGO 2D

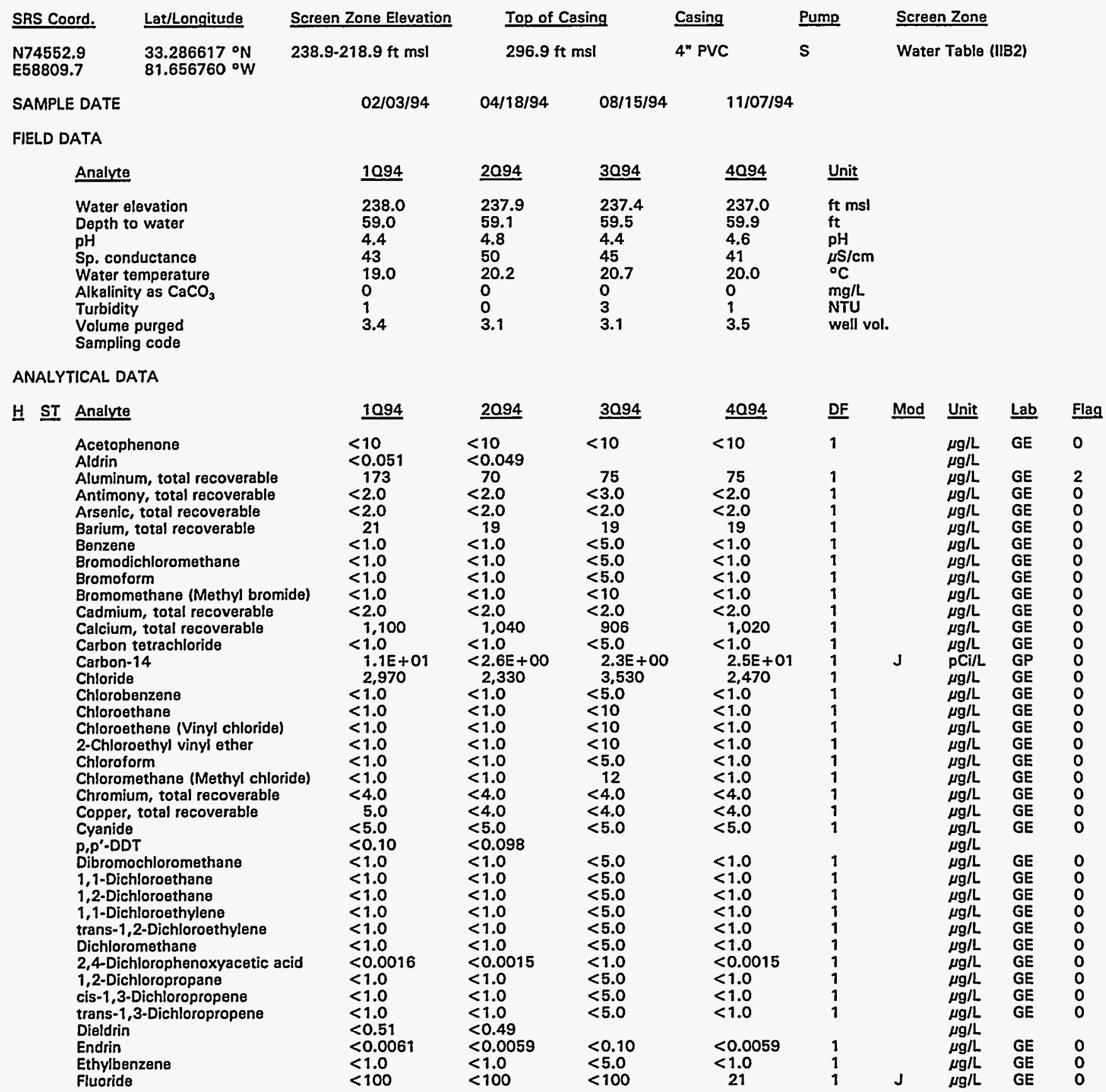

Note: Flagging, dilution factors, modifiers, and laboratories are for fourth quarter 1994 data only. See Appendix B for flagging criteria. - = exceeded holding time for fourth quarter 1994.

- =xceeded screening level or final PDWS for fourth quarter 1994. 


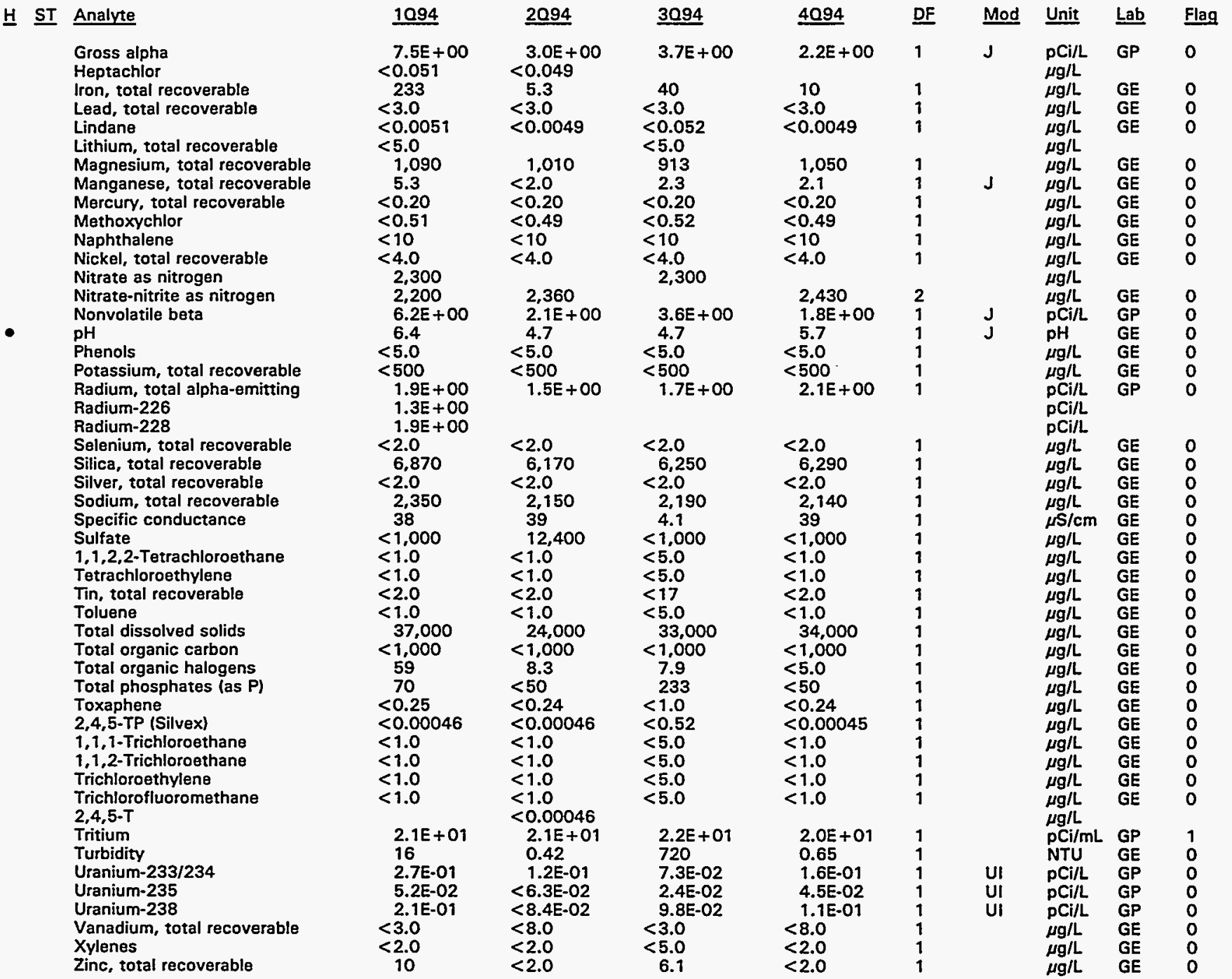

Note: Flagging, dilution factors, modifiers, and laboratories are for fourth quarter 1994 data only. See Appendix B for flagging criteria. - = exceeded holding time for fourth quarter 1994.

- = exceeded screening level or final PDWS for fourth quarter 1994. 
WELL BGO 3D

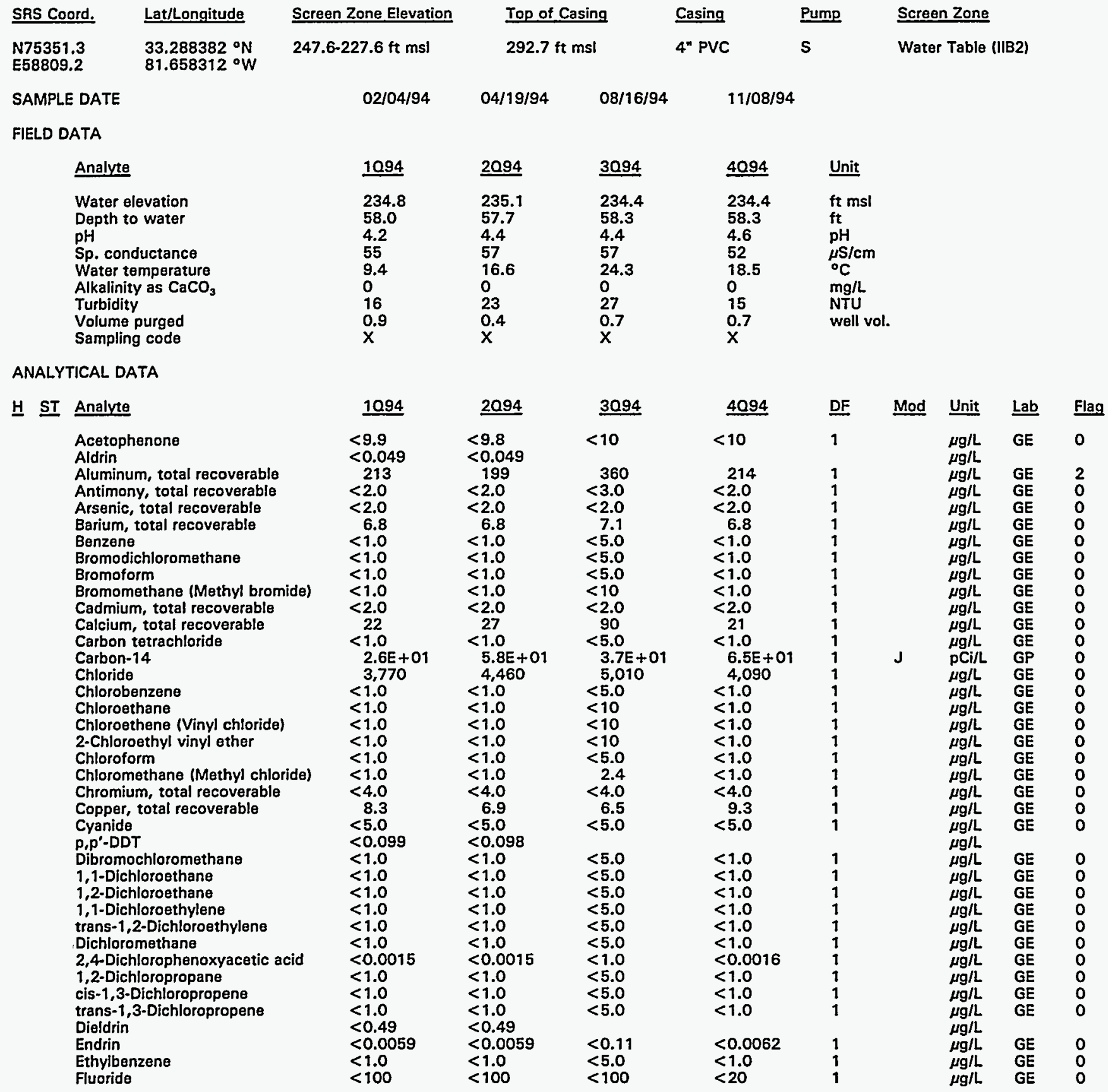

Note: Flagging, dilution factors, modifiers, and laboratories are for fourth quarter 1994 data only. See Appendix B for flagging criteria. - = exceeded holding time for fourth quarter 1994.

- = exceeded screening level or final PDWS for fourth quarter 1994. 
H ST Analyte

Gross alpha

Heptachlor

Iron, total recoverable

Lead, total recoverable

Lindane

Lithium, total recoverable

Magnesium, total recoverable

Manganese, total recoverable

Mercury, total recoverable

Methoxychlor

Nickel, total recoverable

Nitrate as nitrogen

Nitrate-nitrite as nitrogen

Nonvolatile beta

- $\quad \mathrm{pH}$

Phenols

Potassium, total recoverable

Radium, total alpha-emitting

Selenium, total recoverable

Silica, total recoverable

Silver, total recoverable

Sodium, total recoverable

Specific conductance

Sulfate

1,1,2,2-Tetrachloroethane

Tetrachloroethylene

Tin, total recoverable

Toluene

Total dissolved solids

Total organic carbon

Total organic halogens

Total phosphates (as P)

Toxaphene

2,4,5-TP (Silvex)

1,1,1-Trichloroethane

1,1,2-Trichloroethane

Trichloroethylene

Trichlorofluoromethane

2,4,5-T

- Tritium

Turbidity

Uranium-233/234

Uranium-235

Uranium-238

Vanadium, total recoverable

Xylenes

Zinc, total recoverable
1094

$2.4 E+00$

$<0.049$

55

$<3.0$

$<0.0049$

$<5.0$

371

17
$<0.20$

$<0.49$

$<9.9$

$<4.0$

2,600

$1.8 \mathrm{E}+00$

4.5

$<5.0$

$<500$

$3.0 \mathrm{E}+00$

$<2.0$

6,580

$<2.0$

$<, 890$
47

47

1,400

$<1.0$

$<1.0$

3.3

$<1.0$

34,000

$<1,000$

22
$<50$

$<0.24$

$<0.00045$

$<1.0$

$<1.0$

$<1.0$

$<1.0$

3.7E + 01

12

$<5.9 E-02$

$<1.0 E-02$

$<4.9 \mathrm{E}-02$

$<8.0$

$<2.0$

5.2

$\underline{2094}$
$3.8 E+00$
$<0.049$
42
$<3.0$
$<0.0049$

381
17
$<0.20$
$<0.49$
$<9.8$
$<4.0$

3094

$5.5 \mathrm{E}+00$

951

3.4
$<0.055$

$<5.0$

283

14
$<0.20$

$<0.55$

$<0.55$
$<4.0$

2,320

2,400

$2.1 \mathrm{E}+00$

4.6

$<5.0$

$<500$

$<3.0 \mathrm{E}-01$

$<2.0$

6,680

$<2.0$

5,320

48

1,250

$<1.0$

$<1.0$

$<2.0$

$<1.0$

25,000

$<1,000$

$<1,000$
$<50$

$<0.24$

$<0.00045$

$<1.0$

$<1.0$

$<1.0$

$<1.00045$

4.5E + 01

16

2.4E-01

$<0.0 E+00$

2.4E-01

$<8.0$

$<2.0$
7.3
$5.5 \mathrm{E}+00$

4.5

$<5.0$

$<500$

1. $6 E+00$

$<2.0$

4,780

$<2.0$

3,510

50

2,010
$<5,0$

$<5.0$
$<5.0$

$<17$

$<5.0$

$<1,000$

$<1,000$

29

174

$<1.1$

$<0.52$

1.3

$4.5 E+01$

33

1.0E-01

$0.0 E+00$

2.6E-01

$<3.0$

$<5.0$

16
4094 무

4. $1 E+00$

72

$<3.0$

377

$<0.20$

$<0.52$

$<10$

$<4.0$

2,230

3. $2 E+00$

4.6

$<5.0$

$<500$

3.4E+ +00

$<2.0$

6,710

$<2.0$

5,430

49

$<1,000$

$<1.0$

$<1.0$

$<1.0$

28,000

$<1,000$

16
$<50$

$<0.25$

$<0.00046$

1.9
$<1.0$

$<1.0$

$4.9 E+01$

19

6.3E-02

$-5.5 \mathrm{E}-03$

4.0E-02

$<8.0$

$<2.0$

5.6

DF
1
1
1
1
1
1
1
1
1
1
2
1
1
1
1
1
1
1
1
1
1
1
1
1
1
1
1
1
1
1
1
1
1
1
1
1
1
1
1
1
1
1
1
1
1
1
1
1
1
1
1
1
1
1
1
1
1
1
1
1

Mod Unit Lab Flag

pCi/L GP 0

$\mu g / L$ GE 0

$\begin{array}{lll}\mu \mathrm{g} / \mathrm{L} & \mathrm{GE} & 0 \\ \mu \mathrm{g} / \mathrm{L} & \mathrm{GE} & 0\end{array}$

$\mu \mathrm{g} / \mathrm{L}$

$\mu g / L$ GE 0

$\mu \mathrm{g} / \mathrm{L}$ GE 0

$\mu g / L \quad G E \quad 0$

$\mu \mathrm{g} / \mathrm{L}$

$\mu g / L \quad G E$

$\mu g / L \quad G E$

$\mu \mathrm{g} / \mathrm{L}$

$\mathrm{pCi} / \mathrm{L}$
$\mathrm{pH}$

$\mathrm{pH}$
$\mu \mathrm{g} / \mathrm{L}$

$\mu g / L$

pCi/L

$\mu g / L$

$\mu g / L$

$\mu \mathrm{g} / \mathrm{L}$

$\mu S / \mathrm{cm} G$

ugh

$\mu \mathrm{g} / \mathrm{L}$

$\mu g / L$

$\mu g / L$

$\mu g / L$

$\mu g / L$

\rfloor

$\mu g / L$

$\mu g / L$

$\mu \mathrm{g} / \mathrm{L}$

$\mu \mathrm{g} / \mathrm{L}$

$\mu \mathrm{g} / \mathrm{L}$

$\mu g / L$

$\mu g /$

$\mu \mathrm{g} / \mathrm{L}$

$\mu \mathrm{g} / \mathrm{L}$

pCi/mL GP

UI

NCill

$\mathrm{PCi} / \mathrm{L}$

pCi/

$\mu \mathrm{g} / \mathrm{L}$

$\mu \mathrm{g} / \mathrm{L}$

$\mu \mathrm{g} / \mathrm{L}$

Note: Flagging, dilution factors, modifiers, and laboratories are for fourth quarter 1994 data only. See Appendix B for flagging criteria. - = exceeded holding time for fourth quarter 1994.

- = exceeded screening level or final PDWS for fourth quarter 1994. 


\section{WELL BGO 4D}

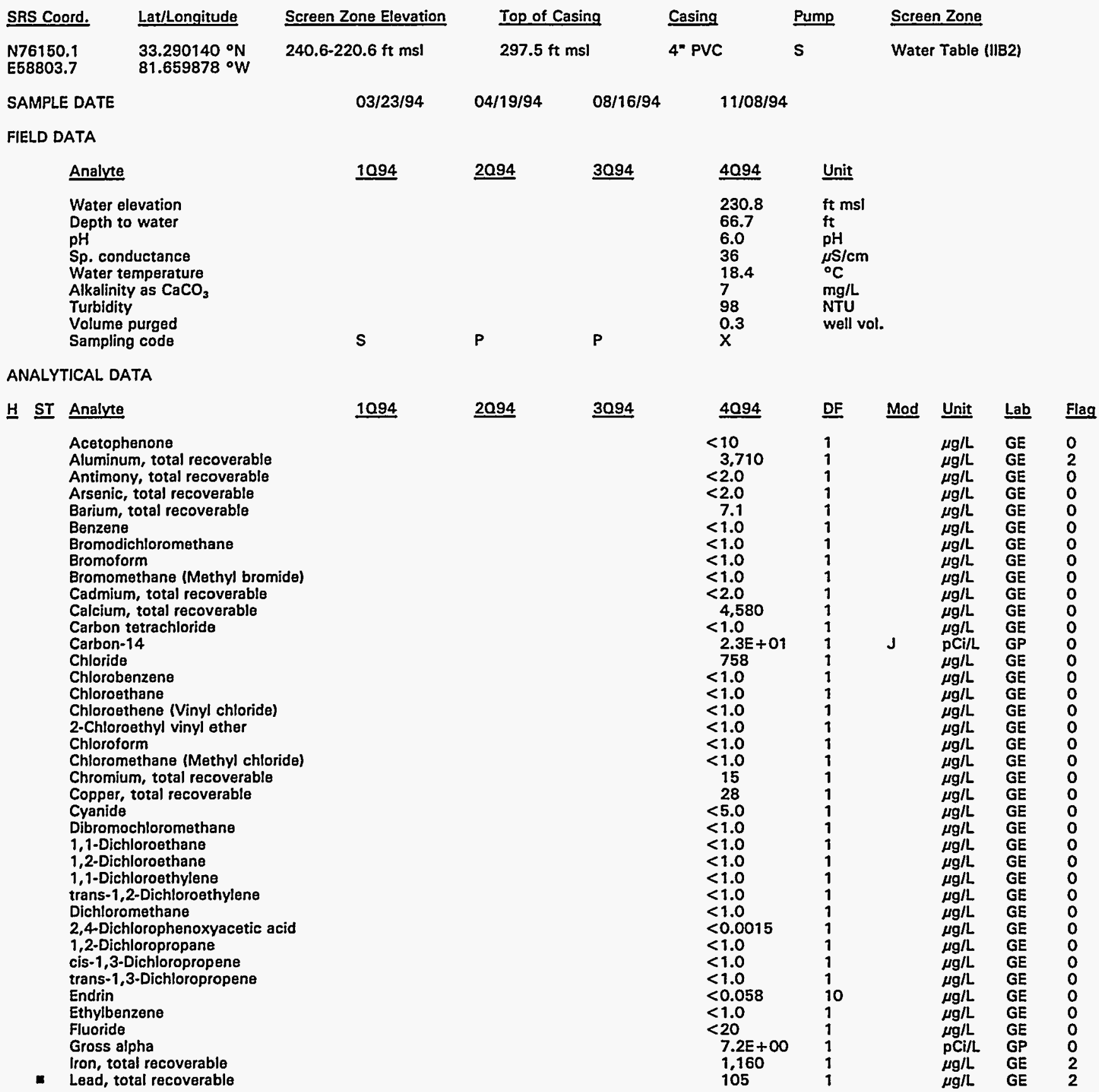

Note: Flagging, dilution factors, modifiers, and laboratories are for fourth quarter 1994 data only. See Appendix B for flagging criteria. - = exceeded holding time for fourth quarter 1994.

- = exceeded screening level or final PDWS for fourth quarter 1994. 
Well BGO 4D continued

ANALYTICAL DATA

\section{H ST Analyte}

Lindane

Magnesium, total recoverable

Manganese, total recoverable

Mercury, total recoverable

Methoxychlor

Naphthalene

Nickel, total recoverable

Nitrate-nitrite as nitrogen

Nonvolatile beta

- $\quad \mathrm{pH}$

Phenols

Potassium, total recoverable

Radium, total alpha-emitting

Selenium, total recoverable

Silica, total recoverable

Silver, total recoverable

Sodium, total recoverable

Specific conductance

Sulfate

$1,1,2,2$-Tetrachloroethane

Tetrachloroethylene

Tin, total recoverable

Toluene

Total dissolved solids

Total organic carbon

Total organic halogens

Total phosphates (as P)

Toxaphene

2,4,5-TP (Silvex)

1,1,1-Trichloroethane

1,1,2-Trichloroethane

Trichloroethylene

Trichlorofluoromethane

Tritium

Turbidity

Uranium-233/234

Uranium-235

Uranium-238

Vanadium, total recoverable

Xylenes

Zinc, total recoverable

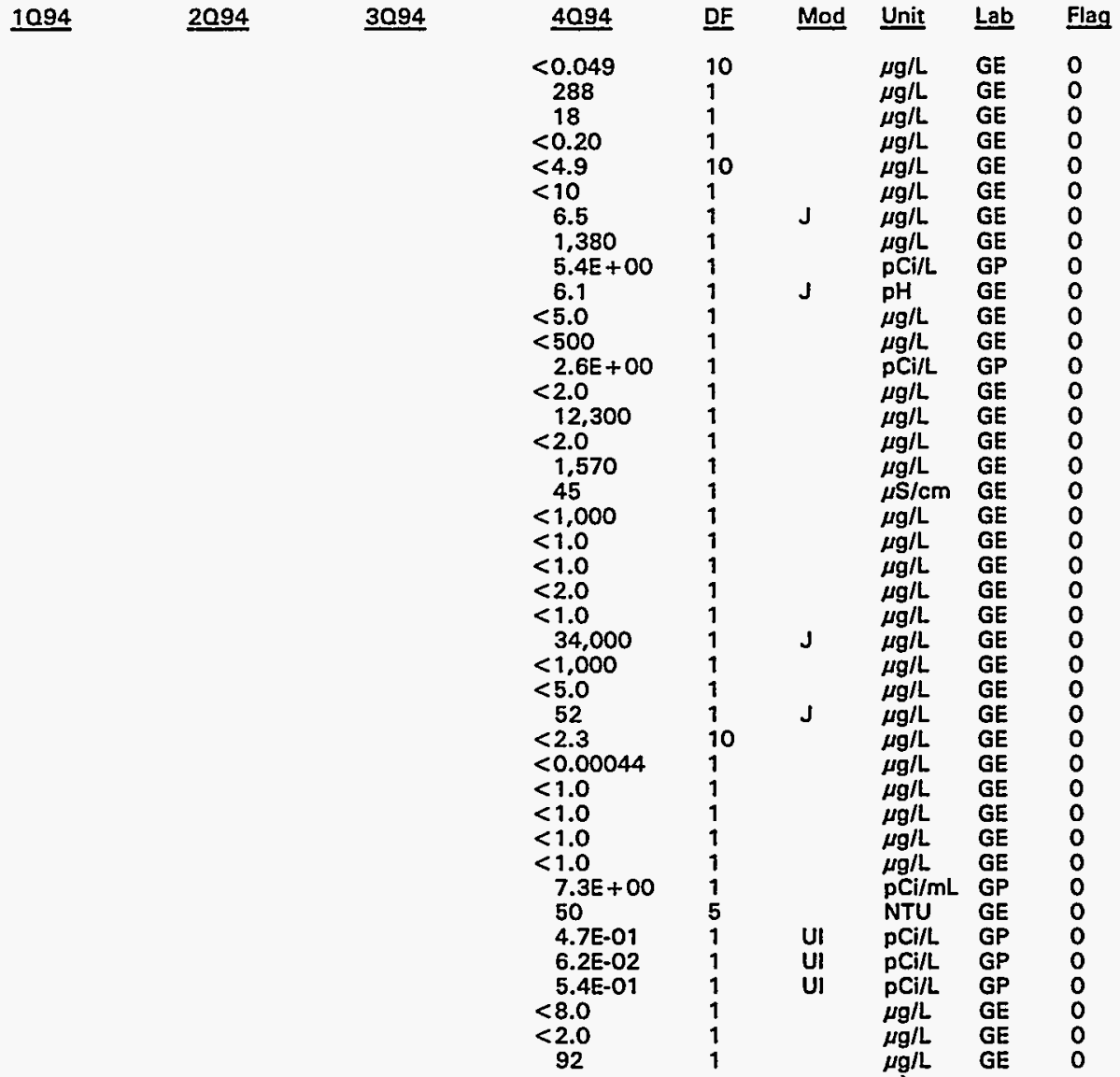

Note: Flagging, dilution factors, modifiers, and laboratories are for fourth quarter 1994 data only. See Appendix B for flagging criteria. - = exceeded holding time for fourth quarter 1994.

a = exceeded screening level or final PDWS for fourth quarter 1994. 


\section{WELL BGO 5C}

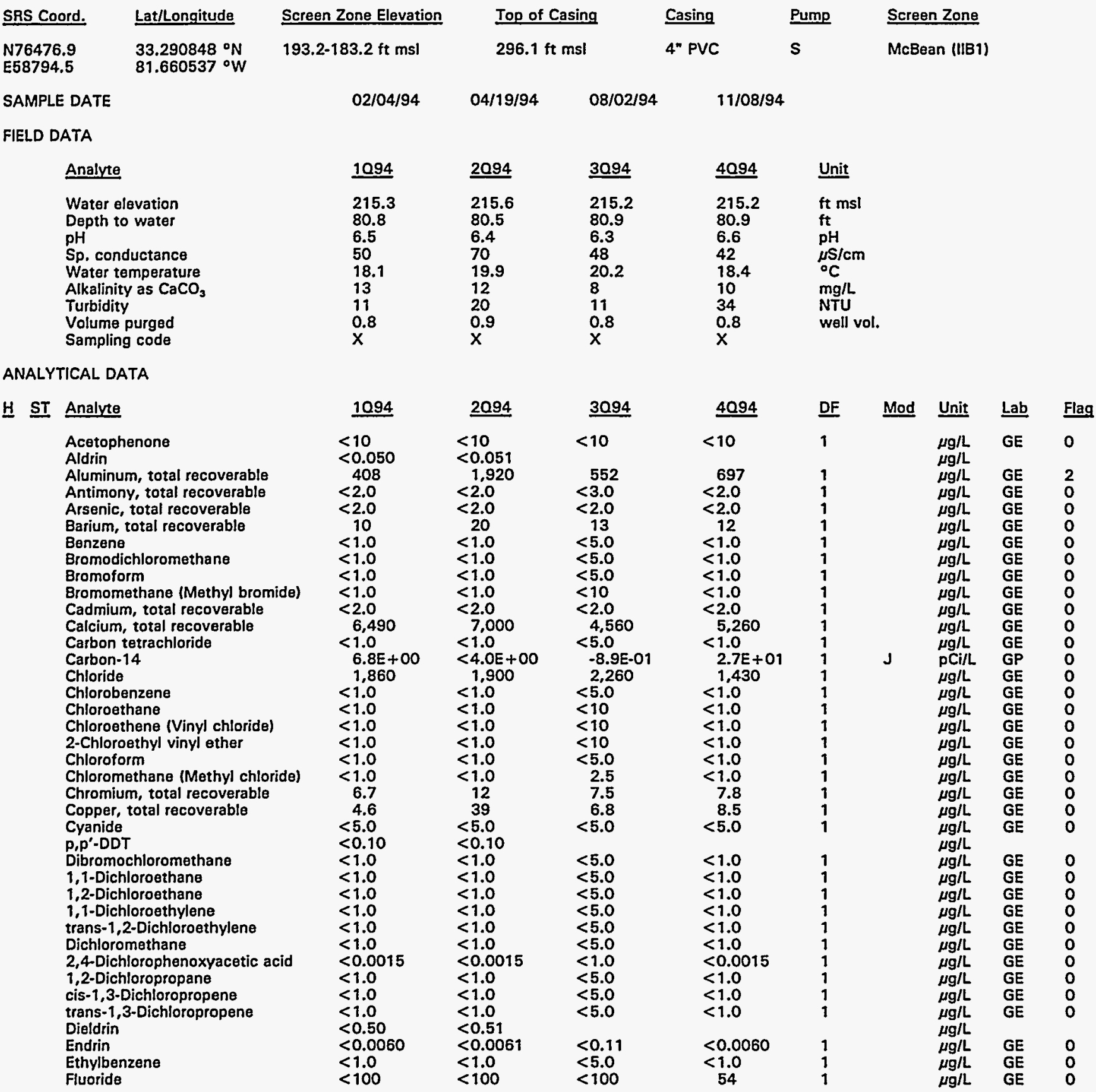

Note: Flagging, dilution factors, modifiers, and laboratories are for fourth quarter 1994 data only. See Appendix B for flagging criteria. - = exceeded holding time for fourth quarter 1994.

- = exceeded screening level or final PDWS for fourth quarter 1994. 
H ST Analyte

Gross alpha

Heptachlor

Iron, total recoverable

Lead, total recoverable

Lindane

Lithium, total recoverable

Magnesium, total recoverable

Manganese, total recoverable

Mercury, total recoverable

Methoxychior

Naphthalene

Nickel, total recoverable

Nitrate as nitrogen

Nitrate-nitrite as nitrogen

Nonvolatile beta

- $\quad \mathrm{pH}$

Phenols

Potassium, total recoverable

Radium, total alpha-emitting

Selenium, total recoverable

Silica, total recoverable

Silver, total recoverable

Sodium, total recoverable

Specific conductance

Sulfate

1,1,2,2-Tetrachloroethane

Tetrachloroethylene

Tin, total recoverable

Toluene

Total dissolved solids

Total organic carbon

Total organic halogens

Total phosphates (as P)

Toxaphene

2,4,5-TP (Silvex)

1,1,1-Trichloroethane

1,1,2-Trichloroethane

Trichloroethylene

Trichlorofluoromethane

2,4,5-T

- Tritium

Turbidity

Uranium-233/234

Uranium-235

Uranium-238

Vanadium, total recoverable

Xylenes

Zinc, total recoverable

\begin{tabular}{|c|c|c|}
\hline 1094 & $\underline{2094}$ & 3094 \\
\hline $\begin{array}{l}1.6 E+00 \\
<0.050\end{array}$ & $\begin{aligned} & 1.5 E+00 \\
< & 0.051\end{aligned}$ & $9.0 E-01$ \\
\hline 44 & 338 & 363 \\
\hline $\begin{array}{l}<3.0 \\
<0.0050\end{array}$ & 9.0 & 3.6 \\
\hline $\begin{array}{l}<0.0050 \\
<5.0\end{array}$ & $<0.0051$ & $\begin{array}{l}<0.053 \\
<5.0\end{array}$ \\
\hline $\begin{array}{l}412 \\
17\end{array}$ & $\begin{array}{l}588 \\
108\end{array}$ & $\begin{array}{l}358 \\
16\end{array}$ \\
\hline$<0.20$ & $<0.20$ & $<0.20$ \\
\hline$<0.50$ & $<0.51$ & $<0.53$ \\
\hline$<10$ & $<10$ & $<10$ \\
\hline$<4.0$ & 7.1 & $\begin{array}{l}4.1 \\
1,190\end{array}$ \\
\hline 970 & 990 & \\
\hline $\begin{array}{l}2.0 E+00 \\
6.5\end{array}$ & $\begin{array}{l}1.9 E+00 \\
6.3\end{array}$ & $\begin{array}{l}6.3 \mathrm{E}-01 \\
6.3\end{array}$ \\
\hline$<5.0$ & $<5.0$ & $<5.0$ \\
\hline $\begin{array}{l}<500 \\
1.8 E+00\end{array}$ & $\begin{array}{l}<500 \\
<-2.0 \mathrm{E}-01\end{array}$ & $\begin{array}{l}<500 \\
8.0 E-01\end{array}$ \\
\hline$<2.0$ & $<2.0$ & $<2.0$ \\
\hline $\begin{aligned} & 13,500 \\
< & 2.0\end{aligned}$ & $\begin{array}{l}15,000 \\
<2.0\end{array}$ & $\begin{aligned} & 10,700 \\
&<2.0\end{aligned}$ \\
\hline $\begin{array}{l}1,890 \\
47\end{array}$ & $\begin{array}{l}1,830 \\
40\end{array}$ & $\begin{array}{l}1,620 \\
37\end{array}$ \\
\hline$<1,000$ & $<1,000$ & $<1,000$ \\
\hline$<1.0$ & $<1.0$ & $<5.0$ \\
\hline$<1.0$ & $<1.0$ & $<5.0$ \\
\hline$<2.0$ & $<2.0$ & $<17$ \\
\hline$<1.0$ & $<1.0$ & $<5.0$ \\
\hline $\begin{array}{l}49,000 \\
1,060\end{array}$ & $\begin{array}{r}34,000 \\
<1,000\end{array}$ & $\begin{array}{r}50,000 \\
<1,000\end{array}$ \\
\hline 6.0 & $<5.0$ & 13 \\
\hline 130 & 1,000 & 0.17 \\
\hline$<0.24$ & $<0.24$ & $<1.1$ \\
\hline$<0.00044$ & $<0.00045$ & $<0.52$ \\
\hline$<1.0$ & $<1.0$ & $<5.0$ \\
\hline$<1.0$ & $<1.0$ & $<5.0$ \\
\hline$<1.0$ & $<1.0$ & $<5.0$ \\
\hline$<1.0$ & $<1.0$ & $<5.0$ \\
\hline $2.9 E+01$ & $\begin{array}{c}<0.00045 \\
2.9 E+01\end{array}$ & $3.1 E+01$ \\
\hline 24 & 217 & 190 \\
\hline$<7.9 E-02$ & $<0.0 E+00$ & 1.2E-01 \\
\hline$<7.9 E-02$ & $\begin{array}{l}<-6.3 E-03 \\
<2.5 E-02\end{array}$ & $\begin{array}{l}0.0 E+00 \\
5.4 E-02\end{array}$ \\
\hline$<8.0$ & $<8.0$ & $<3.0$ \\
\hline$<2.0$ & $<2.0$ & $<5.0$ \\
\hline & & \\
\hline
\end{tabular}

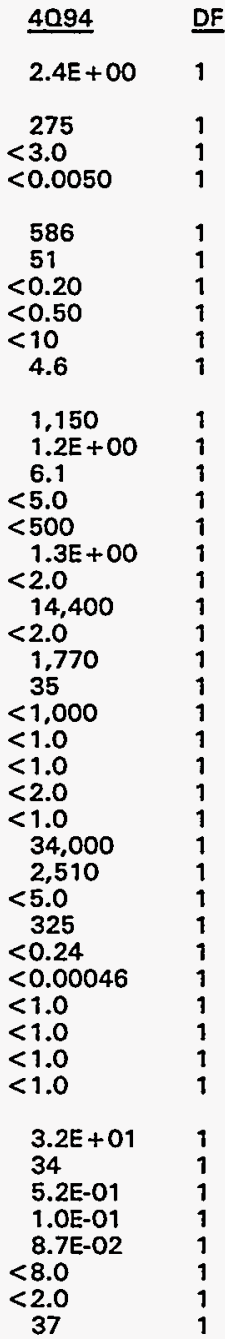

Mod

Unit Lab Flag

$\mathrm{pCi} / \mathrm{L}$ GP 0

$\mu \mathrm{g} / \mathrm{L} \quad \mathrm{GE} \quad 1$

$\mu \mathrm{g} / \mathrm{L} \quad \mathrm{GE} \quad 0$

$\mu \mathrm{g} / \mathrm{L}$

$\mu g / L \quad$ GE 0

$\mu g / L$ GE 2

$\mu \mathrm{g} / \mathrm{L}$

$\mu g / L \quad G E$

$\mu \mathrm{g} / \mathrm{L} \quad \mathrm{GE}$

$J \quad \mu \mathrm{g} / \mathrm{L} \quad \mathrm{GE}$

$\mu \mathrm{g} / \mathrm{L}$

UI $\mu \mathrm{g} / \mathrm{L}$ GE $\quad 0$

J $\mathrm{pCH}$

$\underset{\mu g / L}{\mathrm{pH}}$

$\mu \mathrm{g} / \mathrm{L}$

$\mu g$

$\mu \mathrm{g} / \mathrm{L}$

$\mu \mathrm{g} / \mathrm{L}$

$\mu \mathrm{g} / \mathrm{L}$

$\mu \mathrm{S} / \mathrm{cm} \quad \mathrm{GE}$

$\mu \mathrm{g} / \mathrm{L} \quad \mathrm{GE}$

$\mu \mathrm{g} / \mathrm{L}$

$\mu g / L$

gg/L

$\mu g / L$

$\mu \mathrm{g} / \mathrm{L} \quad \mathrm{GE}$

$\mu \mathrm{g} / \mathrm{L} \quad \mathrm{GE}$

$\mu \mathrm{g} / \mathrm{L} \quad \mathrm{GE}$

$\mu \mathrm{g} / \mathrm{L} \quad \mathrm{GE}$

$\mu \mathrm{g} / \mathrm{L} \quad \mathrm{GE}$

$\mu g / L$

$\mu g$

$\mu \mathrm{g} / \mathrm{L} \quad \mathrm{G}$

$\mu g / L \quad G E$

$\underset{\mu g / L}{\mu g} \quad \mathrm{GE}$

$\mu \mathrm{g} / \mathrm{L}$

$\mathrm{pCi} / \mathrm{mL} \quad \mathrm{GP}$

NTU GE

บ1

UI
$\mathrm{pC}$

$\mathrm{pCi} / L$

$\mu \mathrm{g} / \mathrm{L}$

$\underset{\mu g / L}{\mu g / L}$ 
WELL BGO 5D

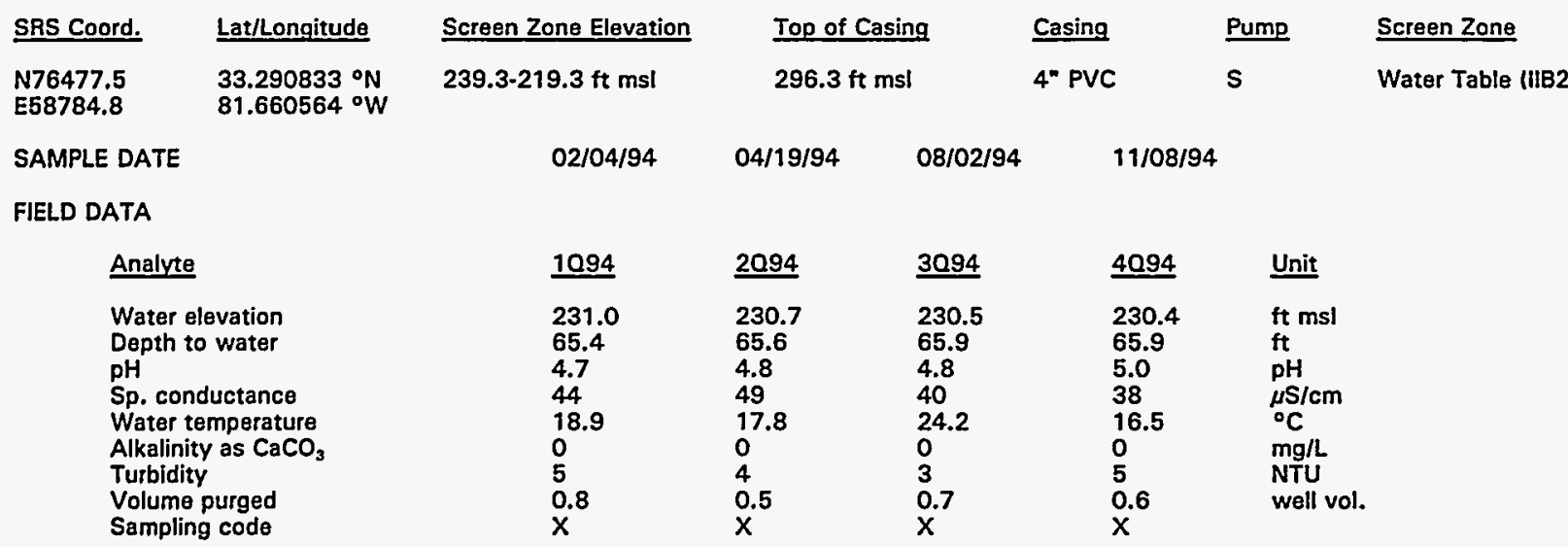

ANALYTICAL DATA

H ST Analyte

Acetophenone

Aldrin

Aluminum, total recoverable

Antimony, total recoverable

Arsenic, total recoverable

Barium, total recoverable

Benzene

Bromodichloromethane

Bromoform

Bromomethane (Methyl bromide)

Cadmium, total recoverable

Calcium, total recoverable

Carbon tetrachloride

Carbon-14

Chloride

Chlorobenzene

Chloroethane

Chloroethene (Vinyl chloride)

2-Chloroethyl vinyl ether

Chloroform

Chloromethane (Methyl chloride)

Chromium, total recoverable

Copper, total recoverable

Cyanide

$p, p^{\prime}-D D T$

Dibromochloromethane

1,1-Dichloroethane

1,2-Dichloroethane

1,1-Dichloroethylene

trans-1,2-Dichloroethylene

Dichloromethane

2,4-Dichlorophenoxyacetic acid

1,2-Dichloropropane

cis-1,3-Dichloropropen

trans-1,3-Dichloropropene

Dieldrin

Endrin

Ethylbenzene

Fluoride

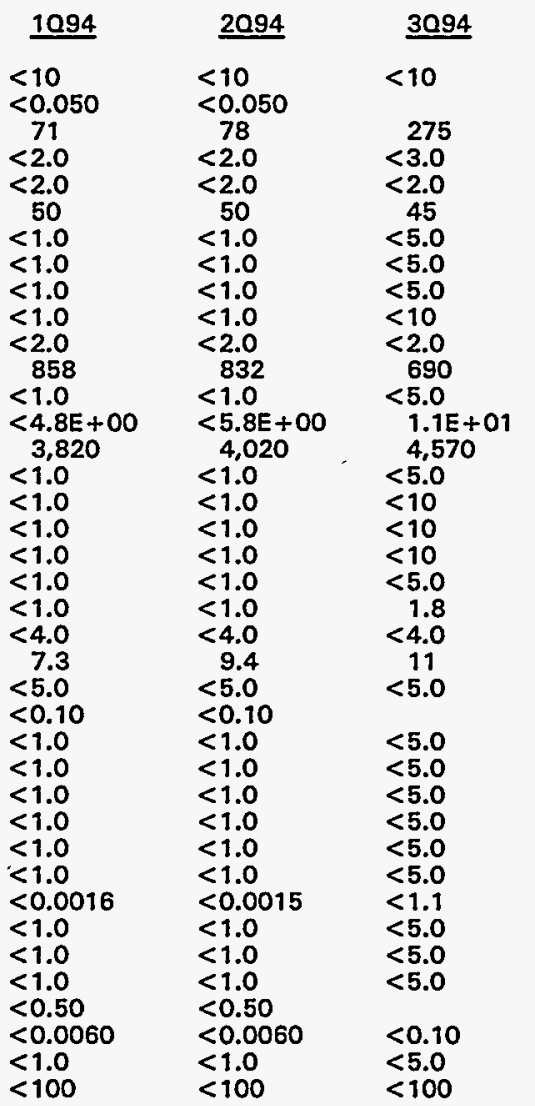

$\begin{aligned} & 4094 \\ &<9.8 \\ & \\ & \quad 61 \\ &<2.0 \\ &<2.0 \\ & 47 \\ &<1.0 \\ &<1.0 \\ &<1.0 \\ &<1.0 \\ &<2.0 \\ & 696 \\ &<1.0 \\ & 2.5 E+01 \\ & 4.040 \\ &<1.0 \\ &<1.0 \\ &<1.0 \\ &<1.0 \\ &<1.0 \\ &<1.0 \\ &<4.0 \\ &<15 \\ &<5.0 \\ &<1.0 \\ &<1.0 \\ &<1.0 \\ &<1.0 \\ &<1.0 \\ &<1.0 \\ &<0.0015 \\ &<1.0 \\ &<1.0 \\ &<1.0 \\ &<0.0059 \\ &<1.0 \\ &<20 \\ &\end{aligned}$

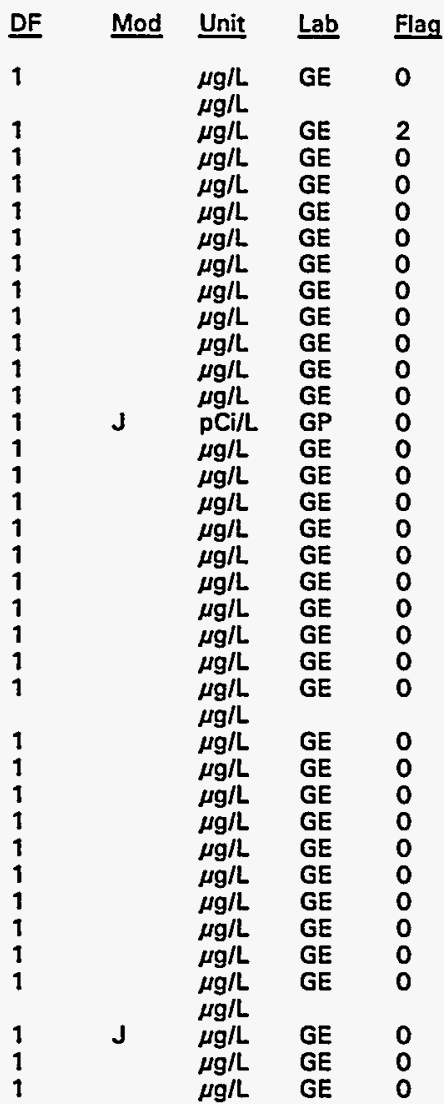

Note: Flagging, dilution factors, modifiers, and laboratories are for fourth quarter 1994 data only. See Appendix B for flagging criteria. - = exceeded holding time for fourth quarter 1994.

- = exceeded screening level or final PDWS for fourth quarter 1994: 


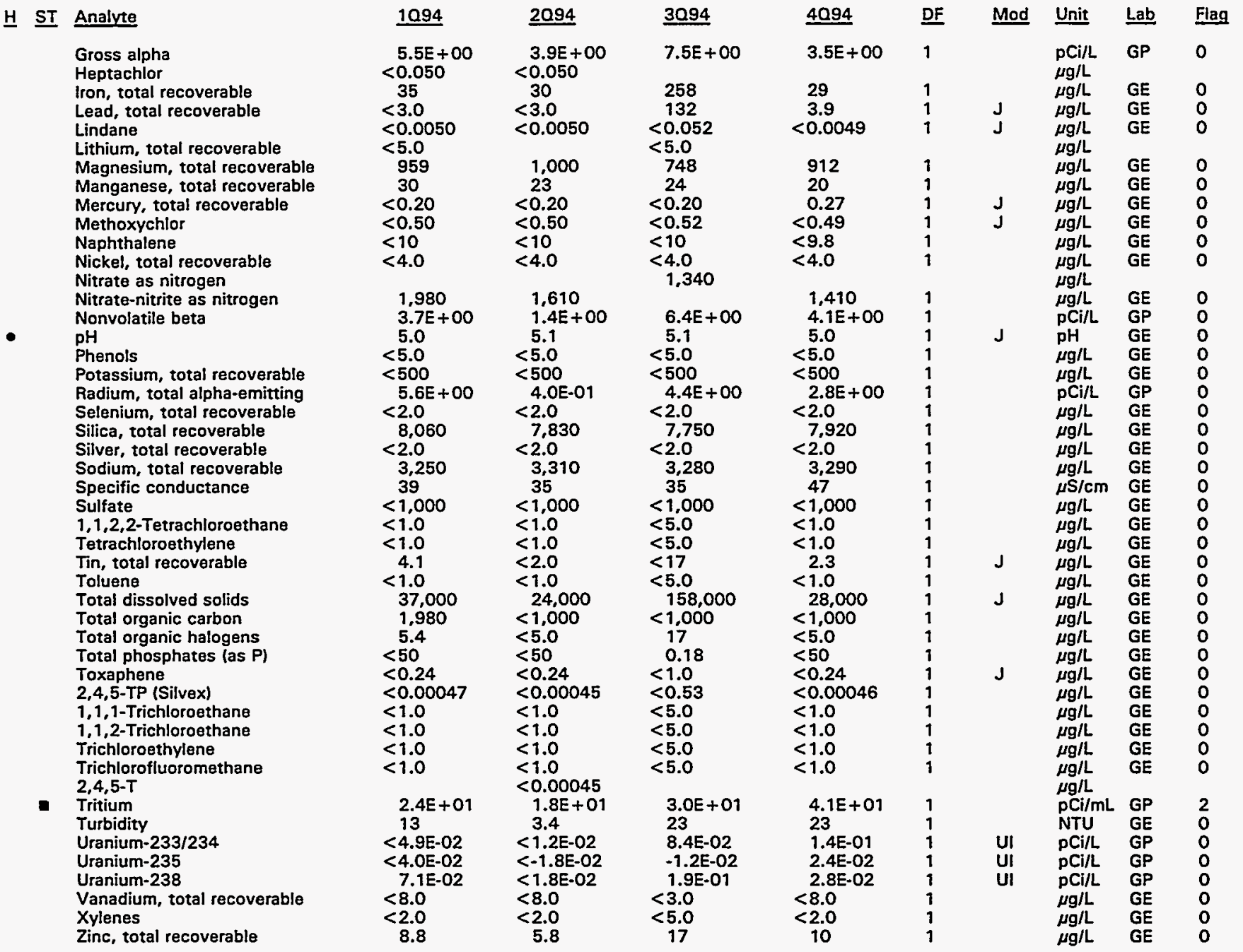

Note: Flagging, dilution factors, modifiers, and laboratories are for fourth quarter 1994 data only. See Appendix B for flagging criteria. - = exceeded holding time for fourth quarter 1994.

- = exceeded screening level or final PDWS for fourth quarter 1994. 


\section{WELL BGO 6A}

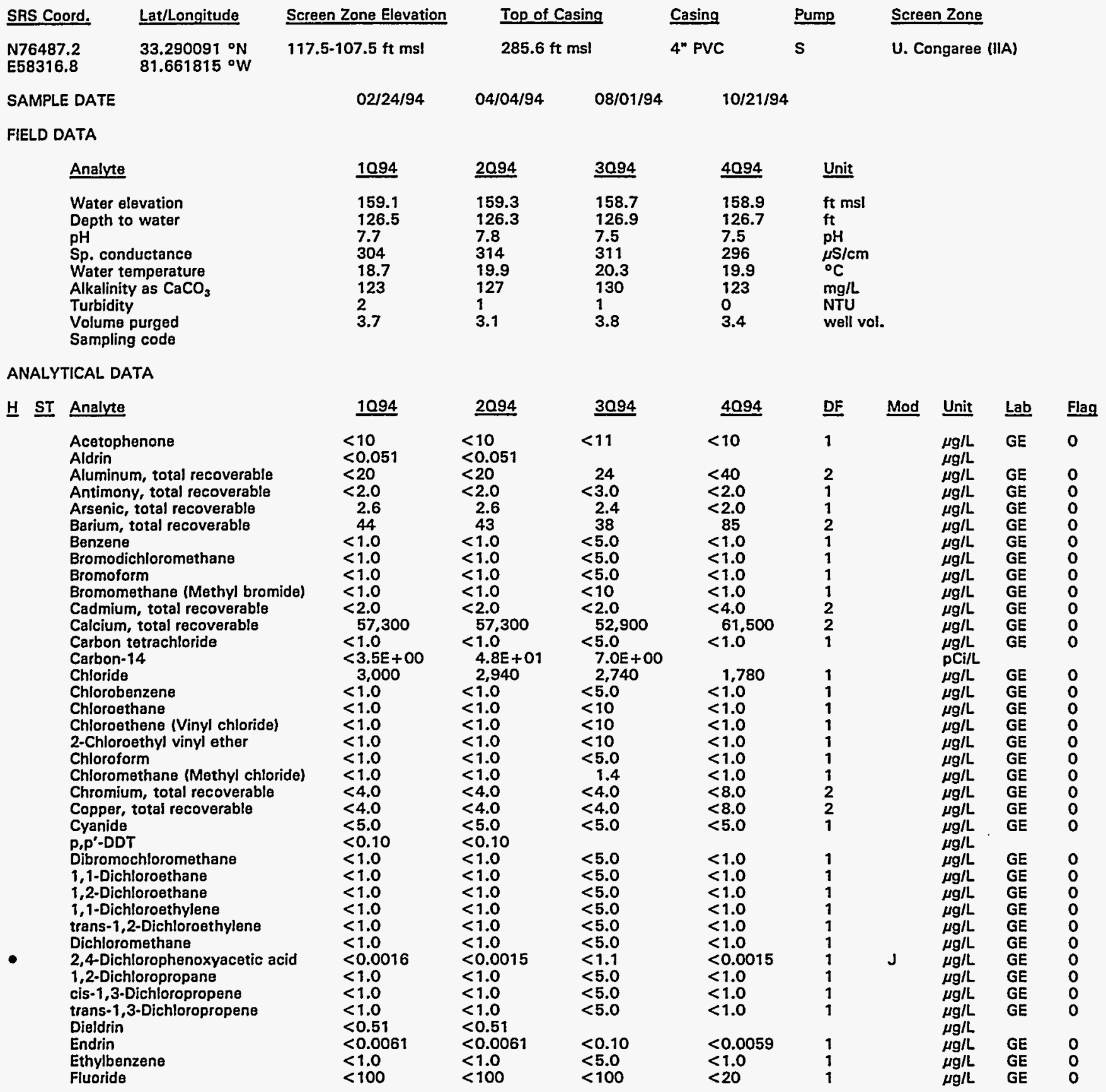

Note: Flagging, dilution factors, modifiers, and laboratories are for fourth quarter 1994 data only. See Appendix B for flagging criteria. - = exceeded holding time for fourth quarter 1994.

- = exceeded screening level or final PDWS for fourth quarter 1994. 
H ST Analyte

Gross alpha

Heptachlor

Iron, total recoverable

Lead, total recoverable

Lindane

Lithium, total recoverable

Magnesium, total recoverable

Manganese, total recoverable

Mercury, total recoverable

Methoxychlor

Naphthalen

Nitrate as nitrogen

Nitrate-nitrite as nitrogen

Nonvolatile beta

$\mathrm{pH}$

Phenols

Potassium, total recoverable

Radium, total alpha-emitting

Selenium, total recoverable

Silica, total recoverable

Silver, total recoverable

Sodium, total recoverable

Specific conductance

Sulfate

1,1,2,2-Tetrachloroethane

Tetrachloroethylene

Tin, total recoverable

Toluene

Total dissolved solids

Total organic carbon

Total organic halogens

Total phosphates (as P)

Toxaphene

- 2,4,5-TP (Silvex)

1,1,1-Trichloroethane

1,1,2-Trichloroethane

Trichloroethylene

Trichlorofluoromethane

$2,4,5-\mathrm{T}$

Tritium

- Turbidity

Uranium-233/234

Uranium-235

Uranium-238

Vanadium, total recoverable

Xylenes

Zinc, total recoverable
1094

$<2.6 E-01$

$<0.051$

$<3.0$

$<0.0051$

$<5.0$

1,470

4.6

$<0.20$

$<0.51$

$<10$

$<4.0$

$<50$

2.1E+00

2.1
7.5
$<5.0$

$<5.0$

835

6.0E-01

$<2.0$

41,400

$<2.0$

2,290

297

7,920

$<1.0$

$<1.0$

$<1.0$

208,000

$<1,000$

$<1,000$
$<50$

$<0.25$

$<0.00047$

$<1.0$

$<1.0$

$<1.0$

$<1.0$

1.4E-02

1.1

8.0E-01

$1.9 E+00$

1. $9 E+00$

$<8.0$

$<2.0$

$<2.0$

$\frac{20.94}{1.0 E+00}$
$<0.051$

3094

7.1E-01

45

$<3.0$

$<0.0051$

1,490

3.4

$<0.20$

$<0.51$

$<10$

$<4.0$

$<50$

$1.1 \mathrm{E}+00$

7.5

$<5.0$

739

$<1.0 E-01$

$<2.0$

40,900

$<2.0$

2,270

300

8,400

$<1.0$

$<1.0$

$<2.0$

$<1.0$

205,000

1,750

$<5.0$

$<50$

$<0.24$

$<0.00046$

$<1.0$

$<1.0$

$<1.0$

$<1.0$

0.36

$<1.3 \mathrm{E}-01$

0.22

2.2E-01

$<2.5 \mathrm{E}-02$

1.6E-01

$<8.0$

$<2.0$

$<2.0$
$<55$

$<3.0$

$<0.052$

7.6

1,260

3.8
$<0.20$

$<0.20$

$<10$

$<4.0$

$<20$

$1.0 E+00$

7.5
$<5.0$

619

1.0E-01

$<2.0$

37,600

$<2.0$

1,950

298

8,990

$<5.0$

$<5.0$

25

$<5.0$

205,000

$<1,000$

$<6.7$

99

$<1.0$

$<5.0$

$<5.0$

$<5.0$

$<5.0$

2.7E-01

0.51

2.2E-01

$-1.4 \mathrm{E}-02$

9.9E-02

$<3.0$

$<5.0$

$<49$
4094 DF Mod Unit Lab Flag

$1.1 E+00$

91

$<3.0$

$<0.0049$

2,940

5.5

$<0.20$

$<0.49$

$<10$

$<8.0$

$<50$

$1.8 E+00$

7.6

$<5.0$

1,580

$0.0 \mathrm{E}+\infty$

$<2.0$

83,600

$<4.0$

4,380

301

7,380

$<1.0$

$<1.0$

$<4.0$

$<1.0$

208,000

1,880

$<5.0$

$<50$

$<0.24$

0.0081

$<1.0$

$<1.0$

$<1.0$

$$
\text { 4.1E-01 }
$$

0.20

$<16$

$<2.0$

$<4.0$

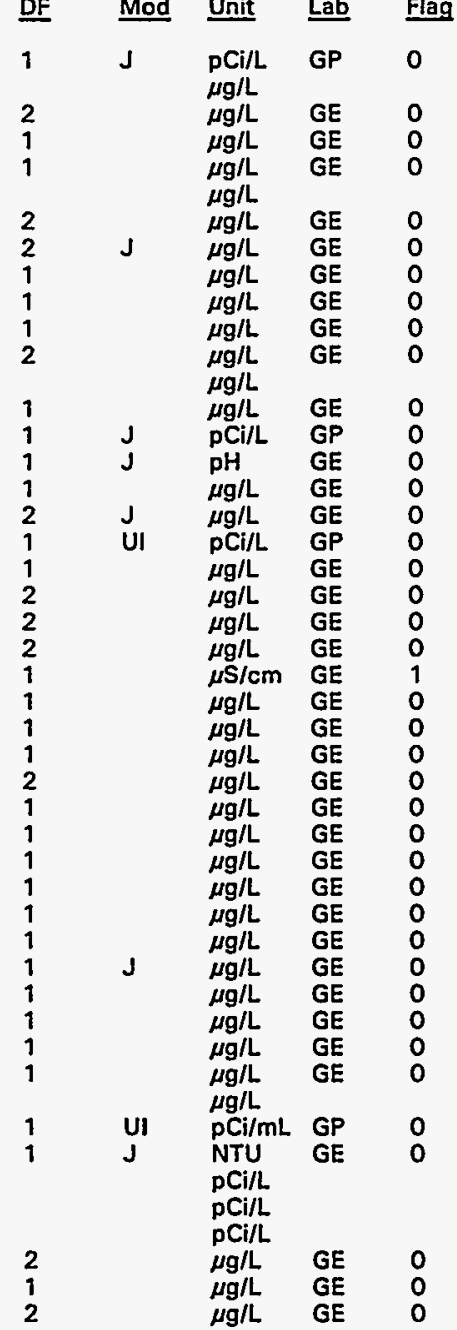

Note: Flagging, dilution factors, modifiers, and laboratories are for fourth quarter 1994 data only. See Appendix B for flagging criteria.

- = exceeded holding time for fourth quarter 1994.

I = exceeded screening level or final PDWS for fourth quarter 1994. 
WELL BGO 6B

\section{SRS Coord. Lat/Longitude \\ N76553.2 \\ E58346.5 \\ $33.290286^{\circ} \mathrm{N}$
$81.661865^{\circ} \mathrm{W}$}

SAMPLE DATE

FIELD DATA

Analyte
Water elevation
Depth to water
pH
Sp. conductance
Water temperature
Alkalinity as $\mathrm{CaCO}_{3}$
Turbidity
Volume purged
Sampling code

ANALYTICAL DATA

H ST Analyte

Acetophenone
Aldrin
Aluminum, total recoverable
Antimony, total recoverable
Arsenic, total recoverable
Barium, total recoverable
Benzene
Bromodichloromethane
Bromoform
Bromomethane (Methyl bromide)
Cadmium, total recoverable
Calcium, total recoverable
Carbon tetrachloride
Chloride
Chlorobenzene
Chloroethane
Chloroethene (Vinyl chloride)
2-Chloroethyl vinyl ether
Chloroform
Chloromethane (Methyl chloride)
Chromium, total recoverable
Copper, total recoverable
Cyanide
p,p'-DDT
Dibromochloromethane
1,1-Dichloroethane
1,2-Dichloroethane
1,1-Dichloroethylene
trans-1,2-Dichloroethylene
Dichloromethane
$2,4-$ Dichlorophenoxyacetic acid
1,2-Dichloropropane
cis-1,3-Dichloropropene
trans-1,3-Dichloropropene
Dieldrin
Endrin
Ethylbenzene
Fluoride
Gross alpha

\begin{tabular}{|c|c|c|c|c|}
\hline Screen Zone Elevation & Top of Casing & Casing & Pump & Screen Zone \\
\hline 149.7-139.7 ft msl & $286.8 \mathrm{ft} \mathrm{msl}$ & 4" PVC & $\mathbf{S}$ & M. B/McBean (IIB1) \\
\hline $01 / 25 / 94$ & $04 / 05 / 94$ & & & \\
\hline
\end{tabular}

$\begin{array}{lllll}1094 & \underline{2094} & \underline{3094} & \underline{4094} & \text { Unit } \\ 219.0 & 218.3 & 218.0 & 218.6 & \mathrm{ft} \mathrm{msl} \\ 67.8 & 68.5 & 68.9 & 68.2 & \mathrm{ft} \\ 9.5 & 8.6 & 8.9 & 8.7 & \mathrm{pH} \\ 192 & 210 & 185 & 180 & \mathrm{fS} / \mathrm{cm} \\ 18.8 & 18.3 & 20.3 & 19.0 & { }^{\circ} \mathrm{C} \\ 70 & 73 & 70 & 71 & \mathrm{mg} / \mathrm{L} \\ 6 & 2 & 2 & 1 & \mathrm{NTU} \\ 0.8 & 0.8 & 0.8 & 1.0 & \text { well vol. } \\ X & X & X & X & \end{array}$

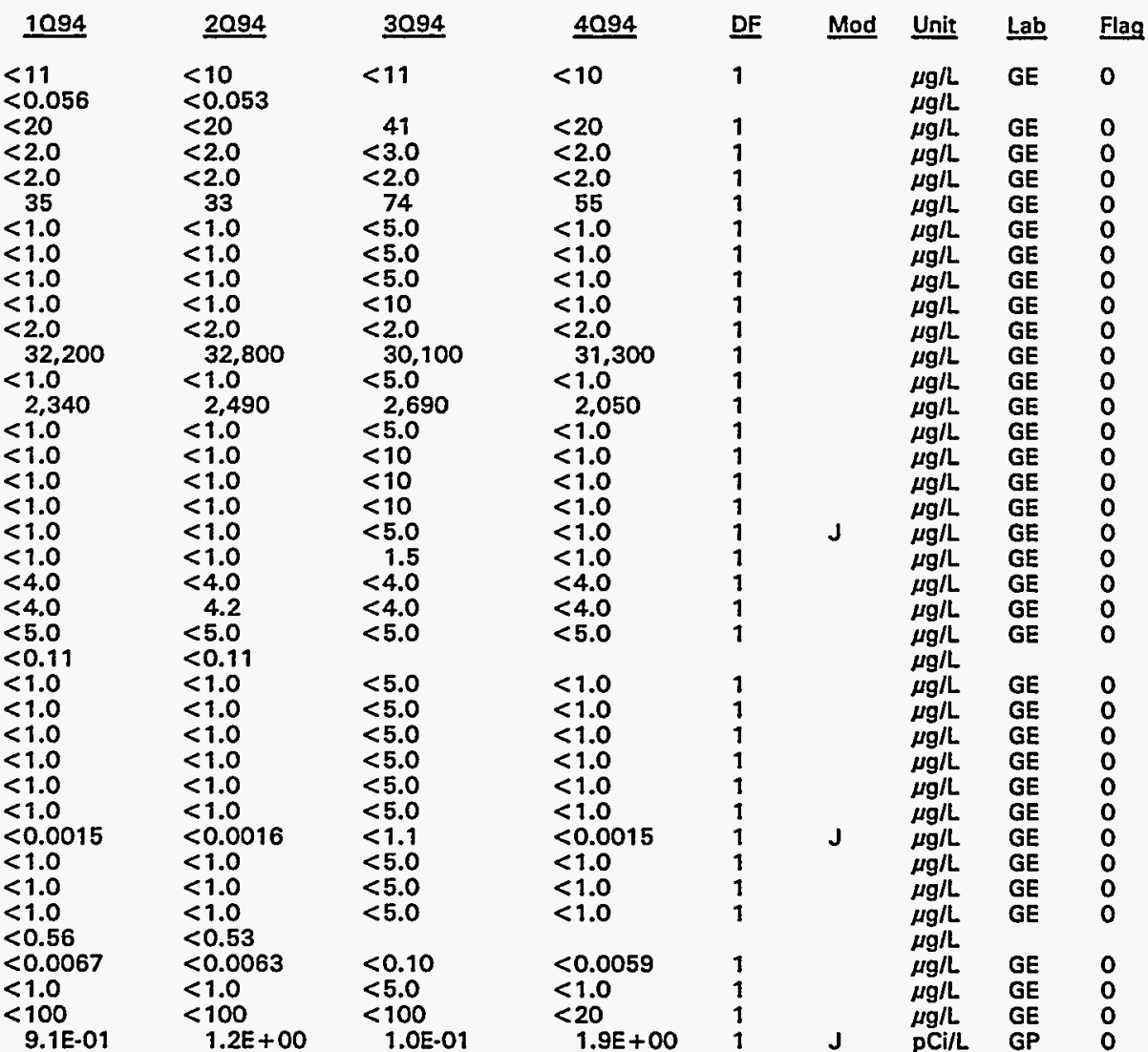

Note: Flagging, dilution factors, modifiers, and laboratories are for fourth quarter 1994 data only. See Appendix B for flagging criteria. - = exceeded holding time for fourth quarter 1994.

n = exceeded screening level or final PDWS for fourth quarter 1994. 
H ST Analyte

Heptachlor

Iron, total recoverable

Lead, total recoverable

Lindane

Lithium, total recoverable

Magnesium, total recoverable

Manganese, total recoverable

Mercury, total recoverable

Methoxychlor

Naphthalene

Nickel, total recoverable

Nitrate as nitrogen

Nitrate-nitrite as nitrogen

Nonvolatile bete

- $\mathrm{pH}$

Phenols

Potassium, total recoverable

Radium, total alpha-emitting

Selenium, total recoverable

Silica, total recoverable

Silver, total recoverable

Sodium, total recoverable

Specific conductance

Sulfate

1,1,2,2-Tetrachloroethane

Tetrachloroethylene

Tin, total recoverable

Toluene

Total dissolved solids

Total organic carbon

Total organic halogens

Total phosphates (as $\mathrm{P}$ )

Toxaphene

2,4,5-TP (Silvex)

1,1,1-Trichloroethane

1,1,2-Trichloroethane

Trichloroethylene

Trichlorofluoromethane

2,4,5-T

- Tritium

Turbidity

Uranium-233/234

Uranium-235

Uranium-238

Vanadium, total recoverable

Xylenes

\begin{tabular}{|c|c|}
\hline 10.94 & 2094 \\
\hline $\begin{array}{c}<0.056 \\
348 \\
7.3\end{array}$ & $\begin{array}{c}<0.053 \\
99 \\
<3.0\end{array}$ \\
\hline$<0.0056$ & $<0.0053$ \\
\hline $\begin{array}{l}866 \\
5.3\end{array}$ & $\begin{array}{l}828 \\
3.1\end{array}$ \\
\hline$<0.20$ & $<0.20$ \\
\hline$<0.56$ & $<0.53$ \\
\hline $\begin{array}{l}<11 \\
<4.0\end{array}$ & $\begin{array}{l}<10 \\
<4.0\end{array}$ \\
\hline 320 & 630 \\
\hline $\begin{array}{l}1.6 E+00 \\
6.8\end{array}$ & $\begin{array}{l}3.7 E+00 \\
8.5\end{array}$ \\
\hline$<5.0$ & $<5.0$ \\
\hline $\begin{array}{l}2,240 \\
9.0 \mathrm{E}-01\end{array}$ & $\begin{array}{l}2,230 \\
5.0 \mathrm{E}-01\end{array}$ \\
\hline $\begin{array}{l}<2.0 \\
19,300\end{array}$ & $\begin{array}{l}<2.0 \\
18.600\end{array}$ \\
\hline$<2.0$ & $<2.0$ \\
\hline $\begin{array}{l}4,790 \\
193\end{array}$ & $\begin{array}{l}4,570 \\
193\end{array}$ \\
\hline 5,100 & 4,940 \\
\hline$<1.0$ & $<1.0$ \\
\hline$<1.0$ & $<1.0$ \\
\hline 3.1 & 4.2 \\
\hline$<1.0$ & $<1.0$ \\
\hline $\begin{aligned} & 127,000 \\
&< 1,000 \\
& 10,300\end{aligned}$ & $\begin{aligned} & 126,000 \\
< & 1,560 \\
< & 5.0\end{aligned}$ \\
\hline $\begin{array}{l}10,300 \\
<50\end{array}$ & $\begin{array}{l}<5.0 \\
<50\end{array}$ \\
\hline$<0.27$ & $<0.25$ \\
\hline$<0.00046$ & $<0.00047$ \\
\hline$<1.0$ & $<1.0$ \\
\hline$<1.0$ & $<1.0$ \\
\hline$<1.0$ & $<1.0$ \\
\hline$<1.0$ & $<1.0$ \\
\hline $2.1 E$ & $\begin{array}{l}0.38 \\
2.4 E+01\end{array}$ \\
\hline 1.2 & 0.60 \\
\hline$<-6.9 \mathrm{E}-0$ & 2.2E-01 \\
\hline $\begin{array}{l}<0.0 \mathrm{E}+00 \\
<-1.4 \mathrm{E}-02\end{array}$ & $<-1.2 \mathrm{E}-03$ \\
\hline $\begin{array}{l}<-1.4 \\
<8.0\end{array}$ & $\begin{array}{l}\text { 7E-01 } \\
0\end{array}$ \\
\hline$<2.0$ & $<2.0$ \\
\hline
\end{tabular}

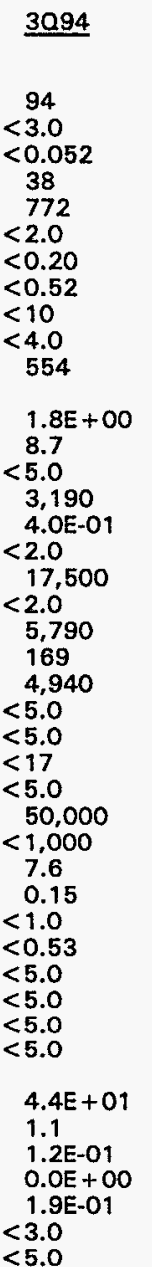

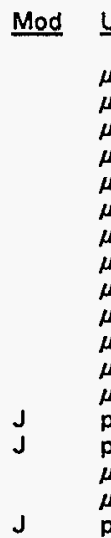



$\mu g / L$

$\mu / L$ GE 0

$\mu \mathrm{g} / \mathrm{L}$ GE 0

$\mu g / L$

$\mu g / L$

$\mu \mathrm{g} / \mathrm{L}$

$\mu g / L$

$\mu g / L$

$\mu g / L$

$\mu \mathrm{g} / \mathrm{L}$
$\mathrm{pCi} / \mathrm{L}$
$\mathrm{pH}$

$\mu g / L$

pCi/L

$1.0 \mathrm{E}+00$

$<2.0$

18,900

4,620

179

3,440

$<1.0$

$<1.0$

$<1.0$

119,000

1,460

$<5.0$

$<50$

$<0.24$

$<0.00045$

$<1.0$

$<1.0$

$<1.0$

$<1.0$

$3.7 E+01$

0.49

2. OE-O1

5.1E-02

8.2E-02

$<8.0$

$<8.0$

Pill

$\mu \mathrm{g} / \mathrm{L}$

$\mu g / L$

.




\section{WELL BGO 6C}

\begin{tabular}{|c|c|c|c|c|c|c|c|}
\hline SRS Coord. & Lat/Longitude & Screan Zone Elevation & Top of & & Casing & Pump & Screen Zone \\
\hline $\begin{array}{l}\text { N76487.1 } \\
\text { E58307.0 }\end{array}$ & $\begin{array}{l}33.290075 \circ \mathrm{N} \\
81.661841^{\circ} \mathrm{W}\end{array}$ & $168.0-158.0 \mathrm{ft} \mathrm{msl}$ & 285.6 & & 4" PVC & $\mathbf{s}$ & McBean (IIB1) \\
\hline SAMPLE DA & & $01 / 25 / 94$ & $04 / 04 / 94$ & $08 / 01 / 94$ & $10 / 21 / 94$ & & \\
\hline FIELD DATA & & & & & & & \\
\hline An & & 1094 & $\underline{2094}$ & 3094 & 4099 & $\underline{\text { Unit }}$ & \\
\hline $\begin{array}{l}\text { Wa } \\
\text { Der } \\
\text { pH } \\
\text { Sp. } \\
\text { Wa } \\
\text { Alk } \\
\text { Tur } \\
\text { Vol }\end{array}$ & $\begin{array}{l}\text { avation } \\
\text { water } \\
\text { uctance } \\
\text { mperature } \\
\text { as } \mathrm{CaCO}_{3} \\
\text { ourged }\end{array}$ & $\begin{array}{l}220.1 \\
65.5 \\
7.5 \\
136 \\
19.6 \\
53 \\
0 \\
2.6\end{array}$ & $\begin{array}{l}220.1 \\
65.5 \\
7.7 \\
149 \\
20.5 \\
52 \\
0 \\
2.8\end{array}$ & $\begin{array}{l}219.8 \\
65.8 \\
7.4 \\
141 \\
21.0 \\
52 \\
0 \\
2.7\end{array}$ & $\begin{array}{l}219.9 \\
65.7 \\
7.2 \\
126 \\
20.2 \\
52 \\
0 \\
2.7\end{array}$ & $\begin{array}{l}\mathrm{ft} \mathrm{msl} \\
\mathrm{ft} \\
\mathrm{pH} \\
\mu \mathrm{S} / \mathrm{cm} \\
{ }^{\circ} \mathrm{C} \\
\mathrm{mg} / \mathrm{L} \\
{ }_{\mathrm{NTU}} \\
\text { well vol. }\end{array}$ & \\
\hline
\end{tabular}

ANALYTICAL DATA

\section{H ST Analyte}

Acetophenone

Aldrin

Aluminum, total recoverable

Antimony, total recoverable

Arsenic, total recoverable

Barium, total recoverable

Benzene

Bromodichloromethane

Bromoform

Cadmium, total recoverable

Calcium, total recoverable

Carbon tetrachloride

Carbon-14

Chloride

Chlorobenzene

Chloroethane

Chloroethene (Vinyl chloride)

2-Chloroethyl vinyl ether

Chloroform

Chloromethane (Methyl chloride)

Chromium, total recoverable

Copper, total recoverable

Cyanide

P.P'-DDT

Dibromochloromethane

1,1-Dichloroethan

1,2-Dichloroethane

1,1-Dichloroethylene

trans-1, 2-Dichloroethylene

Dichloromethane

2,4-Dichlorophenoxyacetic acid

1,2-Dichloropropane

cis-1,3-Dichloropropene

trans-1,3-Dichloropropen

Dieldrin

Endrin

Ethylbenzene

Fluoride

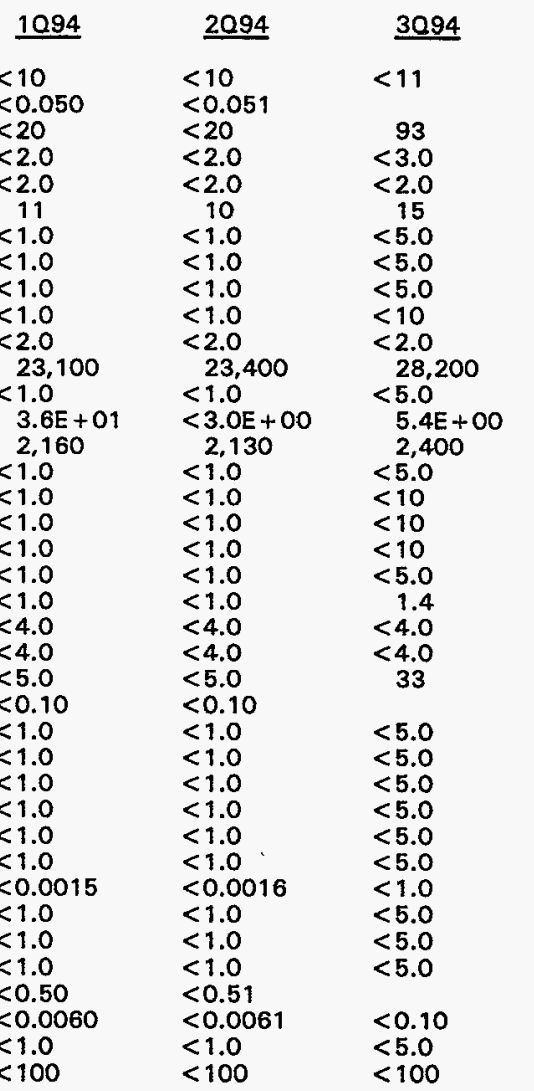

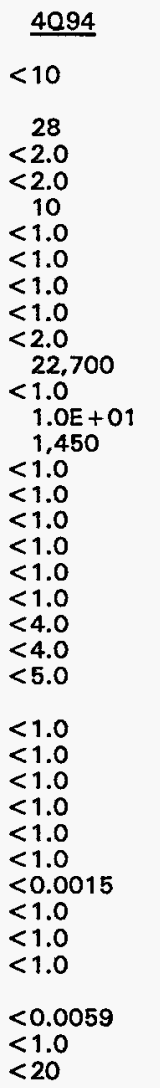

\begin{tabular}{|c|c|c|c|c|}
\hline$\underline{D F}$ & Mod & Unit & $\underline{\text { Lab }}$ & Flag \\
\hline 1 & & $\begin{array}{l}\mu \mathrm{g} / \mathrm{L} \\
\mu \mathrm{g} / \mathrm{L}\end{array}$ & GE & 0 \\
\hline $\begin{array}{l}1 \\
1\end{array}$ & $\mathbf{J}$ & $\begin{array}{l}\mu \mathrm{g} / \mathrm{L} \\
\mu \mathrm{g} / \mathrm{L}\end{array}$ & $\begin{array}{l}\mathrm{GE} \\
\mathrm{GE}\end{array}$ & $\begin{array}{l}1 \\
0\end{array}$ \\
\hline 1 & & $\mu g / L$ & $\overline{\mathrm{GE}}$ & 0 \\
\hline 1 & & $\mu g / L$ & GE & 0 \\
\hline 1 & & $\mu \mathrm{g} / \mathrm{L}$ & GE & 0 \\
\hline 1 & & $\mu \mathrm{g} / \mathrm{L}$ & $\overrightarrow{G E}$ & 0 \\
\hline 1 & & $\mu \mathrm{g} / \mathrm{L}$ & $\overline{G E}$ & 0 \\
\hline 1 & & $\mu g / L$ & $\overline{G E}$ & 0 \\
\hline 1 & & $\mu \mathrm{g} / \mathrm{L}$ & GE & 0 \\
\hline 1 & & $\mu g / L$ & GE & 0 \\
\hline 1 & & $\mu \mathrm{g} / \mathrm{L}$ & GE & 0 \\
\hline 1 & U1 & $\mathrm{pCi} / \mathrm{L}$ & GP & 0 \\
\hline 1 & & $\mu \mathrm{g} / \mathrm{L}$ & GE & 0 \\
\hline 1 & & $\mu \mathrm{g} / \mathrm{L}$ & GE & 0 \\
\hline 1 & & $\mu g / L$ & GE & 0 \\
\hline 1 & & $\mu g / L$ & $\mathrm{GE}$ & 0 \\
\hline 1 & & $\mu \mathrm{g} / \mathrm{L}$ & GE & 0 \\
\hline 1 & & $\mu \mathrm{g} / \mathrm{L}$ & $\overline{G E}$ & 0 \\
\hline 1 & & $\mu \mathrm{g} / \mathrm{L}$ & GE & 0 \\
\hline 1 & & $\mu \mathrm{g} / \mathrm{L}$. & GE & 0 \\
\hline 1 & & $\mu g / L$ & $\overline{G E}$ & 0 \\
\hline 1 & & $\begin{array}{l}\mu \mathrm{g} / \mathrm{L} \\
\mu \mathrm{g} / \mathrm{L}\end{array}$ & GE & 0 \\
\hline 1 & & $\mu \mathrm{g} / \mathrm{L}$ & GE & 0 \\
\hline 1 & & $\mu \mathrm{g} / \mathrm{L}$ & $\mathrm{GE}$ & 0 \\
\hline 1 & & $\mu \mathrm{g} / \mathrm{L}$ & GE & 0 \\
\hline 1 & & $\mu \mathrm{g} / \mathrm{L}$ & $\mathrm{GE}$ & 0 \\
\hline 1 & & $\mu \mathrm{g} / \mathrm{L}$ & GE & 0 \\
\hline 1 & & $\mu \mathrm{g} / \mathrm{L}$ & GE & 0 \\
\hline 1 & $\mathbf{J}$ & $\mu \mathrm{g} / \mathrm{L}$ & GE & 0 \\
\hline 1 & & $\mu g / L$ & GE & 0 \\
\hline 1 & & $\mu \mathrm{g} / \mathrm{L}$ & $\mathrm{GE}$ & 0 \\
\hline 1 & & $\begin{array}{l}\mu \mathrm{g} / \mathrm{L} \\
\mu \mathrm{g} / \mathrm{L}\end{array}$ & GE & 0 \\
\hline 1 & & $\mu \mathrm{g} / \mathrm{L}$ & GE & 0 \\
\hline & & $\mu \mathrm{g} / \mathrm{L}$ & GE & 0 \\
\hline 1 & & $\mu \mathrm{g} / \mathrm{L}$ & GE & 0 \\
\hline
\end{tabular}

Note: Flagging, dilution factors, modifiers, and laboratories are for fourth quarter 1994 data only. See Appendix B for flagging criteria. - = exceeded holding time for fourth quarter 1994.

- = exceeded screening level or final PDWS for fourth quarter 1994. 


\section{H ST Analyte}

Gross alpha

Heptachlor

Iron, total recoverable

Lead, total recoverable

Lindane

Lithium, total recoverable

Magnesium, total recoverable

Manganese, total recoverable

Mercury, total recoverable

Methoxychlor

Nickel, total recoverable

Nitrate as nitrogen

Nitrate-nitrite as nitrogen

Nonvolatile beta

- $\quad \mathrm{pH}$

Phenols

Potassium, total recoverable

Radium, total alpha-emitting

Selenium, total recoverable

Silica, total recoverable

Silver, total recoverable

Sodium, total recoverable

Specific conductance

Sulfate

1,1,2,2-Tetrachloroethane

Tetrachloroethylene

Tin, total recoverable

Toluene

Total activity

Total dissolved solids

Total organic carbon

Total organic halogens

Total phosphates (as P)

Toxaphene

- 2,4,5-TP (Silvex)

1,1,1-Trichloroethane

1,1,2-Trichloroethane

Trichloroethylene

Trichlorofluoromethane

2,4,5-T

- Tritium

Turbidity

Uranium-233/234

Uranium-235

Uranium-238

Vanadium, total recoverable

Xylenes

Zinc, total recoverable

$\frac{1094}{3.3 E+00}$
$<0.050$
$<4.0$
$<3.0$
$<0.0050$
$<5.0$
543
$<2.0$
$<0.20$
$<0.50$
$<10$
$<4.0$

\section{4}

$\frac{3094}{6.0 E-01}$
24
$<3.0$
$<0.052$
$<5.0$
529
$<2.0$
$<0.20$
$<0.52$
$<10$
$<4.0$
928

720

$2.0 E+00$

6.6

$<5.0$

$<500$

6.0E-01

$<2.0$

11,400

$<2.0$

1,990

127

$<1,000$

$<1.0$

$<1.0$

$<1.0$

$9.7 E+05$

79,000

$<1,000$

$<5.0$

$<50$

$<0.24$

$<1.0$

$<1.0$

$<1.0$

$<1.0$

$8.9 E+02$

$<0.10$

$<1.3 \mathrm{E}-01$

$<0.0 E+00$

$<4.6 \mathrm{E}-02$

$<8.0$

$<2.0$

3.6

$\begin{aligned} &<3.8 \mathrm{E}-01 \\ &<0.051 \\ &<4.0 \\ &<3.0 \\ &<0.0051 \\ & 509 \\ &<2.0 \\ &<<0.20 \\ &<<.51 \\ &<10 \\ &<4.0\end{aligned}$

1,040

$<4.0 \mathrm{E}-01$

7.4

$<5.0$

$<500$

$<0.0 E+00$

$<2.0$

11,300

$<2.0$

1,940

131

$<1,000$

$<1.0$

$<1.0$

$<2.0$

$<1.0$

9.7 +05

92,000

$<1,000$

$<5.0$

70

$<0.25$
$<0.00047$

$<1.0$

$<1.0$

$<1.0$

$<1.0$

0.39

$9.8 E+02$

$<0.10$

$<2.8 \mathrm{E}-02$

$<-2.1 E-02$

$<7.0 E-02$

$<8.0$

$<2.0$
6.5E-01

7.4

$<5.0$

$<500$

5.0E-01

$<2.0$

10,900

$<2.0$

2,040

121

$<1,000$

$<5.0$

$<5.0$

$<17$

$<5.0$

$1.2 \mathrm{E}+06$

69,000

$<1,000$

9.3

0.20

$<1.0$

$<0.52$

$<5.0$

$<5.0$

$<5.0$

$<5.0$

$1.3 E+03$

0.48

3.5E-02

$-5.5 \mathrm{E}-03$

4.0E-02

$<3.0$

$<5.0$

12

\begin{tabular}{|c|c|}
\hline 40994 & $\underline{D F}$ \\
\hline $9.0 \mathrm{E}-02$ & 1 \\
\hline $\begin{aligned} & 16 \\
< & 3.0 \\
< & 0.0050\end{aligned}$ & $\begin{array}{l}1 \\
1 \\
1\end{array}$ \\
\hline $\begin{aligned} & 518 \\
< & 2.0 \\
< & 0.20 \\
< & 0.50 \\
< & 10\end{aligned}$ & $\begin{array}{l}1 \\
1 \\
1 \\
1 \\
1\end{array}$ \\
\hline
\end{tabular}

Mod

UI

Unit Lab

$\mathrm{PCi} / \mathrm{L}$
$\mu \mathrm{g} / \mathrm{L}$

$\mu g / L$ GE 0

$\mu g / L \quad G E$ O

$\mu g / L \quad G E$

$\mu g / L \quad G E$

$\mu g / L \quad G E$

$\mu g / L \quad G E$

$\mu g / L$

$\mu g / L \quad G E$

$\mu \mathrm{g} / \mathrm{L}$

$\mu g / L$

930

$1.5 \mathrm{E}+00$

7.3

$<5.0$

$<500$

1.0E-01

$<2.0$

11,400

$<2.0$

1,960

69

$<1,000$

$<1.0$

$<1.0$

$<2.0$

$<1.0$

88,000

$<1,000$

$<5.0$

150

$<0.24$

0.0040

$<1.0$

$<1.0$

$<1.0$

$<1.0$

1.1E+03

$<0.10$

2.5E-01

$-1.3 \mathrm{E}-02$

1.6E-01

$<8.0$

$<2.0$

2.4

$\mu \mathrm{pC}$

Note: Flagging, dilution factors, modifiers, and laboratories are for fourth quarter 1994 data only. See Appendix B for flagging criteria. - = exceeded holding time for fourth quarter 1994.

n = exceeded screening level or final PDWS for fourth quarter 1994. 


\section{WELL BGO 6D}

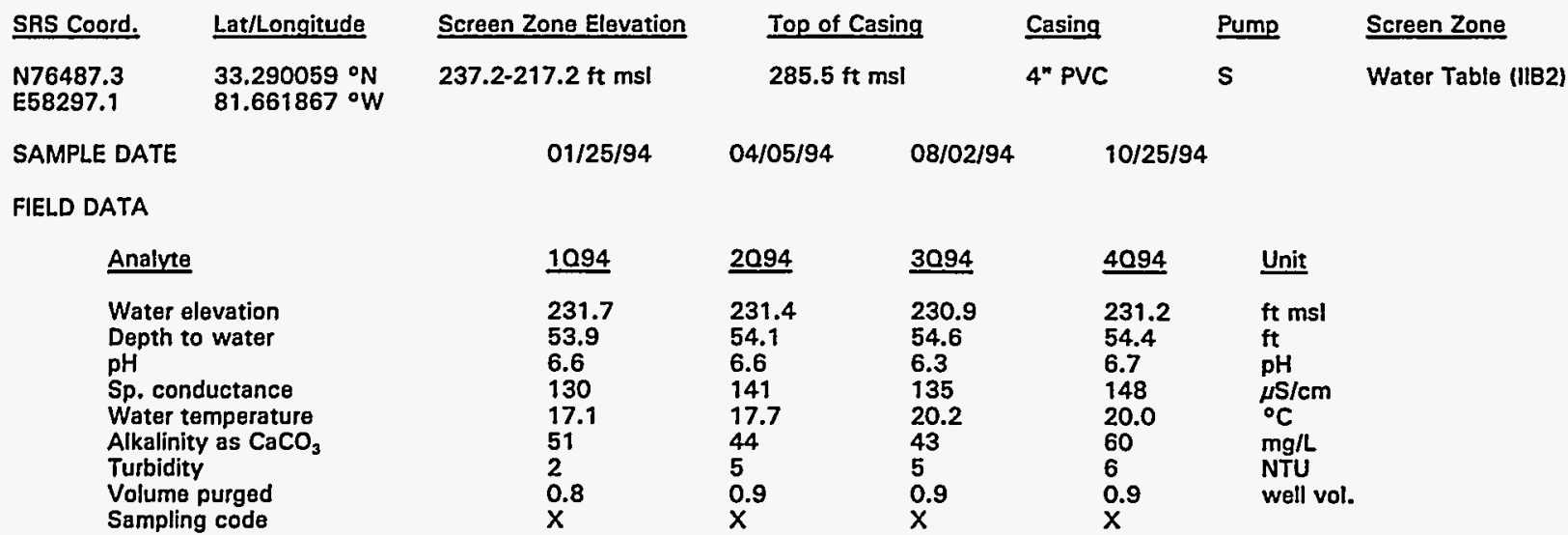

ANALYTICAL DATA

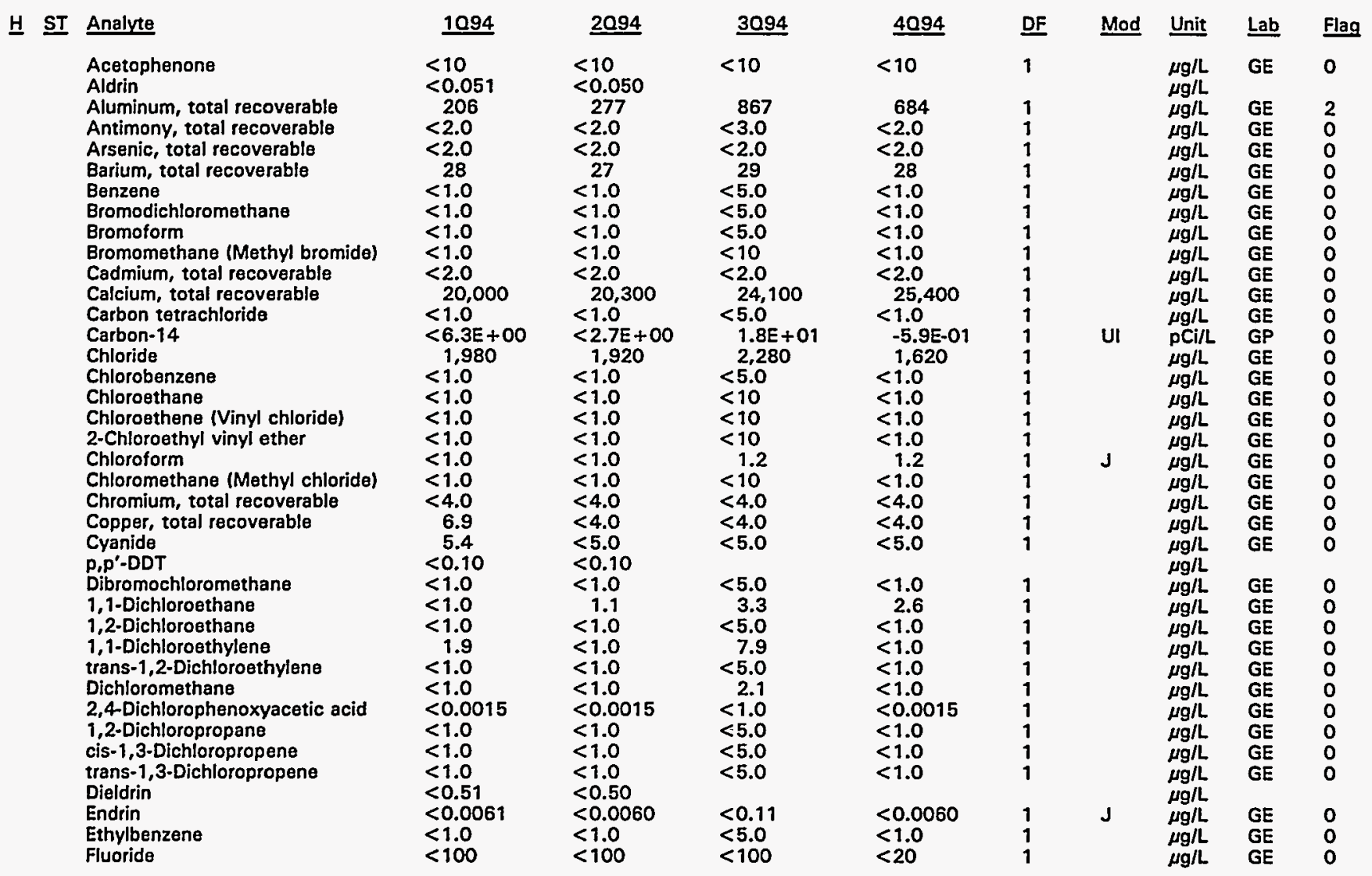

Note: Flagging, dilution factors, modifiers, and laboratories are for fourth quarter 1994 data only. See Appendix B for flagging criteria. - = exceeded holding time for fourth quarter 1994 .

- = exceeded screening level or final PDWS for fourth quarter 1994. 
H ST Analyte

Gross alpha

Heptachlor

Iron, total recoverable

Lead, total recoverable

Lindane

Lithium, total recoverable

Magnesium, total recoverable

Manganese, total recoverable

Mercury, total recoverable

Methoxychlor

Naphthalene

Nickel, total recoverable

Nitrate as nitrogen

Nitrate-nitrite as nitrogen

Nonvolatile beta

- $\quad$ H

Phenols

Potassium, total recoverable

Radium, total alpha-emitting

Selenium, total recoverable

Silica, total recoverable

Silver, total recoverable

Sodium, total recoverable

Specific conductance

Sulfate

1,1,2,2-Tetrachloroethane

- Tetrachloroethylene

Tin, total recoverable

Toluene

Total activity

Total dissoived solids

Total organic carbon

Total organic halogens

Total phosphates (as P)

Toxaphene

2,4,5-TP (Silvex)

1,1,1-Trichloroethane

1,1,2-Trichloroethane

- Trichloroethylene

Trichlorofluoromethane

2,4,5-T

- Tritium

Turbidity

Uranium-233/234

Uranium-235

Uranium-238

Vanadium, total recoverable

Xylenes

Zinc, total recoverable

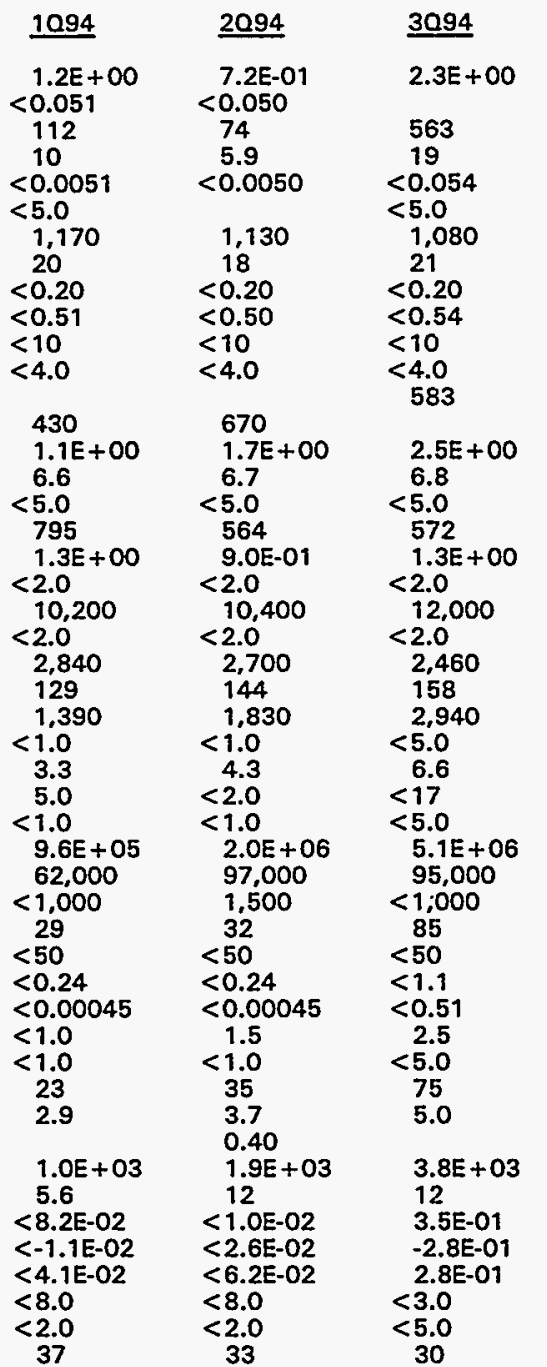

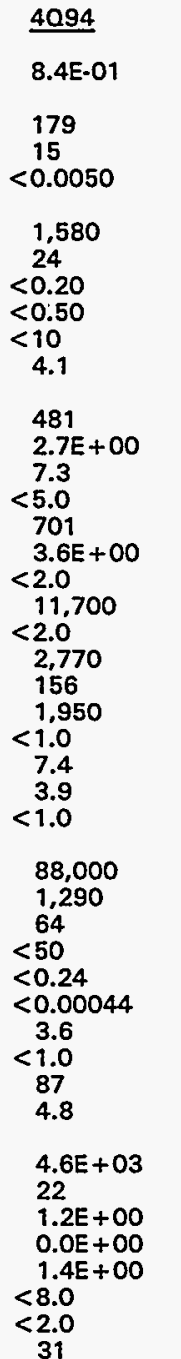

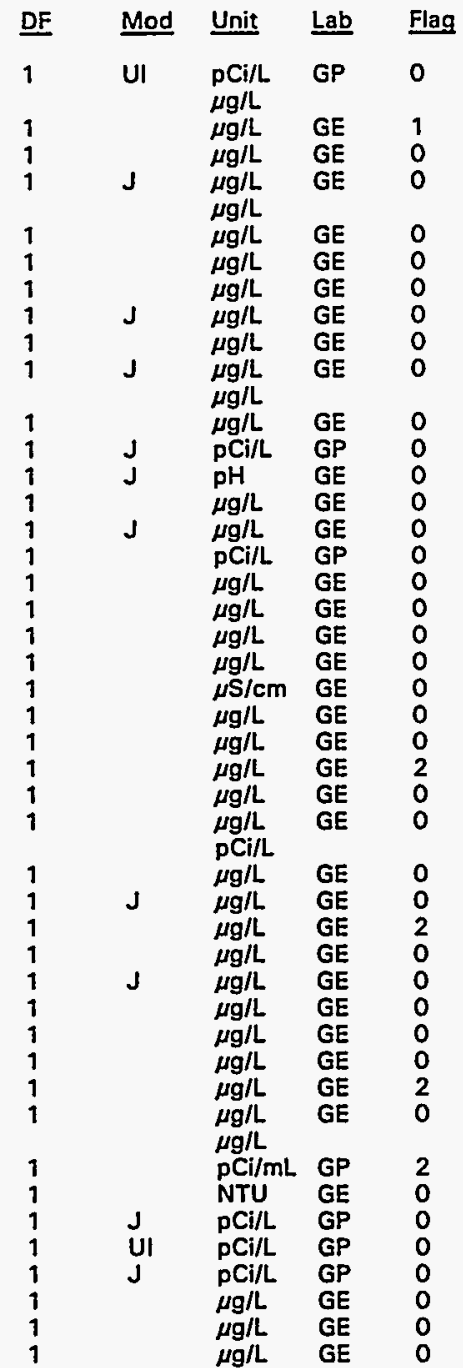

Note: Flagging, dilution factors, modifiers, and laboratories are for fourth quarter 1994 data only. See Appendix B for flagging criteria. - = exceeded holding time for fourth quarter 1994.

- =xceeded screening level or final PDWS for fourth quarter 1994. 
WELL BGO 7D

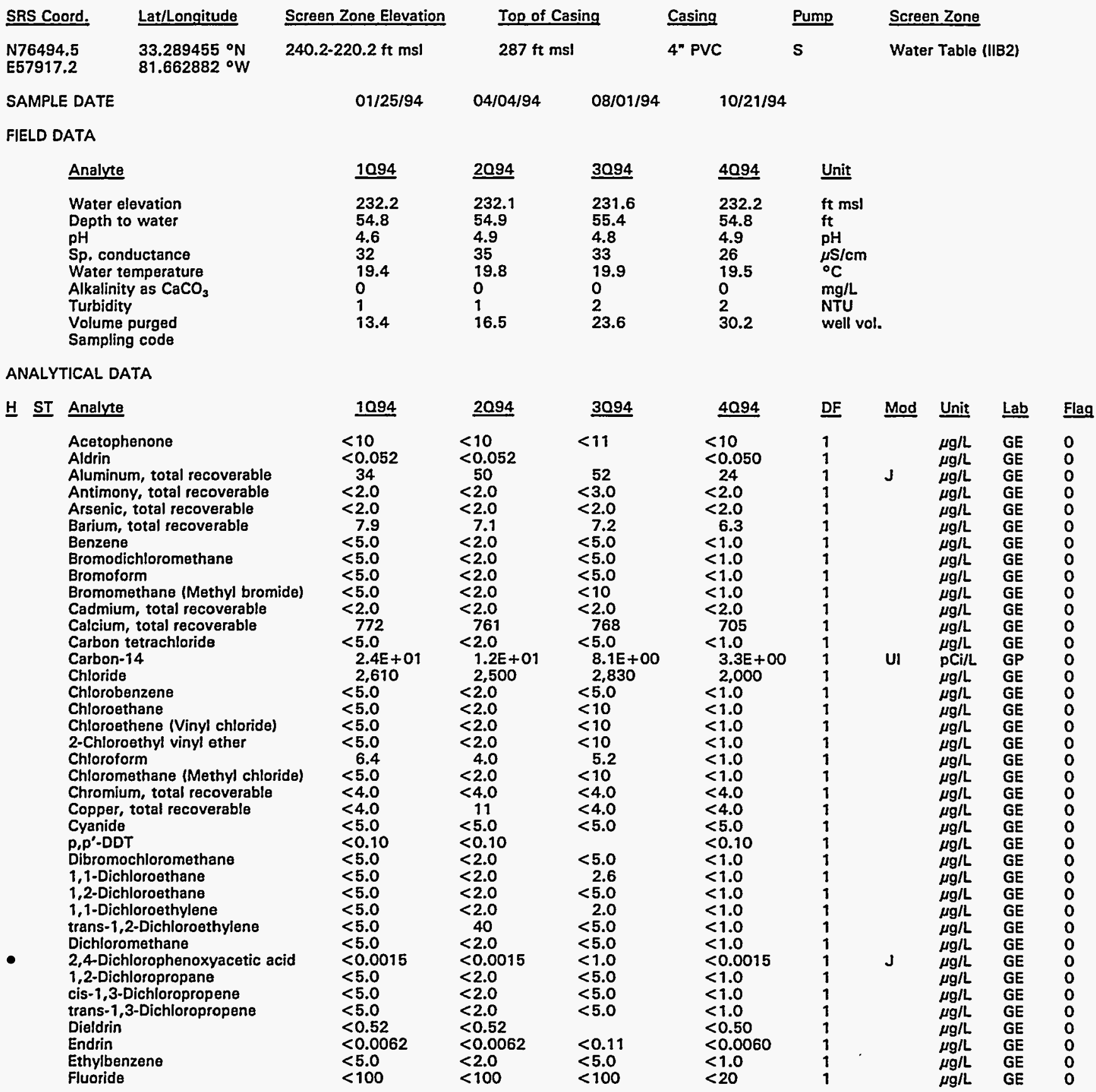

Note: Flagging, dilution factors, modifiers, and laboratories are for fourth quarter 1994 data only. See Appendix B for flagging criteria. - = exceeded holding time for fourth quarter 1994.

n = exceeded screening level or final PDWS for fourth quarter 1994. 


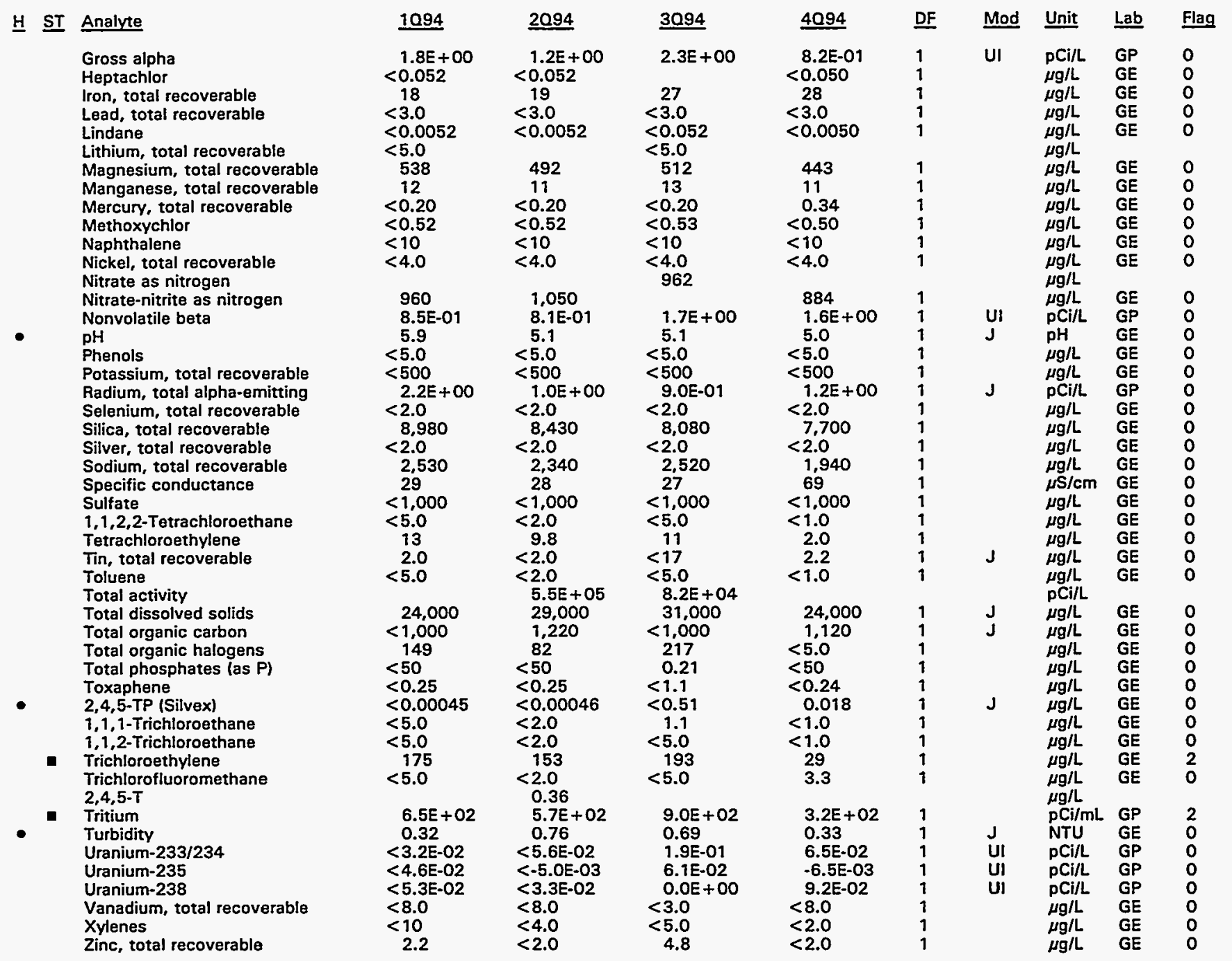

Note: Flagging, dilution factors, modifiers, and laboratories are for fourth quarter 1994 data only. See Appendix B for flagging criteria. - = exceeded holding time for fourth quarter 1994.

- = exceeded screening level or final PDWS for fourth quarter 1994. 


\section{WELL BGO 8AR}

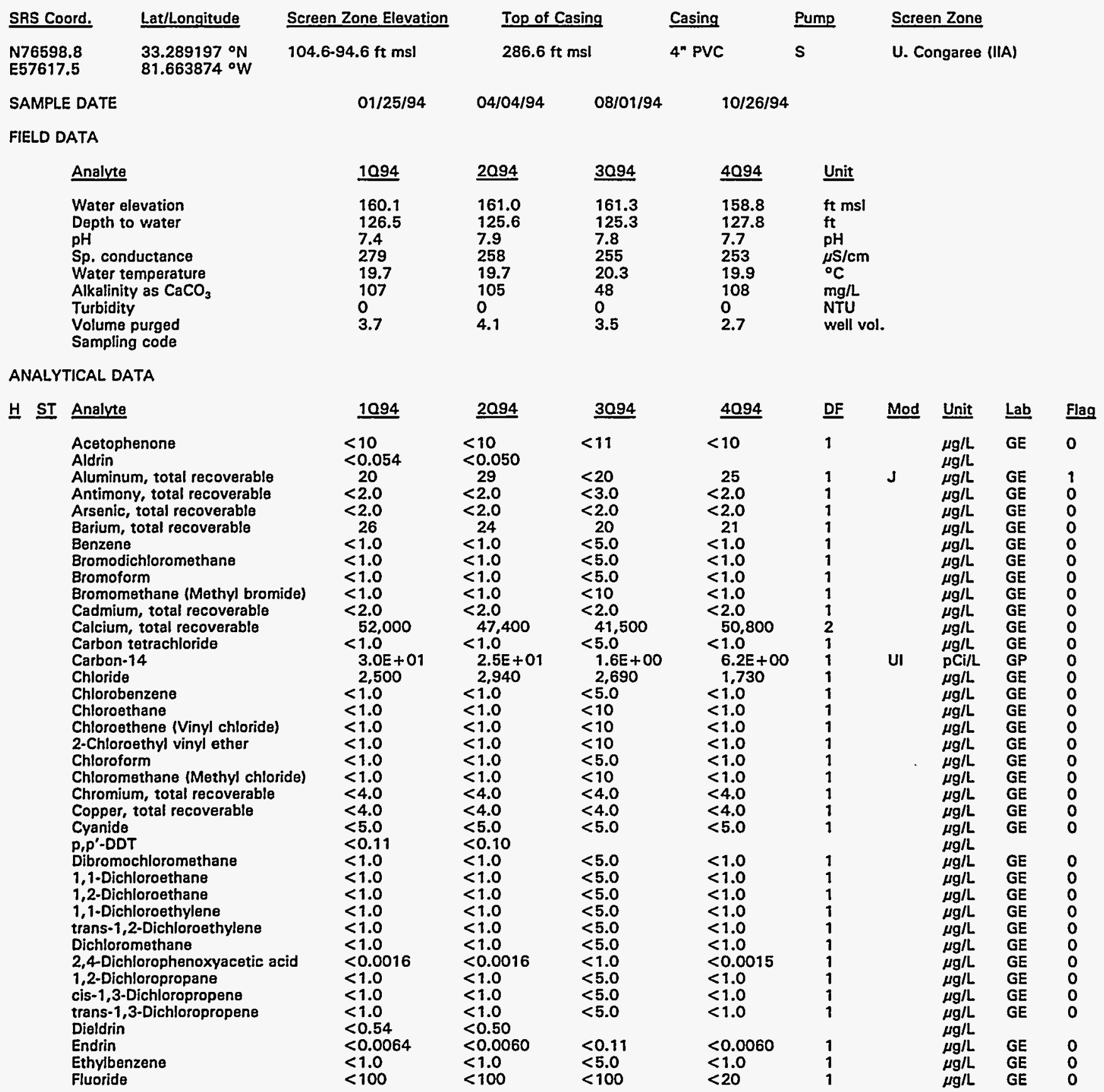

Note: Flagging, dilution factors, modifiers, and laboratories are for fourth quarter 1994 data only. See Appendix B for flagging criteria. - = exceeded holding time for fourth quarter 1994.

m = exceeded screening level or final PDWS for fourth quarter 1994. 


\section{H ST Analyte}

Gross alpha

Heptachlor

Iron, total recoverable

Lead, total recoverable

Lindane

Lithium, total recoverable

Magnesium, total recoverable

Manganese, total recoverable

Mercury, total recoverable

Methoxychlor

Nickhthalene

Nitrate as nitrogen

Nitrate-nitrite as nitrogen

Nonvolatile beta

$\mathrm{pH}$

Phenols

Potassium, total recoverable

Radium, total alpha-emitting

Radium-226

Radium-228

Selenium, total recoverable

Silica, total recoverable

Silver, total recoverable

Sodium, total recoverable

Specific conductance

Sulfate

1,1,2,2-Tetrachloroethane

Tetrachloroethylene

Tin, total recoverable

Toluene

Total dissolved solids

Total organic carbon

Total organic halogens

Total phosphates (as P)

Toxaphene

2,4,5-TP (Silvex)

Tributyl phosphate

1,1,1-Trichloroethane

1,1,2-Trichloroethane

Trichloroethylene

Trichlorofluoromethane

2,4,5-T

Tritium

Turbidity

Uranium-233/234

Uranium-235

Uranium-238

Vanadium, total recoverable

Xylenes

Zinc, total recoverable

\begin{tabular}{|c|c|c|c|}
\hline 1094 & $\underline{2094}$ & 3094 & 4094 \\
\hline $\begin{array}{l}<2.5 E-01 \\
<0.054\end{array}$ & $\begin{array}{l}<-2.5 E-02 \\
<0.050\end{array}$ & 4.9E-03 & 7.3E-01 \\
\hline $\begin{array}{l}13 \\
<3.0\end{array}$ & $\begin{aligned} & 25 \\
< & 3.0\end{aligned}$ & $\begin{array}{r}<55 \\
4.8\end{array}$ & $\begin{array}{r}5.2 \\
<3.0\end{array}$ \\
\hline $\begin{array}{c}<0.0054 \\
5.9\end{array}$ & $<0.0050$ & $\begin{array}{c}<0.052 \\
8.8\end{array}$ & $<0.0050$ \\
\hline $\begin{array}{l}1,380 \\
12\end{array}$ & $\begin{array}{l}1,200 \\
24\end{array}$ & $\begin{array}{l}1,050 \\
11\end{array}$ & $\begin{array}{l}1,280 \\
4.5\end{array}$ \\
\hline$<0.20$ & $<0.20$ & $<0.20$ & $<0.20$ \\
\hline$<0.54$ & $<0.50$ & $<0.53$ & $<0.50$ \\
\hline $\begin{array}{c}<10 \\
5.1\end{array}$ & $\begin{array}{l}<10 \\
<4.0\end{array}$ & $\begin{array}{l}<10 \\
<4.0\end{array}$ & $\begin{array}{l}<10 \\
<4.0\end{array}$ \\
\hline$<50$ & $\begin{array}{l}<20 \\
<50\end{array}$ & $<20$ & $<50$ \\
\hline $\begin{array}{l}1.7 E+00 \\
6.3\end{array}$ & $\begin{array}{l}8.8 E+00 \\
7.8\end{array}$ & $\begin{array}{l}7.5 \mathrm{E}-01 \\
7.9\end{array}$ & $\begin{array}{l}8.0 \mathrm{E}-01 \\
7.8\end{array}$ \\
\hline$<5.0$ & $<5.0$ & $<5.0$ & $<5.0$ \\
\hline $\begin{array}{l}2,040 \\
7.0 E-01\end{array}$ & $\begin{array}{l}1,250 \\
4.0 E-01 \\
2.1 E-01\end{array}$ & $\begin{array}{l}1.120 \\
2.0 \mathrm{E}-01\end{array}$ & $\begin{array}{l}1,190 \\
1.2 E+00\end{array}$ \\
\hline & $1.1 E+00$ & & \\
\hline$<2.0$ & $\begin{array}{l}<2.0 \\
32,200\end{array}$ & $\begin{array}{l}<2.0 \\
29,300\end{array}$ & $\begin{array}{l}<2.0 \\
33,500\end{array}$ \\
\hline 3.7 & $<2.0$ & $<2.0$ & $<2.0$ \\
\hline $\begin{array}{l}2,320 \\
267\end{array}$ & $\begin{array}{l}2,120 \\
245\end{array}$ & $\begin{array}{l}1,830 \\
238\end{array}$ & $\begin{array}{l}2,080 \\
259\end{array}$ \\
\hline 6,300 & 8,740 & 7,860 & 6,880 \\
\hline$<1.0$ & $<1.0$ & $<5.0$ & $<1.0$ \\
\hline$<1.0$ & $<1.0$ & $<5.0$ & $<1.0$ \\
\hline 8.8 & $<2.0$ & $<17$ & $<2.0$ \\
\hline$<1.0$ & $<1.0$ & $<5.0$ & $<1.0$ \\
\hline $\begin{aligned} & 182,000 \\
< & 1,000\end{aligned}$ & $\begin{array}{l}164,000 \\
1,240\end{array}$ & $\begin{aligned} & 171,000 \\
&<<1,000\end{aligned}$ & $\begin{array}{l}176,000 \\
1,780\end{array}$ \\
\hline 47 & 14 & $<6.7$ & $<5.0$ \\
\hline$<50$ & $<50$ & 2,350 & $<50$ \\
\hline $\begin{array}{l}<0.26 \\
<0.00047\end{array}$ & $\begin{array}{l}<0.24 \\
<0.00048\end{array}$ & $\begin{array}{l}<1.1 \\
<0.52\end{array}$ & $\begin{array}{l}<0.24 \\
<0.00045\end{array}$ \\
\hline $\begin{array}{l}<10 \\
<1.0\end{array}$ & $\begin{array}{l}<10 \\
<1.0\end{array}$ & $\begin{array}{l}<10 \\
<5.0\end{array}$ & $\begin{array}{l}<10 \\
<1.0\end{array}$ \\
\hline$<1.0$ & $<1.0$ & $<5.0$ & $<1.0$ \\
\hline$<1.0$ & $<1.0$ & $<5.0$ & $<1.0$ \\
\hline 3.4 & $<\begin{array}{c}<1.0 \\
0.35\end{array}$ & $<5.0$ & $<1.0$ \\
\hline$<0.0 E+00$ & & 3.9E-01 & $-3.3 E-01$ \\
\hline 0.12 & 0.37 & 0.39 & $<0.10$ \\
\hline $\begin{array}{l}<-1.6 \mathrm{E}-02 \\
<-1.6 \mathrm{E}-02\end{array}$ & $\begin{array}{l}1.3 E-01 \\
2\end{array}$ & 1.4E-01 & 5.8E-02 \\
\hline $\begin{array}{l}<-7.6 E-02 \\
<3.8 E-02\end{array}$ & $\begin{array}{c}<-9.5 \mathrm{E}-03 \\
9.3 \mathrm{E}-02\end{array}$ & $\begin{array}{l}-1.0 \mathrm{E}-02 \\
1.5 \mathrm{E}-01\end{array}$ & $\begin{array}{l}1.1 \mathrm{E}-02 \\
6.9 \mathrm{E}-02\end{array}$ \\
\hline$<8.0$ & $<3.0$ & $<3.0$ & $<8.0$ \\
\hline$<2.0$ & $<2.0$ & $<5.0$ & $<2.0$ \\
\hline$<2.0$ & 6.2 & $<49$ & $<2.0$ \\
\hline
\end{tabular}

Note: Flagging, dilution factors, modifiers, and laboratories are for fourth quarter 1994 data only. See Appendix B for flagging criteria. - = exceeded holding time for fourth quarter 1994.

- = exceeded screening level or final PDWS for fourth quarter 1994. 
WELL BGO 8C

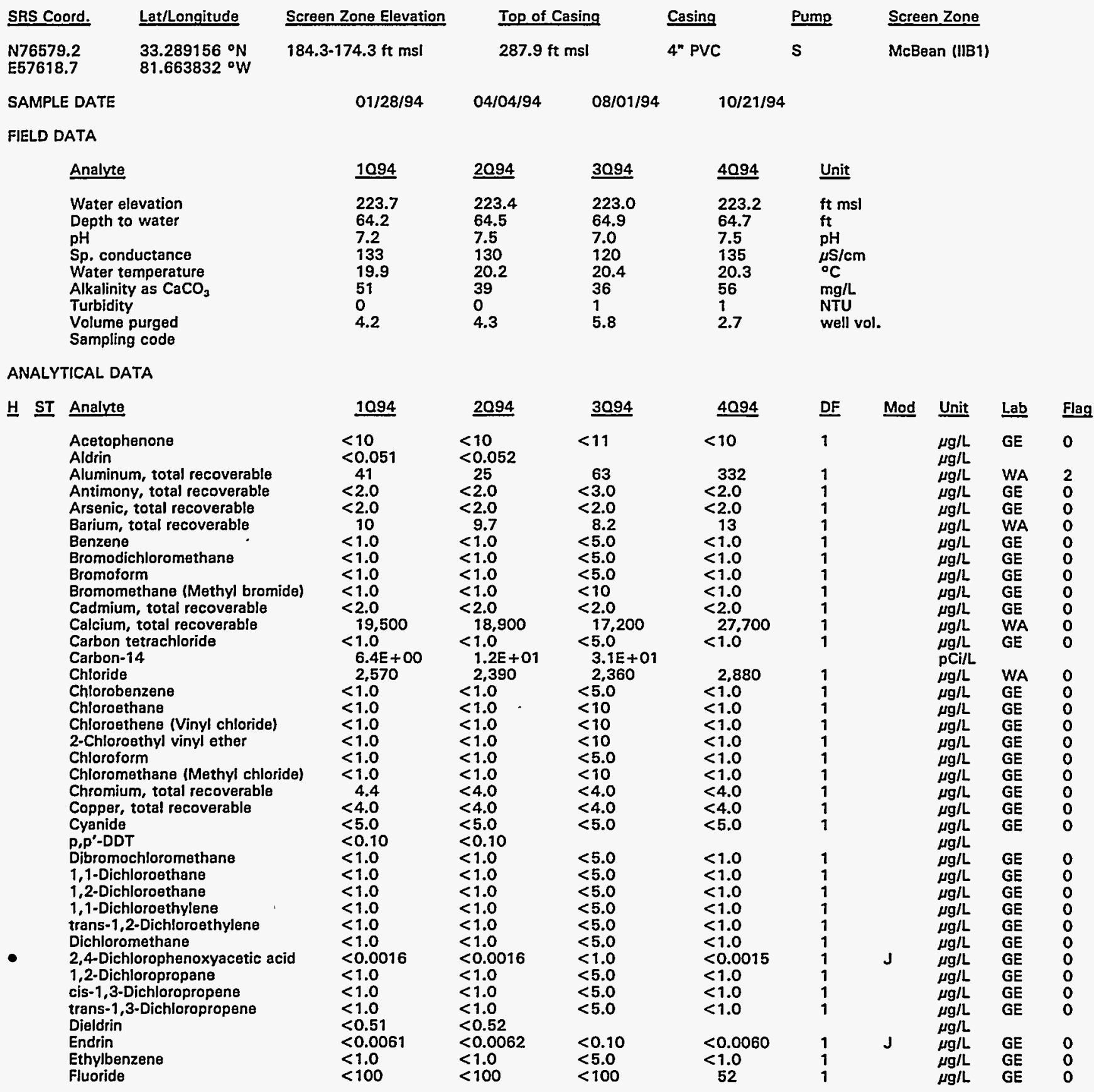

Note: Flagging, dilution factors, modifiers, and laboratories are for fourth quarter 1994 data only. See Appendix B for flagging criteria. - = exceeded holding time for fourth quarter 1994.

- = exceeded screening level or final PDWS for fourth quarter 1994. 


\begin{tabular}{|c|c|c|c|c|c|c|c|c|c|c|}
\hline$\underline{H} \underline{S T}$ & Analyte & 1094 & $\underline{2094}$ & 3094 & 4094 & $\underline{\text { DF }}$ & Mod & Unit & Lab & Flag \\
\hline 0 & 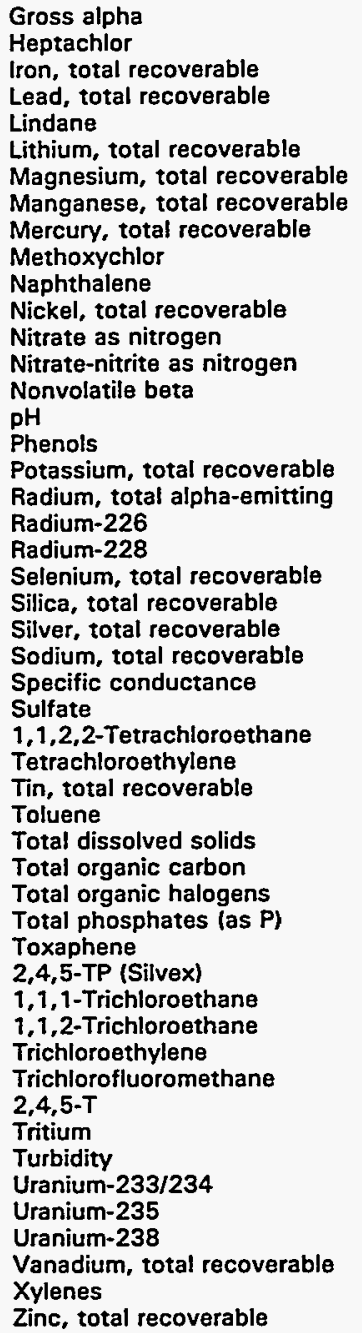 & 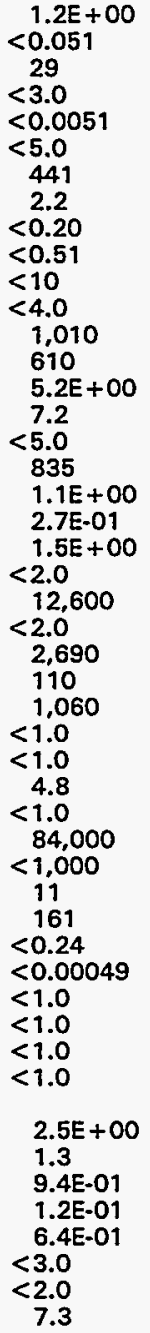 & $\begin{array}{rl}< & 2.0 \\
12,700 & \\
<2.0 & 2.750 \\
& 114 \\
<1,000 \\
<1.0 \\
<1.0 \\
<2.0 \\
<1.0 \\
64.000 \\
<1.000 \\
<5.0 \\
100 \\
<0.25 \\
<0.00048 \\
<1.0 \\
<1.0 \\
<1.0 \\
<1.0 \\
\quad 0.35 \\
1.5 \mathrm{E}+00 \\
<0.10 \\
<4.4 \mathrm{E}-02 \\
<1.5 \mathrm{E}-02 \\
<4.4 \mathrm{E}-02 \\
<8.0 \\
<2.0 \\
3.2\end{array}$ & 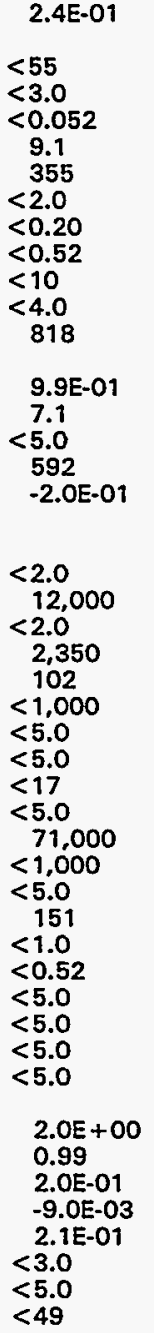 & 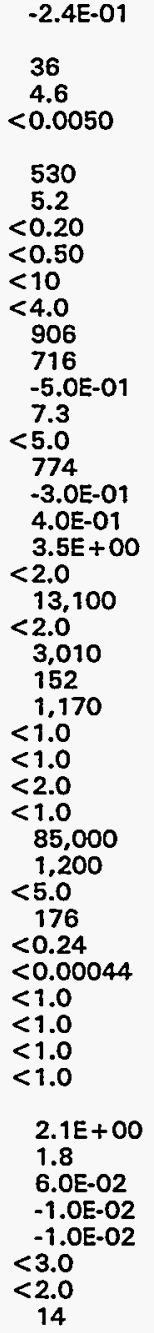 & $\begin{array}{l}1 \\
1 \\
1 \\
1 \\
1 \\
1 \\
1 \\
1 \\
1 \\
1 \\
1 \\
1 \\
1 \\
1 \\
1 \\
1 \\
1 \\
1 \\
1 \\
1 \\
1 \\
1 \\
1 \\
1 \\
1 \\
1 \\
1 \\
1 \\
1 \\
1 \\
1 \\
1 \\
1 \\
1 \\
1 \\
1 \\
1 \\
1 \\
1 \\
1\end{array}$ & $\begin{array}{l}\text { UI } \\
\text { J } \\
\text { J UI }\end{array}$ & 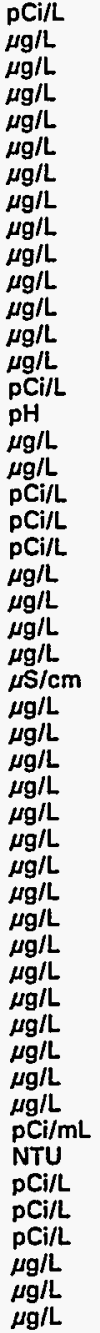 & $\begin{array}{l}\text { GP } \\
\text { WA } \\
\text { WA } \\
\text { GE } \\
\text { WA } \\
\text { WA } \\
\text { GE } \\
\text { GE } \\
\text { GE } \\
\text { GE } \\
\text { WA } \\
\text { GE } \\
\text { TM } \\
\text { GE } \\
\text { GE } \\
\text { GE } \\
\text { GP } \\
\text { TM } \\
\text { TM } \\
\text { GE } \\
\text { GE } \\
\text { GE } \\
\text { WA } \\
\text { WA } \\
\text { WA } \\
\text { GE } \\
\text { GE } \\
\text { GE } \\
\text { GE } \\
\text { WA } \\
\text { GE } \\
\text { GE } \\
\text { WA } \\
\text { GE } \\
\text { GE } \\
\text { GE } \\
\text { GE } \\
\text { GE } \\
\text { GE } \\
\text { GP } \\
\text { GE } \\
\text { TM } \\
\text { TM } \\
\text { TM } \\
\text { WA } \\
\text { GE } \\
\text { WA }\end{array}$ & $\begin{array}{l}0 \\
0 \\
0 \\
0 \\
0 \\
0 \\
0 \\
0 \\
0 \\
0 \\
0 \\
0 \\
0 \\
0 \\
0 \\
0 \\
0\end{array}$ \\
\hline
\end{tabular}

Note: Flagging, dilution factors, modifiers, and laboratories are for fourth quarter 1994 data only. See Appendix B for flagging criteria. - = exceeded holding time for fourth quarter 1994.

n = exceeded screening level or final PDWS for fourth quarter 1994. 


\section{WELL BGO 8D}

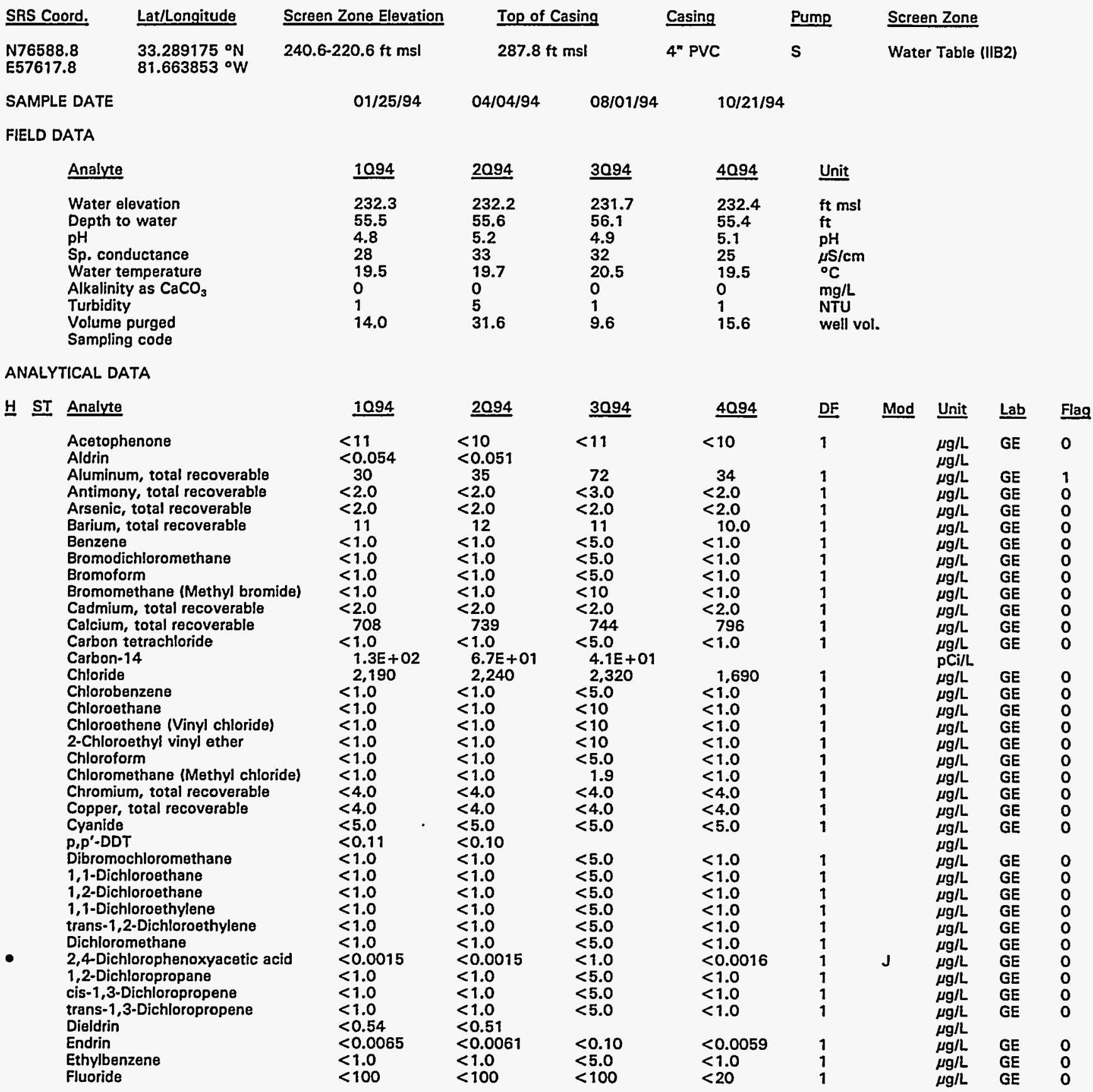

Note: Flagging, dilution factors, modifiers, and laboratories are for fourth quarter 1994 data only. See Appendix B for flagging criteria. - = exceeded holding time for fourth quarter 1994.

- = exceeded screening level or final PDWS for fourth quarter 1994. 


\section{H ST Analvte}

Gross alpha

Heptachlor

Iron, total recoverable

Lead, total recoverable

Lindan

Lithium, total recoverable

Magnesium, total recoverable

Manganese, total recoverable

Mercury, total recoverable

Methoxychlor

Naphthalene

Nickel, total recoverable

Nitrate as nitrogen

Nitrate-nitrite as nitrogen

Nonvolatile beta

- $\quad \mathrm{pH}$

Phenols

Potassium, total recoverable

Radium, total alpha-emitting

Selenium, total recoverable

Silica, total recoverable

Silver, total recoverable

Sodium, total recoverable

Specific conductance

Sulfate

1,1,2,2-Tetrachloroethane

Tetrachloroethylene

Tin, total recoverable

Toluene

Total dissolved solids

Total organic carbon

Total organic halogens

Total phosphates (as P)

Toxaphene

- 2,4,5-TP (Silvex)

1,1,1-Trichloroethane

1,1,2-Trichloroethane

Trichloroethylene

Trichlorofluoromethane

2,4,5-T

- Tritium

Uranium-233/234

Uranium-235

Uranium-238

Vanadium, total recoverable

Xylenes

Zinc, total recoverable

$\begin{aligned} & 1094 \\ & 1.2 \mathrm{E}+00 \\ &< 0.054 \\ & 14< \\ &<3.0 \\ &<0.0054 \\ &<5.0 \\ & 466 \\ & 12 \\ &<0.20 \\ &<0.54 \\ &<11 \\ &<4.0\end{aligned}$

$\begin{array}{ll}\frac{2094}{2.0 E+00} & \underline{3094} \\ <0.051 & 1.8 E+00 \\ 35 & \\ <3.0 & <8 \\ <0.0051 & <3.0 \\ & <0.052 \\ 477 & 7.1 \\ 11 & 464 \\ <0.20 & <.2 \\ <0.51 & <0.20 \\ <10 & <10 \\ <4.0 & <4.0 \\ & 990 \\ 1,220 & \end{array}$

1,130
$1.6 E+00$

6.2

$<5.0$

$<500$

$2.3 E+00$

$<2.0$

7.650

$<2.0$

2,420

27

$<1,000$

$<1.0$

$<1.0$

$<2.0$

$<1.0$

22,000

$<1,000$

$<5.0$

$<50$

$<0.26$

$<0.00046$

$<1.0$

$<1.0$

1.2

4.4

$1.2 \mathrm{E}+01$

$<0.10$

$<6.1 E-02$

$<-8.3 E-03$

$<7.7 E-02$

$<8.0$

$<2.0$

$<2.0$
1. $6 \mathrm{E}+00$

5.1

5.8

$<500$

1. $6 E+\infty$

$<2.0$

7,570

$<2.0$

2,420

1,190

$<1.0$

$<1.0$

$<2.0$

$<1.0$

14,000

$<1,000$

7.3

$<50$

$<0.24$

$<0.00046$

$<1.0$

$<1.0$

1.5

7.5

0.37
$1.7 E+01$

5.0

1.4E-01

$<0.0 E+00$

1.6E-01

$<8.0$

$<2.0$

$<2.0$
$1.2 E+\infty 0$

5.2

$<5.0$

$<500$

$<2.0$

7,620

$<2.0$

2,620

27

1,130

$<5.0$

$<5.0$

$<17$

$<5.0$

26,000

$<1,000$

9.2

$<50$

$<0.52$

$<5.0$

$<5.0$

1.8

5.1

$1.8 E+01$

0.50

2.8E-01

9.3E-02

1.9E-01

$<3.0$

$<5.0$

$<49$

\begin{tabular}{|c|c|c|c|c|c|}
\hline 4094 & $\underline{\mathrm{DF}}$ & Mod & Unit & Lab & Flag \\
\hline $1.4 E+00$ & 1 & $\mathbf{J}$ & $\begin{array}{l}\mathrm{pCi} / \mathrm{L} \\
\mu \mathrm{g} / \mathrm{L}\end{array}$ & GP & 0 \\
\hline 19 & 1 & & $\mu \mathrm{g} / \mathrm{L}$ & GE & 0 \\
\hline & 1 & & $\mu \mathrm{g} / \mathrm{L}$ & GE & 0 \\
\hline$<0.0050$ & 1 & & $\underset{\mu \mathrm{g} / \mathrm{L}}{\mu \mathrm{L}}$ & GE & 0 \\
\hline 413 & 1 & & $\mu \mathrm{g} / \mathrm{L}$ & GE & 0 \\
\hline 11 & 1 & & $\mu g / L$ & GE & 0 \\
\hline$<0.20$ & 1 & & $\mu g / L$ & GE & 0 \\
\hline$<0.50$ & 1 & & $\mu \mathrm{g} / \mathrm{L}$ & GE & 0 \\
\hline$<10$ & 1 & & $\mu g / L$ & GE & 0 \\
\hline$<4.0$ & 1 & & $\begin{array}{l}\mu \mathrm{g} / \mathrm{L} \\
\mu \mathrm{g} / \mathrm{L}\end{array}$ & GE & 0 \\
\hline 1.270 & 1 & & $\mu g / L$ & GE & 0 \\
\hline $2.0 E+\infty 0$ & 1 & $J$ & $\mathrm{pCi} / \mathrm{L}$ & GP & 0 \\
\hline $\begin{array}{r}5.1 \\
-5.0\end{array}$ & 1 & $J$ & $\mathrm{pH}$ & GE & 0 \\
\hline$<5.0$ & 1 & & $\mu g / L$ & GE & 0 \\
\hline $\begin{array}{l}<500 \\
8.0 E-01\end{array}$ & 1 & & $\mu \mathrm{g} / \mathrm{L}$ & GE & 0 \\
\hline $\begin{aligned} & 8.0 \mathrm{E}-01 \\
< & 2.0\end{aligned}$ & 1 & $J$ & $\mathrm{pCi/L}$ & GP & 0 \\
\hline $\begin{array}{l}<2.0 \\
7,410\end{array}$ & $\begin{array}{l}1 \\
1\end{array}$ & & $\begin{array}{l}\mu \mathrm{g} / \mathrm{L} \\
\mu \mathrm{g} / \mathrm{L}\end{array}$ & $\begin{array}{l}\text { GE } \\
\text { GE }\end{array}$ & $\begin{array}{l}0 \\
0\end{array}$ \\
\hline$<2.0$ & 1 & & $\mu \mathrm{g} / \mathrm{L}$ & GE & 0 \\
\hline 2,270 & 1 & & $\mu g / L$ & GE & 0 \\
\hline 26 & 1 & & $\mu \mathrm{S} / \mathrm{cm}$ & GE & 0 \\
\hline$<1,000$ & 1 & & $\mu \mathrm{g} / \mathrm{L}$ & GE & 0 \\
\hline$<1.0$ & 1 & & $\mu \mathrm{g} / \mathrm{L}$ & GE & 0 \\
\hline$<1.0$ & 1 & & $\mu g / L$ & GE & 0 \\
\hline$<2.0$ & 1 & & $\mu \mathrm{g} / \mathrm{L}$ & GE & 0 \\
\hline$<1.0$ & 1 & & $\mu \mathrm{g} / \mathrm{L}$ & GE & 0 \\
\hline 26,000 & 1 & & $\mu g / L$ & GE & 0 \\
\hline $\begin{aligned} & 1,180 \\
&<5.0\end{aligned}$ & $\begin{array}{l}1 \\
1\end{array}$ & $J$ & $\underset{\mu \mathrm{g} / \mathrm{L}}{\mu \mathrm{g} / \mathrm{L}}$ & $\begin{array}{l}\text { GE } \\
\text { GE }\end{array}$ & $\begin{array}{l}0 \\
0\end{array}$ \\
\hline$<50$ & 1 & & $\mu \mathrm{g} / \mathrm{L}$ & GE & 0 \\
\hline$<0.24$ & 1 & & $\mu \mathrm{g} / \mathrm{L}$ & GE & 0 \\
\hline$<0.00047$ & 1 & J & $\mu \mathrm{g} / \mathrm{L}$ & GE & 0 \\
\hline$<1.0$ & 1 & & $\mu \mathrm{g} / \mathrm{L}$ & GE & 0 \\
\hline$<1.0$ & 1 & & $\mu g / L$ & GE & 0 \\
\hline$<1.0$ & 1 & & $\mu \mathrm{g} / \mathrm{L}$ & GE & 0 \\
\hline 6.0 & 1 & & $\begin{array}{l}\mu \mathrm{g} / \mathrm{L} \\
\mu \mathrm{g} / \mathrm{L}\end{array}$ & GE & 1 \\
\hline $2.9 E+02$ & 1 & & $\mathrm{pCi} / \mathrm{mL}$ & GP & 2 \\
\hline 1.6 & 1 & $J$ & $\begin{array}{l}\mathrm{NTU} \\
\mathrm{pCi} / \mathrm{L} \\
\mathrm{pCi} / \mathrm{L} \\
\mathrm{pCi} / \mathrm{L}\end{array}$ & GE & 0 \\
\hline$<8.0$ & 1 & & $\mu \mathrm{g} / \mathrm{L}$ & GE & 0 \\
\hline$<2.0$ & 1 & & $\mu \mathrm{g} / \mathrm{L}$ & GE & 0 \\
\hline$<2.0$ & 1 & & $\mu g / L$ & GE & 0 \\
\hline
\end{tabular}

Note: Flagging, dilution factors, modifiers, and laboratories are for fourth quarter 1994 data only. See Appendix B for flagging criteria. - = exceeded holding time for fourth quarter 1994.

- = exceeded screening level or final PDWS for fourth quarter 1994. 
WELL BGO 9AA

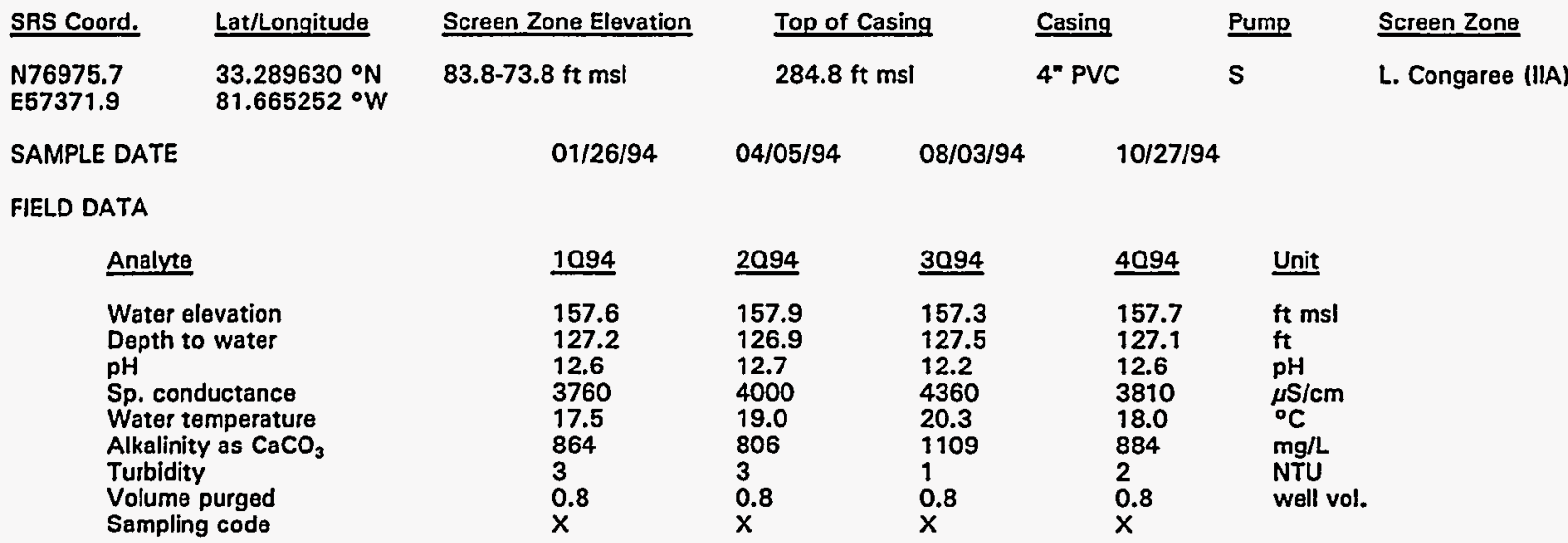

ANALYTICAL DATA

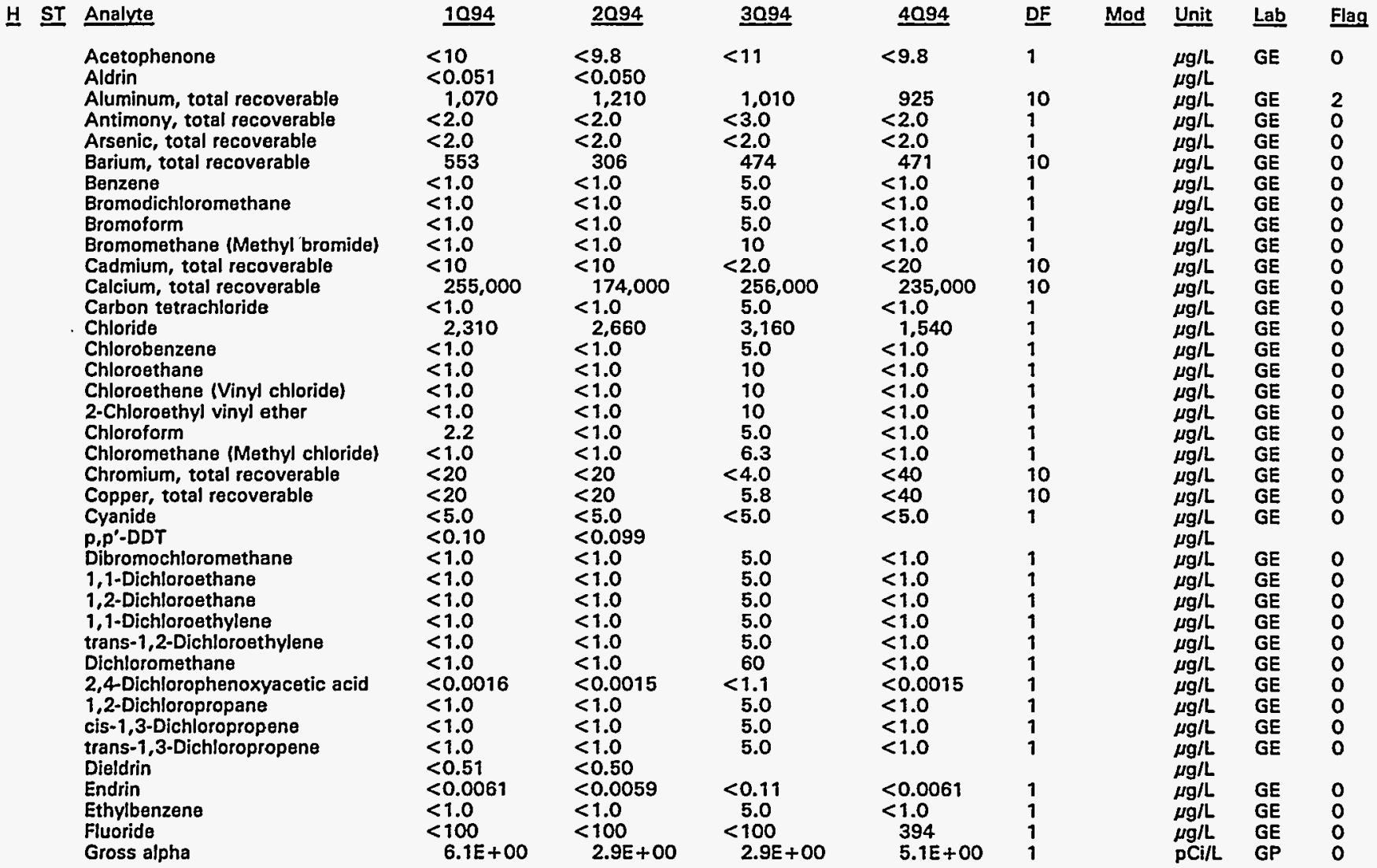

Note: Flagging, dilution factors, modifiers, and laboratories are for fourth quarter 1994 data only. See Appendix B for flagging criteria. - = exceeded holding time for fourth quarter 1994.

- = exceeded screening level or final PDWS for fourth quarter 1994. 


\section{H ST Analyte}

Heptachlor

Iron, total recoverable

Lead, total recoverable

Lindane

Lithium, total recoverable

Magnesium, total recoverable

Manganese, total recoverable

Mercury, total recoverable

Methoxychlor

Naphthalene

Nickel, total recoverable

Nitrate as nitrogen

Nitrate-nitrite as nitrogen

Nonvolatile beta

- $\mathrm{pH}$

Phenols

Potassium, total recoverable

Radium, total alpha-emitting

Selenium, total recoverable

Silica, total recoverable

Silver, total recoverable

Sodium, total recoverable

Specific conductance

Sulfate

1,1,2,2-Tetrachloroethane

Tetrachloroethylene

Tin, total recoverable

Toluene

Total dissolved solids

Total organic carbon

Total organic halogens

Total phosphates (as P)

Toxaphene

2,4,5-TP (Silvex)

1,1,1-Trichloroethane

1,1,2-Trichloroethane

Trichloroethylene

Trichlorofluoromethane

2,4,5-T

Tritium

Turbidity

Uranium-233/234

Uranium-235

Uranium-238

Vanadium, total recoverable

Xylenes

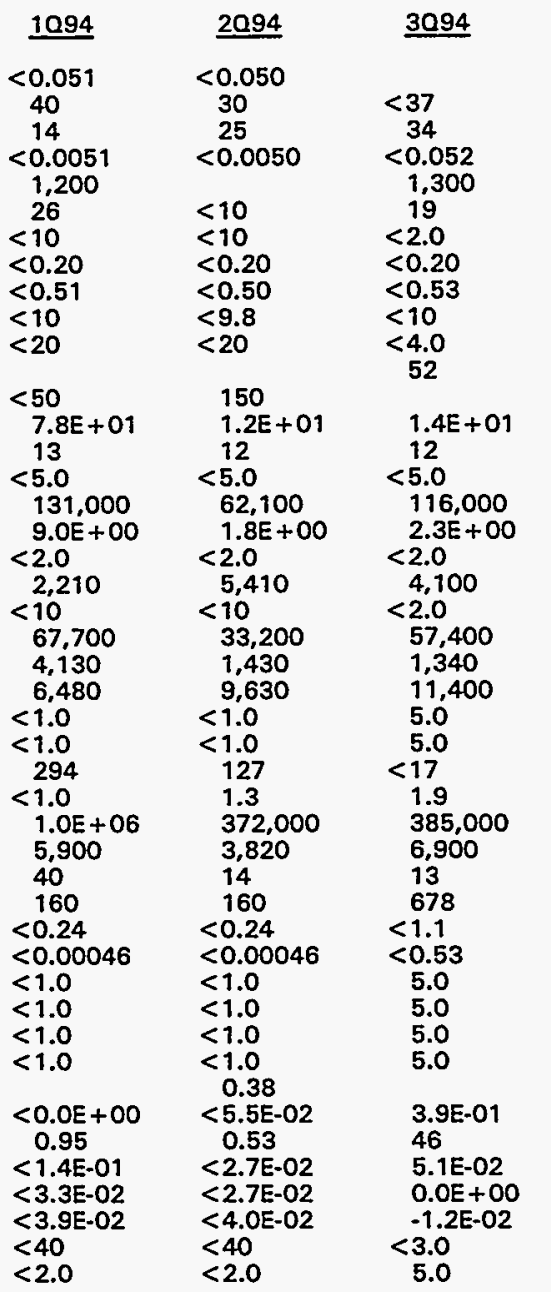

$\quad 4094$
$<40$
26
$<0.0051$
$<20$
$<20$
$<0.20$
$<0.51$
$<9.8$
$<40$
$<50$
$1.3 \mathrm{E}+01$
12
$<5.0$
97,200
$2.0 \mathrm{E}+00$
$<2.0$
$<1,000$
$<20$
48,700
1,580
7,350
$<1.0$
$<1.0$
188
2.1
394,000
4,500
$<5.0$
$<50$
$<0.24$
0.066
$<1.0$
$<1.0$
$<1.0$
$<1.0$
$-1.5 \mathrm{E}-02$
0.25
$7.4 \mathrm{E}-02$
$-4.4 \mathrm{E}-03$
$7.4 \mathrm{E}-02$
$<80$
$<2.0$

$<$

$<$

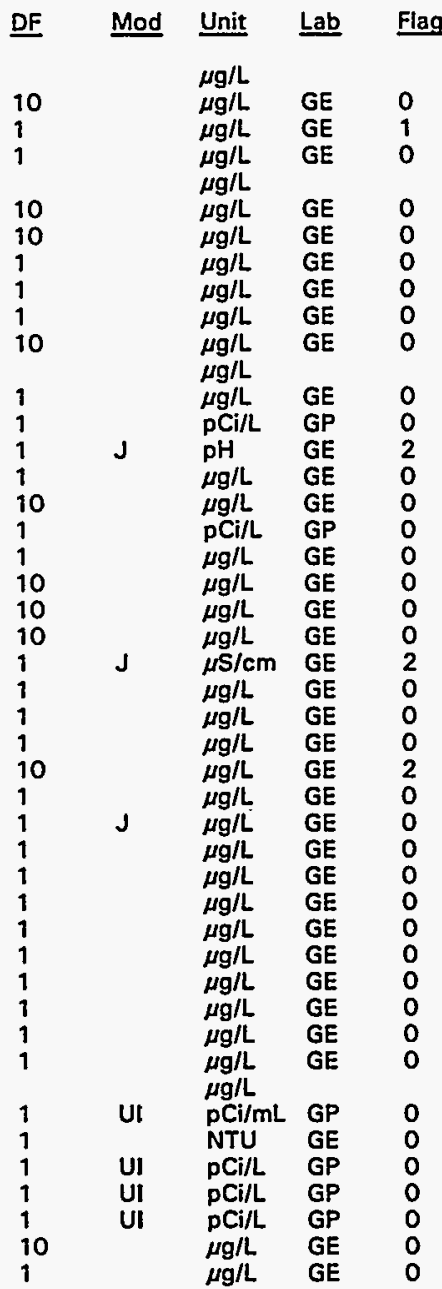

Note: Flagging, dilution factors, modifiers, and laboratories are for fourth quarter 1994 data only. See Appendix B for flagging criteria. - = exceeded holding time for fourth quarter 1994.

- = exceeded screening level or final PDWS for fourth quarter 1994. 


\section{WELL BGO 9D}

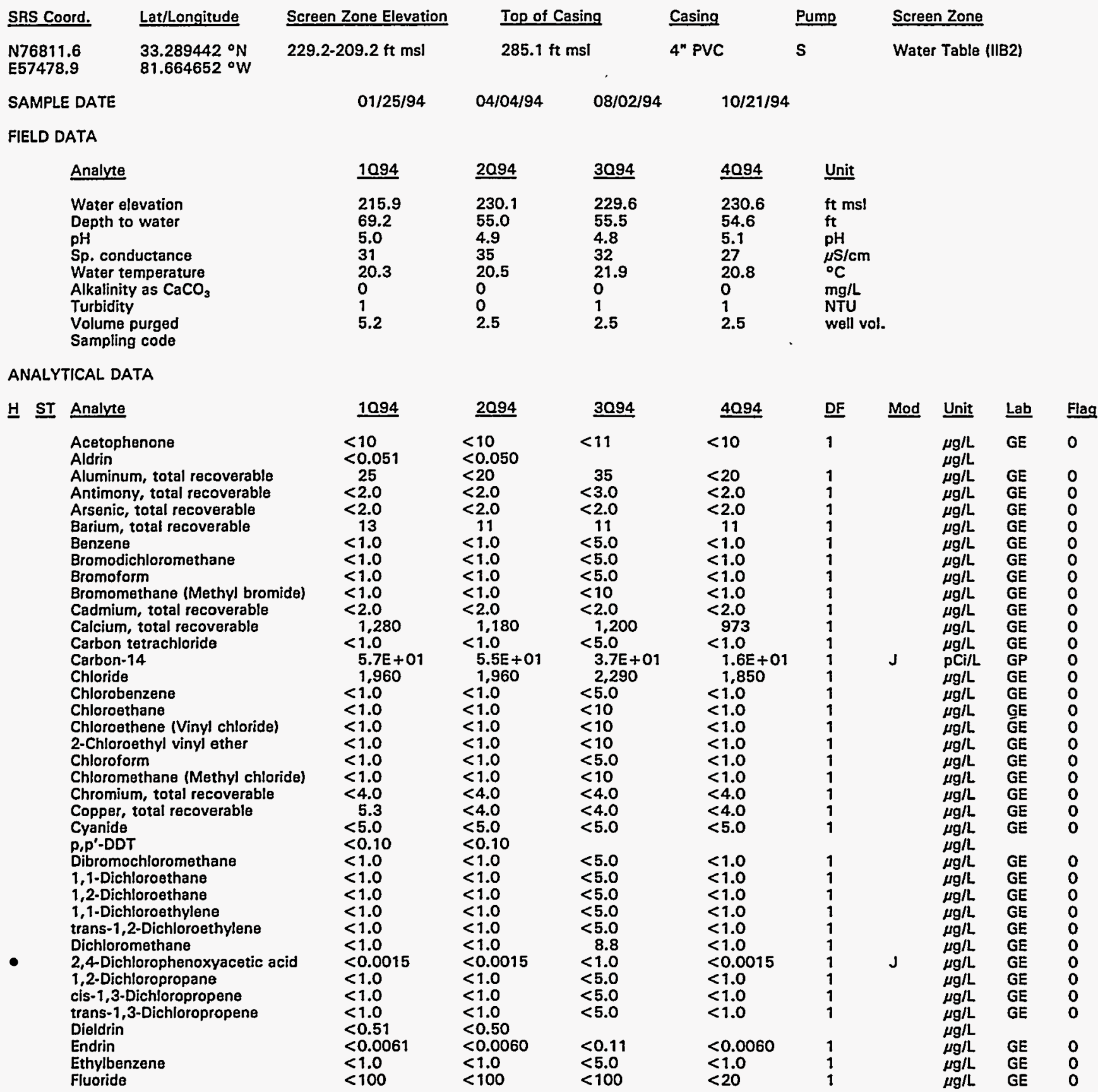

Note: Flagging, dilution factors, modifiers, and laboratories are for fourth quarter 1994 data only. See Appendix B for flagging criteria. - = exceeded holding time for fourth quarter 1994.

- = exceeded screening level or final PDWS for fourth quarter 1994. 
ANALYTICAL DATA

H ST Analyte

Gross alpha

Heptachlor

Iron, total recoverable

Lead, total recoverable

Lindane

Lithium, total recoverable

Magnesium, total recoverable

Manganese, total recoverable

Mercury, total recoverable

Methoxychlor

Naphthalene

Nickel, total recoverable

Nitrate as nitrogen

Nitrate-nitrite as nitrogen

Nonvolatile beta

$\mathrm{pH}$

Phenols

Potassium, total recoverable

Radium, total alpha-emitting

Selenium, total recoverable

Silica, total recoverable

Silver, total recoverable

Sodium, total recoverable

Specific conductance

Sulfate

$1,1,2,2$-Tetrachloroethane

Tetrachloroethylene

Tin, total recoverable

Toluene

Total activity

Total dissolved solids

Total organic carbon

Total organic halogens

Total phosphates (as P)

Toxaphene

2,4,5-TP (Silvex)

1,1,1-Trichloroethane

1,1,2-Trichloroethan

Trichloroethylene

Trichlorofluoromethane

2,4,5-T

- Tritium

Uranium-233/234

Uranium-235

Uranium-238

Vanadium, total recoverable

Xylenes

Zinc, total recoverable

\subsection{4}

1.7E + 00

$<0.051$

10

$<3.0$

$<0.0051$

$<5.0$

560

24

$<0.20$

$<0.51$

$<10$

$<4.0$

1,590

5.9E-01

5.3

$<5.0$

$<500$

$3.5 E+00$

$<2.0$

8,730

$<2.0$

2,240

29

$<1,000$

$<1.0$

$<1.0$

$<2.0$

$<1.0$

2.1E+ 05

29,000

$<1,000$

$<1,000$
24
$<50$

$<50$

$<0.24$

$<0.00045$

$<1.0$

$<1.0$

$<1.0$

2. $4 E+02$

$<0.10$

$<1.9 \mathrm{E}-01$

$<0.0 E+00$

$<6.2 \mathrm{E}-02$

$<8.0$

$<2.0$

$\begin{aligned} & \frac{2094}{} \\ & 3.6 \mathrm{E}+00 \\ &< 0.050 \\ & 6.2<3.0 \\ &< 0.0050 \\ & 527 \\ & 20<0.20 \\ &<0.50\end{aligned}$

$\underline{3094}$

$2.8 E+00$

77

$<3.0$
$<0.053$

$<5.0$

500
21

21
$<0.20$

$<0.53$

$<10$

$<4.0$

$<10$

1,450

$1.4 \mathrm{E}+00$

5.3

$<5.0$

$<500$

1. $1 E+00$

$<2.0$

8.470

$<2.0$

2,390

28

$<1,000$

$<1.0$

$<1.0$

$<2.0$

1.6E + 05

34,000

$<1,000$

$<5.0$

$<50$

$<0.24$

$<0.00045$

$<1.0$

$<1.0$

$<1.0$

0.41

1. $6 E+02$

0.18

$<1.2 \mathrm{E}-02$

$<0.0 E+00$

1.OE-01

$<8.0$

$<2.0$
2. $2 E+00$

5.7

$<5.0$

$<500$

9.0E-01

$<2.0$

7,930

$<2.0$

1,930

28

$<1,000$

$<5.0$
$<5.0$

$<17$

$<5.0$

1.4E + 05

$<5,000$

$<1,000$

9.0

$<50$

$<1.1$
$<0.52$

$<0.52$
$<5.0$

$<5.0$

$<5.0$

$<5.0$

1.0

2.9E-02

$-7.3 \mathrm{E}-03$

1.6E-02

$<3.0$

$<5.0$

$<19$
$2.5 E+02$

$\begin{array}{lll}\frac{4094}{1.9 E+00} & \text { DF } & \text { Mod } \\ 13 & 1 & J\end{array}$

Unit $\quad \underline{\text { Lab }} \quad \underline{\text { Flag }}$

$\mathrm{pCi} / \mathrm{L} \quad \mathrm{GP} \quad \mathrm{O}$

$\mu g / L$

$\mu \mathrm{g} / \mathrm{L} \quad \mathrm{GE}$

$\mu \mathrm{g} / \mathrm{L}$ GE

$\mu g / L \quad G E$

$\mu \mathrm{g} / \mathrm{L}$

$\mu \mathrm{g} / \mathrm{L}$

$\mu g / L$

$\mu g / L$

$\mu \mathrm{g} / \mathrm{L}$

$\mu g / L$

$\mu g / L$

$\mu g / L$

u

$1.3 E+00$

5.3

$<5.0$

$<500$

$1.8 \mathrm{E}+00$

$<2.0$

8,410

$<2.0$

2,780

29

$<1,000$

$<1.0$

$<1.0$

2.7
$<1.0$

31,000

1,040

$<5.0$

$<50$

$<0.24$

$<0.00044$

$<1.0$

$<1.0$

$<1.0$

$<1.0$

1.6E + 02

$<0.10$

1.4E-01

6.1E-03

1.4E-01

$<8.0$

$<2.0$

7.3

$\mu \mathrm{g} / \mathrm{L}$

$\mu \mathrm{g} / \mathrm{L} / \mathrm{L}$

$\mu \mathrm{g} / \mathrm{L}$

$\mu \mathrm{g} / \mathrm{L}$

$\mu \mathrm{S} / \mathrm{cm}$

$\mu \mathrm{g} / \mathrm{L}$

$\mu g / L$

$\mu \mathrm{g} / \mathrm{L} \quad \mathrm{GE}$

$\mu \mathrm{g} / \mathrm{L} \quad \mathrm{GE}$

pCill

$\mathrm{pCi} / \mathrm{L}$

$\mathrm{J} \quad \mu \quad \mu g / L$

$\mu \mathrm{g} / \mathrm{L}$

$\mu \mathrm{g} / \mathrm{L}$.

$\mu \mathrm{g} / \mathrm{L}$

$\mu g / L$

$\mu g / L$

$\mu g / L$

$\mu \mathrm{g} / \mathrm{L}$

pCi/mL GP

PCi/L

PCill

PCi/L

$\mathrm{pCi} / \mathrm{L}$
$\mu \mathrm{g} / \mathrm{L}$

$\mu g / L$

$\mu g / L$

$g / L$

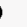

Note: Flagging, dilution factors, modifiers, and laboratories are for fourth quarter 1994 data only. See Appendix B for flagging criteria. - = exceeded holding time for fourth quarter 1994.

- = exceeded screening level or final PDWS for fourth quarter 1994. 
WELL BGO 10AA

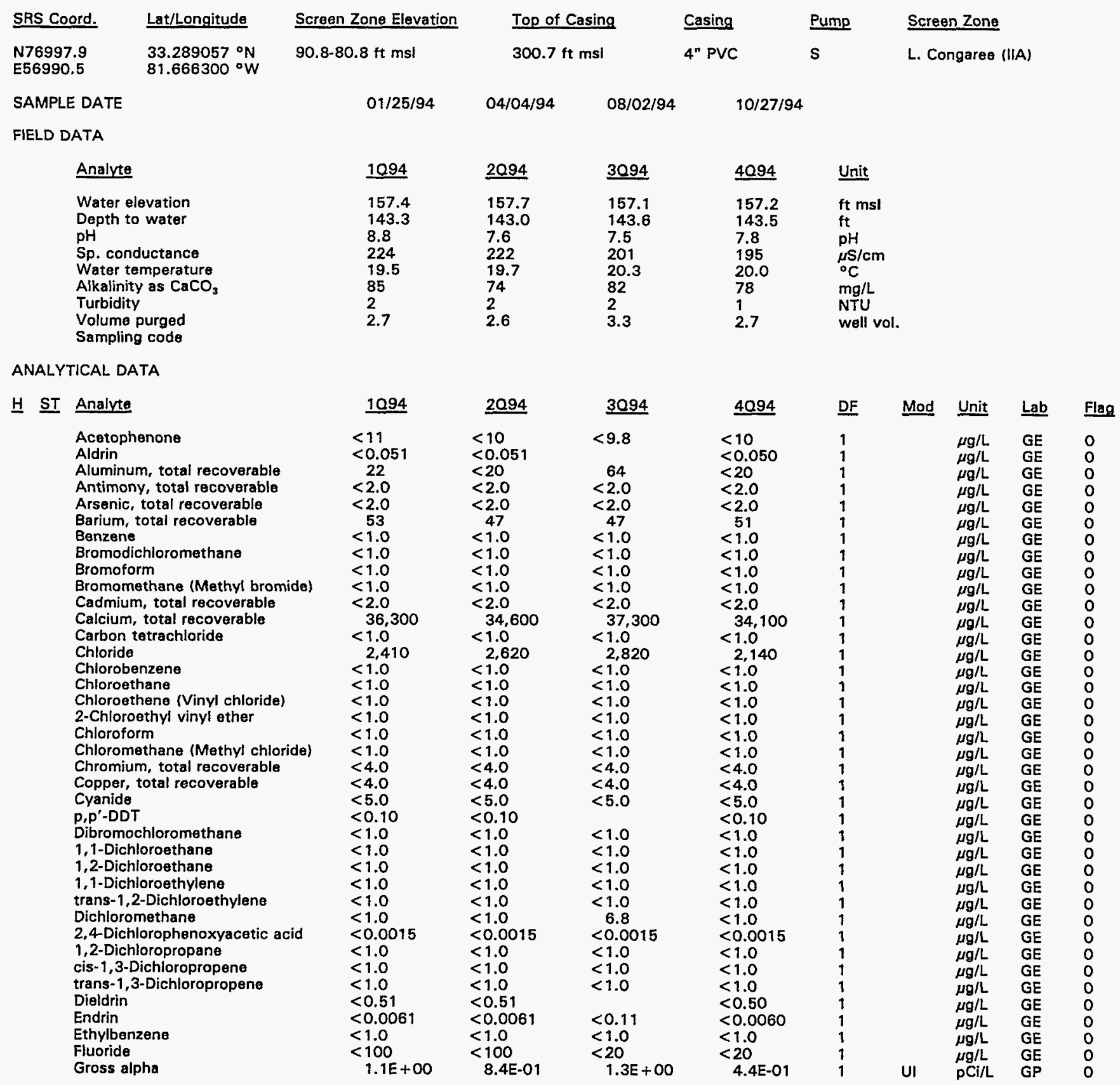

Note: Flagging, dilution factors, modifiers, and laboratories are for fourth quarter 1994 data only. See Appendix B for flagging criteria. - In exceeded holding time for fourth quarter 1994.

a = exceeded screening level or final PDWS for fourth quarter 1994. 
H ST Analyte

Heptachlor

Iron, total recoverable

Lead, total recoverable

Lindane

Lithium, total recoverable

Magnesium, total recoverable

Manganese, total recoverable

Mercury, total recoverable

Methoxychlor

Naphthalene

Nickel, total recoverable

Nitrate as nitrogen

Nitrate-nitrite as nitrogen

Nonvolatile beta

- $\mathrm{pH}$

Phenols

Potassium, total recoverable

Radium, total alpha-emitting

Radium-226

Radium-228

Selenium, total recoverable

Silica, total recoverable

Silver, total recoverable

Sodium, total recoverable

Specific conductance

Sulfate

1,1,2,2-Tetrachloroethane

Tetrachloroethylene

Tin, total recoverable

Toluene

Total dissolved solids

Total organic carbon

Total organic halogens

Total phosphates las P

Toxaphene

2,4,5-TP (Silvex)

1,1,1-Trichloroethane

1,1,2-Trichloroethane

Trichloroethylene

Trichlorofluoromethane

2,4,5-T

Tritium

Turbidity

Uranium-233/234

Uranium-235

Uranium-238

Vanadium, total recoverable

Xylenes

\begin{tabular}{|c|c|c|c|}
\hline 1094 & $\underline{2094}$ & 3094 & 4094 \\
\hline $\begin{array}{c}<0.051 \\
66 \\
5.6 \\
<0.0051 \\
38\end{array}$ & $\begin{array}{c}<0.051 \\
45 \\
5.7 \\
<0.0051\end{array}$ & $\begin{array}{l}88 \\
3.0 \\
<0.053 \\
23\end{array}$ & $\begin{array}{c}<0.050 \\
29 \\
4.7 \\
<0.0050\end{array}$ \\
\hline $\begin{aligned} & 38 \\
& 811 \\
& 5.7 \\
&<0.20 \\
&<0.51 \\
&<11 \\
&<4.0\end{aligned}$ & $\begin{aligned} & 772 \\
& 4.9 \\
< & 0.20 \\
< & 0.51 \\
< & 10 \\
< & 4.0\end{aligned}$ & $\begin{array}{l}793 \\
6.8 \\
<0.20 \\
<0.50 \\
<9.8 \\
<4.0 \\
<20\end{array}$ & $\begin{aligned} & 774 \\
& 4.8 \\
< & 0.20 \\
< & 0.50 \\
< & 10 \\
< & 4.0\end{aligned}$ \\
\hline $\begin{array}{l}<50 \\
3.1 E+00 \\
6.5 \\
<5.0 \\
4.730 \\
1.6 E+00\end{array}$ & $\begin{array}{l}<50 \\
3.8 E+\infty 0 \\
7.7 \\
<5.0 \\
4.240 \\
1.0 E+\infty 0\end{array}$ & $\begin{array}{l}<50 \\
1.9 E+00 \\
7.4 \\
<5.0 \\
2,820 \\
5.0 \mathrm{E}-01 \\
1.5 \mathrm{E}-01 \\
1.2 \mathrm{E}+00\end{array}$ & $\begin{array}{c}<50 \\
2.9 E+00 \\
7.7 \\
<5.0 \\
3,430 \\
6.0 \mathrm{E}-01\end{array}$ \\
\hline $\begin{array}{c}<2.0 \\
31,800 \\
<2.0 \\
4,170 \\
207 \\
5,470\end{array}$ & $\begin{array}{c}<2.0 \\
30,100 \\
<2.0 \\
4,040 \\
202 \\
6,430\end{array}$ & $\begin{array}{c}<2.0 \\
29,900 \\
<2.0 \\
3,060 \\
196 \\
7,690\end{array}$ & $\begin{array}{c}<2.0 \\
29,300 \\
<2.0 \\
3,430 \\
198 \\
5,550\end{array}$ \\
\hline $\begin{array}{l}<1.0 \\
<1.0\end{array}$ & & $\begin{array}{l}<1.0 \\
<1.0\end{array}$ & $\begin{array}{l}<1.0 \\
<1.0\end{array}$ \\
\hline $\begin{array}{r}8.5 \\
<1.0\end{array}$ & $\begin{array}{r}7.3 \\
<1.0\end{array}$ & $\begin{array}{r}5.8 \\
<1.0\end{array}$ & $\begin{array}{r}6.2 \\
<1.0\end{array}$ \\
\hline $\begin{aligned} & 143,000 \\
&< 1,000 \\
& 23\end{aligned}$ & $\begin{array}{l}132,000 \\
1,220\end{array}$ & $\begin{array}{l}138,000 \\
1,410 \\
9,2\end{array}$ & $\begin{array}{l}139,000 \\
1,420 \\
<5.0\end{array}$ \\
\hline $\begin{array}{l}510 \\
<0.25 \\
<0.00046\end{array}$ & $\begin{aligned} & 520 \\
&< 0.24 \\
&<0.00045\end{aligned}$ & $\begin{aligned} & 759 \\
&<0.24 \\
&<0.00044\end{aligned}$ & $\begin{array}{l}92 \\
<0.24 \\
<0.00044\end{array}$ \\
\hline$<1.0$ & $<1.0$ & $<1.0$ & $<1.0$ \\
\hline$<1.0$ & $<1.0$ & $<1.0$ & $<1.0$ \\
\hline $\begin{array}{l}<1.0 \\
<1.0\end{array}$ & $\begin{array}{l}<1.0 \\
<1.0 \\
0.35\end{array}$ & $\begin{array}{r}1.8 \\
<1.0\end{array}$ & $\begin{array}{l}<1.0 \\
<1.0\end{array}$ \\
\hline $\begin{array}{l}<0.0 E+00 \\
1.6\end{array}$ & $\begin{array}{c}<0.0 E+00 \\
0.75\end{array}$ & $\begin{array}{l}2.3 E+01 \\
1.4\end{array}$ & $\begin{array}{l}-1.9 \mathrm{E}-01 \\
0.44\end{array}$ \\
\hline$<1.0 E-01$ & 9.6E-02 & 8.4E-02 & 1.1E-01 \\
\hline$<6.2 \mathrm{E}-02$ & $<-5.1 E-03$ & -2.2E-02 & 1.1E-01 \\
\hline$<8.0$ & $<8.0$ & $<3.0$ & $<8.0$ \\
\hline$<2.0$ & $<2.0$ & $<2.0$ & $<2.0$ \\
\hline
\end{tabular}

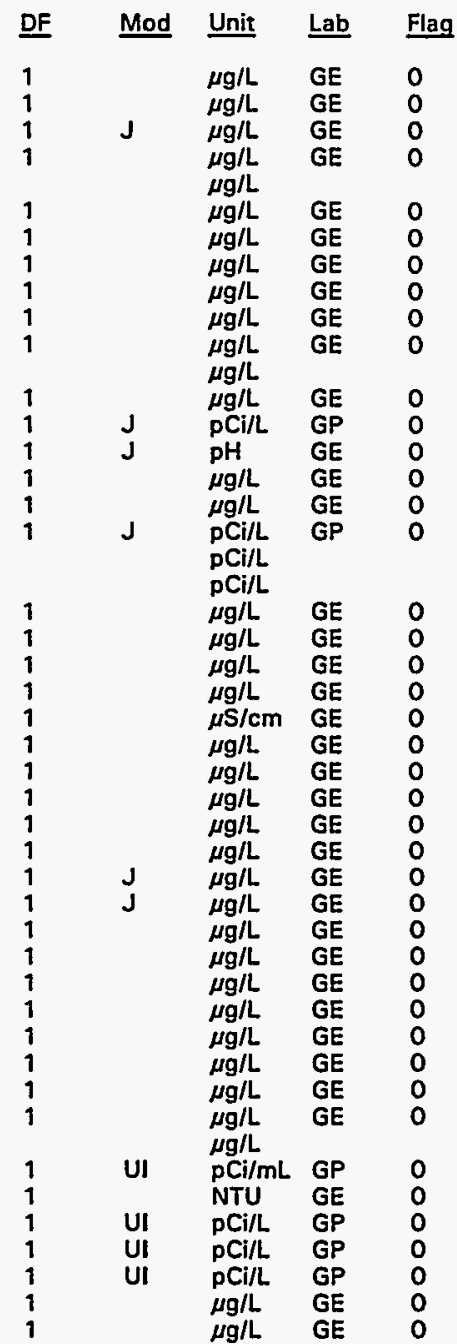

Note: Flagging, dilution factors, modifiers, and laboratories are for fourth quarter 1994 data only. See Appendix B for flagging criteria. $\bullet=$ exceeded holding time for fourth quarter 1994.

- = exceeded screening level or final PDWS for fourth quarter 1994. 


\section{WELL BGO 10AR}

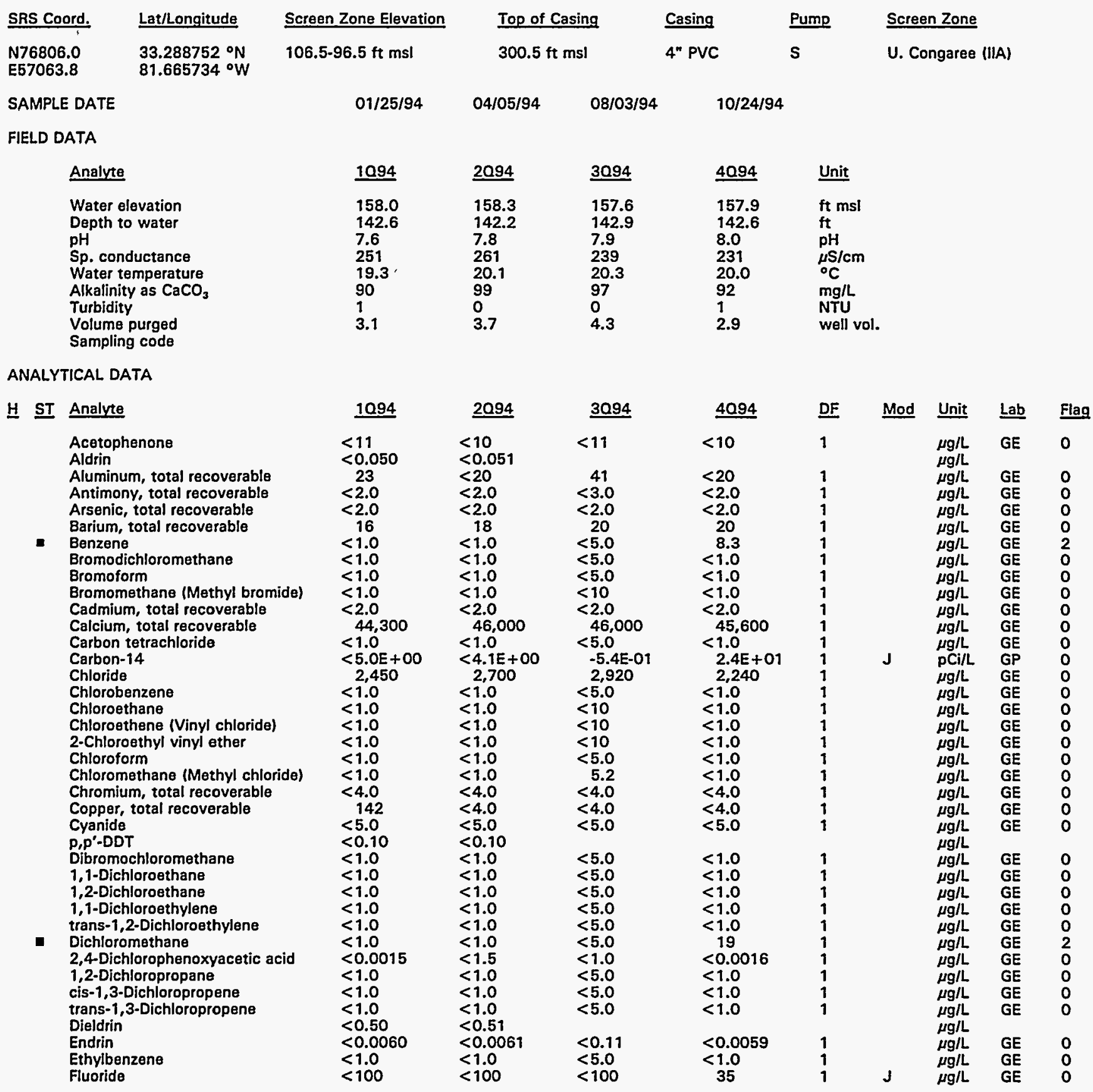

Note: Flagging, dilution factors, modifiers, and laboratories are for fourth quarter 1994 data only. See Appendix B for flagging criteria. - = exceeded holding time for fourth quarter 1994.

- = exceeded screening level or final PDWS for fourth quarter 1994. 


\section{H ST Analyte}

Gross alpha

Heptachlor

Iron, total recoverable

Lead, total recoverable

Lindane

Lithium, total recoverable

Magnesium, total recoverable

Manganese, total recoverable

Mercury, total recoverable

Methoxychlor

Naphthalene

Nickel, total recoverable

Nitrate as nitrogen

Nitrate-nitrite as nitrogen

Nonvolatile beta

$\mathrm{pH}$

Phenols

Potassium, total recoverable

Radium, total alpha-emitting

Selenium, total recoverable

Silica, total recoverable

Silver, total recoverable

Sodium, total recoverable

Specific conductance

Sulfate

1,1,2,2-Tetrachloroethane

Tetrachloroethylene

Tin, total recoverable

Toluene

Total dissolved solids

Total organic carbon

Total organic halogens

Total phosphates (as P)

Toxaphene

2,4,5-TP (Silvex)

Tributyl phosphate

1,1,1-Trichloroethane

1,1,2-Trichloroethane

Trichloroethylene

Trichlorofluoromethane

2,4,5-T

Tritium

Turbidity

Uranium-233/234

Uranium-235

Uranium-238

Vanadium, total recoverable

Xylenes

Zinc, total recoverable

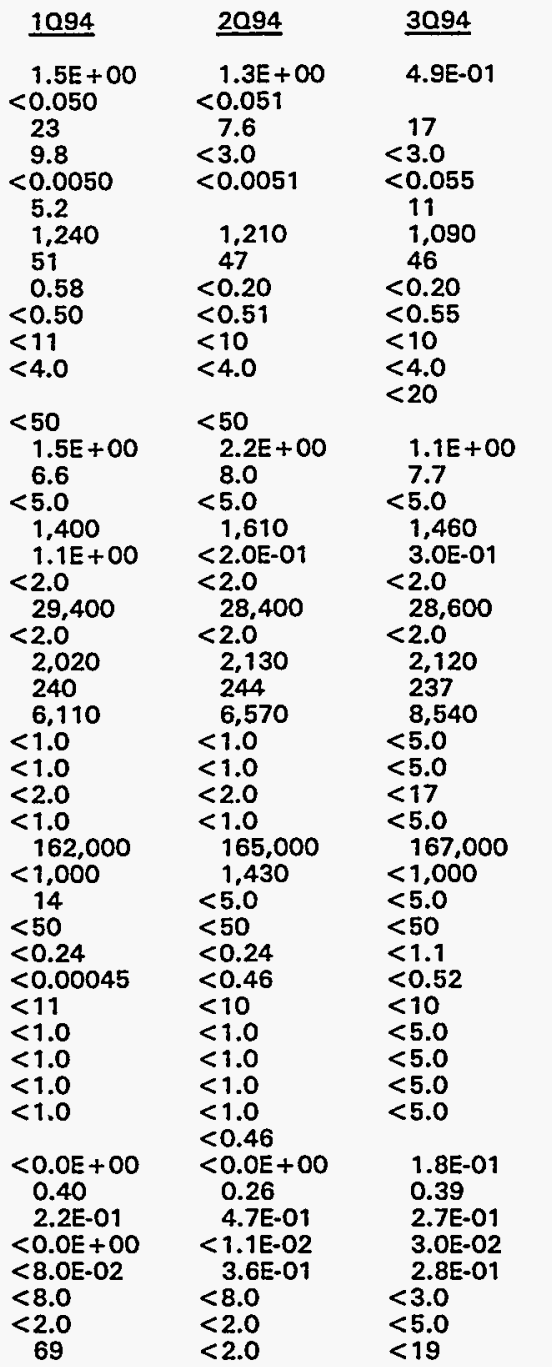

\begin{tabular}{|c|c|}
\hline 4094 & DF \\
\hline $1.6 \mathrm{E}+00$ & 1 \\
\hline 6.9 & 1 \\
\hline $\begin{array}{l}<3.0 \\
<0.0050\end{array}$ & 7 \\
\hline 1,120 & 1 \\
\hline 45 & \\
\hline$<0.20$ & \\
\hline$<0.50$ & 1 \\
\hline $\begin{array}{l}<10 \\
<4.0\end{array}$ & 1 \\
\hline$<50$ & \\
\hline $1.7 E+00$ & \\
\hline 7.9 & 1 \\
\hline$<5.0$ & \\
\hline 1,650 & \\
\hline$-1.0 \mathrm{E}-01$ & 1 \\
\hline $\begin{array}{l}<2.0 \\
28,700\end{array}$ & 7 \\
\hline$<2.0$ & \\
\hline 2,250 & \\
\hline $\begin{array}{l}236 \\
6,190\end{array}$ & 1 \\
\hline$<1.0$ & 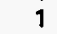 \\
\hline$<1.0$ & \\
\hline 3.3 & 1 \\
\hline$<1.0$ & \\
\hline 164,000 & 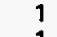 \\
\hline $\begin{array}{l}1,700 \\
<5.0\end{array}$ & 1 \\
\hline$<50$ & \\
\hline$<0.24$ & 1 \\
\hline 0.023 & \\
\hline$<10$ & \\
\hline$<1.0$ & \\
\hline$<1.0$ & \\
\hline$<1.0$ & \\
\hline$<1.0$ & \\
\hline 1.6E-02 & \\
\hline $\begin{array}{l}0.29 \\
7.0 E-01\end{array}$ & \\
\hline $3.2 \mathrm{E}-02$ & \\
\hline 4.3E-01 & \\
\hline$<8.0$ & \\
\hline$<2.0$ & \\
\hline$<2.0$ & \\
\hline
\end{tabular}

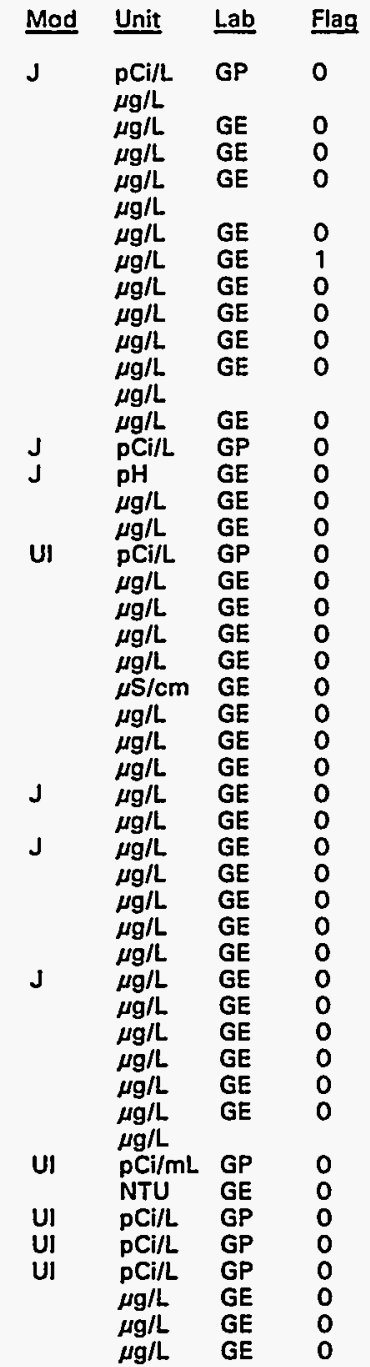

Note: Flagging, dilution factors, modifiers, and laboratories are for fourth quarter 1994 data only. See Appendix B for flagging criteria. - = exceeded holding time for fourth quarter 1994.

- = exceeded screening level or final PDWS for fourth quarter 1994. 
WELL BGO 10B

\begin{tabular}{|c|c|c|c|c|c|c|c|}
\hline SRS Coord. & Lat/Longitude & Screen Zone Elevation & Top of Casin & & Cașing & Pump & Screen Zone \\
\hline $\begin{array}{l}\text { N769882. } 1 \\
\text { E56978.8 }\end{array}$ & $\begin{array}{l}33.289003^{\circ} \mathrm{N} \\
81.666300^{\circ} \mathrm{W}\end{array}$ & $149.0-139.0 \mathrm{ft} \mathrm{ms!}$ & $301 \mathrm{ft} \mathrm{msl}$ & & 4" PVC & $\mathbf{s}$ & Barnwell (IIB1) \\
\hline SAMPLE DA & & $01 / 26 / 94$ & $04 / 05 / 94$ & $08 / 03 / 94$ & $10 / 27 / 94$ & & \\
\hline FIELD DAT & & & & & & & \\
\hline Ans & & 1094 & $\underline{2094}$ & 3094 & 4094 & Unit & \\
\hline $\begin{array}{l}\text { Wa } \\
\text { Dep } \\
\text { pH } \\
\text { Sp. } \\
\text { Wa } \\
\text { Alk } \\
\text { Turl } \\
\text { Vol } \\
\text { San }\end{array}$ & $\begin{array}{l}\text { levation } \\
\text { water } \\
\text { ductance } \\
\text { y merature } \\
\text { y as } \mathrm{CaCO}_{3} \\
\text { purged } \\
\text { g code }\end{array}$ & $\begin{array}{l}220.3 \\
80.8 \\
8.3 \\
215 \\
16.9 \\
82 \\
1 \\
0.9 \\
x\end{array}$ & $\begin{array}{l}220.0 \\
81.1 \\
8.6 \\
253 \\
18.4 \\
95 \\
1 \\
1.0 \\
x\end{array}$ & $\begin{array}{l}219.4 \\
81.6 \\
8.2 \\
198 \\
19.6 \\
80 \\
1 \\
0.9 \\
x\end{array}$ & $\begin{array}{l}219.3 \\
81.8 \\
8.3 \\
235 \\
19.0 \\
94 \\
4 \\
0.9 \\
x\end{array}$ & $\begin{array}{l}\mathrm{ft} \mathrm{msl} \\
\mathrm{ft} \\
\mathrm{pH} \\
\mu \mathrm{S} / \mathrm{cm} \\
{ }^{\circ} \mathrm{C} \\
\mathrm{mg} / \mathrm{L} \\
\mathrm{NTU} \\
\text { well vol. }\end{array}$ & \\
\hline
\end{tabular}

ANALYTICAL DATA

\section{H ST Analyte}

Acetophenone

Aldrin

Aluminum, total recoverable

Antimony, total recoverable

Arsenic, total recoverable

Barium, total recoverable

Benzene

Bromodichloromethane

Bromoform

Bromomethane (Methyl bromide)

Cadmium, total recoverable

Calcium, total recoverable

Carbon tetrachloride

Chloride

Chlorobenzene

Chloroethane

Chloroethene (Vinyl chloride)

2-Chloroethyl vinyl ether

Chloroform

Chloromethane (Methyl chloride)

Chromium, total recoverable

Copper, total recoverable

Cyanide

p,p'-DDT

Dibromochloromethane

1,1-Dichloroethane

1,2-Dichloroethane

1,1-Dichloroethylene

trans-1,2-Dichloroethylene

Dichloromethane

2,4-Dichlorophenoxyacetic acid

1,2-Dichloropropane

cis-1,3-Dichloropropene

trans-1,3-Dichloropropene

Dieldrin

Endrin

Ethylbenzene

Fluotide

Gross alpha

\begin{tabular}{|c|c|c|c|c|}
\hline 1094 & 2094 & 3094 & 4094 & DF \\
\hline $\begin{array}{l}<10 \\
<0.049\end{array}$ & $\begin{array}{l}<10 \\
<0.050\end{array}$ & $<10$ & $<9.8$ & 1 \\
\hline 70 & 60 & 124 & 54 & 1 \\
\hline$<2.0$ & $<2.0$ & $<3.0$ & $<2.0$ & 1 \\
\hline$<2.0$ & $<2.0$ & $<2.0$ & $<2.0$ & $i$ \\
\hline 21 & 18 & 19 & 17 & $i$ \\
\hline$<1.0$ & $<1.0$ & $<5.0$ & $<1.0$ & $i$ \\
\hline$<1.0$ & $<1.0$ & $<5.0$ & $<1.0$ & $i$ \\
\hline$<1.0$ & $<1.0$ & $<5.0$ & $<1.0$ & $i$ \\
\hline$<1.0$ & $<1.0$ & $<10$ & $<1.0$ & $i$ \\
\hline$<2.0$ & $<2.0$ & $<2.0$ & $<2.0$ & 1 \\
\hline 33,200 & 46,300 & $\begin{array}{r}30,200 \\
<5,0\end{array}$ & 60,300 & 2 \\
\hline $\begin{array}{r}<1.0 \\
2,450\end{array}$ & 2,510 & 3,090 & 2,480 & 1 \\
\hline$<1.0$ & $<1.0$ & $<5.0$ & $<1.0$ & $i$ \\
\hline$<1.0$ & $<1.0$ & $<10$ & $<1.0$ & $i$ \\
\hline$<1.0$ & $<1.0$ & $<10$ & $<1.0$ & $i$ \\
\hline$<1.0$ & $<1.0$ & $<10$ & $<1.0$ & 1 \\
\hline$<1.0$ & $<1.0$ & $<5.0$ & $<1.0$ & 1 \\
\hline$<1.0$ & $<1.0$ & 6.9 & $<1.0$ & $i$ \\
\hline$<4.0$ & $<4.0$ & $<4.0$ & $<4.0$ & $i$ \\
\hline$<4.0$ & $<4.0$ & $<4.0$ & $<4.0$ & 1 \\
\hline $\begin{array}{l}<5.0 \\
<0.098\end{array}$ & $<5.0$ & $<5.0$ & $<5.0$ & 1 \\
\hline$<1.0$ & $<1.0$ & $<5.0$ & $<1.0$ & 1 \\
\hline$<1.0$ & $<1.0$ & $<5.0$ & $<1.0$ & $i$ \\
\hline$<1.0$ & $<1.0$ & $<5.0$ & $<1.0$ & \\
\hline$<1.0$ & $<1.0$ & $<5.0$ & $<1.0$ & $j$ \\
\hline$<1.0$ & $<1.0$ & $<5.0$ & $<1.0$ & $i$ \\
\hline$<1.0$ & $<1.0$ & 1.5 & $<1.0$ & $i$ \\
\hline$<0.0015$ & $<0.0015$ & $<1.1$ & $<0.0015$ & \\
\hline$<1.0$ & $<1.0$ & $<5.0$ & $<1.0$ & $i$ \\
\hline$<1.0$ & $<1.0$ & $<5.0$ & $<1.0$ & 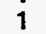 \\
\hline$<1.0$ & $<1.0$ & $<5.0$ & $<1.0$ & \\
\hline$<0.49$ & $<0.50$ & & & \\
\hline$<0.0059$ & $<0.0060$ & $<0.11$ & $<0.0060$ & \\
\hline$<1.0$ & $<1.0$ & $<5.0$ & $<1.0$ & \\
\hline $\begin{array}{l}<100 \\
9.3 \mathrm{E}-01\end{array}$ & $\begin{array}{l}<100 \\
\quad 9.8 \mathrm{E}-01\end{array}$ & $\begin{array}{l}<100 \\
\quad 9.0 \mathrm{E}-01\end{array}$ & $\begin{array}{l}<20 \\
3.3 E-01\end{array}$ & 1 \\
\hline
\end{tabular}

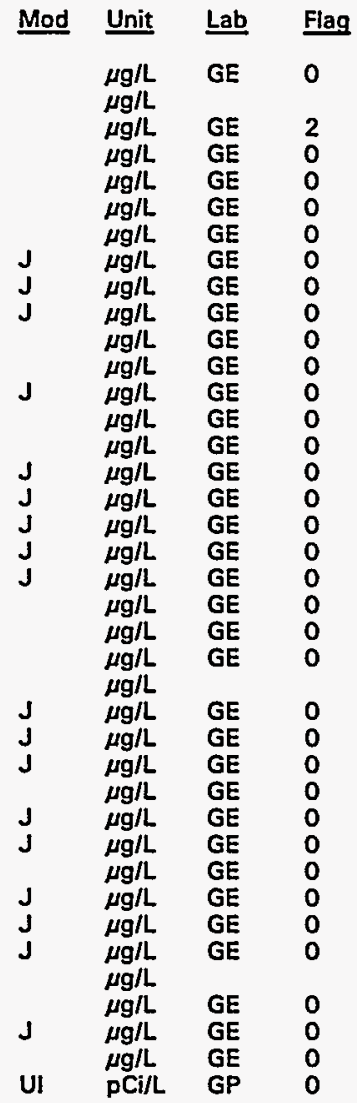

Note: Flagging, dilution factors, modifiers, and laboratories are for fourth quarter 1994 data only. See Appendix B for flagging criteria. - = exceeded holding time for fourth quarter 1994.

- = exceeded screening level or final PDWS for fourth quarter 1994. 
브 ST Analyte

Heptachlor

Iron, total recoverable

Lead, total recoverable

Lindane

Lithium, total recoverable

Magnesium, total recoverable

Manganese, total recoverable

Mercury, total recoverable

Methoxychlor

Naphthalene

Nickel, total recoverable

Nitrate as nitrogen

Nitrate-nitrite as nitrogen

Nonvolatile beta

- $\quad \mathrm{pH}$

Phenols

Potassium, total recoverable

Radium, total alpha-emitting

Selenium, total recoverable

Silica, total recoverable

Silver, total recoverable

Sodium, total recoverable

Specific conductance

Sulfate

1,1,2,2-Tetrachloroethane

Tetrachloroethylene

Tin, total recoverable

Toluene

Total dissolved solids

Total organic carbon

Total organic halogens

Total phosphates (as P)

Toxaphene

2,4,5-TP (Silvex)

1,1,1-Trichloroethane

1,1,2-Trichloroethane

Trichloroethylene

Trichlorofluoromethane

2,4,5-T

Tritium

Turbidity

Uranium-233/234

Uranium-235

Uranium-238

Vanadium, total recoverable

Xylenes

Xylones -

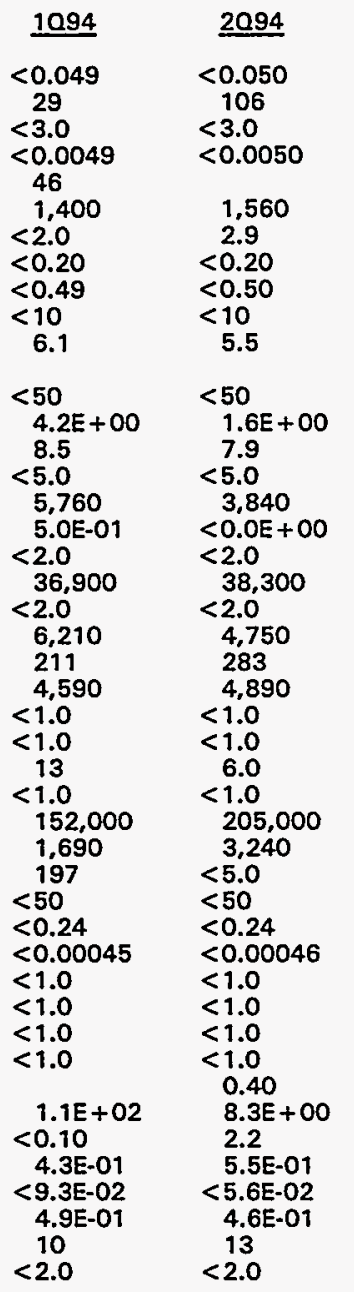

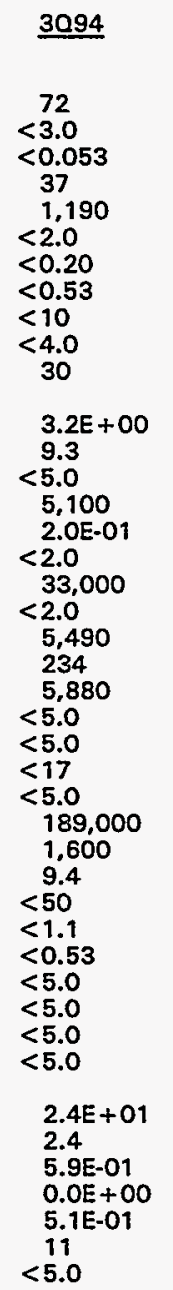

4094

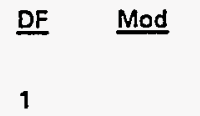

162
$<3.0$

$<0.0050$

1,790

5.3
0.23

0.23

$<0.50$

$<9.8$

$<50$

$7.8 \mathrm{E}-01$

7.9
$<5.0$

$<5.0$

$-1.0 \mathrm{E}-01$

$<2.0$

41,500

$<2.0$

2,930

290

4,520

$<1.0$

$<1.0$

$<2.0$

$<1.0$

209,000

2,630

$<5.0$

$<50$

$<0.24$

$<0.00044$

$<1.0$

$<1.0$

$<1.0$

$<1.0$

$7.0 E+00$

1.3

6.8E-01

4.6E-02

4.0E-01

9.5

$<2.0$

$<2.0$

$<5.0$ 
WELL BGO 10C

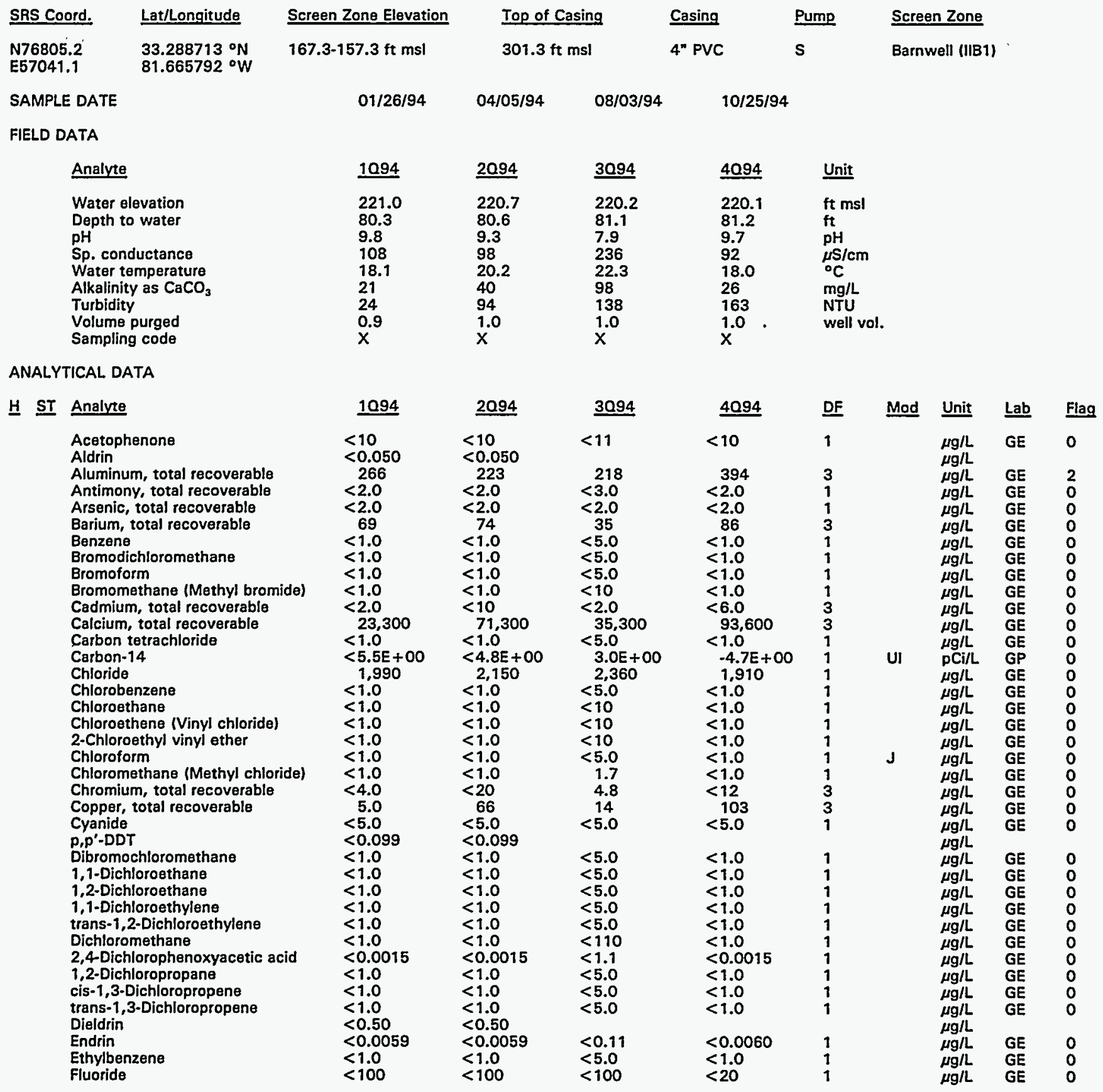

Note: Flagging, dilution factors, modifiers, and laboratories are for fourth quarter 1994 data only. See Appendix B for flagging criteria. - = exceeded holding time for fourth quarter 1994.

- = exceeded screening level or final PDWS for fourth quarter 1994. 
H ST Analyte

Gross alpha

Heptachlor

Iron, total recoverable

Lead, total recoverable

Lindane

Lithium, total recoverable

Magnesium, total recoverable

Manganese, total recoverable

Mercury, total recoverable

Methoxychlor

Naphthalene

Nickel, total recoverable

Nitrate as nitrogen

Nitrate-nitrite as nitrogen

Nonvolatile beta

$\mathrm{pH}$

Phenols

Potassium, total recoverable

Radium, total alpha-emitting

Selenium, total recoverable

Silica, total recoverable

Silver, total recoverable

Sodium, total recoverable

Specific conductance

Sulfate

1,1,2,2-Tetrachloroethane

Tetrachloroethylene

Tin, total recoverable

Toluene

Total dissolved solids

Total organic carbon

Total organic halogens

Total phosphates (as P)

Toxaphene

2,4,5-TP (Silvex)

1,1,1-Trichloroethane

1,1,2-Trichloroethane

Trichloroethylene

Trichlorofluoromethane

2,4,5-T

Tritium

Turbidity

Uranium-233/234

Uranium-235

Uranium-238

Vanadium, total recoverable

Xylenes

Zinc, total recoverable

$\begin{array}{ll}\underline{1094} & \underline{20.94} \\ 1.7 E+00 & 8.7 E-01 \\ <0.050 & <0.050 \\ 74 & 1.240 \\ 5.6 & 33 \\ <0.0050 & <0.0050 \\ 28 & \\ 346 & 1.210 \\ 4.5 & 67 \\ <0.20 & <0.20 \\ <0.50 & <0.50 \\ <10 & <10\end{array}$

$\frac{3094}{5.0 E-01}$
908
15
$<0.054$
$<5.0$
681
15
$<0.20$
$<0.54$
$<10$
$<4.0$
226

$1.0 E+\infty 0$

$1.0 \mathrm{E}+$

2.5E +00

9.5

$<5.0$

5,350

$1.2 \mathrm{E}+00$

$<2.0$

16,200

$<2.0$

5,570

83

1.760

$<1.0$

10

$<1.0$

$<1,000$

5.9

$<50$

$<0.24$

$<0.00046$

$<1.0$

$<1.0$

$<1.0$

$<1.0$

$<5.0$

$<2,500$

$<3.0 E-01$

$<2.0$

23,900

$<10$

3,630

244

3,930

$<1.0$

$<10$

$<10$

161,000

1,340

$<5.0$

190

$<0.24$

$<0.00044$

$<1.0$

$<1.0$

$<1.0$

$<1.0$

0.39

$1.1 E+02$

39

2.2E-01

$<0.0 E+00$

$1.9 E+00$

39

6.9E-01

$<4.5 \mathrm{E}-02$

5.2E-01

$5.0 \mathrm{E}-01$

$<8.0$

$<2.0$
26

$<40$

$<2.0$

124
8.3E-01

7.8
$<5.0$

$<5.0$

$-1.08-01$

$<2.0$

15,600

$<2.0$

2,140

239
4.700

4.700
$<5.0$

$<5.0$

$<17$

$<5.0$

163,000

2,300

5.7

57

$<1.1$

$<0.53$

$<5.0$

$<5.0$

$<5.0$

$<5.0$

2.3 $\mathrm{E}+00$

14

1.1E-01

$-2.6 \mathrm{E}-02$

1.2E-01

$<3.0$

$<5.0$

$\begin{array}{lll}\frac{4094}{1.5 E+00} & \text { DF } & \text { Mod } \\ 2,420 & 3 & \mathrm{~J} \\ 49 & 1 & \end{array}$

Unit Lab Flag

pCi/L GP 0

$\mu \mathrm{g} / \mathrm{L}$

$\mu g / L$ GE 2

$\mu g / L \quad$ GE $\quad 1$

$10.0050 \quad 1 \quad \mu g / L$

1,380

114
$<0.20$

$<0.50$

$<10$

13

298

6.3E-01

7.9

$<5.0$

5.0E-01

$<2.0$

8,570

$<6.0$

3,310

240

3,700

$<1.0$

$<6.0$

171,000

2,870

19

314

$<0.24$

$<0.00044$

$<1.0$

$<1.0$

$<1.0$

$<1.0$

$9.0 \mathrm{E}+00$

6.5

4.5E-01

1.8E-02

3.9E-01

$<24$

$<2.0$

286

$\mu \mathrm{g} / \mathrm{L}$

$\mu g / L$

$\mu g / L$

$\mu g / L$

J $\mu \mathrm{g} / \mathrm{L} \quad \mathrm{GE}$

$\mu \mathrm{g} / \mathrm{L} \quad \mathrm{GE}$

UI PCi/L GP

PCIN GP

UI

$\mu g / L \quad G E$

pCi/L GP

$\mu g / L \quad G E$

$\mu g / L \quad G E$

$\mu \mathrm{g} / \mathrm{L} \quad \mathrm{GE}$

$\mu \mathrm{S} / \mathrm{cm} \quad \mathrm{GE}$

$\mu g / L \quad G E$

$\mu g / L \quad G E$

$\mu g / L$

$\mu g / L$

$\mu g / L$

$\mu g / L$

$\mu \mathrm{g} / \mathrm{L}$

$\mu g / L$

$\mu g / L$
$\mu g / L$
$\mu g / L$

$\mu g / h$

$\mu g / L$

$\mu g / L$

$\mu \mathrm{g} / \mathrm{L}$

$\mathrm{pCi} / \mathrm{mL}$ GP .0

NTU

$\mathrm{pCi} / \mathrm{L}$

$\mathrm{pCi} / \mathrm{L}$

$\mathrm{PCi} / \mathrm{L}$ GP

$\mu g / L \quad G E$

$\underset{\mu \mathrm{g} / \mathrm{L}}{\mathrm{G}} \quad \mathrm{GE}$

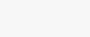

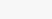

Note: Flagging, dilution factors, modifiers, and laboratories are for fourth quarter 1994 data only. See Appendix B for flagging criteria. - = exceeded holding time for fourth quarter 1994.

u = exceeded screening level or final PDWS for fourth quarter 1994. 
WELL BGO 10DR

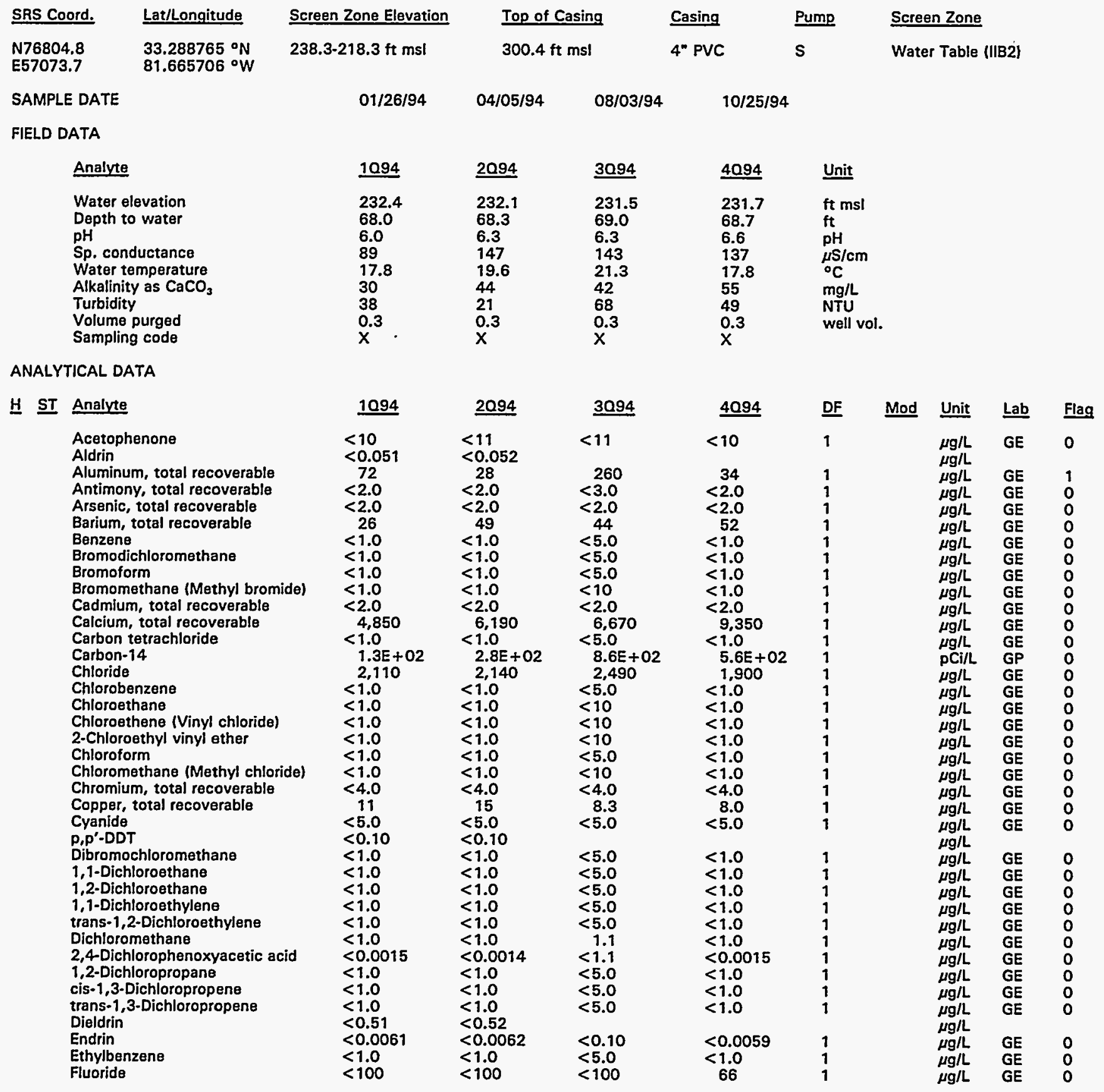

Note: Flagging, dilution factors, modifiers, and laboratories are for fourth quarter 1994 data only. See Appendix B for flagging criteria. - = exceeded holding time for fourth quarter 1994.

- = exceeded screening level or final PDWS for fourth quarter 1994. 
H ST Analyte

Gross alpha

Heptachlor

Iron, total recoverable

Lead, total recoverable

Lindane

Lithium, total recoverable

Magnesium, total recoverable

Manganese, total recoverable

Mercury, total recoverable

Methoxychlor

Naphthalene

Nickel, total recoverable

Nitrate as nitrogen

Nitrate-nitrite as nitrogen

Nonvolatile beta

$\mathrm{pH}$

Phenols

Potassium, total recoverable

Radium, total alpha-emitting

Selenium, total recoverable

Silica, total recoverable

Silver, total recoverable

Sodium, total recoverable

Specific conductance

Sulfate

1,1,2,2-Tetrachloroethane

Tetrachloroethylene

Tin, total recoverable

Toluene

Total activity

Total dissolved solids

Total organic carbon

Total organic halogens

Total phosphates (as P)

Toxaphene

2,4,5-TP (Silvex)

1,1,1-Trichloroethane

1.1,2-Trichloroethane

Trichloroethylene

Trichlorofluoromethane

2,4,5-T

- Tritium

Turbidity

Uranium-233/234

Uranium-235

Uranium-238

Vanadium, total recoverable

Xylenes

Zinc, total recoverable

$\frac{10.94}{1.4 E+00}$
$<0.051$
431
23
$<0.0051$
19
325
24
$<0.20$
$<0.51$
$<10$
11

$2.3 E+00$ $<0.052$

322

19
$<0.0052$

358

358
19

$<0.20$

$<0.52$

$<11$

1,010

3. $O E+00$

6.1
$<5.0$

$<5.0$

$1.7 E+00$

$<2.0$

6,820

$<2.0$

5,520

59

$<1,000$

$<1.0$

$<1.0$

$<2.0$

$<1.0$

$9.4 E+05$

55,000

1,690

8.3

$<50$

$<0.24$

2.2
$<1.0$

$<1.0$

$<1.0$

$<1.0$

$1.0 E+03$

7.8

$<3.8 \mathrm{E}-02$

$<0.0 E+00$

$<0.0 E+\infty$

$<8.0$

$<2.0$

99
1.230
$1.9 E+00$
6.3

$<5.0$

3,250
$1.6 \mathrm{E}+00$

$<2.0$

6,610

$<2.0$

7,730

86

$<1,000$

$<1.0$

$<1.0$

6.3

$<1.0$

1.6E+06

55,000

1,310

15

$<50$

$<0.25$

$<0.00043$

2.5
$<1.0$

$<1.0$

$<1.0$

$<1.0$

0.40
$2.2 E+03$

10.0

$<3.8 E-02$

$<0.0 E+00$

$<2.9 E-02$

$<8.0$

$<2.0$

92

$\frac{3094}{2.8 \mathrm{E}+00}$
950
26
$<0.052$
47
294
16
$<0.20$
$<0.52$
$<10$
7.1
1,080

$6.4 E+00$

7.0

$<5.0$

1,970

1. $O E+00$

$<2.0$

5,190

$<2.0$

5,500

84

$<1,000$

$<5.0$

$<5.0$

$<17$

2.4E+06

36,000

$<1,000$

15

$<50$

$<1.0$

$<0.55$

1.7
$<5.0$

$<5.0$

$<5.0$

$<5.0$

$2.7 E+03$

20

9.0E-01

$0.0 E+00$

4.5E-01

$<3.0$

$<5.0$

69

\begin{tabular}{|c|c|c|}
\hline 4094 & DF & Mod \\
\hline $1.8 E+00$ & 1 & $\mathbf{J}$ \\
\hline $\begin{aligned} & 245 \\
& 13 \\
&< 0.0050\end{aligned}$ & $\begin{array}{l}1 \\
1 \\
1\end{array}$ & \\
\hline $\begin{aligned} & 417 \\
& 16 \\
< & 0.20 \\
< & 0.50 \\
< & 10 \\
& 12\end{aligned}$ & $\begin{array}{l}1 \\
1 \\
1 \\
1 \\
1 \\
1\end{array}$ & \\
\hline $\begin{array}{l}1.130 \\
2.8 E+00 \\
6.5\end{array}$ & $\begin{array}{l}1 \\
1 \\
1\end{array}$ & $\mathbf{J}$ \\
\hline
\end{tabular}

Unit Lab Flag

$\mathrm{pCi} / \mathrm{L} \quad \mathrm{GP} \quad 0$

$\mu \mathrm{g} / \mathrm{L}$

$\mu \mathrm{g} / \mathrm{L} \quad \mathrm{GE} \quad 1$

$\begin{array}{lll}\mu g / L & \text { GE } & 0 \\ \mu g / L & \text { GE } & 0\end{array}$

$\mu \mathrm{g} / \mathrm{L}$

$\mu g / L$

$\mu g / L$

$\mu \mathrm{g} / \mathrm{L}$

$\mu \mathrm{g} / \mathrm{L}$

$\mu g / L$

$\mu g / L \quad G E$

pCi/L GP

6.5
$<5.0$

2,060

$3.9 E+00$

$<2.0$

6,480

$<2.0$

5,830

72

$<1,000$

$<1.0$

$<1.0$

4.8

$<1.0$

51,000

1,750

5.1

$<50$

$<0.24$

$\begin{array}{r}1.3 \\ \hline\end{array}$

$<1.0$

$<1.0$

$<1.0$

$\mathrm{pH}$

$\mu \mathrm{g} / \mathrm{L}$

$\mu \mathrm{g} / \mathrm{L}$
$\mathrm{pCi} / \mathrm{L}$
$\mu \mathrm{g} / \mathrm{L}$

$\mu g / L$

$\mu g / L$

$\mu \mathrm{g} / \mathrm{L}$

$\mu \mathrm{sS/ \textrm {cm }}$

$\mu g / L$

$\mu g / L$
$\mu g / L$
$\mu g / L$

$\mu \mathrm{g} / \mathrm{L}$

$\mathrm{pCi} / \mathrm{L}$

$\mu \mathrm{g} / \mathrm{L}$

$\mu g / L$

$\mu g / L$

$\mu g / L$

$\mu g / L$

J

$\mu g / L$

$\mu g / L$

$\mu g / L$

$\mu g / L$

1.7E +03

pCi/m

10

6.6E-02

$-1.1 \mathrm{E}-02$

4.5E-02

$<8.0$

$<2.0$

NTU

pCi/L

$\mathrm{pCi/L} \quad \mathrm{GP}$

$\mu \mathrm{g} / \mathrm{L}$

$\mu \mathrm{g} / \mathrm{L}$
$\mu \mathrm{g} / \mathrm{L}$

$G E$
$G E$
$G E$
$G E$
$G E$
$G E$
$G E$
$G E$
$G P$
$G E$
$G E$
$G E$
$G P$
$G E$
$G E$
$G E$
$G E$
$G E$
$G E$
$G E$
$G E$
$G E$
$G E$
$G E$
$G E$
$G E$
$G E$
$G E$
$G E$
$G E$
$G E$
$G E$
$G E$
$G E$
$G P$
$G P$
$G E$
$G P$
$G P$
$G P$
$G E$
$G E$
$G E$

1

Note: Flagging, dilution factors, modifiers, and laboratories are for fourth quarter 1994 data only. See Appendix B for flagging criteria. - = exceeded holding time for fourth quarter 1994.

- = exceeded screening level or final PDWS for fourth quarter 1994. 
WELL BGO 11D

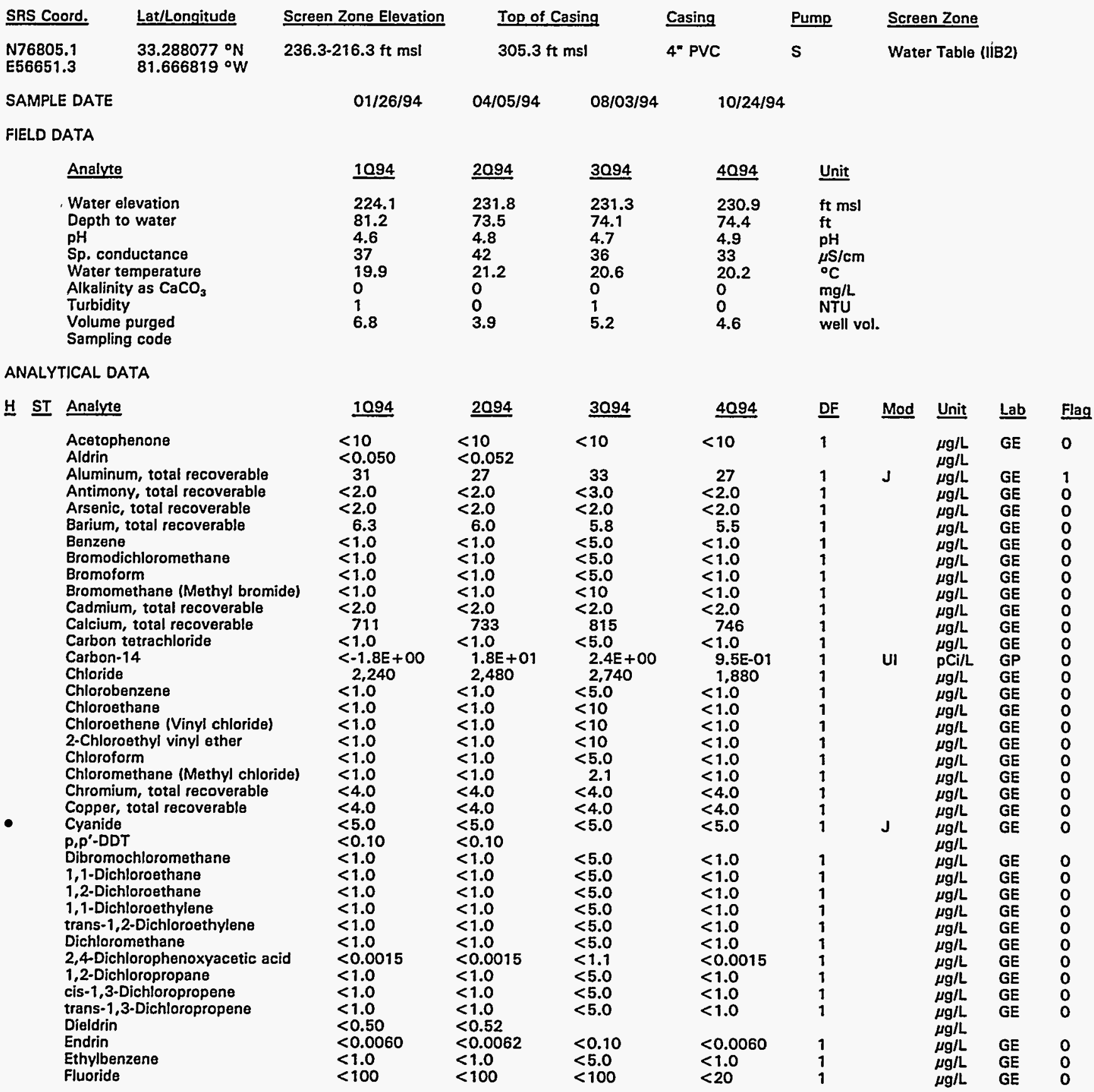

Note: Flagging, dilution factors, modifiers, and laboratories are for fourth quarter 1994 data only. See Appendix B for flagging criteria. - = exceeded holding time for fourth quarter 1994.

m = exceeded screening level or final PDWS for fourth quarter 1994. 
H ST Analyte

Gross alpha

Heptachlor

Iron, total recoverable

Lead, total recoverable

Lindane

Lithium, total recoverable

Magnesium, total recoverable

Manganese, total recoverable

Mercury, total recoverable

Methoxychlor

Naphthalene

Nickel, total recoverable

Nitrate as nitrogen

Nitrate-nitrite as nitrogen

Nonvolatile beta

- $\quad$ pH

Phenols

Potassium, total recoverable

Radium, total alpha-emitting

Selenium, total recoverable

Silica, total recoverable

Silver, total recoverable

Sodium, total recoverable

Specific conductance

Sulfate

$1,1,2,2$-Tetrachloroethane

Tetrachloroethylene

Tin, total recoverable

Toluene

Total activity

Total dissolved solids

Total organic carbon

Total organic halogens

Total phosphates (as P)

Toxaphene

2,4,5-TP (Silvex)

1,1,1-Trichloroethane

1,1,2-Trichloroethane

Trichloroethylene

Trichlorofluoromethane

2,4,5-T

- Tritium

Turbidity

Uranium-233/234

Uranium-235

Uranium-238

Vanadium, total recoverable

Xylenes

Zinc, total recoverable

\section{4 \\ $3.6 E+00$}

$<0.050$

4.6
$<3.0$

$<0.0050$

$<5.0$

632

$<0.20$

$<0.50$

$<10$

$<4.0$

1,960

$2.4 E+00$

5.9
$<5.0$

$<500$

3.7E + 00

$<2.0$

6,900

$<2.0$

3,180

34

$<1.000$

$<1.0$

$<1.0$

2.1
$<1.0$

36,000

$<1,000$

44
$<50$

$<50$
$<0.24$

$<0.00046$

$<1.0$

$<1.0$

$<1.0$

$<1.0$

1.7E+03

$<0.10$

$<9.8 E-02$

$<-1.1 E-02$

$<4.9 E-02$

$<8.0$

$<2.0$

20

$\begin{aligned} & 2094 \\ & 4.6 \mathrm{E}+00 \\ &< 0.052 \\ &< 4.0 \\ &< 3.0 \\ &< 0.0052 \\ & 633 \\ & 4.2 \\ &< 0.20 \\ &< 0.52 \\ &<10\end{aligned}$

3094

$6.3 E+00$

7.8
3.4

$<0.052$

$<5.0$

618
5.1

$<0.20$

$<0.52$

$<10$

$<4.0$

2,140

$2.4 E+00$

4.9
6.9

$<500$

$2.9 E+00$

$<2.0$

6.820

$<2.0$

3,060

36

$<1,000$

$<1.0$

$<1.0$

$<2.0$

$<1.0$

1.5E + 06

36,000

$<1,000$

$<5.0$

$<50$

$<0.25$

$<0.00044$

$<1.0$

$<1.0$

$<1.0$

$<1.0$

0.39

$1.5 E+03$

0.16

7.4E-02

$<9.5 \mathrm{E}-03$

8.3E-02

$<8.0$

$<2.0$

3.2
$2.9 E+00$

5.9
$<5.0$

$<500$

$3.5 \mathrm{E}+00$

$<2.0$

7,240

3,120

37

$<1,000$

$<5.0$

$<5.0$

$<17$

$<5.0$

$7.2 E+05$

$<5,000$

$<1,000$

9.4
$<50$

$<1.0$

$<0.54$

$<5.0$

$<5.0$

$<5.0$

$<5.0$

$1.0 E+03$

$<0.20$

8.8E-02

3.1E-02

6.7E-02

$<3.0$

$<5.0$

$<19$

\begin{tabular}{|c|c|c|}
\hline 4094 & DF & Moo \\
\hline $3.6 E+00$ & 1 & \\
\hline $\begin{array}{l}<4.0 \\
<3.0 \\
<0.0050\end{array}$ & $\begin{array}{l}1 \\
1 \\
1\end{array}$ & \\
\hline $\begin{aligned} & 584 \\
& 4.8 \\
< & 0.20 \\
< & 0.50 \\
< & 10 \\
< & 4.0\end{aligned}$ & $\begin{array}{l}1 \\
1 \\
1 \\
1 \\
1 \\
1\end{array}$ & \\
\hline $\begin{array}{l}2.100 \\
1.5 E+00 \\
5.1 \\
<5.0\end{array}$ & $\begin{array}{l}2 \\
1 \\
1 \\
1\end{array}$ & $\underset{J}{U I}$ \\
\hline
\end{tabular}

Unit Lab Flag

$\mathrm{pCi} / \mathrm{L} \quad \mathrm{GP} \quad \mathrm{O}$

$\mu \mathrm{g} / \mathrm{L}$

$\mu g / L$ GE 0

$\mu \mathrm{g} / \mathrm{L} \quad \mathrm{GE} \quad 0$

$\mu g / L$

$\mu g / L$

$\mu g / L$

$\mu \mathrm{g} / \mathrm{L}$

$\mu g / L$

$\mu g / L$

$\mu \mathrm{g} / \mathrm{L} \quad \mathrm{GE}$

$\mathrm{PCi} / \mathrm{L}$ GP

$\mu \mathrm{g} / \mathrm{L} \quad \mathrm{GE}$

$\mu g / L \quad G E$

$\mathrm{pCi} / \mathrm{L} \quad \mathrm{GP}$

$\mu \mathrm{g} / \mathrm{L} \quad \mathrm{GE}$

$\mu g / L \quad G E$

$\mu \mathrm{g} / \mathrm{L} \quad \mathrm{GE}$

$\mu S / \mathrm{cm}$ GE

$\mu \mathrm{g} / \mathrm{cm}$ GE

$\mu \mathrm{g} / \mathrm{L} \quad \mathrm{GE}$

$\mu g / L \quad G E$

$\mu g / L \quad G E$

$\mu \mathrm{g} / \mathrm{L} \quad \mathrm{GE}$

$<2.0$

$<1.0$

26,000

$<1,000$

$<5.0$

$<50$

$<0.24$

0.0013

$<1.0$

$<1.0$

$<1.0$

$<1.0$

$8.0 E+02$

$<0.10$

1.3E-01

4.4E-03

1.5E-01

$<8.0$

$<2.0$

$<2.0$

$\mu g / L$

$\mu g / L$

pCi/mL GP

NTU

GE 0

GE 0 
WELL BGO 12AR

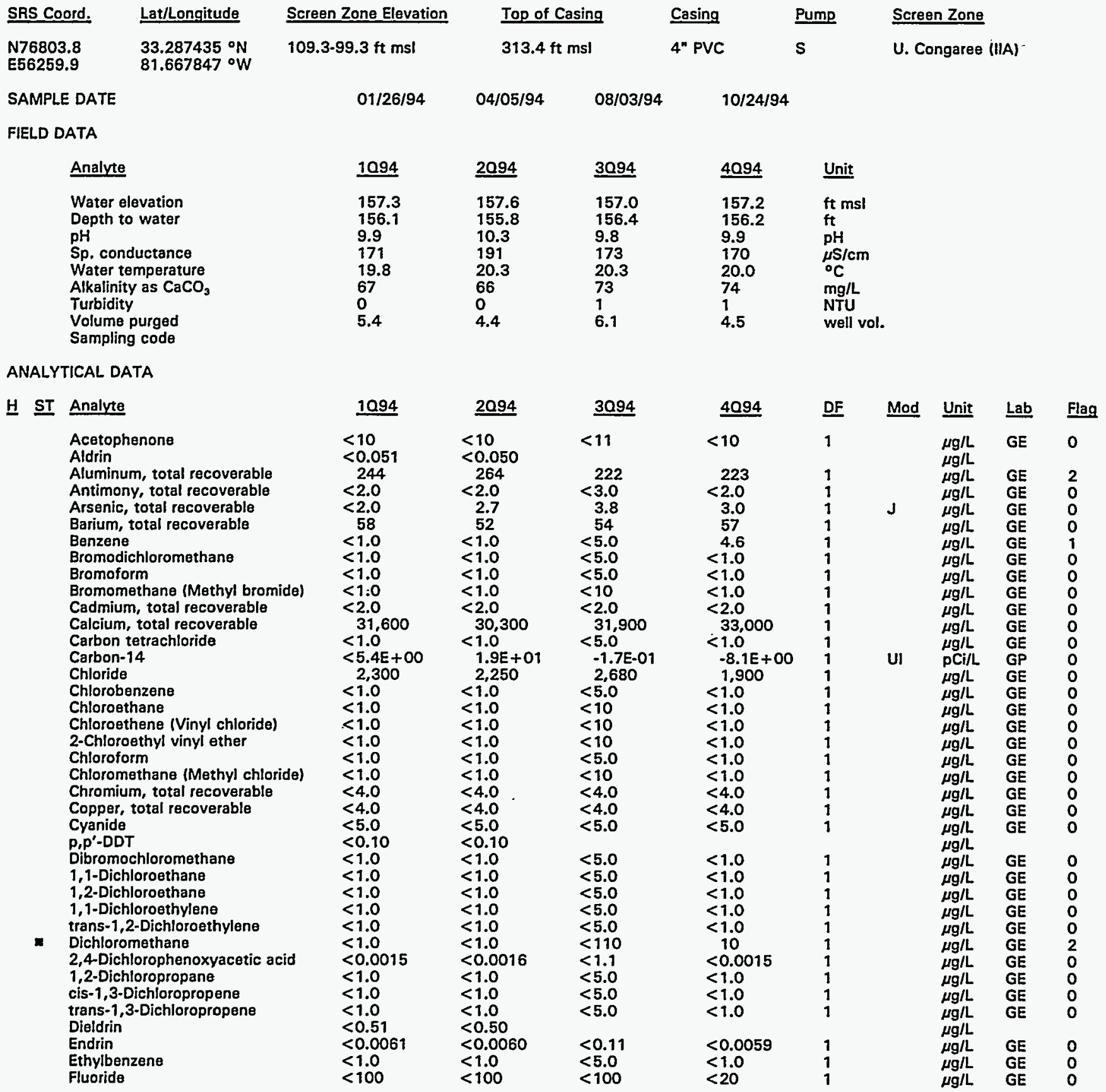

Note: Flagging, dilution factors, modifiers, and laboratories are for fourth quarter 1994 data only. See Appendix B for flagging criteria. - = exceeded holding time for fourth quarter 1994.

- = exceeded screening level or final PDWS for fourth quarter 1994. 


H. ST Analyte
Gross alpha
Heptachlor
Iron, total recoverable
Lead, total recoverable
Lindane
Lithium, total recoverable
Magnesium, total recoverable
Manganese, total recoverable
Mercury, total recoverable
Methoxychlor
Naphthalene
Nickel, total recoverable
Nitrate as nitrogen
Nitrate-nitrite as nitrogen
Nonvolatile beta
pH
Phenols
Potassium, total recoverable
Radium, total alpha-emitting
Selenium, total recoverable
Silica, total recoverable
Silver, total recoverable
Sodium, total recoverable
Specific conductance
Sulfate
1,1,2,2-Tetrachloroethane
Tetrachloroethylene
Tin, total recoverable
Toluene
Total dissolved solids
Total organic carbon
Total organic halogens
Total phosphates (as P)
Toxaphene
$2,4,5-T P$ (Silvex)
Tributyl phosphate
1,1,1-Trichloroethane
1,1,2-Trichloroethane
Trichloroethylene
Trichlorofluoromethane
$2,4,5-T$
Tritium
Turbidity
Uranium-233/234
Uranium-235
Uranium-238
Vanadium, total recoverable
Xylenes
Zinc, total recoverable

\begin{tabular}{|c|c|c|c|c|c|c|c|c|}
\hline 1094 & $\underline{2094}$ & 30994 & 4094 & $\underline{D F}$ & Mod & Unit & $\underline{\text { Lab }}$ & Flag \\
\hline $\begin{array}{l}5.9 \mathrm{E}-01 \\
<0.051\end{array}$ & $\begin{array}{l}1.1 E+00 \\
<0.050\end{array}$ & $1.4 \mathrm{E}-01$ & 3.8E-01 & 1 & Ui & $\begin{array}{l}\mathrm{pCi} / \mathrm{L} \\
\mu \mathrm{g} / \mathrm{L}\end{array}$ & GP & 0 \\
\hline$<4.0$ & $<4.0$ & 19 & 8.0 & 1 & & $\mu \mathrm{g} / \mathrm{L}$ & GE & 0 \\
\hline $\begin{array}{l}<3.0 \\
<0.0051\end{array}$ & $<3.0$ & $\begin{array}{l}<3.0 \\
<0.052\end{array}$ & $<3.0$ & 1 & & $\mu g / L$ & GE & 0 \\
\hline $\begin{array}{l}<0.0051 \\
26\end{array}$ & $<0.0050$ & $\begin{array}{c}<0.052 \\
22\end{array}$ & $<0.0050$ & 1 & & $\underset{\mu g / L}{\mu g / L}$ & GE & 0 \\
\hline 231 & 175 & 238 & 240 & 1 & & $\mu \mathrm{g} / \mathrm{L}$ & GE & 0 \\
\hline$<2.0$ & $<2.0$ & $<2.0$ & $<2.0$ & 1 & & $\mu \mathrm{g} / \mathrm{L}$ & GE & 0 \\
\hline$<0.20$ & $<0.20$ & $<0.20$ & $<0.20$ & 1 & & $\mu \mathrm{g} / \mathrm{L}$ & $\mathrm{GE}$ & 0 \\
\hline$<0.51$ & $<0.50$ & $<0.53$ & $<0.50$ & 1 & & $\mu g / L$ & GE & 0 \\
\hline $\begin{array}{l}<10 \\
<4.0\end{array}$ & $\begin{array}{l}<10 \\
<4.0\end{array}$ & $\begin{array}{l}<10 \\
<4.0\end{array}$ & $\begin{array}{l}<10 \\
<4.0\end{array}$ & $\begin{array}{l}1 \\
1\end{array}$ & & $\underset{\mu \mathrm{g} / \mathrm{L}}{\mu \mathrm{L}}$ & $\begin{array}{l}\mathrm{GE} \\
\mathrm{GE}\end{array}$ & $\begin{array}{l}0 \\
0\end{array}$ \\
\hline$<50$ & 80 & 65 & $<50$ & 1 & & $\underset{\mu \mathrm{g} / \mathrm{L}}{\mu}$ & GE & 0 \\
\hline $2.6 \mathrm{E}+00$ & $4.3 E+00$ & $2.9 E+00$ & 2.7E +00 & 1 & $\mathrm{~J}$ & $\mathrm{pCi/L}$ & GP & 0 \\
\hline 9.4 & 10 & 8.7 & 9.1 & 1 & $J$ & pH & GE & 1 \\
\hline$<5.0$ & $<5.0$ & $<5.0$ & $<5.0$ & 1 & & $\mu g / L$ & GE & 0 \\
\hline $\begin{array}{l}3,600 \\
1.0 E+00\end{array}$ & $\begin{array}{l}3,550 \\
6.0 E-01\end{array}$ & $\begin{array}{l}2,910 \\
7.0 \mathrm{E}-01\end{array}$ & $\begin{array}{l}3,010 \\
2.0 \mathrm{E}-01\end{array}$ & $\begin{array}{l}1 \\
1\end{array}$ & UI & $\begin{array}{l}\mu \mathrm{g} / \mathrm{L} \\
\mathrm{pC} \mathrm{i} / \mathrm{L}\end{array}$ & $\begin{array}{l}\text { GE } \\
G P\end{array}$ & $\begin{array}{l}0 \\
0\end{array}$ \\
\hline$<2.0$ & $<2.0$ & $<2.0$ & $<2.0$ & $i$ & & $\mu \mathrm{g} / \mathrm{L}$ & $\mathrm{GE}$ & 0 \\
\hline $\begin{aligned} & 27,300 \\
< & 2.0\end{aligned}$ & $\begin{aligned} & 25,200 \\
<2.0 & \end{aligned}$ & $\begin{aligned} & 24,500 \\
&<2.0\end{aligned}$ & $\begin{aligned} & 26,100 \\
< & 2.0\end{aligned}$ & $\begin{array}{l}1 \\
1\end{array}$ & & $\begin{array}{l}\mu g / L \\
\mu g / L\end{array}$ & $\begin{array}{l}\mathrm{GE} \\
\mathrm{GE}\end{array}$ & $\begin{array}{l}0 \\
0\end{array}$ \\
\hline $\begin{array}{l}3,050 \\
92\end{array}$ & $\begin{array}{l}3,280 \\
80\end{array}$ & $\begin{array}{l}2,690 \\
108\end{array}$ & $\begin{array}{l}2,900 \\
105\end{array}$ & $\begin{array}{l}1 \\
1\end{array}$ & & $\begin{array}{l}\mu \mathrm{g} / \mathrm{L} \\
\mu \mathrm{S} / \mathrm{cm}\end{array}$ & $\begin{array}{l}\mathrm{GE} \\
\mathrm{GE}\end{array}$ & $\begin{array}{l}0 \\
0\end{array}$ \\
\hline 5,020 & 5,080 & 6,310 & 4,730 & 1 & & $\mu \mathrm{g} / \mathrm{L}$ & $\mathrm{GE}$ & 0 \\
\hline$<1.0$ & $<1.0$ & $<5.0$ & $<1.0$ & 1 & & $\mu \mathrm{g} / \mathrm{L}$ & GE & 0 \\
\hline$<1.0$ & $\begin{array}{r}<1.0 \\
6.5\end{array}$ & $\begin{array}{l}<5.0 \\
<17\end{array}$ & $<1.0$ & 1 & & $\mu g / L$ & GE & 0 \\
\hline $\begin{array}{r}12 \\
<1.0\end{array}$ & $\begin{array}{r}6.5 \\
<1.0\end{array}$ & $\begin{array}{l}<17 \\
<5.0\end{array}$ & $\begin{array}{r}7.3 \\
<1.0\end{array}$ & $\begin{array}{l}1 \\
1\end{array}$ & & $\begin{array}{l}\mu \mathrm{g} / \mathrm{L} \\
\mu \mathrm{g} / \mathrm{L}\end{array}$ & $\begin{array}{l}\mathrm{GE} \\
\mathrm{GE}\end{array}$ & $\begin{array}{l}0 \\
0\end{array}$ \\
\hline & & & $\begin{array}{l}94,000 \\
1,160\end{array}$ & 1 & J & $\begin{array}{l}\mu g / L \\
\mu g / L\end{array}$ & $\begin{array}{l}\mathrm{GE} \\
\mathrm{GE}\end{array}$ & $\begin{array}{l}0 \\
0\end{array}$ \\
\hline $\begin{aligned} & 59 \\
< & 50 \\
< & 0.25\end{aligned}$ & $\begin{array}{l}<5.0 \\
<50 \\
<0.24\end{array}$ & $\begin{array}{r}11 \\
<50 \\
<1.1\end{array}$ & $\begin{array}{l}<5.0 \\
<50 \\
<0.24\end{array}$ & $\begin{array}{l}1 \\
1 \\
1\end{array}$ & & $\begin{array}{l}\mu \mathrm{g} / \mathrm{L} \\
\mu \mathrm{g} / \mathrm{L} \\
\mu \mathrm{g} / \mathrm{L}\end{array}$ & $\begin{array}{l}\mathrm{GE} \\
\mathrm{GE} \\
\mathrm{GE}\end{array}$ & $\begin{array}{l}0 \\
0 \\
0\end{array}$ \\
\hline $\begin{array}{l}<0.25 \\
<0.00045\end{array}$ & $<0.00047$ & $<0.53$ & 0.025 & 1 & J & $\mu \mathrm{g} / \mathrm{L}$ & GE & $\begin{array}{l}0 \\
0\end{array}$ \\
\hline $\begin{array}{l}<10 \\
<1.0\end{array}$ & $\begin{array}{l}<10 \\
<1.0\end{array}$ & $\begin{array}{l}<10 \\
<5.0\end{array}$ & $<10$ & 1 & & $\mu \mathrm{g} / \mathrm{L}$ & $\begin{array}{l}\text { GE } \\
G E\end{array}$ & $\begin{array}{l}0 \\
0\end{array}$ \\
\hline $\begin{array}{l}<1.0 \\
<1.0\end{array}$ & $<1.0$ & $\begin{array}{l}<5.0 \\
<5.0\end{array}$ & $\begin{array}{l}<1.0 \\
<1.0\end{array}$ & 1 & & $\mu \mathrm{g} / \mathrm{L}$ & $\mathrm{GE}$ & 0 \\
\hline 3.0 & 4.3 & 2.7 & 3.2 & $i$ & & $\mu \mathrm{g} / \mathrm{L}$ & $G E$ & 1 \\
\hline$<1.0$ & $\begin{array}{l}<1.0 \\
0.34\end{array}$ & $<5.0$ & $<1.0$ & 1 & & $\begin{array}{l}\mu \mathrm{g} / \mathrm{L} \\
\mu \mathrm{g} / \mathrm{L}\end{array}$ & $\mathrm{GE}$ & 0 \\
\hline$<1.1 E-01$ & $1.3 E+00$ & 5.1E-01 & $3.4 \mathrm{E}-01$ & 1 & UI & $\mathrm{pCi} / \mathrm{mL}$ & GP & 0 \\
\hline 3.0 & 0.44 & 4.2 & 0.64 & 1 & & NTU & GE & $\begin{array}{l}0 \\
0\end{array}$ \\
\hline 2.7E-01 & 1.3E-01 & 1.7E-02 & 2.4E-01 & 1 & UI & $\mathrm{pCi} / \mathrm{L}$ & $\begin{array}{l}\text { GP } \\
\text { GP }\end{array}$ & $\begin{array}{l}0 \\
0\end{array}$ \\
\hline $\begin{array}{l}<0.0 \mathrm{E}+00 \\
<8.4 \mathrm{E}-02\end{array}$ & $\begin{array}{c}<-9.9 \mathrm{E}-03 \\
1.3 \mathrm{E}-01\end{array}$ & $\begin{array}{l}-6.8 \mathrm{E}-03 \\
6.1 \mathrm{E}-02\end{array}$ & $\begin{array}{l}1.9 \mathrm{E}-02 \\
1.6 \mathrm{E}-01\end{array}$ & 1 & UI & $\begin{array}{l}\mathrm{PCI} / \mathrm{L} \\
\mathrm{pCi} / \mathrm{L}\end{array}$ & GP & $\begin{array}{l}0 \\
0\end{array}$ \\
\hline$<8.0$ & $<8.0$ & $<3.0$ & $<8.0$ & 1 & & $\mu g / L$ & GE & 0 \\
\hline 18 & $<2.0$ & $<19$ & $<2.0$ & 1 & & $\mu \mathrm{g} / \mathrm{L}$ & GE & 0 \\
\hline
\end{tabular}

Note: Flagging, dilution factors, modifiers, and laboratories are for fourth quarter 1994 data only. See Appendix B for flagging criteria. - = exceeded holding time for fourth quarter 1994.

- = exceeded screening level or final PDWS for fourth quarter 1994. 


\section{WELL BGO 12CR}

\begin{tabular}{|c|c|c|c|c|c|c|}
\hline SRS Coord. & Lat/Longitude & Screen Zone Elevation & Top of Casing & Casing & Pump & Screen Zone \\
\hline $\begin{array}{l}\text { N76806.0 } \\
\text { E56215.2 }\end{array}$ & $\begin{array}{l}33.287367^{\circ} \mathrm{N} \\
81.667969^{\circ} \mathrm{W}\end{array}$ & $154.0-144.0 \mathrm{ft} \mathrm{msl}$ & $314 \mathrm{ft} \mathrm{ms!}$ & 4" PVC & s & Barnwell (IIB1) \\
\hline ;AMPLE D & & $01 / 26 / 94$ & $4 / 06 / 94$ & & & \\
\hline
\end{tabular}

FIELD DATA

Analyze
Water elevation
Depth to water
pH
Sp. conductance
Water temperature
Alkalinity as $\mathrm{CaCO}_{3}$
Turbidity
Volume purged
Sampling code

ANALYTICAL DATA

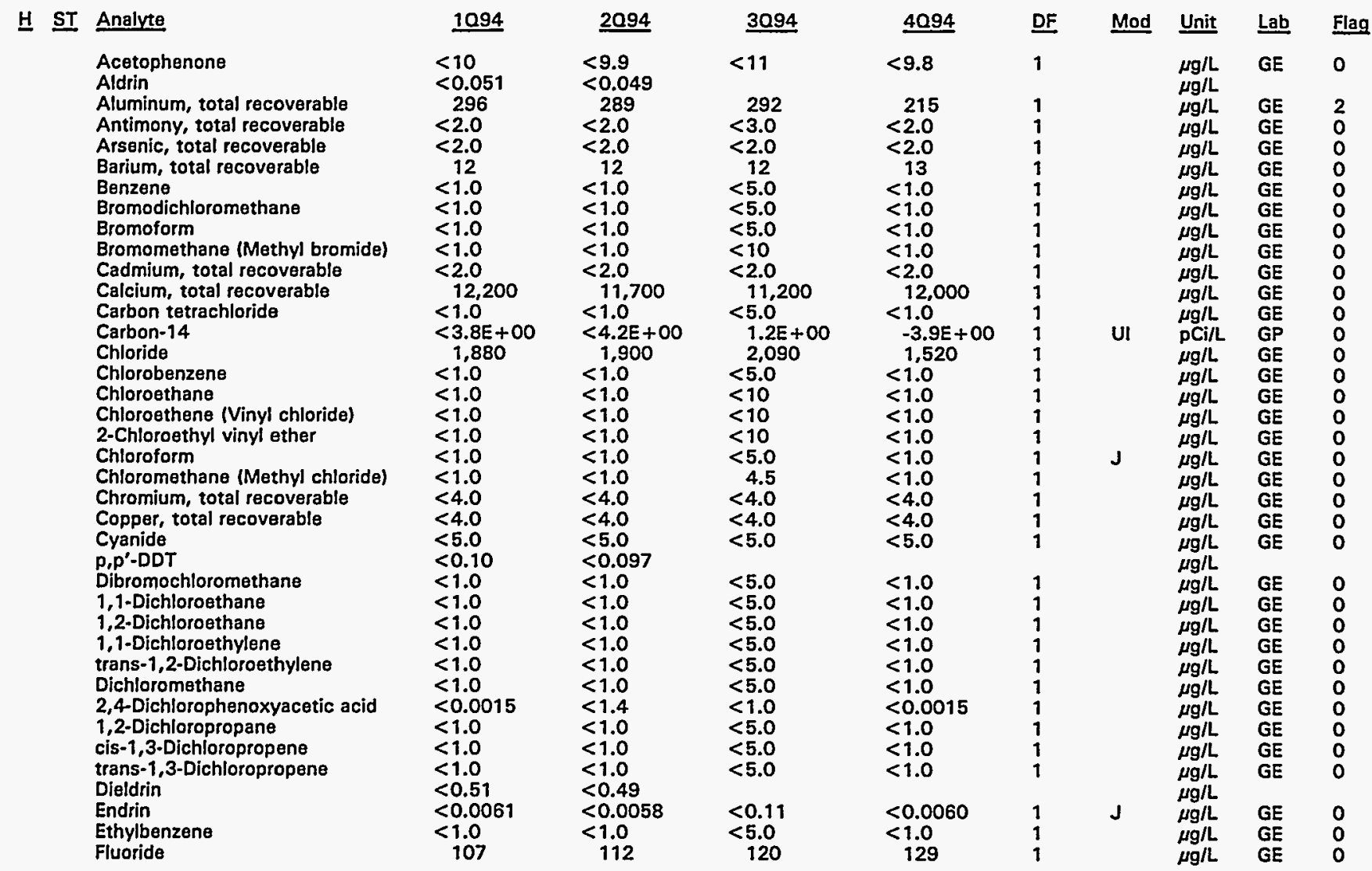

$\begin{array}{lll}1094 & & \underline{2094} \\ 221.7 & & 221.4 \\ 92.3 & & 92.6 \\ 9.7 & & 9.8 \\ 112 & 127 \\ 18.6 & 19.0 \\ 37 & 37 \\ 1 & 1 \\ 0.8 & 0.7 \\ x & x\end{array}$

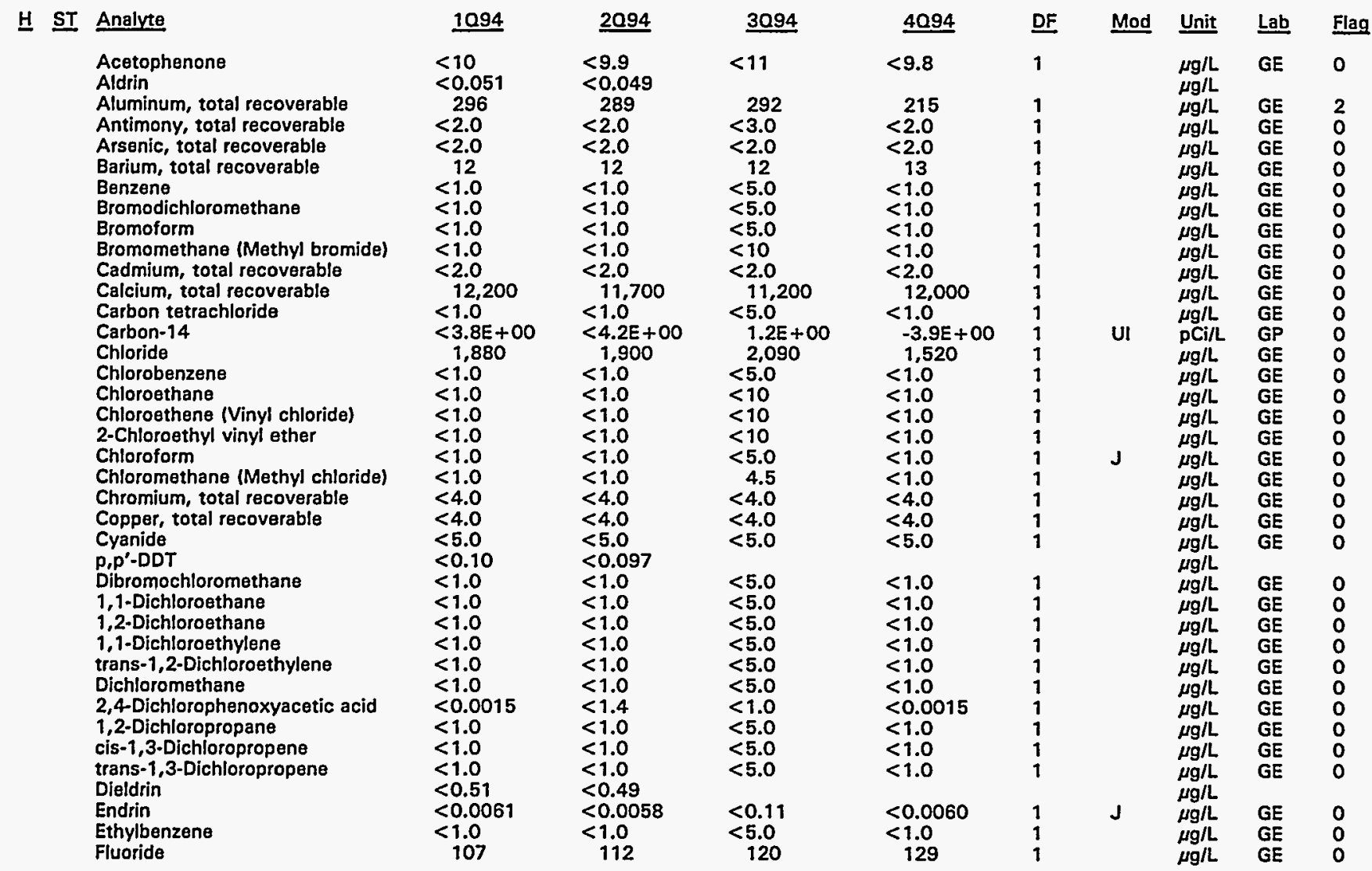

3094
219.4
94.6
9.6
117
20.2
45
2
0.9
$x$

\begin{tabular}{l}
4094 \\
\hline 220.5 \\
93.5 \\
9.7 \\
105 \\
19.4 \\
36 \\
1 \\
0.9 \\
$x$
\end{tabular}

Unit

ft msl

$\mathrm{ft}$

$\mu \mathrm{S} / \mathrm{cm}$

${ }^{\circ} \mathrm{C}$

Ng/L

well vol.

Note: Flagging, dilution factors, modifiers, and laboratories are for fourth quarter 1994 data only. See Appendix B for flagging criteria. - = exceeded holding time for fourth quarter 1994.

- = exceeded screening level or final PDWS for fourth quarter 1994. 
H ST Analyte

Gross alpha

Heptachlor

Iron, total recoverable

Lead, total recoverable

Lindane

Lithium, total recoverable

Magnesium, total recoverable

Manganese, total recoverable

Mercury, total recoverable

Methoxychlor

Naphthalene

Nickel, total recoverable

Nitrate as nitrogen

Nitrate-nitrite as nitrogen

Nonvolatile beta

- $\quad \mathrm{pH}$

Phenols

Potassium, total recoverable

Radium, total alpha-emitting

Selenium, total recoverable

Silica, total recoverable

Silver, total recoverable

Sodium, total recoverable

Specific conductance

Sulfate

1,1,2,2-Tetrachloroethane

Tetrachloroethylene

Tin, total recoverable

Toluene

Total dissolved solids

Total organic carbon

Total organic halogens

Total phosphates (as P)

Toxaphene

2,4,5-TP (Silvex)

Tributyl phosphate

1,1,1-Trichloroethane

1,1,2-Trichloroethane

- Trichloroethylene

Trichlorofluoromethane

2,4,5-T

Tritium

Turbidity

Uranium-233/234

Uranium-235

Uranium-238

Vanadium, total recoverable

Xylenes

Zinc, total recoverable

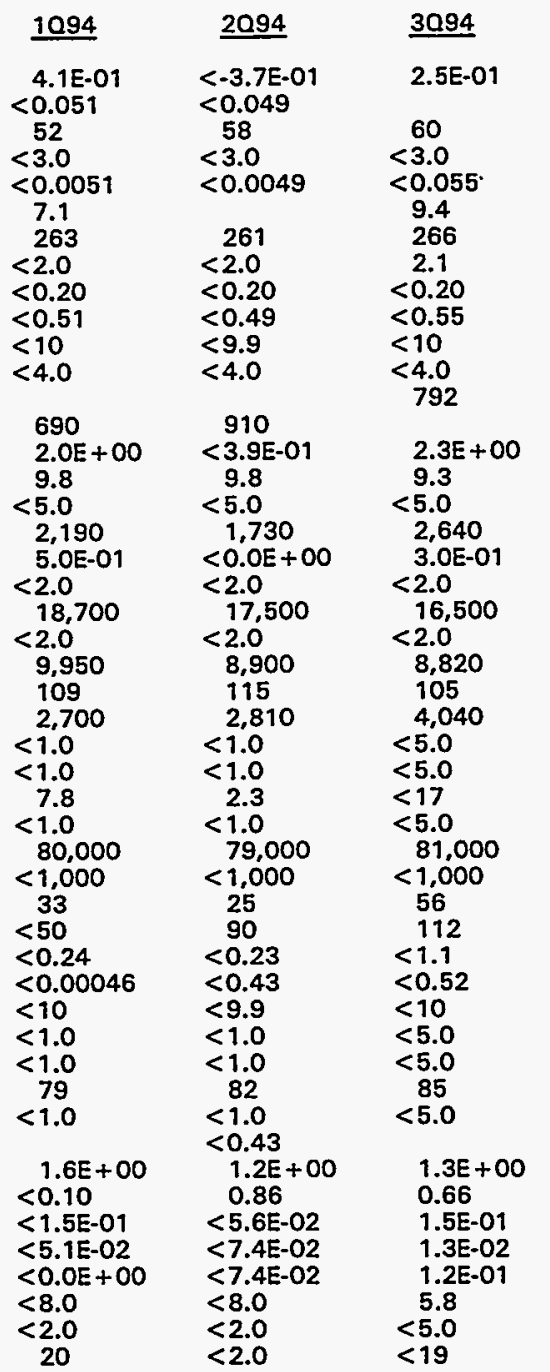

\begin{tabular}{|c|c|}
\hline 4094 & DF \\
\hline 2.0E-01 & 1 \\
\hline $\begin{aligned} & 41 \\
< & 3.0 \\
< & 0.0050\end{aligned}$ & $\begin{array}{l}1 \\
1 \\
1\end{array}$ \\
\hline $\begin{aligned} & 290 \\
< & 2.0 \\
< & 0.20 \\
< & 0.50 \\
< & <.8 \\
< & 4.0\end{aligned}$ & $\begin{array}{l}1 \\
1 \\
1 \\
1 \\
1 \\
1\end{array}$ \\
\hline $\begin{array}{l}825 \\
2.1 E+00 \\
9.4 \\
<5.0 \\
2.010 \\
9.0 E-01\end{array}$ & $\begin{array}{l}1 \\
1 \\
1 \\
1 \\
1\end{array}$ \\
\hline $\begin{array}{l}<2.0 \\
18,200\end{array}$ & 1 \\
\hline $\begin{array}{c}<2.0 \\
8,680 \\
107\end{array}$ & $\begin{array}{l}1 \\
1\end{array}$ \\
\hline & $\begin{array}{l}1 \\
1\end{array}$ \\
\hline $\begin{array}{r}<1.0 \\
5.6\end{array}$ & 7 \\
\hline $\begin{array}{l}<1.0 \\
75,000\end{array}$ & \\
\hline & \\
\hline $\begin{array}{c}99 \\
<0.24\end{array}$ & 1 \\
\hline$<0.00045$ & \\
\hline $\begin{array}{l}<9.8 \\
<1.0\end{array}$ & \\
\hline$<1.0$ & \\
\hline $\begin{array}{r}68 \\
<1.0\end{array}$ & \\
\hline $1.2 E+00$ & \\
\hline $\begin{array}{l}0.61 \\
1.2 \mathrm{E}-01\end{array}$ & \\
\hline$-1.6 \mathrm{E}-02$ & \\
\hline $7.2 E-02$ & \\
\hline$<8.0$ & \\
\hline $\begin{array}{l}<2.0 \\
<20\end{array}$ & \\
\hline$<2.0$ & \\
\hline
\end{tabular}

Mod

Unit Lab Flag

UI $\quad \mathrm{PCi} / \mathrm{L}$ GP 0

$\mu g / L \quad G E \quad 0$

$\mu g \mathrm{~g} / \mathrm{L} \quad \mathrm{GE}$

0
0
0

GE 0

$\mu g / L \quad G E \quad O$

$\mu g / L \quad G E$

$\mu g / L \quad G E$

$\mu \mathrm{g} / \mathrm{L} \quad \mathrm{GE}$

$\mu \mathrm{g} / \mathrm{L} \quad \mathrm{GE}$

$\mu g / L$

$\mu \mathrm{g} / \mathrm{L}$ GE 0

J $\mathrm{PCi} / \mathrm{L}$ GP

$\mu \mathrm{g} / \mathrm{L}$

J

PCIN GP

$\mu g / L$

$\mu g /$

$\mu \mathrm{g} / \mathrm{L}$

$\mu \mathrm{gS} / \mathrm{cm} \quad \mathrm{GE}$

$\mu \mathrm{g} /$

$\mu \mathrm{g} /$

$\mu g / L$

$\mu g / L \quad G E$

J

$\mu \mathrm{g} / \mathrm{L} \quad \mathrm{GE}$

$\mu \mathrm{g} / \mathrm{L}$

$\mu g / L$

J $\mu g / L$ GE

$\mu g / L \quad G E$

$\mu g / L$

$\mu \mathrm{g} / \mathrm{L}$

$\mu \mathrm{g} / \mathrm{L} \quad \mathrm{GE}$

$\mu g / L \quad G E$

$\mu g / L \quad G E$

$\mu \mathrm{g} / \mathrm{L} \quad \mathrm{GE}$

$\mu g / L$

$\mathrm{pCi} / \mathrm{mL} \quad \mathrm{GP}$

NTU GE

U!

pCi/L

ul

pCi/L

$\mu \mathrm{g} / \mathrm{L}$

$\mu g / L$

GE
GP
GP
GP
GE
GE
GE

lag
0
0
0
0
0
0
0
0
0
1
0
0
0
0
0
0
0
0
0
0
0
0
0
0
1
0
0
0
0
0
0
2
0
0
0
0
0
0
0
0
0

Note: Flagging, dilution factors, modifiers, and laboratories are for fourth quarter 1994 data only. See Appendix B for flagging criteria. - = exceeded holding time for fourth quarter 1994.

- = exceeded screening level or final PDWS for fourth quarter 1994. 
WELL BGO 12D

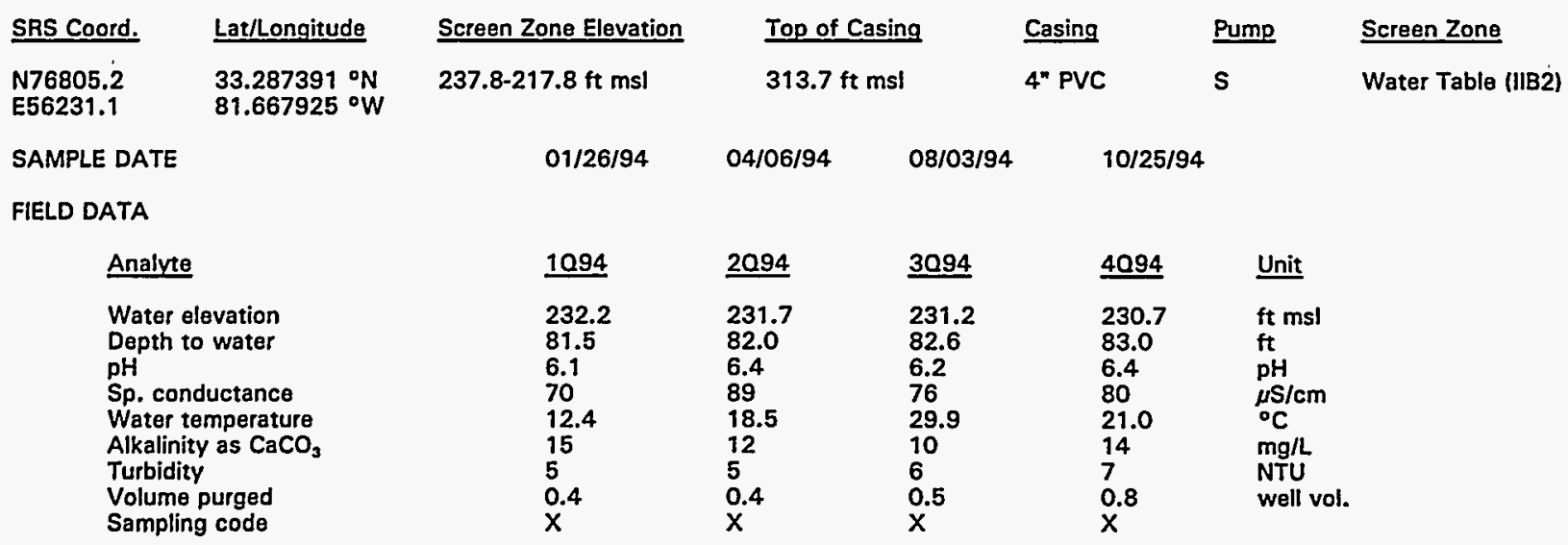

ANALYTICAL DATA

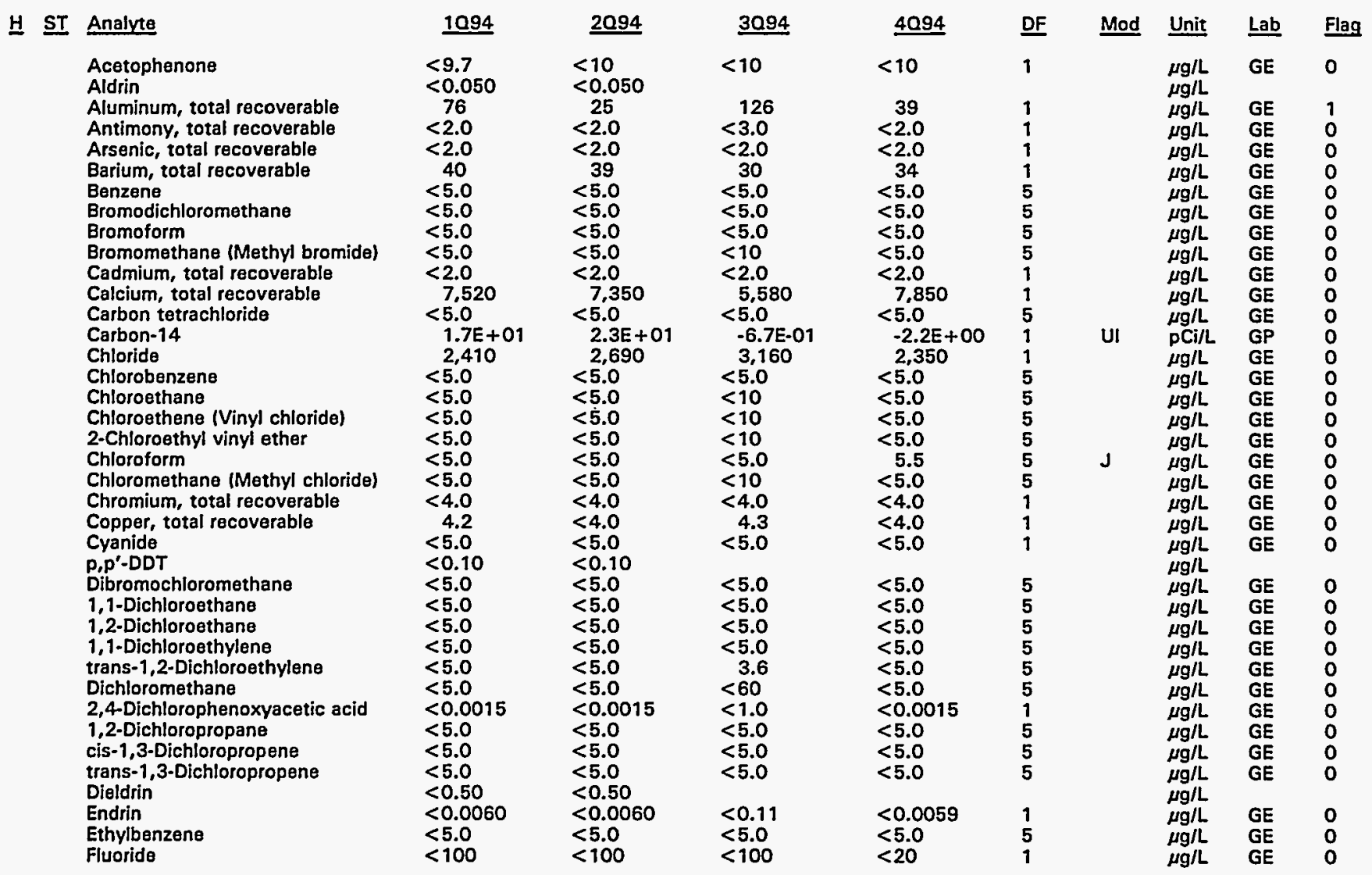

Note: Flagging, dilution factors, modifiers, and laboratories are for fourth quarter 1994 data only. See Appendix B for flagging criteria. - = exceeded holding time for fourth quarter 1994.

- = exceeded screening level or final PDWS for fourth quarter 1994. 
H ST Analyte

Gross alpha

Heptachlor

Iron, total recoverable

Lead, total recoverable

Lindane

Lithium, total recoverable

Magnesium, total recoverable

Manganese, total recoverable

Mercury, total recoverable

Methoxychlor

Naphthalene

Nickel, total recoverable

Nitrate as nitrogen

Nitrate-nitrite as nitrogen

Nonvolatile beta

pH

Phenols

Potassium, total recoverable

Radium, total alpha-emitting

Selenium, total recoverable

Silica, total recoverable

Silver, total recoverable

Sodium, total recoverable

Specific conductance

Sulfate

1,1,2,2-Tetrachloroethane

Tetrachloroethylene

Tin, total recoverable

Toluene

Total dissolved solids

Total organic carbon

Total organic halogens

Total phosphates (as P)

Toxaphene

2,4,5-TP (Silvex)

1,1,1-Trichloroethane

1,1,2-Trichloroethane

- Trichloroethylene

Trichlorofluoromethane

2,4,5-T

Tritium

Turbidity

Uranium-233/234

Uranium-235

Uranium-238

Vanadium, total recoverable

Xylenes

Zinc, total recoverable

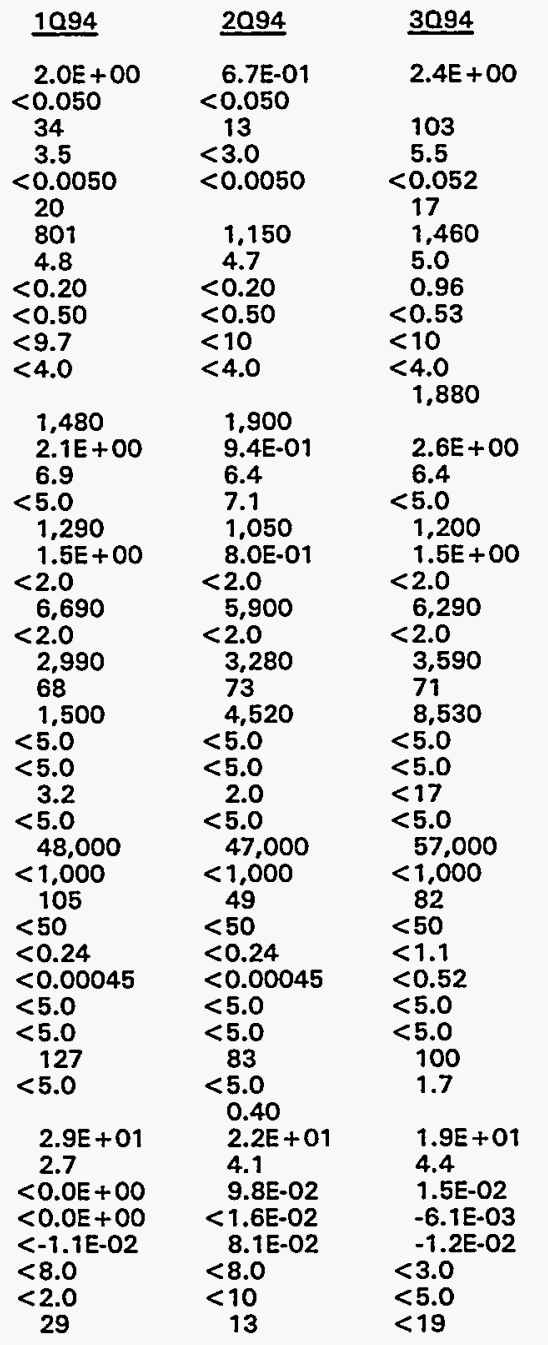

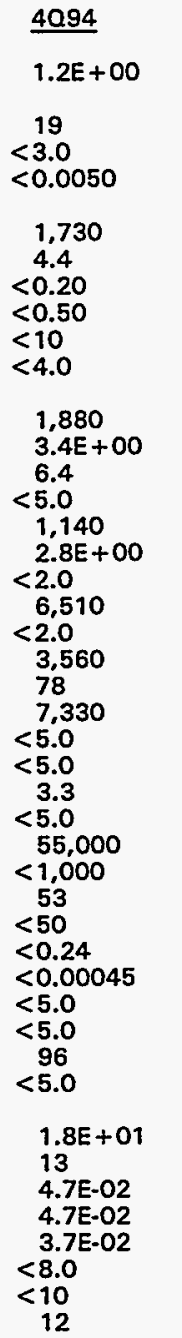

$\begin{array}{llll}\text { Mod } & \text { Unit } & \text { Lab } & \text { Flag } \\ & \begin{array}{lll}\mathrm{pCi} / \mathrm{L} \\ \mu \mathrm{g} / \mathrm{L}\end{array} & \mathrm{GP} & 0 \\ \mu \mathrm{g} / \mathrm{L} & \mathrm{GE} & 0 \\ \mu \mathrm{g} / \mathrm{L} & \mathrm{GE} & 0\end{array}$

$\mu g / L$ GE $O$

$\mu \mathrm{g} / \mathrm{L} \quad \mathrm{GE}$

$\mu \mathrm{g} / \mathrm{L} \quad \mathrm{GE} \quad \mathrm{O}$

$\mu g / L$ GE 0

$\mu g / L \quad G E$

$\mu g / L \quad G E$

$\mu g / L \quad G E$

$\mu \mathrm{g} / \mathrm{L}$

$\begin{array}{lll}\mu \mathrm{g} / \mathrm{L} & \mathrm{GE} & 0 \\ \mathrm{pCi} / \mathrm{L} & \mathrm{GP} & 0\end{array}$

$\mathrm{pH}$

$\mu \mathrm{g} / \mathrm{L}$

$\mu g / L \quad G E$

pCi/L GP

$\mu g / L \quad$ GE

$\mu g / L \quad$ GE

$\mu \mathrm{g} / \mathrm{L} \quad \mathrm{GE}$

$\mu \mathrm{g} / \mathrm{L} \quad \mathrm{GE}$

$\mu g / L \quad G E$

$\mu \mathrm{g} / \mathrm{L} \quad \mathrm{GE}$

$\mu \mathrm{g} / \mathrm{L} \quad \mathrm{GE}$

$\mu \mathrm{g} / \mathrm{L} \quad \mathrm{GE}$

$\mu \mathrm{g} / \mathrm{L} \quad \mathrm{GE}$

$\mu g \mathrm{~g} / \mathrm{L} \quad \mathrm{GE}$

$\mu \mathrm{g} / \mathrm{L} \quad \mathrm{GE}$

$\mu \mathrm{g} / \mathrm{L} \quad \mathrm{GE}$

$\mu \mathrm{g} / \mathrm{L} \quad \mathrm{GE}$

$\mu \mathrm{g} / \mathrm{L} \quad \mathrm{GE}$

$\mu g / L \quad G E$

$\mu \mathrm{g} / \mathrm{L} \quad \mathrm{GE}$

$\mu \mathrm{g} / \mathrm{L} \quad \mathrm{GE}$

$\mu \mathrm{g} / \mathrm{L} \quad \mathrm{GE}$

$\mu g / L$

pCi/mL GP

NTU GE

UI $\mathrm{pCi} / \mathrm{L}$ GP

UI

pCill

pCi/L

$\mu \mathrm{pi} / \mathrm{L}$

$\mu g / L$

$\mu \mathrm{g} / \mathrm{L}$

Note: Flagging, dilution factors, modifiers, and laboratories are for fourth quarter 1994 data only. See Appendix B for flagging criteria. - = exceeded holding time for fourth quarter 1994.

- exceeded screening level or final PDWS for fourth quarter 1994. 


\section{WELL BGO 13DR}

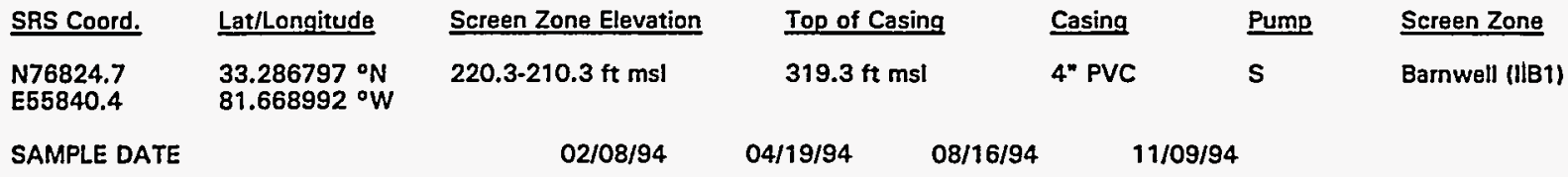

FIELD DATA

Analyte
Water elevation
Depth to water
pH
Sp. conductance
Water temperature
Alkalinity as $\mathrm{CaCO}_{3}$
Turbidity
Volume purged
Sampling code

ANALYTICAL DATA

\begin{tabular}{l}
1094 \\
\hline 231.5 \\
87.8 \\
7.4 \\
107 \\
18.2 \\
36 \\
11 \\
0.6 \\
$x$
\end{tabular}

$\underline{2094}$
231.1
88.2
7.1
82
20.1
20
12
0.6
$x$

$\frac{1094}{<}$
$<9.7$
0.082
22
$<2.0$
$<2.0$
33
$<1.0$
$<1.0$
$<1.0$
$<1.0$
$<2.0$
8.320
$<1.0$
$<2.1 E+00$
2,870
$<1.0$
$<1.0$

$<1.0$

$<1.0$

$<1.0$

$<1.0$

$<1.0$

$<4.0$

7.2

$<5.0$

0.25

$<1.0$

$<1.0$

$<1.0$

$<1.0$

$<1.0$

$<1.0$

$<0.0015$

$<1.0$

$<1.0$

$<1.0$

$<0.50$

0.27

$<1.0$

$<100$

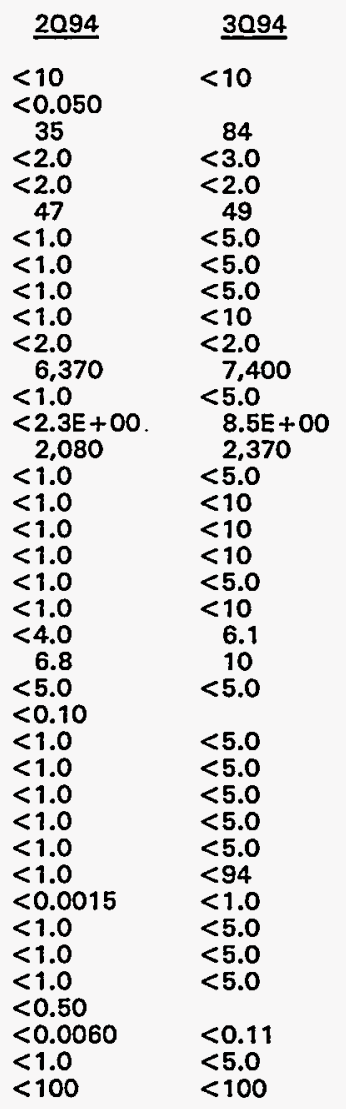

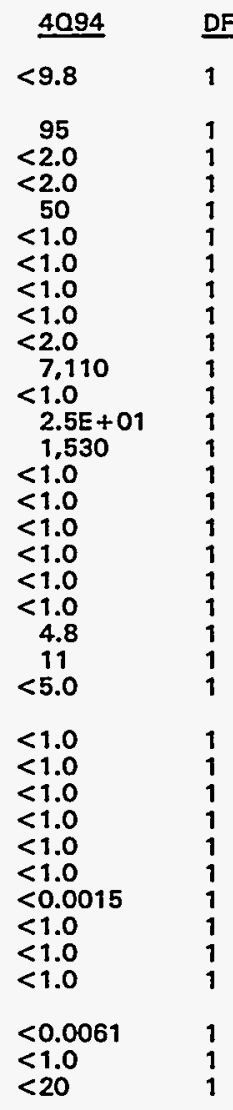

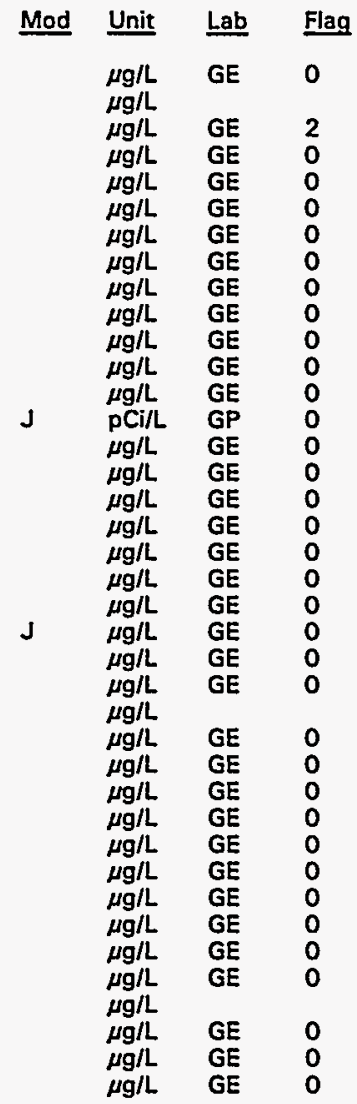

Note: Flagging, dilution factors, modifiers, and laboratories are for fourth quarter 1994 data only. See Appendix B for flagging criteria. - = exceeded holding time for fourth quarter 1994.

- = exceeded screening level or final PDWS for fourth quarter 1994. 


\section{H ST Analyte \\ Gross alpha \\ Heptachlor \\ Iron, total recoverable \\ - Lead, total recoverable \\ Lindane \\ Lithium, total recoverable \\ Magnesium, total recoverable \\ Manganese, total recoverable \\ Mercury, total recoverable \\ Methoxychlor \\ Naphthalene \\ Nickel, total recoverable \\ Nitrate as nitrogen \\ Nitrate-nitrite as nitrogen \\ Nonvolatile beta \\ $\mathrm{pH}$ \\ Phenols \\ Potassium, total recoverable \\ Radium, total alpha-emitting \\ Selenium, total recoverable \\ Silica, total recoverable \\ Silver, total recoverable \\ Sodium, total recoverable \\ Specific conductance \\ Sulfato \\ 1,1,2,2-Tetrachloroethane \\ Tetrachloroethylene \\ Tin, total recoverable \\ Toluene \\ Total dissolved solids \\ Total organic carbon \\ Total organic halogens \\ Total phosphates (as P) \\ Toxaphene \\ 2,4,5-TP (Silvex) \\ Tributyl phosphate \\ 1,1,1-Trichloroethane \\ 1,1,2-Trichloroethane \\ Trichloroethylene \\ Trichlorofluoromethane \\ 2,4,5-T \\ - Tritium \\ Turbidity \\ Uranium-233/234 \\ Uranium-235 \\ Uranium-238 \\ Vanadium, total recoverable \\ Xylenes}

Zinc, total recoverable

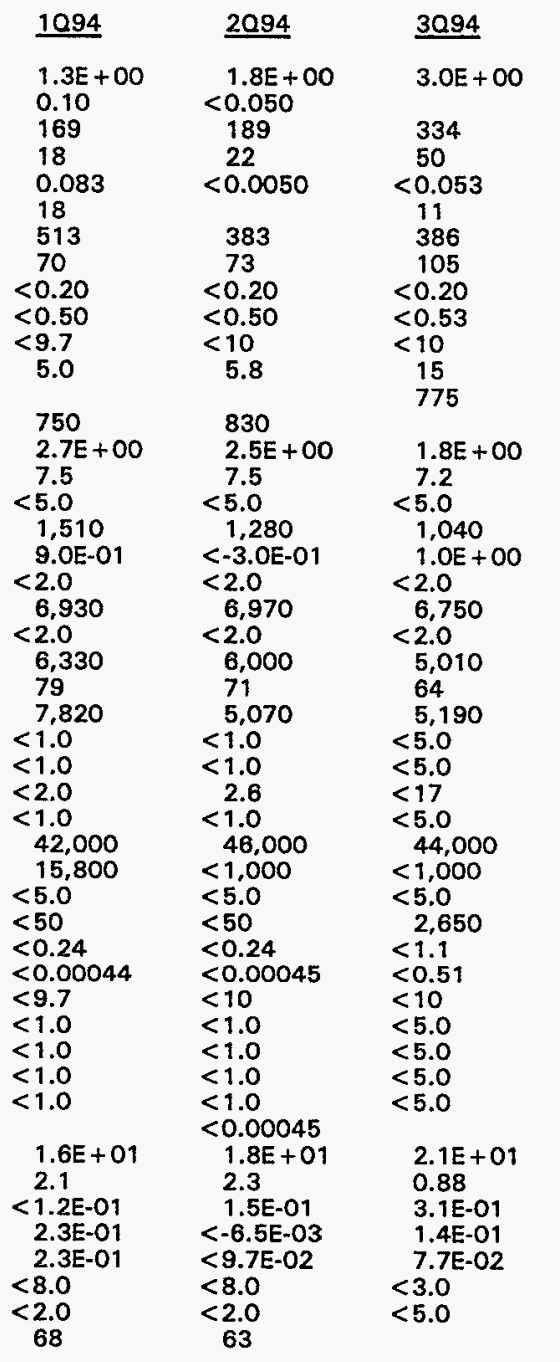

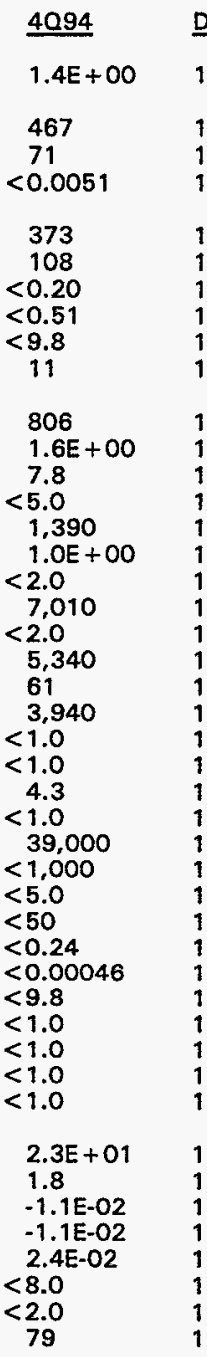

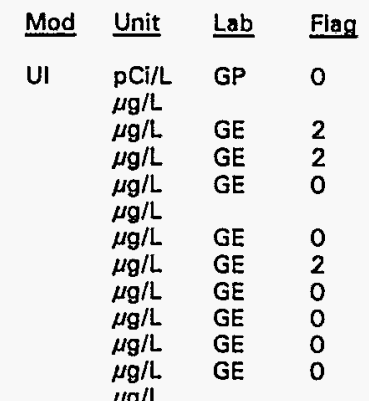

$\mu \mathrm{g} / \mathrm{L} \quad \mathrm{GE}$

UI $\quad \mathrm{pCi} / \mathrm{G} \quad \mathrm{GP}$

$\mu g / L \quad G E$

$\mu g / L \quad G E$

$J \quad \mathrm{pCi} / \mathrm{L} \quad \mathrm{GP}$

$\mu \mathrm{g} / \mathrm{L} \quad \mathrm{GE}$

$\mu g / L \quad G E$

$\mu g / L \quad$ GE

$\mu \mathrm{g} / \mathrm{L} \quad \mathrm{GE}$

$\mu \mathrm{S} / \mathrm{cm}$ GE

$\mu \mathrm{g} / \mathrm{L} \quad \mathrm{GE}$

$\mu \mathrm{g} / \mathrm{L} \quad \mathrm{GE}$

$\mu g / L$

$\mu g / L$

$\checkmark$

$\mu g / L$
$\mu g / L$
$\mu g / L$

$\mu g / L$
$\mu g / L$
$\mu g / L$

$\mu \mathrm{g} / \mathrm{L}$

$\mu \mathrm{g} / \mathrm{L}$

$\mu \mathrm{g} / \mathrm{L}$

$\mu \mathrm{g} / \mathrm{L}$

$\mu \mathrm{g} / \mathrm{L}$

$\mu \mathrm{g} / \mathrm{L}$

$\mu \mathrm{g} / \mathrm{L}$

$\mathrm{pCi} / \mathrm{mL} \quad \mathrm{GP}$

NTU GE

UI

pCi/L

pCi/l

$\mu \mathrm{g} / \mathrm{L}$

$\mu \mathrm{g} / \mathrm{L}$

$\mu \mathrm{g} / \mathrm{L} / \mathrm{L}$ 
WELL BGO 14AR

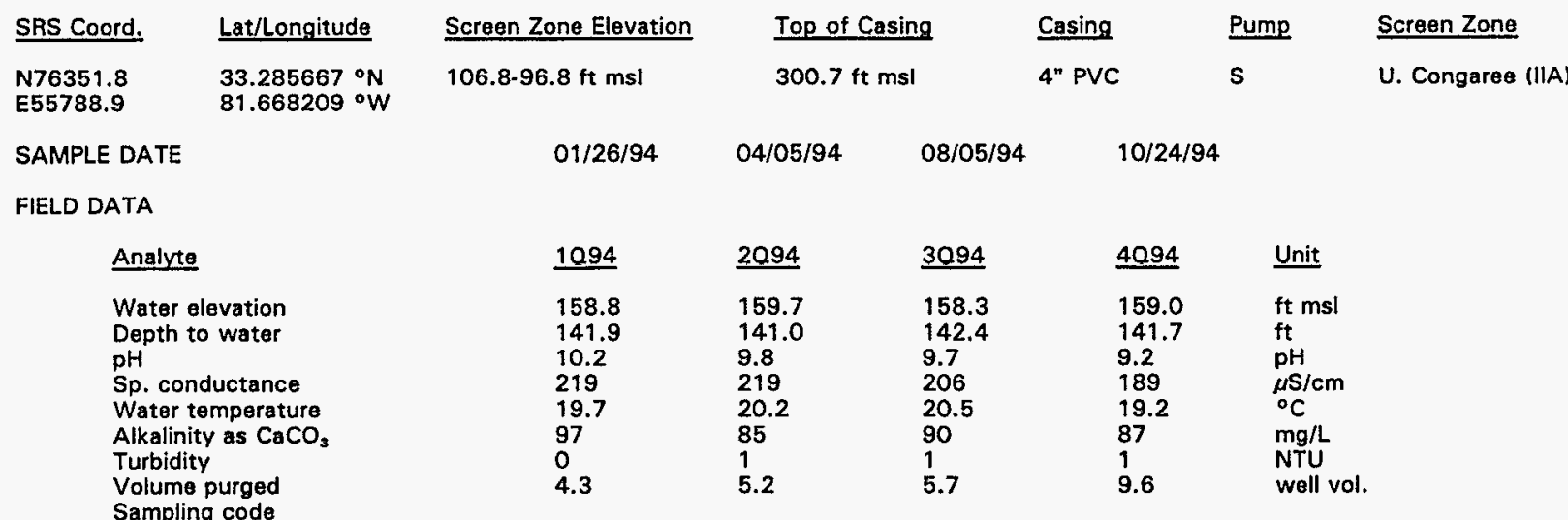

ANALYTICAL DATA

\section{H ST Analyta}

Acetophenone

Aldrin

Aluminum, total recoverable

Antimony, total recoverable

Arsenic, total recoverable

Barium, total recoverable

- Benzene

Bromodichloromethane

Bromoform

Bromomethane (Methyl bromide)

Cadmium, total recoverable

Calcium, total recoverable

Carbon tetrachloride

Carbon-14

Chloride

Chlorobenzene

Chloroethane

Chloroethene (Vinyl chloride)

2-Chloroethyl vinyl ether

Chloroform

Chloromethane (Methyl chloride)

Chromium, total recoverable

Copper, total recoverable

Cyanida

Dibromochloromethane

1,1-Dichloroethane

1,2-Dichloroethane

1,1-Dichloroethylene

trans-1,2-Dichloroethylene

- Dichloromethane

2,4-Dichlorophenoxyacetic acid

1,2-Dichloropropane

cis-1,3-Dichloropropene

trans-1,3-Dichloropropene

Dieldrin

Endrin

Ethylbenzene

Fluoride

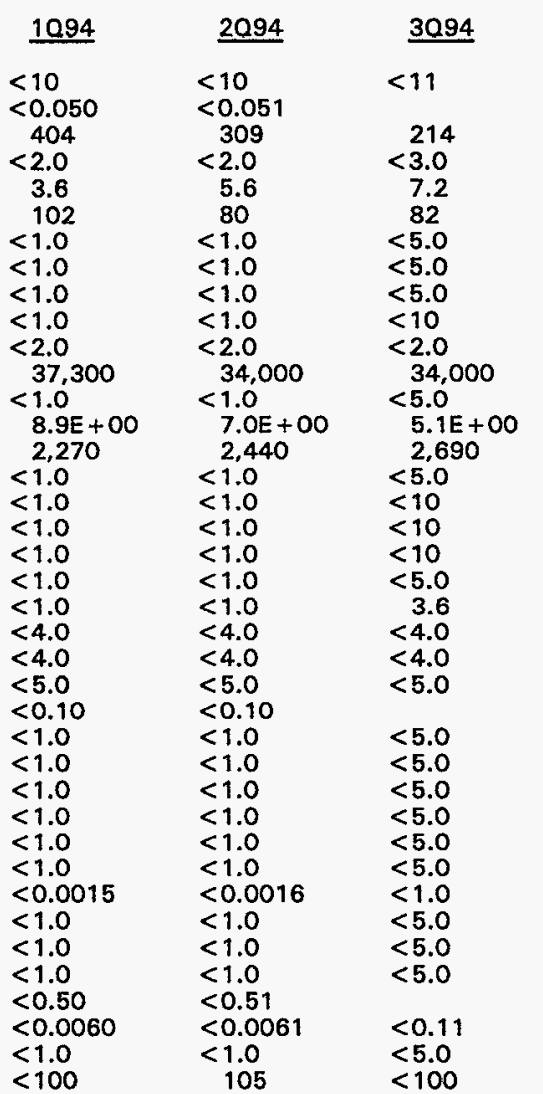

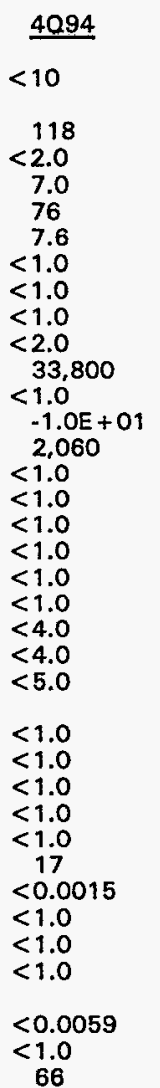

\begin{tabular}{|c|c|c|c|c|}
\hline DF & Mod & Unit & Lab & Flag \\
\hline 1 & & $\begin{array}{l}\mu \mathrm{g} / \mathrm{L} \\
\mu \mathrm{g} / \mathrm{L}\end{array}$ & GE & 0 \\
\hline 1 & & $\mu \mathrm{g} / \mathrm{L}$ & GE & 2 \\
\hline 1 & & $\mu \mathrm{g} / \mathrm{L}$ & $\overline{\mathrm{GE}}$ & 0 \\
\hline 1 & & $\mu \mathrm{g} / \mathrm{L}$ & $\mathrm{GE}$ & 0 \\
\hline 1 & & $\mu \mathrm{g} / \mathrm{L}$ & GE & 0 \\
\hline 1 & & $\mu \mathrm{g} / \mathrm{L}$ & GE & 2 \\
\hline 1 & & $\mu \mathrm{g} / \mathrm{L}$ & $\mathbf{G E}$ & 0 \\
\hline 1 & & $\mu \mathrm{g} / \mathrm{L}$ & GE & 0 \\
\hline 1 & & $\mu \mathrm{g} / \mathrm{L}$ & GE & 0 \\
\hline 1 & & $\mu \mathrm{g} / \mathrm{L}$ & GE & 0 \\
\hline 1 & & $\mu \mathrm{g} / \mathrm{L}$ & GE & 0 \\
\hline 1 & & $\mu \mathrm{g} / \mathrm{L}$ & GE & 0 \\
\hline 1 & UI & $\mathrm{pCi} / \mathrm{L}$ & GP & 0 \\
\hline$i$ & & $\mu g / L$ & GE & 0 \\
\hline 1 & & $\mu g / \mathrm{L}$ & GE & 0 \\
\hline 1 & & $\mu \mathrm{g} / \mathrm{L}$ & GE & 0 \\
\hline 1 & & $\mu g / L$ & GE & 0 \\
\hline 1 & & $\mu \mathrm{g} / \mathrm{L}$ & GE & 0 \\
\hline 1 & & $\mu \mathrm{g} / \mathrm{L}$ & GE & 0 \\
\hline 1 & & $\mu g / L$ & GE & 0 \\
\hline 1 & & $\mu g / L$ & GE & 0 \\
\hline 1 & & $\mu \mathrm{g} / \mathrm{L}$ & GE & 0 \\
\hline 1 & & $\begin{array}{l}\mu \mathrm{g} / \mathrm{L} \\
\mu \mathrm{g} / \mathrm{L}\end{array}$ & GE & 0 \\
\hline 1 & & $\mu g / L$ & GE & 0 \\
\hline 1 & & $\mu \mathrm{g} / \mathrm{L}$ & $\mathrm{GE}$ & 0 \\
\hline 1 & & $\mu \mathrm{g} / \mathrm{L}$ & $\mathrm{GE}$ & 0 \\
\hline 1 & & $\mu g / L$ & GE & 0 \\
\hline 1 & & $\mu g / L$ & GE & 0 \\
\hline 1 & & $\mu g / L$ & GE & 2 \\
\hline 1 & & $\mu g / L$ & GE & 0 \\
\hline 1 & & $\mu \mathrm{g} / \mathrm{L}$ & GE & 0 \\
\hline 1 & & $\mu \mathrm{g} / \mathrm{L}$ & GE & 0 \\
\hline 1 & & $\begin{array}{l}\mu \mathrm{g} / \mathrm{L} \\
\mu \mathrm{g} / \mathrm{L}\end{array}$ & $\mathrm{GE}$ & 0 \\
\hline 1 & & $\mu \mathrm{g} / \mathrm{L}$ & GE & 0 \\
\hline 1 & & $\mu \mathrm{g} / \mathrm{L}$ & $\mathrm{GE}$ & 0 \\
\hline 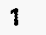 & & $\mu \mathrm{g} / \mathrm{L}$ & $\mathrm{GE}$ & 0 \\
\hline
\end{tabular}

Note: Flagging, dilution factors, modifiers, and laboratories are for fourth quarter 1994 data only. See Appendix B for flagging criteria. - = exceeded holding time for fourth quarter 1994.

- =xceeded screening level or final PDWS for fourth quarter 1994. 
H ST Anaivte

Gross alpha

Heptachlor

Iron, total recoverable

Lead, total recoverable

Lindane

Lithium, total recoverable

Magnesium, total recoverable

Manganese, total recoverable

Mercury, total recoverable

Methoxychlor

Naphthalene

Nickel, total recoverable

Nitrate as nitrogen

Nitrate-nitrite as nitrogen

Nonvolatile beta

$\mathrm{pH}$

Phenols

Potassium, total recoverable

Radium, total alpha-emitting

Selenium, total recoverable

Silica, total recoverable

Silver, total recoverable

Sodium, total recoverable

Specific conductance

Sulfate

1,1,2,2-Tetrachloroethane

Tetrachloroethylene

Tin, total recoverable

Toluene

Total dissolved solids

Total organic carbon

Total organic halogens

Total phosphates (as P)

Toxaphene

2,4,5-TP (Silvex)

Tributyl phosphate

1,1,1-Trichloroethane

1,1,2-Trichloroethane

Trichloroethylene

Trichlorofluoromethane

2,4,5-T

Tritium

Turbidity

Uranium-233/234

Uranium-235

Uranium-238

Vanadium, total recoverable

Xylenes

Zinc, total recoverable

$\begin{aligned} & 1094 \\ & 1.4 E+00 \\ &<0.050 \\ & 9.0 \\ &<3.0 \\ &<0.0050 \\ & 54 \\ & 510 \\ & 11 \\ &<0.20 \\ &<0.50 \\ &<10 \\ &<4.0\end{aligned}$

$\frac{3094}{1.2 E+00}$
22
$<3.0$
$<0.055$
37
534
11
$<0.20$
$<0.55$
$<10$
$<4.0$
237

$1.3 E+01$

10

$<5.0$

8,670

$1.5 E+00$

$<2.0$

24,200

$<2.0$

5.830

207

3,260

$<1.0$

$<1.0$

22
$<1.0$

146,000

146,000
$<1,000$

$<1,00$
49
$<50$

$<0.24$

$<0.00045$

$<10$

$<1.0$

$<1.0$

1.8
$<1.0$

$3.8 E+00$

$<0.10$

$<1.8 \mathrm{E}-01$

$<4.6 E-02$

$<1.7 \mathrm{E}-01$

$<8.0$

$<2.0$

$<2.0$

$\begin{aligned} & 2094 \\ & 2.2 E+00 \\ &<0.051 \\ & 5.4 \\ &<3.0 \\ &<0.0051 \\ & \\ & 533 \\ & 11 \\ &<0.20 \\ &<0.51 \\ &<10 \\ &<4.0\end{aligned}$

$6.9 E+00$

9.3

$5.6 \mathrm{E}+00$

9.8

$<5.0$
7.530

9.0E-01

$<2.0$

24,700

$<2.0$

4.860

191

4,150

$<1.0$

$<1.0$

15

143,000

143,00
1,200

$<5.0$

160

$<0.24$

$<0.00047$

$<10$

$<1.0$

$<1.0$

1.8

$<1.0$

0.39

$2.4 \mathrm{E}+00$

$<5.0$

6,100

9.0E-01

$<2.0$

23,800

$<2.0$

4,340

182

3,800

$<5.0$

$<5.0$

$<17$

$<5.0$

153,000

$<1,000$

9.1

2,150

$<1.1$

$<0.52$

$<10$

$<5.0$

$<5.0$

$<5.0$

2. $2 E+00$

0.92

$<6.6$ E-02

\subsection{3}

5.3E-01

6.6E-02

2.5E-01

1.6E-01

$<8.0$

$<8.0$

$<2.0$
$<2.0$

$<3.0$

$<5.0$

$\begin{aligned} & 40.94 \\ & 1.9 E+00 \\ & \\ & 8.8 \\ &<3.0 \\ &<0.0050 \\ & \\ & 547 \\ & 9.3 \\ &<0.20 \\ &<0.50 \\ &<10 \\ &<4.0\end{aligned}$

Mod

Unit
$\mu \mathrm{gi} / \mathrm{L} / \mathrm{L}$

GE

$\mu \mathrm{g} / \mathrm{L}$ GE

$\mu g / L \quad$ GE $\quad 0$

$\mu \mathrm{g} / \mathrm{L}$

$\mu \mathrm{g} / \mathrm{L} \quad \mathrm{GE} \quad 0$

$\mu \mathrm{g} / \mathrm{L}$ GE 0

$\mu g / L$

$\mu g /$

$\mu \mathrm{g} / \mathrm{L} \quad \mathrm{GE}$

$\mu \mathrm{g} / \mathrm{L} \quad \mathrm{GE}$

$\mu g / L$

176
$4.3 E+00$

9.1

$<5.0$

4,980

$<2.0$

26,100

$<2.0$

3,240

190

2,890

$<1.0$

$<1.0$

9.4

142,000

1,270

$<5.0$

226

$<0.24$

$<0.00045$

$<10$

$<1.0$

$<1.0$

$<1.0$

$<1.0$
$<1.0$

7.0E-01

$<0.10$

4.8E-01

$-6.4 \mathrm{E}-03$

8.2E-02

$<8.0$

$<2.0$

$<2.0$

$\mu \mathrm{g} / \mathrm{L}$ GE 0

$\mathrm{pCi} / \mathrm{L}$ GP 0

HIL GE

$\mu \mathrm{g} / \mathrm{L} \quad \mathrm{GE}$

$\begin{array}{ll}\mu \mathrm{g} / \mathrm{L} & \mathrm{GE} \\ \mathrm{DCi} / \mathrm{L} & \mathrm{GP}\end{array}$

$\mu \mathrm{g} / \mathrm{L} \quad \mathrm{GE}$

$\mu \mathrm{g} / \mathrm{L} \quad \mathrm{GE}$

$\mu g / L \quad G E$

$\mu \mathrm{g} / \mathrm{L} \quad \mathrm{GE}$

$\mu \mathrm{g} / \mathrm{L} \quad \mathrm{GE}$

$\mu g / L \quad G E$

$\mu g / L \quad$ GE

$\mu g / L \quad G E$

$\mu \mathrm{g} / \mathrm{L} \quad \mathrm{GE}$

$\mu g / L \quad G E$

$\mu \mathrm{g} / \mathrm{L} \quad \mathrm{GE}$

$\mu g / L \quad$ GE

$\mu \mathrm{g} / \mathrm{L} \quad \mathrm{GE}$

$\mu \mathrm{g} /$

$\mu \mathrm{g} / \mathrm{L}$

$\mu \mathrm{g} / \mathrm{L}$

$\mu g / L$

$\mu g / L$

$\mu g / L$

GE 0

NTU

UI

pCi/

$\mathrm{pCi} / \mathrm{L}$
$\mu \mathrm{g} / \mathrm{L}$

$\begin{array}{ll}\mu g / L & \mathrm{GE} \\ \mu \mathrm{g} / \mathrm{L} & \mathrm{GE}\end{array}$

Flag



0

0
0
0

0

0

0

0

0

0

0

0

0

0
0

0

0

0

0

0

$9<2.0$


WELL BGO 14CR



Note: Flagging, dilution factors, modifiers, and laboratories are for fourth quarter 1994 data only. See Appendix B for flagging criteria, - = exceeded holding time for fourth quarter 1994.

- = exceeded screening level or final PDWS for fourth quarter 1994. 
H ST Analyte

Gross alpha

Heptachlor

Iron, total recoverable

Lead, total recoverable

Lindane

Lithium, total recoverable

Magnesium, total recoverable

Manganese, total recoverable

Mercury, total recoverable

Methoxychlor

Naphthalene

Nickel, total recoverable

Nitrate as nitrogen

Nitrate-nitrite as nitrogen

Nonvolatile beta

- $\mathrm{pH}$

Phenols

Potassium, total recoverable

Radium, total alpha-emitting

Selenium, total recoverable

Silica, total recoverable

Silver, total recoverable

Sodium, total recoverable

Specific conductance

Sulfate

1,1,2,2-Tetrachloroethane

Tetrachloroethylene

Tin, total recoverable

Toluene

Total dissolved solids

Total organic carbon

Total organic halogens

Total phosphates las P

Toxaphene

2,4,5-TP (Silvex)

Tributyl phosphate

1,1,1-Trichloroethane

1,1,2-Trichloroethane

- Trichloroethylene

Trichlorofluoromethane

2,4,5-T

- Tritium

Turbidity

Uranium-233/234

Uranium-235

Uranium-238

Vanadium, total recoverable

Xylenes

Zinc, total recoverable

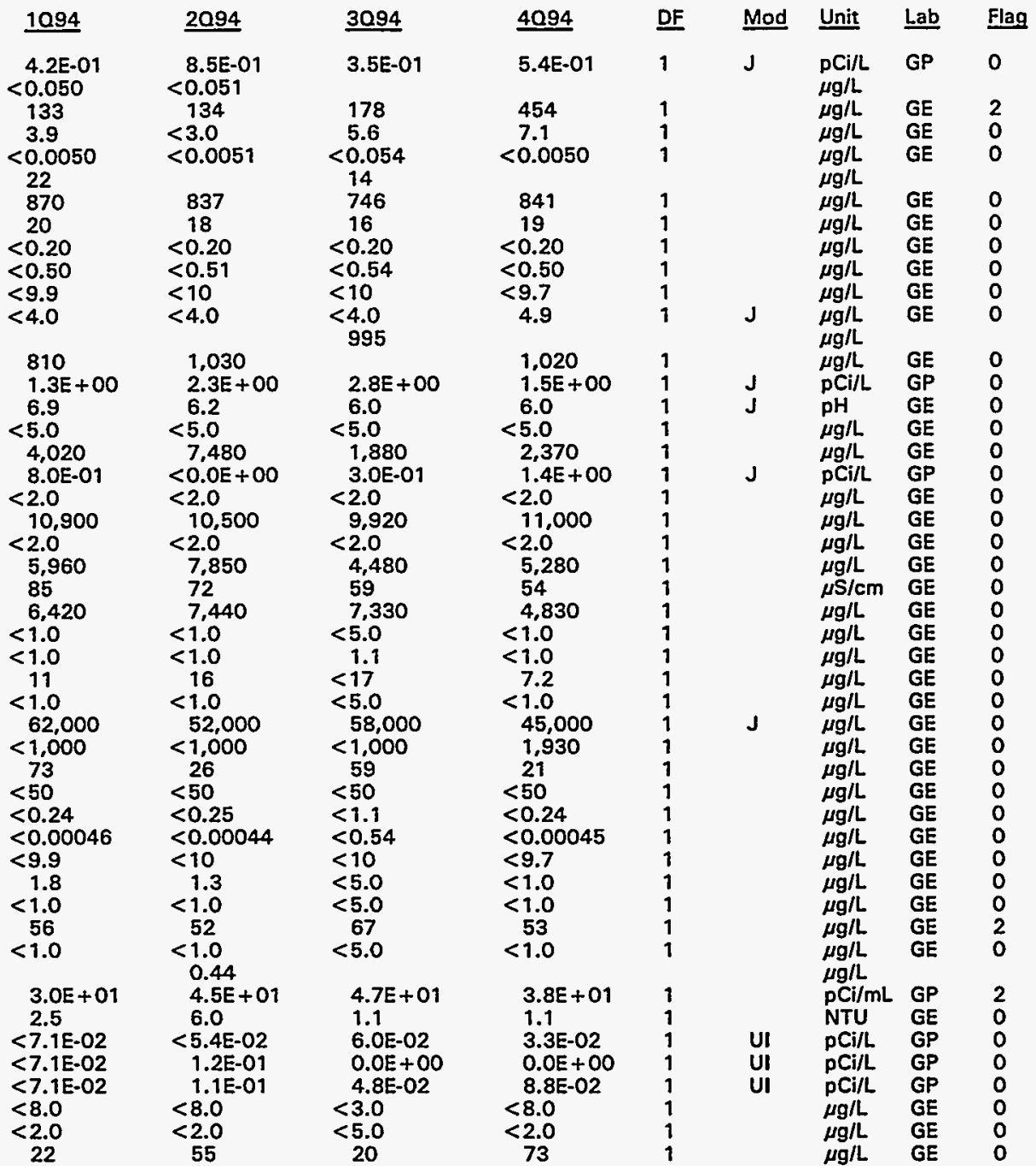

Note: Flagging, dilution factors, modifiers, and laboratories are for fourth quarter 1994 data only. See Appendix B for flagging criteria.

- = exceeded holding time for fourth quarter 1994.

- = exceeded screening level or final PDWS for fourth quarter 1994. 
WELL BGO 14DR

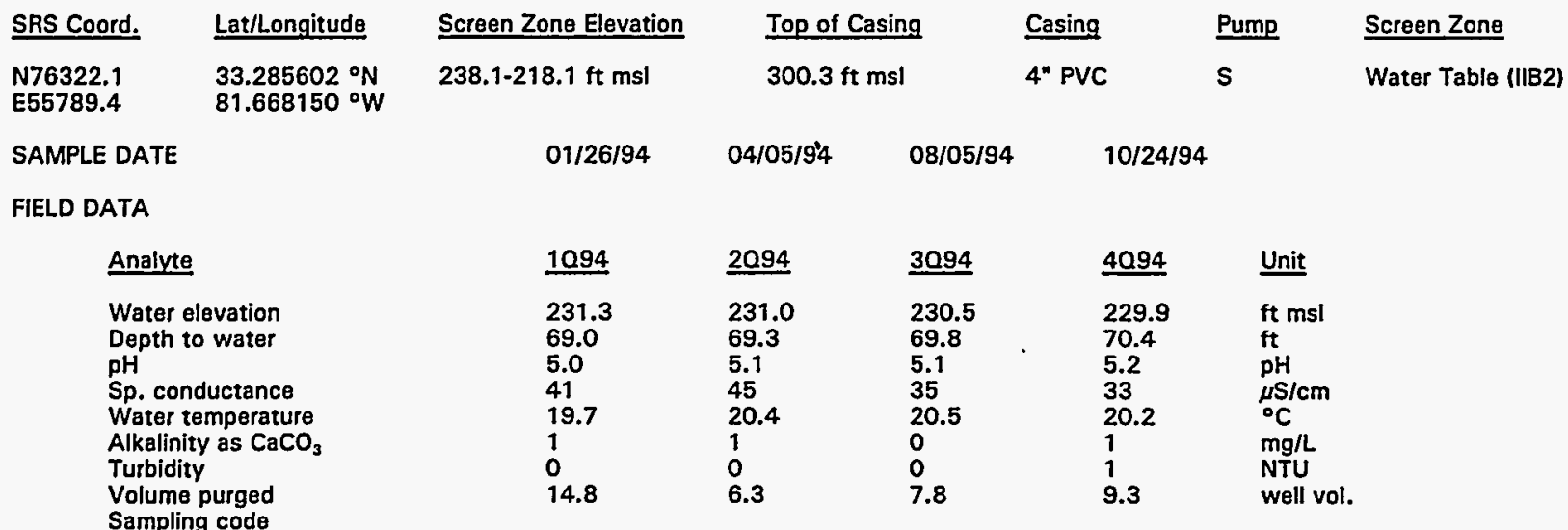

ANALYTICAL DATA

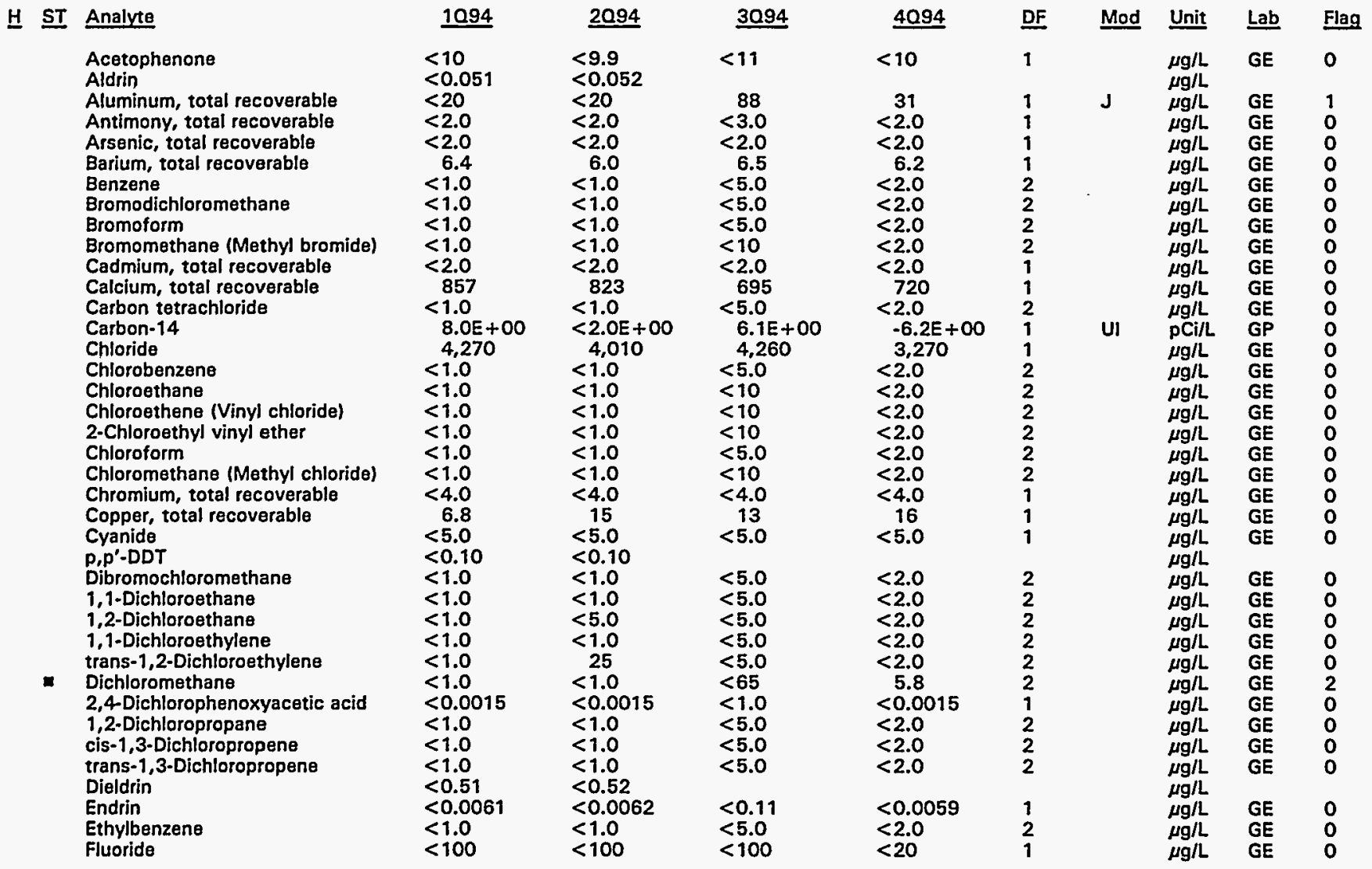

Note: Flagging, dilution factors, modifiers, and laboratories are for fourth quarter 1994 data only. See Appendix B for flagging criteria. - = exceeded holding time for fourth quarter 1994.

- = exceeded screening level or final PDWS for fourth quarter 1994. 
H ST Analyte

Gross alpha

Heptachlor

Iron, total recoverable

Lead, total recoverable

Lindane

Lithium, total recoverable

Magnesium, total recoverable

Manganese, total recoverable

Mercury, total recoverable

Methoxychlor

Naphthalene

Nickel, total recoverable

Nitrate as nitrogen

Nitrate-nitrite as nitrogen

Nonvolatile beta

- $\mathrm{pH}$

Phenols

Potassium, total recoverable

Radium, total alpha-emitting

Selenium, total recoverable

Silica, total recoverable

Silver, total recoverable

Sodium, total recoverable

Specific conductance

Sulfate

1,1,2,2-Tetrachloroethane

Tetrachloroethylene

Tin, total recoverable

Toluene

Total dissolved solids

Total organic carbon

Total organic halogens

Total phosphates (as P)

Toxaphene

2,4,5-TP (Silvex)

Tributyl phosphate

1,1,1-Trichloroethane

1,1,2-Trichloroethane

- Trichloroethylene

Trichlorofluoromethane

2,4,5-T

Tritium

Turbidity

Uranium-233/234

Uranium-235

Uranium-238

Vanadium, total recoverable

Xylenes

Zinc, total recoverable

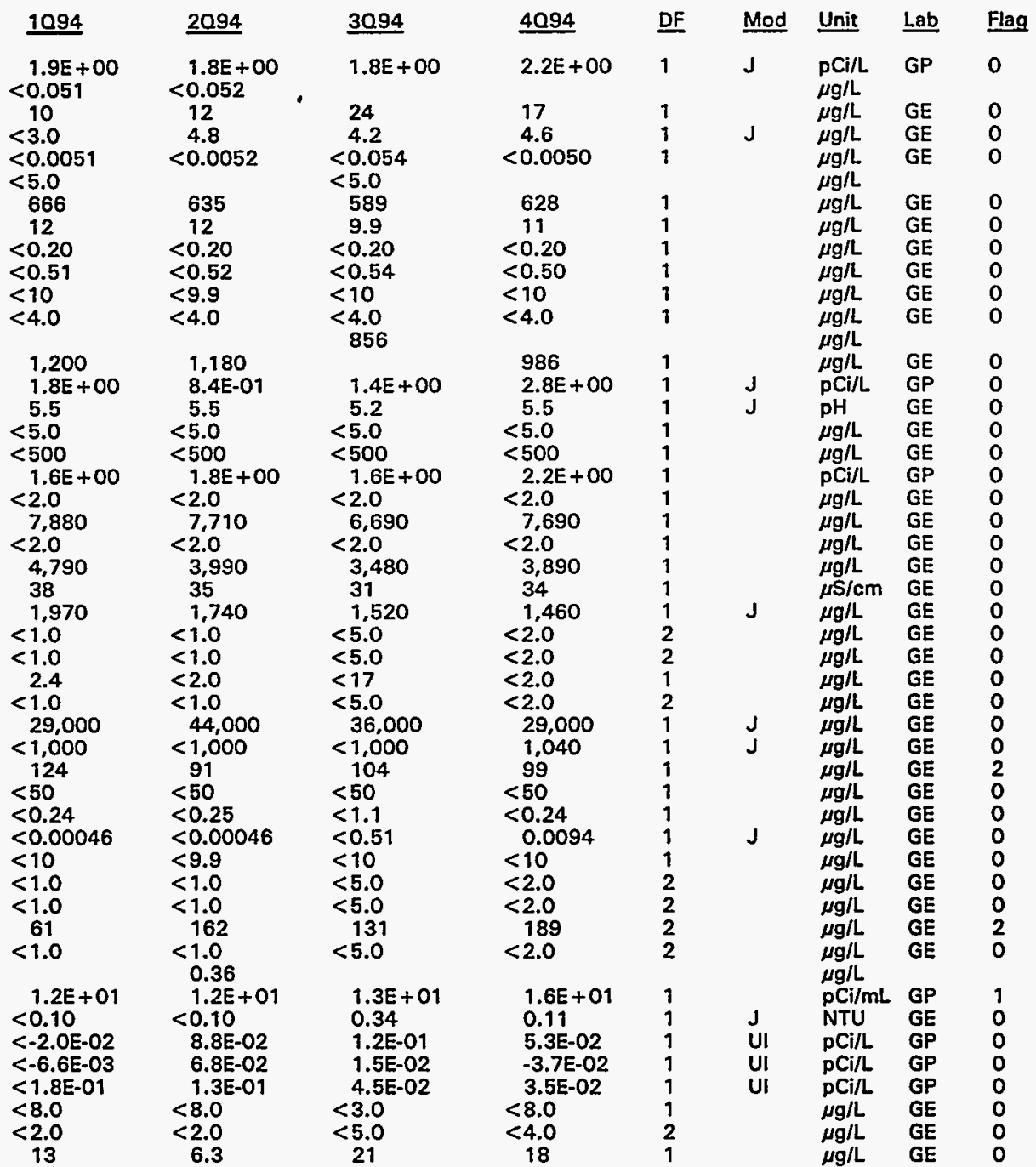

Note: Flagging, dilution factors, modifiers, and laboratories are for fourth quarter 1994 data only. See Appendix B for flagging criteria. - = exceeded holding time for fourth quarter 1994.

- = exceeded screening level or final PDWS for fourth quarter 1994. 


\section{WELL BGO 15D}

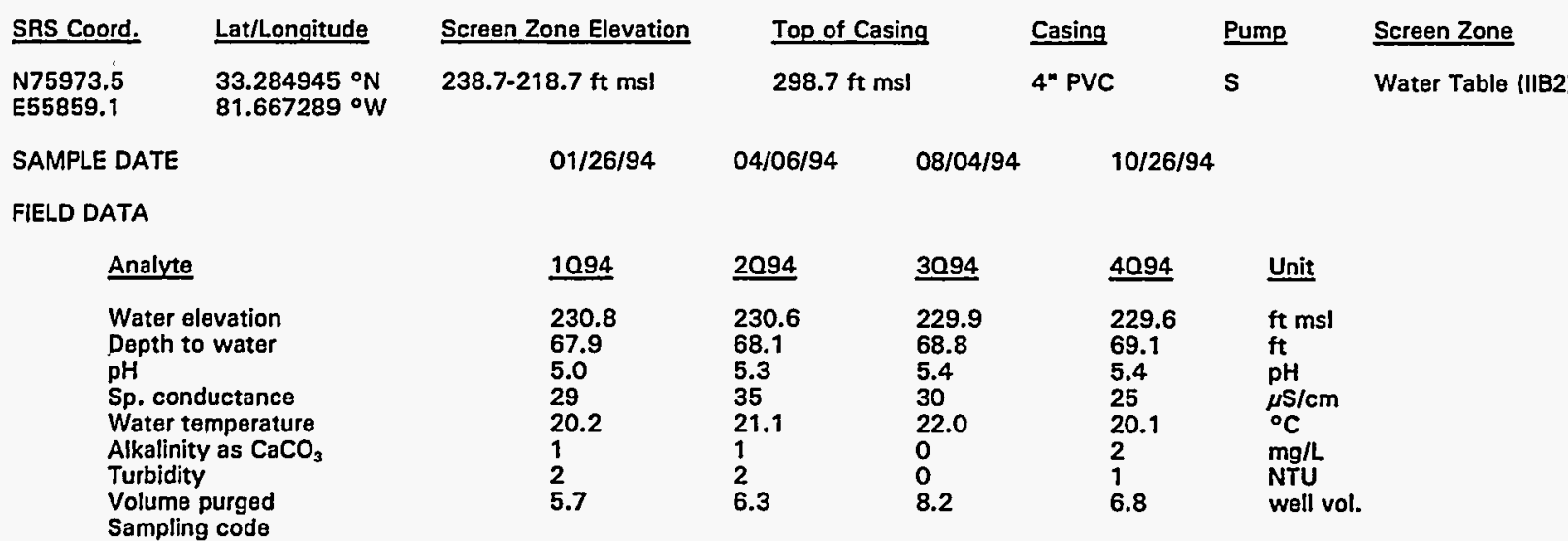

\section{ANALYTICAL DATA}

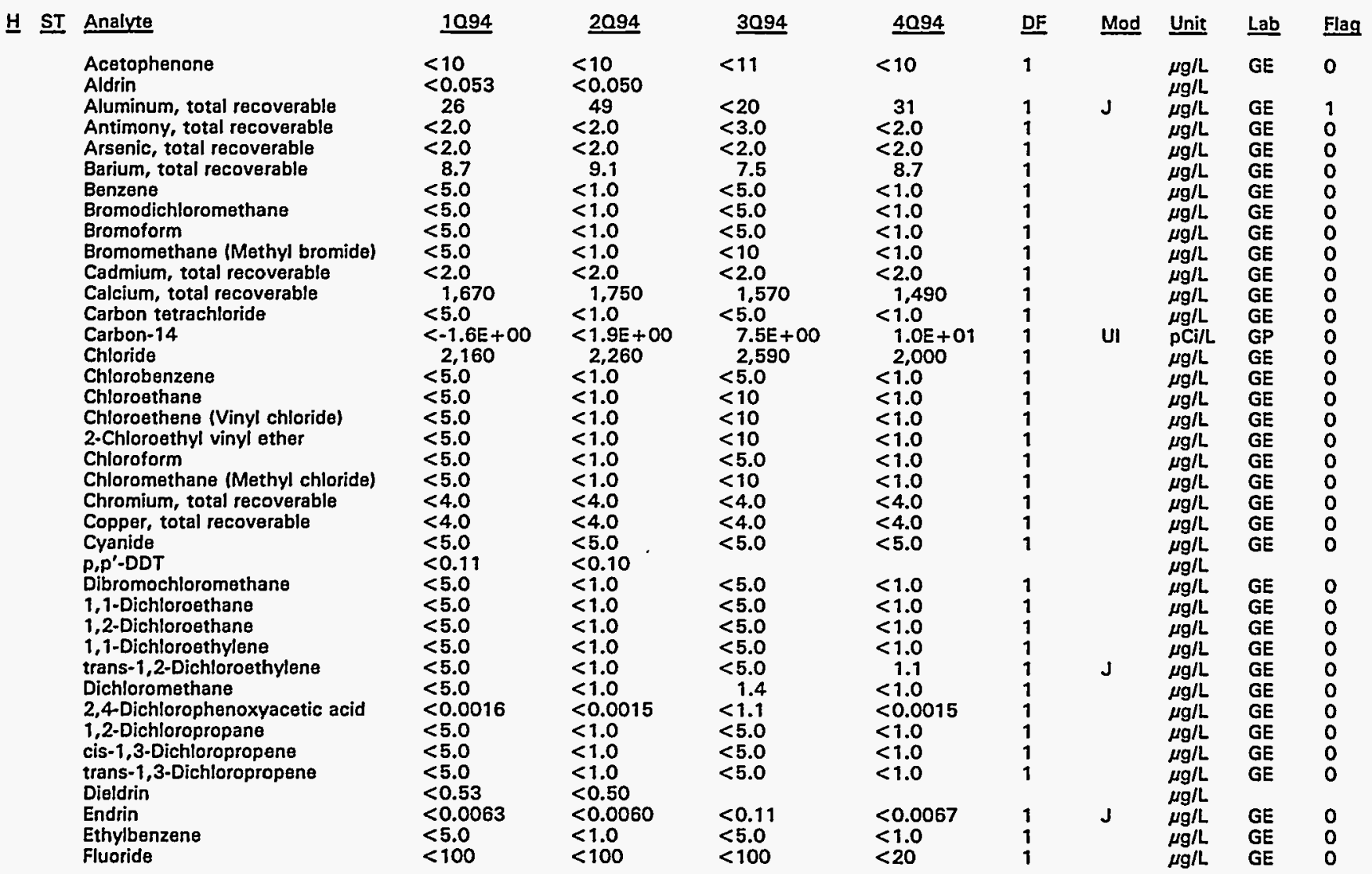

Note: Flagging, dilution factors, modifiers, and laboratories are for fourth quarter 1994 data only. See Appendix B for flagging criteria. - = exceeded holding time for fourth quarter 1994.

m = exceeded screening level or final PDWS for fourth quarter 1994. 
H ST Analyte

Gross alpha

Heptachlor

Iron, total recoverable

Lead, total recoverable

Lindane

Lithium, total recoverable

Magnesium, total recoverable

Manganese, total recoverable

Mercury, total recoverable

Methoxychlor

Naphthalene

Nickel, total recoverable

Nitrate as nitrogen

Nitrate-nitrite as nitrogen

Nonvolatile beta

pH

Phenols

Potassium, total recoverable

Radium, total alpha-emitting

Selenium, total recoverable

Silica, total recoverable

Silver, total recoverable

Sodium, total recoverable

Specific conductance

Sulfate

1,1,2,2-Tetrachloroethane

- Tetrachloroethylene

Tin, total recoverable

Toluene

Total activity

Total dissolved solids

Total organic carbon

Total organic halogens

Total phosphates (as P)

Toxaphene

2,4,5-TP (Silvex)

1,1,1-Trichloroethane

1,1,2-Trichloroethane

- Trichloroethylene

Trichlorofluoromethane

2,4,5-T

- Tritium

Turbidity

Uranium-233/234

Uranium-235

Uranium-238

Vanadium, total recoverable

Xylenes

Zinc, total recoverable

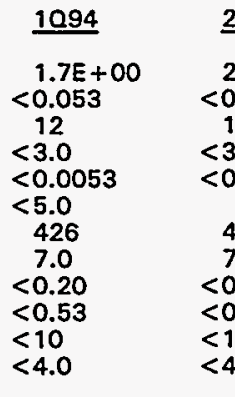

$\quad \underline{3094}$
$2.2 E+00$
10
$<3.0$
$<0.053$
$<5.0$
389
6.6
$<0.20$
$<0.53$
$<10$
$<4.0$
937

$1.6 E+00$

5.6

$<5.0$

$<500$

1.2E +00

$<2.0$

6,800

$<2.0$

2, 190

28

$<1,000$

$<5.0$

$<5.0$

3.2

$<5.0$

2.8E+05

26,000

$<1,000$

55

$<50$

$<0.25$

$<0.00047$

$<5.0$

$<5.0$

67

$<5.0$

2.7E + 02

0.67

$<7$. OE-02

$<0.0 E+00$

$<2.3 E-02$

$<8.0$

$<10$

3.0
2.1E +00

$<0.05$

12
$<3.0$

$<0.0050$

427

7.8
$<0.20$

$<0.50$

$<10$

ๆ,140

$1.9 E+00$

5.4

$<5.0$

$<500$

1.2E +00

$<2.0$

6,930

$<2.0$

2,160

27

$<1,000$

$<1.0$
5.8

5.8

$<2.0$

$<.0$
$2.7 E+05$

26,000

$<1,000$

53

$<50$

$<0.24$

$<0.00046$

$<1.0$

$<1.0$

69

1.4

$<0.00046$

2.6E + 02

2.0

8.9E-02

$<9.0 E-03$

6.5E-02

$<8.0$

$<2.0$

$<2.0$
$1.7 E+00$

5.2

$<5.0$

$<500$

9.0E-01

$<2.0$

6,580

$<2.0$

25

$<1,000$

$<5.0$

5.5
$<17$

50

3.OE + 05

32,000

$<1,000$

69

$<50$

$<1.1$
$<0.54$

$<0.54$

$<5.0$

$<5.0$

70
$<5.0$

3.1E +02

0.21

1.8E-01

3.5E-02

2.2E-01

$<3.0$

$<5.0$
3.7

$\begin{array}{llllll}4094 & \text { DF } & \text { Mod } & \text { Unit } & \text { Lab } & \text { Flag } \\ 2.9 E+00 & 1 & \mathrm{~J} & \begin{array}{l}\mathrm{pCi} / \mathrm{L} \\ \mu \mathrm{g} / \mathrm{L}\end{array} & \mathrm{GP} & 0 \\ 11 & 1 & & \begin{array}{l}\mu \mathrm{g} / \mathrm{L} \\ \mu \mathrm{g} / \mathrm{L}\end{array} & \mathrm{GE} & 0 \\ <3.0 & 1 & & \mathrm{GE} & 0\end{array}$

$\begin{array}{llllll}<0.0056 & 1 & J & \mu g / L & G E & 0\end{array}$

$\begin{array}{lllll}425 & 1 & \mu g / L & G E & 0\end{array}$

7.6

$<0.20$

$<10$

$<4.0$

858
$4.2 E+00$
5.6

5.6

$<5.0$

$<500$

$<2.0$

7.170

$<2.0$

2,090

29

$<1,000$

$<1.0$

6.7

$<2.0$

$<1.0$

32,000

$<1,000$

66

$<50$

$<0.27$

$<0.00045$

$<1.0$

$<1.0$

79

$2.8 E+02$

\subsection{2}

8.OE-02

$0.0 E+00$

6.0E-02

$<8.0$

$<2.0$

$<2.0$

$\mu \mathrm{g} / \mathrm{L} \quad \mathrm{GE}$

$\mu \mathrm{g} / \mathrm{L} \quad \mathrm{GE}$

$\mu \mathrm{g} / \mathrm{L} \quad \mathrm{GE}$

$\mu \mathrm{g} / \mathrm{L} \quad \mathrm{GE}$

$\mu \mathrm{g} / \mathrm{L}$

$\mu \mathrm{g} / \mathrm{L} \quad \mathrm{GE}$

$\mathrm{pCi} / \mathrm{L}$ GP 0

ph

$\mu \mathrm{g} / \mathrm{L}$

$\mu g / L$ GE 0

PCiL GP

$\mu \mathrm{g} / \mathrm{L} \quad \mathrm{G}$

$\mu \mathrm{g} / \mathrm{L}$

$\mu g / L$

G

$\mu S / \mathrm{cm} \quad \mathrm{GE}$

$\mu \mathrm{g} / \mathrm{L}$

$\mu \mathrm{g} / \mathrm{L}$

$\mu g / L \quad G$

$\mu \mathrm{g} / \mathrm{L}$

$\mu \mathrm{g} / \mathrm{L} \quad \mathrm{GE}$

$\mu \mathrm{g} / \mathrm{L} \quad \mathrm{GE}$

$\mu \mathrm{g} / \mathrm{L} \quad \mathrm{GE}$

$\mu \mathrm{g} /$

$\mu \mathrm{g} / \mathrm{L} \quad \mathrm{GE}$

$\mu \mathrm{g} / \mathrm{L} \quad \mathrm{GE}$

Mg/L

$\mu \mathrm{g} / \mathrm{L} \quad \mathrm{GE}$

$\mu \mathrm{g} / \mathrm{L}$

PCi/mL GP

NTU

NT

pCi/L GP

pCill GP

$\mu \mathrm{g} / \mathrm{L} \quad \mathrm{GE}$

$\mu \mathrm{g} / \mathrm{L} \quad \mathrm{GE}$

$\mu g / L$

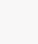

$$
\text { G }
$$

GE

Note: Flagging, dilution factors, modifiers, and laboratories are for fourth quarter 1994 data only. See Appendix B for flagging criteria. - = exceeded holding time for fourth quarter 1994.

- = exceeded screening level or final PDWS for fourth quarter 1994. 
WELL BGO 16AR

\begin{tabular}{|c|c|c|c|c|c|c|c|}
\hline SRS Coord. & Lat/Longitude & Screen Zone Elevation & Top of & & Casing & Pump & Screen Zone \\
\hline $\begin{array}{l}\text { N75743.2 } \\
\text { E56217.1 }\end{array}$ & $\begin{array}{l}33.285020^{\circ} \mathrm{N} \\
81.665899^{\circ} \mathrm{W}\end{array}$ & $113.7-103.7 \mathrm{ft} \mathrm{msl}$ & 303.7 & & 4" PVC & $\mathbf{s}$ & Congaree (IIA) \\
\hline SAMPLE DA & & $02 / 02 / 94$ & $04 / 07 / 94$ & $08 / 10 / 94$ & $11 / 03 / 94$ & & \\
\hline FIELD DATA & & & & & & & \\
\hline & & 1094 & $\underline{2094}$ & 3094 & 4094 & Unit & \\
\hline $\begin{array}{l}\text { Wa } \\
\text { Def } \\
\text { pH } \\
\text { Sp. } \\
\text { Wa } \\
\text { Alk } \\
\text { Tur } \\
\text { Vol } \\
\text { Sar }\end{array}$ & $\begin{array}{l}\text { vation } \\
\text { water } \\
\text { uctance } \\
\text { mperature } \\
\text { as } \mathrm{CaCO}_{3} \\
\end{array}$ & $\begin{array}{l}160.7 \\
143.0 \\
7.7 \\
188 \\
18.9 \\
80 \\
1 \\
3.0\end{array}$ & $\begin{array}{l}161.0 \\
142.8 \\
7.5 \\
200 \\
19.8 \\
78 \\
0 \\
2.7\end{array}$ & $\begin{array}{l}160.2 \\
143.5 \\
7.3 \\
189 \\
20.1 \\
72 \\
1 \\
3.1\end{array}$ & $\begin{array}{l}160.5 \\
143.2 \\
7.6 \\
182 \\
19.3 \\
77 \\
0 \\
3.0\end{array}$ & $\begin{array}{l}\mathrm{ft} \mathrm{msl} \\
\mathrm{ft} \\
\mathrm{pH} \\
\mu \mathrm{S} / \mathrm{cm} \\
{ }^{\circ} \mathrm{C} \\
\mathrm{mg} / \mathrm{L} \\
\text { NTU } \\
\text { well vol. }\end{array}$ & \\
\hline
\end{tabular}

ANALYTICAL DATA

\begin{tabular}{|c|c|c|c|c|c|c|c|c|}
\hline H ST Analyte & 1094 & $\underline{2094}$ & $\underline{3094}$ & 4094 & $\underline{\text { DF }}$ & Mod & Unit & $\underline{\text { Lab }}$ \\
\hline $\begin{array}{l}\text { Acetophenone } \\
\text { Aldrin }\end{array}$ & $\begin{array}{l}<10 \\
<0.052\end{array}$ & $\begin{array}{l}<10 \\
<0.050\end{array}$ & $<11$ & $<10$ & 1 & & $\mu g / L$ & GE \\
\hline $\begin{array}{l}\text { Aluminum, total recoverable } \\
\text { Antimony, total recoverable }\end{array}$ & $\begin{array}{l}<20 \\
<2.0\end{array}$ & $\begin{array}{l}<20 \\
<2.0\end{array}$ & $\begin{array}{l}<20 \\
<3.0\end{array}$ & $\begin{array}{l}<20 \\
<20\end{array}$ & 1 & & $\mu g / L$ & GE \\
\hline Arsenic, total recoverable & $<2.0$ & $<2.0$ & $<2.0$ & $<2.0$ & 1 & & $\mu \mathrm{g} / \mathrm{L}$ & GE \\
\hline $\begin{array}{l}\text { Barium, total recoverable } \\
\text { Benzene }\end{array}$ & $\begin{array}{r}37 \\
<1.0\end{array}$ & $\begin{array}{r}39 \\
<1.0\end{array}$ & $\begin{array}{r}36 \\
<5.0\end{array}$ & $\begin{array}{r}37 \\
<10\end{array}$ & 1 & & $\mu g / L$ & GE \\
\hline Bromodichloromethane & $<1.0$ & $<1.0$ & $<5.0$ & $<1.0$ & 1 & & $\mu g / L$ & GE \\
\hline Bromoform & $<1.0$ & $<1.0$ & $<5.0$ & $<1.0$ & 1 & & $\mu g / \mathrm{L}$ & GE \\
\hline Bromomethane (Methyl bromide) & $<1.0$ & $<1.0$ & $<10$ & $<1.0$ & $i$ & & $\mu g / L$ & GE \\
\hline $\begin{array}{l}\text { Cadmium, total recoverable } \\
\text { Calcium, total recoverable }\end{array}$ & $\begin{array}{l}<2.0 \\
28,800\end{array}$ & $\begin{array}{l}<2.0 \\
29,000\end{array}$ & $\begin{array}{l}<2.0 \\
28,700\end{array}$ & $<2.0$ & $\begin{array}{l}1 \\
1 \\
1\end{array}$ & & $\mu$ & GE \\
\hline $\begin{array}{l}\text { Carbon-14 } \\
\text { Chloride }\end{array}$ & $<-2.9 E+\infty 0$ & $\begin{array}{l}<1.3 E+00 \\
2.030\end{array}$ & $\begin{array}{l}<3.0 \\
2.700\end{array}$ & $2.4 E+01$ & 1 & $\mathbf{J}$ & $\begin{array}{l}\mu \mathrm{gh} / \mathrm{L} \\
\mathrm{PCi} / \mathrm{L}\end{array}$ & $\begin{array}{l}\mathrm{GE} \\
\mathrm{GP} \\
\mathrm{GE}\end{array}$ \\
\hline Chlorobenzene & $<1.0$ & $<1.0$ & $<5.0$ & $<1.0$ & 1 & & $\mu \mathrm{g} / \mathrm{L}$ & $\mathrm{GE}$ \\
\hline $\begin{array}{l}\text { Chloroethane } \\
\text { Chloroethene (Vinyl chloride) }\end{array}$ & $\begin{array}{l}<1.0 \\
<1.0\end{array}$ & $\begin{array}{l}<1.0 \\
<1.0\end{array}$ & $\begin{array}{l}<10 \\
<10\end{array}$ & $\begin{array}{l}<1.0 \\
<1.0\end{array}$ & 1 & & $\mu \mathrm{g} / \mathrm{L}$ & $\begin{array}{l}\text { GE } \\
\text { GE }\end{array}$ \\
\hline $\begin{array}{l}\text { 2-Chloroethyl vinyl other } \\
\text { Chloroform }\end{array}$ & $\begin{array}{l}<1.0 \\
<1.0\end{array}$ & $\begin{array}{l}<1.0 \\
<1.0\end{array}$ & $\begin{array}{l}<10 \\
<5.0\end{array}$ & $\begin{array}{l}<1.0 \\
<1.0\end{array}$ & $\begin{array}{l}1 \\
1\end{array}$ & & $\mu g / L$ & GE \\
\hline Chloromethane (Methyl chloride) & $<1.0$ & $<1.0$ & 5.6 & $<1.0$ & 1 & & $\mu \mathrm{g} / \mathrm{L}$ & GE \\
\hline $\begin{array}{l}\text { Chromium, total recoverable } \\
\text { Copper, total recoverable }\end{array}$ & $\begin{array}{l}<4.0 \\
<4.0\end{array}$ & $\begin{array}{l}<4.0 \\
<4.0\end{array}$ & $\begin{array}{l}<4.0 \\
<4.0\end{array}$ & $\begin{array}{l}<4.0 \\
<4.0\end{array}$ & $\begin{array}{l}1 \\
1\end{array}$ & & $\mu \mathrm{g} / \mathrm{L}$ & $\begin{array}{l}\mathrm{GE} \\
\mathrm{GE}\end{array}$ \\
\hline $\begin{array}{l}\text { Cyanide } \\
p, p^{\circ}-D D T\end{array}$ & $\begin{array}{l}<5.0 \\
<0.10\end{array}$ & $\begin{array}{l}<5.0 \\
<0.10\end{array}$ & $<5.0$ & $<5.0$ & $i$ & & $\mu \mathrm{g} / \mathrm{L}$ & $\mathrm{GE}$ \\
\hline Dibromochloromethane & $<1.0$ & $<1.0$ & $<5.0$ & $<1.0$ & 1 & & $\mu \mathrm{g} / \mathrm{L}$ & GE \\
\hline $\begin{array}{l}\text { 1,1-Dichloroethane } \\
\text { 1,2-Dichloroethane }\end{array}$ & $\begin{array}{l}<1.0 \\
<1.0\end{array}$ & $\begin{array}{l}<1.0 \\
<1.0\end{array}$ & $\begin{array}{l}<5.0 \\
<5.0\end{array}$ & $\begin{array}{l}<1.0 \\
<1.0\end{array}$ & 1 & & $\mu \mathrm{g} / \mathrm{L}$ & GE \\
\hline 1,7-Dichloroethylene & $<1.0$ & $<1.0$ & $<5.0$ & $<1.0$ & 1 & & $\mu \mathrm{g} / \mathrm{L}$ & GE \\
\hline trans-1,2-Dichloroethylene & $<1.0$ & $<1.0$ & $<5.0$ & $<1.0$ & $i$ & & $\mu \mathrm{g} / \mathrm{L}$ & GE \\
\hline 2,4-Dichlorophenoxyacetic acid & $<0.0015$ & $<0.0015$ & $<1.1$ & $<0.0015$ & 1 & & $\mu \mathrm{g} / \mathrm{L}$ & GE \\
\hline $\begin{array}{l}\text { 1,2-Dichloropropane } \\
\text { cis-1,3-Dichloropropene }\end{array}$ & $\begin{array}{l}<1.0 \\
<1.0\end{array}$ & $\begin{array}{l}<1.0 \\
<1.0\end{array}$ & $\begin{array}{l}<5.0 \\
<5.0\end{array}$ & $\begin{array}{l}<1.0 \\
<10\end{array}$ & 1 & & $\mu \mathrm{g} / \mathrm{L}$ & $\begin{array}{l}\text { GE } \\
\text { GE }\end{array}$ \\
\hline $\begin{array}{l}\text { trans-1,3-Dichloropropene } \\
\text { Dieldrin }\end{array}$ & $\begin{array}{l}<1.0 \\
<0.52\end{array}$ & $\begin{array}{l}<1.0 \\
<0.50\end{array}$ & $<5.0$ & $<1.0$ & 1 & & $\begin{array}{l}\mu g / L \\
\mu g / L \\
\mu g / L\end{array}$ & GE \\
\hline $\begin{array}{l}\text { Endrin } \\
\text { Ethylbenzene }\end{array}$ & $\begin{array}{l}<0.0062 \\
<1.0\end{array}$ & $\begin{array}{l}<0.0060 \\
<1.0\end{array}$ & $\begin{array}{l}<0.11 \\
<5.0\end{array}$ & $\begin{array}{l}<0.0057 \\
<1.0\end{array}$ & $\begin{array}{l}1 \\
1\end{array}$ & & $\mu \mathrm{g} / \mathrm{L}$ & $\begin{array}{l}\mathrm{GE} \\
\mathrm{GE}\end{array}$ \\
\hline Fluoride & $<100$ & $<100$ & $<100$ & $<20$ & $i$ & & $\mu \mathrm{g} / \mathrm{L}$ & GE \\
\hline
\end{tabular}

Note: Flagging, dilution factors, modifiers, and laboratories are for fourth quarter 1994 data only. See Appendix B for flagging criteria. - = exceeded holding time for fourth quarter 1994.

- = exceeded screening level or final PDWS for fourth quarter 1994. 
H ST Analyte

Gross alpha

Heptachlor

Iron, total recoverable

Lead, total recoverable

Lindane

Lithium, total recoverable

Magnesium, total recoverable

Manganese, total recoverable

Mercury, total recoverable

Methoxychlor

Naphthalene

Nickel, total recoverable

Nitrate as nitrogen

Nitrate-nitrite as nitrogen

Nonvolatile beta

pH

Phenols

Potassium, total recoverable

Radium, total alpha-emitting

Selenium, total recoverable

Silica, total recoverable

Silver, total recoverable

Sodium, total recoverable

Specific conductance

Sulfate

1,1,2,2-Tetrachloroethane

Tetrachloroethylene

Tin, total recoverable

Toluene

Total dissolved solids

Total organic carbon

Total organic halogens

Total phosphates (as P)

Toxaphen

2,4,5-TP (Silvex)

1,1,1-Trichloroethane

1,1,2-Trichloroethane

Trichloroethylene

Trichlorofluoromethane

2,4,5-T

Tritium

Turbidity

Uranium-233/234

Uranium-235

Uranium-238

Vanadium, total recoverable

Xylenes

Zinc, total recoverable

\begin{tabular}{|c|c|c|}
\hline 1094 & 2094 & 3094 \\
\hline 9.2E-01 & 8.2E-01 & 9.9E-01 \\
\hline $\begin{array}{l}<0.052 \\
8.9\end{array}$ & $<0.050$ & \\
\hline $\begin{array}{r}8.9 \\
<3.0\end{array}$ & $\begin{array}{r}5.0 \\
<3.0\end{array}$ & $\begin{array}{r}8.8 \\
<3.0\end{array}$ \\
\hline $\begin{array}{l}<0.0052 \\
<5.0\end{array}$ & $<0.0050$ & $<0.053$ \\
\hline $\begin{array}{l}<5.0 \\
1,050\end{array}$ & 1,080 & $\begin{array}{l}<5.0 \\
1.000\end{array}$ \\
\hline 10 & 9.4 & 9.8 \\
\hline$<0.20$ & $<0.20$ & $<0.20$ \\
\hline$<0.52$ & $<0.50$ & $\begin{array}{l}<0.53 \\
<10\end{array}$ \\
\hline $\begin{array}{l}<10 \\
<4.0\end{array}$ & $\begin{array}{l}<10 \\
<4.0\end{array}$ & $\begin{array}{r}<10 \\
<4.0 \\
674\end{array}$ \\
\hline 490 & 760 & \\
\hline$<4.3 E-01$ & $1.7 E+00$ & $1.1 E+00$ \\
\hline 7.7 & 7.6 & $\begin{array}{r}7.2 \\
-50\end{array}$ \\
\hline$<5.0$ & $<5.0$ & $\begin{array}{l}<5.0 \\
1.220\end{array}$ \\
\hline $\begin{array}{l}1.470 \\
1.7 E+00\end{array}$ & 4.0E-01 & $\begin{array}{l}1,220 \\
0.0 E+00\end{array}$ \\
\hline$<2.0$ & $<2.0$ & $<2.0$ \\
\hline 23,900 & $\begin{array}{l}23,500 \\
<2,0\end{array}$ & $\begin{aligned} & 21,500 \\
&<2,0\end{aligned}$ \\
\hline 7,870 & 7,010 & 5,720 \\
\hline $\begin{array}{l}183 \\
3.480\end{array}$ & $\begin{array}{l}180 \\
3.110\end{array}$ & $\begin{array}{l}174 \\
3710\end{array}$ \\
\hline$<1.0$ & $<1.0$ & $<5.0$ \\
\hline$<1.0$ & $<1.0$ & $<5.0$ \\
\hline$<2.0$ & 2.2 & $<17$ \\
\hline$<1.0$ & $<1.0$ & $<5.0$ \\
\hline $\begin{array}{l}133,000 \\
<1000\end{array}$ & 131,000 & $\begin{array}{l}121,000 \\
1,100\end{array}$ \\
\hline$<1,0$ & 5.2 & $<5.0$ \\
\hline$<50$ & $<50$ & 3,240 \\
\hline$<0.25$ & $<0.24$ & $<1.1$ \\
\hline$<0.00046$ & $\begin{array}{l}<0.00046 \\
<1.0\end{array}$ & $\begin{array}{l}<0.55 \\
<5.0\end{array}$ \\
\hline$<1.0$ & $<1.0$ & $<5.0$ \\
\hline$<1.0$ & $<1.0$ & $<5.0$ \\
\hline$<1.0$ & $\begin{array}{l}<1.0 \\
<0.00046\end{array}$ & $<5.0$ \\
\hline$<0.0 E+00$ & 3.9E-01 & 2.9E-01 \\
\hline$<0.10$ & $<0.10$ & $<0.20$ \\
\hline 3.8E-01 & $\begin{array}{r}5.0 E-01 \\
<1.1 E-02\end{array}$ & $\begin{array}{l}4.6 \mathrm{E}-01 \\
4.2 \mathrm{E}-02\end{array}$ \\
\hline 2.3E-01 & $2.2 \mathrm{E}-01$ & $\begin{array}{l}4.2 \mathrm{E}-02 \\
5.4 \mathrm{E}-01\end{array}$ \\
\hline$<8.0$ & $<8.0$ & $<3.0$ \\
\hline$<2.0$ & $<2.0$ & $<5.0$ \\
\hline$<2.0$ & $<2.0$ & 10 \\
\hline
\end{tabular}

\begin{tabular}{|c|c|}
\hline 4094 & DF \\
\hline $1.1 E+00$ & 1 \\
\hline $\begin{aligned} & 7.2 \\
< & 3.0 \\
< & 0.0048\end{aligned}$ & $\begin{array}{l}1 \\
1 \\
1\end{array}$ \\
\hline $\begin{array}{l}1.080 \\
8.5 \\
0.60 \\
<0.48 \\
<10 \\
<4.0\end{array}$ & $\begin{array}{l}1 \\
1 \\
1 \\
1 \\
1 \\
1\end{array}$ \\
\hline $\begin{aligned} & 758 \\
& 8.3 \mathrm{E}-01 \\
& 7.5 \\
&<5.0 \\
& 1.120 \\
& 2.0 \mathrm{E}-01 \\
&<2.0 \\
& 22,800 \\
&<2.0 \\
& 5,270 \\
& 182 \\
& 2.160 \\
&<1.0 \\
&<1.0 \\
&<2.0 \\
&<1.0 \\
& 129,000 \\
& 1,580 \\
&<5.0 \\
& 106 \\
&<0.23 \\
&<0.00044 \\
&<1.0 \\
&<1.0 \\
&<1.0 \\
&<1.0\end{aligned}$ & $\begin{array}{l}1 \\
1 \\
1 \\
1 \\
1 \\
1 \\
1 \\
1 \\
1 \\
1 \\
1 \\
1 \\
1 \\
1 \\
1 \\
1 \\
1 \\
1 \\
1 \\
1 \\
1 \\
1 \\
1\end{array}$ \\
\hline $\begin{array}{l}-9.5 E-03 \\
1.7 \\
4.8 \text { E-01 } \\
1.0 \text { E-01 } \\
3.7 \text { E-01 } \\
<8.0 \\
<2.0 \\
<2.0\end{array}$ & 1 \\
\hline
\end{tabular}

Fiag

$\begin{array}{lll}\mu \mathrm{g} / \mathrm{L} & \mathrm{GE} & 0 \\ \mu \mathrm{g} / \mathrm{L} & \mathrm{GE} & 0\end{array}$

$\mu g / L \quad$ GE 0

$\mu \mathrm{g} / \mathrm{L}$

$\mu \mathrm{g} / \mathrm{L} \quad \mathrm{GE} \quad 0$

$\mu g / L$

$\mu g / L \quad G E$

$\mu g / L \quad$ GE

$\mu \mathrm{g} / \mathrm{L} \quad \mathrm{GE}$

$\mu g / L \quad G E$

$\mathrm{pCi} / \mathrm{L} \quad \mathrm{GP}$

$\mu g /$

$\mu \mathrm{g} / \mathrm{L} \quad \mathrm{GE}$

UI $\mathrm{pCi} / \mathrm{L} \quad \mathrm{GP}$

$\mu g / L \quad$ GE

$\mu \mathrm{g} / \mathrm{L}$

$\mu g / L \quad$ GE

$\mu g / L \quad$ GE

$\mu \mathrm{S} / \mathrm{cm}$ GE

$\mu \mathrm{g} / \mathrm{L}$

$\mu \mathrm{g} / \mathrm{L}$

$\mu \mathrm{g} / \mathrm{L}$

$\mu \mathrm{g} / \mathrm{L}$

$\mu \mathrm{g} /$

J $\mu \mathrm{g} /$

$\mu \mathrm{g} / \mathrm{L}$

$\mu g / L$

$\mu \mathrm{g} / \mathrm{L}$

rg/L

$\mu g / L$

$\mu \mathrm{g} /$

$\mu g / L$

$\mu \mathrm{g} / \mathrm{L}$

UI $\mathrm{pCi} / \mathrm{mL}$ GP

NTU

U

UI
pCi

pCi/L

$\mu g / L$

$\mu \mathrm{g} / \mathrm{L}$

Note: Flagging, dilution factors, modifiers, and laboratories are for fourth quarter 1994 data only. See Appendix B for flagging criteria. - = exceeded holding time for fourth quarter 1994.

- = exceeded screening level or final PDWS for fourth quarter 1994. 
WELL BGO 16B

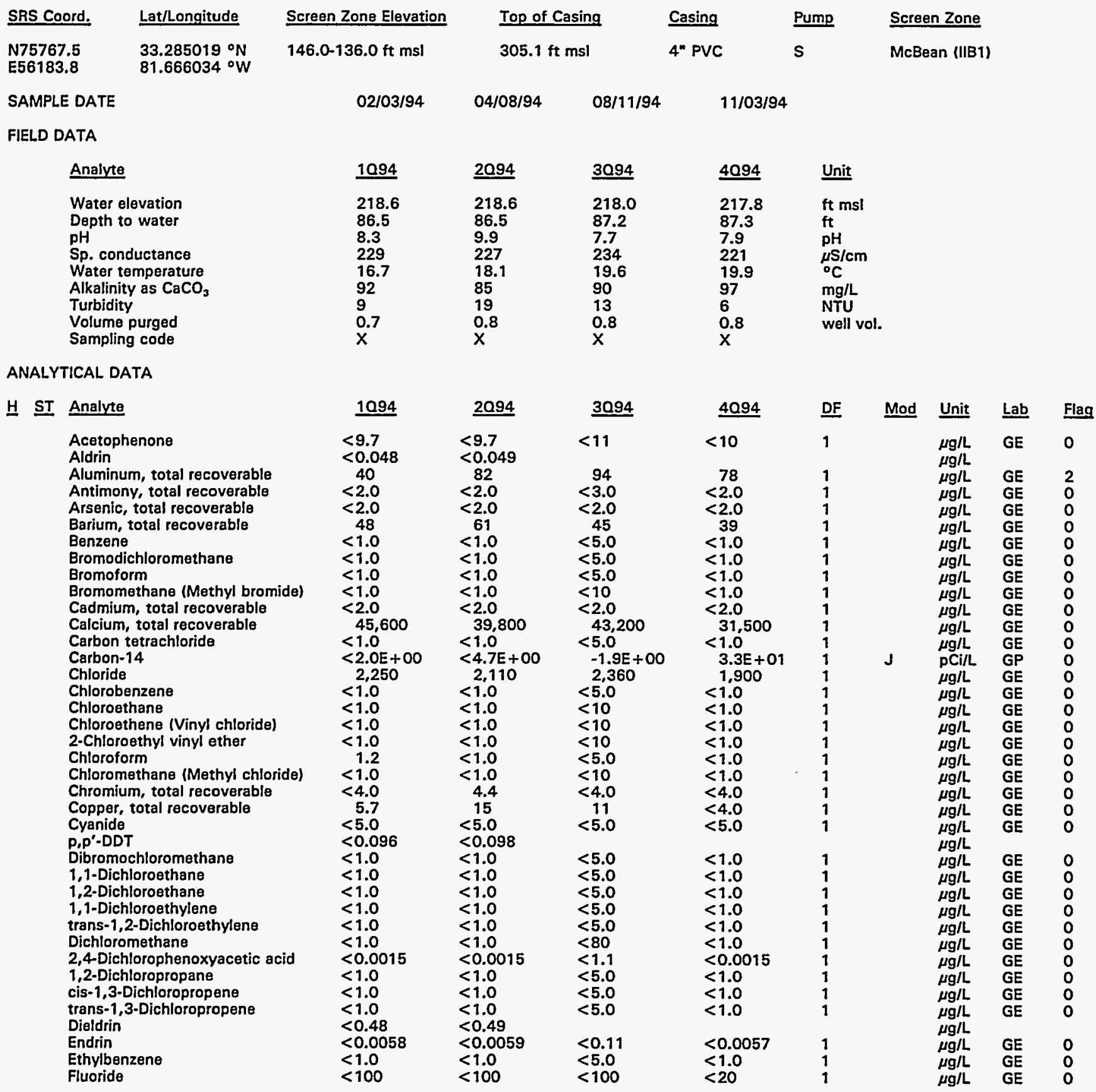

Note: Flagging, dilution factors, modifiers, and laboratories are for fourth quarter 1994 data only. See Appendix B for flagging criteria. - = exceeded holding time for fourth quarter 1994.

- = exceeded screening level or final PDWS for fourth quarter 1994. 
Well BGO 168 continued

\section{ANALYTICAL DATA}

\section{H ST Analyte}

Gross alpha

Heptachlor

Iron, total recoverable

Lead, total recoverable

Lindane

Lithium, total recoverable

Magnesium, total recoverable

Manganese, total recoverable

Mercury, total recoverable

Methoxychlor

Naphthalene

Nickel, total recoverable

Nitrate as nitrogen

Nitrate-nitrite as nitrogen

Nonvolatile beta

- $\mathrm{pH}$

Phenols

Potassium, total recoverable

Radium, total alpha-emitting

Selenium, total recoverable

Silica, total recoverable

Silver, total recoverable

Sodium, total recoverable

Specific conductance

Sulfate

1,1,2,2-Tetrachloroethane

Tetrachioroethylene

Tin, total recoverable

Toluene

Total dissolved solids

Total organic carbon

Total organic halogens

Total phosphates (as P)

Toxaphene

2,4,5-TP (Silvex)

1,1,1-Trichloroethane

1,1,2-Trichloroethane

Trichloroethylene

Trichlorafluoromethane

2,4,5-T

Tritium

Turbidity

Uranium-233/234

Uranium-235

Uranium-238

Vanadium, total recoverable

Xylenes

Zinc, total recoverable

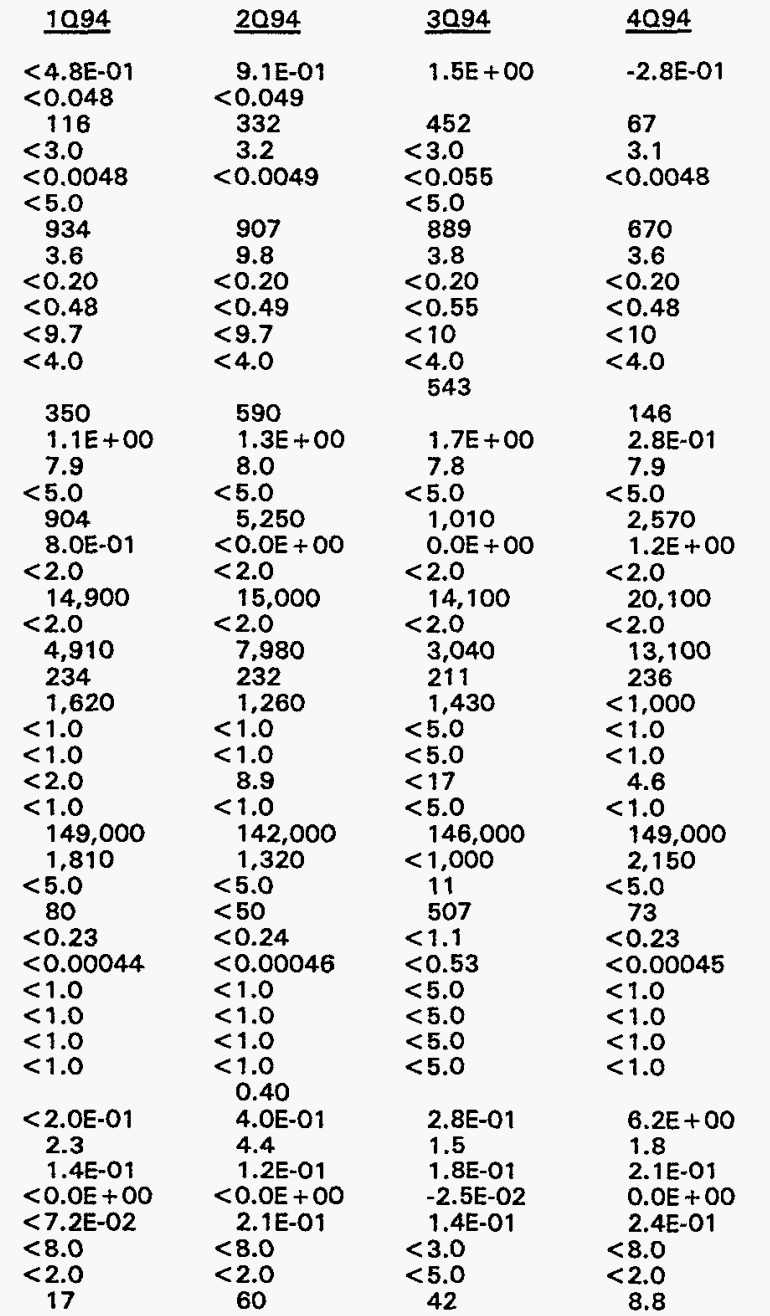

Mod

$\begin{array}{lll}\text { Unit } & \text { Lab } & \text { Flag } \\ \text { pCi/L } & \text { GP } & 0 \\ \mu g / L & & \\ \mu g / L & \text { GE } & 0 \\ \mu g / L & \text { GE } & 0\end{array}$

$\mu \mathrm{g} / \mathrm{L}$ GE

$\mu \mathrm{g} / \mathrm{L}$

$\mu g / L$

$\mu g / L$

$\mu g / L$

$\mu \mathrm{g} / \mathrm{L}$

$\mu \mathrm{g} /$

$\mu \mathrm{g} / \mathrm{L}$

$\mu g / L$

UI $\quad \begin{array}{ll}\mu \mathrm{g} / L \\ \mathrm{~J}\end{array}$

$\mu g / l$

$\mu g / L$

J

$\mu g$

$\mu g / L$

$\mu g / L$

$\mu \mathrm{g} / \mathrm{L}$

$\mu \mathrm{S} / \mathrm{c}$

$\mu \mathrm{g} / \mathrm{L}$

$\mu g / L$

$\mu \mathrm{g} / \mathrm{L}$

$\mu g / L$

J

$\mu \mathrm{g} / \mathrm{L}$

$\mu \mathrm{g} / \mathrm{L}$

J

$\mu \mathrm{g} / \mathrm{L}$

$\mu \mathrm{g} / \mathrm{L}$

$\mu \mathrm{g} / \mathrm{L}$

$\mu \mathrm{g} / \mathrm{L}$

$\mu \mathrm{g} / \mathrm{L}$

$\mu \mathrm{g} / \mathrm{L}$

$\mu \mathrm{g} / \mathrm{L}$

$\mu \mathrm{g} / \mathrm{L}$

pCi/mL GP

PCi

UI

$\mathrm{pCi} / \mathrm{L}$

pill

pCi/L

$\mu \mathrm{g} / \mathrm{L}$

$\mu \mathrm{g} / \mathrm{L}$
$\mu \mathrm{g} / \mathrm{L}$
$\mu \mathrm{g} / \mathrm{L}$

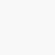

Note: Flagging, dilution factors, modifiers, and laboratories are for fourth quarter 1994 data only. See Appendix B for flagging criteria. - = exceeded holding time for fourth quarter 1994.

- = exceeded screening level or final PDWS for fourth quarter 1994. 
WELL BGO 16D

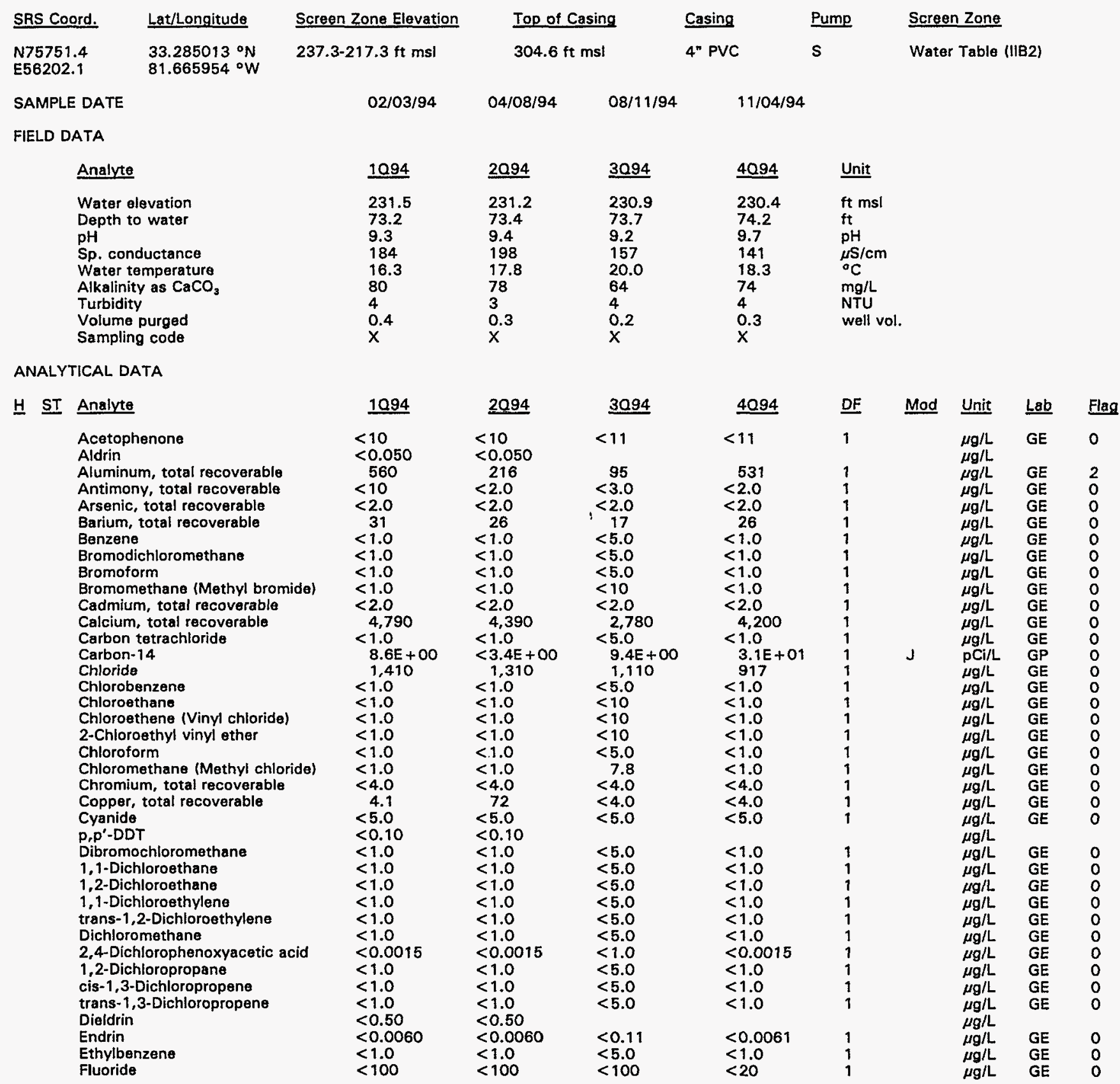

Note: Flagging, dilution factors, modifiers, and laboratories are for fourth quarter 1994 data only. See Appendix B for flagging criteria. - = exceeded holding time for fourth quarter 1994.

- = exceeded screening level or final PDWS for fourth quarter 1994. 
H ST Analyte

Gross alpha

Heptachlor

Iron, total recoverable

Lead, total recoverable

Lindane

Lithium, total recoverable

Magnesium, total recoverable

Manganese, total recoverable

Mercury, total recoverable

Methoxychlor

Naphthalene

Nickel, total recoverable

Nitrate as nitrogen

Nitrate-nitrite as nitrogen

Nonvolatile beta

pH

Phenols

Potassium, total recoverable

Radium, total alpha-emitting

Selenium, total recoverable

Silica, total recoverable

Silver, total recoverable

Sodium, total recoverable

Specific conductance

Sulfate

1,1,2,2-Tetrachloroethane

Tetrachloroethylene

Tin, total recoverable

Toluene

Total activity

Total dissolved solids

Total organic carbon

Total organic halogens

Total phosphates (as P)

Toxaphene

2,4,5-TP (Silvex)

1,1,1-Trichloroethane

1,1,2-Trichloroethane

- Trichloroethylene

Trichlorofluoromethane

2,4,5-T

- Tritium

Turbidity

Uranium-233/234

Uranium-235

Uranium-238

Vanadium, total recoverable

Xylenes

Zinc, total recoverable

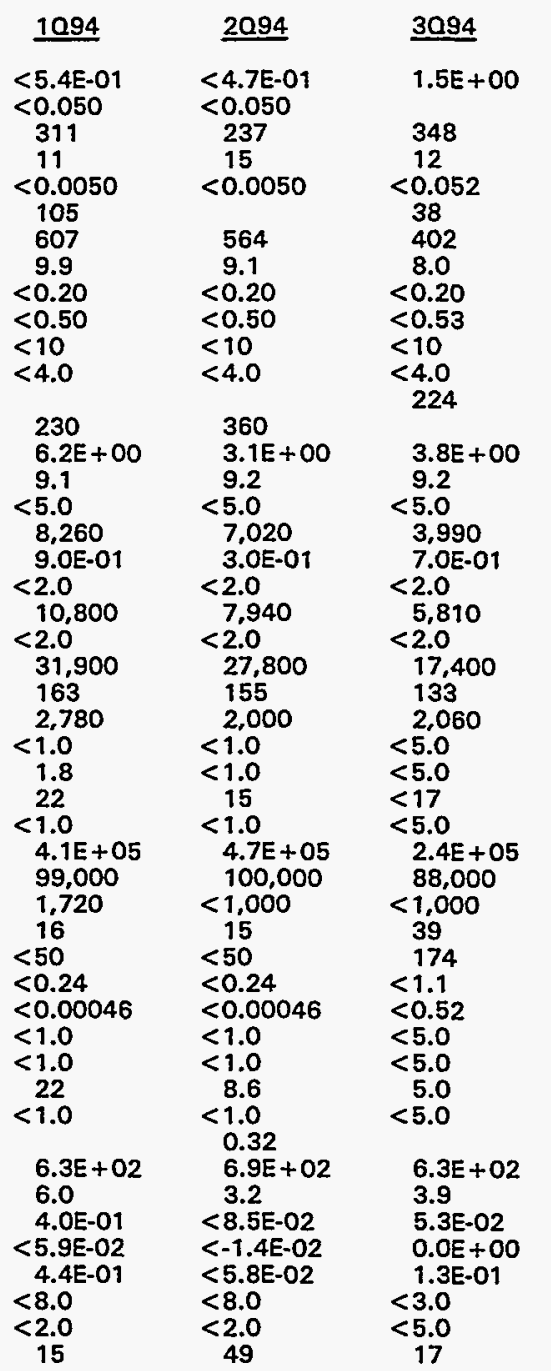

\begin{tabular}{|c|c|c|}
\hline 4094 & $\underline{D F}$ & Mod \\
\hline 8.5E-01 & 1 & $J$ \\
\hline 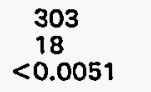 & $\begin{array}{l}1 \\
5 \\
1\end{array}$ & $J$ \\
\hline $\begin{aligned} & 638 \\
& 13 \\
< & 0.20 \\
< & 0.51 \\
< & 11 \\
< & 4.0\end{aligned}$ & $\begin{array}{l}1 \\
1 \\
1 \\
1 \\
1 \\
1\end{array}$ & \\
\hline $\begin{array}{l}277 \\
4.0 \mathrm{E}+00 \\
9.2 \\
<5.0 \\
\mathbf{5}, 570 \\
\mathbf{3 . 0 E - 0 1}\end{array}$ & $\begin{array}{l}1 \\
1 \\
1 \\
1 \\
1 \\
1\end{array}$ & $\begin{array}{l}J \\
\text { UI }\end{array}$ \\
\hline
\end{tabular}

\begin{tabular}{lll} 
Unit & Lab & Flag \\
\cline { 1 - 1 } $\mathrm{pCi} / \mathrm{L}$ & $\mathrm{GP}$ & 0 \\
$\mu \mathrm{g} / \mathrm{L}$ & & \\
$\mu \mathrm{g} / \mathrm{L}$ & $\mathrm{GE}$ & 2 \\
$\mu \mathrm{g} / \mathrm{L}$ & $\mathrm{GE}$ & 0 \\
$\mu \mathrm{g} / \mathrm{L}$ & $\mathrm{GE}$ & 0 \\
$\mu \mathrm{g} / \mathrm{L}$ & & \\
$\mu \mathrm{g} / \mathrm{L}$ & $\mathrm{GE}$ & 0 \\
$\mu \mathrm{g} / \mathrm{L}$ & $\mathrm{GE}$ & 0 \\
$\mu \mathrm{g} / \mathrm{L}$ & $\mathrm{GE}$ & 0 \\
$\mu \mathrm{g} / \mathrm{L}$ & $\mathrm{GE}$ & 0 \\
$\mu \mathrm{g} / \mathrm{L}$ & $\mathrm{GE}$ & 0
\end{tabular}

$<2.0$

10,500

$<2.0$

22,100

124

1.220

$<1.0$

$<1.0$

11

$<1.0$

85,000

$<1,000$

12

$<50$
$<0.24$

$<0.00046$

$<1.0$

$<1.0$

6.8
$<1.0$

$7.3 E+02$

9.8

2.0E-01

7.5E-02

2.2E-01

$<8.0$

$<2.0$

19

$\mu g / L$

$\mu g / L$

gE GE 0

pCi/L GP

$\mu g / L \quad G E$

$\mu \mathrm{g} / \mathrm{L} \quad \mathrm{GE}$

DCill GP

$\mu \mathrm{g} / \mathrm{L} \quad \mathrm{GE}$

$\mu \mathrm{g} / \mathrm{L} \quad \mathrm{GE}$

$\mu \mathrm{g} / \mathrm{L} \quad \mathrm{GE}$

$\mu \mathrm{g} / \mathrm{L} \quad \mathrm{GE}$

$\mu S / \mathrm{cm}$ GE

J

$\mu \mathrm{g} / \mathrm{L} \quad \mathrm{GE}$

$\mu \mathrm{g} / \mathrm{L} \quad \mathrm{GE}$

HG/L GE

$\mu \mathrm{g} / \mathrm{L} \quad \mathrm{GE}$

pCi/L

$\mu \mathrm{g} / \mathrm{L} \quad \mathrm{GE}$

$\mu \mathrm{g} / \mathrm{L} \quad \mathrm{GE}$

$\mu g / L$

$\mu \mathrm{g} / \mathrm{L}$

$\mu \mathrm{g} / \mathrm{L}$

$\mu g / L$

$\mu g / L$

$\mu g / L$

$\mu g / L$

$\mu g / L$

$\mathrm{PCi} / \mathrm{mL} \quad \mathrm{GP}$

PCi/m

$J$

NTU

pCi/L

$\mathrm{PCi} / \mathrm{L}$

$\mu g / L$

$\mu \mathrm{g} / \mathrm{L}$

0

1
1
0
0
0
0
0
0
0
0
0
0
0
1
0
0
0
0
0
0
0
0
0
0
2
0
0
2
0
0
0
0
0
0
0

Note: Flagging, dilution factors, modifiers, and laboratories are for fourth quarter 1994 data only. See Appendix B for flagging criteria. - = exceeded holding time for fourth quarter 1994.

- exceeded screening level or final PDWS for fourth quarter 1994. 


\section{WELL BGO 17DR}

\begin{tabular}{|c|c|c|c|c|c|c|}
\hline SRS Coord. & Lat/Longitude & Screen Zone Elevation & Top of Casing & Casing & Pump & Screen Zone \\
\hline $\begin{array}{l}\text { N75604.0 } \\
\text { E56407.2 }\end{array}$ & $\begin{array}{l}33.285022^{\circ} \mathrm{N} \\
81.665128^{\circ} \mathrm{W}\end{array}$ & $236.9-216.9 \mathrm{ft} \mathrm{msl}$ & $299.2 \mathrm{ft} \mathrm{msl}$ & 4" PVC & $\mathbf{S}$ & Water Table \\
\hline SAMPLE DATE & & $02 / 03 / 94$ & $04 / 08 / 94$ & & & \\
\hline
\end{tabular}

FIELD DATA

Analyte
Water elevation
Depth to water
pH
$\mathrm{Sp}$. conductance
Water temperature
Alkalinity as $\mathrm{CaCO}_{3}$
Turbidity
Volume purged
Sampling code

ANALYTICAL DATA

$\frac{1094}{}$
231.4
67.9
5.6
22
16.9
2
31
0.7
$x$

\begin{tabular}{|c|c|c|}
\hline 1094 & 2094 & 3094 \\
\hline$<10$ & $<10$ & $<10$ \\
\hline $\begin{array}{c}<0.052 \\
126\end{array}$ & $\begin{array}{c}<0.052 \\
93\end{array}$ & 180 \\
\hline$<2.0$ & $<2.0$ & $<3.0$ \\
\hline$<2.0$ & $<2.0$ & $<2.0$ \\
\hline 7.6 & 8.4 & 5.6 \\
\hline$<1.0$ & $<1.0$ & $<5.0$ \\
\hline$<1.0$ & $<1.0$ & $<5.0$ \\
\hline$<1.0$ & $<1.0$ & $<5.0$ \\
\hline$<1.0$ & $<1.0$ & $<10$ \\
\hline$<2.0$ & $<2.0$ & $<2.0$ \\
\hline $\begin{aligned} & 1,130 \\
&<1.0\end{aligned}$ & $\begin{array}{l}1.130 \\
<1.0\end{array}$ & $\begin{array}{r}780 \\
<5.0\end{array}$ \\
\hline $\begin{array}{c}<4.6 E+00 \\
1,210\end{array}$ & $\begin{array}{c}<3.2 E-01 \\
2,470\end{array}$ & $\begin{array}{l}-4.6 E+00 \\
870\end{array}$ \\
\hline$<1.0$ & $<1.0$ & $<5.0$ \\
\hline$<1.0$ & $<1.0$ & $<10$ \\
\hline$<1.0$ & $<1.0$ & $<10$ \\
\hline$<1.0$ & $<1.0$ & $<10$ \\
\hline$<1.0$ & $<1.0$ & $<5.0$ \\
\hline$<1.0$ & $<1.0$ & $<10$ \\
\hline$<4.0$ & $<4.0$ & $<4.0$ \\
\hline$<4.0$ & $<4.0$ & $<4.0$ \\
\hline$<5.0$ & $<5.0$ & $<5.0$ \\
\hline$<0.10$ & $<0.10$ & \\
\hline$<1.0$ & $<1.0$ & $<5.0$ \\
\hline$<1.0$ & $<1.0$ & $<5.0$ \\
\hline$<1.0$ & $<1.0$ & $<5.0$ \\
\hline$<1.0$ & $<1.0$ & $<5.0$ \\
\hline$<1.0$ & $<1.0$ & $<5.0$ \\
\hline$<1.0$ & $<1.0$ & $<80$ \\
\hline$<0.0015$ & $<1.6$ & $<1.1$ \\
\hline$<1.0$ & $<1.0$ & $<5.0$ \\
\hline$<1.0$ & $<1.0$ & $<5.0$ \\
\hline$<1.0$ & $<1.0$ & $<5.0$ \\
\hline$<0.52$ & $<0.52$ & \\
\hline$<0.0062$ & $<0.0062$ & $<0.11$ \\
\hline$<1.0$ & $<1.0$ & $<5.0$ \\
\hline$<100$ & $<100$ & $<100$ \\
\hline
\end{tabular}

$<100$

$\frac{2094}{1233.3}$
65.9
5.6
26
17.3
2
16
0.7
$x$

3094
231.5
67.7
5.2
21
19.7
1
24
0.8
$x$

40.94
231.1
$68.1^{5}$
5.5
21
19.2
1
15
0.9
$x$

Unit

ft msl

$t$

$\mathrm{pH}$

$\mu \mathrm{S} / \mathrm{cm}$

C $\mathrm{C}$

NT/L

well vol.

H. ST Analyte
Acetophenone
Aldrin
Aluminum, total recoverable
Antimony, total recoverable
Arsenic, total recoverable
Barium, total recoverable
Benzene
Bromodichloromethane
Bromoform
Bromomethane (Methyl bromide)
Cadmium, total recoverable
Calcium, total recoverable
Carbon tetrachloride
Carbon-14
Chloride
Chlorobenzene
Chloroethane
Chloroethene (Vinyl chloride)
2-Chloroethyl vinyl ether
Chloroform
Chloromethane (Methyl chloride)
Chromium, total recoverable
Copper, total recoverable
Cyanide
p,p'-DDT
Dibromochloromethane
1,1-Dichloroethane
1,2-Dichlorothane
1,1-Dichlorothylene
trans-1,2-Dichloroethylene
Dichloromethane
2,4-Dichlorophenoxyacetic acid
1,2-Dichloropropane
cis-1,3-Dichloropropene
trans-1,3-Dichloropropene
Dieldrin
Endrin
Ethylbenzene
Fluoride

H ST Analyte

Acetop

Aluminum, total recoverable

Antimony, total recoverable

Barium, total recoverable

romomethane (Methyl bromide)

tetrachloride

Chloride

Chlorobenzen

Chloroethene (Vinyl chloride)

(Methyl chloride)

Copper, total recoverable

$p^{\prime}-D D T$

Dibromochloromethane

,1-Dichloroethane

1-Dichloroethylen

2,4-Dichlorophenoxyacetic acid

Dichloropropen

Fluoride

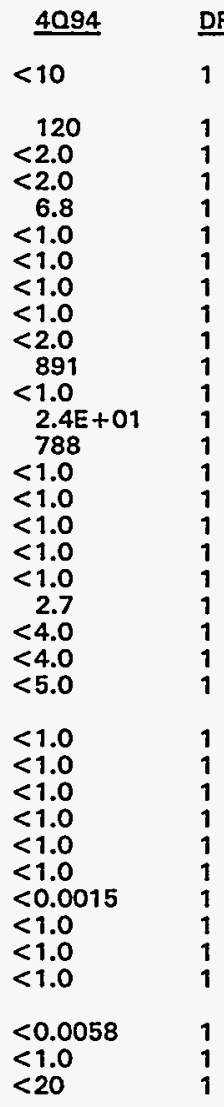

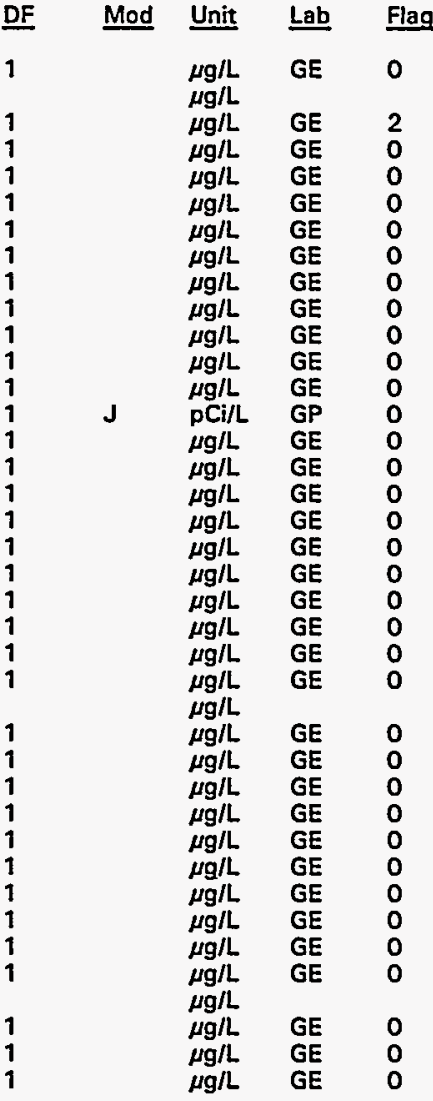

Note: Flagging, dilution factors, modifiers, and laboratories are for fourth quarter 1994 data only. See Appendix B for flagging criteria.

- = exceeded holding time for fourth quarter 1994.

- = exceeded screening level or final PDWS for fourth quarter 1994. 
H ST Analyte

Gross alpha

Heptachlor

Iron, total recoverable

Lead, total recoverable

Lindane

Lithium, total recoverable

Magnesium, total recoverable

Manganese, total recoverable

Mercury, total recoverable

Methoxychlor

Naphthalene

Nickel, total recoverable

Nitrate as nitrogen

Nitrate-nitrite as nitrogen

Nonvolatile beta

- $\quad \mathrm{pH}$

Phenols

Potassium, total recoverable

Radium, total alpha-emitting

Selenium, total recoverable

Silica, total recoverable

Silver, total recoverable

Sodium, total recoverable

Specific conductance

Sulfate

1,1,2,2-Tetrachloroethane

Tetrachloroethylene

Tin, total recoverable

Toluene

Total dissolved solids

Total organic carbon

Total organic halogens

Total phosphates (as P)

Toxaphene

2,4,5-TP (Silvex)

Tributyl phosphate

1,1, 1-Trichloroethane

1,1,2-Trichloroethane

Trichloroethylene

Trichlorofluoromethane

2,4,5-T

Tritium

Turbidity

Uranium-233/234

Uranium-235

Uranium-238

Vanadium, total recoverable

Xylenes

Zinc, total recoverable

$\frac{1094}{2.0 E+00}$
$<0.052$
38
$<3.0$
$<0.0052$
$<5.0$
336
13
$<0.20$
$<0.52$
$<10$
$<4.0$

1.1E +00

$1.4 E+00$

$<0.052$

29
$<3.0$

$<0.0051$

351

$\begin{aligned} & 12 \\ &<0.20\end{aligned}$

$<0.20$

$<10$

$<4.0$

1,140
$2.3 E+00$

$2.3 \mathrm{E}+1$
6.1
65.0

$<5.0$

$<500$

1.7E +00

$<2.0$

7,270

$<2.0$

21

$<1,000$

$<1.0$

$<1.0$

$<1.0$

26,000

$<1,000$

5.8

$<50$

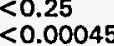

$<10$

$<1.0$

$<1.0$

$<1.0$

$<1.0$

$1.3 E+01$

278

$<1.4 E-02$

$<3.9 \mathrm{E}-03$

$<5.5 \mathrm{E}-02$

$<8.0$

$<2.0$

9.1
1,250

$1.2 E+00$

5.8

$<5.0$

$<500$

$<2.0 E-01$

$<2.0$

7,210

$<2.0$

19

$<1,000$

$<1.0$

$<2.0$

$<1.0$

17,000

$<1,000$

$<5.0$

$<50$

$<0.25$

$<0.47$

$<10$

$<1.0$

$<1.0$

1.3

$<1.0$

$1.4 \mathrm{E}+01$

$1.4 \mathrm{E}+01 \quad 1.4 \mathrm{E}+01$

38

2.1E-01

$<2.2 \mathrm{E}-02$

2.1E-01

$<8.0$

$<2.0$
6.1
529

3.4
$<0.055$

5.3

292

10
$<0.20$

$<0.55$

$<10$

$<4.0$

1.140

9.7E-01

5.7

$<5.0$

7.0E-01

$<2.0$

$\mathbf{5 , 5 5 0}$

$<2.0$

1,020

17
4,980

$\begin{aligned} & 4,980 \\ &<5.0\end{aligned}$

$<5.0$

$<17$

$<5.0$

26,000

$<1,000$

11

171

$<1.1$

$<0.53$

$<10$

$<5.0$

$<5.0$

1.3

1.2

272

2.5E-01

1.3E-02

9.6E-02

$<3.0$

$<5.0$

8.0

\begin{tabular}{|c|c|c|c|c|}
\hline 4094 & $\underline{D F}$ & Mod & $\underline{\text { Unit }}$ & Lat \\
\hline $1.8 E+00$ & 1 & J & $\mathrm{pCi} / \mathrm{L}$ & $G P$ \\
\hline $\begin{array}{r}40 \\
<3.0\end{array}$ & $\begin{array}{l}1 \\
1\end{array}$ & & $\begin{array}{l}\mu \mathrm{g} / \mathrm{L} \\
\mu \mathrm{g} / \mathrm{L}\end{array}$ & $\begin{array}{l}\mathrm{GE} \\
\mathrm{GE}\end{array}$ \\
\hline
\end{tabular}

$\begin{array}{lllll}<0.0048 & 1 & \mu g / L & \text { GE } & 0 \\ & & \mu g / L & \text { GE } & 0\end{array}$

383

11

$<0.20$

$<10$

$<4.0$

1,220

3.2E-01

5.4

$<5.0$

$<500$

6.0E-01

$<2.0$

7.130

$<2.0$

1.130

19

$<1,000$

$<1.0$

$<2.0$

$<1.0$

$<1,000$

$<5.0$

$<50$

$<0.23$

$<0.00045$

$<10$

$<1.0$

$<1.0$

$\begin{aligned} & 1.6 \\ &<1.0\end{aligned}$

$1.4 E+01$

143

2.0E-01

$-1.4 \mathrm{E}-02$

7.8E-02

$<8.0$

$<2.0$

23

$\mu g / L$

$\mu g / L \quad G E$

$\mu \mathrm{g} / \mathrm{L} \quad \mathrm{GE}$

$\mu g / L \quad G E$

$\mu g / L \quad G E$

$\mu \mathrm{g} / \mathrm{L} \quad \mathrm{GE}$

$\mu g / L$

$\mu g / L \quad G E$

UI $\mathrm{pC} i / L$ GP

$\underset{\mu g / L}{\mathrm{pH}} \quad \mathrm{GE}$

$\mu g / L \quad \mathrm{GE}$

J $\mathrm{pCi} / \mathrm{L}$ GP

$\mu \mathrm{g} / \mathrm{L}$

$\mu g / L$

$\mu \mathrm{g} / \mathrm{L}$

$\mu \mathrm{g} / \mathrm{L} \quad \mathrm{GE}$

$\mu \mathrm{S} / \mathrm{cm}$ GE

$\mu g / L \quad G E$

$\mu \mathrm{g} / \mathrm{L}$

$\mu \mathrm{g} / \mathrm{L}$

$\mu g / L$

$\mu \mathrm{g} / \mathrm{L}$

$\mu g / L$

$\mu g / L \quad G E$

$\mu g / L \quad G E$

$\mu g / h$

$\mu g / L$

H $\mathrm{GE}$

$\mu g / L \quad G E$

$\mu \mathrm{g}$

$\mu \mathrm{g} / \mathrm{L} \quad \mathrm{GE}$

$\mu \mathrm{g} / \mathrm{L}$

pCi/mL GP

PCI/mL GP

NTU

UI

pCi/L

PCi/L GP

$\begin{array}{ll}\mu g / L & G E \\ \mu \mathrm{g} / \mathrm{L} & \mathrm{GE}\end{array}$

$\mu \mathrm{g} / \mathrm{L} \quad \mathrm{GE}$

Note: Flagging, dilution factors, modifiers, and laboratories are for fourth quarter 1994 data only. See Appendix B for flagging criteria.

- = exceeded holding time for fourth quarter 1994.

- = exceeded screening level or final PDWS for fourth quarter 1994. 


\section{WELL BGO 18A}

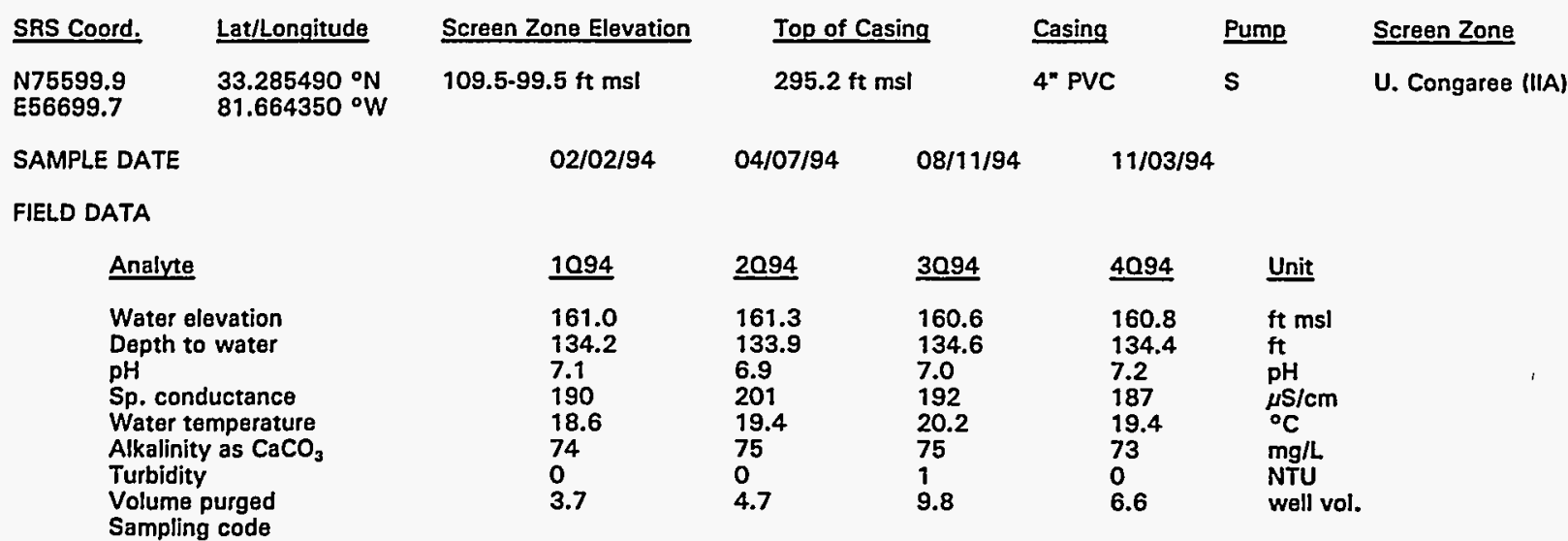

ANALYTICAL DATA

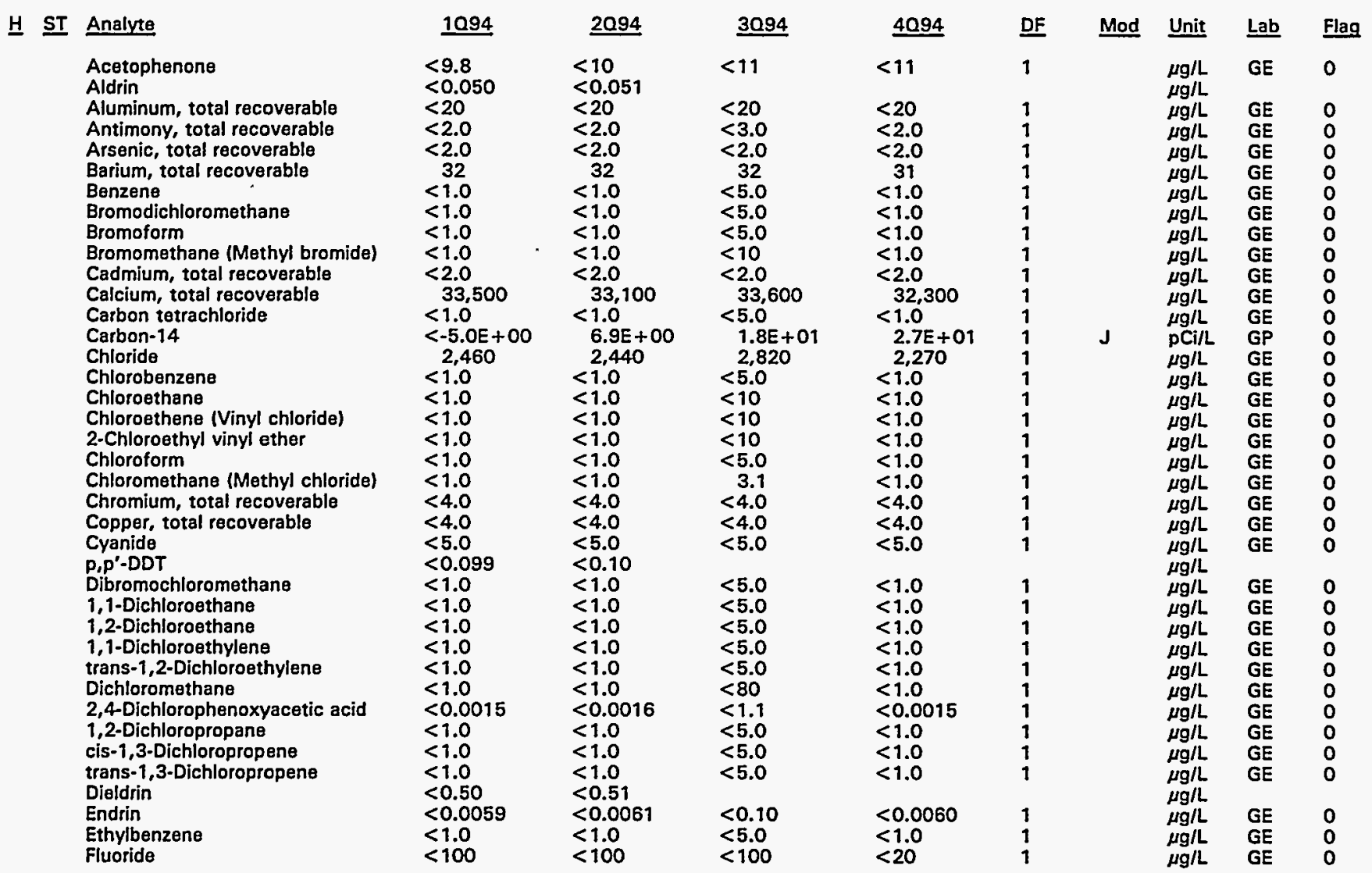

Note: Flagging, dilution factors, modifiers, and laboratories are for fourth quarter 1994 data only. See Appendix B for flagging criteria. - = exceeded holding time for fourth quarter 1994.

- = exceeded screening level or final PDWS for fourth quarter 1994. 
Well BGO 18A continued

ANALYTICAL DATA

H ST Analyte

Gross alpha

Heptachlor

Iron, total recoverable

Lead, total recoverable

Lindane

Lithium, total recoverable

Magnesium, total recoverable

Manganese, total recoverable

Mercury, total recoverable

Methoxychlor

Naphthalene

Nickel, total recoverable

Nitrate as nitrogen

Nitrate-nitrite as nitrogen

Nonvolatile beta

- $\mathrm{pH}$

Phenols

Potassium, total recoverable

Radium, total alpha-emitting

Selenium, total recoverable

Silica, total recoverable

Silver, total recoverable

Sodium, total recoverable

Specific conductance

Sulfate

1,1,2,2-Tetrachlorosthane

Tetrachloroethylene

Tin, total recoverable

Toluene

Total dissolved solids

Total organic carbon

Total organic halogens

Total phosphates (as P)

Toxaphene

2,4,5-TP (Silvex)

1,1,1-Trichloroethane

1,1,2-Trichloroethane

Trichloroethylene

Trichlorofluoromethane

2,4,5-T

Tritium

Turbidity

Uranium-233/234

Uranium-235

Uranium-238

Vanadium, total recoverable

Xylenes

Zinc, total recoverable

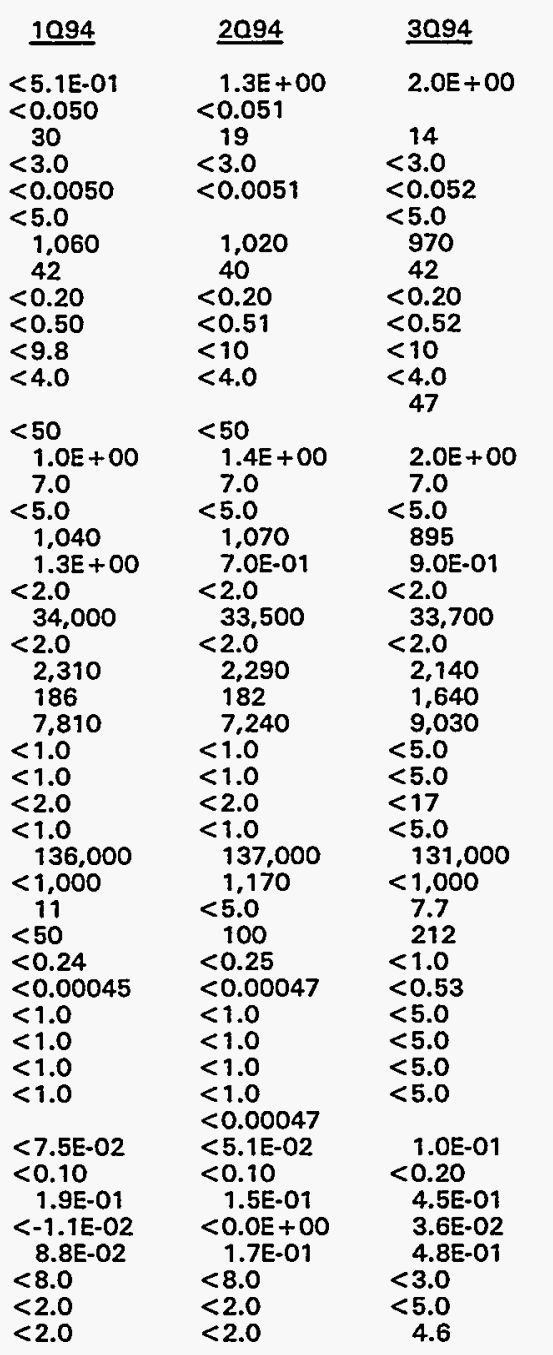

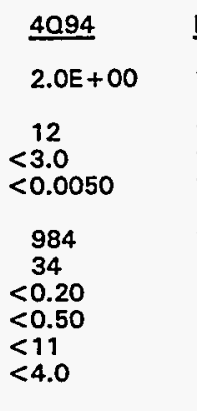

59
$2.2 E+00$
7.1

$<5.0$

1,140

5.0E-01

$<2.0$

34,200

$<2.0$

2,390

187

6,810

$<1.0$

$<1.0$
2.8

2.8
$<1.0$

140,000

1,670

$<5.0$

156

$<0.00045$

$<1.0$

$<1.0$

$<1.0$

$<1.0$

$-9.8 \mathrm{E}-02$

1.1

1.1E-01

8.9E-02

8.9E-02

$<8.0$

$<2.0$

$<2.0$

\begin{tabular}{|c|c|c|c|c|}
\hline DF & Mod & Unit & Lab & Fla \\
\hline 1 & $\mathbf{J}$ & $\begin{array}{l}\mathrm{pCi} / \mathrm{L} \\
\mu \mathrm{g} / \mathrm{L}\end{array}$ & GP & 0 \\
\hline 1 & & $\mu g / L$ & GE & 0 \\
\hline 1 & & $\mu g / L$ & GE & 0 \\
\hline 1 & & $\begin{array}{l}\mu \mathrm{g} / \mathrm{L} \\
\mu \mathrm{g} / \mathrm{L}\end{array}$ & GE & 0 \\
\hline 1 & & $\mu g / L$ & GE & 0 \\
\hline 1 & & $\mu g / L$ & GE & 1 \\
\hline 1 & & $\mu g / L$ & GE & 0 \\
\hline 1 & & $\mu g / L$ & GE & 0 \\
\hline 1 & & $\mu g / L$ & GE & 0 \\
\hline 1 & & $\begin{array}{l}\mu \mathrm{g} / \mathrm{L} \\
\mu \mathrm{g} / \mathrm{L}\end{array}$ & GE & 0 \\
\hline 1 & $J$ & $\mu g / L$ & GE & 0 \\
\hline 1 & $J$ & $\mathrm{pCi} / \mathrm{L}$ & GP & 0 \\
\hline $\begin{array}{l}1 \\
1\end{array}$ & $J$ & $\mathrm{pH}$ & GE & 0 \\
\hline 1 & & $\mu \mathrm{g} / \mathrm{L}$ & GE & 0 \\
\hline 1 & $J$ & $p \mathrm{Ci} / \mathrm{L}$ & GP & 0 \\
\hline 1 & & $\mu g / L$ & $\mathrm{GE}$ & 0 \\
\hline 1 & & $\mu g / L$ & GE & 0 \\
\hline 1 & & $\mu g / L$ & GE & 0 \\
\hline 1 & & $\begin{array}{l}\mu \mathrm{g} / \mathrm{L} \\
\mu \mathrm{S} / \mathrm{cm}\end{array}$ & GE & 0 \\
\hline 1 & & $\mu \mathrm{g} / \mathrm{L}$ & $\mathbf{G E}$ & 0 \\
\hline 1 & & $\mu g / L$ & GE & 0 \\
\hline 1 & & $\mu g / L$ & GE & 0 \\
\hline 1 & $J$ & $\mu g / L$ & GE & 0 \\
\hline 1 & & $\mu g / L$ & GE & 0 \\
\hline 1 & $\mathbf{J}$ & $\begin{array}{l}\mu \mathrm{g} / \mathrm{L} \\
\mu \mathrm{g} / \mathrm{L}\end{array}$ & $\begin{array}{l}\text { GE } \\
\text { GE }\end{array}$ & $\begin{array}{l}0 \\
0\end{array}$ \\
\hline$i$ & & $\mu g / L$ & $\mathbf{G E}$ & 0 \\
\hline 1 & $\mathbf{J}$ & $\mu g / L$ & GE & 0 \\
\hline 1 & & $\mu g / L$ & GE & 0 \\
\hline 1 & & $\mu g / L$ & GE & 0 \\
\hline 1 & & $\mu g / L$ & GE & 0 \\
\hline $\begin{array}{l}1 \\
1\end{array}$ & & $\begin{array}{l}\mu \mathrm{g} / \mathrm{L} \\
\mu \mathrm{g} / \mathrm{L}\end{array}$ & $\begin{array}{l}\mathrm{GE} \\
\mathrm{GE}\end{array}$ & 0 \\
\hline 1 & & $\begin{array}{l}\mu g / L \\
\mu g / L\end{array}$ & GE & 0 \\
\hline 1 & UI & $\mathrm{pCi} / \mathrm{mL}$ & GP & 0 \\
\hline 1 & & NTU & GE & 0 \\
\hline 1 & U1 & $\mathrm{pCi} / \mathrm{L}$ & GP & 0 \\
\hline 1 & U] & $\mathrm{pCi} / \mathrm{L}$ & GP & 0 \\
\hline 1 & U1 & $\mathrm{pCi} / \mathrm{L}$ & GP & 0 \\
\hline $\begin{array}{l}1 \\
1\end{array}$ & & $\mu g / L$ & GE & 0 \\
\hline 1 & & $\mu \mathrm{g} / \mathrm{L}$ & GE & 0 \\
\hline
\end{tabular}

Note: Flagging, dilution factors, modifiers, and laboratories are for fourth quarter 1994 data only. See Appendix B for flagging criteria. - = exceeded holding time for fourth quarter 1994.

- = exceeded screening level or final PDWS for fourth quarter 1994. 


\section{WELL BGO 18D}

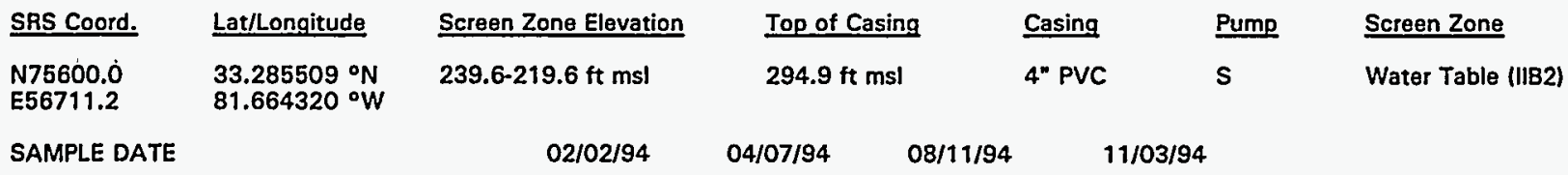

FIELD DATA

Analyte

Water elevation

Depth to water

$\mathrm{pH}$

$\mathrm{Sp}$. conductance

Water temperature

Alkalinity as $\mathrm{CaCO}_{3}$

Turbidity

Volume purged

Sampling code

ANALYTICAL DATA

\section{H ST Analyte}

Acetophenone

Aldrin

Aluminum, total recoverable

Antimony, total recoverable

Arsenic, total recoverable

Barium, total recoverable

Benzene

Bromodichloromethane

Bromoform

Bromomethane (Methyl bromide)

Cadmium, total recoverable

Calcium, total recoverable

Carbon tetrachloride

Carbon-14

Chloride

Chlorobenzene

Chloroethane

Chloroethene (Vinyl chloride)

2-Chloroethyl vinyl ether

Chloroform

Chloromethane (Methyl chloride)

Chromium, total recoverable

Copper, total recoverable

Cyanide

p,p'-DDT

Dibromochloromethane

1,1-Dichloroethane

1,2-Dichloroethane

1,1-Dichloroethylene

trans-1,2-Dichloroethylene

Dichloromethane

2,4-Dichlorophenoxyacetic acid

1,2-Dichloropropane

cis-1,3-Dichloropropene

trans-1,3-Dichloropropene

Dieldrin

Endrin

Ethylbenzene

Fluoride

10.94
232.5
62.5
4.8
26
18.8
0
0
13.6

13.6

2094
232.2
62.7
4.6
29
19.3
0
0
15.9

3094
232.0
63.0
4.8
30
19.9
0
0
20.9

4094
231.6
63.3
5.0
26
19.2
0
0
25.6

Unit

ft msl

pH

$\mu \mathrm{S} / \mathrm{cm}$

${ }^{\circ} \mathrm{C}$

$\mathrm{mg} / \mathrm{L}$

NTUll vol.

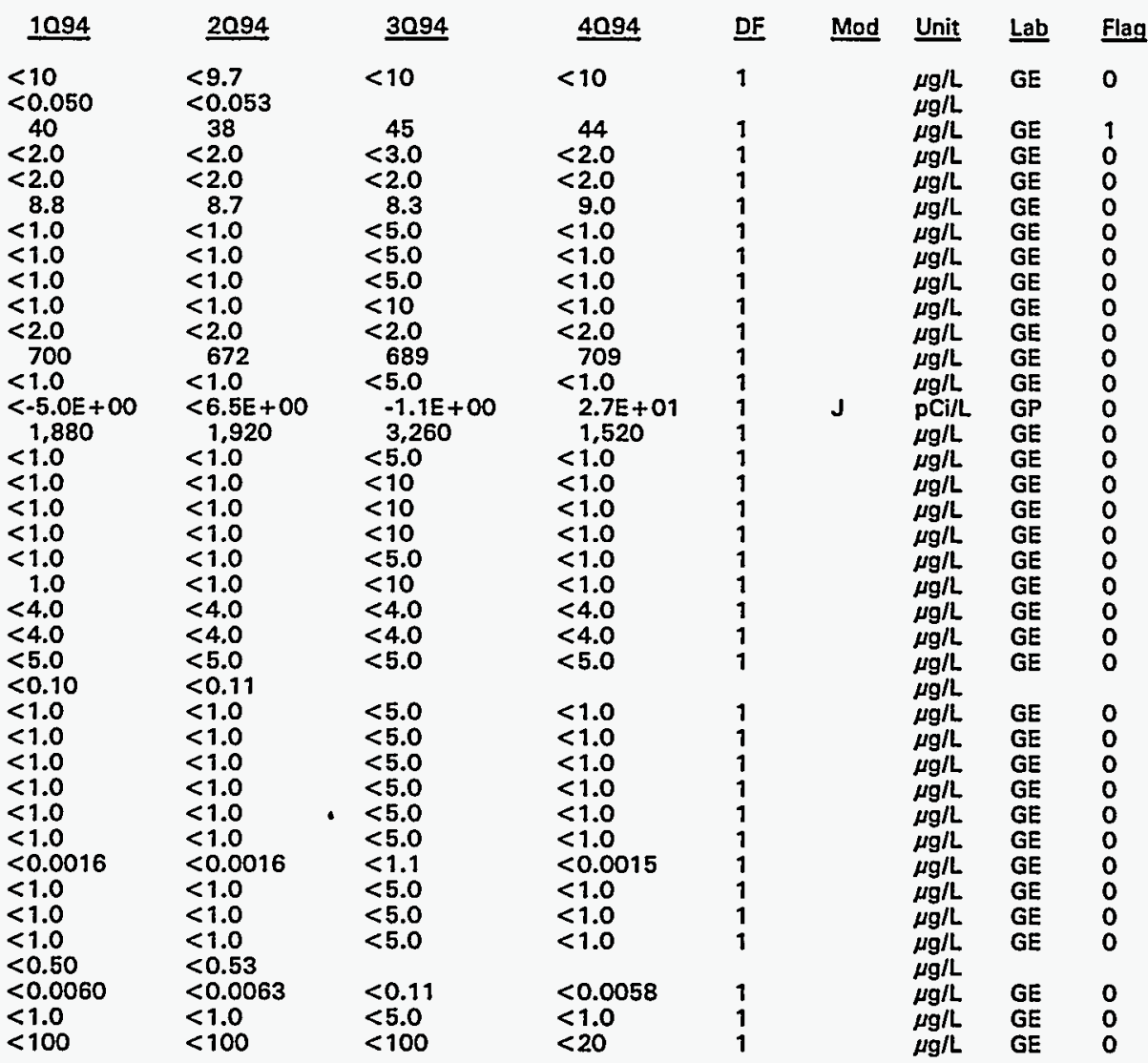

Note: Flagging, dilution factors, modifiers, and laboratories are for fourth quarter 1994 data only. See Appendix B for flagging criteria.

- = exceeded holding time for fourth quarter 1994.

- = exceeded screening level or final PDWS for fourth quarter 1994. 
HE ST Analyte

Gross alpha

Heptachlor

Iron, total recoverable

Lead, total recoverable

Lindane

Lithium, total recoverable

Magnesium, total recoverable

Manganese, total recoverable

Mercury, total recoverable

Methoxychlor

Naphthalene

Nickel, total recoverable

Nitrate as nitrogen

Nitrate-nitrite as nitrogen

Nonvolatile beta

pH

Phenols

Potassium, total recoverable

Radium, total alpha-emitting

Selenium, total recoverable

Silica, total recoverable

Silver, total recoverable

Sodium, total recoverable

Specific conductance

Sulfate

1,1,2,2-Tetrachloroethane

Tetrachloroethylene

Tin, total recoverable

Toluene

Total dissolved solids

Total organic carbon

Total organic halogens

Total phosphates (as P)

Toxaphene

2,4,5-TP (Silvex)

1,1,1-Trichloroethane

1,1,2-Trichloroethane

Trichloroethylene

Trichlorofluoromethane

2,4,5-T

Tritium

Turbidity

Uranium-233/234

Uranium-235

Uranium-238

Vanadium, total recoverable

Xylenes

Zinc, total recoverable

$\begin{aligned} & 1094 \\ & 1.2 E+00 \\ &< 0.050 \\ &< 4.0 \\ &< 3.0 \\ &< 0.0050 \\ &< 5.0 \\ & 310 \\ & 12 \\ &< 0.20 \\ &< 0.50 \\ &<10 \\ &<4.0\end{aligned}$

$\frac{2094}{2.2 E+00}$
$<0.053$

3094

$3.5 E+00$

$<4.0$

$<3.0$

$<0.0053$

300

12

$<0.20$

$<0.53$

$<9.7$

$<4.0$

1,180

$1.4 \mathrm{E}+00$

5.1

$<5.0$

$<500$

$<2.0$

8,190

$<2.0$

1,990

28

$<1,000$

$<1.0$

$<1.0$

$<2.0$

$<1.0$

24,000

$<1,000$

$<5.0$

$<50$

$<0.24$

$<0.00047$

$<1.0$
$<1.0$

$<1.0$

1.1

1.2E +01

$<0.10$

$<7.7$ E-O2

1.5E-01

1.5E-01

$<8.0$

$<2.0$

2.2
1,340

1.2E + 00

5.3

$<5.0$

$<500$

1. $2 E+00$

$<2.0$

8.160

$<2.0$

1,920

24

$<1,000$

$<1.0$

$<1.0$

$<2.0$

$<1.0$

26,000

$<1,000$

6.8

$<50$

$<0.25$
$<0.00047$

$<1.0$

$<1.0$

$<1.0$

$<1.0$

$<0.00047$

1.3E + 01

$<0.10$

1.4E-01

$<0.0 E+00$

$<1.5 E-02$

$<8.0$

$<2.0$
$<2.0$
$<4.0$

$<3.0$

$<0.055$

$<5.0$

296

11
$<0.20$

$<0.55$

$<10$

$<4.0$

1,300

$2.4 E+00$

4.9

$<5.0$

$<500$

1. $6 \mathrm{E}+00$

$<2.0$

7,930

$<2.0$

2,020

23

$<1,000$

$<5.0$

$<5.0$

$<17$

$<5.0$

29,000

$<1,000$

6.4

60

$<1.1$
$<0.53$

$<5.0$

$<5.0$

$<5.0$

$<5.0$

1. $6 \mathrm{E}+01$

$<0.20$

1.5E-01

3.7E-02

9.7E-02

$<3.0$

$<5.0$

\begin{tabular}{|c|c|c|c|c|c|}
\hline 4094 & DF & Mod & Unit & Lab & Flag \\
\hline $2.8 E+00$ & 1 & $J$ & $\begin{array}{l}\mathrm{pCi} / \mathrm{L} \\
\mu \mathrm{g} / \mathrm{L}\end{array}$ & GP & 0 \\
\hline $\begin{array}{l}<4.0 \\
<3.0\end{array}$ & $\begin{array}{l}1 \\
1\end{array}$ & & $\begin{array}{l}\mu \mathrm{g} / \mathrm{L} \\
\mu \mathrm{g} / \mathrm{L}\end{array}$ & $\begin{array}{l}\mathrm{GE} \\
\mathrm{GE}\end{array}$ & $\begin{array}{l}0 \\
0\end{array}$ \\
\hline
\end{tabular}

$<0.0048$

329

12

$<0.20$

$<0.48$

$<10$

$<4.0$

1,430

1. $6 \mathrm{E}+00$

5.3

$<5.0$

$<500$

1.1E+00

$<2.0$

8,160

$<2.0$

2,040

25

$<1,000$

$<1.0$

$<1.0$

$<1.0$

19,000

$<1,000$

$<5.0$

$<50$

$<0.23$

$<0.00044$

$<1.0$

$<1.0$

$<1.0$

$<1.0$

1. $6 E+01$

1.1

3.7E-02

3.7E-O2

1.1E-01

$<8.0$

$<2.0$

2.8

$\begin{array}{lll}\mu g / L & \text { GE } & 0 \\ \mu g / L & \text { GE } & \end{array}$

$\mu \mathrm{g} / \mathrm{L} \quad \mathrm{GE} \quad 0$

$\mu \mathrm{g} / \mathrm{GE}$

$\begin{array}{lll}\mu g / L & \text { GE } & 0 \\ \mu \mathrm{g} / \mathrm{L} & \mathrm{GE} & 0\end{array}$

$\mu \mathrm{g} / \mathrm{L} \quad \mathrm{GE}$

$\mu g / L \quad$ GE

$\mu \mathrm{g} / \mathrm{L} \quad \mathrm{GE}$

$\mu g / L$

$\mu g / L$ GE 0

$\mathrm{pH}$

$\mu \mathrm{g} / \mathrm{L}$



pCi/L GP

$\mu g / L \quad$ GE

$\mu \mathrm{g} / \mathrm{L}$

$\mu \mathrm{g} / \mathrm{L}$

$\mu \mathrm{g} / \mathrm{L}$

$\mu S / \mathrm{cm}$ GE

$\mu \mathrm{g} / \mathrm{L} \quad \mathrm{GE}$

$\mu g / L$ GE

$\mu \mathrm{g} / \mathrm{L}$

$\mu \mathrm{g} / \mathrm{L} \quad \mathrm{GE}$

$\mu \mathrm{g} / \mathrm{L} \quad \mathrm{GE}$

$\mu \mathrm{g} / \mathrm{L} \quad \mathrm{GE}$

$\mu \mathrm{g} / \mathrm{L} \quad \mathrm{GE}$

$\mu \mathrm{g} / \mathrm{L}$

$J$

$\mu \mathrm{g} / \mathrm{L}$

$\mu \mathrm{g} / \mathrm{L}$

$\mu g / L$

$\mu \mathrm{g} / \mathrm{L}$

$\mu \mathrm{g} / \mathrm{L}$

$\mu g / L$

$\mu \mathrm{g} / \mathrm{L}$

NTU

UI
UI

PCi/L

$\mathrm{pCi} / \mathrm{L}$

pCi/L

$\mu g / L$

$\mu g / L$

Note: Flagging, dilution factors, modifiers, and laboratories are for fourth quarter 1994 data only. See Appendix B for flagging criteria. - = exceeded holding time for fourth quarter 1994.

- = exceeded screening level or final PDWS for fourth quarter 1994. 
WELL BGO 19D

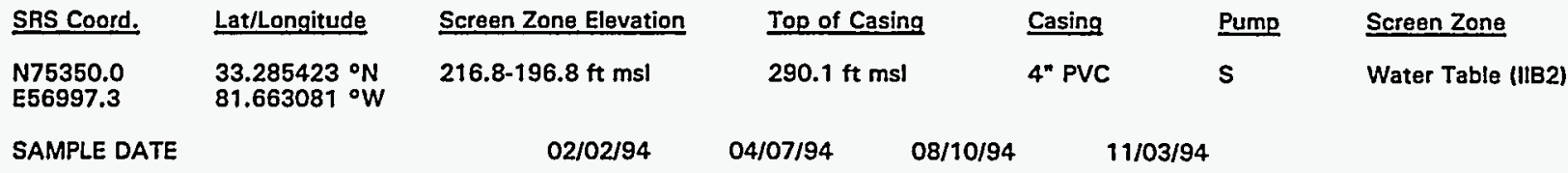

FIELD DATA

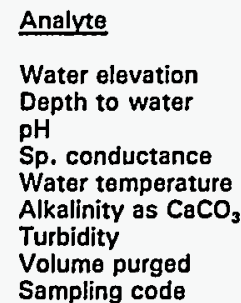

ANALYTICAL DATA

10.94
233.0
57.1
5.7
34
18.0
3
3
6.6

2094
232.8
57.3
6.1
40
19.3
5
2
6.8

3094
232.6
57.5
5.8
35
20.5
4
10
4.5

4094
232.3
57.9
5.7
29
19.6
3
8
4.2

Unit

ft msl

ft

pH

$\mu \mathrm{S} / \mathrm{cm}$

NTU

well vol.

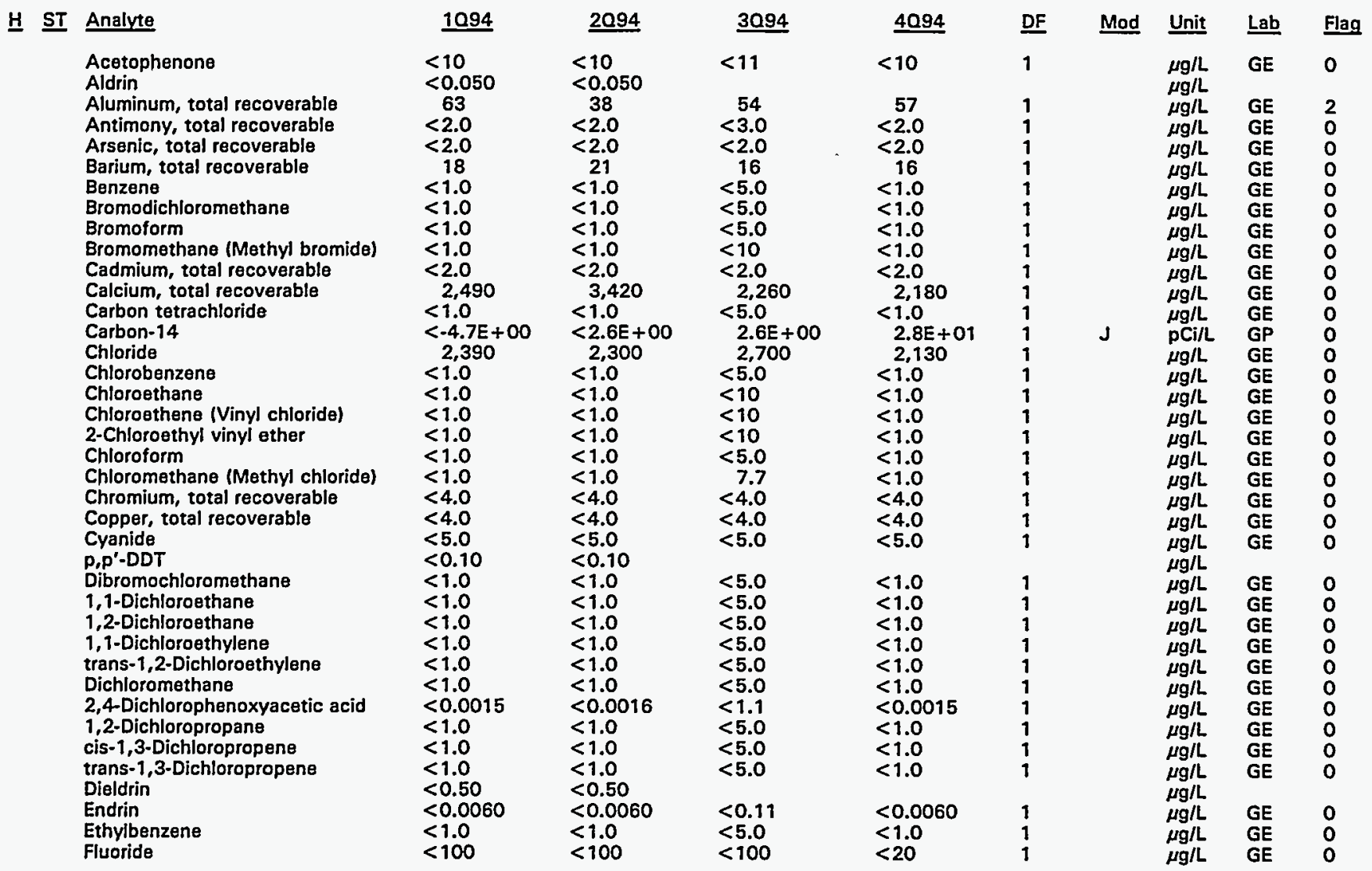

Note: Flagging, dilution factors, modifiers, and laboratories are for fourth quarter 1994 data only. See Appendix B for flagging criteria. - = exceeded holding time for fourth quarter 1994.

- = exceeded screening level or final PDWS for fourth quarter 1994. 
WSRC-TR-94-0610

Unclassified

Well BGO 19D continued

ANALYTICAL DATA

H ST Analyte

Gross alpha

Heptachlor

Iron, total recoverable

Lead, total recoverable

Lindane

Lithium, total recoverable

Magnesium, total recoverable

Manganese, total recoverable

Mercury, total recoverable

Methoxychlor

Naphthalene

Nickel, total recoverable

Nitrate as nitrogen

Nitrate-nitrite as nitrogen

Nonvolatile beta

- $\quad$ pH

Phenols

Potassium, total recoverable

Radium, total alpha-emitting

Selenium, total recoverable

Silica, total recoverable

Silver, total recoverable

Sodium, total recoverable

Specific conductance

Sulfate

1,1,2,2-Tetrachloroethane

Tetrachloroethylene

Tin, total recoverable

Toluene

Total dissolved solids

Total organic carbon

Total organic halogens

Total phosphates (as P)

Toxaphene

2,4,5-TP (Silvex)

1,1,1-Trichloroethane

1,1,2-Trichloroethane

Trichloroethylene

Trichlorofluoromethane

2,4,5-T

- Tritium

Turbidity

Uranium-233/234

Uranium-235

Uranium-238

Vanadium, total recoverable

Xylenes

Zinc, total recoverable

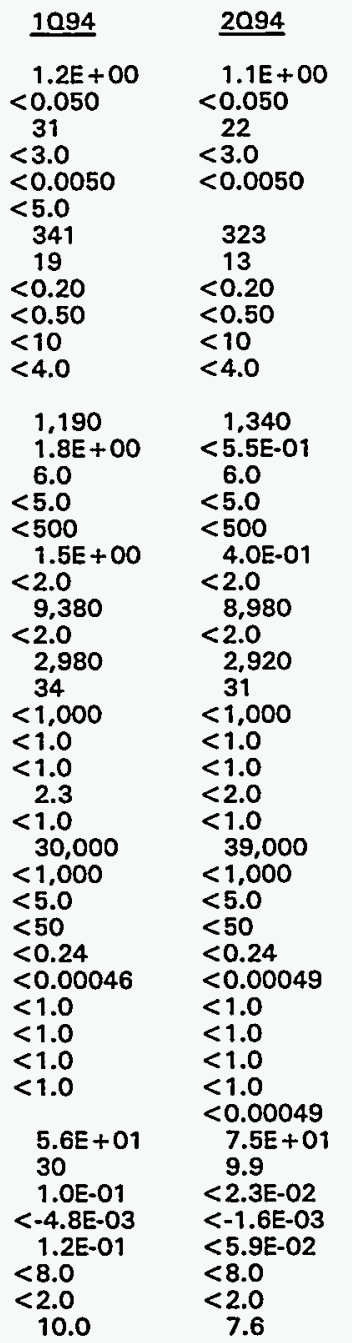

$\underline{3094}$
$2.2 E+00$
87
3.3
$<0.053$
$<5.0$
339
12
$<0.20$
$<0.53$
$<10$
$<4.0$
1,100

1.7E+ +00

5.7

$<5.0$

1.2E+00

$<2.0$

8,770

$<2.0$

2,610

30

$<1,000$

$<5.0$

$<17$

$<5.0$

9,000

$<1,000$

8.4

366

$<1.1$

$<0.53$

$<5.0$

$<5.0$

$<5.0$

$<5.0$

6.7E+01

2.1

4.1E-02

$0.0 E+O 0$

9.2E-02

$<3.0$

$<5.0$
19

\begin{tabular}{|c|c|c|c|c|}
\hline 4094 & DF & Mod & Unit & $\underline{L a b}$ \\
\hline $1.5 E+\infty 0$ & 1 & J & $\mathrm{pCi} / \mathrm{L}$ & GP \\
\hline $\begin{array}{l}36 \\
3.0\end{array}$ & $\begin{array}{l}1 \\
1\end{array}$ & & $\begin{array}{l}\mu g / L \\
\mu g / L\end{array}$ & $\begin{array}{l}\mathrm{GE} \\
\mathrm{GE}\end{array}$ \\
\hline
\end{tabular}

$<0.0050$

357

32
$<0.20$

$<0.50$

$<10$

$<4.0$

1,190

4.8E-01

5.8

$<5.0$

$<500$

$1.2 E+00$

$<2.0$

9,070

$<2.0$

2,420

$<1,000$

$<1.000$

$<1.0$

$<20$

$<2.0$

30,000

$<1,000$

$<5.0$

$<50$

$<0.24$

0.0094

$<1.0$

$<1.0$

$<1.0$

$<1.0$

8.6E + 01

21

5.8E-02

$0.0 E+00$

6.4E-02

$<8.0$

$<2.0$

$\mu g / L \quad G E$

0

$\mu g /$ GE 0

$\mu g / L \quad G E$

$\mu g / L \quad G E$

$\mu \mathrm{g} / \mathrm{L} \quad \mathrm{GE}$

$\mu g / L \quad G E$

$\mu g / L \quad G E$

$\mu \mathrm{g} / \mathrm{L}$

$\mu g / L \quad G E$

UI $\mathrm{pCi} / \mathrm{L}$ GP

$\begin{array}{ll}\mathrm{pH} & \mathrm{GE} \\ \mu \mathrm{g} / \mathrm{L} & \mathrm{GE}\end{array}$

$\mu g / L \quad G E$

J $\mathrm{pCi} / \mathrm{L}$ GP

$\mu g / L \quad G E$

$\mu \mathrm{gg/L} \quad \mathrm{GE}$

$\mu \mathrm{g} / \mathrm{L} \quad \mathrm{GE}$

$\mu \mathrm{g} / \mathrm{L} \quad \mathrm{GE}$

$\mu S / \mathrm{cm}$ GE

$\mu g / L$

$\mu \mathrm{g} / \mathrm{L}$

$\mu g / L$

$\mu \mathrm{g} / \mathrm{L}$

$\mu \mathrm{g} / \mathrm{L}$

$\mu \mathrm{g} / \mathrm{L}$

Hg/L GE

$\mu g / 2$

$\mu g / L \quad$ GE

$\mu g / L \quad$ GE

$\mu \mathrm{g} / \mathrm{L} \quad \mathrm{GE}$

$\mu g / L \quad$ GE

$\mu g / L \quad G E$

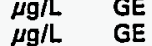

$\mu g / L$

$\mathrm{pCi} / \mathrm{mL} \quad \mathrm{GP}$

NTU GE

UI
UI
UI

pCi/L

PCi/L

$\mathrm{pCi} / \mathrm{L}$ GP

Hg/L GE

$\mu g / L \quad G E$

$\mu g / L \quad$ GE

Note: Flagging, dilution factors, modifiers, and laboratories are for fourth quarter 1994 data only. See Appendix B for flagging criteria. - = exceeded holding time for fourth quarter 1994 .

- exceeded screening level or final PDWS for fourth quarter 1994. 
WELL BGO 2OD

\begin{tabular}{|c|c|c|c|c|c|c|}
\hline SRS Coord. & Lat/Longitude & Screen Zone Elevation & Top of Casing & Casing & Pump & Screen Zone \\
\hline $\begin{array}{l}\text { N74962.2 } \\
\text { E57113.8 }\end{array}$ & $\begin{array}{l}33.284755^{\circ} \mathrm{N} \\
81.662021^{\circ} \mathrm{W}\end{array}$ & $236.3-216.3 \mathrm{ft} \mathrm{msl}$ & $283.7 \mathrm{ft} \mathrm{msl}$ & 4" PVC & $\mathbf{S}$ & Water Table (IIB2) \\
\hline SAMPLE DATE & & $02 / 03 / 94$ & $04 / 08 / 94$ & & & \\
\hline
\end{tabular}

FIELD DATA

Analyte
Water elevation
Depth to water
pH
$\mathrm{Sp}$. conductance
Water temperature
Alkalinity as $\mathrm{CaCO}_{3}$
Turbidity
Volume purged
Sampling code

ANALYTICAL DATA

H ST Analyte

Acetophenone

Aldrin

Aluminum, total recoverable

Antimony, total recoverable

Arsenic, total recoverable

Barium, total recoverable

Benzene

Bromodichloromethane

Bromoform

Bromomethane (Methyl bromide)

Cadmium, total recoverable

Calcium, total recoverable

Carbon tetrachloride

Carbon-14

Chloride

Chlorobenzene

Chloroethane

Chloroethene (Vinyl chloride)

2-Chloroethyl vinyl ether

Chloroform

Chloromethane (Methyl chloride)

Chromium, total recoverable

Copper, total recoverable

Cyanide

$p, p^{\prime}$-DDT

Dibromochloromethane

1,1-Dichloroethane

1,2-Dichloroethane

1,1-Dichloroethylene

trans-1,2-Dichloroethylene

Dichloromethane

2,4-Dichlorophenoxyacetic acid

1,2-Dichloropropane

cis-1,3-Dichloropropen

trans-1,3-Dichloropropene

Dieldrin

Endrin

Ethylbenzene

Fluoride

\begin{tabular}{ll}
10.94 & $\underline{2094}$ \\
\hline 234.3 & 234.2 \\
49.4 & 49.5 \\
5.5 & 5.5 \\
68 & 82 \\
16.0 & 17.2 \\
16 & 3 \\
8 & 5 \\
0.7 & 0.6 \\
$x$ & $x$
\end{tabular}

3094
234.0
49.8
5.5
68
19.5
14
8
0.7
$\times$

$<9.9$

$<0.050$

49
$<2.0$

$<2.0$

46

$<1.0$

$<1.0$

$<1.0$

$<1.0$

$<2.0$

5,330

$<1.0$

$<-6.9 E-01$

1,980

$<1.0$

$<1.0$

$<1.0$

$<1.0$

$<1.0$

$<1.0$

$<4.0$

$<4.0$

$<5.0$

$<0.10$

$<1.0$
$<1.0$

$<1.0$

$<1.0$

$<1.0$

$<1.0$

$<0.0016$

$<1.0$

$<1.0$

$<1.0$

$<0.50$

$<0.0060$

$<1.0$

$<100$

\section{$<10$
$<0.049$}

24
$<20$

$<2.0$

44

$<1.0$

$<1.0$

$<1.0$

$<1.0$

$<2.0$

5,060

$<1.0$

$<-3.7 E-01$

1,960

$<1.0$

$<1.0$

$<1.0$

$<1.0$

$<1.0$

$<4.0$

$<4.0$

$<5.0$

$<0.097$

$<1.0$

$<1.0$

$<1.0$

$<1.0$
$<1.0$

$<1.0$

$<0.0015$

$<1.0$

$<1.0$

$<1.0$

$<0.49$

$<0.0058$

$<1.0$
$<100$

$\quad 3094$
$<11$

42
$<3.0$
$<2.0$
44
$<5.0$
$<5.0$
$<5.0$
$<10$
$<2.0$
5,250
$<5.0$
$-2.8 \mathrm{E}+00$
2.100
$<5.0$

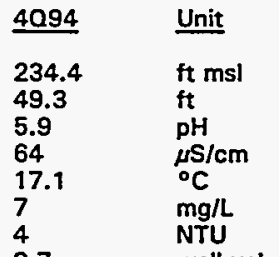

0.7
$\times$

well vol.

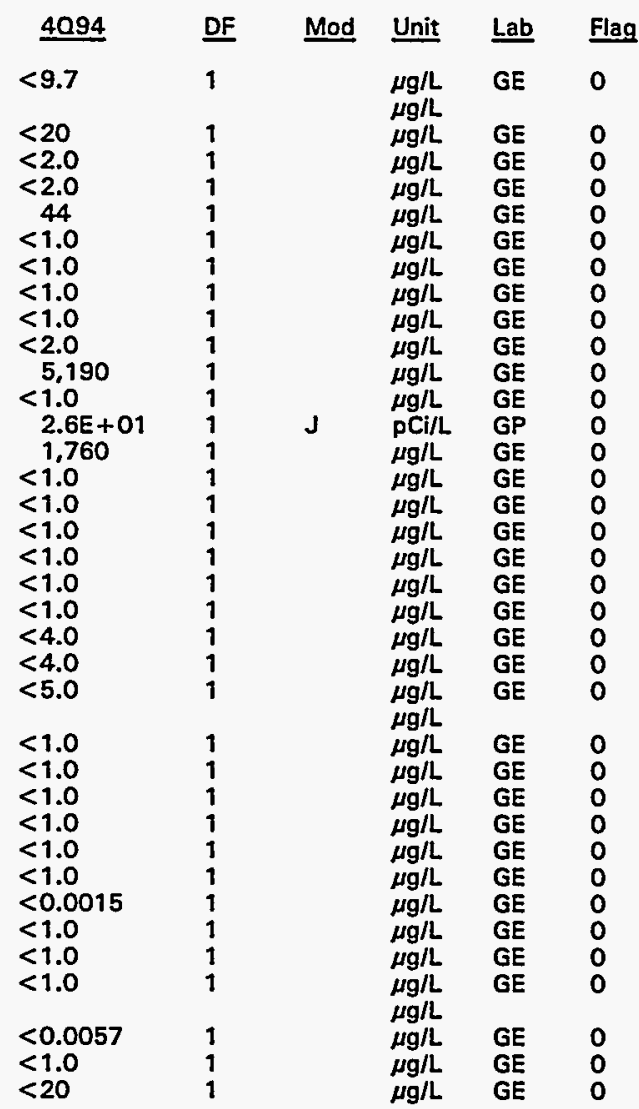

Note: Flagging, dilution factors, modifiers, and laboratories are for fourth quarter 1994 data only. See Appendix B for flagging criteria. - = exceeded holding time for fourth quarter 1994.

- = exceeded screening level or final PDWS for fourth quarter 1994. 


\section{ANALYTICAL DATA}

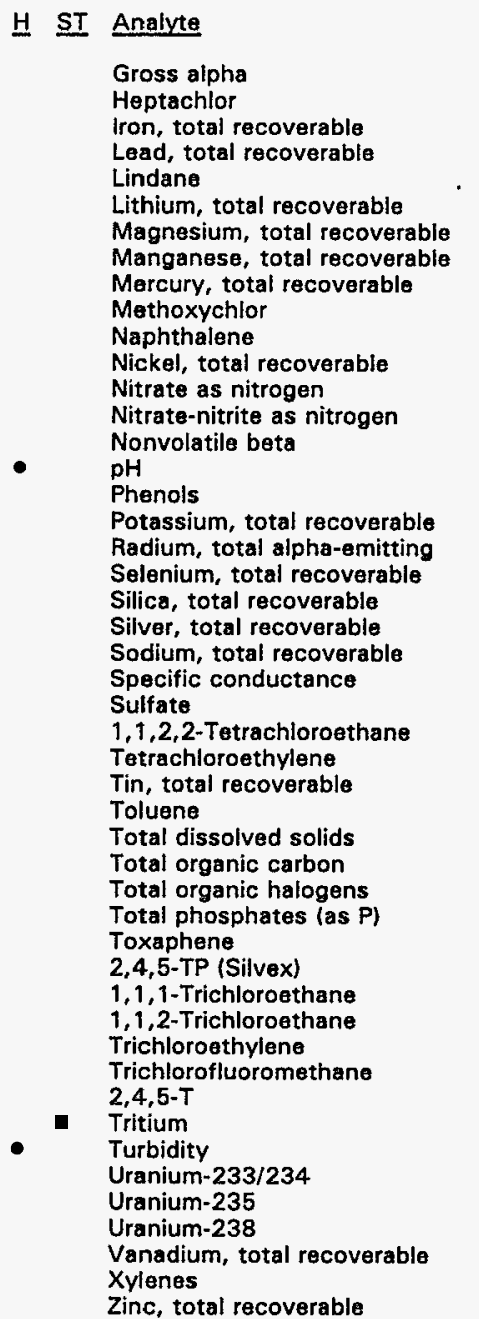

\begin{tabular}{|c|c|}
\hline 1094 & 2094 \\
\hline $\begin{array}{rl} & 1.2 \mathrm{E}+00 \\
< & 0.050 \\
186 & 11 \\
& 11 \\
< & 0.0050 \\
< & 5.0 \\
1.250 & 41 \\
< & 0.20 \\
< & 0.50 \\
< & 9.9 \\
& 5.3\end{array}$ & $\begin{aligned} & 1.5 E+00 \\
&<0.049 \\
& 90 \\
& 6.8 \\
&<0.0049 \\
& \\
& 1.220 \\
& 37 \\
&<0.20 \\
&<0.49 \\
&<10 \\
&<4.0\end{aligned}$ \\
\hline 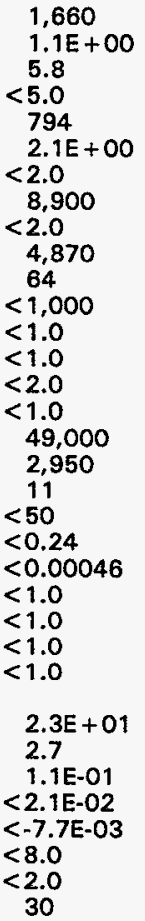 & $\begin{aligned} & 1,980 \\
& 2.1 \mathrm{E}+0 \mathrm{O} \\
& 5.8 \\
&<5.0 \\
& 721 \\
& 7.0 \mathrm{E}-01 \\
&<2.0 \\
& 8.650 \\
&<2.0 \\
& 4,490 \\
& 59 \\
&<1.000 \\
&<1.0 \\
&<1.0 \\
&<2.0 \\
&<1.0 \\
& 38,000 \\
& 1,620 \\
& 6.8 \\
&<50 \\
&<0.23 \\
&<0.00046 \\
&<1.0 \\
&<1.0 \\
&<1.0 \\
&<1.0 \\
& 0.39 \\
& 2.2 \mathrm{E}+01 \\
& 1.3 \\
&<8.5 \mathrm{E}-02 \\
&<3.1 \mathrm{E}-02 \\
&<-1.8 \mathrm{E}-02 \\
&<8.0 \\
&<2.0 \\
& 26 \\
&<\end{aligned}$ \\
\hline
\end{tabular}

\begin{tabular}{|c|c|c|c|c|c|}
\hline 4094 & DF & Mod & $\underline{\text { Unit }}$ & Lab & Fla \\
\hline $1.2 E+00$ & 1 & $\mathrm{~J}$ & $\begin{array}{l}\mathrm{pCi} / \mathrm{L} \\
\mu \mathrm{g} / \mathrm{L}\end{array}$ & GP & 0 \\
\hline 90 & 1 & & $\mu \mathrm{g} / \mathrm{L}$ & GE & 0 \\
\hline 4.6 & 1 & $\mathrm{~J}$ & $\mu g / L$ & GE & 0 \\
\hline$<0.0048$ & 1 & & $\begin{array}{l}\mu \mathrm{g} / \mathrm{L} \\
\mu \mathrm{g} / \mathrm{L}\end{array}$ & GE & 0 \\
\hline 1,300 & 1 & & $\mu g / L$ & $\mathrm{GE}$ & 0 \\
\hline 32 & 1 & & $\mu \mathrm{g} / \mathrm{L}$ & GE & 1 \\
\hline$<0.20$ & 1 & & $\mu \mathrm{g} / \mathrm{L}$ & GE & 0 \\
\hline$<0.48$ & 1 & & $\mu g / L$ & GE & 0 \\
\hline$<9.7$ & 1 & & $\mu \mathrm{g} / \mathrm{L}$ & GE & 0 \\
\hline 4.2 & 1 & $J$ & $\begin{array}{l}\mu \mathrm{g} / \mathrm{L} \\
\mu \mathrm{g} / \mathrm{L}\end{array}$ & GE & 0 \\
\hline 1,730 & 1 & & $\mu \mathrm{g} / \mathrm{L}$ & GE & 0 \\
\hline $1.8 \mathrm{E}+00$ & 1 & $J$ & $\mathrm{pCi} / \mathrm{L}$ & GP & 0 \\
\hline 5.8 & 1 & $J$ & pH & GE & 0 \\
\hline$<5.0$ & 1 & & $\mu g / L$ & GE & 0 \\
\hline$<500$ & 1 & & $\mu g / \mathrm{L}$ & GE & 0 \\
\hline $\begin{array}{l}8.0 \mathrm{E}-01 \\
<2.0\end{array}$ & $\begin{array}{l}1 \\
1\end{array}$ & $J$ & $\mathrm{pCi} / \mathrm{L}$ & GP & 0 \\
\hline 8,540 & 1 & & $\mu \mathrm{g} / \mathrm{L}$ & $\mathrm{GE}$ & 0 \\
\hline$<2.0$ & 1 & & $\mu g / L$ & GE & 0 \\
\hline 4,060 & 1 & & $\mu \mathrm{g} / \mathrm{L}$ & GE & 0 \\
\hline 66 & 1 & & $\mu \mathrm{S} / \mathrm{cm}$ & GE & 0 \\
\hline$<1,000$ & 1 & & $\mu g / L$ & GE & 0 \\
\hline$<1.0$ & 1 & & $\mu \mathrm{g} / \mathrm{L}$ & GE & 0 \\
\hline$<1.0$ & $?$ & & $\mu \mathrm{g} / \mathrm{L}$ & GE & 0 \\
\hline 2.7 & 1 & $J$ & $\mu g / L$ & GE & 0 \\
\hline $\begin{array}{l}<1.0 \\
40,000\end{array}$ & $\begin{array}{l}1 \\
1\end{array}$ & & $\begin{array}{l}\mu \mathrm{g} / \mathrm{L} \\
\mu \mathrm{g} / \mathrm{L}\end{array}$ & $\begin{array}{l}\text { GE } \\
\text { GE }\end{array}$ & $\begin{array}{l}0 \\
0\end{array}$ \\
\hline 2,500 & 1 & & $\mu g / L$ & GE & 0 \\
\hline$<5.0$ & 1 & & $\mu g / L$ & GE & 0 \\
\hline $\begin{array}{l}<50 \\
<0.23\end{array}$ & 1 & $J$ & $\mu \mathrm{g} / \mathrm{L}$ & GE & 0 \\
\hline $\begin{array}{l}<0.23 \\
<0.00045\end{array}$ & $\begin{array}{l}1 \\
1\end{array}$ & & $\mu g / L$ & GE & $\begin{array}{l}0 \\
0\end{array}$ \\
\hline$<1.0$ & 1 & & $\mu g / L$ & $\mathrm{GE}$ & 0 \\
\hline$<1.0$ & 1 & & $\mu \mathrm{g} / \mathrm{L}$ & GE & 0 \\
\hline$<1,0$ & 1 & & $\mu \mathrm{g} / \mathrm{L}$ & GE & 0 \\
\hline$<1.0$ & 1 & & $\begin{array}{l}\mu g / L \\
\mu g / L\end{array}$ & GE & 0 \\
\hline $2.6 \mathrm{E}+01$ & 1 & & $\mathrm{pCi} / \mathrm{mL}$ & GP & 2 \\
\hline 2.8 & 1 & J & NTU & GE & $\overline{0}$ \\
\hline $3.1 \mathrm{E}-\mathrm{O} 2$ & 1 & UI & $\mathrm{pCi} / \mathrm{L}$ & GP & 0 \\
\hline $2.5 E-02$ & 1 & UI & $\mathrm{pCi} / \mathrm{L}$ & $\mathrm{GP}$ & 0 \\
\hline$-3.8 \mathrm{E}-03$ & 1 & UI & $\mathrm{pCi} / \mathrm{L}$ & GP & 0 \\
\hline$<8.0$ & 1 & & $\mu g / L$ & GE & 0 \\
\hline$<2.0$ & 1 & & $\mu_{g} / L$ & GE & 0 \\
\hline 28 & 1 & & $\mu \mathrm{g} / \mathrm{L}$ & GE & \\
\hline
\end{tabular}

Note: Flagging, dilution factors, modifiers, and laboratories are for fourth quarter 1994 data only. See Appendix B for flagging criteria. - = exceeded holding time for fourth quarter 1994.

- =xceeded screening level or final PDWS for fourth quarter 1994. 
WELL BGO 21D

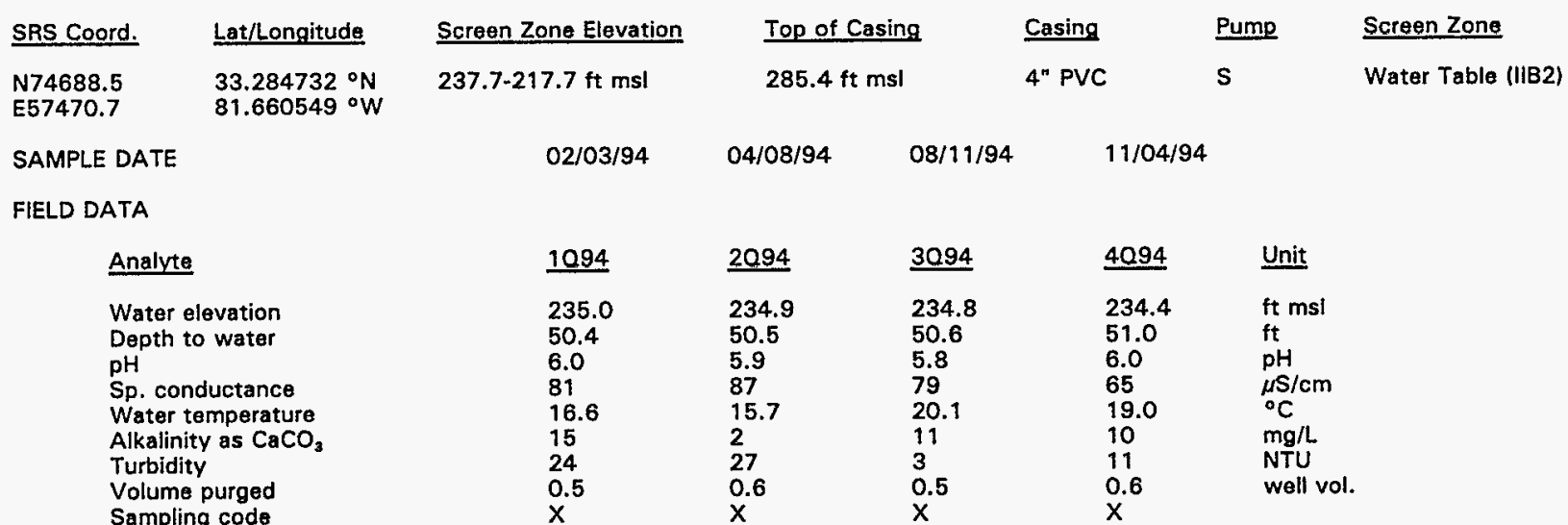

ANALYTICAL DATA

H ST Analyte

Acetophenone

Aldrin

Aluminum, total recoverable Antimony, total recoverable

Arsenic, total recoverable

Barium, total recoverable

Benzene

Bromodichloromethane

Bromoform

Bromomethane (Mathyl bromide)

Cadmium, total recoverable

Calcium, total recoverable

Carbon tetrachloride

Carbon-14

Chloride

Chlorobenzene

Chloroethane

Chloroethene (Vinyl chloride)

2-Chloroethyl vinyl ether

Chloroform

Chloromethane (Methyl chloride)

Chromium, total recoverable

Copper, total recoverable

Cyanide

$p, p^{\prime}$-DDT

1,1-Dichloroethane

1,2-Dichloroethane

1,1-Dichloroethylene

trans-1,2-Dichloroethylene

Dichloromethane

2,4-Dichlorophenoxyacetic acid

1,2-Dichloropropane

cis-1,3-Dichloropropene

trans-1,3-Dichloropropene

Dieldrin

Endrin

Ethylbenzene

Fluoride

\section{4}

$<10.0$

$<0.052$

309

$<2.0$

$<2.0$

50

$<1.0$

$<1.0$

$<1.0$

$<1.0$

$<2.0$

2.140

$<1.0$

$<-2.7 E+00$

4,960

$<1.0$

$<1.0$

$<1.0$

$<1.0$

$<1.0$

$<1.0$

$<4.0$
$<4.0$

$<5.0$

$<0.10$

$<1.0$

$<1.0$

$<1.0$

$<1.0$

$<1.0$

$<1.0$

$<0.0016$

$<1.0$

$<1.0$

$<1.0$

$<0.52$

$<0.0062$

$<1.0$

$<100$
2094

$<11$
$<0.050$

$<0.050$
29

$<2.0$

$<2.0$

45
$<1.0$

$<1.0$

$<1.0$

$<1.0$

$<2.0$

2,020

$<1.0$

$<1.4 \mathrm{E}+00$

5,810

$<1.0$

$<1.0$

$<1.0$

$<1.0$

$<1.0$

1.0

$<1.0$

$<4.0$

$<0.10$

$<0.10$

$<1.0$

$<1.0$

$<1.0$

$<1.0$

$<1.0$

$<0.0016$

$<1.0$

$<1.0$

$<1.0$

$<0.50$

$<0.0060$

$<1.0$

$<100$

$\begin{aligned} & 3094 \\ &<10 \\ & 218 \\ &<3.0 \\ &<2.0 \\ & 40 \\ &<5.0 \\ &<5.0 \\ &<5.0 \\ &<10 \\ &<2.0 \\ & 1.910 \\ &<5.0 \\ &-4.1 E+00 \\ & 5.810 \\ &<5.0 \\ &<10 \\ &<10 \\ &<10 \\ &<5.0 \\ & 3.1 \\ &<4.0 \\ & 4.0 \\ & 8.9 \\ &<5.0 \\ &<5.0 \\ &<5.0 \\ &<5.0 \\ &<5.0 \\ &<5.0 \\ &<1.1 \\ &<5.0 \\ &<5.0 \\ &<5.0 \\ &<0.11 \\ &<5.0 \\ &<100 \\ &\end{aligned}$

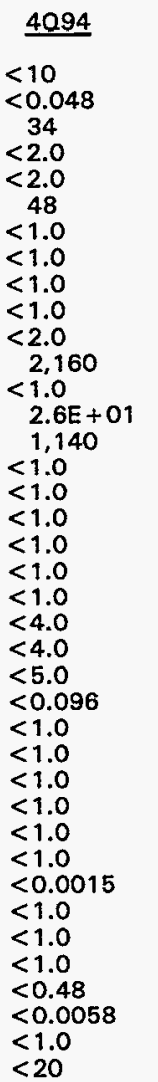

Mod Unit Lab

Flag

$\mu \mathrm{g} / \mathrm{L} \quad \mathrm{GE}$

$\mu g / L \quad G E$

$\mu g / \mathrm{L} \quad \mathrm{GE}$

$\mu g / L \quad G E$

$\mu g / L \quad G E$

$\mu \mathrm{g} / \mathrm{L} \quad \mathrm{GE}$

$\mu \mathrm{g} / \mathrm{L} . \quad \mathrm{GE}$

$\mu \mathrm{g} / \mathrm{L} \quad \mathrm{GE}$

$\mu \mathrm{g} / \mathrm{L} \quad \mathrm{GE}$

$\mu \mathrm{g} / \mathrm{L}$

$\mu \mathrm{g} / \mathrm{L}$

$\mathrm{pCi} / \mathrm{L} \quad \mathrm{GP}$

$\mu \mathrm{g} / \mathrm{L} \quad \mathrm{GE}$

$\mu \mathrm{g} / \mathrm{L} \quad \mathrm{GE}$

$\mu g / L$

$\mu \mathrm{g} /$

$\mu \mathrm{g} / \mathrm{L}$

$\mu g /$

$\mu \mathrm{g} / \mathrm{L}$

$\mu g / L$

$\mu g / L$

$\mu \mathrm{g} /$

$\mu g / L$

$\mu g / L$

$\mu \mathrm{g} / \mathrm{L}$

$\mu g / \mathrm{L}$

$\mu g / L$

$\mu \mathrm{g} / \mathrm{L}$

$\mu g / L$

$\mu g /$

$\mu \mathrm{g} / \mathrm{L}$

$\mu \mathrm{g} / \mathrm{L}$

$\mu{ }_{\mu g / L} / L$

$\mu \mathrm{g} / \mathrm{L}$

0
0
1
0
0
0
0
0
0
0
0
0
0
0
0
0
0
0
0
0
0
0
0
0
0
0
0
0
0
0
0
0
0
0
0
0
0
0
0
0

Note: Flagging, dilution factors, modifiers, and laboratories are for fourth quarter 1994 data only. See Appendix B for flagging criteria.

- = exceeded holding time for fourth quarter 1994.

- exceeded screening level or final PDWS for fourth quarter 1994. 
H ST Analyte

Gross alpha

Heptachlor

Iron, total recoverable

Lead, total recoverable

Lindane

Lithium, total recoverable

Magnesium, total recoverable

Manganese, total recoverable

Mercury, total recoverable

Methoxychlor

Naphthalene

Nickel, total recoverable

Nitrate as nitrogen

Nitrate-nitrite as nitrogen

Nonvolatile beta

- $\quad \mathrm{pH}$

Phenols

Potassium, total recoverable

Radium, total alpha-emitting

Selenium, total recoverable

Silica, total recoverable

Silver, total recoverable

Sodium, total recoverable

Specific conductance

Sulfate

1,1,2,2-Tetrachloroethane

Tetrachloroethylene

Tin, total recoverable

Toluene

Total dissolved solids

Total organic carbon

Total organic halogens

Total phosphates (as P)

Toxaphene

2,4,5-TP (Silvex)

1,1,1-Trichloroethane

1.1,2-Trichloroethane

Trichloroethylene

Trichlorofluoromethane

2,4,5-T

- Tritum

Uurbidity $233 / 234$

Uranium-235

Uranium-238

Vanadium, total recoverable

Xylenes

Zinc, total recoverable

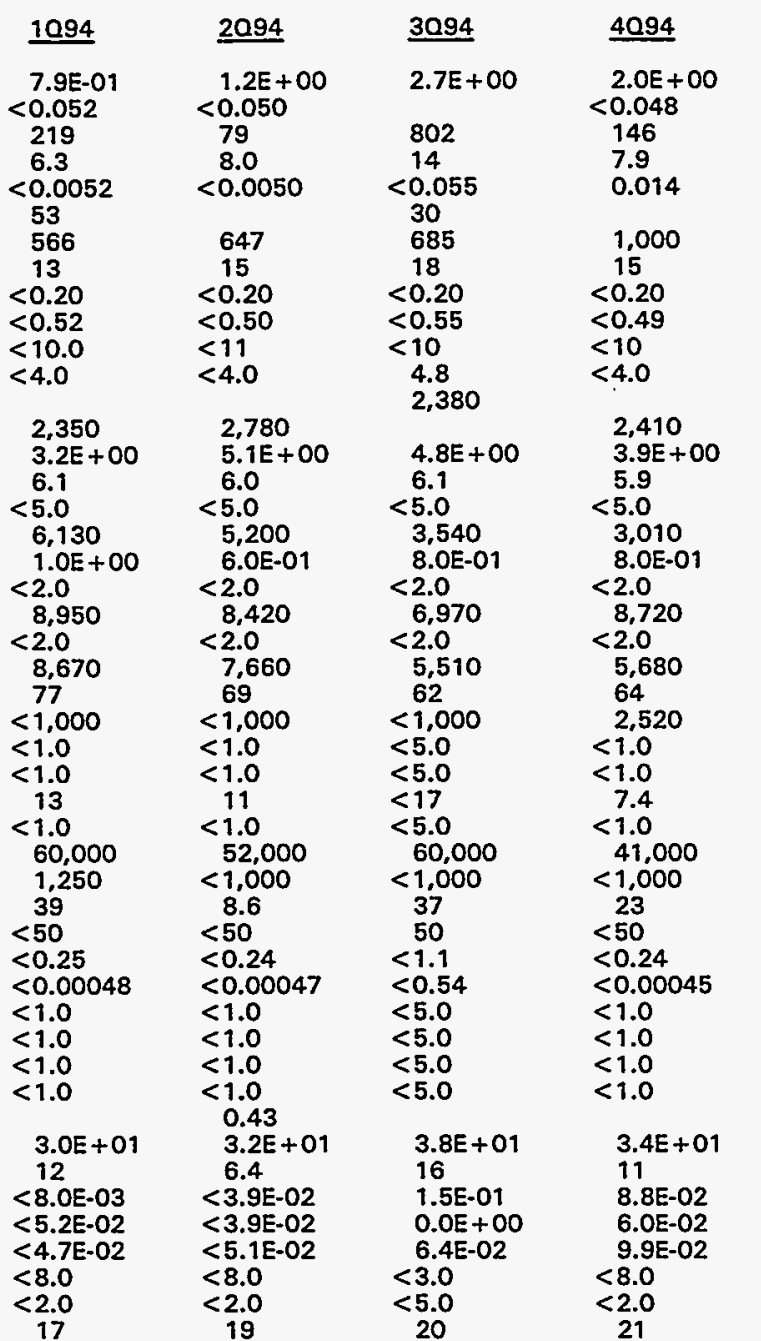

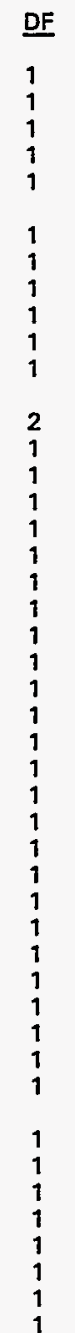

Unit Lab Flag

$\mathrm{pCi} / \mathrm{L} \quad \mathrm{GP} \quad 0$

$\begin{array}{lll}\mu \mathrm{g} / \mathrm{L} & \mathrm{GE} & 0 \\ \mu \mathrm{g} / \mathrm{L} & \mathrm{GE} & 0\end{array}$

$\mu \mathrm{g} / \mathrm{L} \quad \mathrm{GE}$

$\mu g / L \quad G E$

$\mu \mathrm{g} / \mathrm{L}$

$\mu g / L$ GE 0

$\mu g / L \quad G E$ O

$\mu \mathrm{g} / \mathrm{L} \quad \mathrm{GE}$

$\underset{\mu g / L}{\mu \mathrm{GE}}$

$\mu g / L \quad$ GE

$\mu \mathrm{g} / \mathrm{L}$

$\mu g / L \quad G E$

$\mathrm{PCi} / \mathrm{GP}$

J

PHIL GE

glh

$\mu g / L \quad G E$

$\begin{array}{ll}\mathrm{PCi} / \mathrm{L} & \mathrm{GP} \\ \mu \mathrm{g} / \mathrm{L} & \mathrm{GE}\end{array}$

$\mu \mathrm{g} / \mathrm{L} \quad \mathrm{GE}$

$\mu \mathrm{g} / \mathrm{L} \quad \mathrm{GE}$

$\mu g / L \quad G E$

$\mu \mathrm{S} / \mathrm{cm}$ GE

$\mu g / L \quad G E$

$\mu g / L \quad G E$

$\mu g / L \quad G E$

$\mu \mathrm{g} / \mathrm{L}$

$\mu g / L$

$\mu g / L$

$\mu \mathrm{g} / \mathrm{L} \quad \mathrm{GE}$

了

$\mu g /$

$\mu g$

$\mu g / L$

$\mu g / L$

$\mu \mathrm{g} / \mathrm{L}$

$\mu g / L$

$\mu g / L$

$\mathrm{pCi} / \mathrm{mL}$ GP

$\mathrm{J}$

NTU

$\mathrm{pCi} / \mathrm{L} \quad \mathrm{GP}$

$\mathrm{PCI} / \mathrm{GP}$

$\mathrm{pCi} / \mathrm{GP}$

$\mu \mathrm{g} / \mathrm{L} \quad \mathrm{GE}$

$\mu g / L \quad G E$

0
0
0
0

0
0
0
0
0
0
0
0
0
0
0
0
0
0
0
0
0
0
0
0
0
0
0
0
0
0
0
0
0
0
0
0
0
2
0
0
0
0
0
0

Note: Flagging, dilution factors, modifiers, and laboratories are for fourth quarter 1994 data only. See Appendix B for flagging criteria.

- = exceeded holding time for fourth quarter 1994.

- = exceeded screening level or final PDWS for fourth quarter 1994. 


\section{WELL BGO 22DR}

\begin{tabular}{|c|c|c|c|c|c|c|}
\hline SRS Coord. & Lat/Longitude & Screen Zone Elevation & Top of Casing & Casing & Pump & Screen Zone \\
\hline $\begin{array}{l}\text { N74471. } \\
\text { E57831.5 }\end{array}$ & $\begin{array}{l}33.284841^{\circ} \mathrm{N} \\
81.659178{ }^{\circ} \mathrm{W}\end{array}$ & $239.2-219.2 \mathrm{ft} \mathrm{ms} !$ & $286.1 \mathrm{ft} \mathrm{msl}$ & 4" PVC & $\mathbf{S}$ & Water Table (IIB2 \\
\hline SAMPLE DATE & & $02 / 03 / 94$ & $04 / 08 / 94$ & & & \\
\hline
\end{tabular}

FIELD DATA

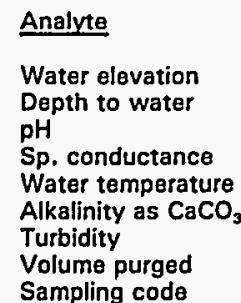

ANALYTICAL DATA

\begin{tabular}{l}
1094 \\
\hline 218.4 \\
67.8 \\
5.4 \\
24 \\
17.3 \\
2 \\
21 \\
$\times$
\end{tabular}

2094
237.0
49.1
5.6
26
17.9
3
219
1.2
$x$

30.94
236.6
49.6
5.5
23
22.5
2
13
1.3
$x$

\begin{tabular}{|c|c|}
\hline 1094 & 2094 \\
\hline $\begin{array}{l}<10 \\
<0.052 \\
883 \\
<2.0 \\
<2.0 \\
9.3 \\
<1.0 \\
<1.0 \\
<1.0 \\
<1.0 \\
<2.0 \\
969 \\
<1.0 \\
2.9 E+01 \\
1.050 \\
<1.0 \\
<1.0 \\
<1.0 \\
<1.0 \\
<1.0 \\
<1.0 \\
<4.0 \\
5.5 \\
<5.0 \\
<0.10 \\
<1.0 \\
<1.0 \\
<1.0 \\
<1.0 \\
<1.0 \\
<1.0 \\
<0.0016 \\
<1.0 \\
<1.0 \\
<1.0 \\
<0.52 \\
<0.0062 \\
<1.0 \\
<100 \\
<1\end{array}$ & 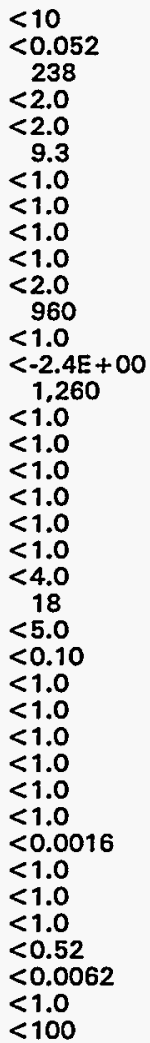 \\
\hline
\end{tabular}

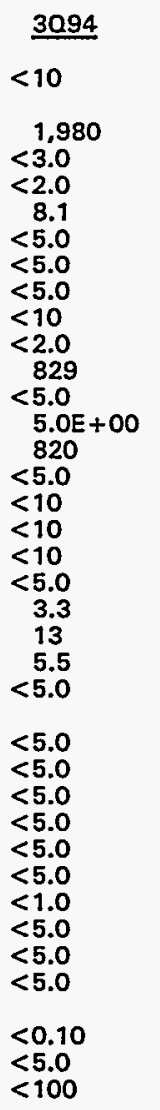

$\begin{array}{ll}4094 & \text { Unit } \\ 236.4 & \mathrm{ft} \mathrm{msl} \\ 49.7 & \mathrm{ft} \\ 5.6 & \mathrm{pH} \\ 19 & \mu S / \mathrm{cm} \\ 20.4 & { }^{\circ} \mathrm{C} \\ 2 & \mathrm{mg} / \mathrm{L} \\ 30 & \mathrm{NTU} \\ 1.2 & \text { well vol. } \\ \mathrm{X} & \end{array}$

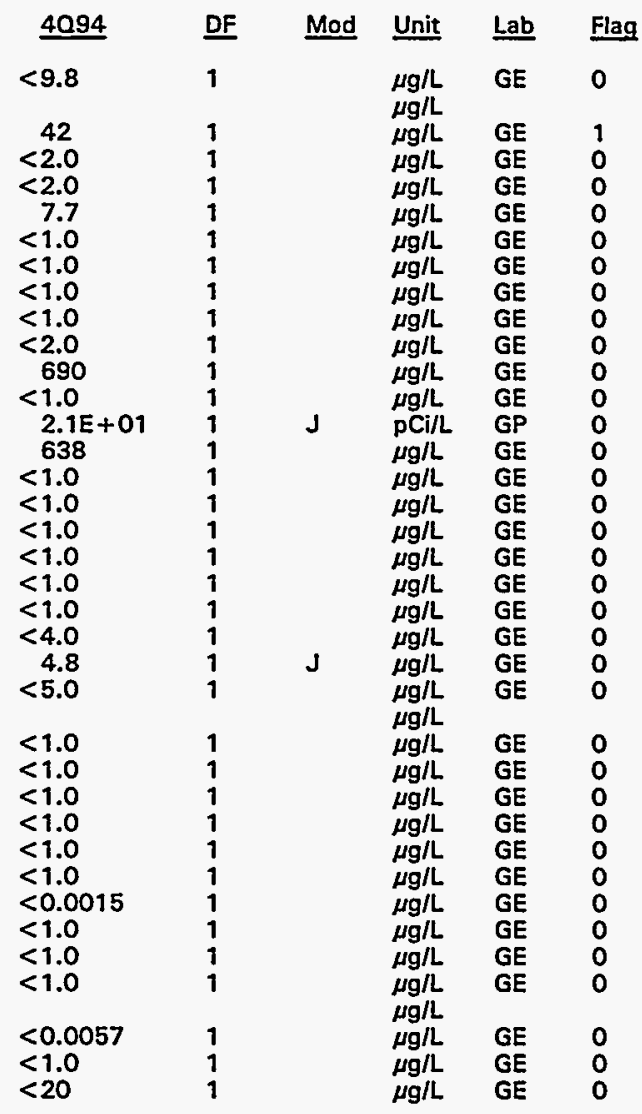

Note: Flagging, dilution factors, modifiers, and laboratories are for fourth quarter 1994 data only. See Appendix B for flagging criteria.

- = exceeded holding time for fourth quarter 1994.

- = exceeded screening level or final PDWS for fourth quarter 1994. 
H ST Analyte

Gross alpha

Heptachlor

Iron, total recoverable

Lead, total recoverable

Lindane

Lithium, total recoverable

Magnesium, total recoverable

Manganese, total recoverable

Mercury, total recoverable

Methoxychlor

Naphthalene

Nickel, total recoverable

Nitrate as nitrogen

Nitrate-nitrite as nitrogen

Nonvolatile beta

- $\quad \mathrm{pH}$

Phenols

Potassium, total recoverable

Radium, total alpha-emitting

Selenium, total recoverable

Silica, total recoverable

Silver, total recoverable

Sodium, total recoverable

Specific conductance

Sulfate

1,1,2,2-Tetrachloroethane

Tetrachloroethylene

Tin, total recoverable

Toluene

Total dissolved solids

Total organic carbon

Total organic halogens

Total phosphates (as P)

Toxaphene

2,4,5-TP (Silvex)

1,1,1-Trichloroethane

1,1,2-Trichloroethane

Trichloroethylene

Trichlorofluoromethane

2,4,5-T

Tritium

Turbidity

Uranium-233/234

Uranium-235

Uranium-238

Vanadium, total recoverable

Xylenes

Zinc, total recoverable

$\frac{1094}{1.4 E+00}$
$<0.052$
354
6.4
$<0.0052$
$<5.0$
766
8.6
$<0.20$
$<0.52$
$<10$
$<4.0$

$\underline{2094}$
$1.4 \mathrm{E}+00$
$<0.052$
118
9.8
$<0.0052$

788
8.1
$<0.20$
$<0.52$
$<10$
4.7

$\frac{30.94}{7.3 E-01}$
5,050
12
$<0.052$
$<5.0$
595
16
$<0.20$
$<0.52$
$<10$
6.8
768

$1.8 E+00$

6.3

$<5.0$

919

$<2.0$

8,010

$<2.0$

1,160

22

$<1,000$

$<1.0$

4.8

$<1.0$

23,000

$<1,000$

$<1,000$
$>5.4$
$<50$

$<0.25$

$<0.00046$

$<1.0$

$<1.0$

$<1.0$

$<1.0$

4. $2 \mathrm{E}+01$

176

$<4.5 \mathrm{E}-02$

$<4.1 \mathrm{E} .02$

$<4.1 \mathrm{E}-02$

$<8.0$

$<2.0$

18

900

$1.5 \mathrm{E}+00$

5.9

$<5.0$

934

$<2.0$

6,680

$<2.0$

1,270

1,370

1,370
$<1.0$

$<1.0$

2.6

$<1.0$

22,000

$<1,000$

$<5.0$

$<50$

$<0.25$

$<0.00047$

$<1.0$

$<1.0$

$<1.0$

$<1.0$

0.42
$3.7 E+01$

77

$<6.7 E-02$

$<-2.0 \mathrm{E} \cdot 02$

$<6.7 E-02$

$<8.0$

$<2.0$

$2.1 E+00$
5.8

5.8
$<5.0$

503

5.OE-01

$<2.0$

$\begin{array}{r}7.570 \\ \hline\end{array}$

$<2.0$

31

$<1,000$

$<5.0$

$<17$

$<5.0$

25,000

$<1,000$

252

$<1.0$

$<0.52$

$<5.0$

$<5.0$

$<5.0$

$<5.0$

2.7E+01

37

3.2E-01

1.5E-02

9.6E-02

11

$<5.0$
23

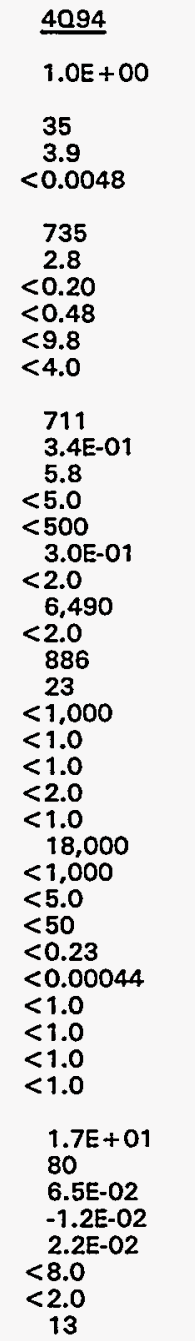

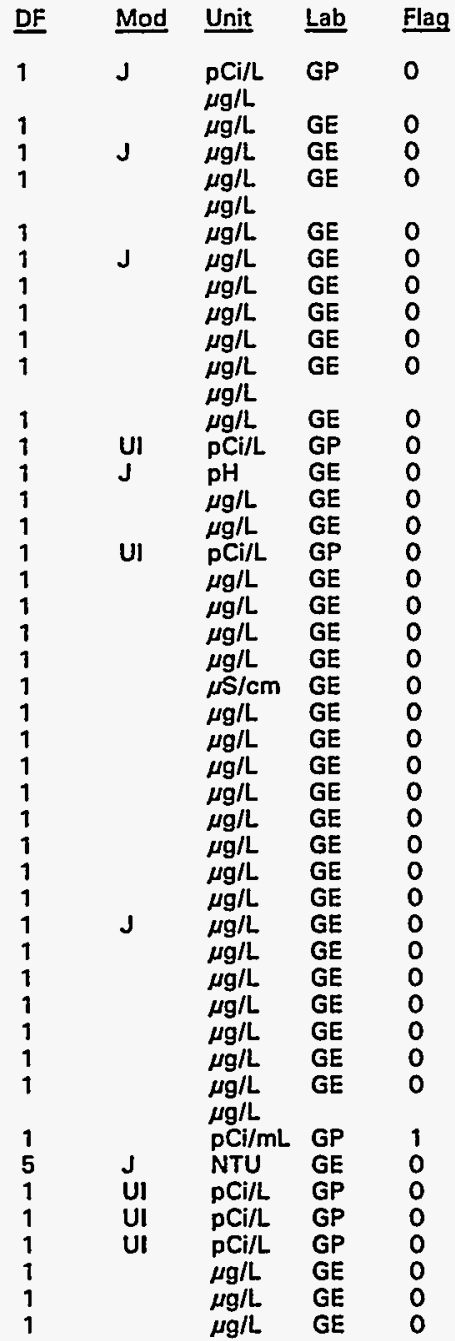

Note: Flagging, dilution factors, modifiers, and laboratories are for fourth quarter 1994 data only. See Appendix B for flagging criteria. - = exceeded holding time for fourth quarter 1994.

- = exceeded screening level or final PDWS for fourth quarter 1994. 
WELL BGO 23D

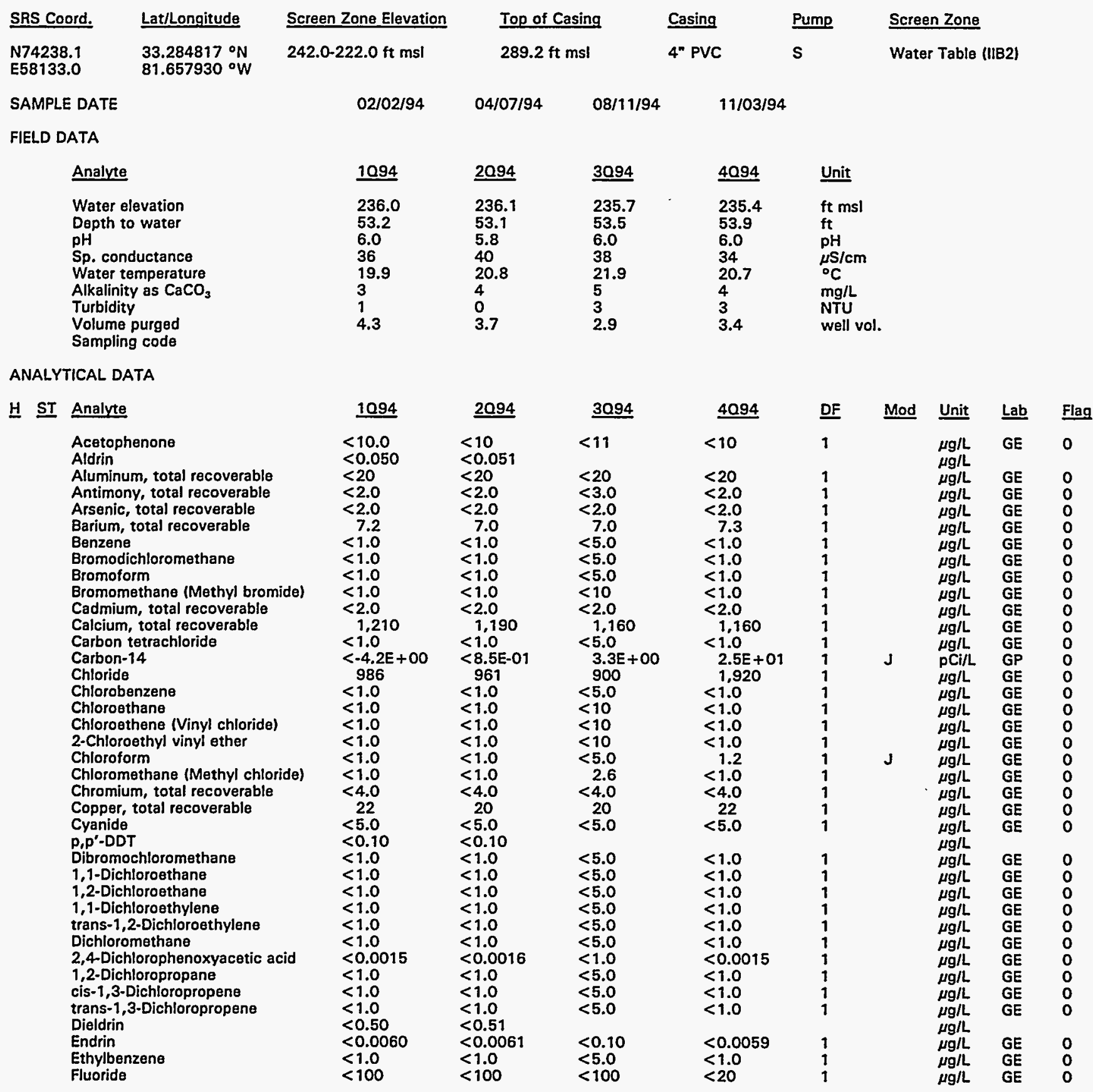

Note: Flagging, dilution factors, modifiers, and laboratories are for fourth quarter 1994 data only. See Appendix B for flagging criteria. - = exceeded holding time for fourth quarter 1994.

- = exceeded screening level or final PDWS for fourth quarter 1994. 
Well BGO 230 continued

ANALYTICAL DATA

H ST Analyte

Gross alpha

Heptachlor

Iron total recoverable

Lead, total recoverable

Lindane

Lithium, total recoverable

Magnesium, total recoverable

Manganese, total recoverable

Mercury, total recoverable

Methoxychior

Naphthalene

Nickel, total recoverable

Nitrate as nitrogen

Nitrate-nitrite as nitrogen

Nonvolatile beta

- $\quad \mathrm{pH}$

Phenols

Potassium, total recoverable

Radium, total alpha-emitting

Selenium, total recoverable

Silica, total recoverable

Silver, total recoverable

Sodium, total recoverable

Specific conductance

1,1,2,2-Tetrachloroethane

1,1,2,2-Tetrachioroethe

Tin, total recoverable

Toluene

Total dissolved solids

Total organic carbon

Total organic halogens

Total phosphates (as P)

Toxaphene

2,4,5-TP (Silvex)

1,1,1-Trichloroethane

1, 1,2-Trichloroethane

Trichloroethylene

Trichlorofluoromethane

2,4,5-T

- Tritium

Turbidity

Uranium-233/234

Uranium-235

Uranium-238

Vanadium, total recoverable

Xylenes

Zinc, total recoverable

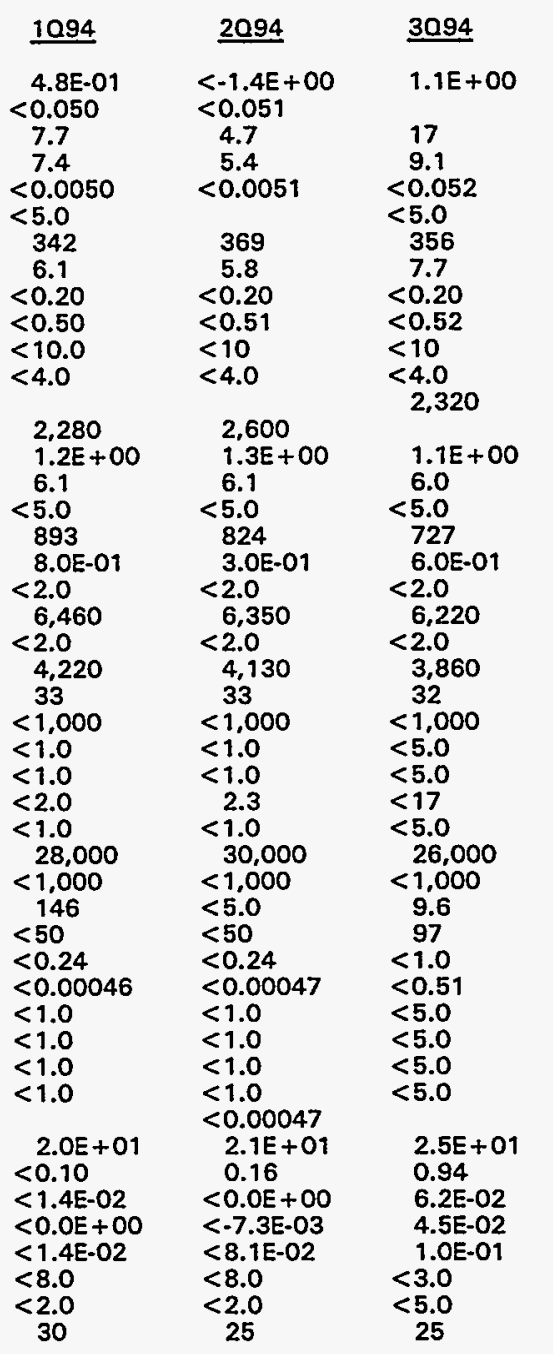

\begin{tabular}{|c|c|}
\hline 4094 & $\underline{D F}$ \\
\hline $6.8 E-01$ & 1 \\
\hline $\begin{aligned} & 5.6 \\
9.1 & <0.0049\end{aligned}$ & $\begin{array}{l}1 \\
1 \\
1\end{array}$ \\
\hline $\begin{aligned} & 385 \\
& 7.4 \\
< & 0.20 \\
< & 0.49 \\
< & 10 \\
< & 4.0\end{aligned}$ & $\begin{array}{l}1 \\
1 \\
1 \\
1 \\
1 \\
1\end{array}$ \\
\hline $\begin{aligned} & 2,380 \\
& 1.2 \mathrm{E}+00 \\
& 6.1 \\
&<5.0 \\
& 596 \\
& 5.0 \mathrm{E}-01 \\
&<2.0 \\
& 6,410 \\
&<2.0 \\
& 3,990 \\
& 34 \\
&<1.000 \\
&<1.0 \\
&<1.0 \\
& 3.1 \\
&<1.0 \\
& 28,000 \\
&<1,000 \\
&<5.0 \\
&<50 \\
&<0.24 \\
&<0.00044 \\
&<1.0 \\
&<1.0 \\
&<1.0 \\
&<1.0\end{aligned}$ & $\begin{array}{l}2 \\
1 \\
1 \\
1 \\
1 \\
1 \\
1 \\
1 \\
1 \\
1 \\
1 \\
1 \\
1 \\
1 \\
1 \\
1 \\
1 \\
1 \\
1 \\
1 \\
1 \\
1 \\
1 \\
1 \\
1 \\
1\end{array}$ \\
\hline $\begin{array}{l}2.7 E+01 \\
1.7 \\
2.1 E-03 \\
-8.2 E-03 \\
8.3 E-02 \\
<8.0 \\
<2.0 \\
22\end{array}$ & 1 \\
\hline
\end{tabular}

Note: Flagging, dilution factors, modifiers, and laboratories are for fourth quarter 1994 data only. See Appendix B for flagging criteria. - = exceeded holding time for fourth quarter 1994.

- exceeded screening level or final PDWS for fourth quarter 1994. 
WELL BGO 24D

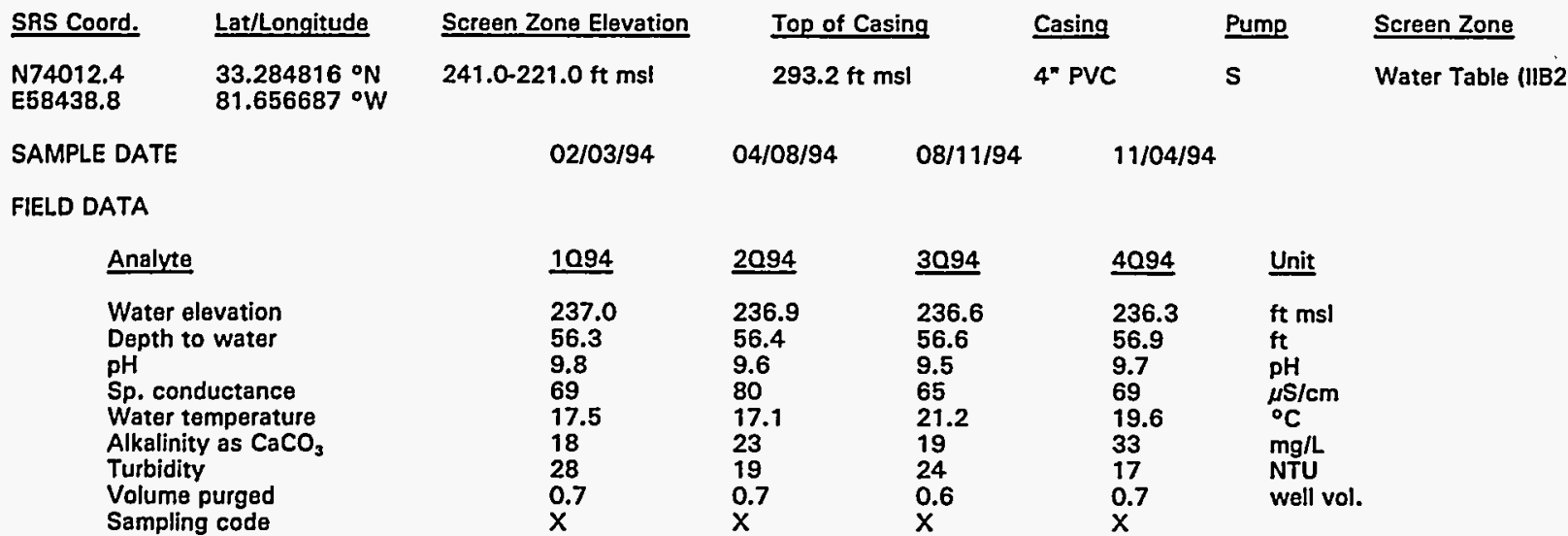

ANALYTICAL DATA

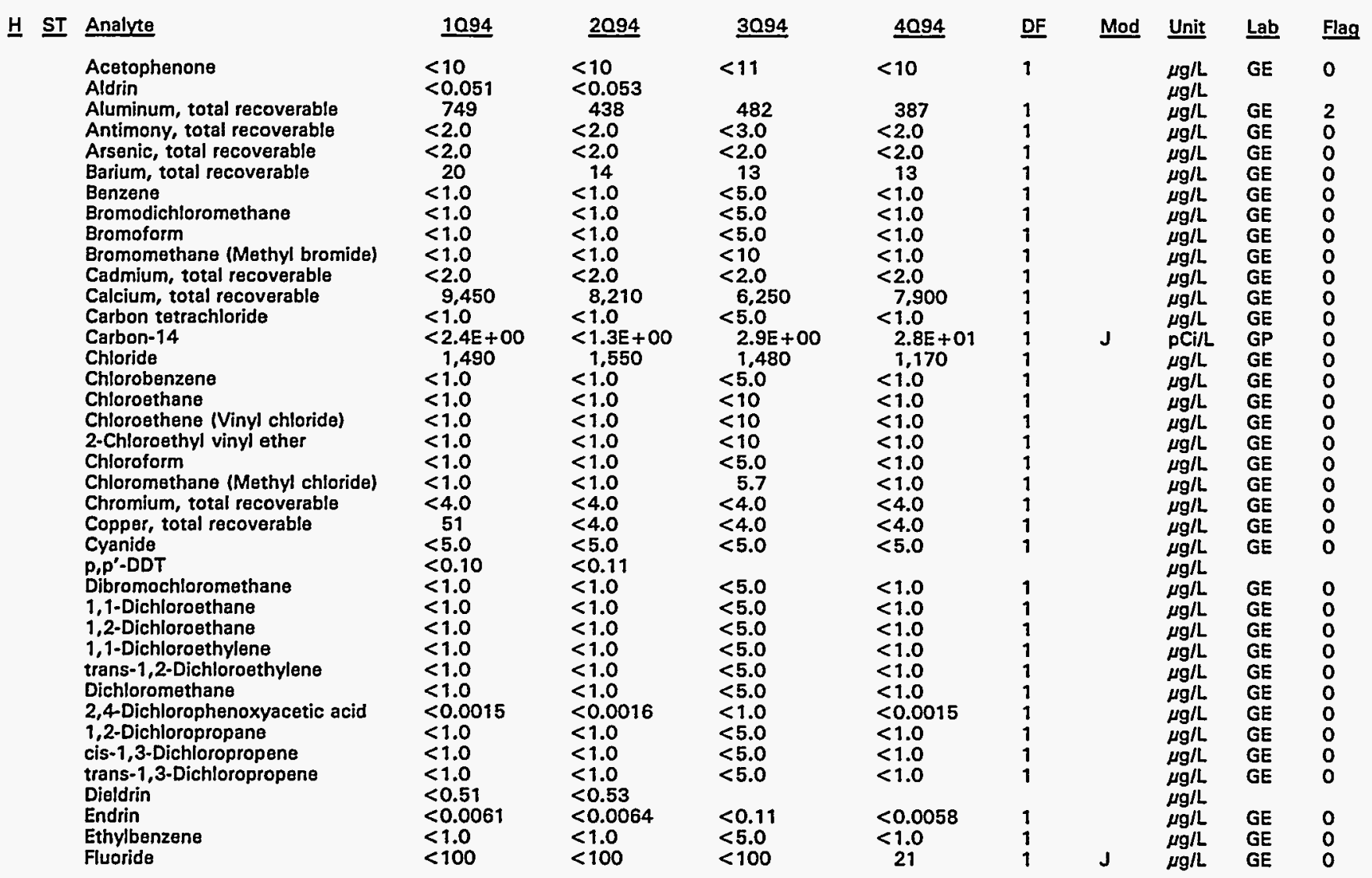

Note: Flagging, dilution factors, modifiers, and laboratories are for fourth quarter 1994 data only. See Appendix B for flagging criteria. - = exceeded holding time for fourth quarter 1994.

- = exceeded screening level or final PDWS for fourth quarter 1994. 


\section{H ST Analyte}

Gross alpha

Heptachlor

Iron, total recoverable

Lead, total recoverable

Lindane

Lithium, total recoverable

Magnesium, total recoverable

Manganese, total recoverable

Mercury, total recoverable

Methoxychlor

Naphthalene

Nickel, total recoverable

Nitrate as nitrogen

Nitrate-nitrite as nitrogen

Nonvolatile beta

pH

Phenols

Potassium, total recoverable

Radium, total alpha-emitting

Selenium total recoverable

Silica, total recoverable

Silver, total recoverable

Sodium, total recoverable

Specific conductance

Sulfate

1,1,2,2-Tetrachloroethane

Tetrachloroethylene

Tin, total recoverable

Toluene

Total dissolved solids

Total organic carbon

Total organic halogens

Total phosphates (as P)

Toxaphene

2,4,5-TP (Silvex)

1,1,1-Trichloroethane

1,1,2-Trichloroethane

Trichloroethylene

Trichlorofluoromethane

2,4,5-T

Tritium

- Turbidity

Uranium-233/234

Uranium-235

Uranium-238

Vanadium, total recoverable

Xylenes

Zinc, total recoverable

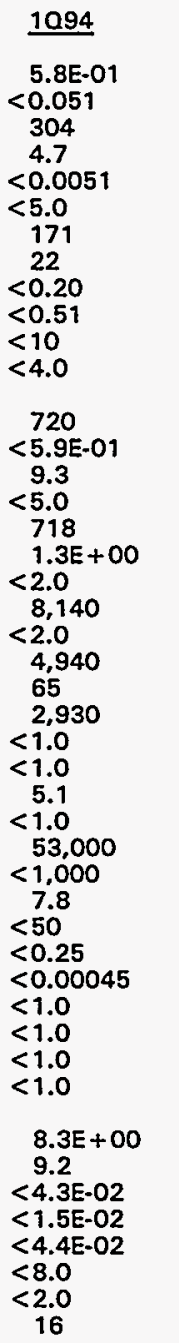

\begin{tabular}{|c|c|}
\hline 2094 & 3094 \\
\hline $\begin{array}{l}5.2 E-01 \\
<0.053\end{array}$ & $9.6 \mathrm{E}-01$ \\
\hline $\begin{array}{l}87 \\
3.4\end{array}$ & $\begin{array}{l}373 \\
20\end{array}$ \\
\hline$<0.0053$ & $\begin{array}{l}<0.053 \\
<5.0\end{array}$ \\
\hline 125 & 118 \\
\hline $\begin{array}{c}11 \\
<0.20\end{array}$ & 19 \\
\hline $\begin{array}{l}<0.20 \\
<0.53\end{array}$ & $<0.20$ \\
\hline $\begin{array}{l}<0.53 \\
<10\end{array}$ & $\begin{array}{l}<0.53 \\
<10\end{array}$ \\
\hline$<4.0$ & $<4.0$ \\
\hline 900 & 740 \\
\hline $\begin{array}{l}1.3 E+00 \\
8.0\end{array}$ & $\begin{array}{l}1.9 E+00 \\
8.9\end{array}$ \\
\hline$<5.0$ & $<5.0$ \\
\hline $\begin{array}{l}610 \\
<1.0 \text {-01 }\end{array}$ & $\begin{array}{l}<500 \\
9.0 \mathrm{E}-01\end{array}$ \\
\hline$<2.0$ & $<2.0$ \\
\hline $\begin{aligned} & 7.070 \\
&<2.0\end{aligned}$ & $\begin{aligned} & 5,420 \\
< & 2.0\end{aligned}$ \\
\hline $\begin{array}{l}4,910 \\
58\end{array}$ & $\begin{array}{l}3,390 \\
56\end{array}$ \\
\hline $\begin{aligned} & 2,770 \\
&<1.0\end{aligned}$ & $\begin{array}{r}3,670 \\
<5,0\end{array}$ \\
\hline$<1.0$ & $\begin{array}{l}<5.0 \\
<5.0\end{array}$ \\
\hline$<2.0$ & $<17$ \\
\hline $\begin{array}{l}<1.0 \\
39.000\end{array}$ & $<5.0$ \\
\hline $\begin{array}{r}39,000 \\
<1,000\end{array}$ & $\begin{array}{r}45,000 \\
<1,000\end{array}$ \\
\hline $\begin{array}{l}<5.0 \\
<50\end{array}$ & $\begin{array}{l}7.6 \\
111\end{array}$ \\
\hline$<0.26$ & $<1.1$ \\
\hline$<0.00047$ & $<0.52$ \\
\hline$<1.0$ & $<5.0$ \\
\hline$<1.0$ & $<5.0$ \\
\hline$<1.0$ & $<5.0$ \\
\hline$<1.0$ & $<5.0$ \\
\hline $\begin{array}{l}0.25 \\
8.4 E+00\end{array}$ & \\
\hline $\begin{array}{l}8.4 E+00 \\
9.0\end{array}$ & $\begin{array}{l}9.6 E+00 \\
6.4\end{array}$ \\
\hline$<3.6 \mathrm{E}-02$ & $1.5 \mathrm{E}-01$ \\
\hline$<0.0 \mathrm{E}+00$ & $0.0 E+00$ \\
\hline$<3.6 \mathrm{E}-02$ & $2.4 \mathrm{E}-01$ \\
\hline$<8.0$ & $<3.0$ \\
\hline $\begin{array}{c}<2.0 \\
11\end{array}$ & $\begin{array}{c}<5.0 \\
40\end{array}$ \\
\hline
\end{tabular}

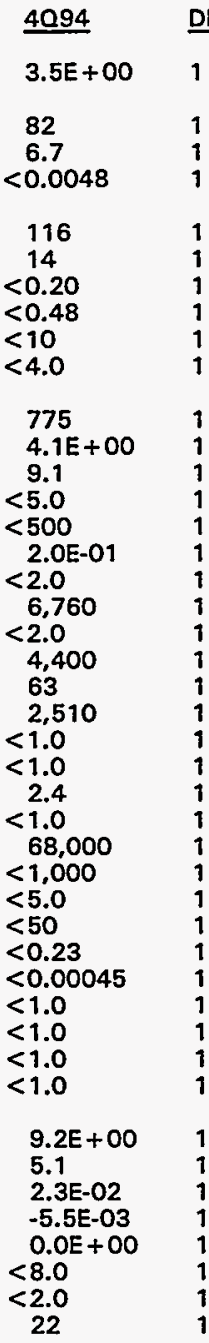

Unit Lab $\quad$ Flag

pCi/L GP 0

$\mu \mathrm{g} / \mathrm{L}$ GE 0

$\mu g / L$ GE

$\begin{array}{lll}\mu g / L & \text { GE } & 0 \\ \mu g / L & \text { GE } & 0\end{array}$

$\mu \mathrm{g} / \mathrm{L}$

$\mu \mathrm{g} / \mathrm{L}$ GE 0

$\mu \mathrm{g} / \mathrm{L} \quad \mathrm{GE}$

$\mu \mathrm{g} / \mathrm{L}$

$\mu g / L$

$\mu g / L$

$\mu g / L$

$\mu \mathrm{g} / \mathrm{L}$

$\mu \mathrm{g} / \mathrm{L}$

pCill GE

J

$\mathrm{pH}$
$\mu \mathrm{g} / \mathrm{L}$

UI

$\mu \mathrm{g} / \mathrm{L}$
$\mathrm{pCi} / \mathrm{L}$
$\mu \mathrm{g} / \mathrm{L}$

$\mu g / L$

$\mu g / L$

$\mu \mathrm{g} / \mathrm{L}$

ISI cm

ug/L

$\mu \mathrm{g} / \mathrm{L}$

$\mu g / L$

$\mu \mathrm{g} /$

J

$\mu g / L \quad G E$

$\mu \mathrm{g} / \mathrm{L}$

$\mu \mathrm{g} /$

$\mu g / L$

$\mu g /$

$\mu \mathrm{g} / \mathrm{L}$

$\mu g /$

$\mu g / L$

$\mu g / L$

$\mu g / L \quad G E$

$\mu \mathrm{g} / \mathrm{L}$

pCi/mL GP

J) NTU GE

$\begin{array}{lll}\text { UI } & \text { pCi/L } & \text { GP } \\ \text { UI } & \text { pCi/L } & \text { GP }\end{array}$

UI
$\mathrm{PC}$

$\mu g / L \quad G E$

$\mu \mathrm{g} / \mathrm{L} \quad \mathrm{GE}$

$\mu g / L \quad G E$

Note: Flagging, dilution factors, modifiers, and laboratories are for fourth quarter 1994 data only. See Appendix B for flagging criteria. - = exceeded holding time for fourth quarter 1994.

- = exceeded screening level or final PDWS for fourth quarter 1994. 


\section{WELL BGO 25A}

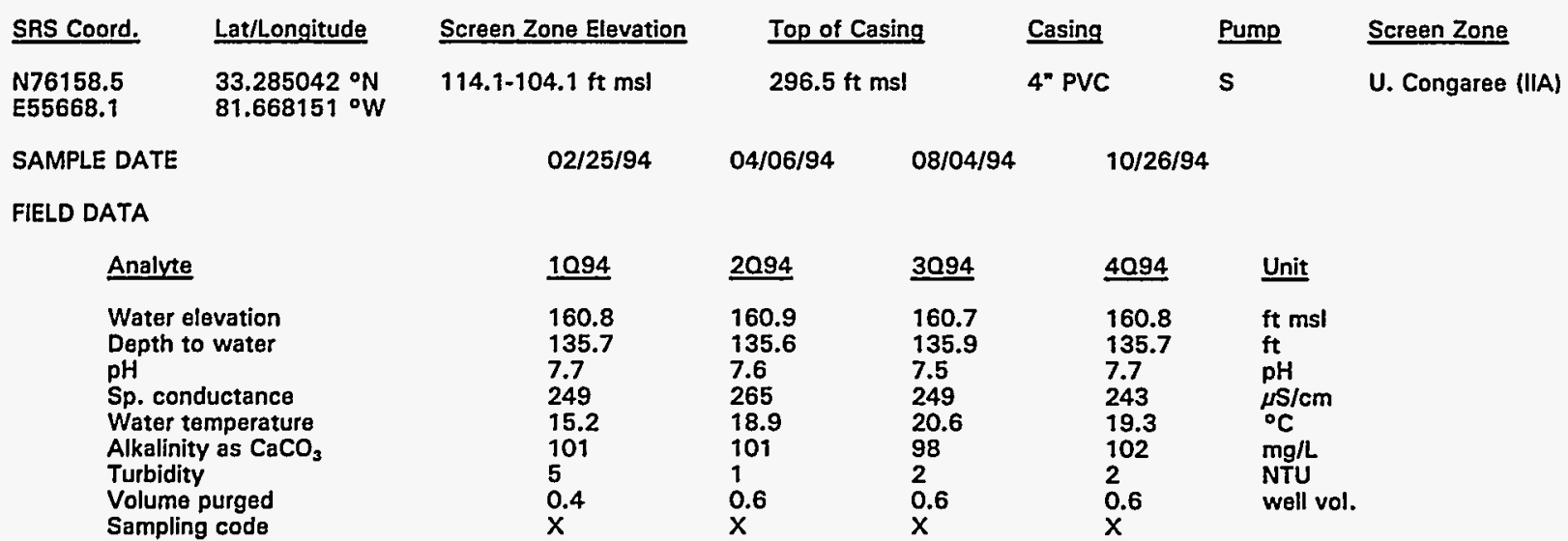

ANALYTICAL DATA

H ST Analyte

Acetophenone

Aldrin

Aluminum, total recoverable

Antimony, total recoverable

Arsenic, total recoverable

Barium, total recoverable

Benzene

Bromodichloromethane

Bromoform

Bromomethane (Methyl bromide)

Cadmium, total recoverable

Calcium, total recoverable

Carbon tetrachloride

Chloride

Chlorobenzene

Chloroethane

Chloroethene (Vinyl chloride)

2-Chloroethyl vinyl ether

Chloroform

Chloromethane (Methyl chloride)

Chromium, total recoverable

Copper, total recoverable

Cyanide

p, ' $^{\prime}$-DDT

Dibromochloromethane

1,1-Dichloroethane

1,2-Dichloroethane

1,1-Dichloroethylene

trans-1,2-Dichloroethylene

Dichloromethane

2,4-Dichlorophenoxyacetic acid

1,2-Dichloropropane

cis-1,3-Dichloropropene

trans-1,3-Dichloropropene

Dieldrin

Endrin

Ethylbenzene

Fluoride

Gross alpha
$<9.7$

$<0.049$

39
$<2.0$

$<2.0$

41

$<1.0$

$<1.0$

$<1.0$

$<1.0$

51,000

$<1.0$

2,560

$<1.0$

$<1.0$

$<1.0$

$<1.0$

$<1.0$

$<1.0$

$<4.0$

11

$<0.097$

$<1.0$

$<1.0$

$<1.0$

$<1.0$

$<1.0$

$<1.0$

$<0.0015$

$<1.0$

$<1.0$

$<1.0$

$<0.49$

$<0.0058$

$<1.0$

$<100$

$<4.1 E-01$

$\begin{aligned} & 2094 \\ &<10 \\ &<0.052 \\ &<20 \\ &<2.0 \\ &<2.0 \\ & 37 \\ &<1.0 \\ &<1.0 \\ &<1.0 \\ &<1.0 \\ &<2.0 \\ & 47.600 \\ &<1.0 \\ & 2,240 \\ &<1.0 \\ &<1.0 \\ &<1.0 \\ &<1.0 \\ &<1.0 \\ &<1.0 \\ &<4.0 \\ & 5.7 \\ &<5.0 \\ &<0.10 \\ &<1.0 \\ &<1.0 \\ &<1.0 \\ &<1.0 \\ &<1.0 \\ &<1.0 \\ &<0.0015 \\ &<1.0 \\ &<1.0 \\ &<1.0 \\ &<0.52 \\ &<0.0062 \\ &<1.0 \\ &<100 \\ &<-7.6 \mathrm{E}-02 \\ &\end{aligned}$

3094
$\quad<10$
37
$<3.0$
2.0
38
$<5.0$
$<5.0$
$<5.0$
$<10$
$<2.0$
47.600
$<5.0$
2,650
$<5.0$
$<10$
$<10$
$<10$
$<5.0$
6.5
$<4.0$
6.8
$<5.0$
$<5.0$
$<5.0$
$<5.0$
$<5.0$
$<5.0$
$<5.0$
$<1.0$
$<5.0$
$<5.0$
$<5.0$
$<0.11$
$<5.0$
$<100$
$6.35-01$

\begin{tabular}{ll}
$\quad$ 4094 & DF \\
$<10$ & 1 \\
\multicolumn{1}{c}{26} & 1 \\
$<2.0$ & 1 \\
$<2.0$ & 1 \\
38 & 1 \\
$<1.0$ & 1 \\
$<1.0$ & 1 \\
$<1.0$ & 1 \\
$<1.0$ & 1 \\
$<2.0$ & 1 \\
47.300 & 2 \\
$<1.0$ & 1 \\
1.740 & 1 \\
$<1.0$ & 1 \\
$<1.0$ & 1 \\
$<1.0$ & 1 \\
$<1.0$ & 1 \\
$<1.0$ & 1 \\
$<1.0$ & 1 \\
$<4.0$ & 1 \\
4.4 & 1 \\
$<5.0$ & 1 \\
$<1.0$ & 1 \\
$<1.0$ & 1 \\
$<1.0$ & 1 \\
$<1.0$ & 1 \\
$<1.0$ & 1 \\
$<1.0$ & 1 \\
$<0.0015$ & 1 \\
$<1.0$ & 1 \\
$<1.0$ & 1 \\
$<1.0$ & 1 \\
$<0.0060$ & 1 \\
$<1.0$ & 1 \\
$<20$ & 1 \\
$6.2 \mathrm{E}-01$ & 1
\end{tabular}

Mod

$\begin{array}{lll}\mu g / \mathrm{L} & \text { GE } & 0 \\ \mu \mathrm{g} / \mathrm{L} & & \end{array}$

$J$

$\mu g / L$

$\mu g / L$

$\mu g / L$
$\mu g / L$
$\mu g / L$

$\mu g / L$

$\mu g / L$

$\mu g / L$

$\mu g / L$

$\mu \mathrm{g} / \mathrm{L}$

$\mu g / L$

$\mu g / L$

$\mu g / L$

$\mu g / L$

$\mu \mathrm{g} / \mathrm{L}$

$\mu \mathrm{g} / \mathrm{L}$

$J$

$\mu g / L / L$

$\mu g / L$

$\mu g / L$

$\mu g / L$

$\mu g / L$

$\mu \mathrm{g} / \mathrm{L}$

$\mu g / L$

$\mu \mathrm{g} / \mathrm{L}$

$\mu g / L$

$\mu g / L$

$\mu g / L$

$\mu g / L \quad G E$

$\mu \mathrm{g} / \mathrm{L}$

uI $\mathrm{pCi} / \mathrm{L}$

$\mathrm{GE}$
$\mathrm{GE}$
$\mathrm{GE}$
$\mathrm{GE}$
$\mathrm{GE}$
$\mathrm{GE}$
$\mathrm{GE}$
$\mathrm{GE}$
$\mathrm{GE}$
$\mathrm{GE}$
$\mathrm{GE}$
$\mathrm{GE}$
$\mathrm{GE}$
$\mathrm{GE}$
$\mathrm{GE}$
$\mathrm{GE}$
$\mathrm{GE}$
$\mathrm{GE}$
$\mathrm{GE}$
$\mathrm{GE}$
$\mathrm{GE}$
$\mathrm{GE}$
$\mathrm{GE}$
$\mathrm{GE}$
$\mathrm{GE}$
$\mathrm{GE}$
$\mathrm{GE}$
$\mathrm{GE}$
$\mathrm{GE}$
$\mathrm{GE}$
$\mathrm{GE}$
$\mathrm{GE}$
$\mathrm{GE}$
$\mathrm{GP}$

0
0
0
0
0
0
0
0
0
0
0
0
0
0
0
0
0
0
0
0
0
0
0
0
0
0
0
0
0
0
0
0
0
0
0
0

Note: Flagging, dilution factors, modifiers, and laboratories are for fourth quarter 1994 data only. See Appendix B for flagging criteria. - = exceeded holding time for fourth quarter 1994.

- = exceeded screening level or final PDWS for fourth quarter 1994. 


\section{ANALYTICAL DATA}

\section{H $\underline{\text { ST Anaiyte }}$}

Heptachlor

Iron, total recoverable

Lead, total recoverable

Lindane

Lithium, total recoverable

Magnesium, total recoverable

Manganese, total recoverable

Mercury, total recoverable

Methoxychlor

Naphthafene

Nickel, total recoverable

Nitrate as nitrogen

Nitrate-nitrite as nitrogen

Nonvolatile beta

- $\mathrm{pH}$

Phenols

Potassium, total recoverable

Radium, total afpha-emitting

Selenium, total recoverable

Silica, total recoverable

Silver, total recoverable

Sodium, total recoverable

Specific conductance

Sulfate

1,1,2,2-Tetrachloroethane

Tetrachloroethylene

Tin, total recoverable

Toluene

Total dissoived solids

Total organic carbon

Total organic halogens

Total phosphates (as P)

Toxaphene

2,4,5-TP (Silvex)

1,1,1-Trichloroethane

1,1,2-Trichloroethane

Trichloroethylene

Trichlorofluoromethane

2,4,5-T

Tritium

Turbidity

Uranium-233/234

Uranium-235

Uranium-238

Vanadium, total recoverable

Xylenes

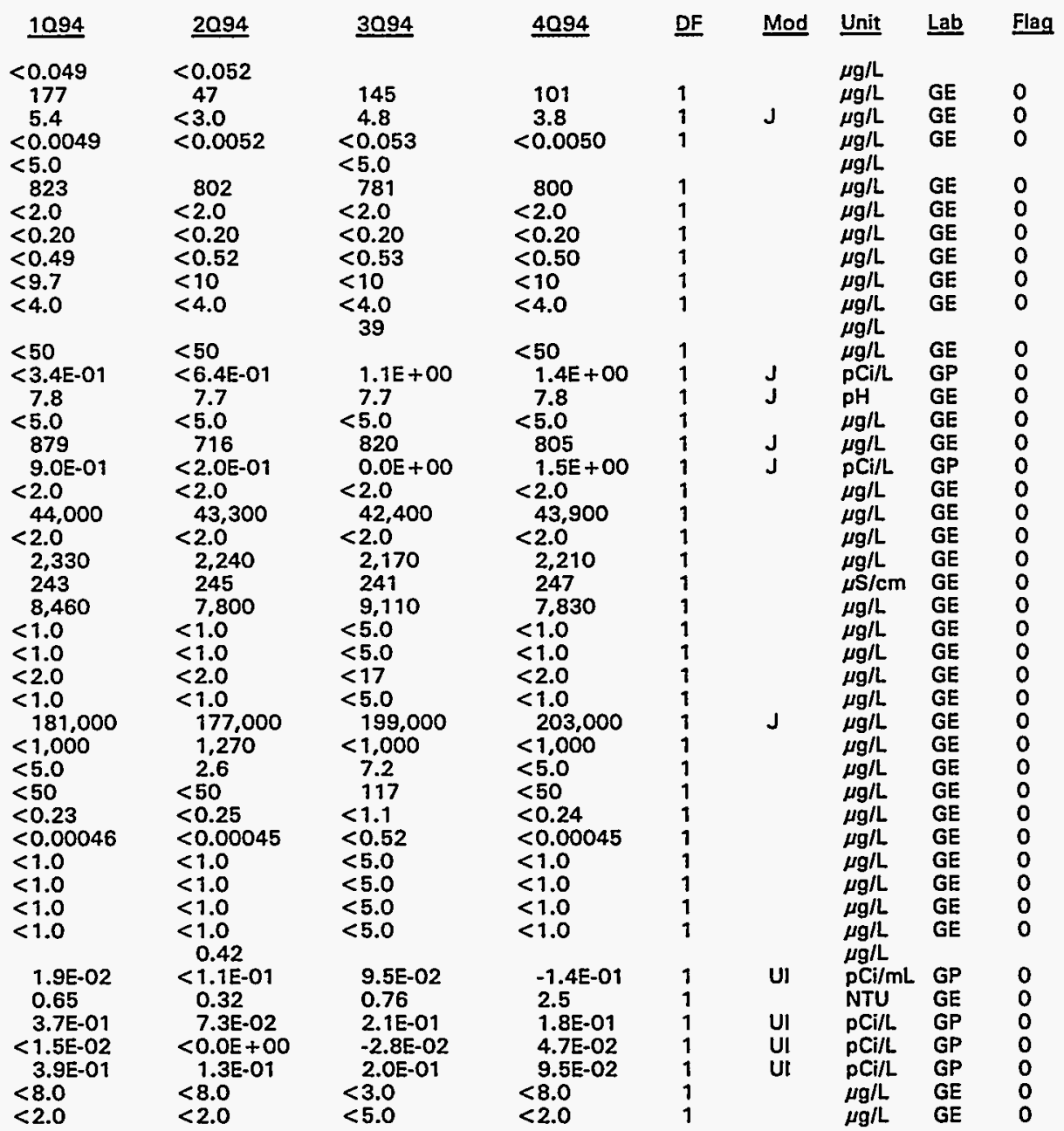

Note: Flagging, dilution factors, modifiers, and laboratories are for fourth quarter 1994 data only. See Appendix B for flagging criteria. - = exceeded holding time for fourth quarter 1994.

- = exceeded screening level or final PDWS for fourth quarter 1994. 


\section{WELL BGO 26A}

\begin{tabular}{|c|c|c|c|c|c|c|c|}
\hline SRS Coord. & Lat/Longitude & Screen Zone Elevation & Top of & & Casing & Pump & Screen Zone \\
\hline $\begin{array}{l}\text { N76144.6 } \\
\text { E55014.2 }\end{array}$ & $\begin{array}{l}33.2839455^{\circ} \mathrm{N} \\
81.669846^{\circ} \mathrm{W}\end{array}$ & $91.0-81.0 \mathrm{ft} \mathrm{msl}$ & 287.2 & & 4" PVC & s & M. Congaree (IIA) \\
\hline SAMPLE DA & & $03 / 22 / 94$ & $04 / 11 / 94$ & $09 / 28 / 94$ & $10 / 25 / 9$ & & \\
\hline FIELD DATA & & & & & & & \\
\hline An: & & 1094 & 20.94 & $\underline{3094}$ & 4094 & Unit & \\
\hline $\begin{array}{l}\text { Wa } \\
\text { Dep } \\
\text { pH } \\
\text { Sp. } \\
\text { Wa } \\
\text { Alk } \\
\text { Turt } \\
\text { Volt } \\
\text { San }\end{array}$ & $\begin{array}{l}\text { evation } \\
\text { water } \\
\text { luctance } \\
\text { mperature } \\
\text { as } \mathrm{CaCO}_{3} \\
\text { purged } \\
\text { code }\end{array}$ & $\begin{array}{l}159.4 \\
127.8\end{array}$ & $\begin{array}{l}159.4 \\
127.8\end{array}$ & $\begin{array}{l}158.7 \\
128.5\end{array}$ & $\begin{array}{l}164.9 \\
122.3\end{array}$ & $\begin{array}{l}\mathrm{ft} \mathrm{ms!} \\
\mathrm{ft} \\
\mathrm{pH} \\
\mu \mathrm{S} / \mathrm{cm} \\
{ }^{\circ} \mathrm{C} \\
\mathrm{mg} / \mathrm{L} \\
\text { NTU } \\
\text { well vol. }\end{array}$ & \\
\hline
\end{tabular}

\section{WELL BGO 26D}

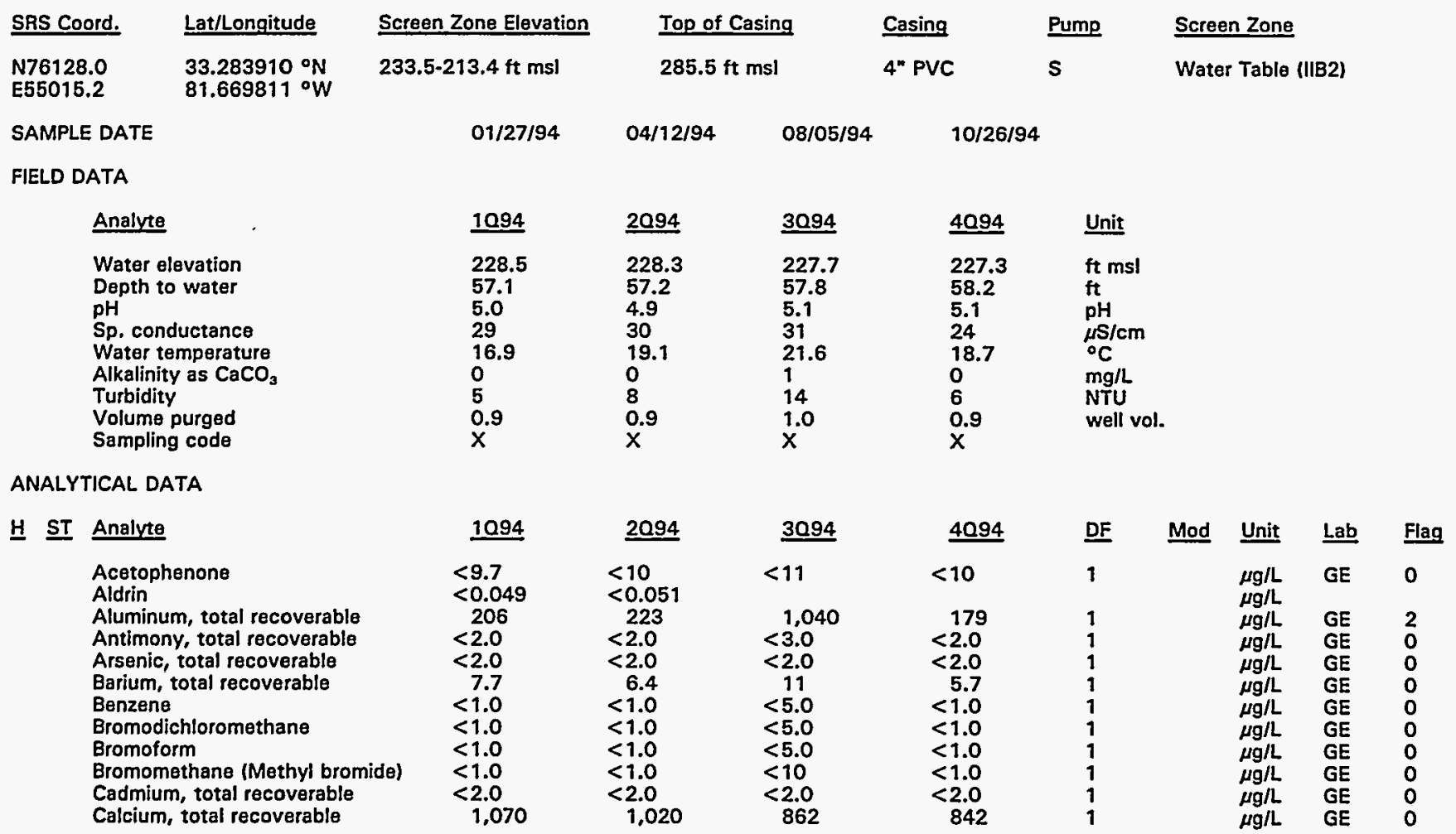

Note: Flagging, dilution factors, modifiers, and laboratories are for fourth quarter 1994 data only. See Appendix B for flagging criteria. - = exceeded holding time for fourth quarter 1994.

- = exceeded screening level or final PDWS for fourth quarter 1994. 
H $\underline{\text { ST }}$ Analyte

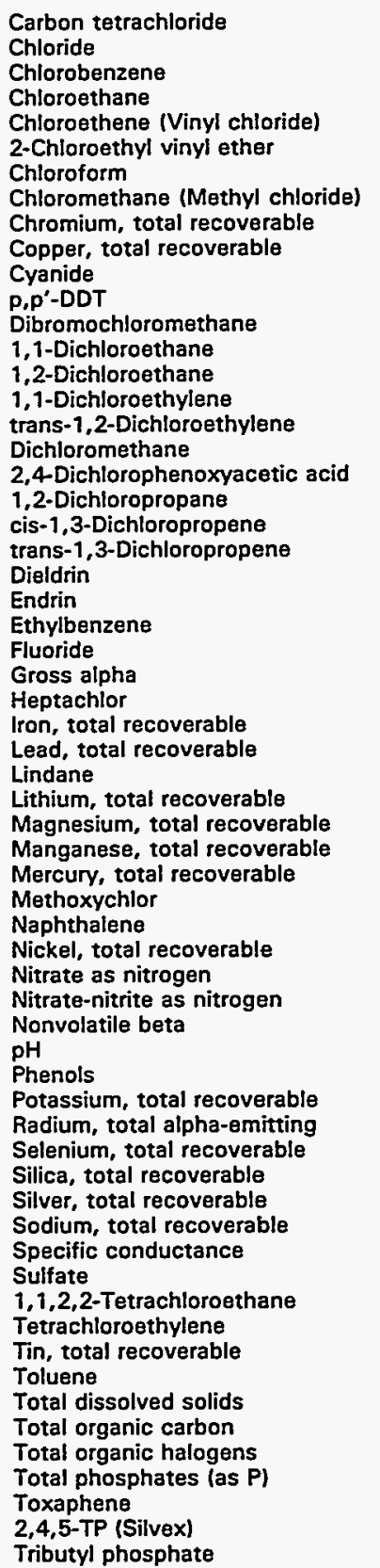

\begin{tabular}{|c|c|c|}
\hline 1094 & 2094 & 3094 \\
\hline$<1.0$ & $<1.0$ & $<5.0$ \\
\hline $\begin{array}{l}2,340 \\
<1.0\end{array}$ & $\begin{array}{l}2,400 \\
<1.0\end{array}$ & $\begin{aligned} & 2,510 \\
&<5.0\end{aligned}$ \\
\hline$<1.0$ & $<1.0$ & $<10$ \\
\hline$<1.0$ & $<1.0$ & $<10$ \\
\hline$<1.0$ & $<1.0$ & $<10$ \\
\hline$<1.0$ & $<1.0$ & $<5.0$ \\
\hline$<1.0$ & $<1.0$ & 3.5 \\
\hline$<4.0$ & $<4.0$ & $<4.0$ \\
\hline 67 & 37 & 321 \\
\hline $\begin{array}{l}<5.0 \\
<0.098\end{array}$ & $\begin{array}{l}<5.0 \\
<0.10\end{array}$ & $<5.0$ \\
\hline$<1.0$ & $<1.0$ & $<5.0$ \\
\hline$<1.0$ & $<1.0$ & $<5.0$ \\
\hline$<1.0$ & $<1.0$ & $<5.0$ \\
\hline$<1.0$ & $<1.0$ & $<5.0$ \\
\hline$<1.0$ & $<1.0$ & $<5.0$ \\
\hline$<1.0$ & $<1.0$ & $<5.0$ \\
\hline$<0.0015$ & $<0.0016$ & $<1.0$ \\
\hline$<1.0$ & $<1.0$ & $<5.0$ \\
\hline$<1.0$ & $<1.0$ & $<5.0$ \\
\hline $\begin{array}{l}<1.0 \\
<0.49\end{array}$ & $\begin{array}{l}<1.0 \\
<0.51\end{array}$ & $<5.0$ \\
\hline$<0.0059$ & $<0.0061$ & $<0.11$ \\
\hline$<1.0$ & $<1.0$ & $<5.0$ \\
\hline $\begin{array}{l}<100 \\
3.3 E+00\end{array}$ & $\begin{array}{l}<100 \\
1.8 E+00\end{array}$ & $\begin{array}{l}<100 \\
2.0 E+00\end{array}$ \\
\hline$<0.049$ & $<0.051$ & \\
\hline $\begin{array}{l}170 \\
12\end{array}$ & $\begin{array}{l}151 \\
97\end{array}$ & $\begin{array}{l}988 \\
59\end{array}$ \\
\hline$<0.0049$ & $<0.0051$ & $<0.052$ \\
\hline$<5.0$ & & $<5.0$ \\
\hline 527 & 430 & 469 \\
\hline $\begin{array}{c}16 \\
<0.20\end{array}$ & 12 & 19 \\
\hline $\begin{array}{l}<0.20 \\
<0.49\end{array}$ & $\begin{array}{r}0.24 \\
<0.51\end{array}$ & $\begin{array}{l}<0.20 \\
<0.53\end{array}$ \\
\hline$<9.7$ & $<10$ & $<10$ \\
\hline$<4.0$ & $<4.0$ & $\begin{array}{r}<4.0 \\
897\end{array}$ \\
\hline 1,010 & 910 & \\
\hline 3. $6 E+00$ & $1.6 E+00$ & 7.4E-01 \\
\hline $\begin{array}{l}5.8 \\
<10\end{array}$ & $\begin{array}{r}5.6 \\
<5.0\end{array}$ & $\begin{array}{r}5.2 \\
<5.0\end{array}$ \\
\hline$<500$ & $<500$ & $<500$ \\
\hline $1.5 E+\infty 0$ & 5.0E-01 & $1.0 E+00$ \\
\hline$<2.0$ & $<2.0$ & $<2.0$ \\
\hline 8,270 & 7,840 & 6,150 \\
\hline$<2.0$ & $\begin{array}{l}<2.0 \\
2,140\end{array}$ & $<2.0$ \\
\hline 28 & 26 & 24 \\
\hline$<1,000$ & $<1,000$ & $<1,000$ \\
\hline$<1.0$ & $<1.0$ & $<5.0$ \\
\hline 1.1 & 1.3 & 1.2 \\
\hline 4.0 & $<2.0$ & $<17$ \\
\hline$<1.0$ & $<1.0$ & $<5.0$ \\
\hline $\begin{array}{r}29,000 \\
<1,000\end{array}$ & $\begin{aligned} & 22,000 \\
&<1,000\end{aligned}$ & $\begin{array}{r}38,000 \\
<1,000\end{array}$ \\
\hline 12 & 10 & 25 \\
\hline $\begin{array}{l}<50 \\
<0.24\end{array}$ & $\begin{array}{l}<50 \\
<0.24\end{array}$ & $\begin{aligned} & 1.740 \\
&<1.1\end{aligned}$ \\
\hline$<0.00045$ & $\begin{array}{l}<0.00047 \\
<10\end{array}$ & $<0.52$ \\
\hline$<9.1$ & $<10$ & $<10$ \\
\hline
\end{tabular}

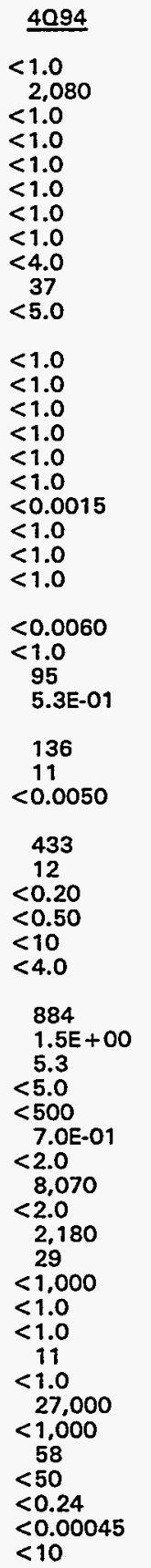

\begin{tabular}{|c|c|c|c|c|}
\hline DF & Mod & Unit & $\underline{L a b}$ & Flag \\
\hline 1 & & $\mu g / L$ & GE & 0 \\
\hline 1 & & $\mu g / L$ & GE & 0 \\
\hline 1 & & $\mu \mathrm{g} / \mathrm{L}$ & GE & 0 \\
\hline 1 & & $\mu \mathrm{g} / \mathrm{L}$ & GE & 0 \\
\hline 1 & & $\mu g / L$ & GE & 0 \\
\hline 1 & & $\mu \mathrm{g} / \mathrm{L}$ & GE & 0 \\
\hline 1 & & $\mu \mathrm{g} / \mathrm{L}$ & $\mathrm{GE}$ & 0 \\
\hline 1 & & $\mu g / L$ & GE & 0 \\
\hline 1 & & $\mu \mathrm{g} / \mathrm{L}$ & GE & 0 \\
\hline 1 & & $\mu \mathrm{g} / \mathrm{L}$ & GE & 0 \\
\hline 1 & & $\begin{array}{l}\mu \mathrm{g} / \mathrm{L} \\
\mu \mathrm{g} / \mathrm{L}\end{array}$ & GE & 0 \\
\hline 1 & & $\mu g / L$ & GE & 0 \\
\hline 1 & & $\mu \mathrm{g} / \mathrm{L}$ & GE & 0 \\
\hline 1 & & $\mu \mathrm{g} / \mathrm{L}$ & GE & 0 \\
\hline 1 & & $\mu g / L$ & GE & 0 \\
\hline 1 & & $\mu g / L$ & GE & 0 \\
\hline 1 & & $\mu \mathrm{g} / \mathrm{L}$ & GE & 0 \\
\hline 1 & & $\mu \mathrm{g} / \mathrm{L}$ & GE & 0 \\
\hline 1 & & $\mu \mathrm{g} / \mathrm{L}$ & GE & 0 \\
\hline 1 & & $\mu g / L$ & GE & 0 \\
\hline 1 & & $\begin{array}{l}\mu \mathrm{g} / \mathrm{L} \\
\mu \mathrm{g} / \mathrm{L}\end{array}$ & GE & 0 \\
\hline 1 & & $\mu \mathrm{g} / \mathrm{L}$ & GE & 0 \\
\hline 1 & & $\mu g / L$ & GE & 0 \\
\hline $\begin{array}{l}1 \\
1\end{array}$ & UI & $\begin{array}{l}\mu g / L \\
\mathrm{pCi} / \mathrm{L}\end{array}$ & GE & $\begin{array}{l}0 \\
0\end{array}$ \\
\hline 1 & & $\mu \mathrm{g} / \mathrm{L}$ & & \\
\hline 1 & & $\begin{array}{l}\mu g / L \\
\mu g / L\end{array}$ & $\begin{array}{l}\mathrm{GE} \\
\mathrm{GE}\end{array}$ & $\begin{array}{l}0 \\
0\end{array}$ \\
\hline 1 & & $\mu \mathrm{g} / \mathrm{L}$ & GE & 0 \\
\hline 1 & & $\mu g / \mathrm{L}$ & GE & 0 \\
\hline 1 & & $\mu \mathrm{g} / \mathrm{L}$ & GE & 0 \\
\hline 1 & & $\mu g / L$ & GE & 0 \\
\hline 1 & & ${ }_{\mu \mathrm{g} / \mathrm{L} / \mathrm{L}}$ & $\begin{array}{l}\text { GE } \\
\text { GE }\end{array}$ & $\begin{array}{l}0 \\
0\end{array}$ \\
\hline$i$ & & $\mu \mathrm{g} / \mathrm{L}$ & GE & 0 \\
\hline 1 & & $\mu \mathrm{g} / \mathrm{L}$ & GE & 0 \\
\hline 1 & UI & pCi/L & GP & 0 \\
\hline 1 & J & $\mathrm{pH}$ & GE & 0 \\
\hline 1 & & $\underset{\mu \mathrm{g} / \mathrm{L}}{\mu}$ & $\begin{array}{l}\text { GE } \\
\text { GE }\end{array}$ & $\begin{array}{l}0 \\
0\end{array}$ \\
\hline 1 & $J$ & $\mathrm{pCi} / \mathrm{L}$ & GP & 0 \\
\hline $\begin{array}{l}1 \\
1\end{array}$ & & $\mu g / L$ & GE & 0 \\
\hline $\begin{array}{l}1 \\
1\end{array}$ & & $\mu g / L$ & GI & 0 \\
\hline $\begin{array}{l}1 \\
1\end{array}$ & & $\mu g / L$ & GE & 0 \\
\hline$i$ & & $\begin{array}{l}\mu \mathrm{g} / \mathrm{L} \\
\mu \mathrm{S} / \mathrm{cm}\end{array}$ & $\begin{array}{l}\mathrm{GE} \\
\mathrm{GE}\end{array}$ & 0 \\
\hline 1 & & $\mu \mathrm{g} / \mathrm{L}$ & GE & 0 \\
\hline 1 & & $\mu \mathrm{g} / \mathrm{L}$ & GE & 0 \\
\hline 1 & & $\mu \mathrm{g} / \mathrm{L}$ & GE & 0 \\
\hline 1 & & $\mu g / L$ & GE & 1 \\
\hline 1 & & $\mu \mathrm{g} / \mathrm{L}$ & GE & 0 \\
\hline 1 & J & $\mu g / L$ & $\mathrm{GE}$ & 0 \\
\hline 1 & & $\mu g / L$ & GE & 0 \\
\hline $\begin{array}{l}1 \\
1\end{array}$ & & $\mu g \mathrm{~g} / \mathrm{L}$ & $\begin{array}{l}G E \\
G E\end{array}$ & $\begin{array}{l}2 \\
0\end{array}$ \\
\hline 1 & & $\mu \mathrm{g} / \mathrm{L}$ & $\mathrm{GE}$ & 0 \\
\hline 1 & & $\mu g / L$ & GE & 0 \\
\hline 1 & & $\mu \mathrm{g} / \mathrm{L}$ & GE & 0 \\
\hline
\end{tabular}

Note: Flagging, dilution factors, modifiers, and laboratories are for fourth quarter 1994 data only. See Appendix B for flagging criteria. - = exceeded holding time for fourth quarter 1994.

- = exceeded screening level or final PDWS for fourth quarter 1994. 
HE ST Analyte

1,1,1-Trichloroethane

1,1,2-Trichloroethane

- Trichloroethylene

Trichlorofluoromethane

2,4,5-T

Tritium

Turbidity

Uranium-233/234

Uranium-235

Uranium-238

Vanadium, total recoverable

Xylenes

$\begin{array}{ll}\underline{1094} & \underline{2094} \\ <1.0 & <1.0 \\ <1.0 & <1.0 \\ 3.8 & 4.7 \\ <1.0 & <1.0 \\ & <0.00047 \\ 6.5 \mathrm{E}+00 & 6.9 \mathrm{E}+00 \\ 11 & 23 \\ <3.7 \mathrm{E}-02 & <0.0 \mathrm{E}+00 \\ <0.0 \mathrm{E}+00 & <5.7 \mathrm{E}-02 \\ <4.3 \mathrm{E}-02 & <4.9 \mathrm{E}-02 \\ <8.0 & <8.0 \\ <2.0 & <2.0\end{array}$

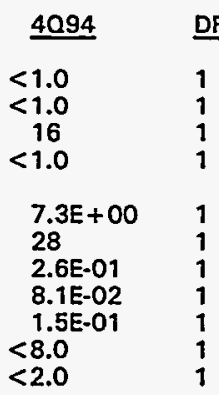

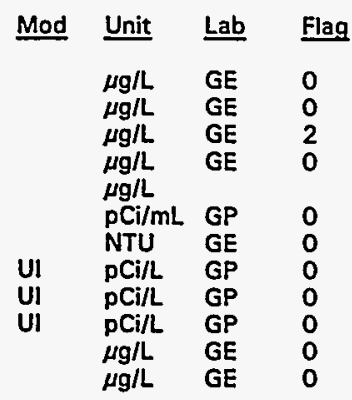

\section{WELL BGO 27C}

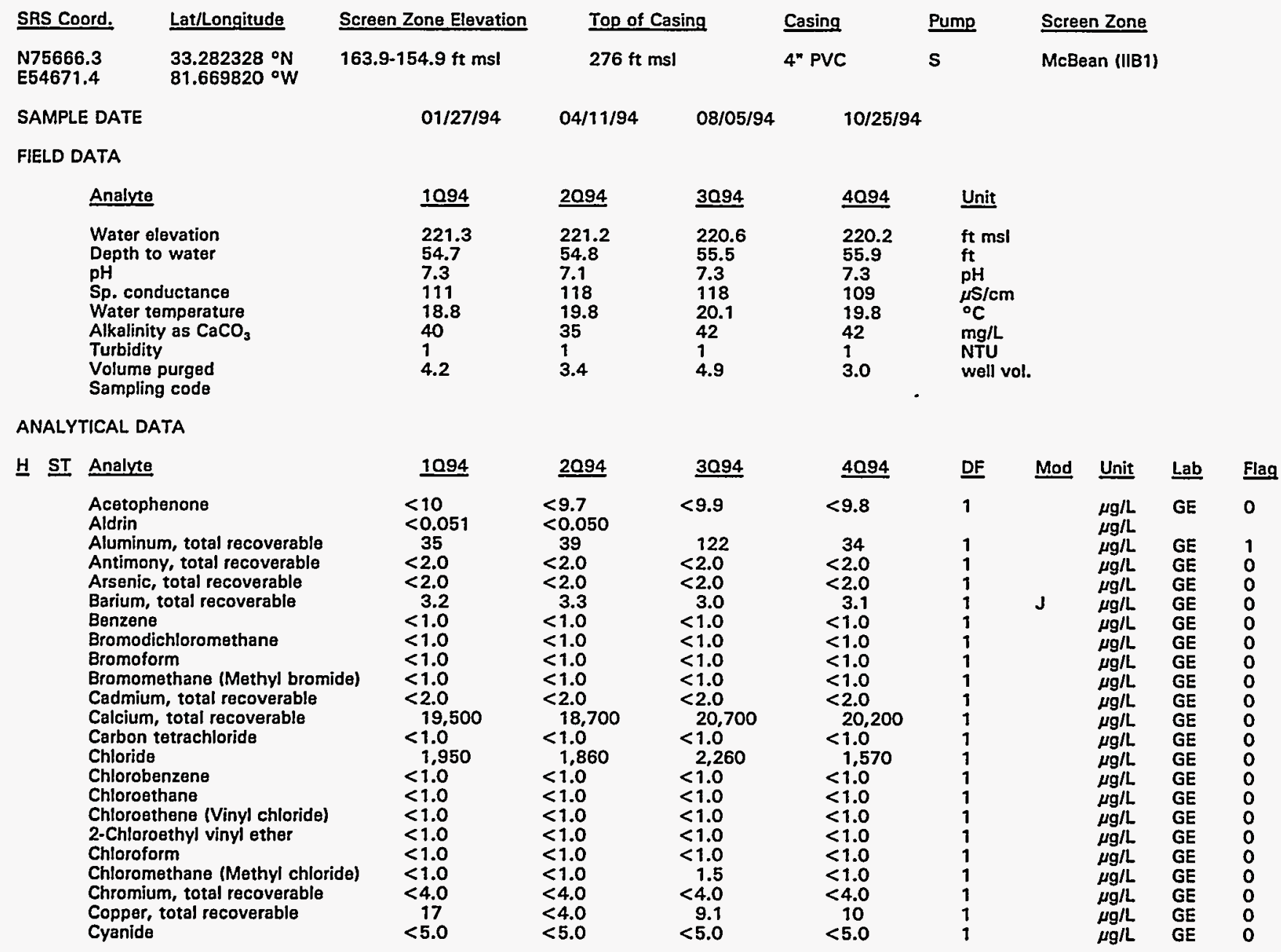

Note: Flagging, dilution factors, modifiers, and laboratories are for fourth quarter 1994 data only. See Appendix B for flagging criteria. - = exceeded holding time for fourth quarter 1994.

m = exceeded screening level or final PDWS for fourth quarter 1994. 


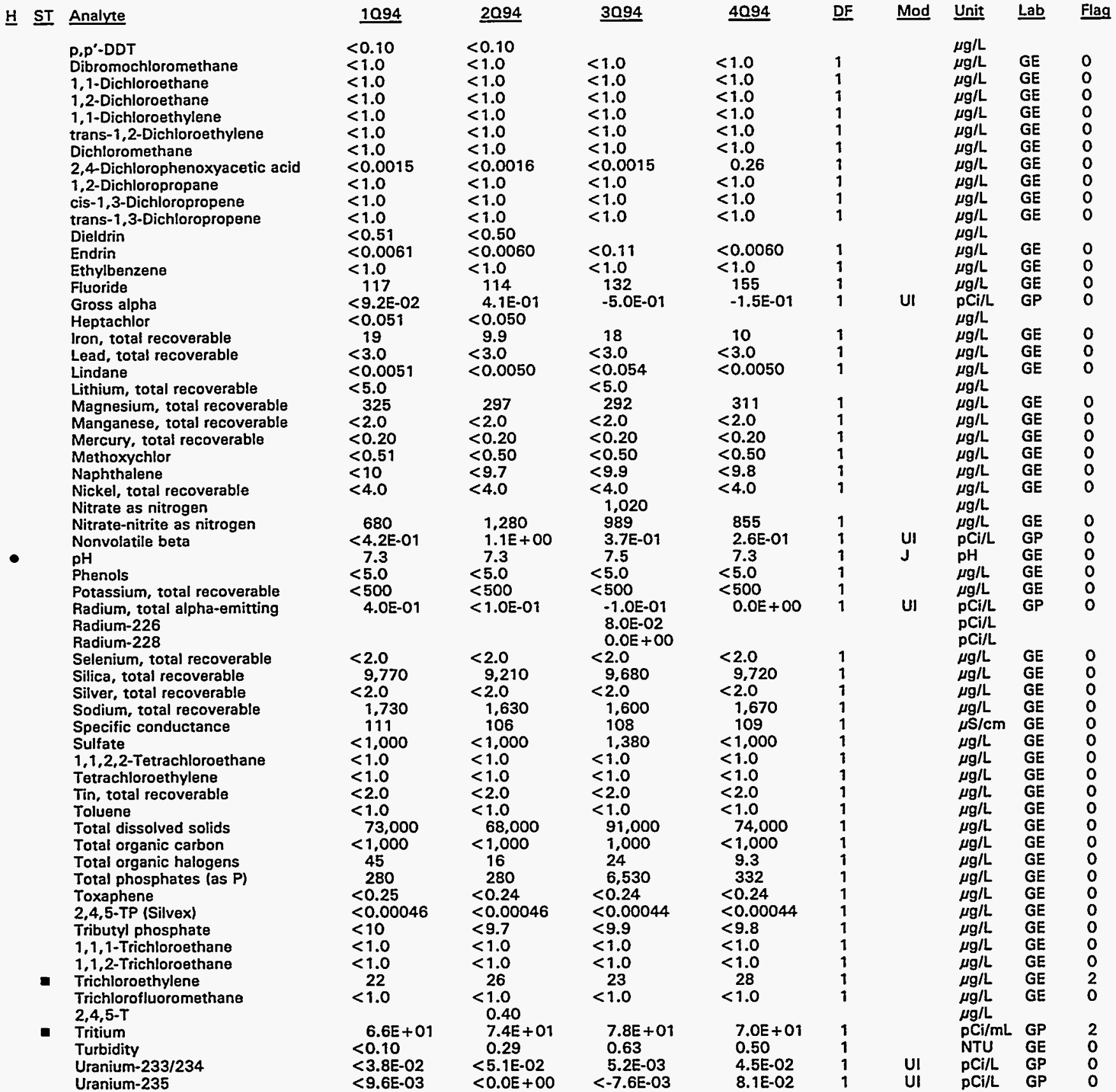

Note: Flagging, dilution factors, modifiers, and laboratories are for fourth quarter 1994 data only. See Appendix B for flagging criteria. - = exceeded holding time for fourth quarter 1994.

- = exceeded screening level or final PDWS for fourth quarter 1994. 
Well BGO 27C continued

ANALYTICAL DATA

\begin{tabular}{|c|c|c|c|c|c|c|c|c|}
\hline H ST Analyte & 1094 & $\underline{2094}$ & 3094 & 4094 & $\underline{D F}$ & Mod & Unit & Lab \\
\hline $\begin{array}{l}\text { Uranium-238 } \\
\text { Vanadium, total recoverable } \\
\text { Xylenes }\end{array}$ & $\begin{array}{l}<-4.7 E-03 \\
<8.0 \\
<2.0\end{array}$ & $\begin{array}{l}<5.5 E-02 \\
<8.0 \\
<2.0\end{array}$ & $\begin{aligned} & -1.2 \mathrm{E}-02 \\
< & 3.0 \\
< & 2.0\end{aligned}$ & $\begin{aligned} & 9.8 \mathrm{E}-03 \\
< & 8.0 \\
< & 2.0\end{aligned}$ & $\begin{array}{l}1 \\
1 \\
1\end{array}$ & UI & $\begin{array}{l}\mathrm{pCi} / \mathrm{L} \\
\mu \mathrm{g} / \mathrm{L} \\
\mu \mathrm{g} / \mathrm{L}\end{array}$ & $\begin{array}{l}\mathrm{GP} \\
\mathrm{GE} \\
\mathrm{GE}\end{array}$ \\
\hline
\end{tabular}

\section{WELL BGO 27D}

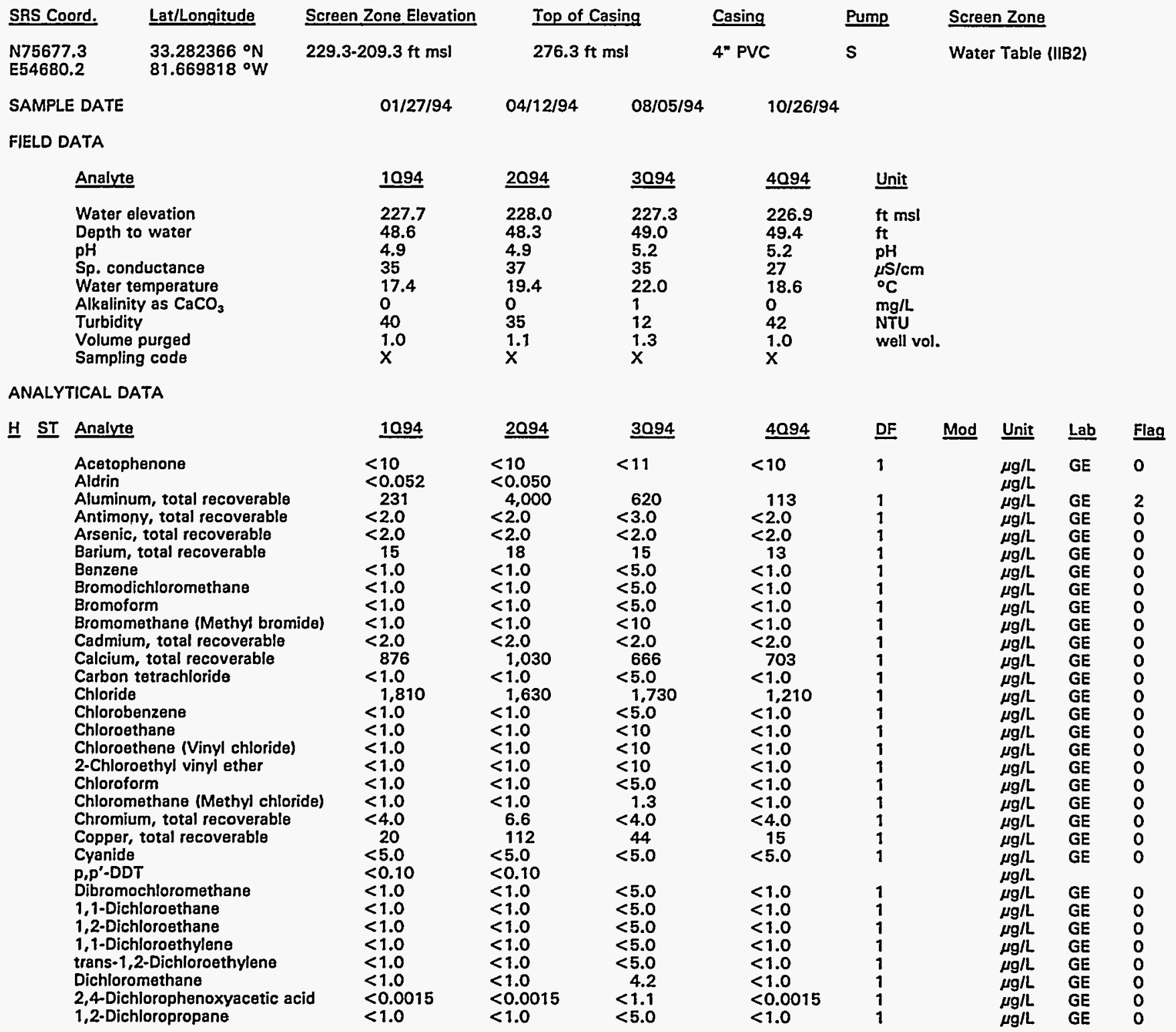

Note: Flagging, dilution factors, modifiers, and laboratories are for fourth quarter 1994 data only. See Appendix B for flagging criteria.

- = exceeded holding time for fourth quarter 1994.

m = exceeded screening level or final PDWS for fourth quarter 1994. 
ANALYTICAL DATA

H ST Analvte

cis-1,3-Dichloropropene trans-1,3-Dichloropropene

Dieldrin

Endrin

Ethylbenzene

Fluoride

Gross alpha

Heptachlor

Iron, total recoverable

Lead, total recoverable

Lindane

Lithium, total recoverable

Magnesium, total recoverable

Manganese, total recoverable

Mercury, total recoverable

Methoxychlor

Naphthalene

Nickel, total recoverable

Nitrate as nitrogen

Nitrate-nitrite as nitrogen

Nonvolatile beta

- $\mathrm{pH}$

Phenols

Potassium, total recoverable

Radium, total alpha-emitting

Selenium, total recoverable

Silica, total recoverable

Silver, total recoverable

Sodium, total recoverable

Specific conductance

Sulfate

1,1,2,2-Tetrachloroethane

Tetrachloroethylene

Tin, total recoverable

Toluene

Total dissolved solids

Total organic carbon

Total organic halogens

Total phosphates (as P)

Toxaphene

2,4,5-TP (Silvex)

Tributyl phosphate

1,1,1-Trichloroethane

1,1,2-Trichloroethane

Trichloroethylene

Trichlorofluoromethane

2,4,5-T

- Tritium

Turbidity

Uranium-233/234

Uranium-235

Uranium-238

Vanadium, total recoverable

Xylenes

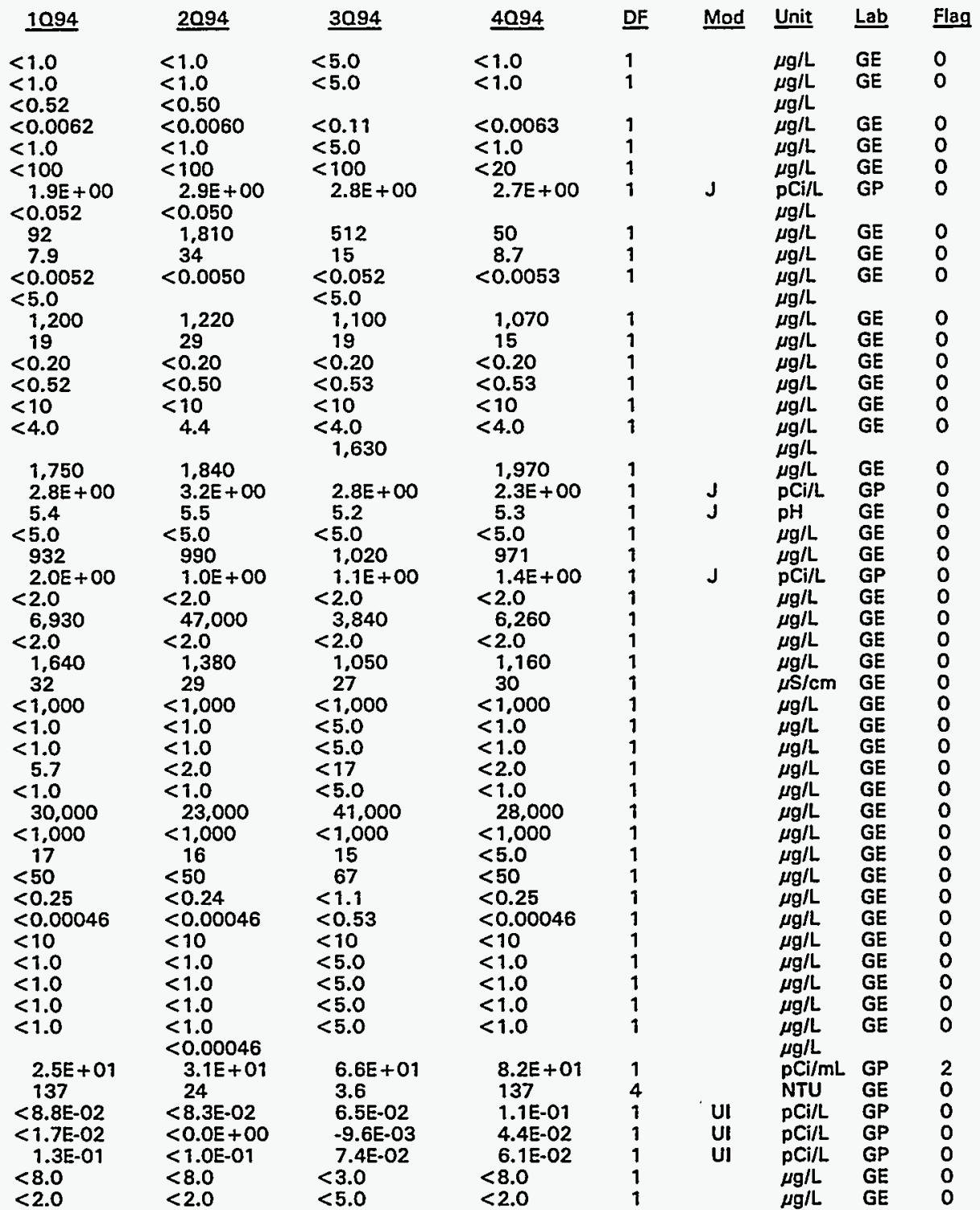

Note: Flagging, dilution factors, modifiers, and laboratories are for fourth quarter 1994 data only. See Appendix B for flagging criteria. - = exceeded holding time for fourth quarter 1994.

- = exceeded screening level or final PDWS for fourth quarter 1994. 
WELL BGO 28D

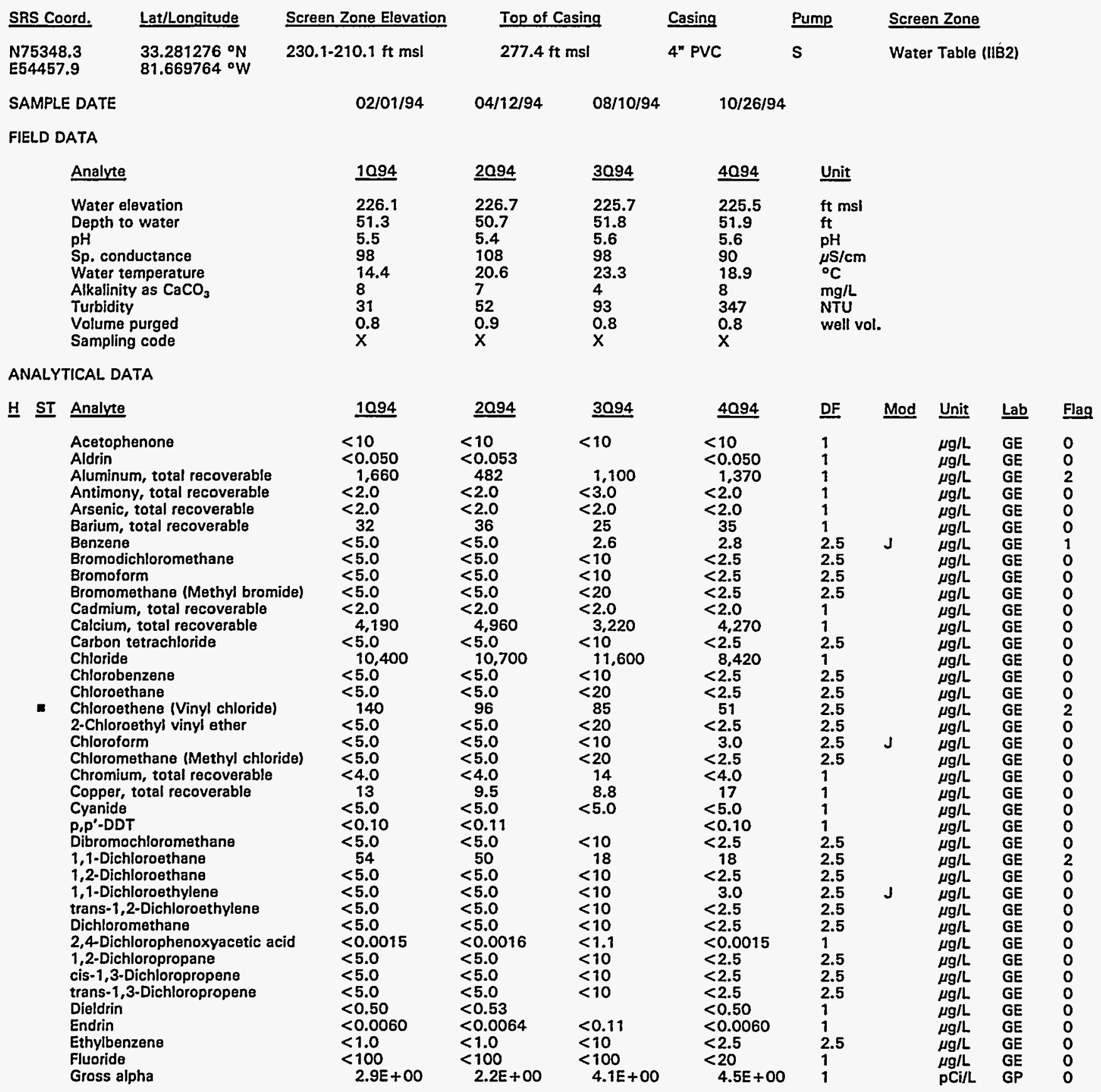

Note: Flagging, dilution factors, modifiers, and laboratories are for fourth quarter 1994 data only. See Appendix B for flagging criteria. - = exceeded holding time for fourth quarter 1994.

- = exceeded screening level or final PDWS for fourth quarter 1994. 


\section{ANALYTICAL DATA}

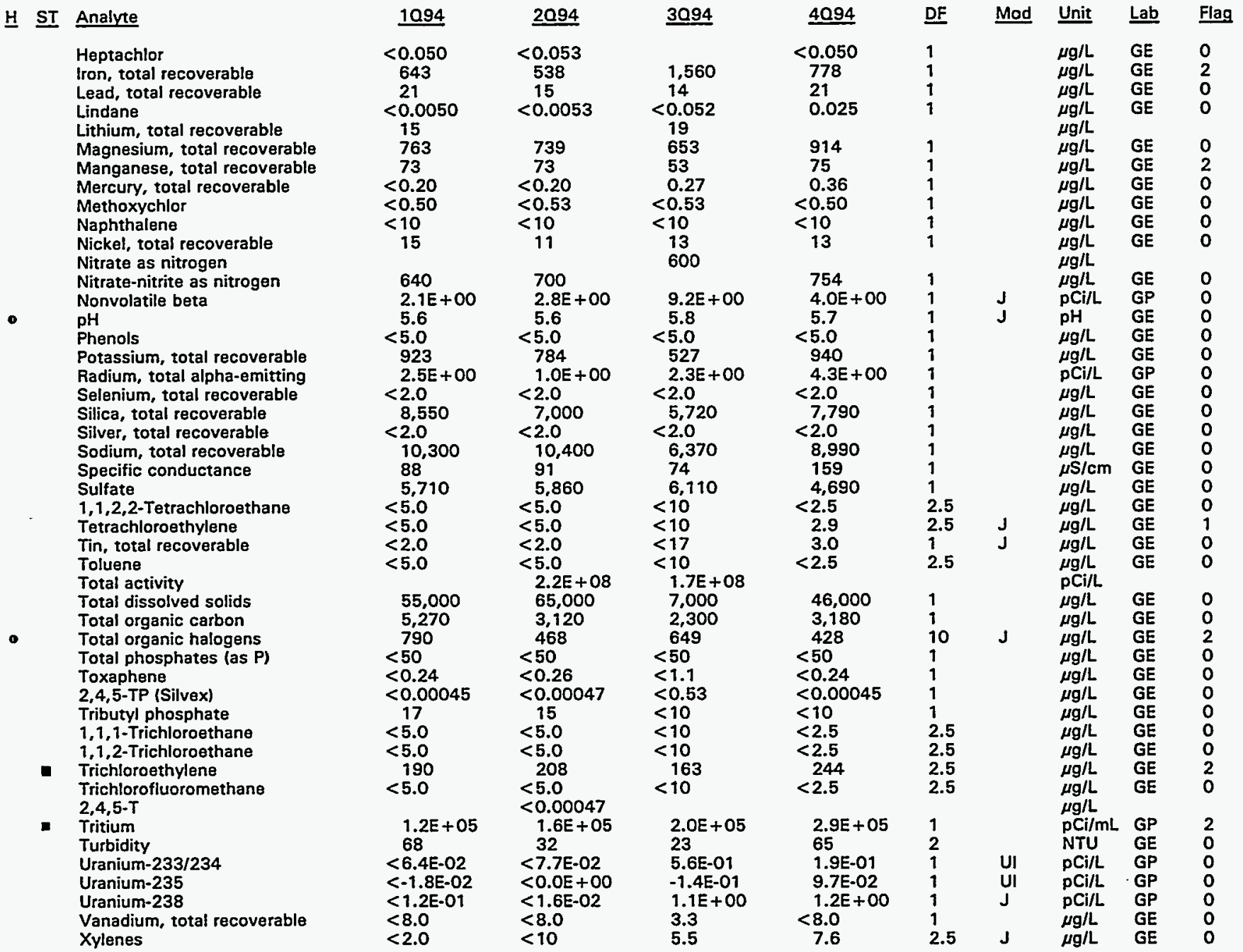

Note: Flagging, dilution factors, modifiers, and laboratories are for fourth quarter 1994 data only. See Appendix B for flagging criteria. - = exceeded holding time for fourth quarter 1994.

a = exceeded screening level or final PDWS for fourth quarter 1994. 
WELL BGO 29A

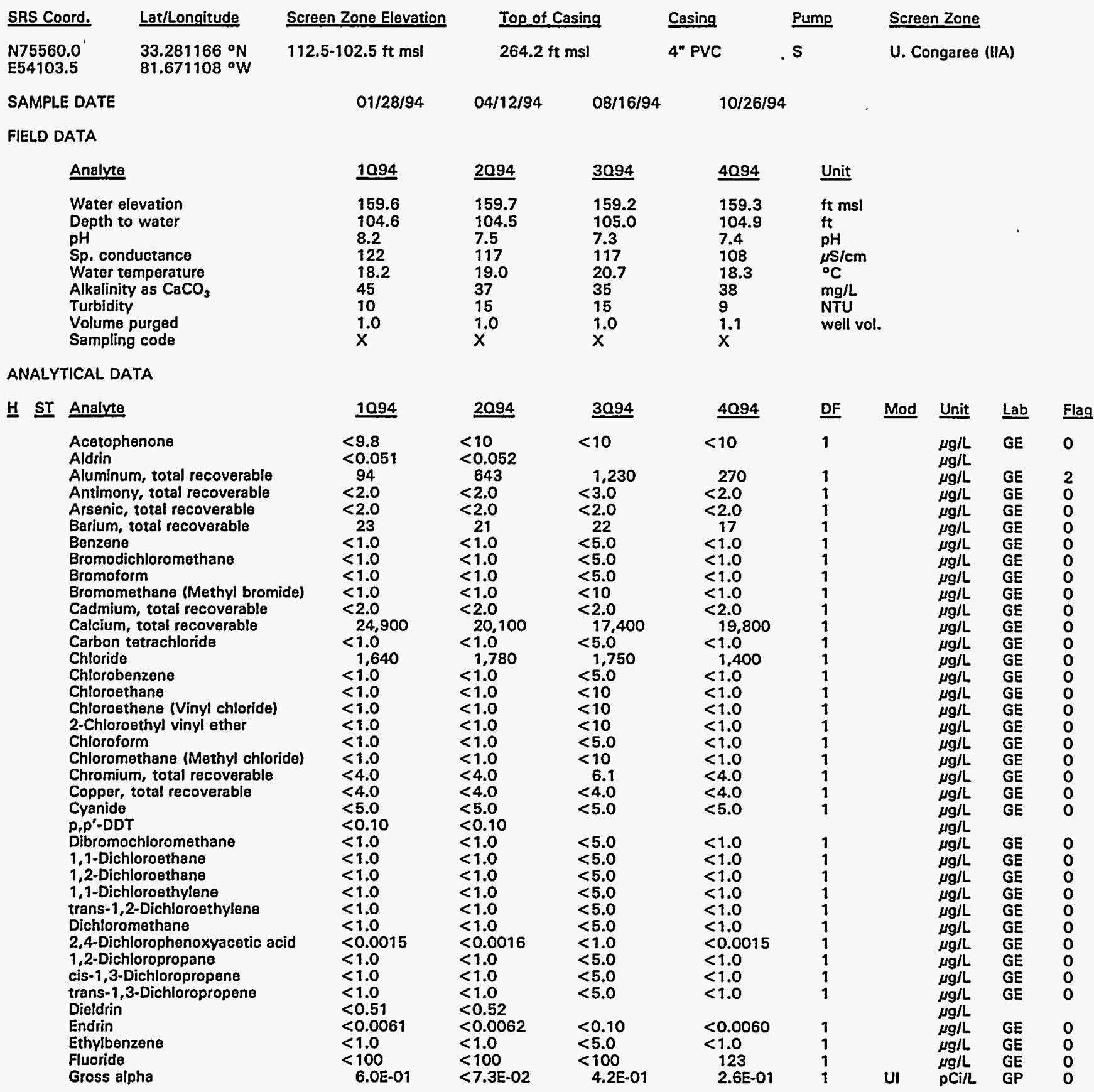

Note: Flagging, dilution factors, modifiers, and laboratories are for fourth quarter 1994 data only. See Appendix B for flagging criteria. - = exceeded holding time for fourth quarter 1994.

- = exceeded screening level or final PDWS for fourth quarter 1994. 


\section{ANALYTICAL DATA}

\section{H $\underline{\text { ST }}$ Analyte}

Heptachlor

Iron, total recoverable

Lead, total recoverable

Lindane

Lithium, total recoverable

Magnesium, total recoverable

Manganese, total recoverable

Mercury, total recoverable

Methoxychlor

Naphthalene

Nickel, total recoverable

Nitrate as nitrogen

Nitrate-nitrite as nitrogen

Nonvolatile beta

- $\mathrm{pH}$

Phenols

Potassium, total recoverable

Radium, total alpha-emitting

Selenium, total recoverable

Silica, total recoverable

Silver, total recoverable

Sodium, total recoverable

Specific conductance

Sulfate

1,1,2,2-Tetrachloroethane

Tetrachloroethylene

Tin, total recoverable

Toluene

Total dissolved solids

Total organic carbon

Total organic halogens

Total phosphates (as P)

Toxaphene

2,4,5-TP (Silvex)

Tributyl phosphate

1,1,1-Trichloroethane

1,1,2-Trichloroethane

Trichloroethylene

Trichlorofluoromethane

2,4,5-T

Tritium

Turbidity

Uranium-233/234

Uranium-235

Uranium-238

Vanadium, total recoverable

Xylenes

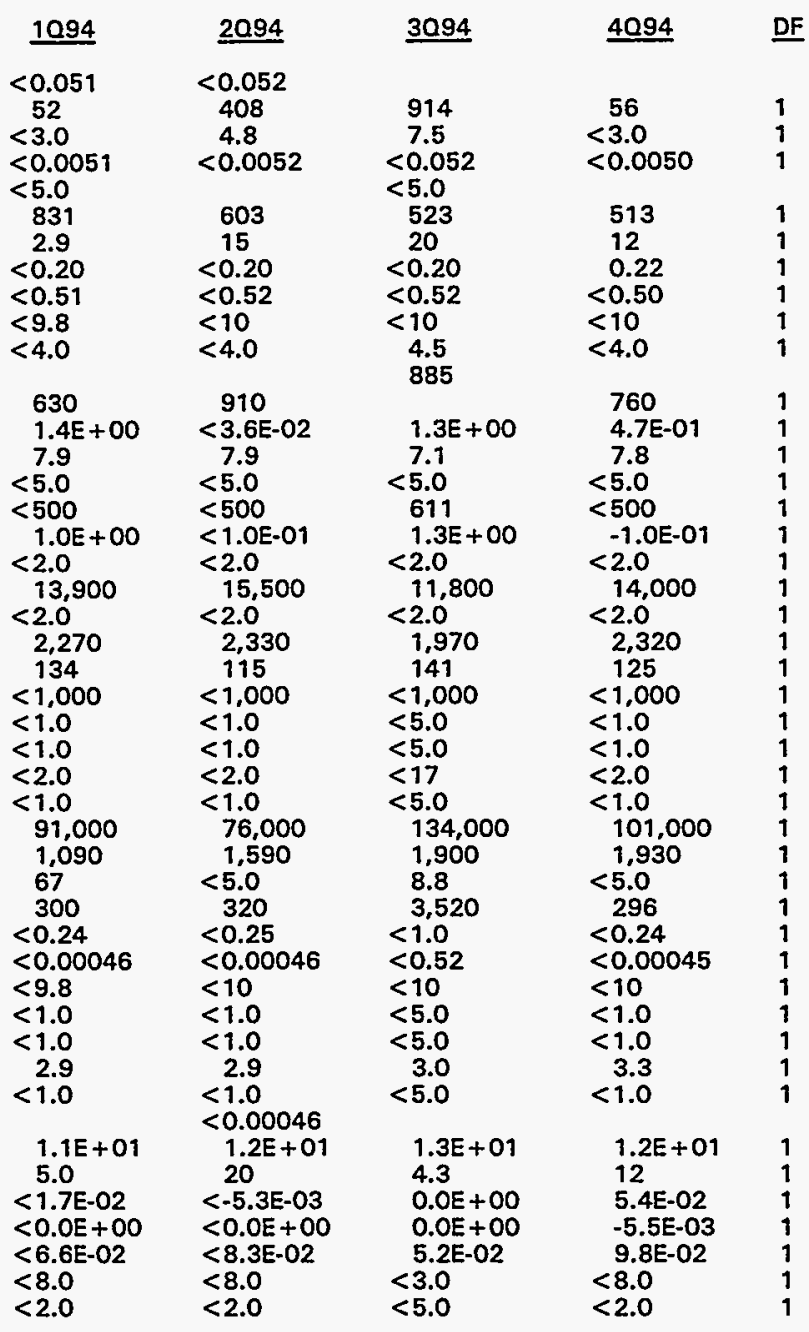

\begin{tabular}{|c|c|c|c|}
\hline Mad & $\underline{\text { Unit }}$ & $\underline{\text { Lab }}$ & Fla \\
\hline \multirow{7}{*}{$\mathbf{J}$} & $\mu g / L$ & & \\
\hline & $\mu \mathrm{g} / \mathrm{L}$ & GE & 0 \\
\hline & $\mu g / L$ & GE & 0 \\
\hline & $\mu \mathrm{g} / \mathrm{L}$ & & \\
\hline & $\mu \mathrm{g} / \mathrm{L}$ & GE & 0 \\
\hline & $\mu \mathrm{g} / \mathrm{L}$ & GE & $\begin{array}{l}0 \\
0\end{array}$ \\
\hline & $\mu \mathrm{g} / \mathrm{L}$ & $\overline{G E}$ & 0 \\
\hline & $\mu g / L$ & $\mathrm{GE}$ & 0 \\
\hline & $\begin{array}{l}\mu \mathrm{g} / \mathrm{L} \\
\mu \mathrm{g} / \mathrm{L}\end{array}$ & & 0 \\
\hline & $\mu \mathrm{g} / \mathrm{L}$ & $\mathrm{GE}$ & 0 \\
\hline U1 & pCi/L & GP & 0 \\
\hline \multirow[t]{2}{*}{$\vec{J}$} & $\mathrm{pH}$ & GE & 0 \\
\hline & $\underset{\mu \mathrm{g} / \mathrm{L}}{\mu}$ & $\begin{array}{l}\mathrm{GE} \\
\mathrm{GE}\end{array}$ & $\begin{array}{l}0 \\
0\end{array}$ \\
\hline \multirow[t]{8}{*}{ U: } & pCi/L & $G P$ & 0 \\
\hline & $\underset{\mu \mathrm{g} / \mathrm{L}}{\mu \mathrm{g} / \mathrm{L}}$ & $\begin{array}{l}\mathrm{GE} \\
\mathrm{GE}\end{array}$ & 0 \\
\hline & $\mu \mathrm{g} / \mathrm{L}$ & $\mathrm{GE}$ & 0 \\
\hline & $\mu \mathrm{g} / \mathrm{L}$ & GE & 0 \\
\hline & $\begin{array}{l}\mu \mathrm{S} / \mathrm{cm} \\
\mu \mathrm{g} / \mathrm{L}\end{array}$ & $\begin{array}{l}\mathrm{GE} \\
\mathrm{GE}\end{array}$ & $\begin{array}{l}0 \\
0\end{array}$ \\
\hline & $\mu \mathrm{g} / \mathrm{L}$ & $\mathrm{GE}$ & 0 \\
\hline & $\begin{array}{l}\mu g / L \\
\mu g / L\end{array}$ & $\begin{array}{l}\mathrm{GE} \\
\mathrm{GE}\end{array}$ & $\begin{array}{l}0 \\
0\end{array}$ \\
\hline & $\mu \mathrm{g} / \mathrm{L}$ & GE & 0 \\
\hline \multirow[t]{10}{*}{$\mathrm{J}$} & $\mu \mathrm{g} / \mathrm{L}$ & GE & 0 \\
\hline & $\mu \mathrm{g} / \mathrm{L}$ & GE & 0 \\
\hline & $\mu \mathrm{g} / \mathrm{L}$ & $\mathrm{GE}$ & 0 \\
\hline & $\mu g / L$ & GE & 0 \\
\hline & $\begin{array}{l}\mu \mathrm{g} / \mathrm{L} \\
\mu \mathrm{g} / \mathrm{L}\end{array}$ & $\begin{array}{l}\mathrm{GE} \\
\mathrm{GE}\end{array}$ & 0 \\
\hline & $\mu g / L$ & GE & 0 \\
\hline & $\begin{array}{c}\mu \mathrm{g} / \mathrm{L} \\
\mu \mathrm{g} / \mathrm{L}\end{array}$ & $\begin{array}{l}\text { GE } \\
\text { GE }\end{array}$ & $\begin{array}{l}0 \\
1\end{array}$ \\
\hline & $\mu g / L$ & GE & 0 \\
\hline & $\begin{array}{l}\mu \mathrm{g} / \mathrm{L} \\
\mathrm{g} \mathrm{C} / \mathrm{mL}\end{array}$ & GP & 1 \\
\hline & NTU & $\mathrm{GE}$ & \\
\hline \multirow{4}{*}{$\begin{array}{l}\text { UI } \\
\text { II } \\
\text { II }\end{array}$} & $\mathrm{pCi} / \mathrm{L}$ & GP & 0 \\
\hline & $\mathrm{pCi} / \mathrm{L}$ & GP & \\
\hline & $\mathrm{pCi} / \mathrm{L}$ & GP & \\
\hline & $\underset{\mu \mathrm{g} / \mathrm{L}}{\mu \mathrm{g}}$ & $\begin{array}{l}\mathrm{GE} \\
\mathrm{GE}\end{array}$ & 0 \\
\hline
\end{tabular}

Note: Flagging, dilution factors, modifiers, and laboratories are for fourth quarter 1994 data only. See Appendix B for flagging criteria. - = exceeded holding time for fourth quarter 1994.

- = exceeded screening level or final PDWS for fourth quarter 1994. 
WELL BGO 29C

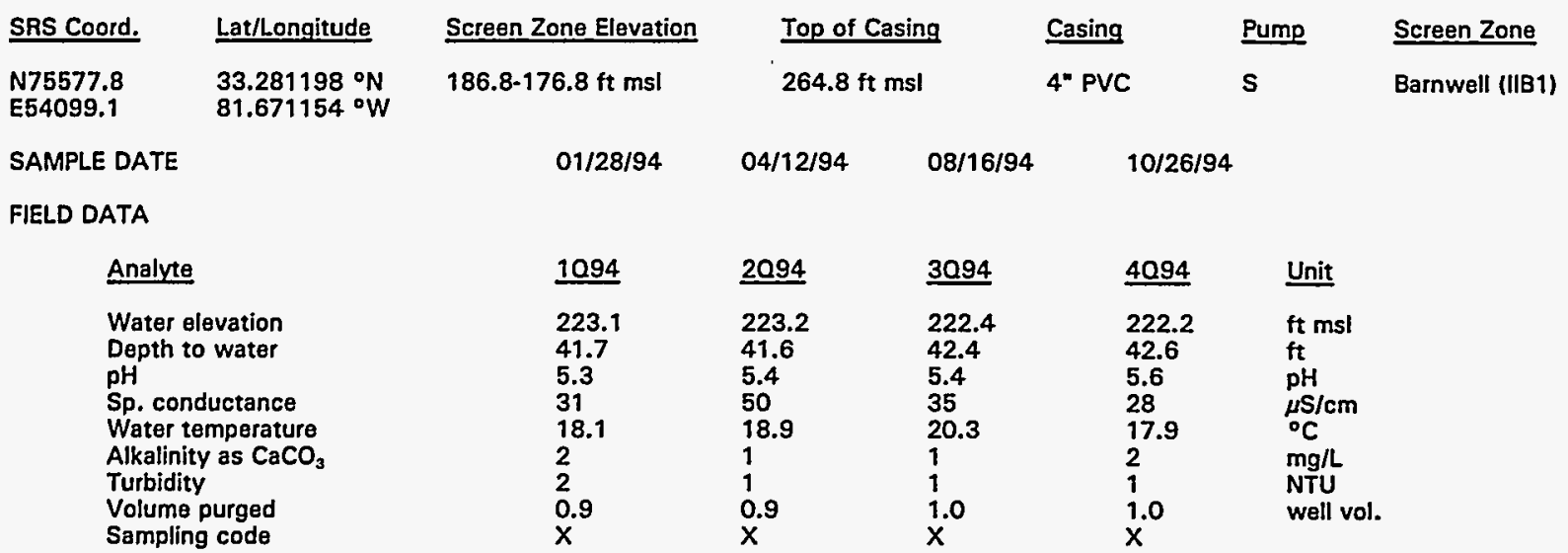

ANALYTICAL DATA

H $\underline{\text { ST }}$ Analyte

Acetophenone
Aldrin
Aluminum, total recoverable
Antimony, total recoverable
Arsenic, total recoverable
Barium, total recoverable
Benzene
Bromodichloromethane
Bromoform
Bromomethane (Methyl bromide)
Cadmium, total recoverable
Calcium, total recoverable
Carbon tetrachloride
Chloride
Chlorobenzene
Chloroethane
Chloroethene (Vinyl chloride)
2-Chloroethyl vinyl ether
Chloroform
Chloromethane (Methyl chloride)
Chromium, total recoverable
Copper, total recoverable
Cyanide
p,p'-DDr
Dibromochloromethane
1,1-Dichloroethane
1,2-Dichloroethane
1,1-Dichloroethylene
trans-1,2-Dichloroethylene
Dichloromethane
2,4-Dichlorophenoxyacetic acid
1,2-Dichloropropane
cis-1,3-Dichloropropene
trans-1,3-Dichloropropene
Dieldrin
Endrin
Ethylbenzene
Fluoride
Gross alpha

\begin{tabular}{lll}
1094 & $\underline{2094}$ & $\underline{3094}$ \\
\hline 10 & $<10$ & $<10$ \\
$<0.049$ & $<0.050$ & \\
101 & $<99$ & 54 \\
$<2.0$ & $<2.0$ & $<3.0$ \\
$<2.0$ & $<2.0$ & $<2.0$ \\
21 & 18 & 19 \\
$<1.0$ & $<1.0$ & $<5.0$ \\
$<1.0$ & $<1.0$ & $<5.0$ \\
$<1.0$ & $<1.0$ & $<5.0$ \\
$<1.0$ & $<1.0$ & $<10$ \\
$<2.0$ & $<2.0$ & $<2.0$ \\
2,710 & 2,780 & 3,310 \\
$<1.0$ & $<1.0$ & $<5.0$ \\
2,170 & 2.180 & 2,570 \\
$<1.0$ & $<1.0$ & $<5.0$ \\
$<1.0$ & $<1.0$ & $<10$ \\
$<1.0$ & $<1.0$ & $<10$ \\
$<1.0$ & $<1.0$ & $<10$ \\
$<1.0$ & $<1.0$ & $<5.0$ \\
$<1.0$ & $<1.0$ & 3.0 \\
$<4.0$ & $<4.0$ & $<4.0$ \\
$<4.0$ & $<4.0$ & $<4.0$ \\
$<5.0$ & $<5.0$ & $<5.0$ \\
$<0.098$ & $<0.10$ & $<5.0$ \\
$<1.0$ & $<1.0$ & $<5.0$ \\
$<1.0$ & $<1.0$ & $<5.0$ \\
$<1.0$ & $<1.0$ & $<5.0$ \\
$<1.0$ & $<1.0$ & $<5.0$ \\
$<1.0$ & $<1.0$ & $<5.0$ \\
$<1.0$ & $<1.0$ & $<5.0$ \\
$<0.0015$ & $<0.0016$ & $<1.0$ \\
$<1.0$ & $<1.0$ & $<5.0$ \\
$<1.0$ & $<1.0$ & $<5.0$ \\
$<1.0$ & $<1.0$ & $<5.0$ \\
$<0.49$ & $<0.50$ & \\
$<0.0059$ & $<0.0060$ & $<0.10$ \\
$<1.0$ & $<1.0$ & $<5.0$ \\
$<100$ & $<100$ & $<100$ \\
$<2.9 E-01$ & $1.0 E+00$ & $1.6 E+00$
\end{tabular}

\begin{tabular}{|c|c|c|}
\hline Mod & Unit & ㄴa \\
\hline & $\mu g / L$ & $\mathrm{GE}$ \\
\hline & $\mu g / L$ & GE \\
\hline & $\mu \mathrm{g} / \mathrm{L}$ & $\mathrm{GE}$ \\
\hline & ${ }_{\mu \mathrm{g} / \mathrm{L}} / \mathrm{L}$ & $\mathrm{GE}$ \\
\hline & $\mu \mathrm{g} / \mathrm{L}$ & GE \\
\hline & $\mu \mathrm{g} / \mathrm{L}$ & $\mathrm{GE}$ \\
\hline & $\mu \mathrm{g} / \mathrm{L}$ & GE \\
\hline & $\begin{array}{l}\mu \mathrm{g} / \mathrm{L} \\
\mu \mathrm{g} / \mathrm{L}\end{array}$ & $\begin{array}{l}\mathrm{GE} \\
\mathrm{GE}\end{array}$ \\
\hline & $\mu \mathrm{g} / \mathrm{L}$ & $\mathrm{GE}$ \\
\hline & $\mu g / L$ & GE \\
\hline & $\mu \mathrm{g} / \mathrm{L}$ & GE \\
\hline & $\mu g / L$ & $\mathrm{GE}$ \\
\hline & $\mu \mathrm{g} / \mathrm{L}$ & GE \\
\hline & $\begin{array}{l}\mu \mathrm{g} / \mathrm{L} \\
\mu \mathrm{g} / \mathrm{L}\end{array}$ & $\mathrm{GE}$ \\
\hline & $\mu \mathrm{g} / \mathrm{L}$ & GE \\
\hline & ${ }_{\mu \mathrm{g} / \mathrm{L}}$ & $\begin{array}{l}\mathrm{GE} \\
\mathrm{GE}\end{array}$ \\
\hline & $\mu g / L$ & GE \\
\hline & $\mu \mathrm{g} / \mathrm{L}$ & GE \\
\hline & $\mu \mathrm{g} / \mathrm{L}$ & $\mathrm{GE}$ \\
\hline & $\underset{\mu \mathrm{g} / \mathrm{L}}{\mu}$ & $\begin{array}{l}\mathrm{GE} \\
\mathrm{GE}\end{array}$ \\
\hline & $\mu \mathrm{g} / \mathrm{L}$ & GE \\
\hline & $\mu g / L$ & $\mathrm{GE}$ \\
\hline & $\mu \mathrm{g} / \mathrm{L}$ & GE \\
\hline & $\begin{array}{l}\mu g / L \\
\mu g / L\end{array}$ & $\begin{array}{l}\mathrm{GE} \\
\mathrm{GE}\end{array}$ \\
\hline & $\mu \mathrm{g} / \mathrm{L}$ & $\mathrm{GE}$ \\
\hline & $\mu \mathrm{g} / \mathrm{L}$ & \\
\hline & $\mu \mathrm{g} / \mathrm{L}$ & $\mathrm{GE}$ \\
\hline & $\mu \mathrm{g} / \mathrm{L}$ & GE \\
\hline $\mathbf{J}$ & $\mathbf{p C i} / \mathrm{L}$ & \\
\hline
\end{tabular}

Note: Flagging, dilution factors, modifiers, and laboratories are for fourth quarter 1994 data only. See Appendix B for flagging criteria. - = exceeded holding time for fourth quarter 1994.

- = exceeded screening level or final PDWS for fourth quarter 1994. 
Well BGO $29 \mathrm{C}$ continued

ANALYTICAL DATA

H ST Analyte

Heptachlor

Iron, total recoverable

Lead, total recoverable

Lindane

Lithium, total recoverable

Magnesium, total recoverable

Manganese, total recoverable

Mercury, total recoverable

Methoxychlor

Naphthalene

Nickel, total recoverable

Nitrate as nitrogen

Nitrate-nitrite as nitrogen

Nonvolatile beta

-

$\mathrm{pH}$

Potassium, total recoverable

Potassium, total recoverable

Selenium, total recoverable

Silica, total recoverable

Silver, total recoverable

Sodium, total recoverable

Specific conductance

Sulfate

1,1,2,2-Tetrachloroethane

Tetrachloroethylene

Tin, total recoverable

Toluene

Total dissolved solids

Total organic carbon

Total organic halogens

Total phosphates (as P)

Toxaphene

2,4,5-TP (Silvex)

Tributyl phosphate

1,1,1-Trichloroethane

1,1,2-Trichloroethane

Trichloroethylene

Trichlorofluoromethane

2,4,5-T

- Tritium

Turbidity

Uranium-233/234

Uranium-235

Uranium-238

Vanadium, total recoverable

Xylenes

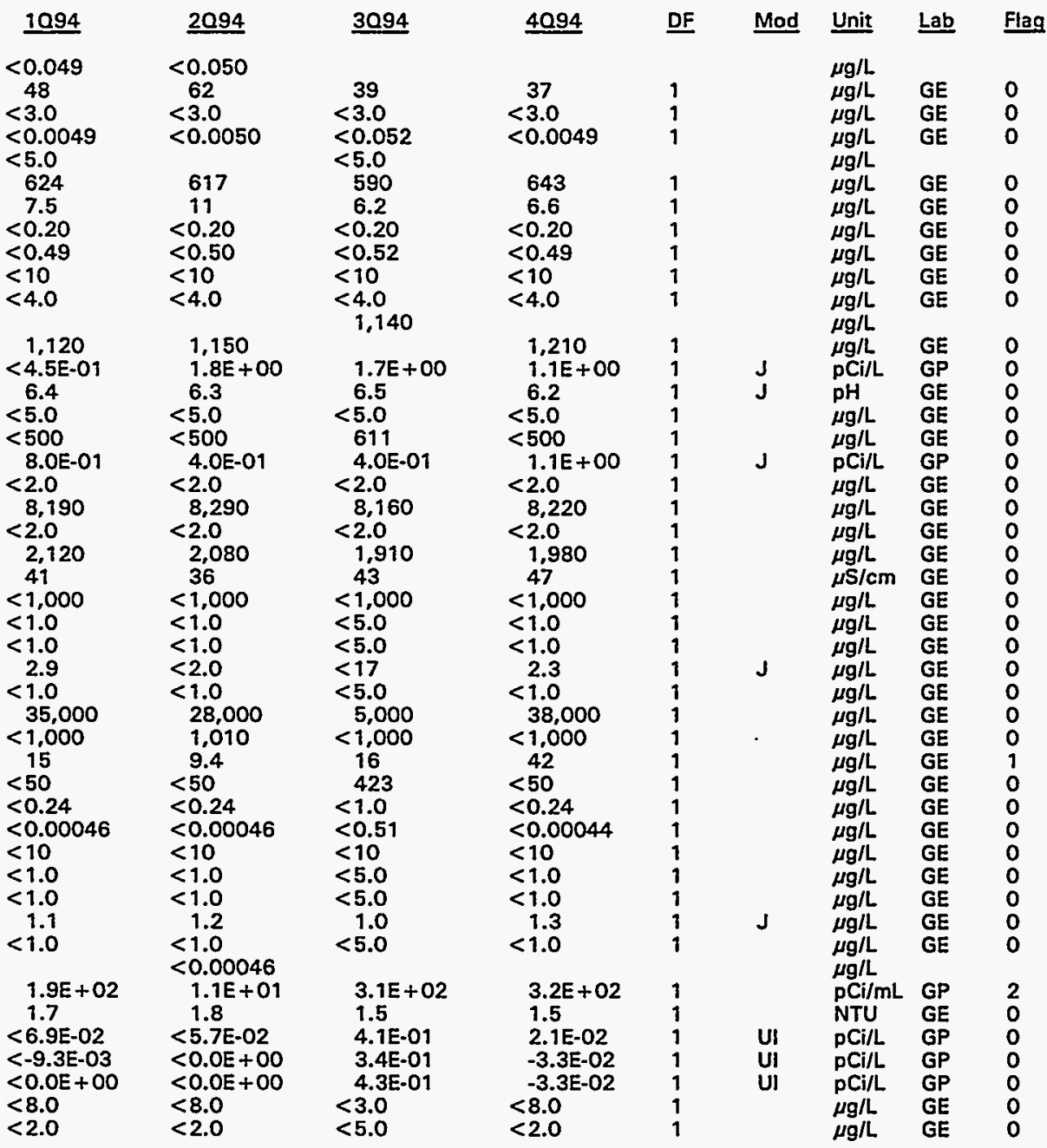

Note: Flagging, dilution factors, modifiers, and laboratories are for fourth quarter 1994 data only. See Appendix B for flagging criteria. 0 = exceeded holding time for fourth quarter 1994.

I = exceeded screening level or final PDWS for fourth quarter 1994. 
WELL BGO 29D

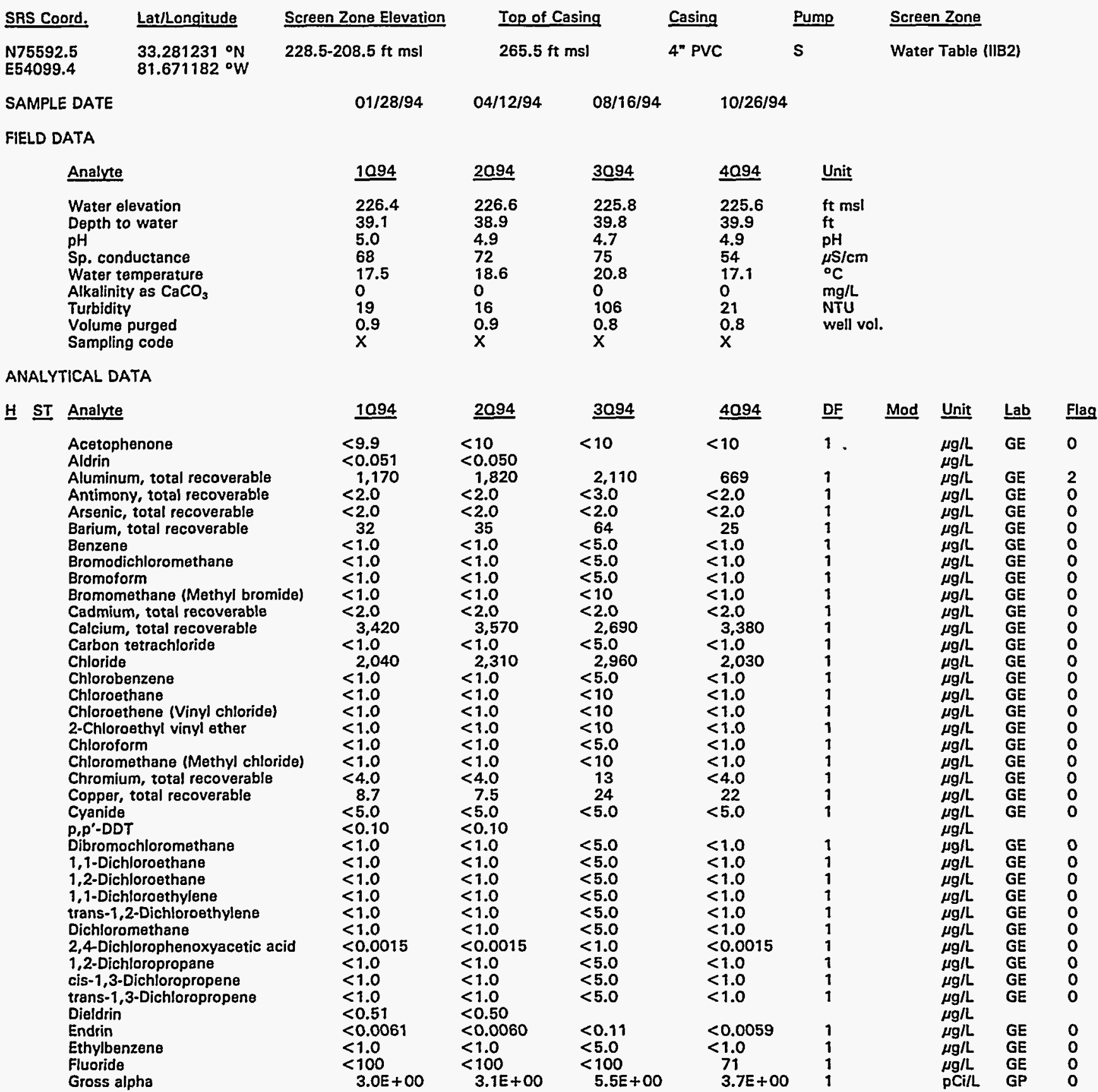

Note: Flagging, dilution factors, modifiers, and laboratories are for fourth quarter 1994 data only. See Appendix B for flagging criteria. - = exceeded holding time for fourth quarter 1994

m = exceeded screening level or final PDWS for fourth quarter 1994. 
ANALYTICAL DATA

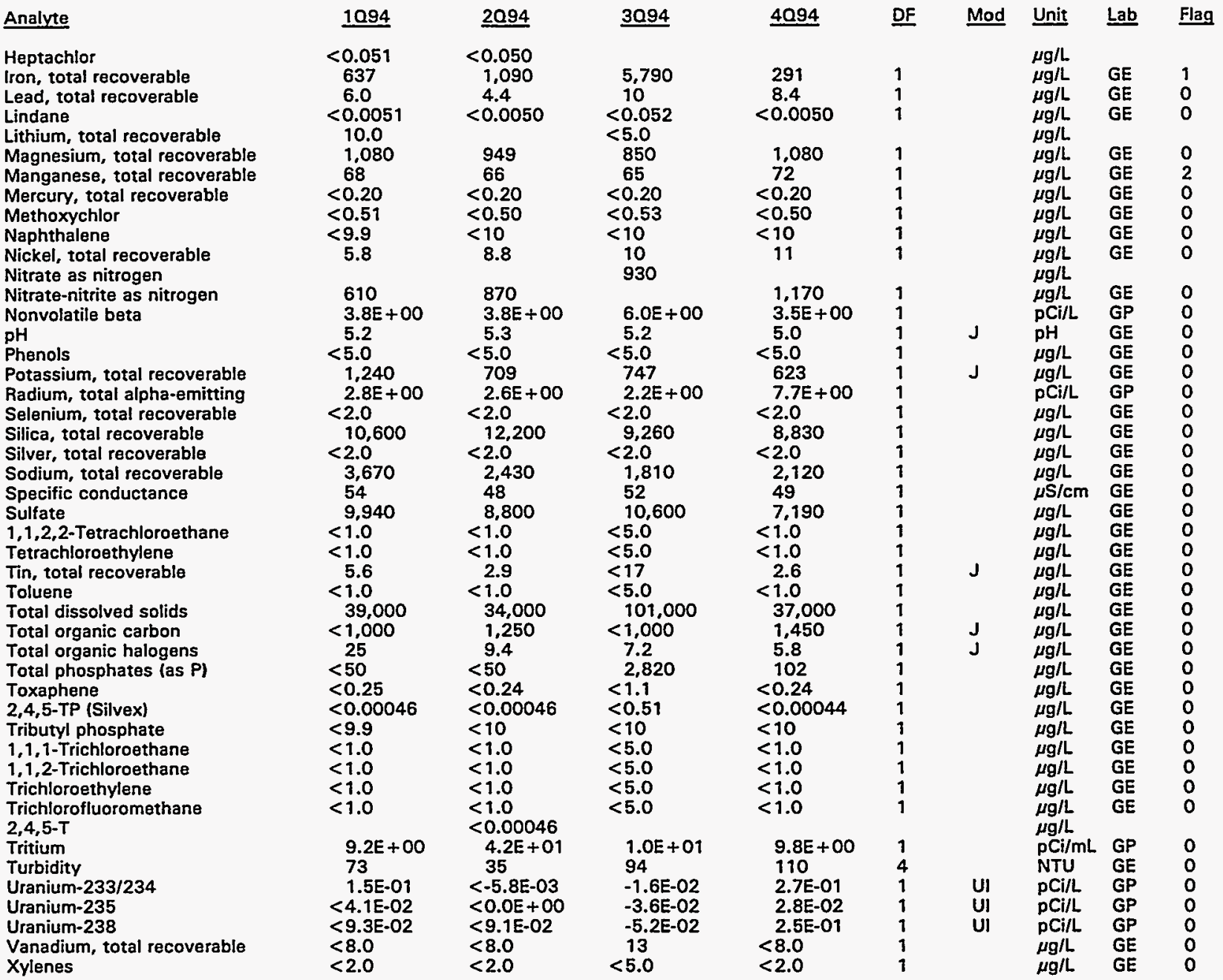

Note: Flagging, dilution factors, modifiers, and laboratories are for fourth quarter 1994 data only. See Appendix B for flagging criteria. - = exceeded holding time for fourth quarter 1994.

n = exceeded screening level or final PDWS for fourth quarter 1994. 


\section{WELL BGO 30C}

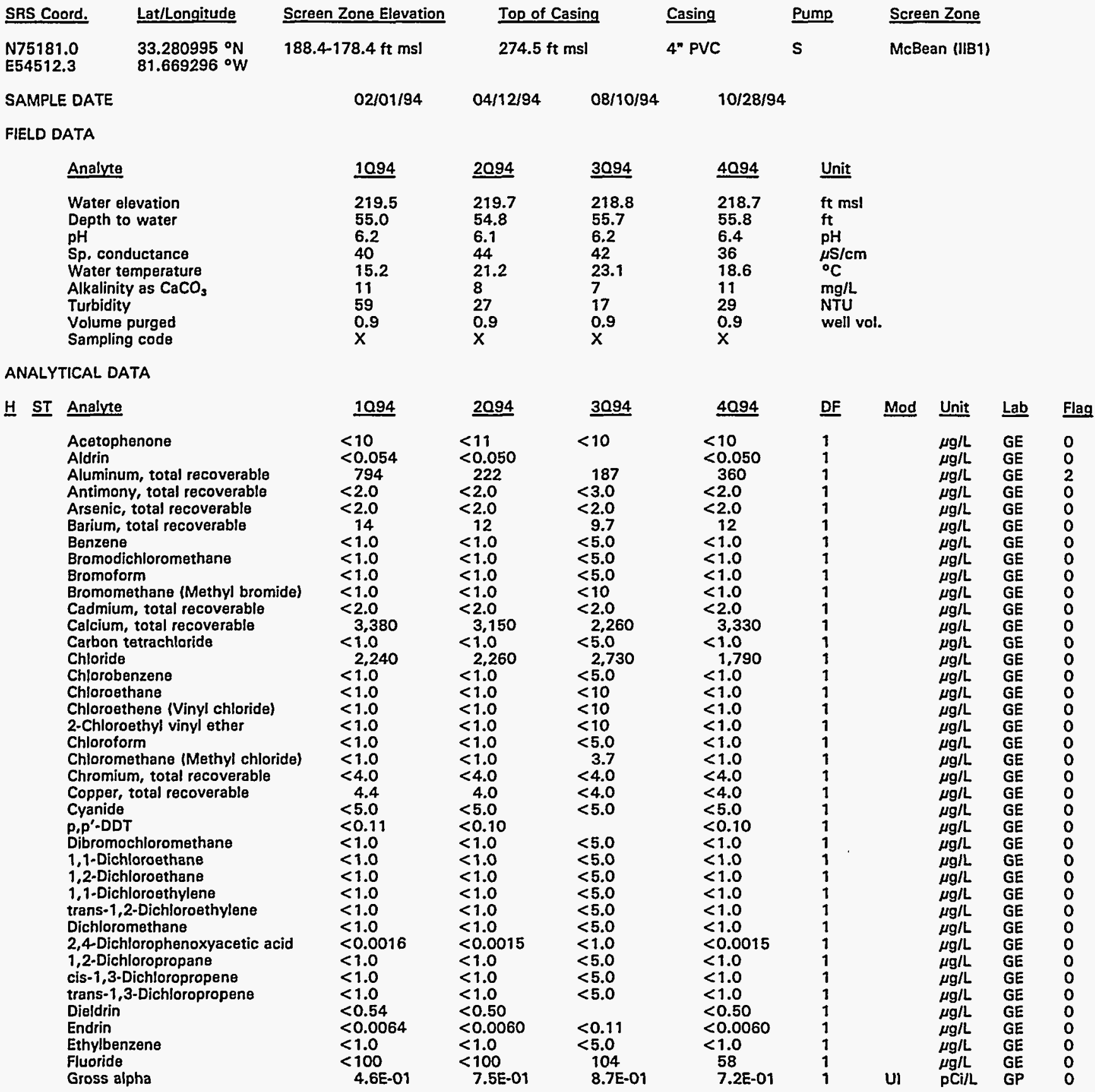

Note: Flagging, dilution factors, modifiers, and laboratories are for fourth quarter 1994 data only. See Appendix B for flagging criteria. - = exceeded holding time for fourth quarter 1994.

- = exceeded screening level or final PDWS for fourth quarter 1994. 
Well BGO $30 \mathrm{C}$ continued

\section{ANALYTICAL DATA}

H. ST Analyte
Heptachlor
Iron, total recoverable
Lead, total recoverable
Lindane
Lithium, total recoverable
Magnesium, total recoverable
Manganese, total recoverable
Mercury, total recoverable
Methoxychlor
Naphthalene
Nickel, total recoverable
Nitrate as nitrogen
Nitrate-nitrite as nitrogen
Nonvolatile beta
pH
Phenols
Potassium, total recoverable
Radium, total alpha-emitting
Selenium, total recoverable
Silica, total recoverable
Silver, total recoverable
Sodium, total recoverable
Specific conductance
Sulfate
1,1,2,2-Tetrachloroethane
Tetrachloroethylene
Tin, total recoverable
Toluene
Total activity
Total dissolved solids
Total organic carbon
Total organic halogens
Total phosphates las P)
Toxaphene
$2,4,5-T P$ (Silvex)
Tributyl phosphate
1,1,1-Trichloroethane
1,1,2-Trichloroethane
Trichloroethylene
Trichlorofluoromethane
$2,4,5-T$
Tritium
Turbidity
Uranium-233/234
Uranium-235
Uranium-238
Vanadium, total recoverable
Xylenes

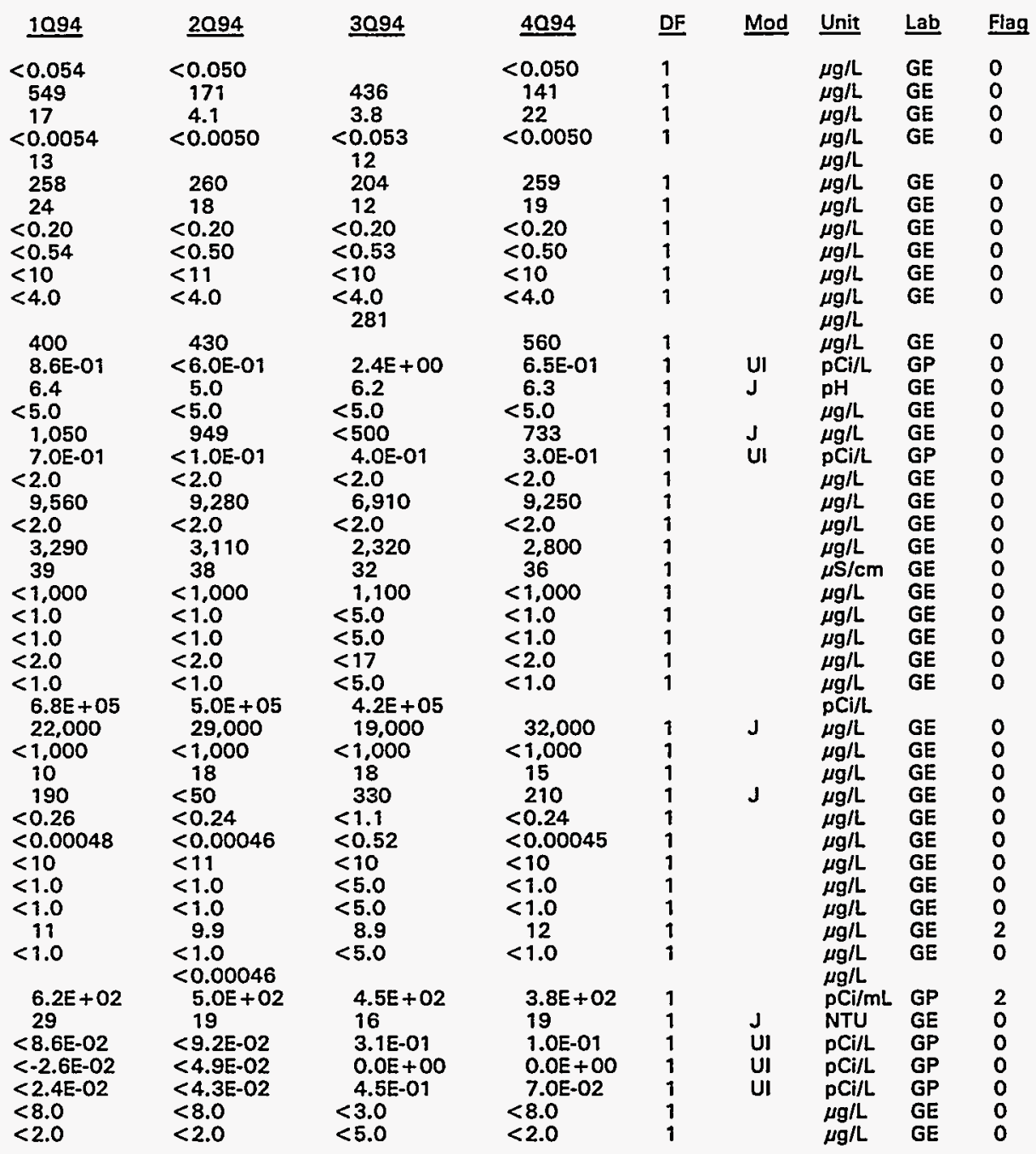

Note: Flagging, dilution factors, modifiers, and laboratories are for fourth quarter 1994 data only. See Appendix B for flagging criteria. - = exceeded holding time for fourth quarter 1994.

- = exceeded screening level or final PDWS for fourth quarter 1994. 
WELL BGO 30D

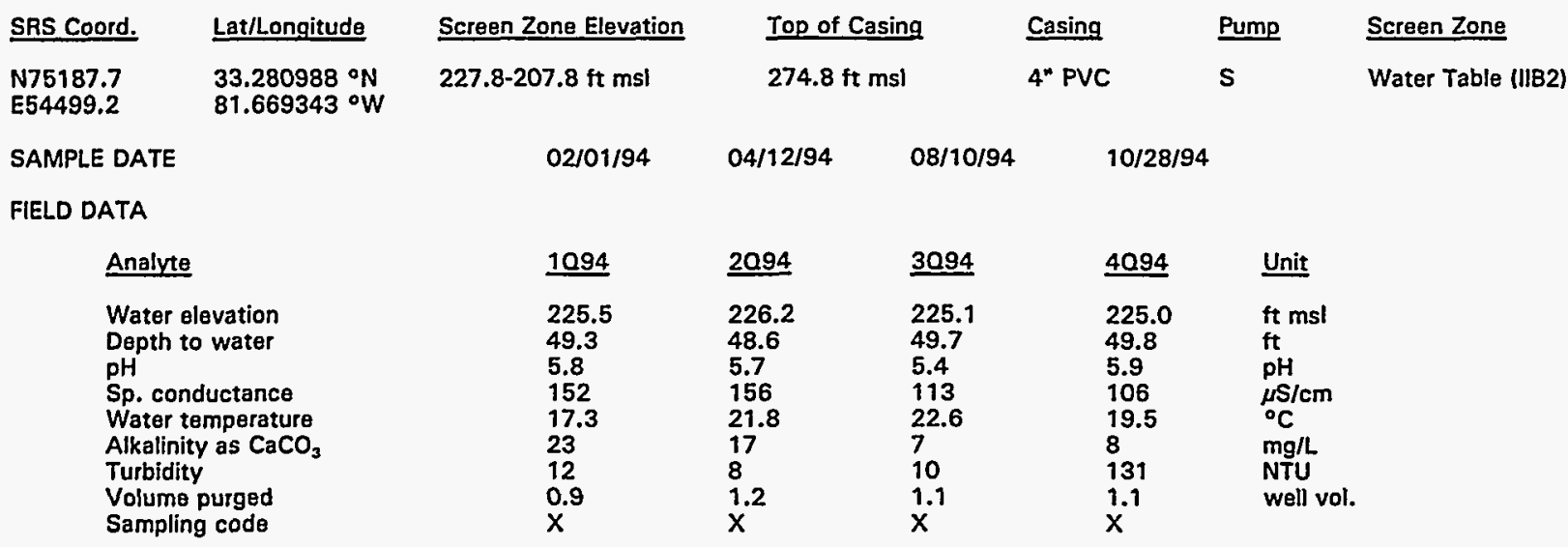

ANALYTICAL DATA

H. Analyte
Acetophenone
Aldrin
Aluminum, total recoverable
Antimony, total recoverable
Arsenic, total recoverable
Barium, total recoverable
Benzene
Bromodichloromethane
Bromoform
Bromomethane (Methyl bromide)
Cadmium, total recoverable
Calcium, total recoverable
Carbon tetrachloride
Chloride
Chlorobenzene
Chloroethane
Chloroethene (Vinyl chloride)
2-Chloroethyl vinyl ether
Chloroform
Chloromethane (Methyl chloride)
Chromium, total recoverable
Copper, total recoverable
Cyanide
p,p'-DDT
Dibromochloromethane
1,1-Dichloroethane
1,2-Dichloroethane
$1,1-$ Dichloroethylene
trans-1,2-Dichloroethylene
Dichloromethane
$2,4-$ Dichlorophenoxyacetic acid
$1,2-$ Dichloropropane
cis-1,3-Dichloropropene
trans-1,3-Dichloropropene
Dieldrin
Endrin
Ethylbenzene
Fluoride
Gross alpha

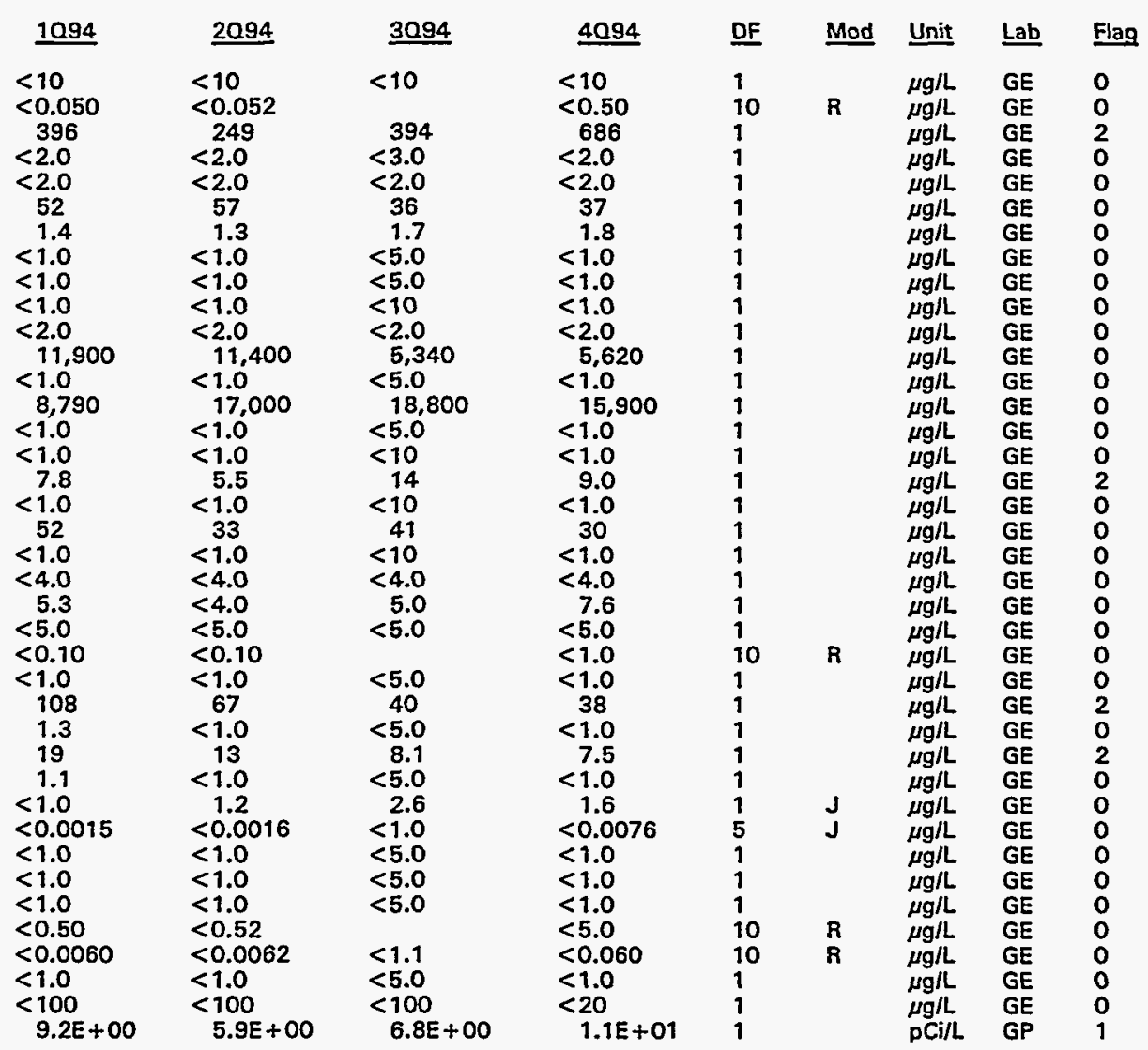

\footnotetext{
Note: Flagging, dilution factors, modifiers, and laboratories are for fourth quarter 1994 data only. See Appendix B for flagging criteria. - = exceeded holding time for fourth quarter 1994.

- = exceeded screening level or final PDWS for fourth quarter 1994.
} 


\section{ANALYTICAL DATA}

\section{H ST Analyze}

Heptachlor

Iron, total recoverable

Lead, total recoverable

Lindane

Lithium, total recoverable

Magnesium, total recoverable

Manganese, total recoverable

Mercury, total recoverable

Methoxychlor

Naphthalene

Nickel, total recoverable

Nitrate as nitrogen

Nitrate-nitrite as nitrogen

Nonvolatile beta

pH

Phenols

Potassium, total recoverable

Radium, total alpha-emitting

Selenium, total recoverable

Silica, total recoverable

Silver, total recoverable

Sodium, total recoverable

Specific conductance

Sulfate

1,1,2,2-Tetrachloroethane

Tetrachloroethylene

Tin, total recoverable

Toluene

Total activity

Total dissolved solids

Total organic carbon

Total organic halogens

Total phosphates (as P)

Toxaphene

2,4,5-TP (Silvex)

Tributyl phosphate

1,1,1-Trichloroethane

1,1,2-Trichloroethane

- Trichloroethylene

Trichlorofluoromethane

2,4,5-T

- Tritium

Turbidity

Uranium-233/234

Uranium-235

Uranium-238

Vanadium, total recoverable

Xylenes

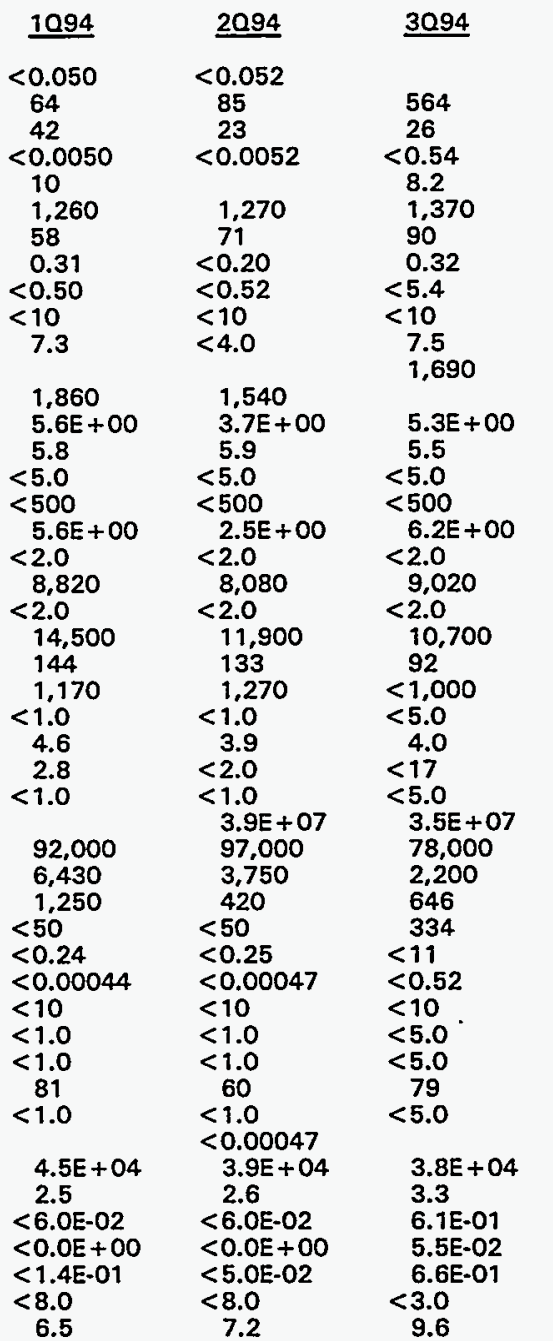

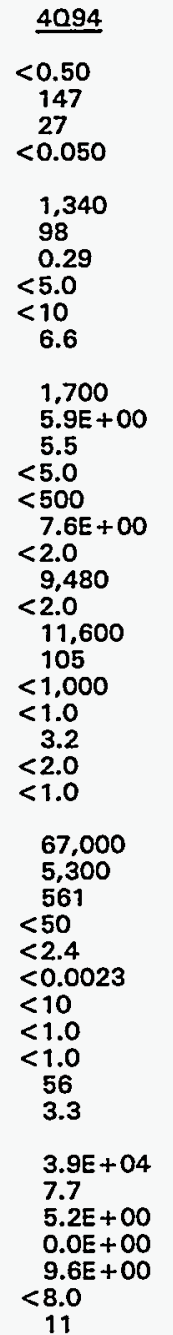

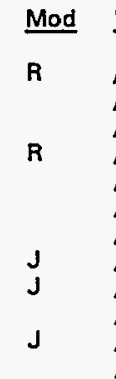

\begin{tabular}{cll} 
Unit & Lab & Flag \\
\cline { 1 - 2 }$\mu \mathrm{g} / \mathrm{L}$ & $\mathrm{GE}$ & 0 \\
$\mu \mathrm{g} / \mathrm{L}$ & $\mathrm{GE}$ & 0 \\
$\mu \mathrm{g} / \mathrm{L}$ & $\mathrm{GE}$ & 1 \\
$\mu \mathrm{g} / \mathrm{L}$ & $\mathrm{GE}$ & 0 \\
$\mu \mathrm{g} / \mathrm{L}$ & & \\
$\mu \mathrm{g} / \mathrm{L}$ & $\mathrm{GE}$ & 0 \\
$\mu \mathrm{g} / \mathrm{L}$ & $\mathrm{GE}$ & 2 \\
$\mu \mathrm{g} / \mathrm{L}$ & $\mathrm{GE}$ & 0 \\
$\mu \mathrm{g} / \mathrm{L}$ & $\mathrm{GE}$ & 0 \\
$\mu \mathrm{g} / \mathrm{L}$ & $\mathrm{GE}$ & 0
\end{tabular}

$\mu g / L \quad G E$

$\mu \mathrm{g} / \mathrm{L}$

$\mu \mathrm{g} / \mathrm{L}$

J

PH GE

$\mu \mathrm{g} / \mathrm{L} \quad \mathrm{GE}$

$\mu g / L \quad G$

$\mu \mathrm{g} / \mathrm{L} \quad \mathrm{GE}$

$\mu g / L \quad G E$

$\mu g / L \quad G E$

$\mu g / L \quad G E$

$\mu \mathrm{g} / \mathrm{L} \quad \mathrm{GE}$

$\mu \mathrm{S} / \mathrm{cm} \quad \mathrm{GE}$

$\mu \mathrm{g} / \mathrm{L} \quad \mathrm{GE}$

$\mu \mathrm{g} / \mathrm{L} \quad \mathrm{GE}$

$\mu g / L \quad G E$

$\mu g / L \quad G E$

$\mu g / L$

$\mathrm{pCi} / \mathrm{L}$
$\mu \mathrm{g} / \mathrm{L}$

$\mu \mathrm{g} / \mathrm{L} / \mathrm{L}$

$\mu \mathrm{g} / \mathrm{L}$

$\mu \mathrm{g} / \mathrm{L}$

$\mu g / L$

$\mu g / L$

$\underset{\mu g / L}{\mu g / L}$

$\mu \mathrm{g} / \mathrm{h}$

$\mu \mathrm{g} / \mathrm{h}$

$\underset{\mu g / L}{\mu g / L}$

pCi/mL GP

J

NTU

UI

ag

Note: Flagging, dilution factors, modifiers, and laboratories are for fourth quarter 1994 data only. See Appendix B for flagging criteria. $\bullet=$ exceeded holding time for fourth quarter 1994.

- = exceeded screening level or final PDWS for fourth quarter 1994. 
WELL BGO 31C

\begin{tabular}{|c|c|c|c|c|c|c|c|}
\hline SRS Coord. & Lat/Longitude & Screen Zone Elevation & \multicolumn{2}{|c|}{ Top of Casing } & Casing & Pump & \multirow{2}{*}{$\frac{\text { Screen Zone }}{\text { McBean (IIB1) }}$} \\
\hline $\begin{array}{l}\text { N74978.0 } \\
\text { E54816.2 }\end{array}$ & $\begin{array}{l}33.2810422^{\circ} \mathrm{N} \\
81.668101{ }^{\circ} \mathrm{W}\end{array}$ & $186.4-176.4 \mathrm{ft} \mathrm{msl}$ & \multicolumn{2}{|c|}{$273.1 \mathrm{ft} \mathrm{msl}$} & 4" PVC & $\mathbf{s}$ & \\
\hline \multicolumn{2}{|c|}{ SAMPLE DATE } & $02 / 01 / 94$ & $04 / 12 / 94$ & $08 / 10 / 94$ & $10 / 28 / 94$ & & \\
\hline \multicolumn{8}{|l|}{ FIELD DATA } \\
\hline \multicolumn{2}{|c|}{ Analyte } & 1094 & $\underline{2094}$ & 3094 & $\underline{4094}$ & Unit I & \\
\hline \multicolumn{2}{|c|}{$\begin{array}{l}\text { Water elevation } \\
\text { Depth to water } \\
\text { pH } \\
\text { Sp. conductance } \\
\text { Water temperature } \\
\text { Alkalinity as } \mathrm{CaCO}_{3} \\
\text { Turbidity } \\
\text { Volume purged } \\
\text { Sampling code }\end{array}$} & $\begin{array}{l}225.3 \\
47.8 \\
5.4 \\
27 \\
15.9 \\
1 \\
13 \\
0.9 \\
\times\end{array}$ & $\begin{array}{l}226.0 \\
47.1 \\
5.1 \\
31 \\
22.0 \\
0 \\
4 \\
0.8 \\
\times\end{array}$ & $\begin{array}{l}224.9 \\
48.2 \\
5.3 \\
29 \\
22.1 \\
1 \\
7 \\
0.8 \\
x\end{array}$ & $\begin{array}{l}224.9 \\
48.2 \\
5.5 \\
25 \\
18.4 \\
2 \\
13 \\
0.9 \\
\times\end{array}$ & $\begin{array}{l}\mathrm{ft} \mathrm{msl} \\
\mathrm{ft} \\
\mathrm{pH} \\
\mu \mathrm{S} / \mathrm{cm} \\
{ }^{\circ} \mathrm{C} \\
\mathrm{mg} / \mathrm{L} \\
\mathrm{NTU} \\
\text { well vol. }\end{array}$ & \\
\hline
\end{tabular}

ANALYTICAL DATA

H ST Analyte

Acetophenone

Aldrin

Aluminum, total recoverable

Antimony, total recoverable

Arsenic, total recoverable

Barium, total recoverable

Benzene

Bromodichloromethane

Bromoform

Bromomethane (Methyl bromide)

Cadmium, total recoverable

Calcium, total recoverable

Carbon tetrachloride

Chloride

Chlorobenzene

Chloroethane

Chloroethene (Vinyl chloride)

2-Chloroethyl vinyl ether

Chloroform

Chloromethane (Methyl chloride)

Chromium, total recoverable

Copper, total recoverable

Cyanide

P.p'-DDT

Dibromochloromethane

1,1-Dichloroethane

1,2-Dichloroethane

1,1-Dichloroethylene

trans-1,2-Dichloroethylene

Dichloromethane

2,4-Dichlorophenoxyacetic acid

1,2-Dichloropropane

cis-1,3-Dichloropropene

trans-1,3-Dichloropropene

Dieldrin

Endrin

Ethylbenzene

Fluoride

Gross alpha

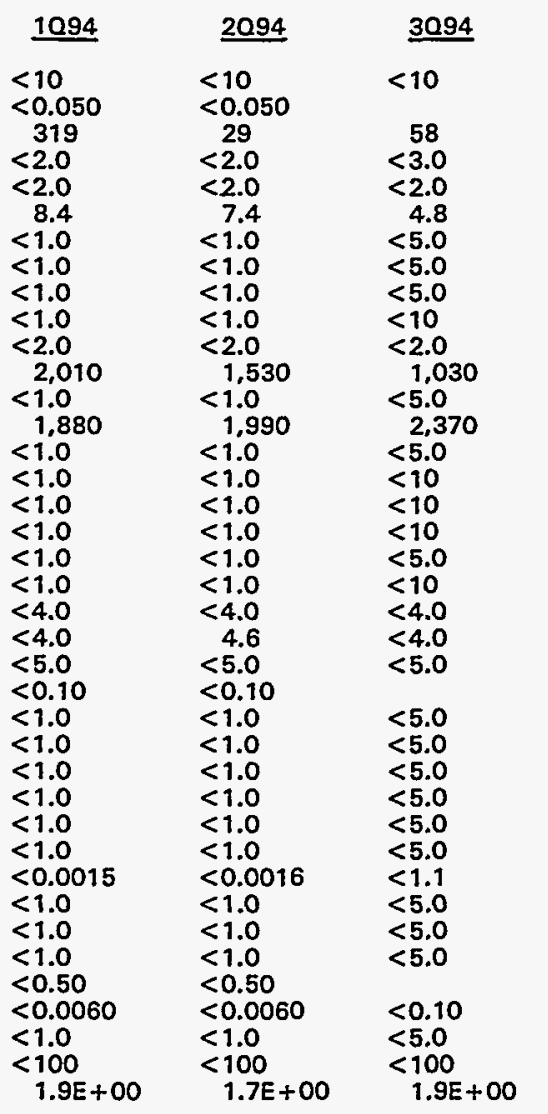

$\begin{aligned} & 4094 \\ &<10 \\ &<0.050 \\ & 141 \\ &<2.0 \\ &<2.0 \\ & \quad 7.6 \\ &<1.0 \\ &<1.0 \\ &<1.0 \\ &<1.0 \\ &<2.0 \\ & 11.520 \\ &<1.0 \\ & 1.520 \\ &<1.0 \\ &<1.0 \\ &<1.0 \\ &<1.0 \\ &<1.0 \\ &<1.0 \\ &<4.0 \\ &<4.0 \\ &<5.0 \\ &<0.10 \\ &<1.0 \\ &<1.0 \\ &<1.0 \\ &<1.0 \\ &<1.0 \\ &<1.0 \\ &<0.0015 \\ &<1.0 \\ &<1.0 \\ &<1.0 \\ &<0.50 \\ &<0.0060 \\ &<1.0 \\ &<20 \\ & 2.8 E+00 \\ &\end{aligned}$

\begin{tabular}{|c|c|c|c|}
\hline DF & Mod & Unit & Lab \\
\hline 1 & & $\mu g / L$ & GE \\
\hline 1 & & $\mu \mathrm{g} / \mathrm{L}$ & GE \\
\hline 1 & & $\mu g / L$ & GE \\
\hline 1 & & $\mu \mathrm{g} / \mathrm{L}$ & GE \\
\hline 1 & & $\mu \mathrm{g} / \mathrm{L}$ & GE \\
\hline 1 & & $\mu g / L$ & GE \\
\hline 1 & & $\mu \mathrm{g} / \mathrm{L}$ & GE \\
\hline 1 & & $\mu \mathrm{g} / \mathrm{L}$ & GE \\
\hline 1 & & $\mu g / L$ & GE \\
\hline 1 & & $\mu g / L$ & GE \\
\hline 1 & & $\mu \mathrm{g} / \mathrm{L}$ & GE \\
\hline 1 & & $\mu \mathrm{g} / \mathrm{L}$ & $\mathbf{G E}$ \\
\hline 1 & & $\mu \mathrm{g} / \mathrm{L}$ & GE \\
\hline 1 & & $\mu g / L$ & GE \\
\hline 1 & & $\mu \mathrm{g} / \mathrm{L}$ & GE \\
\hline 1 & & $\mu \mathrm{g} / \mathrm{L}$ & GE \\
\hline 1 & & $\mu \mathrm{g} / \mathrm{L}$ & GE \\
\hline 1 & & $\mu g / L$ & GE \\
\hline 1 & & $\mu \mathrm{g} / \mathrm{L}$ & GE \\
\hline 1 & & $\mu g / L$ & GE \\
\hline 1 & & $\mu \mathrm{g} / \mathrm{L}$ & GE \\
\hline 1 & & $\mu g / L$ & GE \\
\hline 1 & & $\mu g / L$ & GE \\
\hline 1 & & $\mu \mathrm{g} / \mathrm{L}$ & GE \\
\hline 1 & & $\mu g / L$ & GE \\
\hline 1 & & $\mu \mathrm{g} / \mathrm{L}$ & GE \\
\hline 1 & & $\mu \mathrm{g} / \mathrm{L}$ & GE \\
\hline 1 & & $\mu \mathrm{g} / \mathrm{L}$ & GE \\
\hline 1 & & $\mu \mathrm{g} / \mathrm{L}$ & GE \\
\hline 1 & & $\mu \mathrm{g} / \mathrm{L}$ & GE \\
\hline 1 & & $\mu \mathrm{g} / \mathrm{L}$ & GE \\
\hline 1 & & $\mu g / L$ & GE \\
\hline 1 & & $\mu \mathrm{g} / \mathrm{L}$ & GE \\
\hline 1 & & $\mu \mathrm{g} / \mathrm{L}$ & GE \\
\hline 1 & & $\mu g / L$ & GE \\
\hline 1 & & $\mu \mathrm{g} / \mathrm{L}$ & GE \\
\hline 1 & & $\mu \mathrm{g} / \mathrm{L}$ & GE \\
\hline 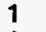 & & $\mu \mathrm{g} / \mathrm{L}$ & GE \\
\hline 1 & \rfloor & $\mathrm{pC} \mathrm{i} / \mathrm{L}$ & GP \\
\hline
\end{tabular}

Note: Flagging, dilution factors, modifiers, and laboratories are for fourth quarter 1994 data only. See Appendix B for flagging criteria. - = exceeded holding time for fourth quarter 1994.

- = exceeded screening level or final PDWS for fourth quarter 1994. 


H ST Analyte
Heptachlor
Iron, total recoverable
Lead, total recoverable
Lindane
Lithium, total recoverable
Magnesium, total recoverable
Manganese, total recoverable
Mercury, total recoverable
Methoxychlor
Naphthalene
Nickel, total recoverable
Nitrate as nitrogen
Nitrate-nitrite as nitrogen
Nonvolatile beta
pH
Phenols
Potassium, total recoverable
Radium, total alpha-emitting
Selenium, total recoverable
Silica, total recoverable
Silver, total recoverable
Sodium, total recoverable
Specific conductance
Sulfate
1,1,2,2-Tetrachloroethane
Tetrachloroethylene
Tin, total recoverable
Toluene
Total activity
Total dissolved solids
Total organic carbon
Total organic halogens
Total phosphates (as P)
Toxaphene
$2,4,5-T P$ (Silvex)
Tributyl phosphate
1,1,1-Trichloroethane
1,1,2-Trichloroethane
Trichloroethylene
Trichlorofluoromethane
$2,4,5-T$
Tritium
Turbidity
Uranium-233/234
Uranium-235
Uranium-238
Vanadium, total recoverable
Xylenes
-

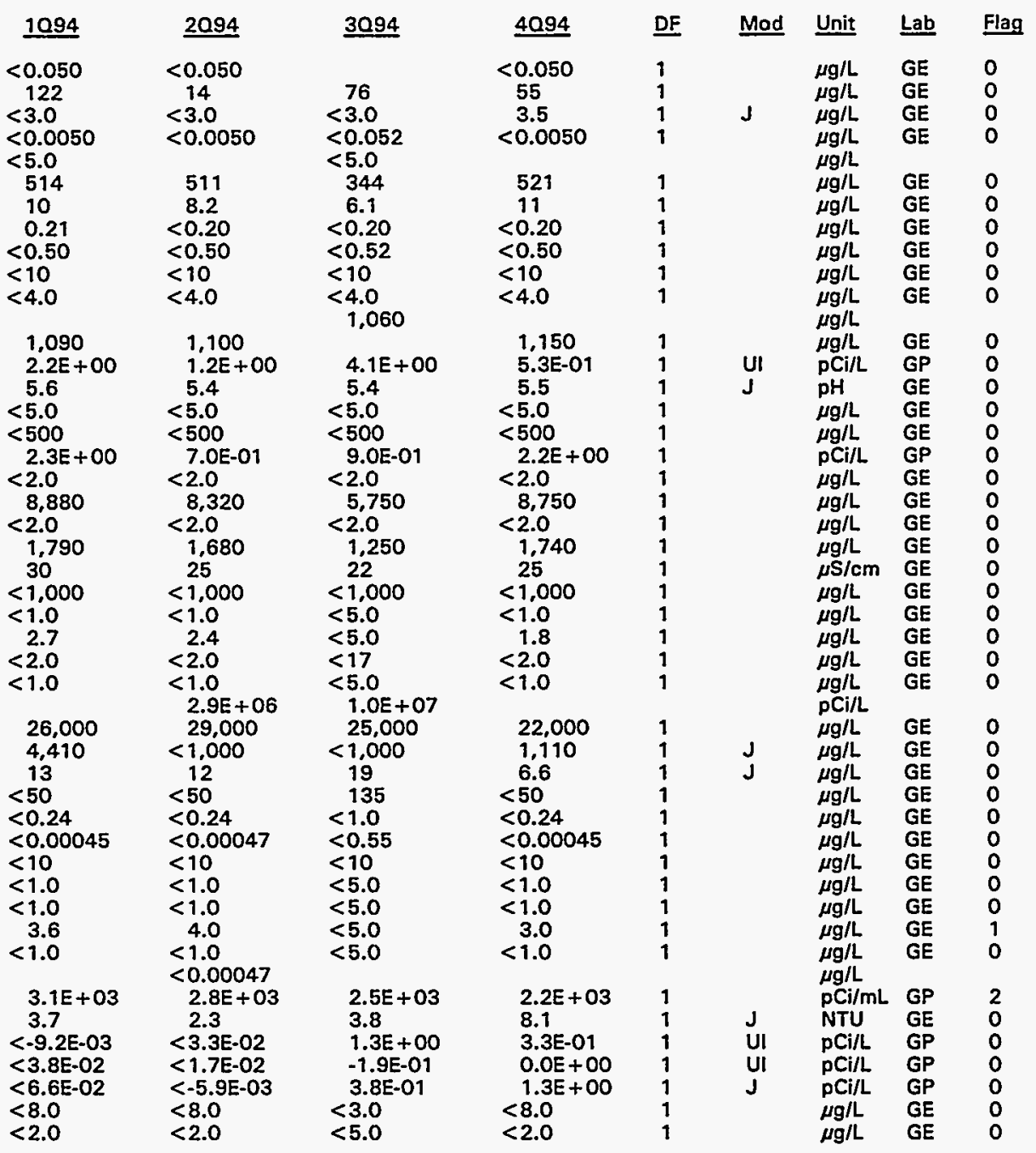

Note: Flagging, dilution factors, modifiers, and laboratories are for fourth quarter 1994 data only. See Appendix B for flagging criteria. - = exceeded holding time for fourth quarter 1994.

- = exceeded screening level or final PDWS for fourth quarter 1994. 
WELL BGO 31D

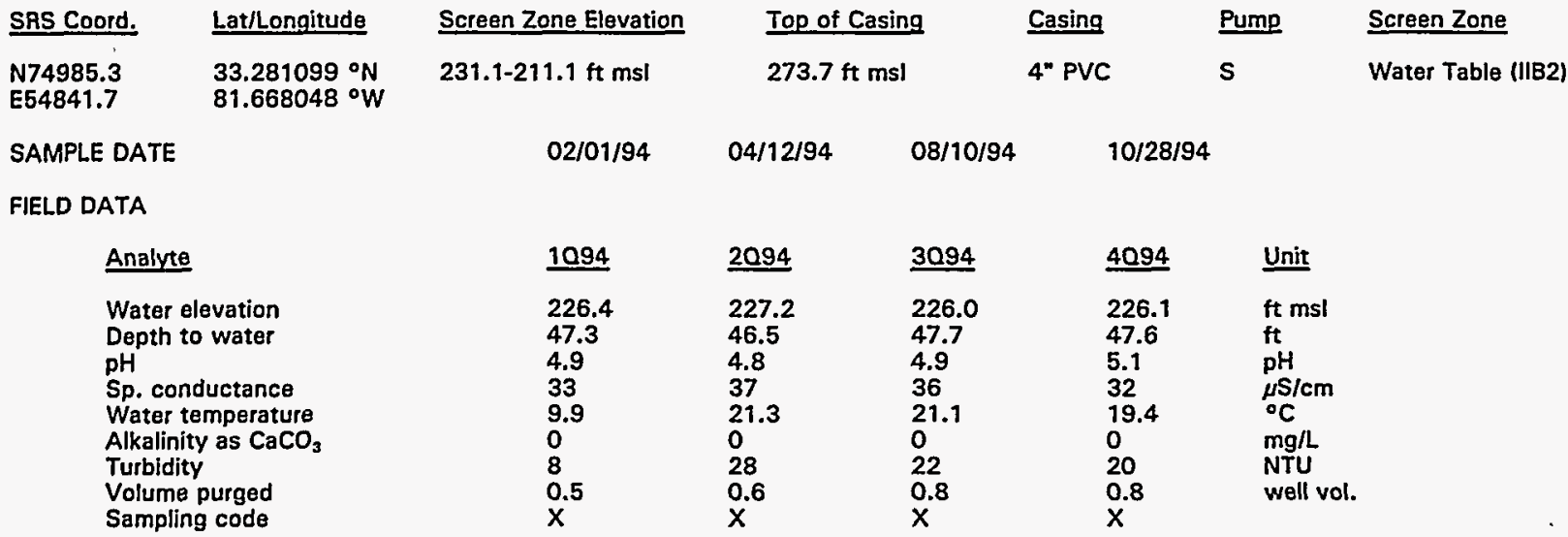

ANALYTICAL DATA

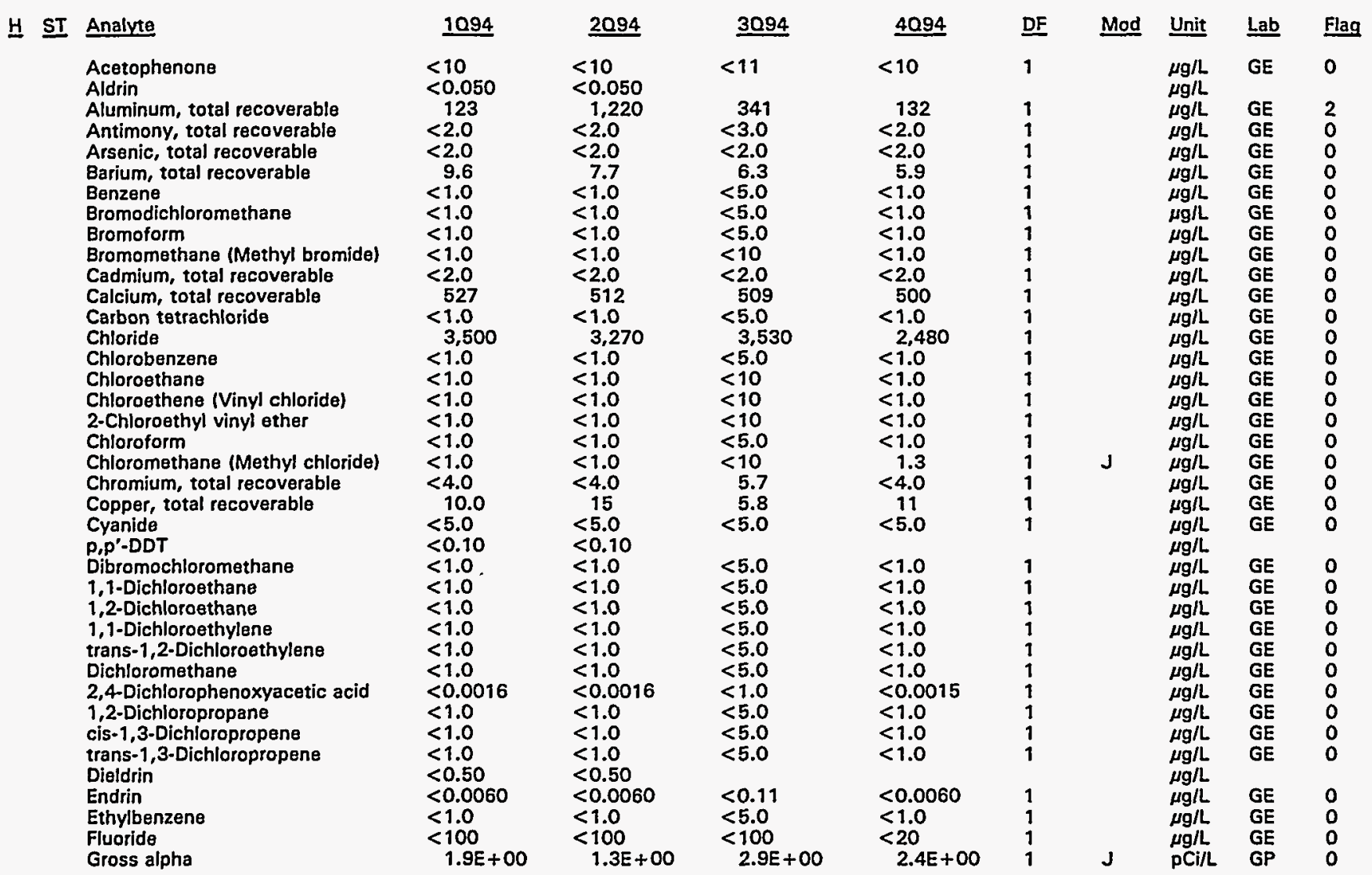

Note: Flagging, dilution factors, modifiers, and laboratories are for fourth quarter 1994 data only. See Appendix B for flagging criteria. - = exceeded holding time for fourth quarter 1994.

- = exceeded screening level or final PDWS for fourth quarter 1994. 


H. ST Analyte
Heptachlor
lron, total recoverable
Lead, total recoverable
Lindane
Lithium, total recoverable
Magnesium, total recoverable
Manganese, total recoverable
Mercury, total recoverable
Methoxychlor
Naphthalene
Nickel, total recoverable
Nitrate as nitrogen
Nitrate-nitrite as nitrogen
Nonvolatile beta
pH
Phenols
Potassium, total recoverable
Radium, total alpha-emitting
Selenium, total recoverable
Silica, total recoverable
Silver, total recoverable
Sodium, total recoverable
Specific conductance
Sulfate
1,1,2,2-Tetrachloroethane
Tetrachloroethylene
Tin, total recoverable
Toluene
Total dissolved solids
Total organic carbon
Total organic halogens
Total phosphates (as P)
Toxaphene
$2,4,5-T P$ (Silvex)
Tributyl phosphate
$1,1,1-$ Trichloroethane
T,1,2-Trichloroethane
Trichloroethylene
Trichlorofluoromethane
$2,4,5-T$
Tritium
Turbidity
Uranium-233/234
Uranium-235
Uranium-238
Vanadium, total recoverable
Xylenes

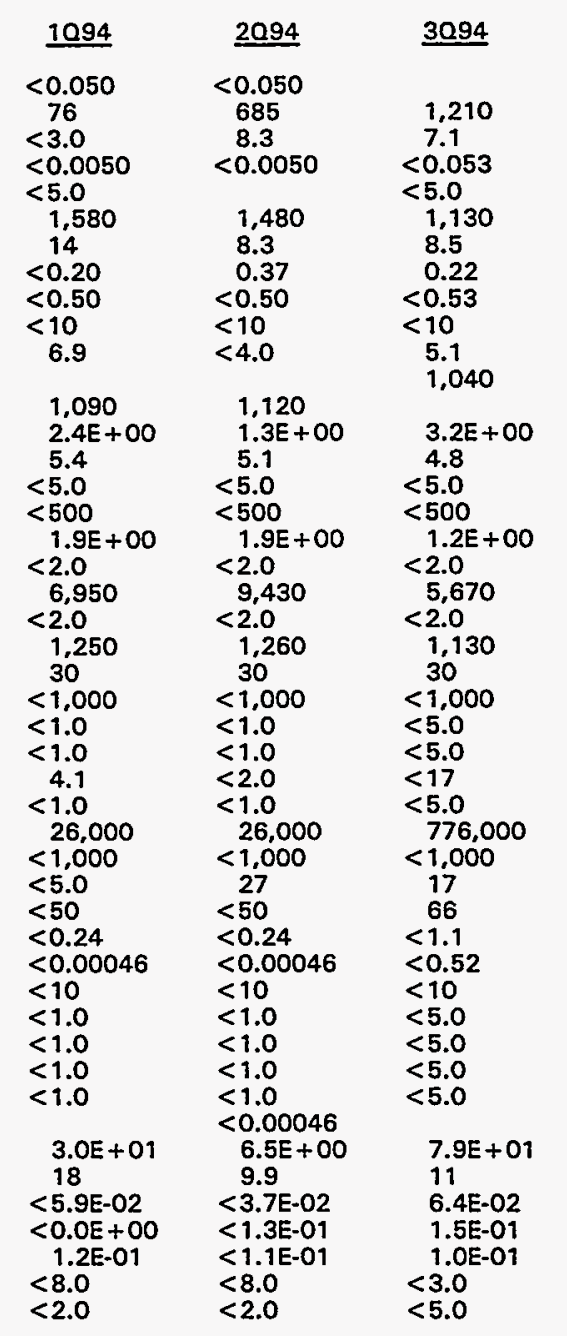

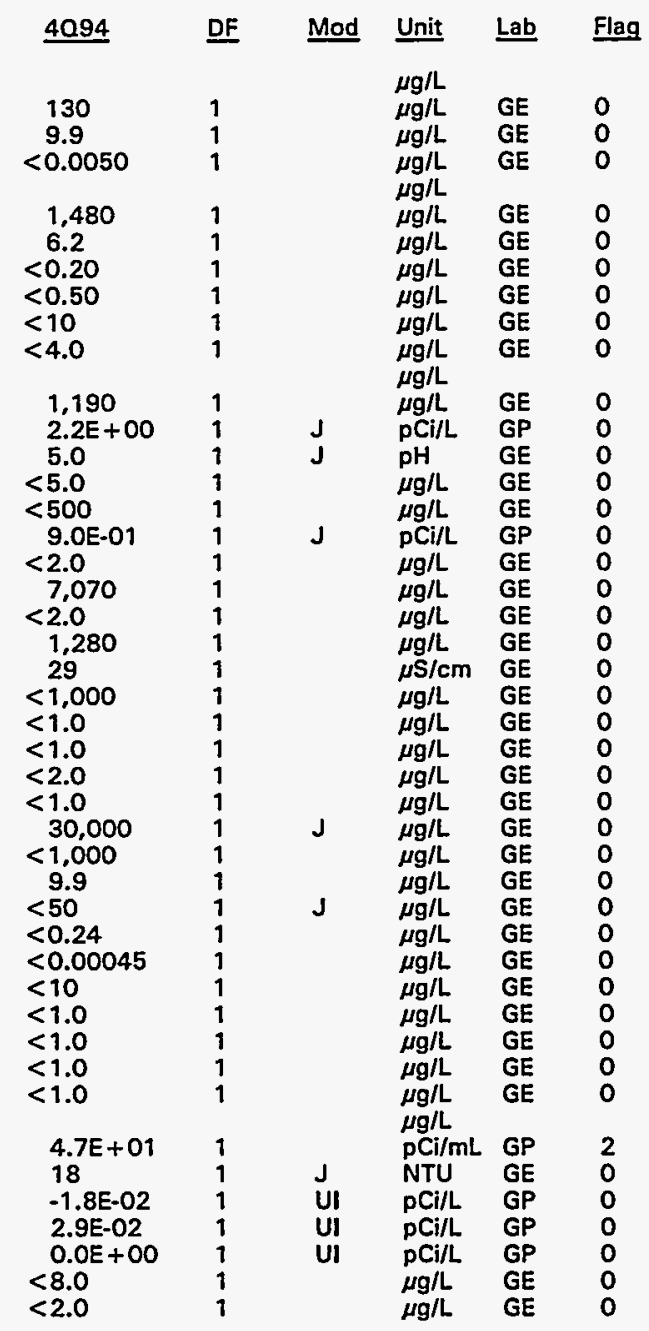

Note: Flagging, dilution factors, modifiers, and laboratories are for fourth quarter 1994 data only. See Appendix B for flagging criteria. - = exceeded holding time for fourth quarter 1994.

- = exceeded screening level or final PDWS for fourth quarter 1994. 
WELL BGO 32D

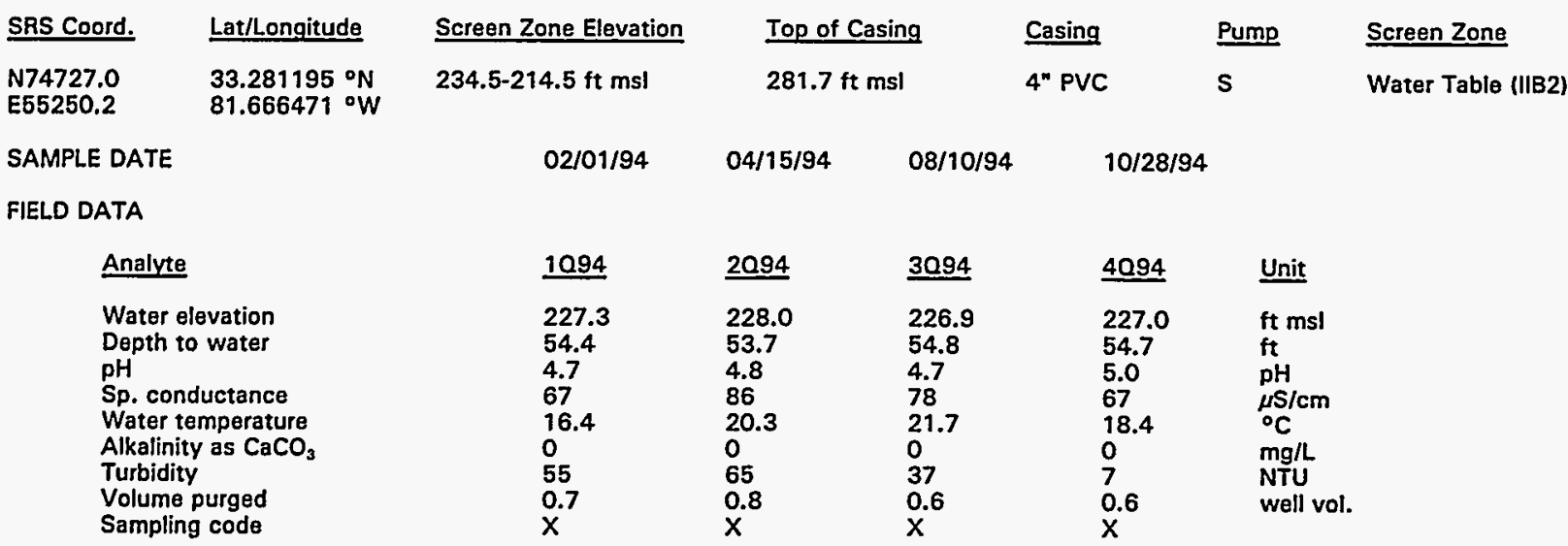

ANALYTICAL DATA

H ST Analyte

Acetophenone

Aldrin

Aluminum, total recoverable

Antimony, total recoverable

Arsenic, total recoverable

Barium, total recoverable

Benzene

Bromodichloromethane

Bromoform

Bromomethane (Methyl bromide)

Cadmium, total recoverable

Calcium, total recoverable

Carbon tetrachloride

Chloride

Chlorobenzene

Chloroethane

Chloroethene (Vinyl chloride)

2-Chloroethyl vinyl ether

Chloroform

Chloromethane (Methyl chloride)

Chromium, total recoverable

Copper, total recoverable

Cyanide

p.p'-DDT

Dibromochloromethane

1,1-Dichloroethane

1,2-Dichloroethane

1,1-Dichloroethylene

trans-1,2-Dichloroethylene

Dichloromethane

2,4-Dichlorophenoxyacetic acid

1,2-Dichloropropane

cis-1,3-Dichloropropene

trans-1,3-Dichloropropene

Dieldrin

Endrin

Ethylbenzene

Fluoride

Gross alpha

$\begin{array}{lll}1094 & \underline{2094} & \underline{3094} \\ <9.9 & <10 & <11 \\ <0.051 & <0.051 & \\ 910 & 855 & 1,900 \\ <2.0 & <2.0 & <3.0 \\ <2.0 & <2.0 & <2.0 \\ 129 & 890 & 51 \\ <1.0 & <1.0 & <5.0 \\ <1.0 & <1.0 & <5.0 \\ <1.0 & <1.0 & <5.0 \\ <1.0 & <1.0 & <10 \\ <2.0 & <2.0 & <2.0 \\ 818 & 794 & 591 \\ <1.0 & <1.0 & <5.0 \\ 5,780 & 6,730 & 8,070 \\ <1.0 & <1.0 & <5.0 \\ <1.0 & <1.0 & <10 \\ <1.0 & <1.0 & <10 \\ <1.0 & <1.0 & <10 \\ 1.4 & <1.0 & <5.0 \\ <1.0 & <1.0 & 4.1 \\ <4.0 & <4.0 & 7.6 \\ 259 & 308 & 184 \\ <5.0 & <5.0 & <5.0 \\ <0.10 & <0.10 & <5.0 \\ <1.0 & <1.0 & <5.0 \\ <1.0 & <1.0 & <5.0 \\ <1.0 & <1.0 & <5.0 \\ <1.0 & <1.0 & <5.0 \\ <1.0 & <1.0 & <5.0 \\ <1.0 & <1.0 & <5.0 \\ <0.0015 & <0.0016 & <1.1 \\ <1.0 & <1.0 & <5.0 \\ <1.0 & <1.0 & <5.0 \\ <1.0 & <1.0 & <5.0 \\ <0.51 & <0.01 & \\ <0.0061 & <0.0061 & <0.11 \\ <1.0 & <1.0 & <5.0 \\ <100 & <100 & <100 \\ 1.2 E+01 & 1.5 E+01 & 2.1 E+01\end{array}$

\begin{tabular}{|c|c|c|c|c|c|}
\hline 4094 & DF & Mod & $\underline{\text { Unit }}$ & $\underline{\mathrm{Lab}}$ & Flag \\
\hline$<10$ & 1 & & $\begin{array}{l}\mu g / L \\
\mu g / L\end{array}$ & GE & 0 \\
\hline 826 & 1 & & $\mu \mathrm{g} / \mathrm{L}$ & GE & 2 \\
\hline$<2.0$ & 1 & & $\mu \mathrm{g} / \mathrm{L}$ & GE & 0 \\
\hline$<2.0$ & 1 & & $\mu g / L$ & GE & 0 \\
\hline 34 & 1 & & $\mu g / L$ & GE & 0 \\
\hline $\begin{array}{l}<1.0 \\
<1.0\end{array}$ & 1 & & $\mu g / L$ & GE & 0 \\
\hline$<1.0$ & 1 & & $\mu \mathrm{g} / \mathrm{L}$ & GE & 0 \\
\hline$<1.0$ & 1 & & $\mu \mathrm{g} / \mathrm{L}$ & GE & 0 \\
\hline$<1.0$ & 1 & & $\mu g / L$ & GE & 0 \\
\hline$<2.0$ & 1 & & $\mu g / L$ & GE & 0 \\
\hline $\begin{array}{r}758 \\
<1.0\end{array}$ & 1 & & $\mu g / L$ & GE & 0 \\
\hline $\begin{array}{l}<1.0 \\
4.310\end{array}$ & 1 & & $\mu \mathrm{g} / \mathrm{L}$ & $\overline{G E}$ & 0 \\
\hline $\begin{aligned} & 4.310 \\
< & 1.0\end{aligned}$ & 1 & & $\mu \mathrm{g} / \mathrm{L}$ & GE & 0 \\
\hline $\begin{array}{l}<1.0 \\
<1.0\end{array}$ & $\begin{array}{l}1 \\
1\end{array}$ & & $\mu \mathrm{g} / \mathrm{L}$ & GE & 0 \\
\hline$<1.0$ & 1 & & $\begin{array}{l}\mu g / L \\
\mu g / L\end{array}$ & $\begin{array}{l}\mathrm{GE} \\
\mathrm{GE}\end{array}$ & $\begin{array}{l}0 \\
0\end{array}$ \\
\hline$<1.0$ & 1 & & $\mu g / L$ & $\mathrm{GE}$ & 0 \\
\hline$<1.0$ & 1 & & $\mu \mathrm{g} / \mathrm{L}$ & GE & 0 \\
\hline$<1.0$ & 1 & & $\mu g / L$ & GE & 0 \\
\hline$<4.0$ & 1 & & $\mu \mathrm{g} / \mathrm{L}$ & GE & 0 \\
\hline $\begin{array}{r}234 \\
<5.0\end{array}$ & $\begin{array}{l}1 \\
1\end{array}$ & & $\mu \mathrm{g} / \mathrm{L}$ & GE & 0 \\
\hline$<5.0$ & & & $\mu g / L$ & & \\
\hline$<1.0$ & 1 & & $\mu \mathrm{g} / \mathrm{L}$ & GE & 0 \\
\hline $\begin{array}{l}<1.0 \\
<1.0\end{array}$ & 1 & & $\mu g / L$ & GE & 0 \\
\hline $\begin{array}{l}<1.0 \\
<1.0\end{array}$ & 1 & & $\mu g / L$ & GE & 0 \\
\hline $\begin{array}{l}<1.0 \\
<1.0\end{array}$ & $\begin{array}{l}1 \\
1\end{array}$ & & $\mu g / L$ & GE & 0 \\
\hline $\begin{array}{l}<1.0 \\
<1.0\end{array}$ & 1 & & $\mu \mathrm{g} / \mathrm{h}$ & $\mathrm{GE}$ & $\stackrel{0}{0}$ \\
\hline $\begin{array}{l}<1.0 \\
<0.0015\end{array}$ & 1 & & $\underset{\mu g / L}{\mu g / L}$ & $\begin{array}{l}\mathrm{GE} \\
\mathrm{GE}\end{array}$ & $\begin{array}{l}0 \\
0\end{array}$ \\
\hline$<1.0$ & 1 & & $\mu \mathrm{g} / \mathrm{L}$ & $\mathrm{GE}$ & 0 \\
\hline$<1.0$ & 1 & & $\mu g / L$ & $\overline{G E}$ & 0 \\
\hline$<1.0$ & 1 & & $\mu g / L$ & GE & 0 \\
\hline$<0.0059$ & 1 & & $\mu g / L$ & & 0 \\
\hline$<1.0$ & 1 & & $\mu g / L$ & GE & 0 \\
\hline$<20$ & 1 & & $\mu g / L$ & GE & 0 \\
\hline $1.0 E+01$ & & & pCi/L & GP & \\
\hline
\end{tabular}

Note: Flagging, dilution factors, modifiers, and laboratories are for fourth quarter 1994 data only. See Appendix B for flagging criteria. - = exceeded holding time for fourth quarter 1994.

- = exceeded screening level or final PDWS for fourth quarter 1994. 


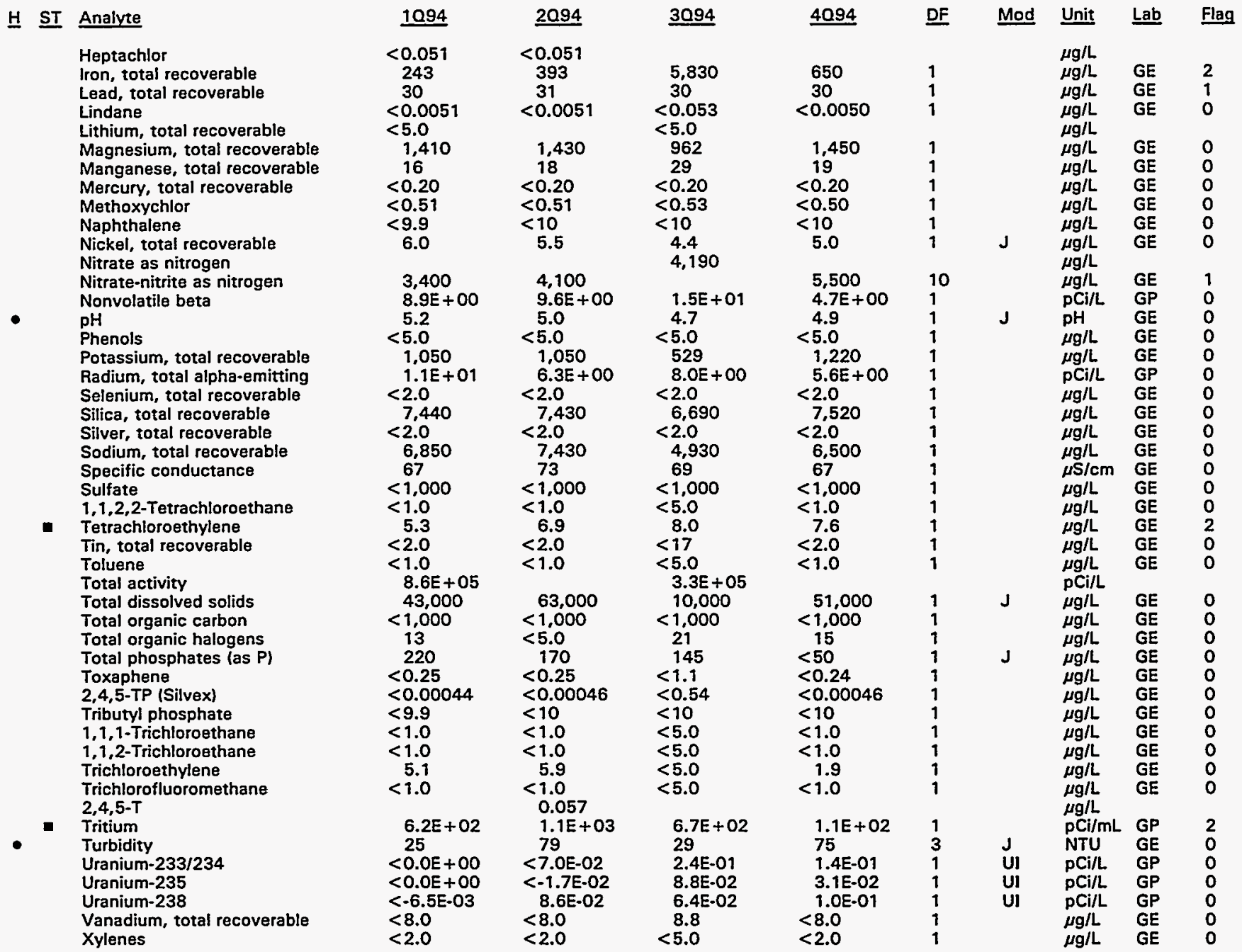

Note: Flagging, dilution factors, modifiers, and laboratories are for fourth quarter 1994 data only. See Appendix B for flagging criteria. - = exceeded holding time for fourth quarter 1994.

- = exceeded screening level or final PDWS for fourth quarter 1994. 
WELL BGO 33C

\begin{tabular}{|c|c|c|c|c|c|c|}
\hline SRS Coord. & Lat/Longitude & Screen Zone Elevation & Top of Casing & Casing & Pump & Screen Zone \\
\hline $\begin{array}{l}\text { N74479.7 } \\
\text { E55681.4 }\end{array}$ & $\begin{array}{l}33.281351^{\circ} \mathrm{N} \\
81.664855{ }^{\circ} \mathrm{W}\end{array}$ & $187.8-177.8 \mathrm{ft} \mathrm{msl}$ & $279.4 \mathrm{ft} \mathrm{msl}$ & 4" PVC & s & McBean (IIB1) \\
\hline PLE D & & $01 / 27 / 94$ & $04 / 11 / 94$ & & & \\
\hline
\end{tabular}

FIELD DATA

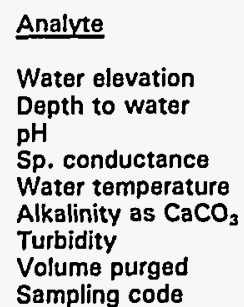

ANALYTICAL DATA

\begin{tabular}{|c|c|c|c|c|}
\hline 1094 & 2094 & 3094 & 4094 & Unit \\
\hline $\begin{array}{l}225.0 \\
54.4 \\
5.7 \\
55 \\
19.3 \\
5 \\
1 \\
7.1\end{array}$ & $\begin{array}{l}225.5 \\
53.9 \\
5.7 \\
59 \\
19.9 \\
4 \\
1 \\
7.0\end{array}$ & $\begin{array}{l}224.6 \\
54.8 \\
5.6 \\
57 \\
20.0 \\
5 \\
1 \\
6.4\end{array}$ & $\begin{array}{l}224.6 \\
54.8 \\
5.6 \\
52 \\
19.6 \\
5 \\
1 \\
6.5\end{array}$ & $\begin{array}{l}\mathrm{ft} \text { msl } \\
\mathrm{ft} \\
\mathrm{pH} \\
\mu \mathrm{S} / \mathrm{cm} \\
{ }^{\circ} \mathrm{C} \\
\mathrm{mg} / \mathrm{L} \\
\text { NTU } \\
\text { Well vol. }\end{array}$ \\
\hline
\end{tabular}

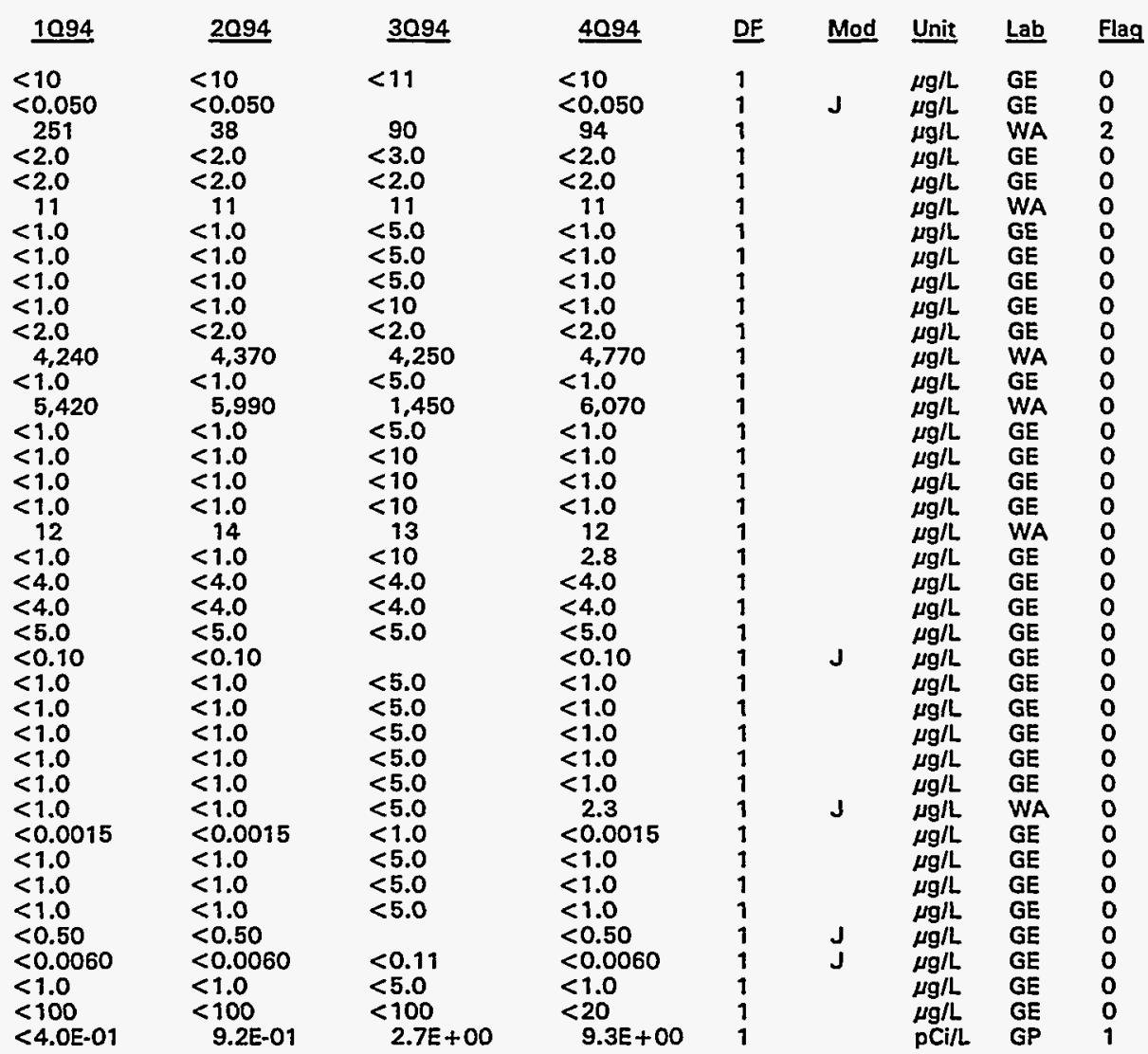

Note: Flagging, dilution factors, modifiers, and laboratories are for fourth quarter 1994 data only. See Appendix B for flagging criteria. - = exceeded holding time for fourth quarter 1994.

- = exceeded screening level or final PDWS for fourth quarter 1994. 
ANALYTICAL DATA

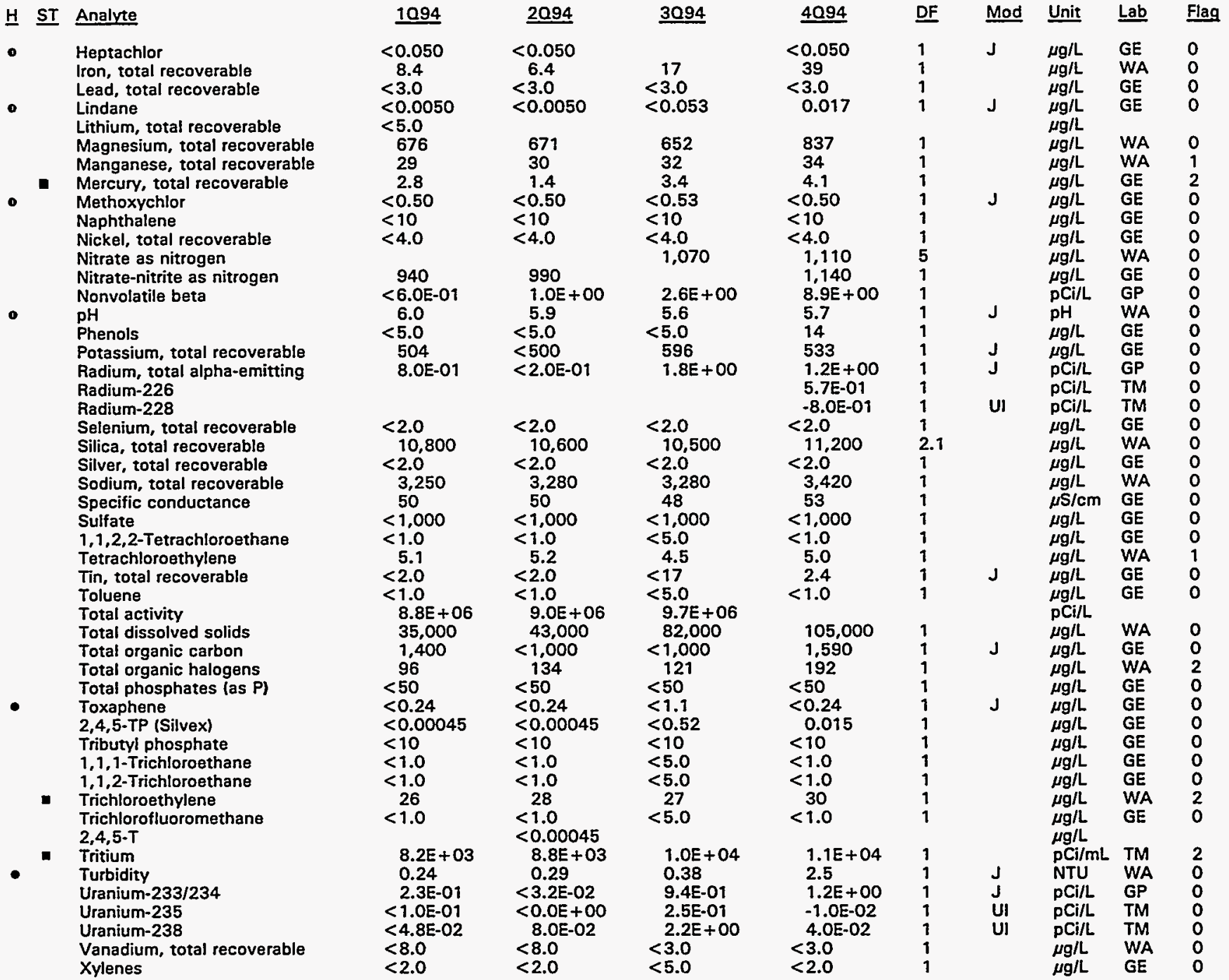

Note: Flagging, dilution factors, modifiers, and laboratories are for fourth quarter 1994 data only. See Appendix B for flagging criteria. - = exceeded holding time for fourth quarter 1994.

- = exceeded screening level or final PDWS for fourth quarter 1994. 


\section{WELL BGO 33D}

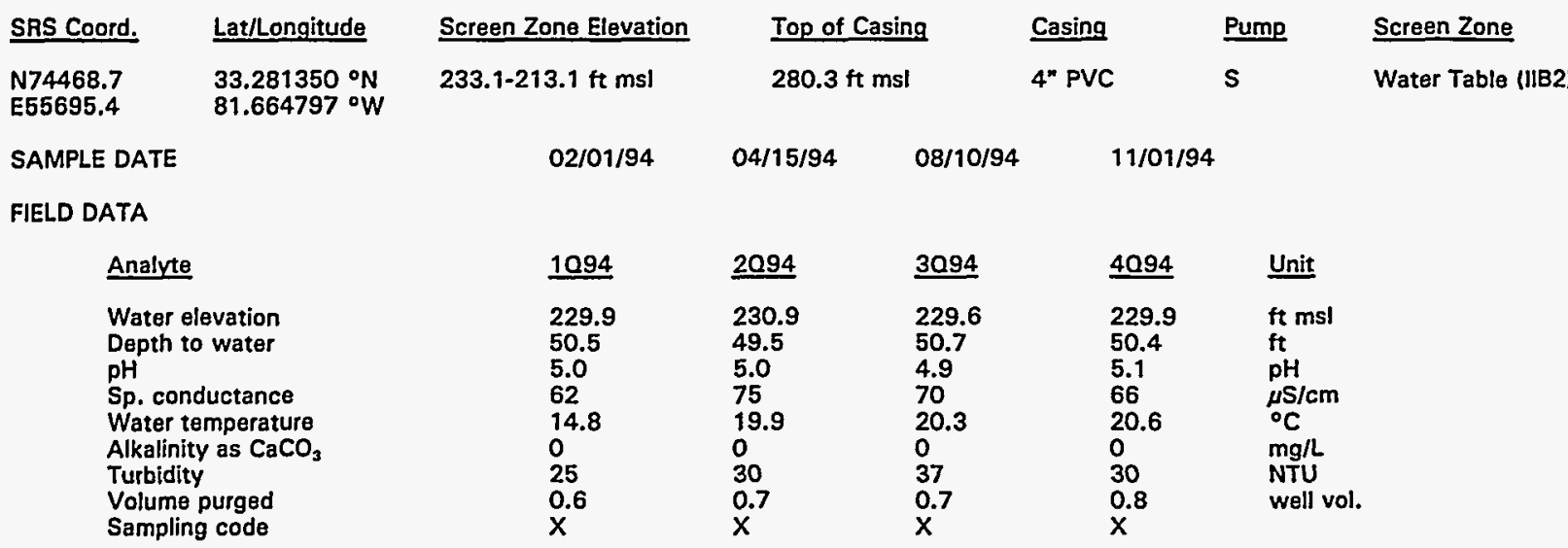

ANALYTICAL DATA

H ST Analyte

Acetophenone

Aldrin

Aluminum, total recoverable

Antimony, total recoverable

Arsenic, total recoverable

Barium, total recoverable

Benzene

Bromodichloromethane

Bromoform

Bromomethane (Methyl bromide)

Cadmium, total recoverable

Calcium, total recoverable

Carbon tetrachloride

Chloride

Chlorobenzene

Chloroethane

Chloroethene (Vinyl chloride)

2-Chloroethyl vinyl ether

Chloroform

Chloromethane (Methyl chloride)

Chromium, total recoverable

Copper, total recoverable

Cyanide

$p, p^{\prime}$-DDT

Dibromochloromethane

1,1-Dichloroethane

1,2-Dichloroethane

1,1-Dichloroethylene

trans-1,2-Dichloroethylene

Dichloromethane

2,4-Dichlorophenoxyacetic acid

1,2-Dichloropropane

cis-1,3-Dichloropropen

trans-1,3-Dichloropropene

Dieldrin

Endrin

Ethylbenzene

Fluoride

Gross alpha
$<9.7$

$<0.050$

2,380

$<2.0$
$<2.0$

19

$<1.0$

$<1.0$

$<1.0$

$<1.0$

$<2.0$

1,330

$<1.0$

8,500

$<1.0$

$<1.0$

$<1.0$

$<1.0$

$<1.0$

$<1.0$

$<4.0$

20
$<5.0$

$<5.0$
$<0.10$

$<1.0$

$<1.0$

$<1.0$

$<1.0$

$<1.0$

$<1.0$

$<0.0015$

$<1.0$

$<1.0$

$<1.0$

$<0.50$

$<0.0060$

$<1.0$

$<100$

$3.4 E+00$
2094

$<10$

332

$<2.0$

$<2.0$

$<1.0$

$<1.0$

$<1.0$

$<1.0$

$<2.0$

1,390

$<1.0$

8,640

$<1.0$

$<1.0$

$<1.0$

$<1.0$

$<1.0$

$<1.0$

$<4.0$

67

$<5.0$

$<0.10$

$<1.0$

$<1.0$

$<1.0$

$<1.0$

$<1.0$

$<1.0$

$<0.0016$

$<1.0$

$<1.0$

$<1.0$

$<0.50$

$<0.0060$

$<1.0$

$<100$

$5.4 E+00$

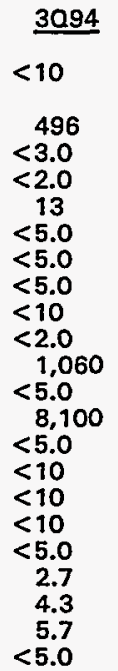

4099

$<10$

322
$<2.0$

$<2.0$
$<2.0$

16

$<1.0$

$<1.0$

$<1.0$

$<1.0$

1,500

$<1.0$

6,860

$<1.0$

$<1.0$

$<1.0$

$<1.0$

$<1.0$

$<1.0$

$<4.0$

71
$<5.0$

$<5.0$

$<5.0$

$<5.0$

$<5.0$

$<5.0$

$<5.0$

$<1.0$

$<5.0$

$<5.0$
$<5.0$

(

$<0.11$

$<5.0$
$<100$

$4.5 E+00$
$<1.0$

$<1.0$

$<1.0$

$<1.0$

$<1.0$

$<1.0$

$<0.0015$

$<1.0$

$<1.0$

$<1.0$

$<0.0060$

$<1.0$

$<20$

2. $3 E+00$
Unit Lab Flag

$\mu g / L$ GE 0

$\mu g / L$

$\mu g / L$

$\mu g / L$

$\mu g / L$
$\mu g / L$

$\mu \mathrm{g} / \mathrm{L}$

$\mu \mathrm{g} / \mathrm{L}$

$\underset{\mu g / L}{\mu g / L}$

$\underset{\mu g / L}{\mu g / L}$

$\mu \mathrm{g} / \mathrm{L}$

$\mu \mathrm{g} / \mathrm{L}$

$\mu g / L$

$\mu g / L$

$\mu g / L$

$\mu g / L$

$\mu \mathrm{g} / \mathrm{L}$

$\mu g / L$

$\mu \mathrm{g} / \mathrm{L}$

$\mu g / L$

$\mu \mathrm{g} / \mathrm{L}$

$\mu g / L$

$\mu g / L$

$\mu \mathrm{g} / \mathrm{L}$

$\mu g / L$

$\mu g / L$

$\mu g / L \quad G E$

$\mu g / L$

$\mu \mathrm{g} / \mathrm{L}$

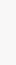

Note: Flagging, dilution factors, modifiers, and laboratories are for fourth quarter 1994 data only. See Appendix B for flagging criteria. - = exceeded holding time for fourth quarter 1994.

E = exceeded screening level or final PDWS for fourth quarter 1994. 


\section{ANALYTICAL DATA}

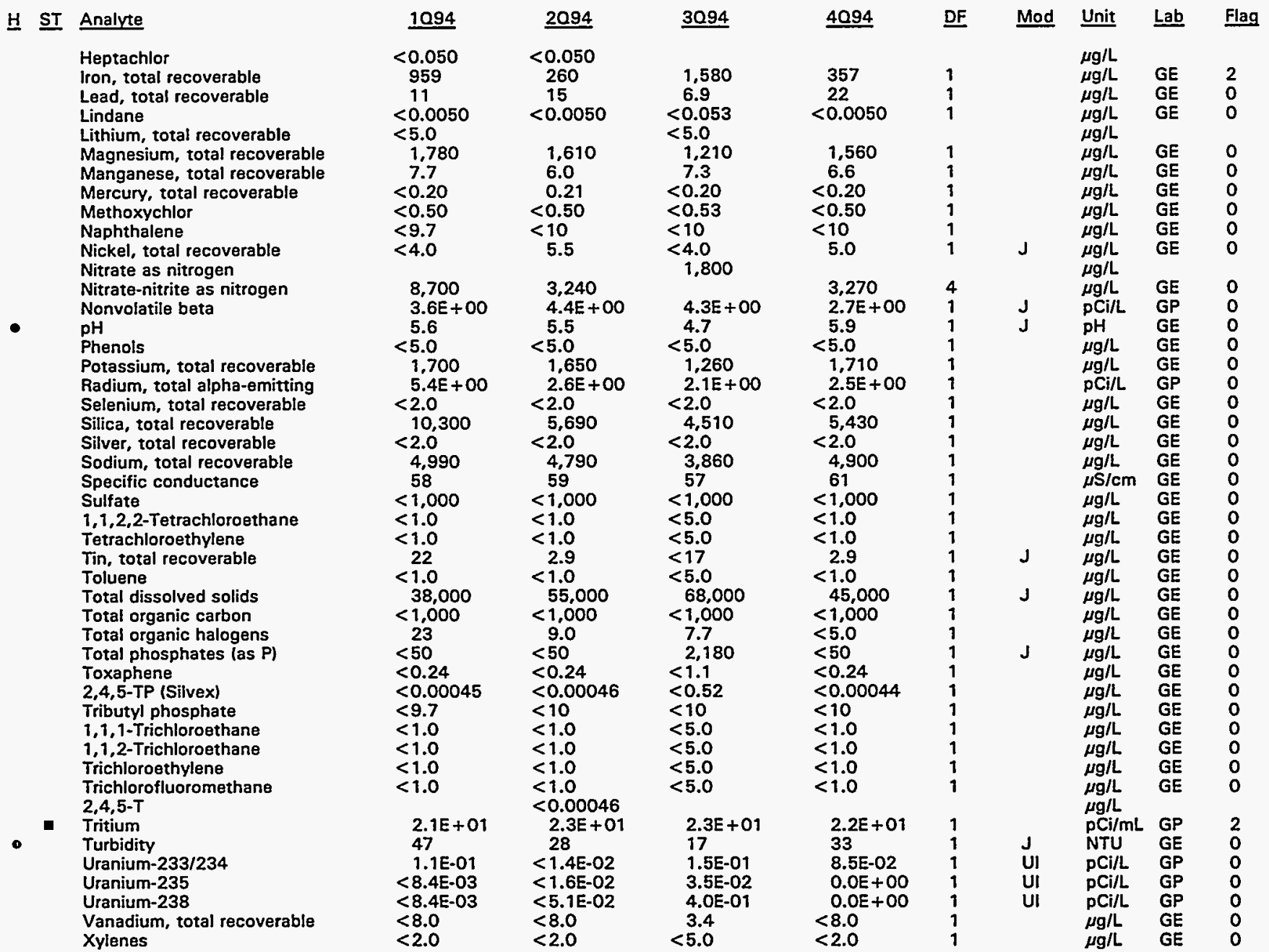

Note: Flagging, dilution factors, modifiers, and laboratories are for fourth quarter 1994 data only. See Appendix B for flagging criteria. - = exceeded holding time for fourth quarter 1994.

I = exceeded screening level or final PDWS for fourth quarter 1994. 
WELL BGO 34D

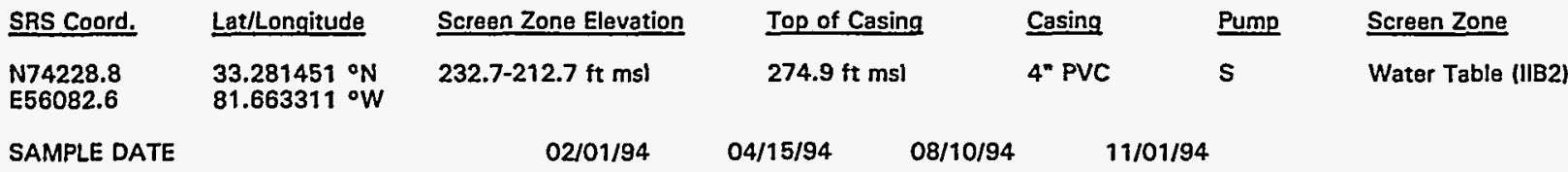

FIELD DATA

Analyte
Water elevation
Depth to water
pH
Sp. conductance
Water temperature
Alkalinity as $\mathrm{CaCO}_{3}$
Turbidity
Volume purged
Sampling code

ANALYTICAL DATA

H ST Analyte

Acetophenone

Aldrin

Aluminum, total recoverable

Antimony, total recoverable

Arsenic, total recoverable

Barium, total recoverable

Benzene

Bromodichloromethane

Bromoform

Bromomethane (Methyl bromide)

Cadmium, total recoverable

Calcium, total recoverable

Carbon tetrachloride

Chloride

Chlorobenzene

Chloroethane

Chloroethene (Vinyl chloride)

2-Chloroethyl vinyl ether

Chloroform

Chloromethane (Methyl chloride)

Chromium, total recoverable

Copper, total recoverable

Cyanide

p,p'-DDT

Dibromochloromethane

1,1-Dichloroethane

1,2-Dichlorosthane

1,1-Dichloroethylene

trans-1,2-Dichloroethylene

Dichloromethane

2,4-Dichlorophenoxyacetic acid

1,2-Dichloropropane

cis-1,3-Dichloropropene

trans-1,3-Dichloropropene

Dieldrin

Endrin

Ethylbenzene

Fluoride

Gross alpha

\begin{tabular}{lll}
1094 & $\underline{2094}$ & $\underline{3094}$ \\
\hline 232.3 & 233.7 & 232.2 \\
42.6 & 41.3 & 42.7 \\
5.7 & 7.8 & 5.7 \\
33 & 100 & 38 \\
15.1 & 19.9 & 19.7 \\
2 & 27 & 5 \\
5 & 5 & 4 \\
1.0 & 0.9 & 1.1 \\
$x$ & $x$ & $x$
\end{tabular}

\section{4}

$<10$

$<0.053$

$<0.053$
143
$<2.0$

$<2.0$
$<2.0$

$<2.0$
26

$<1.0$

$<1.0$

$<1.0$

$<1.0$

$<2.0$

2,330

3,440

$<1.0$

$<1.0$

$<1.0$

$<1.0$

$<1.0$

$<1.0$

$<4.0$
9.8
$<5.0$

$<0.11$

$<0.11$

$<1.0$

$<1.0$

$<1.0$

$<1.0$

$<1.0$

$<0.0015$

$<1.0$

$<1.0$

$<1.0$

$<0.53$

$<0.0064$

$<1.0$

$<100$

$1.7 E+00$

$\quad 2094$
$<10$
$<0.050$
146
$<2.0$
$<2.0$
49
$<1.0$
$<1.0$
$<1.0$
$<1.0$
$<2.0$
14.200
$<1.0$
3.630
$<1.0$
$<1.0$
$<1.0$
$<1.0$
$<1.0$
$<1.0$
$<4.0$
$<4.0$
$<5.0$
$<0.10$
$<1.0$
$<1.0$
$<1.0$
$<1.0$
$<1.0$
$<1.0$
$<0.0016$
$<1.0$
$<1.0$
$<1.0$
$<0.50$
$<0.0060$
$<1.0$
$<100$
$1.56+00$

$\quad 3094$
$\quad<11$
$\quad 262$
$<3.0$
$<2.0$
21
$<5.0$
$<5.0$
$<5.0$
$<10$
$<2.0$
3.280
$<5.0$
3.850
$<5.0$
$<10$
$<10$
$<10$
$<5.0$
2.6
$<4.0$
$<4.0$
$<5.0$
$<5.0$
$<5.0$
$<5.0$
$<5.0$
$<5.0$
$<5.0$
$<1.1$
$<5.0$
$<5.0$
$<5.0$
$<0.11$
$<5.0$
$<100$
$1.9 E+00$

$\underline{\text { Unit }}$

ft msl

$\mathrm{pH}$

$\mu \mathrm{S} / \mathrm{cm}$

Ng/L

well vol.

$\begin{array}{ll}19.0 & \mathrm{mg} / \mathrm{L} \\ 1 & \text { NTU } \\ 24 & \text { well vol. } \\ 0.9 & \end{array}$

\begin{tabular}{|c|c|c|c|c|}
\hline$\underline{4094}$ & $\underline{D F}$ & Mod & Unit & $L a b$ \\
\hline$<10$ & 1 & & $\begin{array}{c}\mu \mathrm{g} / \mathrm{L} \\
\mu \mathrm{g} / \mathrm{L}\end{array}$ & GE \\
\hline 261 & 1 & & $\mu \mathrm{g} / \mathrm{L}$ & GE \\
\hline$<2.0$ & 1 & & $\mu g / L$ & GE \\
\hline$<2.0$ & 1 & & $\mu g / L$ & GE \\
\hline 19 & 1 & & $\mu \mathrm{g} / \mathrm{L}$ & GE \\
\hline $\begin{array}{l}<1.0 \\
<1.0\end{array}$ & 1 & & $\mu \mathrm{g} / \mathrm{L}$ & $\begin{array}{l}\mathrm{GE} \\
\mathrm{GE}\end{array}$ \\
\hline$<1.0$ & $i$ & & $\mu \mathrm{g} / \mathrm{L}$ & $\mathrm{GE}$ \\
\hline$<1.0$ & 1 & & $\mu \mathrm{g} / \mathrm{L}$ & GE \\
\hline$<2.0$ & 1 & & $\mu g / L$ & GE \\
\hline $\begin{array}{l}2,430 \\
<1.0\end{array}$ & 1 & & $\underset{\mu g / L}{\mu g / L}$ & $\begin{array}{l}\mathrm{GE} \\
\mathrm{GE}\end{array}$ \\
\hline$<250$ & $i$ & & $\mu \mathrm{g} / \mathrm{L}$ & $\mathrm{GE}$ \\
\hline$<1.0$ & 1 & & $\mu \mathrm{g} / \mathrm{L}$ & GE \\
\hline$<1.0$ & 1 & & $\mu g / L$ & GE \\
\hline$<1.0$ & 1 & & $\mu g / L$ & GE \\
\hline$<1.0$ & 1 & & $\mu \mathrm{g} / \mathrm{L}$ & GE \\
\hline$<1.0$ & 1 & & $\mu g / L$ & GE \\
\hline$<1.0$ & 1 & & $\mu \mathrm{g} / \mathrm{L}$ & GE \\
\hline$<4.0$ & 1 & & $\mu \mathrm{g} / \mathrm{L}$ & $\mathrm{GE}$ \\
\hline $\begin{array}{r}4.8 \\
-5.8\end{array}$ & 1 & $J$ & $\mu \mathrm{g} / \mathrm{L}$ & GE \\
\hline$<5.0$ & 1 & & $\underset{\mu \mathrm{g} / \mathrm{L}}{\mu \mathrm{g} / \mathrm{L}}$ & \\
\hline$<1.0$ & 1 & & $\mu \mathrm{g} / \mathrm{L}$ & GE \\
\hline$<1.0$ & 1 & & $\mu \mathrm{g} / \mathrm{L}$ & GE \\
\hline$<1.0$ & 1 & & $\mu \mathrm{g} / \mathrm{L}$ & GE \\
\hline$<1.0$ & 1 & & $\mu g / L$ & GE \\
\hline$<1.0$ & 1 & & $\mu g / L$ & GE \\
\hline$<1.0$ & 1 & & $\mu \mathrm{g} / \mathrm{L}$ & GE \\
\hline$<0.0015$ & 1 & & $\mu \mathrm{g} / \mathrm{L}$ & GE \\
\hline$<1.0$ & 1 & & $\mu g / L$ & GE \\
\hline$<1.0$ & 1 & & $\mu \mathrm{g} / \mathrm{L}$ & GE \\
\hline$<1.0$ & 1 & & $\mu g / L$ & GE \\
\hline$<0.0063$ & 1 & $J$ & $\mu \mathrm{g} / \mathrm{L}$ & \\
\hline$<1.0$ & 1 & & $\mu \mathrm{g} / \mathrm{L}$ & GE \\
\hline$<20$ & 1 & & $\mu \mathrm{g} / \mathrm{L}$ & GE \\
\hline $1.3 E+00$ & 1 & $J$ & $\mathrm{Ci} / \mathrm{L}$ & GP \\
\hline
\end{tabular}

Note: Flagging, dilution factors, modifiers, and laboratories are for fourth quarter 1994 data only. See Appendix B for flagging criteria. - = exceeded holding time for fourth quarter 1994.

- = exceeded screening level or final PDWS for fourth quarter 1994. 


\section{ANALYTICAL DATA}

H SI

Heptachlor

Iron, total recoverable

Lead, total recoverable

Lindane

Lithium, total recoverable

Magnesium, total recoverable

Manganese, total recoverable

Mercury, total recoverable

Methoxychlor

Naphthalene

Nickel, total recoverable

Nitrate as nitrogen

Nitrate-nitrite as nitrogen

Nonvolatile beta

- $\mathrm{pH}$

Phenols

Potassium, total recoverable

Radium, total alpha-emitting

Selenium, rotal recoverable

Silica, total recoverable

Silver, total recoverable

Sodium, total recoverable

Specific conductance

Sulfate

1,1,2,2-Tetrachloroethane

Tetrachloroethylene

Tin, total recoverable

Toluene

Total dissolved solids

Total organic carbon

Total organic halogens

Total phosphates (as P)

Toxaphene

2,4,5-TP (Silvex)

Tributyl phosphate

1,1,1-Trichloroethane

1,1,2-Trichloroethane

Trichloroethylene

Trichlorofluoromethane

2,4,5-T

- Tritium

Turbidity

Uranium-233/234

Uranium-235

Uranium-238

Vanadium, tota! recoverable

Xylenes

$\begin{array}{ccc}1094 & \underline{2094} & \underline{3094} \\ <0.053 & <0.050 & \\ 21 & 36 & 36 \\ 8.9 & <3.0 & 9.9 \\ <0.0053 & <0.0050 & <0.054 \\ <5.0 & & <5.0 \\ 788 & 1.210 & 713 \\ 3.6 & 2.6 & 5.2 \\ <0.20 & <0.20 & <0.20 \\ <0.53 & <0.50 & <0.54 \\ <10 & <10 & <10 \\ <4.0 & <4.0 & <4.0 \\ 1.050 & 940 & 864 \\ 1.75+00 & 1.85+00 & \end{array}$

1.7E+ +00

5.9

$<5.0$

$<500$

2. $0 E+00$

$<2.0$

6,620

$<2.0$

1,750

32

$<1,000$

$<1.0$

$<1.0$

9.2

$<1.0$

26,000

$<1,000$

19
$<50$

$<0.26$

$<0.00045$

$<10$

$<1.0$

$<1.0$

$<1.0$

$<1.0$

2.3E +01

0.93

$<-1.1 \mathrm{E}-03$

$<O . O E+00$

$<4.2 \mathrm{E}-02$

$<8.0$

$<2.0$
$1.8 \mathrm{E}+00$

7.8

$<5.0$

$<500$
$1.3 \mathrm{E}+00$

$<2.0$

6,580

$<2.0$

1,910

92

$<1,000$

$<1.0$

$<1.0$

$<2.0$

$<1.0$

60,000

$<1,000$

$<5.0$

$<50$

$<0.24$

$<0.00047$

$<10$

$<1.0$

$<1.0$

$<1.0$

$<1.0$

$<0.00047$

2. $6 E+00$

5.3

$<5.0$

$<500$

8.0E-01

$<2.0$

6,910

$<2.0$

1,560

36

$<1,000$

$<5.0$

$<5.0$

$<17$

$<5.0$

$<1,000$
$<1,000$

9.3

170

$<1.1$

$<0.55$

$<10$

$<5.0$

$<5.0$

$<5.0$

$<5.0$

$2.5 \mathrm{E}+01$

1.6

$<1.5 E-02$

$<0.0 E+00$

$<3.5 E-02$

$<8.0$
$2.5 E+01$

1.5

1.3E-01

7.5E-02

$<3.0$
$<5.0$

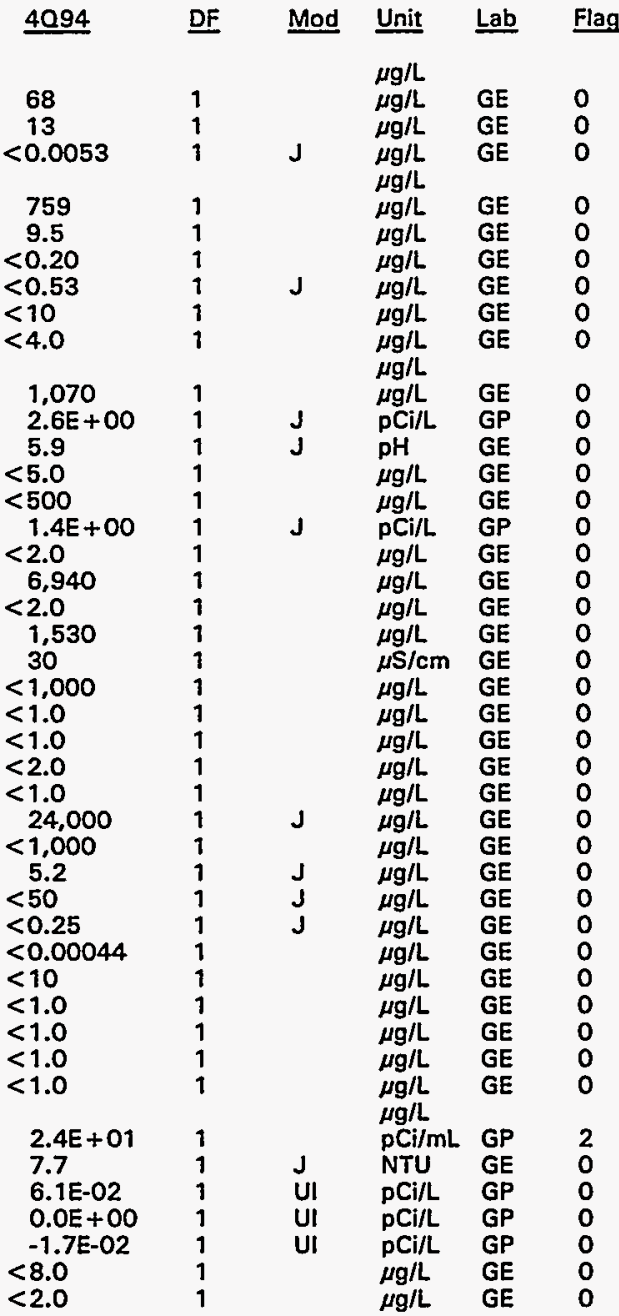

Note: Flagging, dilution factors, modifiers, and laboratories are for fourth quarter 1994 data only. See Appendix 8 for flagging criteria. - = exceeded holding time for fourth quarter 1994.

n = exceeded screening level or final PDWS for fourth quarter 1994. 


\section{WELL BGO 35C}

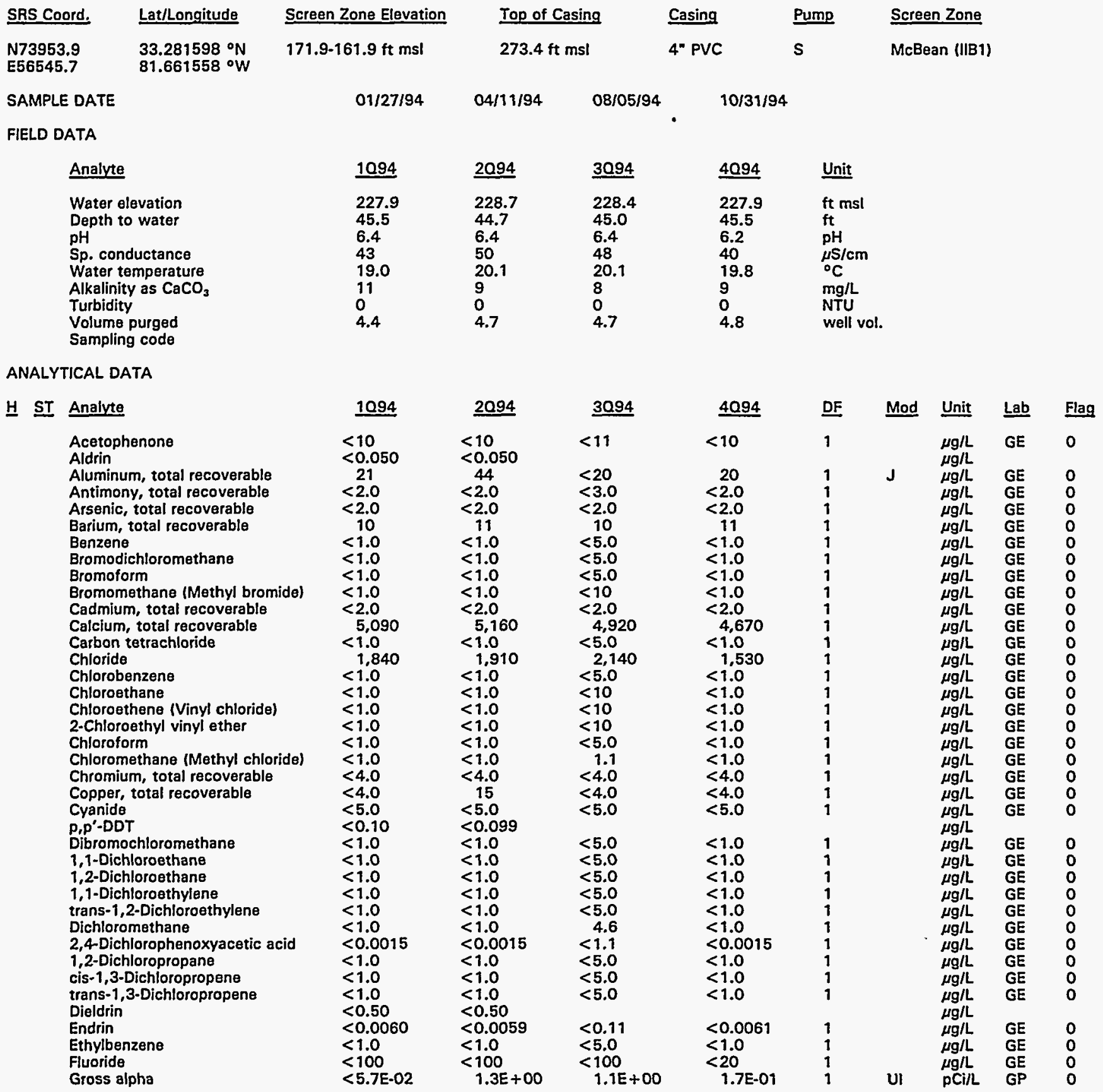

Note: Flagging, dilution factors, modifiers, and laboratories are for fourth quarter 1994 data only. See Appendix B for flagging criteria. $\bullet=$ exceeded holding time for fourth quarter 1994.

- = exceeded screening level or final PDWS for fourth quarter 1994. 
Well BGO 35C continued

\section{ANALYTICAL DATA}

H $\underline{\text { ST Analyte }}$

Heptachlor

Iron, total recoverable

Lead, total recoverable

Lindane

Lithium, total recoverable

Magnesium, total recoverable

Manganese, total recoverable

Mercury, total recoverable

Methoxychlor

Naphthalene

Nickel, total recoverable

Nitrate as nitrogen

Nitrate-nitrite as nitrogen

Nonvolatile beta

-

Potassium, total recoverabl

Radium, total alpha-emitting

Radium-226

Radium-228

Selenium, total recoverable

Silica, total recoverable

Silver, total recoverable

Sodium, total recoverable

Specific conductance

Sulfate

1,1,2,2-Tetrachloroethane

Tetrachloroethylene

Tin, total recoverable

Toluene

Total dissoived solids

Total organic carbon

Total organic halogens

Total phosphates (as P)

Toxaphene

2,4,5-TP (Silvex)

Tributyl phosphate

1,1,1-Trichloroethane

1,1,2-Trichloroethane

- Trichloroethylene

Trichlorofluoromethane

$2,4,5-T$

- Tritium

Turbidity

Uranium-233/234

Uranium-235

Uranium-238

Vanadium, total recoverable

Xylenes

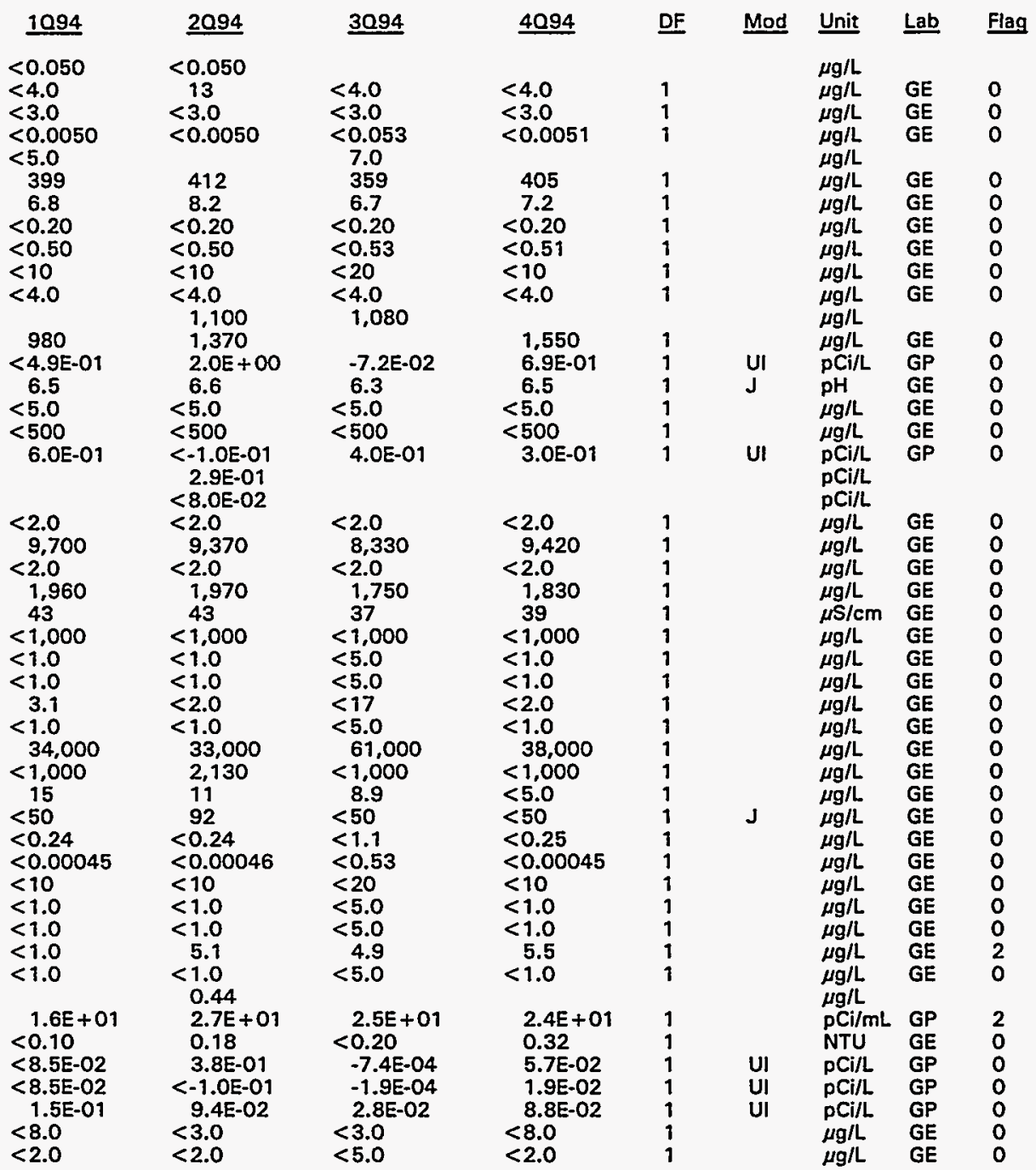

Note: Flagging, dilution factors, modifiers, and laboratories are for fourth quarter 1994 data only. See Appendix B for flagging criteria. - = exceeded holding time for fourth quarter 1994.

- = exceeded screening level or final PDWS for fourth quarter 1994. 
WELL BGO 35D

\begin{tabular}{|c|c|c|c|c|c|c|c|}
\hline Lat/Lonaitude & Lat/Longitude & Screen Zone Elevation & \multicolumn{2}{|c|}{ Top of Casing } & Casing & Pump & \multirow{3}{*}{$\frac{\text { Screen Zone }}{\text { Water Table (IIB2) }}$} \\
\hline $\begin{array}{l}\text { N73946.0 } \\
\text { E56556.5 }\end{array}$ & $\begin{array}{l}33.281599^{\circ} \mathrm{N} \\
81.661514^{\circ} \mathrm{W}\end{array}$ & 239.4-219.4 ft msl & \multicolumn{2}{|c|}{$273.5 \mathrm{ft} \mathrm{msl}$} & 4" PVC & $\mathbf{S}$ & \\
\hline SAMPLE DA & & $02 / 01 / 94$ & $04 / 15 / 94$ & $08 / 10 / 94$ & $11 / 01 / 94$ & & \\
\hline \multicolumn{8}{|l|}{ FIELD DATA } \\
\hline \multicolumn{2}{|c|}{ Analyte } & 1094 & $\underline{2094}$ & $\underline{3094}$ & $\underline{4 Q 94}$ & Unit & \\
\hline \multicolumn{2}{|c|}{$\begin{array}{l}\text { Water elevation } \\
\text { Depth to water } \\
\text { pH } \\
\text { Sp. conductance } \\
\text { Water temperature } \\
\text { Alkalinity as } \mathrm{CaCO}_{3} \\
\text { Turbidity } \\
\text { Volume purged } \\
\text { Sampling code }\end{array}$} & $\begin{array}{l}233.6 \\
39.9 \\
4.6 \\
37 \\
15.2 \\
0 \\
30 \\
0.8 \\
x\end{array}$ & $\begin{array}{l}235.1 \\
38.4 \\
4.8 \\
45 \\
20.5 \\
0 \\
38 \\
1.1 \\
x\end{array}$ & $\begin{array}{l}234.1 \\
39.4 \\
4.7 \\
39 \\
20.2 \\
0 \\
55 \\
0.8 \\
x\end{array}$ & $\begin{array}{l}234.4 \\
39.1 \\
4.7 \\
61 \\
21.2 \\
0 \\
33 \\
0.9 \\
x\end{array}$ & $\begin{array}{l}\text { ft msl } \\
\text { ft } \\
\mathrm{pH} \\
\text { pS/cm } \\
{ }^{\circ} \mathrm{C} \\
\mathrm{mg} / \mathrm{l} \\
\text { NTU } \\
\text { well vol. }\end{array}$ & \\
\hline
\end{tabular}

ANALYTICAL DATA

H ST Analyte

Acetophenone

Aldrin

Aluminum, total recoverable

Antimony, total recoverable

Arsenic, total recoverable

Barium, total recoverable

Benzene

Bromodichloromethane

Bromoform

Cadmium, total recoverable

Calcium, total recoverable

Carbon tetrachloride

Chloride

Chlorobenzene

Chloroethane

Chloroethene (Vinyl chloride)

2-Chloroethyl vinyl ether

Chloroform

Chloromethane (Methyl chloride)

Chromium, total recoverable

Copper, total recoverable

Cyanide

$p, p^{\prime}$-DDT

Dibromochloromethane

1,1-Dichloroethane

1,2-Dichloroethane

1,1-Dichloroethylene

trans-1,2-Dichloroethylene

Dichloromethane

2,4-Dichlorophenoxyacetic acid

1,2-Dichloropropane

cis-1,3-Dichloropropene

trans-1,3-Dichloropropene

Dieldrin

Endrin

Ethylbenzene

Fluoride

Gross alpha

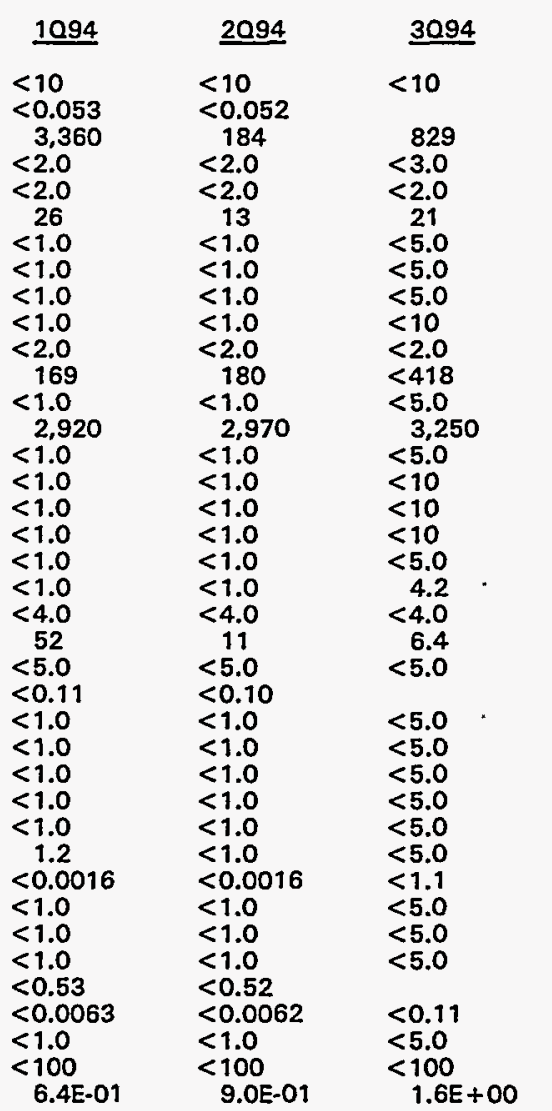

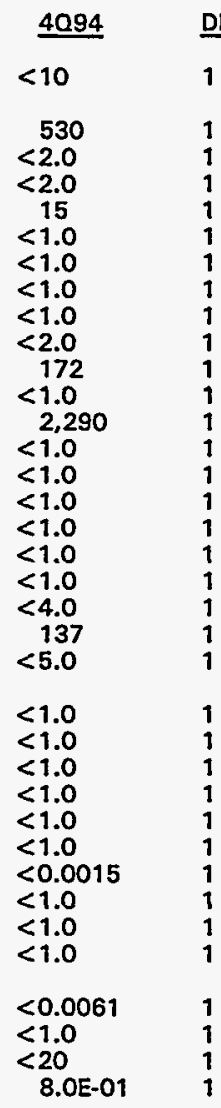

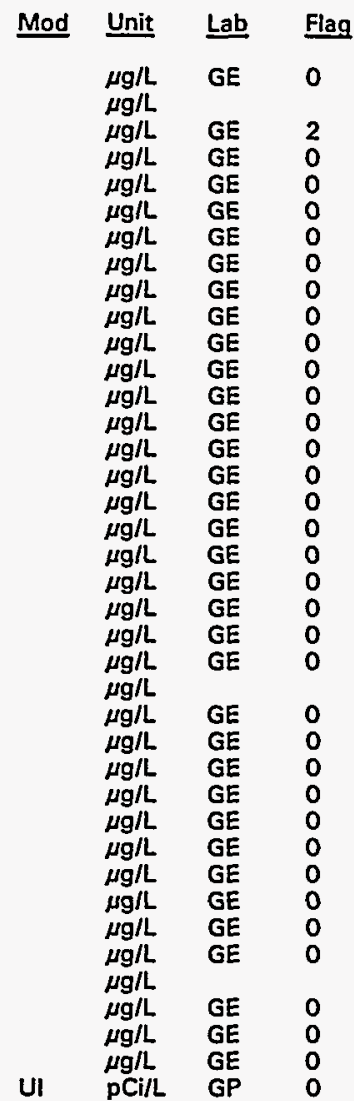

Note: Flagging, dilution factors, modifiers, and laboratories are for fourth quarter 1994 data only. See Appendix B for flagging criteria. - = exceeded holding time for fourth quarter 1994.

- = exceeded screening level or final PDWS for fourth quarter 1994. 


\section{ANALYTICAL DATA}

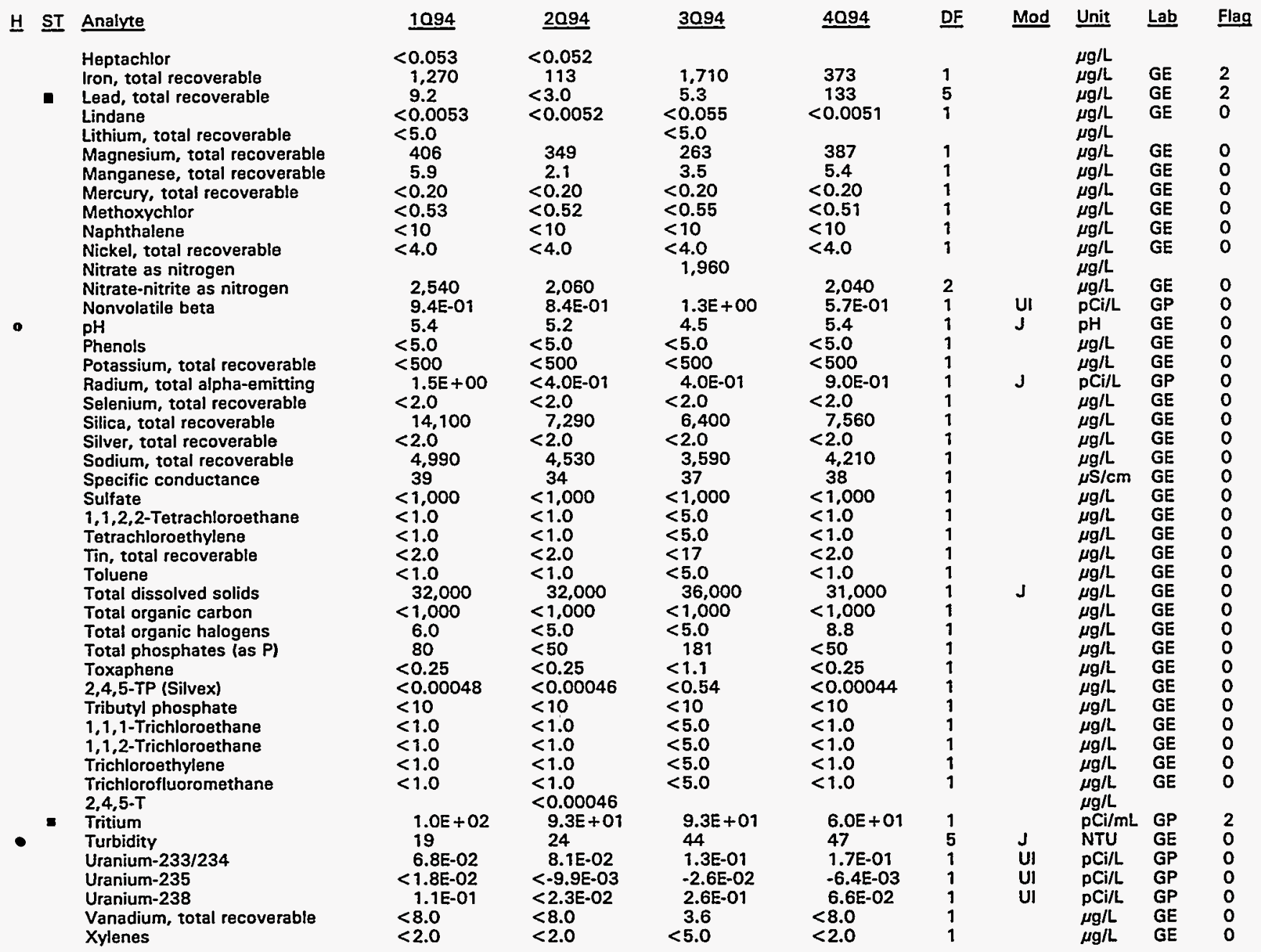

Note: Flagging, dilution factors, modifiers, and laboratories are for fourth quarter 1994 data only. See Appendix B for flagging criteria. - = exceeded holding time for fourth quarter 1994 .

- = exceeded screening level or final PDWS for fourth quarter 1994. 
WELL BGO 36D

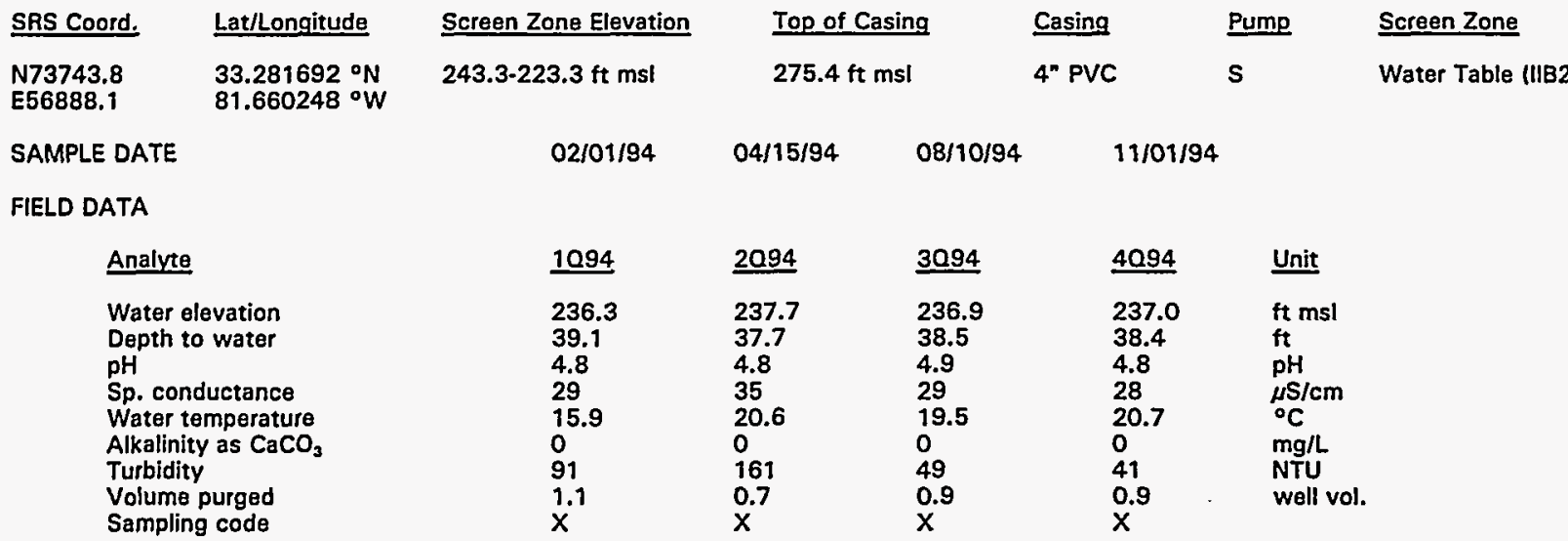

ANALYTICAL DATA

H ST Analyte

Acetophenone

Aldrin

Aluminum, total recoverable

Antimony, total recoverable

Arsenic, total recoverable

Barium, total recoverable

Benzene

Bromodichloromethane

Bromoform

Bromomethane (Methyl bromide)

Cadmium, total recoverable

Calcium, total recoverable

Carbon tetrachloride

Chloride

Chlorobenzene

Chloroethane

Chloroethene (Vinyl chloride)

2-Chloroethyl vinyl ether

Chloroform

Chloromethane (Methyl chloride)

Chromium, total recoverable

Copper, total recoverable

Cyanide

P, ${ }^{\prime}$-DDT

Dibromochloromethane

1,1-Dichloroethane

1,2-Dichloroethane

1,1-Dichloroethylene

trans-1,2-Dichloroethylene

Dichloromethane

2,4-Dichlorophenoxyacetic acid

1,2-Dichloropropane

cis-1,3-Dichloropropene

trans-1,3-Dichloropropene

Dieldrin

Endrin

Ethylbenzene

Fluoride

Gross alpha
$<10$

$<0.050$

$<2.0$

$<2.0$

17
$<1.0$

$<1.0$

$<1.0$

$<1$.

$<2.0$

$<1.0$

3,480

$<1.0$

$<1.0$

$<1.0$

$<1.0$

$<1.0$

$<1.0$

$<4.0$

147
$<5.0$

$<0.10$

$<1.0$

$<1.0$

$<1.0$

$<1.0$

$<1.0$

$<1.0$

$<0.0015$

$<1.0$

$<1.0$
$<0.50$

$<0.0060$

$<1.0$

$<100$

$1.5 E+00$
$<10$

$<0.049$

1,050

$<2.0$

17

$<1.0$

$<1.0$

$<1.0$

$<1.0$

$<2.0$

82

$<1.0$

3,650

$<1.0$

$<1.0$

$<1.0$

$<1.0$

$<1.0$

$<1.0$

$<4.0$

192

$<5.0$

$<0.097$

$<1.0$

$<1.0$

$<1.0$

$<1.0$

$<10$

$<1.0$

$<0.0015$

$<1.0$

$<1.0$

$<1.0$

$<0.49$

$<0.0058$

$<1.0$

$<100$

$1.9 E+00$

$\quad 3094$
$<10$
$\quad 1.520$
$<3.0$
$<2.0$
12
$<5.0$
$<5.0$
$<5.0$
$<10$
$<2.0$
$<418$
$<5.0$
3,820
$<5.0$
$<10$
$<10$
$<10$
$<5.0$
4.9
5.7
121
$<5.0$
$<5.0$
$<5.0$
$<5.0$
$<5.0$
$<5.0$
$<5.0$
$<5$
$<1.0$
$<5.0$
$<5.0$
$<5.0$
$<0.11$
$<5.0$
$<100$
$3.5 E+00$

$\begin{aligned} & 4094 \\ &<10 \\ & 950 \\ &<2.0 \\ &<2.0 \\ & 15 \\ &<1.0 \\ &<1.0 \\ &<1.0 \\ &<1.0 \\ &<2.0 \\ & 103 \\ &<1.0 \\ & 2.670 \\ &<1.0 \\ &<1.0 \\ &<1.0 \\ &<1.0 \\ &<1.0 \\ &<1.0 \\ &<4.0 \\ & 63 \\ &<5.0 \\ &<1.0 \\ &<1.0 \\ &<1.0 \\ &<1.0 \\ &<1.0 \\ &<1.0 \\ &<0.0015 \\ &<1.0 \\ &<1.0 \\ &<1.0 \\ &<0.0060 \\ &<1.0 \\ & 21.7 \\ &\end{aligned}$

Mod Unit Lab Flag

$\mu g / L$

$\mu g / L$

$\mu g / L$
$\mu g / L$

$\mu g / L$

$\mu g / L$

$\mu g / L$

$\mu g / L$

$\mu g / L$

$\mu g / L$
$\mu g / L$
$\mu g / L$

$\mu g / L$

$\mu \mathrm{g} / \mathrm{L}$

$\mu g / L$

$\mu \mathrm{g} / \mathrm{L}$

$\mu \mathrm{g} / \mathrm{L}$

$\mu g / L$

$\mu g / L$

$\mu g / L$
$\mu g / L$
$\mu g / L$

g/L

$\mu g / L$
$\mu g / L$
$\mu g / L$

$\mu \mathrm{g} /$

$\mu g / L$

$\mu g / L$

$\mu g / L$

$\mu \mathrm{g} / \mathrm{L}$
$\mu \mathrm{g} / \mathrm{L}$

gl

$\mu g / L$

$\mu g / L$

$\mu \mathrm{g} / \mathrm{L}$

$\mu \mathrm{g} / \mathrm{L}$

$\mu g / L$

GE 0

GE

GE

Note: Flagging, dilution factors, modifiers, and laboratories are for fourth quarter 1994 data only. See Appendix B for flagging criteria. - = exceeded holding time for fourth quarter 1994.

= exceeded screening level or final PDWS for fourth quarter 1994. 


\section{Analyte}

Heptachlor

Iron, total recoverable

Lead, total recoverable

Lindane

Lithium, total recoverable

Magnesium, total recoverable

Manganese, total recoverable

Mercury, total recoverable

Methoxychlor

Naphthalene

Nickel, total recoverable

Nitrate as nitrogen

Nitrate-nitrite as nitrogen

Nonvolatile beta

- $\mathrm{pH}$

Phenols

Potassium, total recoverable

Radium, total alpha-emitting

Selenium, total recoverable

Silica, total recoverable

Silver, total recoverable

Sodium, total recoverable

Specific conductance

Sulfate

1,1,2,2-Tetrachloroethane

Tetrachloroethylene

Tin, total recoverable

Toluene

Total dissolved solids

Total organic carbon

Total organic halogens

Total phosphates (as P)

Toxaphene

2,4,5-TP (Silvex)

Tributyl phosphate

1,1,1-Trichloroethane

1,1,2-Trichloroethane

Trichloroethylene

Trichlorofluoromethane

2,4,5-T

- Tritium

Turbidity

Uranium-233/234

Uranium-235

Uranium-238

Vanadium, total recoverable

Xylenes

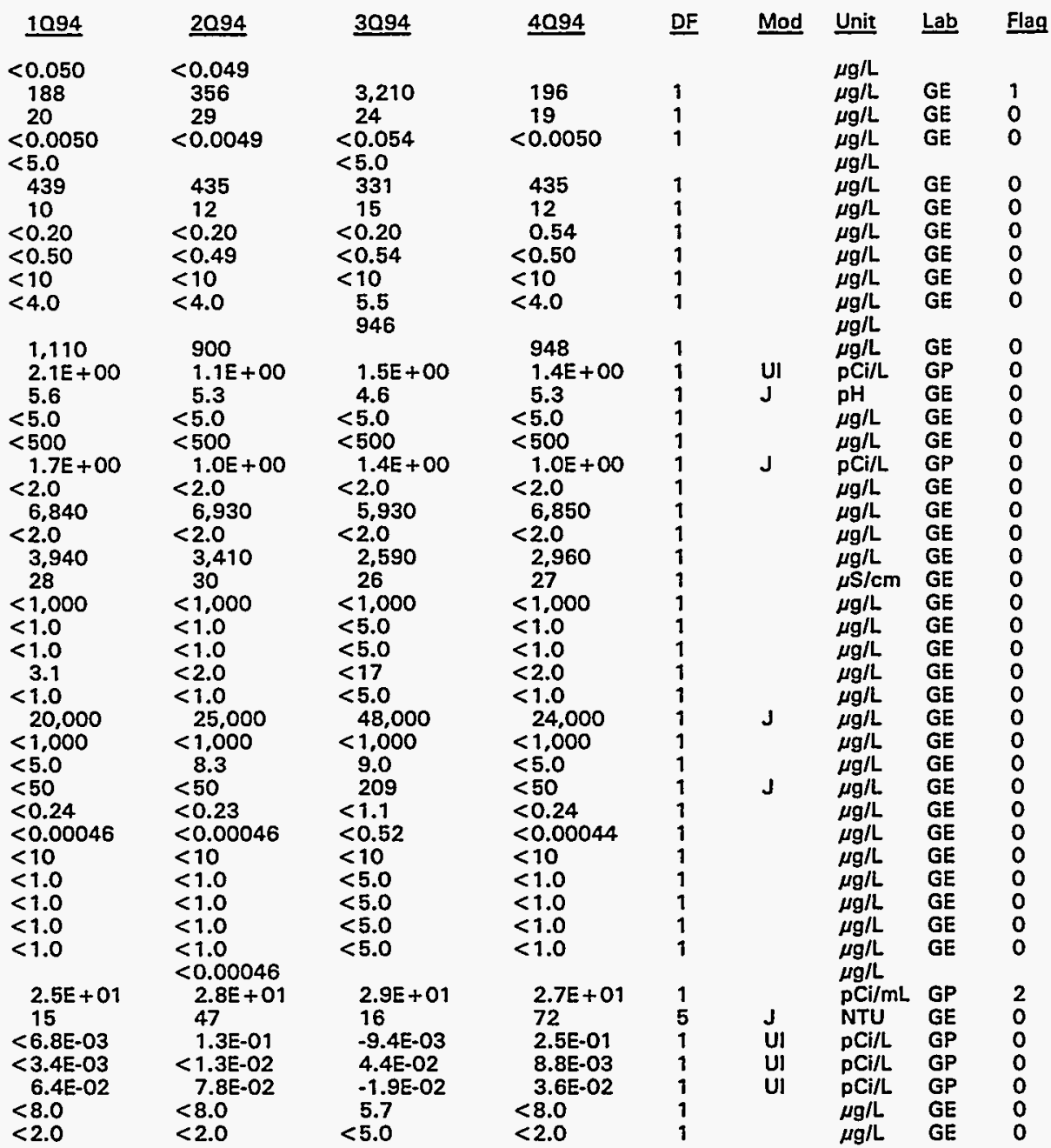

Note: Flagging, dilution factors, modifiers, and laboratories are for fourth quarter 1994 data only. See Appendix B for flagging criteria. - = exceeded holding time for fourth quarter 1994.

- = exceeded screening level or final PDWS for fourth quarter 1994. 
WELL BGO 37C

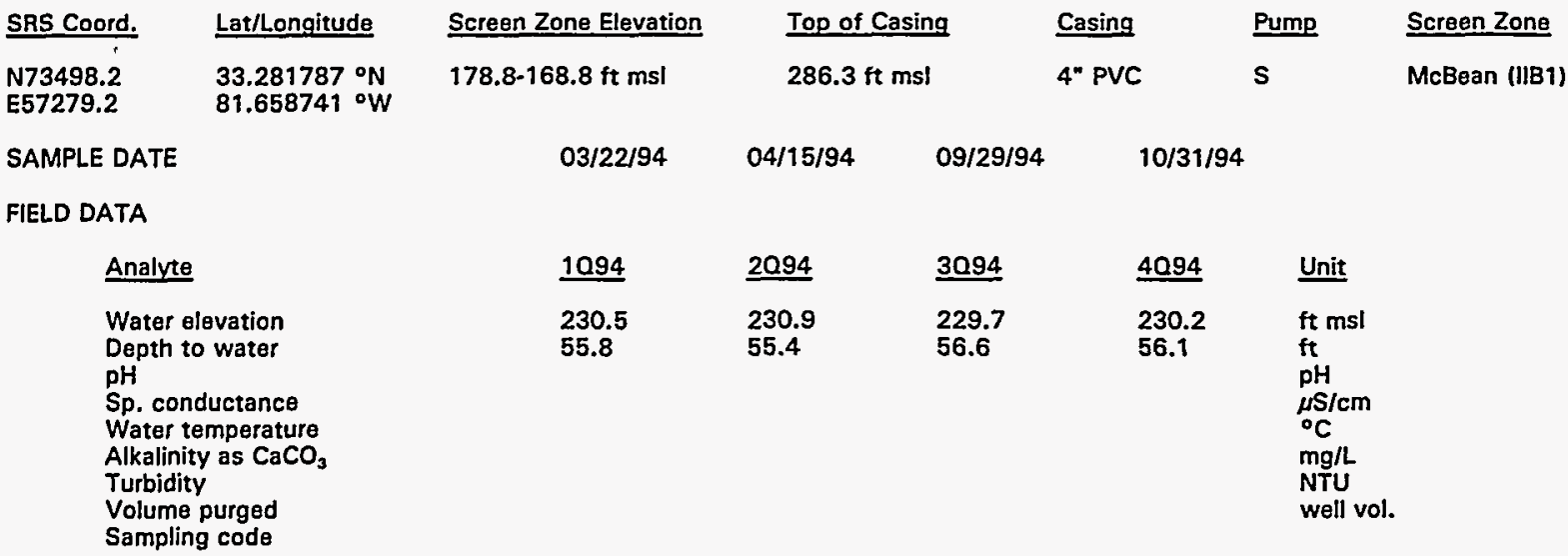

\section{WELL BGO 37D}

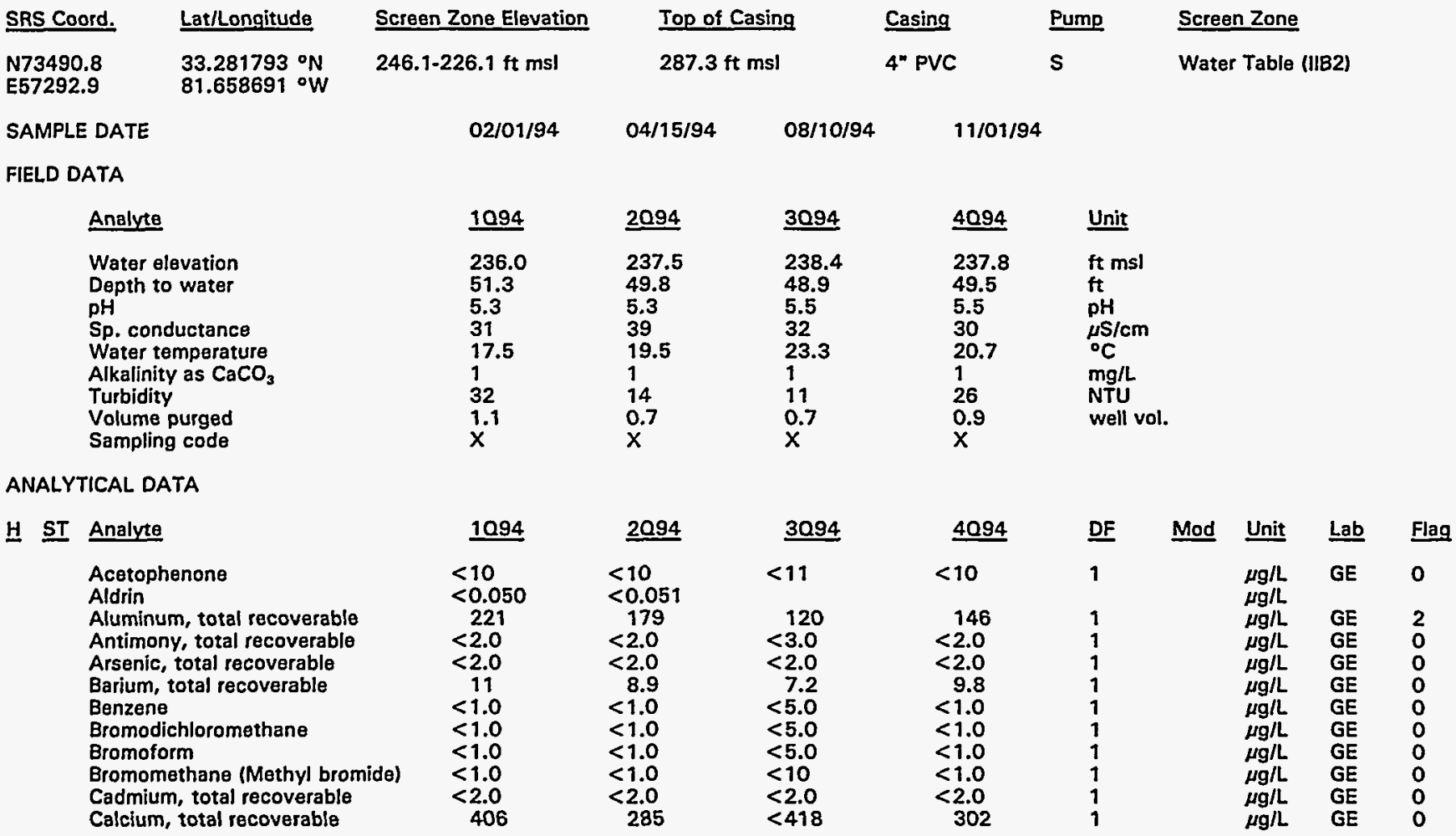

Note: Flagging, dilution factors, modifiers, and laboratories are for fourth quarter 1994 data only. See Appendix B for flagging criteria. - = exceeded holding time for fourth quarter 1994.

- exceeded screening level or final PDWS for fourth quarter 1994. 


\section{Analyte}

Carbon tetrachloride

Chloride

Chlorobenzene

Chloroethane

Chloroethene (Vinyl chloride)

2-Chloroethyl vinyl ether

Chloroform

Chloromethane (Methyl chloride)

Chromium, total recoverable

Copper, total recoverable

Cyanide

p,p'-DDT

Dibromochloromethane

1,1-Dichloroethane

1,2-Dichloroethane

1,1-Dichloroethylene

trans-1,2-Dichloroethylene

Dichloromethane

2,4-Dichlorophenoxyacetic acid

1,2-Dichloropropane

cis-1,3-Dichloropropene

trans-1,3-Dichloropropene

Dieldrin

Endrin

Ethylbenzene

Fluoride

Gross alpha

Heptachlor

Iron, total recoverable

Lead, total recoverable

Lindane

Lithium, total recoverable

Magnesium, total recoverable

Manganese, total recoverable

Mercury, total recoverable

Methoxychlor

Naphthalene

Nickel, total recoverable

Nitrate as nitrogen

Nitrate-nitrite as nitrogen

Nonvolatile beta

pH

Phenols

Potassium, total recoverable

Radium, total alpha-emitting

Selenium, total recoverable

Silica, total recoverable

Silver, total recoverable

Sodium, total recoverable

Specific conductance

Sulfate

1,1,2,2-Tetrachloroethane

Tetrachloroethylene

Tin, total recoverable

Toluene

Total dissolved solids

Total organic carbon

Total organic halogens

Total phosphates (as P)

Toxaphene

2,4,5-TP (Silvex)

Tributyl phosphate

\begin{tabular}{|c|c|c|c|c|c|c|c|c|}
\hline 1094 & 2094 & 3094 & 4094 & DF & Mod & Unit & $\underline{L a b}$ & Fla \\
\hline $\begin{array}{l}<1.0 \\
1,510 \\
<1.0 \\
<1.0 \\
<1.0 \\
<1.0 \\
<1.0 \\
<1.0 \\
<4.0 \\
25 \\
<5.0 \\
<0.10\end{array}$ & $\begin{array}{l}<1.0 \\
1.740 \\
<1.0 \\
<1.0 \\
<1.0 \\
<1.0 \\
<1.0 \\
<1.0 \\
<4.0 \\
13 \\
<5.0 \\
<0.10\end{array}$ & $\begin{aligned} &<5.0 \\
& 8.880 \\
&<5.0 \\
&<10 \\
&<10 \\
&<10 \\
&<5.0 \\
& 2.1 \\
&<4.0 \\
& \quad 9.1 \\
&<5.0\end{aligned}$ & $\begin{array}{l}<1.0 \\
\quad 1.260 \\
<1.0 \\
<1.0 \\
<1.0 \\
<1.0 \\
<1.0 \\
<1.0 \\
<4.0 \\
15 \\
<5.0\end{array}$ & $\begin{array}{l}1 \\
1 \\
1 \\
1 \\
1 \\
1 \\
1 \\
1 \\
1 \\
1 \\
1\end{array}$ & & $\begin{array}{l}\mu g / L \\
\mu g / L \\
\mu g / L \\
\mu g / L \\
\mu g / L \\
\mu g / L \\
\mu g / L \\
\mu g / L \\
\mu g / L \\
\mu g / L \\
\mu g / L \\
\mu g / L\end{array}$ & $\begin{array}{l}\text { GE } \\
\text { GE } \\
\text { GE } \\
\text { GE } \\
\text { GE } \\
\text { GE } \\
\text { GE } \\
\text { GE } \\
\text { GE } \\
\text { GE } \\
\text { GE }\end{array}$ & $\begin{array}{l}0 \\
0 \\
0 \\
0 \\
0 \\
0 \\
0 \\
0 \\
0 \\
0 \\
0\end{array}$ \\
\hline $\begin{array}{l}<1.0 \\
<1.0 \\
<1.0 \\
<1.0 \\
<1.0 \\
<1.0 \\
<0.0015 \\
<1.0 \\
<1.0 \\
<1.0 \\
<0.50\end{array}$ & $\begin{array}{l}<1.0 \\
<1.0 \\
<1.0 \\
<1.0 \\
<1.0 \\
<1.0 \\
<0.0015 \\
<1.0 \\
<1.0 \\
<1.0 \\
<0.51\end{array}$ & $\begin{array}{l}<5.0 \\
<5.0 \\
<5.0 \\
<5.0 \\
<5.0 \\
<5.0 \\
<1.0 \\
<5.0 \\
<5.0 \\
<5.0\end{array}$ & $\begin{array}{l}<1.0 \\
<1.0 \\
<1.0 \\
<1.0 \\
<1.0 \\
<1.0 \\
<0.0015 \\
<1.0 \\
<1.0 \\
<1.0\end{array}$ & $\begin{array}{l}1 \\
1 \\
1 \\
1 \\
1 \\
1 \\
1 \\
1 \\
1 \\
1\end{array}$ & & $\begin{array}{l}\mu g / L \\
\mu g / L \\
\mu g / L \\
\mu g / L \\
\mu g / L \\
\mu g / L \\
\mu g / L \\
\mu g / L \\
\mu g / L \\
\mu g / L \\
\mu g / L\end{array}$ & $\begin{array}{l}\text { GE } \\
\text { GE } \\
\text { GE } \\
\text { GE } \\
\text { GE } \\
\text { GE } \\
\text { GE } \\
\text { GE } \\
\text { GE } \\
\text { GE }\end{array}$ & $\begin{array}{l}0 \\
0 \\
0 \\
0 \\
0 \\
0 \\
0 \\
0 \\
0 \\
0\end{array}$ \\
\hline $\begin{array}{l}<0.0060 \\
<1.0 \\
<100 \\
4.4 E-01 \\
<0.050\end{array}$ & $\begin{array}{l}<0.0061 \\
<1.0 \\
<100 \\
<1.6 \mathrm{E}-01 \\
<0.051\end{array}$ & $\begin{array}{l}<5.0 \\
<100 \\
1.4 E+00\end{array}$ & $\begin{array}{l}<0.0060 \\
<1.0 \\
<20 \\
\quad 7.1 E-01\end{array}$ & $\begin{array}{l}1 \\
1 \\
1 \\
1\end{array}$ & UI & $\begin{array}{l}\mu \mathrm{g} / \mathrm{L} \\
\mu \mathrm{g} / \mathrm{L} \\
\mu \mathrm{g} / \mathrm{L} \\
\mathrm{pCi} / \mathrm{L} \\
\mu \mathrm{g} / \mathrm{L}\end{array}$ & $\begin{array}{l}\text { GE } \\
\text { GE } \\
\text { GE } \\
\text { GP }\end{array}$ & $\begin{array}{l}0 \\
0 \\
0 \\
0\end{array}$ \\
\hline $\begin{aligned} & 224 \\
& 15 \\
< & 0.0050 \\
< & 5.0\end{aligned}$ & $\begin{array}{l}82 \\
5.7 \\
<0.0051\end{array}$ & $\begin{array}{r}339 \\
6.0 \\
<5.0\end{array}$ & $\begin{array}{c}73 \\
9.6 \\
<0.0050\end{array}$ & $\begin{array}{l}1 \\
1 \\
1\end{array}$ & & $\begin{array}{l}\mu g / L \\
\mu g / L \\
\mu g / L \\
\mu g / L\end{array}$ & $\begin{array}{l}\text { GE } \\
\text { GE } \\
\text { GE }\end{array}$ & $\begin{array}{l}0 \\
0 \\
0\end{array}$ \\
\hline $\begin{aligned} & 333 \\
& 20 \\
< & 0.20 \\
< & 0.50 \\
< & 10 \\
& 4.7\end{aligned}$ & $\begin{aligned} & 285 \\
& 12 \\
< & 0.20 \\
< & 0.51 \\
< & 10 \\
< & 4.0\end{aligned}$ & $\begin{aligned} & 260 \\
& 9.8 \\
< & 0.20 \\
< & 10 \\
< & 4.0 \\
& 1,900\end{aligned}$ & $\begin{aligned} & 290 \\
& 14 \\
< & 0.20 \\
< & 0.50 \\
< & 10 \\
< & 4.0\end{aligned}$ & $\begin{array}{l}1 \\
1 \\
1 \\
1 \\
1 \\
1\end{array}$ & & $\begin{array}{l}\mu g / L \\
\mu g / L \\
\mu g / L \\
\mu g / L \\
\mu g / L \\
\mu g / L \\
\mu g / L\end{array}$ & $\begin{array}{l}\mathrm{GE} \\
\mathrm{GE} \\
\mathrm{GE} \\
\mathrm{GE} \\
\mathrm{GE} \\
\mathrm{GE}\end{array}$ & $\begin{array}{l}0 \\
0 \\
0 \\
0 \\
0 \\
0\end{array}$ \\
\hline $\begin{array}{l}2,360 \\
<3.1 \mathrm{E}-01 \\
6.1 \\
<5.0 \\
<500 \\
1.3 \mathrm{E}+00 \\
<2.0 \\
6,350 \\
<2.0 \\
4,890 \\
34 \\
<1,000 \\
<1.0 \\
<1.0 \\
2.4 \\
<1.0 \\
24,000 \\
<1,000 \\
<5.0 \\
<50 \\
<0.24 \\
<0.00045 \\
<10\end{array}$ & $\begin{aligned} & 2,160 \\
&<-1.7 \mathrm{E}-01 \\
& 5.8 \\
&<5.0 \\
&<500 \\
&<1.0 \mathrm{E}-01 \\
&<2.0 \\
& 6.290 \\
&<2.0 \\
& 4,380 \\
& 30 \\
&<1.000 \\
&<1.0 \\
&<1.0 \\
&<2.0 \\
&<1.0 \\
& 27,000 \\
&<1,000 \\
&<5.0 \\
&<50 \\
&<0.25 \\
&<0.00044 \\
&<10\end{aligned}$ & $\begin{array}{rl} & 8.1 \mathrm{E}-01 \\
5.0 & < \\
<5.0 \\
<500 \\
& 5.0 \mathrm{E}-01 \\
<2.0 & 4,590 \\
< & 2.0 \\
& 3,440 \\
& 27 \\
<1,000 \\
<5.0 \\
<5.0 \\
<17 \\
<5.0 \\
5,000 \\
<1,000 \\
<5.0 \\
305 \\
<0.52 \\
<10\end{array}$ & $\begin{aligned} & 2,100 \\
& 8.7 \mathrm{E}-01 \\
& 5.9 \\
&<5.0 \\
&<500 \\
& 7.0 \mathrm{E}-01 \\
&<2.0 \\
& 6,110 \\
&<2.0 \\
& 4,300 \\
& 33 \\
&<1.000 \\
&<1.0 \\
&<1.0 \\
&<2.0 \\
&<1.0 \\
& 28,000 \\
&<1,000 \\
&<5.0 \\
&<50 \\
&<0.24 \\
&<0.00045 \\
&<10\end{aligned}$ & $\begin{array}{l}2 \\
1 \\
1 \\
1 \\
1 \\
1 \\
1 \\
1 \\
1 \\
1 \\
1 \\
1 \\
1 \\
1 \\
1 \\
1 \\
1 \\
1 \\
1 \\
1 \\
1 \\
1 \\
1\end{array}$ & $\begin{array}{l}\text { U! } \\
J\end{array}$ & $\begin{array}{l}\mu g / L \\
\mathrm{pCi} / \mathrm{L} \\
\mathrm{pH} \\
\mu \mathrm{g} / \mathrm{L} \\
\mu \mathrm{g} / \mathrm{L} \\
\mathrm{pCi} / \mathrm{L} \\
\mu \mathrm{g} / \mathrm{L} \\
\mu \mathrm{g} / \mathrm{L} \\
\mu \mathrm{g} / \mathrm{L} \\
\mu \mathrm{g} / \mathrm{L} \\
\mu \mathrm{S} / \mathrm{cm} \\
\mu \mathrm{g} / \mathrm{L} \\
\mu \mathrm{g} / \mathrm{L} \\
\mu \mathrm{g} / \mathrm{L} \\
\mu \mathrm{g} / \mathrm{L} \\
\mu \mathrm{g} / \mathrm{L} \\
\mu \mathrm{g} / \mathrm{L} \\
\mu \mathrm{g} / \mathrm{L} \\
\mu \mathrm{g} / \mathrm{L} \\
\mu \mathrm{g} / \mathrm{L} \\
\mu \mathrm{g} / \mathrm{L} \\
\mu g / \mathrm{L} \\
\mu \mathrm{g} / \mathrm{L}\end{array}$ & $\begin{array}{l}\mathbf{G E} \\
\mathbf{G P} \\
\mathbf{G E} \\
\mathbf{G E} \\
\mathbf{G E} \\
\mathbf{G P} \\
\mathbf{G E} \\
\mathbf{G E} \\
\mathbf{G E} \\
\mathbf{G E} \\
\mathbf{G E} \\
\mathbf{G E} \\
\mathbf{G E} \\
\mathbf{G E} \\
\mathbf{G E} \\
\mathbf{G E} \\
\mathbf{G E} \\
\mathbf{G E} \\
\mathbf{G E} \\
\mathbf{G E} \\
\mathbf{G E} \\
\mathbf{G E} \\
\mathbf{G E}\end{array}$ & $\begin{array}{l}0 \\
0 \\
0 \\
0 \\
0 \\
0 \\
0 \\
0 \\
0 \\
0 \\
0 \\
0 \\
0 \\
0 \\
0 \\
0 \\
0 \\
0 \\
0 \\
0 \\
0\end{array}$ \\
\hline
\end{tabular}

Note: Flagging, dilution factors, modifiers, and laboratories are for fourth quarter 1994 data only. See Appendix B for flagging criteria. - = exceeded holding time for fourth quarter 1994.

a exceeded screening level or final PDWS for fourth quarter 1994. 
Well BGO 37D continued

ANALYTICAL DATA

H ST Analyte

1,1,1-Trichloroethane

1,1,2-Trichloroethane

Trichloroethylene

Trichlorofluoromethane

2,4,5-T

- Tritium

Uranium-233/234

Uranium-235

Uranium-238

Vanadium, total recoverable

Xylenes

\begin{tabular}{|c|c|c|}
\hline 1094 & 2094 & 3094 \\
\hline $\begin{array}{l}<1.0 \\
<1.0 \\
<1.0 \\
<1.0\end{array}$ & $\begin{array}{l}<1.0 \\
<1.0 \\
<1.0 \\
<1.0 \\
<0.00044\end{array}$ & $\begin{array}{l}<5.0 \\
<5.0 \\
<5.0 \\
<5.0\end{array}$ \\
\hline $\begin{aligned} & 3.0 E+01 \\
& 25 \\
& 1.4 E-01 \\
&<1.3 E-02 \\
&< 5.9 E-03 \\
&< 8.0 \\
&< 2.0\end{aligned}$ & $\begin{aligned} & 3.5 E+01 \\
& 30 \\
< & 2.3 E-02 \\
< & 0.0 E+00 \\
< & 2.7 E-02 \\
< & 8.0 \\
< & 2.0\end{aligned}$ & $\begin{array}{l}3.3 E+01 \\
15 \\
3.7 E-02 \\
-5.6 E-02 \\
-1.4 E-02 \\
<3.0 \\
<5.0\end{array}$ \\
\hline
\end{tabular}

\begin{tabular}{|c|c|}
\hline 4094 & $\underline{D}$ \\
\hline $\begin{array}{l}<1.0 \\
<1.0 \\
<1.0 \\
<1.0\end{array}$ & $\begin{array}{l}1 \\
1 \\
1\end{array}$ \\
\hline $\begin{array}{l}3.1 \mathrm{E}+01 \\
68 \\
-2.4 \mathrm{E}-02 \\
6.3 \mathrm{E}-02 \\
5.1 \mathrm{E}-02 \\
<8.0 \\
<2.0\end{array}$ & \\
\hline
\end{tabular}

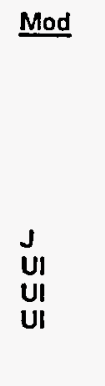

\begin{tabular}{ll} 
Unit & La \\
\hline$\mu g / L$ & $\mathrm{GE}$ \\
$\mu \mathrm{g} / \mathrm{L}$ & $\mathrm{GE}$ \\
$\mu \mathrm{g} / \mathrm{L}$ & $\mathrm{G}$ \\
$\mu \mathrm{g} / \mathrm{L}$ & $\mathrm{GE}$ \\
$\mu \mathrm{g} / \mathrm{L}$ & \\
$\mathrm{pCi/mL}$ & $\mathrm{GP}$ \\
$\mathrm{NTU}$ & $\mathrm{G}$ \\
$\mathrm{pCi} / \mathrm{L}$ & $\mathrm{GP}$ \\
$\mathrm{pCi/L}$ & $\mathrm{GP}$ \\
$\mathrm{pCi} / \mathrm{L}$ & $\mathrm{GP}$ \\
$\mu \mathrm{g} / \mathrm{L}$ & $\mathrm{GE}$ \\
$\mu \mathrm{g} / \mathrm{L}$ & $\mathrm{GE}$
\end{tabular}
Flag

0

0

0
0

0

2

0

0

0

\section{WELL BGO 38D}

\section{SRS Coord. \\ N73329.3 \\ E57557.5 \\ SAMPLE DATE \\ FIELD DATA

Analyte
Water elevation
Depth to water
pH
Sp. conductance
Water temperature
Alkalinity as CaCO
Turbidity
Volume purged
Sampling code

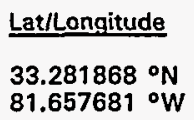

ANALYTICAL DATA

H ST Analyte

Acetophenone

Aldrin

Aluminum, total recoverable

Antimony, total recoverable

Arsenic, total recoverable

Barium, total recoverable

Benzene

Bromodichloromethane

Bromoform

Bromomethane (Methyl bromide)

Screen Zone Elevation

242.3-222.3 ft ms

$02 / 01 / 94$

$04 / 15 / 94$

\section{Top of Casing}

$291.6 \mathrm{ft} \mathrm{msl}$

$08 / 09 / 94$

$\begin{array}{lll}\text { Casing } & \text { Pump } & \text { Screen Zone } \\ \text { 4" PVC }^{\text {" PV }} & \text { S } & \text { Water Table (IIB2) }\end{array}$

Cadmium, total recoverable

Calcium, total recoverable

Carbon tetrachloride

Chloride

Chlorobenzene

Chloroethane

Chloroethene (Vinyl chloride)

2-Chloroethyl vinyl ether

Chloroform

Chloromethane (Methyl chloride)

Chromium, total recoverable

Copper, total recoverable

Cyanide

\begin{tabular}{|c|c|c|c|c|}
\hline 1094 & 2094 & 3094 & 4094 & Unit \\
\hline $\begin{array}{l}233.9 \\
57.7 \\
4.4 \\
32 \\
16.3 \\
0 \\
26 \\
1.1 \\
x\end{array}$ & $\begin{array}{l}235.0 \\
56.6 \\
4.4 \\
42 \\
20.3 \\
0 \\
21 \\
0.8 \\
\times\end{array}$ & $\begin{array}{l}235.2 \\
56.4 \\
4.4 \\
37 \\
21.7 \\
0 \\
37 \\
0.9 \\
x\end{array}$ & $\begin{array}{l}234.8 \\
56.8 \\
4.5 \\
34 \\
20.6 \\
0 \\
45 \\
1.0 \\
x\end{array}$ & $\begin{array}{l}\mathrm{ft} \mathrm{msl} \\
\mathrm{ft} \\
\mathrm{pH} \\
\mu \mathrm{S} / \mathrm{cm} \\
{ }^{\circ} \mathrm{C} \\
\mathrm{mg} / \mathrm{L} \\
\text { NTU } \\
\text { well vol. }\end{array}$ \\
\hline
\end{tabular}

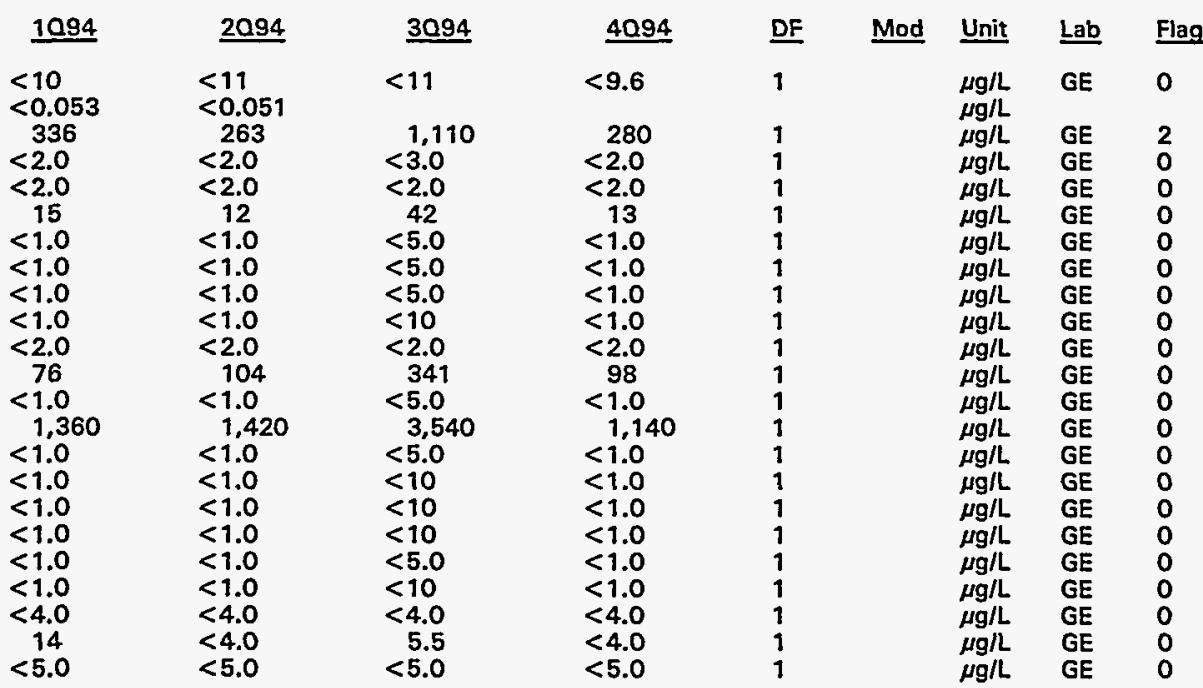

Note: Flagging, dilution factors, modifiers, and laboratories are for fourth quarter 1994 data only. See Appendix B for flagging criteria. - = exceeded holding time for fourth quarter 1994.

= exceeded screening level or final PDWS for fourth quarter 1994. 


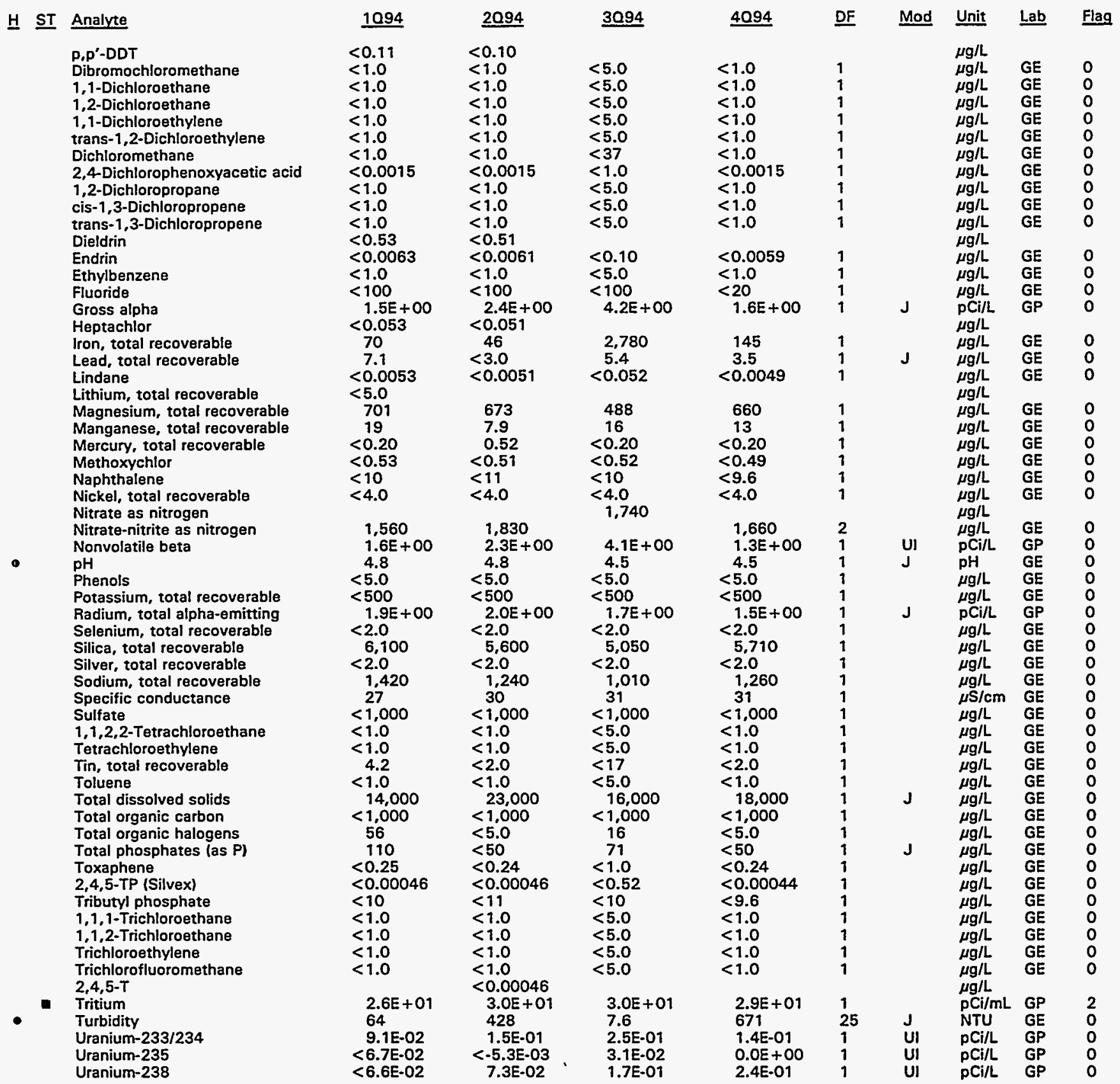

Note: Flagging, dilution factors, modifiers, and laboratories are for fourth quarter 1994 data only. See Appendix B for flagging criteria. - = exceeded holding time for fourth quarter 1994.

- = exceeded screening level or final PDWS for fourth quarter 1994. 
Well BGO 38D continued

ANALYTICAL DATA

\begin{tabular}{|c|c|c|c|c|c|c|c|c|}
\hline H ST Analyte & 1094 & $\underline{2094}$ & $\underline{3094}$ & 40994 & $\underline{D F}$ & Mod & $\underline{\text { Unit }}$ & $\underline{\text { Lab }}$ \\
\hline $\begin{array}{l}\text { Vanadium, total recoverable } \\
\text { Xylenes }\end{array}$ & $\begin{array}{l}<8.0 \\
<2.0\end{array}$ & $\begin{array}{l}<8.0 \\
<2.0\end{array}$ & $\begin{array}{r}6.0 \\
<5.0\end{array}$ & $\begin{array}{l}<8.0 \\
<2.0\end{array}$ & $\begin{array}{l}1 \\
1\end{array}$ & & $\begin{array}{l}\mu g / L \\
\mu g / L\end{array}$ & $\begin{array}{l}\text { GE } \\
\text { GE }\end{array}$ \\
\hline
\end{tabular}

WELL BGO 39D

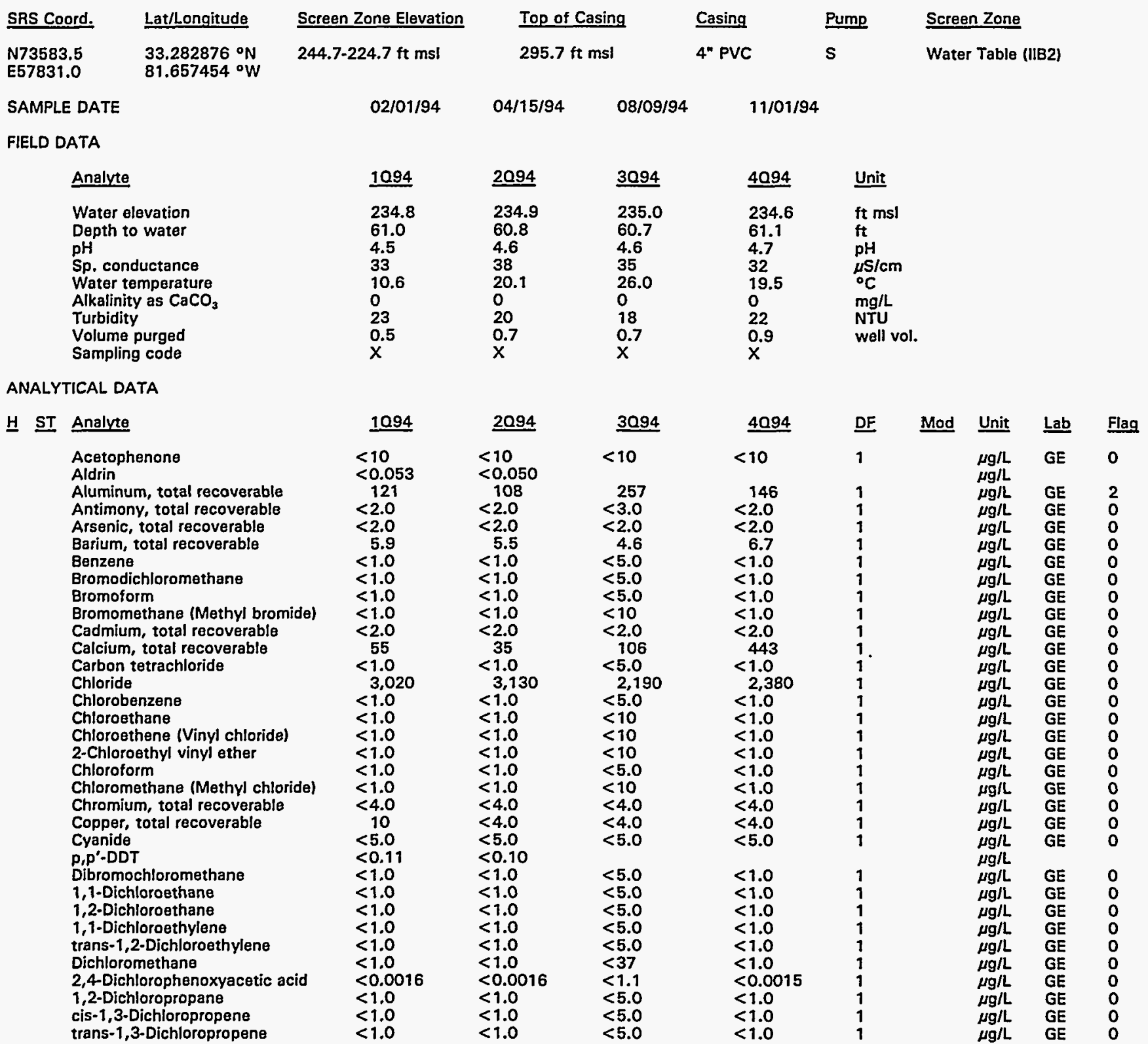

Note: Flagging, dilution factors, modifiers, and laboratories are for fourth quarter 1994 data only. See Appendix B for flagging criteria. - = exceeded holding time for fourth quarter 1994.

- = exceeded screening level or final PDWS for fourth quarter 1994. 


\section{ANALYTICAL DATA}

HT Analyte
Dieldrin
Endrin
Ethylbenzene
Fluoride
Gross alpha
Heptachlor
Iron, total recoverable
Lead, total recoverable
Lindane
Lithium, total recoverable
Magnesium, total recoverable
Manganese, total recoverable
Mercury, rotal recoverable
Methoxychlor
Naphthalene
Nickel, total recoverable
Nitrate as nitrogen
Nitrate-nitrite as nitrogen
Nonvolatile beta
pH
Phenols
Potassium, total recoverable
Radium, total alpha-emitting
Selenium, total recoverable
Silica, total recoverable
Silver, total recoverable
Sodium, total recoverable
Specific conductance
Sulfate
$1,1,2,2-$ Tetrachloroethane
Tetrachloroethylene
Tin, total recoverable
Toluene
Total dissolved solids
Total organic carbon
Total organic halogens
Total phosphates (as P)
Toxaphene
$2,4,5-T P$ (Silvex)
Tributyl phosphate
1,1,1-Trichloroethane
T,1,2-Trichloroethane
Trichloroethylene
Trichlorofluoromethane
$2,4,5-T$
Tritium
Turbidity
Uranium-233/234
Uranium-235
Uranium-238
Vanadium, total recoverable
Xylenes
-
-

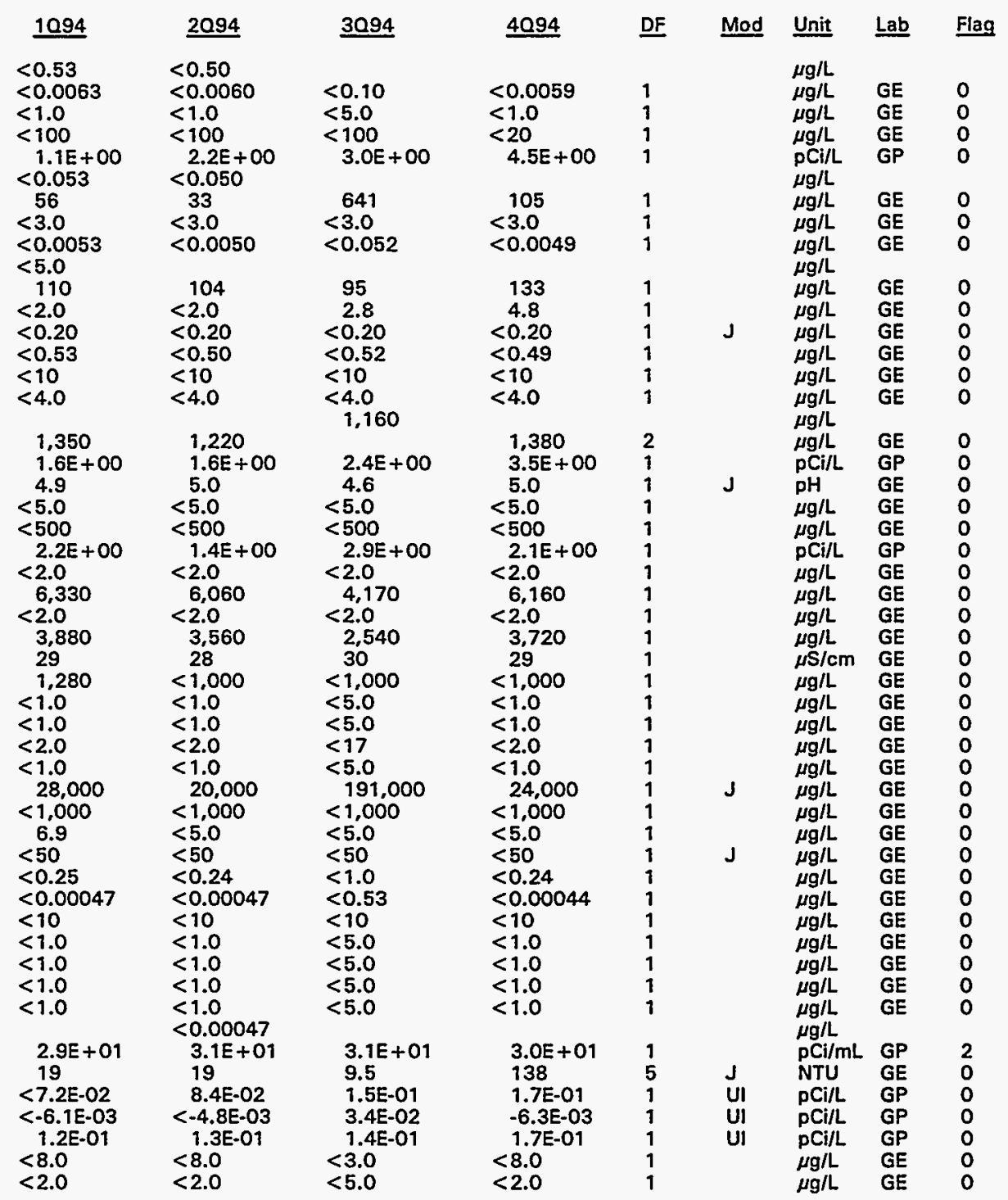

Note: Flagging, dilution factors, modifiers, and laboratories are for fourth quarter 1994 data only. See Appendix B for flagging criteria. - = exceeded holding time for fourth quarter 1994.

= exceeded screening level or final PDWS for fourth quarter 1994. 


\section{WELL BGO 40D}

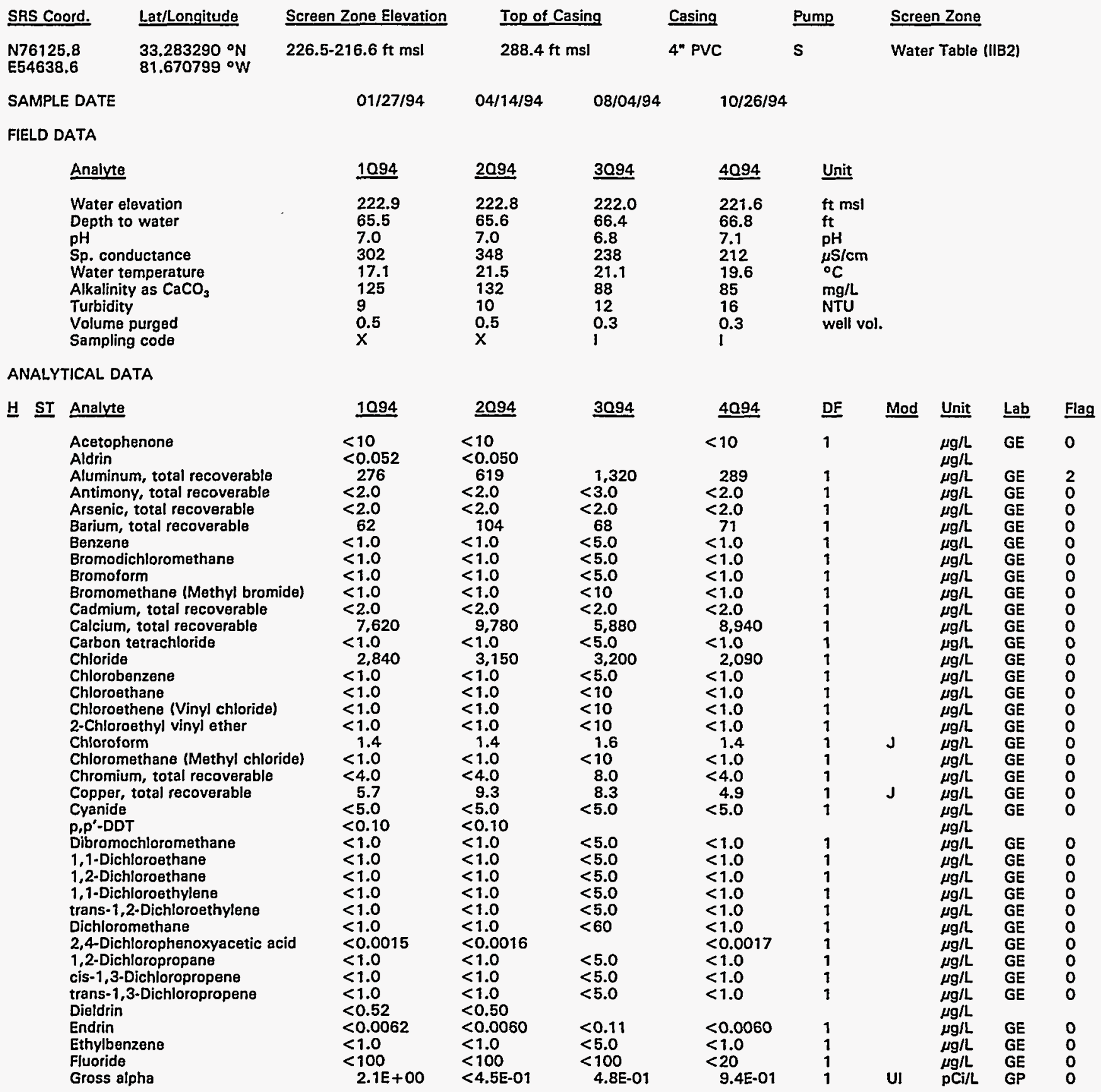

Note: Flagging, dilution factors, modifiers, and laboratories are for fourth quarter 1994 data only. See Appendix B for flagging criteria. - = exceeded holding time for fourth quarter 1994.

- exceeded screening level or final PDWS for fourth quarter 1994. 


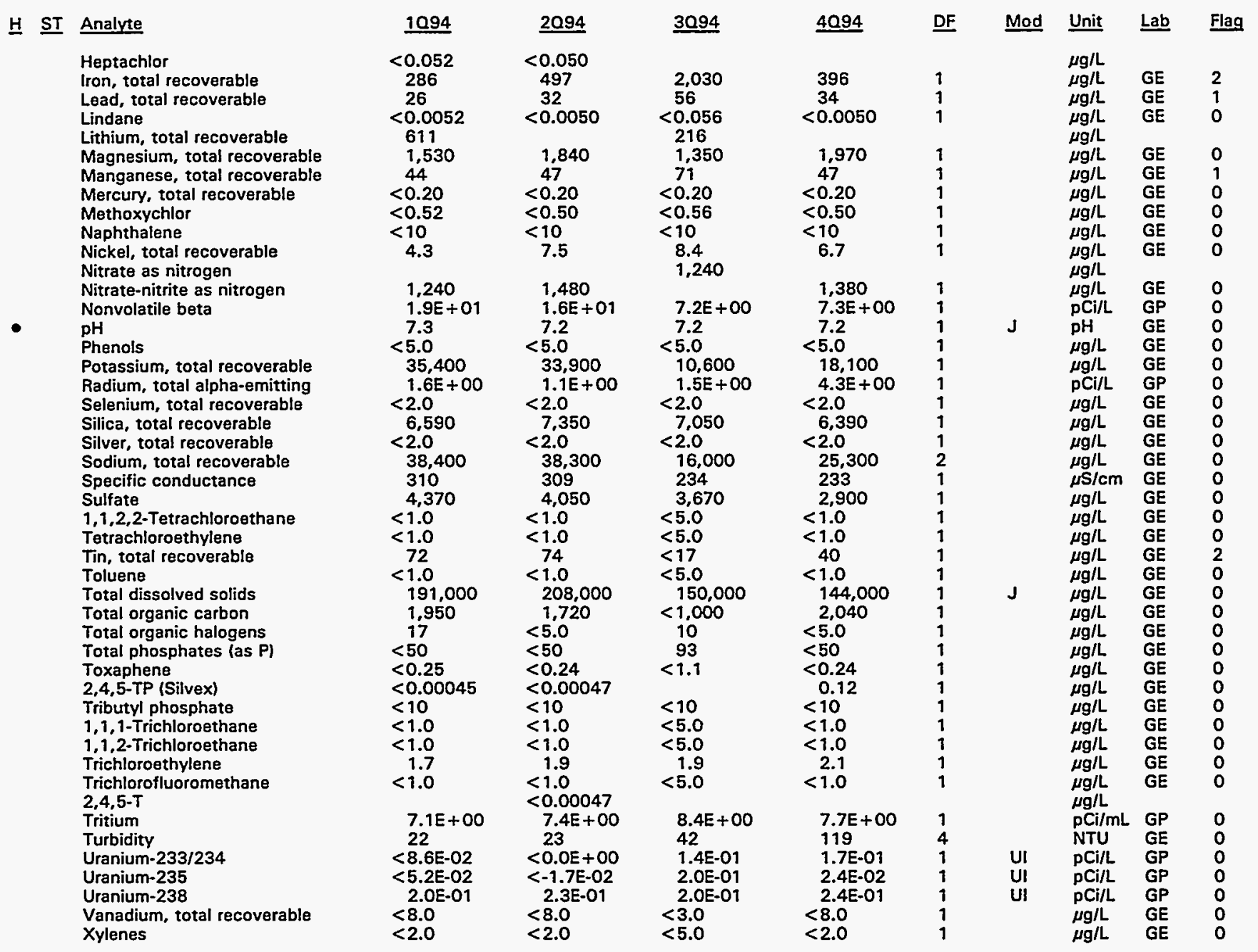

Note: Flagging, dilution factors, modifiers, and laboratories are for fourth quarter 1994 data only. See Appendix B for flagging criteria. - = exceeded holding time for fourth quarter 1994.

- exceeded screening level or final PDWS for fourth quarter 1994. 


\section{WELL BGO 41A}

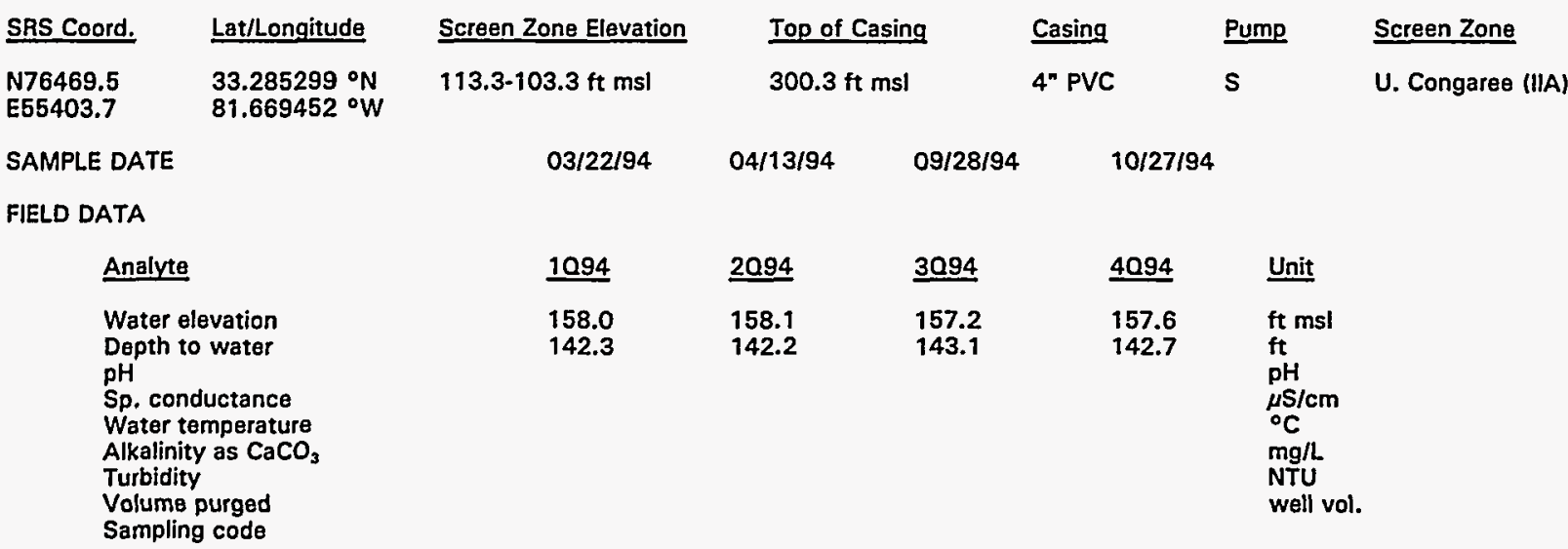

\section{WELL BGO 42C}

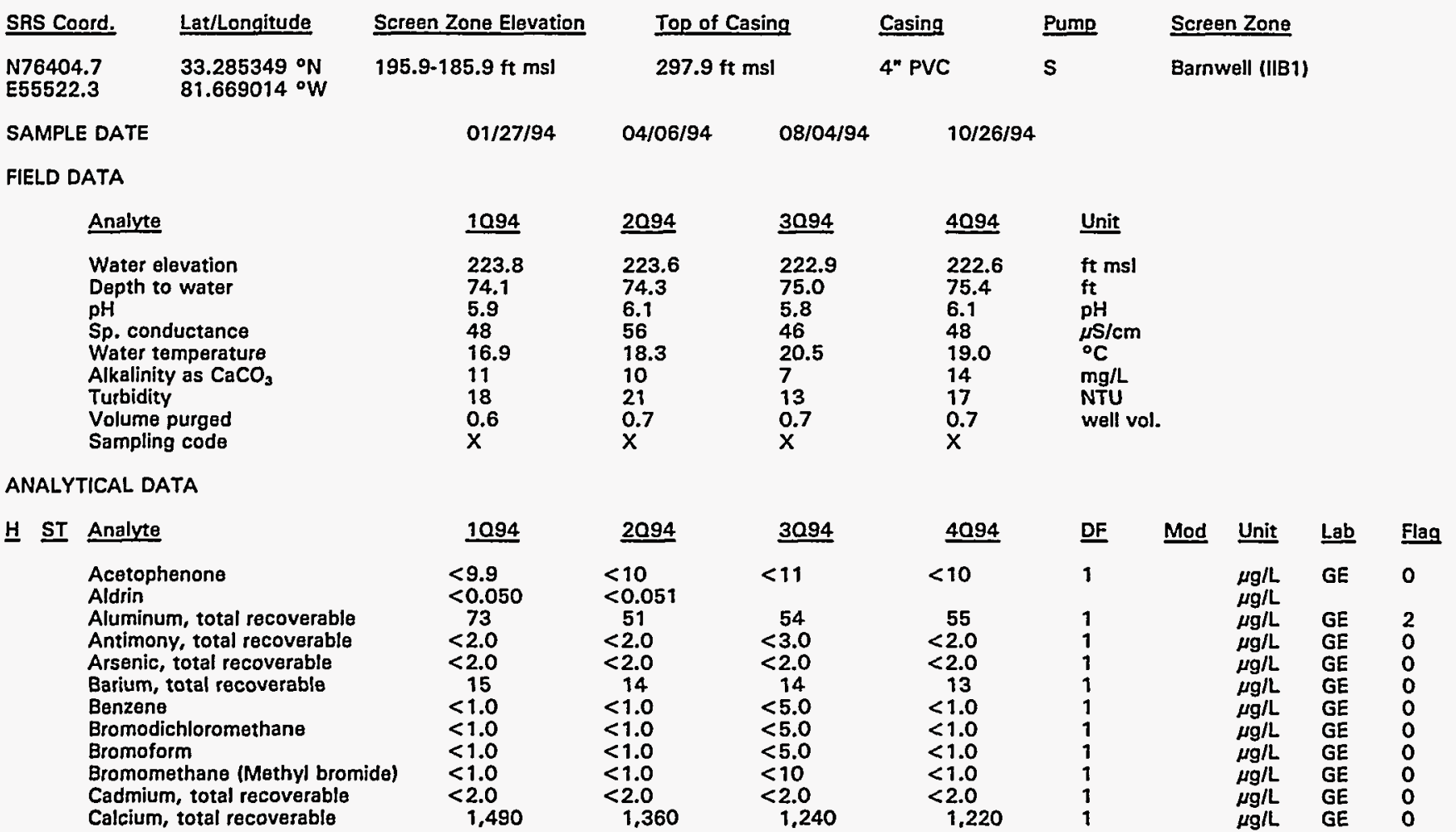

Note: Flagging, dilution factors, modifiers, and laboratories are for fourth quarter 1994 data only. See Appendix B for flagging criteria. - = exceeded holding time for fourth quarter 1994.

- = exceeded screening level or final PDWS for fourth quarter 1994. 
Well BGO 42C continued ANALYTICAL DATA

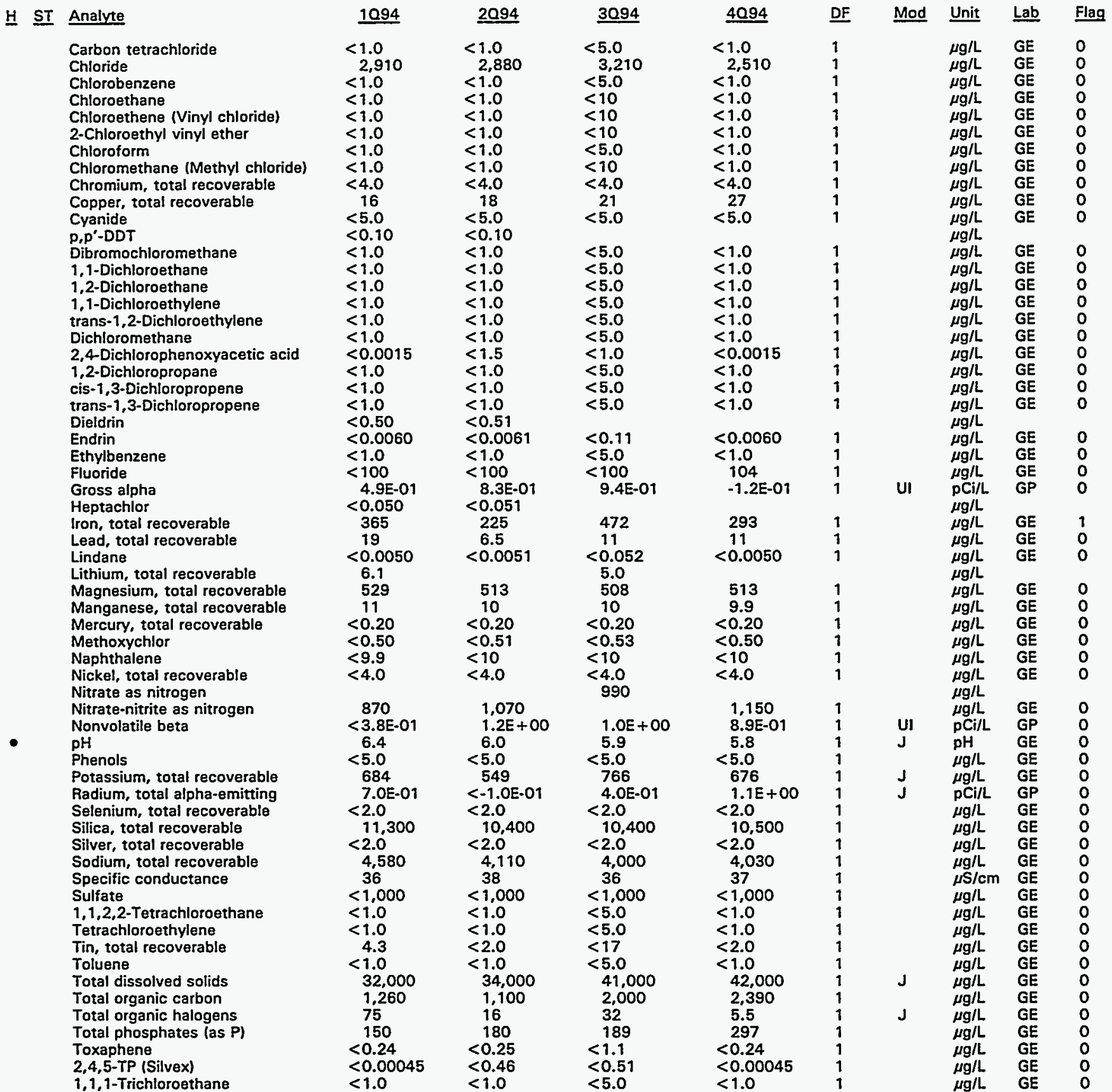

Note: Flagging, dilution factors, modifiers, and laboratories are for fourth quarter 1994 data only. See Appendix B for flagging criteria. - = exceeded holding time for fourth quarter 1994.

- = exceeded screening level or final PDWS for fourth quarter 1994. 
ANALYTICAL DATA

\section{H ST Analyte}

1,1,2-Trichloroethane

- Trichloroethylene

Trichlorofluoromethane

2,4,5-T

Tritium

Turbidity

Uranium-233/234

Uranium-235

Uranium-238

Vanadium, total recoverable

Xylenes

\begin{tabular}{|c|c|c|}
\hline 1094 & $\underline{2094}$ & 3094 \\
\hline $\begin{array}{r}<1.0 \\
36 \\
<1.0\end{array}$ & $\begin{array}{c}<1.0 \\
28 \\
<1.0 \\
<0.46\end{array}$ & $\begin{array}{r}<5.0 \\
34 \\
<5.0\end{array}$ \\
\hline $\begin{array}{l}6.6 \mathrm{E}+00 \\
1.7 \\
1.0 \mathrm{E}-01 \\
1.0 \mathrm{E}-01 \\
1.2 \mathrm{E}-01 \\
<8.0 \\
<2.0\end{array}$ & $\begin{aligned} & 6.5 \mathrm{E}+00 \\
& 3.2 \\
< & -1.2 \mathrm{E}-02 \\
< & 1.4 \mathrm{E}-02 \\
< & 1.5 \mathrm{E}-02 \\
< & 8.0 \\
< & 2.0\end{aligned}$ & $\begin{array}{l}7.1 E+00 \\
3.3 \\
1.9 E-01 \\
0.0 E+00 \\
3.5 E-01 \\
<3.0 \\
<5.0\end{array}$ \\
\hline
\end{tabular}

$\begin{array}{ll}\begin{array}{ll}4094 \\ <1.0\end{array} & 1 \\ 32 & 1 \\ <1.0 & 1 \\ 6.5 \mathrm{E}+00 & 1 \\ 2.5 & 1 \\ 7.0 \mathrm{E}-02 & 1 \\ 1.0 \mathrm{E}-02 & 1 \\ 4.5 \mathrm{E}-02 & 1 \\ <8.0 & 1 \\ <2.0 & 1\end{array}$

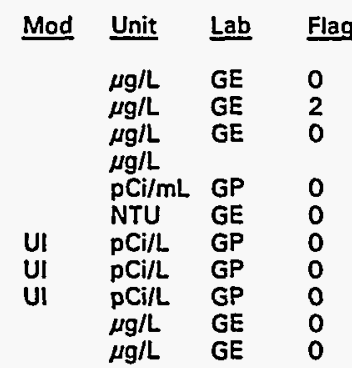

\section{WELL BGO 43A}

\begin{tabular}{|c|c|c|c|c|c|c|c|}
\hline SRS Coord. & Lat/Longitude & Screen Zone Elevation & Top of & & Casing & Pump & Screen Zone \\
\hline $\begin{array}{l}\text { N77061.4 } \\
\text { E56253.4 }\end{array}$ & $\begin{array}{l}33.287994^{\circ} \mathrm{N} \\
81.668364^{\circ} \mathrm{W}\end{array}$ & $115.9-105.9 \mathrm{ft} \mathrm{msl}$ & 314.9 & & 4" PVC & s & U. Congaree (IIA) \\
\hline SAMPLE DA & & $03 / 22 / 94$ & $04 / 13 / 94$ & $09 / 28 / 94$ & $10 / 27 / 94$ & & \\
\hline FIELD DATA & & & & & & & \\
\hline Ana & & 1094 & 2094 & 3094 & 4094 & Unit & \\
\hline $\begin{array}{l}\text { Wat } \\
\text { Dep } \\
\text { pH } \\
\text { Sp. } \\
\text { Wat } \\
\text { Alke } \\
\text { Turt } \\
\text { Volt } \\
\text { Sant }\end{array}$ & $\begin{array}{l}\text { wation } \\
\text { water } \\
\text { uctance } \\
\text { mperature } \\
\text { as } \mathrm{CaCO}_{3} \\
\text { urged } \\
\text { code }\end{array}$ & $\begin{array}{l}160.1 \\
154.8\end{array}$ & $\begin{array}{l}160.1 \\
154.8\end{array}$ & $\begin{array}{l}158.1 \\
156.8\end{array}$ & $\begin{array}{l}158.2 \\
156.7\end{array}$ & $\begin{array}{l}\text { ft msl } \\
\mathrm{ft} \\
\text { pH } \\
\mu \mathrm{H} / \mathrm{cm} \\
{ }^{\circ} \mathrm{C} \\
\mathrm{mg} / \mathrm{L} \\
\text { NTU } \\
\text { well vol. }\end{array}$ & \\
\hline
\end{tabular}

Note: Flagging, dilution factors, modifiers, and laboratories are for fourth quarter 1994 data only. See Appendix B for flagging criteria. - = exceeded holding time for fourth quarter 1994.

- = exceeded screening level or final PDWS for fourth quarter 1994. 


\section{WELL BGO 43AA}

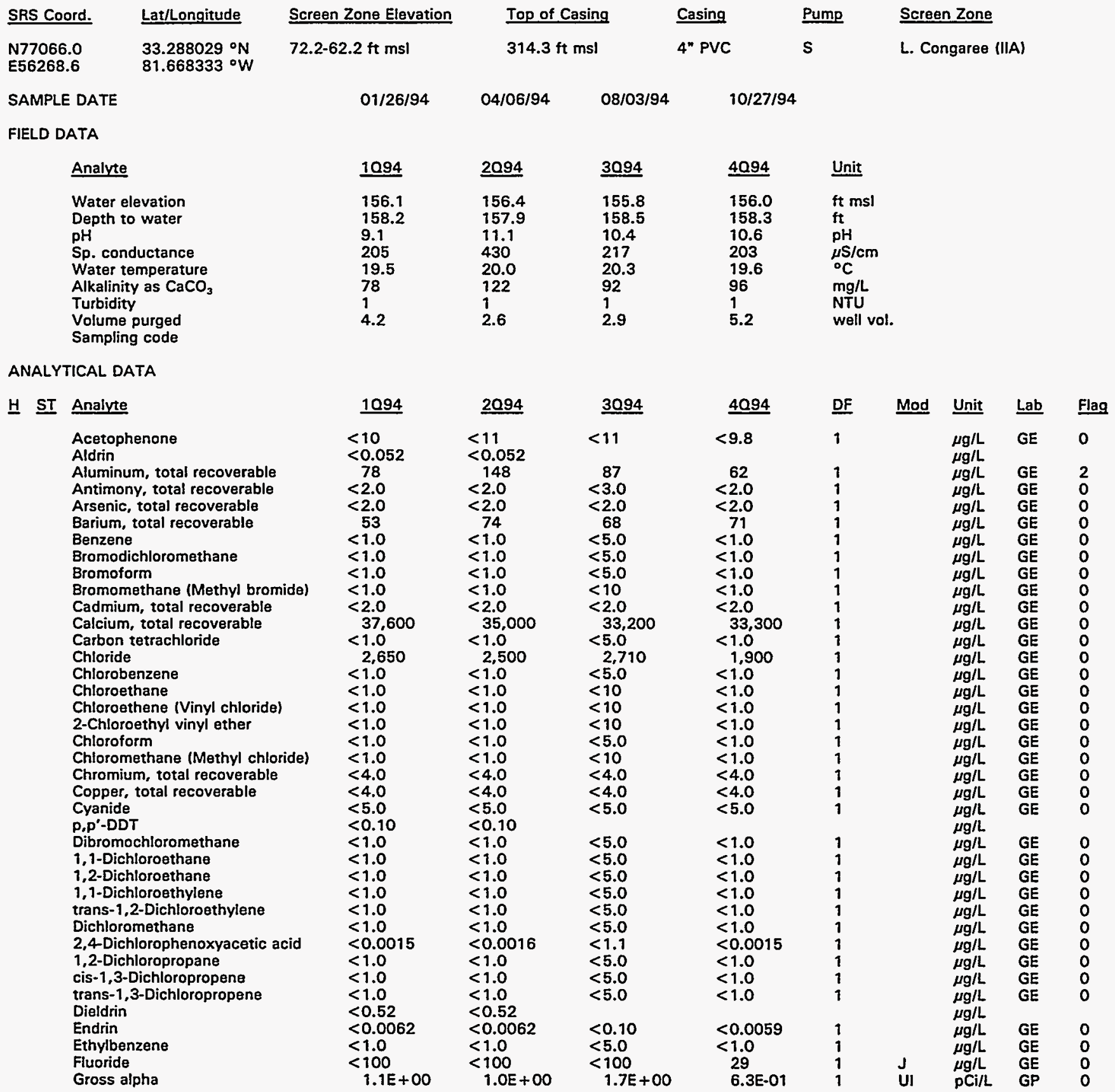

Note: Flagging, dilution factors, modifiers, and laboratories are for fourth quarter 1994 data only. See Appendix B for flagging criteria. - = exceeded holding time for fourth quarter 1994.

n = exceeded screening level or final PDWS for fourth quarter 1994. 


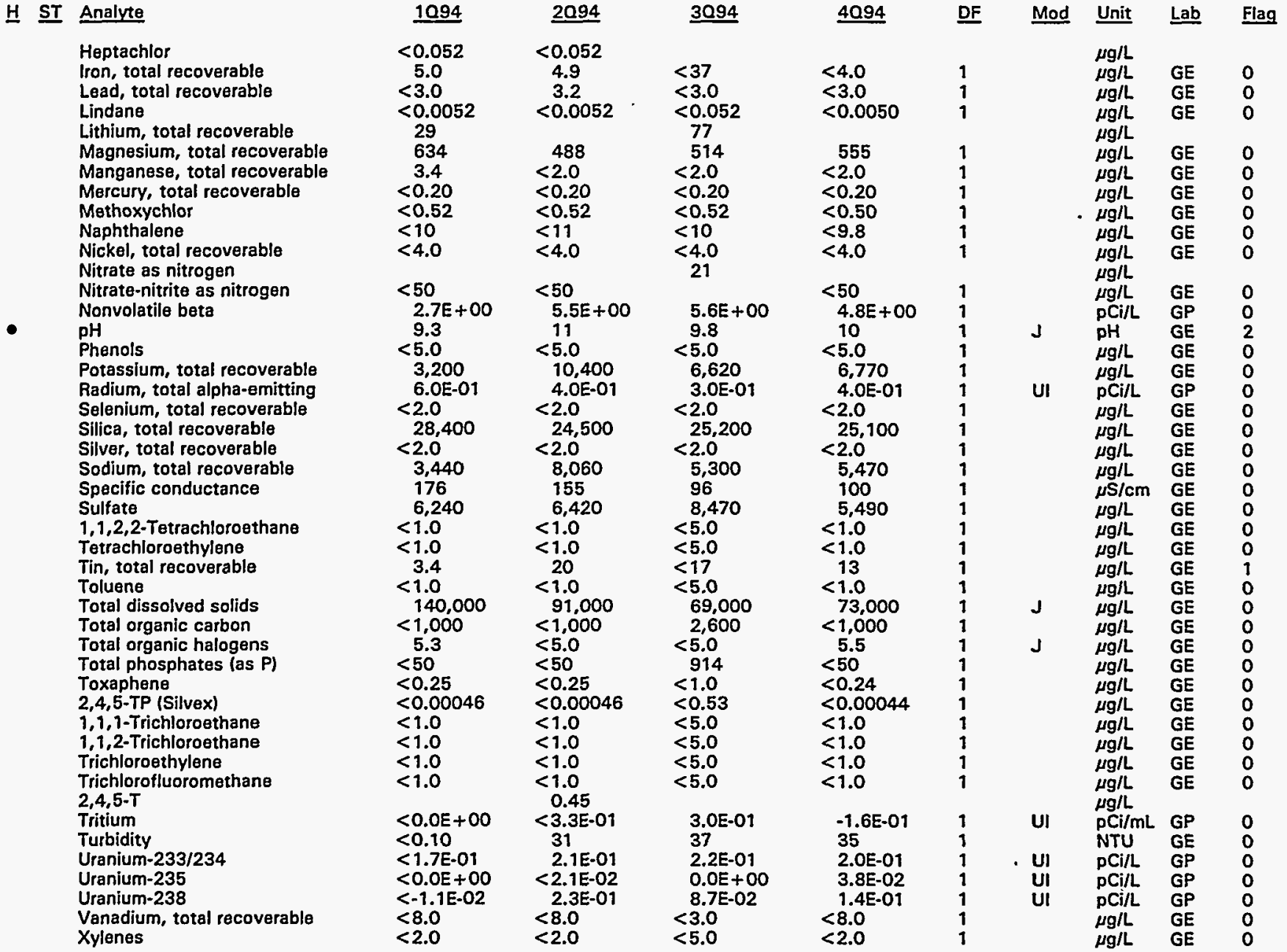

Note: Flagging, dilution factors, modifiers, and laboratories are for fourth quarter 1994 data only. See Appendix B for flagging criteria. - = exceeded holding time for fourth quarter 1994.

- = exceeded screening level or final PDWS for fourth quarter 1994. 
WELL BGO 43CR

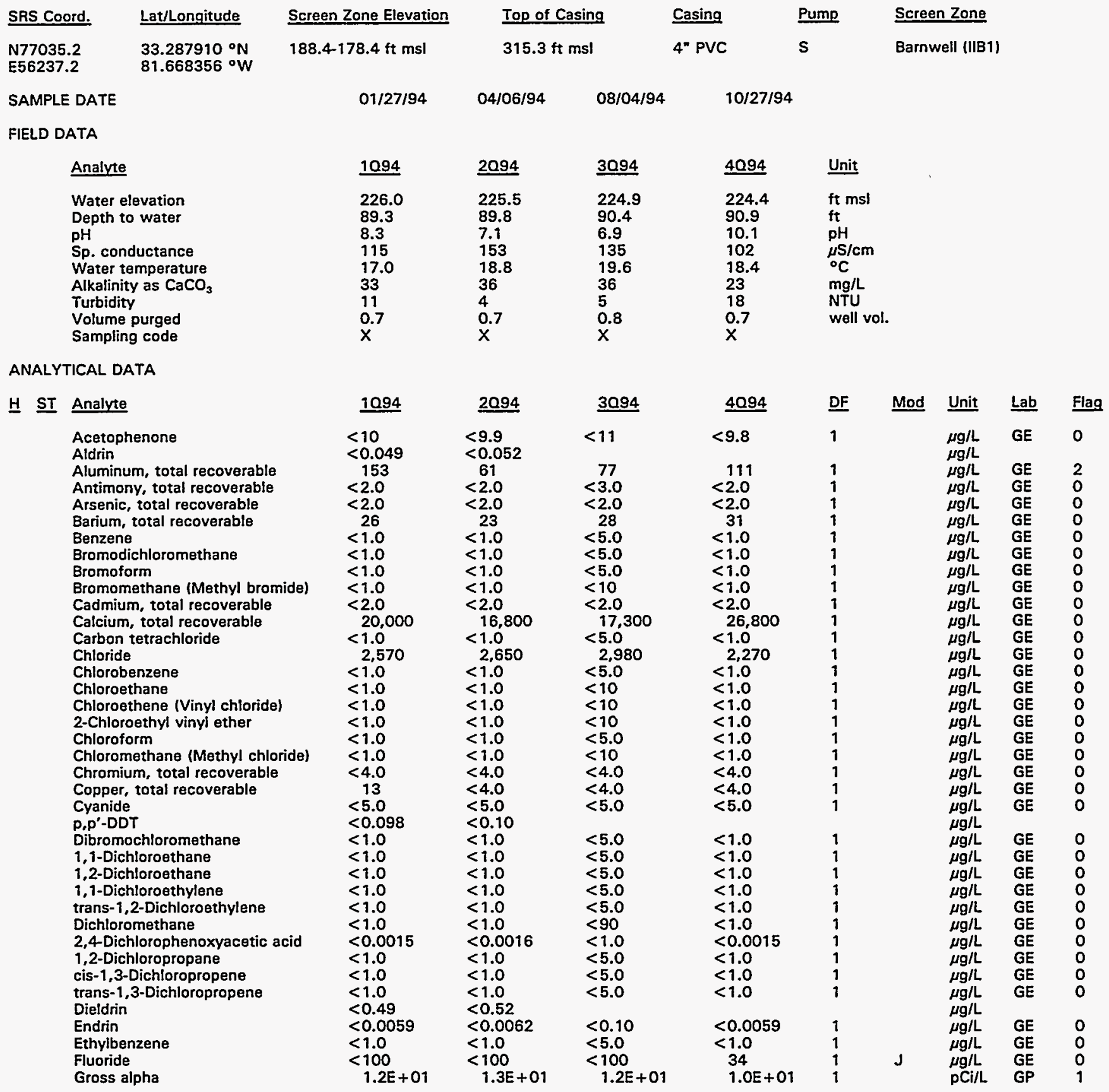

Note: Flagging, dilution factors, modifiers, and laboratories are for fourth quarter 1994 data only. See Appendix B for flagging criteria. - = exceeded holding time for fourth quarter 1994.

- = exceeded screening level or final PDWS for fourth quarter 1994. 
H ST Analyze

Heptachlor

Iron, total recoverable

Lead, total recoverable

Lindane

Lithium, total recoverable

Magnesium, total recoverable

Manganese, total recoverable

Mercury, total recoverable

Methoxychlor

Naphthalene

Nickel, total recoverable

Nitrate as nitrogen

Nitrate-nitrite as nitrogen

Nonvolatile beta

$\mathrm{pH}$

Phenols

Potassium, total recoverable

Radium, total alpha-emitting

Selenium, total recoverable

Silica, total recoverable

Silver, total recoverable

Sodium, total recoverable

Specific conductance

Sulfate

1,1,2,2-Tetrachloroethane

Tetrachloroethylene

Tin, total recoverable

Toluene

Total dissolved solids

Total organic carbon

Total organic halogens

Total phosphates (as P)

Toxaphene

2,4,5-TP (Silvex)

1,1,1-Trichloroethane

1,1,2-Trichloroethane

Trichloroethylene

Trichlorofluoromethane

2,4,5-T

Tritium

Turbidity

Uranium-233/234

Uranium-235

Uranium-238

Vanadium, total recoverable

Xylenes

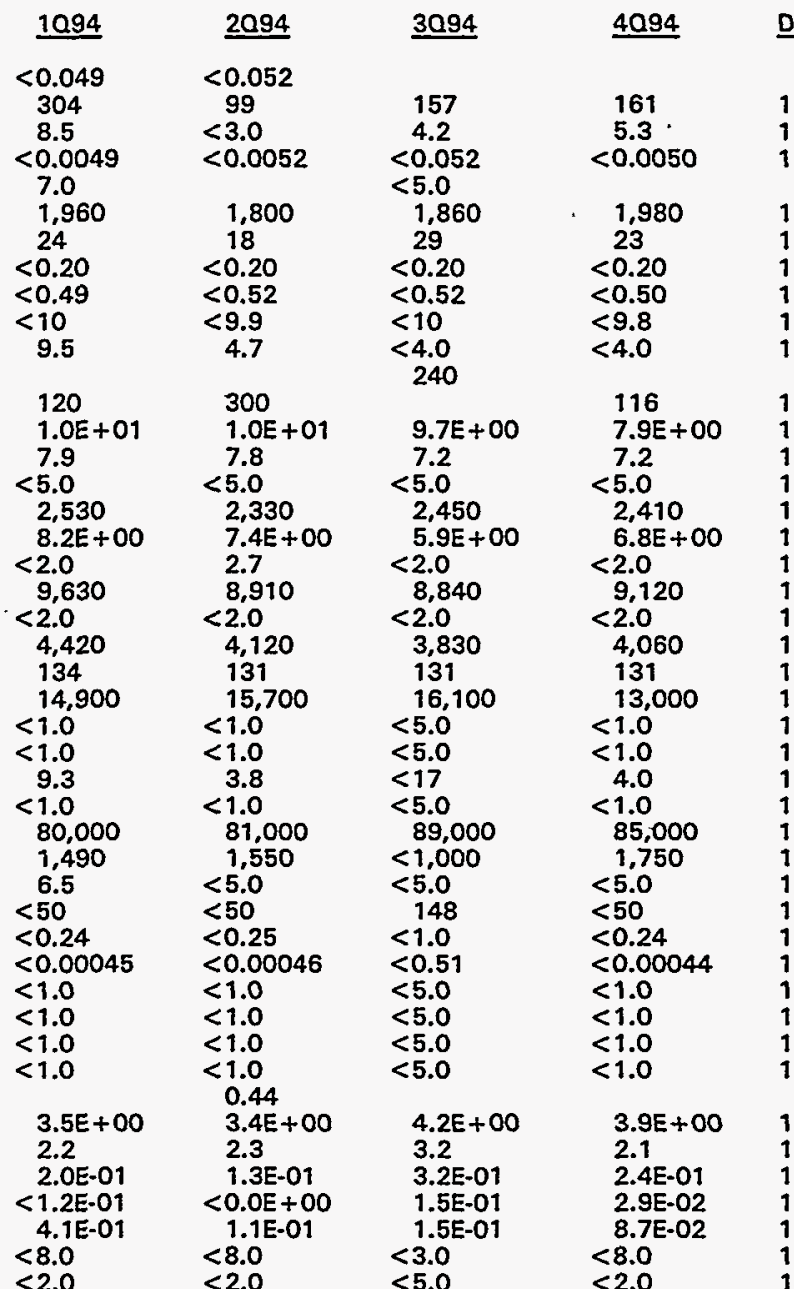

$<2.0$

$<5.0$

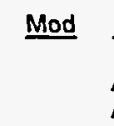

Unit
$\mu g / L$
$\mu g / L$

$\mu g / L \quad G E \quad 1$

$\begin{array}{lll}\mu g / L & G E & 0 \\ \mu g / L & G E & 0\end{array}$

(1)

$\mu g / L$

$\mu g / L$

$\mu g / L$

$\mu g / h$

$\mu \mathrm{g} / \mathrm{L}$

$\mu \mathrm{g} / \mathrm{L}$

$\mu g / L$

$\mu \mathrm{g} / \mathrm{L}$

J

pCil

pH

$\mu \mathrm{g} / \mathrm{L}$

pCi/L

$\mu \mathrm{g} / \mathrm{L}$

$\mu g / L$

$\mu \mathrm{g} / \mathrm{L}$

$\mu g / L$

$\mu \mathrm{S} / \mathrm{cm}$

$\mu g / L$

$\mu g / L$

$\mu g / L$

$\mu g / L$

$\mu g / L$

$J$

$\mu g / L$

$\mu g /$

$\underset{\mu g / L}{\mu g / L}$

$\mu g / L$

$\mu g / \mathrm{L}$

$\mu g / L$

$\mu g / L$
$\mu g / L$
$\mu g / L$

$\mu g / L$

$\mathrm{pCi} / \mathrm{mL}$ GP

NTU GE

uI

$\mathrm{pCi} /$

pCi/L

,

$\mu g / L$
Lab Flag

GE 0

GE

GE $\quad 0$

GE

GE $\quad 0$

GP 0

$G E$

GE

GP

GE

GE

$\mathrm{GE}$

GE

GE

GE

GE

.

GP 0

0
0

0
0
0
0
0

0
0

0

Note: Flagging, dilution factors, modifiers, and laboratories are for fourth quarter 1994 data only. See Appendix B for flagging criteria. - = exceeded holding time for fourth quarter 1994.

- = exceeded screening level or final PDWS for fourth quarter 1994. 
WELL BGO 43D

\begin{tabular}{|c|c|c|c|c|c|c|}
\hline SRS Coord. & Lat/Longitude & Screen Zone Elevation & Top of Casing & Casing & Pump & Screen Zone \\
\hline $\begin{array}{l}N 77056.7 \\
E 56238.8\end{array}$ & $\begin{array}{l}33.287960^{\circ} \mathrm{N} \\
81.668394^{\circ} \mathrm{W}\end{array}$ & 208.2-198.2 ft msl & $315.3 \mathrm{ft} \mathrm{msl}$ & $4^{\prime \prime} \mathrm{PVC}$ & s & vell (IIB1) \\
\hline AMPL & & $01 / 26 / 94$ & $04 / 06 / 94$ & & & \\
\hline
\end{tabular}

FIELD DATA

Analyte
Water elevation
Depth to water
pH
Sp. conductance
Water temperature
Alkalinity as $\mathrm{CaCO}_{3}$
Turbidity
Volume purged
Sampling code

ANALYTICAL DATA

\section{H ST Anaiyte}

Acetophenone

Aldrin

Aluminum, total recoverable Antimony, total recoverable

Arsenic, total recoverable

Barium, total recoverable

Benzene

Bromodichloromethane

Bromoform

Bromomethane (Methyl bromide)

Cadmium, total recoverable

Calcium, total recoverable

Carbon tetrachloride

Chloride

Chlorobenzene

Chloroethane

Chloroethene (Vinyl chloride)

2-Chloroethyl vinyl ether

Chloroform

Chloromethane (Methyl chloride)

Chromium, total recoverable

Copper, total recoverable

Cyanide

p, $p^{\prime}$-DDT

Dibromochloromethane

1,i-Dichloroethane

1,2-Dichloroethane

1,1-Dichloroethylene

trans-1,2-Dichloroethylene

Dichloromethane

2,4-Dichlorophenoxyacetic acid

1,2-Dichloropropane

cis-1,3-Dichloropropene

trans-1,3-Dichloropropene

Dieldrin

Endrin

Ethylbenzene

Fluoride

Gross alpha

1094
232.2
83.1
5.1
62
18.9
1
1
5.6

$\underline{2094}$
231.6
83.8
5.9
80
19.1
4
1
4.7

3094
231.0
84.3
5.5
69
19.3
1
2
7.9

1094

$<10$

$<0.051$

28
$<2.0$

$<2.0$

12

$<1.0$

$<1.0$

$<1.0$

$<1.0$

$<2.0$

4,440

$<1.0$

3,060

$<1.0$

$<1.0$

$<1.0$

$<1.0$

$<1.0$

$<1.0$

$<4.0$

$<4.0$

$<5.0$

$<0.10$

$<1.0$

$<1.0$

$<1.0$

$<1.0$

$<1.0$
$<0.0015$

$<1.0$

$<1.0$

$<1.0$

$<0.51$

$<0.0061$

$<1.0$

$<100$

$6.8 \mathrm{E}+00$

2094
$<10$
$<0.050$
21
$<2.0$
$<2.0$
12
$<1.0$
$<1.0$
$<1.0$
$<1.0$
$<2.0$
51.000
$<1.0$
3.170
$<1.0$
$<1.0$
$<1.0$
$<1.0$
$<1.0$
$<1.0$
$<4.0$
$<4.0$
$<5.0$
$<0.10$
$<1.0$
$<1.0$
$<1.0$
$<1.0$
$<1.0$
$<1.0$
$<0.0016$
$<1.0$
$<1.0$
$<1.0$
$<0.50$
$<0.0060$
$<1.0$
$<100$
$5.7 E+00$

$\quad 3094$
$\quad<11$
$\quad 50$
$<3.0$
$<2.0$
13
$<5.0$
$<5.0$
$<5.0$
$<10$
$<2.0$
5.600
$<5.0$
3.370
$<5.0$
$<10$
$<10$
$<10$
$<5.0$
$<10$
$<4.0$
$<4.0$
$<5.0$
$<5.0$
$<5.0$
$<5.0$
$<5.0$
$<5.0$
$<110$
$<1.0$
$<5.0$
$<5.0$
$<5.0$
$<0.11$
$<5.0$
$<100$
$5.6 E+00$

$\begin{aligned} & 4094 \\ &<10 \\ & 21 \\ &<2.0 \\ &<2.0 \\ & 14 \\ &<1.0 \\ &<1.0 \\ &<1.0 \\ &<1.0 \\ &<2.0 \\ & 61.190 \\ &<1.0 \\ & 2,550 \\ &<1.0 \\ &<1.0 \\ &<1.0 \\ &<1.0 \\ &<1.0 \\ &<1.0 \\ &<4.0 \\ &<4.0 \\ &<5.0 \\ &<1.0 \\ &<1.0 \\ &<1.0 \\ &<1.0 \\ &<1.0 \\ &<1.0 \\ &<0.0015 \\ &<1.0 \\ &<1.0 \\ &<1.0 \\ &<0.0059 \\ &<1.0 \\ &<20 \\ & 4.4 E+00\end{aligned}$

Unit

ft msl

ft

$\stackrel{\mathrm{pH}}{\mu \mathrm{S} / \mathrm{cm}}$

${ }^{\circ} \mathrm{C}$

mg/L

well vol. 


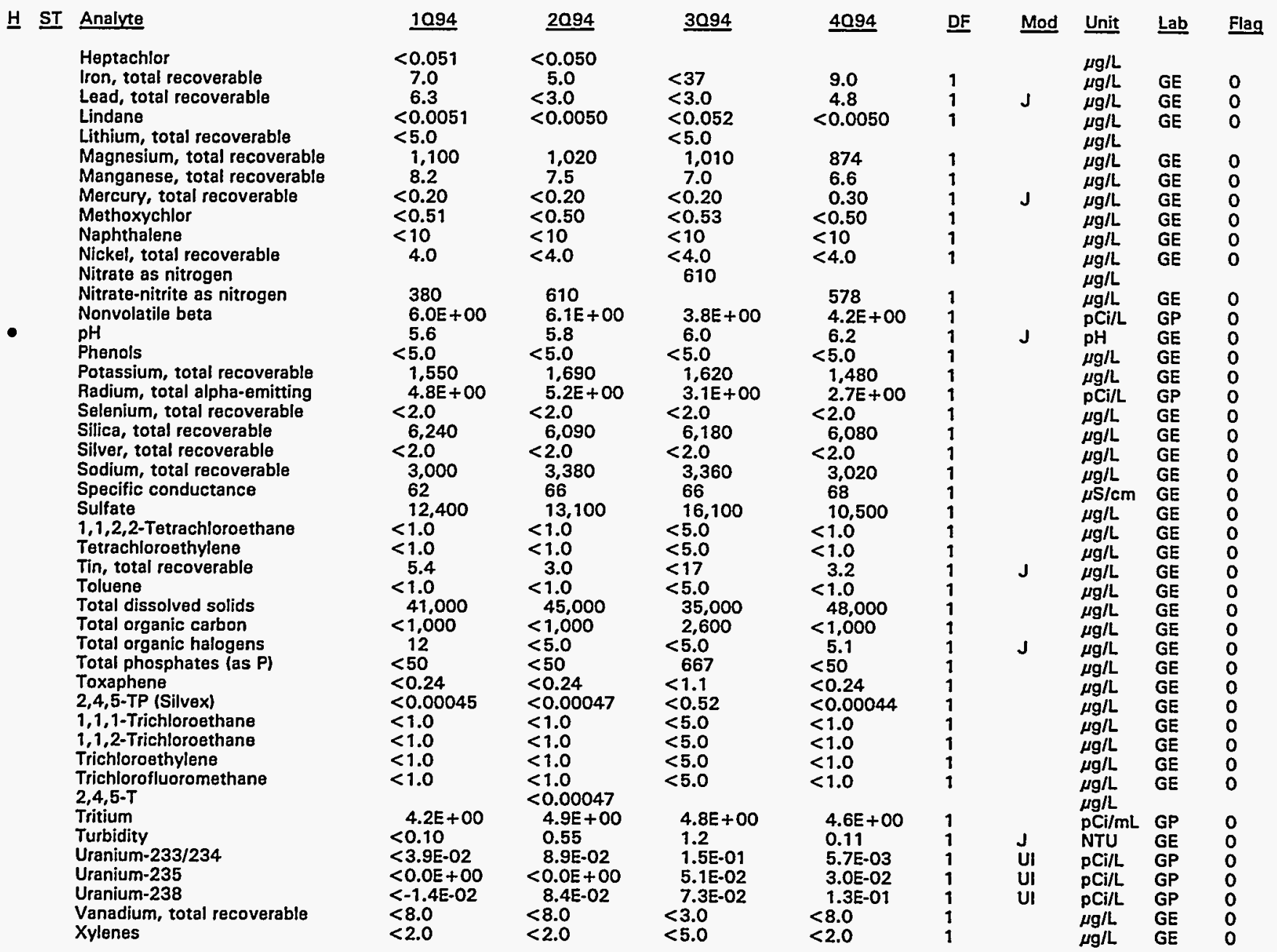

Note: Flagging, dilution factors, modifiers, and laboratories are for fourth quarter 1994 data only. See Appendix B for flagging criteria. - = exceeded holding time for fourth quarter 1994.

- = exceeded screening level or final PDWS for fourth quarter 1994. 
WELL BGO 44A

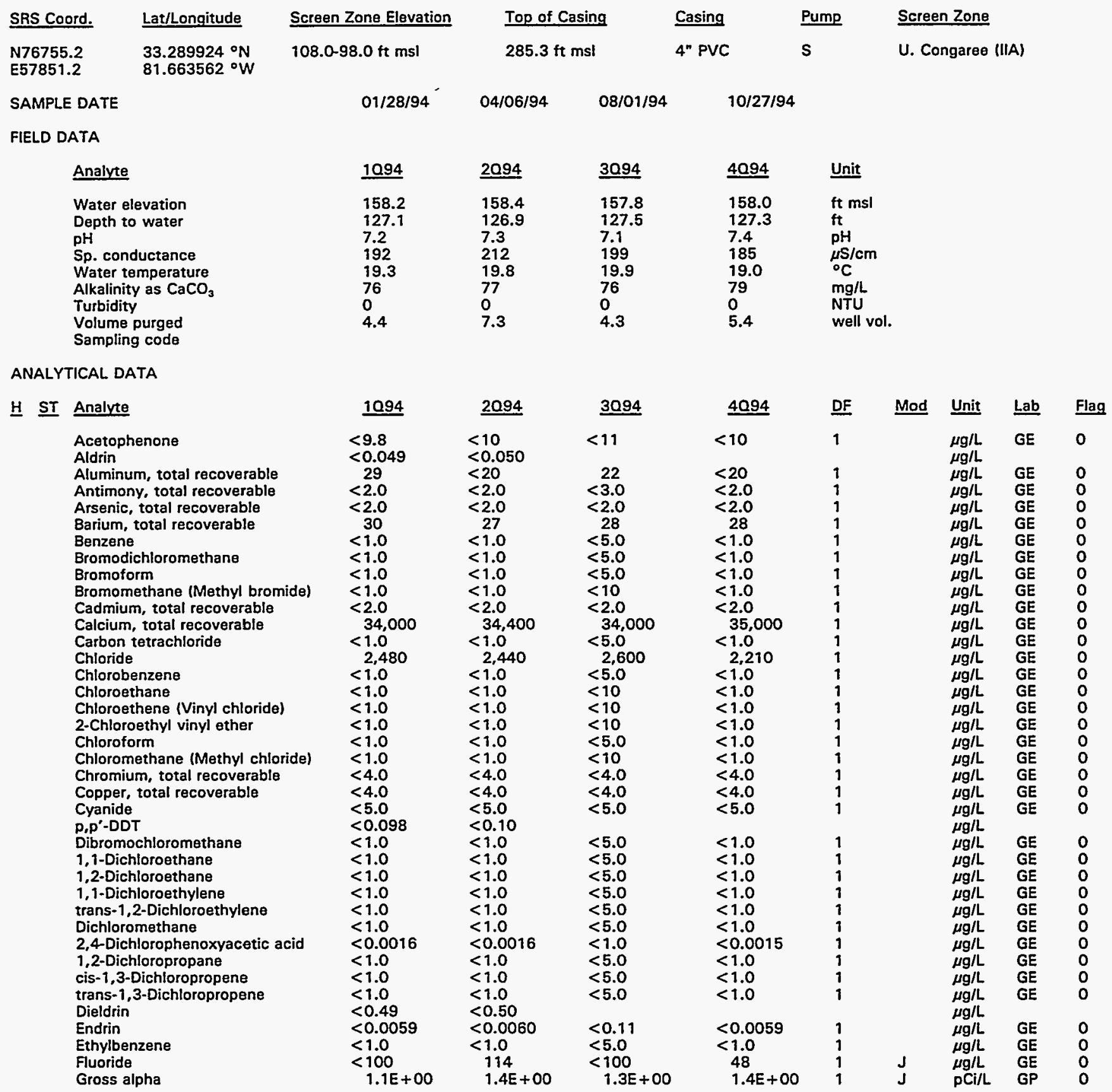

Note: Flagging, dilution factors, modifiers, and laboratories are for fourth quarter 1994 data only. See Appendix B for flagging criteria. $\bullet=$ exceeded holding time for fourth quarter 1994.

- = exceeded screening level or final PDWS for fourth quarter 1994. 


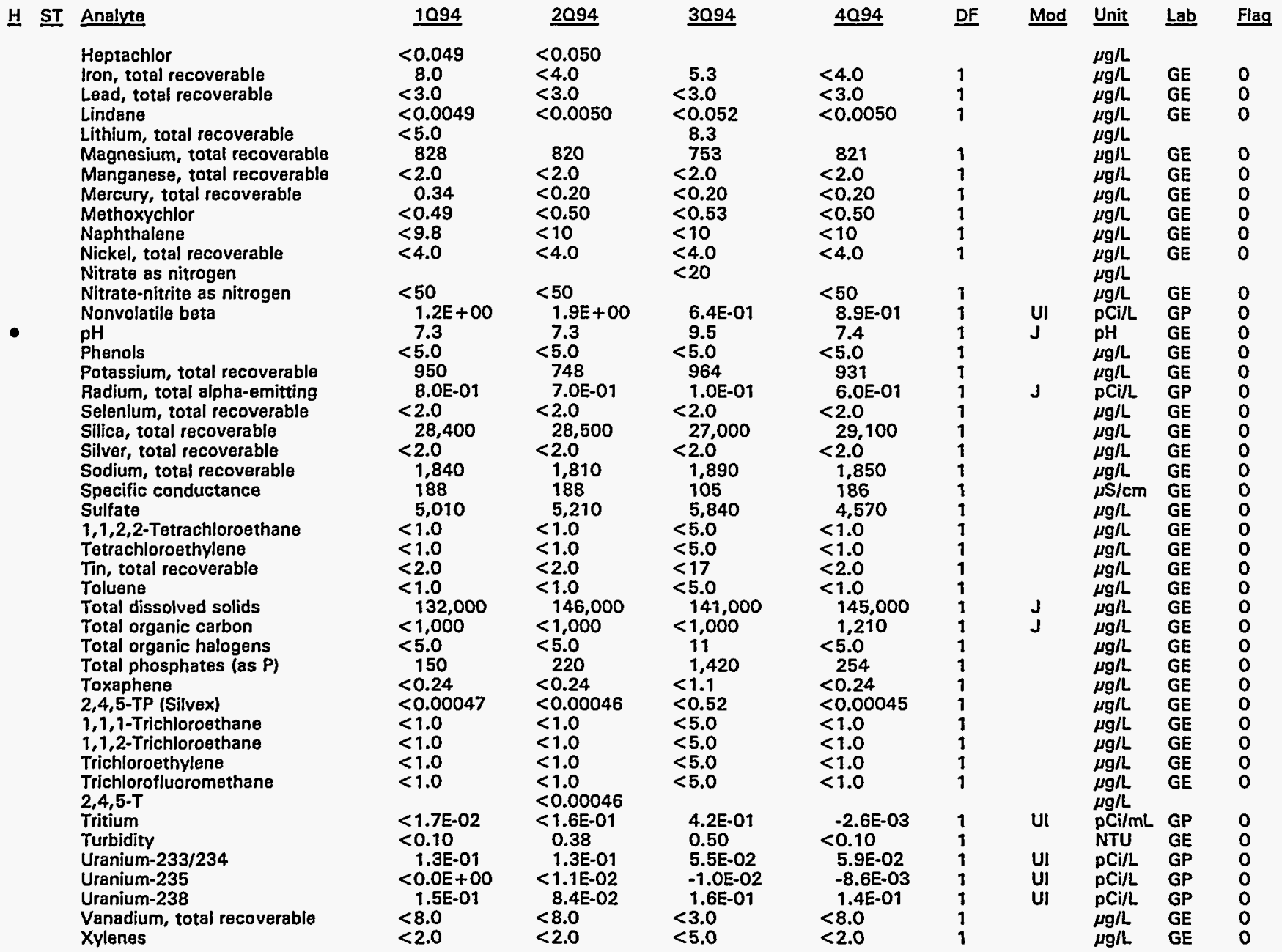

Note: Flagging, dilution factors, modifiers, and laboratories are for fourth quarter 1994 data only. See Appendix B for flagging criteria. - = exceeded holding time for fourth quarter 1994.

= exceeded screening level or final PDWS for fourth quarter 1994. 
WELL BGO 44AA

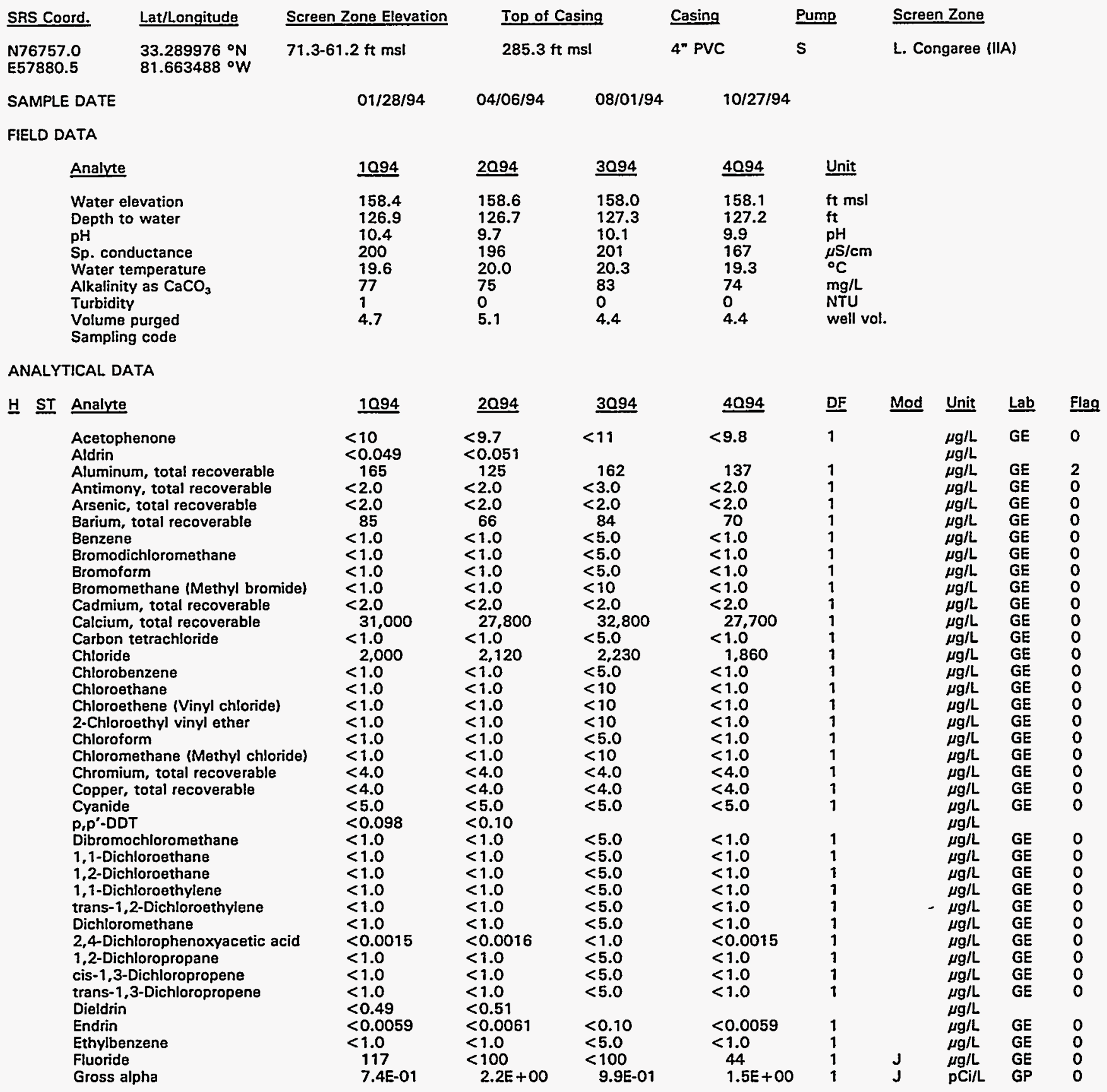

Note: Flagging, dilution factors, modifiers, and laboratories are for fourth quarter 1994 data only. See Appendix B for flagging criteria. - = exceeded holding time for fourth quarter 1994.

- = exceeded screening level or final PDWS for fourth quarter 1994. 


\section{H ST Analyte}

Heptachlor

Iron, total recoverable

Leed, total recoverable

Lindane

Lithium, total recoverable

Magnesium, total recoverable

Manganese, total recoverable Mercury, total recoverable

Methoxychlor

Naphthalene

Nickel, total recoverable

Nitrate as nitrogen

Nitrate-nitrite as nitrogen

Nonvolatile beta

$\mathrm{pH}$

Phenols

Potassium, total recoverable

Radium, total alpha-emitting

Selenium, total recoverable

Silica, total recoverable

Silver, total recoverable

Sodium, total recoverable

Specific conductance

Sulfate

1,1,2,2-Tetrachloroethane

Tetrachloroethylene

Tin, total recoverable

Toluene

Total dissolved solids

Total organic carbon

Total organic halogens

Total phosphates (as P)

Toxaphene

2,4,5-TP (Silvex)

1,1,1-Trichloroethane

1,1,2-Trichloroethane

Trichloroethylene

Trichlorofluoromethane

2,4,5-T

Tritium

Turbidity

Uranium-233/234

Uranium-235

Uranium-238

Vanadium, total recoverable

Xylenes

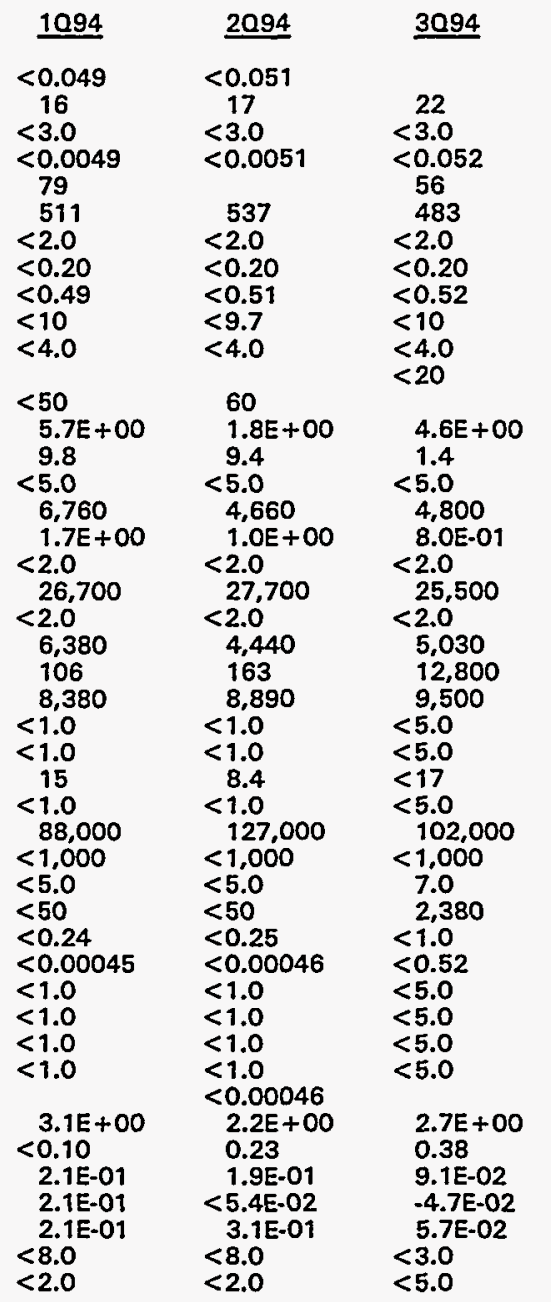

$4094 \quad$ DF

Mod Unit Láb Flag

17

$<3.0$
$<0.0049$

528

$<2.0$

$<0.49$

$<9.8$

$<4.0$

$<50$

$3.4 E+00$

9.5

$<5.0$

4,060

4.0E-01

$<2.0$

27.800

$<2.0$

4,130

155

7,940

$<1.0$

$<1.0$

7.6

$<1.0$

$<1,000$

$<5.0$

$<50$

$<0.24$

$<0.00046$

$<1.0$

$<1.0$

$<1.0$

$<1.0$

$3.0 E+00$

$<0.10$

2.8E-01

$-2.3 \mathrm{E}-02$

3.3E-02

$<8.0$

$<2.0$ $\mu g / L$

$\mu g / L \quad G E \quad 0$

$\mu g / \mathrm{L}$ GE

$\mu$ /L

$\mu g / L \quad G E$

$\mu g / L$

$\mu g / L$

$\mu g / L$

$\mu g / L$

$\mu g / L$

$\underset{\mu g / L}{\mu g / L}$

J

pH GE

$\mu g / 2$ GE

UI

PCi/L GP

$\mu g / L \quad G E$

$\mu g / L \quad G E$

$\mu \mathrm{g} / \mathrm{L} \quad \mathrm{GE}$

$\mu S / \mathrm{cm}$ GE

$\mu g / L \quad G E$

$\mu \mathrm{g} / \mathrm{L} \quad \mathrm{GE}$

$\mu g / L \quad G E$

$\mu g / L \quad$ GE

$\mu \mathrm{g} / \mathrm{L}$

$\mu g / L$

$\mu g / L$

$\mu \mathrm{g} / \mathrm{L}$

$\mu g / L$

$\mu g / L$

$\mu g / L$

$\mu \mathrm{g} / \mathrm{L}$

$\mu \mathrm{g} / \mathrm{L}$

$\mu g / L$

$\mu \mathrm{g} / \mathrm{L}$

$\mu \mathrm{g} / \mathrm{L}$

$\mathrm{pCi} / \mathrm{mL}$ GP

NTU GE

pCi/L GP

PCiLl

$\mathrm{PCi} / \mathrm{L} \quad \mathrm{GP}$

$\mu \mathrm{g} / \mathrm{L}$

Note: Flagging, dilution factors, modifiers, and laboratories are for fourth quarter 1994 data only. See Appendix B for flagging criteria. - = exceeded holding time for fourth quarter 1994.

- = exceeded screening level or final PDWS for fourth quarter 1994. 
WELL BGO 44B

\begin{tabular}{|c|c|c|c|c|c|c|}
\hline SRS Coord. & Lat/Longitude & Screen Zone Elevation & Top of Casing & Casing & Pump & Screen Zone \\
\hline $\begin{array}{l}\text { N76756.0 } \\
\text { E57865.8 }\end{array}$ & $\begin{array}{l}33.289950^{\circ} \mathrm{N} \\
81.663525 \circ \mathrm{W}\end{array}$ & $158.1-148.1 \mathrm{ft} \mathrm{msl}$ & $285.2 \mathrm{ft} \mathrm{msl}$ & 4" PVC & $\mathbf{s}$ & McBean (IIB I) \\
\hline SAMPLE DATE & & $02 / 08 / 94$ & $04 / 06 / 94$ & & & \\
\hline
\end{tabular}

FIELD DATA

Analyte
Water elevation
Depth to water
pH
Sp. conductance
Water temperature
Alkalinity as $\mathrm{CaCO}_{3}$
Turbidity
Volume purged
Sampling code

ANALYTICAL DATA

\section{H ST Analyte}

Acetophenone

Aldrin

Aluminum, total recoverable Antimony, total recoverable

Arsenic, total recoverable

Barium, total recoverable

Benzene

Bromodichloromethane

Bromoform

Bromomethane (Methyl bromide)

Cadmium, total recoverable

Calcium, total recoverable

Carbon tetrachloride

Chioride

Chlorobenzene

Chloroethane

Chloroethene (Vinyi chloride)

2-Chloroethyl vinyl ether

Chloroform

Chloromethane (Methyl chloride)

Chromium, total recoverable

Copper, total recoverable

Cyanide

P.P'-DDT

Dibromochloromethane

1.1-Dichloroethane

1,2-Dichloroethane

1,1-Dichloroethylene

trans-1,2-Dichloroethylene

Dichloromethane

2,4-Dichlorophenoxyacetic acid

1,2-Dichloropropane

cis-1,3-Dichloropropene

trans-1,3-Dichloropropene

Dieldrin

Endrin

Ethylbenzene

Fluoride

Gross alpha

1094
221.2
64.0
11.3
399
17.5
90
127
0.8
$x$

$\underline{2094}$
221.2
64.0
8.4
244
19.5
121
211
0.8
$x$

1094

$<10$

$<0.053$

$<4.0$

15

50

$<1.0$

$<1.0$

$<1.0$

$<1.0$

$<2.0$

63,500

$<1.0$

2,410

$<1.0$

$<1.0$

$<1.0$

$<1.0$

$<1.0$

$<1.0$

$<4.0$

$<4.0$

$<5.0$

$<0.11$

$<1.0$

$<1.0$

$<1.0$

$<1.0$

$<1.0$

$<1.0$

$<0.0014$

$<1.0$

$<1.0$

$<1.0$

$<0.53$

$<0.006$

$<1.0$

$3.8 \mathrm{E}+00$

\begin{tabular}{|c|c|}
\hline 2094 & 3094 \\
\hline $\begin{array}{l}<9.8 \\
<0.050\end{array}$ & $<11$ \\
\hline 250 & 129 \\
\hline$<2.0$ & $<3.0$ \\
\hline 3.8 & 2.5 \\
\hline 138 & 108 \\
\hline $\begin{array}{l}<1.0 \\
<10\end{array}$ & $<5.0$ \\
\hline $\begin{array}{l}<1.0 \\
<1.0\end{array}$ & $<5.0$ \\
\hline $\begin{array}{l}<1.0 \\
<1.0\end{array}$ & $\begin{array}{l}<5.0 \\
<10\end{array}$ \\
\hline$<4.0$ & $<2.0$ \\
\hline $\begin{aligned} & 114,000 \\
< & 1.0\end{aligned}$ & $\begin{aligned} & 60.500 \\
< & 5.0\end{aligned}$ \\
\hline 2,220 & 2,650 \\
\hline$<1.0$ & $<5.0$ \\
\hline$<1.0$ & $<10$ \\
\hline $\begin{array}{l}<1.0 \\
<1.0\end{array}$ & $<10$ \\
\hline $\begin{array}{l}<1.0 \\
<1.0\end{array}$ & $<10$ \\
\hline $\begin{array}{l}<1.0 \\
<1.0\end{array}$ & $<5.0$ \\
\hline $\begin{array}{l}<1.0 \\
<8.0\end{array}$ & $\begin{array}{l}<10 \\
<4.0\end{array}$ \\
\hline$<8.0$ & $<4.0$ \\
\hline$<5.0$ & $<5.0$ \\
\hline$<0.10$ & \\
\hline$<1.0$ & $<5.0$ \\
\hline$<1.0$ & $<5.0$ \\
\hline$<1.0$ & $<5.0$ \\
\hline$<1.0$ & $<5.0$ \\
\hline$<1.0$ & $<5.0$ \\
\hline$<1.0$ & 1.7 \\
\hline$<0.0016$ & $<1.1$ \\
\hline$<1.0$ & $<5.0$ \\
\hline$<1.0$ & $<5.0$ \\
\hline$<1.0$ & $<5.0$ \\
\hline $\begin{array}{l}<0.50 \\
<0.0060\end{array}$ & \\
\hline$<1.0$ & $<5.0$ \\
\hline$<100$ & $<100$ \\
\hline $1.5 E+00$ & $1.2 E+O C$ \\
\hline
\end{tabular}

4094
220.9
64.3
8.6
271
14.7
120
86
0.8
$x$

Unit

$\mathrm{ft} \mathrm{msl}$

$\mathrm{ft}$

pH

$\mu \mathrm{S} / \mathrm{cm}$

$\mathrm{mg} / \mathrm{L}$

NTU

well vol.

\begin{tabular}{|c|c|c|c|c|}
\hline 4094 & $\underline{D F}$ & Mod & Unit & $\underline{\text { Lab }}$ \\
\hline$<10$ & 1 & & $\mu \mathrm{g} / \mathrm{L}$ & GE \\
\hline$<0.050$ & 1 & & $\mu \mathrm{g} / \mathrm{L}$ & $\mathrm{GE}$ \\
\hline 81 & 1 & & $\mu \mathrm{g} / \mathrm{L}$ & $\mathrm{GE}$ \\
\hline 4.8 & 1 & & $\mu g / L$ & GE \\
\hline 3.2 & 1 & $J$ & $\mu g / L$ & GE \\
\hline 71 & 1 & & $\mu g / L$ & $\mathrm{GE}$ \\
\hline$<1.0$ & 1 & & $\mu \mathrm{g} / \mathrm{L}$ & GE \\
\hline$<1.0$ & 1 & & $\mu \mathrm{g} / \mathrm{L}$ & $\overline{G E}$ \\
\hline$<1.0$ & 1 & & $\mu \mathrm{g} / \mathrm{L}$ & GE \\
\hline$<1.0$ & 1 & & $\mu \mathrm{g} / \mathrm{L}$ & GE \\
\hline$<2.0$ & 1 & & $\mu \mathrm{g} / \mathrm{L}$ & GE \\
\hline 47,000 & 1 & & $\mu \mathrm{g} / \mathrm{L}$ & GE \\
\hline$<1.0$ & 1 & & $\mu \mathrm{g} / \mathrm{L}$ & GE \\
\hline 2,070 & 1 & & $\mu g / L$ & GE \\
\hline$<1.0$ & 1 & & $\mu \mathrm{g} / \mathrm{L}$ & GE \\
\hline$<1.0$ & 1 & & $\mu \mathrm{g} / \mathrm{L}$ & GE \\
\hline$<1.0$ & 1 & & $\mu g / L$ & GE \\
\hline$<1.0$ & 1 & & $\mu g / L$ & GE \\
\hline$<1.0$ & 1 & & $\mu g / L$ & GE \\
\hline$<1.0$ & 1 & & $\mu g / L$ & GE \\
\hline$<4.0$ & 1 & & $\mu g / L$ & GE \\
\hline$<4.0$ & 1 & & $\mu \mathrm{g} / \mathrm{L}$ & GE \\
\hline$<5.0$ & 1 & & $\mu g / L$ & GE \\
\hline$<0.10$ & 1 & & $\mu g / L$ & GE \\
\hline$<1.0$ & 1 & & $\mu \mathrm{g} / \mathrm{L}$ & $\mathrm{GE}$ \\
\hline$<1.0$ & 1 & & $\mu \mathrm{g} / \mathrm{L}$ & GE \\
\hline$<1.0$ & 1 & & $\mu \mathrm{g} / \mathrm{L}$ & GE \\
\hline$<1.0$ & 1 & & $\mu \mathrm{g} / \mathrm{L}$ & $\mathrm{GE}$ \\
\hline$<1.0$ & 1 & & $\mu \mathrm{g} / \mathrm{L}$ & GE \\
\hline$<1.0$ & 1 & & $\mu \mathrm{g} / \mathrm{L}$ & GE \\
\hline$<0.0015$ & 1 & & $\mu g / L$ & GE \\
\hline$<1.0$ & 1 & & $\mu \mathrm{g} / \mathrm{L}$ & GE \\
\hline$<1.0$ & 1 & & $\mu \mathrm{g} / \mathrm{L}$ & GE \\
\hline$<1.0$ & 1 & & $\mu g / L$ & GE \\
\hline$<0.50$ & 1 & & $\mu \mathrm{g} / \mathrm{L}$ & GE \\
\hline$<0.0060$ & 1 & & $\mu g / L$ & GE \\
\hline$<1.0$ & 1 & & $\mu g / L$ & GE \\
\hline$<20$ & 1 & & $\mu \mathrm{g} / \mathrm{L}$ & GE \\
\hline 8.3E-01 & 1 & UI & $\mathrm{pCi} / \mathrm{L}$ & GP \\
\hline
\end{tabular}

Note: Flagging, dilution factors, modifiers, and laboratories are for fourth quarter 1994 data only. See Appendix B for flagging criteria. - = exceeded holding time for fourth quarter 1994.

$\mathbf{u}=$ exceeded screening level or final PDWS for fourth quarter 1994. 
HI ST Analyte

Heptachlor

Iron, total recoverable

Lead, total recoverable

Lindane

Lithium, total recoverable

Magnesium, total recoverable

Manganese, total recoverable

Mercury, total recoverable

Methoxychlor

Naphthalene

Nickel, total recoverable

Nitrate as nitrogen

Nitrate-nitrite as nitrogen

- $\mathrm{pH}$

pH

Potassium, total recoverable

Radium, total alpha-emitting

Selenium, total recoverable

Silica, total recoverable

Silver, total recoverable

Sodium, total recoverable

Specific conductance

Sulfate

1,1,2,2-Tetrachloroethane

Tetrachloroethylene

Tin, total recoverable

Toluene

Total activity

Total dissolved solids

Total organic carbon

Total organic halogens

Total phosphates (as P)

Toxaphene

2,4,5-TP (Silvex)

$1,1,1-$ Trichlorosthane

1,1,2-Trichloroethane

Trichloroethylene

Trichlorofluoromethane

2,4,5-T

- Tritium

Uranium-233/234

Uranium-235

Uranium-235

Vanadium, total recoverable

Xylenes

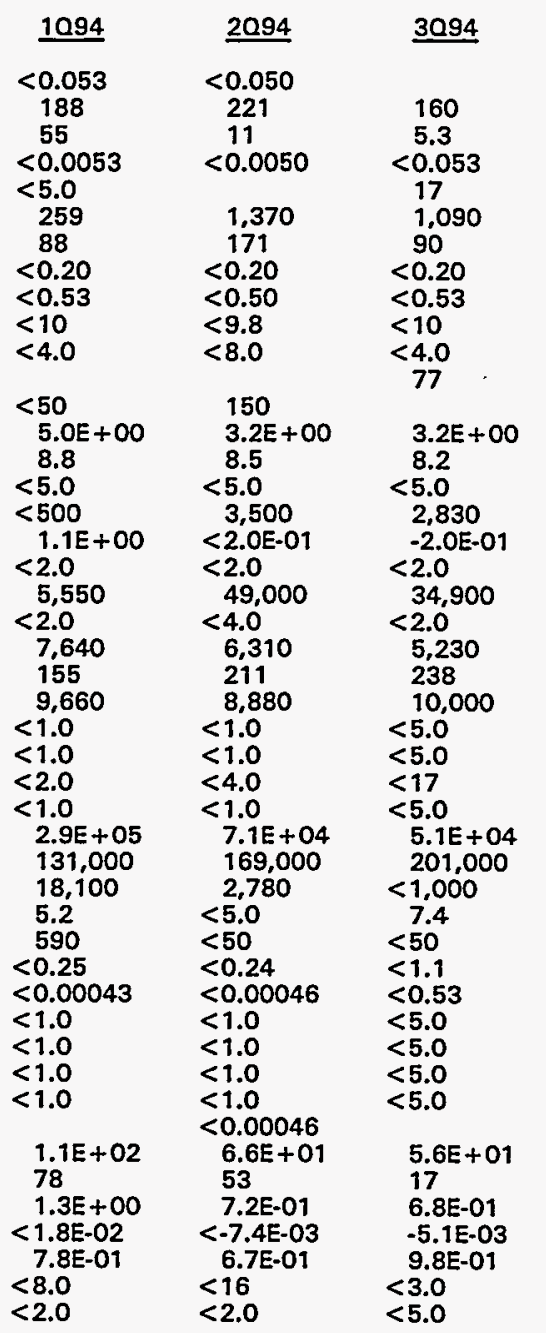

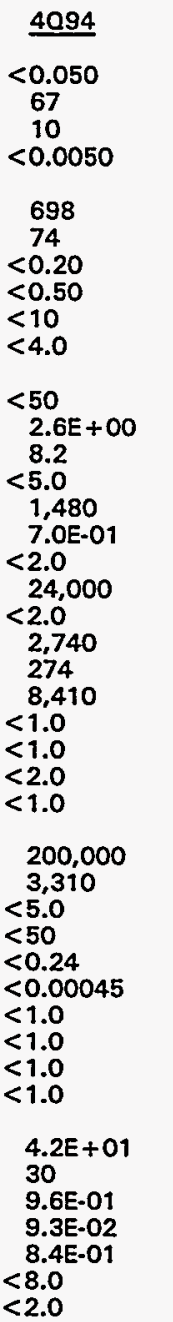

\begin{tabular}{|c|c|c|c|}
\hline DF & Mod & Unit & Lab \\
\hline 1 & & $\mu g / L$ & GE \\
\hline 1 & & $\mu g / L$ & GE \\
\hline 1 & & $\mu g / L$ & GE \\
\hline 1 & & $\begin{array}{l}\mu \mathrm{g} / \mathrm{L} \\
\mu \mathrm{g} / \mathrm{L}\end{array}$ & GE \\
\hline 1 & & $\mu \mathrm{g} / \mathrm{L}$ & $\mathrm{GE}$ \\
\hline 1 & & $\mu g / L$ & $\mathrm{GE}$ \\
\hline 1 & & $\mu \mathrm{g} / \mathrm{L}$ & GE \\
\hline 1 & & $\mu g / L$ & GE \\
\hline 1 & & $\mu \mathrm{g} / \mathrm{L}$ & GE \\
\hline 1 & & $\begin{array}{l}\mu \mathrm{g} / \mathrm{L} \\
\mu \mathrm{g} / \mathrm{L}\end{array}$ & GE \\
\hline 1 & & $\mu \mathrm{g} / \mathrm{L}$ & GE \\
\hline 1 & $\mathrm{~J}$ & PCi/L & GP \\
\hline $\begin{array}{l}1 \\
1\end{array}$ & $J$ & $\mathrm{pH}$ & $\begin{array}{l}\text { GE } \\
\text { GE }\end{array}$ \\
\hline 1 & & $\mu g / L$ & $\mathrm{GE}$ \\
\hline 1 & $J$ & $\mathrm{pCi} / \mathrm{L}$ & GP \\
\hline 1 & & $\mu g / L$ & GE \\
\hline 1 & & $\mu \mathrm{g} / \mathrm{L}$ & GE \\
\hline 1 & & $\mu \mathrm{g} / \mathrm{L}$ & GE \\
\hline$i$ & & $\begin{array}{l}\mu \mathrm{g} / \mathrm{L} \\
\mu \mathrm{S} / \mathrm{cm}\end{array}$ & $\begin{array}{l}\text { GE } \\
\text { GE }\end{array}$ \\
\hline 1 & & $\mu \mathrm{g} / \mathrm{L}$ & GE \\
\hline 1 & & $\mu g / L$ & GE \\
\hline 1 & & $\mu g / L$ & GE \\
\hline 1 & & $\mu \mathrm{g} / \mathrm{L}$ & GE \\
\hline 1 & & $\begin{array}{l}\mu \mathrm{g} / \mathrm{L} \\
\mathrm{pCi} / \mathrm{L}\end{array}$ & \\
\hline 1 & & $\mu \mathrm{g} / \mathrm{L}$ & GE \\
\hline 1 & & $\mu g / L$ & GE \\
\hline 1 & & $\mu g / L$ & GE \\
\hline 1 & & $\mu \mathrm{g} / \mathrm{L}$ & GE \\
\hline 1 & & $\mu g / L$ & GE \\
\hline 1 & & $\mu g / L$ & GE \\
\hline 1 & & $\mu \mathrm{g} / \mathrm{L}$ & GE \\
\hline 1 & & $\mu g / L$ & GE \\
\hline 1 & & $\begin{array}{l}\mu \mathrm{g} / \mathrm{L} \\
\mu \mathrm{g} / \mathrm{L}\end{array}$ & GE \\
\hline 1 & & $\mathrm{pCi} / \mathrm{mL}$ & $G P$ \\
\hline 1 & $J_{11}$ & NTU & GE \\
\hline 1 & UI & pCi/L & GP \\
\hline 1 & UI & PCi/L & GP \\
\hline $\begin{array}{l}1 \\
1\end{array}$ & UI & $\begin{array}{l}\mathrm{pCi} / \mathrm{L} \\
\mu \mathrm{g} / \mathrm{L}\end{array}$ & $\begin{array}{l}\text { GP } \\
\text { GE }\end{array}$ \\
\hline 1 & & $\mu g / L$ & GE \\
\hline
\end{tabular}

Note: Flagging, dilution factors, modifiers, and laboratories are for fourth quarter 1994 data only. See Appendix B for flagging criteria. - = exceeded holding time for fourth quarter 1994.

- = exceeded screening level or final PDWS for fourth quarter 1994. 
WELL BGO 44C

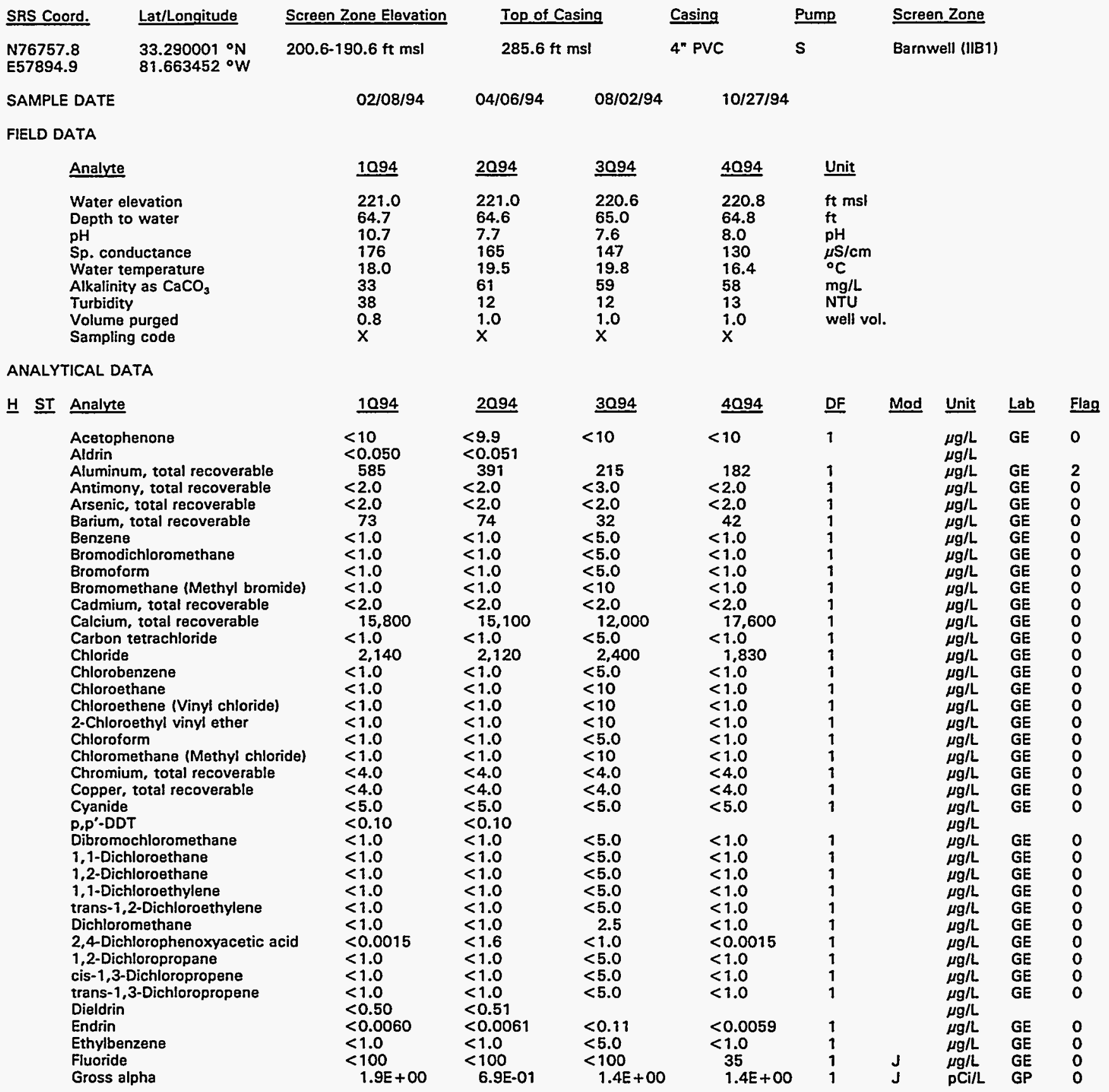

Note: Flagging, dilution factors, modifiers, and laboratories are for fourth quarter 1994 data only. See Appendix B for flagging criteria. - = exceeded holding time for fourth quarter 1994.

m = exceeded screening level or final PDWS for fourth quarter 1994. 


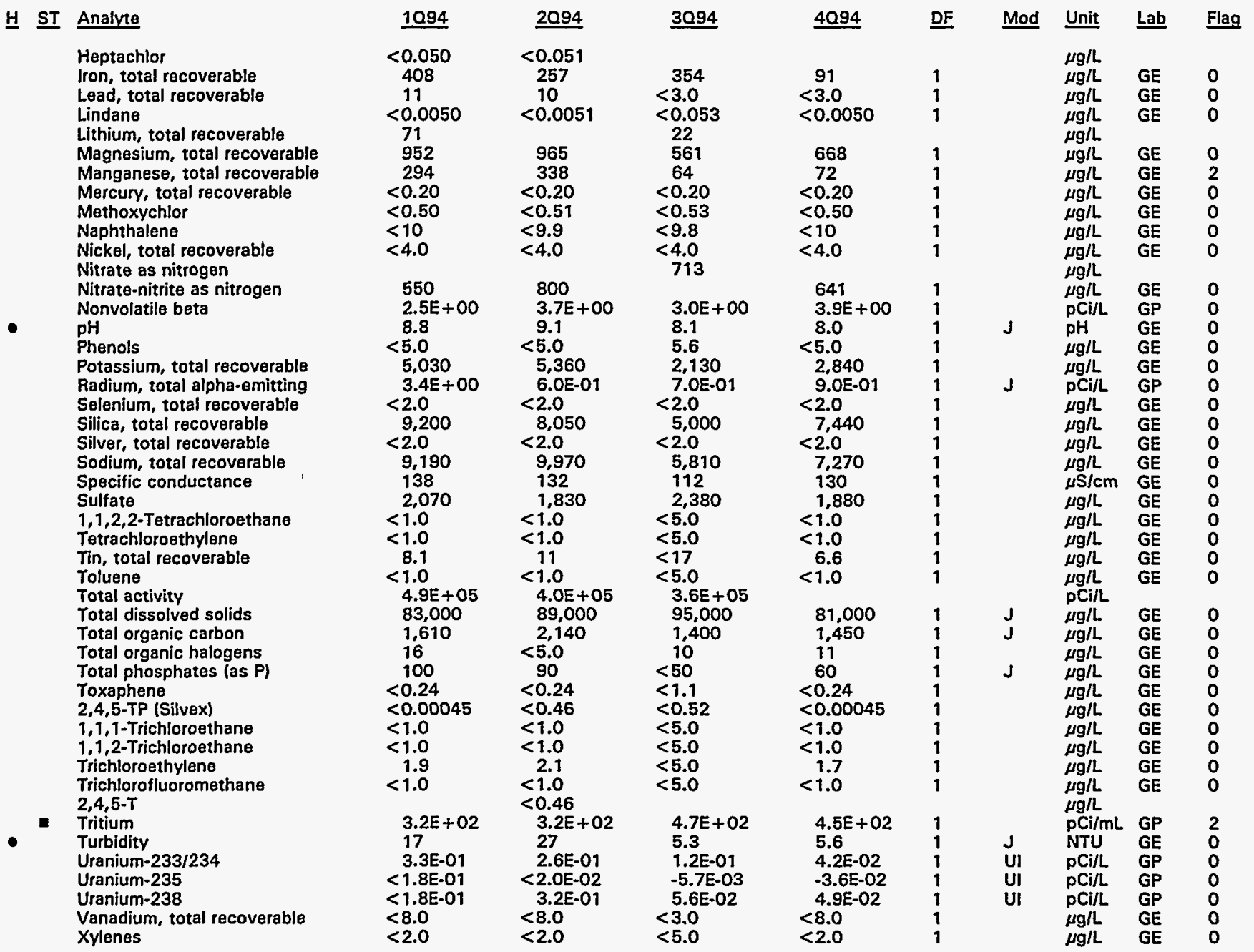

Note: Flagging, dilution factors, modifiers, and laboratories are for fourth quarter 1994 data only. See Appendix B for flagging criteria. - = exceeded holding time for fourth quarter 1994.

- = exceeded screening level or final PDWS for fourth quarter 1994. 
WELL BGO 44D

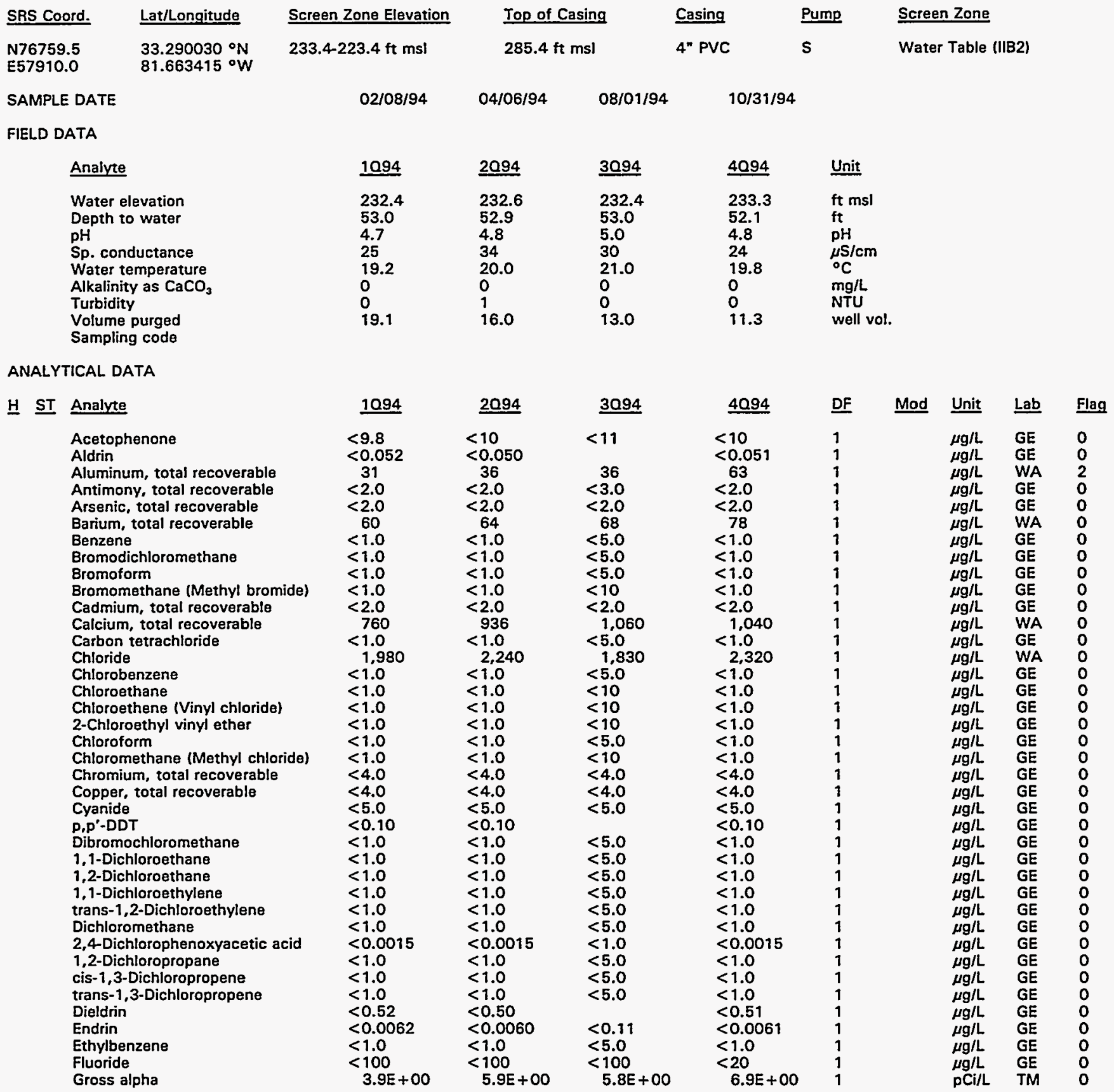

Note: Flagging, dilution factors, modifiers, and laboratories are for fourth quarter 1994 data only. See Appendix B for flagging criteria. - = exceeded holding time for fourth quarter 1994.

m = exceeded screening level or final PDWS for fourth quarter 1994. 
H ST Analyte

Heptachlor

Iron, total recoverable

Lead, total recoverable

Lindane

Lithium, total recoverable

Magnesium, total recoverable

Manganese, total recoverable

Mercury, total recoverable

Methoxychlor

Naphthalene

Nickel, total recoverable

Nitrate as nitrogen

Nitrate-nitrite as nitrogen

Nonvolatile beta

$\mathrm{pH}$

Phenols

Potassium, total recoverable

Radium, total alpha-emitting

Radium-226

Radium-228

Selenium, total recoverable

Silica, total recoverable

Silver, total recoverable

Sodium, total recoverable

Specific conductance

Sulfate

1,1,2,2-Tetrachloroethane

Tetrachloroethylene

Tin, total recoverable

Toluene

Total activity

Total dissolved solids

Total organic carbon

Total organic halogens

Total phosphates (as P)

Toxaphene

2,4,5-TP (Silvex)

1,1,1-Trichloroethane

1,1,2-Trichloroethane

Trichloroethylene

Trichlorofluoromethane

2,4,5-T

- Tritium

Turbidity

Uranium-233/234

Uranium-235

Uranium-238

Vanadium, total recoverable

Xylenes

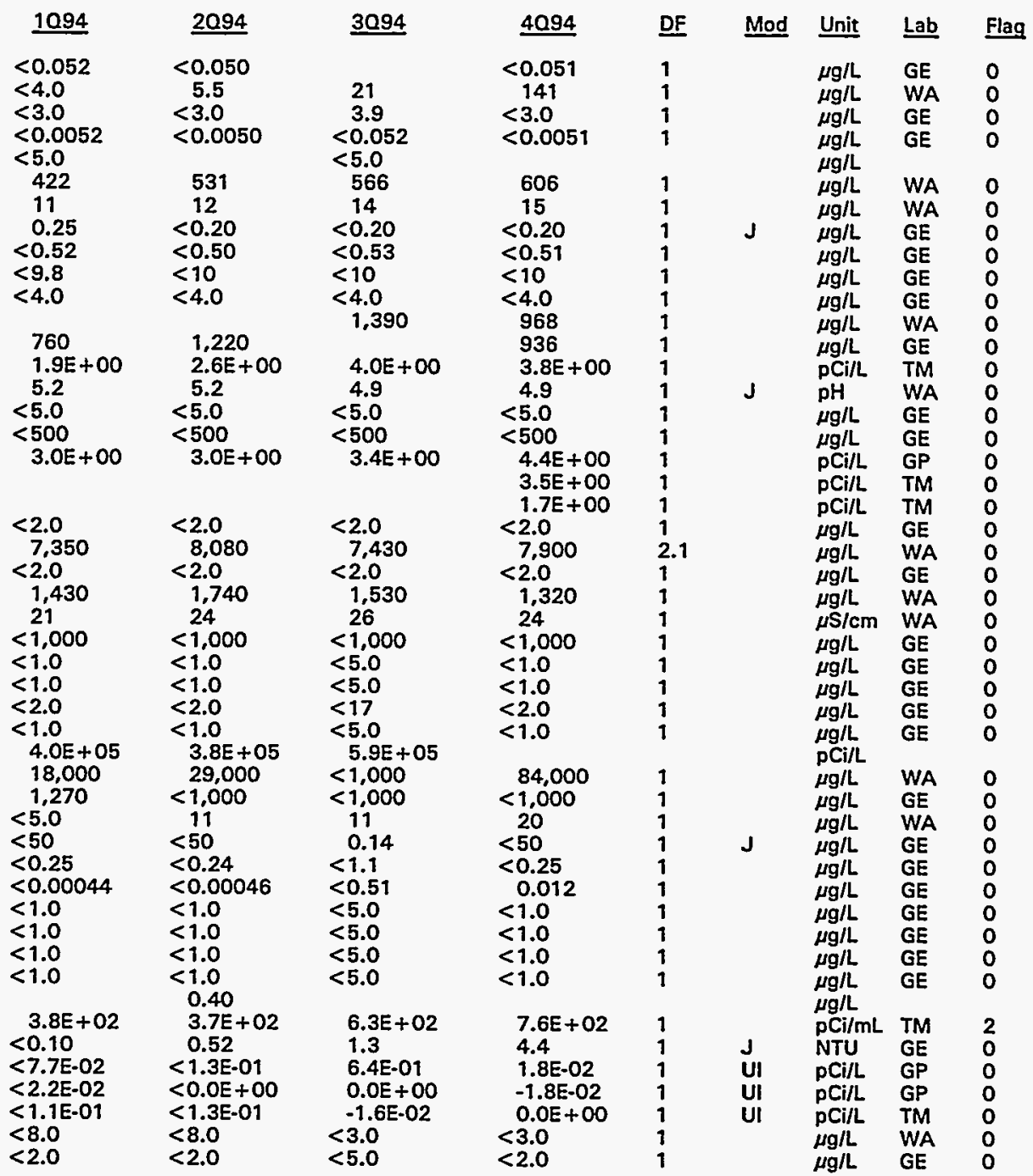

Note: Flagging, dilution factors, modifiers, and laboratories are for fourth quarter 1994 data only. See Appendix B for flagging criteria. - = exceeded holding time for fourth quarter 1994.

m = exceeded screening level or final PDWS for fourth quarter 1994. 
WELL BGO 45A

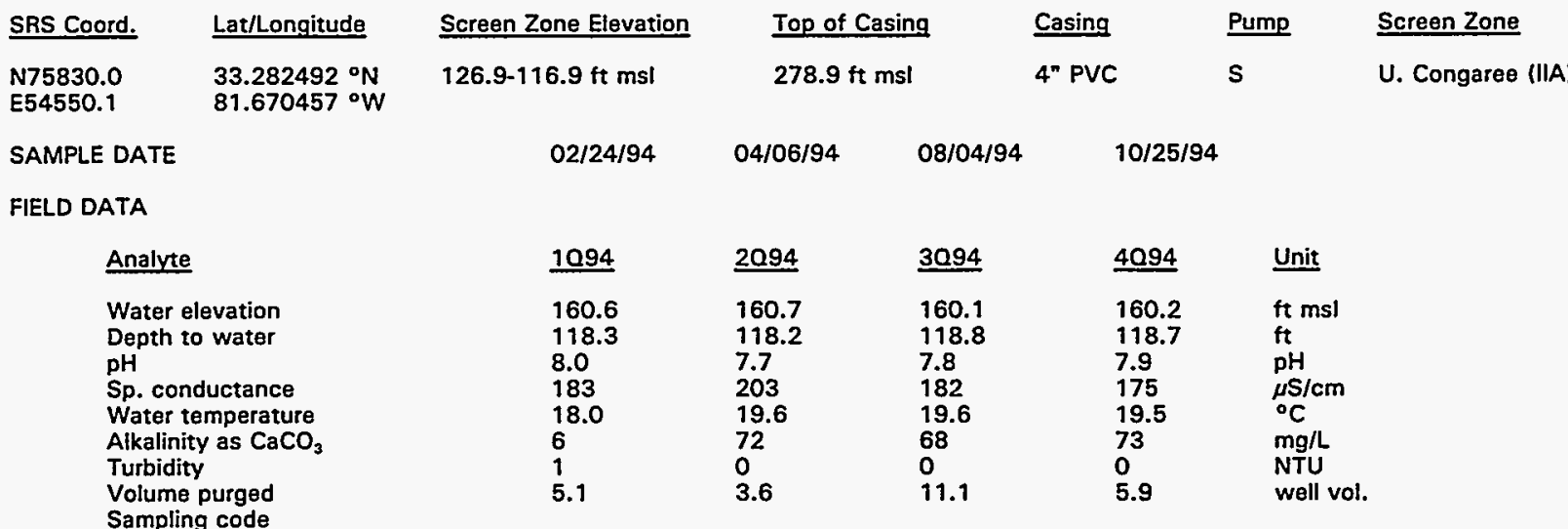

ANALYTICAL DATA

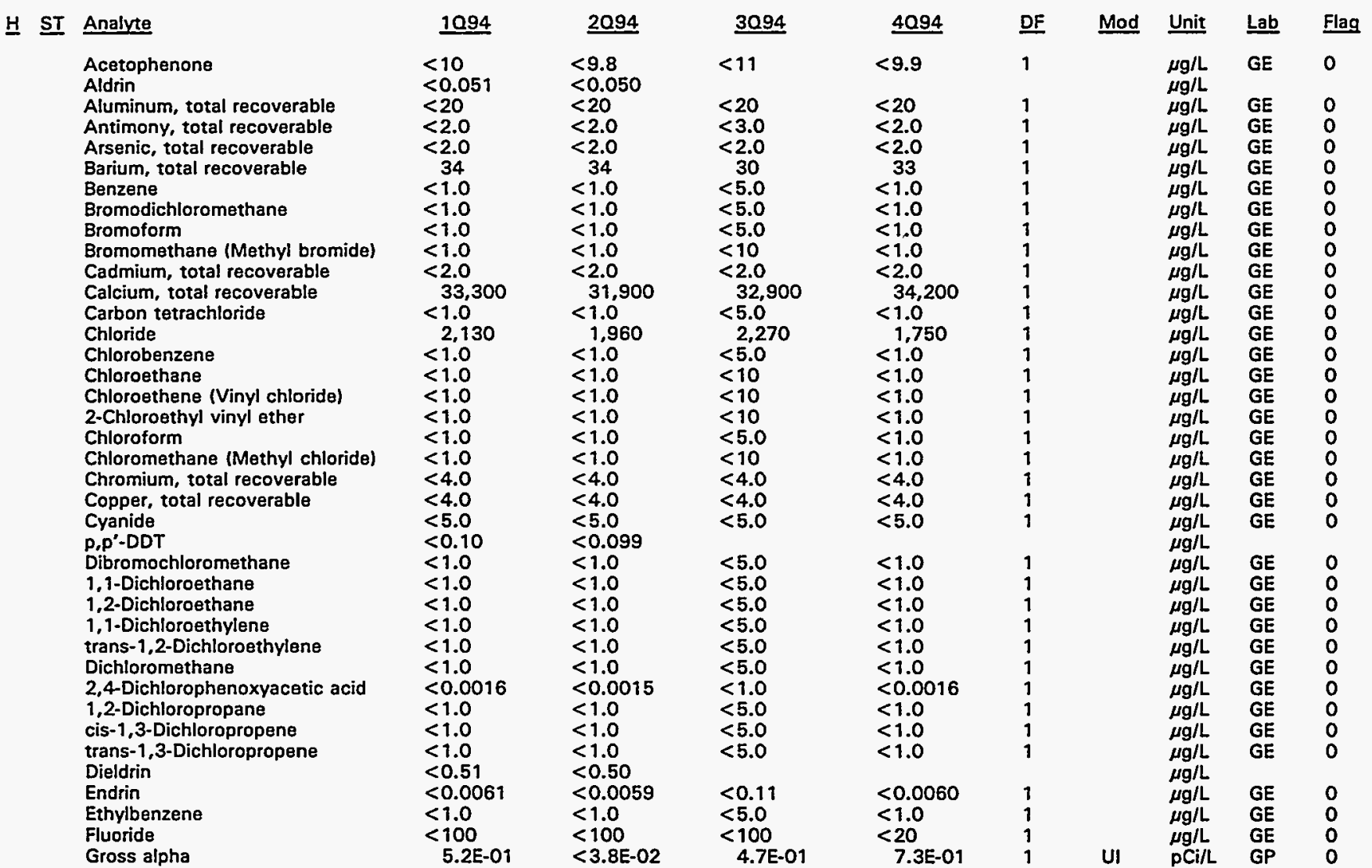

Note: Flagging, dilution factors, modifiers, and laboratories are for fourth quarter 1994 data only. See Appendix B for flagging criteria. 0 = exceeded holding time for fourth quarter 1994.

n = exceeded screening level or final PDWS for fourth quarter 1994. 
H ST Analyte

Heptachlor

Iron, total recoverable

Lead, total recoverable

Lindane

Lithium, total recoverable

Magnesium, total recoverable

Manganese, total recoverable

Mercury, total recoverable

Methoxychlor

Naphthalene

Nickel, total recoverable

Nitrate as nitrogen

Nitrate-nitrite as nitrogen

Nonvolatile beta

$\mathrm{pH}$

Phenols

Potassium, total recoverable

Radium, total alpha-emitting

Selenium, total recoverable

Silica, total recoverable

Silver, total recoverable

Sodium, total recoverable

Specific conductance

Sulfate

1,1,2,2-Tetrachloroethane

Tetrachloroethylene

Tin, total recoverable

Toluene

Total dissolved solids

Total organic carbon

Total organic halogens

Total phosphates (as P)

Toxaphene

2,4,5-TP (Silvex)

Tributyl phosphate

1,1,1-Trichloroethane

1,1,2-Trichloroethane

Trichloroethylene

Trichlorofluoromethane

2,4,5-T

Tritium

Turbidity

Uranium-233/234

Uranium-235

Uranium-238

Vanadium, total recoverable

Xylenes

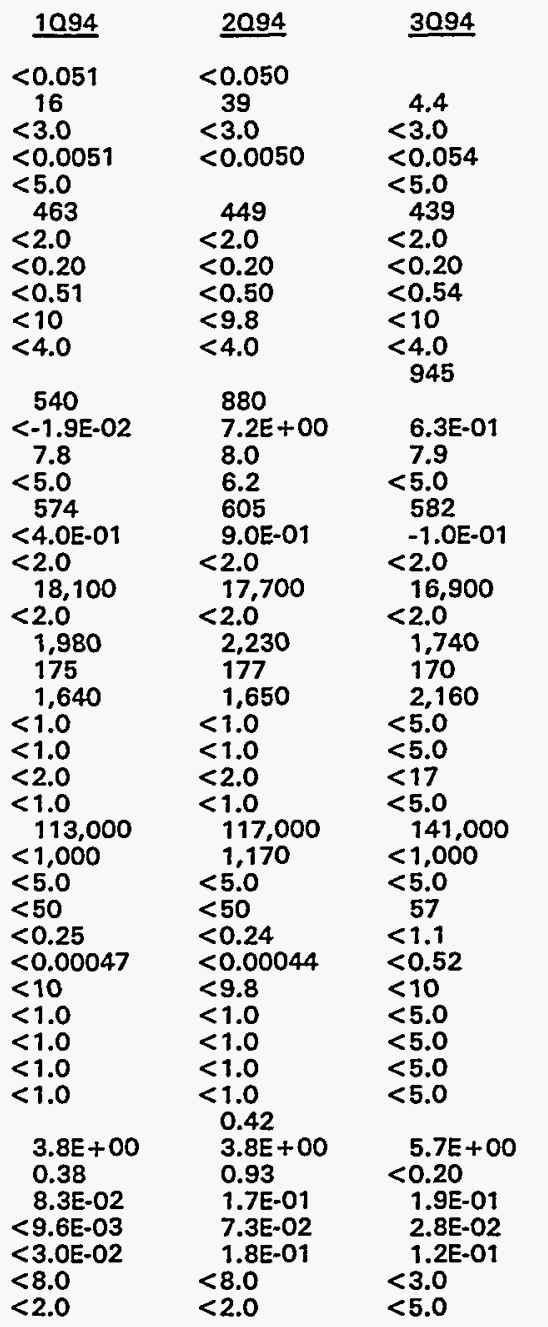

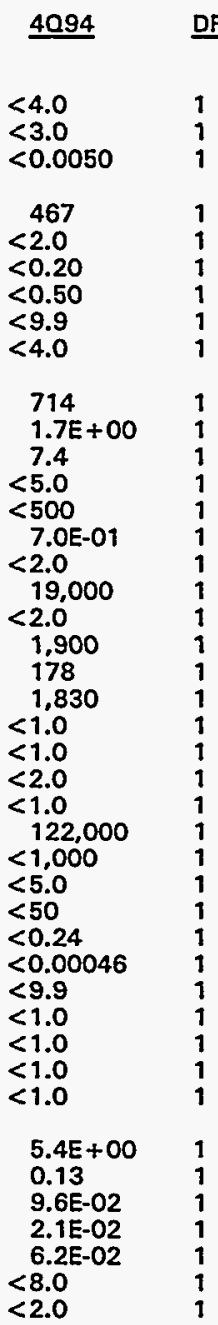

Flag

$\begin{array}{lll}\mu \mathrm{g} / \mathrm{L} & & \\ \mu \mathrm{g} / \mathrm{L} & \mathrm{GE} & 0 \\ \mu \mathrm{g} / \mathrm{L} & \mathrm{GE} & 0 \\ \mu \mathrm{g} / \mathrm{L} & \mathrm{GE} & 0 \\ \mu \mathrm{g} / \mathrm{L} & & \\ \mu \mathrm{g} / \mathrm{L} & \mathrm{GE} & 0 \\ \mu \mathrm{g} / \mathrm{L} & \mathrm{GE} & 0 \\ \mu \mathrm{g} / \mathrm{L} & \mathrm{GE} & 0 \\ \mu \mathrm{g} / \mathrm{L} & \mathrm{GE} & 0 \\ \mu \mathrm{g} / \mathrm{L} & \mathrm{GE} & 0\end{array}$

$\mu \nu, \mu g / L \quad G E \quad 0$

J $\mathrm{pCi} / \mathrm{L}$ GP

J $\mathrm{pH}$ GE

$\mu g / L \quad G E$

$\mu \mathrm{g} / \mathrm{L} \quad \mathrm{GE}$

J

$\mu g / L \quad G E$

$\mu g / L \quad \mathrm{GE}$

$\mu g / L \quad G E$

$\mu \mathrm{S} / \mathrm{cm} \quad \mathrm{GE}$

$\mu g / L \quad G E$

$\mu g / L \quad G E$

$\mu \mathrm{g} / \mathrm{L} \quad \mathrm{GE}$

$\mu \mathrm{g} / \mathrm{L} \quad \mathrm{GE}$

$\mu \mathrm{gIL} \quad \mathrm{GE}$

J

$\mu \mathrm{g} / \mathrm{L}$

$\mu g / L \quad G E$

$\mu g / L$ GE

$\mu g \mathrm{~g} / \mathrm{L} \quad \mathrm{GE}$

$\mu \mathrm{g} / \mathrm{L} \quad \mathrm{GE}$

$\mu g / L \quad G E$

$\mu g / L \quad G E$

$\mu \mathrm{g} / \mathrm{L} \quad \mathrm{GE}$

$\mu \mathrm{g} / \mathrm{L} \quad \mathrm{GE}$

$\mu \mathrm{g} / \mathrm{L}$

pCi/mL GP

NTU GE

PCi/L GP

$\mathrm{pCi} / \mathrm{L}$ GP

PCi/L GP

$\mu \mathrm{g} / \mathrm{L} \quad \mathrm{GE}$

$\mu \mathrm{g} / \mathrm{L}$

Note: Flagging, dilution factors, modifiers, and laboratories are for fourth quarter 1994 data only. See Appendix B for flagging criteria. - = exceeded holding time for fourth quarter 1994.

- = exceeded screening level or final PDWS for fourth quarter 1994. 
WELL BGO 45B

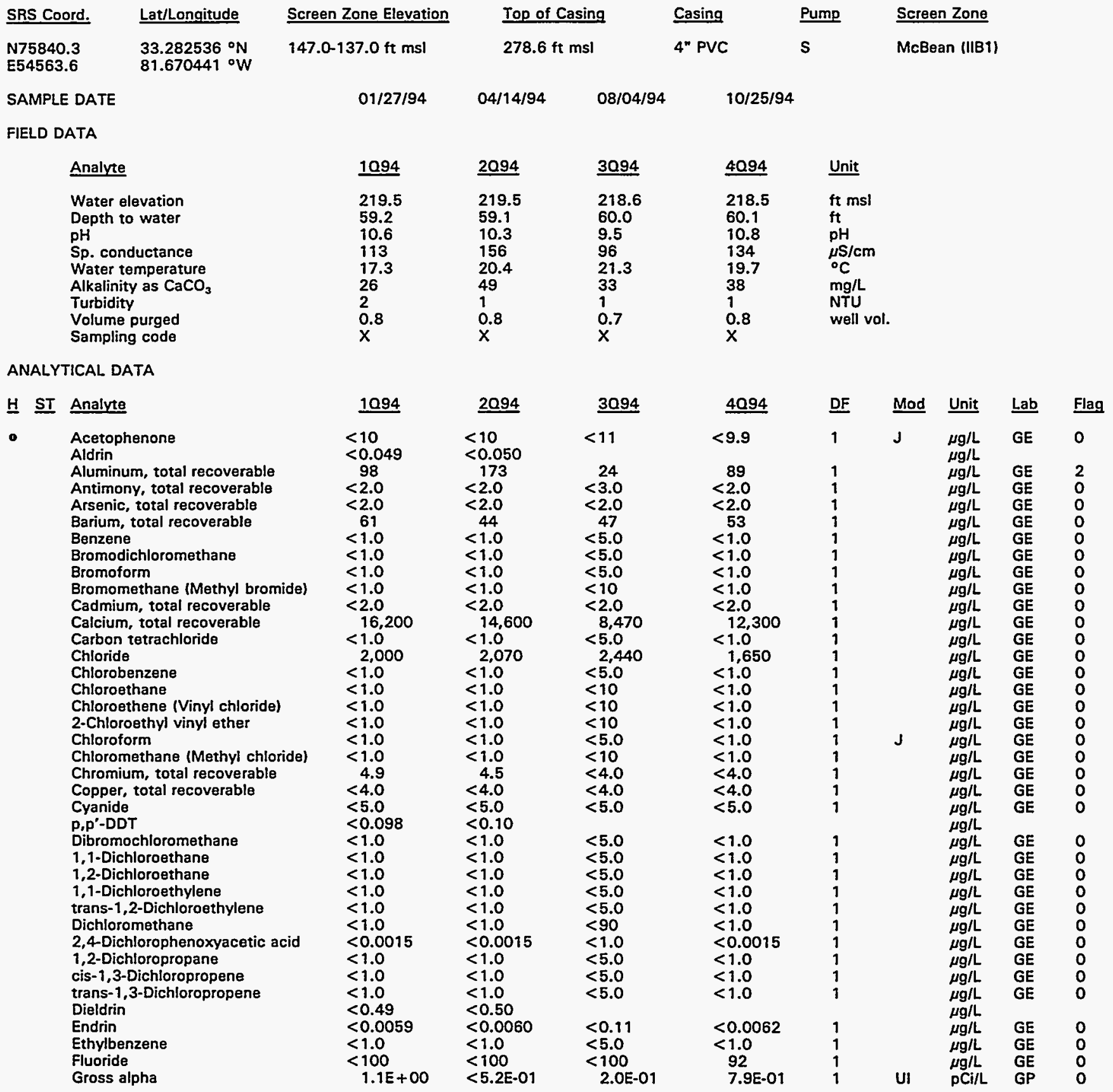

Note: Flagging, dilution factors, modifiers, and laboratories are for fourth quarter 1994 data only. See Appendix B for flagging criteria. - = exceeded holding time for fourth quarter 1994.

w = exceeded screening level or final PDWS for fourth quarter 1994. 


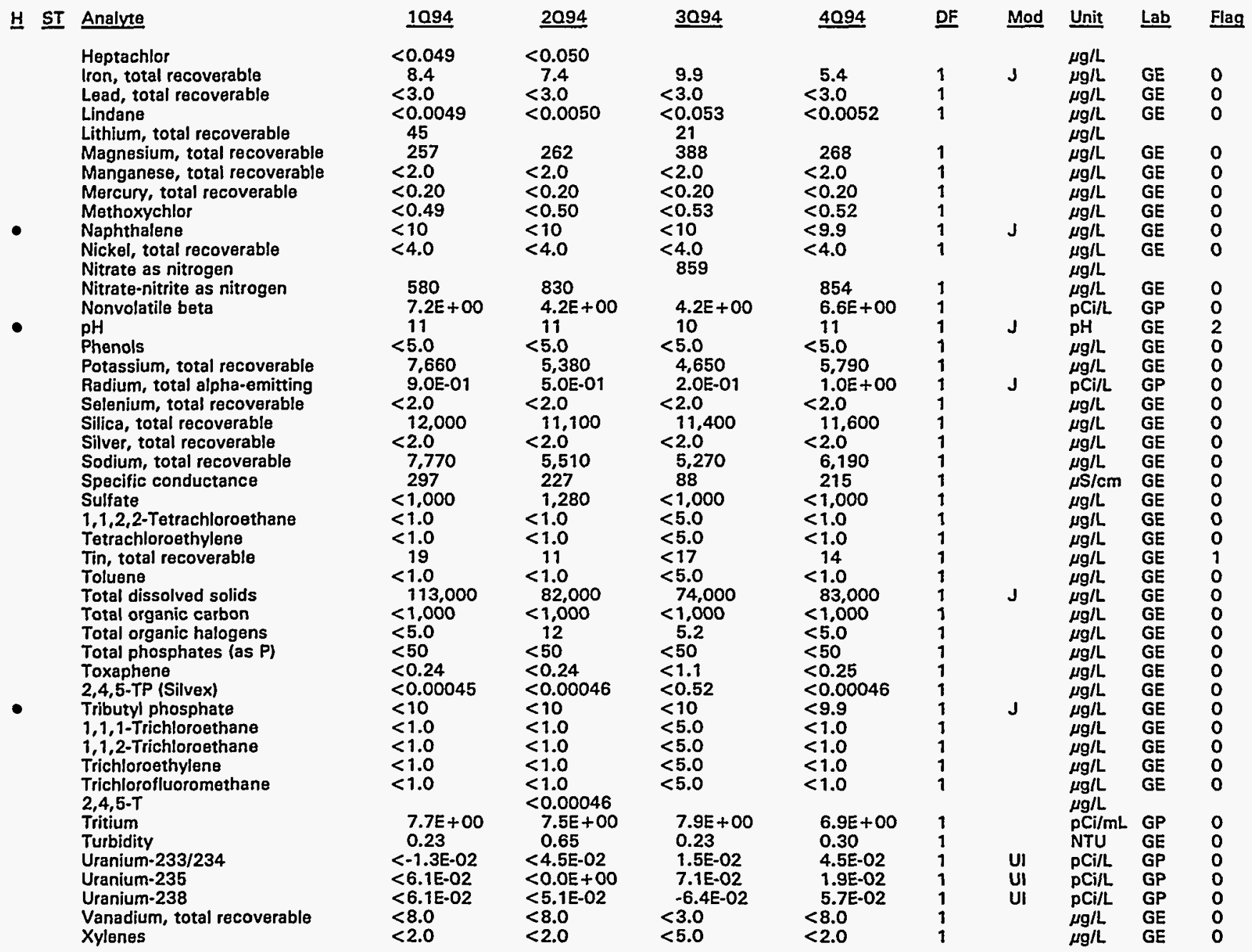

Note: Flagging, dilution factors, modifiers, and laboratories are for fourth quarter 1994 data only. See Appendix B for flagging criteria. - = exceeded holding time for fourth quarter 1994.

- = exceeded screening level or final PDWS for fourth quarter 1994. 
WELL BGO 45C

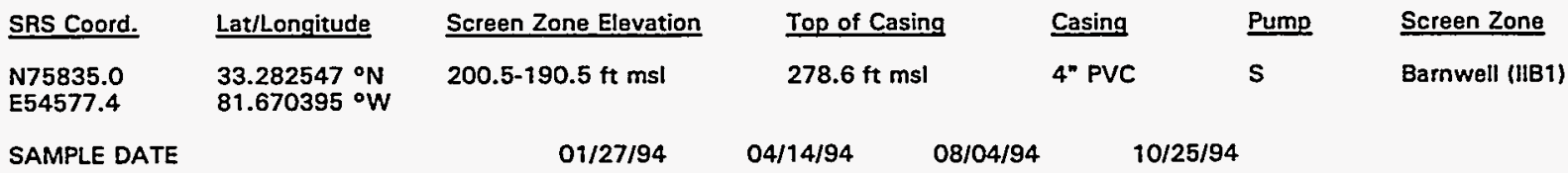

FIELD DATA

Analyte
Water elevation
Depth to water
pH
Sp. conductance
Water temperature
Alkalinity as $\mathrm{CaCO}_{3}$
Turbidity
Volume purged
Sampling code

ANALYTICAL DATA

\section{H ST Analyte}

Acetophenone
Aldrin
Aluminum, total recoverable
Antimony, total recoverable
Arsenic, total recoverable
Barium, total recoverable
Benzene
Bromodichloromethane
Bromoform
Bromomethane (Methyl bromide)
Cadmium, total recoverable
Calcium, total recoverable
Carbon tetrachloride
Chloride
Chlorobenzene
Chloroethane
Chloroethene (Vinyl chloride)
2-Chloroethyl vinyl ether
Chloroform
Chloromethane (Methyl chloride)
Chromium, total recoverable
Copper, total recoverable
Cyanide
p,p'-DDT
Dibromochloromethane
1,1-Dichloroethane
1,2-Dichloroethane
1,1-Dichloroethylene
trans-1,2-Dichloroethylene
Dichloromethane
2,4-Dichlorophenoxyacetic acid
1,2-Dichloropropane
cis-1,3-Dichloropropene
trans-1,3-Dichloropropene
Dieldrin
Endrin
Ethylbenzene
Fluoride
Gross alpha

1094
223.4
55.2
5.5
32
16.9
3
2
0.9
$x$

$\underline{2094}$
223.4
55.2
5.4
46
20.7
4
4
1.0
$x$

1094

$<10$

$<0.050$

55
$<2.0$

$<2.0$

9.4

$<1.0$

$<1.0$

$<1.0$

$<1.0$

$<2.0$

2,060

$<1.0$

2,400

$<1.0$

$<1.0$

$<1.0$

$<1.0$

$<1.0$

$<1.0$

$<4.0$

17

$<5.0$

$<0.10$

$<1.0$

$<1.0$

$<1.0$

$<1.0$

$<0.0016$

$<1.0$

$<1.0$

$<1.0$

$<0.50$

$<0.0060$

$<1.0$

$<100$

$<1.7 E-01$

\section{$\underline{2094}$}

$<10$
$<0.051$

47
$<20$

$<2.0$

8.7

$<1.0$

$<1.0$

$<1.0$

$<1.0$

$<2.0$

2,050

$<1.0$

2,460

$<1.0$

$<1.0$

$<1.0$

$<1.0$

$<1.0$

$<1.0$

$<4.0$

16

$<5.0$

$<0.10$

$<1.0$

$<1.0$

$<1.0$

$<1.0$

$<0.0016$

$<1.0$

$<1.0$

$<1.0$

$<0.51$

$<0.0061$

$<1.0$

$<100$

$<2.3 E-01$

3094
222.6
56.1
5.4
35
20.1
2
2
1.0
$x$

$\underline{4094}$
222.2
56.4
5.6
29
18.9
3
1
1.0
$x$

Unit

ft msl

ft

$\mathrm{pH}$

${ }_{\circ \mathrm{C}} \mathrm{c} / \mathrm{cm}$

$\mathrm{mg} / \mathrm{L}$

NTU

well vol.

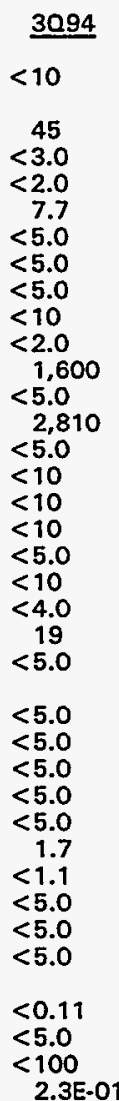

$\begin{array}{ll}\begin{array}{ll}4094 \\ <10\end{array} & \text { DF } \\ <0.050 & 1 \\ 45 & 1 \\ <2.0 & 1 \\ <2.0 & 1 \\ 8.2 & 1 \\ <1.0 & 1 \\ <1.0 & 1 \\ <1.0 & 1 \\ <1.0 & 1 \\ <2.0 & 1 \\ 1.700 & 1 \\ <1.0 & 1 \\ 2.200 & 1 \\ <1.0 & 1 \\ <1.0 & 1 \\ <1.0 & 1 \\ <1.0 & 1 \\ <1.0 & 1 \\ <1.0 & 1 \\ <4.0 & 1 \\ 24 & 1 \\ <5.0 & 1 \\ <0.10 & 1 \\ <1.0 & 1 \\ <1.0 & 1 \\ <1.0 & 1 \\ <1.0 & 1 \\ <1.0 & 1 \\ <1.0 & 1 \\ <0.0015 & 1 \\ <1.0 & 1 \\ <1.0 & 1 \\ <1.0 & 1 \\ <0.50 & 1 \\ <0.0060 & 1 \\ <1.0 & 1 \\ <20 & 1 \\ 3.3 \mathrm{E}-01 & 1 \\ & \end{array}$

DF
1
1
1
1
1
1
1
1
1
1
1
1
1
1
1
1
1
1
1
1
1
1
1
1
1
1
1
1
1
1
1
1
1
1
1
1
1
1

\section{Mod}

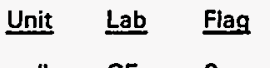

$\mu \mathrm{g} / \mathrm{L}$ GE 0 $\mu \mathrm{g} / \mathrm{L} \quad \mathrm{GE}$ $\mu g / L$ $\mu g / L$ $\mu \mathrm{g} / \mathrm{L}$ $\mu g / L$ $\mu g / L$ $\mu g / L$ $\mu g / L$ $\mu g / L$ $\mu g / L$ $\mu g / L$ $\mu g / L$ $\mu \mathrm{g} / \mathrm{L}$ $\mu \mathrm{g} / \mathrm{L}$ $\mu g / L$ $\mu \mathrm{g} / \mathrm{L}$ $\mu g / L$ $\mu g / L$ $\underset{\mu g / L}{\mu g / L}$ $\mu g / L$ $\mu g / L$ $\mu \mathrm{g} / \mathrm{L}$ $\underset{\mu g / L}{\mu g / L}$ $\mu g / L$ $\mu \mathrm{g} / \mathrm{L}$ $\mu g / L$ $\mu \mathrm{g} / \mathrm{L}$ $\mu \mathrm{g} / \mathrm{L}$ $\mu g / L$ $\mu \mathrm{g} / \mathrm{L}$ $\mu g / L$ $\mu \mathrm{g} / \mathrm{L}$ $\mu \mathrm{g} / \mathrm{L}$

0
0
0
0
0
0
0
0
0
0
0
0
0
0
0
0
0
0
0
0
0
0
0
0
0
0
0
0
0
0
0
0
0
0
0
0
0
0
0
0
0
0
0
0
0

Note: Flagging, dilution factors, modifiers, and laboratories are for fourth quarter 1994 data only. See Appendix B for flagging criteria. 0 = exceeded holding time for fourth quarter 1994.

a = exceeded screening level or final PDWS for fourth quarter 1994. 
Iron total recoverable

Lead, total recoverable

Lindane

Lithium, total recoverable

Magnesium, total recoverable

Manganese, total recoverable

- Mercury, total recoverable

Methoxychlor

Naphthalene

Nickel, total recoverable

Nitrate as nitrogen

Nitrate-nitrite as nitrogen

Nonvolatile beta

pH

Phenols

Potassium, total recoverable

Radium, total alpha-emitting

Selenium, total recoverable

Silica, total recoverable

Silver, total recoverable

Sodium, total recoverable

Specific conductance

Sulfate

1,1,2,2-Tetrachloroethane

Tetrachloroethylens

Tin, total recoverable

Toluene

Total activity

Total dissolved solids

Total organic carbon

Total organic halogens

Total phosphates (as P)

Toxaphene

2,4,5-TP (Silvex)

Tributyl phosphate

1,1,1-Trichloroethane

1,1,2-Trichloroethane

Trichloroethylene

Trichlorofluoromethane

2,4,5-T

- Tritium

Turbidity

Uranium-233/234

Uranium-235

Uranium-238

Vanadium, total recoverable

Xylenes

\begin{tabular}{|c|c|c|}
\hline 10.94 & $\underline{2094}$ & 3094 \\
\hline$<0.050$ & $<0.051$ & \\
\hline 31 & 35 & 70 \\
\hline 17 & 14 & 14 \\
\hline$<0.0050$ & $<0.0051$ & $<0.054$ \\
\hline $\begin{array}{l}6.1 \\
551 \\
16\end{array}$ & $\begin{array}{l}525 \\
14\end{array}$ & $\begin{array}{c}<5.0 \\
482 \\
12\end{array}$ \\
\hline$<0.20$ & $<0.20$ & $<0.20$ \\
\hline$<0.50$ & $<0.51$ & $<0.54$ \\
\hline$<10$ & $<10$ & $<10$ \\
\hline$<4.0$ & $<4.0$ & $\begin{array}{c}<4.0 \\
804\end{array}$ \\
\hline 780 & 830 & \\
\hline $1.5 E+00$ & $<4.1 E-01$ & $1.0 E+00$ \\
\hline $\begin{array}{r}6.4 \\
<5.0\end{array}$ & $\begin{array}{r}6.4 \\
<5.0\end{array}$ & $\begin{array}{r}5.5 \\
<5.0\end{array}$ \\
\hline $\begin{array}{l}1.160 \\
2.9 E+00\end{array}$ & $\begin{array}{l}927 \\
<0.0 E+00\end{array}$ & 553 \\
\hline$<2.0$ & $<2.0$ & $<2.0$ \\
\hline 8,010 & 7,670 & 6,600 \\
\hline$<2.0$ & $<2.0$ & $<2.0$ \\
\hline $\begin{array}{l}2,680 \\
33\end{array}$ & $\begin{array}{l}2,500 \\
32\end{array}$ & $\begin{array}{l}2,300 \\
29\end{array}$ \\
\hline$<1,000$ & $<1,000$ & $<1,000$ \\
\hline$<1.0$ & $<1.0$ & $<5.0$ \\
\hline$<1.0$ & $<1.0$ & $<5.0$ \\
\hline $\begin{array}{r}3.5 \\
<1.0\end{array}$ & $\begin{array}{l}<2.0 \\
<1.0\end{array}$ & $\begin{array}{l}<17 \\
<5.0\end{array}$ \\
\hline & & $\begin{array}{l}5.2 E+05 \\
60,000\end{array}$ \\
\hline $\begin{array}{c}<1,000 \\
8.9\end{array}$ & $\begin{array}{l}<1,000 \\
<5.0\end{array}$ & $\begin{array}{c}<1,000 \\
9.0\end{array}$ \\
\hline $\begin{array}{l}<50 \\
<0.24\end{array}$ & $\begin{array}{l}<50 \\
<0.25\end{array}$ & $\begin{array}{l}<50 \\
<1.1\end{array}$ \\
\hline$<0.00047$ & $<0.00047$ & $<0.53$ \\
\hline $\begin{array}{l}<10 \\
<1.0\end{array}$ & $\begin{array}{l}<10 \\
<1.0\end{array}$ & $\begin{array}{l}<10 \\
<5.0\end{array}$ \\
\hline$<1.0$ & $<1.0$ & $<5.0$ \\
\hline$<1.0$ & $<1.0$ & $<5.0$ \\
\hline$<1.0$ & $<1.0$ & $<5.0$ \\
\hline & $<0.00047$ & \\
\hline $\begin{array}{l}. .4 E+02 \\
2.0\end{array}$ & 1.6 & $\begin{array}{l}5.6 \mathrm{E}+02 \\
1.7\end{array}$ \\
\hline 6.3E-02 & $<4.1 E-02$ & 5.0E-01 \\
\hline $\begin{array}{l}<8.7 E-03 \\
<5.4 \mathrm{E}-02\end{array}$ & $<-3.9 E-03$ & 2.3E-02 \\
\hline$<8.0$ & $<8.0$ & $<3.0$ \\
\hline$<2.0$ & $<2.0$ & $<5.0$ \\
\hline
\end{tabular}

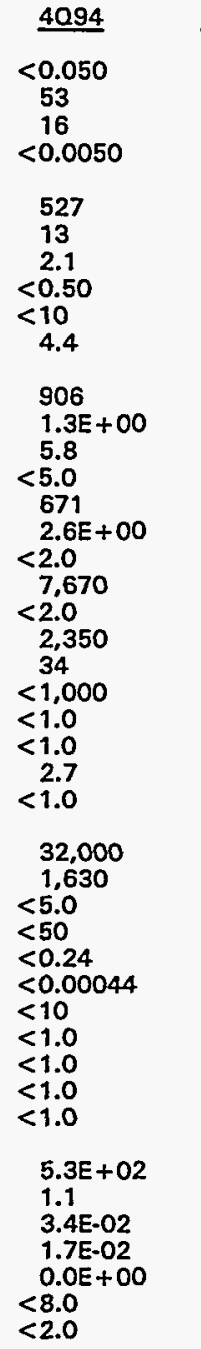

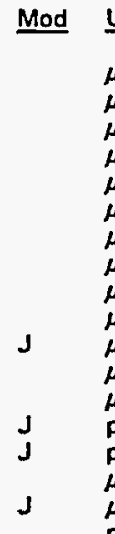

\begin{tabular}{lll} 
Unit & Lab & Flag \\
\hline$\mu g / L$ & GE & 0 \\
$\mu g / L$ & $G E$ & 0 \\
$\mu g / L$ & $G E$ & 0 \\
$\mu g / L$ & $G E$ & 0
\end{tabular}

$\mu \mathrm{g} / \mathrm{L} \quad \mathrm{GE}$

酸

$\mu g / L$

$\mu g / L$

$\mu \mathrm{g} / \mathrm{L} \quad \mathrm{GE}$

$\mu g / L \quad G E$

$\mu \mathrm{g} / \mathrm{L} \quad \mathrm{GE}$

$\mu g / L$

$\mu \mathrm{g} / \mathrm{L} \quad \mathrm{GE}$

PCi/L GP

pH GE

$\mu \mathrm{g} / \mathrm{L}$

$\mu g / L \quad G E$

PCi/L GP

$\mu g / L \quad G E$

$\mu g / L \quad G E$

$\mu g / L \quad G E$

$\mu g / L \quad G E$

$\mu \mathrm{g} / \mathrm{L} \quad \mathrm{GE}$

$\mu \mathrm{g} / \mathrm{L}$

$\mu g / L$

$J \quad \mu g / L \quad G E$

$\mu g / L$

pCi/h

$J$

$\mu \mathrm{g} / \mathrm{L}$

Mg/L GE

$\mu \mathrm{g} / \mathrm{L} \quad \mathrm{GE}$

$\mu \mathrm{g} / \mathrm{L} \quad \mathrm{GE}$

$\mu g / L \quad G E$

$\mu g / L \quad G E$

$\mu \mathrm{g} / \mathrm{L} \quad \mathrm{GE}$

$\mu g / L \quad G E$

$\mu g / L \quad G E$

$\mu g / L \quad G E$

$\mu \mathrm{g} / \mathrm{L}$

$\mathrm{pCi} / \mathrm{mL}$ GP

NTU GE

UI $p C i / L$ GP

UI

pCi/L

$\mathrm{pCi} / \mathrm{L}$

$\mu g / L$

$$
0
$$

0

2

0

o

0

0
0

0

0

0
0

0

0

0
0

o

0
0

0

0

0

0

0
0

2

2

0

o

○

Note: Flagging, dilution factors, modifiers, and laboratories are for fourth quarter 1994 data only. See Appendix B for flagging criteria.

- = exceeded holding time for fourth quarter 1994.

a = exceeded screening level or final PDWS for fourth quarter 1994. 
WELL BGO 45D

\begin{tabular}{|c|c|c|c|c|c|c|}
\hline SRS Coord. & Lat/Longitude & Screen Zone Elevation & Top of Casing & Casing & Pump & Screen Zone \\
\hline $\begin{array}{l}\text { N75854.3 } \\
\text { E54585.6 }\end{array}$ & $\begin{array}{l}33.282603{ }^{\circ} \mathrm{N} \\
81.670411^{\circ} \mathrm{W}\end{array}$ & $229.6-209.6 \mathrm{ft} \mathrm{msl}$ & $278.6 \mathrm{ft} \mathrm{ms!}$ & $4^{n}$ PVC & $s$ & Water $T$ \\
\hline SAMPLE DA & & $01 / 26 / 94$ & $04 / 06 / 94$ & & & \\
\hline
\end{tabular}

FIELD DATA

Analyte

Water elevation

Depth to water

$\mathrm{pH}$

$\mathrm{Sp}$. conductance

Water temperature

Alkalinity as $\mathrm{CaCO}_{3}$

Turbidity

Volume purged

Sampling code

\section{ANALYTICAL DATA}

\section{H $\underline{\text { ST Analyte }}$}

Acetophenone

Aldrin

Aluminum, total recoverable

Antimony, total recoverable

Arsenic, total recoverable

Barium, total recoverable

Benzene

Bromadichloromethane

Bromaform

Bromomethane (Methyl bromide)

Cadmium, total recoverable

Calcium, total recoverable

Carbon tetrachloride

Chloride

Chlorobenzene

Chloroethane

Chloroethene (Vinyl chloride)

2-Chloroethyl vinyl ether

Chloroform

Chloromethane (Methyl chloride)

Chromium, total recoverable

Copper, total recoverable

Cyanide

p,p'-DDT

Dibromochloromethane

1.1-Dichloroethane

1.2-Dichloroethane

1,1-Dichloroethylene

trans-1,2-Dichloroethylene

Dichloromethane

2,4-Dichlorophenoxyacetic acid

1,2-Dichloropropane

cis-1,3-Dichloropropene

trans-1,3-Dichloropropene

Dieldrin

Endrin

Ethylbenzene

Fluoride

Gross alpha

$\begin{array}{lll}1094 & \underline{2094} & \text { 3094 } \\ 227.8 & 228.1 & 227.3 \\ 50.8 & 50.5 & 51.3 \\ 4.9 & 5.3 & 5.0 \\ 26 & 35 & 28 \\ 18.7 & 20.1 & 19.2 \\ 0 & 1 & 0 \\ 1 & 1 & 1 \\ 8.9 & 2.7 & 11.0\end{array}$

\section{4}

$\frac{4094}{<10}$

$<10$
$<0.051$

27
$<2.0$

$<2.0$
$<2.0$

$<2.0$
8.3

$<1.0$

$<1.0$

$<1.0$

$<1.0$

$<2.0$

840

$<1.0$

1,990

$<1.0$

$<1.0$

$<1.0$

$<1.0$

$<1.0$

$<4.0$

11

$<5.0$

$<0.10$

$<1.0$

$<1.0$

$<1.0$

$<1.0$

$<1.0$

$<1.0$

$<0.0015$

$<1.0$

$<1.0$

$<1.0$

$<0.51$

$<0.0061$

$<1.0$

$<100$

$1.1 E+00$
$<11$

45
$<3.0$

0.05

44
$<2.0$

$<2.0$

9.8

$<1.0$

$<1.0$

$<1.0$

$<1.0$

1,760

$<1.0$

2,140

$<1.0$

$<1.0$

$<1.0$

$<1.0$

$<1.0$

$<1.0$

$<4.0$

$<4.0$

$<5.0$

$<0.10$

$<1.0$

$<1.0$

$<1.0$

$<1.0$

$<1.0$

$<1.0$

$<1.5$

$<1.0$

$<1.0$

$<1.0$

$<0.51$

$<0.0062$

$<1.0$

$<100$

$<8.8 \mathrm{E}-01$

$<4.0$
$<5.0$

$<5.0$

$<5.0$

$<5.0$

$<5.0$

$<5.0$

$<34$

$<1.1$

$<5.0$

$<5.0$

$<5.0$

$<5.0$
27
$<2.0$

$<2.0$
$<2.0$

$<2.0$

$<1.0$

$<1.0$

$<1.0$

$<1.0$

$<2.0$

1,110

$<1.0$

1,820

$<1.0$

$<1.0$

$<1.0$

$<1.0$

$<1.0$

$<1.0$

$<4.0$

$<4.0$

$<4.0$
$<5.0$

$<0.11$

$<100$

8.1E-01
$<1.0$

$<1.0$

$<1.0$

$<1.0$

$<1.0$

$<1.0$

$<0.0015$

$<1.0$

$<1.0$

$<1.0$

$<0.0062$

$<1.0$

$<20$

$1.4 E+00$

4094
226.9
51.7
5.2
25
19.4
0
1
7.3

Unit

ft mst

tt

$\mathrm{pH}$

${ }^{\mu} \mathrm{C} / \mathrm{cm}$

mg/L

well vol.

\begin{tabular}{|c|c|c|c|c|}
\hline DF & Mod & Unit & Lab & Flac \\
\hline 1 & & $\begin{array}{l}\mu g / L \\
\mu g / L\end{array}$ & GE & 0 \\
\hline 1 & $\mathbf{J}$ & $\mu \mathrm{g} / \mathrm{L}$ & GE & 1 \\
\hline 1 & & $\mu g / L$ & GE & 0 \\
\hline 1 & & $\mu g / L$ & GE & 0 \\
\hline 1 & & $\mu g / L$ & GE & 0 \\
\hline 1 & & $\mu \mathrm{g} / \mathrm{L}$ & GE & 0 \\
\hline 1 & & $\mu g / L$ & GE & 0 \\
\hline 1 & & $\mu g / L$ & GE & 0 \\
\hline 1 & & $\mu \mathrm{g} / \mathrm{L}$ & GE & 0 \\
\hline 1 & & $\mu g / L$ & GE & 0 \\
\hline 1 & & $\mu g / L$ & GE & 0 \\
\hline 1 & & $\mu g / L$ & GE & 0 \\
\hline 1 & & $\mu \mathrm{g} / \mathrm{L}$ & GE & 0 \\
\hline 1 & & $\mu g / L$ & GE & 0 \\
\hline 1 & & $\mu g / L$ & GE & 0 \\
\hline 1 & & $\mu g / L$ & GE & 0 \\
\hline 1 & & $\mu g / L$ & GE & 0 \\
\hline 1 & & $\mu g / L$ & GE & 0 \\
\hline$i$ & & $\mu g h$ & 단 & 0 \\
\hline$i$ & & ga/L & GF & 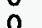 \\
\hline 1 & & $\mu g / L$ & GE & 0 \\
\hline 1 & & II & $G E$ & 0 \\
\hline 1 & & $\mu \mathrm{g} / \mathrm{L}$ & GE & 0 \\
\hline 1 & & $\mu \mathrm{g} / \mathrm{L}$ & GE & 0 \\
\hline 1 & & $\mu g / L$ & GE & 0 \\
\hline 1 & & $\mu \mathrm{g} / \mathrm{L}$ & GE & 0 \\
\hline 1 & & $\mu g / L$ & GE & 0 \\
\hline 1 & & $\mu g / L$ & GE & 0 \\
\hline 1 & & $\mu g / L$ & GE & 0 \\
\hline 1 & & $\mu \mathrm{g} / \mathrm{L}$ & GE & 0 \\
\hline 1 & & $\begin{array}{l}\mu g / L \\
\mu g / L\end{array}$ & $\mathrm{GE}$ & 0 \\
\hline 1 & & $\mu g / L$ & GE & 0 \\
\hline 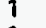 & & $\mu g / L$ & GE & 0 \\
\hline$\because$ & & $\mu g / L$ & GE & 0 \\
\hline 1 & $J$ & $\mathrm{pCi} / \mathrm{L}$ & GP & 0 \\
\hline
\end{tabular}

Note: Flagging, dilution factors, modifiers, and laboratories are for fourth quarter 1994 data only. See Appendix B for flagging criteria. $-=$ exceeded holding time for fourth quarter 1994.

a = exceeded screening level or final PDWS for fourth quarter 1994. 


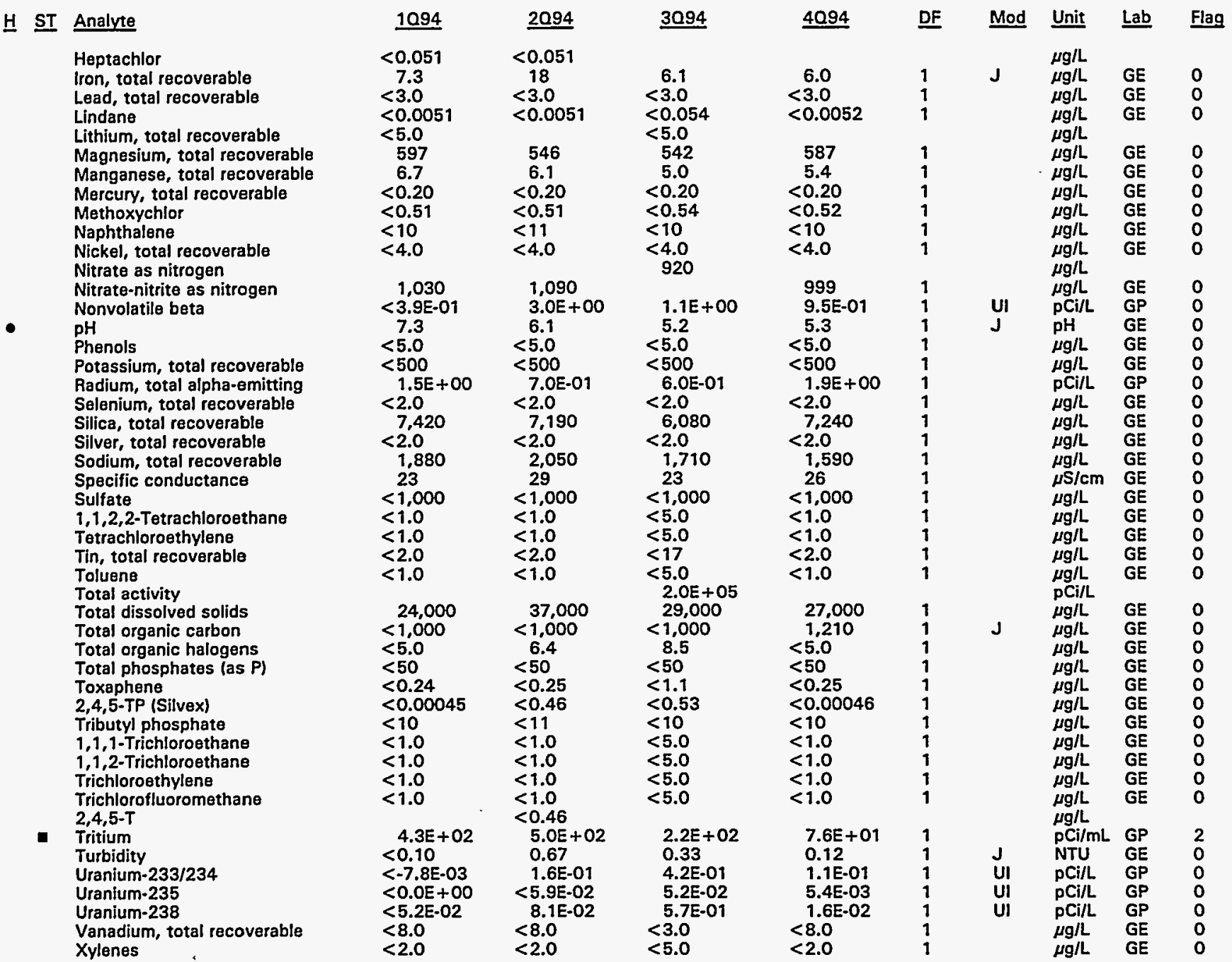

Note: Flagging, dilution factors, modifiers, and laboratories are for fourth quarter 1994 data only. See Appendix B for flagging criteria. - = exceeded holding time for fourth quarter 1994.

I = exceeded screening level or final PDWS for fourth quarter 1994. 
WELL BGO 46B

\begin{tabular}{|c|c|c|c|c|c|c|c|c|c|c|}
\hline \multirow{2}{*}{$\begin{array}{l}\text { SRS Coord. } \\
\text { N75012.1 } \\
\text { E54444.7 }\end{array}$} & Lat/Longitude & Screen Zone Elevation & \multicolumn{2}{|c|}{ Top of Casing } & Casing & Pump & \multicolumn{4}{|c|}{ Screen Zone } \\
\hline & $\begin{array}{l}33.280511^{\circ} \mathrm{N} \\
81.669146^{\circ} \mathrm{W}\end{array}$ & $150.4-140.4 \mathrm{ft} \mathrm{msl}$ & 265.4 & & 4" PVC & s & $\mathrm{McBe}$ & an (IIB1) & & \\
\hline \multicolumn{2}{|c|}{ SAMPLE DATE } & $02 / 21 / 94$ & $04 / 12 / 94$ & $08 / 12 / 94$ & $10 / 27 / 94$ & & & & & \\
\hline \multicolumn{11}{|c|}{ FIELD DATA } \\
\hline & Analyte & 1094 & $\underline{2094}$ & 3094 & 4094 & $\underline{\text { Unit }}$ & & & & \\
\hline & $\begin{array}{l}\text { Water elevation } \\
\text { Depth to water } \\
\text { pH } \\
\text { Sp. conductance } \\
\text { Water temperature } \\
\text { Alkalinity as } \mathrm{CaCO}_{3} \\
\text { Turbidity } \\
\text { Volume purged } \\
\text { Sampling code }\end{array}$ & $\begin{array}{l}217.8 \\
47.6 \\
6.4 \\
59 \\
19.7 \\
18 \\
1 \\
2.7\end{array}$ & $\begin{array}{l}218.0 \\
47.4 \\
6.3 \\
64 \\
20.1 \\
16 \\
0 \\
6.6\end{array}$ & $\begin{array}{l}217.3 \\
48.1 \\
\\
P\end{array}$ & $\begin{array}{l}217.1 \\
48.3 \\
P\end{array}$ & $\begin{array}{l}\mathrm{ft} \mathrm{ms} ! \\
\mathrm{ft} \\
\mathrm{pH} \\
\mu \mathrm{S} / \mathrm{cm} \\
{ }^{\circ} \mathrm{C} \\
\mathrm{mg} / \mathrm{L} \\
\text { NTU } \\
\text { well vol. }\end{array}$ & & & & \\
\hline \multicolumn{11}{|c|}{ ANALYTICAL DATA } \\
\hline \multirow[t]{2}{*}{ H $\underline{\text { ST }}$} & Analyte & 1094 & $\underline{2094}$ & $\underline{3094}$ & $\underline{4094}$ & DF & Mod & $\underline{\text { Unit }}$ & $\underline{\text { Lab }}$ & Flag \\
\hline & $\begin{array}{l}\text { Acetophenone } \\
\text { Aldrin } \\
\text { Aluminum, total recoverable } \\
\text { Antimony, total recoverable } \\
\text { Arsenic, total recoverable } \\
\text { Barium, total recoverable } \\
\text { Benzene } \\
\text { Bromodichloromethane } \\
\text { Bromoform } \\
\text { Bromomethane (Methyl bromide) } \\
\text { Cadmium, total recoverable } \\
\text { Calcium, total recoverable } \\
\text { Carbon tetrachloride } \\
\text { Chloride } \\
\text { Chlorobenzene } \\
\text { Chloroethane } \\
\text { Chloroethene (Vinyl chloride) } \\
\text { 2-Chloroethyl vinyl ether } \\
\text { Chloroform } \\
\text { Chloromethane (Methyl chloride) } \\
\text { Chromium, total recoverable } \\
\text { Copper, total recoverable } \\
\text { Cyanide } \\
\text { p,p'-DDT } \\
\text { Dibromochloromethane } \\
\text { 1,1-Dichloroethane } \\
\text { 1,2-Dichloroethane } \\
\text { i,1-Dichloroethylene } \\
\text { trans-1,2-Dichloroethylene } \\
\text { Dichloromethane } \\
\text { 2,4-Dichlorophenoxyacetic acid } \\
1,2-\text { Dichloropropane } \\
\text { cis-1,3-Dichloropropene } \\
\text { irans-1,3-Dichloropropene } \\
\text { ieldrin } \\
\text { Endrin } \\
\text { Ethylbenzene } \\
\text {-luoride } \\
\text { Gross alpha }\end{array}$ & $\begin{array}{l}<10 \\
<0.051 \\
<20 \\
<2.0 \\
<2.0 \\
3.3 \\
<1.0 \\
<1.0 \\
<1.0 \\
<1.0 \\
<2.0 \\
<8.080 \\
<1.0 \\
<1.990 \\
<1.0 \\
<1.0 \\
<1.0 \\
<1.0 \\
<1.0 \\
<1.0 \\
<4.0 \\
<4.0 \\
<5.0 \\
<0.10 \\
<1.0 \\
<1.0 \\
<1.0 \\
<1.0 \\
<1.0 \\
<1.0 \\
<0.0015 \\
<1.0 \\
<1.0 \\
<1.0 \\
<0.51 \\
<0.0061 \\
<1.0 \\
<4.2 \mathrm{E}-01\end{array}$ & $\begin{array}{l}<10 \\
<0.051 \\
21 \\
<2.0 \\
<2.0 \\
<3.0 \\
<1.0 \\
<1.0 \\
<1.0 \\
<1.0 \\
<2.0 \\
9.120 \\
<1.0 \\
<1.030 \\
<1.0 \\
<1.0 \\
<1.0 \\
<1.0 \\
<1.0 \\
<1.0 \\
<4.0 \\
<4.0 \\
<5.0 \\
<0.10 \\
<1.0 \\
<1.0 \\
<1.0 \\
<1.0 \\
<1.0 \\
<1.0 \\
<0.0016 \\
<1.0 \\
<1.0 \\
<1.0 \\
<0.51 \\
<0.0061 \\
<1.0 \\
157 \\
1.2 E+00\end{array}$ & & & & & $\begin{array}{l}\mu g / L \\
\mu g / L \\
\mu g / L \\
\mu g / L \\
\mu g / L \\
\mu g / L \\
\mu g / L \\
\mu g / L \\
\mu g / L \\
\mu g / L \\
\mu g / L \\
\mu g / L \\
\mu g / L \\
\mu g / L \\
\mu g / L \\
\mu g / L \\
\mu g / L \\
\mu g / L \\
\mu g / L \\
\mu g / L \\
\mu g / L \\
\mu g / L \\
\mu g / L \\
\mu g / L \\
\mu g / L \\
\mu g / L \\
\mu g / L \\
\mu g / L \\
\mu g / L \\
\mu g / L \\
\mu g / L \\
\mu g / L \\
\mu g / L \\
\mu g / L \\
\mu g / L \\
\mu g / L \\
\mu g / L \\
\mu g / L \\
\mu g / L \\
p C i / L\end{array}$ & & \\
\hline
\end{tabular}
Note: Flagging, dilution factors, modifiers, and laboratories are for fourth quarter 1994 data only. See Appendix B for flagging criteria.
$-=$ exceeded holding time for fourth quarter 1994 .

- = exceeded screening level or final PDWS for fourth quarter 1994. 
Heptachlor

Iron, total recoverable

Lead, total recoverable

Lindane

Lithium, total recoverable

Magnesium, total recoverable

Manganese, total recoverable

Mercury, total recoverable

Methoxychlor

Naphthalene

Nickel, total recoverable

Nitrate as nitrogen

Nitrate-nitrite as nitrogen

Nonvolatile beta

$\mathrm{pH}$

Phenols

Potassium, total recoverable

Radium, total alpha-emitting

Radium-226

Radium-228

Selenium, total recoverable

Silica, total recoverable

Silver, total recoverable

Sodium, total recoverable

Specific conductance

Sulfate

1,1,2,2-Tetrachloroethane

Tetrachloroethylene

Tin, total recoverable

Toluene

Total dissolved solids

Total organic carbon

Total organic halogens

Total phosphates (as P)

Toxaphene

2,4,5-TP (Silvex)

Tributyl phosphate

1,1,1-Trichloroethane

1,1,2-Trichloroethane

Trichloroethylene

Trichlorofluoromethane

2,4,5-T

Tritium

Turbidity

Uranium-233/234

Uranium-235

Uranium-238

Vanadium, total recoverable

Xylenes

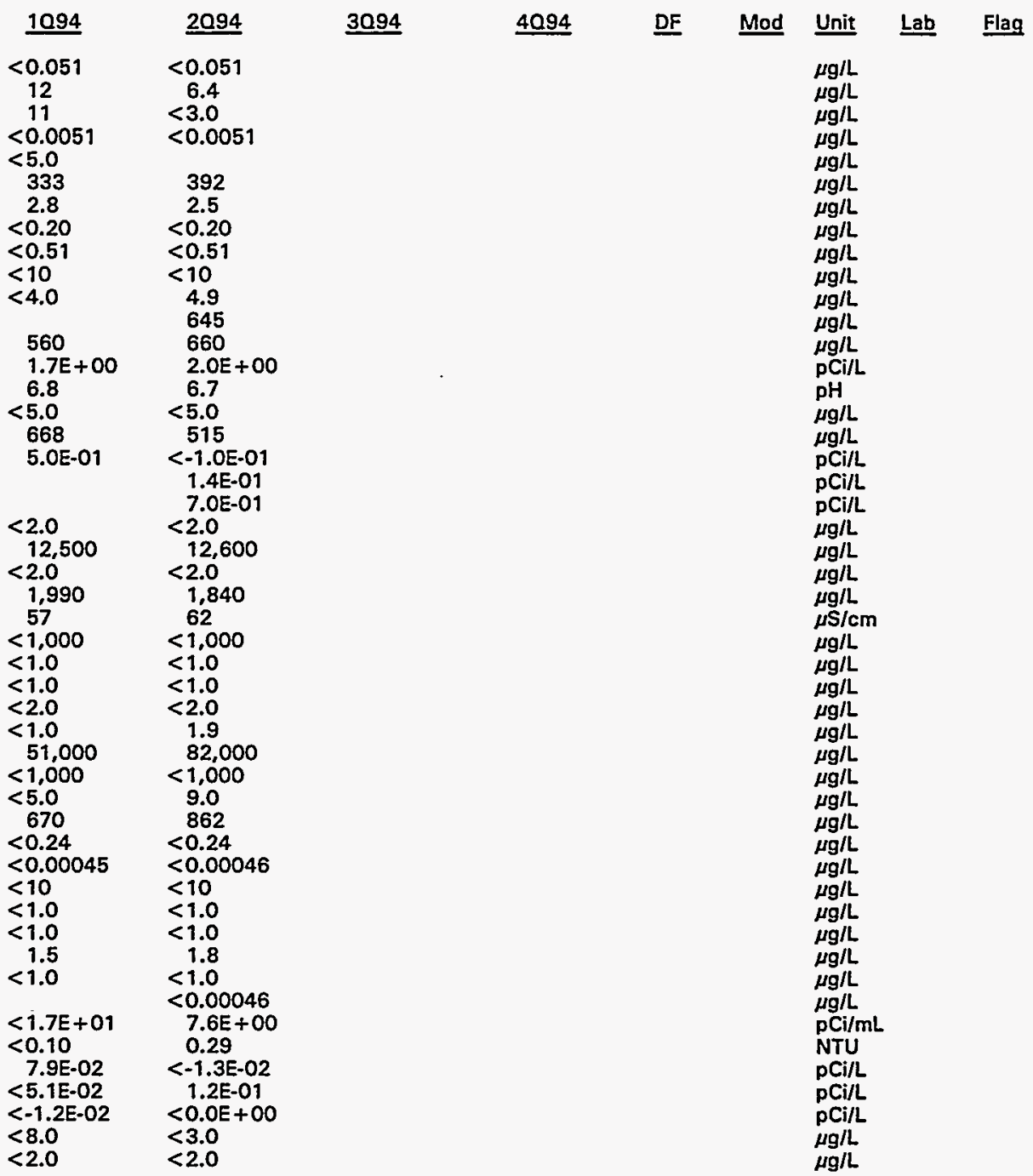

Note: Flagging, dilution factors, modifiers, and laboratories are for fourth quarter 1994 data only. See Appendix B for flagging criteria. - = exceeded holding time for fourth quarter 1994.

- = exceeded screening level or final PDWS for fourth quarter 1994. 
WELL BGO 46C

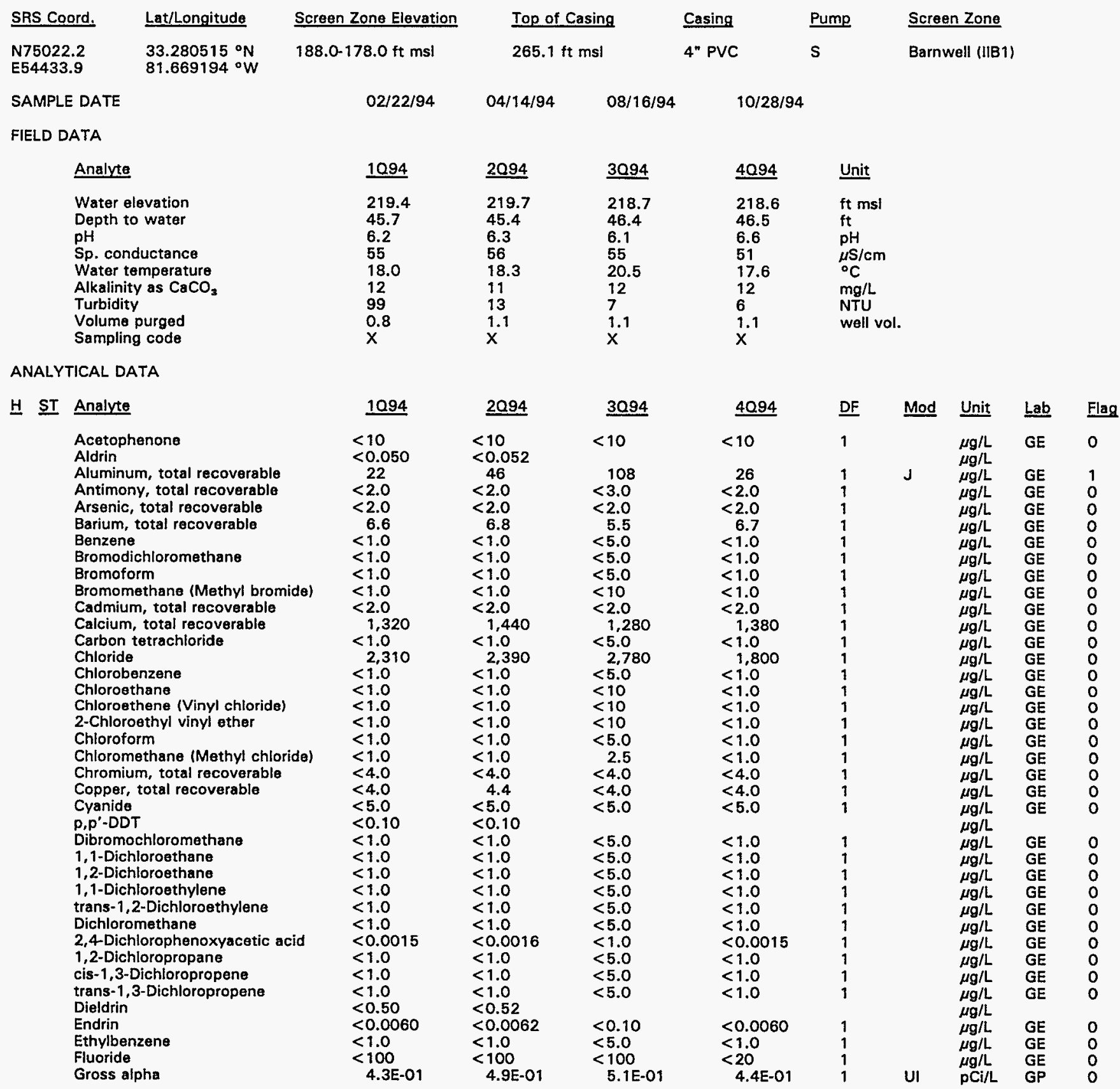

Note: Flagging, dilution factors, modifiers, and laboratories are for fourth quarter 1994 data only. See Appendix B for flagging criteria. - = exceeded holding time for fourth quarter 1994.

n = exceeded screening level or final PDWS for fourth quarter 1994. 


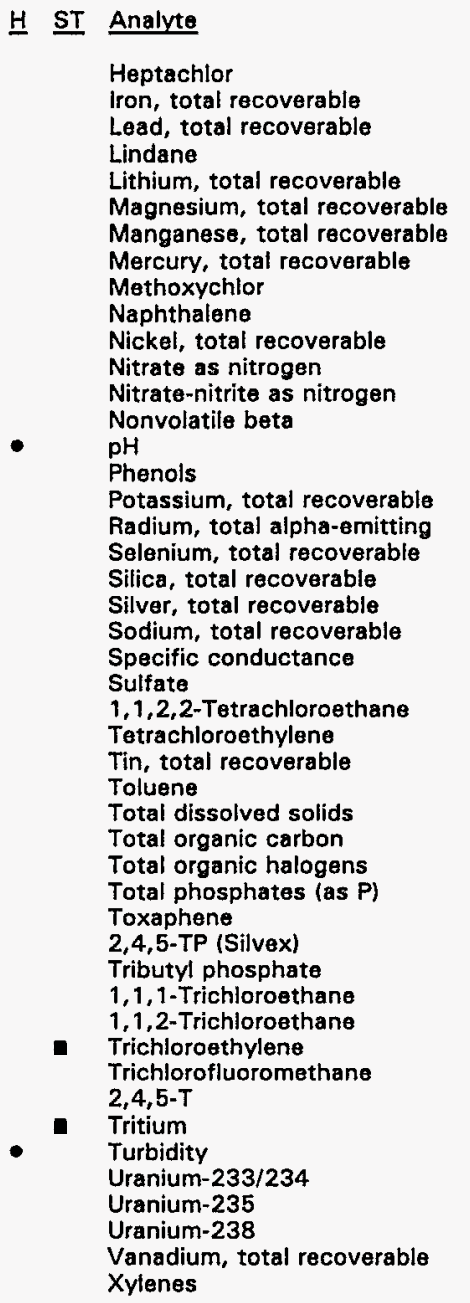

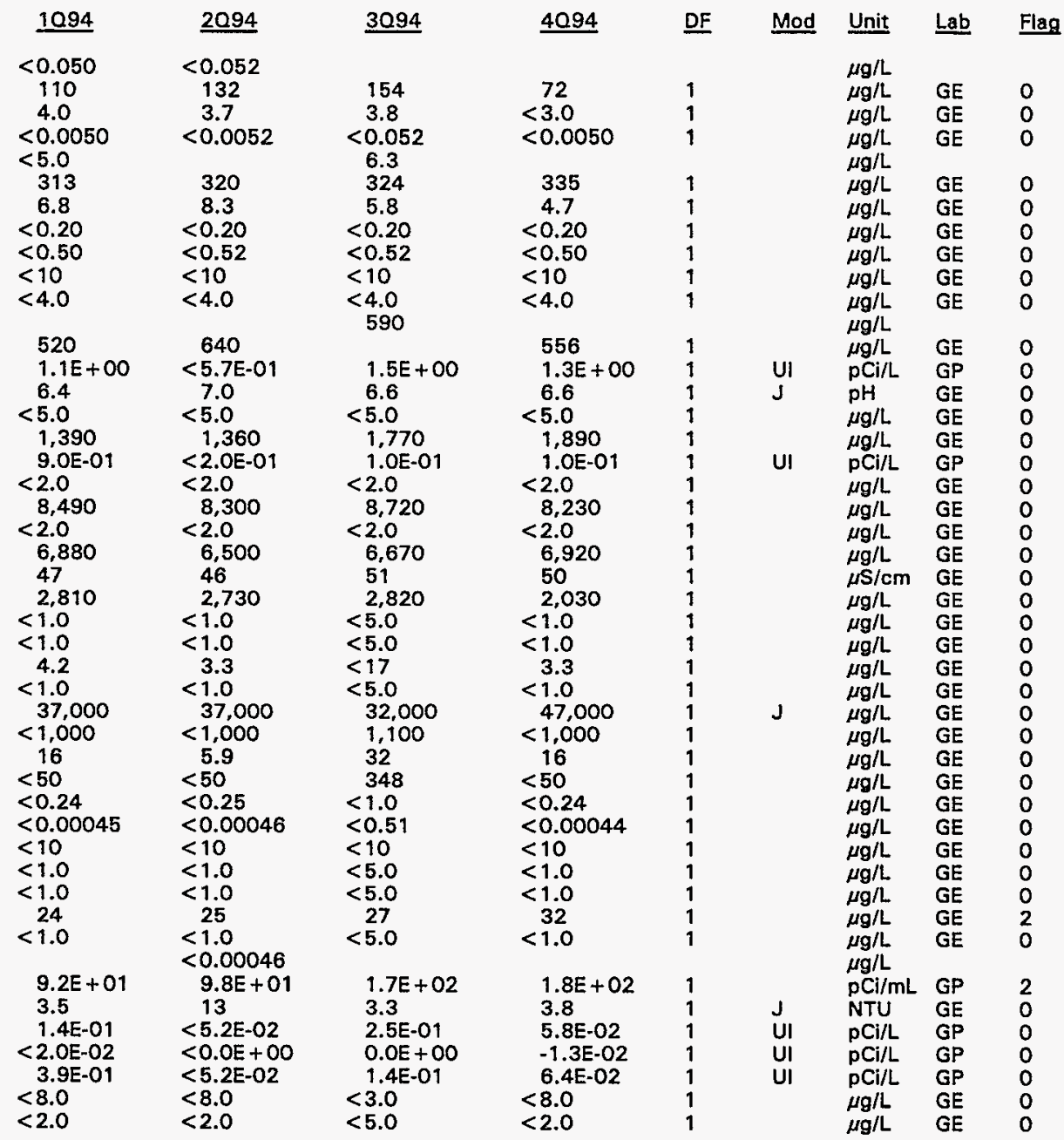

Note: Flagging, dilution factors, modifiers, and laboratories are for fourth quarter 1994 data only. See Appendix B for flagging criteria. - = exceeded holding time for fourth quarter 1994

n = exceeded screening level or final PDWS for fourth quarter 1994. 


\section{WELL BGO 46D}

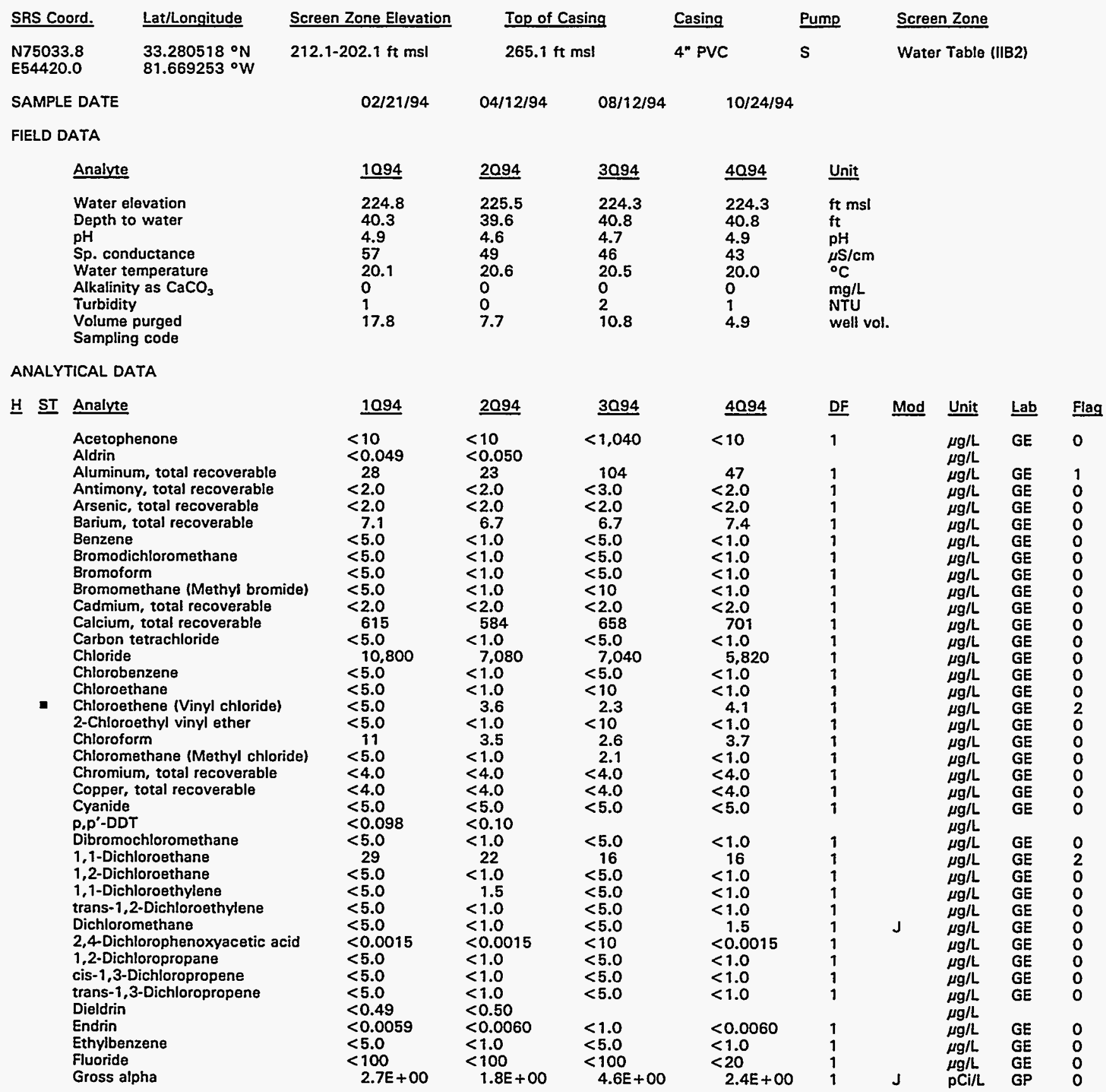

Note: Flagging, dilution factors, modifiers, and laboratories are for fourth quarter 1994 data only. See Appendix B for flagging criteria. - = exceeded holding time for fourth quarter 1994.

- = exceeded screening level or final PDWS for fourth quarter 1994. 


\section{H $\underline{\text { ST Analyte }}$}

Heptachlor

Iron, total recoverable

Lead, total recoverable

Lindane

Lithium, total recoverable

Magnesium, total recoverable

Manganese, total recoverable

Mercury, total recoverable

Methoxychlor

Nickel, total recoverable

Nickel, total recove
Nitrate as nitrogen

Nitrate-nitrite as nitrogen

Nonvolatile beta

pH

Phenols

Potassium, total recoverable

Radium, total alpha-emitting

Selenium, total recoverable

Silica, total recoverable

Silver, total recoverable

Sodium, total recoverable

Specific conductance

Sulfate

$1,1,2,2$-Tetrachloroethane

- Tetrachloroethylene

Tin, total recoverable

Toluene

Total activity

Total dissolved solids

Total organic carbon

Total organic halogens

Total phosphates (as P)

Toxaphene

2,4,5-TP (Silvex)

Tributyl phosphate

1,1,1-Trichloroethane

1,1,2-Trichloroethane

- Trichloroethylene

Trichlorofluoromethane

2,4,5-T

- Tritium

Turbidity

Uranium-233/234

Uranium-235

Uranium-238

Vanadium, total recoverable

Xylenes

\begin{tabular}{|c|c|}
\hline 1094 & 2094 \\
\hline $\begin{array}{l}<0.049 \\
5.1 \\
<3.0 \\
<0.0049\end{array}$ & $\begin{aligned}< & 0.050 \\
& 5.3 \\
< & 3.0 \\
< & 0.0050\end{aligned}$ \\
\hline $\begin{array}{l}<5.0 \\
428 \\
17 \\
0.71 \\
<0.49 \\
<10 \\
<4.0\end{array}$ & $\begin{array}{l}429 \\
17 \\
0.31 \\
<0.50 \\
<10 \\
<4.0\end{array}$ \\
\hline $\begin{aligned} & 900 \\
& 3.3 \mathrm{EE}+00 \\
& 5.1 \\
&< 5.0 \\
&< 500 \\
& 1.7 \mathrm{E}+00 \\
&< 2.0 \\
& 7,870 \\
&<2.0 \\
& 7,800 \\
& 56 \\
&< 1,000 \\
&< 5.0 \\
& 6.0 \\
&<2.0 \\
&<5.0\end{aligned}$ & $\begin{aligned} & 780 \\
& 1.1 E+00 \\
& 5.1 \\
&<5.0 \\
&<500 \\
& 9.0 \mathrm{E}-01 \\
&<2.0 \\
& 7.760 \\
&<2.0 \\
& 5,890 \\
& 43 \\
&<1,000 \\
&<1.0 \\
& 8.3 \\
&<2.0 \\
&<1.0\end{aligned}$ \\
\hline $\begin{aligned} & 41,000 \\
& 1,930 \\
& 191 \\
&< 50 \\
&< 0.24 \\
&< 0.00046 \\
&< 10 \\
&<5.0 \\
&<5.0 \\
& 48 \\
&<5.0\end{aligned}$ & $\begin{aligned} & 3.8 E+07 \\
& 33,000 \\
& 2,300 \\
& 199 \\
&<50 \\
&< 0.24 \\
&< 0.00046 \\
&< 10 \\
&< 1.0 \\
&< 1.0 \\
& 44 \\
&< 1.0\end{aligned}$ \\
\hline $\begin{aligned} & 3.4 E+04 \\
< & 0.10 \\
& 3.7 E-01 \\
< & 1.1 E-01 \\
& 1.2 E+00 \\
< & 8.0 \\
< & 10\end{aligned}$ & $\begin{array}{c}3.9 \mathrm{E}+04 \\
<0.10 \\
1.4 \mathrm{E}-01 \\
<4.9 \mathrm{E}-03 \\
9.8 \mathrm{E}-02 \\
<8.0 \\
3.3\end{array}$ \\
\hline
\end{tabular}

$\underline{4094}$

13
$<3.0$

$<0.0050$

526
16

16
0.25

$<0.50$

$<10$

$<4.0$

921
$2.0 \mathrm{E}+00$
5.0

$<5.0$

$<500$

$2.5 \mathrm{E}+00$

$<2.0$

$1.7 E+00$

$<2.0$

8,390
$<2.0$

5,250

43

$<1,000$

$<5.0$

8.2

$<17$

$<5.0$

$4.0 \mathrm{E}+07$

65,000

2,600

353
69

$<10$

$<5.1$

$<10$

$<5.0$

$<5.0$

$<5.0$

4.5E +04

0.77

$1.7 E+00$

$-5.3 E-02$

7.5E-01

$<3.0$

3.2

8,840

$<2.0$

6,260

47

$<1,000$

$<1.0$

3.3

$<1.0$

25,000
4,290

161

$\begin{array}{r}161 \\ <50 \\ \hline\end{array}$

$<0.24$

$<0.00044$

$<10$

$<1.0$

$<1.0$

34

1.7

$5.0 E+04$

$<0.10$

1. $1 E+02$

4.7E +00

2.3E + 02

$<8.0$

8.3

DF
1
1
1
1
1
1
1
1
1
1
1
1
1
1
1
1
1
1
1
1
1
1
1
1
1
1
1
1
4
1
1
1
1
1
1
1
1
1
1
1
1
1
1

Mod

Lab Flag

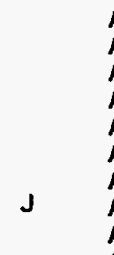

$\mu g / L \quad$ LE

$\begin{array}{lll}\mu \mathrm{g} / \mathrm{L} & \mathrm{GE} & 0 \\ \mu \mathrm{g} / \mathrm{L} & \mathrm{GE} & 0 \\ \mu \mathrm{g} / \mathrm{L} & \mathrm{GE} & 0\end{array}$

$\mu g / L$

$\mu g / L$ GE $O$

$\begin{array}{lll}\mu g / L & \text { GE } & 0 \\ \mu g / L & \text { GE } & 0\end{array}$

$\begin{array}{lll}\mu \mathrm{g} / \mathrm{L} & \mathrm{GE} & 0 \\ \mu \mathrm{g} / \mathrm{L} & \mathrm{GE} & 0\end{array}$

$\mu g / L \quad G E$

$\mu g / L \quad G E$

$\mu g / L$

$\mu g / L \quad G E$

J) $\mathrm{PCI}$ GP 0

$\begin{array}{lll}\mathrm{pH} & \mathrm{GE} & 0 \\ \mu \mathrm{g} / \mathrm{L} & \mathrm{GE} & 0\end{array}$

$\mu \mathrm{g} / \mathrm{L}$ GE 0

PCi/L GP 0

$\mu g / L$ GE 0

$\mu g / L \quad$ GE $\quad 0$

$\mu \mathrm{g} / \mathrm{L} \quad \mathrm{GE} \quad 0$

$\mu \mathrm{S} / \mathrm{cm}$ GE 0

$\mu \mathrm{g} / \mathrm{L}$ GE 0

$\mu g / L \quad \mathrm{GE} \quad 0$

J $\mu g / h$

$\begin{array}{lll}\mu g / L & \mathrm{GE} & 0 \\ \mu \mathrm{g} / \mathrm{L} & \mathrm{GE} & 0\end{array}$

$\underset{\mu \mathrm{g} / \mathrm{L}}{\mathrm{PCi}} \mathrm{GE} \quad 0$

J

$\mu g / L$ GE 0

$\begin{array}{lll}\mu g / L & G E & 2 \\ \mu g / L & G E & 0\end{array}$

$\mu g / L$ GE 0

$\mu g / L \quad G E$

$\mu g / L \quad G E$

$\mu \mathrm{g} / \mathrm{L} \quad \mathrm{GE} \quad 0$

$\mu \mathrm{g} / \mathrm{L}$ GE

$\mu \mathrm{g} / \mathrm{L}$ GE 0

$\mu \mathrm{g} / \mathrm{L}$

pCi/mL GP

NTU GE

GP

$\begin{array}{ll}\mathrm{pCi} / \mathrm{L} & \mathrm{GP} \\ \mathrm{pCi} / \mathrm{L} & \mathrm{GP}\end{array}$

$\mu g / L \quad G E$

$\mu \mathrm{g} / \mathrm{L} \quad \mathrm{GE}$

Note: Flagging, dilution factors, modifiers, and laboratories are for fourth quarter 1994 data only. See Appendix B for flagging criteria. - = exceeded holding time for fourth quarter 1994.

- = exceeded screening level or final PDWS for fourth quarter 1994. 


\section{WELL BGO 47A}

$\begin{array}{lll}\text { SRS Coord. } & & \text { Lat/Longitude } \\ \text { N74728.8 } & & 33.280650^{\circ} \mathrm{N} \\ \text { E54914.0 } & 81.667360^{\circ} \mathrm{W}\end{array}$

SAMPLE DATE

FIELD DATA

\section{Screen Zone Elevation}

96.8-86.8 ft ms

\section{Top of Casing \\ $266.9 \mathrm{ft} \mathrm{ms}$}

$\begin{array}{ll}\text { Casing } & \text { Pump } \\ 4^{n} \text { PVC } & S\end{array}$

$10 / 20 / 94$

\section{Analyte}

Water elevation Depth to water pH

Sp. conductance Water temperature Alkalinity as $\mathrm{CaCO}$ Turbidity

Volume purged

Sampling code

$$
02 / 25 / 94
$$

$04 / 11 / 94$

$08 / 12 / 94$

3094
161.5
105.4
7.4
159
20.1
54
1
6.7

Acetophenone

Aldrin

Aluminum, total recoverable

Antimony, total recoverable

Arsenic, total recoverable

Barium, total recoverable

Benzene

Bromodichloromethane

Bromoform

Bromomethane (Methyl bromide)

Cadmium, total recoverable

Calcium, total recoverable

Carbon tetrachloride

Chloride

Chlorobenzene

Chloroethane

Chloroethene (Vinyl chloride)

2-Chloroethyl vinyl ether

Chloroform

Chloromethane (Methyl chloride)

Chromium, total recoverable

Copper, total recoverable

Cyanide

p,p'-DDT

Dibromochloromethane

1,1-Dichloroethane

1,2-Dichloroethane

1,1-Dichloroethylene

trans-1,2-Dichloroethylene

Dichloromethane

2,4-Dichlorophenoxyacetic acid

1,2-Dichloropropane

cis-1,3-Dichloropropene

trans-1,3-Dichloropropene

Dieldrin

Endrin

Ethylbenzene

Fluoride

Gross alpha

$\begin{array}{ll}1094 & \underline{2094} \\ 162.2 & 162.3 \\ 104.7 & 104.6 \\ 7.4 & 7.2 \\ 163 & 161 \\ 18.7 & 20.1 \\ 5 & 49 \\ 3 & 1 \\ 9.4 & 5.3\end{array}$

\section{4}

$<10$

\section{4}

$<10$

$<0.050$
88

$<2.0$

3.7

42

$<1.0$

$<1.0$

$<1.0$

$<1.0$

$<2.0$
25,300

$<1.0$

2,820

$<1.0$

$<1.0$

$<1.0$
$<1.0$

1.3

$<1.0$

$<4.0$

$<4.0$

$<5.0$

$<0.10$

$<1.0$

$<1.0$

$<1.0$

$<1.0$

$<1.0$

$<1.0$

$<0.0015$

$<1.0$

$<10$

$<0.50$

$<0.0060$

$<1.0$

143

8.8E-01
$<0.050$

44
$<10$

$<10$
41

$<1.0$

$<1.0$

$<1.0$

$<1.0$

$<2.0$

24,800

$<1.0$

2,490

$<1.0$

$<1.0$

$<1.0$

$<1.0$

$<1.0$

$<1.0$

$<4.0$

12

$<5.0$

$<0.099$

$<1.0$

$<1.0$
$<1.0$

$<1.0$

$<1.0$

$<1.0$

$<1.0$

$<0.0016$

$<1.0$

$<1.0$

$<1.0$

$<0.50$

$<0.0059$

$<1.0$

129

9.8E-01
3094

$<10$

51

$<.8$
3.0

43

$<5.0$

$<5.0$

$<5.0$

$<10$

$<2.0$

25,100

3,140

$<5.0$

$<5.0$

$<10$

$<10$

$<5.0$

$<10$

$<4.0$

$<4.0$

$<5.0$

$<5.0$

$<5.0$

$<5.0$

$<5.0$

$<5.0$

$<5.0$

$<1.0$

$<5.0$

$<5.0$

$<5.0$

$<0.10$

$<5.0$

1.9E + 00

4094
161.9
105.0
7.3
148
20.0
52
1
5.0

Unit

ft msi

$\mathrm{ft}$

$\mathrm{pH}$

$\mu \mathrm{S} / \mathrm{cm}$

${ }^{\circ} \mathrm{C}$

NTI L

well vol.
Screen Zone

U. Congaree (IIA) 


\section{H ST Analyte}

Heptachlor

Iron, total recoverable

Lead, total recoverable

Lindane

Lithium, total recoverable

Magnesium, total recoverable

Manganese, total recoverable

Mercury, total recoverable

Methoxychlor

Naphthalene

Nickel, total recoverable

Nitrate as nitrogen

Nitrate-nitrite as nitrogen

- $\quad$ pH

pH

Potassium, total recoverable

Radium, total alpha-emitting

Selenium, total recoverable

Silica, total recoverable

Silver, total recoverable

Sodium, total recoverable

Specific conductance

Sulfate

1,1,2,2-Tetrachloroethane

Tetrachloroethylene

Tin, total recoverable

Toluene

Total dissolved solids

Total organic carbon

Total organic halogens

Total phosphates (as P)

Toxaphene

2,4,5-TP (Silvex)

Tributyl phosphate

1,1,1-Trichloroethane

1,1,2-Trichloroethane

Trichloroethylene

Trichlorofluoromethane

2,4,5-T

Tritium

Turbidity

Uranium-233/234

Uranium-235

Uranium-238

Vanadium, total recoverable

Xylenes

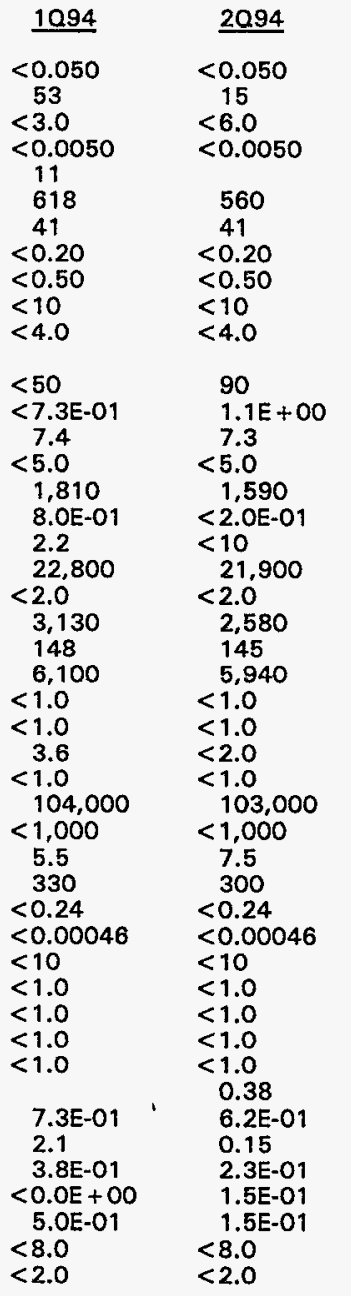

3094



52
$<3.0$
$<0.052$
12
542
40
$<0.20$
$<0.52$
$<10$
$<4.0$
$<20$

$2.8 E+00$

7.4
$<5.0$

1,720

7.0E-01

$<2.0$

21,400

$<2.0$

2,340

139

6,730

$<5.0$

$<5.0$

$<17$

$<5.0$
107,000

$<1,000$

11

806

$<1.0$

$<0.52$

$<10$

$<5.0$

$<5.0$

$<5.0$

$<5.0$

$1.5 \mathrm{E}+00$

0.43

2.2E-01

$0.0 E+00$

2.5E-01

$<3.0$

$<5.0$
4094 DF $\underline{\text { Mod Unit }} \underline{\text { Lab }}$ Flag

9.4

$<3.0$

$<0.0049$

566

40
$<0.20$

$<0.49$

$<10$

$<4.0$

$<50$

$1.8 \mathrm{E}+00$

7.2
$<5.0$

1,550

9.0E-01

$<2.0$

22,900

$<2.0$

2,200

148

5,830

$<1.0$

$<1.0$

3.2

$<1.0$

100,000

1,850

112

359

$<0.24$

$<0.00045$

$<10$

$<1.0$

$<1.0$

$<1.0$

$<1.0$

7.5E-01

0.11

$<8.0$
$<2.0$

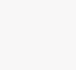


WSRC-TR-94-0610

Unclassified

WELL BGO 47C

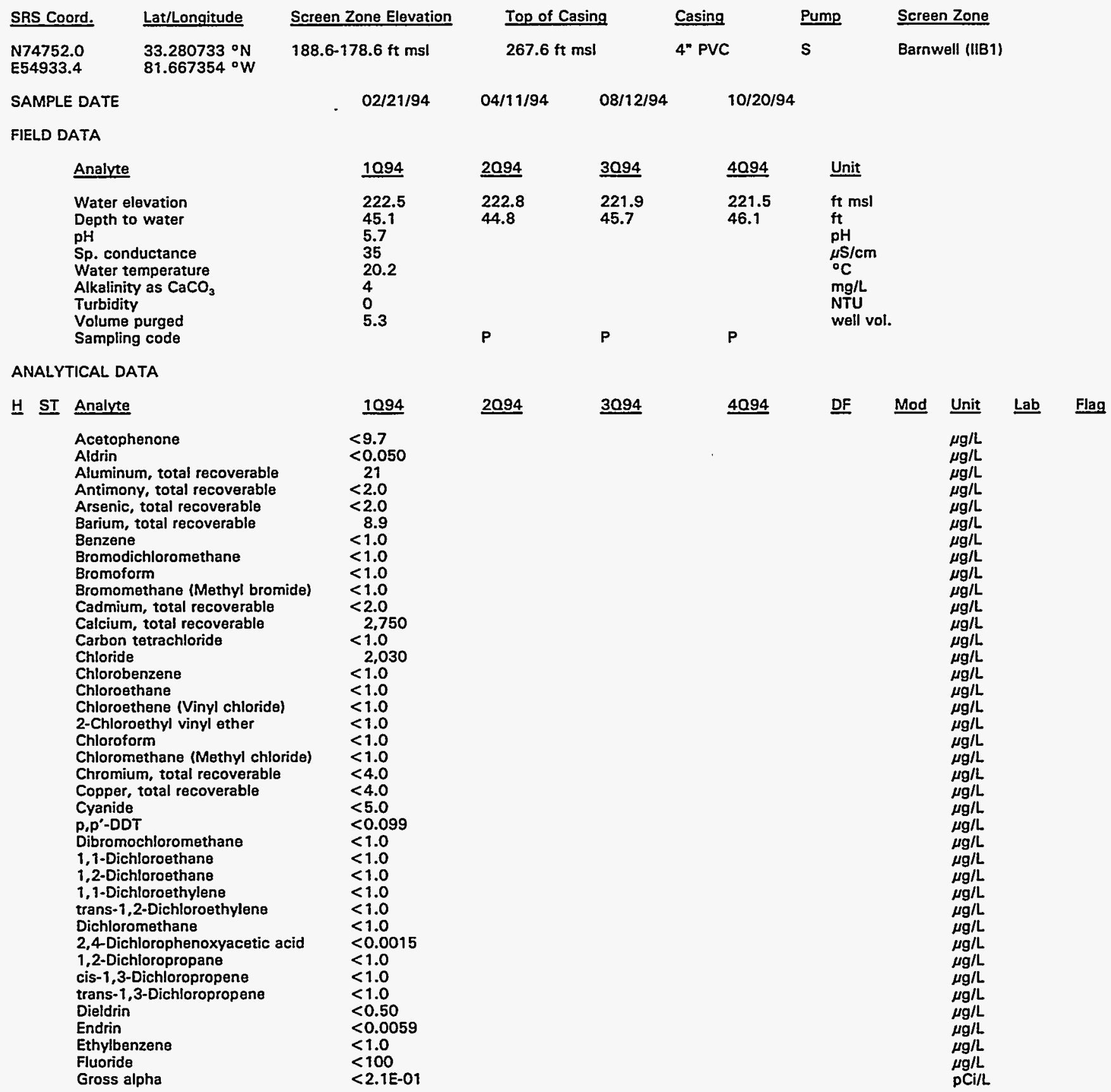

Note: Flagging, dilution factors, modifiers, and laboratories are for fourth quarter 1994 data only. See Appendix $B$ for flagging criteria. - = exceeded holding time for fourth quarter 1994.

- = exceeded screening level or final PDWS for fourth quarter 1994. 
Heptachlor

Iron, total recoverable

Lead, total recoverable

Lindane

Lithium, total recoverable

Magnesium, total recoverable

Manganese, total recoverable

Mercury, total recoverable

Methoxychlor

Naphthalene

Nickel, total recoverable

Nitrate-nitrite as nitrogen

Nonvolatile beta

pH

Phenols

Potassium, total recoverable

Radium, total alpha-emitting

Selenium, total recoverable

Silica, total recoverable

Silver, total recoverable

Sodium, total recoverable

Specific conductance

Sulfate

$1,1,2,2$-Tetrachloroethane

Tetrachloroethylene

Tin, rotal recoverable

Toluene

Total dissolved solids

Total organic carbon

Total organic halogens

Total phosphates (as P)

Toxapheno

2,4,5-TP (Silvex)

Tributyl phosphate

1,1,1-Trichloroethane

1,1,2-Trichloroethane

Trichloroethylene

Trichlorofluoromethane

Tritium

Turbidity

Uranium-233/234

Uranium-235

Uranium-238

Vanadium, total recoverable

Xylenes

\begin{tabular}{l}
1094 \\
\hline$<.050$ \\
$<4.0$ \\
$<3.0$ \\
$<0.0050$ \\
$<5.0$ \\
635 \\
7.7 \\
$<0.20$ \\
$<0.50$ \\
$<9.7$ \\
$<4.0$ \\
1,310 \\
$<1.5 E-01$ \\
6.0 \\
$<5.0$ \\
694 \\
$9.0 E-01$ \\
$<2.0$ \\
9,350 \\
$<2.0$ \\
1,860 \\
36 \\
$<1.000$ \\
$<1.0$ \\
$<1.0$ \\
$<2.0$ \\
$<1.0$ \\
34,000 \\
$<1,000$ \\
39 \\
$<50$ \\
$<0.24$ \\
$<0.00045$ \\
$<9.7$ \\
$<1.0$ \\
$<1.0$ \\
3.0 \\
$<1.0$ \\
$3.8 E+02$ \\
$<0.10$ \\
$8.8 E-01$ \\
$<0.0 E+00$ \\
$2.1 E+00$ \\
$<8.0$ \\
$<2.0$ \\
\end{tabular}

$\underline{2094}$

$\underline{3094}$

4094

$\underline{\mathrm{DF}}$

Mod Unit Lab Flag

$\mu g / L$

$\mu g / L$

$\mu g / L$

$\mu g / L$

$\mu g / L$

$\mu \mathrm{g} / \mathrm{L}$

$\mu g / L$

$\mu g / L$

$\mu g / L$

$\mu \mathrm{g} / \mathrm{L}$
$\mathrm{pCi} / \mathrm{L}$

$\mathrm{pH}$

$\mu \mathrm{g} / \mathrm{L}$

$\mu g / L$

$\mu \mathrm{pC} / \mathrm{L}$

$\mu g / L$

$\mu g / L$

$\mu \mathrm{g} / \mathrm{L}$

$\mu \mathrm{g} / \mathrm{L}$

$\mu \mathrm{g} / \mathrm{L}$

$\mu g / L$

$\mu g / h$

$\mu g / L$

$\mu \mathrm{g} / \mathrm{L}$

$\underset{\mu g / L}{\mu g / L}$

$\mu \mathrm{g} / \mathrm{L}$

$\mu \mathrm{g} / \mathrm{L}$

$\mu \mathrm{g} / \mathrm{L}$

$\mu g / L$

$\mu \mathrm{g} / \mathrm{L}$

$\mu g / L$

$\mathrm{pCi} / \mathrm{mL}$

NTU

pCi/L

pCi/L

pCi/L

$\mu \mathrm{gg} / \mathrm{L}$

$<8.0$

Note: Flagging, dilution factors, modifiers, and laboratories are for fourth quarter 1994 data only. See Appendix B for flagging criteria. - = exceeded holding time for fourth quarter 1994.

I = exceeded screening level or final PDWS for fourth quarter 1994. 


\section{WELL BGO 47D}

\section{SRS Coord. Lat/Longitude \\ N74739.7 \\ E54922.9 $81.667357 \circ \mathrm{W}$}

\section{Screen Zone Elevation \\ 213.4-203.4 ft msl}

SAMPLE DATE

FIELD DATA

$02 / 21 / 94$

$04 / 11 / 94$

$08 / 12 / 94$
Pump
Screen Zone

Water Table (IIB2)

\section{Top of Casing}

$267.4 \mathrm{ft} \mathrm{ms}$
4" PVC S

$10 / 20 / 94$

\section{Analyte \\ Water elevation Depth to water \\ Sp. conductance \\ Water temperature \\ Alkalinity as $\mathrm{CaCO}_{3}$ \\ urbidity \\ Volume purged \\ Sampling code}

10.94
225.7
41.7
5.3
41
20.0
1
0
8.3

$\begin{array}{ll}2094 & \underline{3094} \\ 226.5 & 225.2 \\ 40.9 & 42.2 \\ 5.2 & 5.1 \\ 47 & 44 \\ 20.6 & 20.6 \\ 2 & 1 \\ 0 & 0 \\ 12.7 & 11.0\end{array}$

4094
225.3
42.1
5.3
38
20.5
0
0
11.5

Unit

ft msl

$\mathrm{ft}$

$\mu \mathrm{S} / \mathrm{cm}$

mg/L

well vol.

ANALYTICAL DATA

HT Analyte
Acetophenone
Aldrin
Aluminum, total recoverable
Antimony, total recoverable
Arsenic, total recoverable
Barium, total recoverable
Benzene
Bromodichloromethane
Bromoform
Bromomethane (Methyl bromide)
Cadmium, total recoverable
Calcium, total recoverable
Carbon tetrachloride
Chloride
Chlorobenzene
Chloroethane
Chlorosthene (Vinyl chloride)
2-Chloroethyl vinyl ether
Chloroform
Chloromethane (Methyl chloride)
Chromium, total recoverable
Copper, total recoverable
Cyanide
p,p'-DDT
Dibromochloromethane
1,1-Dichloroethane
1,2-Dichloroethane
1,1-Dichloroethylene
trans-1,2-Dichloroethylene
Dichloromethane
2,4-Dichlorophenoxyacetic acid
1,2-Dichloropropane
cis-1,3-Dichloropropene
trans-1,3-Dichloropropene
Dieldrin
Endrin
Ethylbenzene
Fluoride
Gross alpha

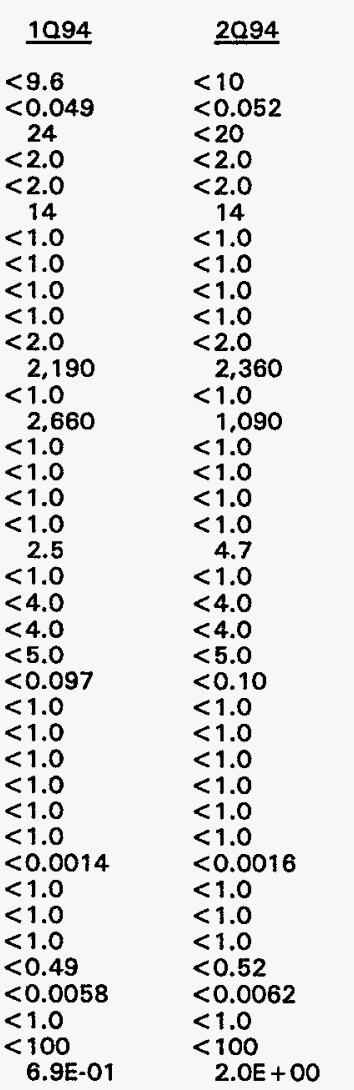

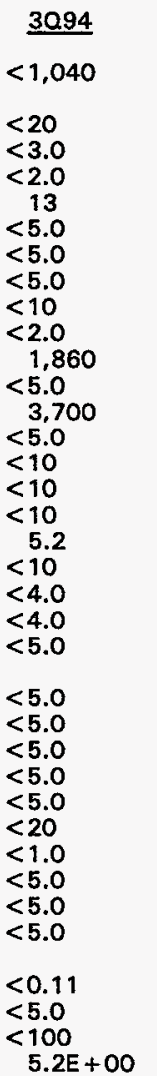

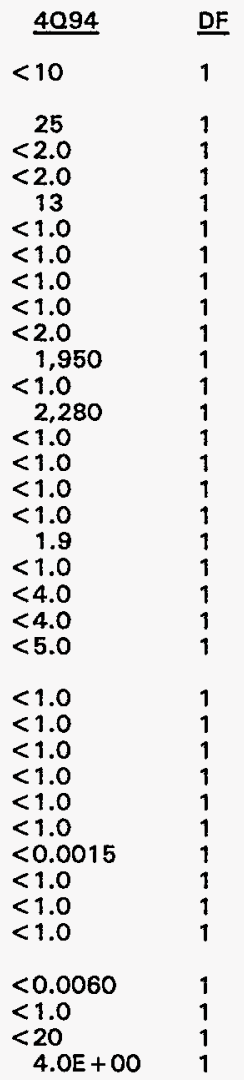

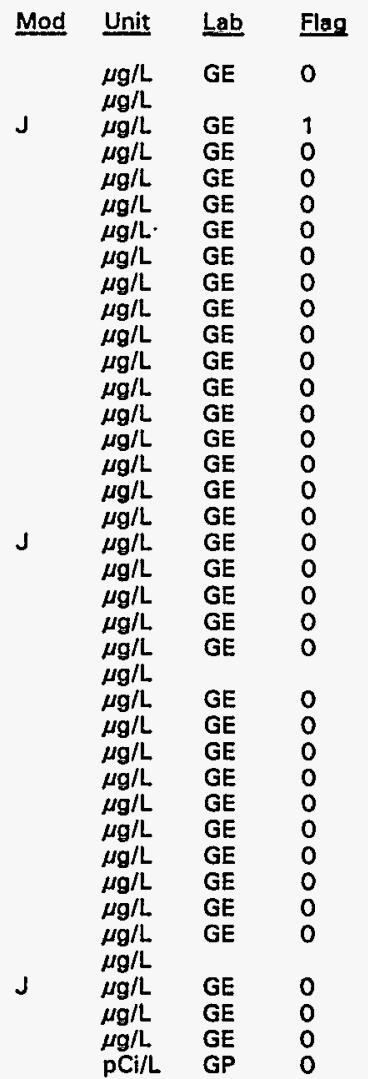

Note: Flagging, dilution factors, modifiers, and laboratories are for fourth quarter 1994 data only. See Appendix B for flagging criteria. - = exceeded holding time for fourth quarter 1994.

- = exceeded screening level or final PDWS for fourth quarter 1994. 
ANALYTICAL DATA

H $\underline{\text { ST Analyte }}$

Heptachlor

Iron total recoverable

Lead, total recoverable

Lindane

Lithium, total recoverable

Magnesium, total recoverable

Manganese, total recoverable

Mercury, total recoverable

Methoxychlor

Naphthalene

Nickel, total recoverable

Nitrate as nitrogen

Nitrate-nitrite as nitrogen

Nonvolatile beta

- $\mathrm{pH}$

Phenols

Potassium, total recoverable

Radium, total alpha-emitting

Selenium, total recoverable

Silica, total recoverable

Silver, total recoverable

Sodium, total recoverable

Specific conductance

Sulfate

1,1,2,2-Tetrachloroethane

Tetrachloroethylene

Tin, total recoverable

Toluene

Total activity

Total dissolved solids

Total organic carbon

Total organic halogens

Total phosphates (as P)

Toxaphene

2,4,5-TP (Silvex)

Tributyl phosphate

$1,1,1$-Trichloroethane

1,1,2-Trichloroethane

- Trichloroethylene

Trichlorofluoromethane

2,4,5-T

Tritium

Turbidity

Uranium-233/234

Uranium-235

Uranium-238

Vanadium, total recoverable

Xylenes

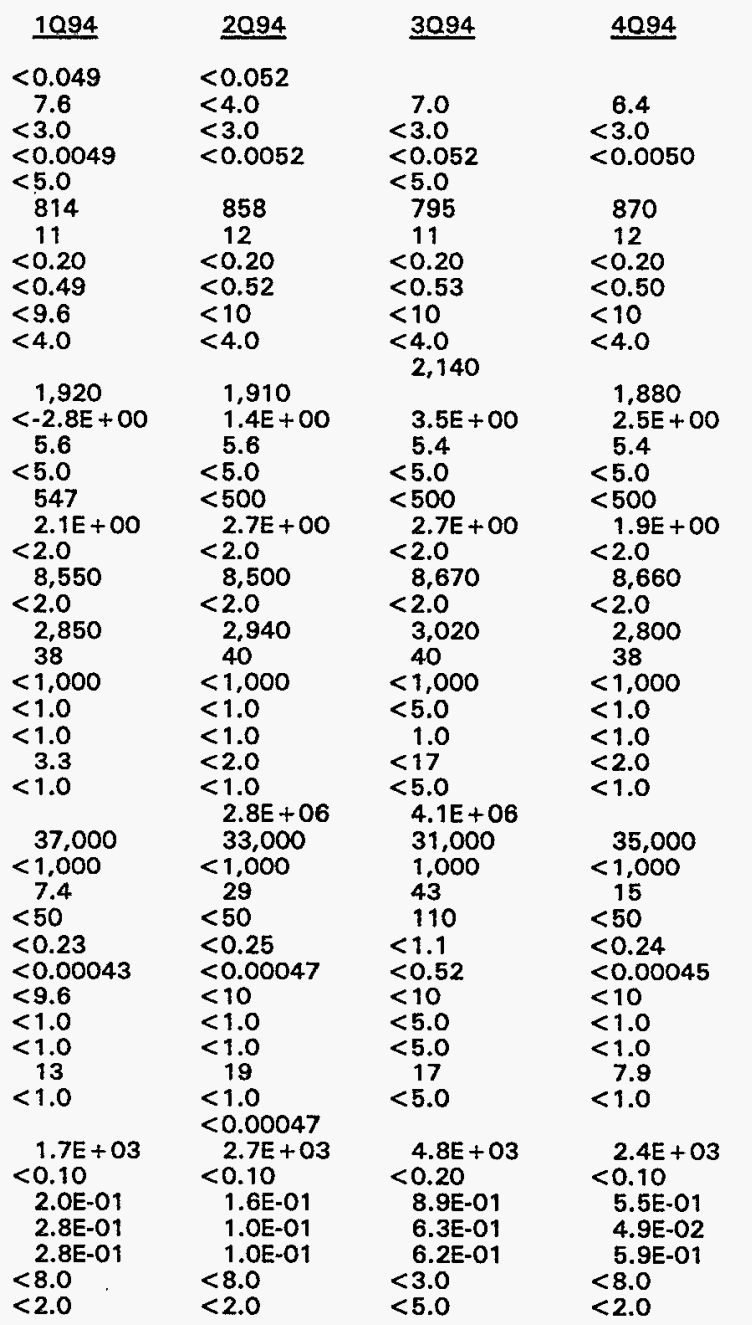

\begin{tabular}{|c|c|c|}
\hline$\underline{\mathrm{DF}}$ & Mod & Unit \\
\hline & & $\mu g / L$ \\
\hline 1 & $J$ & $\mu \mathrm{g} / \mathrm{L}$ \\
\hline 1 & & $\mu \mathrm{g} / \mathrm{L}$ \\
\hline 1 & $J$ & $\mu \mathrm{g} / \mathrm{L}$ \\
\hline 1 & & $\mu \mathrm{g} / \mathrm{L}$ \\
\hline 1 & & Pgil \\
\hline 1 & & mall \\
\hline 1 & $J$ & $\mu \mathrm{g} / \mathrm{L}$ \\
\hline 1 & & $\mu \mathrm{g} / \mathrm{L}$ \\
\hline 1 & & $\mu \mathrm{g} / \mathrm{L}$ \\
\hline 2 & & $\mu_{\mathrm{g} / \mathrm{L}}$ \\
\hline 1 & $J$ & $\mathrm{pCi} / \mathrm{L}$ \\
\hline 1 & J & $\mathrm{pH}$ \\
\hline 1 & & $\mu \mathrm{g} / \mathrm{L}$ \\
\hline 1 & & $\mu \mathrm{g} / \mathrm{L}$ \\
\hline 1 & & $\mathrm{pCi} / \mathrm{L}$ \\
\hline 1 & & $\mu \mathrm{g} / \mathrm{L}$ \\
\hline 1 & & $\mu g / L$ \\
\hline 1 & & $\mu g / L$ \\
\hline 1 & & $\mu \mathrm{g} / \mathrm{L}$ \\
\hline 1 & & $\mu \mathrm{S} / \mathrm{cm}$ \\
\hline 1 & & $\mu \mathrm{g} / \mathrm{L}$ \\
\hline 1 & & $\mu \mathrm{g} / \mathrm{L}$ \\
\hline 1 & & $\mu \mathrm{g} / \mathrm{L}$ \\
\hline 1 & & $\mu \mathrm{g} / \mathrm{L}$ \\
\hline 1 & & $\begin{array}{l}\mu \mathrm{g} / \mathrm{L} \\
\mathrm{pCi} / \mathrm{L}\end{array}$ \\
\hline 1 & $J$ & $\mu \mathrm{g} / \mathrm{L}$ \\
\hline 1 & & $\mu \mathrm{g} / \mathrm{L}$ \\
\hline 1 & & $\mu \mathrm{g} / \mathrm{L}$ \\
\hline 1 & & $\mu \mathrm{g} / \mathrm{L}$ \\
\hline 1 & $J$ & $\mu \mathrm{g} / \mathrm{L}$ \\
\hline 1 & & $\mu g / L$ \\
\hline 1 & & $\mu \mathrm{g} / \mathrm{L}$ \\
\hline 1 & & $\mu \mathrm{g} / \mathrm{L}$ \\
\hline 1 & & $\mu \mathrm{a} / \mathrm{L}$ \\
\hline 1 & & $\mu \mathrm{g} / \mathrm{L}$ \\
\hline & & $\mu \mathrm{g} / \mathrm{L}$ \\
\hline 1 & & $\mathrm{pCi} / \mathrm{mL}$ \\
\hline 1 & $J$ & NTU \\
\hline 1 & UI & $\mathrm{pCi} / \mathrm{L}$ \\
\hline 1 & UI & $\mathrm{pCi} / \mathrm{L}$ \\
\hline 1 & UI & $\mathrm{pCi} / \mathrm{L}$ \\
\hline 1 & & \\
\hline
\end{tabular}

Lab Flag

GE $\quad 0$

GE

GE

GE

GE

GE

GE $\quad 0$

GE

GP

$G E$

Note: Flagging, dilution factors, modifiers, and laboratories are for fourth quarter 1994 data only. See Appendix B for flagging criteria. - = exceeded holding time for fourth quarter 1994.

= exceeded screening level or final PDWS for fourth quarter 1994. 
WSRC-TR-94-0610

Unclassified

WELL BGO 48C

\begin{tabular}{|c|c|c|c|c|c|c|}
\hline SRS Coord. & Lat/Longitude & Screen Zone Elevation & Top of Casing & Casing & Pump & Screen Zone \\
\hline $\begin{array}{l}N 74599.6 \\
\text { E55124.4 }\end{array}$ & $\begin{array}{l}33.280708^{\circ} \mathrm{N} \\
81.666555^{\circ} \mathrm{W}\end{array}$ & $186.7-176.7 \mathrm{ft} \mathrm{msl}$ & $276.6 \mathrm{ft} \mathrm{msl}$ & $4^{n} \mathrm{PVC}$ & $s$ & Barnwell (IIB1) \\
\hline SAMPLE DATE & & $02 / 21 / 94$ & $04 / 11 / 94$ & & & \\
\hline
\end{tabular}

FIELD DATA

Analyte
Water elevation
Depth to water
pH
Sp. conductance
Water temperature
Alkalinity as $\mathrm{CaCO}_{3}$
Turbidity
Volume purged
Sampling code

ANALYTICAL DATA

Analyte
Acetophenone
Aldrin
Aluminum, total recoverable
Antimony, total recoverable
Arsenic, total recoverable
Barium, total recoverable
Benzene
Bromodichloromethane
Bromoform
Bromomethane (Methyl bromide)
Cadmium, total recoverable
Calcium, total recoverable
Carbon tetrachloride
Chloride
Chlorobenzene
Chloroethane
Chloroethene (Vinyl chloride)
2-Chloroethyl vinyl ether
Chloroform
Chloromethane (Methyl chloride)
Chromium, total recoverable
Copper, total recoverable
Cyanide
p,p'-DDT
Dibromochloromethane
1,1-Dichloroethane
1,2-Dichloroethane
1,1-Dichloroethylene
trans-1,2-Dichloroethylene
Dichloromethane
2,4-Dichlorophenoxyacetic acid
1,2-Dichloropropane
cis-1,3-Dichloropropene
trans-1,3-Dichloropropene
Dieldrin
Endrin
Ethylbenzene
Fluoride
Gross alpha

$\begin{array}{lllll}1094 & \underline{2094} & \underline{3094} & \underline{4094} & \text { Unit } \\ 223.0 & 223.5 & 222.6 & 222.6 & \mathrm{ft} \mathrm{msl} \\ 53.6 & 53.1 & 54.0 & 54.0 & \mathrm{ft} \\ 5.4 & 5.0 & 5.3 & 5.3 & \mathrm{pH} \\ 35 & 39 & 38 & 32 & \mu \mathrm{S} / \mathrm{cm} \\ 20.1 & 20.6 & 20.8 & 20.3 & { }^{\circ} \mathrm{C} \\ 1 & 1 & 1 & 1 & \mathrm{mg} / \mathrm{L} \\ 1 & 1 & 1 & 0 & \mathrm{NTU} \\ 6.6 & 5.2 & 6.2 & 11.5 & \text { well vol. }\end{array}$

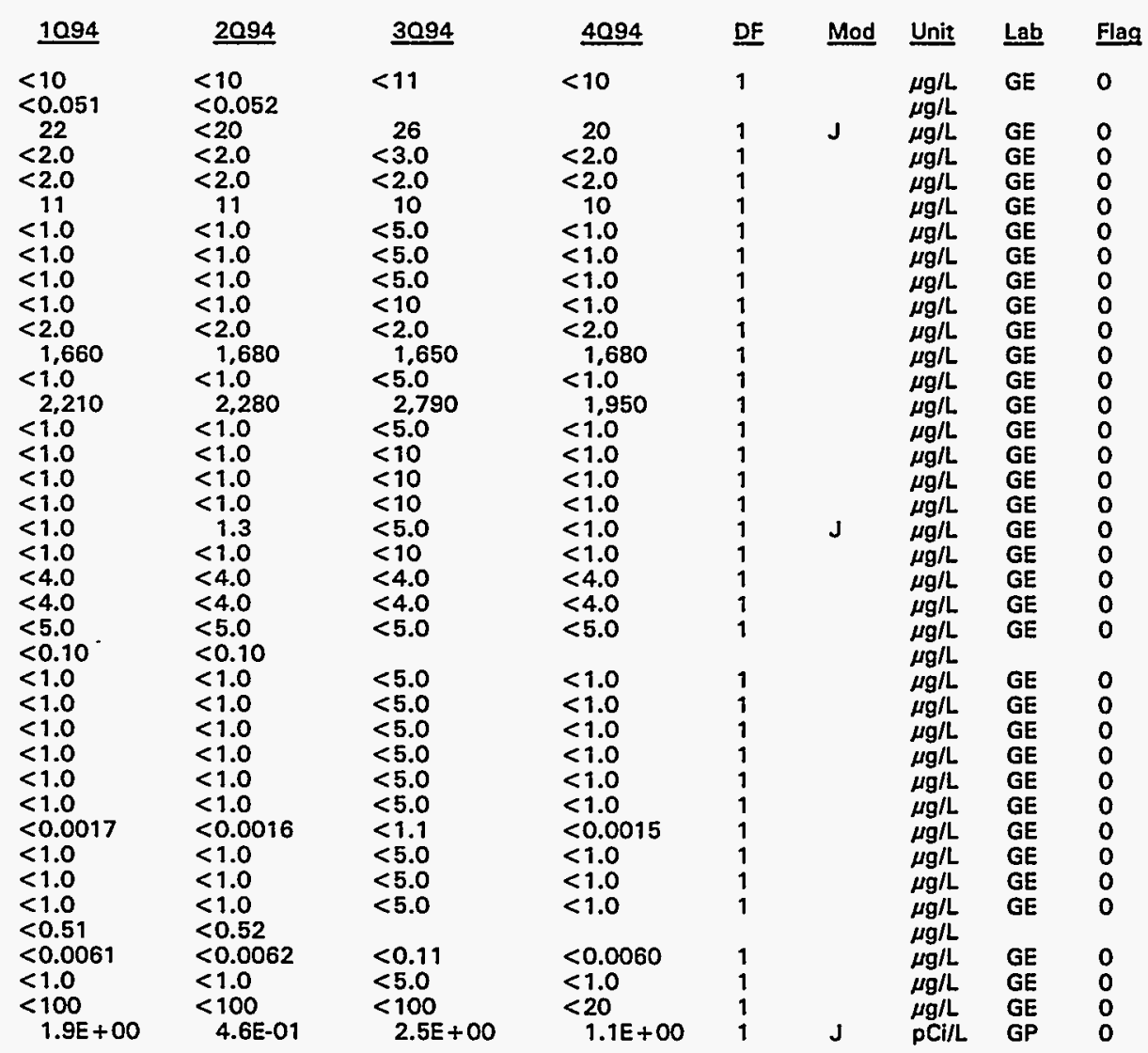

Note: Flagging, dilution factors, modifiers, and laboratories are for fourth quarter 1994 data only. See Appendix B for flagging criteria. - = exceeded holding time for fourth quarter 1994.

a = exceeded screening level or final PDWS for fourth quarter 1994. 
H ST Analyte

Heptachlor

Iron, total recoverable

Lead, total recoverable

Lindane

Lithium, total recoverable

Magnesium, total recoverable

Manganese, total recoverable

Mercury, total recoverable

Methoxychlor

Naphthalene

Nickel, total recoverable

Nitrate as nitrogen

Nitrate-nitrite as nitrogen

Nonvolatile beta

- $\quad \mathrm{pH}$

Phenols

Potassium, total recoverable

Radium, total alpha-emitting

Selenium, total recoverable

Silica, total recoverable

Silver, total recoverable

Sodium, total recoverable

Specific conductance

Sulfate

1,1,2,2-Tetrachloroethane

Tetrachloroethylene

Tin, total recoverable

Toluene

Total activity

Total dissolved solids

Total organic carbon

Total organic halogens

Total phosphates (as P)

Toxaphene

2,4,5-TP (Silvex)

Tributyl phosphate

1,1,1-Trichloroethane

1,1,2-Trichloroethane

Trichloroethylene

Trichlorofluoromethane

2,4,5-T

- Tritium

Uranium-233/234

Uranium-235

Uranium-238

Vanadium, total recoverable

Xylenes

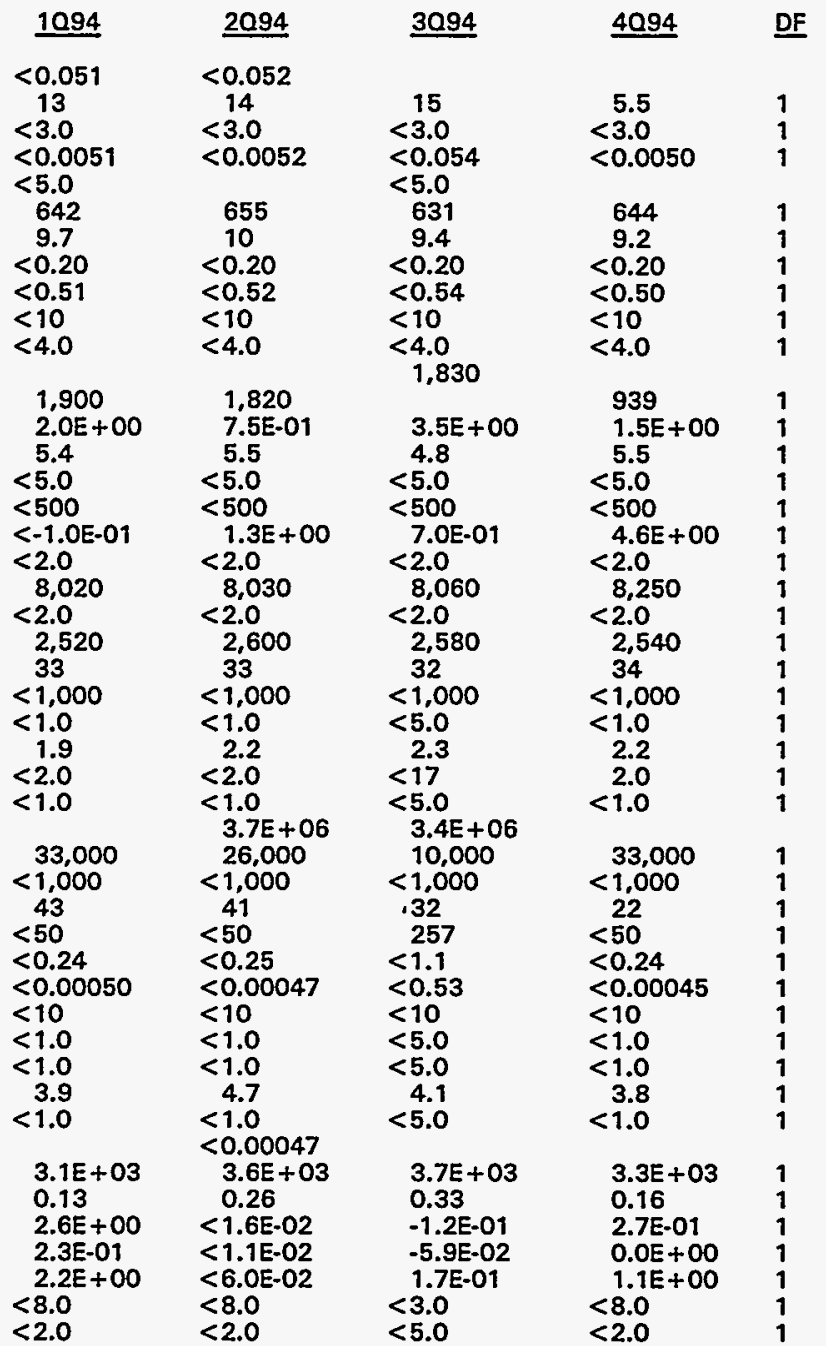

Mod Unit Lab Flag

J $\underset{\mu g / L}{\mu g / L}$ GE 0

$\begin{array}{lll}\mu \mathrm{g} / \mathrm{L} & \mathrm{GE} & 0 \\ \mu \mathrm{g} / \mathrm{L} & \mathrm{GE} & 0\end{array}$

$\mu g / L$

$\mu \mathrm{g} / \mathrm{L}$ GE 0

$\mu \mathrm{g} / \mathrm{L}$ GE 0

$\mu g / L$ GE 0

$\mu g / L \quad G$

$\underset{\mu \mathrm{g} / \mathrm{L}}{\mu \mathrm{GE}}$

$\mu g / L$

$\mu \mathrm{g} / \mathrm{L} \quad \mathrm{GE}$

PCIR GE 0

$\begin{array}{llll}\text { UI } & \mathrm{PCi} / \mathrm{L} & \mathrm{GP} & 0 \\ \mathrm{pH} & \mathrm{GE} & 0\end{array}$

$\mu \mathrm{g} / \mathrm{L}$ GE $\quad$

$\mu g / L \quad G E$

pCi/L GP

$\mu \mathrm{g} / \mathrm{L}$

$\mu \mathrm{g} / \mathrm{L}$

$\mu \mathrm{g} / \mathrm{L} \quad \mathrm{GE}$

Ug/L GE

$\mu \mathrm{S} / \mathrm{cm} \quad \mathrm{GE}$

$\mu \mathrm{g} / \mathrm{L} \quad \mathrm{GE}$

$\mu \mathrm{g} / \mathrm{L} \quad \mathrm{GE}$

$\mu g / L \quad G E$

$\mu \mathrm{g} / \mathrm{L} \quad \mathrm{GE}$

$\mu g / L$

pCin

$\mu g / L$

$\mu g / L$

$\mu g / L$

$\mu g / L$

$\mu g / L$

$\mu g / L$

$\mu g / L$

$\mu g / L \quad G E$

$\mu \mathrm{g} / \mathrm{L} \quad \mathrm{GE}$

$\mu g / L$

$\begin{array}{llll} & \mu \mathrm{g} \mathrm{Ci} / \mathrm{mL} & \mathrm{GP} & \mathbf{2} \\ \mathrm{J} & \mathrm{NTU} & \mathrm{GE} & 0 \\ \text { UI } & \mathrm{pCi} / \mathrm{L} & \mathrm{GP} & 0 \\ \text { UI } & \mathrm{pCi} / \mathrm{L} & \mathrm{GP} & 0 \\ \mathrm{~J} & \mathrm{pCi} / \mathrm{L} & \mathrm{GP} & 0 \\ & \mu \mathrm{g} / \mathrm{L} & \mathrm{GE} & 0 \\ & \mu \mathrm{g} / \mathrm{L} & \mathrm{GE} & 0\end{array}$

Note: Flagging, dilution factors, modifiers, and laboratories are for fourth quarter 1994 data only. See Appendix B for flagging criteria. - = exceeded holding time for fourth quarter 1994.

- =xceeded screening level or final PDWS for fourth quarter 1994. 
WELL BGO 48D

\begin{tabular}{|c|c|c|c|c|c|c|c|}
\hline SRS Coord. & Lat/Longitude & Screen Zone Elevation & Top o & & Casing & Pump & Screen Zone \\
\hline $\begin{array}{l}\text { N74586.4 } \\
\text { E55121.0 }\end{array}$ & $\begin{array}{l}33.280673^{\circ} \mathrm{N} \\
81.666538^{\circ} \mathrm{W}\end{array}$ & $212.0-202.0 \mathrm{ft} \mathrm{ms}$ & 276.9 & & 4" PVC & $\mathbf{s}$ & Water Table (IIB2) \\
\hline SAMPLE DA & & $02 / 21 / 94$ & $04 / 11 / 94$ & $08 / 09 / 94$ & $10 / 20 / 94$ & & \\
\hline IELD DAT & & & & & & & \\
\hline An: & & 1094 & 2094 & 3094 & 4094 & Unit & \\
\hline $\begin{array}{l}\text { Wa } \\
\text { Dep } \\
\text { pH } \\
\text { Sp. } \\
\text { Wa } \\
\text { Alk } \\
\text { Tur } \\
\text { Vol }\end{array}$ & $\begin{array}{l}\text { evation } \\
\text { water } \\
\text { luctance } \\
\text { mperature } \\
\text { as } \mathrm{CaCO}_{3} \\
\text { purged }\end{array}$ & $\begin{array}{l}226.2 \\
50.7 \\
4.7 \\
57 \\
20.5 \\
0 \\
2 \\
17.5\end{array}$ & $\begin{array}{l}226.8 \\
50.1 \\
4.4 \\
65 \\
21.0 \\
0 \\
1 \\
14.5\end{array}$ & $\begin{array}{l}225.6 \\
51.3 \\
4.6 \\
61 \\
21.4 \\
0 \\
1 \\
11.9\end{array}$ & $\begin{array}{l}225.7 \\
51.2 \\
4.6 \\
55 \\
20.9 \\
0 \\
1 \\
4.8\end{array}$ & $\begin{array}{l}\mathrm{ft} \mathrm{msl} \\
\mathrm{ft} \\
\mathrm{pH} \\
\mu \mathrm{S} / \mathrm{cm} \\
{ }^{\circ} \mathrm{C} \\
\mathrm{mg} / \mathrm{L} \\
\mathrm{NTU} \\
\text { well vol. }\end{array}$ & \\
\hline
\end{tabular}

ANALYTICAL DATA

H ST Analyte

Acetophenone

Aldrin

Aluminum, total recoverable

Antimony, total recoverable

Arsenic, total recoverable

Barium, total recoverable

Benzene

Bromodichloromethane

Bromoform

Bromomethane (Methyl bromide)

Cadmium, total recoverable

Calcium, total recoverable

Carbon tetrachloride

Chloride

Chlorobenzene

Chloroethane

Chloroethene (Vinyl chloride)

2-Chlorosthyl vinyl ether

Chloroform

Chloromethane (Methyl chloride)

Chromium, total recoverable

Copper, total recoverable

Cyanide

Pysid

Dibromochloromethane

1,1-Dichloroethane

1,2-Dichloroethane

1,1-Dichloroethylene

trans-1,2-Dichloroethylene

Dichloromethane

2,4-Dichlorophenoxyacetic acid

1,2-Dichloropropane

cis-1,3-Dichloropropene

trans-1,3-Dichloropropene

Dieldrin

Endrin

Ethylbenzene

Fluoride

Gross alpha

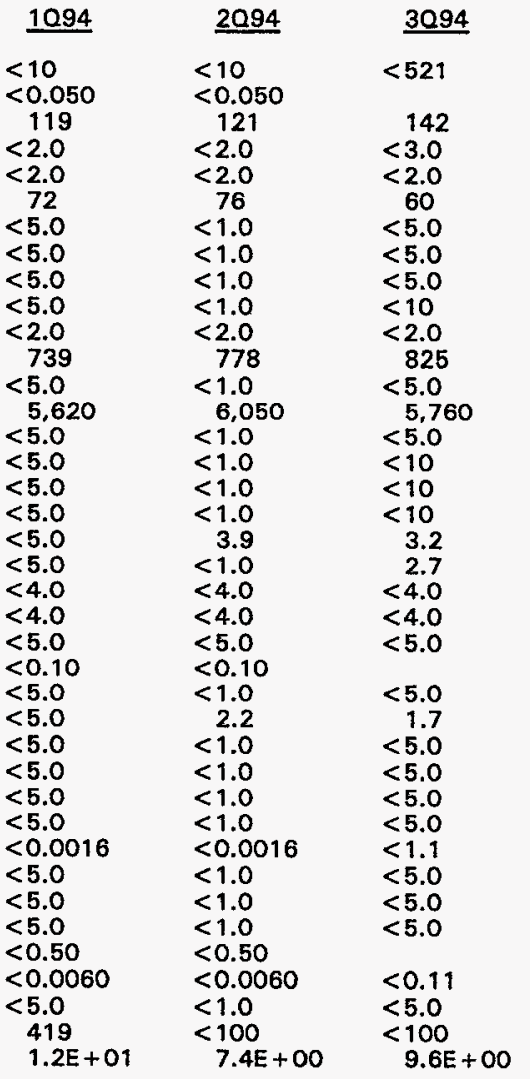

$\begin{aligned} & 4094 \\ & 10 \\ &<<.050 \\ & 119 \\ &<2.0 \\ &<2.0 \\ & 47 \\ &<5.0 \\ &<5.0 \\ &<5.0 \\ &<5.0 \\ &<2.0 \\ & 808 \\ &<5.0 \\ & 4.030 \\ &<5.0 \\ &<5.0 \\ &<5.0 \\ &<5.0 \\ & \quad 9.5 \\ &<5.0 \\ &<4.0 \\ &<4.0 \\ &<5.0 \\ &<0.10 \\ &<5.0 \\ &<5.0 \\ &<5.0 \\ &<5.0 \\ &<5.0 \\ &<5.0 \\ &<0.0015 \\ &<5.0 \\ &<5.0 \\ &<5.0 \\ &<0.50 \\ &<0.0060 \\ &<5.0 \\ &<20 \\ & 1.46+01 \\ &\end{aligned}$

\begin{tabular}{|c|c|c|c|}
\hline DF & Mod & Unit & Lab \\
\hline 1 & & $\mu g / L$ & $\mathrm{GE}$ \\
\hline 1 & & $\mu \mathrm{g} / L$ & $\overline{\mathrm{GE}}$ \\
\hline 1 & & $\mu \mathrm{g} / L$ & $\mathrm{GE}$ \\
\hline 1 & & $\mu g / L$ & GE \\
\hline 1 & & $\mu \mathrm{g} / \mathrm{L}$ & GE \\
\hline 1 & & $\mu g / L$ & GE \\
\hline 5 & & $\mu \mathrm{g} / \mathrm{L}$ & GE \\
\hline 5 & & $\mu g / L$ & GE \\
\hline 5 & & $\mu g / L$ & GE \\
\hline 5 & & $\mu \mathrm{g} / \mathrm{L}$ & GE \\
\hline 1 & & $\mu g / L$ & GE \\
\hline 1 & & $\mu g / L$ & GE \\
\hline 5 & & $\mu \mathrm{g} / \mathrm{L}$ & $\mathrm{GE}$ \\
\hline 1 & & $\mu \mathrm{g} / \mathrm{L}$ & GE \\
\hline 5 & & $\mu \mathrm{g} / \mathrm{L}$ & GE \\
\hline 5 & & $\mu g / L$ & GE \\
\hline 5 & & $\mu g / L$ & GE \\
\hline 5 & & $\mu g / L$ & GE \\
\hline 5 & $\mathrm{~J}$ & $\mu g / L$ & GE \\
\hline $\begin{array}{l}5 \\
1\end{array}$ & & $\mu \mathrm{g} / \mathrm{L}$ & $\begin{array}{l}\mathrm{GE} \\
\mathrm{GE}\end{array}$ \\
\hline 1 & & $\mu g / L$ & $\mathrm{GE}$ \\
\hline 1 & & $\mu \mathrm{g} / \mathrm{L}$ & GE \\
\hline 1 & & $\mu \mathrm{g} / \mathrm{L}$ & $\mathrm{GE}$ \\
\hline 5 & & $\mu \mathrm{g} / \mathrm{L}$ & $\overline{G E}$ \\
\hline 5 & & $\mu g / L$ & GE \\
\hline 5 & & $\mu g / L$ & $\mathrm{GE}$ \\
\hline 5 & & $\mu g / L$ & GE \\
\hline 5 & & $\mu g / L$ & GE \\
\hline 5 & & $\mu g / L$ & GE \\
\hline 1 & & $\mu g / L$ & GE \\
\hline 5 & & $\mu g / L$ & $\mathrm{GE}$ \\
\hline 5 & & $\mu g / L$ & $\mathrm{GE}$ \\
\hline- & & $\mu g / L$ & $\mathrm{GE}$ \\
\hline 1 & & $\mu \mathrm{g} / \mathrm{L}$ & GE \\
\hline 1 & & $\mu g / L$ & GE \\
\hline 5 & & $\mu g / L$ & GE \\
\hline 1 & & $\mu \mathrm{g} / \mathrm{L}$ & GE \\
\hline 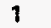 & & $p C \mathrm{i} / \mathrm{h}$ & GP \\
\hline
\end{tabular}

Note: Flagging, dilution factors, modifiers, and laboratories are for fourth quarter 1994 data only. See Appendix B for flagging criteria. - = exceeded holding time for fourth quarter 1994.

- = exceeded screening level or final PDWS for fourth quarter 1994. 


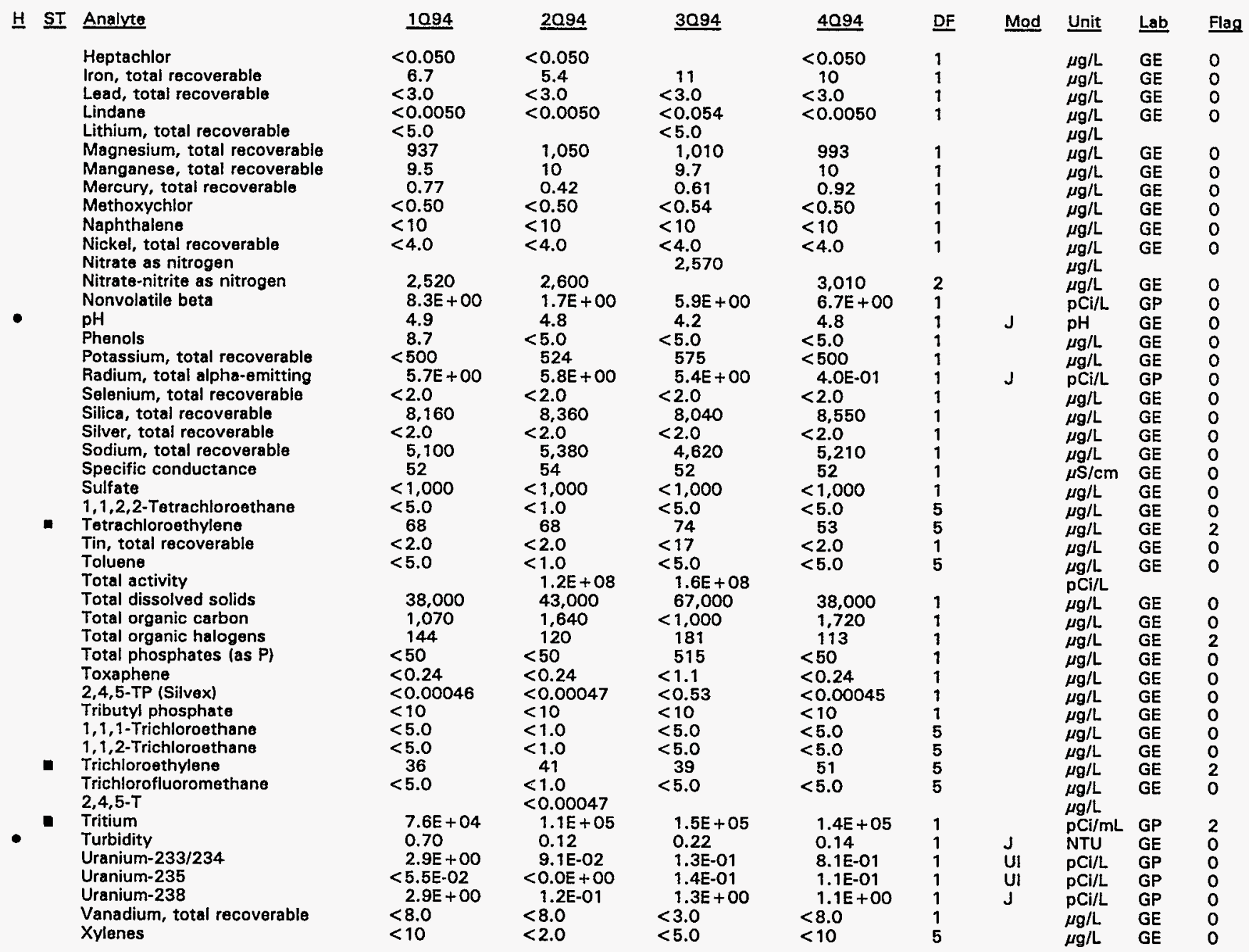

Note: Flagging, dilution factors, modifiers, and laboratories are for fourth quarter 1994 data only. See Appendix B for flagging criteria. - = exceeded holding time for fourth quarter 1994.

m = exceeded screening level or final PDWS for fourth quarter 1994. 


\section{WELL BGO 49A}

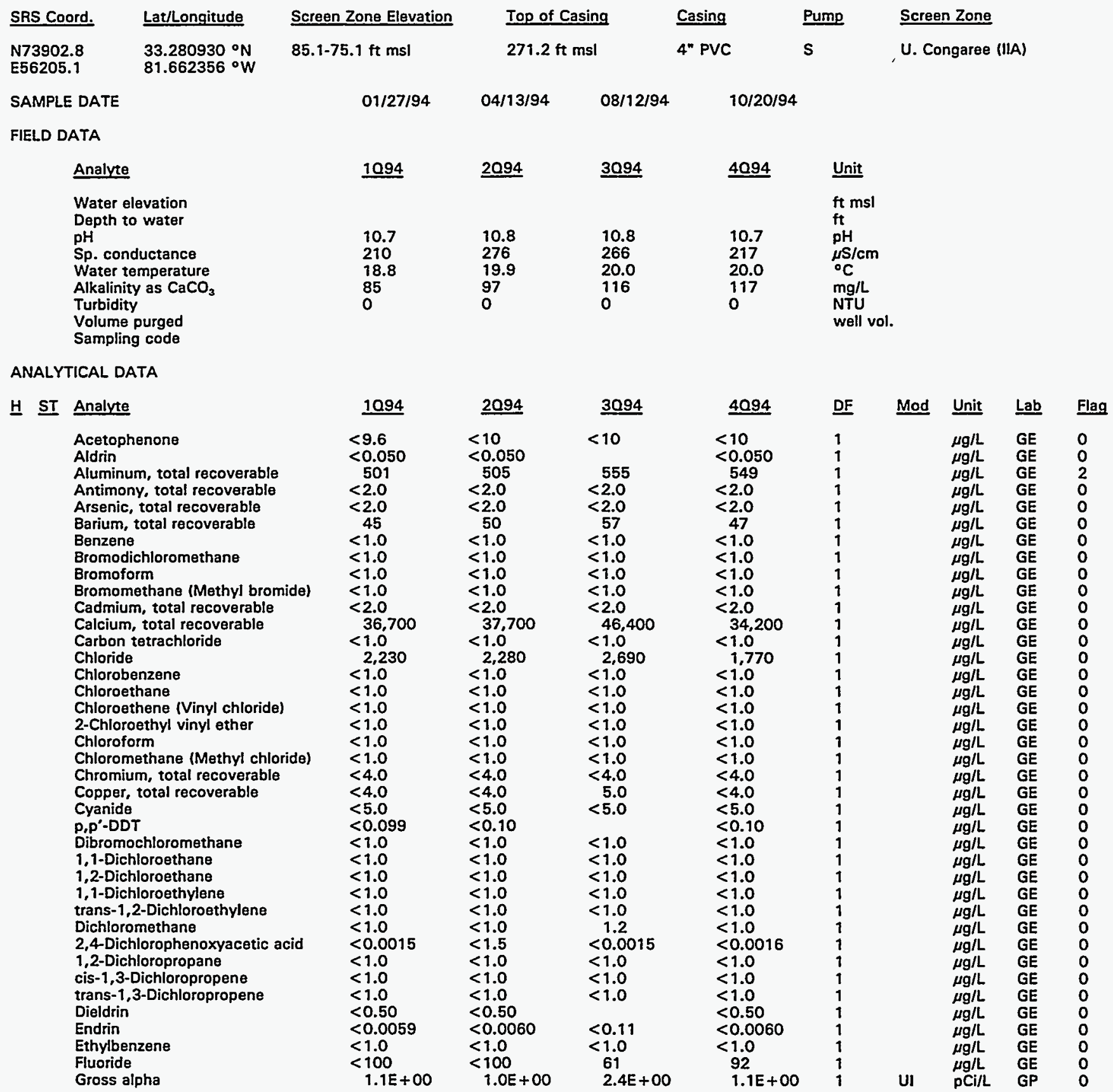

Note: Flagging, dilution factors, modifiers, and laboratories are for fourth quarter 1994 data only. See Appendix B for flagging criteria. - = exceeded holding time for fourth quarter 1994.

- =xceeded screening level or final PDWS for fourth quarter 1994. 
HI ST Analyte

Heptachlor

Iron, total recoverable

Lead, total recoverable

Lindane

Lithium, total recoverable

Magnesium, total recoverable

Manganese, total recoverable

Mercury, total recoverable

Methoxychlor

Naphthalene

Nickel, total recoverable

Nitrate as nitrogen

Nitrate-nitrite as nitrogen

Nonvolatile beta

$\mathrm{pH}$

Phenols

Potassium, total recoverable

Radium, total alpha-emitting

Radium-226

Radium-228

Selenium, total recoverable

Silica, total recoverable

Silver, total recoverable

Sodium, total recoverable

Specific conductance

Sulfate

1,1,2,2-Tetrachloroethane

Tetrachloroethylene

Tin, total recoverable

Toluene

Total dissolved solids

Total organic carbon

Total organic halogens

Total phosphates (as P)

Toxaphene

2,4,5-TP (Silvex)

Tributyl phosphate

1,1,1-Trichloroethane

1,1;2-Trichloroethane

Trichloroethylene

Trichlorofluoromethane

2,4,5-T

Tritium

Turbidity

Uranium-233/234

Uranium-235

Uranium-238

Vanadium, total recoverable

Xylenes

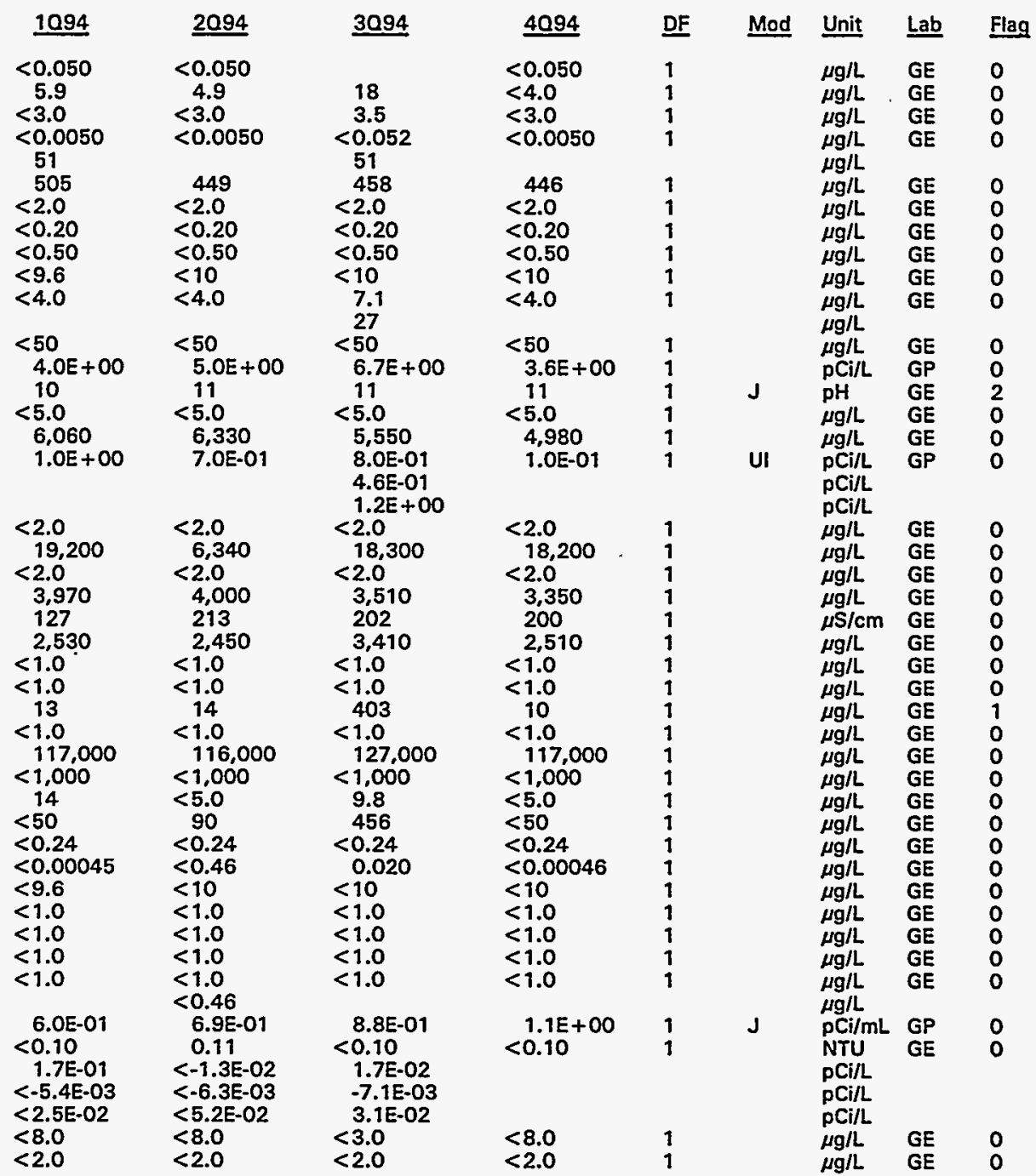

Note: Flagging, dilution factors, modifiers, and laboratories are for fourth quarter 1994 data only. See Appendix B for flagging criteria. - = exceeded holding time for fourth quarter 1994.

- = exceeded screening level or final PDWS for fourth quarter 1994. 


\section{WELL BGO 49C}

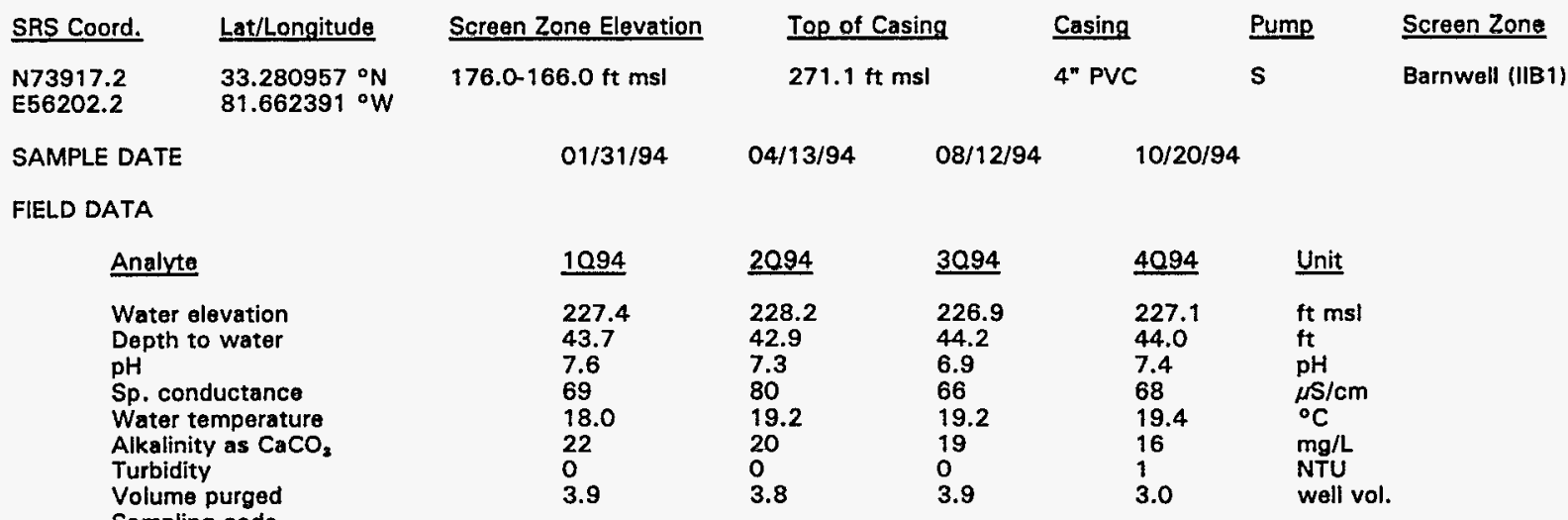

ANALYTICAL DATA

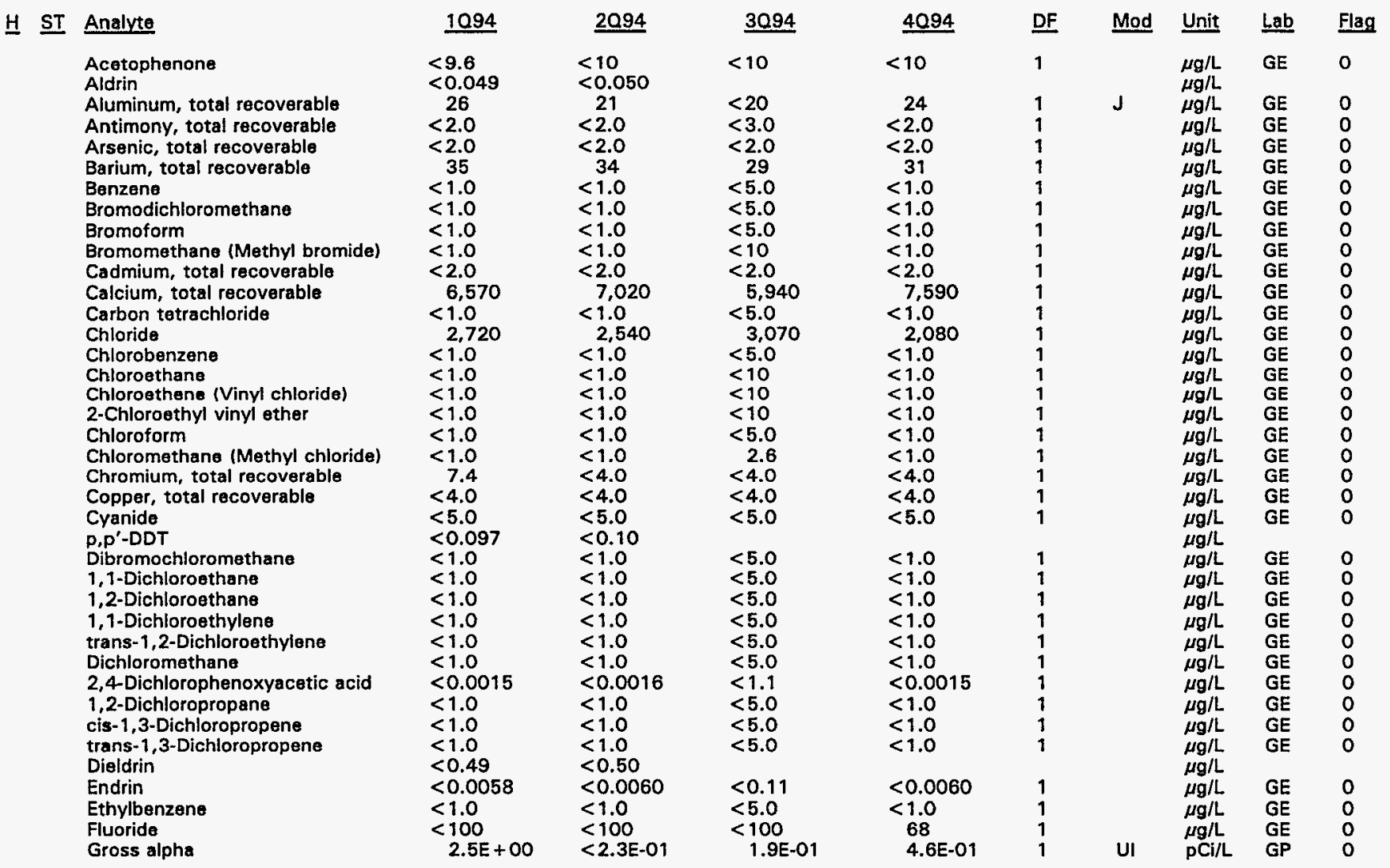

Note: Flagging, dilution factors, modifiers, and laboratories are for fourth quarter 1994 data only. See Appendix B for flagging criteria. - = exceeded holding time for fourth quarter 1994.

- = exceeded screening level or final PDWS for fourth quarter 1994. 


\section{H SI Analyte}

Heptachlor

Iron, total recoverable

Lead, total recoverable

Lindane

Lithium, total recoverable

Magnesium, total recoverable

Manganese, total recoverable

Mercury, total recoverable

Methoxychlo

Naphthalene

Nickel, total recoverable

Nitrate as nitrogen

Nitrate-nitrite as nitrogen

Nonvolatile beta

- $\quad \mathrm{pH}$

Phenols

Potassium, total recoverable

Radium, total alpha-emitting

Radium-226

Radium-228

Selenium, total recoverable

Silica, total recoverable

Silver, total recoverable

Sodium, total recoverable

Specific conductance

Sulfate

$1,1,2,2$-Tetrachloroethane

Tetrachloroethylene

Tin, total recoverable

Toluene

Total dissolved solids

Total organic carbon

Total organic halogens

Total phosphates (as P)

Toxaphene

2,4,5-TP (Silvex)

Tributyl phosphate

1,1,1-Trichloroethane

1,1,2-Trichloroethane

Trichloroethylene

Trichlorofluoromethane

2,4,5-T

- Tritium

Turbidity

Uranium-233/234

Uranium-235

Uranium-238

Vanadium, total recoverable

Xylenes

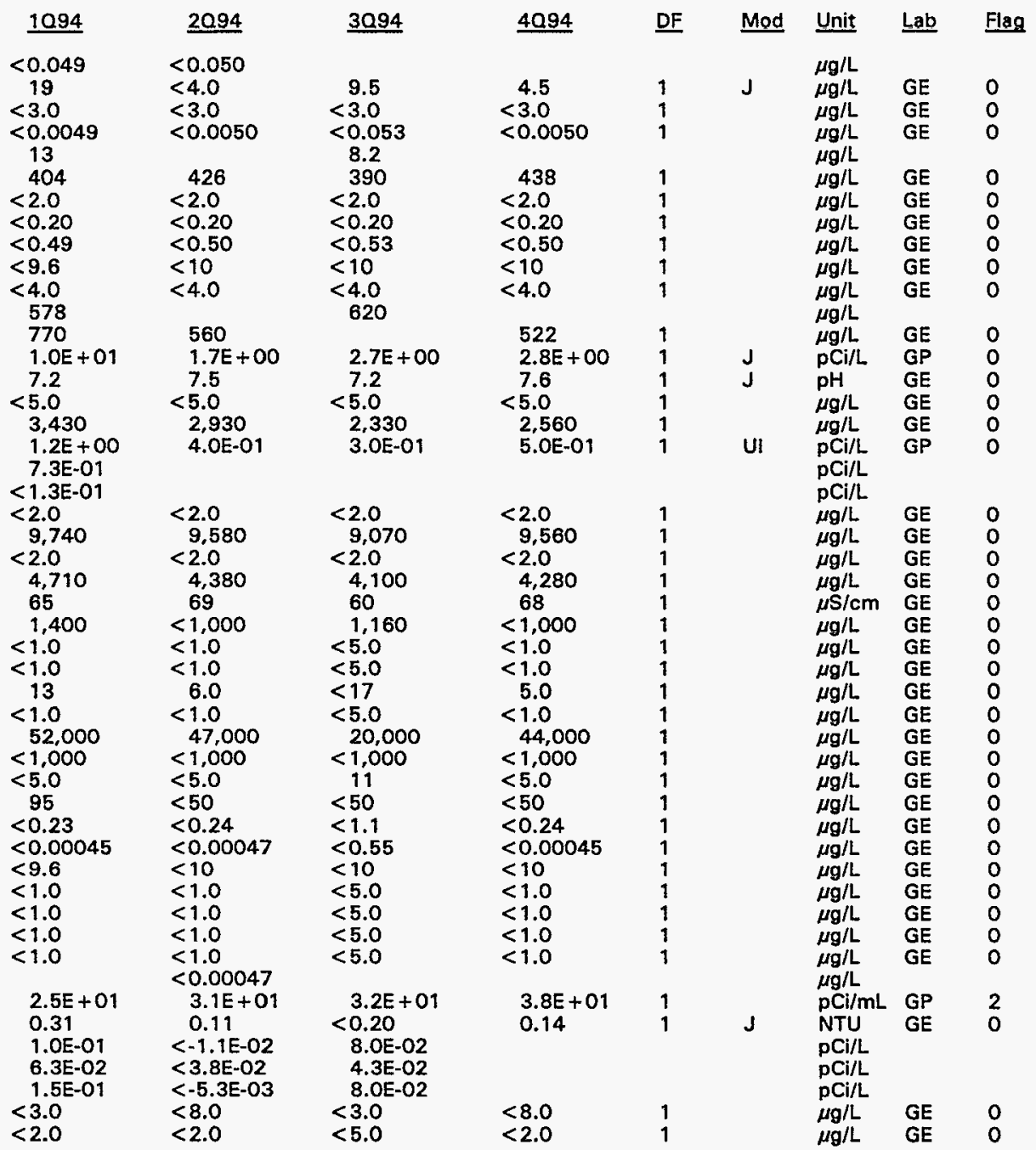

Note: Flagging, dilution factors, modifiers, and laboratories are for fourth quarter 1994 data only. See Appendix B for flagging criteria. - II exceeded holding time for fourth quarter 1994.

- = exceeded screening level or final PDWS for fourth quarter 1994. 


\section{WELL BGO 49D}

\begin{tabular}{|c|c|c|c|c|c|c|c|}
\hline SRS Coord. & Lat/Longitude & Screen Zone Elevation & \multicolumn{2}{|l|}{ Top of Casing } & Casing & Pump & \multirow{3}{*}{$\begin{array}{l}\text { Screen Zone } \\
\text { Water Table (IIB2) }\end{array}$} \\
\hline $\begin{array}{l}\text { N73931.5 } \\
\text { E56198.8 }\end{array}$ & $\begin{array}{l}33.280983^{\circ} \mathrm{N} \\
81.662428{ }^{\circ} \mathrm{W}\end{array}$ & $238.5-218.5 \mathrm{ft} \mathrm{msl}$ & \multicolumn{2}{|l|}{$271.5 \mathrm{ft} \mathrm{mst}$} & 4" PVC & $\mathbf{S}$ & \\
\hline \multicolumn{2}{|c|}{ SAMPLE DATE } & $01 / 28 / 94$ & $04 / 14 / 94$ & $8 / 16 / 94$ & $10 / 21 / 94$ & & \\
\hline \multicolumn{8}{|l|}{ FIELD DATA } \\
\hline \multicolumn{2}{|c|}{ Analyte } & 1094 & 2094 & 3094 & 4094 & Unit & \\
\hline \multicolumn{2}{|c|}{$\begin{array}{l}\text { Water elevation } \\
\text { Depth to water } \\
\text { pH } \\
\text { Sp. conductance } \\
\text { Water temperature } \\
\text { Alkalinity as } \mathrm{CaCO}_{3} \\
\text { Turbidity } \\
\text { Volume purged } \\
\text { Sampling code }\end{array}$} & $\begin{array}{l}233.1 \\
38.4 \\
5.1 \\
29 \\
17.0 \\
1 \\
161 \\
0.8 \\
x\end{array}$ & $\begin{array}{l}234.6 \\
36.9 \\
5.4 \\
39 \\
17.7 \\
1 \\
10 \\
0.9 \\
\times\end{array}$ & $\begin{array}{l}33.0 \\
38.5 \\
.1 \\
32 \\
9.0 \\
8 \\
.8\end{array}$ & $\begin{array}{l}233.0 \\
38.5 \\
5.3 \\
27 \\
17.2 \\
1 \\
48 \\
0.8 \\
\times\end{array}$ & $\begin{array}{l}\text { ft msl } \\
\mathrm{ft} \\
\mathrm{pH} \\
\mu \mathrm{s} / \mathrm{cm} \\
{ }^{\circ} \mathrm{C} \\
\mathrm{mg} / \mathrm{L} \\
\text { NTU } \\
\text { well vol. }\end{array}$ & \\
\hline
\end{tabular}

\section{ANALYTICAL DATA}

H $\underline{\text { ST }}$ Analyte

Acetophenone

Aldrin

Aluminum, total recoverable

Antimony, total recoverable

Arsenic, total recoverable

Barium, total recoverable

Benzene

Bromodichloromethane

Bromoform

Bromomethane (Methyl bromide)

Cadmium, total recoverable

Calcium, total recoverable

Carbon tetrachloride

Chloride

Chlorobenzene

Chloroethane

Chloroethene (Vinyl chloride)

2-Chloroethyl vinyl ether

Chloroform

Chloromethane (Methyl chloride)

Chromium, total recoverable

Copper, total recoverable

Cyanide

P.P०-DDT

Dibromochloromethane

1,1-Dichloroethane

1,2-Dichloroethane

1,1-Dichloroethylene

trans-1,2-Dichloroethylene

Dichloromethane

- 2,4-Dichlorophenoxyacetic acid

1,2-Dichloropropan

cis-1,3-Dichloropropene

trans-1,3-Dichloropropene

Dieldrin

Endrin

Ethylbenzene

Fluoride

Gross alpha

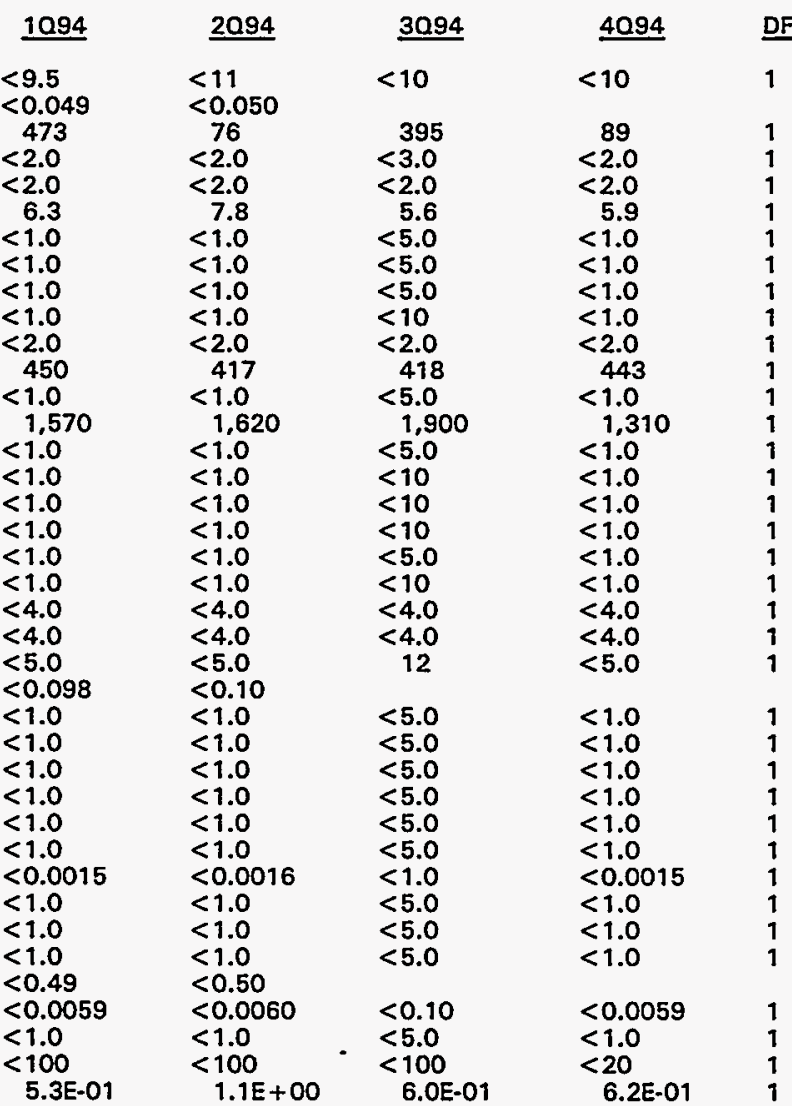

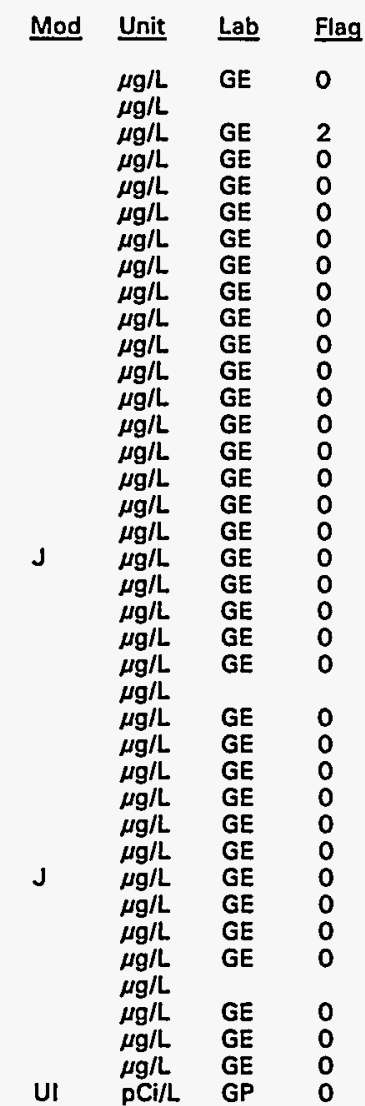

Note: Flagging, dilution factors, modifiers, and laboratories are for fourth quarter 1994 data only. See Appendix B for flagging criteria. - = exceeded holding time for fourth quarter 1994.

- = exceeded screening level or final PDWS for fourth quarter 1994. 


\section{H ST Analyte}

Heptachlor

Iron, total recoverable

Lead, total recoverable

Lindane

Lithium, total recoverable

Magnesium, total recoverable

Manganese, total recoverable

Mercury, total recoverable

Methoxychlor

Naphthalene

Nickel, total recoverable

Nitrate as nitrogen

Nitrate-nitrite as nitrogen

Nonvolatile beta

- $\quad \mathrm{pH}$

Phenols

Potassium, total recoverable

Radium, total alpha-emitting

Selenium, total recoverable

Silica, total recoverable

Silver, total recoverable

Sodium, total recoverable

Specific conductance

Sulfate

1,1,2,2-Tetrachloroethane

Tetrachloroethylene

Tin, total recoverable

Toluene

Total dissolved solids

Total organic carbon

Total organic halogens

Total phosphates (as P)

Toxaphene

- 2,4,5-TP (Silvex)

Tributyl phosphate

$1,1,1-$ Trichloroethane

1,1,2-Trichloroethane

Trichloroethylene

Trichlorofluoromethane

2,4,5-T

- Tritium

Turbidity

Uranium-233/234

Uranium-235

Uranium-238

Vanadium, total recoverable

Xylenes

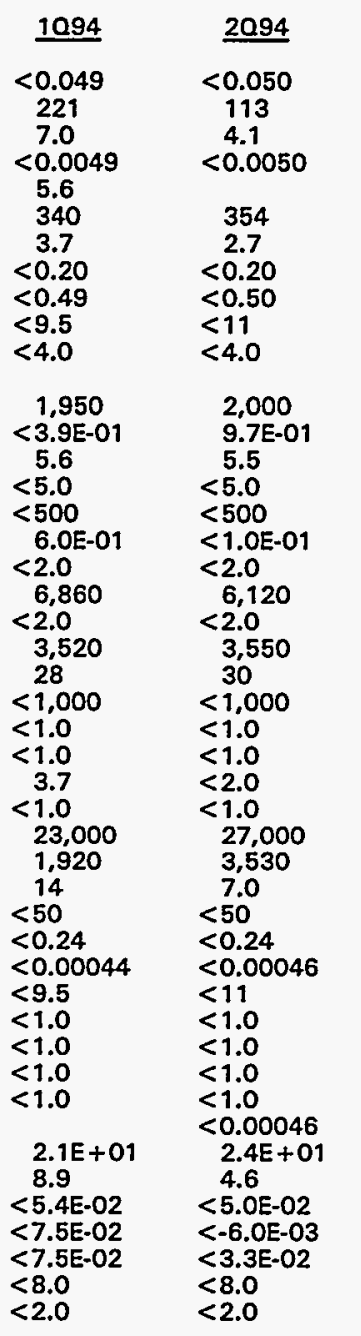

3094

$4094 \quad$ DF

Mod

$\begin{array}{ll} & 504 \\ & 5.1 \\ < & 0.052 \\ < & 5.0\end{array}$

80
5.2

$<0.0049$

341

3.5

$<0.52$

$<10$
$<4.0$

1,900

1.1E+00

5.5

$<5.0$

$<500$

6.0E-01

$<2.0$

7.220

$<2.0$

3,240

29

$<1,000$

$<5.0$

$<5.0$

$<17$

$<5.0$

5,000

2,200

9.6

188

$<1.0$

$<0.52$

$<10$

$<5.0$

$<5.0$

$<5.0$

$<5.0$

2.7E +01

1.8

1.2E-01

$0.0 E+00$

$6.5 \mathrm{E}-02$

$<3.0$
$<5.0$
352
2.3

$<0.20$

$<0.49$

$<10$

$<4.0$

1,890

9.1E-01

6.1
$<5.0$

$<500$

6.0E-01

$<2.0$

6,070

$<2.0$

3,330

$<1,000$

$<1.0$

$<1.0$

$<1.0$

$<1.0$

22,000

$<1,000$

$<5.0$

$<50$

$<0.24$

$<0.00044$

$<10$

$<1.0$

$<1.0$

$<1.0$

$<1.0$

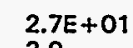

2.9

$<8.0$

\section{DF}

Unit Lab Flag

$\mu \mathrm{g} / \mathrm{L}$

$\mu g / L$ GE 0

Hg/L GE

$\mu \mathrm{g} / \mathrm{L}$

J

$\mu g / L$ GE $\quad 0$

$\mu g / L$ GE 0

$\mu g / L$ GE 0

$\mu g / L$ GE 0

$\mu \mathrm{g} / \mathrm{L} \quad \mathrm{GE}$

$\mu g / L$

$\mu \mathrm{g} / \mathrm{L} \quad \mathrm{GE}$

UI $\quad$ pCi/L GP

$\mu \mathrm{g} / \mathrm{L} \quad \mathrm{GE}$

$\mu \mathrm{g} / \mathrm{L} \quad \mathrm{GE}$

J

pCi/L GP 0

$\mu \mathrm{g} / \mathrm{L}$ GE 0

$\mu g / L \quad G E$

$\mu g / L \quad G E$

$\mu \mathrm{g} / \mathrm{L}$

$\mu S / \mathrm{cm}$ GE

$\mu \mathrm{g} / \mathrm{L} \quad \mathrm{GE}$

$\mu \mathrm{g} / \mathrm{L} \quad \mathrm{GE}$

$\mu \mathrm{g} / \mathrm{L} \quad \mathrm{GE}$

$\mu \mathrm{g} / \mathrm{L} \quad \mathrm{GE}$

$\mu \mathrm{g} / \mathrm{L} \quad \mathrm{GE}$

$\mu g / L \quad G E$

$\mu g / L \quad G E$

$\mu g / L$

$\mu \mathrm{g} / \mathrm{L}$

$\mu g / L \quad G E$

$\mu g / L \quad G E$

$\mu g / L \quad G E$

$\mu g / L \quad G E$

$\mu g / L \quad G E$

$\mu g / L \quad G E$

$\mu \mathrm{g} / \mathrm{L} \quad \mathrm{GE}$

$\mu g / L$

$\mu \mathrm{g} / \mathrm{L}$

$<2.0$

$<8.0$

$<8.0$

NTU

GP $\quad 2$

pCi/L

$\mathrm{pCi} / \mathrm{L}$

$\mu g / L$ GE 0

$\mu g / L$ GE

Note: Flagging, dilution factors, modifiers, and laboratories are for fourth quarter 1994 data only. See Appendix B for flagging criteria. - = exceeded holding time for fourth quarter 1994.

- = excéeded screening level or final PDWS for fourth quarter 1994. 
WELL BGO 50A

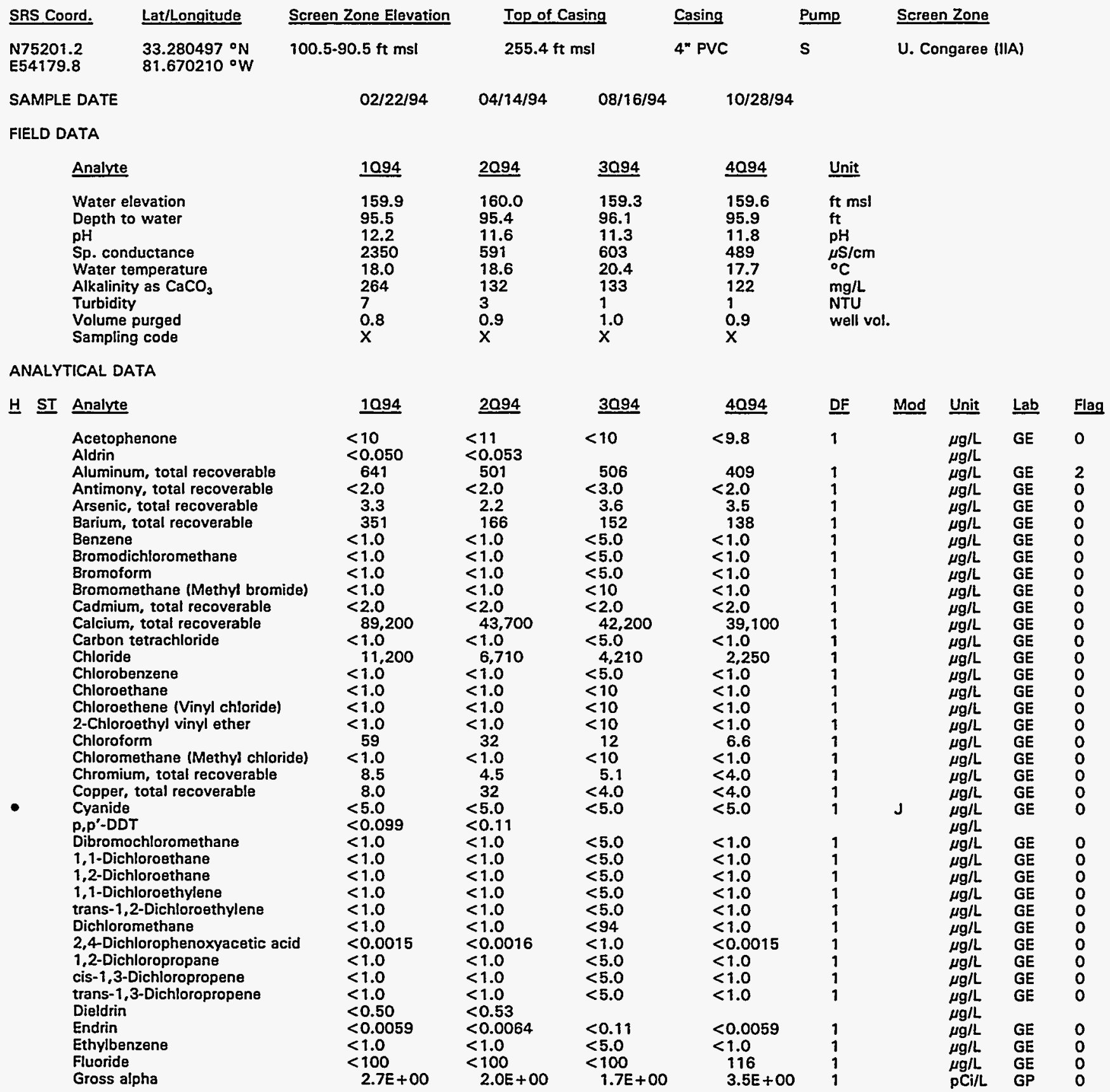

Note: Flagging, dilution factors, modifiers, and laboratories are for fourth quarter 1994 data only. See Appendix B for flagging criteria. - = exceeded holding time for fourth quarter 1994.

- = exceeded screening level or final PDWS for fourth quarter 1994. 


\section{H ST Analyte}

Heptachlor

Iron, total recoverable

Lead, total recoverable

Lindane

Lithium, total recoverable

Magnesium, total recoverable

Manganese, total recoverable

Mercury, total recoverable

Methoxychlor

Naphthalene

Nickel, total recoverable

Nitrate as nitrogen

Nitrate-nitrite as nitrogen

Nonvolatile beta

pH

Phenols

Potassium, total recoverable

Radium, total alpha-emitting

Selenium, total recoverable

Silica, total recoverable

Silver, total recoverable

Sodium, total recoverable

Specific conductance

Sulfate

1,1,2,2-Tetrachloroethane

Tetrachloroethylene

Tin, total recoverable

Toluene

Total dissolved solids

Total organic carbon

Total organic halogens

Total phosphates (as P)

Toxaphene

2,4,5-TP (Silvex)

Tributyl phosphate

1,1,1-Trichloroethane

1,1,2-Trichloroethane

Trichloroethylene

Trichlorofluoromethane

$2,4,5-T$

Tritium

Turbidity

Uranium-233/234

Uranium-235

Uranium-238

Vanadium, total recoverable

Xylenes

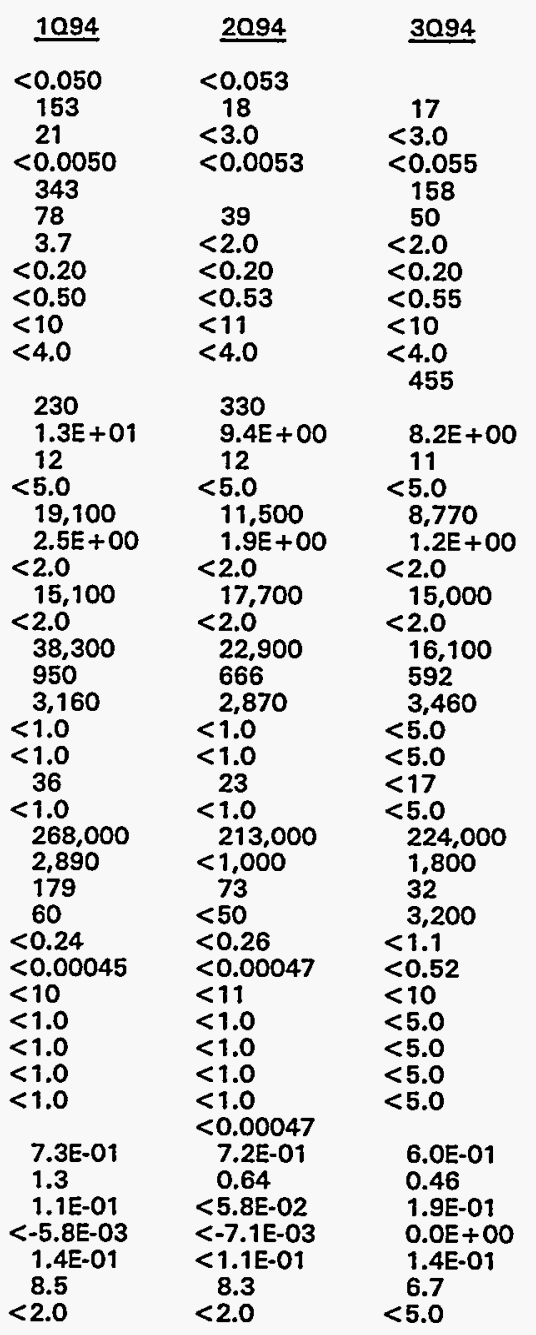

4094

DF

8.8
$<3.0$

$<0.0050$

63
$<20$

$<0.20$

$<0.50$

$<9.8$

$<4.0$

$2.1 \mathrm{E}+00$

12
$<5.0$

7,910

1.1E+00

$<2.0$

17,400

$<2.0$

14,500

512

2,680
$<1.0$

$<1.0$

15
$<1.0$

169,000

$<1,000$

9.8
$<50$

$<0.24$

$<0.00045$

$<9.8$

$<1.0$

$<1.0$

$<1.0$

$<1.0$

1.3E-01

0.66

5.6E-02

2.8E-02

$5.6 \mathrm{E}-02$

$<8.0$

$<2.0$
Mod

Unit Lab Flag

$\mu g / L \quad$ GE $\quad 0$

$\mu g / L \quad G E \quad 0$

$\mu g / L \quad G E$

$\mu g / L \quad G E$

$\mu g / L \quad G E$

$\mu g / L \quad G E$

$\begin{array}{ll}\mu g / L & G E \\ \mu g / L & G E\end{array}$

$\mu \mathrm{g} / \mathrm{L} \quad \mathrm{GE}$

$\mu g / L$

$\mu g / L$ GE 0

$\mathrm{pH}$ * GE 2

UGIL GE

$\mu g / L$ GE 0

J

$\mathrm{pCi} / \mathrm{L}$ GP 0

$\mu \mathrm{g} / \mathrm{L} \quad \mathrm{GE}$

$\mu g / L \quad \mathrm{GE}$

$\mu \mathrm{g} / \mathrm{L} \quad \mathrm{GE}$

$\mu S / \mathrm{cm} \quad \mathrm{GE}$

$\mu g / L \quad G E$

$\mu \mathrm{g} / \mathrm{L} \quad \mathrm{GE}$

$\mu g / L$

$\mu g / L$

J

$\mu \mathrm{g} / \mathrm{L} \quad \mathrm{GE}$

$\mu \mathrm{g} / \mathrm{L} \quad \mathrm{GE}$

$\mu g / L \quad$ GE

$\mu \mathrm{g} / \mathrm{L} \quad \mathrm{GE}$

$\mu \mathrm{g} / \mathrm{L} \quad \mathrm{GE}$

$\mu \mathrm{g} / \mathrm{L} \quad \mathrm{GE}$

$\mu \mathrm{g} / \mathrm{L} \quad \mathrm{GE}$

$\underset{\mu g / L}{\mu g / L} \quad \mathrm{GE}$

$\mu \mathrm{g} / \mathrm{L} \quad \mathrm{GE}$

$\mu g / L \quad G E$

$\mu \mathrm{g} / \mathrm{L}$

$\mathrm{pCi} / \mathrm{mL}$ GP

NTU GE

NTU

pCi/L

$\mathrm{pCi} /$
$\mathrm{pCi} /$
$\mu \mathrm{g} / \mathrm{h}$

$\mu g / L$

0

0

0

Note: Flagging, dilution factors, modifiers, and laboratories are for fourth quarter 1994 data only. See Appendix B for flagging criteria. - = exceeded holding time for fourth quarter 1994.

m = exceeded screening level or final PDWS for fourth quarter 1994. 
WSRC-TR-94-0610

Unclassified

WELL BGO 50C

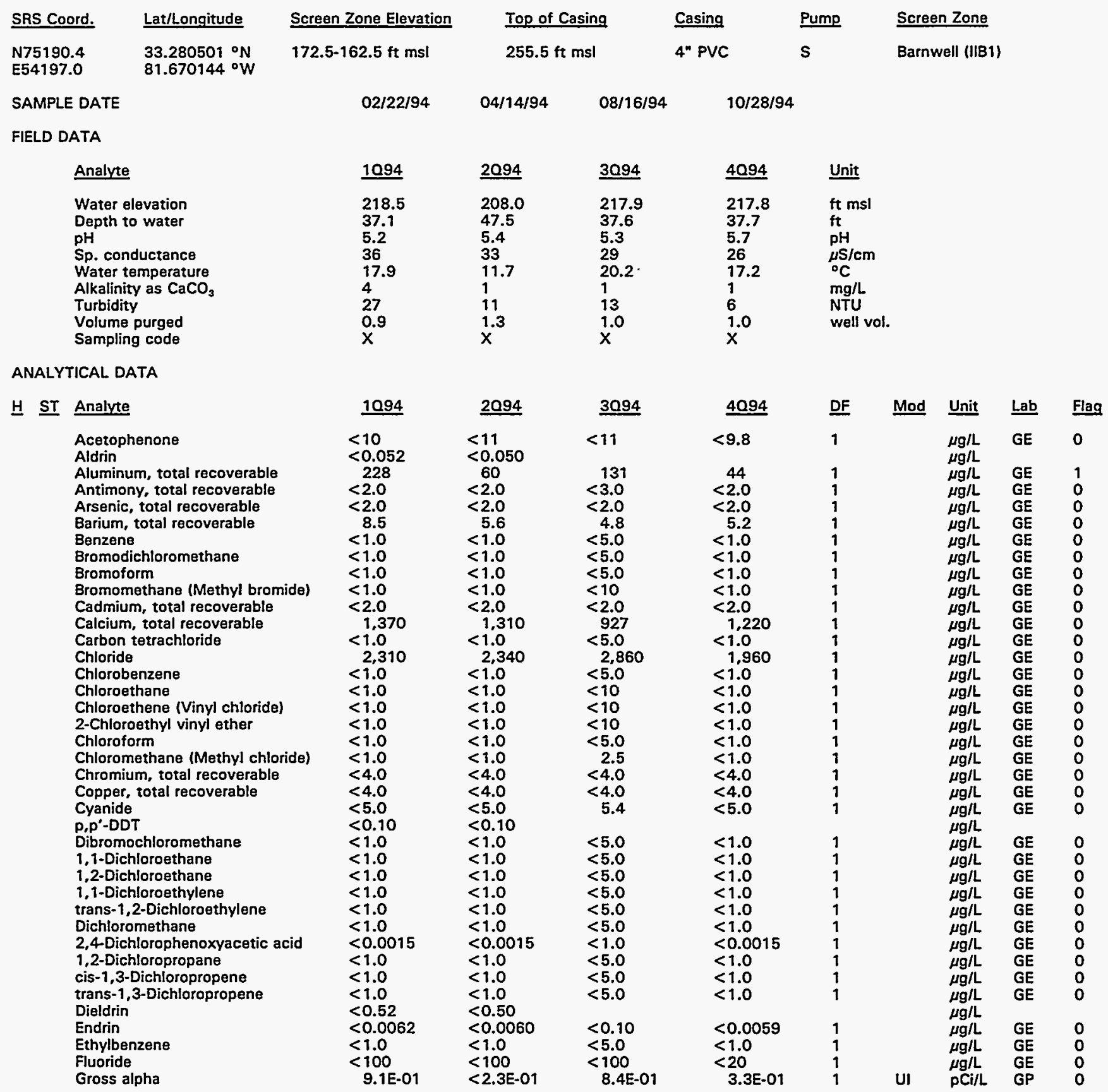

Note: Flagging, dilution factors, modifiers, and laboratories are for fourth quarter 1994 data only. See Appendix B for flagging criteria. - = exceeded holding time for fourth quarter 1994.

- = exceeded screening level or final PDWS for fourth quarter 1994. 
H ST Analyte

Heptachlor
lron, total recoverable
Lead, total recoverable
Lindane
Lithium, total recoverable
Magnesium, total recoverable
Manganese, total recoverable
Mercury, total recoverable
Methoxychlor
Naphthalene
Nickel, total recoverable
Nitrate as nitrogen
Nitrate-nitrite as nitrogen
Nonvolatile beta
pH
Phenols
Potassium, total recoverable
Radium, total alpha-emitting
Selenium, total recoverable
Silica, total recoverable
Silver, total recoverable
Sodium, total recoverable
Specific conductance
Sulfate
1,1,2,2-Tetrachloroethane
Tetrachloroethylene
Tin, total recoverable
Toluene
Total dissolved solids
Total organic carbon
Total organic halogens
Total phosphates (as P)
Toxaphene
$2,4,5-T P$ (Silvex)
Tributyl phosphate
1,1,1-Trichloroethane
1,1,2-Trichloroethane
Trichloroethylene
Trichlorofluoromethane
$2,4,5-T$
Tritium
Turbidity
Uranium-233/234
Uranium-235
Uranium-238
Vanadium, total recoverable
Xylenes

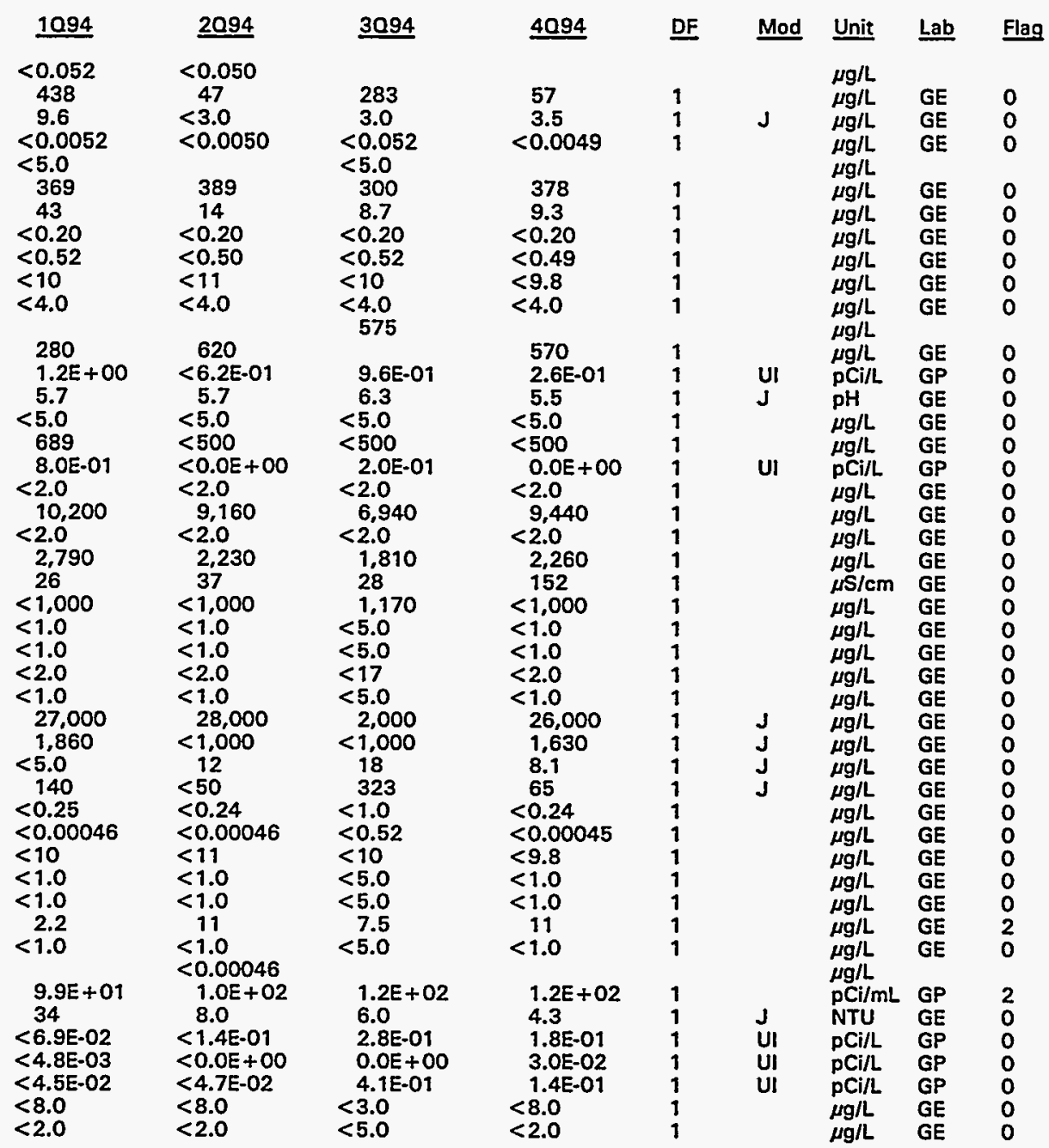

Note: Flagging, dilution factors, modifiers, and laboratories are for fourth quarter 1994 data only. See Appendix B for flagging criteria. - = exceeded holding time for fourth quarter 1994.

= exceeded screening level or final PDWS for fourth quarter 1994. 


\section{WELL BGO 50D}

\section{SRS Coord. \\ N75181.3 \\ E54209.1}

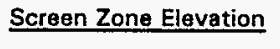

228.0-208.0 $\mathrm{tt} \mathrm{msl}$

$02 / 21 / 94$

\section{Top of Casing \\ $256 \mathrm{ft} \mathrm{msl}$}

$\begin{array}{ll}\text { Casing } & \text { Pump } \\ \text { 4" PVC }^{\text {"PV }} & \mathrm{s}\end{array}$

$10 / 24 / 94$

FIELD DATA

Analyte
Water elevation
Depth to water
pH
Sp. conductance
Water temperature
Alkalinity as $\mathrm{CaCO}_{3}$
Turbidity
Volume purged
Sampling code

$\begin{array}{ll}1094 & 2094 \\ 224.8 & 225.4 \\ 31.2 & 30.6 \\ 6.1 & 6.0 \\ 63 & 74 \\ 20.0 & 20.0 \\ 18 & 14 \\ 1 & 1 \\ 4.5 & 4.3\end{array}$

ANALYTICAL DATA

\section{H ST Analyte}

Acetophenone

Aldrin

Aluminum, total recoverable

Antimony, total recoverable

Arsenic, total recoverable

Barium, total recoverable

Benzene

Bromodichloromethane

Bromoform

Bromomethane (Methyl bromide)

Cadmium, total recoverable

Calcium, total recoverable

Carbon tetrachloride

Chloride

Chlorobenzene

Chloroethane

Chloroethene (Vinyl chloride)

2-Chloroethyl vinyl ether

Chloroform

Chloromethane (Methyl chloride)

Chromium, total recoverable

Copper, total recoverable

Cyanide

p.p'-DDT

Dibromochloromethane

1,1-Dichloroethane

1,2-Dichloroethane

1,1-Dichloroethylene

trans-1,2-Dichloroethylene

Dichloromethane

2,4-Dichlorophenoxyacetic acid

3,2-Dichloropropane

cis-1,3-Dichloropropene

trans-1,3-Dichloropropene

Dieldrin

Endrin

Ethylbenzene

Fluoride

Gross alpha

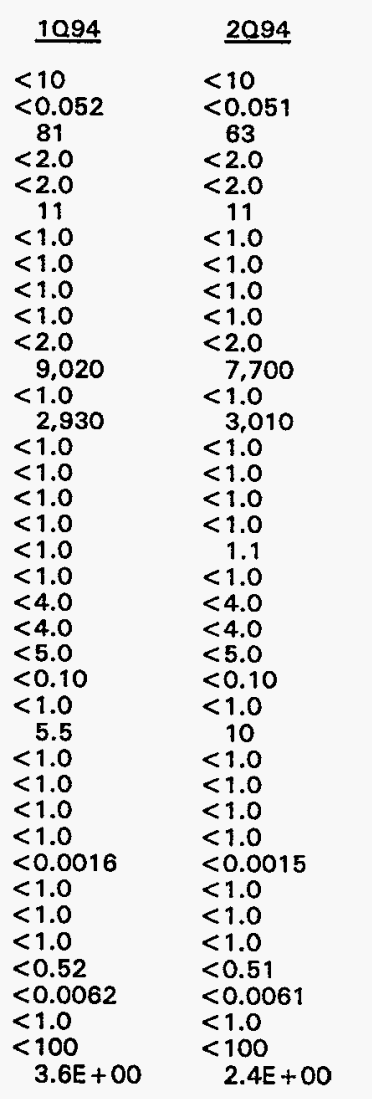

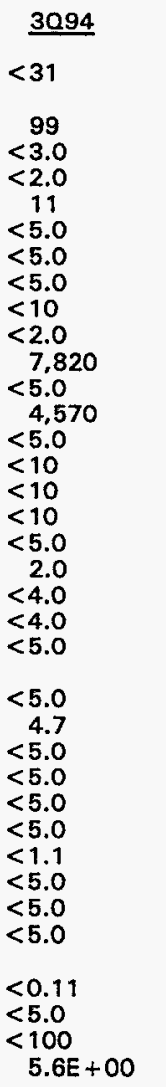

$\underline{\text { Unit }}$

ft msi

$\mathrm{ft}$

$\mathrm{pH}$
$\mu \mathrm{S} / \mathrm{cm}$

${ }^{\circ} \mathrm{C}$

$\mathrm{mg} / \mathrm{L}$

well vol.
Screen Zone

Water Table (IIB2)
20.6

5

18

3.8

$\begin{array}{ll}31.9 & \mathrm{ft} \\ 6.3 & \mathrm{pH} \\ 65 & \mu \mathrm{s} / \mathrm{cm} \\ 19.7 & { }^{\circ} \mathrm{C} \\ 18 & \mathrm{mg} / \mathrm{L} \\ 1 & \mathrm{NTU} \\ 3.8 & \text { well vol }\end{array}$

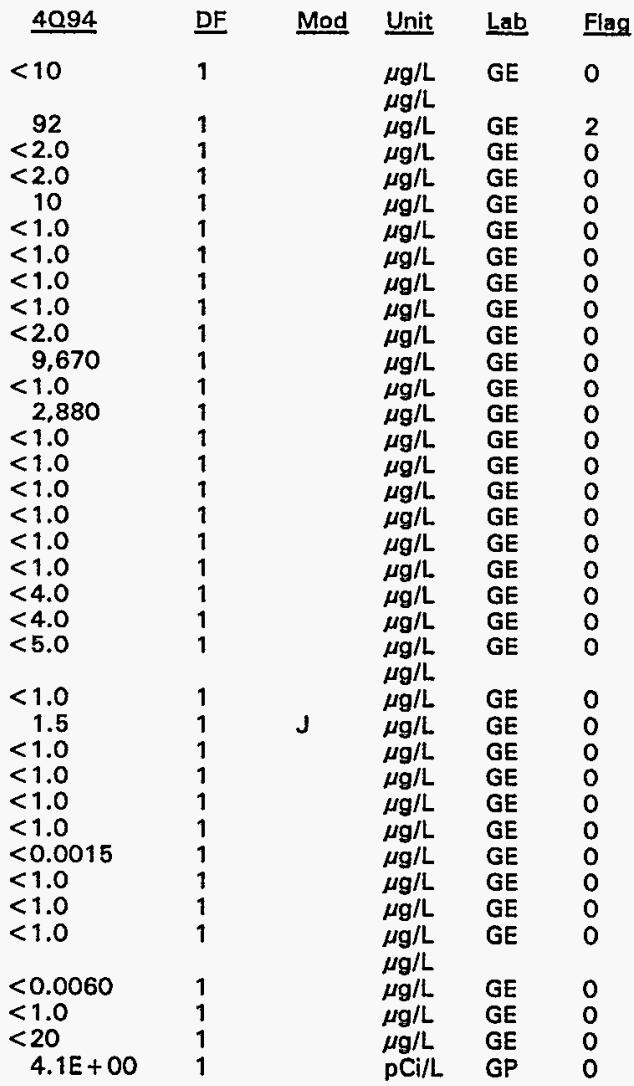

Note: Flagging, dilution factors, modifiers, and laboratories are for fourth quarter 1994 data only. See Appendix B for flagging criteria. - II exceeded holding time for fourth quarter 1994.

- = exceeded screening level or final PDWS for fourth quarter 1994. 


\section{ANALYTICAL DATA}

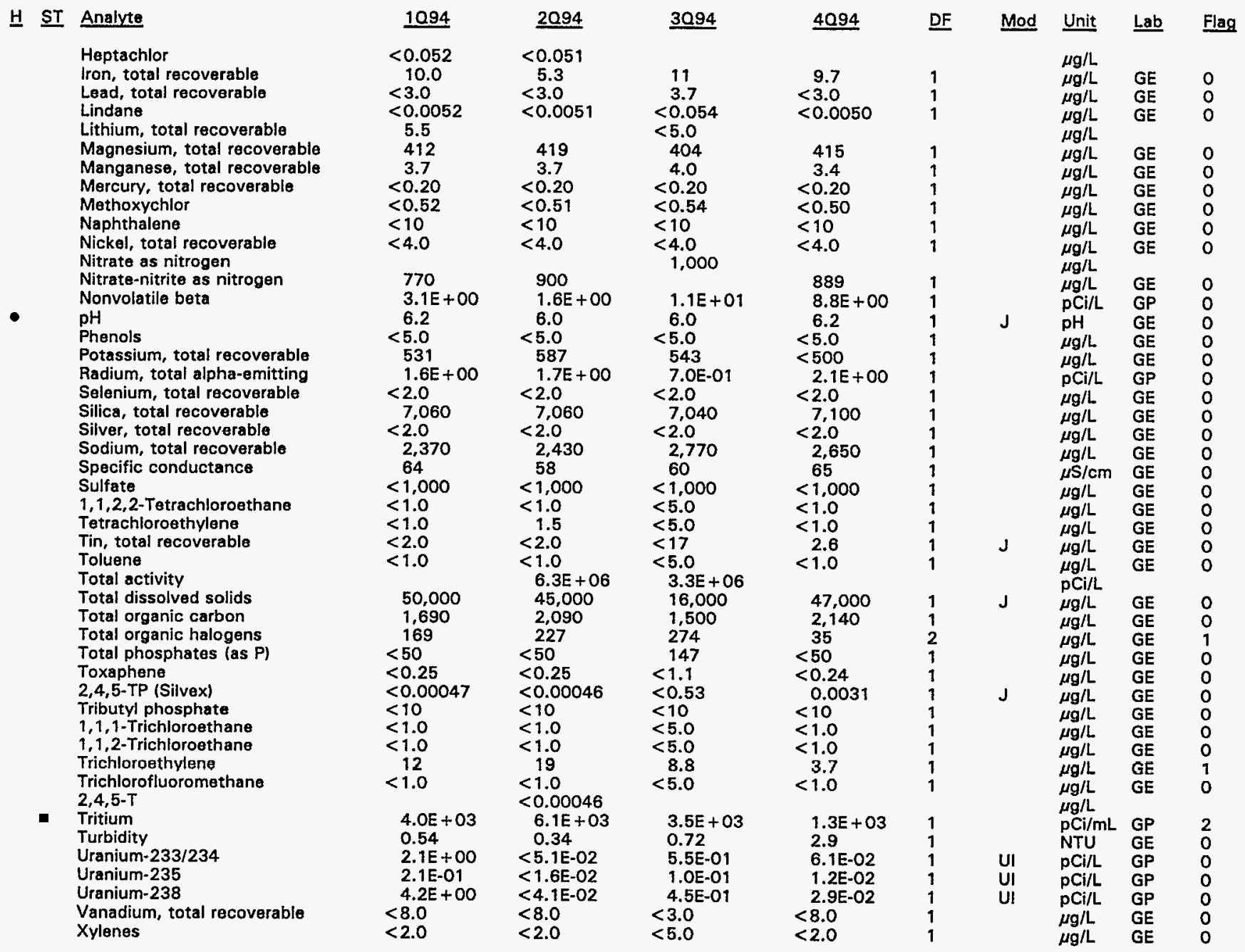

Note: Flagging, dilution factors, modifiers, and laboratories are for fourth quarter 1994 data only. See Appendix B for flagging criteria. - = exceeded holding time for fourth quarter 1994.

- = exceeded screening level or final PDWS for fourth quarter 1994. 


\section{WELL BGX 1A}

\begin{tabular}{|c|c|c|c|c|c|c|c|}
\hline SRS Coord. & Lat/Longitude & Screen Zone Elevation & Top of & & Casing & Pump & Screen Zone \\
\hline $\begin{array}{l}\text { N76831.9 } \\
\text { E58590.4 }\end{array}$ & $\begin{array}{l}33.291300^{\circ} \mathrm{N} \\
81.661764^{\circ} \mathrm{W}\end{array}$ & $124.1-114.1 \mathrm{ft} \mathrm{msl}$ & 291.2 & & 4" PVC & $\mathbf{S}$ & U. Congaree (IIA) \\
\hline SAMPLE DA & & $03 / 22 / 94$ & $04 / 13 / 94$ & $09 / 28 / 94$ & $11 / 07$ & & \\
\hline FIELD DATA & & & & & & & \\
\hline Ana & & 1094 & $\underline{2094}$ & $\underline{3094}$ & $\underline{4094}$ & $\underline{\text { Un }}$ & \\
\hline $\begin{array}{l}\text { Wat } \\
\text { Dep } \\
\text { pH } \\
\text { Sp. } \\
\text { Wat } \\
\text { Alka } \\
\text { Turt } \\
\text { Volu } \\
\text { Sam }\end{array}$ & $\begin{array}{l}\text { evation } \\
\text { water } \\
\text { fuctance } \\
\text { imperature } \\
\text { as } \mathrm{CaCO}_{3} \\
\text { purged } \\
\text { code }\end{array}$ & $\begin{array}{l}158.1 \\
133.1\end{array}$ & $\begin{array}{l}158.2 \\
133.1\end{array}$ & $\begin{array}{l}157.4 \\
133.8\end{array}$ & $\begin{array}{l}157.9 \\
133.4\end{array}$ & $\begin{array}{l}\mathrm{ft} \\
\mathrm{ft} \\
\mathrm{pH} \\
\mu \mathrm{S} \\
{ }^{\circ} \mathrm{C} \\
\mathrm{mg} \\
\mathrm{NT} \\
\text { we }\end{array}$ & \\
\hline
\end{tabular}

WELL BGX 1C

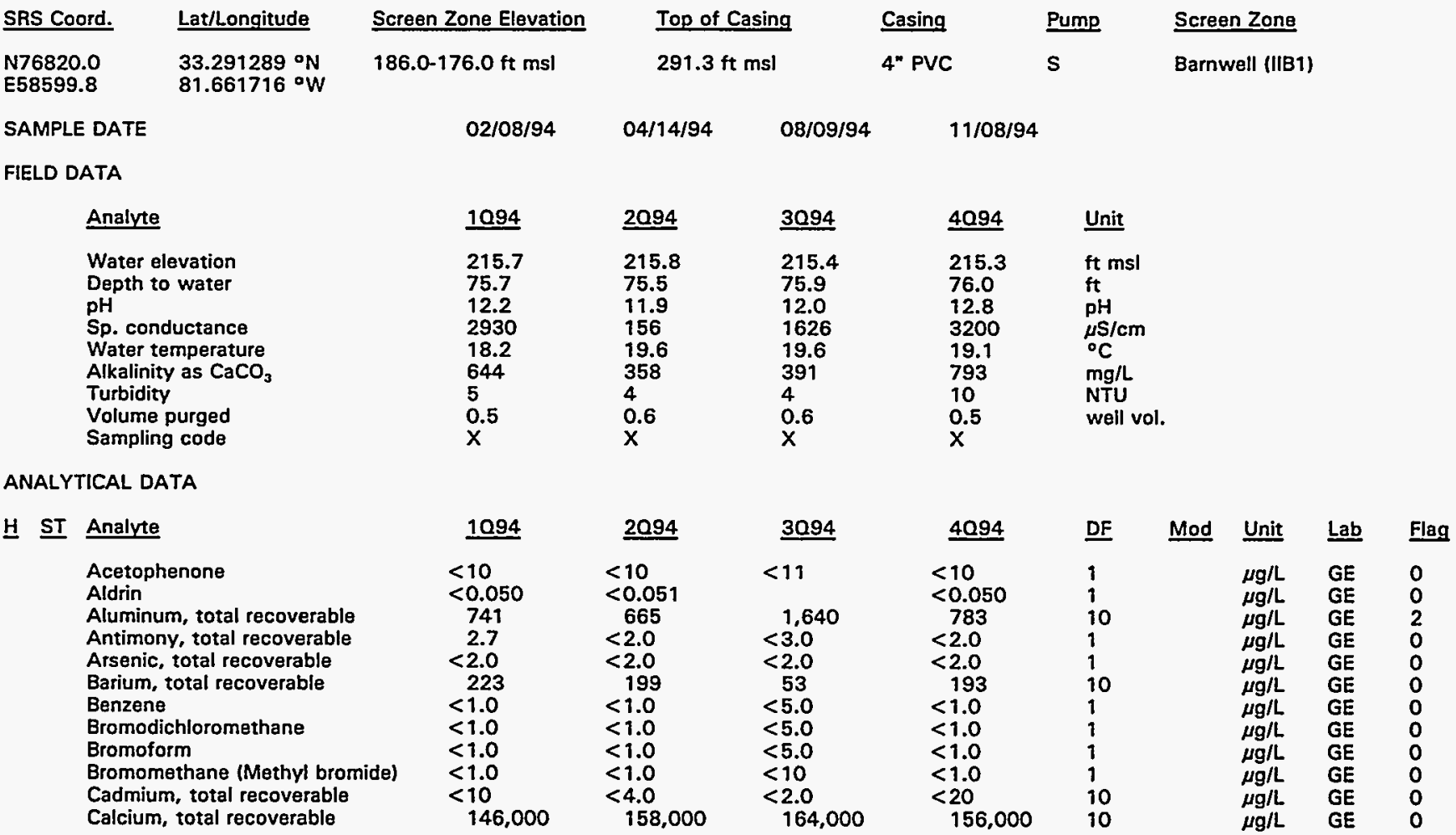

Note: Flagging, dilution factors, modifiers, and laboratories are for fourth quarter 1994 data only. See Appendix B for flagging criteria. - = exceeded holding time for fourth quarter 1994.

- = exceeded screening level or final PDWS for fourth quarter 1994. 
H $\underline{\text { ST }}$ Analyte

Carbon tetrachloride

Chloride

Chlorobenzene

Chloroethane

Chloroethene (Vinyl chloride)

2-Chloroethyl vinyl ether

Chloroform

Chloromethane (Methyl chloride)

Chromium, total recoverable

Copper, total recoverable

Cyanide

$p, p^{\prime}$-DDT

Dibromochloromethane

1,1-Dichloroethane

1,2-Dichloroethane

1, 1-Dichloroethylene

trans-1,2-Dichloroethylene

Dichloromethane

2,4-Dichlorophenoxyacetic acid

1,2-Dichloropropane

cis-1,3-Dichloropropene

trans-1,3-Dichloropropene

Dieldrin

Endrin

Ethylbenzene

Fluoride

Gross alpha

Heptachlor

Iron, total recoverable

Lead, total recoverable

Lindane

Lithium, total recoverable

Magnesium, total recoverable

Manganese, total recoverable

Mercury, total recoverable

Methoxychlor

Naphthalene

Nickel, total recoverable

Nitrate as nitrogen

Nitrate-nitrite as nitrogen

Nonvolatile beta

pH

Phenols

Potassium, total recoverable

Radium, total alpha-emitting

Selenium, total recoverable

Silica, total recoverable

Silver, total recoverable

Sodium, total recoverable

Specific conductance

Sulfate

1,1,2,2-Tetrachloroethane

Tetrachloroethylene

Tin, total recoverable

Toluene

Total activity

Total dissolved solids

Total organic carbon

Total organic halogens

Total phosphates (as P)

Toxaphene

2,4,5-TP (Silvex)

\begin{tabular}{|c|c|c|}
\hline 1094 & $\underline{2094}$ & 3094 \\
\hline$<1.0$ & $<1.0$ & $<5.0$ \\
\hline $\begin{array}{r}827 \\
<1.0\end{array}$ & $\begin{array}{r}935 \\
<1.0\end{array}$ & $\begin{array}{r}3,350 \\
<50\end{array}$ \\
\hline$<1.0$ & $<1.0$ & $\begin{array}{l}<5.0 \\
<10\end{array}$ \\
\hline$<1.0$ & $<1.0$ & $<10$ \\
\hline$<1.0$ & $<1.0$ & $<10$ \\
\hline$<1.0$ & $<1.0$ & $<5.0$ \\
\hline$<1.0$ & $<1.0$ & $<10$ \\
\hline$<20$ & $<8.0$ & 14 \\
\hline$<20$ & $<8.0$ & 5.6 \\
\hline $\begin{array}{l}<5.0 \\
<0.10\end{array}$ & $\begin{array}{l}<5.0 \\
<0.10\end{array}$ & $<5.0$ \\
\hline$<1.0$ & $<1.0$ & $<5.0$ \\
\hline$<1.0$ & $<1.0$ & $<5.0$ \\
\hline$<1.0$ & $<1.0$ & $<5.0$ \\
\hline$<1.0$ & $<1.0$ & $<5.0$ \\
\hline$<1.0$ & $<1.0$ & $<5.0$ \\
\hline$<1.0$ & $<1.0$ & $<5.0$ \\
\hline$<0.0016$ & $<0.0016$ & $<1.1$ \\
\hline$<1.0$ & $<1.0$ & $<5.0$ \\
\hline $\begin{array}{l}<1.0 \\
<1.0\end{array}$ & $<1.0$ & \\
\hline $\begin{array}{l}<1.0 \\
<0.50\end{array}$ & $\begin{array}{l}<1.0 \\
<0.51\end{array}$ & $<5.0$ \\
\hline$<0.0060$ & $<0.0061$ & $<0.11$ \\
\hline$<1.0$ & $<1.0$ & $<5.0$ \\
\hline $\begin{array}{l}118 \\
7.0 E+00\end{array}$ & $\begin{aligned}< & 100 \\
& 1.8 E+00\end{aligned}$ & $<100$ \\
\hline$<0.050$ & $<0.051$ & \multirow{9}{*}{$\begin{array}{l}3,480 \\
22 \\
<0.054 \\
<5.0 \\
777 \\
51 \\
<0.20 \\
<0.54 \\
<10 \\
12 \\
1,100\end{array}$} \\
\hline $\begin{array}{l}25 \\
21\end{array}$ & $\begin{array}{l}11 \\
8.8\end{array}$ & \\
\hline$<0.0050$ & $<0.0051$ & \\
\hline 300 & 170 & \\
\hline $\begin{array}{l}<10 \\
<0.20\end{array}$ & $\begin{array}{l}<4.0 \\
<0.20\end{array}$ & \\
\hline$<0.50$ & $<0.51$ & \\
\hline$<10$ & $<10$ & \\
\hline$<20$ & $<8.0$ & \\
\hline 270 & 380 & \\
\hline $1.7 E+01$ & $3.5 E+00$ & $5.4 \mathrm{E}+00$ \\
\hline$<5.0$ & $\begin{array}{r}12 \\
<5.0\end{array}$ & $\begin{array}{r}6.0 \\
<5.0\end{array}$ \\
\hline $\begin{array}{l}10,100 \\
2.1 E+\infty 0\end{array}$ & $\begin{array}{l}5,710 \\
1.5 \mathrm{E}+00\end{array}$ & 672 \\
\hline$<2.0$ & $<2.0$ & $<2.0$ \\
\hline 7,050 & 6,600 & 9,320 \\
\hline$<10$ & $<4.0$ & $<2.0$ \\
\hline $\begin{array}{l}7,360 \\
1.800\end{array}$ & 5,220 & 2,050 \\
\hline$<1,000$ & $\begin{array}{r}1,140 \\
<1,000\end{array}$ & $\begin{array}{c}50 \\
<1,000\end{array}$ \\
\hline$<1.0$ & $<1.0$ & $<1.000$ \\
\hline$<1.0$ & $<1.0$ & $<5.0$ \\
\hline$<10$ & 4.7 & $<17$ \\
\hline$<1.0$ & $\begin{array}{c}<1.0 \\
1.5 E+06\end{array}$ & $\begin{array}{l}<5.0 \\
1.9 E+06\end{array}$ \\
\hline 430,000 & 412,000 & 57,000 \\
\hline$<1,000$ & $<1,000$ & $<1,000$ \\
\hline $\begin{array}{l}5.4 \\
100\end{array}$ & $\begin{array}{c}<5.0 \\
60\end{array}$ & $\begin{array}{l}7.5 \\
348\end{array}$ \\
\hline$<0.24$ & $<0.24$ & $<1.1$ \\
\hline$<0.0$ & $<0.00046$ & $<0.53$ \\
\hline
\end{tabular}

\begin{tabular}{|c|c|c|c|c|}
\hline$\underline{\mathrm{DF}}$ & Mod & $\underline{\text { Unit }}$ & $\underline{\text { Lab }}$ & Flag \\
\hline 1 & & $\mu \mathrm{g} / \mathrm{L}$ & $\mathrm{GE}$ & 0 \\
\hline 1 & & $\mu g / L$ & $G E$ & 0 \\
\hline 1 & & & $\overline{G E}$ & 0 \\
\hline$i$ & & $\mu \mathrm{g} / \mathrm{L}$ & $\mathrm{GE}$ & 0 \\
\hline 1 & & $\mu g / L$ & $\mathrm{GE}$ & 0 \\
\hline 1 & & & $\mathrm{GE}$ & 0 \\
\hline 1 & & $\mu g / L$ & GE & 0 \\
\hline 1 & & $\mu g / L$ & $\mathrm{GE}$ & 0 \\
\hline 10 & & $\mu g / L$ & $\mathrm{GE}$ & 0 \\
\hline 10 & & $\mu g / h$ & GE & 0 \\
\hline 1 & & $\mu g / L$ & GE & 0 \\
\hline & & $\mu g / L$ & GE & 0 \\
\hline$i$ & & ${ }_{\mu \mathrm{g} / \mathrm{L}}$ & $\begin{array}{l}\mathrm{GE} \\
\mathrm{GE}\end{array}$ & $\begin{array}{l}0 \\
0\end{array}$ \\
\hline$i$ & & $\mu g / L$ & $\mathrm{GE}$ & 0 \\
\hline 1 & & pg/L & $\begin{array}{l}\text { GE } \\
\text { GE }\end{array}$ & 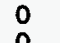 \\
\hline$i$ & & $\underset{\mu g / L}{\mu g / L}$ & $\begin{array}{l}\mathrm{GE} \\
\mathrm{GE}\end{array}$ & $\begin{array}{l}0 \\
0\end{array}$ \\
\hline 1 & & $\mu \mathrm{g} / \mathrm{L}$ & GE & 0 \\
\hline 1 & & $\underset{\mu \mathrm{g} / \mathrm{L}}{\mu}$ & $\begin{array}{l}\mathrm{GE} \\
\mathrm{GE}\end{array}$ & $\begin{array}{l}0 \\
0\end{array}$ \\
\hline 1 & & $\mu \mathrm{g} / \mathrm{L}$ & GE & 0 \\
\hline 1 & & $\mu \mathrm{g} / \mathrm{L}$ & $\mathrm{GE}$ & 0 \\
\hline & & $\mu \mathrm{g} / \mathrm{L}$ & GE & 0 \\
\hline$\frac{1}{1}$ & & $\mu \mathrm{g} / \mathrm{L} / \mathrm{L}$ & $\begin{array}{c}\mathrm{GE} \\
\mathrm{GP}\end{array}$ & $\begin{array}{l}0 \\
0\end{array}$ \\
\hline 1 & & $\mu \mathrm{g} / \mathrm{L}$ & $\mathrm{GE}$ & 0 \\
\hline 10 & & ${ }_{\mu g / L}$ & $\begin{array}{l}G E \\
G E\end{array}$ & $\stackrel{0}{0}$ \\
\hline$i$ & & ${ }_{\mu \mathrm{g} / \mathrm{L}}$ & GE & 0 \\
\hline 10 & & $\begin{array}{l}\mu \mathrm{gg} / \mathrm{L} \\
\mu / \mathrm{L}\end{array}$ & GE & 0 \\
\hline 10 & & $\mu \mathrm{g} / \mathrm{L}$ & GE & 0 \\
\hline 1 & J & $\mu g / L$ & GE & 0 \\
\hline $\begin{array}{l}1 \\
10\end{array}$ & & & $\begin{array}{l}\text { GE } \\
\text { GE }\end{array}$ & $\begin{array}{l}0 \\
0\end{array}$ \\
\hline 1 & & $\begin{array}{l}\mu \mathrm{g} / \mathrm{L} \\
\mathrm{pCi} / \mathrm{L}\end{array}$ & $\begin{array}{l}\text { GE } \\
G P\end{array}$ & $\begin{array}{l}0 \\
0\end{array}$ \\
\hline $\begin{array}{l}1 \\
1\end{array}$ & J & $\mathrm{pH}$ & GE & 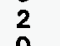 \\
\hline 10 & & $\begin{array}{l}\mu g / L \\
\mu g / L\end{array}$ & GE & $\begin{array}{l}0 \\
0\end{array}$ \\
\hline 1 & & $\mu \mathrm{g} / \mathrm{L}$ & GE & 0 \\
\hline 10 & & $\mu g / L$ & GE & 0 \\
\hline $\begin{array}{l}10 \\
10\end{array}$ & & $\mu \mathrm{g} / \mathrm{h}$ & $\begin{array}{l}\text { GE } \\
\text { GE }\end{array}$ & $\begin{array}{l}0 \\
0 \\
0\end{array}$ \\
\hline 1 & & $\mu \mathrm{s} / \mathrm{cm}$ & GE & 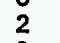 \\
\hline 1 & & $\begin{array}{r}\mu g / L \\
\mu q L\end{array}$ & $\begin{array}{l}\mathrm{GE} \\
\mathrm{GE}\end{array}$ & $\begin{array}{l}0 \\
0\end{array}$ \\
\hline 1 & & $\mu g / L$ & $G E$ & 0 \\
\hline 10 & & $\underset{\mu \mathrm{g} / \mathrm{L}}{\mu}$ & $\begin{array}{l}\text { GE } \\
\text { GE }\end{array}$ & $\begin{array}{l}0 \\
0\end{array}$ \\
\hline 1 & & $\mu g / L$ & GE & 0 \\
\hline 1 & & $\mu \mathrm{g} / \mathrm{L}$ & GE & 0 \\
\hline 1 & & $\mu g / L$ & $\begin{array}{l}\text { GE } \\
G E\end{array}$ & 0 \\
\hline$i$ & $\mathrm{~J}$ & $\mu g / L$ & $\mathrm{GE}$ & 0 \\
\hline & & & GE & 0 \\
\hline
\end{tabular}

Note: Flagging, dilution factors, modifiers, and laboratories are for fourth quarter 1994 data only. See Appendix B for flagging criteria. - = exceeded holding time for fourth quarter 1994.

- = exceeded screening level or final PDWS for fourth quarter 1994. 
Well BGX 1C continued

ANALYTICAL DATA

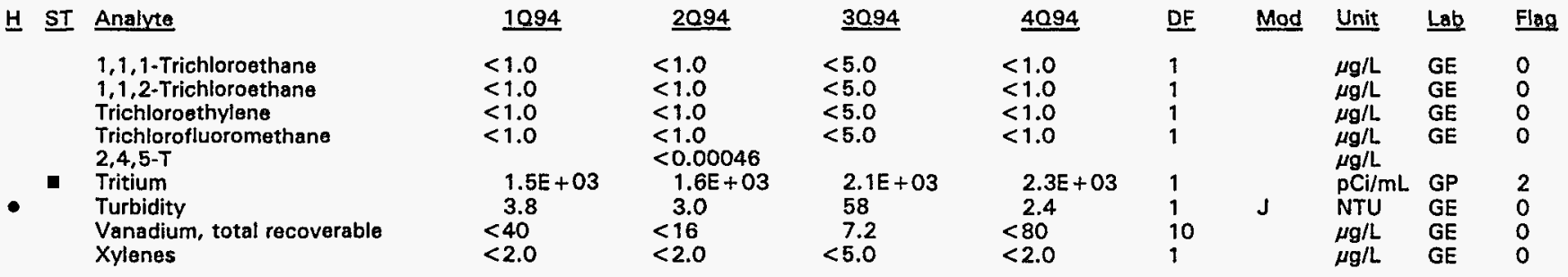

\section{WELL BGX 1D}

\section{SRS Coord. \\ N76809.5 \\ E58608.6 \\ Lat/Longitude \\ $33.291280^{\circ} \mathrm{N}$ $81.661673^{\circ} \mathrm{W}$}

\section{Screen Zone Elevation \\ 234.7-214.7 ft msi}

$02 / 08 / 94$

$04 / 14 / 94$

\section{Top of Casing}

$291.3 \mathrm{ft} \mathrm{msl}$

08/09/94

$\begin{array}{ll}\text { Casing } & \text { Pump } \\ 4^{n \text { PVC }} & \mathrm{S}\end{array}$

Screen Zone

Water Table (IIB2)

FIELD DATA

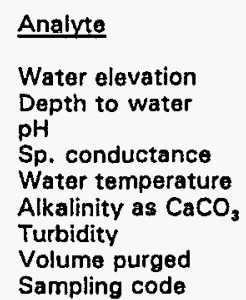

ANALYTICAL DATA

Analyte
Acetophenone
Aldrin
Aluminum, total recoverable
Antimony, total recoverable
Arsenic, total recoverable
Barium, total recoverable
Benzene
Bromodichloromethane
Bromoform
Bromomethane (Methyl bromide)
Cadmium, total recoverable
Calcium, total recoverable
Carbon tetrachloride
Chloride
Chlorobenzene
Chloroethane
Chloroethene (Vinyl chloride)
2-Chloroethyl vinyl ether
Chloroform
Chloromethane (Methyl chloride)
Chromium, total recoverable
Copper, total recoverable
Cyanide
p,p'-DDT
Dibromochloromethane
1,1-Dichloroethane

1094
229.7
61.6
5.9
131
18.5
21
43
0.7
$x$

2094
229.8
61.5
5.4
109
19.8
4
45
0.4
$x$

3094
229.2
62.1
5.2
42
23.3
4
6
0.1
$X$

1094

$<10$

385

$<2.0$

$<2.0$

32
$<1.0$

$<1.0$

$<1.0$

$<1.0$

$<2.0$

5,390

3,080

$<1.0$

$<1.0$

$<1.0$

<

1.0

$<1.0$

$<4.0$

$<5.0$

$<0.10$

$<1.0$
$<1.0$

\section{$\underline{20.94}$}

$<10$

165

$<2.0$

$<2.0$

27

$<1.0$

$<1.0$

$<1.0$

$<1.0$

$<2.0$

6,020

$<1.0$

3,080
$<1.0$

$<1.0$
$<1.0$

$<1.0$

$<1.0$

$<1.0$

$<1.0$

$<4.0$

$<4.0$

$<5.0$

$<0.10$

$<1.0$

$<1.0$

30.94
$<11$

802
$<3.0$
$<2.0$
202
$<5.0$
$<5.0$
$<5.0$
$<10$
$<2.0$
164,000
$<5.0$
1.460
$<5.0$
$<10$
$<10$
$<10$
$<5.0$
2.5
55.8
$<4.0$
$<5.0$
$<5.0$
$<5.0$

$\quad 4094$
$<10$

229
$<2.0$
$<2.0$
19
$<1.0$
$<1.0$
$<1.0$
$<1.0$
$<2.0$
4.250
$<1.0$
2.470
$<1.0$
$<1.0$
$<1.0$
$<1.0$
$<1.0$
$<1.0$
$<4.0$
4.2
$<5.0$
$<1.0$
$<1.0$

4094
229.4
61.9
5.7
41
20.9
3
8
0.3
$x$

Unit

ft msl

$\mathrm{pH}$

$\mu \mathrm{S} / \mathrm{cm}$

${ }^{\circ} \mathrm{C}$

$\mathrm{mg} / \mathrm{L}$

well vol.

.


Well BGX 1D continued

ANALYTICAL DATA

H ST Analyte

2-Dichlorosthane

1,1-Dichloroethylene

trans-1,2-Dichloroethylene

Dichloromethane

2.4-Dichlorophenoxyacetic acid

1,2-Dichloropropane

cis-1,3-Dichloropropene

trans-1,3-Dichloropropene

Dibidrin

Endrin

Ethylbenzene

Fluoride

Gross alpha

Heptachlor

Iron, total recoverable

Lead, total recoverable

Lindane

Lithium, total recoverable

Magnesium, total recoverable

Manganese, total recoverable

Mercury, total recoverable

Methoxychlor

Naphthalene

Nickel, total recoverable

Nitrate as nitrogen

Nitrate-nitrite as nitrogen

Nonvolatile bete

- $\mathrm{pH}$

Phenols

Potassium, total recoverable

Radium, total alpha-emitting

Selenium, total recoverable

Silica, total recoverable

Silver, total recoverable

Sodium, total recoverable

Specific conductance

Sulfate

1,1,2,2-Tetrachioroethane

Tetrachioroethylene

Tin, total recoverable

Toluene

Total activity

Total dissolved solids

Total organanic carbon

Total organic halogens

Total phosphates (as P)

Toxaphene

2,4,5-TP (Silvex)

$1,1,1$-Trichloroethane

1,1,2-Trichloroethane

Trichloroethylene

Trichlorofluoromethane

2,4,5-T

- Tritium

Vanadium, total recoverable

Xylenes

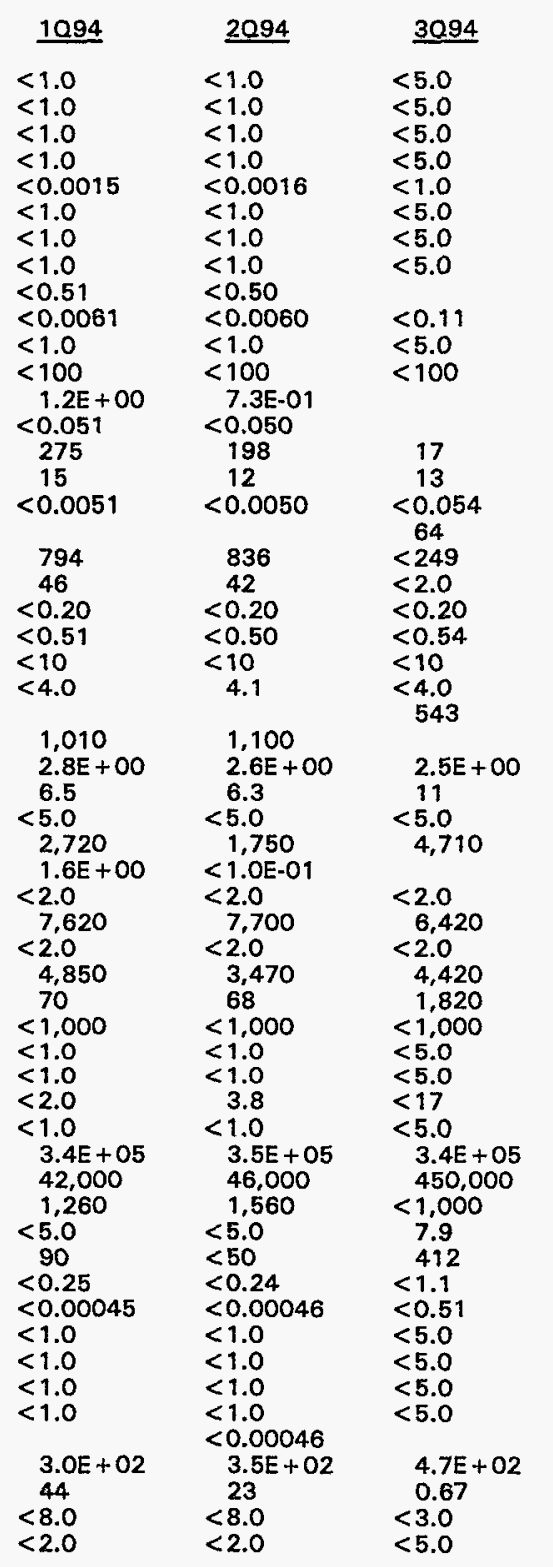

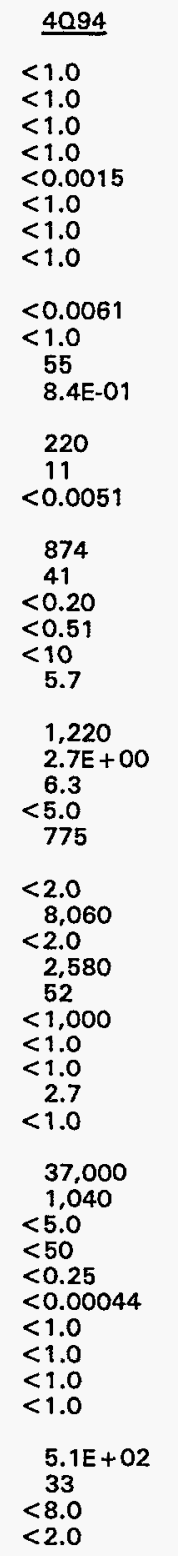

\section{DF Mod}

Unit Lab

$\mu \mathrm{g} / \mathrm{L} \quad \mathrm{GE}$

$\mu \mathrm{g} / \mathrm{L} \quad \mathrm{GE}$

$\mu \mathrm{g} / \mathrm{L} \quad \mathrm{GE}$

$\mu \mathrm{g} / \mathrm{L}$

$\mu \mathrm{g} / \mathrm{L}$

$\mu \mathrm{g} / \mathrm{L}$

$\mu g / L$

$\mu \mathrm{g} / \mathrm{L}$

$\mu, \mu g / L$

$\mu \mathrm{g} / \mathrm{L}$

UI $\quad \mathrm{pCi} / \mathrm{L}$

$\mu g / L$

$\mu g / L$

$\mu g / L$

$\mu g / L$

$\mu \mathrm{g} / \mathrm{L}$

$\mu \mathrm{g} / \mathrm{L}$

$\mu g / L$
$\mu g / L$
$\mu g / L$

$\mu \mathrm{g} / \mathrm{L}$

$\mu \mathrm{g} / \mathrm{L}$

$\mu g / L$

$\mu g / L$

pCi/L

PH

$\mu \mathrm{g} / \mathrm{L}$

$\mu \mathrm{g} / \mathrm{L}$

$\mathrm{pCi} / \mathrm{L}$

$\mu \mathrm{g} / \mathrm{L}$

$\mu g / L$

$\mu \mathrm{S} / \mathrm{cm}$

$\mu \mathrm{g} / \mathrm{L}$

$\mu g / L$

$\mu g / L$

$\mu \mathrm{g} / \mathrm{L}$

$\mu g / L$

$\mu \mathrm{pCi} / \mathrm{L}$

$\mathrm{pCi} / \mathrm{L}$
$\mu \mathrm{g} / \mathrm{L}$

$\mu \mathrm{g} / \mathrm{L}$

$\mu g / L$
$\mu g / L$
$\mu g / L$

$\mu g / L$

$\mu g / L$

$\mu \mathrm{g} / \mathrm{L}$

$\mu \mathrm{g} / \mathrm{L}$

ug/L

$\mu \mathrm{g}$

$\mu g / L$

$\mathrm{pCi} / \mathrm{mL} \quad \mathrm{GP}$

NTU

$\mu g h$

$\mu g / L$
Flag

0
0

Note: Flagging, dilution factors, modifiers, and laboratories are for fourth quarter 1994 data only. See Appendix B for flagging criteria. - = exceeded holding time for fourth quarter 1994.

- = exceeded screening level or final PDWS for fourth quarter 1994. 


\section{WELL BGX 2B}

\begin{tabular}{|c|c|c|c|c|c|c|c|c|}
\hline SRS Coord. & Lat/Longitude & Screen Zone Elevation & \multicolumn{2}{|c|}{ Top of Casing } & Casing & \multicolumn{2}{|l|}{ Pump } & \multirow{3}{*}{$\frac{\text { Screen Zone }}{\text { McBean (IIB1) }}$} \\
\hline $\begin{array}{l}\text { N77203.4 } \\
\text { E58256.5 }\end{array}$ & $\begin{array}{l}33.291577^{\circ} \mathrm{N} \\
81.663365{ }^{\circ} \mathrm{W}\end{array}$ & $147.2-137.2 \mathrm{ft} \mathrm{msl}$ & \multicolumn{2}{|c|}{$291.3 \mathrm{ft} \mathrm{msl}$} & 4" PVC & \multirow{2}{*}{\multicolumn{2}{|c|}{$\mathbf{S}$}} & \\
\hline SAMPLE DA & & $02 / 08 / 94$ & $04 / 14 / 94$ & $08 / 09 / 94$ & $11 / 08 / 94$ & & & \\
\hline \multicolumn{9}{|l|}{ FIELD DATA } \\
\hline \multicolumn{2}{|c|}{ Analyte } & 1094 & 2094 & 3094 & 4094 & & Unit & \\
\hline \multicolumn{2}{|c|}{$\begin{array}{l}\text { Water elevation } \\
\text { Depth to water } \\
\text { pH } \\
\text { Sp. conductance } \\
\text { Water temperature } \\
\text { Alkalinity as } \mathrm{CaCO}_{3} \\
\text { Turbidity } \\
\text { Volume purged } \\
\text { Sampling code }\end{array}$} & $\begin{array}{l}212.5 \\
78.8 \\
7.3 \\
272 \\
17.8 \\
102 \\
213 \\
1.0 \\
x\end{array}$ & $\begin{array}{l}212.4 \\
78.9 \\
7.6 \\
261 \\
19.1 \\
99 \\
17 \\
1.0 \\
x\end{array}$ & $\begin{array}{l}212.1 \\
79.2 \\
7.7 \\
238 \\
19.1 \\
101 \\
11 \\
1.0 \\
x\end{array}$ & $\begin{array}{l}211.8 \\
79.6 \\
8.3 \\
216 \\
18.4 \\
95 \\
67 \\
1.0 \\
\times\end{array}$ & & $\begin{array}{l}\mathrm{ft} \mathrm{msl} \\
\mathrm{ft} \\
\mathrm{pH} \\
u \mathrm{~S} / \mathrm{cm} \\
{ }^{\circ} \mathrm{C} \\
\mathrm{mg} / \mathrm{L} \\
\text { NTU } \\
\text { well vol. }\end{array}$ & \\
\hline
\end{tabular}

\section{ANALYTICAL DATA}

HI Analyte
Acetophenone
Aldrin
Aluminum, total recoverable
Antimony, total recoverable
Arsenic, total recoverable
Barium, total recoverable
Benzene
Bromodichloromethane
Bromoform
Bromomethane (Methy] bromide)
Cadmium, total recoverable
Calcium, total recoverable
Carbon tetrachloride
Chloride
Chlorobenzene
Chloroethane
Chloroethene (Vinyl chloride)
2-Chloroethyl vinyl ether
Chloroform
Chloromethane (Methyl chloride)
Chromium, total recoverable
Copper, total recoverable
Cyanide
p,p'-DDT
Dibromochloromethane
1,1-Dichloroethane
1,2-Dichloroethane
1,1-Dichloroethylene
trans-1,2-Dichloroethylene
Dichloromethane
2,4-Dichlorophenoxyacetic acid
1,2-Dichloropropane
cis-1,3-Dichloropropene
trans-1,3-Dichloropropene
Dieldrin
Endrin
Ethylbenzene
Fluoride
Gross alpha

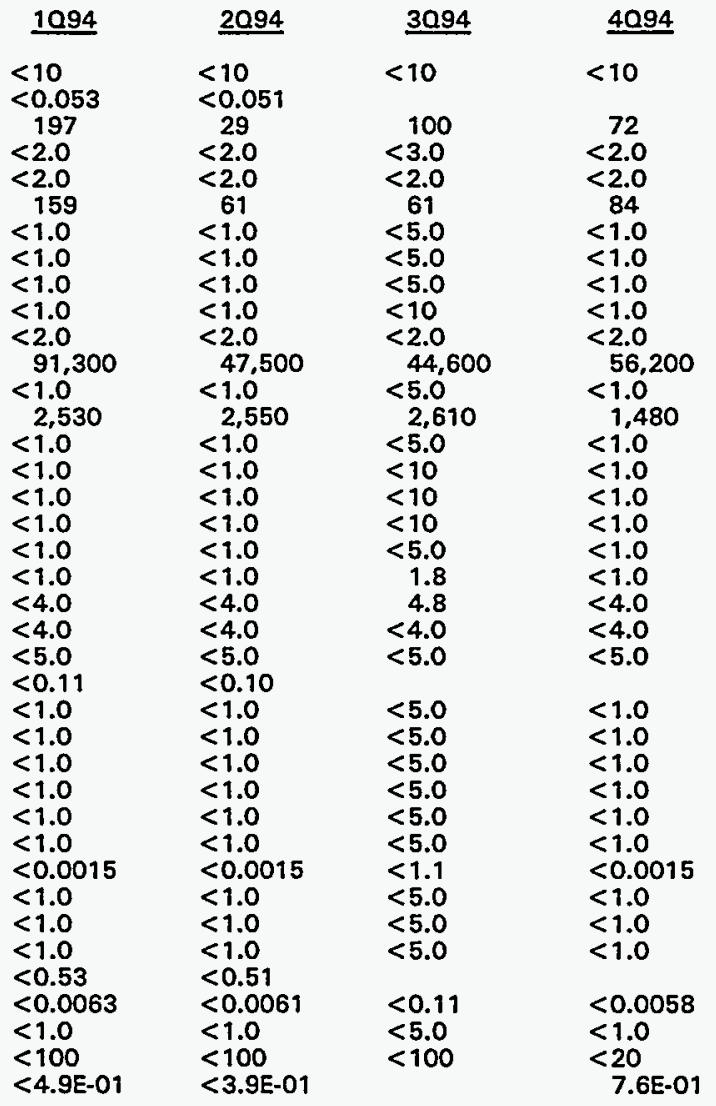

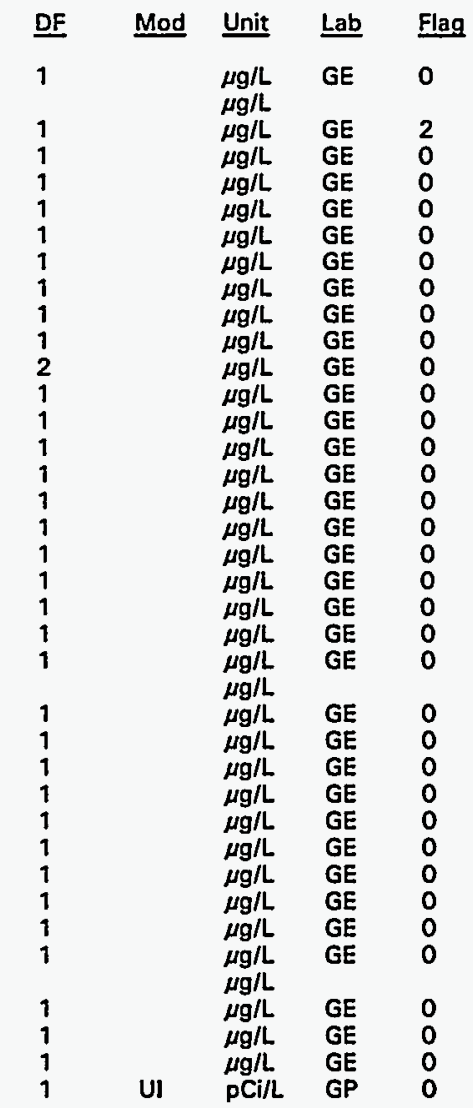

Note: Flagging, dilution factors, modifiers, and laboratories are for fourth quarter 1994 data only. See Appendix B for flagging criteria. - = exceeded holding time for fourth quarter 1994.

- = exceeded screening level or final PDWS for fourth quarter 1994. 


\section{H ST Analyte}

Heptachlor

Iron, total recoverable

Lead, total recoverable

Lindane

Magnesium, total recoverable

Manganese, total recoverable

Mercury, total recoverable

Methoxychlor

Naphthalene

Nickel, total recoverable

Nitrate as nitrogen

Nitrate-nitrite as nitrogen

Nonvolatile beta

$\mathrm{pH}$

Phenols

Potassium, total recoverable

Radium, total alpha-emitting

Selenium, total recoverable

Silica, total recoverable

Silver, total recoverable

Sodium, total recoverable

Specific conductance

Sulfate

1,1,2,2-Tetrachloroethane

Tetrachloroethylene

Tin, total recoverable

Toluene

Total dissolved solids

Total organic carbon

Total organic halogens

Total phosphates (as P)

Toxaphene

2,4,5-TP (Silvex)

1,1,1-Trichloroethane

1,1,2-Trichloroethane

Trichloroethylene

Trichlorofluoromethane

2,4,5-T

Tritium

Turbidity

Vanadium, total recoverable

Xylenes

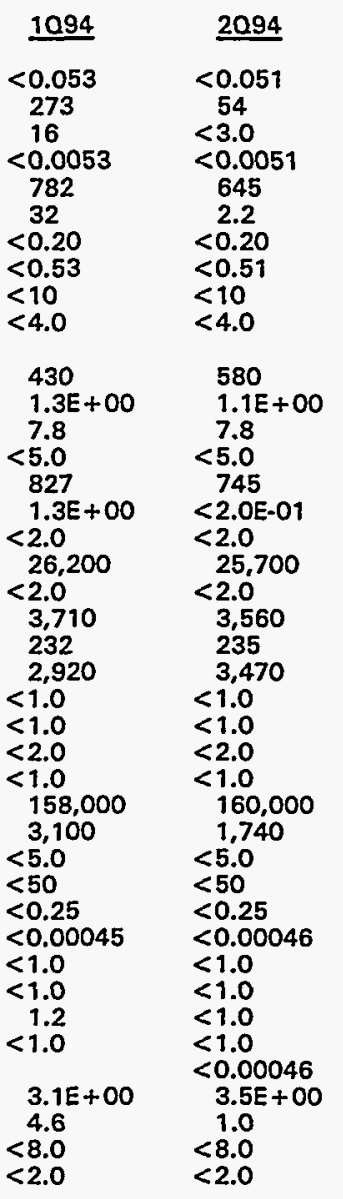

\section{4}

4094

115

$<.1$

602

6.8
4.8

$<0.20$

$<10$

$<4.0$

636

3.5E-01

7.6

$<.0$
810

$<2.0$

$<2.0$
24,500

$<2.0$

3,330

217

3,950

$<5.0$

$<5.0$

$<17$

$<5.0$

149,000

3,700

8.3

3,460

$<1.1$

$<0.53$

$<5.0$

$<5.0$

$<5.0$

$<5.0$

$6.3 E+00$

1.7

$<3.0$

$<5.0$

$\quad \underline{4094}$
120
5.9
$<0.0049$
666
9.3
$<0.20$
$<0.49$
$<10$
$<4.0$
731
$1.5 \mathrm{E}+00$
7.8
$<5.0$
766

$<2.0$
26,900
$<2.0$
3,470
231
2,830
$<1.0$
$<1.0$
$<2.0$
$<1.0$
152,000
1,860
$<5.0$
71
$<0.23$
$<0.00045$
$<1.0$
$<1.0$
$<1.0$
$<1.0$
6.05
4.7
$<8.0$
$<2.0$

DF Mod Unit Lab Flag

$\mu g / L$

$\mu g / L$ GE

$\mu g / L \quad G E$

$\mu g / L \quad G E$

$\mu g / L \quad G E$

$\mu g / L \quad G E$

$\mu g / L \quad G E$

$\mu \mathrm{g} / \mathrm{h} . \quad \mathrm{GE}$

$\mu g / L \quad G E$

PCIL GE 0

PCIL GP 0

PH GE

$\mu g / L \quad \mathrm{GE}$

pCi/L

$\mu g / L \quad G E$

$\mu g / L \quad G E$

$\mu \mathrm{g} / \mathrm{L} \quad \mathrm{GE}$

$\mu S / \mathrm{cm}$ GE

$\mu g / L \quad G E$

$\mu \mathrm{g} / \mathrm{L} \quad \mathrm{GE}$

$\mu \mathrm{g} / \mathrm{L} \quad \mathrm{GE}$

$\mu g / L \quad G E$

$\underset{\mu g / L}{\mu g} \quad \mathrm{GE}$

$\mu g / L \quad G E$

$\mu g / L \quad G E$

$\mu g / L \quad G E$

$\begin{array}{ll}\mu \mathrm{g} / \mathrm{L} & \mathrm{GE} \\ \mu \mathrm{g} / \mathrm{L} & \mathrm{GE}\end{array}$

$\mu \mathrm{g} / \mathrm{L} \quad \mathrm{GE}$

$\mu \mathrm{g} / \mathrm{L} \quad \mathrm{GE}$

$\mu g / L \quad G E$

$\mu g / L \quad G E$

$\mathrm{pCi} / \mathrm{mL}$ GP

NTU GE

$\mu g / L \quad G E$

$\mu \mathrm{g} / \mathrm{L}$

0
0
0
0
0
0
0
0
0
0
0
0
0
0
0
0
0
0
0
0
0
0
0
0
0
0
0
0
0
0
0
0
0
0
0
0
0
0
0
0

Note: Flagging, dilution factors, modifiers, and laboratories are for fourth quarter 1994 data only. See Appendix B for flagging criteria. - = exceeded holding time for fourth quarter 1994.

- = exceeded screening level or final PDWS for fourth quarter 1994. 


\section{WELL BGX 2D}

\section{SRS Coord. Lat/Longitude \\ N77192.4 \\ E58265.6 \\ $33.291567 \circ \mathrm{N}$
$81.663320^{\circ} \mathrm{W}$}

SAMPLE DATE

FIELD DATA

\section{Screen Zone Elevation \\ 191.1-181.1 ft msl}

$02 / 08 / 94$

\section{Top of Casing}

$291.1 \mathrm{ft} \mathrm{msl}$

$\begin{array}{ll}\text { Casing } & \text { Pump } \\ \text { 4" PVC } & 5\end{array}$

$11 / 08 / 94$

\section{Analyte}

Water elevation

Depth to water

$\mathrm{pH}$

Sp. conductance

Water temperature

Alkalinity as $\mathrm{CaCO}_{3}$

Turbidity

Volume purged

Sampling code

\begin{tabular}{lll}
1094 & & 2094 \\
\hline 215.1 & & 215.2 \\
76.0 & & 75.9 \\
5.3 & & 5.4 \\
37 & 75 \\
18.1 & 19.2 \\
3 & 3 \\
5 & 2 \\
0.9 & 0.9 \\
$X$ & $x$
\end{tabular}

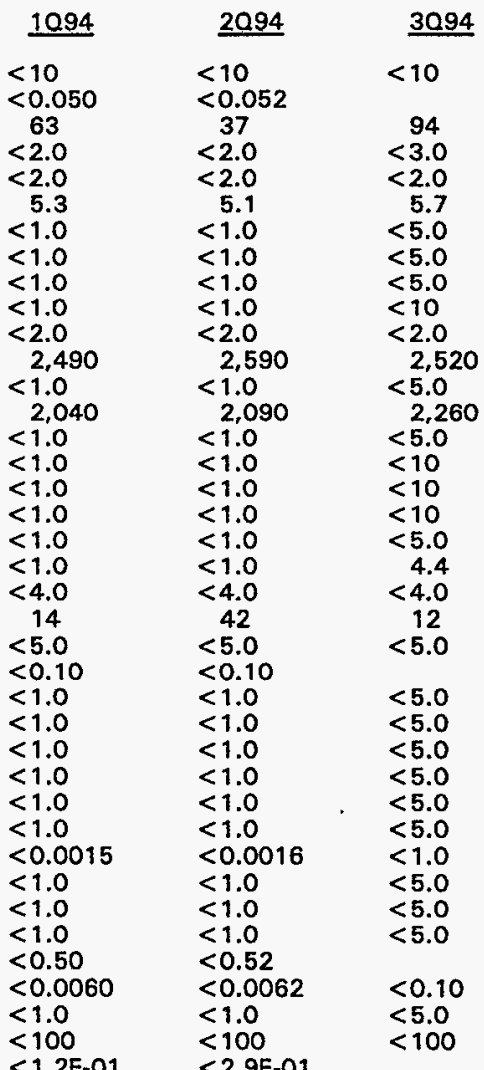

ANALYTICAL DATA

H $\underline{\text { ST }}$ Analyte

Acetophenone

Aldrin

Aluminum, total recoverable

Antimony, total recoverable

Arsenic, total recoverable

Barium, total recoverable

Benzene

Bromodichloromethane

Bromoform

Bromomethane (Methyl bromide)

Cadmium, total recoverable

Calcium, total recoverable

Carbon tetrachloride

Chloride

Chlorobenzene

Chloroethane

Chloroethene (Vinvl chloride)

2-Chloroethyl vinyl ether

Chloroform

Chloromethane (Methyl chloride)

Chromium, total recoverable

Copper, total recoverable

Cyanide

p.p'-DDT

Dibromochloromethane

1,1-Dichloroethane

1,2-Dichloroethane

1,1-Dichloroethylene

trans-1,2-Dichloroethylene

Dichloromethane

2,4-Dichlorophenoxyacetic acid

1,2-Dichloropropane

cis-1,3-Dichloropropene

trans-1,3-Dichloropropene

Dieldrin

Endrin

Ethylbenzene

Fluoride

Gross alpha

3094
214.8
76.3
5.5
35
19.2
1
2
0.9
$x$

$04 / 14 / 94 \quad 08 / 09 / 94$

4094
219.6
71.5
5.8
32
18.7
2
2
0.7
$x$

Unit

ft $\mathrm{msl}$

$\mathrm{pt}$

$\mu S / \mathrm{cm}$

Mg/L

well vol.
Screen Zone

Barnwell (IIB1)

\begin{tabular}{|c|c|c|c|c|c|}
\hline 4094 & $\underline{\mathrm{DF}}$ & Mod & Unit & $\underline{L a b}$ & Flag \\
\hline$<9.9$ & 1 & & $\mu g / L$ & $\mathrm{GE}$ & 0 \\
\hline $\begin{array}{r}51 \\
20\end{array}$ & 1 & & $\mu g / L$ & GE & 2 \\
\hline $\begin{array}{l}<2.0 \\
<2.0\end{array}$ & $\begin{array}{l}1 \\
1\end{array}$ & & $\mu g / L$ & GE & 0 \\
\hline 5.3 & 1 & & $\mu \mathrm{g} / \mathrm{L}$ & GE & $\begin{array}{l}0 \\
0\end{array}$ \\
\hline$<1.0$ & 1 & & $\mu g / L$ & $\mathrm{GE}$ & 0 \\
\hline$<1.0$ & 1 & & $\mu \mathrm{g} / \mathrm{L}$ & $\mathrm{GE}$ & 0 \\
\hline$<1.0$ & 1 & & $\mu g / L$ & GE & 0 \\
\hline$<1.0$ & 1 & & $\mu g / \mathrm{L}$ & $\mathrm{GE}$ & 0 \\
\hline$<2.0$ & 1 & & $\mu \mathrm{g} / \mathrm{L}$ & $\overrightarrow{G E}$ & 0 \\
\hline 2,570 & 1 & & $\mu \mathrm{g} / \mathrm{L}$ & GE & 0 \\
\hline$<1.0$ & 1 & & $\mu g / L$ & GE & 0 \\
\hline 1,640 & 1 & & $\mu \mathrm{g} / \mathrm{L}$ & $\mathrm{GE}$ & 0 \\
\hline$<1.0$ & 1 & & $\mu \mathrm{g} / \mathrm{L}$ & GE & 0 \\
\hline$<1.0$ & 1 & & $\mu \mathrm{g} / \mathrm{L}$ & GE & 0 \\
\hline$<1.0$ & 1 & & $\mu \mathrm{g} / \mathrm{L}$ & GE & 0 \\
\hline$<1.0$ & 1 & & $\mu \mathrm{g} / \mathrm{L}$ & GE & 0 \\
\hline$<1.0$ & 1 & & $\mu \mathrm{g} / \mathrm{L}$ & GE & 0 \\
\hline$<1.0$ & 1 & & $\mu g / L$ & GE & 0 \\
\hline$<4.0$ & 1 & & $\mu \mathrm{g} / \mathrm{L}$ & GE & 0 \\
\hline 18 & 1 & & $\mu \mathrm{g} / \mathrm{L}$ & GE & 0 \\
\hline$<5.0$ & 1 & & $\mu \mathfrak{g} / \mathrm{L}$ & GE & 0 \\
\hline$<1.0$ & 1 & & $\mu \mathrm{g} / \mathrm{L}$ & GE & 0 \\
\hline$<1.0$ & 1 & & $\mu \mathrm{g} / \mathrm{L}$ & GE & 0 \\
\hline$<1.0$ & 1 & & $\mu \mathrm{g} / \mathrm{L}$ & GE & 0 \\
\hline$<1.0$ & 1 & & $\mu \mathrm{g} / \mathrm{L}$ & GE & 0 \\
\hline$<1.0$ & 1 & & $\mu g / L$ & GE & 0 \\
\hline$<1.0$ & 1 & & $\mu g / L$ & GE & 0 \\
\hline$<0.0015$ & 1 & & $\mu \mathrm{g} / \mathrm{L}$ & GE & 0 \\
\hline$<1.0$ & 1 & & $\mu \mathrm{g} / \mathrm{L}$ & GE & 0 \\
\hline$<1.0$ & 1 & & $\mu \mathrm{g} / \mathrm{L}$ & GE & 0 \\
\hline$<1.0$ & 1 & & $\begin{array}{l}\mu g / L \\
\mu g / L\end{array}$ & GE & 0 \\
\hline$<0.0059$ & 1 & & $\mu \mathrm{g} / \mathrm{L}$ & GE & 0 \\
\hline$<1.0$ & 1 & & $\mu \mathrm{g} / \mathrm{L}$ & GE & 0 \\
\hline$<20$ & 1 & & $\mu \mathrm{g} / \mathrm{L}$ & GE & 0 \\
\hline 2.6E-01 & 1 & UI & $\mathrm{pCi} / \mathrm{L}$ & GP & 0 \\
\hline
\end{tabular}

Note: Flagging, dilution factors, modifiers, and laboratories are for fourth quarter 1994 data only. See Appendix B for flagging criteria. - = exceeded holding time for fourth quarter 1994.

- = exceeded screening level or final PDWS for fourth quarter 1994. 
Well BGX 2D continued

ANALYTICAL DATA

\section{H ST Analvte}

Heptachlor

Iron total recoverable

Lead, total recoverable

Lindane

Magnesium, total recoverable

Manganese, total recoverable

Mercury, total recoverable

Methoxychlor

Naphthalene

Nickel, total recoverable

Nitrate as nitrogen

Nitrate-nitrite as nitrogen

Nonvolatile beta

- $\mathrm{pH}$

Phenols

Potassium, total recoverable

Radium, total alpha-emitting

Selenium, total recoverable

Silica, total recoverable

Silver, total recoverable

Sodium, total recoverable

Specific conductance

Sulfate

1,1,2,2-Tetrachloroethane

Tetrachloroethylene

Tin, total recoverable

Toluene

Total dissolved solids

Total organic carbon

Total organic halogens

Total phosphates (as P)

Toxaphene

2,4,5-TP (Silvex)

1,1,1-Trichloroethane

1,1,2-Trichloroethane

- Trichloroethylene

Trichlorofluoromethane

2,4,5-T

- Tritium

Turbidity

Vanadium, total recoverable

Xylenes

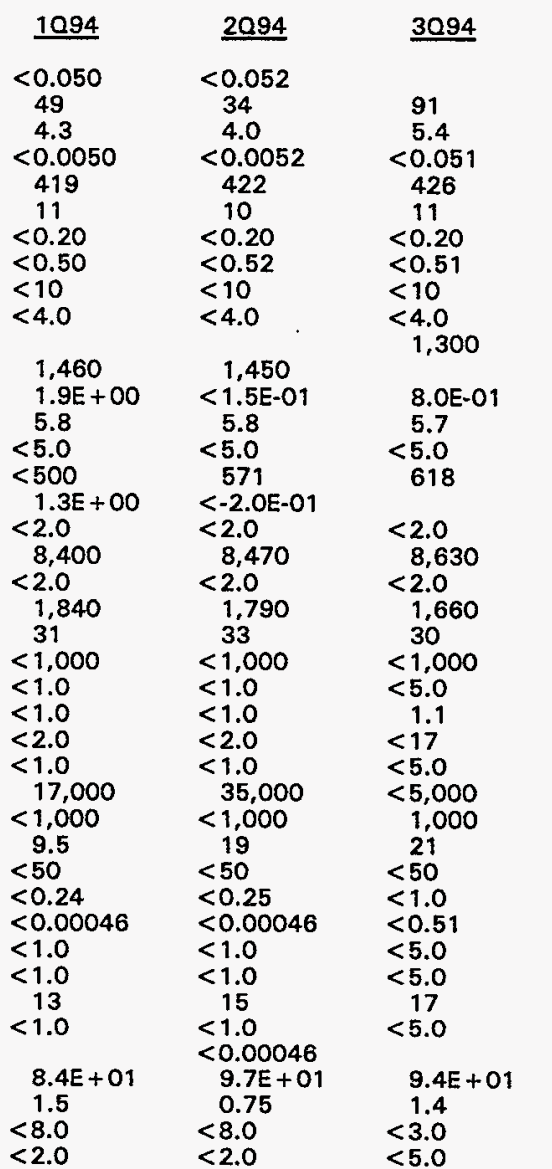

4094

44
5.4
$<0.0049$
439
9.6
$<0.20$
$<0.49$
$<9.9$
$<4.0$

1,450
$8.1 \mathrm{E}-01$
5.6
$<5.0$
$<500$
$<2.0$
8,660
$<2.0$
1.680
32
$<1.000$
$<1.0$
$<1.0$
$<2.0$
$<1.0$
28,000
$<1.000$
15
$<50$
$<0.24$
$<0.00044$
$<1.0$
$<1.0$
16
$<1.0$
$9.2 \mathrm{E}+01$
17
$<8.0$
$<2.0$

$\underline{\text { Mod Unit Lab Flag }}$

$\mu g / L$

$\mu \mathrm{g} / \mathrm{L} \quad \mathrm{GE}$

$\mu \mathrm{g} / \mathrm{L} \quad \mathrm{GE}$

$\mu g / L \quad G E$

$\mu \mathrm{g} / \mathrm{L}$

$\mu g / L$

$\mu \mathrm{g} / \mathrm{L}$

$\mu \mathrm{g} / \mathrm{L} \quad \mathrm{GE}$

$\mu \mathrm{g} / \mathrm{L} \quad \mathrm{GE}$

$\mu \mathrm{g} / \mathrm{L} \quad \mathrm{GE}$

$\mu g / L$

$\mu \mathrm{gCi} / \mathrm{L}$

UI

$\mu g / L \quad$ GE

$\mu g / L \quad G E$

pCilL

$\mu g / L \quad G E$

$\mu \mathrm{g} / \mathrm{L} \quad \mathrm{GE}$

$\mu \mathrm{g} / \mathrm{L} \quad \mathrm{GE}$

$\mu \mathrm{g} / \mathrm{L}$

$\mu \mathrm{S} / \mathrm{cm}$

$\mu \mathrm{g} / \mathrm{L}$

$\mu \mathrm{g} / \mathrm{L}$

$\mu \mathrm{g} / \mathrm{L}$

$\mu \mathrm{g} / \mathrm{L}$

$\mu g / L$

$\mu g / \mathrm{L}$

$\mu g / L$

$\mu g / L$

$\mu g / L$

$\mu \mathrm{g} / \mathrm{L}$

$\mu g / L$

$\mu \mathrm{g} / \mathrm{L}$

$\mu \mathrm{g} / \mathrm{L}$

$\mathrm{pCi} / \mathrm{mL}$

NTU

$\mu \mathrm{g} / \mathrm{L}$

$\mu g / L$
(1)

0

0

0

0

o

Note: Flagging, dilution factors, modifiers, and laboratories are for fourth quarter 1994 data only. See Appendix B for flagging criteria. - = exceeded holding time for fourth quarter 1994.

- = exceeded screening level or final PDWS for fourth quarter 1994. 


\section{WELL BGX 3D}

$\begin{array}{ll}\text { SRS Coord. } & \text { Lat/Longitude } \\ N 77577.0 & 33.291626^{\circ} \mathrm{N} \\ \text { E57780.1 } & 81.665346^{\circ} \mathrm{W}\end{array}$

SAMPLE DATE

FIELD DATA

Analyte
Water elevation
Depth to water
pH
Sp. conductance
Water temperature
Alkalinity as $\mathrm{CaCO}_{3}$
Turbidity
Volume purged
Sampling code

\section{ANALYTICAL DATA}

\section{브 ST Analyte}

Acetophenone
Aldrin
Aluminum, total recoverable
Antimony, total recoverable
Arsenic, total recoverable
Barium, total recoverable
Benzene
Bromodichloromethane
Bromoform
Bromomethane (Methyl bromide)
Cadmium, total recoverable
Calcium, total recoverable
Carbon tetrachloride
Chloride
Chlorobenzene
Chloroethane
Chloroethene (Vinyl chloride)
2-Chloroethyl vinyl ether
Chloroform
Chloromethane (Methyl chloride)
Chromium, total recoverable
Copper, total recoverable
Cyanide
P,p'-DDT
Dibromochloromethane
1,1-Dichloroethane
1,2-Dichloroethane
1,1-Dichloroethylene
trans-1,2-Dichloroethylene
Dichloromethane
2,4-Dichlorophenoxyacetic acid
1,2-Dichloropropane
cis-1,3-Dichloropropene
trans-1,3-Dichloropropene
Dieldrin
Endrin
Ethylbenzene
Fluoride
Gross alpha

\section{Screen Zone Elevation \\ $221.6-201.6 \mathrm{ft} \mathrm{msl}$}

$01 / 31 / 94$

$04 / 13 / 94$

\section{Top of Casing}

$291.2 \mathrm{ft} \mathrm{msl}$

$\begin{array}{ll}\text { Casing } & \text { Pump } \\ \text { 4" PVC } & \mathrm{s}\end{array}$

Screen Zone

Barnwell (IIB1)

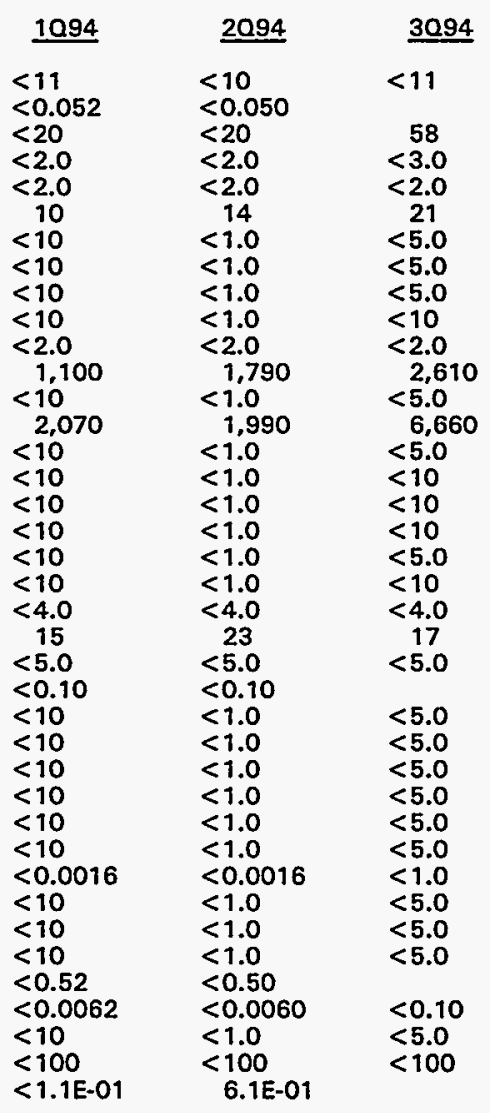

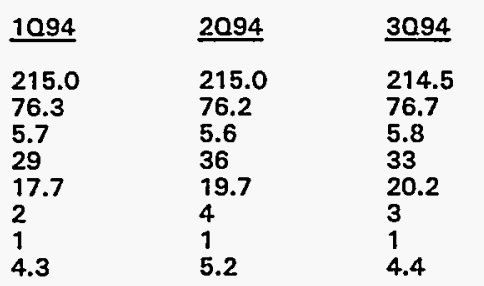

4094
214.6
76.6
5.8
28
19.2
4
0
5.8

Unit

ft msl

ft

$\mu \mathrm{S} / \mathrm{cm}$

${ }^{\circ} \mathrm{C}$

Mg/L

well vol.

nit
msi
M/cm
NTU
well vol.




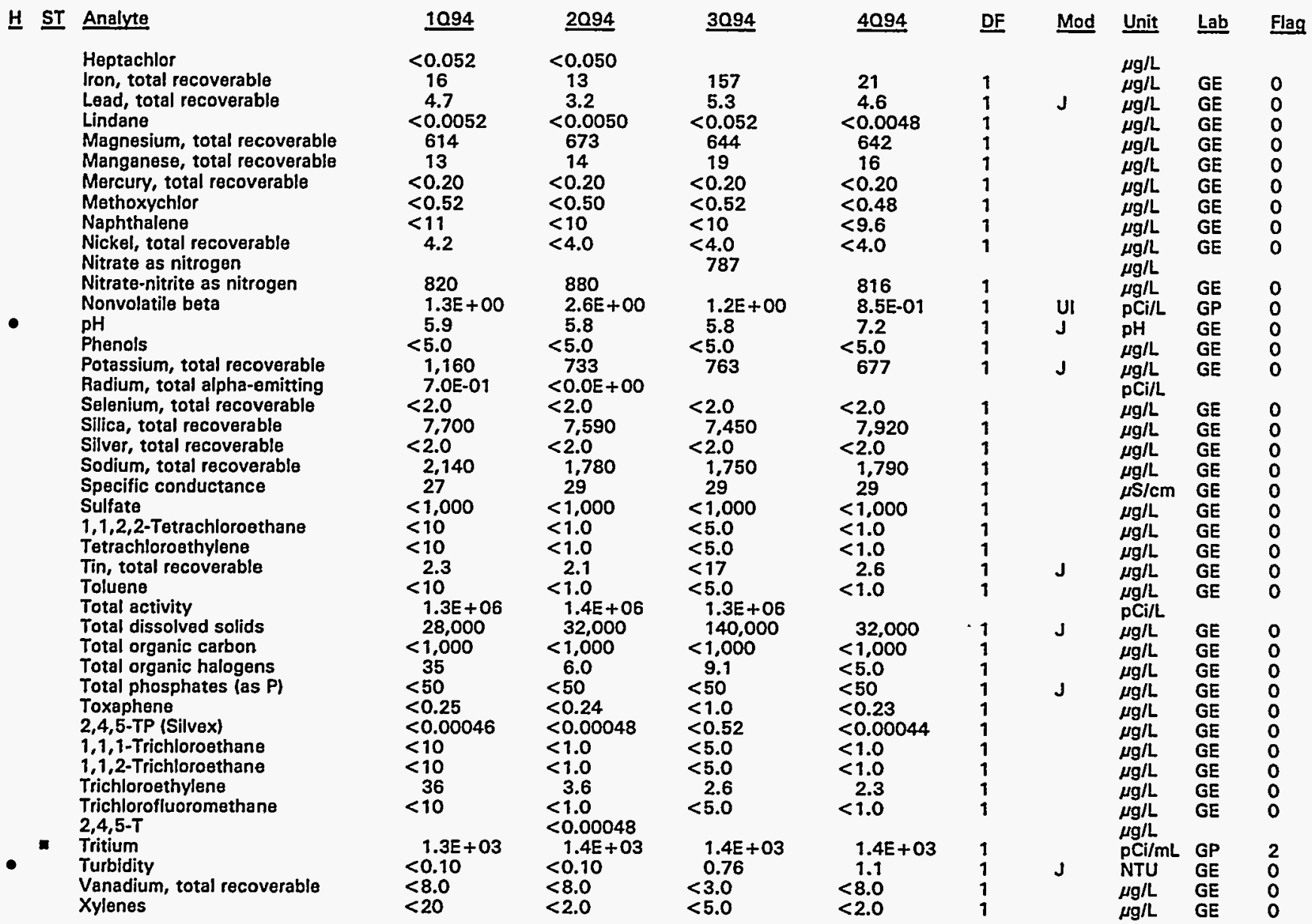

Note: Flagging, dilution factors, modifiers, and laboratories are for fourth quarter 1994 data only. See Appendix B for flagging criteria. - = exceeded holding time for fourth quarter 1994.

- = exceeded screening level or final PDWS for fourth quarter 1994. 
WELL BGX 4A

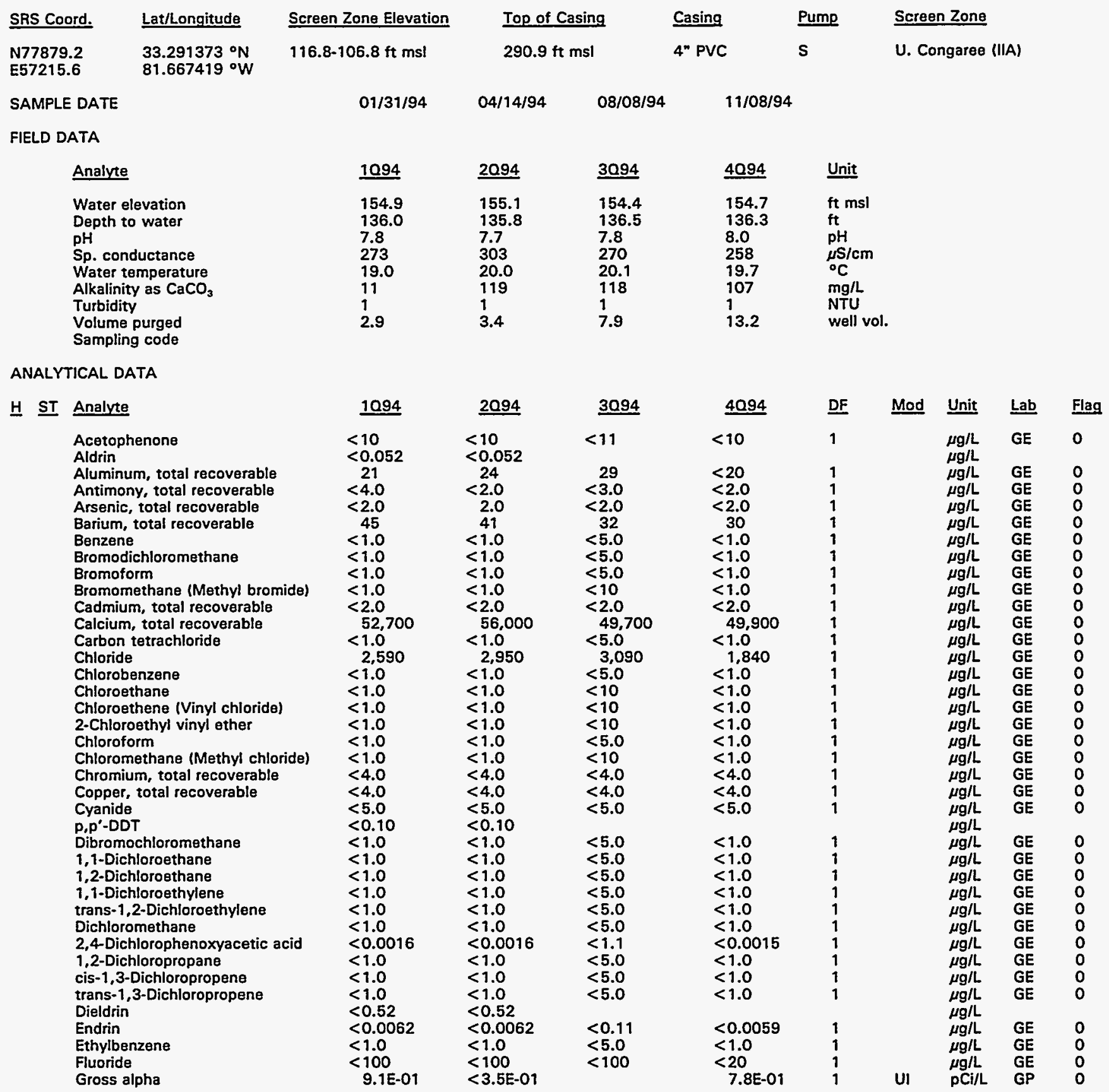

Note: Flagging, dilution factors, modifiers, and laboratories are for fourth quarter 1994 data only. See Appendix B for flagging criteria. - = exceeded holding time for fourth quarter 1994.

- = exceeded screening level or final PDWS for fourth quarter 1994. 


\section{H ST Analyze}

Heptachlor

Iron, total recoverable

Lead, total recoverable

Lindane

Magnesium, total recoverable

Manganese, total recoverable

Mercury, total recoverable

Methoxychlor

Naphthalene

Nickel, total recoverable

Nitrate as nitrogen

Nitrate-nitrite as nitrogen

Nonvolatile beta

$\mathrm{pH}$

Phenols

Potassium, total recoverable

Radium, total alpha-emitting

Radium-226

Radium-228

Selenium, total recoverable

Silica, total recoverable

Silver, total recoverable

Sodium, total recoverable

Specific conductance

Sulfate

1,1,2,2-Tetrachloroethane

Tetrachloroethylene

Tin, total recoverable

Toluene

Total dissolved solids

Total organic carbon

Total organic halogens

Total phosphates (as $P$ )

Toxaphene

2,4,5-TP (Silvex)

1,1,1-Trichloroethane

1,1,2-Trichloroethane

Trichloroethylene

Trichlorofluoromethane

2,4,5-T

Tritium

Turbidity

Vanadium, total recoverable

Xylenes

\begin{tabular}{|c|c|c|}
\hline 1094 & $\underline{2094}$ & 3094 \\
\hline$<0.052$ & $<0.052$ & \\
\hline $\begin{array}{r}31 \\
<3.0\end{array}$ & $\begin{array}{r}21 \\
<3.0\end{array}$ & $\begin{array}{r}34 \\
<3.0\end{array}$ \\
\hline$<0.0052$ & $<0.0052$ & $<0.055$ \\
\hline $\begin{array}{l}1,340 \\
9.4\end{array}$ & $\begin{array}{l}1,310 \\
12\end{array}$ & $\begin{array}{l}1,120 \\
11\end{array}$ \\
\hline$<0.20$ & $<0.20$ & $<0.20$ \\
\hline$<0.52$ & $<0.52$ & $<0.55$ \\
\hline $\begin{array}{l}<10 \\
<4.0\end{array}$ & $<10$ & $<10$ \\
\hline$<4.0$ & $\begin{array}{l}<4.0 \\
<20\end{array}$ & $\begin{array}{c}<4.0 \\
34\end{array}$ \\
\hline$<50$ & $<50$ & \\
\hline $\begin{array}{l}1.9 E+00 \\
7.7\end{array}$ & $5.1 E+00$ & $1.4 \mathrm{E}+00$ \\
\hline 6.3 & $\begin{array}{r}7.7 \\
<5.0\end{array}$ & $\begin{array}{r}7.3 \\
<5.0\end{array}$ \\
\hline 2,300 & 1,590 & 1,290 \\
\hline & $\begin{array}{c}<-1.0 \mathrm{E}-01 \\
2.1 \mathrm{E}-01 \\
1.5 \mathrm{E}+00\end{array}$ & \\
\hline$<4.0$ & $<2.0$ & $<2.0$ \\
\hline $\begin{aligned} & 37,400 \\
&<2,0\end{aligned}$ & 35,000 & 33,600 \\
\hline $\begin{array}{l}<2.0 \\
3,120\end{array}$ & $\begin{array}{l}<2.0 \\
2.550\end{array}$ & $<2.0$ \\
\hline $\begin{array}{l}278 \\
8,320\end{array}$ & 267 & 263 \\
\hline $\begin{array}{l}8,320 \\
<1.0\end{array}$ & 10,200 & 8.080 \\
\hline$<1.0$ & $<1.0$ & $<5.0$ \\
\hline 6.1 & $<2.0$ & $\begin{array}{l}<5.0 \\
<17\end{array}$ \\
\hline$<1.0$ & $<1.0$ & $<5.0$ \\
\hline $\begin{array}{l}193,000 \\
1,110\end{array}$ & $\begin{array}{l}211,000 \\
1,260\end{array}$ & $\begin{array}{l}194,000 \\
<1,000\end{array}$ \\
\hline$<5.0$ & 13 & 14 \\
\hline $\begin{array}{l}<50 \\
<0.25\end{array}$ & $\begin{array}{l}<50 \\
<0.25\end{array}$ & $\begin{aligned} & 766 \\
&<1.1\end{aligned}$ \\
\hline$<0.00047$ & $<0.00046$ & $<0.54$ \\
\hline$<1.0$ & $<1.0$ & $<5.0$ \\
\hline$<1.0$ & $<1.0$ & $<5.0$ \\
\hline$<1.0$ & $<1.0$ & $<5.0$ \\
\hline$<1.0$ & $\begin{array}{l}<1.0 \\
<0.00046\end{array}$ & $<5.0$ \\
\hline$<5.7 E-02$ & $2.8 E-01$ & 2.0E-01 \\
\hline 0.14 & 0.40 & 0.21 \\
\hline $\begin{array}{l}<8.0 \\
<2.0\end{array}$ & $\begin{array}{l}<3.0 \\
<2.0\end{array}$ & $\begin{array}{l}<3.0 \\
<5.0\end{array}$ \\
\hline
\end{tabular}

\begin{tabular}{|c|c|}
\hline 4094 & $\underline{\mathrm{DF}}$ \\
\hline $\begin{aligned} & 19 \\
&<3.0 \\
&<0.0049 \\
& 1,160 \\
& 9.6 \\
&<0.20 \\
&<0.49 \\
&<10 \\
&<4.0\end{aligned}$ & $\begin{array}{l}1 \\
1 \\
1 \\
1 \\
1 \\
1 \\
1 \\
1 \\
1\end{array}$ \\
\hline $\begin{array}{l}<50 \\
1.0 E+00 \\
7.7 \\
<5.0 \\
1.060\end{array}$ & $\begin{array}{l}1 \\
1 \\
1 \\
1 \\
1\end{array}$ \\
\hline
\end{tabular}

Mod

Unit Lab Flag

$<2.0$

33,600

$<2.0$

2,060

258

6,590

$<1.0$

$<2.0$

$<1.0$

173,000

1,320

$<5.0$

69
$<0.24$

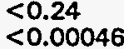

$<1.0$

$<1.0$

$<1.0$

$<1.0$

$\begin{array}{ll} & -1.3 E-01 \\ < & 0.37 \\ < & 8.0\end{array}$

$\begin{aligned} & 0.37 \\ < & 8.0 \\ < & 2.0\end{aligned}$

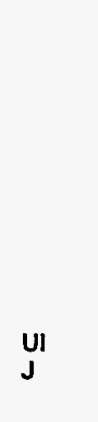

$\mu \mathrm{g} / \mathrm{L}$ GE 0

$\mu \mathrm{g} / \mathrm{L}$ GE 0

$\mu \mathrm{g} / \mathrm{L}$ GE 0

$\mu g / L$ GE $\quad 0$

$\mu g / L$ GE 0

$\mu g / L$ GE 0

$\mu g / L \quad$ GE $\quad 0$

$\mu \mathrm{g} / \mathrm{L}$

$\mu g / L \quad$ GE 0

PCi/L GP 0

GE 0

Mg/L GE 0

$\mu \mathrm{g} / \mathrm{L} \quad \mathrm{GE}$

$\mathrm{pCi} / \mathrm{L}$

pCi/L

$\mu \mathrm{g} / \mathrm{L} \quad \mathrm{CE}$

$\mu g / L \quad G E$

$\mu g / L \quad G E$

$\mu \mathrm{S} / \mathrm{cm} \quad \mathrm{GE}$

$\mu \mathrm{g} / \mathrm{L} \quad \mathrm{GE}$

$\mu g / L \quad G E$

$\mu g / L \quad G E$

$\mu \mathrm{g} / \mathrm{L}$

$\mu \mathrm{g} / \mathrm{h}$

J

J $\mu g /$

J

Ug/L

$\mu \mathrm{g} / \mathrm{L} \quad \mathrm{GE}$

$\mu g / L \quad G E$

$\mu g / L \quad G E$

$\mu g / L \quad G E$

$\mu g / L \quad G E$

$\mu g / L$

UI $\mathrm{pCi} / \mathrm{mL}$ GP

NTU GE

$\mu g / L \quad G E$

$\mu g / L \quad G E$ $\mu g / L \quad G E$

$\mu g / L \quad G E$



Note: Flagging, dilution factors, modifiers, and laboratories are for fourth quarter 1994 data only. See Appendix B for flagging criteria. - = exceeded holding time for fourth quarter 1994.

a = exceeded screening level or final PDWS for fourth quarter 1994. 
WELL BGX 4C

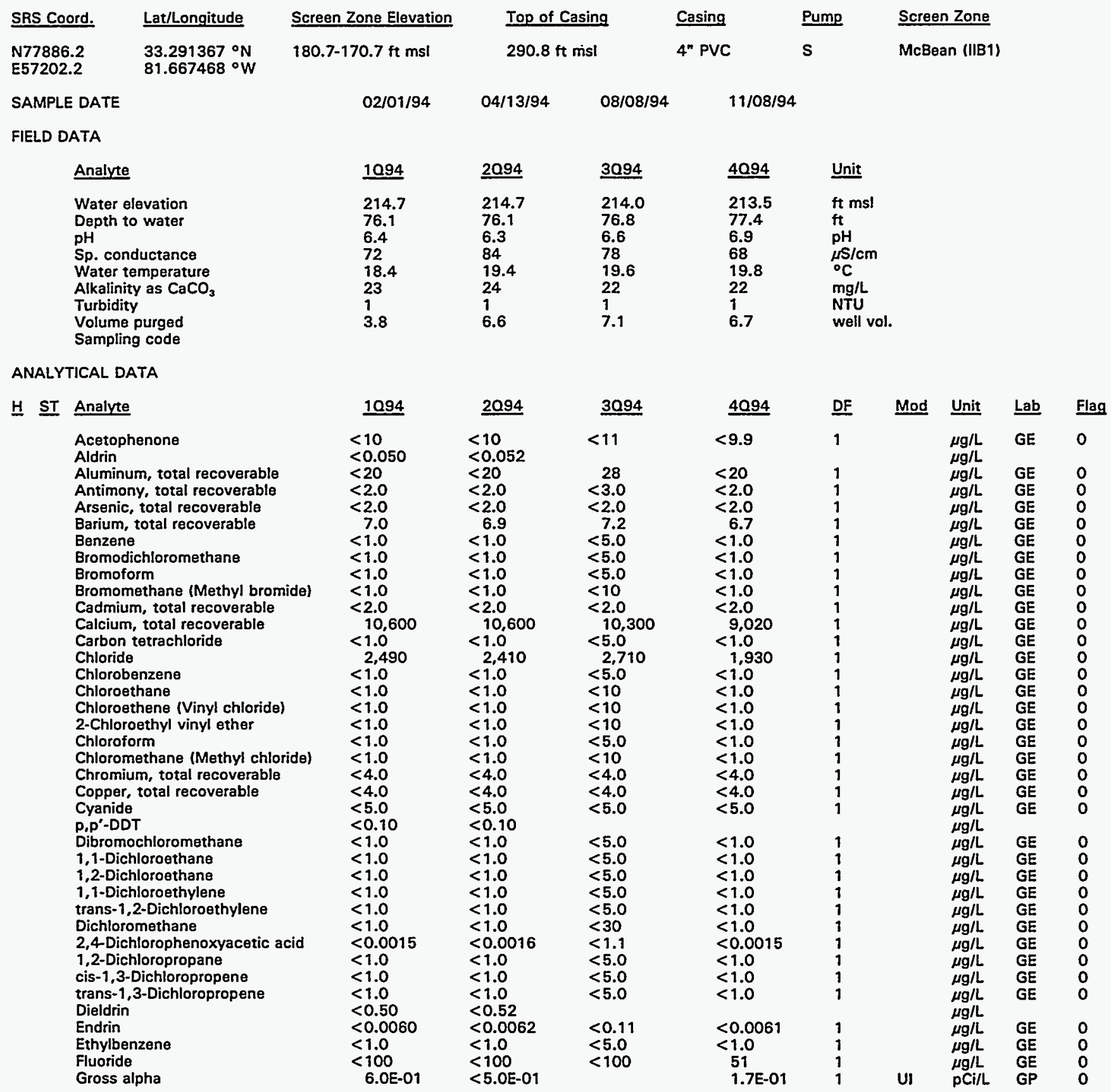

Note: Flagging, dilution factors, modifiers, and laboratories are for fourth quarter 1994 data only. See Appendix B for flagging criteria. - = exceeded holding time for fourth quarter 1994.

- = exceeded screening level or final PDWS for fourth quarter 1994. 


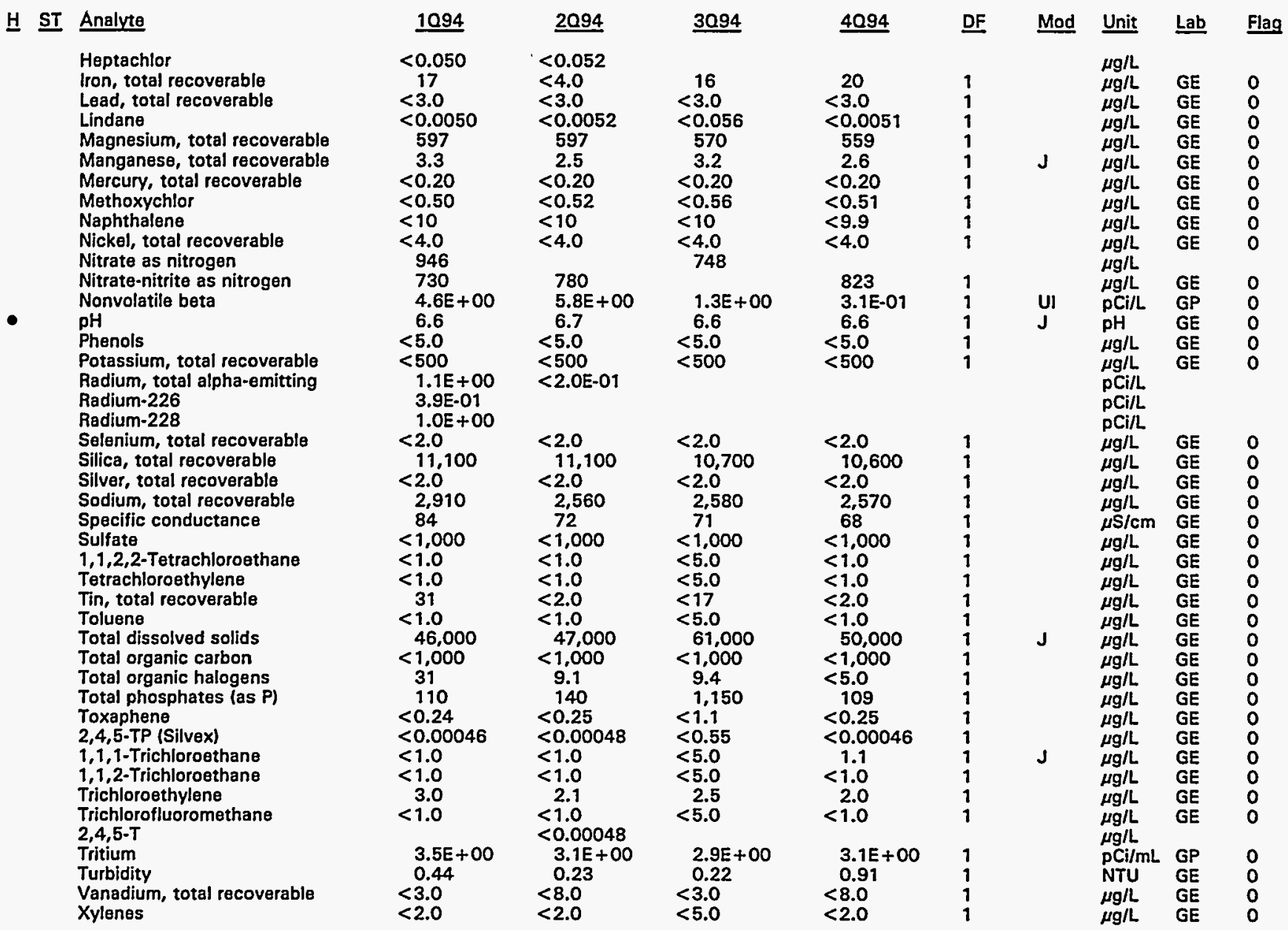

Note: Flagging, dilution factors, modifiers, and laboratories are for fourth quarter 1994 data only. See Appendix B for flagging criteria. - = exceeded holding time for fourth quarter 1994.

m = exceeded screening level or final PDWS for fourth quarter 1994. 
WELL BGX 4D

\begin{tabular}{|c|c|c|c|c|c|c|c|c|c|c|c|}
\hline \multirow{2}{*}{\multicolumn{2}{|c|}{$\begin{array}{l}\text { SRS Coord. } \\
\text { N77893.9 } \\
\text { E57186.2 }\end{array}$}} & Lat/Longitude & Screen Zone Elevation & \multicolumn{2}{|c|}{ Top of Casing } & \multirow{2}{*}{$\frac{\text { Casing }}{4^{\prime \prime} \text { PVC }}$} & Pump & \multicolumn{4}{|c|}{ Screen Zone } \\
\hline & & $\begin{array}{l}33.291358^{\circ} \mathrm{N} \\
81.667525^{\circ} \mathrm{W}\end{array}$ & $23.8-203.8 \mathrm{ft} \mathrm{msl}$ & $290.9 f$ & & & $\mathrm{~s}$ & \multicolumn{4}{|c|}{ Barnwell (IIB1) } \\
\hline \multicolumn{3}{|c|}{ SAMPLE DATE } & $01 / 31 / 94$ & $04 / 13 / 94$ & $08 / 08 / 94$ & $11 / 08 / 94$ & & & & & \\
\hline \multicolumn{12}{|c|}{ FIELD DATA } \\
\hline \multicolumn{3}{|c|}{ Analyte } & 1094 & 2094 & 3094 & 4094 & Unit & & & & \\
\hline \multicolumn{3}{|c|}{$\begin{array}{l}\text { Water elevation } \\
\text { Depth to water } \\
\text { pH } \\
\text { Sp. conductance } \\
\text { Water temperature } \\
\text { Alkalinity as } \mathrm{CaCO}_{3} \\
\text { Turbidity } \\
\text { Volume purged } \\
\text { Sampling code }\end{array}$} & $\begin{array}{l}215.9 \\
75.0 \\
5.9 \\
34 \\
17.6 \\
4 \\
0 \\
6.7\end{array}$ & $\begin{array}{l}215.9 \\
75.0 \\
5.7 \\
45 \\
19.8 \\
5 \\
1 \\
4.0\end{array}$ & $\begin{array}{l}215.1 \\
75.8 \\
5.4 \\
33 \\
19.8 \\
1 \\
1 \\
8.8\end{array}$ & $\begin{array}{l}214.6 \\
76.3 \\
6.0 \\
38 \\
19.8 \\
4 \\
1 \\
10.6\end{array}$ & $\begin{array}{l}\mathrm{ft} \mathrm{msl} \\
\mathrm{ft} \\
\mathrm{pH} \\
\mu \mathrm{S} / \mathrm{cm} \\
{ }^{\circ} \mathrm{C} \\
\mathrm{mg} / \mathrm{L} \\
\text { NTU } \\
\text { well vol. }\end{array}$ & & & & \\
\hline \multicolumn{12}{|c|}{ ANALYTICAL DATA } \\
\hline \multirow[t]{2}{*}{$\underline{H} \underline{S T}$} & Ana & & 1094 & $\underline{2094}$ & 3094 & 4094 & DF & Mod & Unit & $\underline{\text { Lab }}$ & Flag \\
\hline & $\begin{array}{l}\text { Ace } \\
\text { Aldr } \\
\text { Alun } \\
\text { Anti } \\
\text { Arse } \\
\text { Bari } \\
\text { Benz } \\
\text { Bron } \\
\text { Bror } \\
\text { Bron } \\
\text { Cad: } \\
\text { Calc } \\
\text { Carb } \\
\text { Chlo } \\
\text { Chlo } \\
\text { Chlo } \\
\text { Chlo } \\
2-C h \\
\text { Chlo } \\
\text { Chlo } \\
\text { Chro } \\
\text { Cop } \\
\text { Cyar } \\
\text { p,p' } \\
\text { Dibr } \\
1,1- \\
1,2- \\
1,1- \\
\text { tran } \\
\text { Dich } \\
2,4 \\
1,2- \\
\text { cis- } \\
\text { tran } \\
\text { Diel } \\
\text { Endr } \\
\text { Ethy } \\
\text { Fluo } \\
\text { Gros }\end{array}$ & $\begin{array}{l}\text { enone } \\
\text { m, total recoverable } \\
\text { y, total recoverable } \\
\text { total recoverable } \\
\text { total recoverable } \\
\text { chloromethane } \\
\text { im } \\
\text { ethane (Methyl bromide) } \\
\text { n, total recoverable } \\
\text { total recoverable } \\
\text { etrachloride } \\
\text { nzene } \\
\text { hane } \\
\text { hene (Vinyl chloride) } \\
\text { ethyl vinyl ether } \\
\text { rm } \\
\text { ethane (Methyl chloride) } \\
\text { m, total recoverable } \\
\text { total recoverable } \\
\text { chloromethane } \\
\text { loroethane } \\
\text { loroethane } \\
\text { loroethylene } \\
\text { 2-Dichloroethylene } \\
\text { methane } \\
\text { lorophenoxyacetic acid } \\
\text { loropropane } \\
\text { Dichloropropene } \\
\text { 3-Dichloropropene } \\
\text { zene }\end{array}$ & $\begin{array}{l}<10 \\
<0.054 \\
40 \\
<2.0 \\
<2.0 \\
7.6 \\
<1.0 \\
<1.0 \\
<1.0 \\
<1.0 \\
<2.0 \\
21,790 \\
<1.0 \\
21.460 \\
<1.0 \\
<1.0 \\
<1.0 \\
<1.0 \\
<1.0 \\
<1.0 \\
<4.0 \\
<4.0 \\
<5.0 \\
<0.11 \\
<1.0 \\
<1.0 \\
<1.0 \\
<1.0 \\
<1.0 \\
<1.0 \\
<0.0015 \\
<1.0 \\
<1.0 \\
<1.0 \\
<0.54 \\
<0.0064 \\
<1.0 \\
<100 \\
<1.1 \mathrm{E}-01\end{array}$ & $\begin{array}{l}<10 \\
<0.052 \\
23 \\
<2.0 \\
<2.0 \\
<.1 \\
<1.0 \\
<1.0 \\
<1.0 \\
<1.0 \\
<2.0 \\
2,420 \\
<1.0 \\
2,430 \\
<1.0 \\
<1.0 \\
<1.0 \\
<1.0 \\
<1.0 \\
<1.0 \\
<4.0 \\
5.1 \\
<5.0 \\
<0.10 \\
<1.0 \\
<1.0 \\
<1.0 \\
<1.0 \\
<1.0 \\
<1.0 \\
<0.0016 \\
<1.0 \\
<1.0 \\
<1.0 \\
<0.52 \\
<0.0062 \\
<1.0 \\
<100 \\
7.7 \mathrm{E}-01\end{array}$ & $\begin{aligned} &<11 \\
& 44 \\
&<3.0 \\
&<2.0 \\
&<8.2 \\
&<5.0 \\
&<5.0 \\
&<5.0 \\
&<10 \\
&<2.0 \\
&<1.480 \\
&<5.0 \\
&<2.950 \\
&<5.0 \\
&<10 \\
&<10 \\
&<10 \\
&<5.0 \\
&<3.6 \\
&<4.0 \\
&<4.0 \\
&<5.0 \\
&<5.0 \\
&<5.0 \\
&<5.0 \\
&<5.0 \\
&<5.0 \\
&<40 \\
&<1.1 \\
&<5.0 \\
&<5.0 \\
&<5.0 \\
&<0.11 \\
&<5.0 \\
&<100\end{aligned}$ & $\begin{array}{l}<9.9 \\
<0.050 \\
61 \\
<2.0 \\
<2.0 \\
>9.0 \\
<1.0 \\
<1.0 \\
<1.0 \\
<1.0 \\
<2.0 \\
2,190 \\
<1.0 \\
2,570 \\
<1.0 \\
<1.0 \\
<1.0 \\
<1.0 \\
<1.0 \\
<1.0 \\
<4.0 \\
<4.0 \\
<5.0 \\
<0.099 \\
<1.0 \\
<1.0 \\
<1.0 \\
<1.0 \\
<1.0 \\
<1.0 \\
<0.0015 \\
<1.0 \\
<1.0 \\
<1.0 \\
<0.50 \\
<0.0059 \\
<1.0 \\
64 \\
-1.0 \mathrm{E}-01\end{array}$ & $\begin{array}{l}1 \\
1 \\
1 \\
1 \\
1 \\
1 \\
1 \\
1 \\
1 \\
1 \\
1 \\
1 \\
1 \\
1 \\
1 \\
1 \\
1 \\
1 \\
1 \\
1 \\
1 \\
1 \\
1 \\
1 \\
1 \\
1 \\
1 \\
1 \\
1 \\
1 \\
1 \\
1 \\
1 \\
1 \\
1 \\
1 \\
1 \\
1 \\
1\end{array}$ & & $\begin{array}{l}\mu g / L \\
\mu g / L \\
\mu g / L \\
\mu g / L \\
\mu g / L \\
\mu g / L \\
\mu g / L \\
\mu g / L \\
\mu g / L \\
\mu g / L \\
\mu g / L \\
\mu g / L \\
\mu g / L \\
\mu g / L \\
\mu g / L \\
\mu g / L \\
\mu g / L \\
\mu g / L \\
\mu g / L \\
\mu g / L \\
\mu g / L \\
\mu g / L \\
\mu g / L \\
\mu g / L \\
\mu g / L \\
\mu g / L \\
\mu g / L \\
\mu g / L \\
\mu g / L \\
\mu g / L \\
\mu g / L \\
\mu g / L \\
\mu g / L \\
\mu g / L \\
\mu g / L \\
\mu g / L \\
\mu g / L \\
\mu g / L \\
p C i / L\end{array}$ & $\begin{array}{l}\mathrm{GE} \\
\mathrm{GE} \\
W A \\
\mathrm{GE} \\
\mathrm{GE} \\
W A \\
\mathrm{GE} \\
\mathrm{GE} \\
\mathrm{GE} \\
\mathrm{GE} \\
\mathrm{GE} \\
\mathrm{GE} \\
\mathrm{GE} \\
\mathrm{WA} \\
\mathrm{GE} \\
\mathrm{GE} \\
\mathrm{GE} \\
\mathrm{GE} \\
\mathrm{GE} \\
\mathrm{GE} \\
\mathrm{GE} \\
\mathrm{GE} \\
\mathrm{GE} \\
\mathrm{GE} \\
\mathrm{GE} \\
\mathrm{GE} \\
\mathrm{GE} \\
\mathrm{GE} \\
\mathrm{GE} \\
\mathrm{GE} \\
\mathrm{GE} \\
\mathrm{GE} \\
\mathrm{GE} \\
\mathrm{GE} \\
\mathrm{GE} \\
\mathrm{GE} \\
\mathrm{GE} \\
\mathrm{GE} \\
\mathrm{TM}\end{array}$ & $\begin{array}{l}0 \\
0 \\
2 \\
0 \\
0 \\
0 \\
0 \\
0 \\
0 \\
0 \\
0 \\
0 \\
0 \\
0 \\
0 \\
0 \\
0 \\
0 \\
0 \\
0 \\
0 \\
0 \\
0 \\
0 \\
0 \\
0 \\
0 \\
0 \\
0 \\
0 \\
0 \\
0 \\
0 \\
0 \\
0 \\
0 \\
0 \\
0 \\
0\end{array}$ \\
\hline
\end{tabular}

Note: Flagging, dilution factors, modifiers, and laboratories are for fourth quarter 1994 data only. See Appendix B for flagging criteria. - = exceeded holding time for fourth quarter 1994.

- = exceeded screening level or final PDWS for fourth quarter 1994. 
HE ST Analyte

Heptachlor

Iron, total recoverable

Lead, total recoverable

Lindane

Magnesium, total recoverable

Manganese, total recoverable

Mercury, total recoverable

Methoxychlor

Naphthalene

Nickel, total recoverable

Nitrate as nitrogen

Nitrate-nitrite as nitrogen

Nonvolatile beta

pH

Phenols

Potassium, total recoverable

Radium, total alpha-emitting

Selenium, total recoverable

Silica, total recoverable

Silver, total recoverable

Sodium, total recoverable

Specific conductance

Sulfate

1,1,2,2-Tetrachloroethane

Tetrachloroethylene

Tin, total recoverable

Toluene

Total dissolved solids

Total organic carbon

Total organic halogens

Total phosphates (as P)

Toxaphene

2,4,5-TP (Silvex)

1,1,1-Trichloroethane

1,1,2-Trichloroethane

Trichloroethylene

Trichlorofluoromethane

2,4,5-T

- Tritium

Turbidity

Vanadium, total recoverable

Xylenes

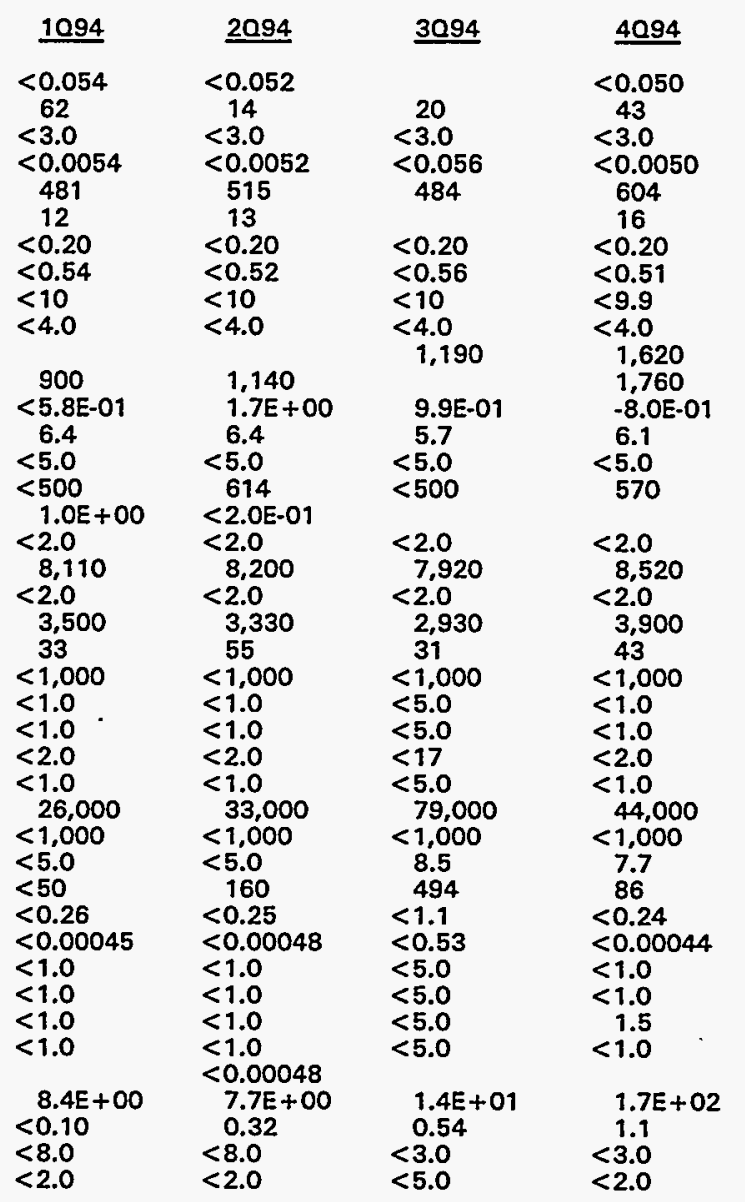

\begin{tabular}{|c|c|c|c|}
\hline DF & Mod & Unit & Lab \\
\hline 1 & \multirow{11}{*}{$\mathbf{J}$} & $\mu \mathrm{g} / \mathrm{L}$ & GE \\
\hline 1 & & $\mu \mathrm{g} / \mathrm{L}$ & GE \\
\hline 1 & & $\mu \mathrm{g} / \mathrm{L}$ & GE \\
\hline 1 & & $\mu \mathrm{g} / \mathrm{L}$ & GE \\
\hline 1 & & $\mu g / L$ & GE \\
\hline 1 & & $\mu \mathrm{g} / \mathrm{L}$ & WA \\
\hline 1 & & $\mu \mathrm{g} / \mathrm{L}$ & GE \\
\hline 1 & & $\mu g / L$ & GE \\
\hline 1 & & $\mu g / L$ & GE \\
\hline 1 & & $\mu \mathrm{g} / \mathrm{L}$ & $\mathrm{GE}$ \\
\hline 5 & & $\mu g / L$ & WA \\
\hline 1 & \multirow{4}{*}{$\begin{array}{l}\text { UI } \\
\mathbf{J}\end{array}$} & $\mu \mathrm{g} / \mathrm{L}$ & GE \\
\hline 1 & & $\mathrm{pCi} / \mathrm{L}$ & $T M$ \\
\hline 1 & & $\mathrm{pH}$ & GE \\
\hline 1 & & $\mu \mathrm{g} / \mathrm{L}$ & GE \\
\hline 1 & \multirow[t]{15}{*}{$\mathbf{J}$} & $\begin{array}{l}\mu \mathrm{g} / \mathrm{L} \\
\mathrm{pCi} / \mathrm{L}\end{array}$ & GE \\
\hline$i$ & & $\mu \mathrm{g} / \mathrm{L}$ & GE \\
\hline 2.1 & & $\mu g / L$ & WA \\
\hline 1 & & $\mu \mathrm{g} / \mathrm{L}$ & GE \\
\hline 1 & & $\mu \mathrm{g} / \mathrm{L}$ & WA \\
\hline 1 & & $\mu \mathrm{S} / \mathrm{cm}$ & WA \\
\hline 1 & & $\mu \mathrm{g} / \mathrm{L}$ & $\mathrm{GE}$ \\
\hline 1 & & $\mu g / L$ & GE \\
\hline 1 & & $\mu g / L$ & GE \\
\hline 1 & & $\mu \mathrm{g} / \mathrm{L}$ & GE \\
\hline 1 & & $\mu g / L$ & GE \\
\hline 1 & & $\mu g / L$ & WA \\
\hline 1 & & $\mu g / L$ & GE \\
\hline 1 & & $\mu \mathrm{g} / \mathrm{L}$ & WA \\
\hline 1 & & $\mu \mathrm{g} / \mathrm{L}$ & WA \\
\hline 1 & \multirow[t]{4}{*}{$J$} & $\mu g / L$ & GE \\
\hline 1 & & $\mu g / L$ & GE \\
\hline 1 & & $\mu \mathrm{g} / \mathrm{L}$ & GE \\
\hline 1 & & $\mu g / L$ & GE \\
\hline 1 & \multirow[t]{6}{*}{$J$} & $\mu g / L$ & GE \\
\hline 1 & & $\mu g / L$ & GE \\
\hline 1 & & pCi/mL & $T M$ \\
\hline 1 & & NTU & GE \\
\hline 1 & & $\mu g / L$ & WA \\
\hline 1 & & $\mu g / L$ & GE \\
\hline
\end{tabular}

Note: Flagging, dilution factors, modifiers, and laboratories are for fourth quarter 1994 data only. See Appendix B for flagging criteria. - = exceeded holding time for fourth quarter 1994.

- exceeded screening level or final PDWS for fourth quarter 1994. 
WELL BGX 5D

\begin{tabular}{|c|c|c|c|c|c|c|}
\hline SRS Coord. & Lat/Longitude & Screen Zone Elevation & Top of Casing & Casing & Pump & $\underline{\text { Screen Zone }}$ \\
\hline $\begin{array}{l}\text { N78402.0 } \\
\text { E57308.6 }\end{array}$ & $\begin{array}{l}33.2926811^{\circ} \mathrm{N} \\
81.668190^{\circ} \mathrm{W}\end{array}$ & $215.0-195.0 \mathrm{ft} \mathrm{msl}$ & $285 \mathrm{ft} \mathrm{ms!}$ & 4" PVC & $\mathbf{s}$ & Barnwell (IIB1) \\
\hline SAMPL: & & $01 / 31 / 94$ & $04 / 13 / 94$ & & & \\
\hline
\end{tabular}

FIELD DATA

Analyte
Water elevation
Depth to water
pH
$\mathrm{Sp}$. conductance
Water temperature
Alkalinity as $\mathrm{CaCO}_{3}$
Turbidity
Volume purged
Sampling code

ANALYTICAL DATA

Acetophenone

Aldrin

Aluminum, total recoverable

Antimony, total recoverable

Arsenic, total recoverable

Barium, total recoverable

Benzene

Bromodichloromethane

Bromoform

Bromomethane (Methyl bromide)

Cadmium, total recoverable

Calcium, total recoverable

Carbon tetrachloride

Chloride

Chlorobenzene

Chloroethane

Chloroethene (Vinyl chloride)

2-Chloroethyl vinyl ether

Chloroform

Chloromethane (Methyl chloride)

Chromium, total recoverable

Copper, total recoverable

Cyanide

$p, p^{\prime}$-DDT

Dibromochloromethane

1,1-Dichloroethane

1,2-Dichloroethane

1,1-Dichloroethylene

trans-1,2-Dichloroethylene

Dichloromethane

2,4-Dichlorophenoxyacetic acid

1,2-Dichloropropane

cis-1,3-Dichloropropene

trans-1,3-Dichloropropene

Dieldrin

Endrin

Ethylbenzene

Fluoride

Gross alpha

\begin{tabular}{|c|c|c|}
\hline 1094 & $\underline{2094}$ & $\underline{3094}$ \\
\hline $\begin{array}{l}<9.7 \\
<0.048\end{array}$ & $\begin{array}{l}<10 \\
<0.051\end{array}$ & $<11$ \\
\hline 92 & 97 & 114 \\
\hline$<2.0$ & $<2.0$ & $<3.0$ \\
\hline$<2.0$ & $<2.0$ & $<2.0$ \\
\hline 24 & 24 & 24 \\
\hline$<1.0$ & $<1.0$ & $<5.0$ \\
\hline$<1.0$ & $<1.0$ & $<5.0$ \\
\hline$<1.0$ & $<1.0$ & $<5.0$ \\
\hline$<1.0$ & $<1.0$ & $<10$ \\
\hline$<2.0$ & $<2.0$ & $<2.0$ \\
\hline 2,050 & 2,270 & 2,230 \\
\hline$<1.0$ & $<1.0$ & $<5.0$ \\
\hline 4,050 & 4,040 & $\begin{array}{r}4,110 \\
\end{array}$ \\
\hline $\begin{array}{l}<1.0 \\
<1.0\end{array}$ & $\begin{array}{l}<1.0 \\
<1.0\end{array}$ & $\begin{array}{l}<5.0 \\
<10\end{array}$ \\
\hline$<1.0$ & $<1.0$ & $<10$ \\
\hline$<1.0$ & $<1.0$ & $<10$ \\
\hline$<1.0$ & $<1.0$ & $<5.0$ \\
\hline$<1.0$ & $<1.0$ & $<10$ \\
\hline$<4.0$ & $<4.0$ & $<4.0$ \\
\hline$<4.0$ & $<4.0$ & $<4.0$ \\
\hline$<5.0$ & $<5.0$ & 67 \\
\hline$<0.095$ & $<0.10$ & \\
\hline$<1.0$ & $<1.0$ & $<5.0$ \\
\hline$<1.0$ & $<1.0$ & $<5.0$ \\
\hline$<1.0$ & $<1.0$ & $<5.0$ \\
\hline$<1.0$ & $<1.0$ & $<5.0$ \\
\hline$<1.0$ & $<1.0$ & $<5.0$ \\
\hline$<1.0$ & $<1.0$ & $<5.0$ \\
\hline$<0.0015$ & $<0.0016$ & $<1.1$ \\
\hline$<1.0$ & $<1.0$ & $<5.0$ \\
\hline$<1.0$ & $<1.0$ & $<5.0$ \\
\hline$<1.0$ & $<1.0$ & $<5.0$ \\
\hline$<0.48$ & $<0.51$ & \\
\hline$<0.0057$ & $<0.0061$ & $<0.11$ \\
\hline$<1.0$ & $<1.0$ & $<5.0$ \\
\hline$<100$ & $<100$ & $<100$ \\
\hline $4.2 \mathrm{E}+0$ & $3.6 E+\infty 0$ & \\
\hline
\end{tabular}

\begin{tabular}{l}
3094 \\
\hline 208.5 \\
76.6 \\
5.0 \\
59 \\
20.4 \\
1 \\
1 \\
4.1
\end{tabular}

4094
207.8
77.2
5.2
54
19.6
1
1
3.8

$\underline{\text { Unit }}$

ft ms!

$\mathrm{ft}$

$\underset{\mu \mathrm{S} / \mathrm{cm}}{\mathrm{pH}}$

${ }^{\circ} \mathrm{C}$

$\mathrm{mg} / \mathrm{L}$

NTU

3.4

4.1

4.

well vol.

\begin{tabular}{|c|c|c|c|c|}
\hline 4094 & $\underline{\mathrm{DF}}$ & Mod & Unit & $\underline{\operatorname{Lab}}$ \\
\hline$<10$ & 1 & & $\mu g / L$ & GE \\
\hline 114 & 1 & & $\mu \mathrm{g} / \mathrm{L}$ & GE \\
\hline$<2.0$ & 1 & & $\mu \mathrm{g} / \mathrm{L}$ & GE \\
\hline$<2.0$ & 1 & & $\mu \mathrm{g} / \mathrm{L}$ & GE \\
\hline 23 & 1 & & $\mu \mathrm{g} / \mathrm{L}$ & GE \\
\hline$<1.0$ & 1 & & $\mu \mathrm{g} / \mathrm{L}$ & GE \\
\hline$<1.0$ & 1 & & $\mu g / L$ & GE \\
\hline$<1.0$ & 1 & & $\mu \mathrm{g} / \mathrm{L}$ & GE \\
\hline$<1.0$ & 1 & & $\mu \mathrm{g} / \mathrm{L}$ & $\mathrm{GE}$ \\
\hline$<2.0$ & 1 & & $\mu g / L$ & GE \\
\hline 2,280 & 1 & & $\mu \mathrm{g} / \mathrm{L}$ & $\overline{G E}$ \\
\hline$<1.0$ & 1 & & $\mu \mathrm{g} / \mathrm{L}$ & GE \\
\hline 3,200 & 1 & & $\mu \mathrm{g} / \mathrm{L}$ & GE \\
\hline$<1.0$ & 1 & & $\mu g / L$ & GE \\
\hline$<1.0$ & 1 & & $\mu \mathrm{g} / \mathrm{h}$ & GE \\
\hline$<1.0$ & 1 & & $\mu \mathrm{g} / \mathrm{L}$ & GE \\
\hline$<1.0$ & 1 & & $\mu g / L$ & GE \\
\hline$<1.0$ & 1 & & $\mu g / \mathrm{L}$ & GE \\
\hline$<1.0$ & 1 & & $\mu \mathrm{g} / \mathrm{L}$ & GE \\
\hline$<4.0$ & 1 & & $\mu \mathrm{g} / \mathrm{L}$ & GE \\
\hline 143 & 1 & & $\mu \mathrm{g} / \mathrm{L}$ & GE \\
\hline$<5.0$ & 1 & - & $\mu \mathrm{g} / \mathrm{L}$ & GE \\
\hline$<1.0$ & 1 & & $\mu g / L$ & $\mathrm{GE}$ \\
\hline$<1.0$ & $i$ & & $\mu \mathrm{g} / \mathrm{L}$ & $\mathrm{GE}$ \\
\hline$<1.0$ & 1 & & $\mu g / \mathrm{L}$ & $\mathrm{GE}$ \\
\hline$<1.0$ & 1 & & $\mu \mathrm{g} / \mathrm{L}$ & GE \\
\hline$<1.0$ & 1 & & $\mu \mathrm{g} / \mathrm{L}$ & GE \\
\hline$<1.0$ & 1 & & $\mu g / L$ & GE \\
\hline$<0.0015$ & $i$ & & $\mu \mathrm{g} / \mathrm{L}$ & $\mathrm{GE}$ \\
\hline$<1.0$ & 1 & & $\mu \mathrm{g} / \mathrm{L}$ & GE \\
\hline$<1.0$ & 1 & & $\mu g / L$ & GE \\
\hline$<1.0$ & 1 & & $\mu g / L$ & GE \\
\hline$<0.0059$ & 1 & & $\mu g / L$ & GE \\
\hline$<1.0$ & 1 & & $\mu \mathrm{g} / \mathrm{L}$ & $\overline{G E}$ \\
\hline 31 & 1 & $J$ & $\mu \mathrm{g} / \mathrm{L}$ & GE \\
\hline $7.1 E+00$ & 1 & & $\mathrm{pCi} / \mathrm{L}$ & GP \\
\hline
\end{tabular}

Note: Flagging, dilution factors, modifiers, and laboratories are for fourth quarter 1994 data only. See Appendix B for flagging criteria. - = exceeded holding time for fourth quarter 1994.

- = exceeded screening level or final PDWS for fourth quarter 1994. 
Heptachlor

Iron, total recoverable

Lead, total recoverable

Lindane

Magnesium, total recoverable

Manganese, total recoverable

Mercury, total recoverable

Methoxychlor

Naphthalene

Nickel, total recoverable

Nitrate as nitrogen

Nitrate-nitrite as nitrogen

Nonvolatile beta

pH

Phenols

Potassium, total recoverable

Radium, total alpha-emitting

Selenium, total recoverable

Silica, total recoverable

Silver, total recoverable

Sodium, total recoverable

Specific conductance

Sulfate

$1,1,2,2$-Tetrachloroethane

Tetrachloroethylene

Tin, total recoverable

Toluene

Total dissolved solids

Total organic carbon

Total organic halogens

Total phosphates (as P)

Toxaphene

2,4,5-TP (Silvex)

1,1,1-Trichloroethane

1,1,2-Trichloroethane

Trichloroethylene

Trichlorofluoromethane

2,4,5-T

- Tritium

Turbidity

Vanadium, total recoverable

Xylenes

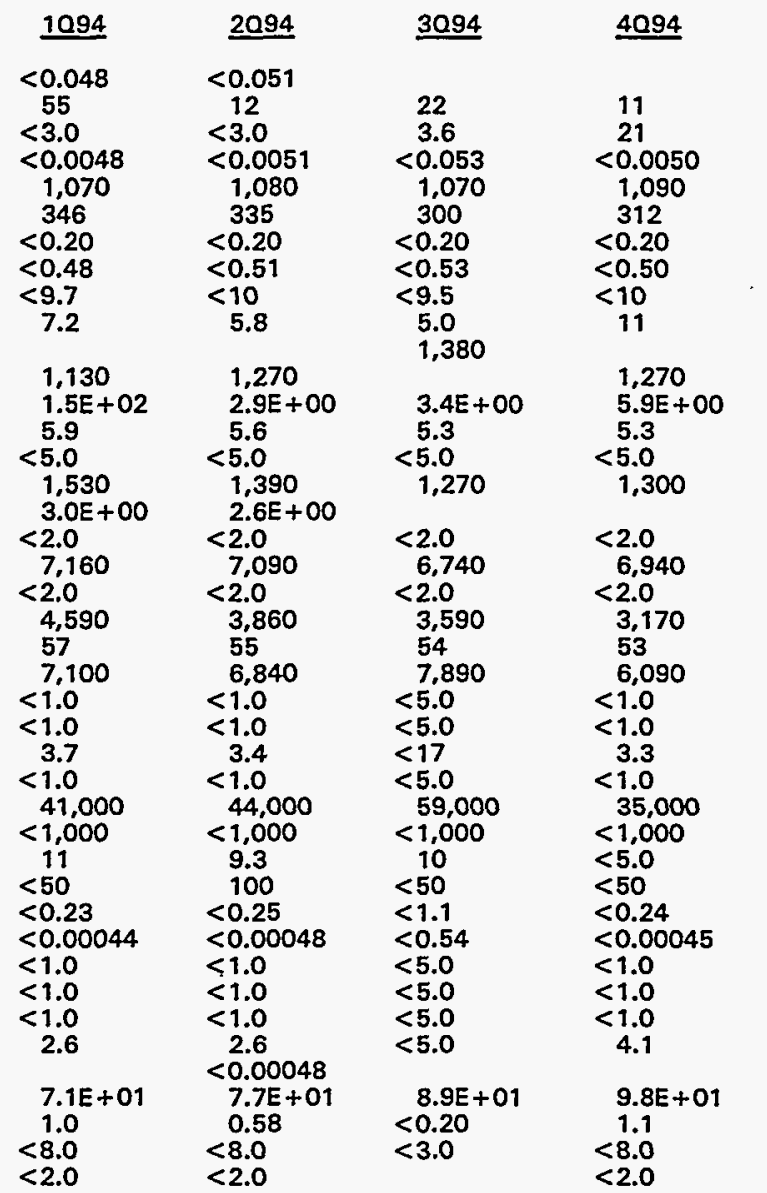

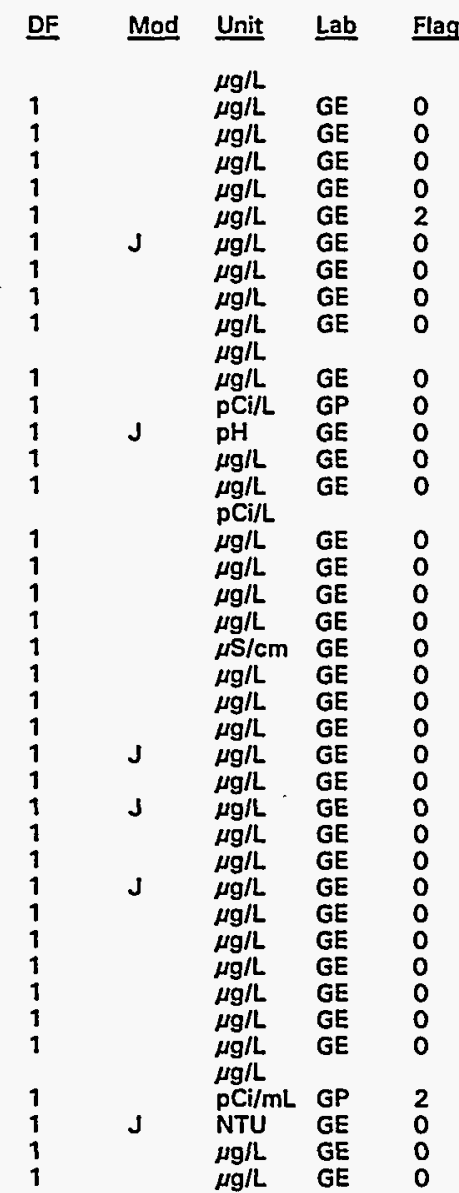

Note: Flagging, dilution factors, modifiers, and laboratories are for fourth quarter 1994 data only. See Appendix B for flagging criteria. - = exceeded holding time for fourth quarter 1994.

- = exceeded screening level or final PDWS for fourth quarter 1994. 
WELL BGX 6D

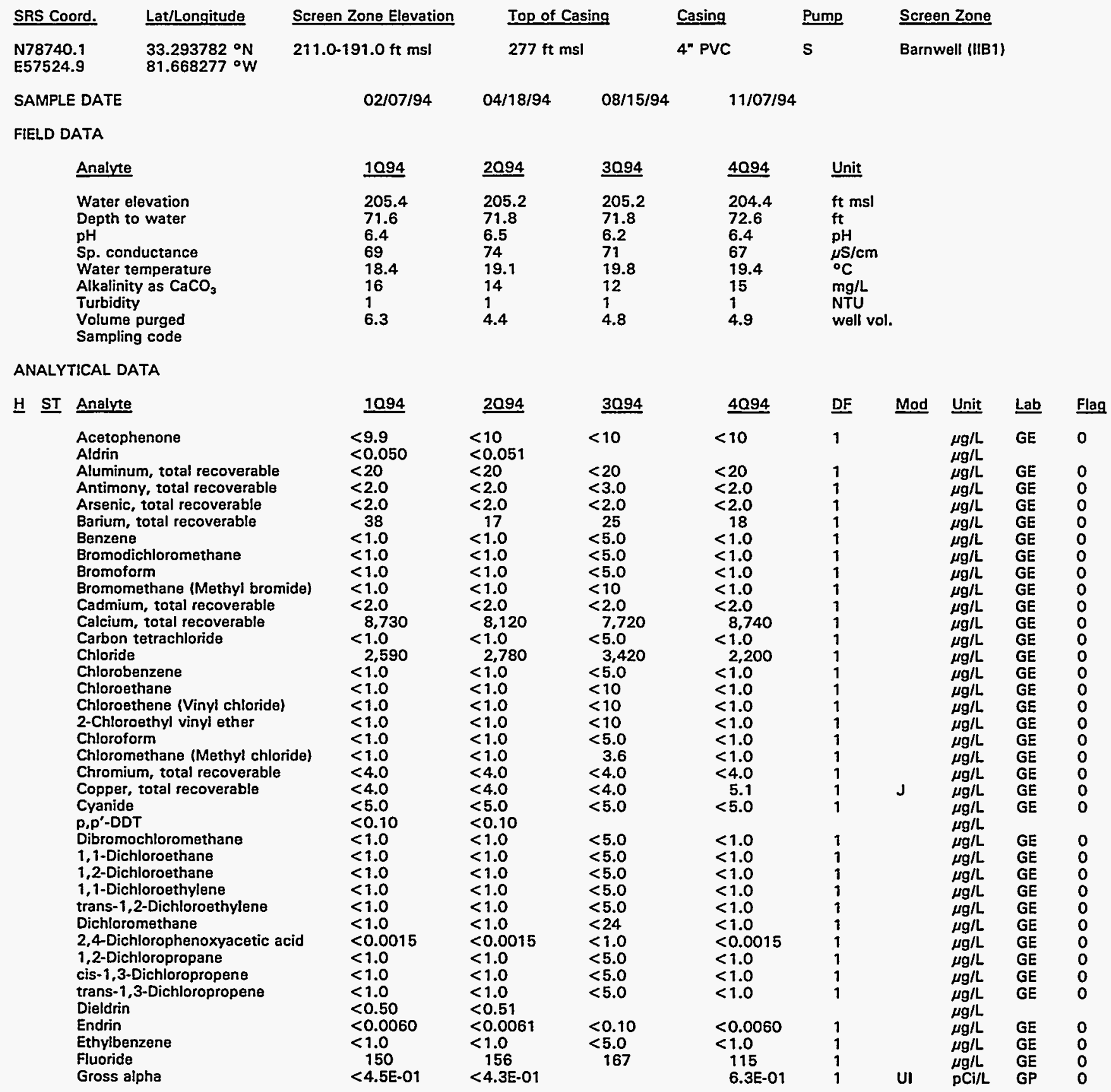

Note: Flagging, dilution factors, modifiers, and laboratories are for fourth quarter 1994 data only. See Appendix B for flagging criteria. - = exceeded holding time for fourth quarter 1994.

m = exceeded screening level or final PDWS for fourth quarter 1994. 


\section{ANALYTICAL DATA}

HI ST

Heptachlor

Iron, total recoverable

Lead, total recoverable

Lindane

Lithium, total recoverable

Magnesium, total recoverable

Manganese, total recoverable

Mercury, total recoverable

Methoxychlor

Naphthalene

Nickel, total recoverable

Nitrate as nitrogen

Nitrate-nitrite as nitrogen

Nonvolatile beta

$\mathrm{pH}$

Phenols

Potassium, total recoverable

Radium, total alpha-emitting

Selenium, total recoverable

Silica, total recoverable

Silver, total recoverable

Sodium, total recoverable

Specific conductance

Sulfate

1,1,2,2-Tetrachloroethane

Tetrachloroethylene

Tin, total recoverable

Toluene

Total dissolved solids

Total organic carbon

Total organic halogens

Total phosphates (as P)

Toxaphene

2,4,5-TP (Silvex)

1,1,1-Trichloroethane

1,1,2-Trichloroethane

Trichloroethylene

Trichlorofluoromethane

2,4,5-T

Tritium

Turbidity

Vanadium, total recoverable

Xylenes

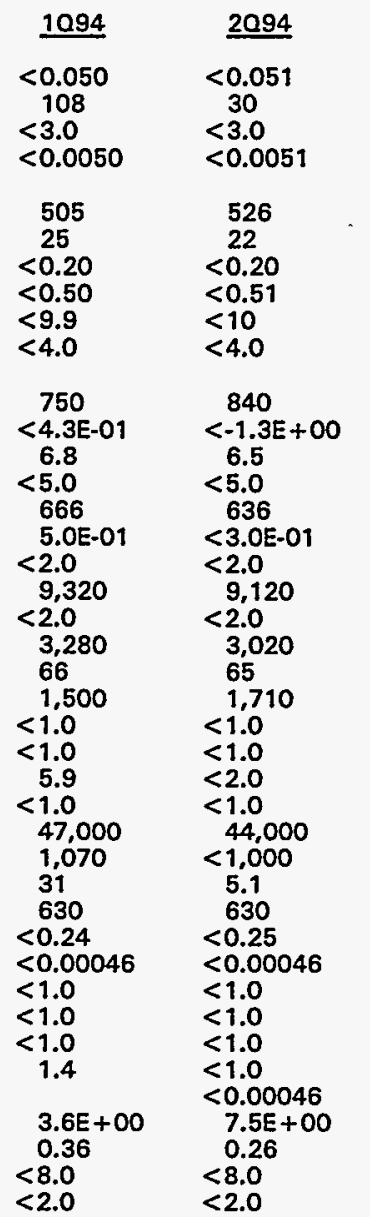

3094

4094

DF

Mod

$\begin{array}{ll} & 30 \\ & 4.2 \\ < & 0.052 \\ < & 5.0\end{array}$

35
13

$<5.0$

479
20

$<0.20$

$<0.52$

$<10$

$<4.0$

7.0E-01

6.5
$<5.0$

509

$<2.0$

9,030

$<2.0$

3,260

66

2,360

$<5.0$

$<5.0$

$<17$

$<5.0$

44,000

$<1,000$

7.4

937

$<1.0$

$<0.52$

$<5.0$

$<5.0$

$<5.0$

$<5.0$

$9.3 E+\infty$

$\begin{aligned} & 0.32 \\ &<3.0\end{aligned}$

$<0.0050$

509
20

$<0.20$

$<0.50$

$<10$

$<4.0$

870
$6.2 \mathrm{E}-0$

6.7
$<5.0$

$<5.0$

519

$<2.0$

9,480

2,850
67

1,860

$<1.0$

$<1.0$

$<2.0$

$<1.0$

53,000

$<1,000$

$<5.0$

565

$<0.24$

$<0.00046$

$<1.0$

$<1.0$

$<1.0$

$<1.0$

$1.2 E+01$

0.83

$<8.0$

$<2.0$

1 Hod

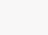

Unit Lab Flag

$\begin{array}{lll}\mu \mathrm{g} / \mathrm{L} & \\ \mu \mathrm{g} / \mathrm{L} & \mathrm{GE} & 0 \\ \mu \mathrm{g} / \mathrm{L} & \mathrm{GE} & 0\end{array}$

$\mu \mathrm{g} / \mathrm{L}$ GE 0

$\mu g / L$

$\mu \mathrm{g} / \mathrm{L}$ GE 0

$\mu g / L \quad G E \quad 0$

$\mu \mathrm{g} / \mathrm{L} \quad \mathrm{GE}$

$\mu \mathrm{g} / \mathrm{L} \quad \mathrm{GE}$

$\mu g / L$

$\mu \mathrm{g} / \mathrm{L}$ GE 0

pH

$\mu$ /L GE

$\mu g / L$ GE

pCi/L

$\mu \mathrm{g} / \mathrm{L} \quad \mathrm{GE}$

$\mu g / L \quad G E$

$\mu \mathrm{g} / \mathrm{L} \quad \mathrm{GE}$

$\mu \mathrm{g} / \mathrm{L}$

$\mu \mathrm{S} / \mathrm{cm}$ GE

$\mu g / L \quad G E$

$\mu g / L \quad$ GE

$\mu g / L \quad G E$

$\mu g / L$

$\mu g / L$

$\mu g / L$

$\mu g / L$

$\mu \mathrm{g} / \mathrm{L}$

$\mu \mathrm{g} /$

$\mu g / L$

$\mu g / L$

$\mu g / L$

$\mu \mathrm{g}$

$\mu g / L$

$\mathrm{pCi} / \mathrm{mL} \quad \mathrm{GP}$

NTU

$\mu g / L \quad G E$

$\mu g / L$



Note: Flagging, dilution factors, modifiers, and laboratories are for fourth quarter 1994 data only. See Appendix B for flagging criteria. - = exceeded holding time for fourth quarter 1994.

= exceeded screening level or final PDWS for fourth quarter 1994. 
WELL BGX 7D

\begin{tabular}{|c|c|c|c|c|c|c|}
\hline SRS Coord. & Lat/Longitude & Screen Zone Elevation & Top of Casing & Casing & Pump & Screen Zone \\
\hline $\begin{array}{l}\text { N78349.3 } \\
\text { E58312.8 }\end{array}$ & $\begin{array}{l}33.294203^{\circ} \mathrm{N} \\
81.665443^{\circ} \mathrm{W}\end{array}$ & $214.1-194.1 \mathrm{ft} \mathrm{msl}$ & $279.2 \mathrm{ft} \mathrm{msl}$ & 4" PVC & $\mathbf{S}$ & Barnwell (IIB1) \\
\hline SAMPLE DATE & & $02 / 07 / 94$ & $04 / 18 / 94$ & & & \\
\hline
\end{tabular}

FIELD DATA

Anaivte

Water elevation

Depth to water

$\mathrm{pH}$

Sp. conductance

Water temperature

Alkalinity as $\mathrm{CaCO}_{3}$

Turbidity

Volume purged

Sampling code

ANALYTICAL DATA

HI ST Analyte

Acetophenone

Aldrin

Aluminum, total recoverable

Antimony, total recoverable

Arsenic, total recoverable

Barium, total recoverable

Benzene

Bromodichloromethane

Bromoform

Bromomethane (Methyl bromide)

Cadmium, total recoverable

Calcium, total recoverable

Carbon tetrachloride

Chloride

Chlorobenzene

Chloroethane

Chloroethene (Vinyl chloride)

2-Chloroethyl vinyl ether

Chloroform

Chloromethane (Mathyl chloride)

Chromium, total recoverable

Copper, total recoverable

Cyanide

p.p'-DDT

Dibromochloromethan

1,1-Dichloroethane

1,2-Dichloroethan

1,1-Dichloroethylene

trans-1,2-Dichloroethylene

Dichloromethane

2,4-Dichlorophenoxyacetic acid

1,2-Dichloropropane

cis-1,3-Dichloropropene

trans-1,3-Dichloropropene

Dieldrin

Endrin

Ethylbenzene

Fluoride

Gross alpha

$\begin{array}{ll}1094 & \underline{2094} \\ 205.3 & 205.2 \\ 74.0 & 74.0 \\ 5.1 & 5.3 \\ 21 & 24 \\ 18.3 & 19.3 \\ 0 & 0 \\ 1 & 0 \\ 5.7 & 7.0\end{array}$

3094
205.1
74.1
5.0
23
19.6
0
1
6.7

4094
204.5
74.7
4.9
20
18.9
0
1
6.8

Unit

ft ms!

$\mathrm{pH}$

${ }^{\circ} \mathrm{C}$

mg/L

well vol.

\begin{tabular}{|c|c|c|c|c|c|c|c|c|}
\hline 1094 & 20.94 & 3094 & 4094 & DF & Mod & Unit & $\underline{\text { Lab }}$ & Fla \\
\hline $\begin{array}{l}<9.9 \\
<0.051\end{array}$ & $\begin{array}{l}<10 \\
<0.052\end{array}$ & $<11$ & $<10$ & 1 & & $\underset{\mu \mathrm{g} / \mathrm{L}}{\mu}$ & GE & 0 \\
\hline & 25 & 36 & 34 & 1 & & $\mu \mathrm{g} / \mathrm{L}$ & GE & 1 \\
\hline$<2.0$ & $<2.0$ & $<3.0$ & $<2.0$ & 1 & & $\mu \mathrm{g} / \mathrm{L}$ & GE & 0 \\
\hline$<2.0$ & $<2.0$ & $<2.0$ & $<2.0$ & 1 & & $\mu \mathrm{g} / \mathrm{L}$ & $\mathrm{GE}$ & 0 \\
\hline 7.2 & 6.7 & 7.2 & 6.4 & 1 & & $\mu \mathrm{g} / \mathrm{L}$ & $\mathrm{GE}$ & 0 \\
\hline$<1.0$ & $<1.0$ & $<5.0$ & $<1.0$ & 1 & & $\mu \mathrm{g} / \mathrm{L}$ & $\overline{G E}$ & $=$ \\
\hline$<1.0$ & $<1.0$ & $<5.0$ & $<1.0$ & 1 & & $\mu \mathrm{g} / \mathrm{L}$ & GE & \\
\hline$<1.0$ & $<1.0$ & $<5.0$ & $<1.0$ & $j$ & & $\mu g / L$ & GE & 0 \\
\hline$<1.0$ & $<1.0$ & $<10$ & $<1.0$ & 1 & & $\mu \mathrm{g} / \mathrm{L}$ & GE & 0 \\
\hline$<2.0$ & $<2.0$ & $<2.0$ & $<2.0$ & 1 & & $\mu \mathrm{g} / \mathrm{L}$ & GE & 0 \\
\hline 497 & 440 & 418 & 380 & 1 & & $\mu g / L$ & $\mathrm{GE}$ & 0 \\
\hline$<1.0$ & $<1.0$ & $<5.0$ & $<1.0$ & $i$ & & $\mu \mathrm{g} / \mathrm{L}$ & GE & \\
\hline 1,870 & 1,860 & 2,260 & 1,570 & 1 & & $\mu \mathrm{g} / \mathrm{L}$ & $\overline{G E}$ & 0 \\
\hline$<1.0$ & $<1.0$ & $<5.0$ & $<1.0$ & 1 & & $\mu \mathrm{g} / \mathrm{L}$ & GE & \\
\hline$<1.0$ & $<1.0$ & $<10$ & $<1.0$ & 1 & & $\mu g / L$ & GE & 0 \\
\hline$<1.0$ & $<1.0$ & $<10$ & $<1.0$ & 1 & & $\mu \mathrm{g} / \mathrm{L}$ & $\mathrm{GE}$ & 0 \\
\hline$<1.0$ & $<1.0$ & $<10$ & $<1.0$ & 1 & & $\mu \mathrm{g} / \mathrm{L}$ & GE & 0 \\
\hline$<1.0$ & $<1.0$ & $<5.0$ & $<1.0$ & 1 & & $\mu \mathrm{g} / \mathrm{L}$ & GE & 0 \\
\hline$<1.0$ & $<1.0$ & 1.8 & $<1.0$ & 1 & & $\mu \mathrm{g} / \mathrm{L}$ & GE & 0 \\
\hline$<4.0$ & $<4.0$ & $<4.0$ & $<4.0$ & $i$ & & $\mu \mathrm{g} / \mathrm{L}$ & $\overline{G E}$ & 0 \\
\hline$<4.0$ & $<4.0$ & $<4.0$ & $<4.0$ & 1 & & $\mu \mathrm{g} / \mathrm{L}$ & GE & 0 \\
\hline$<5.0$ & $<5.0$ & $<5.0$ & $<5.0$ & 1 & & $\mu \mathrm{g} / \mathrm{h}$ & $\mathrm{GE}$ & \\
\hline $\begin{array}{l}<0.10 \\
<1.0\end{array}$ & $\begin{array}{l}<0.10 \\
<1.0\end{array}$ & $<5,0$ & $<1.0$ & 1 & & $\mu g / L$ & & \\
\hline$<1.0$ & $<1.0$ & $<5.0$ & $<1.0$ & 1 & & $\mu g / L$ & $G E$ & 0 \\
\hline$<1.0$ & $<1.0$ & $<5.0$ & $<1.0$ & $i$ & & $\mu \mathrm{g} / \mathrm{L}$ & GE & \\
\hline$<1.0$ & $<1.0$ & $<5.0$ & $<1.0$ & 1 & & $\mu \mathrm{g} / \mathrm{L}$ & GE & 0 \\
\hline$<1.0$ & $<1.0$ & $<5.0$ & $<1.0$ & $i$ & & $\mu g / L$ & GE & \\
\hline$<1.0$ & $<1.0$ & $<5.0$ & $<1.0$ & 1 & & $\mu \mathrm{g} / \mathrm{L}$ & GE & 0 \\
\hline$<0.0015$ & $<0.0015$ & $<1.0$ & $<0.0015$ & 1 & & $\mu \mathrm{g} / \mathrm{L}$ & $\mathrm{GE}$ & 0 \\
\hline$<1.0$ & $<1.0$ & $<5.0$ & $<1.0$ & 1 & & $\mu g / L$ & GE & \\
\hline$<1.0$ & $<1.0$ & $<5.0$ & $<1.0$ & 1 & & $\mu g / L$ & GE & \\
\hline $\begin{array}{l}<1.0 \\
<0.51\end{array}$ & $\begin{array}{l}<1.0 \\
<0.52\end{array}$ & $<5.0$ & $<1.0$ & $i$ & & $\mu \mathrm{g} / \mathrm{L}$ & GE & \\
\hline$<0.0061$ & $<0.0062$ & $<0.11$ & $<0.0059$ & 1 & & $\mu g / h$ & GE & 0 \\
\hline$<1.0$ & $<1.0$ & $<5.0$ & $<1.0$ & 1 & & $\mu g / L$ & GE & \\
\hline$<100$ & $<100$ & $<100$ & $<20$ & 1 & & $\mu g / L$ & GE & \\
\hline $1.0 E+00$ & 9.3E-01 & & $1.0 E+00$ & 1 & $J$ & pCi/L & GP & \\
\hline
\end{tabular}

Note: Flagging, dilution factors, modifiers, and laboratories are for fourth quarter 1994 data only. See Appendix B for flagging criteria. - = exceeded holding time for fourth quarter 1994.

- exceeded screening level or final PDWS for fourth quarter 1994. 
Analyte

Heptachlor

Iron, total recoverable

Lead, total recoverable

Lindane

Magnesium, total recoverable

Manganese, total recoverable

Mercury, total recoverable

Methoxychlor

Nickel, total recoverable

Nitrate as nitrogen

Nitrate-nitrite as nitrogen

Nonvolatile beta

pH

Phenols

Potassium, total recoverable

Radium, total alpha-emitting

Selenium, total recoverable

Silica, total recoverable

Silver, total recoverable

Sodium, total recoverable

Specific conductance

Sulfate

1,1,2,2-Tetrachloroethane

Tetrachloroethylene

Tin, total recoverable

Toluene

Total activity

Total dissolved solids

Total organic carbon

Total organic halogens

Total phosphates (as P)

Toxaphene

2,4,5-TP (Silvex)

1,1,1-Trichloroethane

1,1,2-Trichloroethane

Trichloroethylene

Trichlorofluoromethane

2,4,5-T

Tritium

Turbidity

Vanadium, total recoverable

Xylenes

\begin{tabular}{|c|c|}
\hline 1094 & $\underline{2094}$ \\
\hline $\begin{aligned} &< 0.051 \\
& 18 \\
&<3.0 \\
&<0.0051 \\
& 372 \\
& 11 \\
&<0.20 \\
&<0.51 \\
&<9.9 \\
&<4.0\end{aligned}$ & $\begin{array}{c}<0.052 \\
45 \\
3.6 \\
<0.0052 \\
377 \\
10 \\
<0.20 \\
<0.52 \\
<10 \\
<4.0\end{array}$ \\
\hline $\begin{aligned} & 550 \\
& 1.9 E+00 \\
& 5.3 \\
&<5.0 \\
&<500 \\
& 1.1 E+00 \\
&<2.0 \\
& 6,560 \\
&<2.0 \\
& 1,550 \\
& 20 \\
&<1,000 \\
&<1.0 \\
&<1.0 \\
& 5.4 \\
&<1.0\end{aligned}$ & $\begin{aligned} &< 50 \\
& 1.6 E+00 \\
& 5.4 \\
&<5.0 \\
&<500 \\
& 6.0 \mathrm{E}-01 \\
&<2.0 \\
& 6,480 \\
&<2.0 \\
& 1,560 \\
& 19 \\
&<1.000 \\
&<1.0 \\
&<1.0 \\
&<2.0 \\
&<1.0\end{aligned}$ \\
\hline $\begin{aligned} & 15,000 \\
< & <1,000 \\
< & 5.0 \\
<50 & <0.25 \\
< & <0.00046 \\
< & <1.0 \\
< & <1.0 \\
< & 1.0 \\
< & <.0\end{aligned}$ & $\begin{aligned} & 9.4 E+05 \\
& 15,000 \\
& 1,010 \\
< & 5.0 \\
< & 50 \\
< & 0.25 \\
< & 0.00046 \\
< & 1.0 \\
< & 1.0 \\
< & 1.0 \\
< & 1.0\end{aligned}$ \\
\hline $\begin{aligned} & 1.1 E+03 \\
< & 0.10 \\
< & 8.0 \\
< & 2.0\end{aligned}$ & $\begin{array}{l}9.0 E+02 \\
0.18 \\
<8.0 \\
<2.0\end{array}$ \\
\hline
\end{tabular}

3094

4094

DF $\underline{\text { Mod }}$

Lab

Flag

$<68$

$<0.054$

348

12

$<0.20$

$<0.54$

$<10$

$<4.0$

660

$3.1 E+00$

11
$<5.0$

$<5.0$
582

$<2.0$

6.470

$<2.0$

21

$<1,000$

$<5.0$

$<5.0$

$<17$

$<5.0$

1.1E+06

8,000

$<1,000$

5.5

$<50$

$<1.1$

$<5.0$

$<5.0$

$<5.0$

$<5.0$

$1.2 E+03$

0.39

$<3.0$
17
7.2

369

7.2

$<0.20$

$<0.49$

$<10$

$<4.0$

572

1. $6 \mathrm{E}+00$

5.3
$<5.0$

516

$<2.0$

6,650

$<2.0$

1,430

18

$<1,000$

$<1.0$

$<1.0$

17,000

$<1,000$

$<5.0$

$<50$

$<0.24$

$<0.00046$

$<1.0$

$<1.0$

$<1.0$

$<1.0$

1.1E + 03

0.21

$<8.0$

$<2.0$
$<0.0049$ $\mu g / L \quad G E$

$\mu g / L \quad G E$

$\mu \mathrm{g} / \mathrm{L} \quad \mathrm{GE}$

$\mu g / L \quad G E$

$\mu g / L \quad$ GE

$\mu \mathrm{g} / \mathrm{L} \quad \mathrm{GE}$

$\mu g / L$ GE

$\mu g / L$

pCi/L GP

pH GE

$\underset{\mu g / L}{\mu g} \quad$ GE

pCi/L

$\mu \mathrm{g} / \mathrm{L}$

$\mu g /$

$\mu \mathrm{g} / \mathrm{L}$

$\mu g / L$

$\mu \mathrm{S} / \mathrm{c}$

$\mu \mathrm{g} / \mathrm{L}$

$\mu \mathrm{g}$

$\mu g / L \quad G E$

$\mu \mathrm{g} / \mathrm{L} \quad \mathrm{GE}$

$\mu g / L \quad G E$

pCi/L

$\mu g / L \quad G E$

$\mu g / L \quad$ GE

$\mu g / L$ GE

$\mu g / L$

$\mu g h$

$\mu g /$

$\mu g / L$

$\mu \mathrm{g} /$

$\mu \mathrm{g}$

$\mu \mathrm{g} / \mathrm{L}$

pCi/m

$\mathrm{pCi} / \mathrm{mL}$ GP

NTU GE

$\mu g / L \quad G E$ $\mu g / L \quad G E$

Note: Flagging, dilution factors, modifiers, and laboratories are for fourth quarter 1994 data only. See Appendix B for flagging criteria. - = exceeded holding time for fourth quarter 1994.

- = exceeded screening level or final PDWS for fourth quarter 1994. 


\section{WELL BGX 8DR}

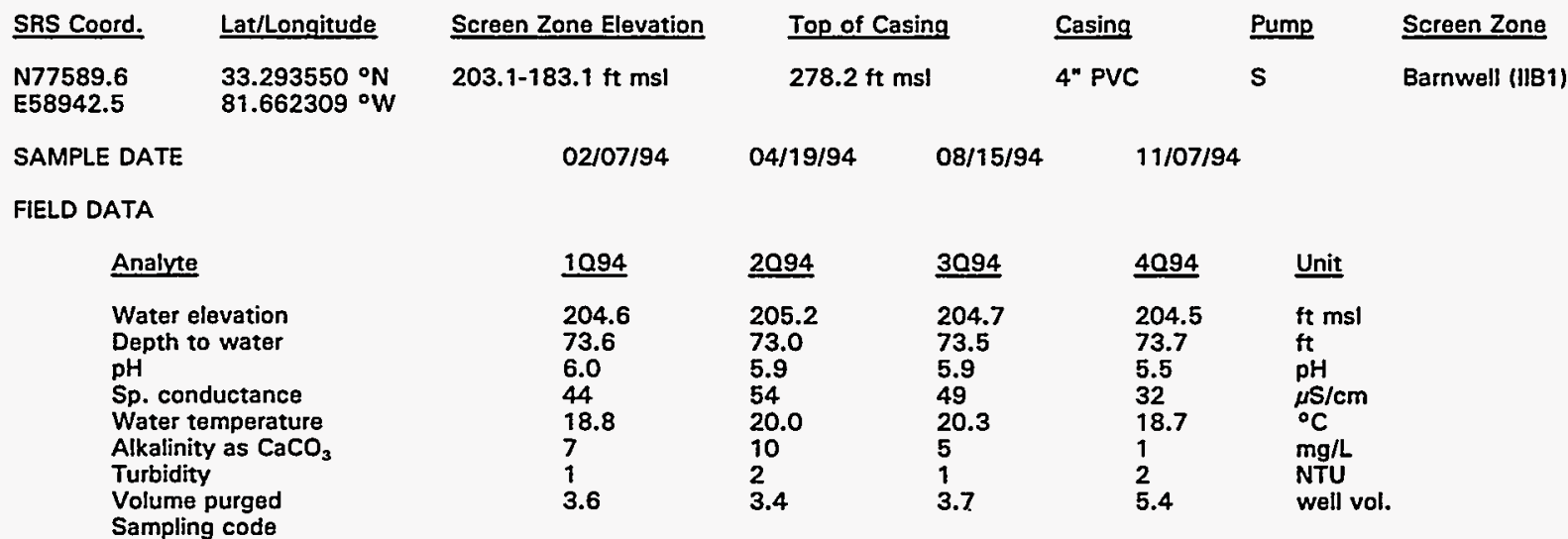

ANALYTICAL DATA

\section{HI ST Analyte}

Acetophenone

Aldrin

Aluminum, total recoverable

Antimony, total recoverable

Arsenic, total recoverable

Barium, total recoverable

Benzene

Bromodichloromethane

Bromoform

Bromomethane (Methyl bromide)

Cadmium, total recoverable

Calcium, total recoverable

Carbon tetrachloride

Chloride

Chlorobenzene

Chloroethane

Chloroethene (Vinyl chloride)

2-Chloroethyl vinyl ether

Chloroform

Chloromethane (Methyl chloride)

Chromium, total recoverable

Copper, total recoverable

Cyanide

P.-DDT

Dibromochloromethane

1,1-Dichloroethane

1,2-Dichloroethane

1,1-Dichloroethylene

trans-1,2-Dichloroethylen

Dichloromethane

2,4-Dichlorophenoxyacetic acid

1,2-Dichloropropane

cis-1,3-Dichloropropene

trans-1,3-Dichloropropene

Dieldrin

Endrin

Ethylbenzene

Fluoride

Gross alpha

\begin{tabular}{|c|c|c|}
\hline 1094 & 2094 & 3094 \\
\hline $\begin{aligned}<10 \\
<0.050 \\
84 \\
<2.0 \\
<2.0 \\
125 \\
<1.0 \\
<1.0 \\
<1.0 \\
<1.0 \\
<2.0 \\
37.700 \\
<1.0 \\
21.330 \\
<1.0 \\
<1.0 \\
<1.0 \\
<1.0 \\
<1.0 \\
<1.0 \\
<4.0 \\
<4.0 \\
<5.0 \\
<0.10 \\
<1.0 \\
<1.0 \\
<1.0 \\
<1.0 \\
<1.0 \\
<1.0 \\
<0.0015 \\
<1.0 \\
<1.0 \\
<1.0 \\
<0.50 \\
<0.0060 \\
<1.0 \\
<100 \\
1.05+00\end{aligned}$ & $\begin{array}{l}<10 \\
<0.050 \\
85 \\
<2.0 \\
<2.0 \\
275 \\
<1.0 \\
<1.0 \\
<1.0 \\
<1.0 \\
<2.0 \\
76.700 \\
<1.0 \\
21.530 \\
<1.0 \\
<1.0 \\
<1.0 \\
<1.0 \\
<1.0 \\
<1.0 \\
<4.0 \\
7.2 \\
<5.0 \\
<0.10 \\
<1.0 \\
<1.0 \\
<1.0 \\
<1.0 \\
<1.0 \\
<1.0 \\
<0.0015 \\
<1.0 \\
<1.0 \\
<1.0 \\
<0.50 \\
<0.0060 \\
<1.0 \\
<100 \\
1.2 E \\
<100\end{array}$ & $\begin{array}{l}<10 \\
\\
96 \\
<3.0 \\
<2.0 \\
110 \\
<5.0 \\
<5.0 \\
<5.0 \\
<10 \\
<2.0 \\
34.400 \\
<5.0 \\
2.940 \\
<5.0 \\
<10 \\
<10 \\
<10 \\
<5.0 \\
1.4 \\
<4.0 \\
<4.0 \\
<5.0 \\
<5.0 \\
<5.0 \\
<5.0 \\
<5.0 \\
<5.0 \\
<5.0 \\
<1.0 \\
<5.0 \\
<5.0 \\
<5.0 \\
<0.11 \\
<5.0 \\
<100 \\
<5\end{array}$ \\
\hline
\end{tabular}

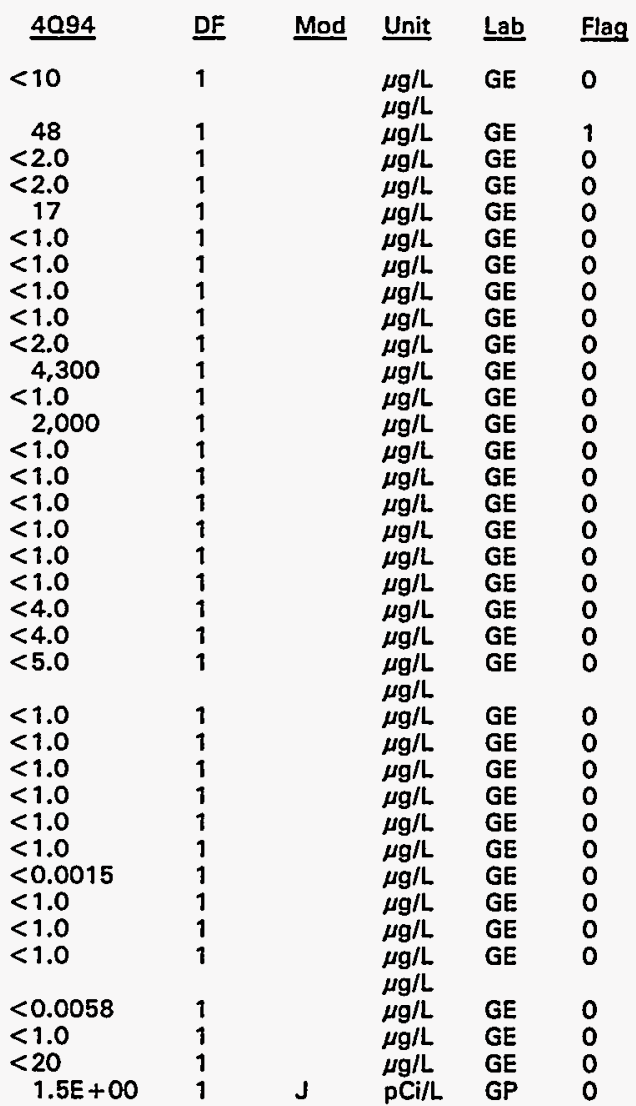

Note: Flagging, dilution factors, modifiers, and laboratories are for fourth quarter 1994 data only. See Appendix B for flagging criteria. - = exceeded holding time for fourth quarter 1994 .

- = exceeded screening level or final PDWS for fourth quarter 1994. 
Well BGX 8DR continued

\section{ANALYTICAL DATA}

\section{H ST Analyte}

Heptachlor

Iron, total recoverable

Lead, total recoverable

Lindane

Magnesium, total recoverable

Manganese, total recoverable

Mercury, total recoverable

Methoxychlor

Naphthalene

Nickel, total recoverable

Nitrate as nitrogen

Nitrate-nitrite as nitrogen

Nonvolatile beta

$\mathrm{pH}$

Phenols

Potassium, total recoverable

Radium, total alpha-emitting

Selenium, total recoverable

Silica, total recoverable

Silver, total recoverable

Sodium, total recoverable

Specific conductance

Sulfate

1,1,2,2-Tetrachloroethane

Tetrachloroethylene

Tin, total recoverable

Toluene

Total activity

Total dissolved solids

Total organic carbon

Total organic halogens

Total phosphates (as P)

Toxaphene

2,4,5-TP (Silvex)

1,1,1-Trichloroethane

1,1,2-Trichlorosthane

Trichloroethylene

Trichlorofluoromethane

$2,4,5-T$

Tritium

Turbidity

Vanadium, total recoverable

Xylenes

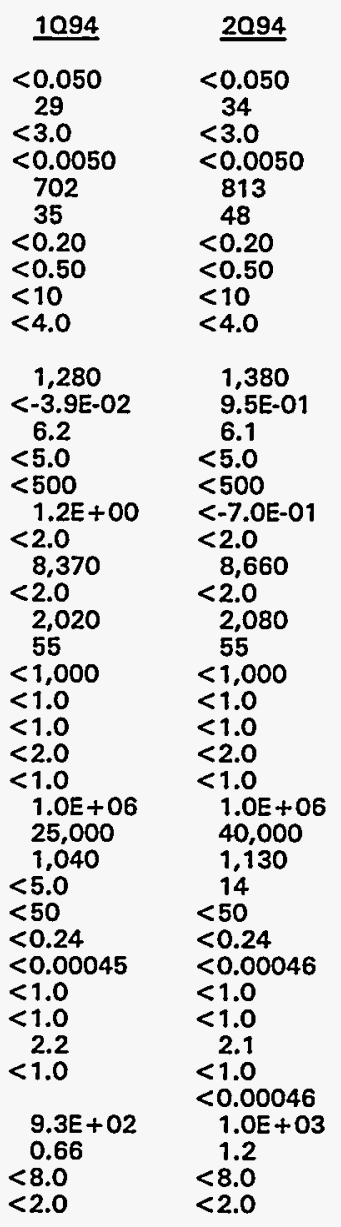

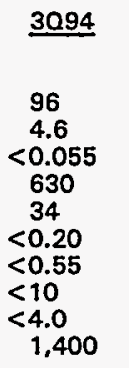

1. $5 E+\infty 0$

6.2

$<5.0$

$<500$

$<2.0$

8,200

2.0

1,880

51

$<1,000$

$<5.0$

$<5.0$

$<17$

$<5.0$

$1.0 E+06$

39,000

$<1,000$

6.2
430

430
$<1.1$

$<0.52$

$<5.0$

$<5.0$

1.9
$<5.0$

$<5.0$

$1.1 E+03$

0.55

$<3.0$

$<5.0$

$\begin{array}{lll}\begin{array}{lll}4094 \\ \end{array} & \text { DF } & \text { Mod } \\ 22 & 1 & \\ <3.0 & 1 & \\ <0.0049 & 1 & \\ 594 & 1 & \\ 22 & 1 & \\ <0.20 & 1 & \\ <0.49 & 1 & \\ <10 & 1 & \\ <4.0 & 1 & \\ 1,460 & 1 & \\ 1.3 E+\infty & 1 & \text { UI } \\ 5.9 & 1 & J \\ <5.0 & 1 & \end{array}$

Unit Lab $\quad$ Flag

$\mu \mathrm{g} / \mathrm{L}$

$\mu g / L \quad G E$

$\mu g / L \quad G E$

$\mu \mathrm{g} / \mathrm{L} \quad \mathrm{GE}$

$\mu g / L \quad G E$

$\mu \mathrm{g} / \mathrm{L} \quad \mathrm{GE}$

$\mu g / L \quad G E$

$\mu \mathrm{g} / \mathrm{L} \quad \mathrm{GE}$

$\mu g / L \quad G E$

$\mu g / L \quad G E$

PCi/L GP

pH GE

$\mu \mathrm{g} / \mathrm{L} \quad \mathrm{GE}$

$\mu g / L \quad G E$

$\mathrm{pCi} / \mathrm{L}$

$\mu g / L \quad G E$

$\mu g / L \quad G E$

$\mu \mathrm{g} / \mathrm{L} \quad \mathrm{GE}$

$\mu \mathrm{g} / \mathrm{L} \quad \mathrm{GE}$

$\mu \mathrm{sS/cm} \quad \mathrm{GE}$

$\mu g / L \quad G E$

$\mu g / L$ GE

$\mu \mathrm{g} / \mathrm{L}$ GE

$\mu \mathrm{g} / \mathrm{L}$

J

$\mu \mathrm{g} / \mathrm{L}$

PCIL GE

$\underset{\mu g / L}{\mathrm{~g} / \mathrm{L}} \quad \mathrm{GE}$

$\mu \mathrm{g} / \mathrm{L} \quad \mathrm{GE}$

$\mu \mathrm{g} / \mathrm{L} \quad \mathrm{GE}$

$\mu g / L$ GE

$\mu \mathrm{g} / \mathrm{L}$

$\mu \mathrm{g} / \mathrm{L}$

$\mu \mathrm{g} / \mathrm{L} \quad \mathrm{GE}$

$\mu \mathrm{g} / \mathrm{L}$

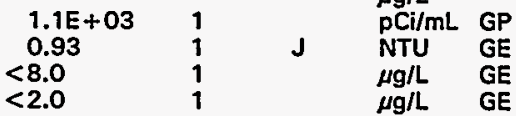

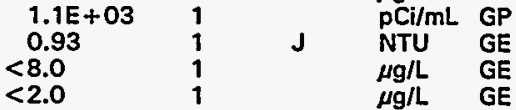

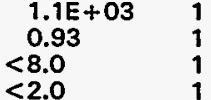

(a)

0

0

0

0

0

0
0

0

0

0

0

0
0

0

0

0

0
0
0

Note: Flagging, dilution factors, modifiers, and laboratories are for fourth quarter 1994 data only. See Appendix B for flagging criteria. - = exceeded holding time for fourth quarter 1994.

- = exceeded screening level or final PDWS for fourth quarter 1994. 
WELL BGX 9D

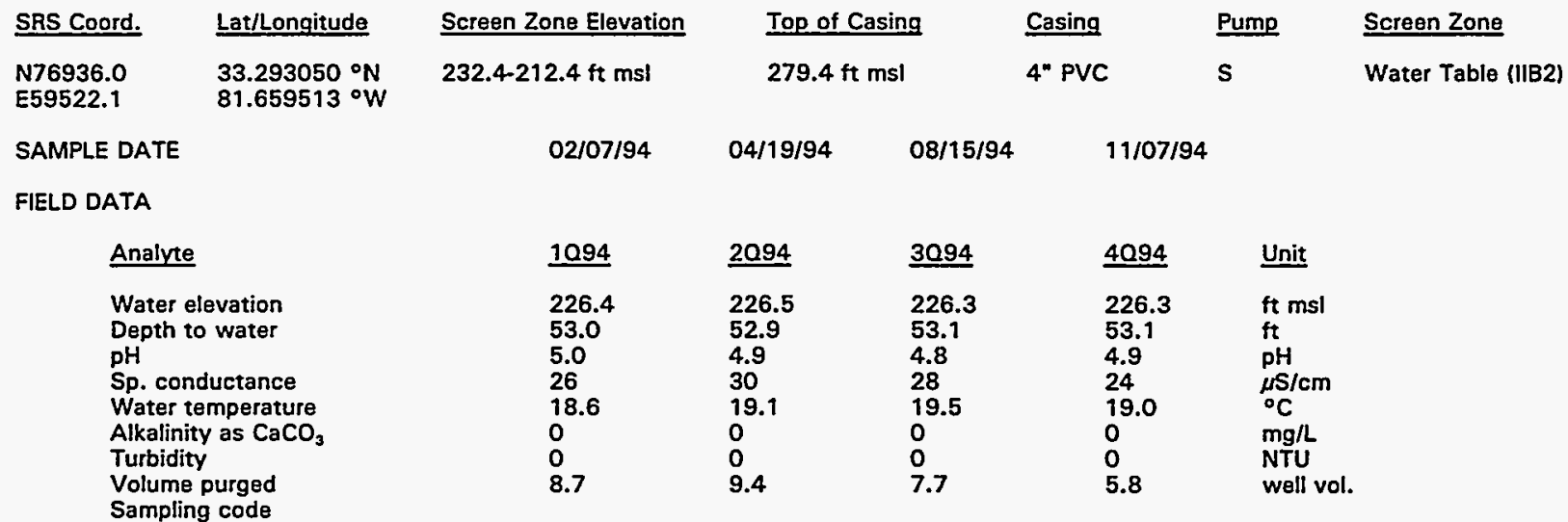

\section{ANALYTICAL DATA}

\section{H ST Analyte}

\section{Acetophenone}

Aldrin

Aluminum, total recoverable

Antimony, total recoverable

Arsenic, total recoverable

Barium, total recoverable

Benzene

Bromodichloromethane

Bromoform

Bromomethane (Methyl bromide)

Cadmium, total recoverable

Calcium, total recoverable

Carbon tetrachloride

Chloride

Chlorobenzene

Chloroethane

Chloroethene (Vinyl chloride)

2-Chloroethyl vinyl ether

Chloroform

Chloromethane (Methyl chloride)

Chromium, total recoverable

Copper, total recoverable

Cyanide

p,p'-DDT

Dibromochloromethane

1,1-Dichloroethane

1,2-Dichloroethane

1,1-Dichloroethylene

trans-1,2-Dichloroethylene

Dichloromethane

2,4-Dichlorophenoxyacetic acid

1,2-Dichloropropane

cis-1,3-Dichloropropene

trans-1,3-Dichloropropene

Dieldrin

Endrin

Ethylbenzene

Fluoride

Gross alpha

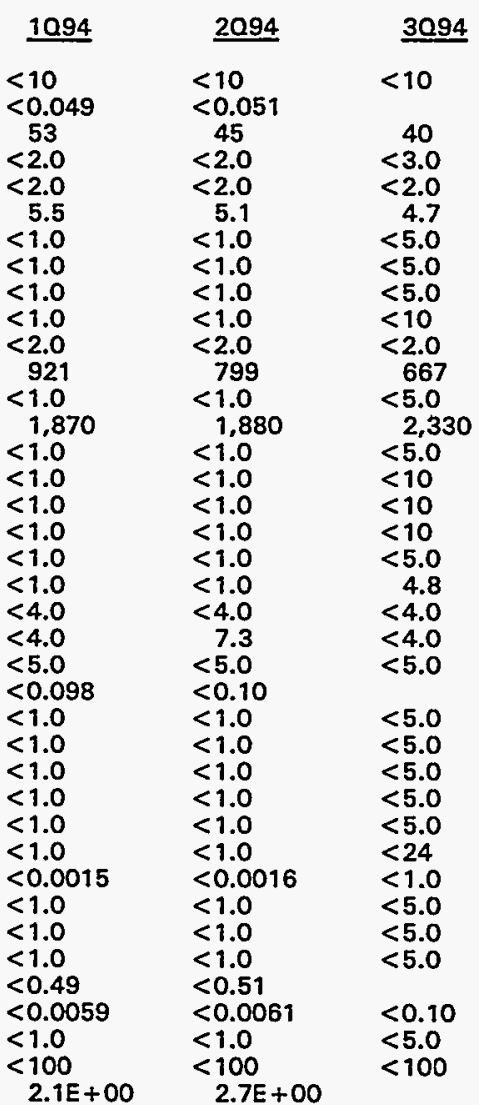

\begin{tabular}{|c|c|c|c|c|c|}
\hline 4094 & DF & Mod & Unit & $\underline{\text { Lab }}$ & Flag \\
\hline$<9.9$ & 1 & & $\underset{\mu \mathrm{g} / \mathrm{L}}{\mu \mathrm{L}}$ & GE & 0 \\
\hline 43 & 1 & & $\mu \mathrm{g} / \mathrm{L}$ & GE & 1 \\
\hline$<2.0$ & $i$ & & $\mu g / L$ & $\overline{G E}$ & 0 \\
\hline$<2.0$ & 1 & & $\mu \mathrm{g} / \mathrm{L}$ & GE & 0 \\
\hline 4.8 & 1 & J & $\mu g / L$ & GE & 0 \\
\hline$<1.0$ & 1 & & $\mu \mathrm{g} / \mathrm{L}$ & $\overrightarrow{G E}$ & 0 \\
\hline$<1.0$ & 1 & & $\mu g / L$ & GE & 0 \\
\hline$<1.0$ & 1 & & $\mu \mathrm{g} / \mathrm{L}$ & GE & 0 \\
\hline$<1.0$ & 1 & & $\mu \mathrm{g} / \mathrm{L}$ & GE & 0 \\
\hline$<2.0$ & 1 & & $\mu \mathrm{g} / \mathrm{L}$ & GE & 0 \\
\hline 670 & 1 & & $\mu \mathrm{g} / \mathrm{L}$ & GE & 0 \\
\hline$<1.0$ & 1 & & $\mu \mathrm{g} / \mathrm{L}$ & GE & 0 \\
\hline 1,560 & 1 & & $\mu g / L$ & GE & 0 \\
\hline$<1.0$ & 1 & & $\mu \mathrm{g} / \mathrm{L}$ & GE & 0 \\
\hline$<1.0$ & 1 & & $\mu g / L$ & GE & 0 \\
\hline$<1.0$ & 1 & & $\mu \mathrm{g} / \mathrm{L}$ & GE & 0 \\
\hline$<1.0$ & 1 & & $\mu \mathrm{g} / \mathrm{L}$ & GE & 0 \\
\hline$<1.0$ & 1 & & $\mu \mathrm{g} / \mathrm{L}$ & GE & 0 \\
\hline$<1.0$ & 1 & & $\mu g / L$ & GE & 0 \\
\hline$<4.0$ & 1 & & $\mu \mathrm{g} / \mathrm{L}$ & GE & 0 \\
\hline$<4.0$ & 1 & & $\mu \mathrm{g} / \mathrm{L}$ & GE & 0 \\
\hline$<5.0$ & 1 & & $\mu g / L$ & $\overrightarrow{G E}$ & 0 \\
\hline$<1.0$ & 1 & & $\mu \mathrm{g} / \mathrm{L}$ & GE & 0 \\
\hline$<1.0$ & 1 & & $\mu \mathrm{g} / \mathrm{L}$ & GE & 0 \\
\hline$<1.0$ & 1 & & $\mu g / L$ & GE & 0 \\
\hline$<1.0$ & 1 & & $\mu g / L$ & GE & 0 \\
\hline$<1.0$ & 1 & & $\mu \mathrm{g} / \mathrm{L}$ & GE & 0 \\
\hline$<1.0$ & 1 & & $\mu \mathrm{g} / \mathrm{L}$ & GE & 0 \\
\hline$<0.0016$ & 1 & & $\mu g / L$ & GE & 0 \\
\hline$<1.0$ & 1 & & $\mu g / L$ & GE & 0 \\
\hline$<1.0$ & 1 & & $\mu \mathrm{g} / \mathrm{L}$ & GE & 0 \\
\hline$<1.0$ & 1 & & $\mu \mathrm{g} / \mathrm{L}$ & GE & 0 \\
\hline$<0.0064$ & 1 & & $\mu \mathrm{g} / \mathrm{L}$ & GE & 0 \\
\hline$<1.0$ & 1 & & $\mu \mathrm{g} / \mathrm{L}$ & $\overrightarrow{G E}$ & 0 \\
\hline$<20$ & 1 & & $\mu \mathrm{g} / \mathrm{L}$ & GE & 0 \\
\hline $1.9 E+00$ & 1 & $J$ & $\mathrm{pCi} / \mathrm{L}$ & $G P$ & 0 \\
\hline
\end{tabular}

Note: Flagging, dilution factors, modifiers, and laboratories are for fourth quarter 1994 data only. See Appendix B for flagging criteria. - = exceeded holding time for fourth quarter 1994.

- =xceeded screening level or final PDWS for fourth quarter 1994. 


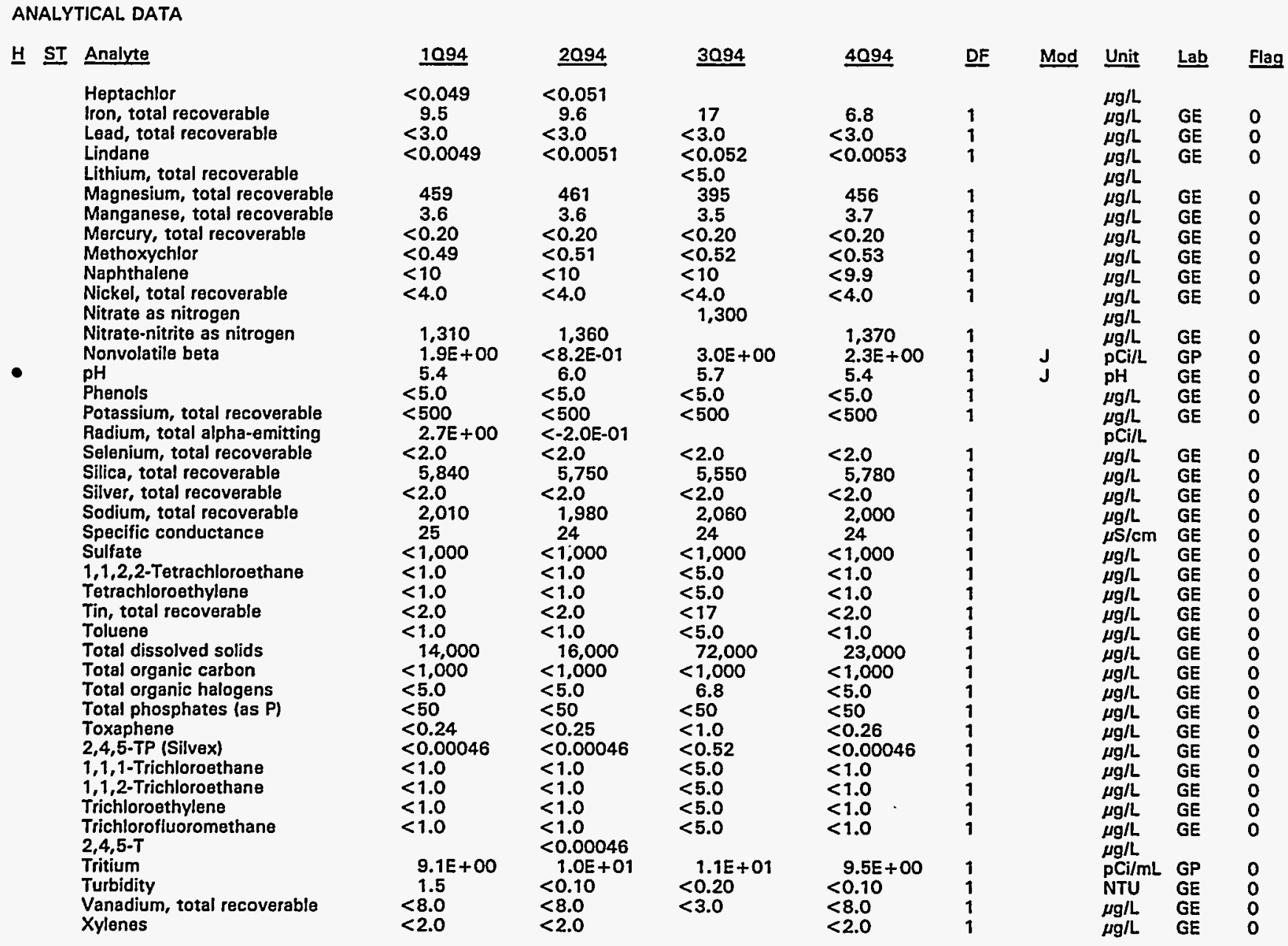

Note: Flagging, dilution factors, modifiers, and laboratories are for fourth quarter 1994 data only. See Appendix B for flagging criteria. - = exceeded holding time for fourth quarter 1994.

w = exceeded screening level or final PDWS for fourth quarter 1994. 
WELL BGX 10D

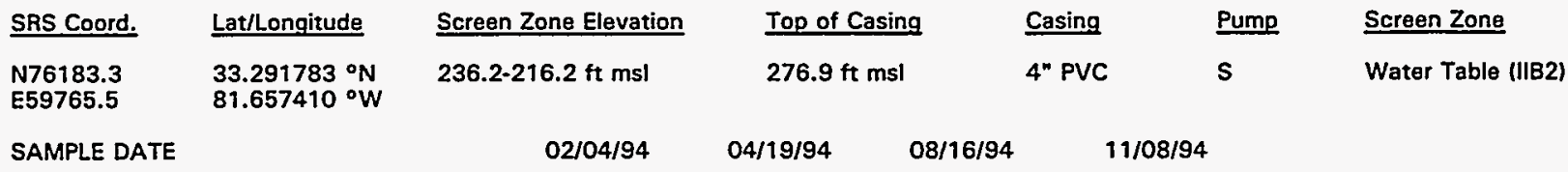

FIELD DATA

Analyte
Water elevation
Depth to water
pH
Sp. conductance
Water temperature
Alkalinity as $\mathrm{CaCO}_{3}$
Turbidity
Volume purged
Sampling code

ANALYTICAL DATA

HI ST Analyte

Acetophenone
Aldrin
Aluminum, total recoverable
Antimony, total recoverable
Arsenic, total recoverable
Barium, total recoverable
Benzene
Bromodichloromethane
Bromoform
Bromomethane (Methyl bromide)
Cadmium, total recoverable
Calcium, total recoverable
Carbon tetrachloride
Chloride
Chlorobenzene
Chloroethane
Chloroethene (Vinyl chloride)
2-Chloroethyl vinyl ether
Chloroform
Chloromethane (Methyl chloride)
Chromium, total recoverable
Copper, total recoverable
Cyanide
p,p'-DDT
Dibromochloromethane
1,1-Dichloroethane
1,2-Dichloroethane
1,1-Dichloroethylene
trans-1,2-Dichloroethylene
Dichloromethane
2,4-Dichlorophenoxyacetic acid
1,2-Dichloropropane
cis-1,3-Dichloropropene
trans-1,3-Dichloropropene
Dieldrin
Endrin
Ethylbenzene
Fluoride
Gross alpha

\begin{tabular}{|c|c|}
\hline 1094 & 2094 \\
\hline $\begin{array}{l}225.6 \\
51.3 \\
5.7 \\
29 \\
16.5 \\
3 \\
20 \\
0.3 \\
x\end{array}$ & $\begin{array}{l}225.3 \\
51.6 \\
6.1 \\
39 \\
18.8 \\
4 \\
14 \\
0.5 \\
x\end{array}$ \\
\hline
\end{tabular}

$<10$

$<0.049$

179
$<2.0$

$<2.0$

12

$<1.0$

$<1.0$

$<1.0$

$<1.0$

$<2.0$

950

$<1.0$

1,700

$<1.0$

$<1.0$

$<1.0$

$<1.0$

$<1.0$

$<4.0$

37

$<5.0$

$<0.098$

$<1.0$

$<1.0$

$<1.0$

$<1.0$

$<1.0$

$<1.0$

$<0.0015$

$<1.0$

$<1.0$

$<1.0$

$<0.49$

$<0.0059$

$<1.0$

$<100$

$1.4 E+00$

$<10$
$<0.053$

159
$<2.0$

$<2.0$

11
$<1.0$

$<1.0$

$<1.0$

$<1.0$

$<2.0$

918
$<1.0$

$<1.0$

$<1.730$

$<1.0$

$<1.0$

$<1.0$

$<1.0$

$<1.0$

$<4.0$

38

$<5.0$

$<0.11$

$<1.0$

$<1.0$

$<1.0$

$<1.0$

$<1.0$

$<0.0016$

$<1.0$

$<1.0$

$<1.0$

$<0.53$

$<0.0063$

$<1.0$

$<100$

$1.1 \mathrm{E}+00$

3094
225.1
51.8
6.0
37
19.8
6
22
0.5
$x$

3094

$<10$

1,180

$<3.0$

$<2.0$

69
$<5.0$

$<5.0$

$<5.0$

$<10$

$<2.0$

848
$<5.0$

2,070

$<5.0$

$<10$

$<10$

$<10$

$<5.0$

$<10$
5.4

5.4
34
$<5.0$

$<5.0$

$<5.0$

$<5.0$

$<5.0$

$<5.0$

$<25$

$<1.1$

$<5.0$

$<5.0$

$<5.0$

$<0.11$

$<5.0$

$<100$

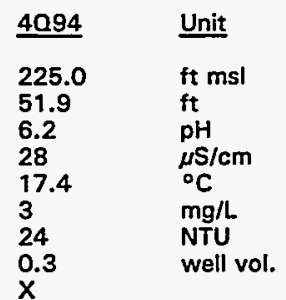

\begin{tabular}{|c|c|c|c|c|c|}
\hline 4094 & $\underline{D F}$ & Mod & Unit & Lab & Fiag \\
\hline$<10$ & 1 & & $\mu \mathrm{g} / \mathrm{L}$ & GE & 0 \\
\hline 445 & 1 & & $\mu g / L$ & GE & 2 \\
\hline$<2.0$ & 1 & & $\mu \mathrm{g} / \mathrm{L}$ & GE & 0 \\
\hline$<2.0$ & 1 & & $\mu \mathrm{g} / \mathrm{L}$ & GE & 0 \\
\hline 14 & $i$ & & $\mu g / L$ & $\mathrm{GE}$ & 0 \\
\hline$<1.0$ & 1 & & $\mu \mathrm{g} / \mathrm{L}$ & GE & 0 \\
\hline$<1.0$ & 1 & & $\mu \mathrm{g} / \mathrm{L}$ & GE & 0 \\
\hline$<1.0$ & 1 & & $\mu \mathrm{g} / \mathrm{L}$ & $\mathbf{G E}$ & 0 \\
\hline$<1.0$ & 1 & & $\mu \mathrm{g} / \mathrm{L}$ & $\mathrm{GE}$ & 0 \\
\hline$<2.0$ & 1 & & $\mu \mathrm{g} / \mathrm{L}$ & GE & 0 \\
\hline 873 & 1 & & $\mu \mathrm{g} / \mathrm{L}$ & GE & 0 \\
\hline$<1.0$ & 1 & & $\mu g / L$ & GE & 0 \\
\hline 1,250 & 1 & & $\mu \mathrm{g} / \mathrm{L}$ & $\mathrm{GE}$ & 0 \\
\hline$<1.0$ & 1 & & $\mu \mathrm{g} / \mathrm{L}$ & GE & 0 \\
\hline$<1.0$ & 1 & & $\mu \mathrm{g} / \mathrm{L}$ & GE & 0 \\
\hline$<1.0$ & 1 & & $\mu g / L$ & GE & 0 \\
\hline$<1.0$ & 1 & & $\mu \mathrm{g} / \mathrm{L}$ & GE & 0 \\
\hline$<1.0$ & 1 & & $\mu \mathrm{g} / \mathrm{L}$ & GE & 0 \\
\hline$<1.0$ & 1 & & $\mu g / L$ & GE & 0 \\
\hline$<4.0$ & 1 & & $\mu \mathrm{g} / \mathrm{L}$ & GE & 0 \\
\hline 59 & 1 & & $\mu \mathrm{g} / \mathrm{L}$ & GE & 0 \\
\hline$<5.0$ & 1 & & $\mu \mathrm{g} / \mathrm{L}$ & GE & 0 \\
\hline$<1.0$ & 1 & & $\begin{array}{l}\mu \mathrm{g} / \mathrm{L} \\
\mu \mathrm{g} / \mathrm{L}\end{array}$ & GE & 0 \\
\hline$<1.0$ & 1 & & $\mu \mathrm{g} / \mathrm{L}$ & GE & 0 \\
\hline$<1.0$ & 1 & & $\mu \mathrm{g} / \mathrm{L}$ & GE & 0 \\
\hline$<1.0$ & 1 & & $\mu \mathrm{g} / \mathrm{L}$ & $\mathrm{GE}$ & 0 \\
\hline$<1.0$ & 1 & & $\mu \mathrm{g} / \mathrm{L}$ & GE & 0 \\
\hline$<1.0$ & 1 & & $\mu \mathrm{g} / \mathrm{L}$ & GE & 0 \\
\hline$<0.0015$ & 1 & & $\mu \mathrm{g} / \mathrm{L}$ & GE & 0 \\
\hline$<1.0$ & 1 & & $\mu \mathrm{g} / \mathrm{L}$ & GE & 0 \\
\hline$<1.0$ & 1 & & $\mu g / L$ & GE & 0 \\
\hline$<1.0$ & 1 & & $\mu \mathrm{g} / \mathrm{L}$ & GE & 0 \\
\hline & & & $\mu \mathrm{a} / \mathrm{L}$ & & 0 \\
\hline$<1.0$ & $i$ & & $\mu g / L$ & $\mathrm{GE}$ & 0 \\
\hline$<20$ & 1 & & $\mu \mathrm{g} / \mathrm{L}$ & $\mathrm{GE}$ & $\mathbf{0}$ \\
\hline $1.3 E+\infty 0$ & 1 & $J$ & pCi/L & GP & 0 \\
\hline
\end{tabular}

Note: Flagging, dilution factors, modifiers, and laboratories are for fourth quarter 1994 data only. See Appendix B for flagging criteria. $\bullet=$ exceeded holding time for fourth quarter 1994.

- = exceeded screening level or final PDWS for fourth quarter 1994. 


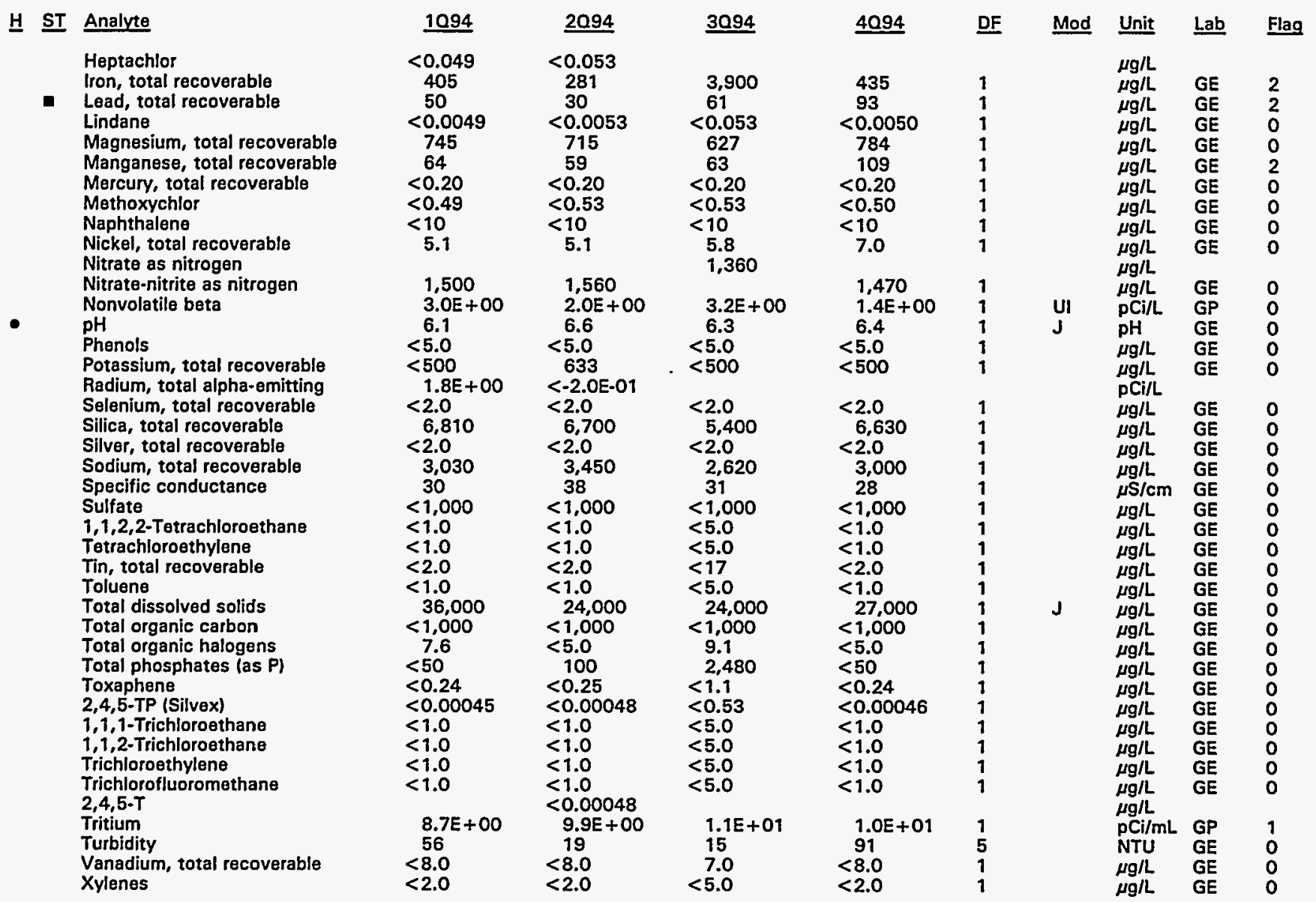

Note: Flagging, dilution factors, modifiers, and laboratories are for fourth quarter 1994 data only. See Appendix B for flagging criteria. - = exceeded holding time for fourth quarter 1994.

- = exceeded screening level or final PDWS for fourth quarter 1994. 
WELL BGX 11D

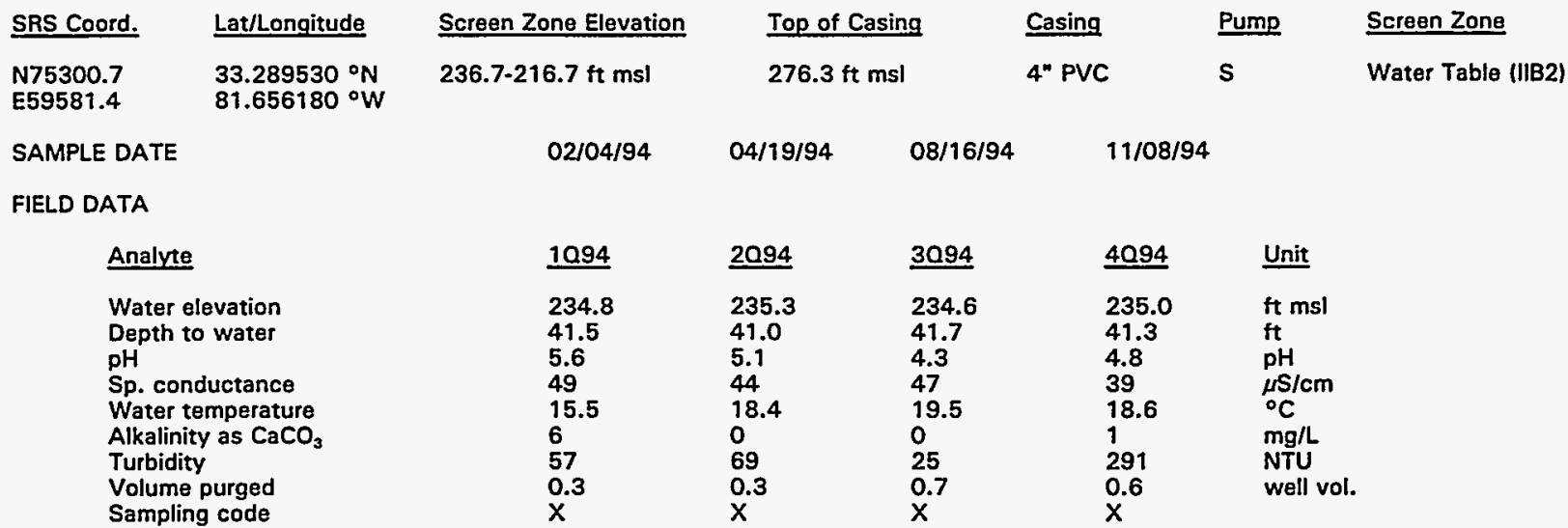

ANALYTICAL DATA

H ST Analyte

Acetophenone

Aldrin

Aluminum, total recoverable

Antimony, total recoverable

Arsenic, total recoverable

Barium, total recoverable

Benzene

Bromodichloromethane

Bromoform

Bromomethane (Methyl bromide)

Cadmium, total recoverable

Calcium, total recoverable

Carbon tetrachloride

Chloride

Chlorobenzene

Chloroethane

Chloroethene (Vinyl chloride)

2-Chloroethyl vinyl ether

Chloroform

Chloromethane (Methyl chloride)

Chromium, total recoverable

Copper, total recoverable

Cyanide

p,p'-DDT

Dibromochloromethane

1, i-Dichloroethane

1,2-Dichloroethane

1,1-Dichloroethylene

trans-1,2-Dichloroethylene

Dichloromethane

2,4-Dichlorophenoxyacetic acid

1,2-Dichloropropane

cis-1,3-Dichloropropene

trans-1,3-Dichloropropene

Dieldrin

Endrin

Ethylbenzene

Fluoride

Gross alpha
109

$<10$

$<0.050$

539
$<2.0$

$<2.0$

12

$<1.0$

$<1.0$

$<1.0$

$<1.0$
$<2.0$

2,790

$<1.0$

3,190

$<1.0$

$<1.0$

$<1.0$

$<1.0$

$<1.0$

$<4.0$

4.1

$<5.0$
$<0.10$

$<0.10$

$<1.0$

$<1.0$

$<1.0$

$<1.0$

$<1.0$

$<0.0015$

$<1.0$

$<1.0$

$<1.0$

$<0.50$

$<0.0060$

$<1.0$

$<100$

$3.0 E+00$

$\begin{aligned} & 2094 \\ &<=9 \\ &< 0.050 \\ & 1.460 \\ &<2.0 \\ &<2.0 \\ & 22 \\ &<1.0 \\ &<1.0 \\ &<1.0 \\ &<1.0 \\ &<2.0 \\ & 21.590 \\ &<1.0 \\ & 3.150 \\ &<1.0 \\ &<1.0 \\ &<1.0 \\ &<1.0 \\ &<1.0 \\ &<1.0 \\ &<4.0 \\ & 71.9 \\ &<5.0 \\ &<0.10 \\ &<1.0 \\ &<1.0 \\ &<1.0 \\ &<1.0 \\ &<1.0 \\ &<1.0 \\ &<0.0015 \\ &<1.0 \\ &<1.0 \\ &<1.0 \\ &<0.50 \\ &<0.0060 \\ &<1.0 \\ &<100 \\ & 4.4 E+00\end{aligned}$

$<10$

2,440

$<3.0$

$<2.0$

45
$<5.0$

$<5.0$

$<5.0$

$<10$

$<2.0$

1,370

3,790

$<5.0$

$<10$

$<10$

$<10$

$<5.0$

1.9
21

5.0

$<5.0$

$<5.0$

$<5.0$

$<5.0$

$<5.0$

$<5.0$

$<25$

$<1.0$

$<5.0$

$<5.0$

$<5.0$

$<0.11$

$<5.0$

$<100$

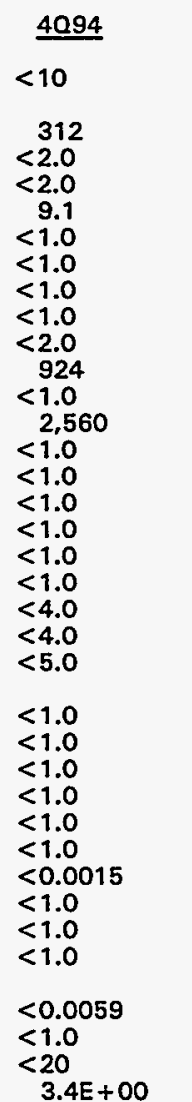

\begin{tabular}{|c|c|c|c|}
\hline DF & Mod & Unit & La: \\
\hline 1 & & $\underset{\mu g / L}{\mu g / L}$ & GE \\
\hline 1 & & $\mu \mathrm{g} / \mathrm{L}$ & GE \\
\hline 1 & & $\mu g / L$ & GE \\
\hline 1 & & $\mu \mathrm{g} / \mathrm{L}$ & GE \\
\hline 1 & & $\mu g / L$ & GE \\
\hline 1 & J & $\mu g / L$ & GE \\
\hline 1 & $\mathrm{~J}$ & $\mu \mathrm{g} / \mathrm{L}$ & GE \\
\hline 1 & J & $\mu \mathrm{g} / \mathrm{L}$ & GE \\
\hline 1 & 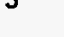 & $\mu g / L$ & $\mathrm{GE}$ \\
\hline 1 & & $\mu \mathrm{g} / \mathrm{L}$ & GE \\
\hline 1 & $\mathrm{~J}$ & $\mu g / L$ & GE \\
\hline 1 & & $\mu g / L$ & $\mathrm{GE}$ \\
\hline 1 & $J$ & $\mu g / L$ & $\mathrm{GE}$ \\
\hline 1 & $J$ & $\mu g / L$ & $\mathrm{GE}$ \\
\hline 1 & $\mathrm{~J}$ & $\mu g / L$ & $\mathrm{GE}$ \\
\hline 1 & $\mathrm{~J}$ & $\mu g / L$ & GE \\
\hline 1 & $J$ & $\mu \mathrm{g} / \mathrm{L}$ & GE \\
\hline 1 & $J$ & $\mu \mathrm{g} / \mathrm{L}$ & GE \\
\hline 1 & & $\mu g / L$ & GE \\
\hline 1 & & $\mu g / L$ & GE \\
\hline 1 & & $\begin{array}{l}\mu g / L \\
\mu g / L\end{array}$ & GE \\
\hline 1 & $J$ & $\mu \mathrm{g} / \mathrm{L}$ & $\mathrm{GE}$ \\
\hline 1 & J & $\mu \mathrm{g} / \mathrm{L}$ & GE \\
\hline 1 & $\mathrm{~J}$ & $\mu g / L$ & GE \\
\hline 1 & $\mathrm{~J}$ & $\mu g / L$ & GE \\
\hline 1 & $\mathrm{~J}$ & $\mu g / L$ & GE \\
\hline 1 & $J$ & $\mu g / L$ & GE \\
\hline 1 & & $\mu \mathrm{g} / \mathrm{L}$ & GE \\
\hline 1 & $J$ & $\mu g / L$ & GE \\
\hline 1 & $J$ & $\mu g / L$ & $\mathbf{G}$ \\
\hline 1 & $J$ & $\begin{array}{l}\mu \mathrm{g} / \mathrm{L} \\
\mu \mathrm{g} / \mathrm{L}\end{array}$ & $\mathbf{G E}$ \\
\hline & & $\mu g / L$ & G \\
\hline 1 & $J$ & $\mu \mathrm{g} / \mathrm{L}$ & \\
\hline 1 & & $g / L$ & \\
\hline
\end{tabular}

Note: Flagging, dilution factors, modifiers, and laboratories are for fourth quarter 1994 data only. See Appendix 8 for flagging criteria. - exceeded holding time for fourth quarter 1994.

n = exceeded screening level or final PDWS for fourth quarter 1994. 


\section{ANALYTICAL DATA}

\section{H ST Analyte}

Heptachlor

Iron, total recoverable

Lead, total recoverable

Lindane

Magnesium, total recoverable

Manganese, total recoverable

Mercury, total recoverable

Methoxychlor

Naphthalene

Nickel, total recoverable

Nitrate as nitrogen

Nitrate-nitrite as nitrogen

Nonvolatile beta

pH

Phenols

Potassium, total recoverable

Radium, total alpha-emitting

Selenium, total recoverable

Silica, total recoverable

Silver, total recoverable

Sodium, total recoverable

Specific conductance

Sulfate

1,1,2,2-Tetrachloroethane

Tetrachloroethylene

Tin, total recoverable

Toluene

Total dissolved solids

Total organic carbon

Total organic halogens

Total phosphates (as P)

Toxaphene

2,4,5-TP (Silvex)

1,1,1-Trichloroethane

1,1,2-Trichloroethane

Trichloroethylene

Trichlorofluoromethane

2,4,5-T

Tritium

Turbidity

Vanadium, total recoverable

Xylenes

\begin{tabular}{|c|c|}
\hline 1094 & 2094 \\
\hline $\begin{array}{l}<0.050 \\
234 \\
8.9 \\
<0.0050 \\
706 \\
2.3 \\
<0.20 \\
<0.50 \\
<10 \\
<4.0\end{array}$ & $\begin{array}{c}<0.050 \\
828 \\
7.0 \\
<0.0050 \\
758 \\
3.0 \\
<0.20 \\
<0.50 \\
<9.9 \\
<4.0\end{array}$ \\
\hline $\begin{array}{rl} & 2,100 \\
3.0 E+00 & 5.8 \\
5 & <.8 \\
<5.0 \\
<500 \\
3.6 E+00 \\
<2.0 \\
6,680 \\
<2.0 \\
2,760 \\
41 \\
<1,000 \\
<1.0 \\
<1.0 \\
7.7 \\
<1.0 \\
38,000 \\
2,050 \\
<5.0 \\
1,210 \\
<0.24 \\
<0.00046 \\
<1.0 \\
<1.0 \\
<1.0 \\
<1.0\end{array}$ & $\begin{aligned} & 1,970 \\
& 8.6 E+00 \\
& 5.6 \\
&<5.0 \\
&<500 \\
&< 0.0 E+00 \\
&<2.0 \\
& 9,120 \\
&< 2.0 \\
& 2,550 \\
& 37 \\
&<1,000 \\
&<1.0 \\
&<1.0 \\
&<2.0 \\
&<1.0 \\
& 30,000 \\
&<1,000 \\
&<5.0 \\
& 360 \\
&<0.24 \\
&<0.00046 \\
&<1.0 \\
&<1.0 \\
&<1.0 \\
&<1.0 \\
&<0.00046\end{aligned}$ \\
\hline $\begin{aligned} & 1.8 E+01 \\
& 334 \\
&= 8.0 \\
&= 2.0\end{aligned}$ & $\begin{aligned} & 2.1 \mathrm{E}+01 \\
& 205<1 \\
&< 8.0 \\
&<2.0\end{aligned}$ \\
\hline
\end{tabular}

$\underline{4094}$

$\begin{array}{ll} & 131 \\ 4.8 \\ <0.0050 \\ 717 \\ <2.0 \\ <0.20 \\ <0.50 \\ <10\end{array}$

$<10$

$<10$

1,880

$4.9 E+\infty 0$

5.7
$<5.0$

$<500$

$<2.0$

6,690

$<2.0$

1,910

41

$<1,000$

$<5.0$

$<5.0$

$<17$

$<5.0$
36,000

1,400

13

3,360

$<1.1$

$<0.52$

$<5.0$

$<5.0$

$<5.0$

$<5.0$

$2.0 \varepsilon+01$

364

19
$<5.0$

\section{1,970 \\ 2. $4 \mathrm{E}+00$ \\ 5.5
$<5.0$}

$<500$

$<2.0$

6,230

$<2.0$

2,660

34

$<1,000$

$<1.0$

$<1.0$

$<2.0$

$<1.0$

28,000

$<1,000$

$<5.0$

$<50$

$<0.24$

$<0.00044$

$<1.0$

$<1.0$

$<1.0$

$<1.0$

$1.9 E+01$

$1.9 E+01$
263
$<8.0$

$<8.0$
DF Mod Unit Lab Flag

$\mu g / L$

$\mu g / L \quad G E$

$\mu g / L \quad G E$

$\mu g / L \quad G E$

$\mu \mathrm{g} / \mathrm{L} \quad \mathrm{GE}$

$\mu g / L \quad G E$

$\mu \mathrm{g} / \mathrm{L} \quad \mathrm{GE}$

$\begin{array}{ll}\mu g / L & \mathrm{GE} \\ \mu \mathrm{g} / \mathrm{L} & \mathrm{GE}\end{array}$

$\mu g / L$

$\mu g / L \quad G E$

J $\mathrm{PCi} / \mathrm{L}$ GP

PH GE 0

$\mu g / L$ GE 0

$\mu \mathrm{g} / \mathrm{L} \quad \mathrm{GE}$

pCi/L

$\mu \mathrm{g} / \mathrm{L}$

$\mu g / L \quad G E$

$\mu g / L \quad G E$

$\mu \mathrm{g} / \mathrm{L} \quad \mathrm{GE}$

$\mu \mathrm{S} / \mathrm{cm}$ GE

$\mu \mathrm{g} / \mathrm{L}$

J

$\mu g / L \quad$ GE

$\mu g / L \quad G E$

$\mu g / L$

$\mu g / L$

$\mu g / L \quad G E$

$\mu g / L \quad G E$

$\mu g / L \quad G E$

$\mu \mathrm{g} / \mathrm{L}$

$\mu \mathrm{g} / \mathrm{L}$

$\mu g / L \quad G E$

$\mu \mathrm{g} / \mathrm{L} \quad \mathrm{GE}$

$\mu g / L$

$\mu \mathrm{g}$

$\mu \mathrm{g}$

$\mu \mathrm{g} / \mathrm{L}$

PCi/mL GP

NTU GE

$\begin{array}{ll}\mu \mathrm{g} / \mathrm{L} & \mathrm{GE} \\ \mu \mathrm{g} / \mathrm{L} & \mathrm{GE}\end{array}$

0
0
0
0
0
0
0
0
0
0
0
0
0
0
0
0
0
0
0
0
0
0
0
0
0
0
0
0
0
0
0
0
0
0
0
0
0
0
0
0
0

Note: Flagging, dilution factors, modifiers, and laboratories are for fourth quarter 1994 data only. See Appendix B for flagging criteria. - = exceeded holding time for fourth quarter 1994.

- = exceeded screening level or final PDWS for fourth quarter 1994. 


\section{WELL BGX 12C}

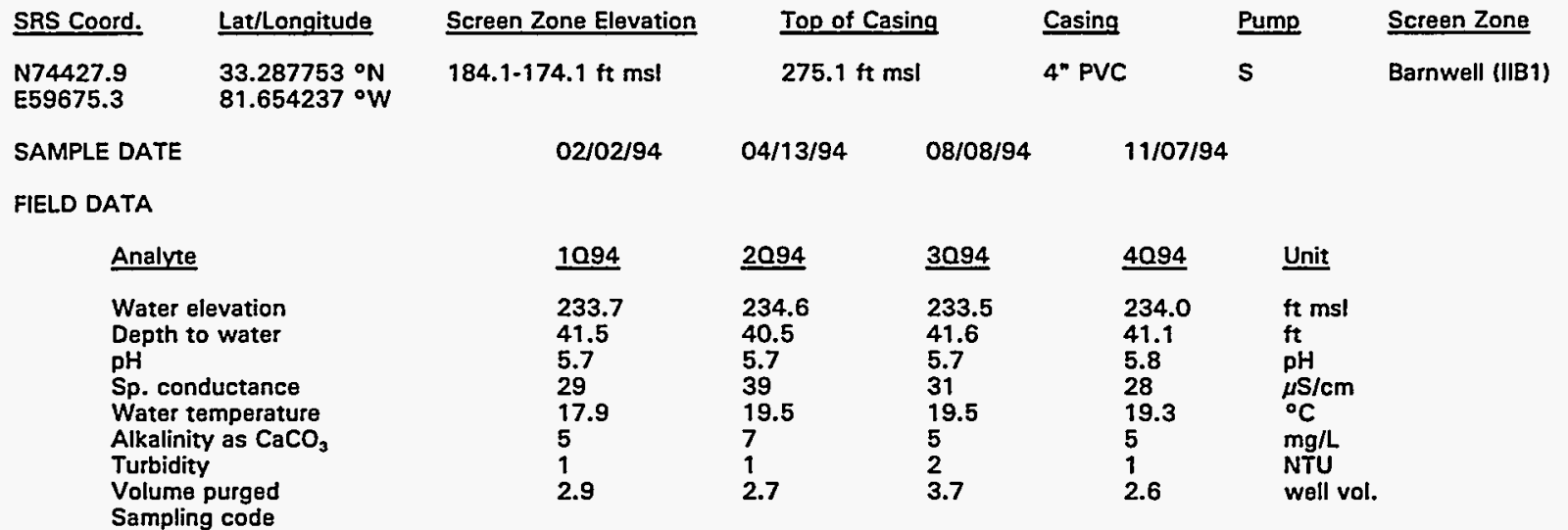

ANALYTICAL DATA

HE ST Analvte

Acetophenone

Aldrin

Aluminum, total recoverable

Antimony, total recoverable

Arsenic, total recoverable

Barium, total recoverable

Benzene

Bromodichloromethan

Bromoform

Bromomethane (Methyl bromide)

Cadmium, total recoverable

Calcium, total recoverable

Carbon tetrachloride

Chloride

Chlorobenzene

Chloroethane

Chloroethene (Vinyl chloride)

2-Chloroethyl vinyl ether

Chloroform

Chloromethane (Methyl chloride)

Chromium, total recoverable

Copper, total recoverable

Cyanide

p,p'-DDT

Dibromochloromethane

1,1-Dichloroethane

1,2-Dichloroethane

1.1-Dichloroethylene

trans-1,2-Dichloroethylene

Dichloromethan

2,4-Dichlorophenoxyacetic acid

1,2-Dichloropropane

cis-1,3-Dichloropropene

trans-1,3-Dichloropropene

Dieldrin

Endrin

Ethylbenzene

Fluoride

Gross alpha

\begin{tabular}{|c|c|c|}
\hline 1094 & 2094 & 3094 \\
\hline$<9.8$ & $<10$ & $<10$ \\
\hline $\begin{array}{c}<0.051 \\
23\end{array}$ & $\begin{array}{l}<0.052 \\
<20\end{array}$ & $<20$ \\
\hline$<2.0$ & $<2.0$ & $<2.0$ \\
\hline$<2.0$ & $<2.0$ & $<2.0$ \\
\hline 5.6 & 8.9 & 7.0 \\
\hline$<1.0$ & $<1.0$ & $<1.0$ \\
\hline$<1.0$ & $<1.0$ & $<1.0$ \\
\hline$<1.0$ & $<1.0$ & $<1.0$ \\
\hline$<1.0$ & $<1.0$ & $<1.0$ \\
\hline$<2.0$ & $<2.0$ & $<2.0$ \\
\hline 814 & 1,090 & 1,110 \\
\hline$<1.0$ & $<1.0$ & $<1.0$ \\
\hline $\begin{aligned} & 2,400 \\
< & 1.0\end{aligned}$ & $\begin{array}{r}2,470 \\
<1.0\end{array}$ & $\begin{aligned} 2,870 \\
<1.0\end{aligned}$ \\
\hline$<1.0$ & $<1.0$ & $<1.0$ \\
\hline$<1.0$ & $<1.0$ & $<1.0$ \\
\hline$<1.0$ & $<1.0$ & $<1.0$ \\
\hline$<1.0$ & $<1.0$ & $<1.0$ \\
\hline$<1.0$ & $<1.0$ & 4.1 \\
\hline$<4.0$ & $<4.0$ & $<4.0$ \\
\hline$<4.0$ & $<4.0$ & $<4.0$ \\
\hline$<5.0$ & $<5.0$ & $<5.0$ \\
\hline$<0.10$ & $<0.10$ & \\
\hline$<1.0$ & $<1.0$ & $<1.0$ \\
\hline$<1.0$ & $<1.0$ & $<1.0$ \\
\hline$<1.0$ & $<1.0$ & $<1.0$ \\
\hline$<1.0$ & $<1.0$ & $<1.0$ \\
\hline$<1.0$ & $<1.0$ & $<1.0$ \\
\hline$<1.0$ & $<1.0$ & $<1.0$ \\
\hline$<0.0016$ & $<0.0016$ & $<0.0015$ \\
\hline$<1.0$ & $<1.0$ & $<1.0$ \\
\hline$<1.0$ & $<1.0$ & $<1.0$ \\
\hline$<1.0$ & $<1.0$ & $<1.0$ \\
\hline$<0.51$ & $<0.52$ & \\
\hline$<0.0061$ & $<0.0062$ & $<0.11$ \\
\hline$<1.0$ & $<1.0$ & $<1.0$ \\
\hline$<100$ & $<100$ & 78 \\
\hline 6.7E-01 & $<1.5 \mathrm{E}-01$ & \\
\hline
\end{tabular}

\begin{tabular}{|c|c|c|c|c|}
\hline 4094 & DF & Mod & Unit & $\underline{\text { Lab }}$ \\
\hline$<10$ & 1 & & $\mu \mathrm{g} / \mathrm{L}$ & GE \\
\hline$<20$ & 1 & & $\mu g / L$ & GE \\
\hline$<2.0$ & 1 & & $\mu \mathrm{g} / \mathrm{L}$ & $\mathrm{GE}$ \\
\hline$<2.0$ & $i$ & & $\mu \mathrm{g} / \mathrm{L}$ & $\mathrm{GE}$ \\
\hline 11 & 1 & & $\mu g / L$ & $\mathrm{GE}$ \\
\hline$<1.0$ & $i$ & & $\mu \mathrm{g} / \mathrm{L}$ & $\mathrm{GE}$ \\
\hline$<1.0$ & $i$ & & $\mu \mathrm{g} / \mathrm{L}$ & $\mathrm{GE}$ \\
\hline$<1.0$ & 1 & & $\mu g / L$ & $\mathrm{GE}$ \\
\hline$<1.0$ & 1 & & $\mu g / L$ & GE \\
\hline$<2.0$ & $i$ & & $\mu \mathrm{g} / \mathrm{L}$ & $\mathrm{GE}$ \\
\hline 1,440 & 1 & & $\mu g / L$ & GE \\
\hline$<1.0$ & 1 & & $\mu g / L$ & $G E$ \\
\hline 1,950 & 1 & & $\mu \mathrm{g} / \mathrm{L}$ & $G E$ \\
\hline$<1.0$ & 1 & & $\mu \mathrm{g} / \mathrm{L}$ & $\overline{G E}$ \\
\hline$<1.0$ & 1 & & $\mu g / L$ & $\mathrm{GE}$ \\
\hline$<1.0$ & 1 & & $\mu g / L$ & GE \\
\hline$<1.0$ & 1 & & $\mu g / L$ & GE \\
\hline$<1.0$ & 1 & & $\mu \mathrm{g} / \mathrm{L}$ & GE \\
\hline$<1.0$ & 1 & & $\mu \mathrm{g} / \mathrm{L}$ & $\mathrm{GE}$ \\
\hline$<4.0$ & 1 & & $\mu g / L$ & $\mathrm{GE}$ \\
\hline$<4.0$ & 1 & & $\mu \mathrm{g} / \mathrm{L}$ & GE \\
\hline$<5.0$ & 1 & & $\mu \mathrm{g} / \mathrm{L}$ & GE \\
\hline$<1.0$ & 1 & & $\mu \mathrm{g} / \mathrm{L}$ & GE \\
\hline$<1.0$ & 1 & & $\mu \mathrm{g} / \mathrm{L}$ & $\overrightarrow{G E}$ \\
\hline$<1.0$ & 1 & & $\mu g / L$ & GE \\
\hline$<1.0$ & 1 & & $\mu g / L$ & GE \\
\hline$<1.0$ & 1 & & $\mu \mathrm{g} / \mathrm{L}$ & $\mathrm{GE}$ \\
\hline$<1.0$ & 1 & & $\mu g / L$ & $\overline{G E}$ \\
\hline$<0.0015$ & 1 & & $\mu g / L$ & GE \\
\hline$<1.0$ & 1 & & $\mu \mathrm{g} / \mathrm{L}$ & GE \\
\hline$<1.0$ & 1 & & $\mu \mathrm{g} / \mathrm{L}$ & GE \\
\hline$<1.0$ & 1 & & $\mu g / L$ & GE \\
\hline$<0.0061$ & 1 & & $\mu q / \mathrm{L}$ & \\
\hline$<1.0$ & 1 & & $\mu g / L$ & GE \\
\hline 22 & 1 & $J$ & $\mu \mathrm{g} / \mathrm{L}$ & GE \\
\hline $5.8 \mathrm{E}-01$ & 1 & Ui & $\mathrm{pCi} / \mathrm{L}$ & GP \\
\hline
\end{tabular}

Note: Flagging, dilution factors, modifiers, and laboratories are for fourth quarter 1994 data only. See Appendix B for flagging criteria. - = exceeded holding time for fourth quarter 1994.

- = exceeded screening level or final PDWS for fourth quarter 1994. 


\section{H ST Analyte}

Heptachlor

Iron, total recoverable

Lead, total recoverable

Lindane

Magnesium, total recoverable

Manganese, total recoverable

Mercury, total recoverable

Methoxychlor

Nickel, total recoverable

Nickel, total recoverate as nitrogen

Nitrate-nitrite as nitrogen

Nonvolatile beta

$\mathrm{pH}$

Phenols

Potassium, total recoverable

Radium, total alpha-emitting

Selenium, total recoverable

Silica, total recoverable

Silver, total recoverable

Sodium, total recoverable

Specific conductance

Sulfate

1,1,2,2-Tetrachloroethane

Tetrachloroethylene

Tin, total recoverable

Toluene

Total dissolved solids

Total organic carbon

Total organic halogens

Total phosphates (as P)

Toxaphene

2,4,5-TP (Silvex)

1,1,1-Trichloroethane

1,1,2-Trichloroethane

Trichloroethylene

Trichlorofluoromethane

2,4,5-T

Tritium

Turbidity

Vanadium, total recoverable

Xylenes

\begin{tabular}{|c|c|c|c|c|c|c|c|}
\hline 1094 & $\underline{2094}$ & 3094 & $\underline{4094}$ & DF & Mod & $\underline{\text { Unit }}$ & Lab \\
\hline $\begin{array}{l}<0.051 \\
17 \\
<3.0 \\
<0.0051 \\
370 \\
19 \\
<0.20 \\
<0.51 \\
<9.8 \\
<4.0\end{array}$ & $\begin{aligned} &< 0.052 \\
& 23 \\
&< 3.0 \\
&< 0.0052 \\
& 341 \\
& 16 \\
&<0.20 \\
&<0.52 \\
&<10 \\
&<4.0\end{aligned}$ & $\begin{array}{c}17 \\
<3.0 \\
<0.056 \\
354 \\
16 \\
<0.20 \\
<0.50 \\
<10 \\
<4.0 \\
151\end{array}$ & $\begin{array}{c}33 \\
<3.0 \\
<0.0051 \\
354 \\
15 \\
<0.20 \\
<0.51 \\
<10 \\
<4.0\end{array}$ & $\begin{array}{l}1 \\
1 \\
1 \\
1 \\
1 \\
1 \\
1 \\
1 \\
1\end{array}$ & & $\begin{array}{l}\mu g / L \\
\mu g / L \\
\mu g / L \\
\mu g / L \\
\mu g / L \\
\mu g / L \\
\mu g / L \\
\mu g / L \\
\mu g / L \\
\mu g / L \\
\mu g / L\end{array}$ & $\begin{array}{l}\mathrm{GE} \\
\mathrm{GE} \\
\mathrm{GE} \\
\mathrm{GE} \\
\mathrm{GE} \\
\mathrm{GE} \\
\mathrm{GE} \\
\mathrm{GE} \\
\mathrm{GE}\end{array}$ \\
\hline $\begin{array}{l}150 \\
<7.2 \mathrm{E}-01 \\
6.0 \\
<5.0 \\
546 \\
2.5 \mathrm{E}+00\end{array}$ & $\begin{aligned} & 130 \\
< & 7.2 E-01 \\
& 6.1 \\
< & 5.0 \\
& 552 \\
< & 2.0 E-01\end{aligned}$ & $\begin{array}{l}138 \\
-2.0 \mathrm{E}-01 \\
5.9 \\
<5.0 \\
<500\end{array}$ & $\begin{array}{l}132 \\
1.2 E+00 \\
5.9 \\
<5.0 \\
548\end{array}$ & $\begin{array}{l}1 \\
1 \\
1 \\
1 \\
1\end{array}$ & $\begin{array}{l}\text { UI } \\
\text { J } \\
\text { J }\end{array}$ & $\begin{array}{l}\mu \mathrm{g} / \mathrm{L} \\
\mathrm{pCI} / \mathrm{L} \\
\mathrm{pH} \\
\mu \mathrm{g} / \mathrm{L} \\
\mu \mathrm{g} / \mathrm{L} \\
\mathrm{pCi} / \mathrm{L}\end{array}$ & $\begin{array}{l}\text { GE } \\
\text { GP } \\
\text { GE } \\
\text { GE } \\
\text { GE }\end{array}$ \\
\hline $\begin{array}{c}<2.0 \\
12,600 \\
<2.0 \\
3,650 \\
27 \\
1,070\end{array}$ & $\begin{array}{c}<2.0 \\
12,400 \\
<2.0 \\
3,760 \\
30 \\
<1,000\end{array}$ & $\begin{array}{c}<2.0 \\
12,300 \\
<2.0 \\
3,150 \\
30 \\
1,110\end{array}$ & $\begin{array}{c}<2.0 \\
12,500 \\
<2.0 \\
3,470 \\
28 \\
<1,000\end{array}$ & $\begin{array}{l}1 \\
1 \\
1 \\
1 \\
1 \\
1\end{array}$ & & $\begin{array}{l}\mu \mathrm{g} / \mathrm{L} \\
\mu \mathrm{g} / \mathrm{L} \\
\mu \mathrm{g} / \mathrm{L} \\
\mu \mathrm{g} / \mathrm{L} \\
\mu \mathrm{S} / \mathrm{cm} \\
\mu \mathrm{g} / \mathrm{L}\end{array}$ & $\begin{array}{l}\mathrm{GE} \\
\mathrm{GE} \\
\mathrm{GE} \\
\mathrm{GE} \\
\mathrm{GE} \\
\mathrm{GE}\end{array}$ \\
\hline $\begin{array}{l}<1.0 \\
<1.0\end{array}$ & $\begin{array}{l}<1.0 \\
<1.0\end{array}$ & $\begin{array}{l}<1.0 \\
<1.0\end{array}$ & $\begin{array}{l}<1.0 \\
<1.0\end{array}$ & $i$ & & & $\begin{array}{l}\text { GE } \\
\text { GE }\end{array}$ \\
\hline $\begin{array}{l}<2.0 \\
<1.0\end{array}$ & $\begin{array}{l}<2.0 \\
<1.0\end{array}$ & $\begin{array}{l}<2.0 \\
<1.0\end{array}$ & $\begin{array}{l}<2.0 \\
<1.0\end{array}$ & 1 & & $\mu \mathrm{g} / \mathrm{L}$ & GE \\
\hline $\begin{aligned} & 25,000 \\
&< 1,000 \\
& 12\end{aligned}$ & $\begin{array}{c}27,000 \\
<1,000 \\
6.1\end{array}$ & $\begin{array}{c}39,000 \\
<1,000 \\
8.1\end{array}$ & $\begin{aligned} & 33,000 \\
< & 1,000 \\
< & 5.0\end{aligned}$ & $\begin{array}{l}1 \\
1 \\
1\end{array}$ & & $\begin{array}{l}\mu \mathrm{g} / \mathrm{L} \\
\mu \mathrm{g} / \mathrm{L} \\
\mu \mathrm{g} / \mathrm{L}\end{array}$ & $\begin{array}{l}\mathrm{GE} \\
\mathrm{GE} \\
\mathrm{GE}\end{array}$ \\
\hline $\begin{array}{l}<50 \\
<0.25 \\
<0.00046\end{array}$ & $\begin{aligned} & 110 \\
< & 0.25 \\
< & 0.00046\end{aligned}$ & $\begin{aligned} & 2,060 \\
< & 0.24 \\
< & 0.00045\end{aligned}$ & $\begin{array}{l}<50 \\
<0.25 \\
<0.00046\end{array}$ & $\begin{array}{l}1 \\
1 \\
1\end{array}$ & & $\mu g g / L$ & GE \\
\hline $\begin{array}{l}<1.0 \\
<1.0\end{array}$ & $\begin{array}{l}<1.0 \\
<1.0\end{array}$ & $\begin{array}{l}<1.0 \\
<1.0\end{array}$ & $\begin{array}{l}<1.0 \\
<1.0\end{array}$ & 1 & & $\mu g / L$ & $\begin{array}{l}\mathrm{GE} \\
\mathrm{GE}\end{array}$ \\
\hline$<1.0$ & $<1.0$ & $<1.0$ & $<1.0$ & 1 & & $\mu \mathrm{g} / \mathrm{L}$ & GE \\
\hline$<1.0$ & $\begin{array}{l}<1.0 \\
<0.00046\end{array}$ & $<1.0$ & $<1.0$ & 1 & & $\mu g / L$ & GE \\
\hline $\begin{array}{l}<0.0 E+00 \\
0.12 \\
<8.0 \\
<2.0\end{array}$ & $\begin{array}{l}<5.4 \mathrm{E}-02 \\
0.38 \\
<8.0 \\
<2.0\end{array}$ & $\begin{aligned} & 6.2 \mathrm{E}-01 \\
& 0.45 \\
< & 3.0 \\
< & 2.0\end{aligned}$ & $\begin{aligned} & 2.0 \mathrm{E}-01 \\
& 0.39 \\
< & 8.0 \\
< & 2.0\end{aligned}$ & $\begin{array}{l}1 \\
1 \\
1 \\
1\end{array}$ & UI & $\begin{array}{l}\mathrm{pCi} / \mathrm{mL} \\
\mathrm{NTU} \\
\mu \mathrm{g} / \mathrm{L} \\
\mu \mathrm{g} / \mathrm{L}\end{array}$ & $\begin{array}{l}\mathrm{GP} \\
\mathrm{GE} \\
\mathrm{GE} \\
\mathrm{GE}\end{array}$ \\
\hline
\end{tabular}

Note: Flagging, dilution factors, modifiers, and laboratories are for fourth quarter 1994 data only. See Appendix B for flagging criteria. - = exceeded holding time for fourth quarter 1994.

- = exceeded screening level or final PDWS for fourth quarter 1994. 
WELL BGX 12D

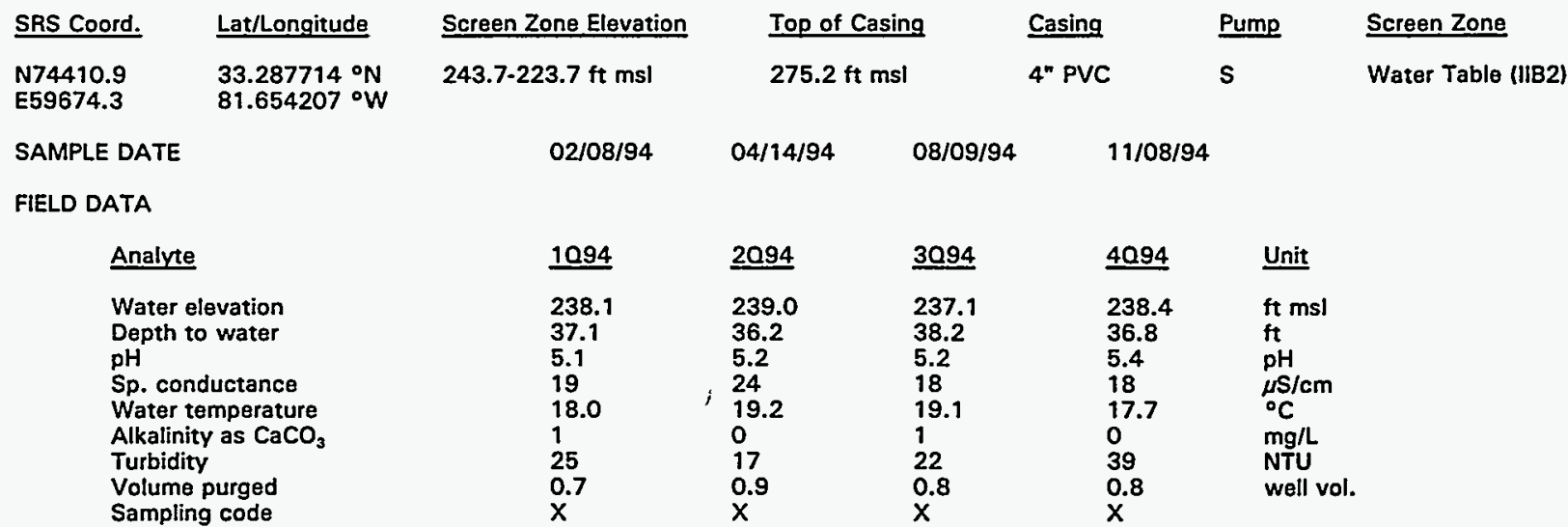

\section{ANALYTICAL DATA}

H․ ST Analyte

Acetophenone

Aldrin

Aluminum, total recoverable

Antimony, total recoverable

Arsenic, total recoverable

Barium, total recoverable

Benzene

Bromodichloromethane

Bromoform

Bromomethane (Methyl bromide)

Cadmium, total recoverable

Calcium, total recoverable

Carbon tetrachloride

Chloride

Chlorobenzene

Chloroethane

Chloroethene (Vinyl chloride)

2-Chloroethyl vinyl ether

Chloroform

Chloromethane (Methyl chloride)

Chromium, total recoverable

Copper, total recoverable

Cyanide

p,p'-DDT

Dibromochloromethane

1,1-Dichloroethane

1,2-Dichloroethane

1,1-Dichloroethylene

trans-1,2-Dichloroethyiene

Dichloromethane

2,4-Dichlorophenoxyacetic acid

1,2-Dichloropropane

cis-1,3-Dichloropropene

trans-1,3-Dichloropropene

Dieldrin

Endrin

Ethylbenzene

Fluoride

Gross alpha
$<10$

$<0.053$

$<2.0$

$<2.0$

19
$<1.0$

$<1.0$

$<1.0$

$<1.0$

$<2.0$

396
$<1.0$

$<1.0$

$<1.0$

$<1.0$

$<1.0$

$<1.0$

$<1.0$

$<1.0$

$<4.0$

9.1

$<5.0$

$<0.11$

$<1.0$

$<1.0$

$<1.0$

$<1.0$

$<1.0$

$<1.0$

$<0.0015$

$<1.0$

$<1.0$

$<1.0$

$<0.53$

$<0.0064$

$<1.0$

$<100$

$1.3 E+00$

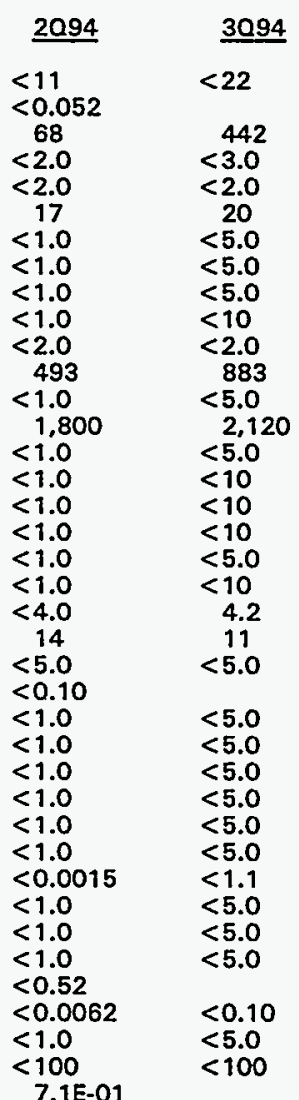

\begin{tabular}{|c|c|c|c|c|}
\hline 4094 & $\underline{\mathrm{DF}}$ & Mad & Unit & $\underline{\text { Lab }}$ \\
\hline$<10$ & 1 & & $\mu \mathrm{g} / \mathrm{L}$ & GE \\
\hline 118 & 1 & & $\mu \mathrm{g} / \mathrm{L}$ & GE \\
\hline$<2.0$ & 1 & & $\mu g / L$ & GE \\
\hline$<2.0$ & 1 & & $\mu \mathrm{g} / \mathrm{L}$ & $\mathrm{GE}$ \\
\hline 20 & $i$ & & $\mu \mathrm{g} / \mathrm{L}$ & $\overrightarrow{G E}$ \\
\hline$<1.0$ & 1 & & $\mu \mathrm{g} / \mathrm{L}$ & $G E$ \\
\hline$<1.0$ & $i$ & & $\mu \mathrm{g} / \mathrm{L}$ & $\mathrm{GE}$ \\
\hline$<1.0$ & $i$ & & $\mu \mathrm{g} / \mathrm{L}$ & $\mathrm{GE}$ \\
\hline$<1.0$ & 1 & & $\mu \mathrm{g} / \mathrm{L}$ & GE \\
\hline$<2.0$ & 1 & & $\mu g / L$ & GE \\
\hline 267 & $i$ & & $\mu \mathrm{g} / \mathrm{L}$ & $\mathrm{GE}$ \\
\hline$<1.0$ & 1 & & $\mu \mathrm{g} / \mathrm{L}$ & $\overrightarrow{G E}$ \\
\hline 1,340 & $i$ & & $\mu \mathrm{g} / \mathrm{L}$ & $\overrightarrow{G E}$ \\
\hline$<1.0$ & 1 & & $\mu \mathrm{g} / \mathrm{L}$ & $\mathrm{GE}$ \\
\hline$<1.0$ & $i$ & & $\mu \mathrm{g} / \mathrm{L}$ & GE \\
\hline$<1.0$ & 1 & & $\mu \mathrm{g} / \mathrm{L}$ & GE \\
\hline$<1.0$ & 1 & & $\mu g / L$ & GE \\
\hline$<1.0$ & 1 & & $\mu \mathrm{g} / \mathrm{L}$ & GE \\
\hline$<1.0$ & 1 & & $\mu g / L$ & $G E$ \\
\hline$<4.0$ & 1 & & $\mu \mathrm{g} / \mathrm{L}$ & $\mathrm{GE}$ \\
\hline 5.1 & $i$ & $J$ & $\mu \mathrm{g} / \mathrm{L}$ & $\overline{G E}$ \\
\hline$<5.0$ & 1 & & $\begin{array}{l}\mu / L \\
\mu \mathrm{g} / \mathrm{L}\end{array}$ & GE \\
\hline$<1.0$ & 1 & & $\mu \mathrm{g} / \mathrm{L}$ & $\mathrm{GE}$ \\
\hline$<1.0$ & 1 & & $\mu \mathrm{g} / \mathrm{L}$ & GE \\
\hline$<1.0$ & $i$ & & $\mu \mathrm{g} / \mathrm{L}$ & $\mathrm{GE}$ \\
\hline$<1.0$ & $i$ & & $\mu \mathrm{g} / \mathrm{L}$ & $\mathrm{GE}$ \\
\hline$<1.0$ & $i$ & & $\mu \mathrm{g} / \mathrm{L}$ & $\mathrm{GE}$ \\
\hline$<1.0$ & 1 & & $\mu g / L$ & $\mathrm{GE}$ \\
\hline$<0.0015$ & 1 & & $\mu \mathrm{g} / \mathrm{L}$ & $\mathrm{GE}$ \\
\hline$<1.0$ & 1 & & $\mu \mathrm{g} / \mathrm{L}$ & GE \\
\hline$<1.0$ & $i$ & & $\mu \mathrm{g} / \mathrm{L}$ & GE \\
\hline$<1.0$ & 1 & & $\mu \mathrm{g} / \mathrm{L}$ & GE \\
\hline$<0.0059$ & 1 & & $\mu \mathrm{g} / \mathrm{L}$ & GE \\
\hline$<1.0$ & 1 & & $\mu \mathrm{g} / \mathrm{L}$ & GE \\
\hline 38 & $i$ & $J$ & $\mu \mathrm{g} / \mathrm{L}$ & $\mathrm{GE}$ \\
\hline 8.0E-01 & $i$ & UI & pCi/L & $G P$ \\
\hline
\end{tabular}

Note: Flagging, dilution factors, modifiers, and laboratories are for fourth quarter 1994 data only. See Appendix B for flagging criteria. - = exceeded holding time for fourth quarter 1994.

a = exceeded screening level or final PDWS for fourth quarter 1994. 


\section{ANALYTICAL DATA}

\section{H ST Analyte}

Heptachlor

Iron, total recoverable

Lead, total recoverable

Lindane

Magnesium, total recoverable

Manganese, total recoverable

Mercury, total recoverable

Methoxychlor

Naphthalene

Nickel, total recoverable

Nitrate as nitrogen

Nitrate-nitrite as nitrogen

Nonvolatile beta

- $\quad \mathrm{pH}$

Phenols

Potassium, total recoverable

Radium, total alpha-emitting

Selenium, total recoverable

Silica, total recoverable

Silver, total recoverable

Sodium, total recoverable

Specific conductance

Sulfate

1,1,2,2-Tetrachloroethane

Tetrachloroethylene

Tin, total recoverable

Toluene

Total dissolved solids

Total organic carbon

Total organic halogens

Total phosphates (as P)

Toxaphene

2,4,5-TP (Silvex)

1,1,1-Trichlorosthane

1,1,2-Trichloroethane

Trichloroethylene

Trichlorofluoromethane

2,4,5-T

Tritium

Turbidity

Vanadium, total recoverable

Xylenes

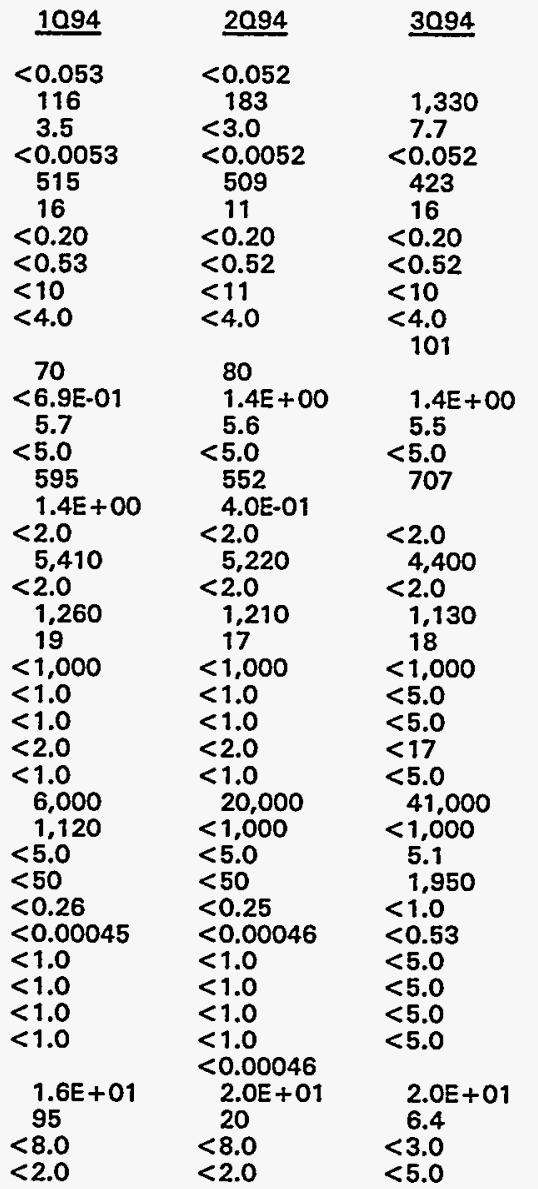

$\quad \underline{4094}$

120
3.5
$<0.0050$
520
14
$<0.20$
$<0.50$
$<10$
$<4.0$

80

$9.9 \mathrm{E}-01$
5.6

5.6
$<5.0$

553

$<2.0$

5,080

$<2.0$

1,650

$<16$

$<1,000$

$<1.0$

$<2.0$

$<1.0$

18,000

$<1,000$

$<5.0$

$<50$

$<0.24$

$<0.00044$

$<1.0$

$<1.0$

$<1.0$

$<1.0$

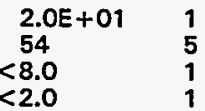

$\mu g / L$

$\mu \mathrm{g} / \mathrm{L}$ GE 0

$\mu g / L$ GE 0

$\mu \mathrm{g} / \mathrm{L} \quad \mathrm{GE} \quad 0$

$\mu g / L \quad G E$

$\mu g / L \quad G E$

$\mu g / L \quad G E$

$\mu g / L \quad G E$

$\mu g / L \quad G E$

$\mu g / L$

$\mu \mathrm{g} / \mathrm{L} \quad \mathrm{GE} \quad 0$

UI $\mathrm{PCI} / L$ GP 0

PH

$\mu \mathrm{g} / \mathrm{L} \quad \mathrm{GE}$

$\mu \mathrm{g} / \mathrm{L} \quad \mathrm{GE}$

pCi/L

$\mu g / L \quad G E$

$\mu \mathrm{g} / \mathrm{L}$

$\mu \mathrm{g} / \mathrm{L} \quad \mathrm{GE}$

$\mu g / L . \quad G E$

$\mu \mathrm{S} / \mathrm{cm}$ GE

$\mu \mathrm{g} / \mathrm{L}$

$\mu g / L$

$\mu \mathrm{g} / \mathrm{L} \quad \mathrm{GE}$

$\mu g / L$

$\mu g / 1$

$\mu \mathrm{g} / \mathrm{L}$

ug/L GE

$\mu g / L \quad G E$

$\mu \mathrm{g} / \mathrm{L} \quad \mathrm{GE}$

$\mu \mathrm{g} / \mathrm{L} \quad \mathrm{GE}$

$\mu g / L \quad G E$

$\mu \mathrm{g} / \mathrm{L} \quad \mathrm{GE}$

$\mu \mathrm{gg} / \mathrm{L} \quad \mathrm{GE}$

$\underset{\mu g / L}{\mu g / L} \quad \mathrm{GE}$

$\mu \mathrm{g} / \mathrm{L}$

$\mathrm{pCi} / \mathrm{mL}$ GP

NTU GE

$\underset{\mu \mathrm{g} / \mathrm{L}}{\mu \mathrm{GE}}$
Flag

政




\section{WELL FSS 1D}

\begin{tabular}{|c|c|c|c|c|c|c|}
\hline SRS Coord. & Lat/Longitude & Screen Zone Elevation & Top of Casing & Casing & Pump & Screen Zone \\
\hline $\begin{array}{l}\text { N75257.6 } \\
\text { E53897.6 }\end{array}$ & $\begin{array}{l}33.280161^{\circ} \mathrm{N} \\
81.671063^{\circ} \mathrm{W}\end{array}$ & $229.9-209.9 \mathrm{f} \mathrm{msl}$ & $266 \mathrm{ft} \mathrm{msl}$ & 4" PVC & $\mathbf{s}$ & Water Table \\
\hline SAMPLE DATE & & $02 / 01 / 94$ & $04 / 27 / 94$ & & & \\
\hline
\end{tabular}

FIELD DATA

Analyte
Water elevation
Depth to water
pH
Sp. conductance
Water temperature
Alkalinity as $\mathrm{CaCO}_{3}$
Turbidity
Volume purged
Sampling code

ANALYTICAL DATA

H ST

Analyte
Acetophenone
Aldrin
Aluminum, total recoverable
Antimony, total recoverable
Arsenic, total recoverable
Barium, total recoverable
Benzene
Bromodichloromethane
Bromoform
Bromomethane (Methyl bromide)
Cadmium, total recoverable
Calcium, total recoverable
Carbon tetrachloride
Carbon-14
Chloride
Chlorobenzene
Chloroethane
Chloroethene (Vinyl chloride)
2-Chloroethyl vinyl ether
Chloroform
Chloromethane (Methyl chloride)
Chromium, total recoverable
Copper, total recoverable
Cyanide
p,p'-DDT
Dibromochloromethane
1,1-Dichloroethane
1,2-Dichloroethane
1,1-Dichloroethylene
trans-1,2-Dichloroethylene
Dichloromethane
2,4-Dichlorophenoxyacetic acid
1,2-Dichloropropane
cis-1,3-Dichloropropene
trans-1,3-Dichloropropene
Dieldrin
Endrin
Ethylbenzene
Fluoride
Gross alpha

\begin{tabular}{l}
1094 \\
\hline 223.4 \\
42.6 \\
5.8 \\
44 \\
15.1 \\
10 \\
28 \\
0.6 \\
$x$
\end{tabular}

20.94
224.1
41.9
5.8
81
18.9
23
28
0.5
$x$

\begin{tabular}{l}
3094 \\
\hline 223.0 \\
43.0 \\
5.8 \\
76 \\
21.0 \\
18 \\
76 \\
0.8 \\
$x$
\end{tabular}

$$
\begin{array}{r}
1094 \\
<10
\end{array}
$$

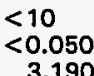$$
\begin{array}{r}
3,190 \\
<2.0
\end{array}
$$$$
<2.0
$$$$
12
$$$$
<1.0
$$$$
<1.0
$$$$
<1.0
$$$$
<1.0
$$$$
<2.0
$$$$
8,920
$$$$
<1.0
$$$$
<-4.9 E+00
$$$$
2,630
$$$$
\begin{aligned}
& <1.0 \\
& <1.0
\end{aligned}
$$$$
<1.0
$$$$
<1.0
$$$$
<1.0
$$$$
<1.0
$$$$
<4.0
$$$$
<5.0
$$$$
<0.10
$$$$
<1.0
$$$$
<1.0
$$$$
<1.0
$$$$
<1.0
$$$$
<1.0
$$$$
<1.0
$$$$
<0.0015
$$$$
<1.0
$$$$
<1.0
$$$$
<1.0
$$$$
<0.50
$$$$
<0.0060
$$$$
<1.0
$$$$
7.3 E-01
$$

$\begin{aligned} & 2094 \\ &<10 \\ & 737 \\ &<2.0 \\ &<2.0 \\ & 5.9 \\ &<1.0 \\ &<1.0 \\ &<1.0 \\ &<1.0 \\ &<2.0 \\ & 6.260 \\ &<1.0 \\ & \\ & 2.780 \\ &<1.0 \\ &<1.0 \\ &<1.0 \\ &<1.0 \\ &<1.0 \\ &<1.0 \\ & 4.3 \\ & 119 \\ &<5.0 \\ &<1.0 \\ &<1.0 \\ &<1.0 \\ &<1.0 \\ &<1.0 \\ &<1.0 \\ &<1.1 \\ &<1.0 \\ &<1.0 \\ &<1.0 \\ &<0.11 \\ &<1.0 \\ &<100 \\ & 2.4 E+00 \\ &\end{aligned}$

$\quad 3094$
$<10$

2,460
$<3.0$
$<2.0$
14
$<5.0$
$<5.0$
$<5.0$
$<10$
$<2.0$
11,700
$<5.0$

$\quad \underline{4094}$
$<9.7$

$\quad 993$
$<2.0$
$<2.0$
7.5
$<1.0$
$<1.0$
$<1.0$
$<1.0$
$<2.0$
18,300
$<1.0$

Unit

ft msl

$\mathrm{ft}$

pH

${ }^{\circ} \mathrm{S} / \mathrm{C}$

mg/L

well vol.

$\begin{array}{lll}4094 & & \text { Unit } \\ 222.9 & & \mathrm{ft} \mathrm{msl} \\ 43.1 & \mathrm{ft} \\ 6.5 & \mathrm{pH} \\ 105 & \mu \mathrm{S} / \mathrm{cm} \\ 18.9 & & { }^{\circ} \mathrm{C} \\ 14 & & \mathrm{mg} / \mathrm{L} \\ 66 & & \text { NTU } \\ 0.9 & \text { well vol. } \\ \mathrm{X} & & \end{array}$

3,230

$\begin{array}{ll} & 3,230 \\ <5.0\end{array}$

$<10$

$<10$

$<10$

$<5.0$

4.5
9.4

348

$<5.0$

$<5.0$

$<5.0$

$<5.0$

$<5.0$

$<5.0$

$<5.0$

$<7.1$

$<5.0$

$<5.0$

$<5.0$

$<0.11$

$<5.0$
$<100$

$2.3 E+00$

$\begin{array}{ll} & 2,340 \\ <1.0 & 1 \\ <1.0 & 1 \\ <1.0 & 1 \\ <1.0 & 1 \\ <1.0 & 1 \\ <1.0 & 1 \\ <4.0 & 1 \\ 191 & 1 \\ <5.0 & 1 \\ <1.0 & 1 \\ <1.0 & 1 \\ <1.0 & 1 \\ <1.0 & 1 \\ <1.0 & 1 \\ <1.0 & 1 \\ <0.0015 & 1 \\ <1.0 & 1 \\ <1.0 & 1 \\ <1.0 & 1 \\ <0.0060 & 1 \\ <1.0 & 1 \\ <20 & 1 \\ <1.1 E+00 & 1\end{array}$

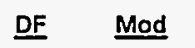

$\begin{array}{lll}\text { Unit } & \text { Lab } & \text { Flag } \\ \begin{array}{c}\mu \mathrm{g} / \mathrm{L} \\ \mu \mathrm{g} / \mathrm{L} \\ \mu \mathrm{g} / \mathrm{L}\end{array} & \text { GE } & 0 \\ \mu \mathrm{gE} & 2\end{array}$

$\mu \mathrm{g} / \mathrm{L} \quad \mathrm{GE}$

$\mu \mathrm{g} / \mathrm{L} \quad \mathrm{GE}$

$\mu g / L \quad G E$

$\mu \mathrm{g} / \mathrm{L} \quad \mathrm{GE}$

$\mu g / L \quad \mathrm{GE}$

$\mu g / L \quad G E$

$\mu \mathrm{g} / \mathrm{L} \quad \mathrm{GE}$

$\mu g / L \quad G E$

pCi/L $\mu \mathrm{g} / \mathrm{L}$ $\mu g / L$

$\mu g / L$

$\mu \mathrm{g} / \mathrm{L}$

$\mu g / L$

$\mu g / L$

$\mu \mathrm{g} / \mathrm{L}$

$\mu \mathrm{g} / \mathrm{L}$

$\mu g / L$

$\mu g / L$

$\mu g / L$

$\mu g / L$

$\mu \mathrm{g} / \mathrm{L}$

$\mu g / L$

$\mu \mathrm{g} / \mathrm{L}$

$\mu g / L$

$\mu g / L$

$\mu g / L$

$\mu g / \mathrm{L}$

$\mu g / L$

J

$\mu g / L$

$\mu \mathrm{g} / \mathrm{L}$

0
0
0
0
0
0
0
0
0
0
0
0
0
0
0
0
0
0
0
0
0
0
0
0
0
0
0
0
0
0
0
0
0
0
0
0
0
0

Note: Flagging, dilution factors, modifiers, and laboratories are for fourth quarter 1994 data only. See Appendix B for flagging criteria. - = exceeded holding time for fourth quarter 1994.

- = exceeded screening level or final PDWS for fourth quarter 1994. 


\section{H ST Analyte}

Heptachlor

Iron, total recoverable

Lead, total recoverable

Lindane

Magnesium, total recoverable

Manganese, total recoverable

Mercury, total recoverable

Methoxychlor

Naphthalene

Nickel, total recoverable

Nitrate as nitrogen

Nitrate-nitrite as nitrogen

Nonvolatile beta

$\mathrm{pH}$

Phenols

Potassium, total recoverable

Radium, total alpha-emitting

Radium-226

Radium-228

Selenium, total recoverable

Silica, total recoverable

Silver, total recoverable

Sodium, total recoverable

Specific conductance

Sulfate

1,1,2,2-Tetrachloroethane

Tetrachloroethylene

Tin, total recoverable

Toluene

Total dissolved solids

Total organic carbon

Total organic halogens

Total phosphates (as P)

Toxaphene

2,4,5-TP (Silvex)

Tributyl phosphate

1,1,1-Trichloroethane

1,1,2-Trichloroethane

Trichloroethylene

Trichlorofluoromethane

Tritium

Turbidity

Uranium-233/234

Uranium-235

Uranium-238

Vanadium, total recoverable

Xylenes

Zinc, total recoverable

\begin{tabular}{|c|c|}
\hline 1094 & $\underline{2094}$ \\
\hline \multicolumn{2}{|l|}{$<0.050$} \\
\hline 572 & 845 \\
\hline $\begin{aligned} & 119 \\
< & 0.0050\end{aligned}$ & $\begin{array}{c}16 \\
<0.055\end{array}$ \\
\hline 567 & 326 \\
\hline $\begin{array}{c}46 \\
<0.20\end{array}$ & $\begin{array}{c}17 \\
<020\end{array}$ \\
\hline $\begin{array}{l}<0.20 \\
<0.50\end{array}$ & $<0.55$ \\
\hline$<10$ & $<10$ \\
\hline 8.7 & $<4.0$ \\
\hline \multirow{6}{*}{$\begin{array}{l}220 \\
1.6 \mathrm{E}+00 \\
6.6 \\
<5.0 \\
<500 \\
8.0 \mathrm{E}-01\end{array}$} & 312 \\
\hline & $2.8 E+00$ \\
\hline & 6.3 \\
\hline & $<5.0$ \\
\hline & $<500$ \\
\hline & $\begin{array}{l}4.4 \mathrm{E}-01 \\
8.0 \mathrm{E}-01\end{array}$ \\
\hline$<2.0$ & $<2.0$ \\
\hline 9,810 & 4,270 \\
\hline$<2.0$ & $<2.0$ \\
\hline $\begin{array}{l}2,280 \\
109\end{array}$ & $\begin{array}{l}1,380 \\
106\end{array}$ \\
\hline 1,210 & 1,720 \\
\hline$<1.0$ & $<1.0$ \\
\hline$<1.0$ & $<1.0$ \\
\hline 2.1 & $<2.0$ \\
\hline$<1.0$ & $<1.0$ \\
\hline 61,000 & 63,000 \\
\hline 1,110 & $<1,000$ \\
\hline & $<5.0$ \\
\hline$<0.24$ & $\begin{array}{r}55 \\
<1.1\end{array}$ \\
\hline$<0.00045$ & $<0.55$ \\
\hline & \\
\hline$<1.0$ & $<1.0$ \\
\hline$<1.0$ & $<1.0$ \\
\hline$<1.0$ & $<1.0$ \\
\hline$<1.0$ & $<1.0$ \\
\hline $9.0 E+00$ & $1.0 E+01$ \\
\hline 28 & 16 \\
\hline 1.2E-01 & $<3.7$ E-02 \\
\hline$<-5.3 E-03$ & $<3.3 \mathrm{E}-02$ \\
\hline$<4.9 E-02$ & $<5.0 \mathrm{OE}-02$ \\
\hline$<8.0$ & $<<.0$ \\
\hline & \\
\hline
\end{tabular}

\section{$\underline{3094}$}

$\underline{4094}$

3,060

$\begin{aligned} & 53 \\ &<0.054\end{aligned}$

376

58

$<0.20$

$<0.54$

$<10$

11
355

$1.4 E+00$

6.5

$<5.0$

$<500$

5.0E-01

135

$<0.0050$

457

28
$<0.20$

$<0.50$

$<9.7$

5.3

321

8.4E-01

6.6

$<5.0$

$<500$

$<2.0$

7,180

$<2.0$

2,540

108

1,610

$<5.0$

$<5.0$

$<5.0$

$<17$

63,000

$<1,000$

11

2,820

$<1.1$

$<0.53$

$<10$

$<5.0$

$<5.0$

$<5.0$

$<5.0$

$1.1 E+01$

19

6.2E-02

$-1.3 \mathrm{E}-02$

7.5E-02

4.4

$<5.0$

$<2.0$

8.110

$<2.0$

3,520

112

1,410

$<1.0$

$<1.0$

$<1.0$

69,000

1,090

$<5.0$

55

$<0.24$

$<0.00045$

$<9.7$

$<1.0$

$<1.0$

$<1.0$

$<1.0$

1.0E +01

17

2.7E-02

$-6.2 \mathrm{E}-03$

2.7E-02

$<8.0$

$<2.0$$$
\text { ( }
$$

\section{DF Mod Unit Lab Flag}

$\begin{array}{llll} & \mu g / L & & \\ & \mu \mathrm{g} / \mathrm{L} & \mathrm{GE} & 0 \\ & \mu \mathrm{g} / \mathrm{L} & \mathrm{GE} & 0 \\ \mathrm{~J} & \mu \mathrm{g} / \mathrm{L} & \mathrm{GE} & 0 \\ & \mu \mathrm{g} / \mathrm{L} & \mathrm{GE} & 0 \\ & \mu \mathrm{g} / \mathrm{L} & \mathrm{GE} & 1 \\ & \mu \mathrm{g} / \mathrm{L} & \mathrm{GE} & 0 \\ \mathrm{~J} & \mu \mathrm{g} / \mathrm{L} & \mathrm{GE} & 0 \\ & \mu \mathrm{g} / \mathrm{L} & \mathrm{GE} & 0 \\ \mathrm{~J} & \mu \mathrm{g} / \mathrm{L} & \mathrm{GE} & 0 \\ & \mu \mathrm{g} / \mathrm{L} & & \\ & \mu \mathrm{g} / \mathrm{L} & \mathrm{GE} & 0 \\ \mathrm{UI} & \mathrm{PCi} / \mathrm{L} & \mathrm{GP} & 0 \\ \mathrm{~J} & \mathrm{pH} & \mathrm{GE} & 0 \\ & \mu \mathrm{g} / \mathrm{L} & \mathrm{GE} & 0 \\ & \mu \mathrm{g} / \mathrm{L} & \mathrm{GE} & 0 \\ & \mathrm{DCC} / \mathrm{L} & & \end{array}$

1
1
1
1
1
1
1
1
1
1
1
1
1
1

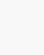

1
1
1
1
1
1
1
1
1
1
1
1
1
1
1
1
1
1
1
1
1
1
1
1
1
1
1
1

$\operatorname{lng}(2)$

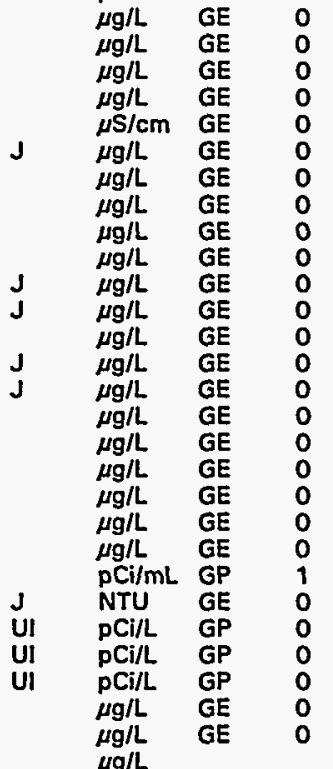

Note: Flagging, dilution factors, modifiers, and laboratories are for fourth quarter 1994 data only. See Appendix B for flagging criteria. - = exceeded holding time for fourth quarter 1994.

- = exceeded screening level or final PDWS for fourth quarter 1994. 
WELL FSS 2D

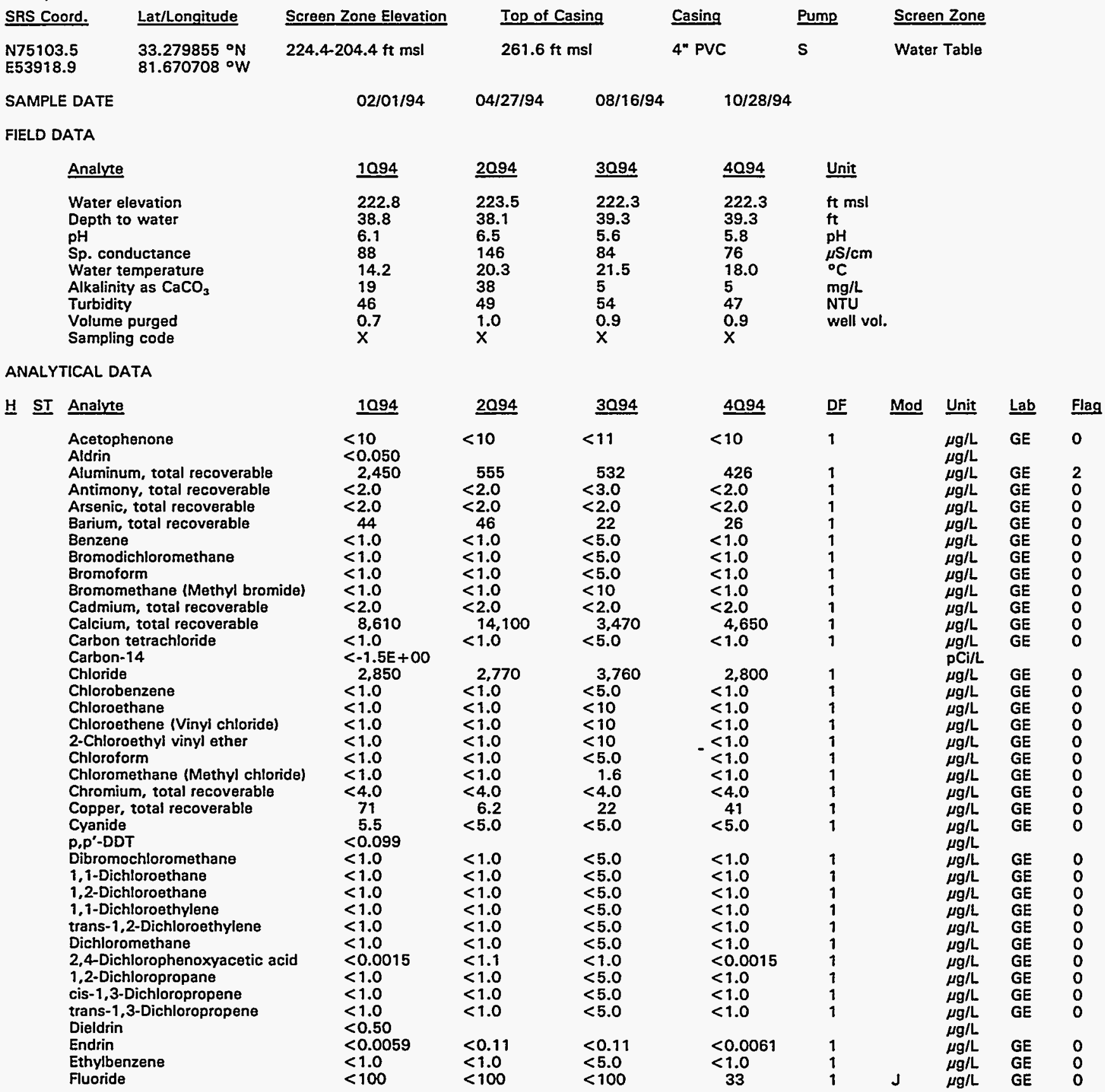

Note: Flagging, dilution factors, modifiers, and laboratories are for fourth quarter 1994 data only. See Appendix B for flagging criteria. - = exceeded holding time for fourth quarter 1994.

- = exceeded screening level or final PDWS for fourth quarter 1994. 
H ST Analyte

Gross alpha

Heptachlor

Iron, total recoverable

Lead, total recoverable

Lindane

Magnesium, total recoverable

Manganese, total recoverable

Mercury, total recoverable

Methoxychlor

Naphthalene

Nickel, total recoverable

Nitrate as nitrogen

Nitrate-nitrite as nitrogen

Nonvolatile beta

- $\mathrm{pH}$

Phenols

Potassium, total recoverable

Radium, total alpha-emitting

Radium-226

Radium-228

Selenium, total recoverable

Silica, total recoverable

Silver, total recoverable

Sodium, total recoverable

Specific conductance

Sulfate

1,1,2,2-Tetrachloroethane

Tetrachloroethylene

Tin, total recoverable

Toluene

Total dissolved solids

Total organic carbon

Total organic halogens

Total phosphates (as P)

Toxaphene

2,4,5-TP (Silvex)

Tributyl phosphate

1,1,1-Trichloroethane

1,1,2-Trichloroethane

Trichloroethylene

Trichlorofluoromethane

- Tritium

Turbidity

Uranium-233/234

Uranium-235

Uranium-238

Vanadium, total recoverable

Xylenes

Zinc, total recoverable

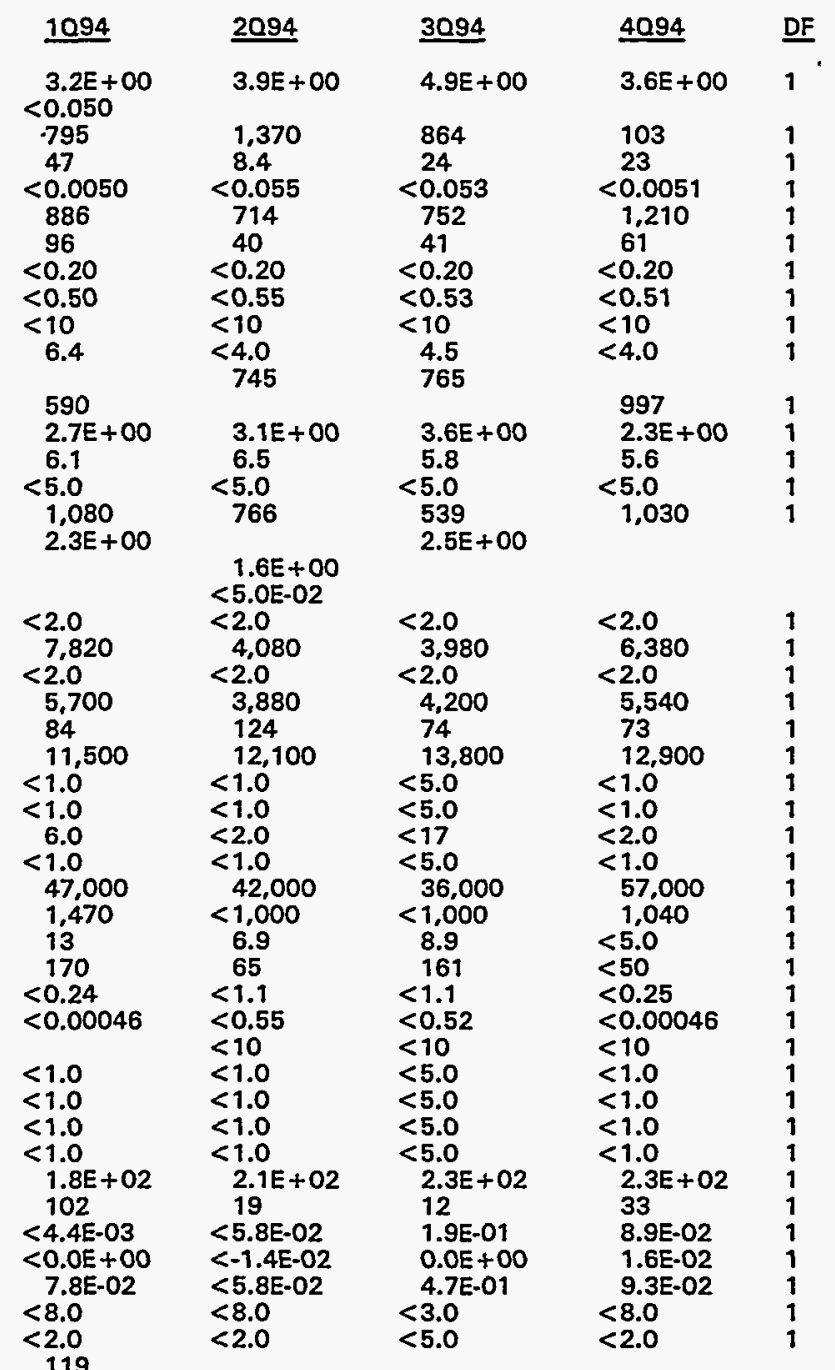

\begin{tabular}{cccc} 
Mod & $\begin{array}{ccc}\text { Unit } \\
\mu C i / L\end{array}$ & Lab & Flag \\
\cline { 1 - 2 }$\mu g / L$ & & 0 \\
$\mu g / L$ & GE & 0 \\
$\mu g / L$ & GE & 0 \\
$\mu g / L$ & GE & 0 \\
$\mu g / L$ & GE & 0 \\
$\mu g / L$ & GE & 2 \\
$\mu g / L$ & GE & 0 \\
$\mu g / L$ & GE & 0 \\
$\mu g / L$ & GE & 0
\end{tabular}

$\mu g / L$ GE 0

$\mu \mathrm{g} / \mathrm{L}$

$\begin{array}{llll} & \mu g / L & \text { GE } & 0 \\ J & \mathrm{pCi} / L & \mathrm{GP} & 0 \\ J & \mathrm{pH} & \mathrm{GE} & 0 \\ \mu g / L & \mathrm{GE} & 0\end{array}$

$\mu g / L \quad$ GE

pCi/L

$\mathrm{pCi} / \mathrm{L}$

Ug/L GE

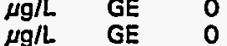

$\mu \mathrm{g} / \mathrm{L}$ GE 0

$\mu g / L$ GE 0

$\mu S / \mathrm{cm}$ GE 0

$\mu \mathrm{g} / \mathrm{L}$

$\mu \mathrm{g} / \mathrm{L}$

$\mu \mathrm{g} / \mathrm{L}$

$\mu \mathrm{g} / \mathrm{h}$

$\mu g / L$

J

$\mu \mathrm{g} / \mathrm{L} \quad \mathrm{GE}$

$\mu g / L . \quad$ GE

J

$\mu g / L \quad G E$

$\mu \mathrm{g} / \mathrm{L} \quad \mathrm{GE}$

$\mu \mathrm{g} / \mathrm{L}$

$\mu g / L$

$\mu g / L$

$\mu g / L$

$\mu g / L$

$\mu \mathrm{g} / \mathrm{L}$

NTU GP

$\mathbf{U}$

PCill

UI

pCi/h

$\mathrm{pCi} / \mathrm{L}$

$\mu g / L$

$\mu \mathrm{g} / \mathrm{L}$

$\mu g / L$

Note: Flagging, dilution factors, modifiers, and laboratories are for fourth quarter 1994 data only. See Appendix B for flagging criteria. - = exceeded holding time for fourth quarter 1994.

- = exceeded screening level or final PDWS for fourth quarter 1994. 
WELL FSS 3D

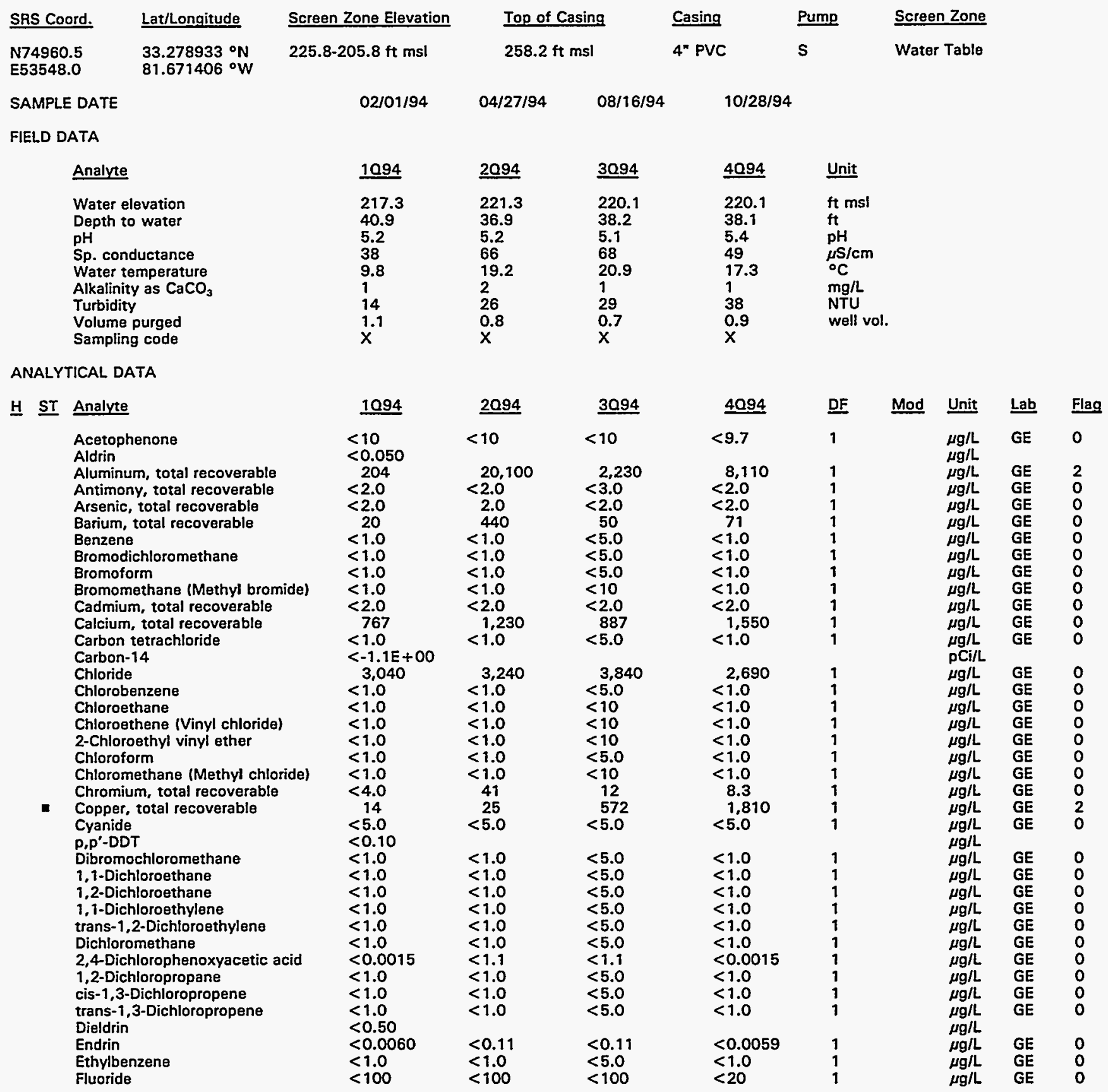

Note: Flagging, dilution factors, modifiers, and laboratories are for fourth quarter 1994 data only. See Appendix B for flagging criteria. - = exceeded holding time for fourth quarter 1994.

- = exceeded screening level or final PDWS for fourth quarter 1994. 


\section{ANALYTICAL DATA}

HE ST Analyte

Gross alpha

Heptachlor

Iron, total recoverable

- Lead, total recoverable

Lindane

Magnesium, total recoverable

Manganese, total recoverable

Mercury, total recoverable

Methoxychlor

Naphthalene

Nickel, total recoverable

Nitrate as nitrogen

Nitrate-nitrite as nitrogen

Nonvolatile beta

- $\mathrm{pH}$

Phenols

Potassium, total recoverable

Radium, total alpha-emitting

Radium-226

Radium-228

Selenium, total recoverable

Silica, total recoverable

Silver, total recoverable

Sodium, total recoverable

Specific conductance

Sulfate

1,1,2,2-Tetrachloroethane

Tetrachloroethylene

Tin, total recoverable

Toluene

Total dissolved solids

Total organic carbon

Total organic halogens

Total phosphates (as P)

Toxaphene

2,4,5-TP (Silvex)

Tributyl phosphate

1,1,1-Trichloroethane

1,1,2-Trichloroethane

Trichloroethylene

Trichlorofluoromethane

- Tritium

Uranium-233/234

Uranium-235

Uranium-238

Vanadium, total recoverable

Xylenes

Zinc, total recoverable

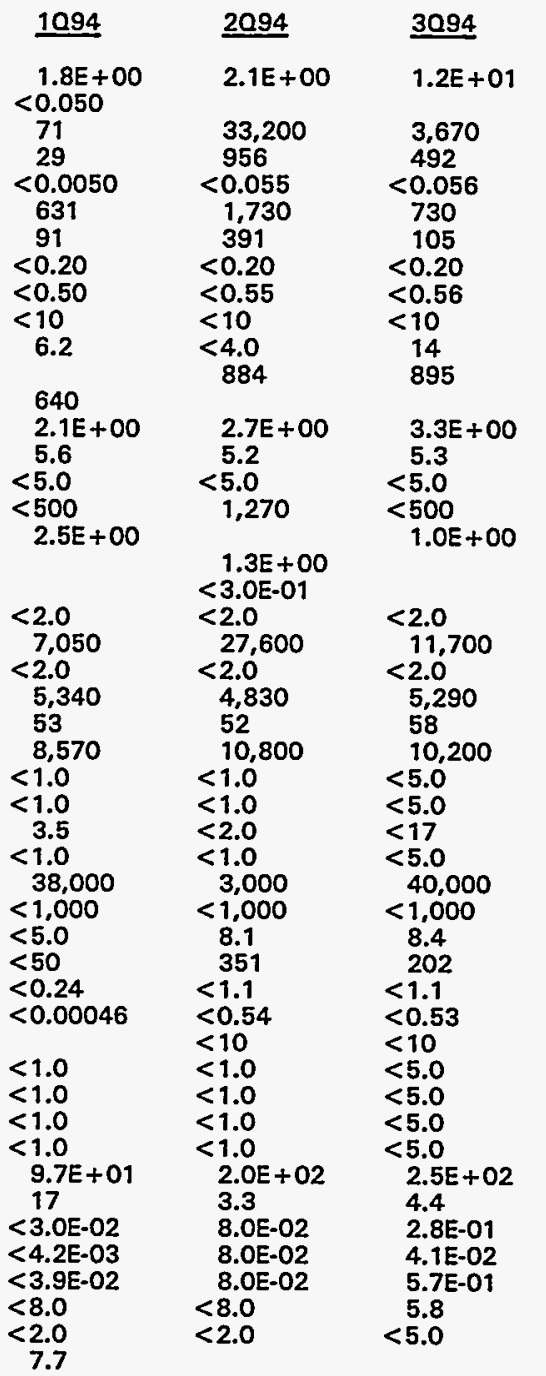

$\frac{4094}{1.5 \mathrm{E}+00}$
668
1.740
$<0.0050$
1,100
600
$<0.20$
$<0.50$
$<9.7$
45
813
$1.8 \mathrm{E}+00$
5.2
$<5.0$
$<500$

$<2.0$

12,400

$<2.0$

5,330

55

8,440

$<1.0$

$<1.0$

$<2.0$

40,000

1,150

32

$<50$

$<0.24$

$<0.00045$

$<9.7$

$<1.0$

$<1.0$

$<1.0$

$<1.0$

$2.6 \mathrm{E}+02$

99

$-8.6 \mathrm{E}-03$

$0.0 E+00$

$9.1 \mathrm{E}-02$

11

$<2.0$

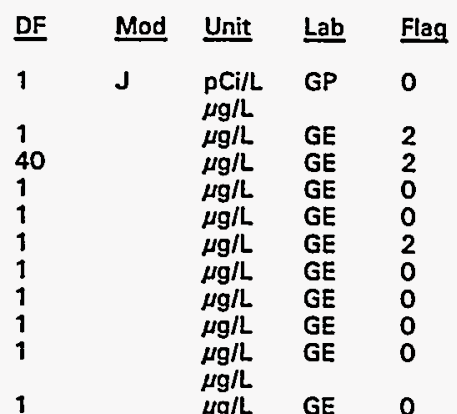

$\mu \mathrm{g} / \mathrm{L} \quad \mathrm{GE} \quad 0$

$\begin{array}{lll}J & \mathrm{pCi} / \mathrm{L} & \mathrm{GP} \\ \mathrm{J} & \mathrm{pH} & \mathrm{GE}\end{array}$

$\mu g / L \quad G E$

$\mu g / L \quad G E$

pCi/L

$\mathrm{pC} i / \mathrm{L}$

$\begin{array}{lll}\mu g / L & \text { GE } & 0 \\ \mu g / L & \text { GE } & 0\end{array}$

H $G$

IS/cm GE

$\mu \mathrm{g} / \mathrm{L}$ GE

$\mu g / L$

$\mu \mathrm{g} / \mathrm{L}$ GE

$\mu \mathrm{g} / \mathrm{L} \quad \mathrm{GE}$

$\mu \mathrm{gg} / \mathrm{L} \quad \mathrm{GE}$

$\begin{array}{lll}j & \mu g / L & G E \\ J & \mu g / L & G E \\ J & g / L & G E\end{array}$

$\mu g / L \quad G E$

J $\mu \rho g / L \quad G E$

$\underset{\mu \mathrm{g} / \mathrm{L}}{\mu \mathrm{G}} \quad \mathrm{GE}$

$\mu \mathrm{g} / \mathrm{L} \quad \mathrm{GE}$

$\mu g / L \quad G E$

$\mu \mathrm{g} / \mathrm{L} \quad \mathrm{GE}$

$\mu \mathrm{g} / \mathrm{L} \quad \mathrm{GE}$

PCi/mL GP

NTU GE

$\mathrm{pCi} / \mathrm{L}$

$\mathrm{pCi} / \mathrm{L}$

pCi/L GP

$\mu \mathrm{g} / \mathrm{L} \quad \mathrm{GE}$

$\mu g / L \quad G E$

$\mu \mathrm{g} / \mathrm{L}$

Note: Flagging, dilution factors, modifiers, and laboratories are for fourth quarter 1994 data only. See Appendix B for flagging criteria. - = exceeded holding time for fourth quarter 1994.

- = exceeded screening level or final PDWS for fourth quarter 1994. 
WELL FSS 4D

\begin{tabular}{|c|c|c|c|c|c|c|}
\hline SRS Coord. & Lat/Longitude & Screen Zone Elevation & Top of Casing & Casing & Pump & Screen Zone \\
\hline $\begin{array}{l}\text { N75537.8 } \\
\text { E52876.1 }\end{array}$ & $\begin{array}{l}33.279114{ }^{\circ} \mathrm{N} \\
81.674297^{\circ} \mathrm{W}\end{array}$ & $222.6-202.6 \mathrm{ft} \mathrm{msl}$ & $291.8 \mathrm{ft} \mathrm{msl}$ & 4" PVC & $s$ & Water Table \\
\hline Al & & $02 / 01 / 94$ & )4/27/94 & & & \\
\hline
\end{tabular}

FIELD DATA

Analyte

Water elevation

Depth to water

$\mathrm{pH}$

Sp. conductance

Water temperature

Alkalinity as $\mathrm{CaCO}_{3}$

Turbidity

Volume purged

Sampling code

ANALYTICAL DATA

H ST Analyte

Acetophenone

Aldrin

Aluminum, total recoverable

Antimony, total recoverable

Arsenic, total recoverable

Barium, total recoverable

Benzene

Bromodichloromethane

Bromoform

Bromomethane (Methyl bromide)

Cadmium, total recoverable

Calcium, total recoverable

Carbon tetrachloride

Carbon-14

Chloride

Chlorobenzene

Chloroethane

Chloroethene (Vinyl chloride)

2-Chloroethyl vinyl ethe

Chloroform

Chloromethane (Methyl chloride)

Chromium, total recoverable

Copper, total recoverable

Cyanide

p,p'-DDT

Dibromochloromethane

1,1-Dichloroethane

1,2-Dichloroethane

1,1-Dichloroethylene

trans-1,2-Dichloroethylene

Dichloromethane

2,4-Dichlorophenoxyacetic acid

1,2-Dichloropropane

cis-1,3-Dichloropropene

trans-1,3-Dichloropropene

Dieldrin

Endrin

Ethylbenzene

Fluoride

\begin{tabular}{l}
1094 \\
\hline 220.0 \\
71.8 \\
5.1 \\
46 \\
13.5 \\
1 \\
31 \\
1.0 \\
$x$
\end{tabular}

2094
220.0
71.8
5.0
54
20.5
0
31
1.1
$x$

30.94
219.2
72.7
5.0
50
20.7
0
34
1.5
$x$

1094

$<10$

$<0.049$

1.010

$<2.0$

$<2.0$

14
$<1.0$

$<1.0$

$<1.0$

$<1.0$

$<2.0$

1,580

$<1.0$

$<1.9 E+00$

4,060

$<1.0$

$<1.0$

$<1.0$

$<1.0$

$<1.0$

$<1.0$

$<4.0$

5.6

$<5.0$

$<0.098$

$<1.0$

$<1.0$

$<1.0$

$<1.0$

$<1.0$

$<1.0$

$<0.0016$

$<1.0$

$<1.0$

$<1.0$

$<0.49$

$<0.0059$

$<i .0$

$<100$
$<10$

418
$<2.0$

$<2.0$

11

$<1.0$

$<1.0$

$<1.0$

$<1.0$

$<2.0$

1,140

$<1.0$

4,190

$<1.0$
$<1.0$

$<1.0$

$<1.0$

$<1.0$

$<1.0$

4.1

28
$<5.0$

$<1.0$

$<1.0$

$<1.0$

$<1.0$

$<1.0$

$<1.0$

$<1.1$

$<1.0$

$<1.0$

$<1.0$

$<0.11$

$<1.0$

$<100$

3094
$<10$

481
$<3.0$
$<2.0$
11
$<5.0$
$<5.0$
$<5.0$
$<10$
$<2.0$
914
$<5.0$

4.670
$<5.0$
$<10$
$<10$
$<10$
$<5.0$
3.1
4.0
$<5.8$
$<5.0$
$<5.0$
$<5.0$
$<5.0$
$<5.0$
$<5.0$
$<5.0$
$<1.0$
$<5.0$
$<5.0$
$<5.0$
$<0.11$
$<5.0$
$<100$

$\begin{aligned} & 4094 \\ &<10 \\ & 147 \\ &<2.0 \\ &<2.0 \\ & 8.3 \\ &<1.0 \\ &<1.0 \\ &<1.0 \\ &<1.0 \\ &<2.0 \\ &<1.200 \\ &<1.0 \\ & \\ & 3,200 \\ &<1.0 \\ &<1.0 \\ &<1.0 \\ &<1.0 \\ &<1.0 \\ &<1.0 \\ &<4.0 \\ & 4.8 \\ &<5.0 \\ &<1.0 \\ &<1.0 \\ &<1.0 \\ &<1.0 \\ &<1.0 \\ &<1.0 \\ &<0.0015 \\ &<1.0 \\ &<1.0 \\ &<1.0 \\ &<0.0059 \\ &<1.0 \\ &<20 \\ &\end{aligned}$

\begin{tabular}{|c|c|c|c|c|}
\hline DF & Mad & Unit & $\underline{\text { Lab }}$ & Flas \\
\hline 1 & \multirow{33}{*}{$\mathbf{J}$} & $\begin{array}{c}\mu \mathrm{g} / \mathrm{L} \\
\mu \mathrm{g} / \mathrm{L}\end{array}$ & GE & 0 \\
\hline 1 & & $\mu \mathrm{g} / \mathrm{L}$ & GE & 2 \\
\hline 1 & & $\mu \mathrm{g} / \mathrm{L}$ & GE & 0 \\
\hline 1 & & $\mu \mathrm{g} / \mathrm{L}$ & GE & 0 \\
\hline 1 & & $\mu \mathrm{g} / \mathrm{L}$ & GE & 0 \\
\hline 1 & & $\mu g / L$ & GE & 0 \\
\hline 1 & & $\mu g / L$ & GE & 0 \\
\hline 1 & & $\mu \mathrm{g} / \mathrm{L}$ & GE & 0 \\
\hline 1 & & $\mu \mathrm{g} / \mathrm{L}$ & GE & 0 \\
\hline 1 & & $\mu g / L$ & GE & 0 \\
\hline 1 & & $\mu g / L$ & GE & 0 \\
\hline 1 & & $\begin{array}{l}\mu \mathrm{g} / \mathrm{L} \\
\mathrm{pCi} / \mathrm{L}\end{array}$ & GE & 0 \\
\hline 1 & & $\mu \mathrm{g} / \mathrm{L}$ & GE & 0 \\
\hline 1 & & $\mu \mathrm{g} / \mathrm{L}$ & GE & 0 \\
\hline $\begin{array}{l}1 \\
1\end{array}$ & & $\mu \mathrm{g} / \mathrm{L}$ & GE & 0 \\
\hline 1 & & $\mu g / L$ & GE & $\begin{array}{l}0 \\
0\end{array}$ \\
\hline 1 & & $\mu \mathrm{g} / \mathrm{h}$ & GE & 0 \\
\hline 1 & & $\mu \mathrm{g} / \mathrm{L}$ & $\overline{G E}$ & 0 \\
\hline 1 & & $\mu \mathrm{g} / \mathrm{L}$ & GE & 0 \\
\hline 1 & & $\mu \mathrm{g} / \mathrm{L}$ & GE & 0 \\
\hline 1 & & $\begin{array}{l}\mu \mathrm{g} / \mathrm{L} \\
\mu \mathrm{g} / \mathrm{L}\end{array}$ & GE & 0 \\
\hline 1 & & $\mu \mathrm{g} / \mathrm{L}$ & GE & 0 \\
\hline 1 & & $\mu \mathrm{g} / \mathrm{L}$ & $\begin{array}{l}\mathrm{GE} \\
\mathrm{GE}\end{array}$ & $\begin{array}{l}0 \\
0\end{array}$ \\
\hline 1 & & $\mu \mathrm{g} / \mathrm{L}$ & $\mathrm{GE}$ & 0 \\
\hline 1 & & $\mu \mathrm{g} / \mathrm{L}$ & GE & 0 \\
\hline 1 & & $\mu g / L$ & GE & 0 \\
\hline 1 & & $\mu g / L$ & GE & 0 \\
\hline 1 & & $\mu \mathrm{g} / \mathrm{L}$ & GE & 0 \\
\hline 1 & & $\mu g / L$ & GE & 0 \\
\hline & & $\begin{array}{l}\mu \mathrm{g} / \mathrm{L} \\
\mu \mathrm{g} / \mathrm{L}\end{array}$ & GE & 0 \\
\hline 1 & & $\mu \mathrm{g} / \mathrm{L}$ & GE & 0 \\
\hline 1 & & $\mu g / L$ & GE & 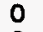 \\
\hline & & $\mu g / L$ & GE & 0 \\
\hline
\end{tabular}

Unit

$\mathrm{ft}$ msl

t

$\mu \mathrm{S} / \mathrm{cm}$



NTU

well vol. 
H ST Analyte

Gross alpha

Heptachlor

Iron, total recoverable

Lead, total recoverable

Lindane

Magnesium, total recoverable

Manganese, total recoverable

Mercury, total recoverable

Methoxychlor

Naphthalene

Nickel, total recoverable

Nitrate as nitrogen

Nitrate-nitrite as nitrogen

Nonvolatile beta

pH

Phenols

Potassium, total recoverable

Radium, total alpha-emitting

Radium-226

Radium-228

Selenium, total recoverable

Silica, total recoverable

Silver, total recoverable

Sodium, total recoverable

Specific conductance

Sulfate

1,1,2,2-Tetrachloroethane

Tetrachloroethylene

Tin, total recoverable

Toluene

Total dissolved solids

Total organic carbon

Total organic halogens

Total phosphates (as P)

Toxaphene

2,4,5-TP (Silvex)

Tributyl phosphate

1,1,1-Trichloroethane

1,1,2-Trichloroethane

Trichloroethylene

Trichlorofluoromethane

Tritium

Turbidity

Uranium-233/234

Uranium-235

Uranium-238

Vanadium, total recoverable

Xylenes

Zinc, total recoverable

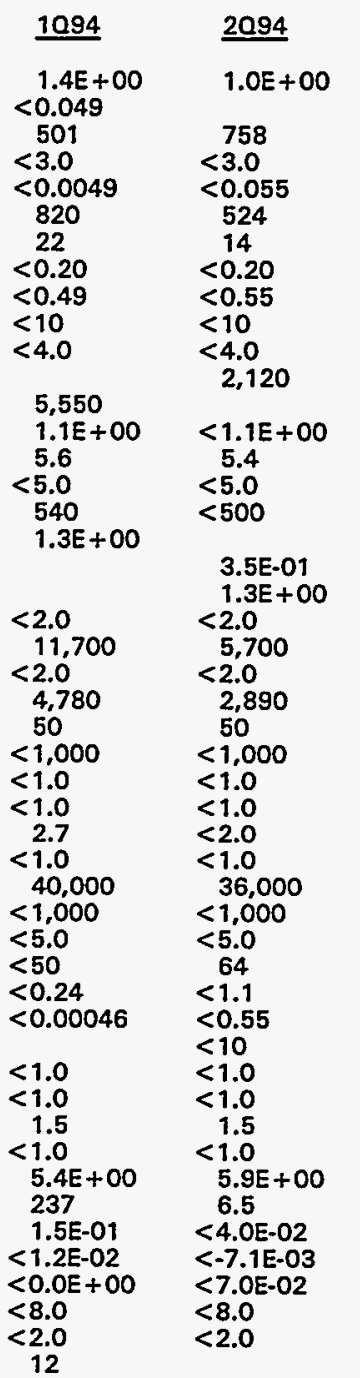

$\frac{3094}{1.0 E+00}$
745
3.4
$<0.054$
588
17
$<0.20$
$<0.54$
$<10$

$\frac{4094}{3.2 E+00} \quad \frac{\mathrm{DF}}{1} \quad \frac{\mathrm{Mod}}{\mathrm{J}}$

Unit Lab Flag

$<3.0$

$<0.0050$

755

$<0.20$

$<0.50$

$<10$

2,180

1.1E+00

6.0

$<500$

5.0E-01

$<2.0$

7,040

$<2.0$

45

$<1,000$

$<5.0$

$<5.0$

$<17$

$<5.0$

28,000

$<1,000$

$<5.0$

3,190

$<1.1$

$<0.52$

$<10$

$<5.0$

$<5.0$

1.2

$<5.0$

$6.6 E+00$

19

3.6E-01

1. $3 E+00$

$<3.0$

$<5.0$

$<10$

$<4.0$

2,960
$3.7 E+00$
5.2

$<500$

$<2.0$

9,230

$<2.0$

4,280

42
$<1,000$

$<1.0$

$<1.0$

$<2.0$

$<1.0$

36,000

1,860

5.0

$<50$

$<0.24$

$<0.00044$

$<10$

$<1.0$

$<1.0$

1.5
$<1.0$

5. $3 E+\infty 0$

21

4.4E-01

O. $0 E+00$

5.1E-01

$<8.0$

$<2.0$

$\underset{\mu \mathrm{gC} / \mathrm{L}}{\mathrm{pC}} \quad \mathrm{GP} \quad 0$

$\mu g / L$ GE 0

$\mu g / L \quad G E$ O

$\mu g / L \quad$ GE $\quad 0$

$\mu \mathrm{g} / \mathrm{L}$ GE 0

$\mu g / L \quad G E$

$\mu g / L$

$\mu g / L$

$\mu \mathrm{g} / \mathrm{L}$

$\mu g / L$

$\mu g / L$

$\mu \mathrm{g} / \mathrm{L}$ GE 0

$\mathrm{pCi} / \mathrm{L}$

pCi/L

pCi/L

$\mu g / L \quad G E$

$\mu \mathrm{g} / \mathrm{L} \quad \mathrm{GE}$

$\mu \mathrm{g} / \mathrm{L} \quad \mathrm{GE}$

$\mu \mathrm{S} / \mathrm{cm}$ GE

$\mu \mathrm{H} / \mathrm{cm} \quad \mathrm{GE}$

$\mu g / L \quad G E$

$\mu g / L \quad G E$

$\mu \mathrm{g} / \mathrm{L}$

$\mathrm{J} \quad \mu \mathrm{g} / \mathrm{g}$

J

$\mu g / L \quad G E$

$\mu \mathrm{g} / \mathrm{L} \quad \mathrm{GE}$

$\mu g / L$ GE

$\mu \mathrm{g} / \mathrm{L} \quad \mathrm{GE}$

$\mu \mathrm{g} / \mathrm{L}$

J

$\mu g /$

$\mu \mathrm{g} / \mathrm{GE}$

$\begin{array}{lll}J & \text { NTU } & \text { GE } \\ \text { UI } & \text { pCi/L } & \text { GP }\end{array}$

UI $\mathrm{pCi} / \mathrm{L}$ GP

PCi/L GP

$\mu g / L \quad$ GE

$\mu \mathrm{g} / \mathrm{L}$

o

Note: Flagging, dilution factors, modifiers, and laboratories are for fourth quarter 1994 data only. See Appendix B for flagging criteria. - = exceeded holding time for fourth quarter 1994.

- = exceeded screening level or final PDWS for fourth quarter 1994. 
WELL HMD 1D

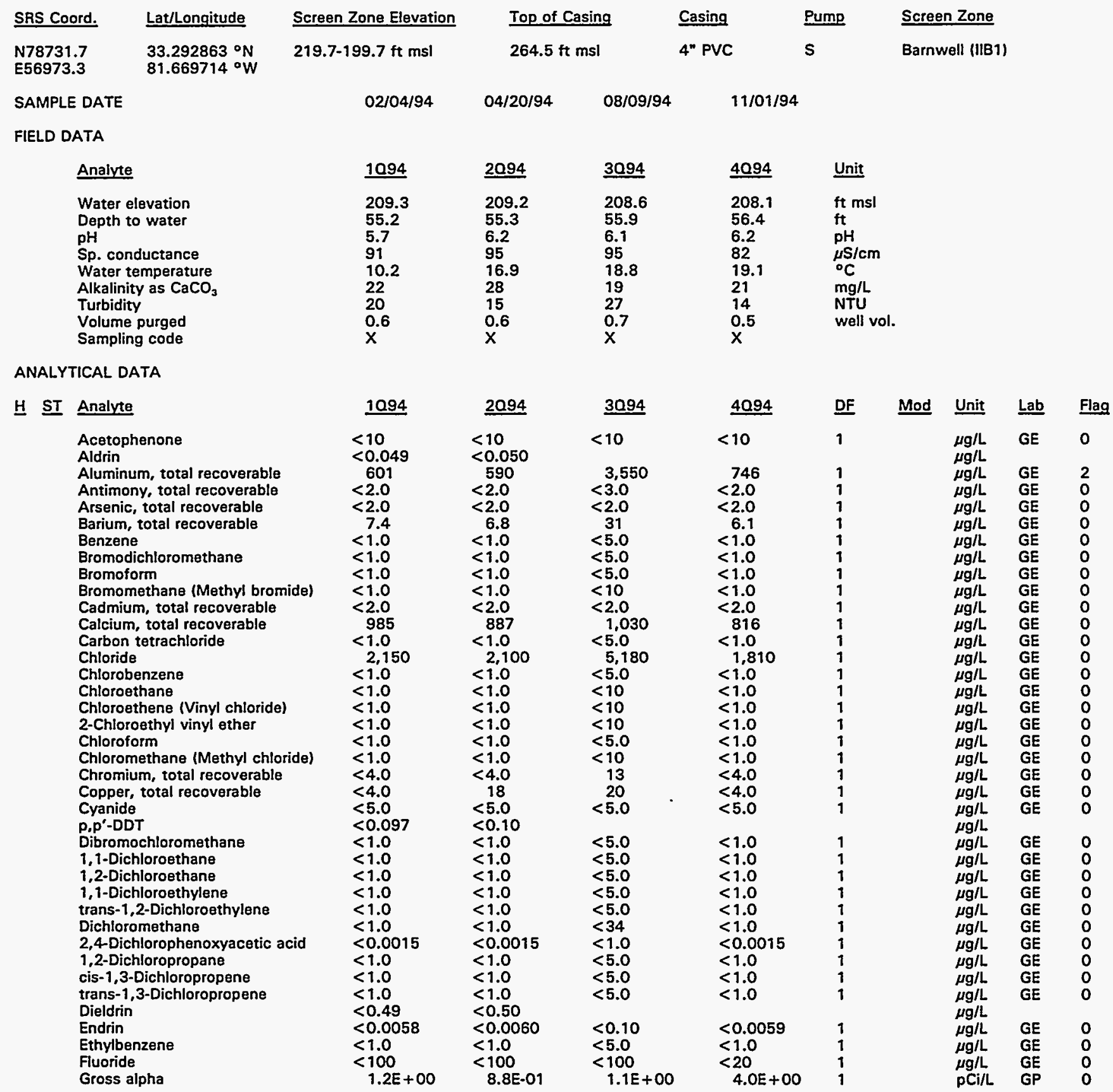

Note: Flagging, dilution factors, modifiers, and laboratories are for fourth quarter 1994 data only. See Appendix B for flagging criteria. - = exceeded holding time for fourth quarter 1994.

m = exceeded screening level or final PDWS for fourth quarter 1994. 


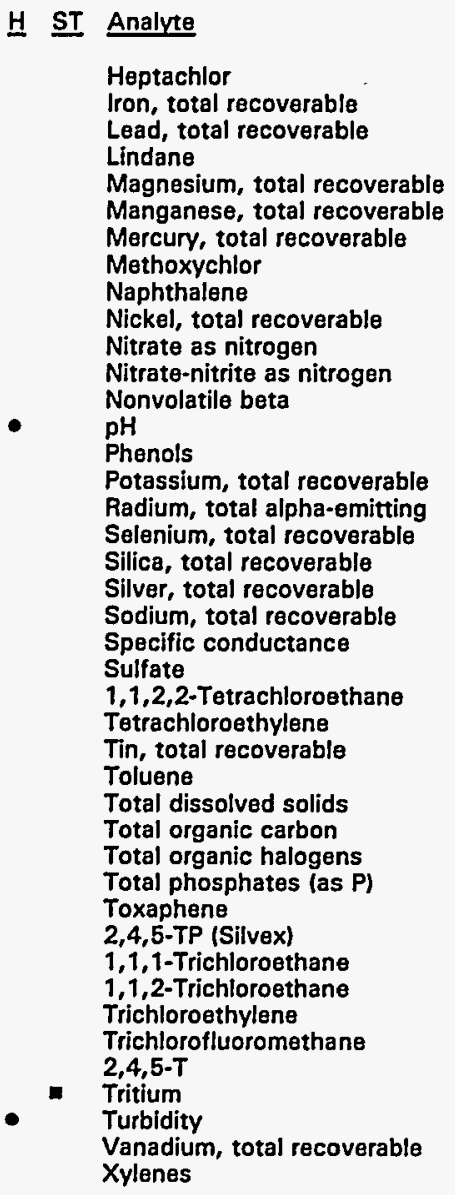

$\underline{3094}$
11,300
63
60.052
294
73
70.20
$<0.52$
$<10$
13
102

4.6E-01

6.2

$<5.0$

$<500$

$<2.0$

7,010

2.3

11,900

77

10,400

$<5.0$

$<5.0$

$<17$

$<5.0$

378,000

$<1,000$

5.7

$<50$

$<1.0$

$<0.52$

$<5.0$

$<5.0$

$<5.0$

$<5.0$

$<1.0$

$<0.00045$

$3.7 E+01 \quad 3.3 E+01$

16

$<8.0$

8.3

10
$<5.0$

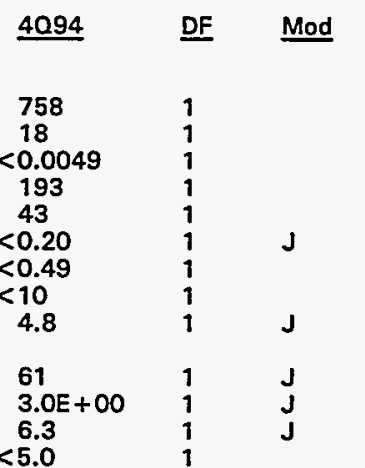

Unit Lab Flag

$\mu \mathrm{g} / \mathrm{L}$

$\mu \mathrm{g} / \mathrm{L} \quad \mathrm{GE}$

$\mu g / L \quad G E$

$\mu g / L \quad G E$

$\mu \mathrm{g} / \mathrm{L}$

$\begin{array}{ll}\mu \mathrm{g} / \mathrm{L} & \mathrm{GE} \\ \mu \mathrm{g} / \mathrm{L} & \mathrm{GE}\end{array}$

$\mu \mathrm{g} / \mathrm{L} \quad \mathrm{GE}$

$\mu \mathrm{g} / \mathrm{L} \quad \mathrm{GE}$

$\mu g / L$

$\mu \mathrm{g} / \mathrm{L} \quad \mathrm{GE}$

$\mathrm{PCi} \quad \mathrm{GP}$

pH

$\mu \mathrm{g} / \mathrm{L} \quad \mathrm{GE}$

$\mu \mathrm{g} / \mathrm{L} \quad \mathrm{GE}$

PCi/L

$\mu \mathrm{g} / \mathrm{L}$

$\mu g / L$

$<2.0$

4,700

15,000

81

9,130

$<1.0$

$<1.0$

$<2.0$

$<1.0$

50,000

1,030

$<5.0$

$<50$

$<0.24$

$<0.00044$

$<1.0$

$<1.0$

$<1.0$

$<1.0$

4. $4 \mathrm{E}+01$

$\begin{aligned} & 4.4 \mathrm{E}+ \\ & 31 \\ &<8.0\end{aligned}$
$<8.0$

$3 E+01$
3
0

$\mu g$

$\mu \mathrm{g} / \mathrm{L}$ GE $\quad 0$

$\mu \mathrm{g} / \mathrm{L}$ GE $\quad 0$

$\mu \mathrm{g} / \mathrm{L}$ GE $\quad 0$

$\mu \mathrm{g} / \mathrm{L}$ GE 0

$\mu g / L \quad G E$

$\mu \mathrm{g} / \mathrm{L} \quad \mathrm{GE}$

$\begin{array}{ll}\mu g / L & \mathrm{GE} \\ \mu \mathrm{g} / \mathrm{L} & \mathrm{GE}\end{array}$

$\mu \mathrm{g} / \mathrm{L} \quad \mathrm{GE}$

$\mu \mathrm{g} / \mathrm{L}$

PCi/mL GP

$\mathrm{pCi} / \mathrm{mL}$ GP

$\begin{array}{ll}N T U & \mathrm{GE} \\ \mu \mathrm{g} / \mathrm{L} & \mathrm{GE}\end{array}$

2
0
0
0
1
0
0
0
0
0
0
0
0
0
0
0
0
0
0
0
0
0
0
0
0
0
0
0
0
0
0
0
0
0
0
0
0
2
0
0
0
0

.

$<2.0-1$

Note: Flagging, dilution factors, modifiers, and laboratories are for fourth quarter 1994 data only. See Appendix B for flagging criteria. - = exceeded holding time for fourth quarter 1994.

- = exceeded screening level or final PDWS for fourth quarter 1994. 
WELL HMD 2D

\begin{tabular}{|c|c|c|c|c|c|c|c|}
\hline SRS Coord. & $\underline{\text { Lat/Longitude }}$ & Screen Zone Elevation & \multicolumn{2}{|l|}{ Top of Casing } & Casing & Pump & \multirow{3}{*}{$\begin{array}{l}\text { Screen Zone } \\
\text { Bamwell (IIBT) }\end{array}$} \\
\hline $\begin{array}{l}\text { N79665.8 } \\
\text { E57269.7 }\end{array}$ & $\begin{array}{l}33.295413^{\circ} \mathrm{N} \\
81.670748{ }^{\circ} \mathrm{W}\end{array}$ & $210.8-190.8 \mathrm{ft} \mathrm{msl}$ & $261.1 \mathrm{ft} \mathrm{msl}$ & & 4" PVC & $\mathbf{s}$ & \\
\hline \multicolumn{2}{|c|}{ SAMPLE DATE } & $02 / 04 / 94$ & $04 / 15 / 94$ & $88 / 08 / 94$ & $10 / 31 / 94$ & & \\
\hline \multicolumn{8}{|l|}{ FIELD DATA } \\
\hline \multicolumn{2}{|c|}{ Analyte } & 1094 & $\underline{2094}$ & 3094 & 4094 & Unit & \\
\hline \multicolumn{2}{|c|}{$\begin{array}{l}\text { Water elevation } \\
\text { Depth to water } \\
\text { pH } \\
\text { Sp. conductance } \\
\text { Water temperature } \\
\text { Alkalinity as } \mathrm{CaCO}_{3} \\
\text { Turbidity } \\
\text { Volume purged } \\
\text { Sampling code }\end{array}$} & $\begin{array}{l}199.6 \\
61.5 \\
5.5 \\
71 \\
18.6 \\
10 \\
1 \\
21.0\end{array}$ & $\begin{array}{l}199.8 \\
61.3 \\
5.8 \\
72 \\
19.8 \\
9 \\
1 \\
12.2\end{array}$ & $\begin{array}{l}199.8 \\
61.3 \\
6.8 \\
73 \\
19.6 \\
10 \\
1.3\end{array}$ & $\begin{array}{l}199.0 \\
62.1 \\
5.8 \\
68 \\
19.1 \\
8 \\
1 \\
13.9\end{array}$ & $\begin{array}{l}\mathrm{ft} \mathrm{msl} \\
\mathrm{ft} \\
\mathrm{pH} \\
\mu \mathrm{S} / \mathrm{cm} \\
{ }^{\circ} \mathrm{C} \\
\mathrm{mg} / \mathrm{L} \\
\mathrm{NTU} \\
\text { well vol. }\end{array}$ & \\
\hline
\end{tabular}

\section{ANALYTICAL DATA}

H ST Analyte

Acetophenone

Aldrin

Aluminum, total recoverable

Antimony, total recoverable

Arsenic, total recoverable

Barium, total recoverable

Benzene

Bromodichloromethane

Bromoform

Bromomethane (Methyl bromide)

Cadmium, total recoverable

Calcium, total recoverable

Carbon tetrachloride

Chloride

Chlorobenzene

Chloroethane

Chloroethene (Vinyl chloride)

2-Chloroethyl vinyl ether

Chloroform

Chloromethane (Methyl chloride)

Chromium, total recoverable

Copper, total recoverable

Cyanide

p.p'-DDT

Dibromochloromethane

1,1-Dichloroethane

1,2-Dichloroethane

1,1-Dichloroethylene

trans-1,2-Dichloroethylene

Dichloromethane

2,4-Dichlorophenoxyacetic acid

1,2-Dichloropropane

cis-1,3-Dichloropropene

trans-1,3-Dichloropropene

Dieldrin

Endrin

Ethylbenzene

Fluoride

Gross alpha

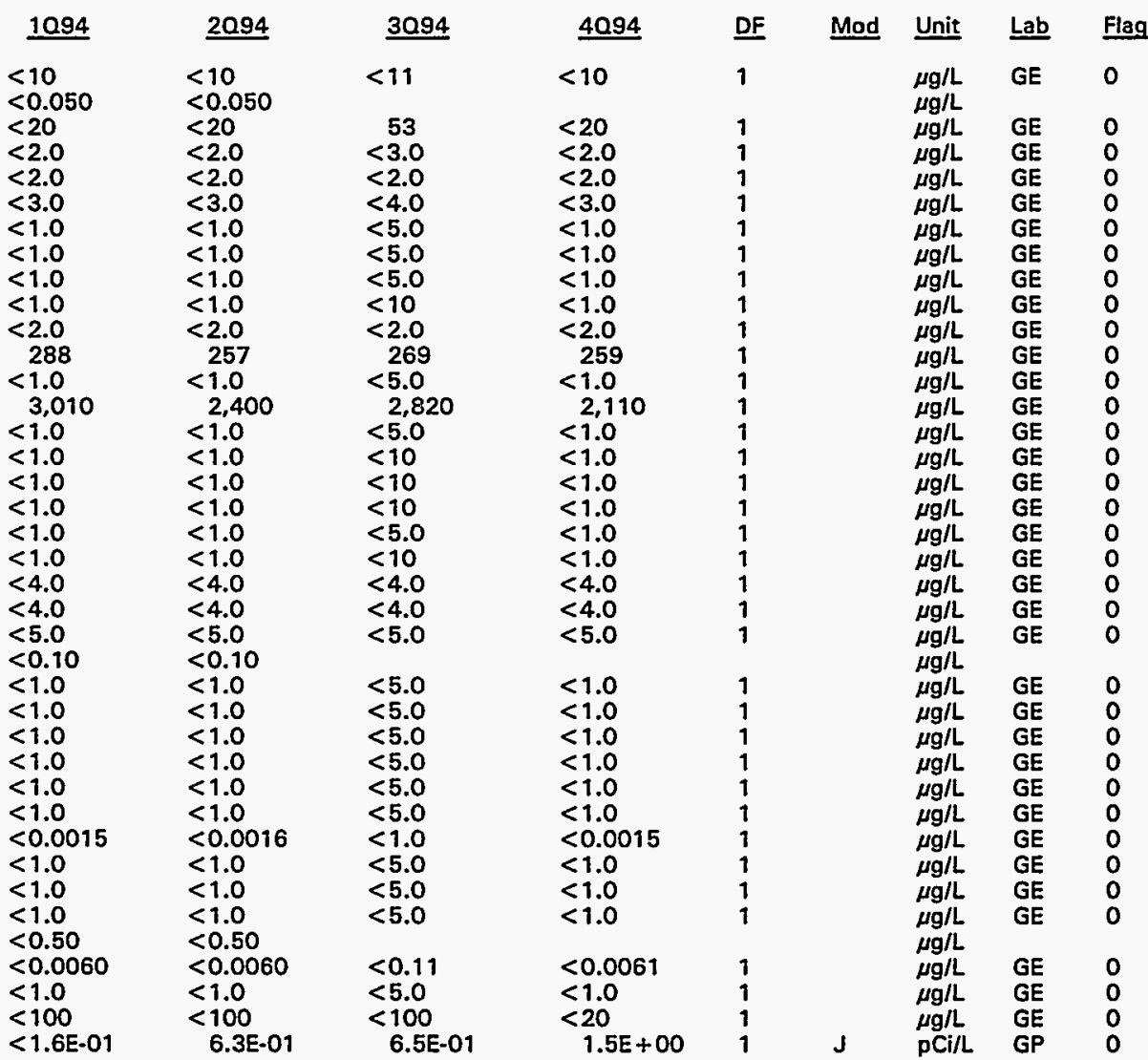

Note: Flagging, dilution factors, modifiers, and laboratories are for fourth quarter 1994 data only. See Appendix B for flagging criteria. - = exceeded holding time for fourth quarter 1994.

II = exceeded screening level or final PDWS for fourth quarter 1994. 


\section{ANALYTICAL DATA}

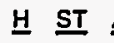

Analyte

Heptachlor

Iron, total recoverable

Lead, total recoverable

Lindane

Magnesium, total recoverable

Manganese, total recoverable

Mercury, total recoverable

Methoxychlor

Naphthalene

Nickel, total recoverable

Nitrate as nitrogen

Nitrate-nitrite as nitrogen

Nonvolatile beta

$\mathrm{pH}$

Phenols

Potassium, total recoverable

Radium, total alpha-emitting

Radium-226

Radium-228

Selenium, total recoverable

Silica, total recoverable

Silver, total recoverable

Sodium, total recoverable

Specific conductance

Sulfate

1,1,2,2-Tetrachloroethane

Tetrachloroethylene

Tin, total recoverable

Toluene

Total dissolved solids

Total organic carbon

Total organic halogens

Total phosphates las P

Toxaphene

2,4,5-TP (Silvex)

1,1,1-Trichloroethane

1,1,2-Trichloroethane

Trichloroethylene

Trichlorofluoromethane

2,4,5-T

Tritium

Turbidity

Vanadium, total recoverable

Xylenes
1094

$<0.050$

58

$<3.0$
$<0.0050$

83

3.8

$<0.20$

$<0.50$

$<10$

$<4.0$

299

280

$1.2 E+00$

7.5

$<5.0$

$<500$

1.1E+00

8.4E-01

7.OE-01

$<2.0$

5,940

$<2.0$

14,100

73

13,600

$<1.0$

$<1.0$

4.8

$<1.0$

55,000

$<1,000$

22
75

75
$<0.24$

$<0.00046$

$<1.0$

$<1.0$

$<1.0$

1.4

1.4E +01

0.36

$<3.0$

$<2.0$ $\underline{2094}$

$<0.050$

77

$<3.0$

$<0.0050$

77

3.9

$<0.20$

$<0.50$

$<10$

$<4.0$

190

6.0

6.

$<500$

$<0.0 E+00$

$<2.0$

5,140

$<2.0$

10,900

64

10,600

$<1.0$

$<1.0$

$<2.0$

$<1.0$

41,000

$<1,000$

12
$<50$

$<0.24$

$<0.00046$

$<1.0$

$<1.0$

$<1.0$

$<1.0$

$<0.00046$

$1.5 \mathrm{E}+0$

0.32

$<8.0$

$<2.0$

\section{$\underline{3094}$}

4094

20

$<3.0$

81

$<0.20$

$<0.56$

$<10$

$<4.0$

222

2.2E-01

6.0

$<5.0$

$<500$

$<2.0$

5,820

$<2.0$

13,400

60

9,960

$<5.0$

$<5.0$

$<17$

$<5.0$

48,000

$<1,000$

8.1

777
$<1.1$

$<0.52$

$<0.52$
$<5.0$

$<5.0$

$<5.0$

1.0

$1.2 E+01$

0.30

$<3.0$

$<5.0$
11

$<3.0$

80

2.8

$<0.20$

$<0.51$

$<10$

$<4.0$

296
$7.6 \mathrm{E}+00$

6.0
$<5.0$

$<500$

$<2.0$

5,980

$<2.0$

12,800

66

9,780

$<1.0$

$<1.0$

$<2.0$

$<1.0$

45,000

$<1,000$

$<5.0$

91

$<0.24$

$<0.00044$

$<1.0$

$<1.0$

$<1.0$

$<1.0$

$1.1 E \div 01$

0.66

$<8.0$

$<2.0$

$\begin{array}{ll}\text { DF } & \text { Mod } \\ 1 & \\ 1 & \\ 1 & \\ 1 & \\ 1 & \text { J } \\ 1 & \\ 1 & \\ 1 & \\ 1 & \\ 1 & \\ 1 & \\ 1 & \text { J }\end{array}$

Unit Lab Flag

$\mu \mathrm{g} / \mathrm{L} \quad \mathrm{GE} \quad \mathrm{O}$

$\begin{array}{lll}\mu \mathrm{g} / \mathrm{L} & \mathrm{GE} & 0 \\ \mu \mathrm{g} / \mathrm{L} & \mathrm{GE} & 0\end{array}$

$\mu \mathrm{g} / \mathrm{L} \quad \mathrm{GE}$

$\mu g / L \quad G E$

$\mu \mathrm{g} / \mathrm{L} \quad \mathrm{GE}$

$\mu g / L \quad G E$

$\mu g / L$ GE

$\mu \mathrm{g} / \mathrm{L} \quad \mathrm{GE}$

$\mu \mathrm{g} / \mathrm{L}$

$\mu g / L \quad G E$

pCill GP

$\begin{array}{lll}\mathrm{pH} & \mathrm{GE} & 0 \\ \mu \mathrm{g} / \mathrm{L} & \mathrm{GE} & 0\end{array}$

$\mu \mathrm{g} / \mathrm{L}$ GE 0

$\mathrm{PCi} / \mathrm{L}$

pCill

pCi/L

$\mu \mathrm{g} / \mathrm{L}$ GE 0

$\mu g / L$

$\mu g / L$

$\mu g / L$

$\mu \mathrm{S} / \mathrm{cm}$ GE

Ugl GE

$\mu g$

$\mu g / h$

$\mu g / L$

$\mu g / L$

$\mu g / L$

$\mu g / L$

$\mu \mathrm{g} /$

$\mu \mathrm{g} / \mathrm{L}$

$\mu g / L$

$\mu \mathrm{g}$

$\mu \mathrm{g}$

$\mu g / L$

pCi/mL GP 1

NTU GE

$\mu \mathrm{g} / \mathrm{L} \quad \mathrm{GE}$

1
0
0
0

Note: Flagging, dilution factors, modifiers, and laboratories are for fourth quarter 1994 data only. See Appendix B for flagging criteria. - = exceeded holding time for fourth quarter 1994.

- = exceeded screening level or final PDWS for fourth quarter 1994. 
WELL HMD 3D

\begin{tabular}{|c|c|c|c|c|c|c|}
\hline SRS Coord. & Lat/Longitude & Screen Zone Elevation & Top of Casing & Casing & Pump & Screen Zone \\
\hline $\begin{array}{l}\text { N79578.7 } \\
\text { E57745.2 }\end{array}$ & $\begin{array}{l}33.295996^{\circ} \mathrm{N} \\
81.669327{ }^{\circ} \mathrm{W}\end{array}$ & $207.7-187.7 \mathrm{ft} \mathrm{msl}$ & $259.5 \mathrm{ft} \mathrm{msl}$ & 4" PVC & s & ell (IIB1) \\
\hline SAMPL & & $02 / 04 / 94$ & $04 / 15 / 94$ & & & \\
\hline
\end{tabular}

FIELD DATA

Analyte
Water elevation
Depth to water
pH
Sp. conductance
Water temperature
Alkalinity as $\mathrm{CaCO}_{3}$
Turbidity
Volume purged
Sampling code

ANALYTICAL DATA
Acetophenone

Aldrin

Aluminum, total recoverable Antimony, total recoverable

Arsenic, total recoverable

Barium, total recoverable

Benzene

Bromodichloromethane

Bromoform

Cadmium, total recoverable

Calcium, total recoverable

Carbon tetrachloride

Chloride

Chlorobenzene

Chloroethane

Chloroethene (Vinyl chloride)

2-Chloroethyl vinyl ether

Chloroform

Chloromethane (Methyl chloride)

Chromium, total recoverable

Copper, total recoverable

Cyanide

p,p'-DDT

Dibromochloromethane

1,1-Dichloroethane

1,2-Dichloroethane

1,1-Dichloroethylene

trans-1,2-Dichloroethylene

Dichloromethane

2,4-Dichlorophenoxyacetic acid

1,2-Dichloropropane

cis-1,3-Dichloropropene

trans-1,3-Dichloropropen

Dieldrin

Endrin

Ethylbenzene

Fluoride

Gross alpha

$\begin{array}{lll}\underline{1094} & & \underline{2094} \\ 199.1 & & 199.2 \\ 60.4 & 60.3 \\ 5.1 & 5.0 \\ 61 & 67 \\ 18.8 & 14.0 \\ 0 & 0 \\ 3 & 2 \\ 12.7 & 10.6\end{array}$

$\underline{1094}$

$<10$

$<0.050$

38
$<2.0$

$<2.0$

9.4

$<1.0$

$<1.0$

$<1.0$

$<1.0$

$<2.0$

966
$<1.0$

2,700

$<1.0$

$<1.0$

$<1.0$

$<1.0$

$<1.0$

$<1.0$

$<4.0$

$<4.0$
6.6
$<5.0$

$<0.10$

$<1.0$

$<1.0$

$<1.0$

$<1.0$

$<1.0$

$<1.0$

$<0.0016$

$<1.0$

$<1.0$

$<1.0$

$<0.50$

$<0.0060$

$<1.0$

$<100$

$\quad 2094$
$\quad 10$
$<10$
$<0.051$
40
$<2.0$
$<2.0$
8.6
$<1.0$
$<1.0$
$<1.0$
$<1.0$
$<2.0$
940
$<1.0$
2,870
$<1.0$
$<1.0$
$<1.0$
$<1.0$
$<1.0$
$<1.0$
$<4.0$
6.8
$<5.0$
$<0.10$
$<1.0$
$<1.0$
$<1.0$
$<1.0$
$<1.0$
$<1.0$
$<0.0015$
$<1.0$
$<1.0$
$<1.0$
$<0.51$
$<0.0061$
$<1.0$
$<100$
$1.66+00$

$\begin{aligned} & 3094 \\ &<10 \\ & 87 \\ &<2.0 \\ &<2.0 \\ & 9.3 \\ &<1.0 \\ &<1.0 \\ &<1.0 \\ &<1.0 \\ &<2.0 \\ & 957 \\ &<1.0 \\ & 3.410 \\ &<1.0 \\ &<1.0 \\ &<1.0 \\ &<1.0 \\ &<1.0 \\ &<1.0 \\ &<4.0 \\ & 8.1 \\ &<5.0 \\ &<1.0 \\ &<1.0 \\ &<1.0 \\ &<1.0 \\ &<1.0 \\ &<1.0 \\ &<0.0015 \\ &<1.0 \\ &<1.0 \\ &<1.0 \\ &<0.11 \\ &<1.0 \\ &<20 \\ & 3.2 E+00\end{aligned}$

\begin{tabular}{l}
\hline 4094 \\
198.6 \\
60.9 \\
5.1 \\
55 \\
20.1 \\
0 \\
1 \\
7.4
\end{tabular}

Unit

$\mathrm{ft} \mathrm{msl}$

$\mathrm{ft}$

$\mathrm{pH}$ $\mu \mathrm{S} / \mathrm{cm}$

$\mathrm{mg} / \mathrm{L}$

NTU

well vol.

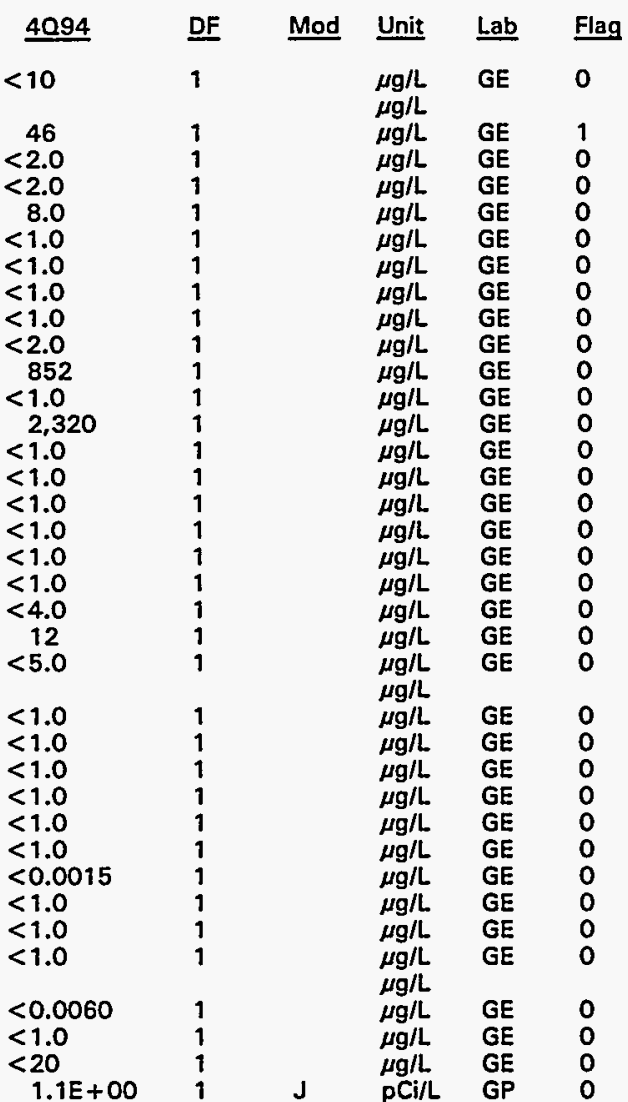

Note: Flagging, dilution factors, modifiers, and laboratories are for fourth quarter 1994 data only. See Appendix B for flagging criteria. - = exceeded holding time for fourth quarter 1994.

- = exceeded screening level or final PDWS for fourth quarter 1994. 


\section{ANALYTICAL DATA}

H ST Analyte

Heptachlor

Iron, total recoverable

Lead, total recoverable

Lindane

Magnesium, total recoverable

Manganese, total recoverable

Mercury, total recoverable

Methoxychlor

Naphthalene

Nickel, total recoverable

Nitrate as nitrogen

Nitrate-nitrite as nitrogen

Nonvolatile beta

$\mathrm{pH}$

Phenols

Potassium, total recoverable

Radium, total alpha-emitting

Selenium, total recoverable

Silica, total recoverable

Silver, total recoverable

Sodium, total recoverable

Specific conductance

Sulfate

1,1,2,2-Tetrachloroethane

Tetrachloroethylene

Tin, total recoverable

Toluene

Total dissolved solids

Total organic carbon

Total organic halogens

Total phosphates (as P)

Toxaphene

2,4,5-TP (Silvex)

1,1,1-Trichloroethane

1,1,2-Trichloroethan

Trichloroethylene

Trichlorofluoromethane

2,4,5-T

- Tritium

Turbidity

Vanadium, total recoverable

Xylenes

\begin{tabular}{|c|c|}
\hline 1094 & $\underline{2094}$ \\
\hline $\begin{aligned} &< 0.050 \\
& 26 \\
&<3.0 \\
&<0.0050 \\
& 619 \\
& 16 \\
&<0.20 \\
&<0.50 \\
&<10 \\
&<4.0\end{aligned}$ & $\begin{array}{c}<0.051 \\
46 \\
<3.0 \\
<0.0051 \\
587 \\
16 \\
<0.20 \\
<0.51 \\
<10 \\
<4.0\end{array}$ \\
\hline $\begin{aligned} & 380 \\
& 1.1 E+00 \\
& 5.1 \\
&<5.0 \\
& 632 \\
& 1.5 E+00 \\
&<2.0 \\
& 6.670 \\
&<2.0 \\
& 7,760 \\
& 59 \\
& 14,400 \\
&<1.0 \\
&<1.0 \\
& 2.3 \\
&<1.0 \\
& 38,000 \\
&<1.000 \\
& 29 \\
&<50 \\
&<0.24 \\
&<0.00046 \\
&<1.0 \\
&<1.0 \\
& 4.9 \\
& 4.9\end{aligned}$ & $\begin{aligned} & 420 \\
& 1.0 E+00 \\
& 5.8 \\
&<5.0 \\
& 516 \\
& 8.0 E-01 \\
&<2.0 \\
& 6,560 \\
&<2.0 \\
& 7,340 \\
& 57 \\
& 14,200 \\
&<1.0 \\
&<1.0 \\
&<2.0 \\
&<1.0 \\
& 46,000 \\
&<1,000 \\
&<5.0 \\
&<50 \\
&<0.25 \\
&<0.00046 \\
&<1.0 \\
&<1.0 \\
&<1.0 \\
& 3.3 \\
&<0.00046\end{aligned}$ \\
\hline $\begin{aligned} & 8.6 E+00 \\
< & 0.10 \\
< & 8.0 \\
< & 2.0\end{aligned}$ & $\begin{array}{l}<0.00046 \\
1.2 \mathrm{E}+01 \\
4.6 \\
<8.0 \\
<2.0\end{array}$ \\
\hline
\end{tabular}

$\underline{3094}$

4094

DF

Mod

$\begin{array}{rr}694 & 38 \\ <3.0 & <3.0\end{array}$

$<70$

19

$<0.20$

$<0.50$

$<10$

$<4.0$

477

$2.0 E+00$

$2.0 \mathrm{E}+00 \quad 9.5 \mathrm{E}-01$

5.4
$<5.0$

527

$<2.0$

6.800

$<2.0$

6,940

57

12,600

$<1.0$

$<1.0$

2.3

$<1.0$

48,000

$<1,000$

15

1,230

$<0.24$

$<0.00045$

$<1.0$

$<1.0$

$<1.0$
4.2

$1.9 \mathrm{E}+01$

4.5

$<3.0$
562
15
$<0.20$

$<0.50$

$<10$

$<4.0$

602
$9.5 \mathrm{E}-01$
5.3
$<5.0$

546

$<2.0$

6,650

$<2.0$

6,990

55

10,400

$<1.0$

$<1.0$

$<2.0$

$<1.0$

39,000

$<1,000$

$<5.0$

$<50$

$<0.24$

$<0.00044$

$<1.0$

$<1.0$

$<1.0$

4.0

$2.9 E+01$

1.9
$<8.0$
$<2.0$

d


WELL HMD 4D

\begin{tabular}{|c|c|c|c|c|c|c|}
\hline SRS Coord. & Lat/Longitude & Screen Zone Elevation & Top of Casing & Casing & Pump & Screen Zone \\
\hline $\begin{array}{l}\text { N79160.4 } \\
\text { E58188.5 }\end{array}$ & $\begin{array}{l}33.295794^{\circ} \mathrm{N} \\
81.667346^{\circ} \mathrm{W}\end{array}$ & $208.9-188.9 \mathrm{ft} \mathrm{msl}$ & $250.9 \mathrm{ft} \mathrm{msl}$ & 4" PVC & s & Barnwell (IIB1) \\
\hline SAMPLE DA & & $02 / 04 / 94$ & $14 / 20 / 94$ & & & \\
\hline
\end{tabular}

FIELD DATA

Analyte

Water elevation

Depth to water

$\mathrm{pH}$

Sp. conductance

Water temperature

Alkalinity as $\mathrm{CaCO}_{3}$

Turbidity

Volume purged

Sampling code

ANALYTICAL DATA

\section{H ST Analyte}

Acetophenone

Aldrin

Aluminum, total recoverable

Antimony, total recoverable

Arsenic, total recoverable

Barium, total recoverable

Benzene

Bromodichloromethane

Bromoform

Bromomethane (Methyl bromide)

Cadmium, total recoverable

Calcium, total recoverable

Carbon tetrachloride

Chloride

Chlorobenzene

Chloroethane

Chloroethene (Vinyl chloride)

2-Chloroethyl vinyl ether

Chloroform

Chloromethane (Methyl chloride)

Chromium, total recoverable

Copper, total recoverable

Cyanide

P.P'-DDT

Dibromochloromethane

1.1-Dichloroethane

1.2-Dichloroethane

1,1-Dichloroethylene

trans-1,2-Dichloroethylene

Dichloromethane

2,4-Dichlorophenoxyacetic acid

1,2-Dichloropropane

cis-1,3-Dichloropropene

trans-1,3-Dichloropropene

Dieldrin

Endrin

Ethylbenzene

Fluoride

Gross alpha

\begin{tabular}{ll} 
1094 & $\underline{2094}$ \\
\hline 192.5 & 199.8 \\
58.4 & 51.1 \\
5.4 & 5.7 \\
22 & 28 \\
17.6 & 18.1 \\
3 & 4 \\
25 & 20 \\
2.5 & 0.8 \\
$x$ & $x$
\end{tabular}

3094
199.7
51.3
5.9
34
25.2
5
9
0.7
$x$

\section{4}

$<10$

$<0.050$
747

$<2.0$

$<2.0$

22

$<1.0$

$<1.0$

$<1.0$

$<1.0$

$<2.0$

1,390

$<1.0$

2,180

$<1.0$

$<1.0$

$<1.0$

$<1.0$

$<1.0$

$<1.0$

$<4.0$

76

$<5.0$

$<0.10$

$<1.0$

$<1.0$

$<1.0$

$<1.0$

$<1.0$

$<1.0$

$<0.0015$

$<1.0$

$<1.0$

$<1.0$

$<0.50$

$<0.0060$

$<1.0$

$<100$

$2.5 E+00$

$\quad 2094$
$<10$
$<0.052$
152
$<2.0$
$<2.0$
20
$<1.0$
$<1.0$
$<1.0$
$<1.0$
$<2.0$
999
$<1.0$
2,350
$<1.0$
$<1.0$
$<1.0$
$<1.0$
$<1.0$
$<1.0$
$<4.0$
27
$<5.0$
$<0.10$
$<1.0$
$<1.0$
$<1.0$
$<1.0$
$<1.0$
$<1.0$
$<0.0015$
$<1.0$
$<1.0$
$<1.0$
$<0.52$
$<0.0062$
$<1.0$
$<100$
$1.6 E+00$

$\begin{aligned} & 30.94 \\ &<22 \\ & \\ & 248 \\ &<3.0 \\ &<2.0 \\ & 20 \\ &<5.0 \\ &<5.0 \\ &<5.0 \\ &<10 \\ &<2.0 \\ & 834 \\ &<5.0 \\ & 3.160 \\ &<5.0 \\ &<10 \\ &<10 \\ &<10 \\ &<5.0 \\ & 4.5 \\ &<4.0 \\ & 16 \\ &<5.0 \\ &<5.0 \\ &<5.0 \\ &<5.0 \\ &<5.0 \\ &<5.0 \\ &<5.0 \\ &<1.1 \\ &<5.0 \\ &<5.0 \\ &<5.0 \\ &<0.11 \\ &<5.0 \\ &<100 \\ & 2.9 E+00\end{aligned}$

$\begin{aligned} & 4094 \\ &< \\ & 246 \\ & 246 \\ &<2.0 \\ &<2.0 \\ & 25 \\ &<1.0 \\ &<1.0 \\ &<1.0 \\ &<1.0 \\ &<2.0 \\ & 984 \\ &<1.0 \\ &<1.760 \\ &<1.0 \\ &<1.0 \\ &<1.0 \\ &<1.0 \\ &<1.0 \\ &<1.0 \\ &<4.0 \\ & 25 \\ &<5.0 \\ &<1.0 \\ &<1.0 \\ &<1.0 \\ &<1.0 \\ &<1.0 \\ &<1.0 \\ &<0.0015 \\ &<1.0 \\ &<1.0 \\ &<1.0 \\ &<0.0059 \\ &<1.0 \\ &<20 \\ &<1.96+00 \\ &\end{aligned}$

\begin{tabular}{|c|c|c|c|}
\hline DF & Mod & Unit & $\underline{\text { Lab }}$ \\
\hline 1 & & ${ }_{\mu \mathrm{g} / \mathrm{L}}$ & GE \\
\hline 1 & & $\mu g / L$ & GE \\
\hline$i$ & & $\mu g / L$ & $\mathrm{GE}$ \\
\hline$i$ & & $\mu g / L$ & $\overrightarrow{\mathrm{GE}}$ \\
\hline 1 & & $\mu g / L$ & GE \\
\hline 1 & & $\mu \mathrm{g} / \mathrm{L}$ & $\overrightarrow{G E}$ \\
\hline 1 & & $\mu \mathrm{g} / \mathrm{L}$ & $\mathrm{GE}$ \\
\hline$i$ & & $\mu \mathrm{g} / \mathrm{L}$ & $\overline{\mathrm{GE}}$ \\
\hline 1 & & $\mu g / L$ & $\mathrm{GE}$ \\
\hline 1 & & $\mu \mathrm{g} / \mathrm{L}$ & GE \\
\hline 1 & & $\mu \mathrm{g} / \mathrm{L}$ & $\mathrm{GE}$ \\
\hline 1 & & $\mu \mathrm{g} / \mathrm{L}$ & $\mathrm{GE}$ \\
\hline 1 & & $\mu \mathrm{g} / \mathrm{L}$ & $\mathrm{GE}$ \\
\hline 1 & & $\mu \mathrm{g} / \mathrm{L}$ & GE \\
\hline 1 & & $\mu g / L$ & GE \\
\hline 1 & & $\mu g / L$ & $\mathrm{GE}$ \\
\hline 1 & & $\mu \mathrm{g} / \mathrm{L}$ & $\overline{G E}$ \\
\hline 1 & & $\mu \mathrm{g} / \mathrm{L}$ & GE \\
\hline 1 & & $\mu g / L$ & GE \\
\hline 1 & & $\mu \mathrm{g} / \mathrm{L}$ & GE \\
\hline 1 & & $\mu \mathrm{g} / \mathrm{L}$ & $G E$ \\
\hline 1 & & $\mu \mathrm{g} / \mathrm{L}$ & GE \\
\hline 1 & & $\mu \mathrm{g} / \mathrm{L}$ & GE \\
\hline 1 & & $\mu \mathrm{g} / \mathrm{L}$ & $\mathrm{GE}$ \\
\hline 1 & & $\mu \mathrm{g} / \mathrm{L}$ & GE \\
\hline 1 & & $\mu \mathrm{g} / \mathrm{L}$ & GE \\
\hline 1 & & $\mu g / L$ & GE \\
\hline 1 & & $\mu \mathrm{g} / \mathrm{L}$ & GE \\
\hline 1 & & $\mu \mathrm{g} / \mathrm{L}$ & $\mathrm{GE}$ \\
\hline 1 & & $\mu g / L$ & GE \\
\hline 1 & & $\mu \mathrm{g} / \mathrm{L}$ & GE \\
\hline$i$ & & $\begin{array}{l}\mu / L \\
\mu g / L\end{array}$ & GE \\
\hline 1 & & $\mu \mathrm{g} / \mathrm{L}$ & GE \\
\hline 1 & & $\mu g / L$ & GE \\
\hline 1 & $\mathrm{~J}$ & $\mathrm{pCi} / \mathrm{L}$ & $\begin{array}{l}\text { GE } \\
\text { GP }\end{array}$ \\
\hline
\end{tabular}

Note: Flagging, dilution factors, modifiers, and laboratories are for fourth quarter 1994 data only. See Appendix B for flagging criteria. - = exceeded holding time for fourth quarter 1994.

- = exceeded screening level or final PDWS for fourth quarter 1994. 


\section{H ST Analyte}

Heptachlor

Iron, total recoverable

Lead, total recoverable

Lindane

Magnesium, total recoverable

Manganese, total recoverable

Mercury, total recoverable

Methoxychlor

Naphthalene

Nickel, total recoverable

Nitrate as nitrogen

Nitrate-nitrite as nitrogen

Nonvolatile beta

- $\quad \mathrm{pH}$

Phenols

Potassium, total recoverable

Radium, total alpha-emitting

Selenium, total recoverable

Silica, total recoverable

Silver, total recoverable

Sodium, total recoverable

Specific conductance

Sulfate

1,1,2,2-Tetrachlorosthane

Tetrachloroethylene

Tin, total recoverable

Toluene

Total dissolved solids

Total organic carbon

Total organic halogens

Total phosphates (as P)

Toxaphene

2,4,5-TP (Silvex)

$1,1,1$-Trichloroethane

1,1,2-Trichloroethane

Trichloroethylene

Trichlorofluoromethane

$2,4,5-T$

Tritium

- Turbidity

Vanadium, total recoverable

Xylenes

$\quad 1094$
$<0.050$
487
41
$<0.0050$
458
36
$<0.20$
$<0.50$
$<10$
7.7

2094

$<0.052$

119

14

$<0.0052$

406

26

$<0.20$

$<0.52$

$<10$

$<4.0$

350

$1.9 E+00$

5.9

$<5.0$

1,560

1. $6 \mathrm{E}+00$

$<2.0$

7,470

$<2.0$

1,070

28

$<1,000$

$<1.0$

$<1.0$

3.3
$<1.0$

24,000

$<1,000$

32
$<50$

$<50$

$<0.24$

$<0.00046$

$<1.0$

$<1.0$

$<1.0$

$<1.0$

440

$2.1 \mathrm{E}+00$

6.3

$<5.0$

1,910

$2.9 E+00$

$<2.0$

6,720

$<2.0$

1,270

29

$<1,000$

$<1.0$

$<1.0$

3.8

$<1.0$

18,000

$<1,000$

$<5.0$

$<50$

$<0.25$

$<0.00046$

$<1.0$

$<1.0$

$<1.0$

$<0.00046$

1.1E+01

66

$<8.0$

$<2.0$

256

$<8.0$

$<2.0$

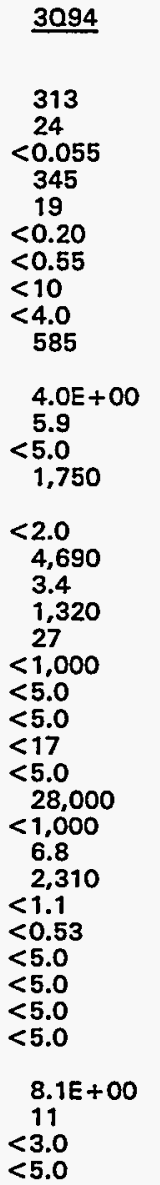

$\quad \underline{4094}$


179
23
$<0.0049$
459
29
$<0.20$
$<0.49$
$<9.9$
$<4.0$

DF

Mod

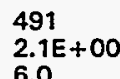

6.0

$<5.0$

2,000

$<2.0$

6,890

$<2.0$

1,250

27

$<1,000$

$<1.0$

$<1.0$

5.5

$<1.0$
23,000

$<1,000$

$<5.0$

$<50$

$<0.24$

$<0.00044$

$<1.0$

$<1.0$

$<1.0$

$<1.0$

$6.1 E+00$

113
$<8.0$

$<8.0$
$<2.0$

$\begin{array}{lll}1 & & \\ 1 & & \\ 1 & & \\ 1 & & \\ 1 & & \\ 1 & J & \\ 1 & & \\ 1 & & \\ 1 & & \\ 1 & & \\ 1 & \mathrm{~J} & \text { P } \\ 1 & \mathrm{~J} & \mathrm{pH} \\ 1 & & \end{array}$

$\mu \mathrm{g} / \mathrm{L} \quad \mathrm{LE}$

$\begin{array}{lll}\mu \mathrm{g} / \mathrm{L} & \mathrm{GE} & 1 \\ \mu \mathrm{g} / \mathrm{L} & \mathrm{GE} & 0\end{array}$

$\mu \mathrm{g} / \mathrm{L} \quad \mathrm{GE} \quad 0$

$\begin{array}{lll}\mu \mathrm{g} / \mathrm{L} & \mathrm{GE} & 1 \\ \mu \mathrm{g} / \mathrm{L} & \mathrm{GE} & 0\end{array}$

$\mu \mathrm{g} / \mathrm{L}$ GE 0

$\mu \mathrm{g} / \mathrm{L} \quad \mathrm{GE}$

$\mu \mathrm{gg} / \mathrm{L}$

$\mu g / L \quad G E$

$\mathrm{pCi} / \mathrm{L}$ GP

$\mathrm{pH}$

$\mu \mathrm{g} / \mathrm{L} \quad \mathrm{GE}$

$\mu \mathrm{g} / \mathrm{L} \quad \mathrm{GE}$

pCi/L

$\mu g / L \quad G E$

$\mu \mathrm{g} / \mathrm{L}$

$\mu g / 2$

$\mu g / L \quad G E$

$\mu \mathrm{S} / \mathrm{cm} \quad \mathrm{GE}$

r

$\mu \mathrm{g} / \mathrm{h}$

$\mu g / L \quad G E$

$\mu \mathrm{g} / \mathrm{L} \quad \mathrm{GE}$

$\mu g / L$

$\mu \mathrm{g} / \mathrm{L}$

$\mu g / L$

$\mu g / L$

GE

$\mu g / L \quad G E$

$\mu \mathrm{g} / \mathrm{L}$

$\mu g / L$

$\mu g / L$

$\mu \mathrm{g} / \mathrm{L}$

$\mu g / L$

$\mu g / h$

$\mu \mathrm{g} / \mathrm{L}$

pCi/mL GP O

$<3.0$
$<5.0$

NTU

$\mu \mathrm{g} / \mathrm{L}$

Note: Flagging, dilution factors, modifiers, and laboratories are for fourth quarter 1994 data only. See Appendix B for flagging criteria. - = exceeded holding time for fourth quarter 1994.

m = exceeded screening level or final PDWS for fourth quarter 1994. 


\section{WELL HSB 85A}

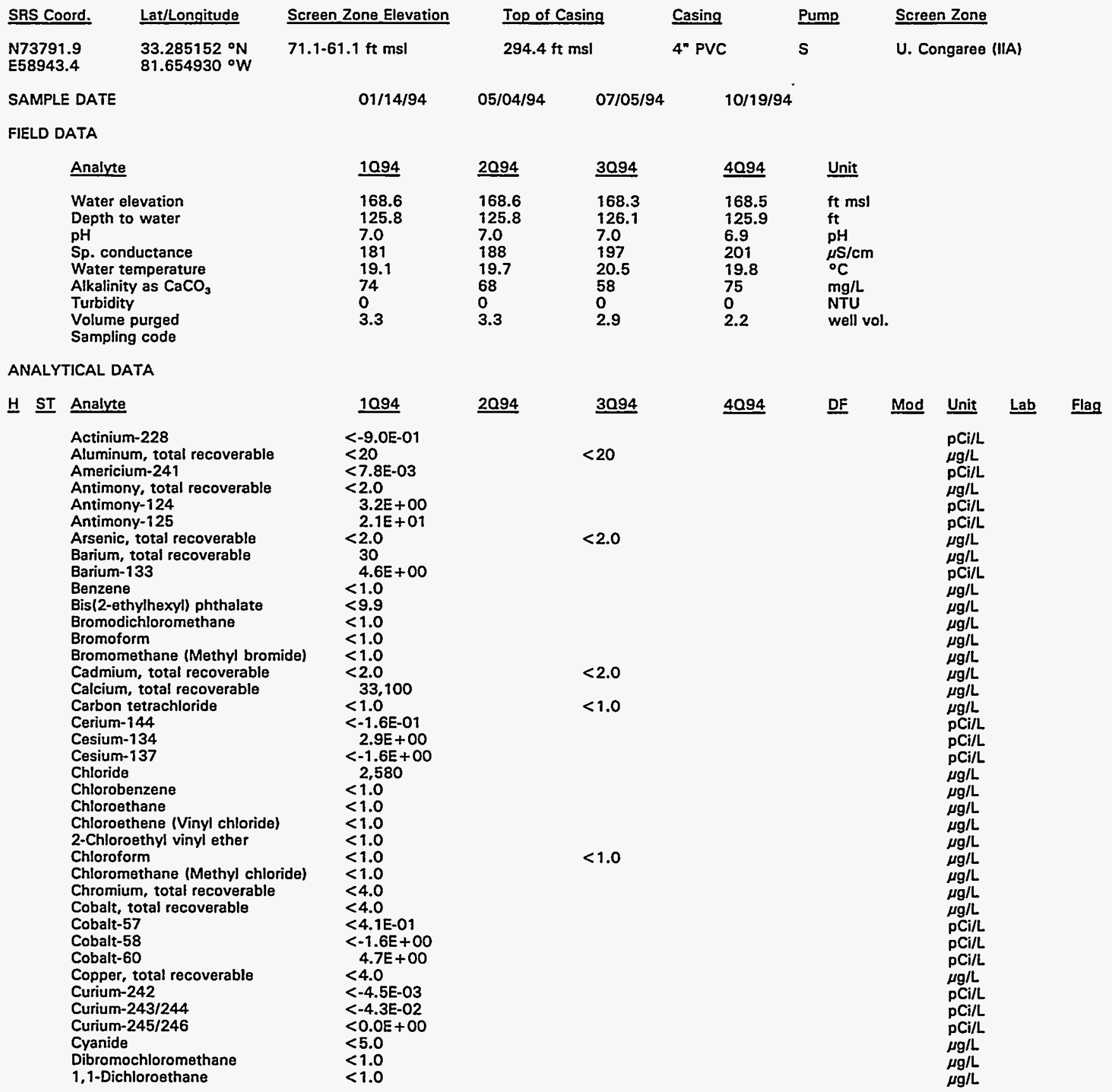

Note: Flagging, dilution factors, modifiers, and laboratories are for fourth quarter 1994 data only. See Appendix B for flagging criteria. - = exceeded holding time for fourth quarter 1994.

a = exceeded screening level or final PDWS for fourth quarter 1994. 
H ST Analyte

1,2-Dichlorosthane

1,1-Dichloroethylene

trans-1,2-Dichloroethylene

Dichloromethane

1,2-Dichloropropane

cis-1,3-Dichloropropene

trans-1,3-Dichloropropene

Ethylbenzene

Europium-152

Europium-154

Europium-155

Fluoride

Gross alpha

lodine-129

Iron, total recoverable

Lead, total recoverable

Lead-212

Magnesium, total recoverable

Manganese, total recoverable

Manganese-54

Mercury, total recoverable

Neptunium-239

Nickel, total recoverable

Nitrate as nitrogen

Nitrate-nitrite as nitrogen

Nonvolatile bete

$\mathrm{pH}$

Phenols

Plutonium-238

Plutonium-239/240

Potassium, total recoverable

Potassium-40

Promethium-14

Promethium-146

Radium, total alpha-emitting

Radium-226

Radium-228

Ruthenium+106

Selenium, total recoverable

Silica, total recoverable

Silver, total recoverable

Sodium, total recoverable

Sodium-22

Specific conductance

Strontium-89

Strontium-90

Sulfate

Technetium-99

1,1,2,2-Tetrachloroethane

Tetrachloroethylene

Thallium, total recoverable

Thorium-228

Thorium-230

Thorium-232

Thorium-234

Tin-113

Toluene

Total dissolved solids

Total organic carbon

Total organic halogens

Total phosphates (as P)

1,1,1-Trichloroethane

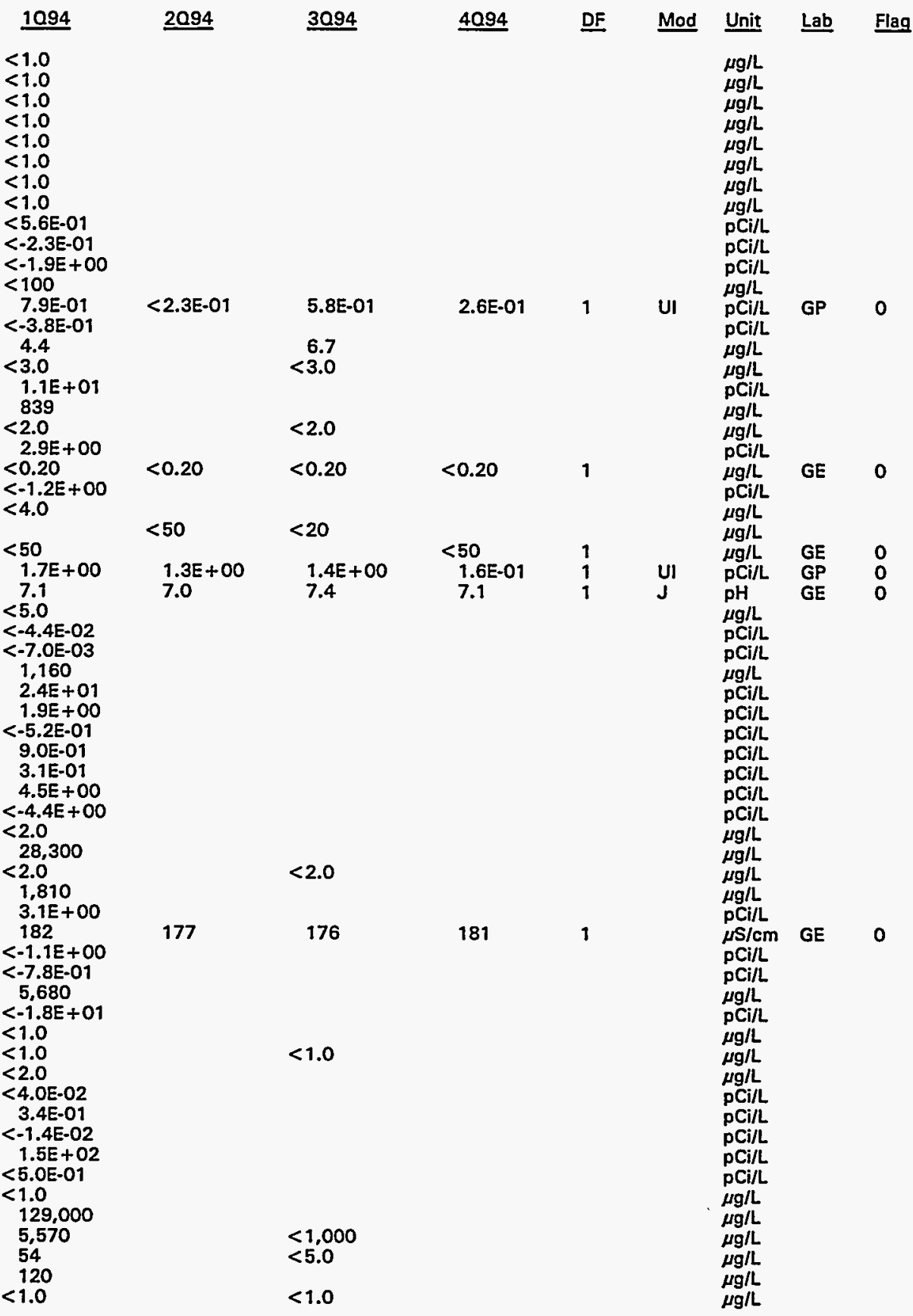

Note: Flagging, dilution factors, modifiers, and laboratories are for fourth quarter 1994 data only. See Appendix B for flagging criteria. - = exceeded holding time for fourth quarter 1994.

- = exceeded screening level or final PDWS for fourth quarter 1994. 


\section{H ST Analyze}

1,1,2-Trichloroethane Trichloroethylene

Trichlorofluoromethane

Tritium

Uranium-233/234

Uranium-235

Uranium-238

Vanadium, total recoverable

Yttrium-88

Zinc, total recoverable

Zinc-65

Zirconium-95

\begin{tabular}{|c|c|c|c|c|c|c|c|}
\hline 1094 & $\underline{2094}$ & 3094 & 4094 & DF & Mod & Unit & $\underline{\text { Lab }}$ \\
\hline $\begin{array}{c}<1.0 \\
<1.0 \\
<1.0 \\
6.8 \mathrm{E}-02 \\
1.1 \mathrm{E}-01 \\
7.9 \mathrm{E}-02 \\
7.9 \mathrm{E}-02 \\
<8.0 \\
<-1.4 \mathrm{E}+00 \\
17 \\
<-1.8 \mathrm{E}-01 \\
2.6 \mathrm{E}+00\end{array}$ & $<1.4 \mathrm{E}-01$ & $\begin{array}{l}<1.0 \\
2.5 E-01\end{array}$ & 3.2E-01 & 1 & UI & $\begin{array}{l}\mu \mathrm{g} / \mathrm{L} \\
\mu \mathrm{g} / \mathrm{L} \\
\mu \mathrm{g} / \mathrm{L} \\
\mathrm{pCi} / \mathrm{mL} \\
\mathrm{pCi} / \mathrm{L} \\
\mathrm{pCi} / \mathrm{L} \\
\mathrm{pCi} / \mathrm{L} \\
\mu \mathrm{g} / \mathrm{L} \\
\mathrm{pCi} / \mathrm{L} \\
\mu \mathrm{g} / \mathrm{L} \\
\mathrm{pCi} / \mathrm{L} \\
\mathrm{pCi} / \mathrm{L}\end{array}$ & GP \\
\hline
\end{tabular}

\section{WELL HSB 85B}

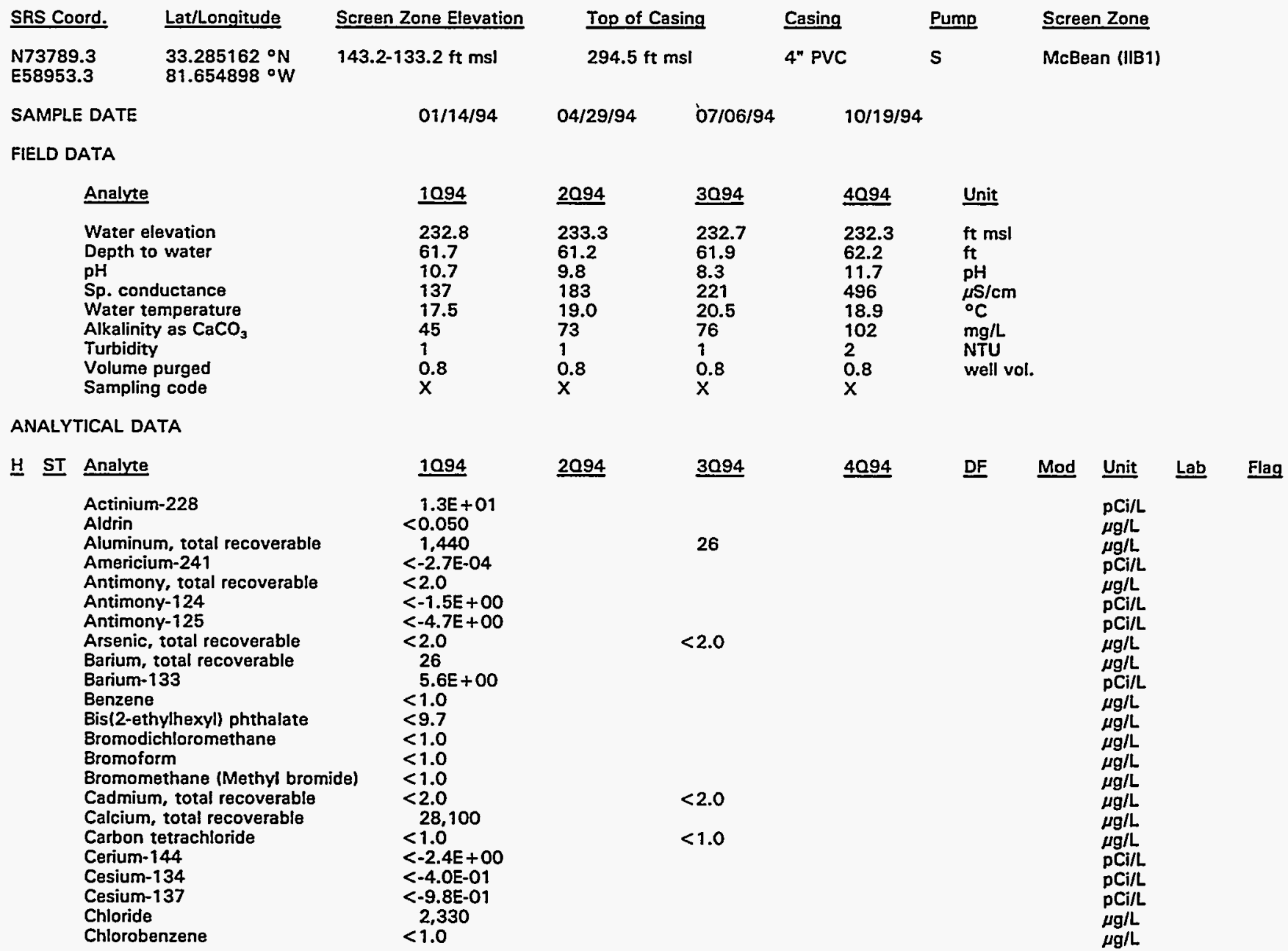

Note: Flagging, dilution factors, modifiers, and laboratories are for fourth quarter 1994 data only. See Appendix B for flagging criteria. - = exceeded holding time for fourth quarter 1994.

- = exceeded screening level or final PDWS for fourth quarter 1994. 
H ST Analyte

Chlorosthane

Chloroethene (Vinyl chloride)

2-Chloroethyl vinyl ether

Chloroform

Chloromethane (Methyl chloride)

Chromium, total recoverable

Cobalt, total recoverable

Cobalt-57

Cobalt-58

Cobalt-60

Copper, total recoverable

Curium-242

Curium-243/244

Curium-245/246

Cyanide

p.p'-DDT

Dibromochloromethane

1,1-Dichloroethane

1,2-Dichloroethane

1,1 -Dichloroethylene

trans-1,2-Dichloroethylene

Dichloromethane

1,2-Dichloropropane

cis-1,3-Dichloropropene

trans-1,3-Dichloropropene

Dieldrin

Ethylbenzene

Europium-152

Europium-154

Europium-155

Fluoride

Gross alpha

Heptachlor

lodine-129

Iron, total recoverable

Lead, total recoverable

Lead-212

Lindane

Magnesium, total recoverable

Manganese, total recoverable

Manganese-54

Mercury, total recoverable

Neptunium-239

Nickel, total recoverable

Nitrate as nitrogen

Nitrate-nitrite as nitrogen

Nonvolatile beta

pH

Phenols

Plutonium-238

Plutonium-239/240

Potassium, total recoverable

Potassium -40

Promethium-144

Promethium-146

Radium, total alpha-emitting

Radium-226

Radium-228

Ruthenium-106

Selenium, total recoverable

Silica, total recoverable

Silver, total recoverable

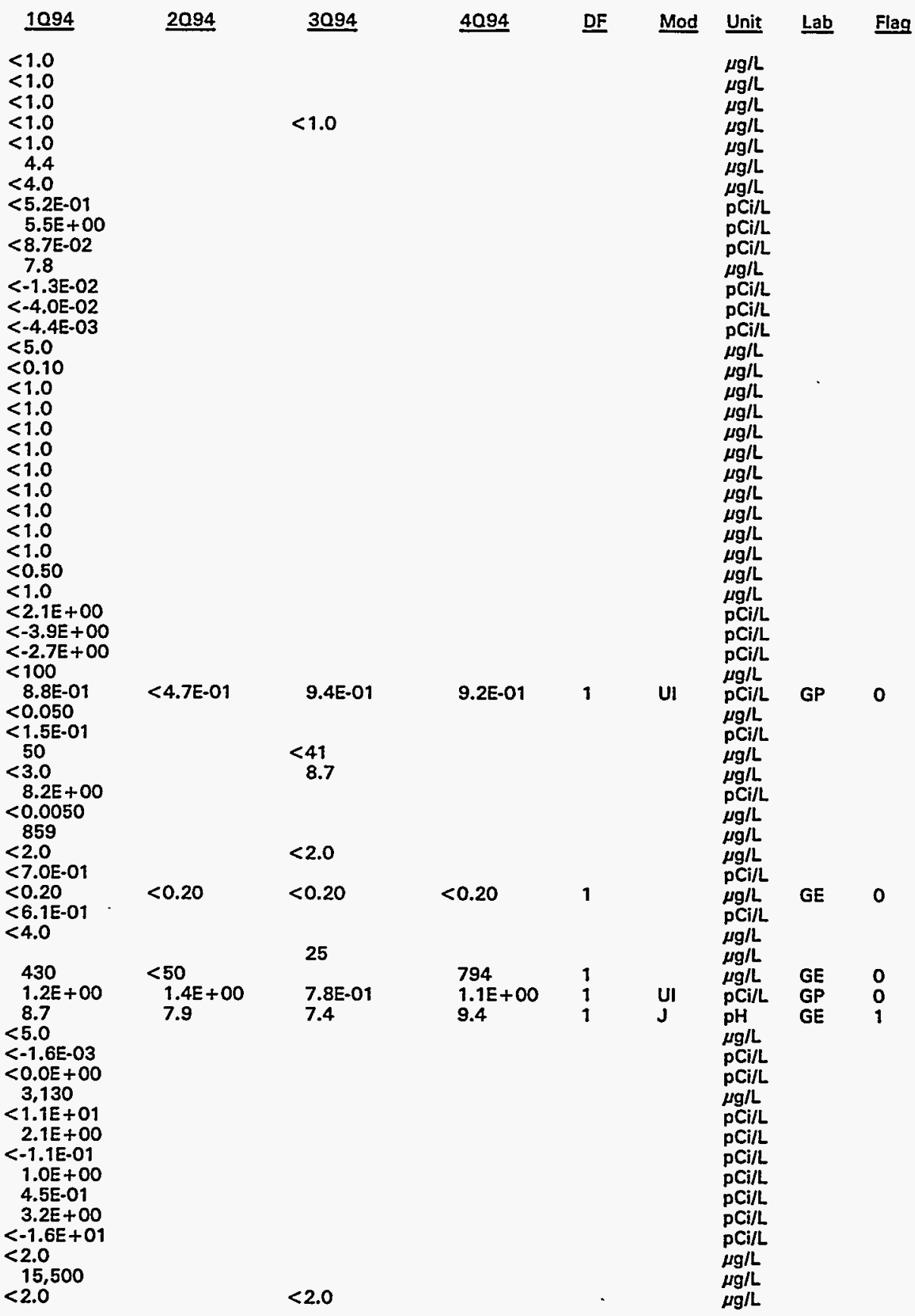

Note: Flagging, dilution factors, modifiers, and laboratories are for fourth quarter 1994 data only. See Appendix B for flagging criteria. - = exceeded holding time for fourth quarter 1994.

- = exceeded screening level or final PDWS for fourth quarter 1994. 
WSRC-TR-94-0610

Unclassified

Well HSB 85B continued

ANALYTICAL DATA

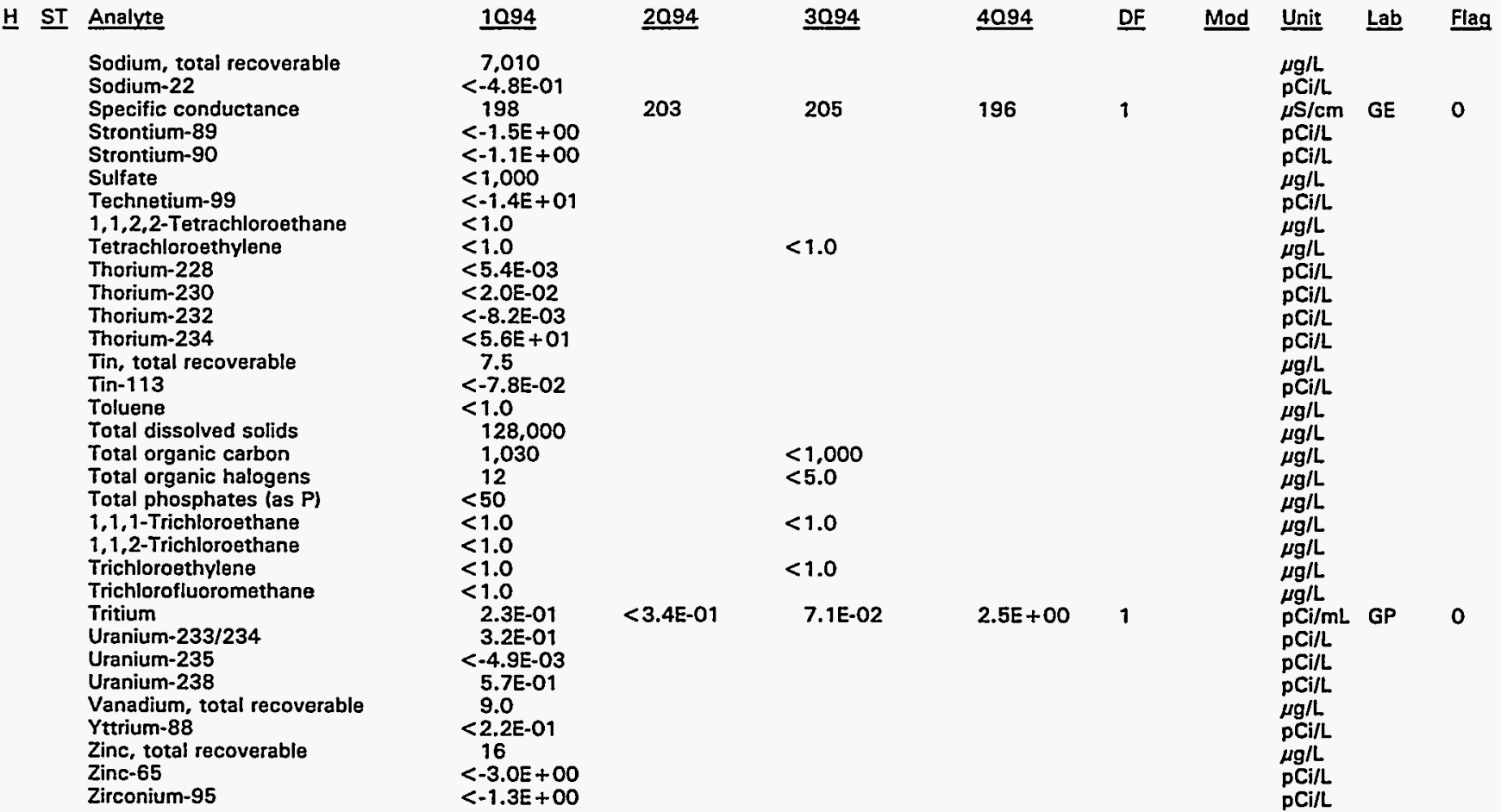

Note: Flagging, dilution factors, modifiers, and laboratories are for fourth quarter 1994 data only. See Appendix B for flagging criteria. - = exceeded holding time for fourth quarter 1994.

- = exceeded screening level or final PDWS for fourth quarter 1994. 
WELL HSB 85C

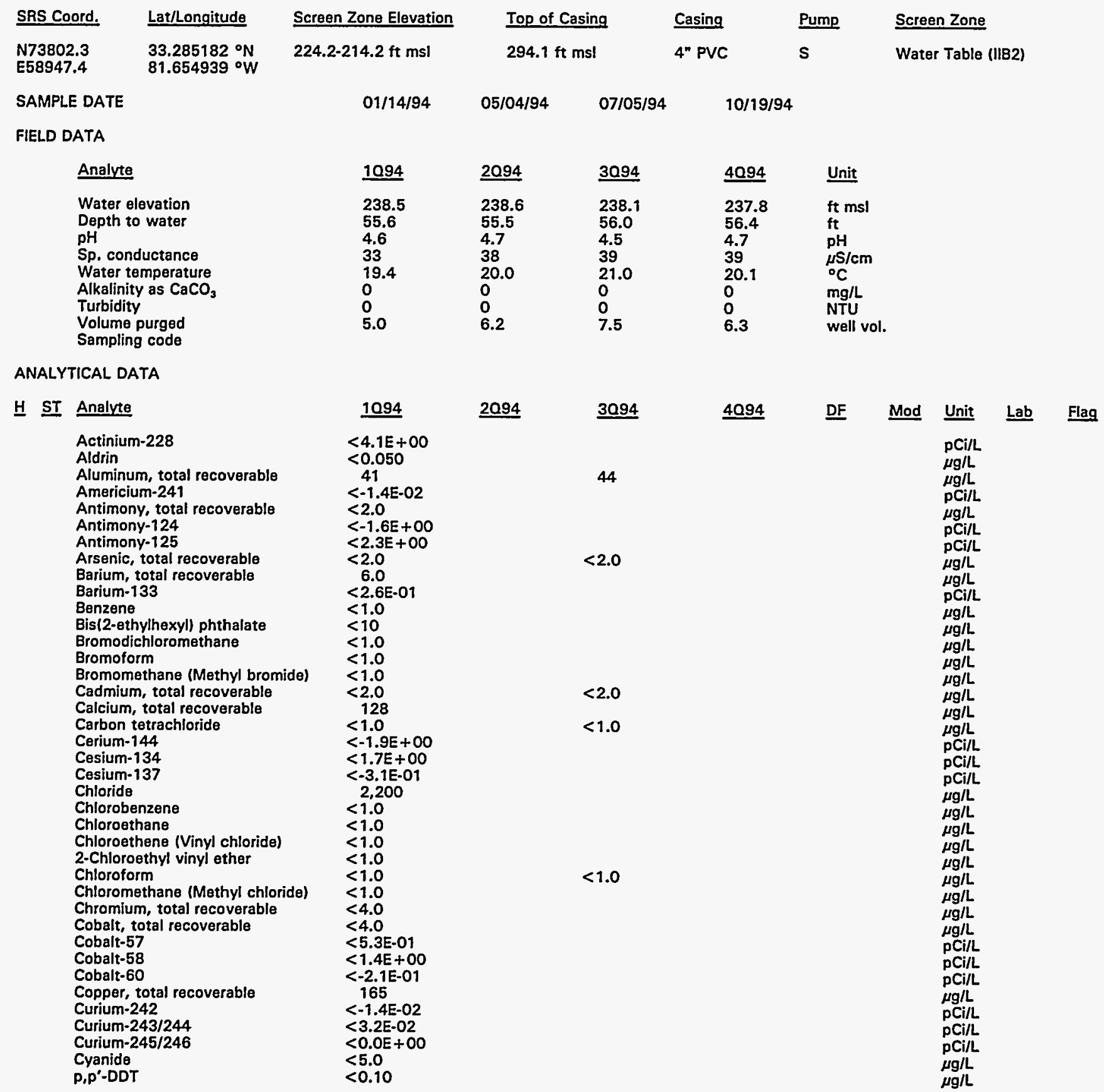

Note: Flagging, dilution factors, modifiers, and laboratories are for fourth quarter 1994 data only. See Appendix B for flagging criteria. - = exceeded holding time for fourth quarter 1994.

- = exceeded screening level or final PDWS for fourth quarter 1994. 
Well HSB 85C continued

ANALYTICAL DATA

H ST Analyze

Dibromochloromethane

1,1-Dichloroethane

1,2-Dichloroethane

1,1-Dichloroethylene

trans-1,2-Dichloroethylene

Dichloromethane

1,2-Dichloropropane

cis-1,3-Dichloropropene

trans-1,3-Dichloropropene

Dieldrin

Ethylbenzene

Europium-152

Europium-154

Europium-155

Fluoride

Gross alpha

Heptachlor

lodine-129

Iron, total recoverable

Lead, total recoverable

Lead-212

Lindane

Magnesium, total recoverable

Manganese, total recoverable

Manganese-54

Mercury, total recoverable

Neptunium-239

Nickel, total recoverable

Nitrate as nitrogen

Nitrate-nitrite as nitrogen

Nonvolatile beta

$\mathrm{pH}$

Phenols

Piutonium-238

Plutonium-239/240

Potassium, total recoverable

Potassium-40

Promethium-144

Promethium-146

Radium, total alpha-emitting

Radium-226

Radium-228

Ruthenium-106

Selenium, total recoverable

Silica, total recoverable

Silver, total recoverable

Sodium, total recoverable

Sodium-22

Specific conductance

Strontium-89

Strontium-90

Sulfate

Technetium-99

1,1,2,2-Tetrachloroethane

Tetrachloroethylene

Thorium-228

Thorium-230

Thorium-232

Thorium-234

Tin, total recoverable

Tin-113

Toluene

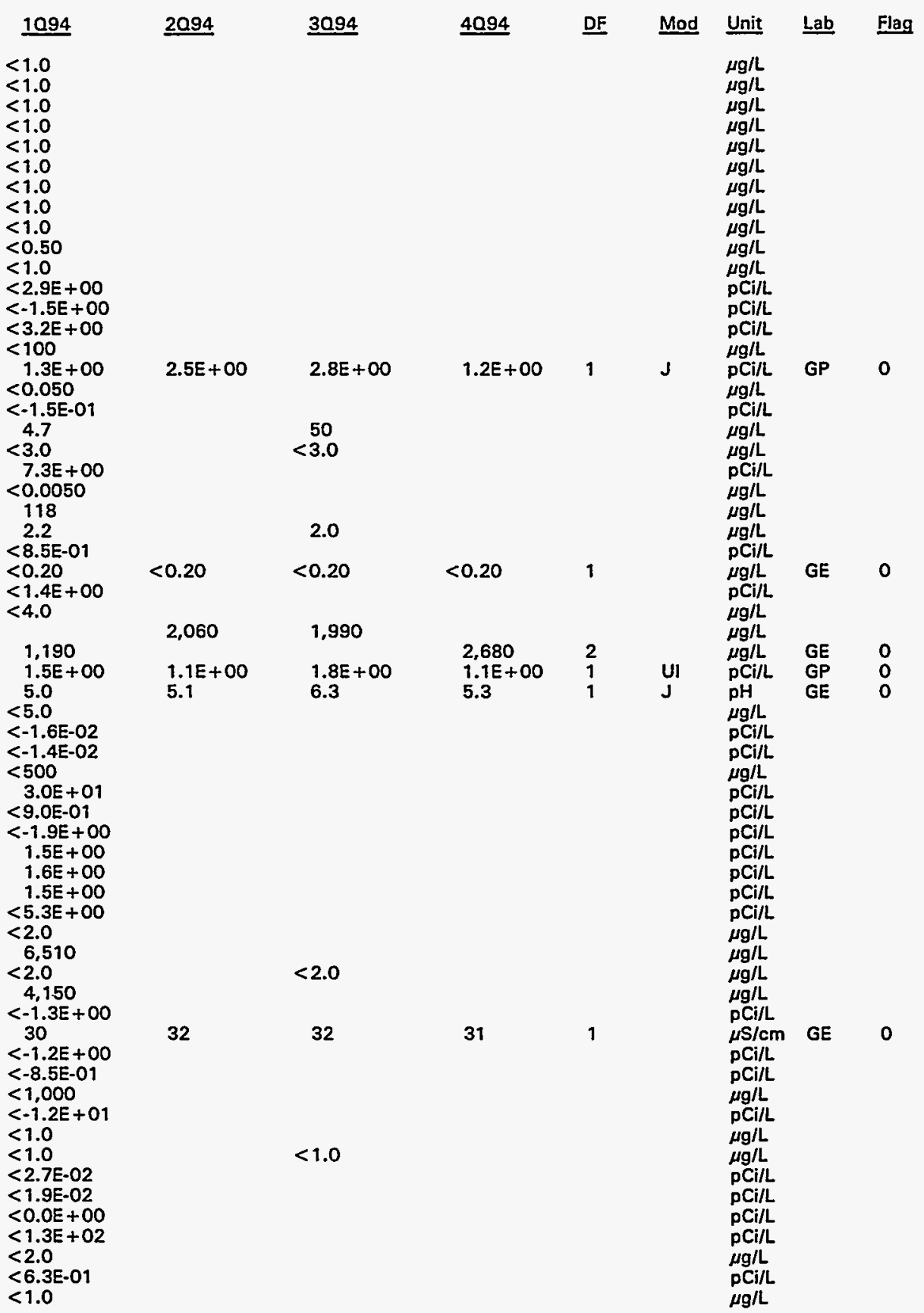

Note: Flagging, dilution factors, modifiers, and laboratories are for fourth quarter 1994 data only. See Appendix B for flagging criteria. - = exceeded holding time for fourth quarter 1994.

- = exceeded screening level or final PDWS for fourth quarter 1994. 
WSRC-TR-94-0610

Unclassified

Well HSB 85C continued

ANALYTICAL DATA

\section{H ST Analyte}

Total dissolved solids

Total organic carbon

Total organic halogens

Total phosphates (as P)

1,1,1-Trichloroethane

1,1,2-Trichloroethane

Trichloroethylene

Trichlorofluoromethane

Tritium

Uranium-233/234

Uranium-235

Uranium-238

Vanadium, total recoverable

Yttrium-88

Zinc, total recoverable

Zinc-65

Zirconium-95

\begin{tabular}{|c|c|c|c|c|c|c|c|}
\hline 1094 & $\underline{2094}$ & 3094 & 4094 & $\underline{\mathrm{DF}}$ & Mod & Unit & Lab \\
\hline $\begin{aligned} & 26,000 \\
& 20,400 \\
&<5.0<50 \\
&< 50 \\
&< 1.0 \\
&< 1.0 \\
&< 1.0 \\
&< 1.0 \\
& 4.5 E+00 \\
&<0.0 E+00 \\
&<-6.6 E-03 \\
&<-6.6 E-03 \\
&<8.0 \\
&<1.0 E-01 \\
& 20 \\
&<1.2 E+00 \\
& 4.6 E+00\end{aligned}$ & $6.7 E+00$ & $\begin{aligned} &<1,000<5.0 \\
&<1.0 \\
&<1.0 \\
& \quad 6.8 E+\infty\end{aligned}$ & $6.8 E+00$ & 1 & & $\begin{array}{l}\mu g / L \\
\mu g / L \\
\mu g / L \\
\mu g / L \\
\mu g / L \\
\mu g / L \\
\mu g / L \\
\mu g / L \\
\text { pCi/mL } \\
\text { pCi/L } \\
\text { pCi/L } \\
\text { pCi/L } \\
\mu g / L \\
\text { pCi/L } \\
\mu \mathrm{g} / L \\
\text { pCi/L } \\
\text { pCi/L }\end{array}$ & GP \\
\hline
\end{tabular}

Note: Flagging, dilution factors, modifiers, and laboratories are for fourth quarter 1994 data only. See Appendix B for flagging criteria.

- = exceeded holding time for fourth quarter 1994.

- = exceeded screening level or final PDWS for fourth quarter 1994. 
WSRC-TR-94-0610

Unclassified

THIS PAGE LEFT BLANK INTENTIONALLY. 


\section{Appendix E}

\section{Data Quality/Usability Assessment}


WSRC-TR-94-0610

Unclassified

THIS PAGE LEFT BLANK INTENTIONALLY. 


\section{Data Quality/Usability Assessment}

Quality assurance/quality control (QAVQ) procedures relating to accuracy and precision of analyses performed on groundwater samples are followed in the field and laboratory and are reviewed prior to publication of results. The review by the Environmental Protection Department/Environmental Monitoring Section (EPD/EMS) of the volume of analytical data acquired each quarter and presented in various reports is an ongoing process; its review of the QA/QC data cannot be completed in time to meet the deadlines for the reports required by the Resource Conservation and Recovery Act and associated regulations. Other site and regulatory personnel can obtain further information on the data quality and usability in a variety of ways, including those described below.

\section{Data Qualification}

The contract laboratories continually assess their own accuracy and precision according to U.S. Environmental Protection Agency (EPA) guidelines. They submit sample- or batch-specific QA QC information either at the same time as analytical results or in quarterly summaries. Properly defined and used result modifiers (also referred to as qualifiers) can be a key component in assessing data usability. Result modifiers designed by EPD/EMS and used by the primary laboratories are presented in Appendix D.

\section{Assessment of Accuracy of the Data}

Accuracy, or the nearness of the reported result to the true concentration of a constituent in a sample, can be assessed in several ways.

A laboratory's general accuracy can be judged by analysis of results obtained from known samples. The non-radionuclide contract laboratories analyze commercial reference samples every quarter at EPD/EMS' request. The results of these analyses are presented in the EPD/EMS groundwater monitoring quarterly reports. The primary laboratories also seek or maintain state certification by participating periodically in performance studies; reference samples and analysis of results are provided by EPA. Results of these studies also are published in the EPD/EMS quarterly reports.

Analysis of blanks provides a tool for assessing the accuracy of both sampling and laboratory analysis. Results for all field blanks for the quarter can be found in the EPD/EMS quarterly reports. Any field or laboratory blanks that exceed established minimums are identified in the same reports, in tables associating them with groundwater samples analyzed in the same batches.

Surrogates, organic compounds similar in chemical behavior to the compounds of interest but not normally found in environmental samples, are used to monitor the effect of the matrix on the accuracy of analyses for organic parameters. For example, for analyses of volatile organics by EPA Method 8240 , three surrogate compounds are added to all samples and blanks in each analytical batch. In analyses of semivolatile organics, three acid compounds and three basel neutral compounds are used. Two surrogates are used in organochlorine pesticides analyses. Percent recoveries for surrogate analyses are calculated by laboratory personnel, reported to EPD/EMS, reviewed, and entered into the database, but they are not published. If recoveries are not within specified limits, the laboratory is expected to reanalyze the samples or attach qualifiers to the data identifying the anomalous results. 
Sample-specific accuracy for both organic and inorganic parameters can be assessed by examination of matrix spike/matrix spike duplicate results. A sample is analyzed unspiked to determine a baseline set of values. A second portion of the sample is spiked with known concentrations of compounds appropriate to the analyses being performed, typically five volatile organic compounds for volatile organics analyses, eleven semivolatile compounds for semivolatiles, six pesticide compounds for pesticides, all metals for metals analyses by SW-846 methods (EPA, 1986), and a known quantity of cyanide for cyanide analysis. The percentage of the spike compound that is recovered (i.e., measured in excess of the value obtained for the unspiked sample) is a direct measure of analytical accuracy. EPA requires matrix spike/matrix spike duplicates to be run at least once per 20 samples of similar matrix.

Matrix spike/matrix spike duplicate results are reported to EPD/EMS but are not published. For organic compounds, according to EPA guidelines, no action is taken on the basis of matrix spike/ matrix spike duplicate data alone (i.e., no result modifiers are assigned solely on the basis of matrix spike results); however, the results can indicate if a laboratory is having a systematic problem in the analysis of one or more analytes.

In the case of inorganic compounds, such as metals, the matrix spike sample analysis provides information about the effect of each sample matrix on the digestion and measurement methodology. Data qualifiers assigned by the laboratories on the basis of the percentage of spike recovery are reported in the published results tables.

\section{Assessment of Precision}

Precision of the analyses, or agreement of a set of replicate results among themselves, is assessed through the use of duplicates initiated by the laboratory and blind replicates provided by EPD/EMS. The results of duplicate and replicate analyses are presented in those results tables of the quarterly reports which report only one quarter of data, usually during first, second, and third quarters. Duplicate and replicate results are not presented in results tables that report more than one quarter of data, generally provided in fourth quarter reports. In this case, the results tables instead present only the highest result for each analyte for each quarter of the year.

The laboratories assess precision by calculating the relative percent difference (RPD) for each pair of laboratory-initiated duplicate results. One of the contract laboratories uses a data qualifier (J3) to modify metals analyses when the RPD for laboratory duplicates is greater than 20 percent.

Additional statistical comparisons of laboratory duplicate and blind replicate results, both intraand interlaboratory, are presented in the EPD/EMS quarterly reports. The calculation used for these reports is the mean relative difference (MRD) which is similar to EPA's RPD except that the MRD is the average of all the RPD values from one laboratory for each compound (intralaboratory MRD) or all the RPD values from all laboratories for each compound (interlaboratory MRD), during one quarter. Because detection limits may vary among samples, the MRD requires calculation of a reference detection limit, which is the detection limit at the 90th percentile of the array of limits in the population of all duplicate and replicate analyses for a given analyte during a particular quarter. The MRD is not method-specific.

\section{Method-Specific Accuracy and Precision}

The contract laboratories' EPA-approved laboratory procedures include QAVC requirements as an integral part of the methods. Thus, knowledge of the method used in obtaining data is an important component of determining data usability. EPA has conducted extensive research and 
development on the methods approved for the analysis of water and waste water; information on the accuracy and precision of a method is available from EPA publications, as is full information on required $Q A / Q C$ procedures. A listing of the methods used by the primary laboratories during fourth quarter 1993 is given below along with the source for the method description. Many, if not all, of these sources include presentations of representative accuracy and precision results.

\section{Methods Used by the Contract Laboratories}

\begin{tabular}{|c|c|c|}
\hline Method & Used to Analyze & Source \\
\hline EPA120.1 & Specific conductance & EPA EMSL, 1983 \\
\hline EPA150.1 & & EPA EMSL, 1983 \\
\hline EPA160.1 & Total dissolved solids & EPA EMSL, 1983 \\
\hline EPA160.2 & Total dissolved solids, total suspended solids & EPA EMSL, 1983 \\
\hline EPA180.1 & Turbidity & EPA EMSL, 1983 \\
\hline EPA200.7 & Metals & EPA EMSL, 1983 \\
\hline EPA204.2 & Antimony & EPA EMSL, 1983 \\
\hline EPA206.2 & Arsenic & EPA EMSL, 1983 \\
\hline EPA239.2 & Lead & EPA EMSL, 1983 \\
\hline EPA245.1 & Mercury & EPA EMSL, 1983 \\
\hline EPA270.2 & Selenium & EPA EMSL, 1983 \\
\hline EPA279.2 & Thallium & EPA EMSL, 1983 \\
\hline EPA300.0 & Chloride, nitrite, sulfate & EPA EMSL, 1991 \\
\hline EPA310.1 & Alkalinity & EPA EMSL, 1983 \\
\hline EPA325.2 & Chloride & EPA EMSL, 1983 \\
\hline EPA335.3 & Cyanide & EPA EMSL, 1983 \\
\hline EPA340.2 & Fluoride & EPA EMSL, 1983 \\
\hline EPA353.1 & Nitrogen, nitrate-nitrite & EPA EMSL, 1983 \\
\hline EPA353.2 & Nitrogen, nitrate, nitrite, or combined & EPA EMSL, 1983 \\
\hline EPA365.1 & Phosphorus, all forms (reported as total phosphates) & EPA EMSL, 1983 \\
\hline EPA365.2 & Phosphorus, all forms (reported as total phosphates) & EPA EMSL, 1983 \\
\hline EPA376.2 & Sulfide & EPA EMSL, 1983 \\
\hline EPA413.1 & Oil \& grease & EPA EMSL, 1983 \\
\hline EPA415.1 & $\begin{array}{l}\text { Dissolved organic carbon, total inorganic carbon, total organic } \\
\text { carbon }\end{array}$ & EPA EMSL, 1983 \\
\hline EPA418.1 & Total petroleum hydrocarbons & EPA EMSL, 1983 \\
\hline EPA420.2 & Phenols & EPA EMSL, 1983 \\
\hline EPA900.0 & Gross alpha, nonvolatile beta & EPA EMSL, 1980 \\
\hline EPA900.1 & Total alpha-emitting radium & EPA EMSL, 1980 \\
\hline EPA906.0 & Tritium & EPA EMSL, 1980 \\
\hline EPA6010 & Metals & EPA, 1986 \\
\hline EPA7041 & Antimony & EPA, 1986 \\
\hline EPA7060 & Arsenic & EPA, 1986 \\
\hline EPA7421 & Lead & EPA, 1986 \\
\hline EPA7470 & Mercury & EPA, 1986 \\
\hline EPA7740 & Selenium & EPA, 1986 \\
\hline EPA7841 & Thallium & EPA, 1986 \\
\hline EPA8010 & Chlorinated volatile organics & EPA, 1986 \\
\hline EPA8080 & Organochlorine pesticides and PCBs & EPA, 1986 \\
\hline EPA8150 & Chlorinated herbicides & EPA, 1986 \\
\hline EPA8240 & GCMS volatiles & EPA, 1986 \\
\hline EPA8270 & GCMS semivolatiles & EPA, 1986 \\
\hline EPA8280 & Dioxins and furans & EPA, 1986 \\
\hline EPA9012 & Cyanide & EPA, 1986 \\
\hline EPA9020 & Total organic halogens & EPA, 1986 \\
\hline EPA9020A & Total organic halogens & EPA, 1986 \\
\hline EPA9030 & Sulfide & EPA, 1986 \\
\hline
\end{tabular}


Method Used to Analyze Source

EPA9060 Dissolved organic carbon, total inorganic carbon, total organic EPA, 1986 carbon

An example of available method-specific QAVQC information is that for the analysis of metals by EPA Method 6010/200.7 (EPA, 1986/EPA EMSL, 1983). The primary laboratories, General Engineering Laboratories (GE) and Roy F. Weston, Inc. (Weston), use this inductively coupled plasma (ICP) atomic emission spectrometric method.

The following precision and accuracy data are based on the experience of seven laboratories that applied the ICP technique to acid-distilled water matrices that had been spiked with various metal concentrates. (Note: Not all seven laboratories analyzed all 14 elements.) The references give results for samples having three concentration ranges; the results here are for samples having the lowest values, similar to actual groundwater results for SRS.

ICP Precision and Accuracy Data

\begin{tabular}{|c|c|c|c|}
\hline Element & True value $(\mu \mathrm{g} / \mathrm{L})$ & $\begin{array}{l}\text { Mean reported } \\
\text { value }(\mu \mathrm{g} / \mathrm{L})\end{array}$ & $\begin{array}{l}\text { Mean percent } \\
\text { RSD }^{\mathrm{a}}\end{array}$ \\
\hline Aluminum & 60 & 62 & 33 \\
\hline Arsenic & 22 & 19 & 23 \\
\hline Beryllium & 20 & 20 & 9.8 \\
\hline Cadmium & 2.5 & 2.9 & 16 \\
\hline Chromium & 10 & 10 & 18 \\
\hline Cobalt & 20 & 20 & 4.1 \\
\hline Copper & 11 & 11 & 40 \\
\hline Iron & 20 & 19 & 15 \\
\hline Lead & 24 & 30 & 32 \\
\hline Manganese & 15 & 15 & 6.7 \\
\hline Nickel & 30 & 28 & 11 \\
\hline Selenium & 6 & 8.5 & 42 \\
\hline Vanadium & 70 & 69 & 2.9 \\
\hline Zinc & 16 & 19 & 45 \\
\hline
\end{tabular}

a Relative standard deviation. In EPA (1986), the column heading is Mean Standard Deviation (\%).

As another example, EPA Method 601/8010 (EPA, 1991/EPA, 1986) is used by both GE and Weston for analyses of halogenated volatile organics. In the presentation of the method in both references, the following table gives method-specific accuracy and precision as functions of concentration. Contract laboratories are expected to achieve or at least approach these limits.

\section{Accuracy and Precision as Functions of Concentration for EPA Method 601/8010}

Parameter

Bromodichloromethane

Bromoform

Bromomethane

Carbon tetrachloride

Chlorobenzene

Chloroethane

2-Chloroethyl vinyl ether ${ }^{f}$
Accuracy as

recovery, $X^{\prime a}(\mu \mathrm{g} / \mathrm{L})$

$1.12 C-1.02^{d}$

$0.96 C-2.05$

$0.76 C-1.27$

$0.98 C-1.04$

$1.00 C-1.23$

$0.99 C-1.53$

$1.00 \mathrm{C}$
Single analyst

precision $(\mu \mathrm{g} / \mathrm{L})^{\mathrm{b}}$

$0.11 \bar{X}+0.04^{\mathrm{e}}$

$0.12 \bar{X}+0.58$

$0.28 \bar{X}+0.27$

$0.15 \bar{X}+0.38$

$0.15 \bar{X}-0.02$

$0.14 \bar{X}-0.13$

$0.20 \bar{X}$
Overall precision $(\mu \mathrm{g} / \mathrm{L})^{\mathrm{C}}$

$0.20 \bar{X}+1.00$

$0.21 \bar{X}+2.41$

$0.36 \bar{X}+0.94$

$0.20 \bar{X}+0.39$

$0.18 \bar{X}+1.21$

$0.17 \bar{X}+0.63$

$0.35 \bar{X}$ 


Parameter
Chloroform
Chloromethane
Dibromochloromethane
1,2-Dichlorobenzene
1,3-Dichlorobenzene
1,4-Dichlorobenzene
1,1-Dichloroethane
1,2-Dichloroethane
1,1-Dichloroethene
trans-1,2-Dichloroethene
Dichloromethane
(Methylene chloride)
1,2-Dichloropropane
cis-1,3-Dichloropropene
trans-1,3-Dichloropropene
1,1,2,2-Tetrachloroethane
Tetrachloroethylene
1,1,1-Trichloroethane
1,1,2-Trichloroethane
Trichloroethylene
Trichlorofluoromethane
Vinyl chloride

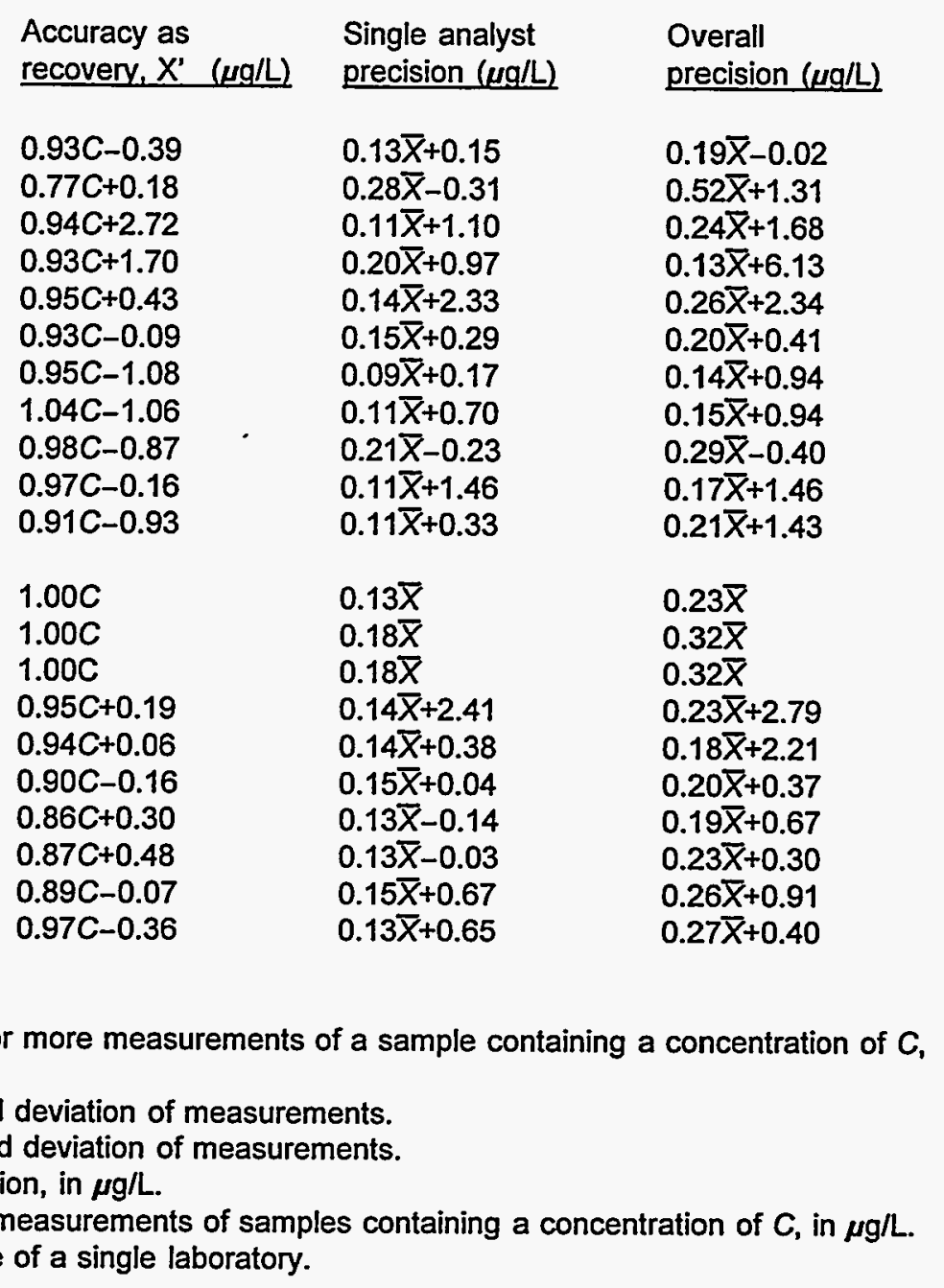

a $X^{\prime}=$ expected recovery for one or more measurements of a sample containing a concentration of $C$, in $\mu \mathrm{g} / \mathrm{L}$.

b Expected single analyst standard deviation of measurements.

c Expected interlaboratory standard deviation of measurements.

d $C=$ true value for the concentration, in $\mu \mathrm{g} / \mathrm{L}$.

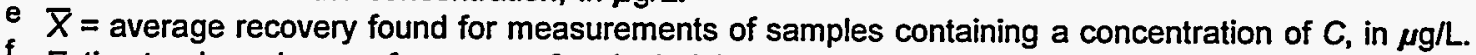

Estimates based on performance of a single laboratory.

\section{References Cited}

EPA (U.S. Environmental Protection Agency), 1986. Test Methods for Evaluating Solid Waste (SW-846), Volumes IA-IC. Washington, DC.

EPA (U.S. Environmental Protection Agency), 1991. Guidelines Establishing Test Procedures for the Analysis of Pollutants, Code of Federal Regulations, Title 40, Part 136, Appendix A. Washington, DC.

EPA EMSL (U.S. Environmental Protection Agency, Environmental Monitoring and Systems Laboratory), 1980. Prescribed Procedures for Measurement of Radioactivity in Drinking Water, EPA-600/4-80-032. Cincinnati, $\mathrm{OH}$.

EPA EMSL (U.S. Environmental Protection Agency, Environmental Monitoring and Systems Laboratory), 1983. Methods for Chemical Analysis of Water and Wastes. Cincinnati, $\mathrm{OH}$.

EPA EMSL (U.S. Environmental Protection Agency, Environmental Monitoring and Systems Laboratory), 1991. Test Method, The Determination of Inorganic Anions in Water by Ion Chromatography-Method 300.0. Cincinnati, $\mathrm{OH}$. 
WSRC-TR-94-0610

Unclassified

THIS PAGE LEFT BLANK INTENTIONALLY. 


\section{Appendix F}

Time Series Plots 
WSRC-TR-94-0610

Unclassified

THIS PAGE LEFT BLANK INTENTIONALLY. 


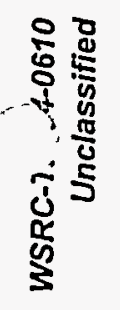
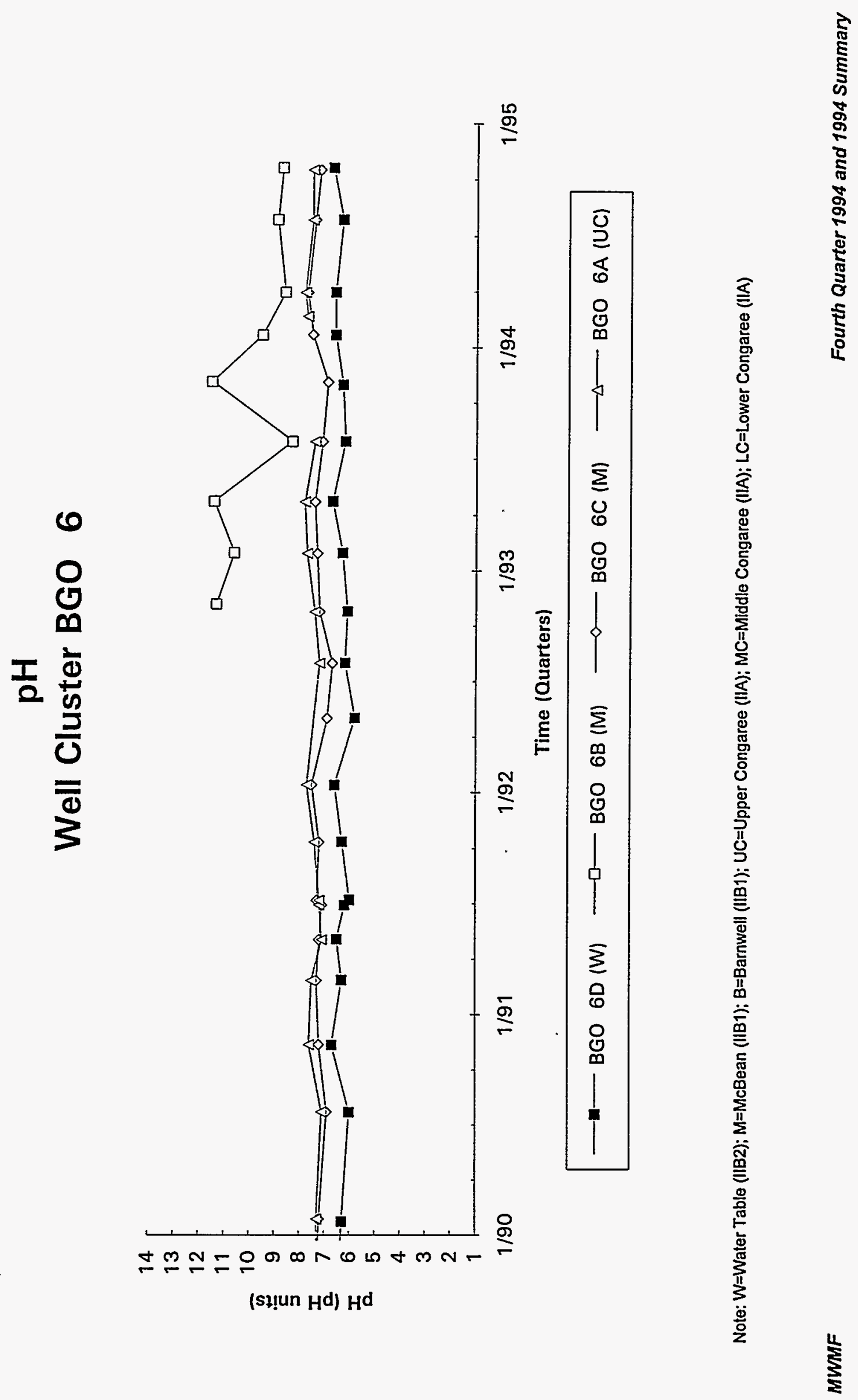


\section{$\mathrm{pH}$ \\ Well Cluster BGO 8}

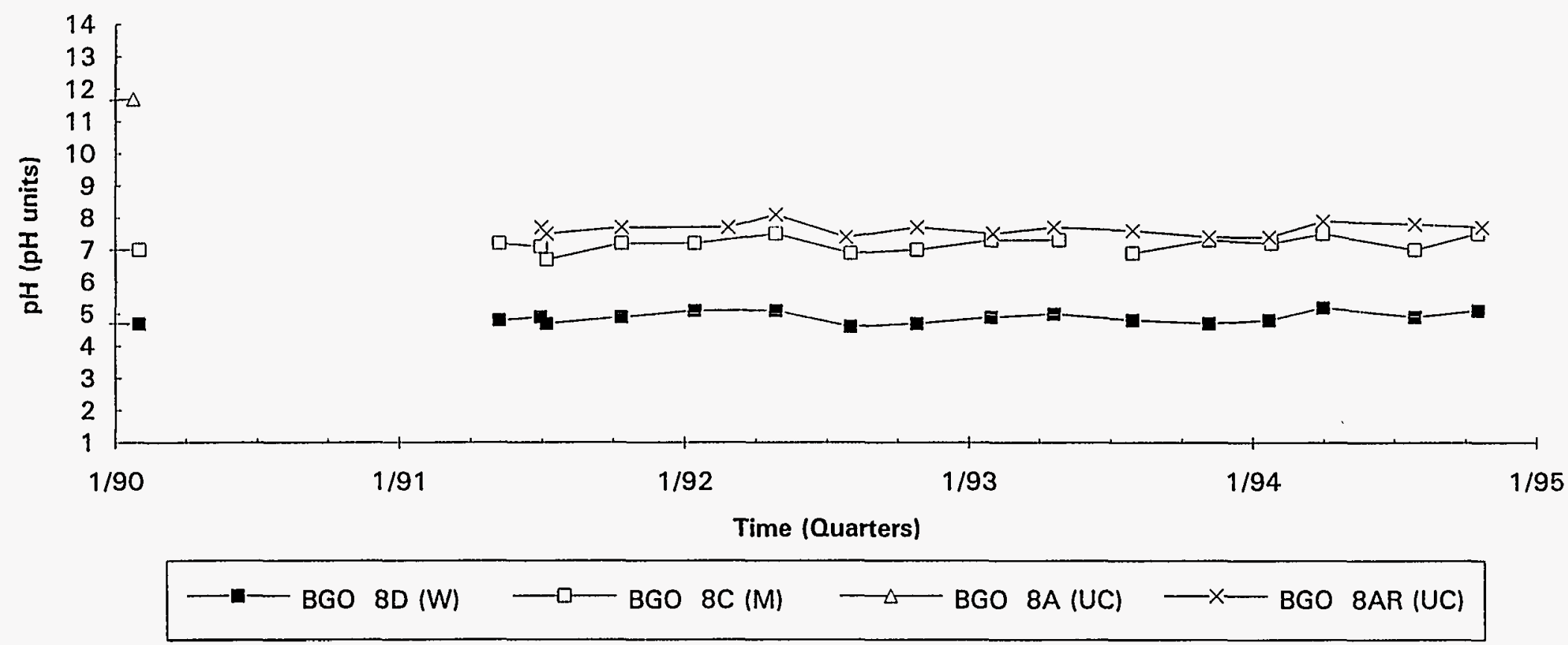

Note: $W=$ Water Table (IIB2); $M=M c B e a n$ (IIB1); B=Barnwell (IIB1); UC=Upper Congaree (IIA); MC=Middle Congaree (IIA); LC=Lower Congaree (IIA) 

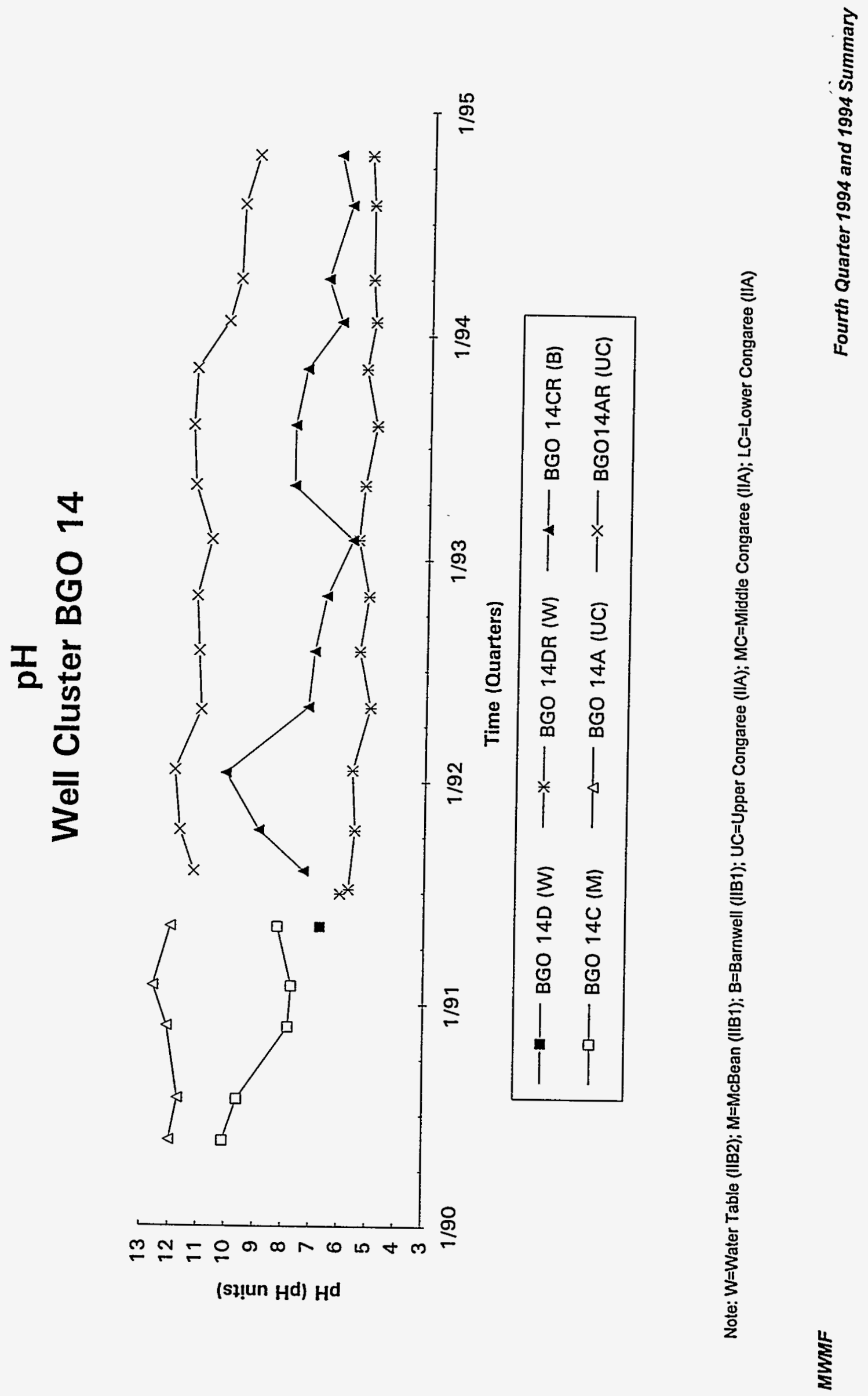


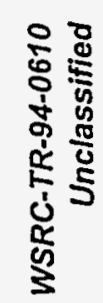
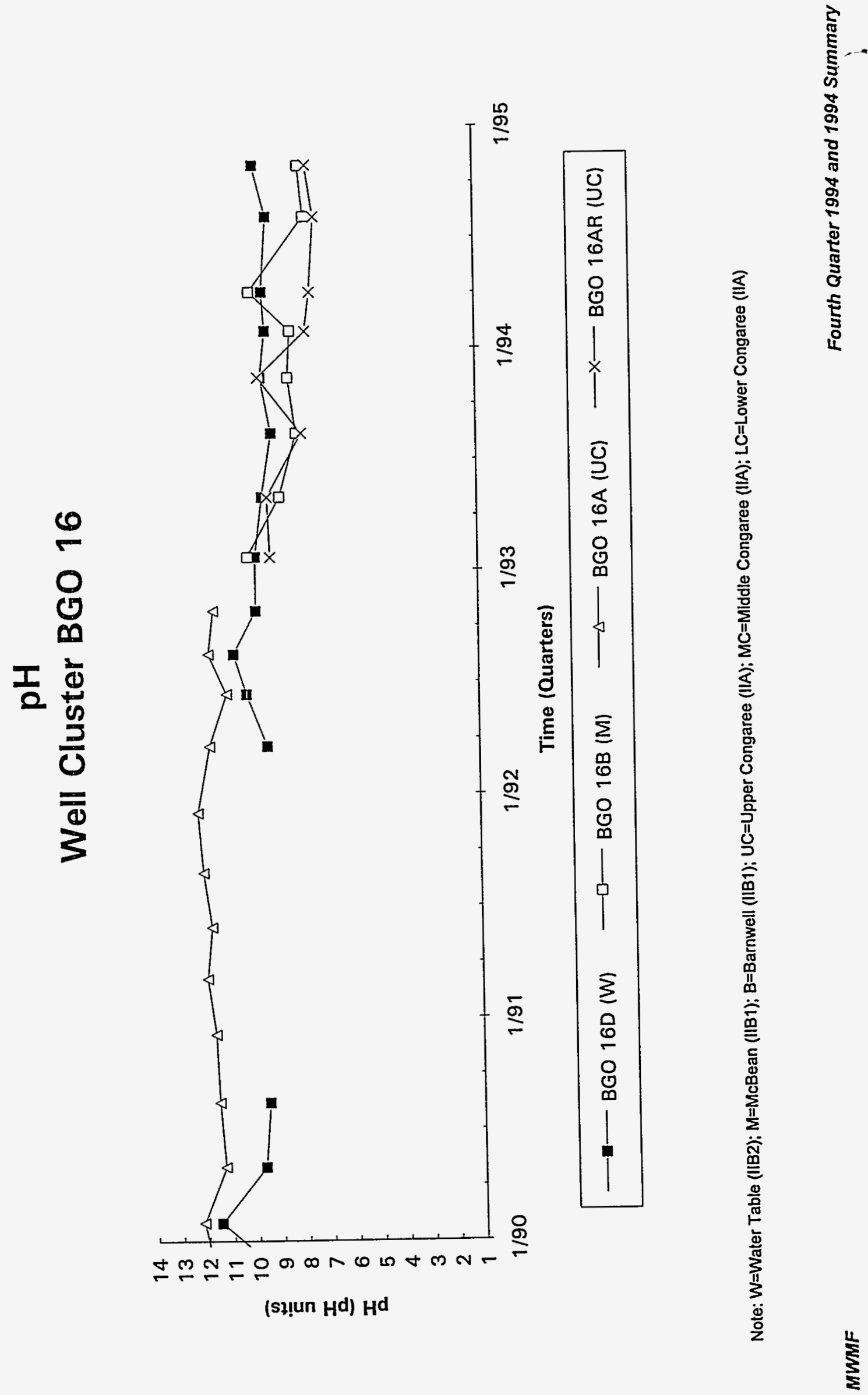


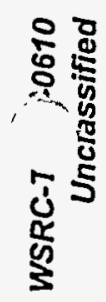
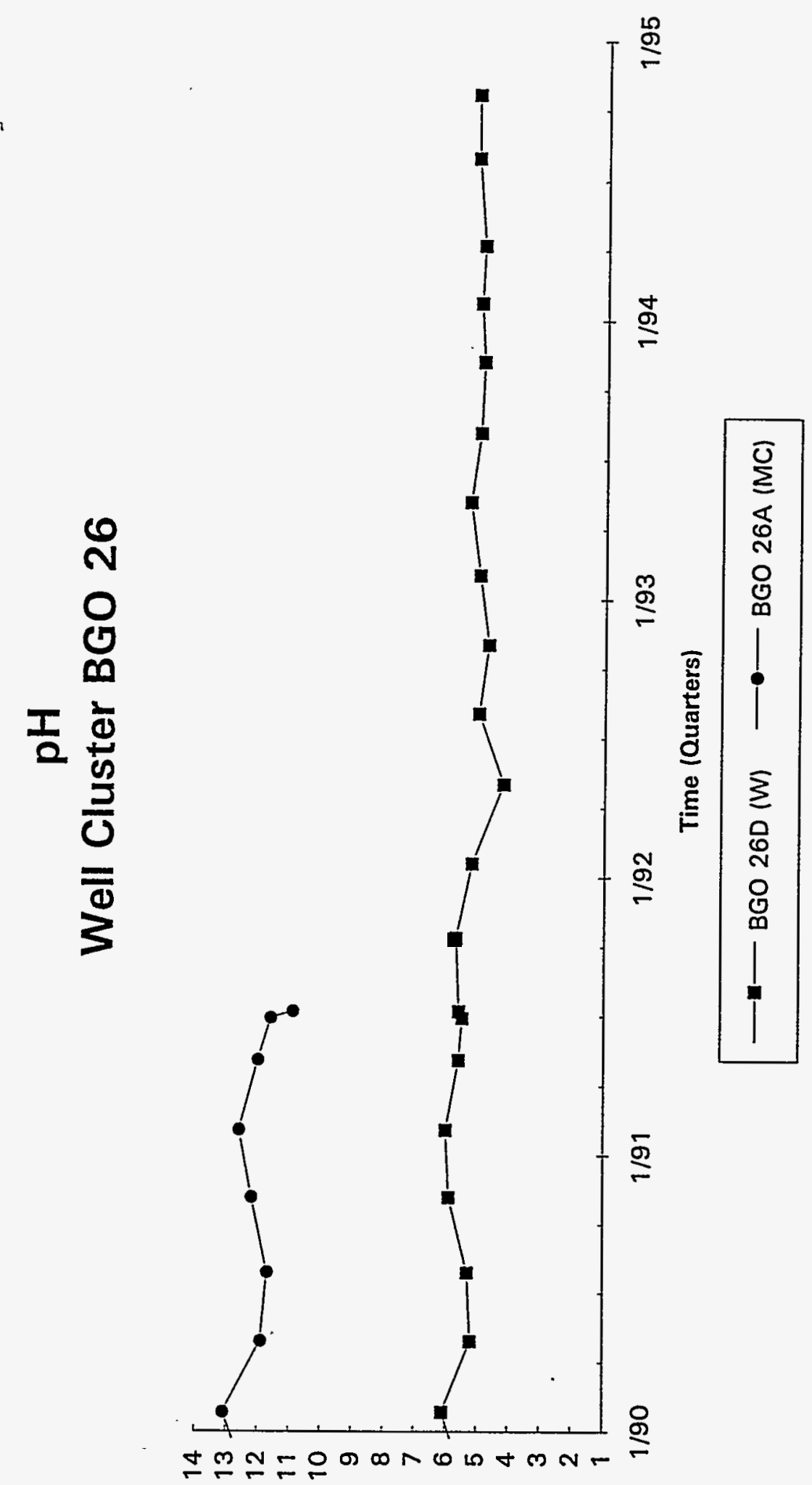

(sł! un $\mathrm{Hd}^{d} \mathrm{Hd}^{d}$ 

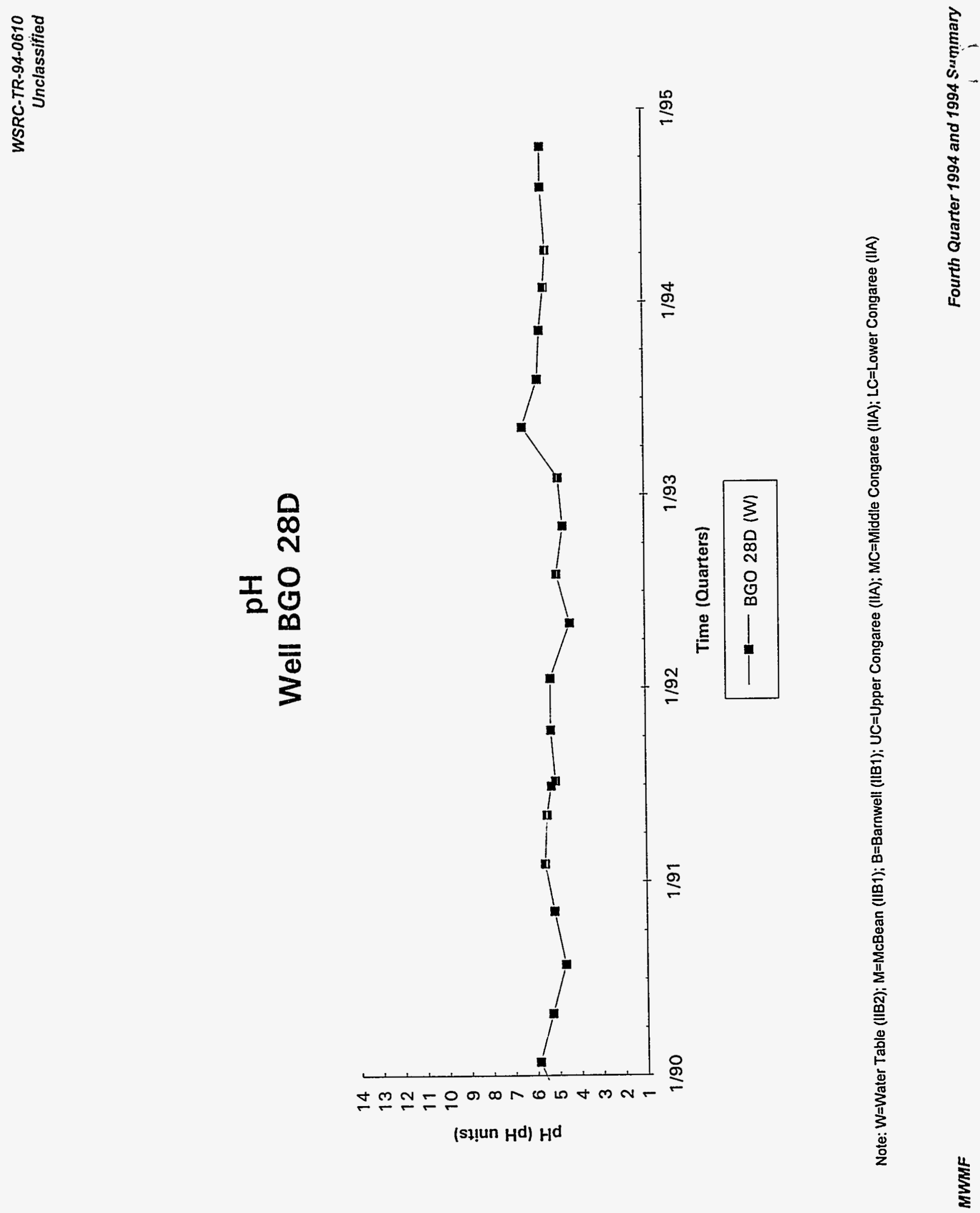


\section{$\mathrm{pH}$ \\ Well Cluster BGO 30}

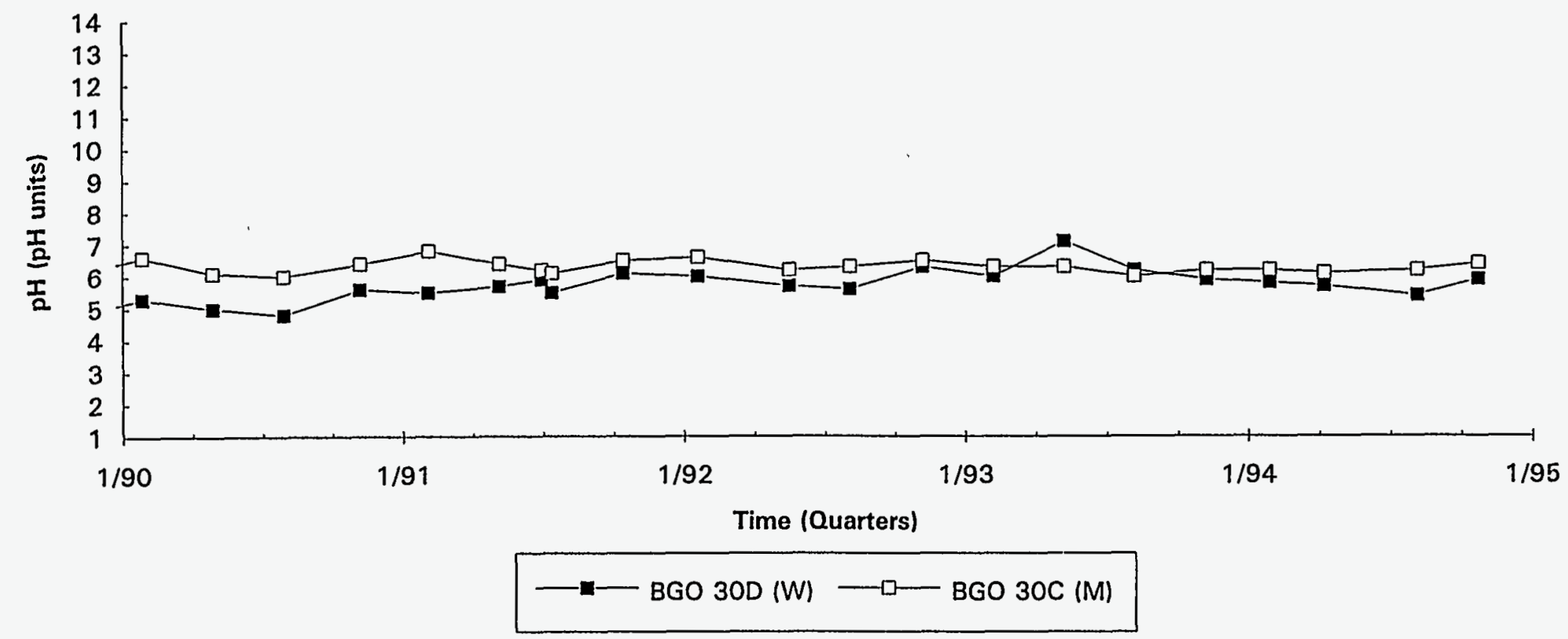

Note: W=Water Table (IIB2); M=MCBean (IIB1); B=Barnwell (IIB1); UC=Upper Congaree (IIA); MC=Middle Congaree (IIA); LC=Lower Congaree (IIA) 

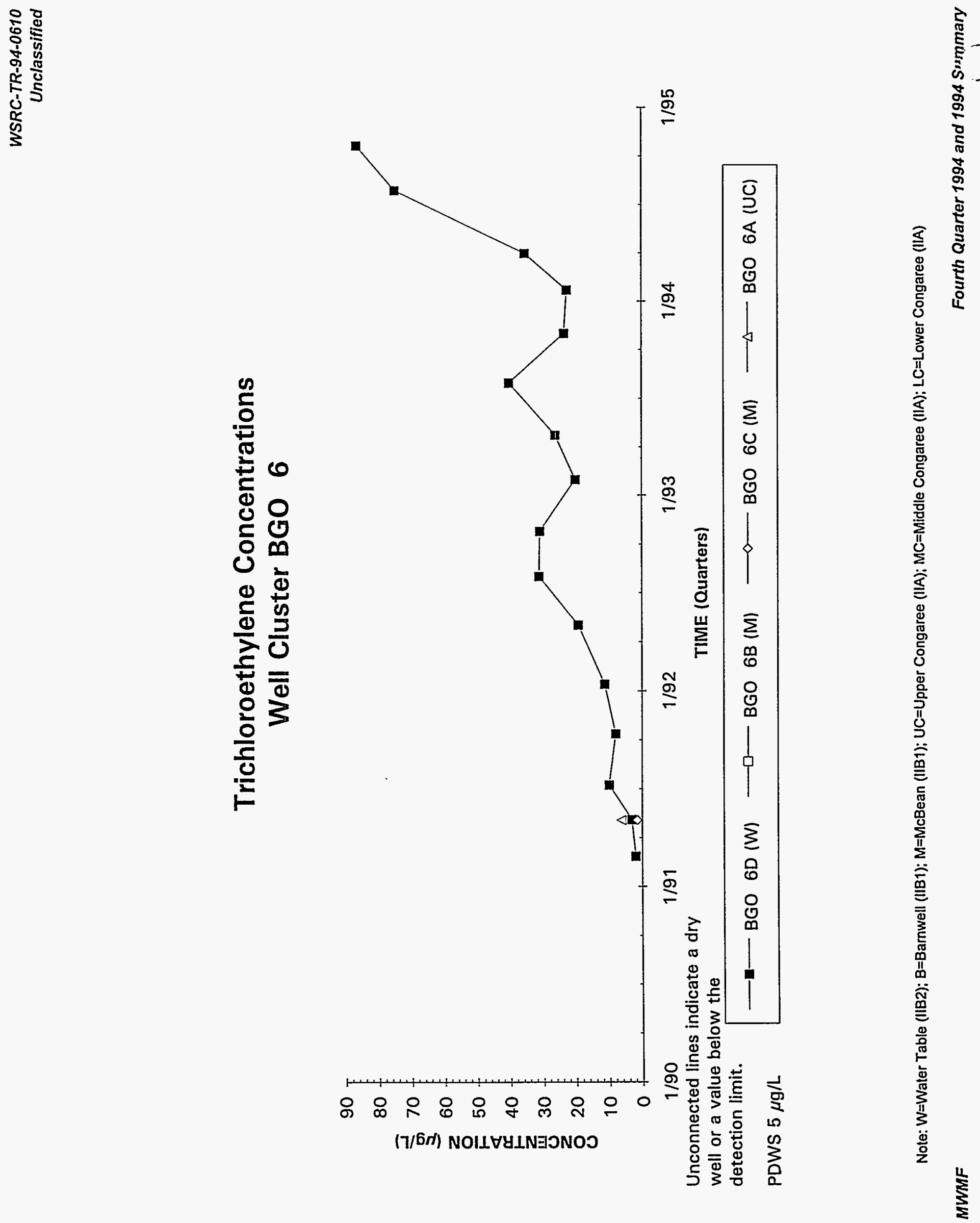

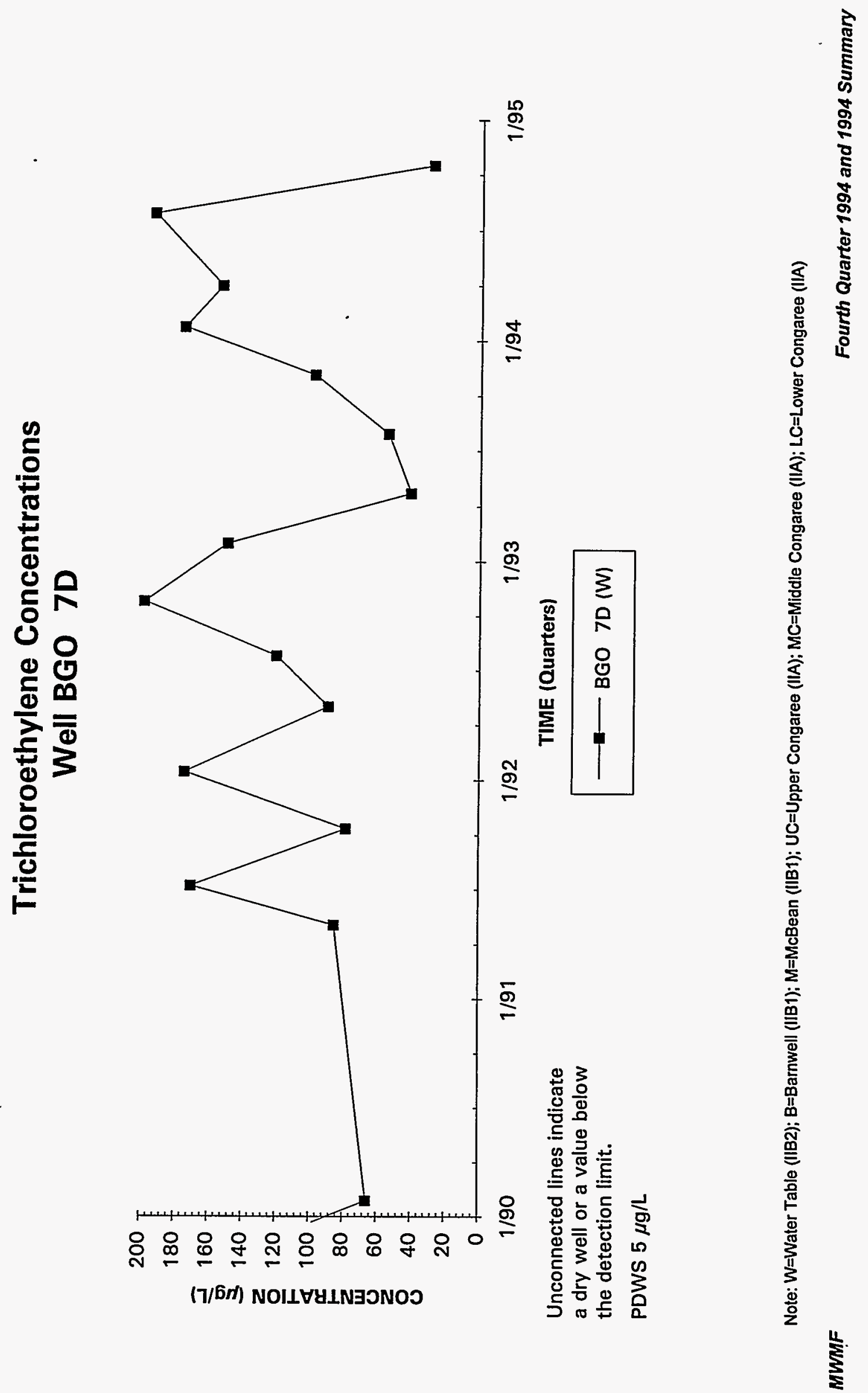


\section{Trichloroethylene Concentrations Well Cluster BGO 8}

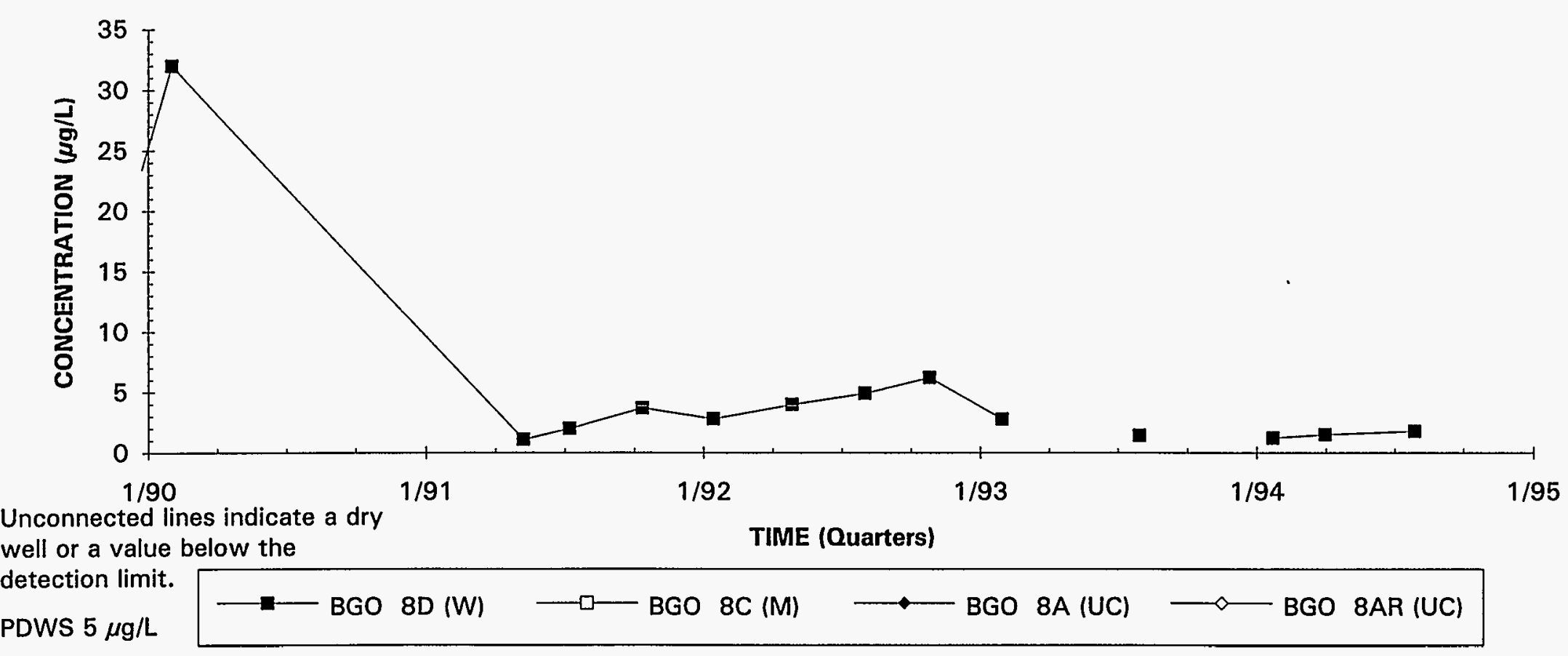

Note: W=Water Table (IIB2); B=Barnwell (IIB1); M=McBean (IIB1); UC=Upper Congaree (IIA); MC=Middle Congaree (IIA); LC=Lower Congaree (IIA) 


\section{Trichloroethylene Concentrations Well Cluster BGO 12}

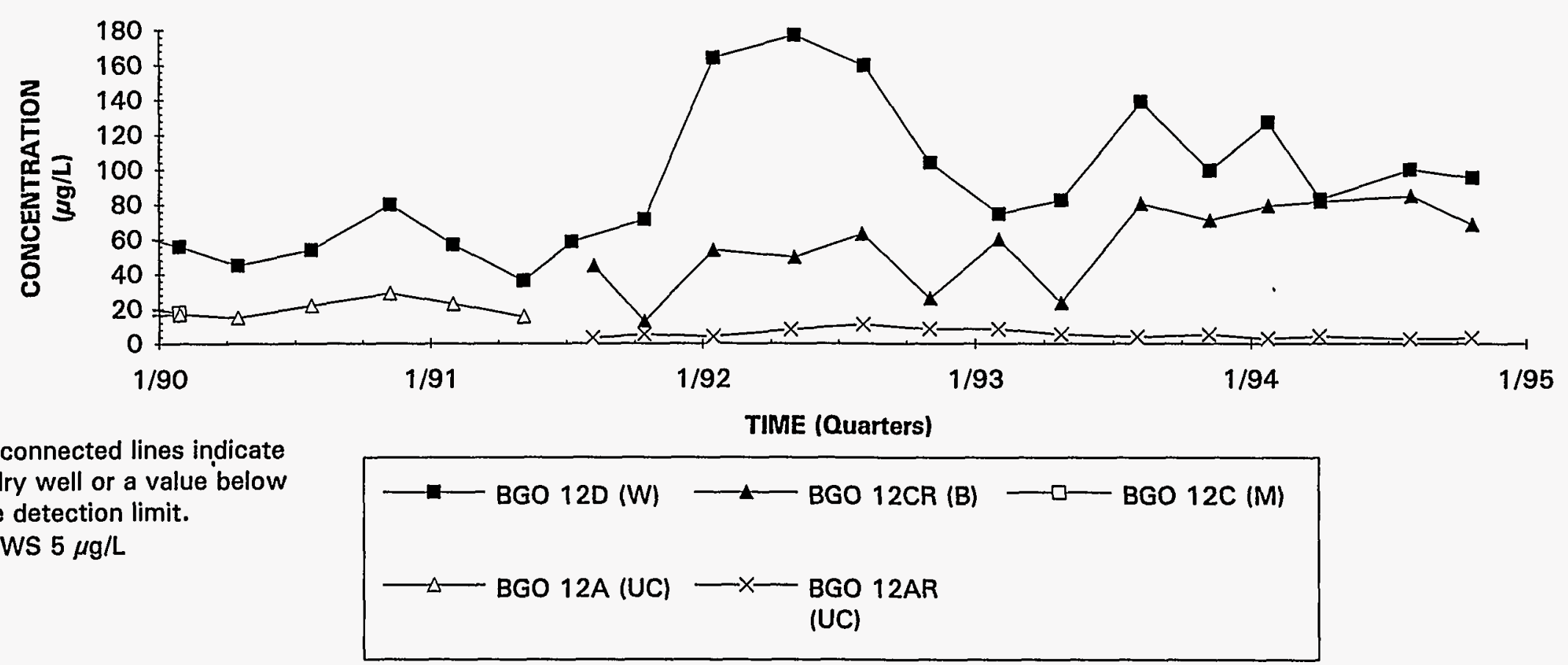

Note: W=Water Table (IIB2); B=Barnwell (IIB1); M=McBean (IIB1); UC=Upper Congaree (IIA); MC=Middle Congaree (IIA); LC=Lower Congaree (IIA) 

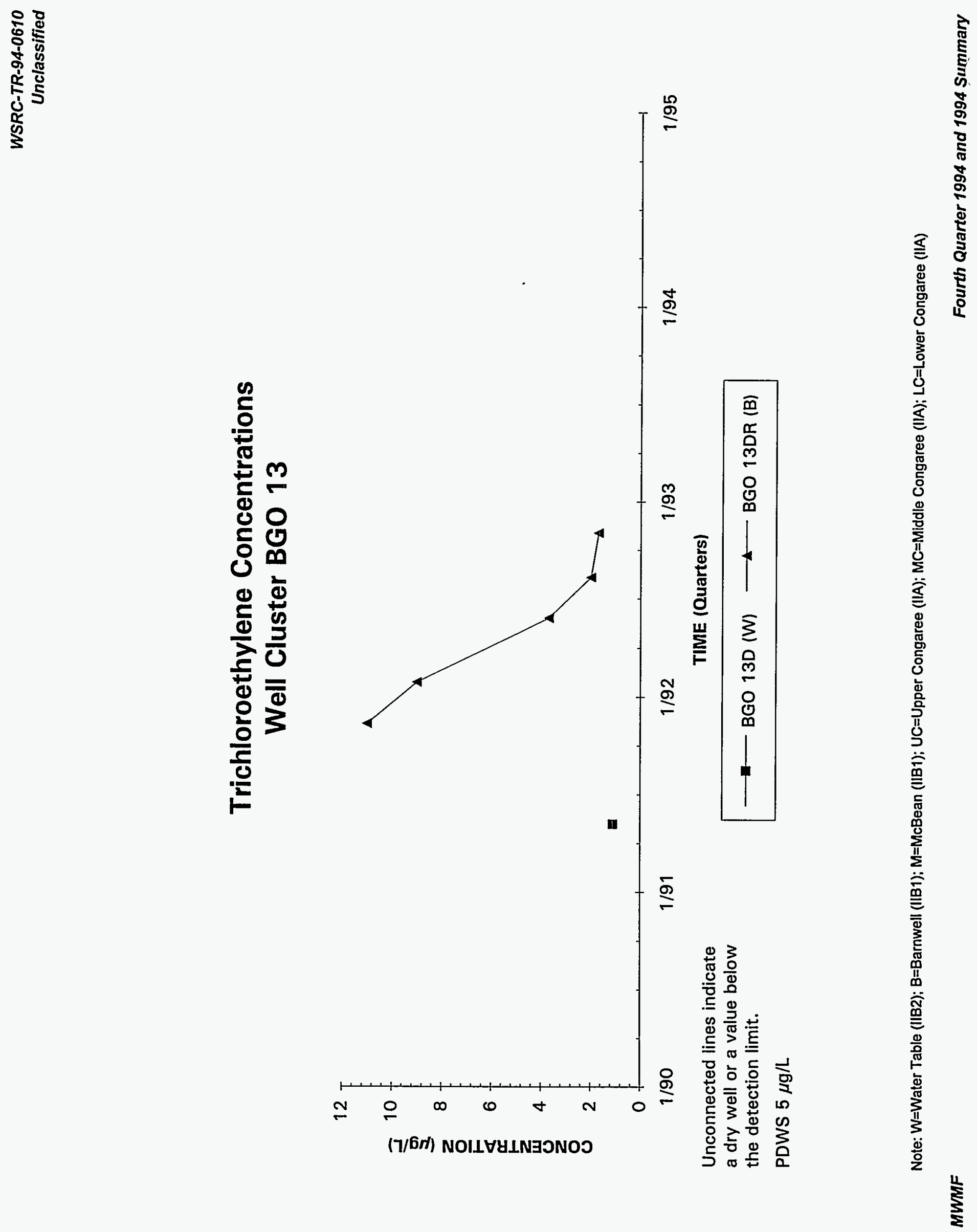


\section{Trichloroethylene Concentrations \\ Well Cluster BGO 14}

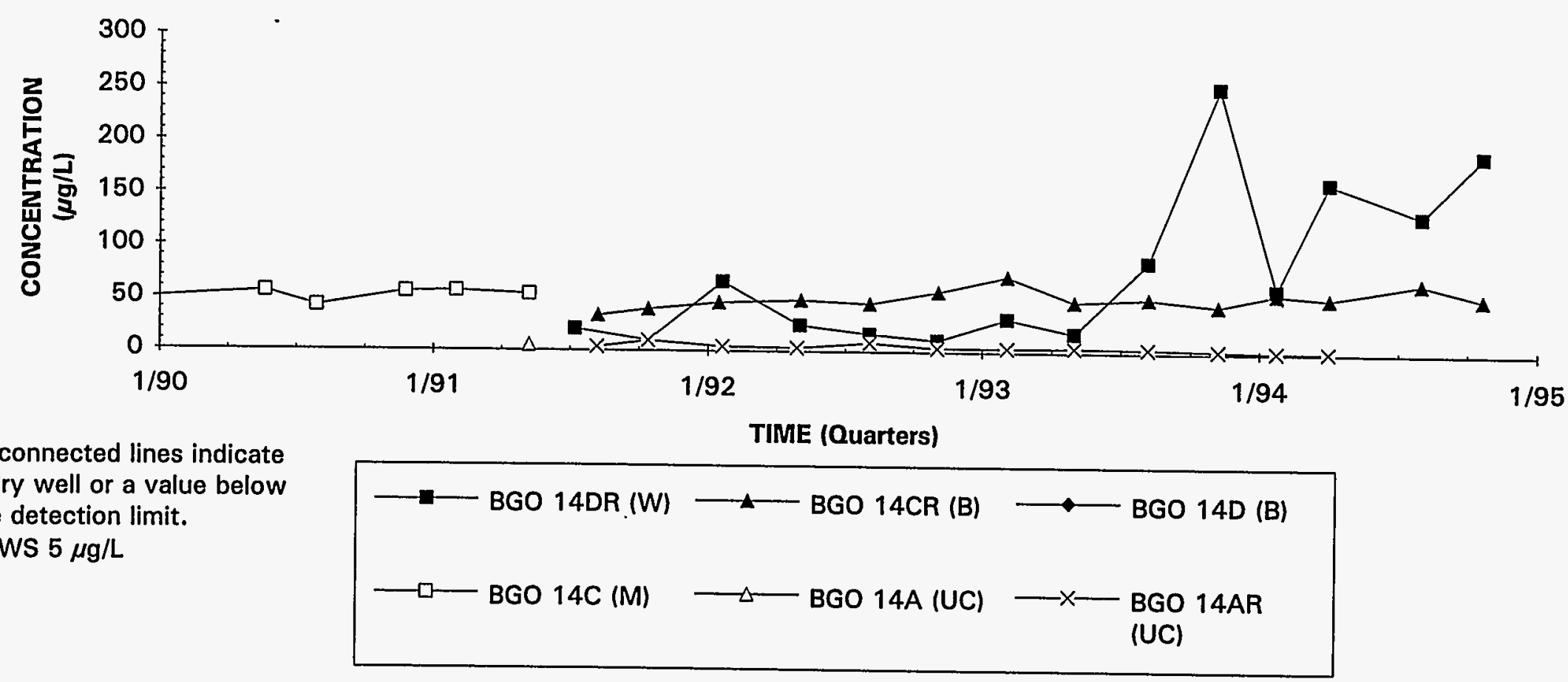

Note: W=Water Table (IIB2); B=Barnwell (IIB1); M=MCBean (IIB1); UC=Upper Congaree (IIA); MC=Middle Congaree (IIA); LC=Lower Congaree (IIA) 

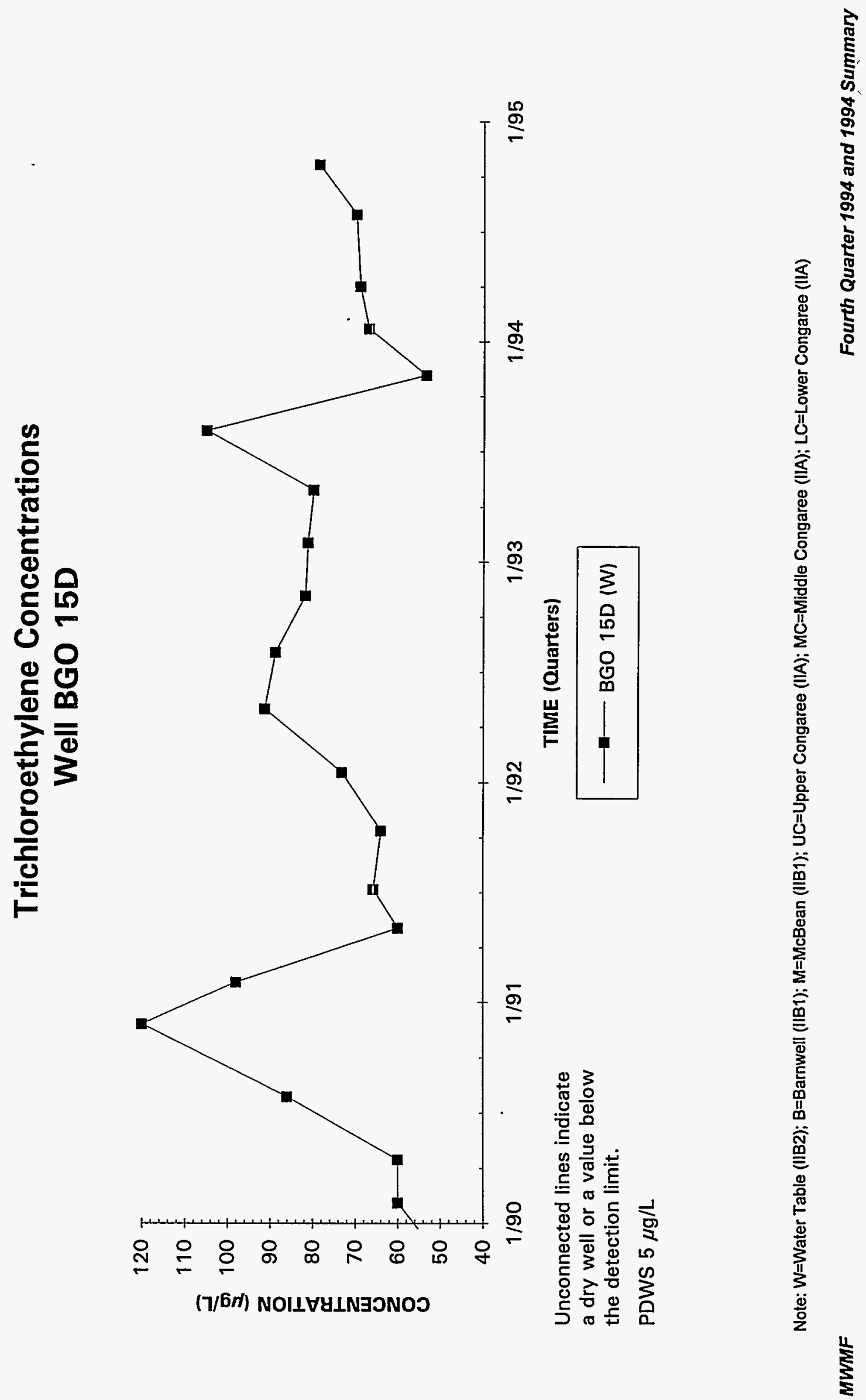


\section{Trichloroethylene Concentrations Well Cluster BGO 16}

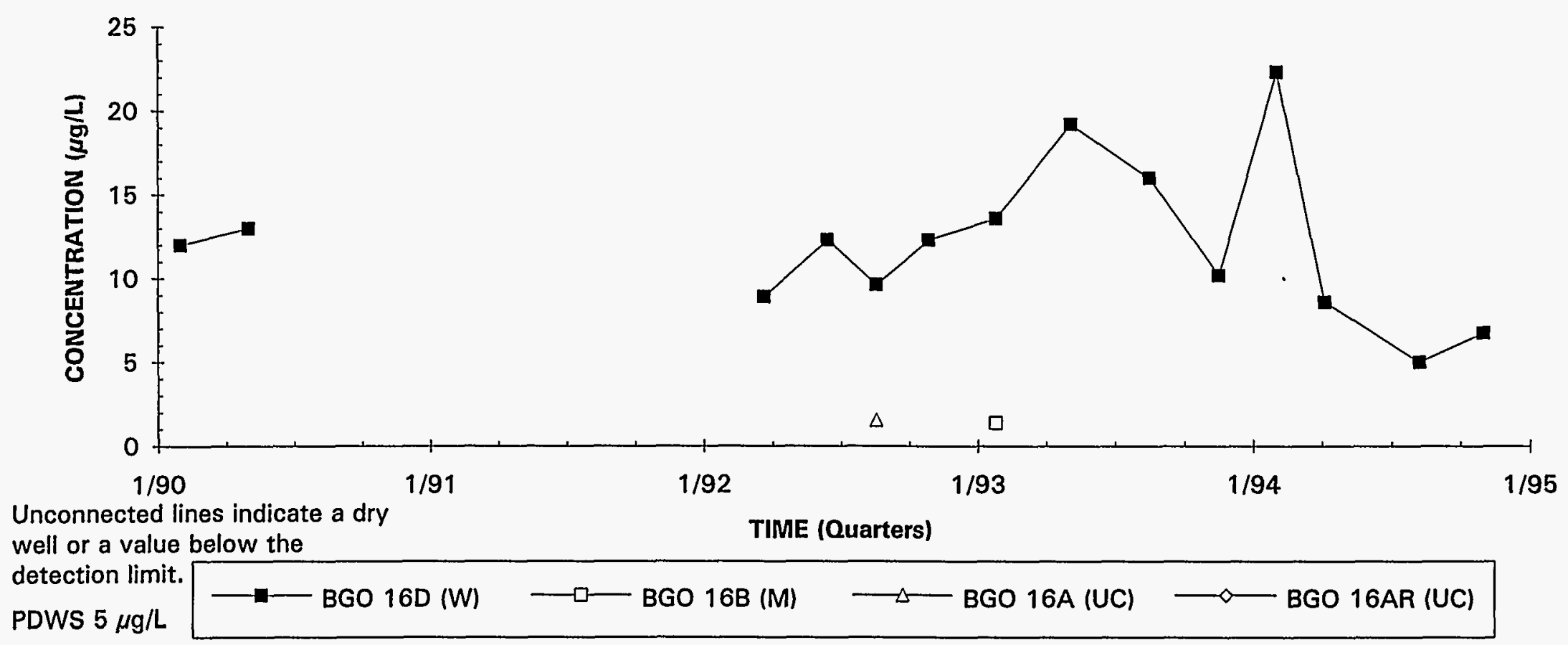

Note: W=Water Table (IIB2); B=Barnwell (IIB1); M=McBean (IIB1); UC=Upper Congaree (IIA); MC=Middle Congaree (IIA); LC=Lower Congaree (IIA) 

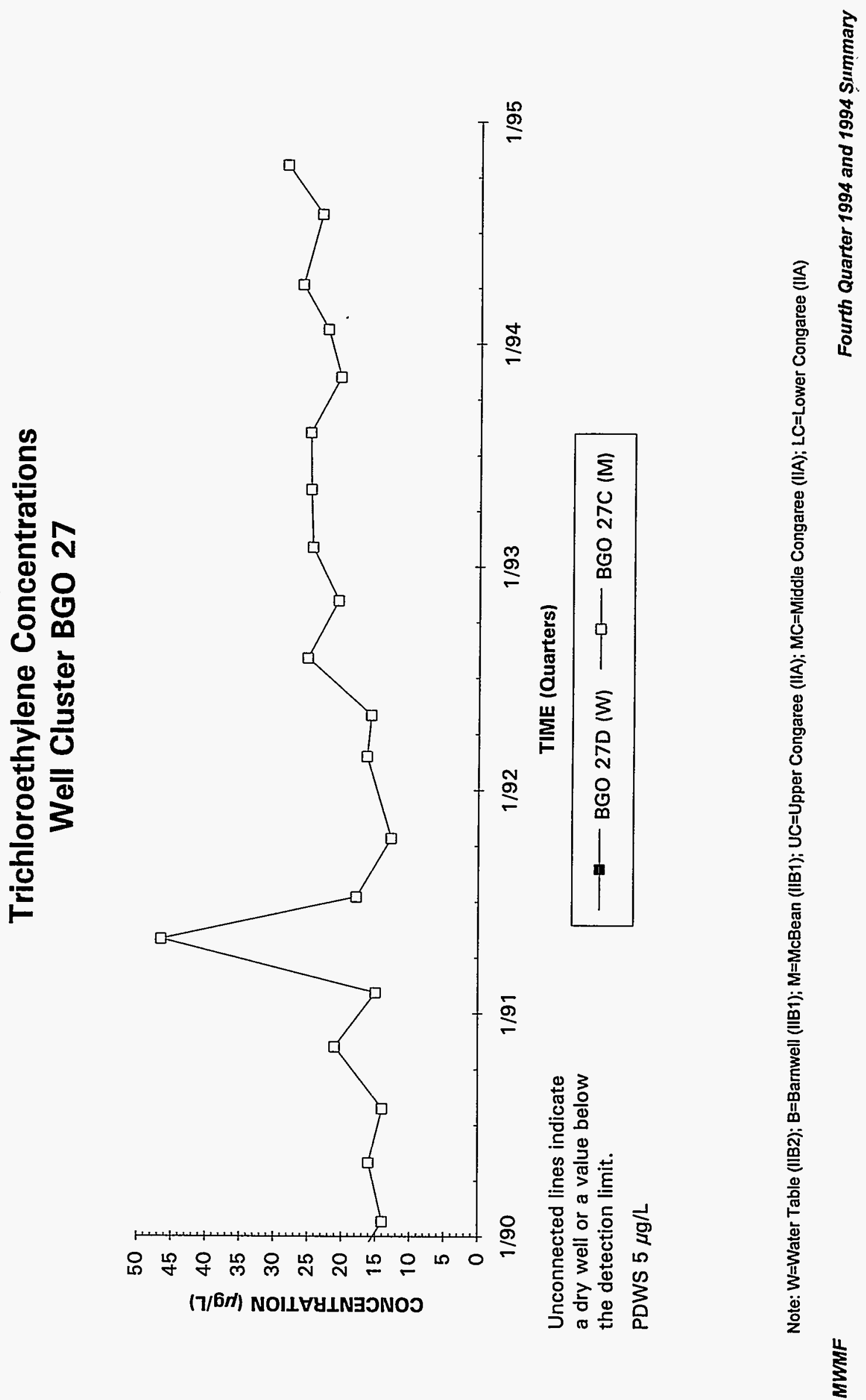

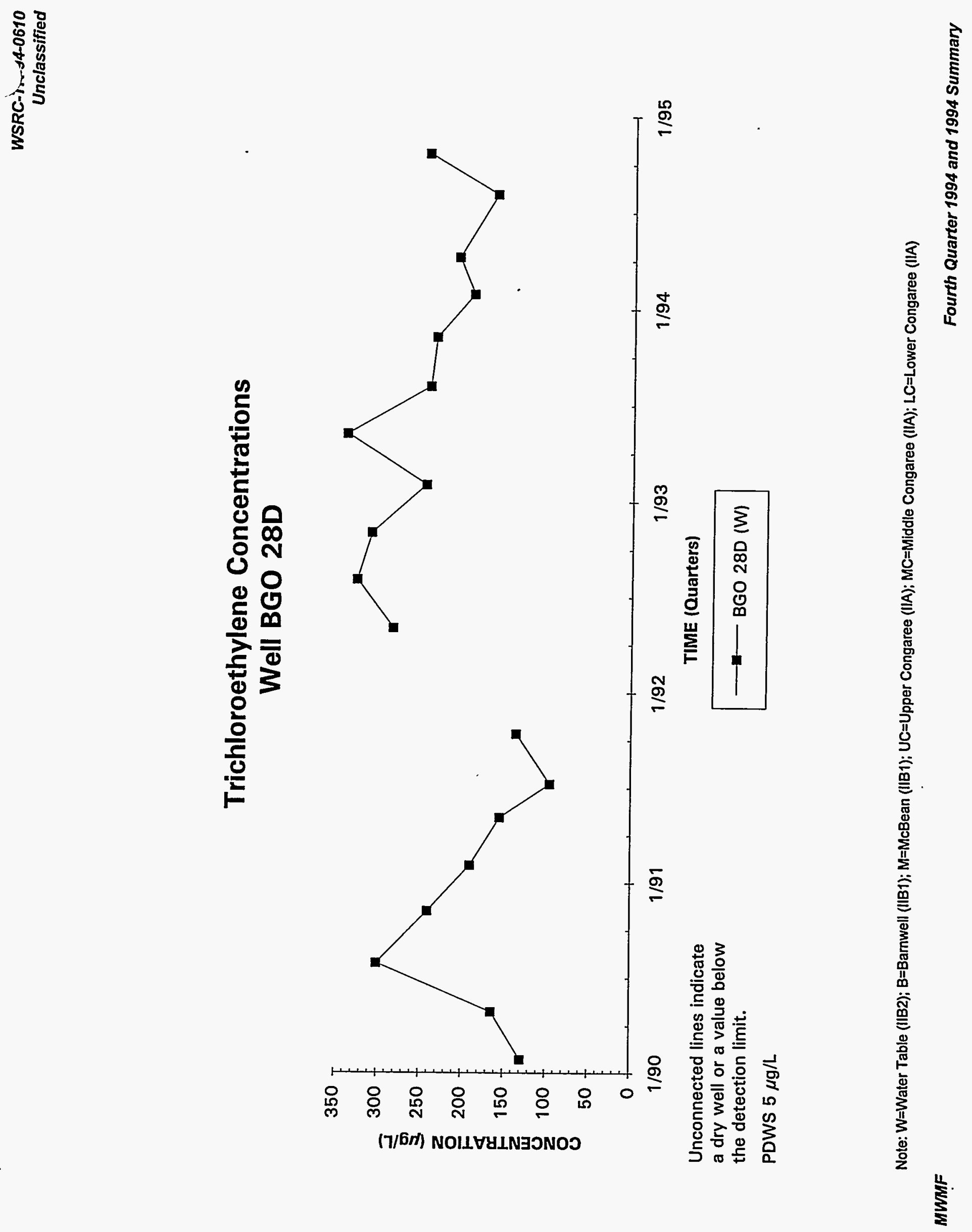


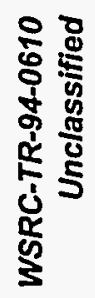

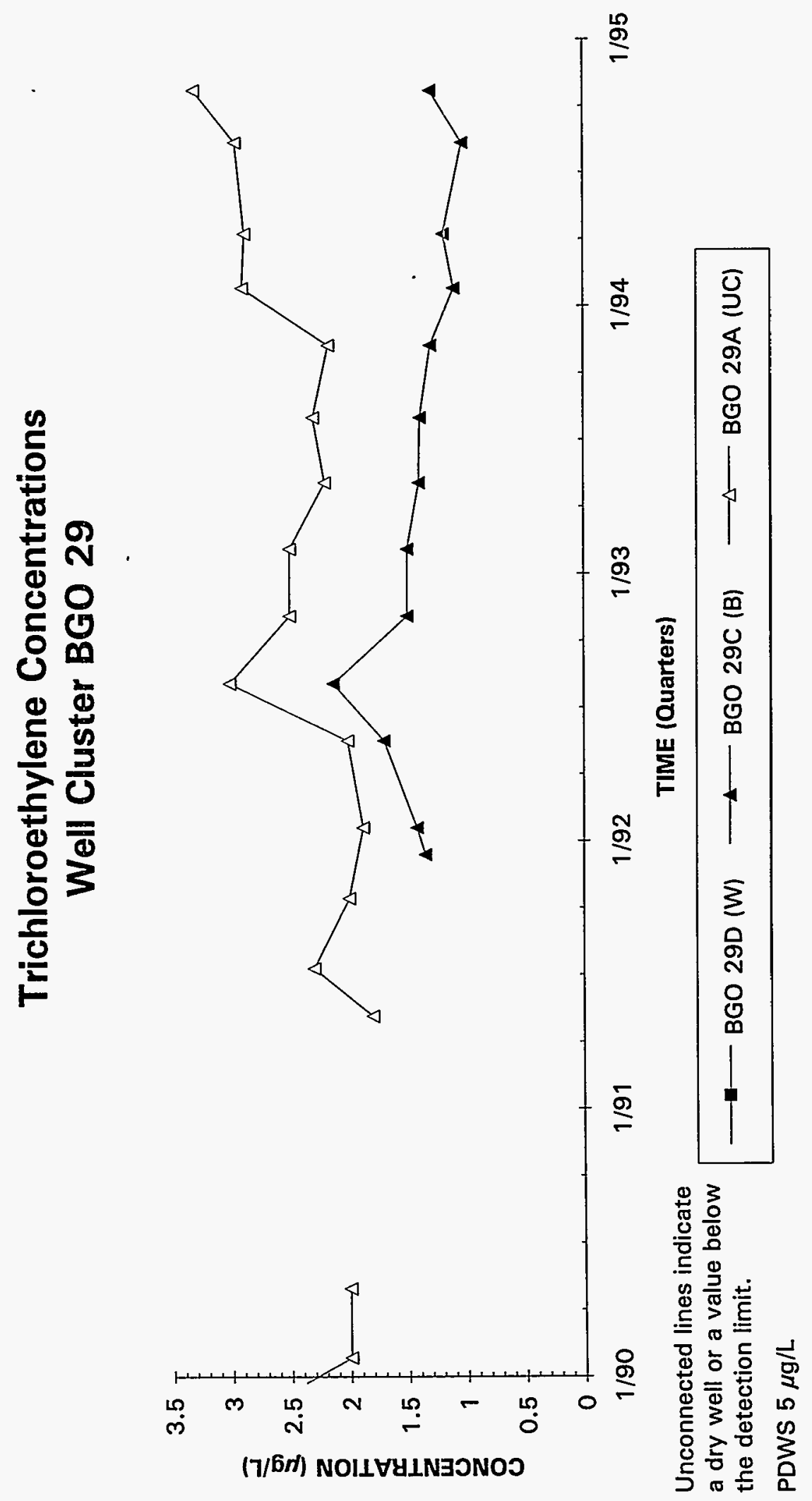



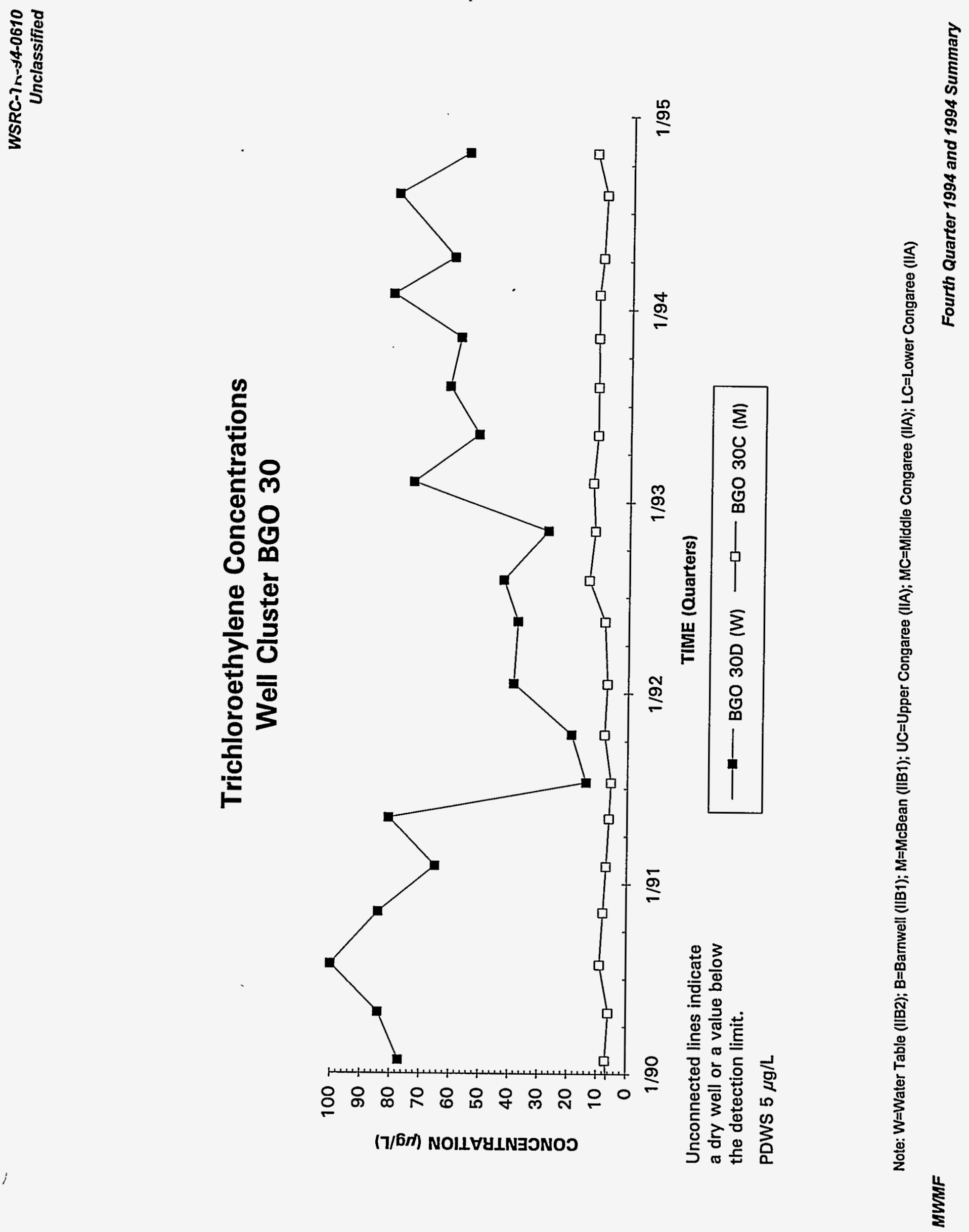

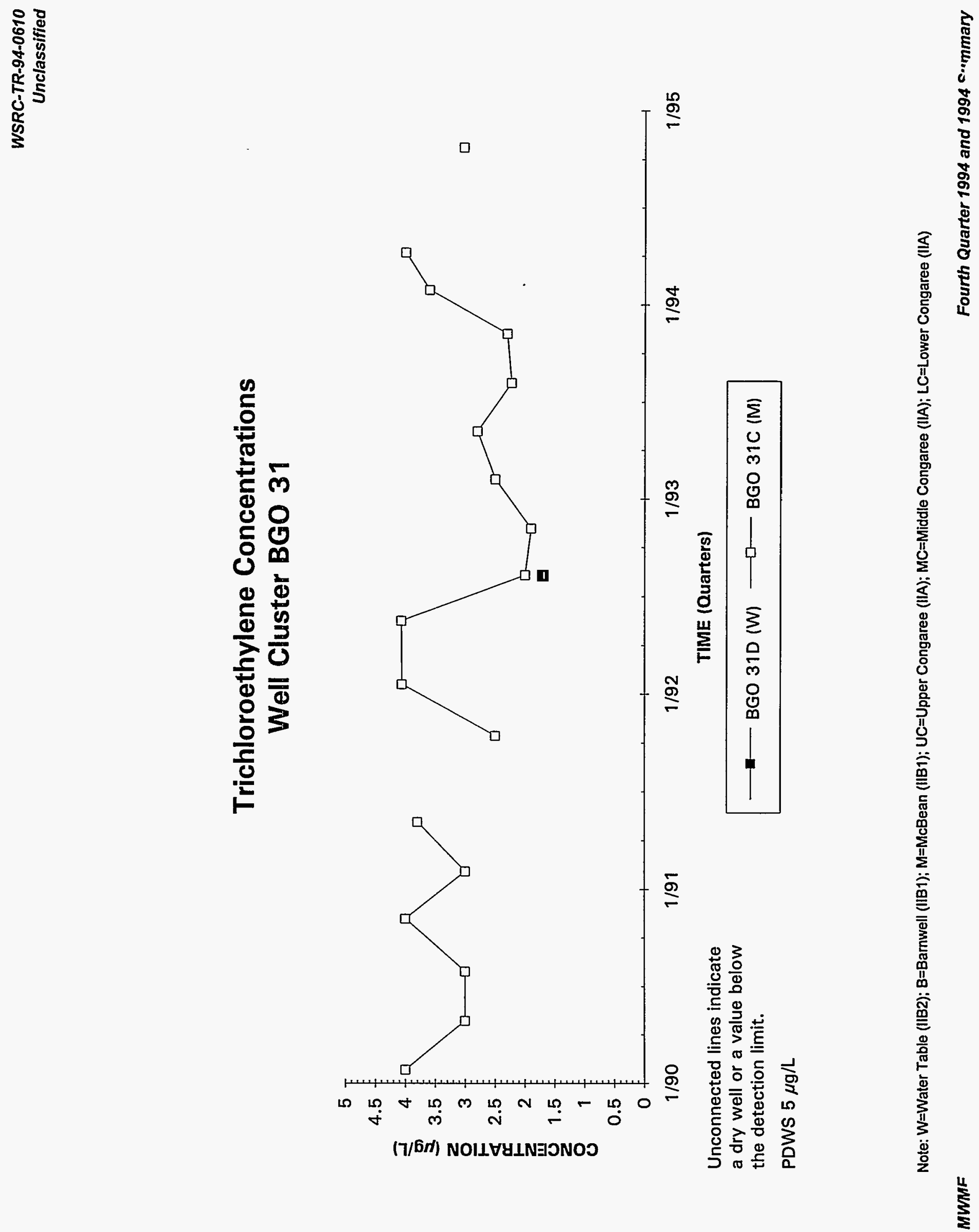


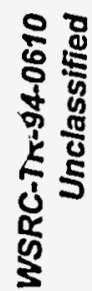
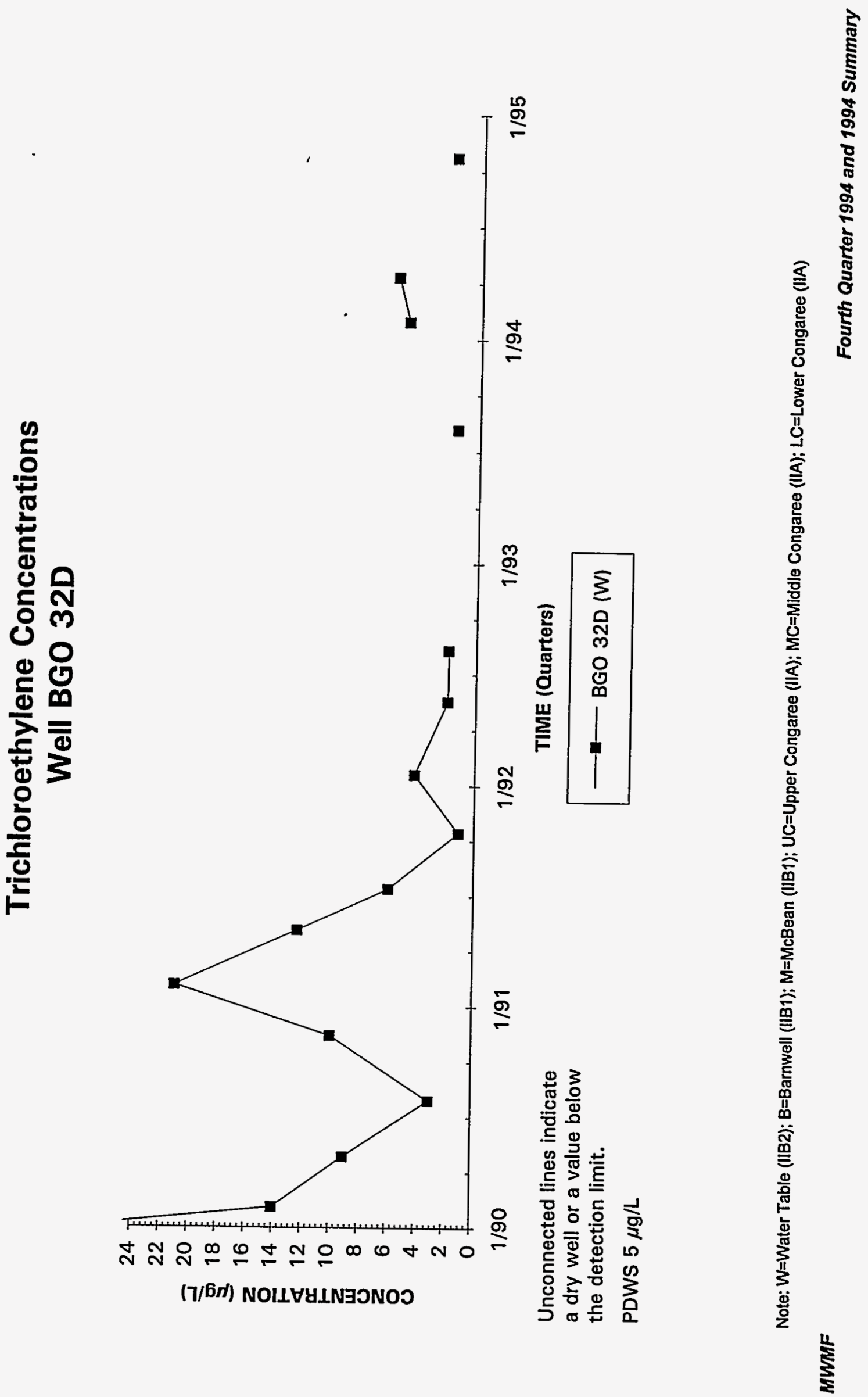

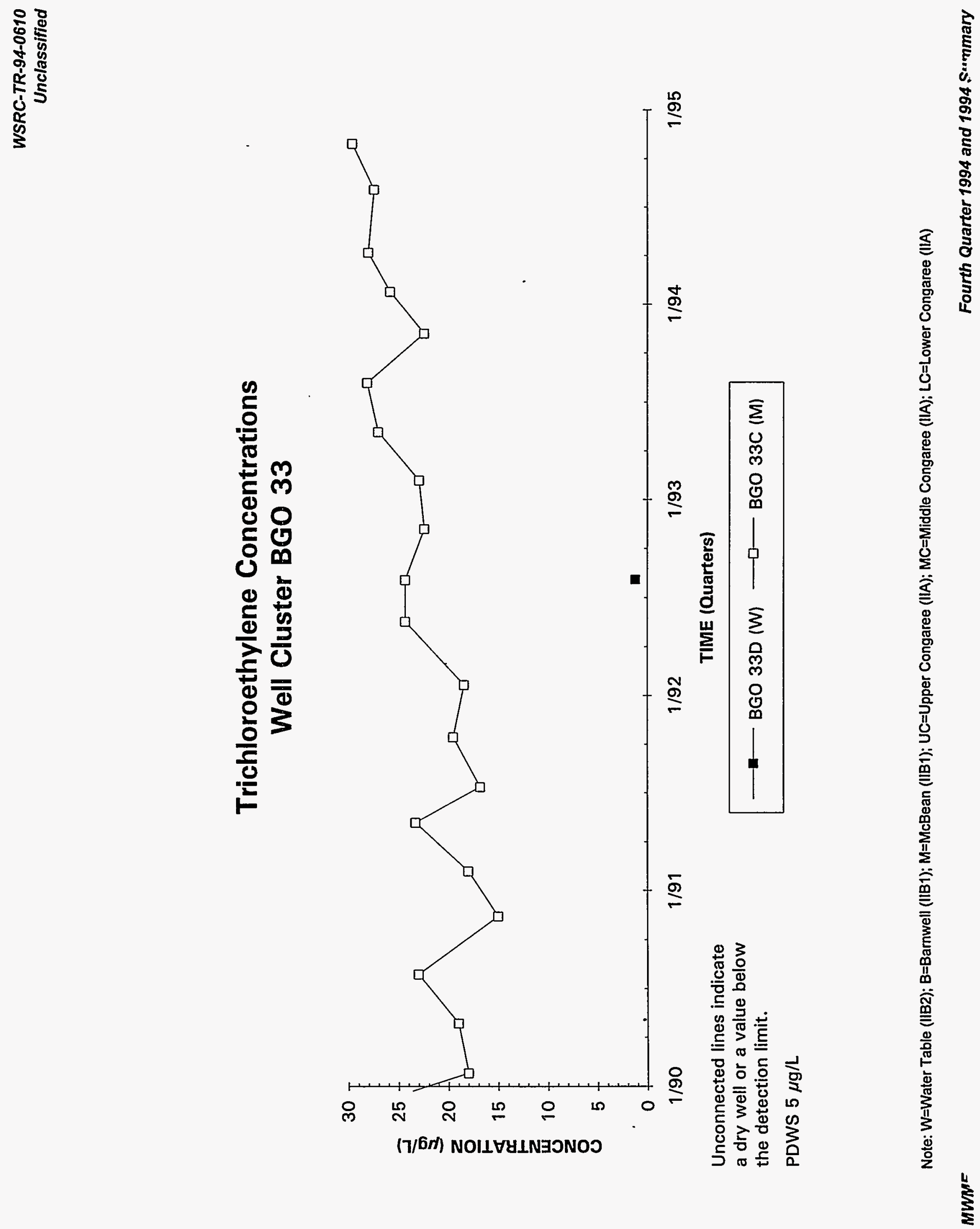

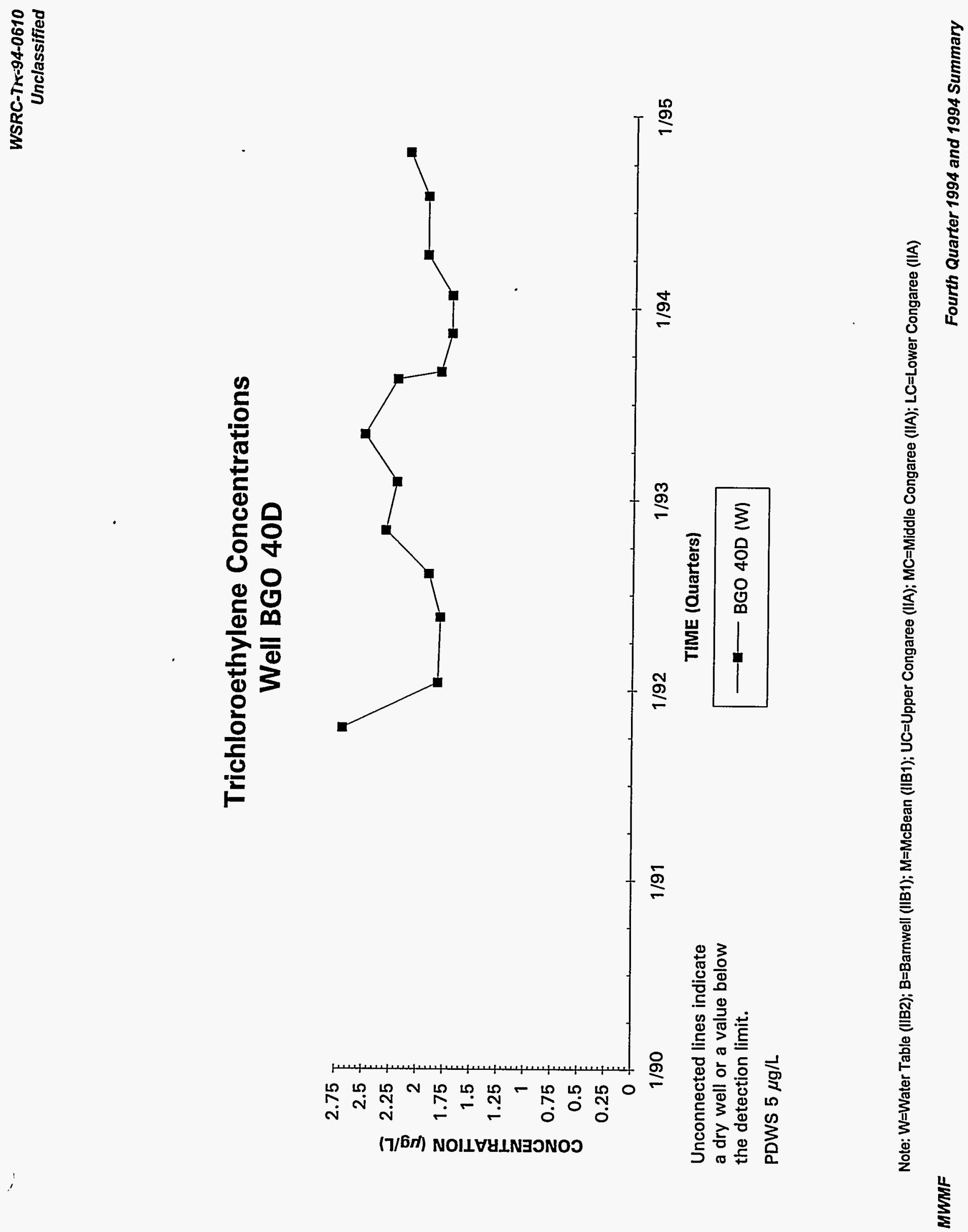

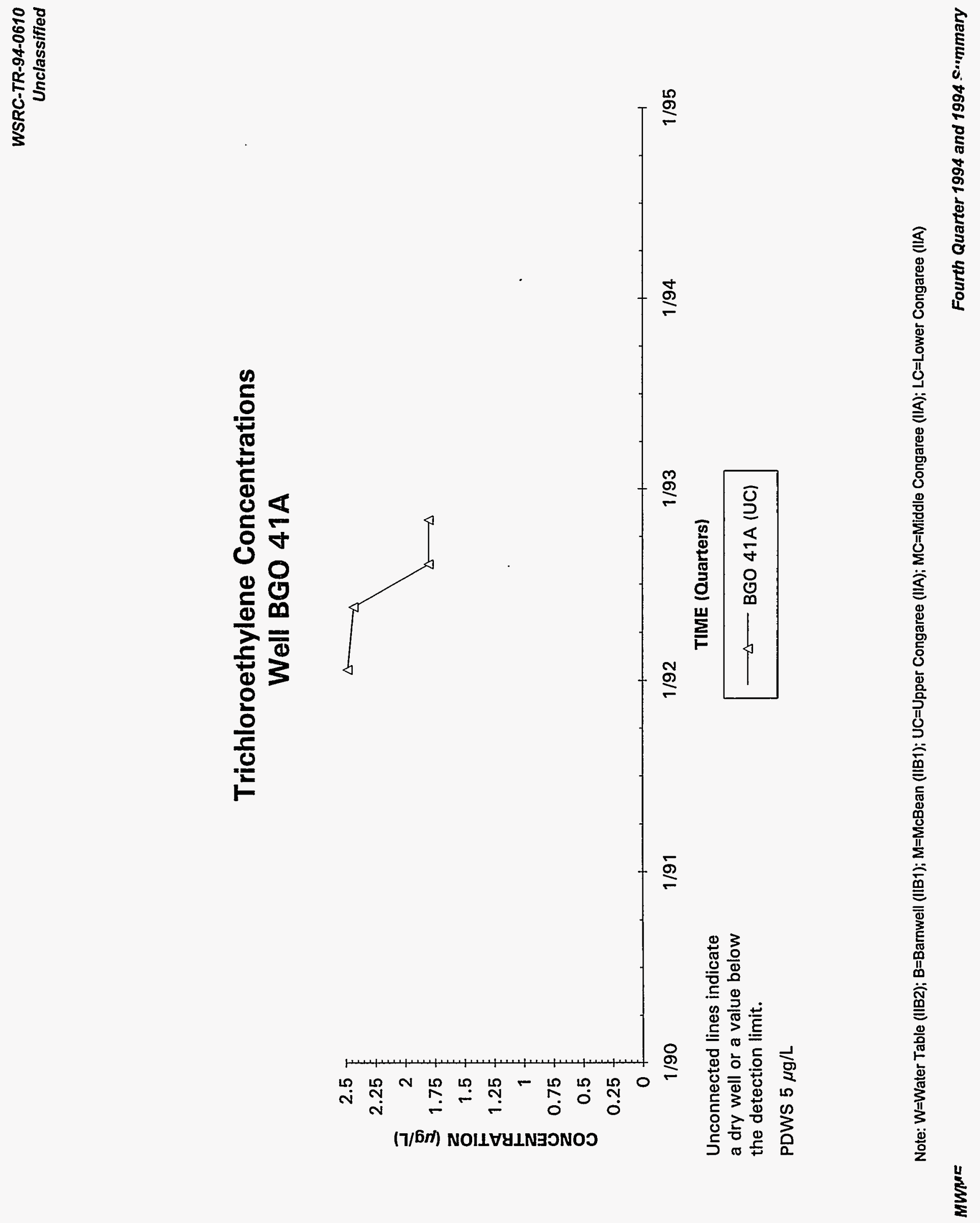


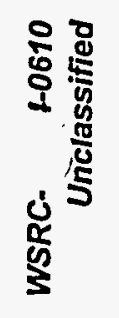
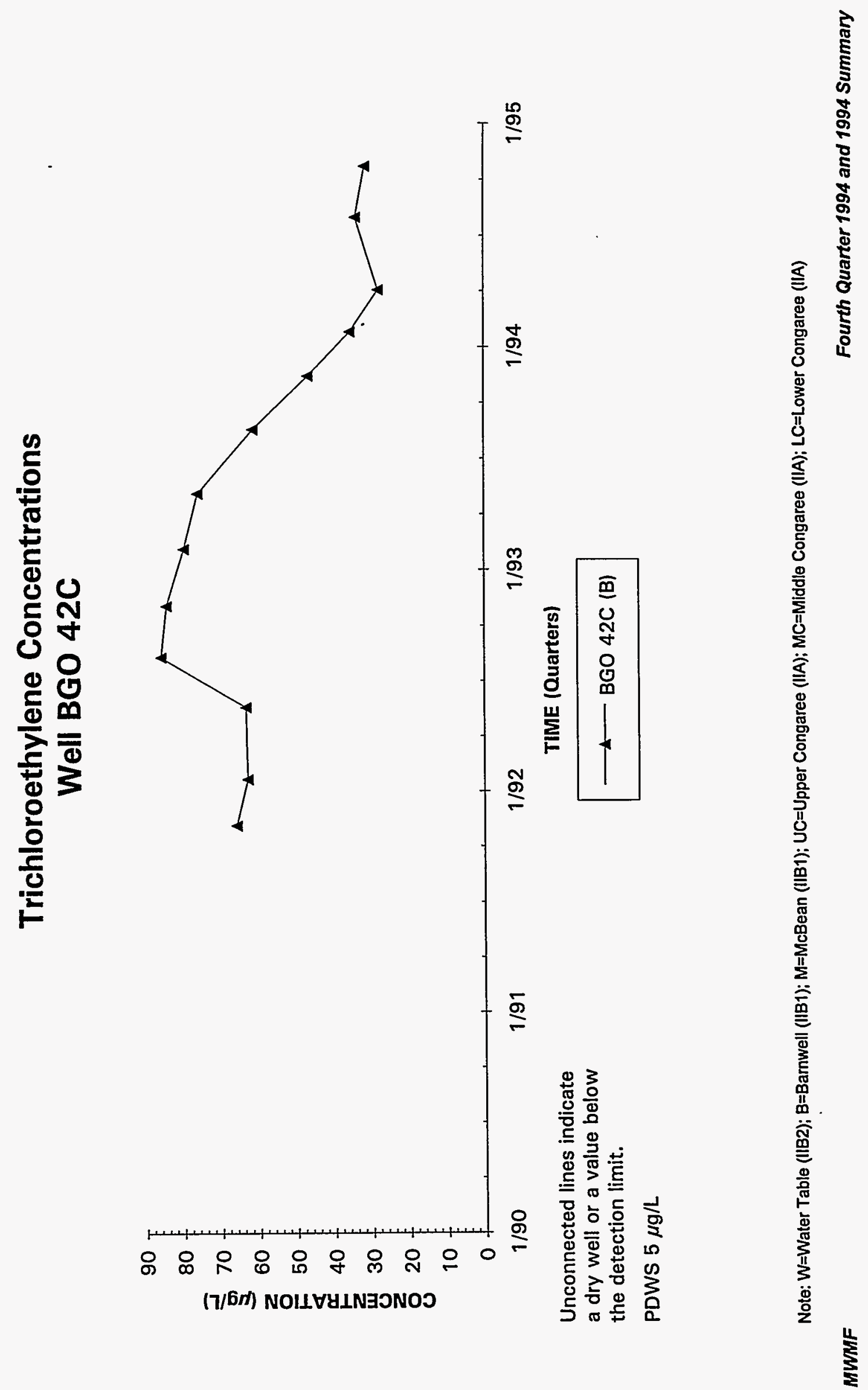


\section{Trichloroethylene Concentrations} Well Cluster BGO 44

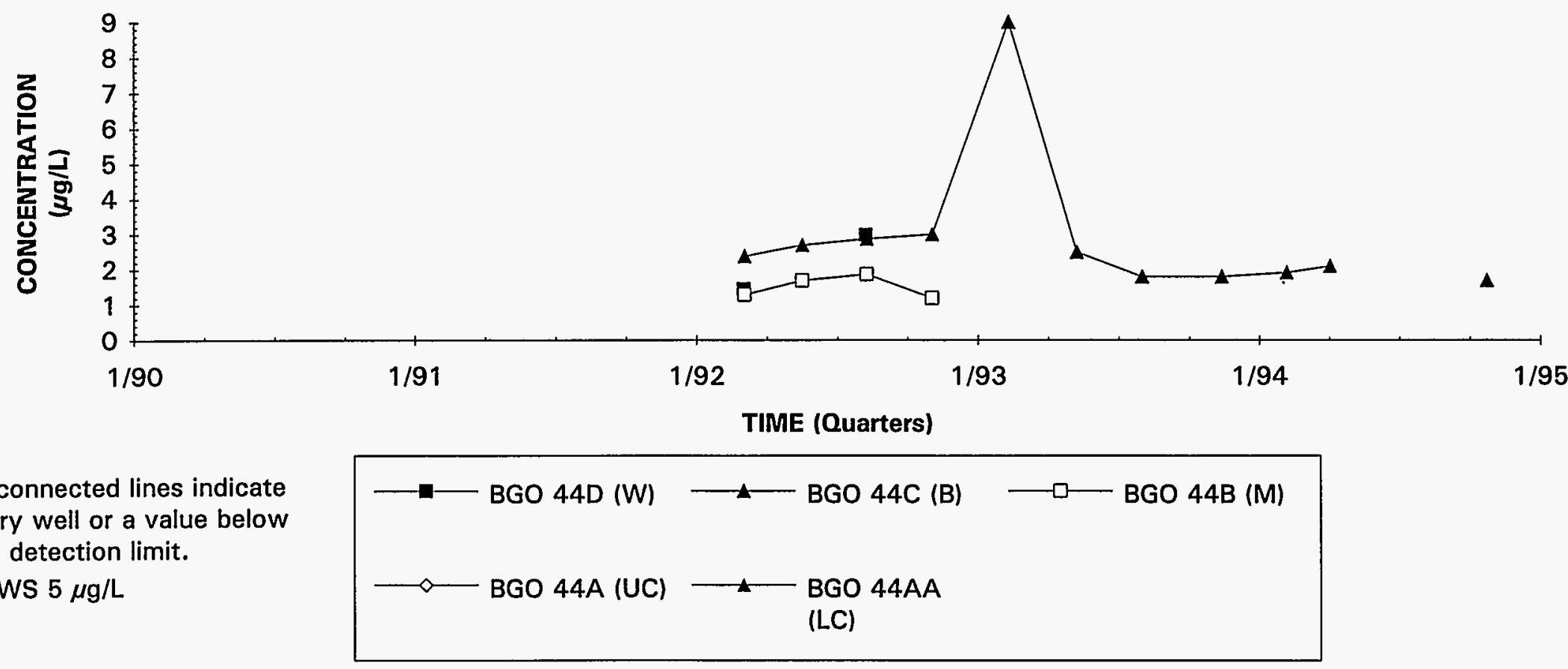

Note: W=Water Table (IIB2); B=Barnwell (IIB1); M=McBean (IIB1); UC=Upper Congaree (IIA); MC=Middle Congaree (IIA); LC=Lower Congaree (IIA) 


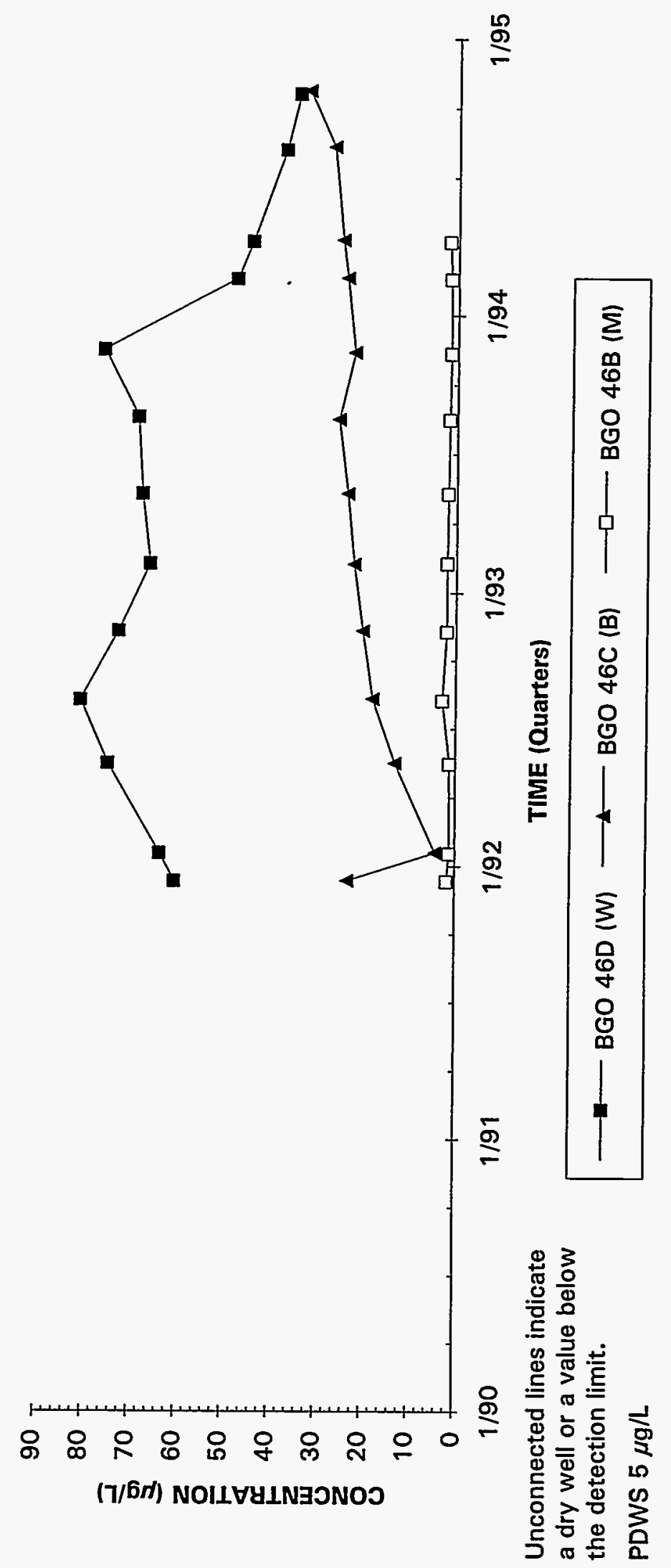



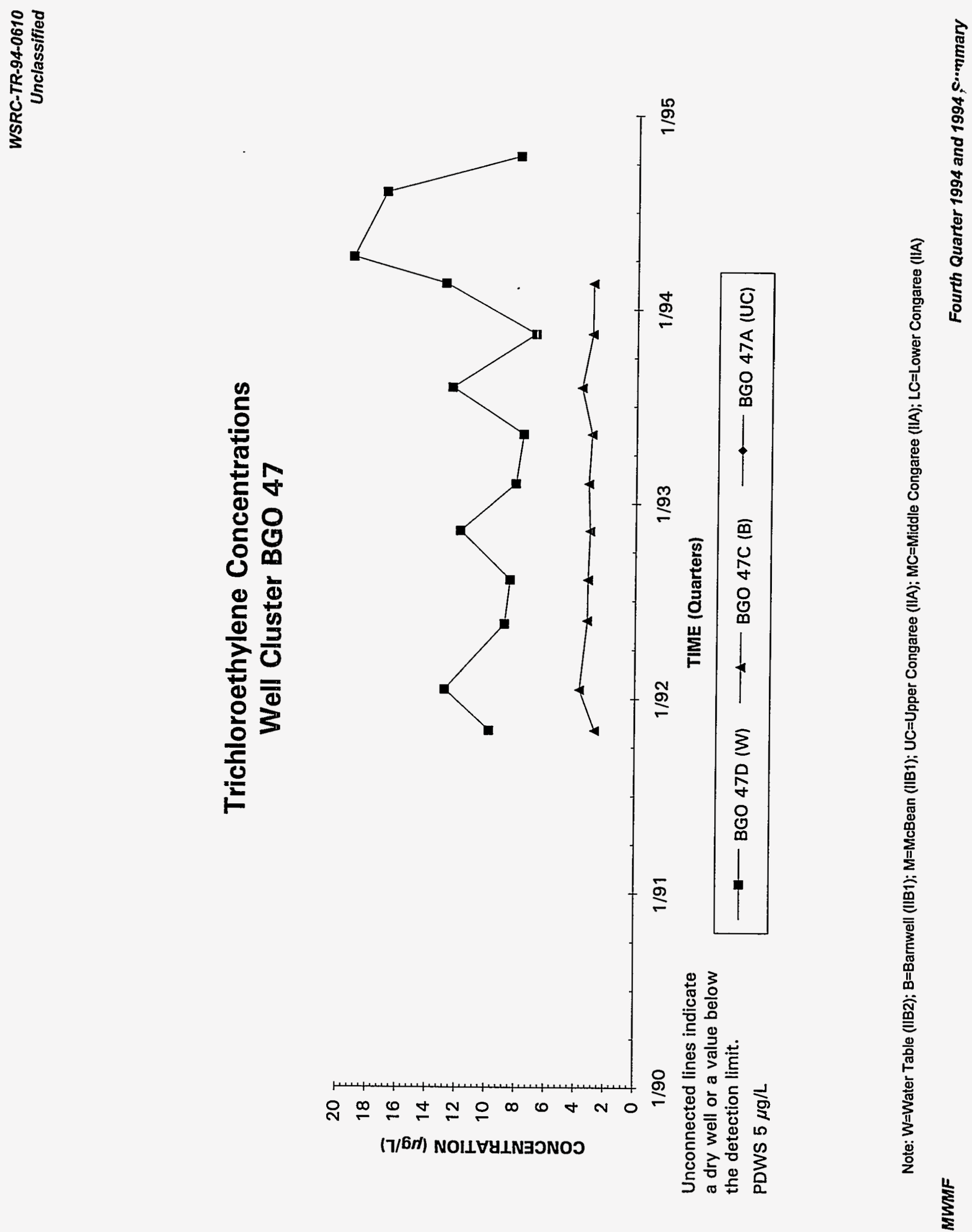


\section{Trichloroethylene Concentrations Well Cluster BGO 48}

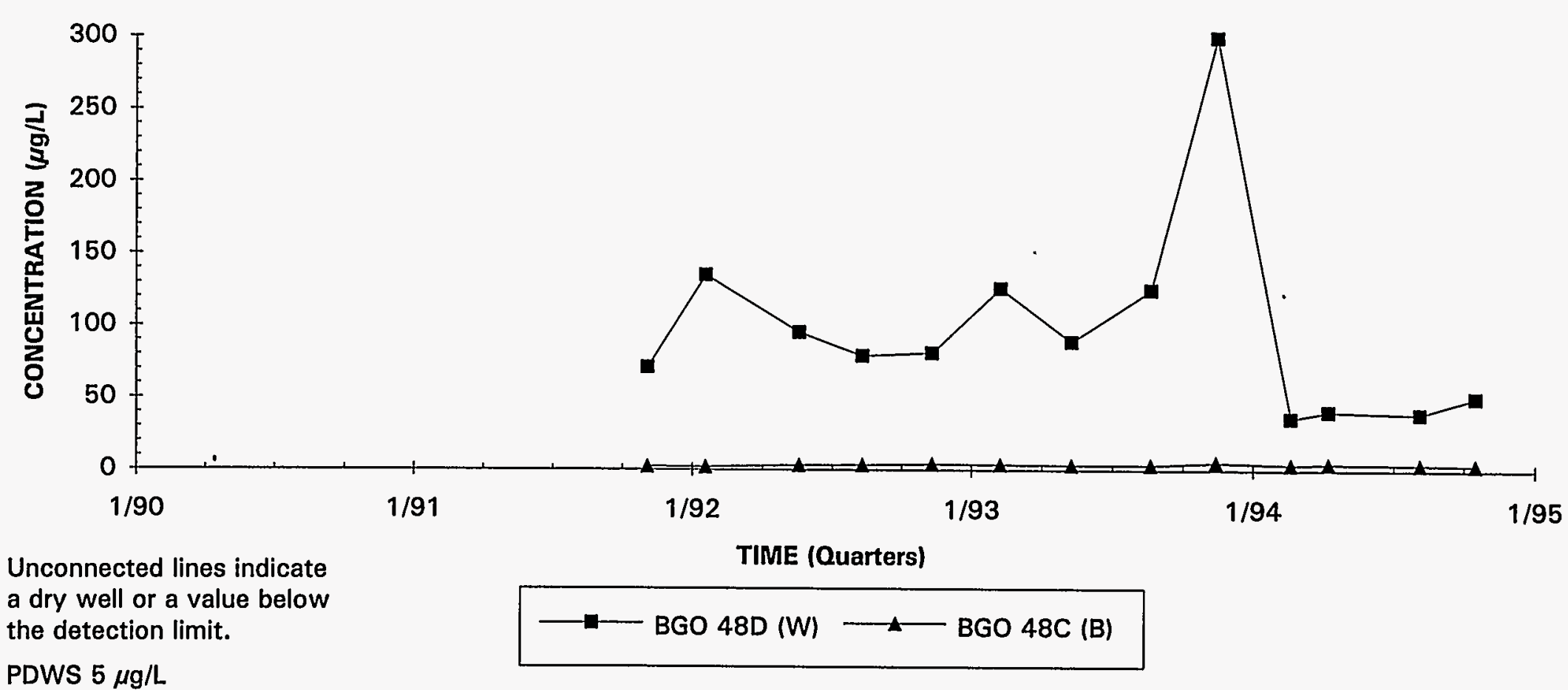

Note: W=Water Table (IIB2); B=Barnwell (IIB1); M=McBean (IIB1); UC=Upper Congaree (IIA); MC=Middle Congaree (IIA); LC=Lower Congaree (IIA) 


\section{Trichloroethylene Concentrations \\ Well Cluster BGO 50}

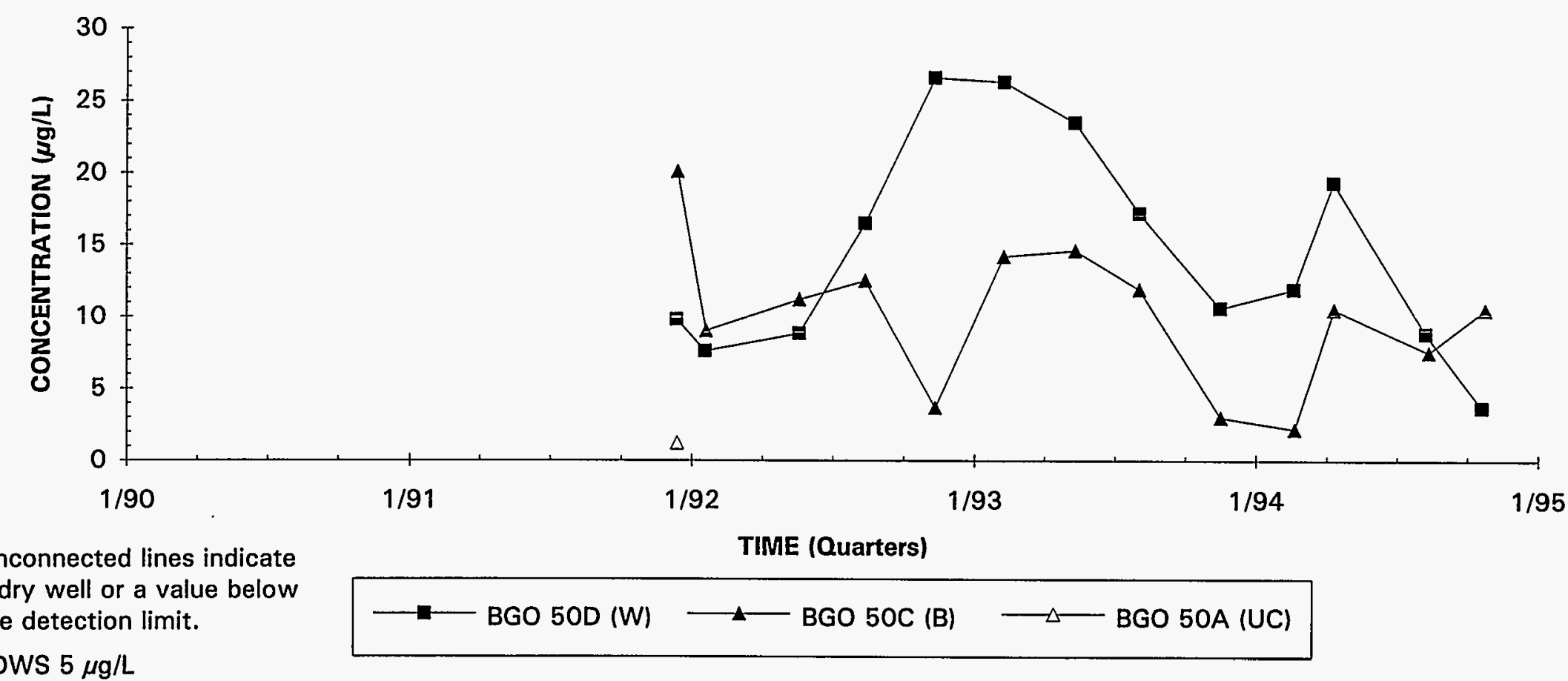

Note: W=Water Table (IIB2); B=Barnwell (IIB1); $M=$ McBean (IIB1); UC=Upper Congaree (IIA); MC=Middle Congaree (IIA); LC=Lower Congaree (IIA) 


\section{Trichloroethylene Concentrations} Well Cluster BGX 2

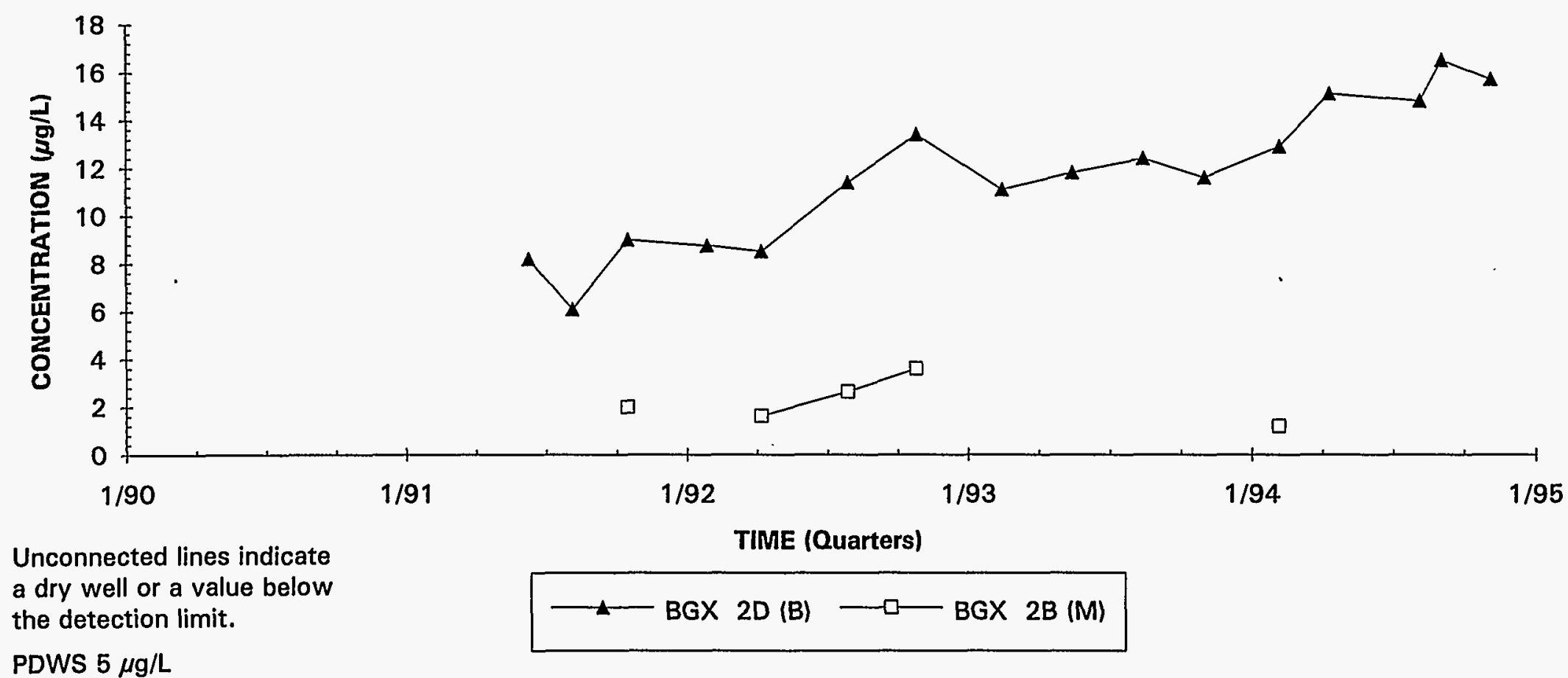

PDWS $5 \mu \mathrm{g} / \mathrm{L}$ 

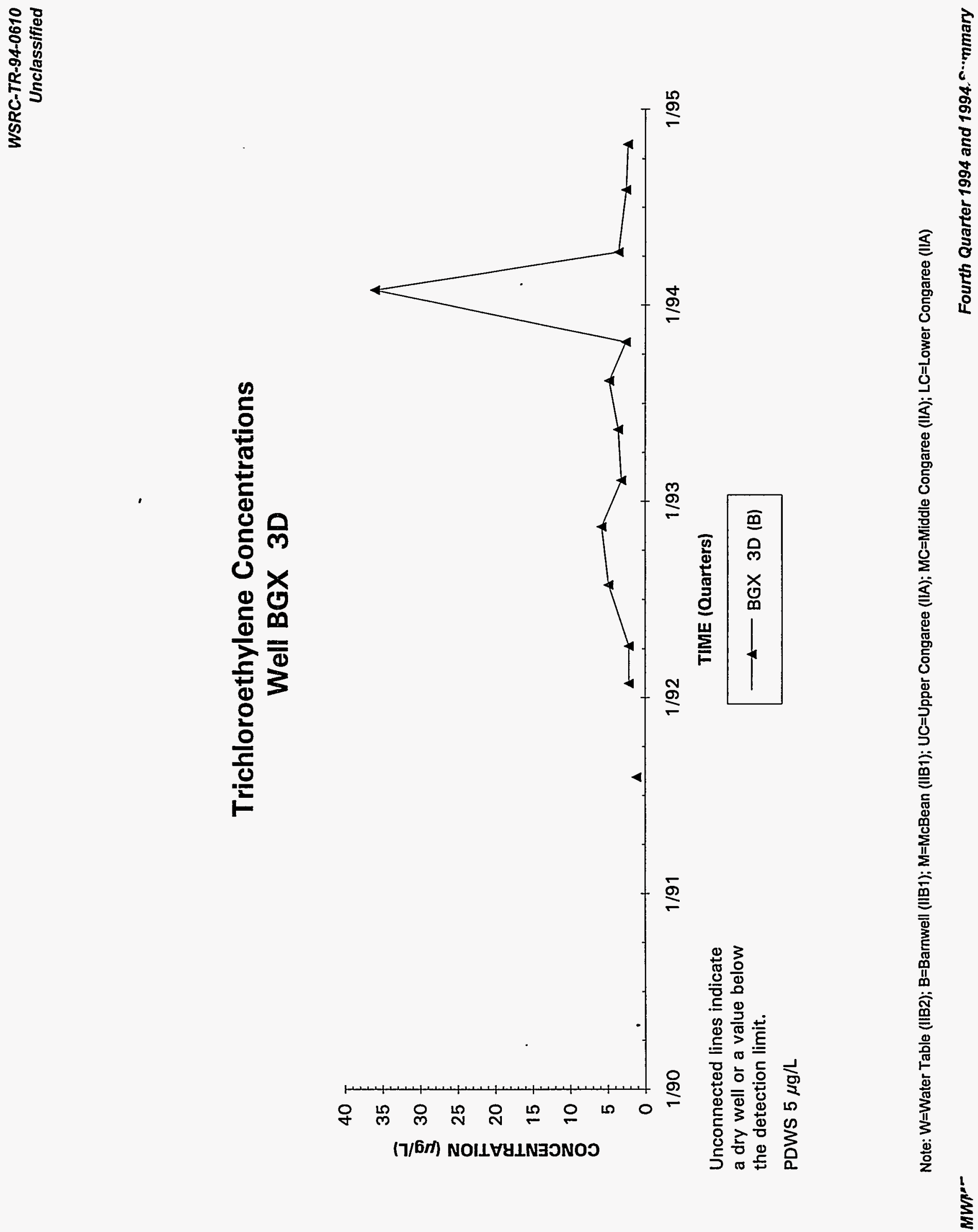


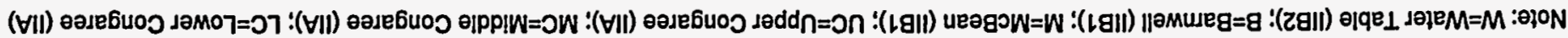

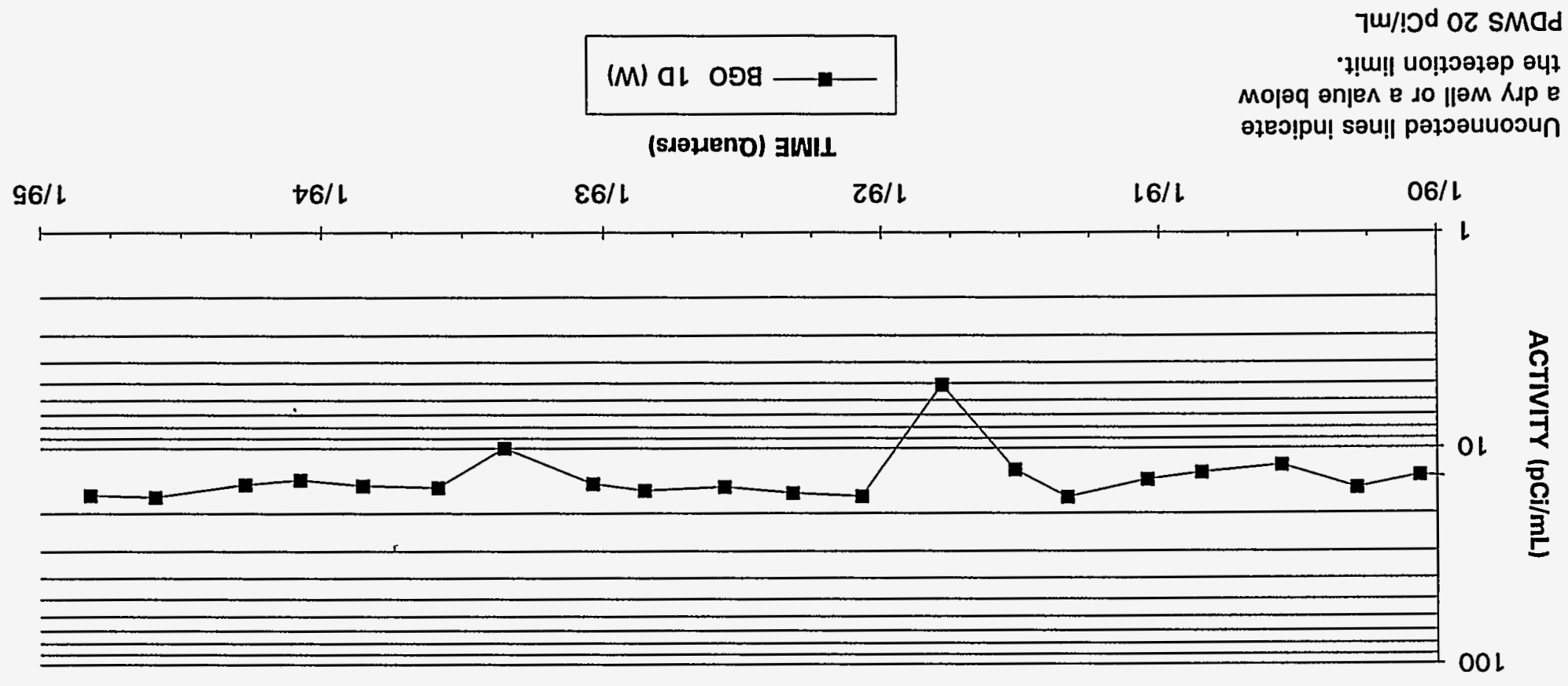

al OSg \|әM

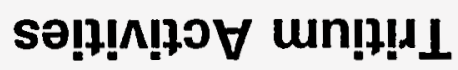




\section{Tritium Activities \\ Well BGO 2D}

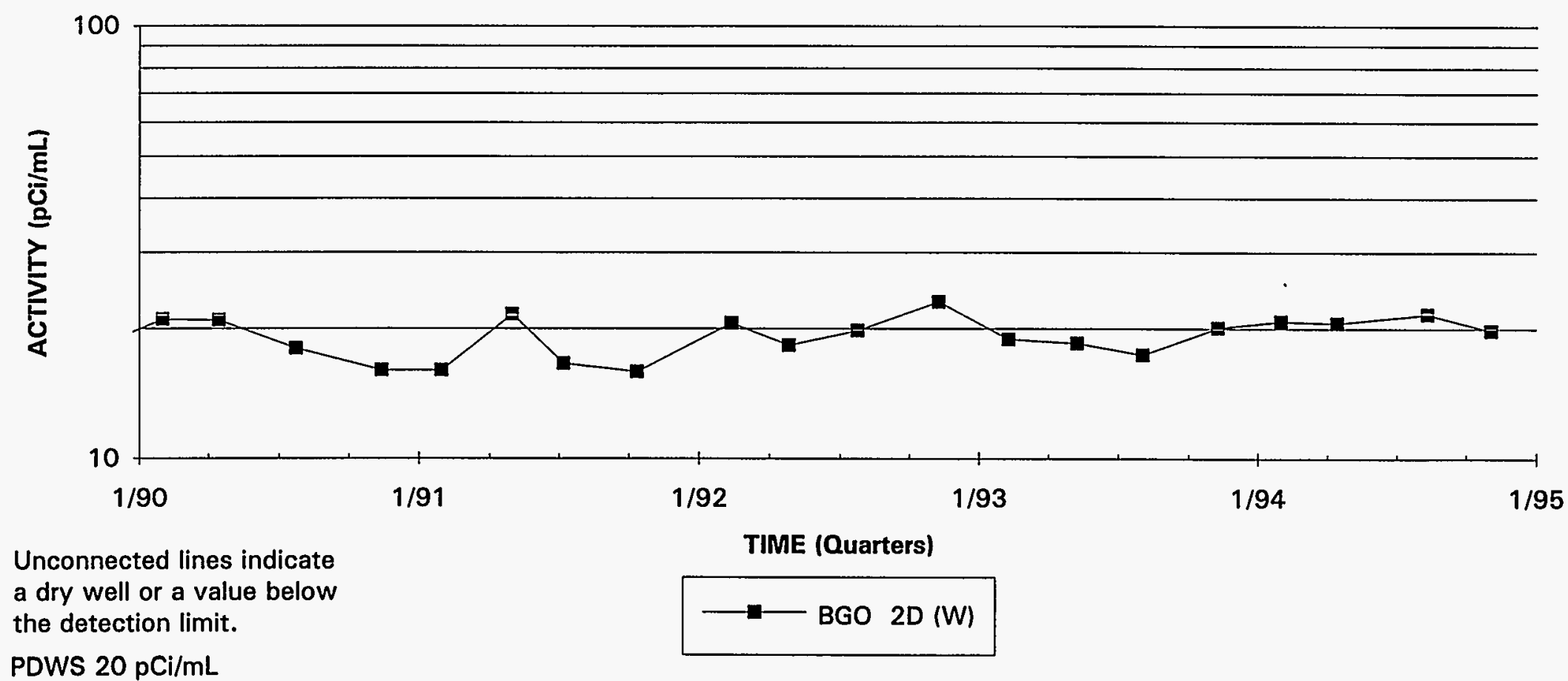

Note: W=Water Table (IIB2); B=Barnwell (IIB1); M=McBean (IIB1); UC=Upper Congaree (IIA); MC=Middle Congaree (IIA); LC=Lower Congaree (IIA) 


\section{Tritium Activities} Well BGO 3D

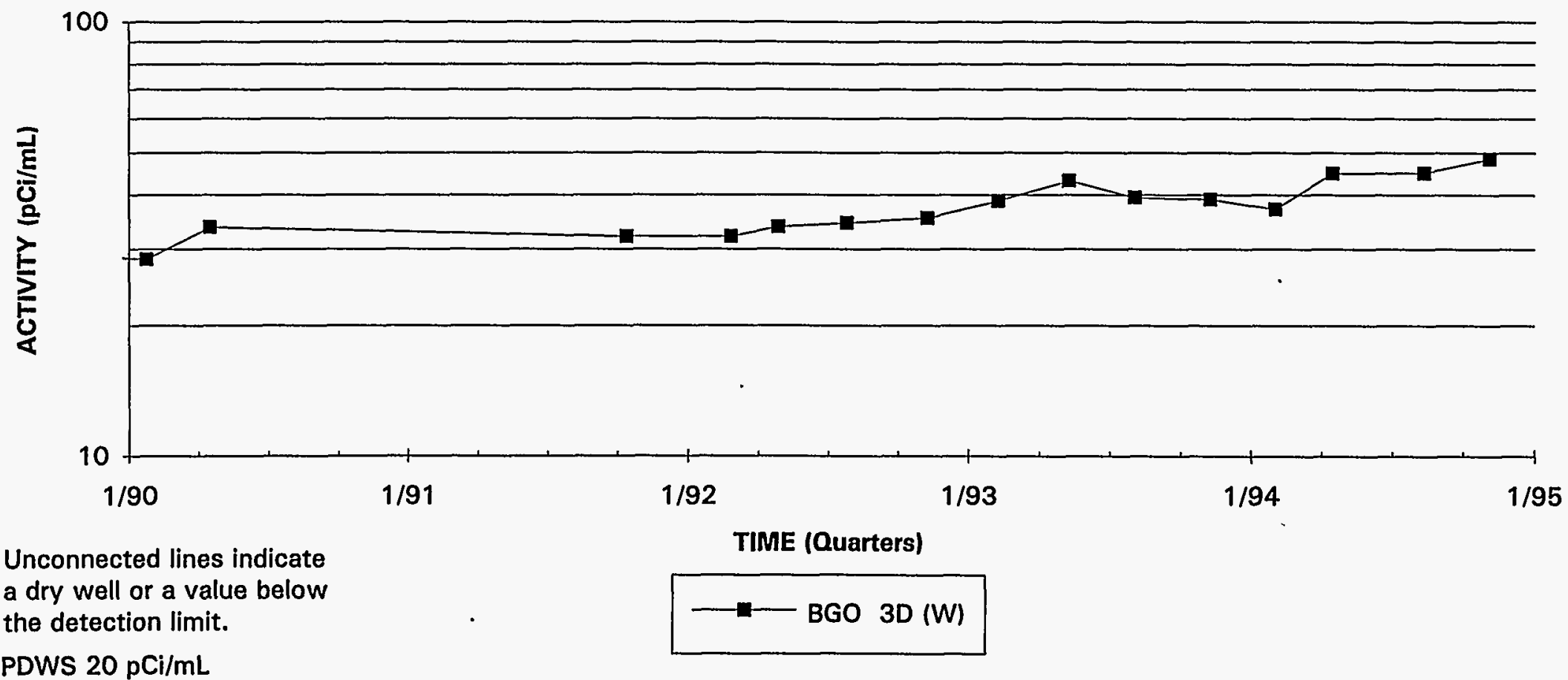

Note: W=Water Table (IIB2); B=Barnwell (IIB1); M=McBean (IIB1); UC=Upper Congaree (IIA); MC=Middle Congaree (IIA); LC=Lower Congaree (IIA) 


\section{Tritium Activities}

Well BGO 4D

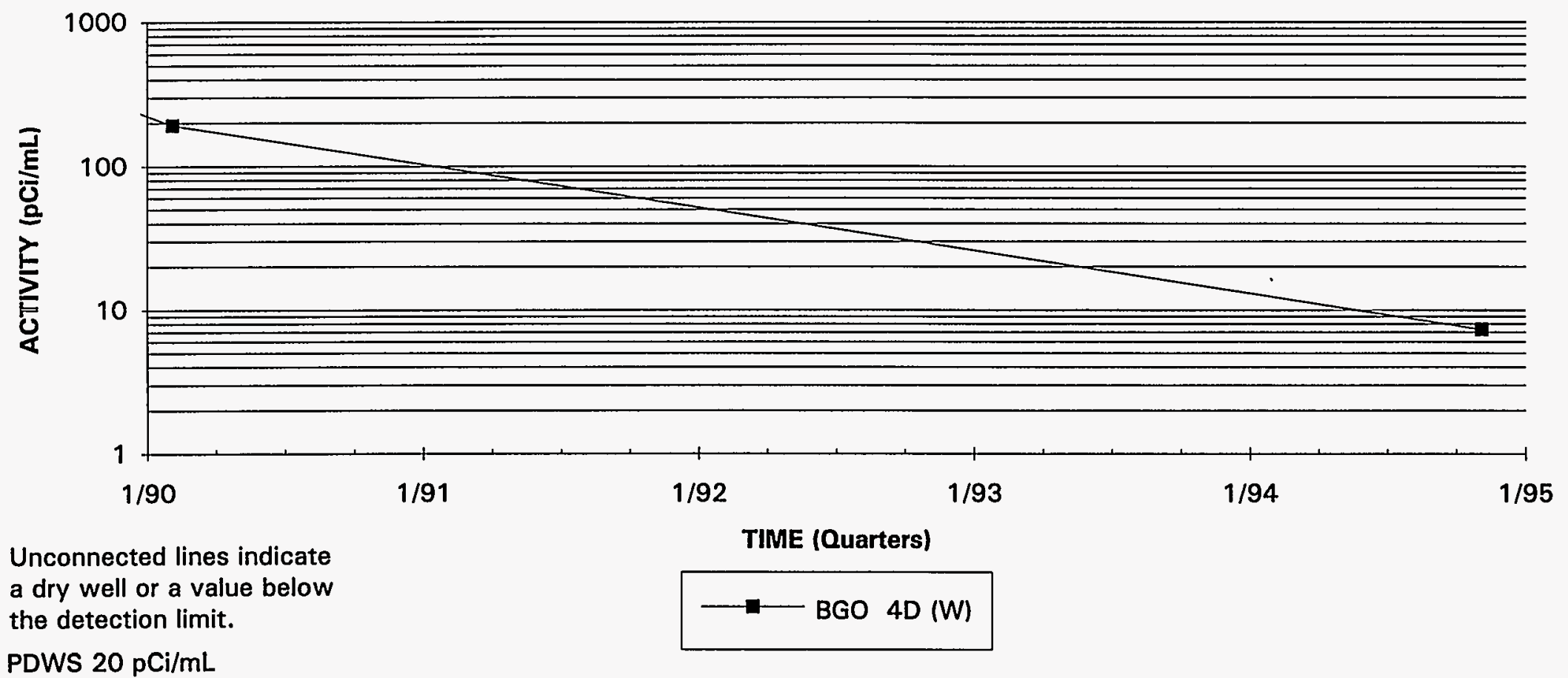

PDWS $20 \mathrm{pCi} / \mathrm{mL}$ 
Tritium Activities Well Cluster BGO 5

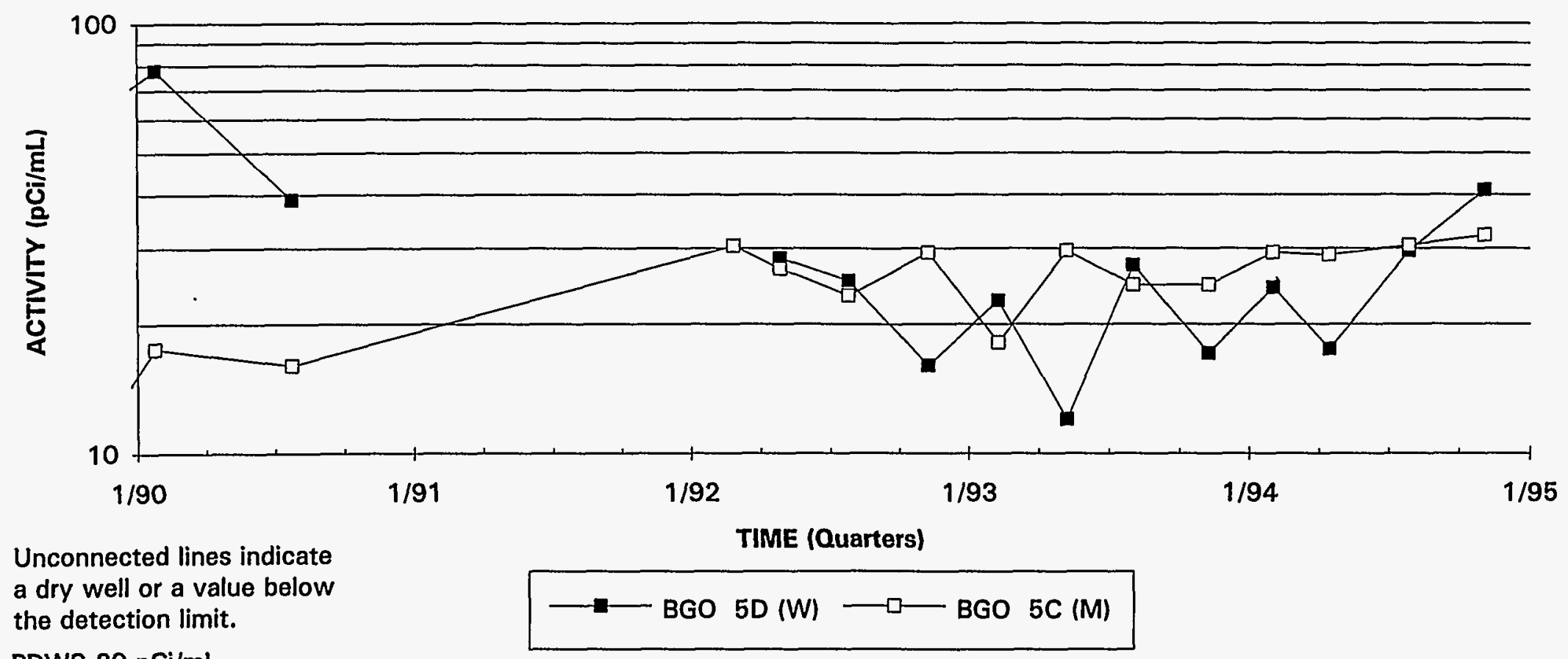

PDWS $20 \mathrm{pCi} / \mathrm{mL}$ 


\section{Tritium Activities} Well Cluster BGO 6

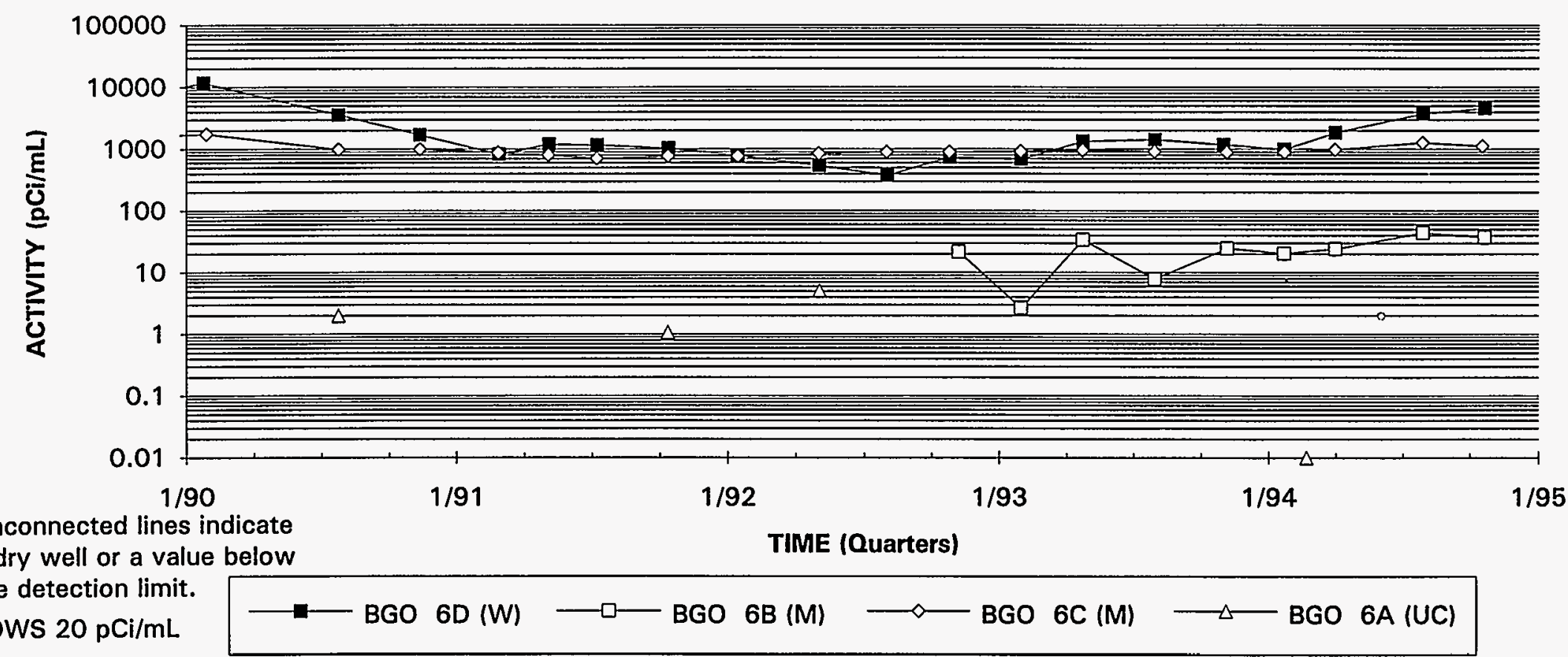

Note: W=Water Table (IIB2); B=Barnwell (IIB1); M=McBean (IIB1); UC=Upper Congaree (IIA); MC=Middle Congaree (IIA); LC=Lower Congaree (IIA) 


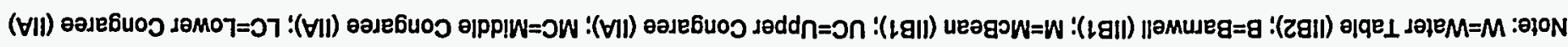

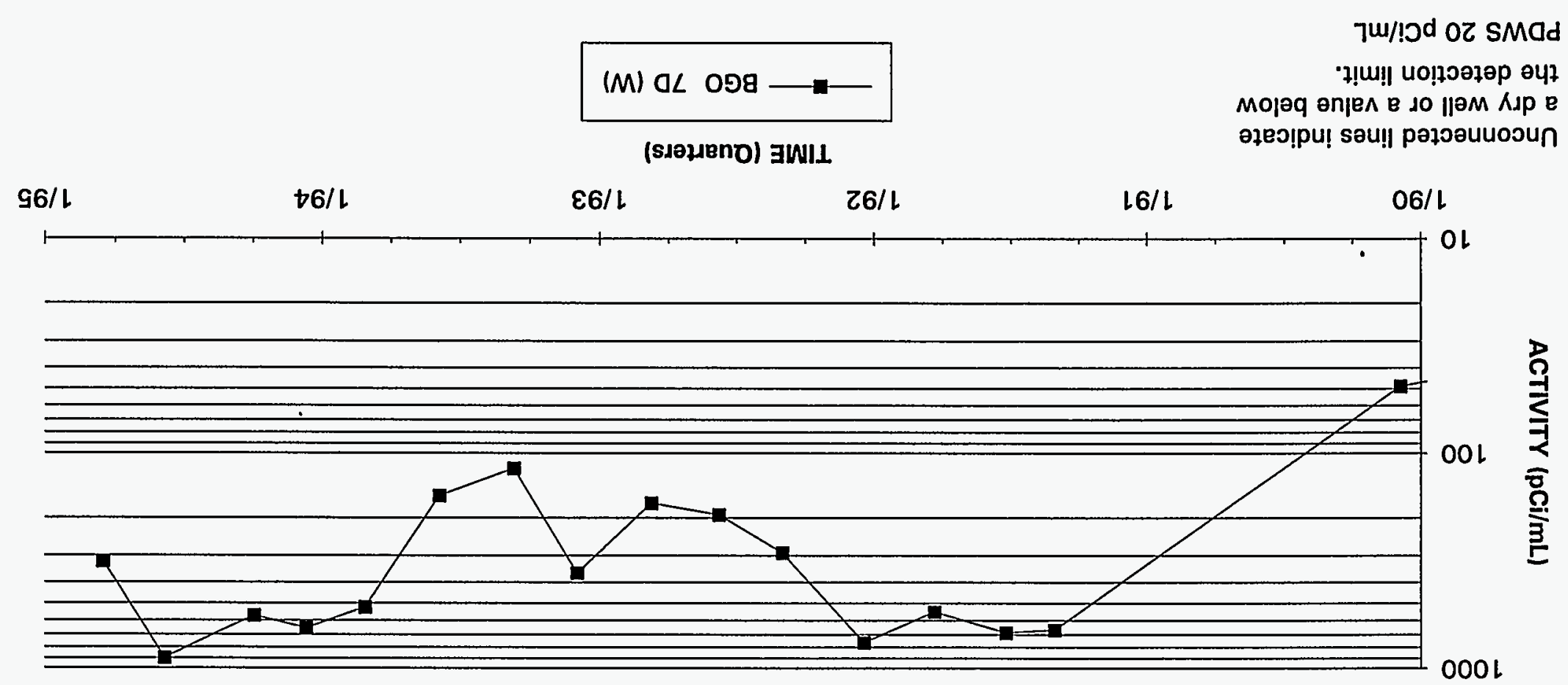

aL ODg \|әM

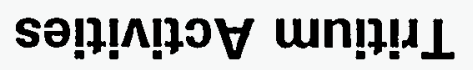




\section{Tritium Activities}

\section{Well Cluster BGO 8}

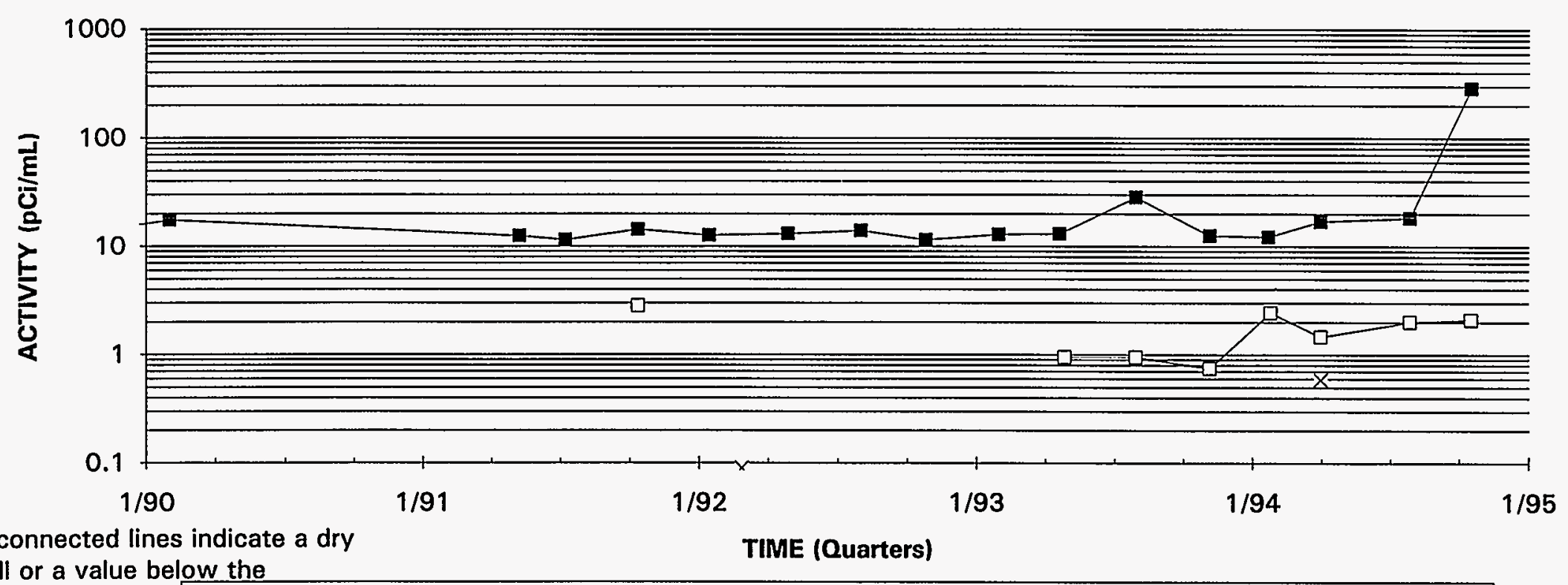

well or a value below the

detection limit.

PDWS $20 \mathrm{pCi} / \mathrm{mL}$

$\rightarrow$ BGO 8D (W)

$\longrightarrow$ BGO 8C (M)

BGO $8 A(U C)-x-B G O$ 8AR (UC)

Note: W=Water Table (IIB2); B=Barnwell (IIB1); M=McBean (IIB1); UC=Upper Congaree (IIA); MC=Middle Congaree (IIA); LC=Lower Congaree (IIA) 


\section{Tritium Activities Well Cluster BGO 9}

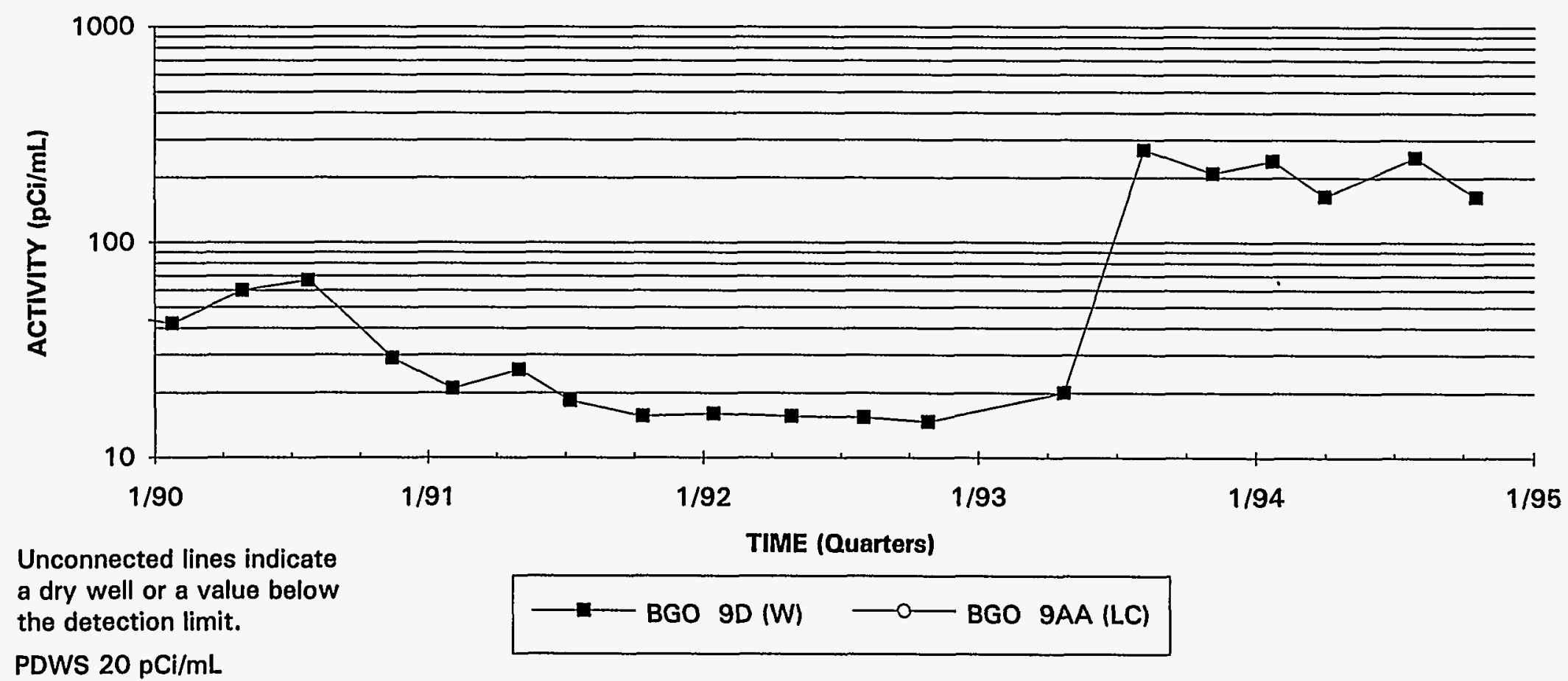

Note: W=Water Table (IIB2); B=Barnwell (IIB1); M=McBean (IIB1); UC=Upper Congaree (IIA); MC=Middle Congaree (IIA); LC=Lower Congaree (IIA) 


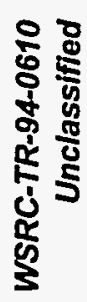
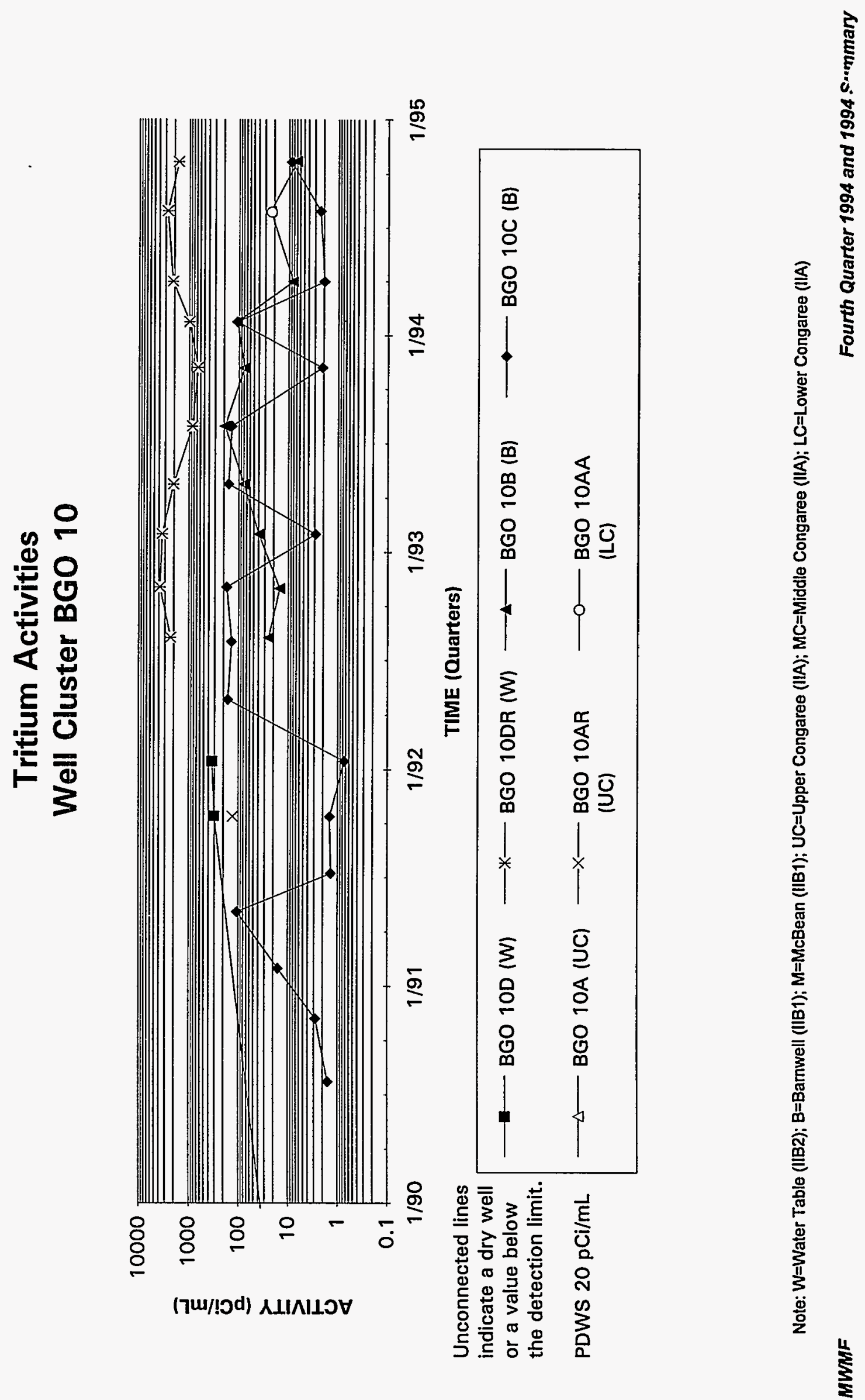


\section{Tritium Activities \\ Well BGO 11D}

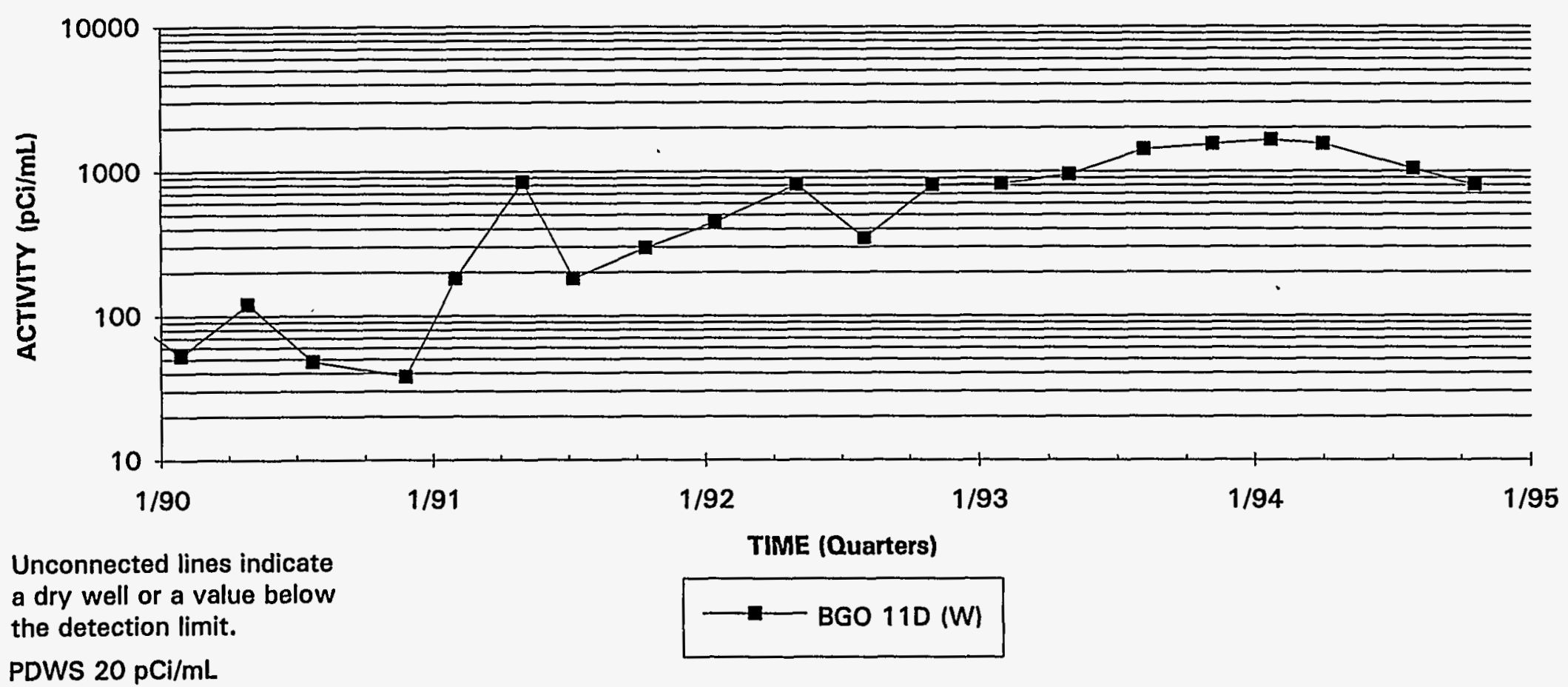

Note: $W=$ Water Table (IIB2); B=Barnwell (IIB1); $M=$ McBean (IIB1); UC=Upper Congaree (IIA); MC=Middle Congaree (IIA); LC=Lower Congaree (IIA) 


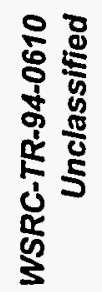
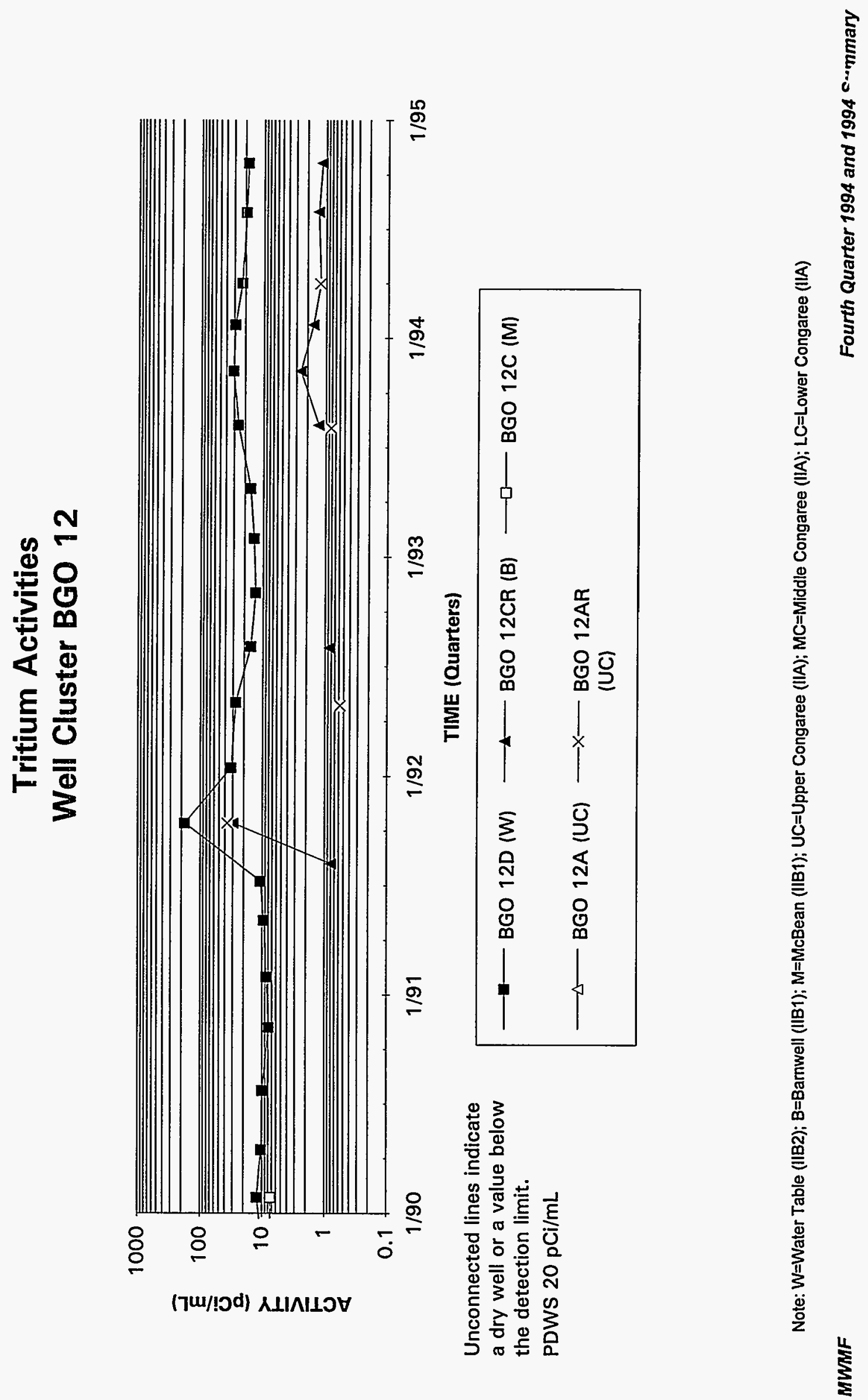


\section{Tritium Activities}

\section{Well Cluster BGO 13}

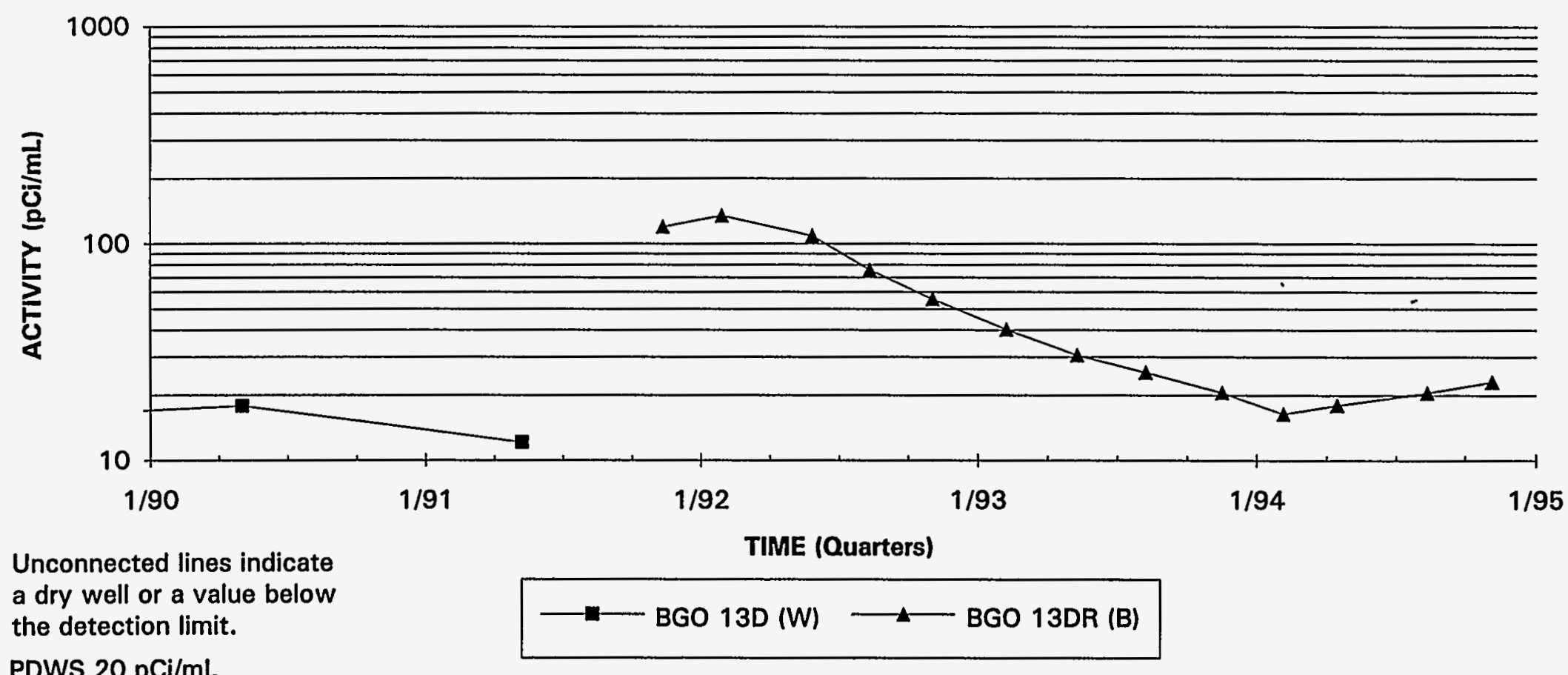

PDWS $20 \mathrm{pCi} / \mathrm{mL}$ 


\section{Tritium Activities}

\section{Well Cluster BGO 14}

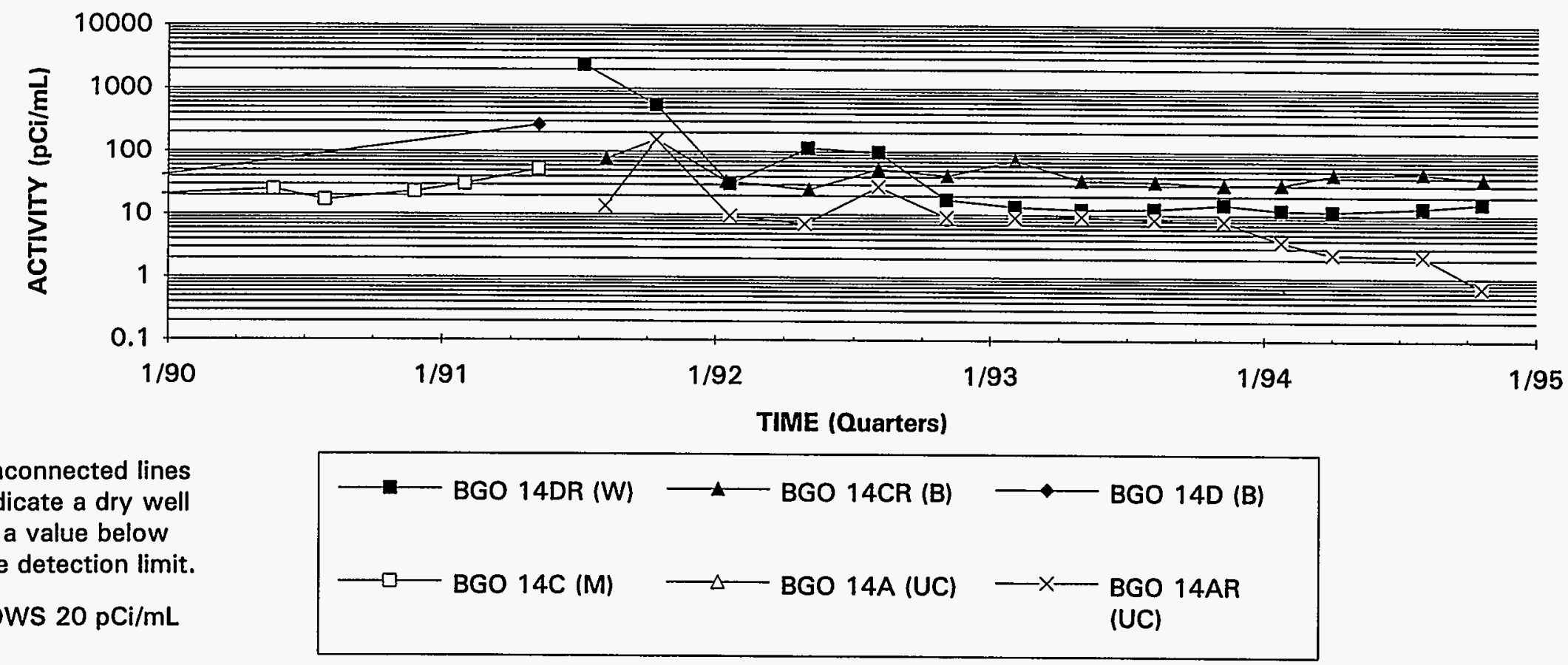

Note: W=Water Table (IIB2); B=Barnwell (IIB1); M=McBean (IIB1); UC=Upper Congaree (IIA); MC=Middle Congaree (IIA); LC=Lower Congaree (IIA) 


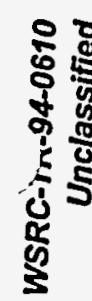
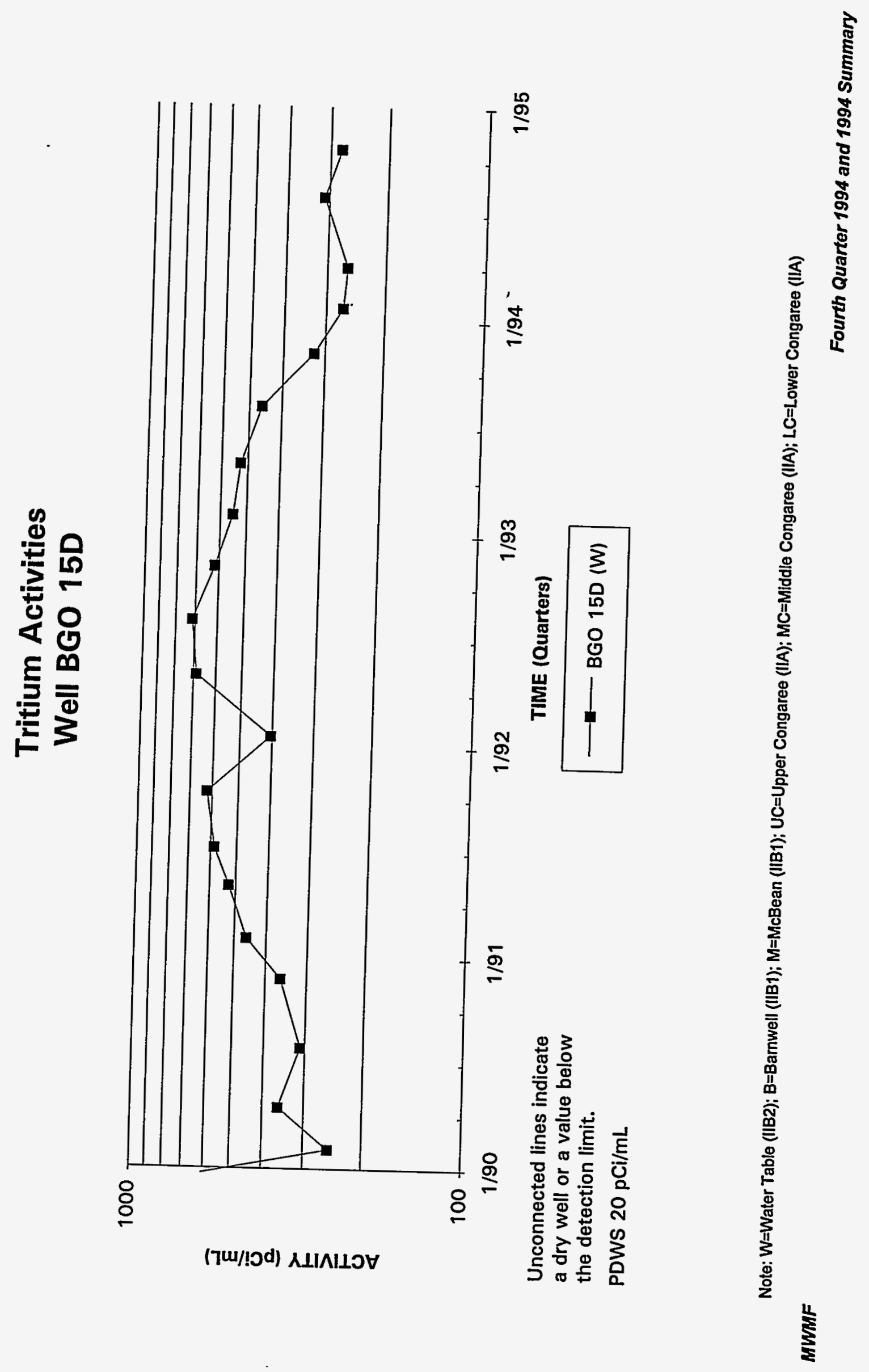


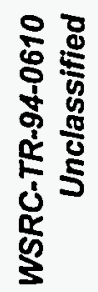
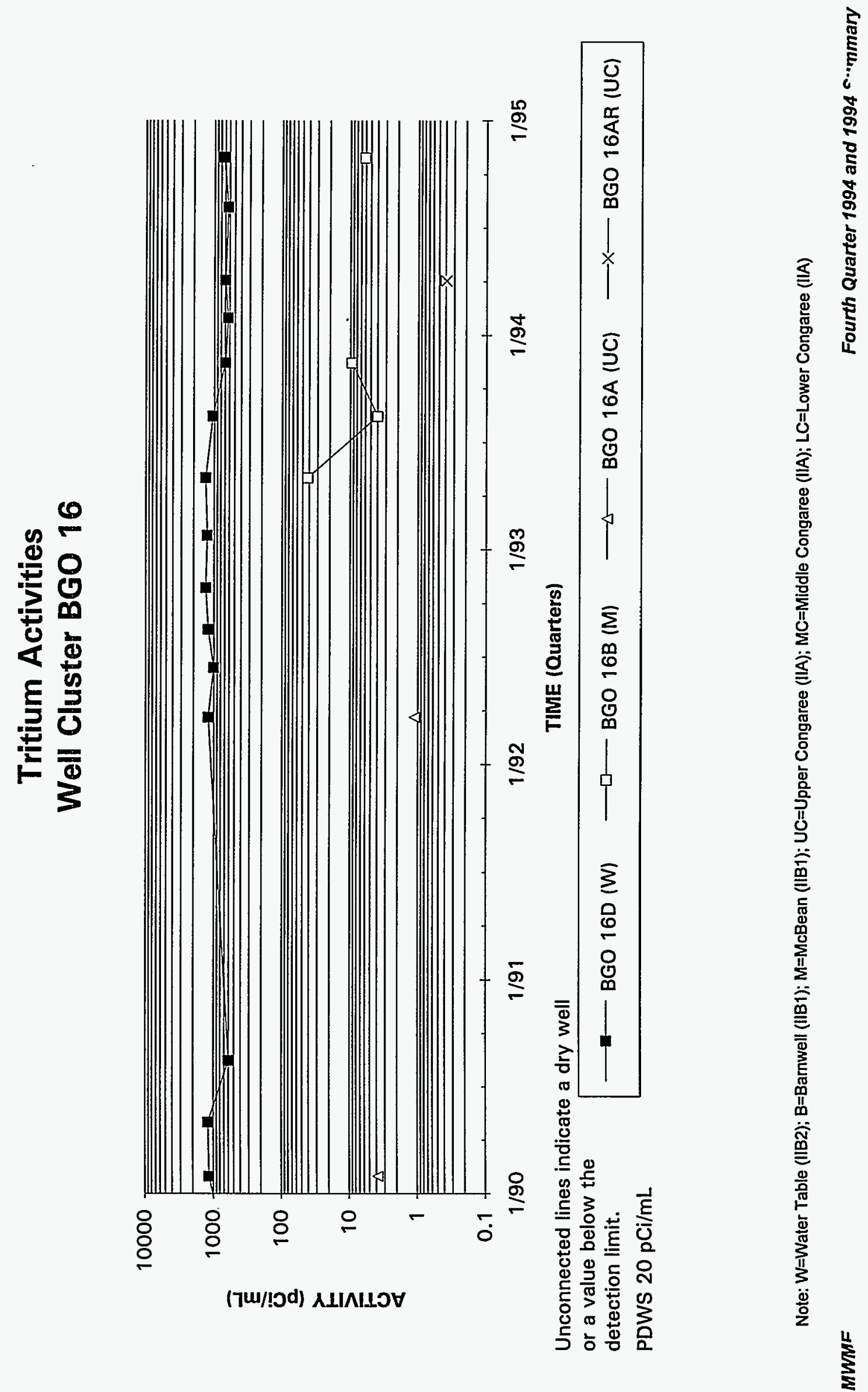


\section{Tritium Activities}

\section{Well Cluster BGO 17}

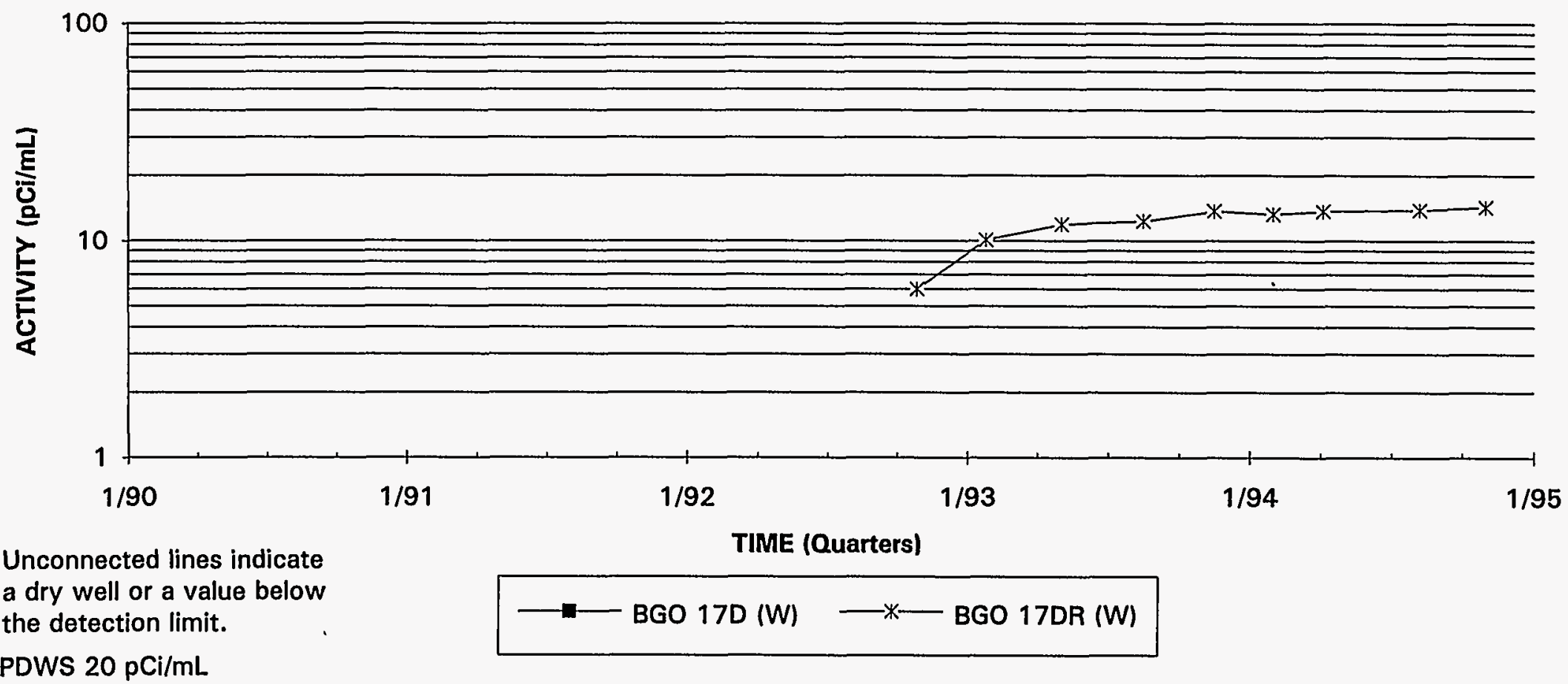

Note: W=Water Table (IIB2); B=Barnwell (IIB1); M=McBean (IIB1); UC=Upper Congaree (IIA); MC=Middle Congaree (IIA); LC=Lower Congaree (IIA) 

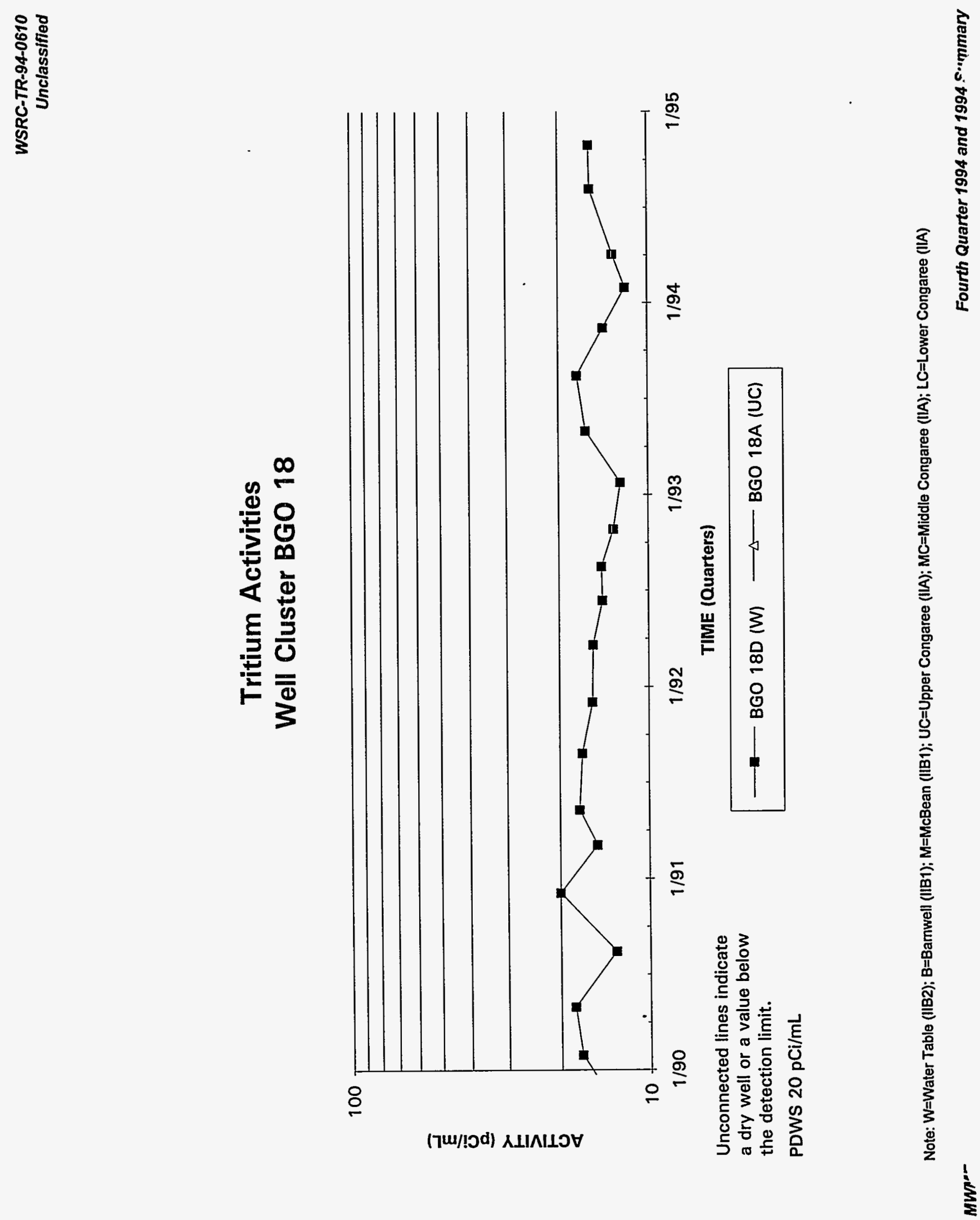


\section{Tritium Activities Well BGO 19D}

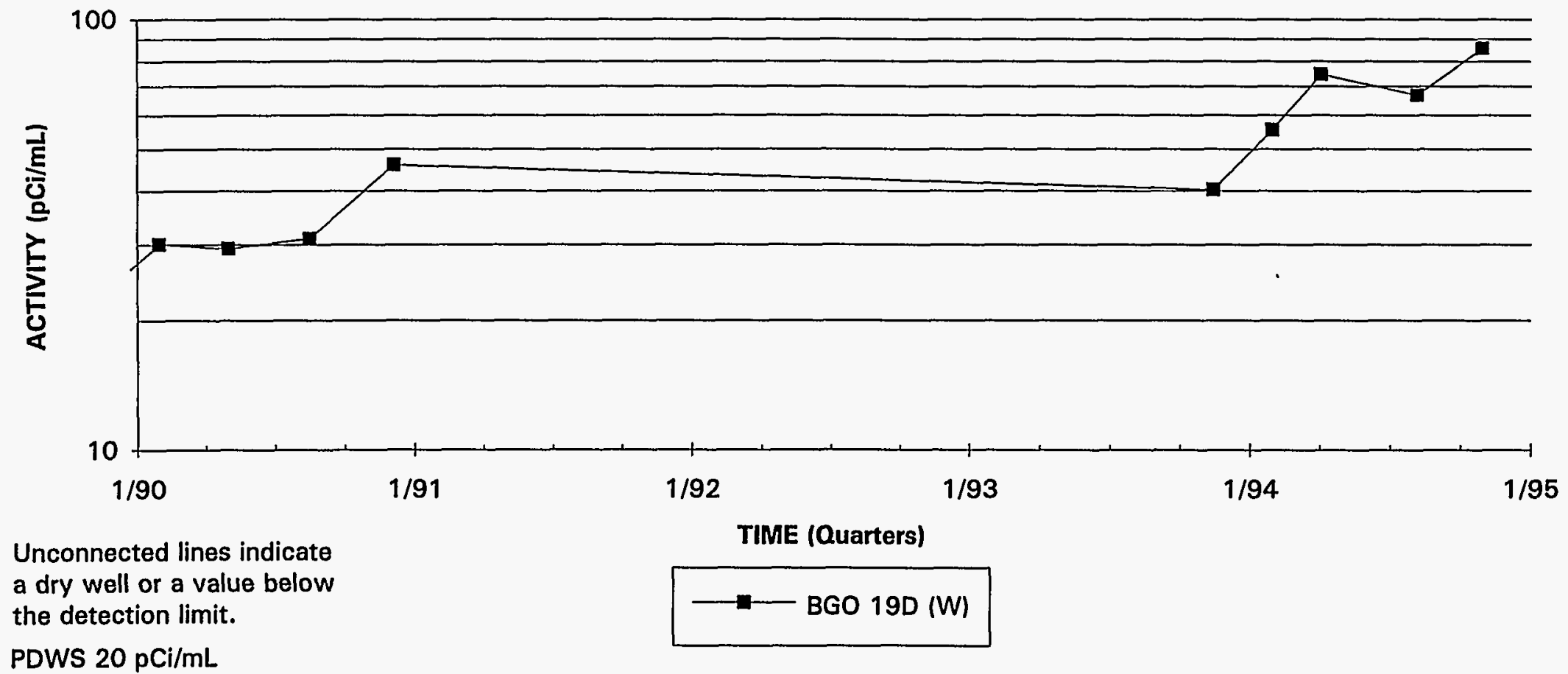




\section{Tritium Activities \\ Well BGO 20D}

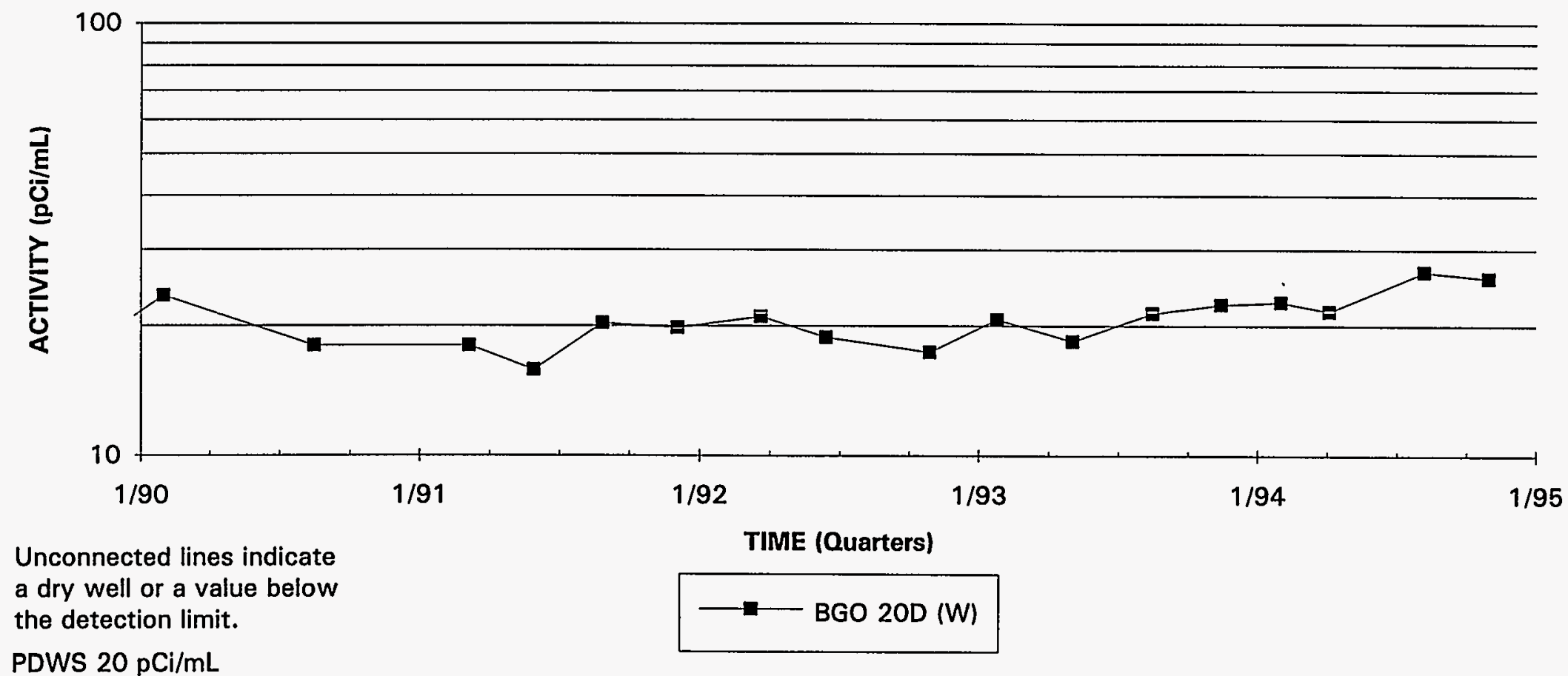

PDWS $20 \mathrm{pCi} / \mathrm{mL}$

Note: W=Water Table (IIB2); B=Barnwell (IIB1); M=McBean (IIB1); UC=Upper Congaree (IIA); MC=Middle Congaree (IIA); LC=Lower Congaree (IIA) 


\section{Tritium Activities}

\section{Well BGO 21D}

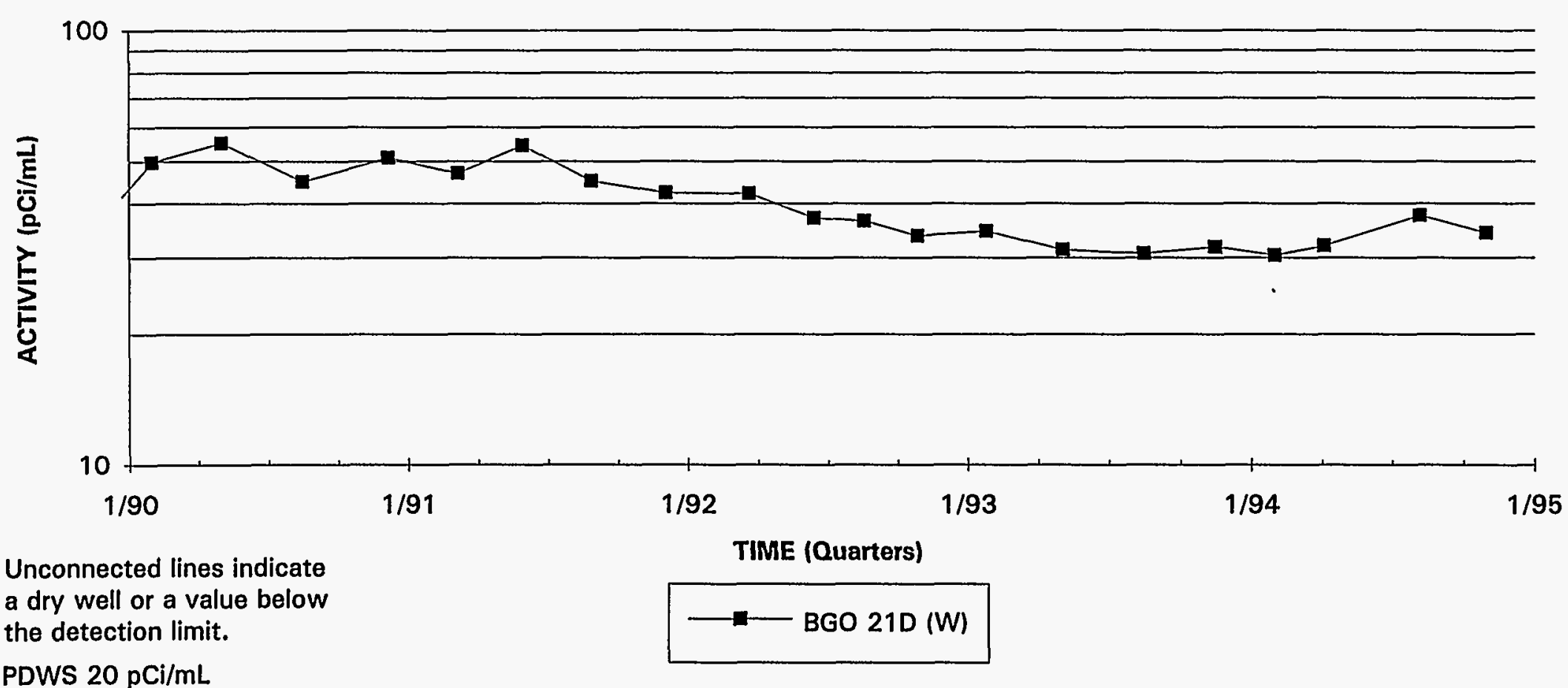

PDWS $20 \mathrm{pCi} / \mathrm{mL}$ 


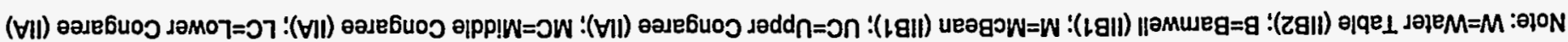

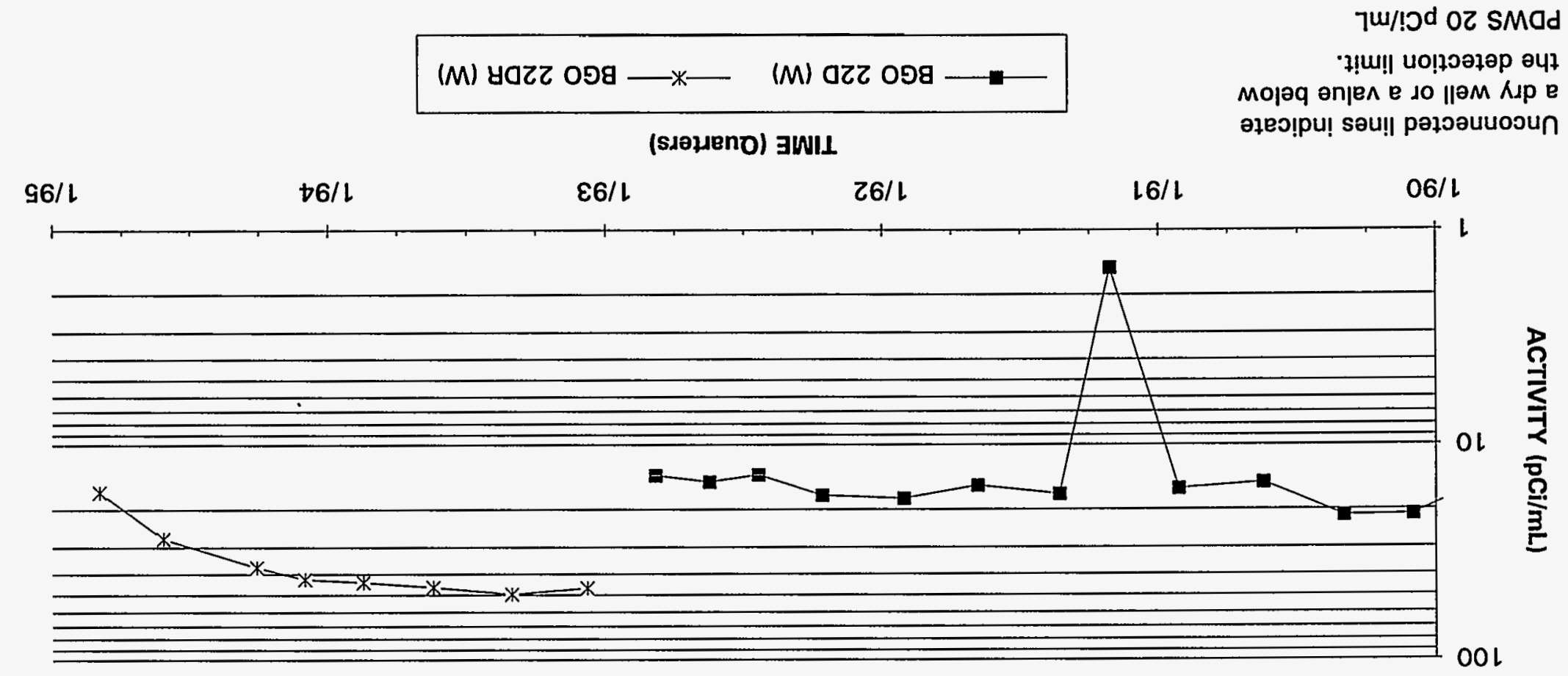

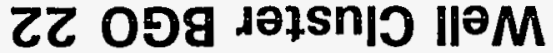

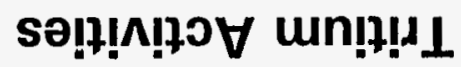




\section{Tritium Activities \\ Well BGO 23D}

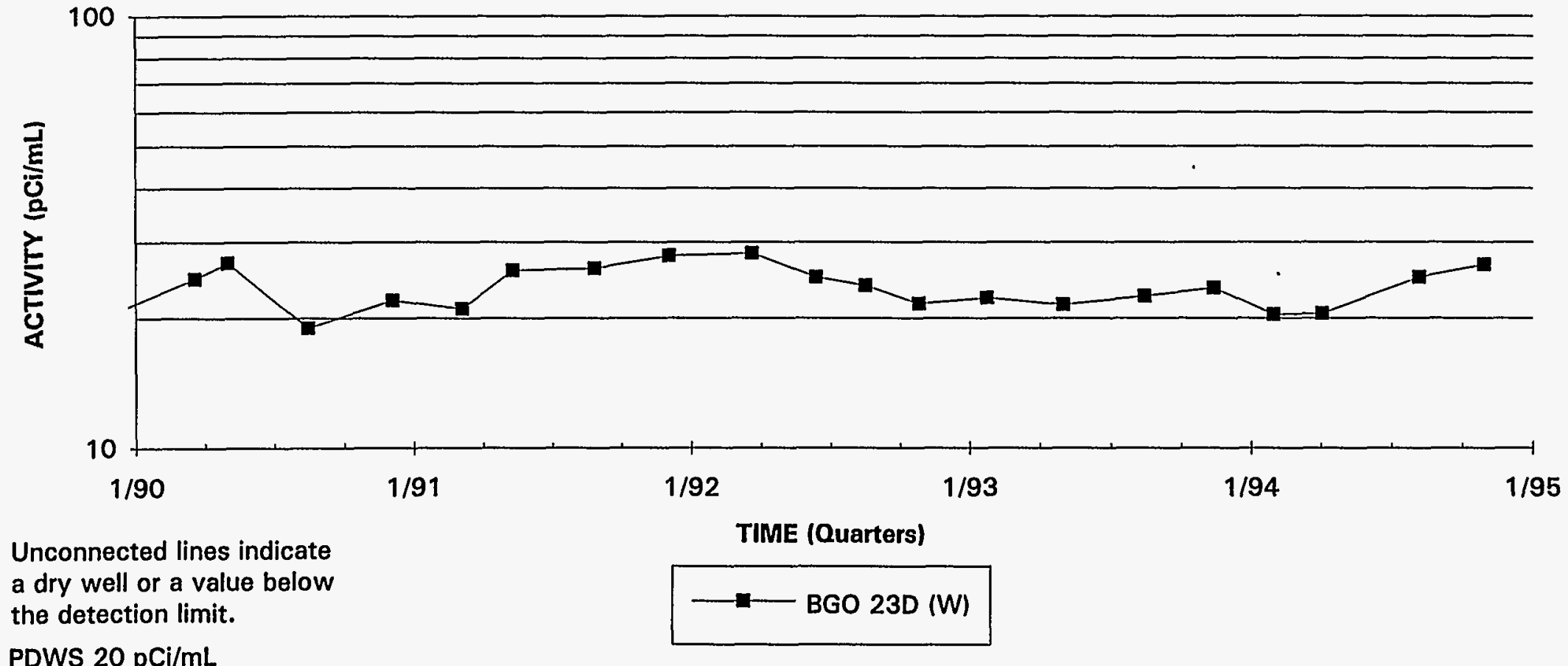

Note: W=Water Table (IIB2); B=Barnwell (IIB1); M=McBean (IIB1); UC=Upper Congaree (IIA); MC=Middle Congaree (IIA); LC=Lower Congaree (IIA) 

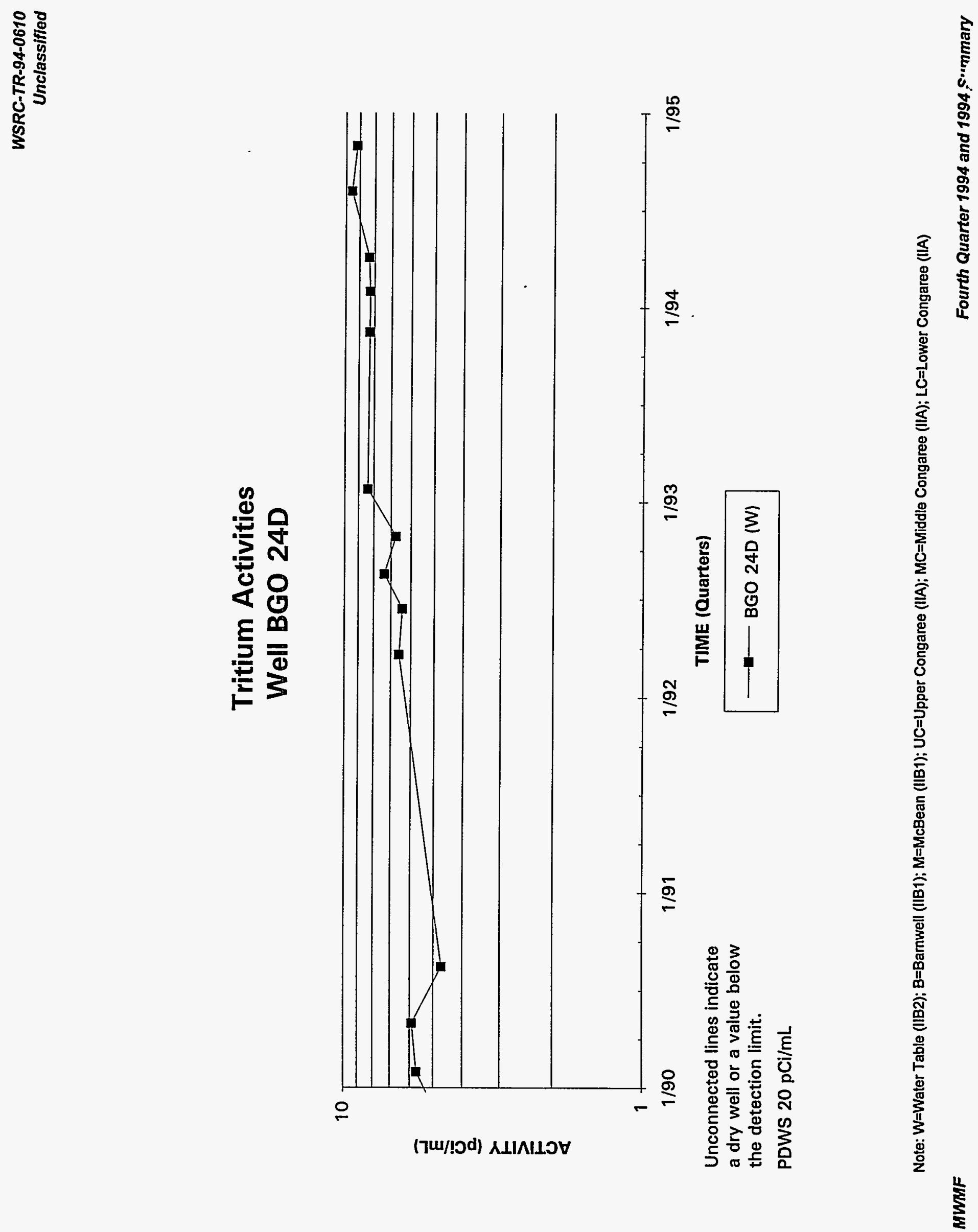


\section{Tritium Activities}

\section{Well BGO 25A}

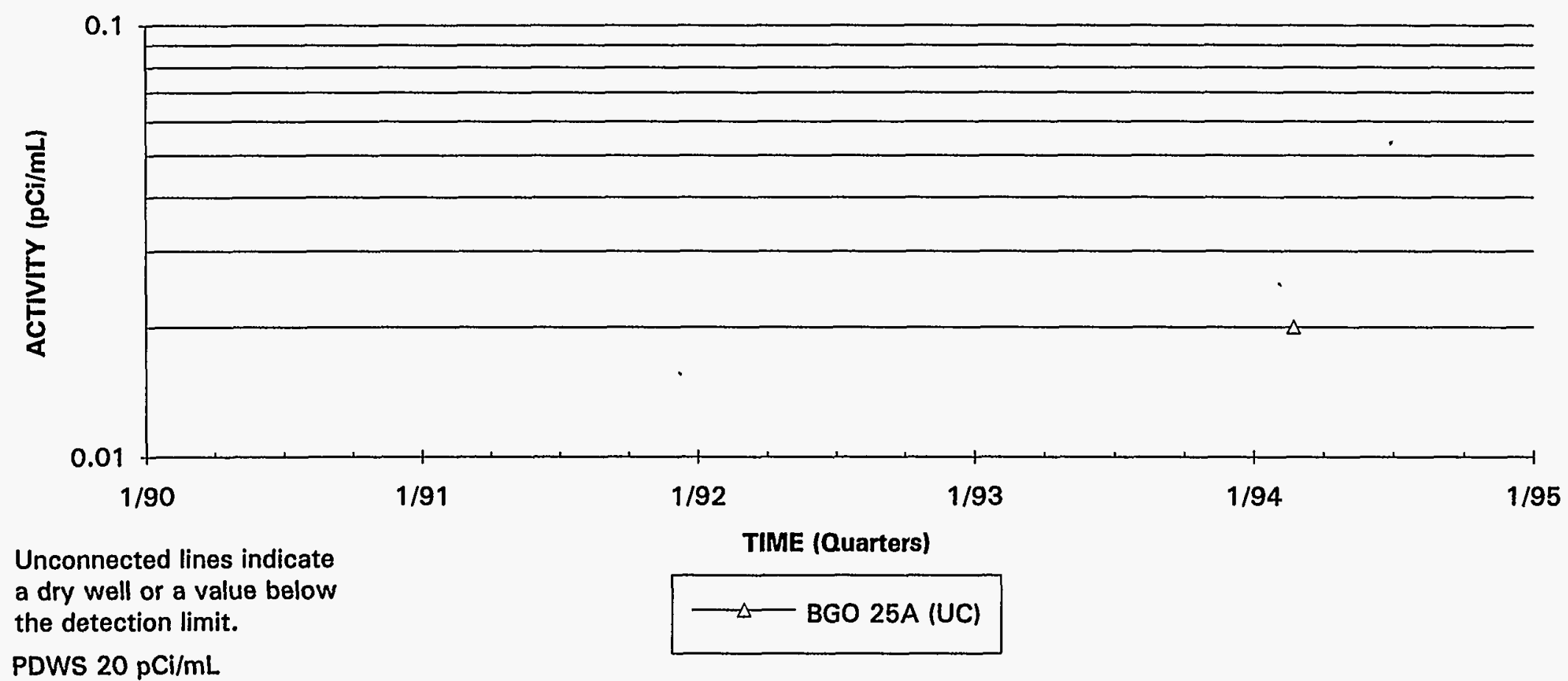

Note: W=Water Table (IIB2); B=Barnwell (IIB1); M=McBean (IIB1); UC=Upper Congaree (IIA); MC=Middle Congaree (IIA); LC=Lower Congaree (IIA) 


\section{Tritium Activities}

\section{Well Cluster BGO 26}

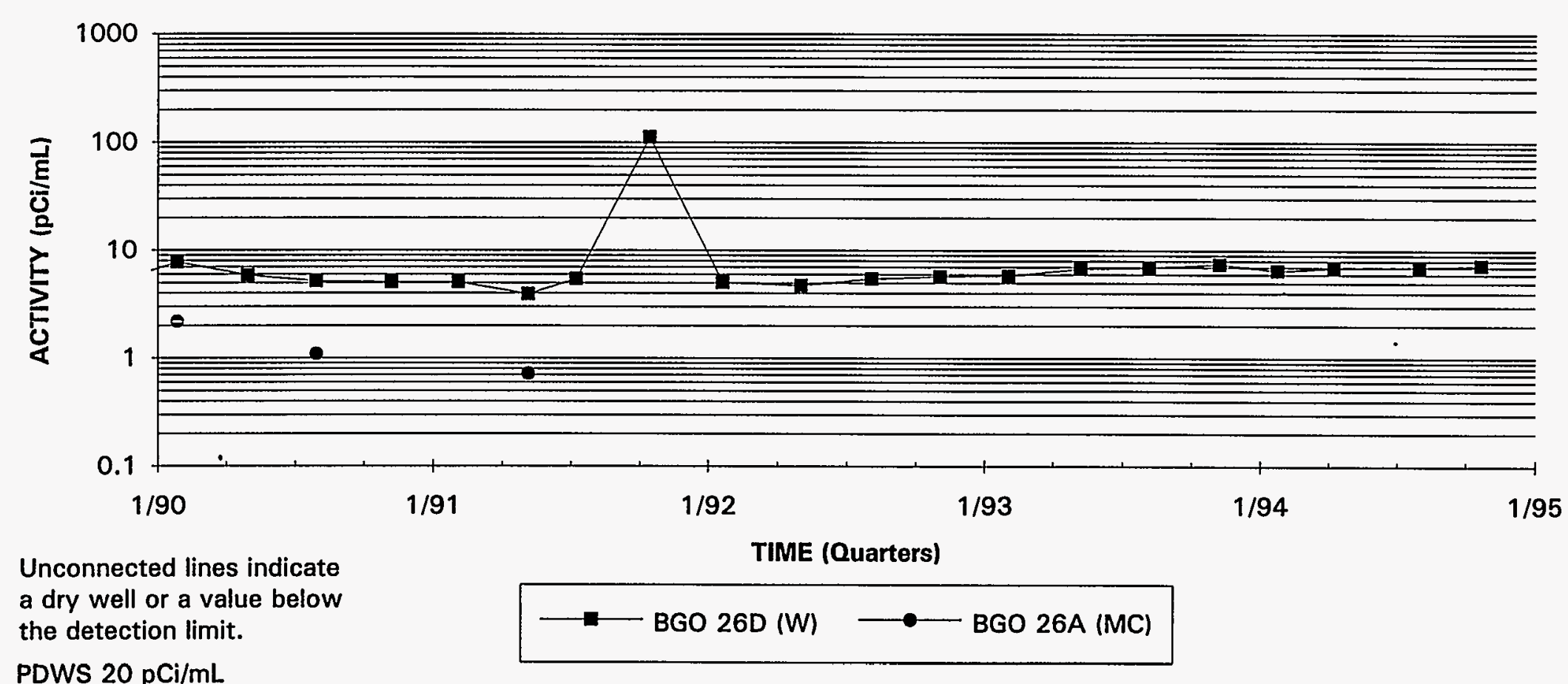

Note: W=Water Table (IIB2); B=Barnwell (IIB1); M=McBean (IIB1); UC=Upper Congaree (IIA); MC=Middle Congaree (IIA); LC=Lower Congaree (IIA) 


\section{Tritium Activities}

Well Cluster BGO 27

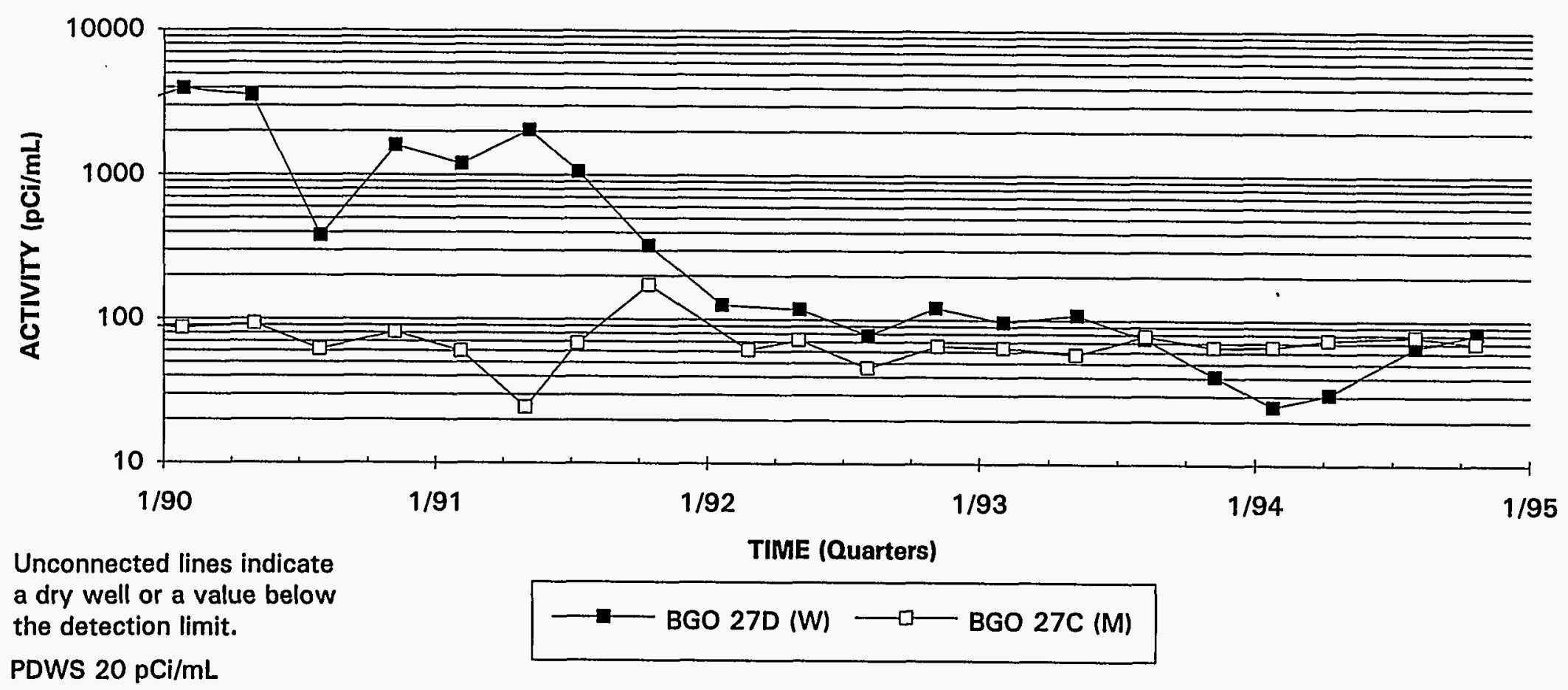

Note: W=Water Table (IIB2); B=Barnwell (IIB1); M=McBean (IIB1); UC=Upper Congaree (IIA); MC=Middle Congaree (IIA); LC=Lower Congaree (IIA) 


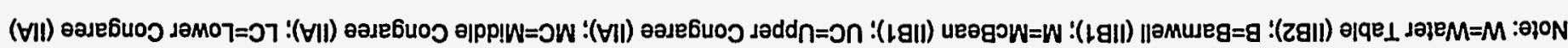

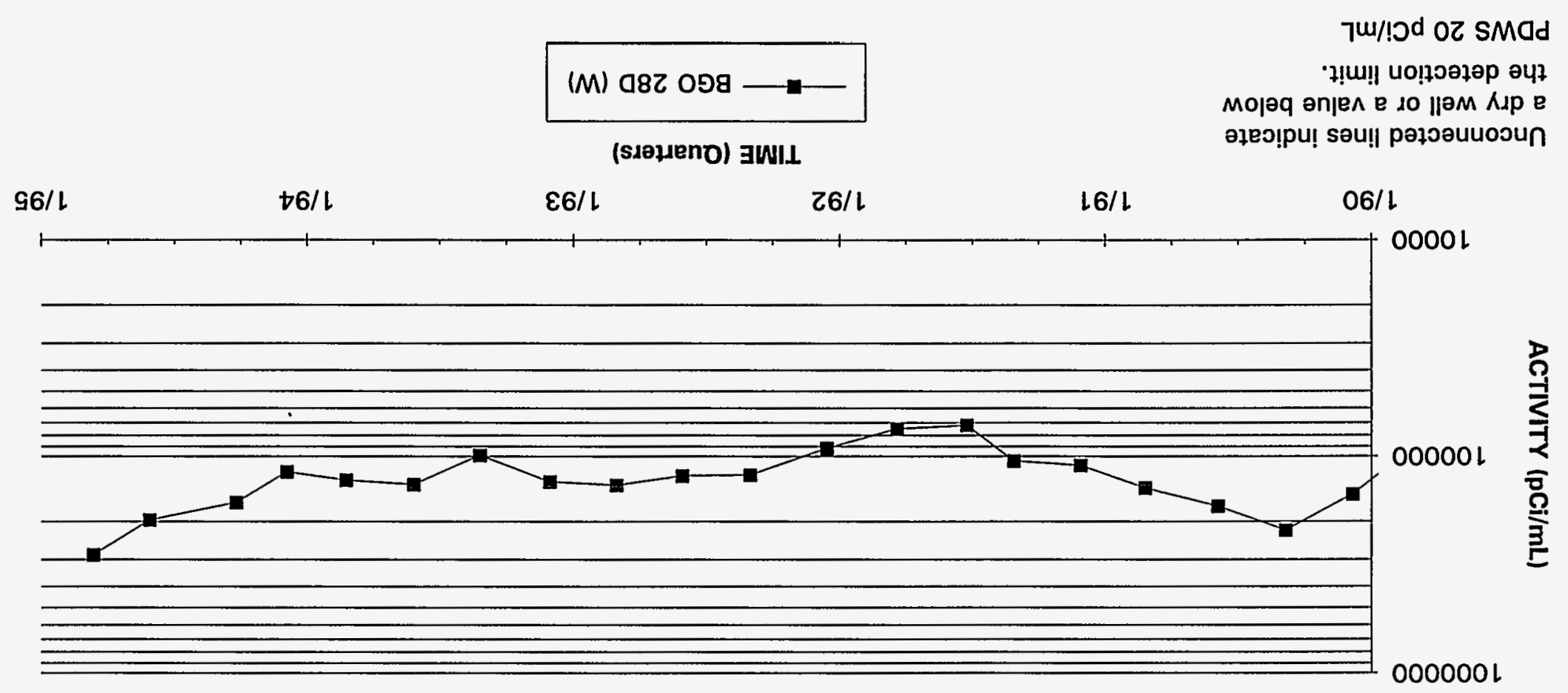

$082009 \| \odot M$

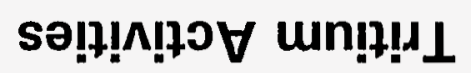




\section{Tritium Activities Well Cluster BGO 29}

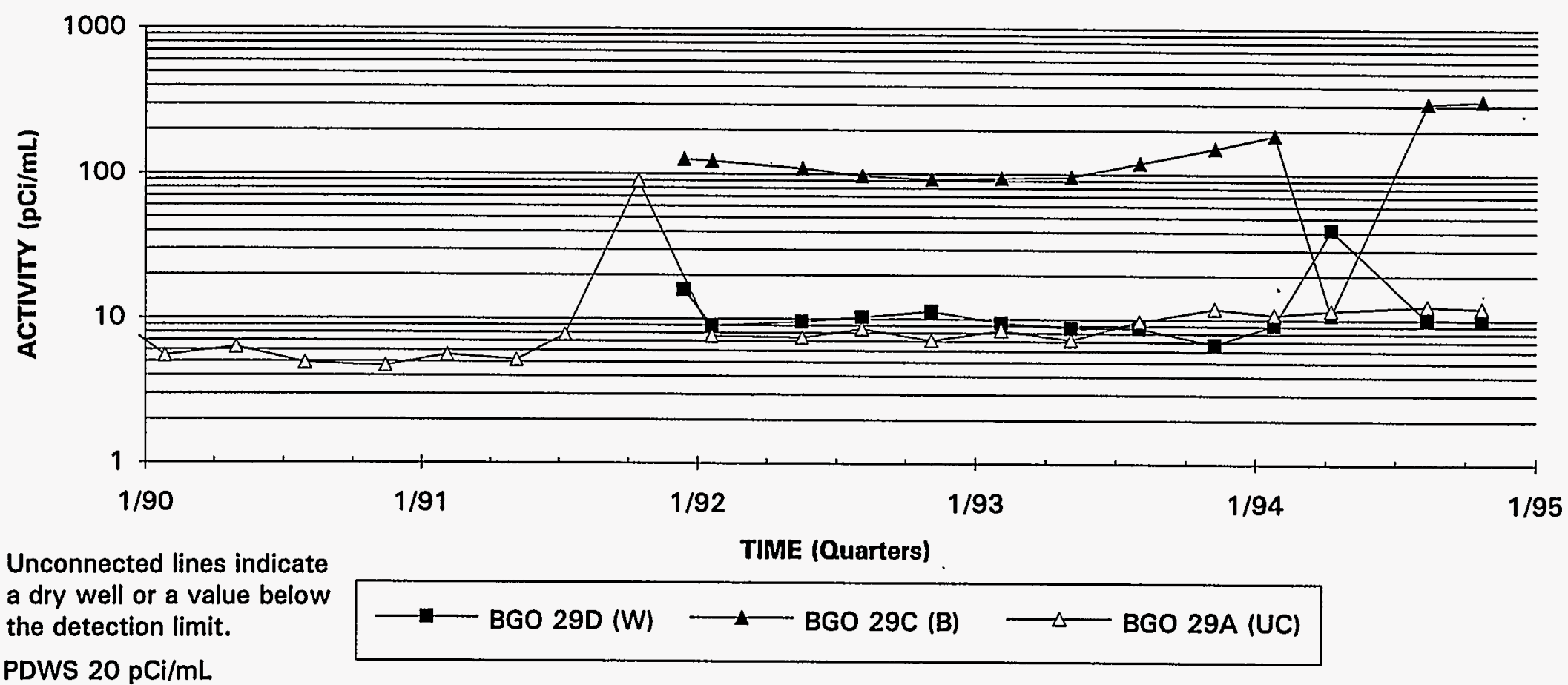

Note: W=Water Table (IIB2); B=Barnwell (IIB1); M=MCBean (IIB1); UC=Upper Congaree (IIA); MC=Middle Congaree (IIA); LC=Lower Congaree (IIA) 


\section{Tritium Activities}

Well Cluster BGO 30

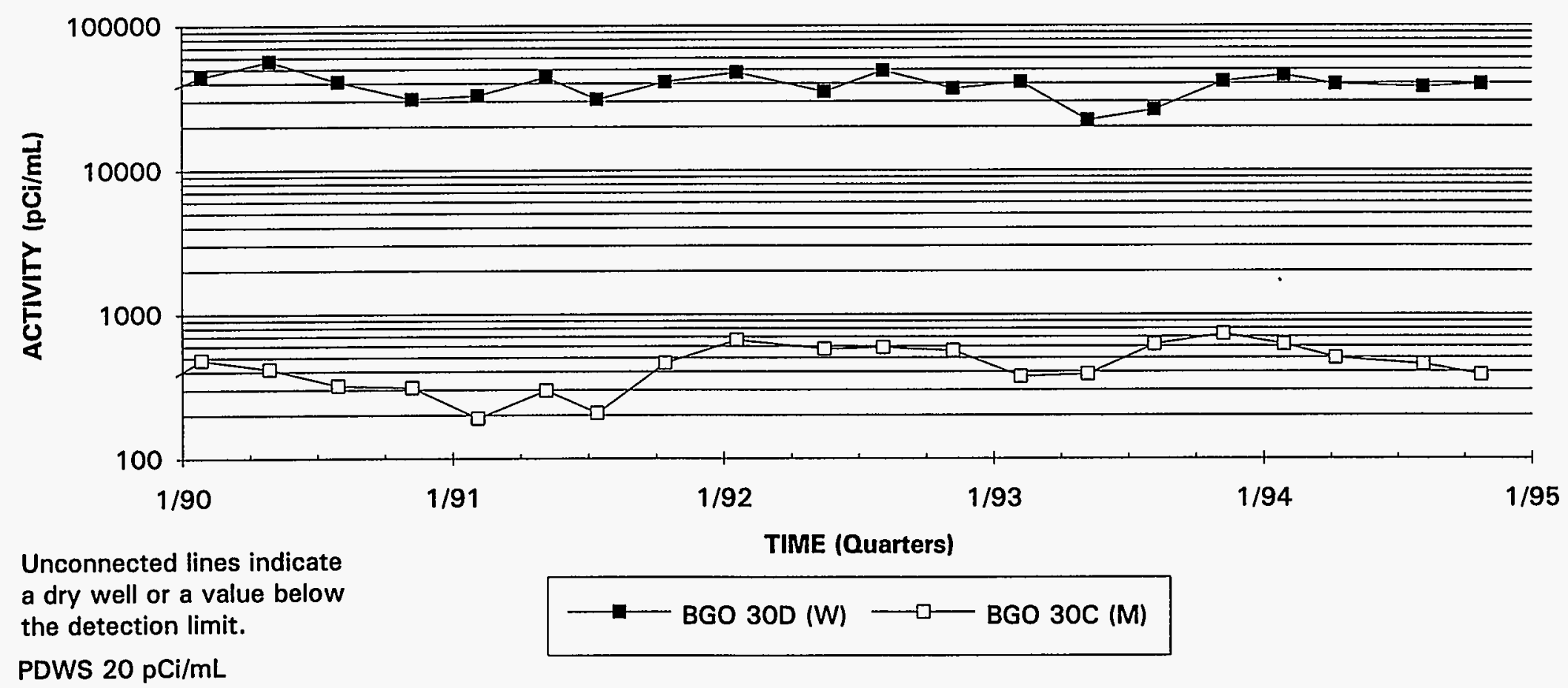

Note: W=Water Table (IIB2); B=Barnwell (IIB1); M=McBean (IIB1); UC=Upper Congaree (IIA); MC=Middle Congaree (IIA); LC=Lower Congaree (IIA) 


\section{Tritium Activities}

\section{Well Cluster BGO 31}

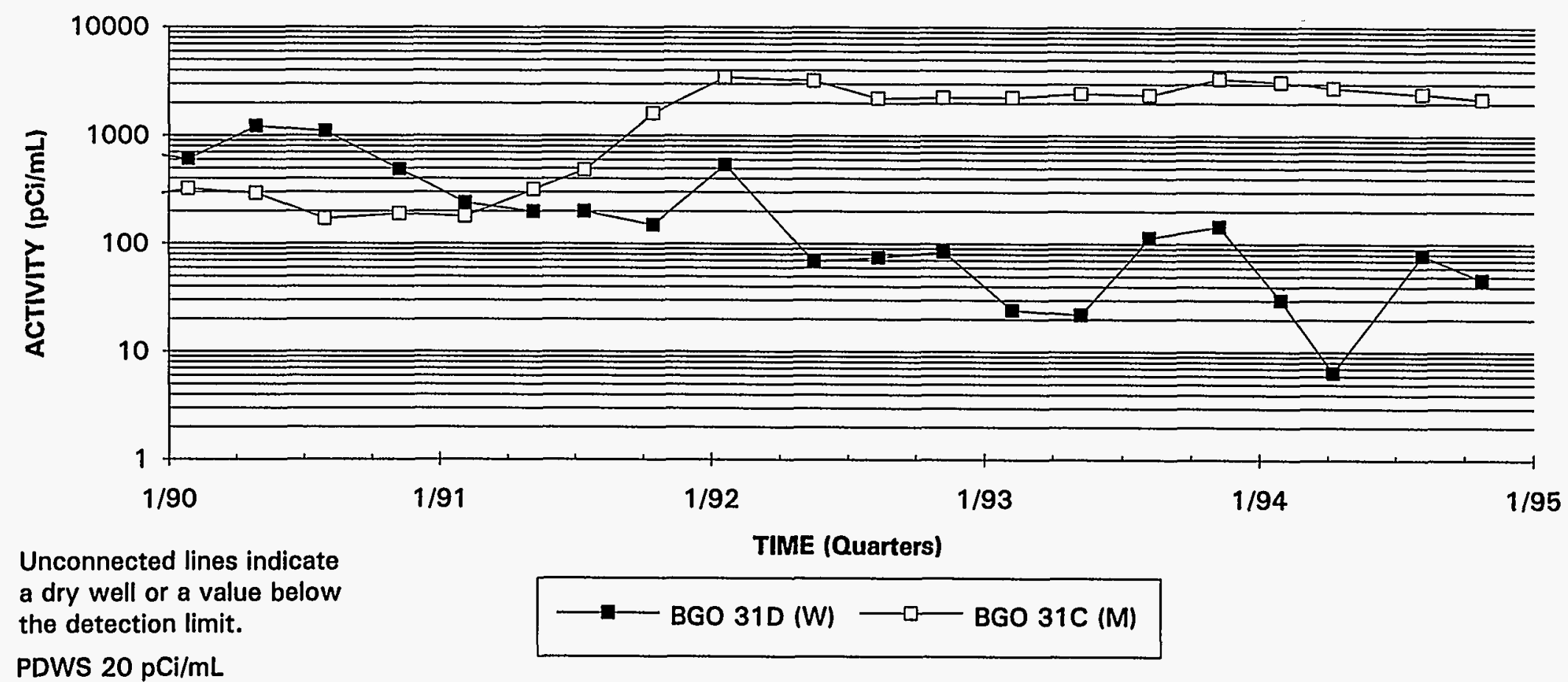

Note: W=Water Table (IIB2); B=Bamwell (IIB1); M=McBean (IIB1); UC=Upper Congaree (IIA); MC=Middle Congaree (IIA); LC=Lower Congaree (IIA) 


\section{Tritium Activities}

Well BGO 32D

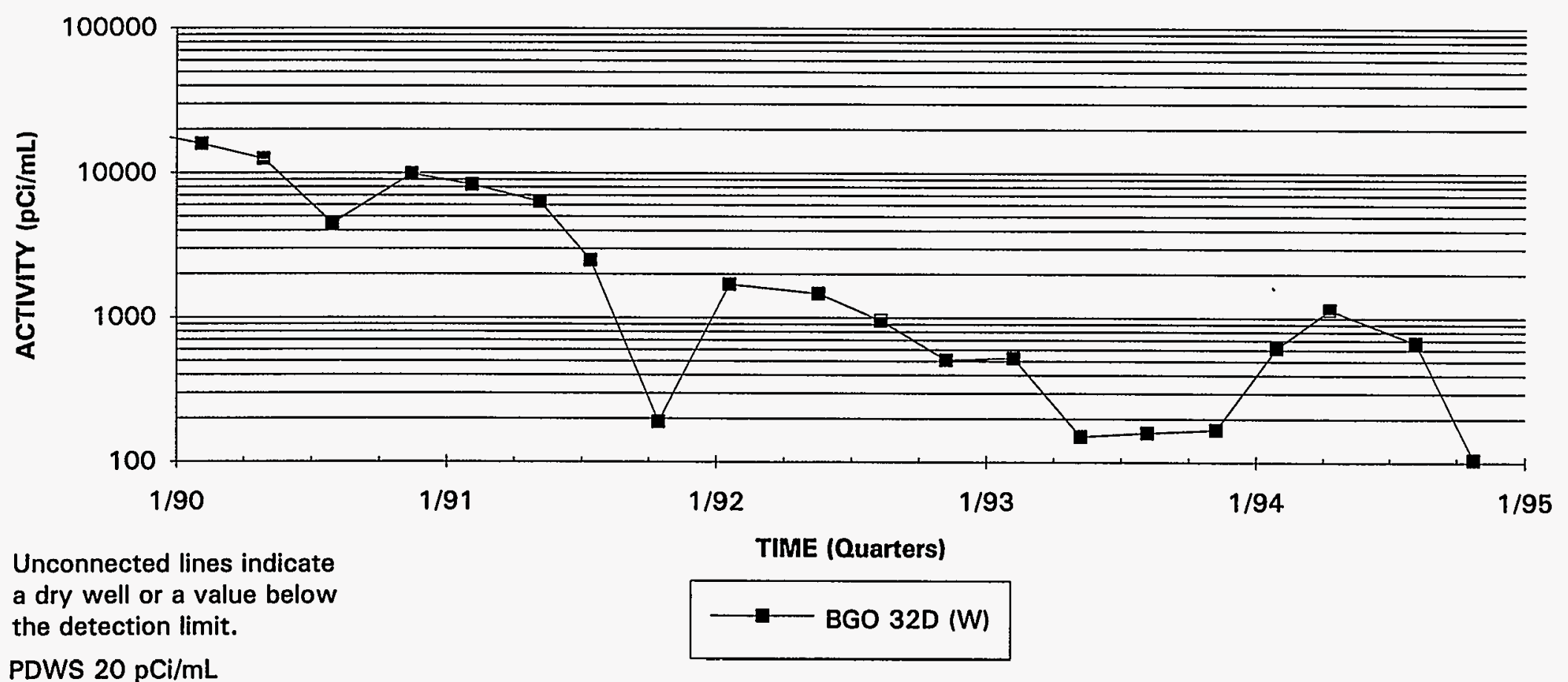

Note: W=Water Table (IIB2); B=Barnwell (IIB1); M=MCBean (IIB1); UC=Upper Congaree (IIA); MC=Middle Congaree (IIA); LC=Lower Congaree (IIA) 


\section{Tritium Activities}

Well Cluster BGO 33

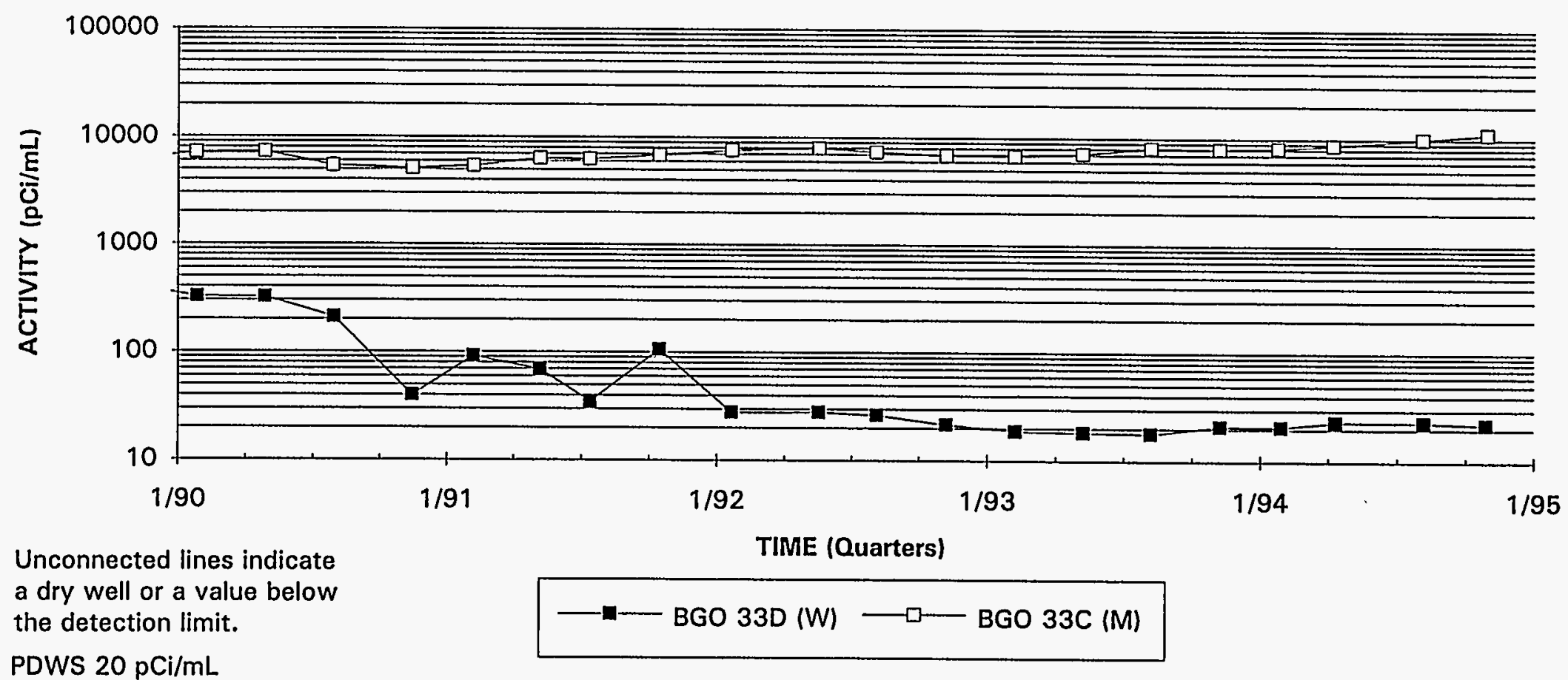

Nole: W=Water Table (IIB2); B=Barnwell (IIB1); M=MCBean (IIB1); UC=Upper Congaree (IIA); MC=Middle Congaree (IIA); LC=Lower Congaree (IIA) 


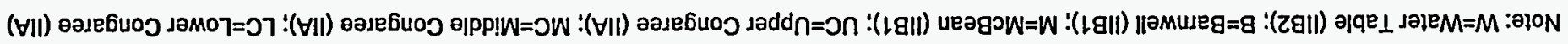

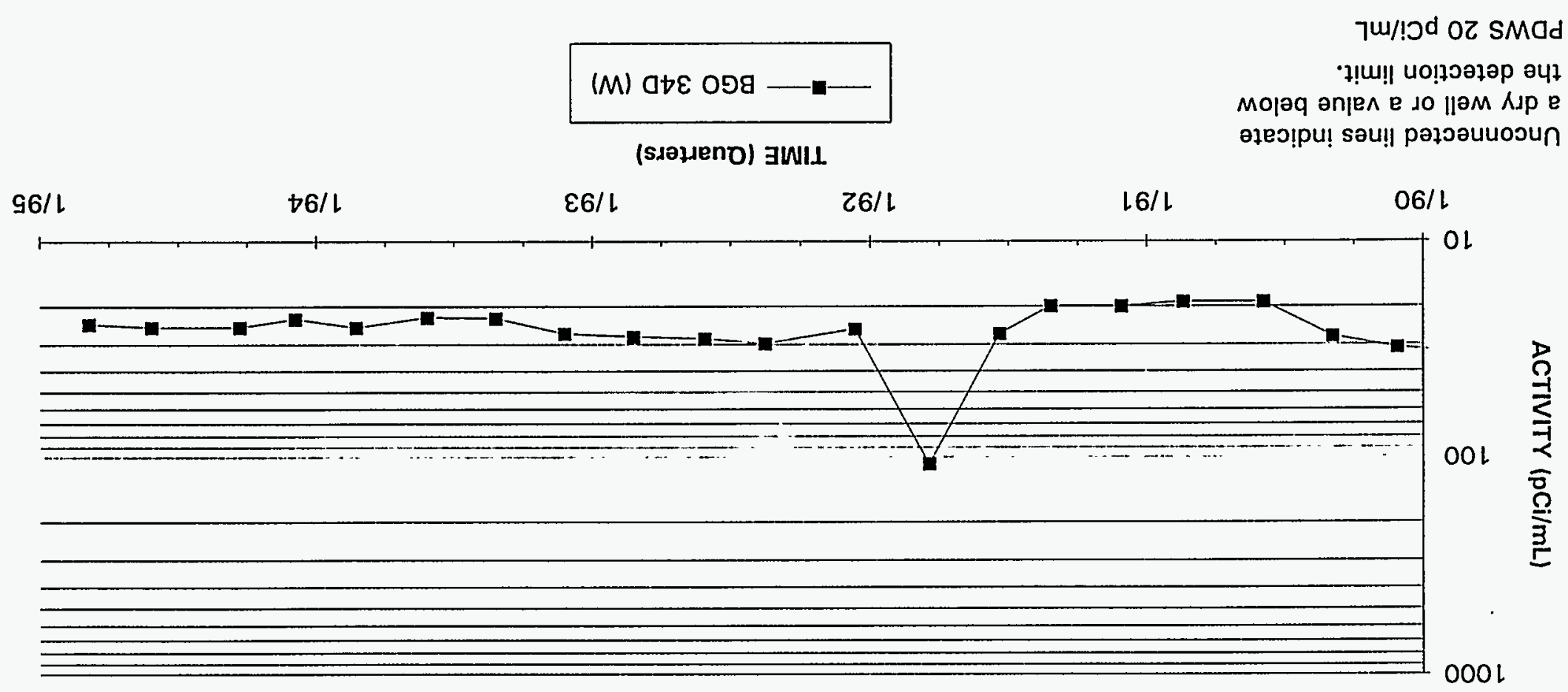

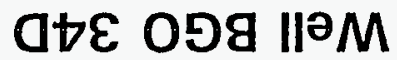

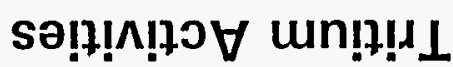




\section{Tritium Activities}

\section{Well Cluster BGO 35}

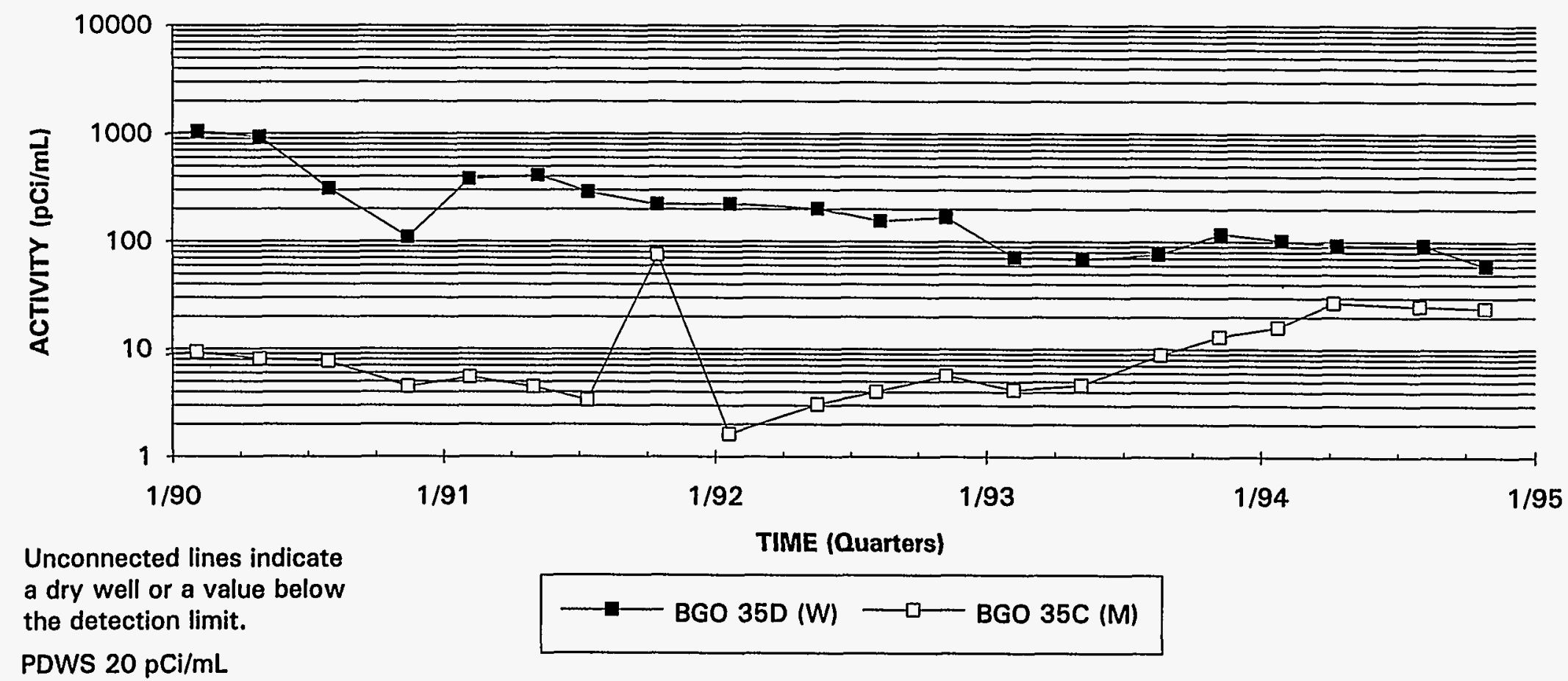

Note: W=Water Table (IIB2); B=Barnwell (IIB1); M=McBean (IIB1); UC=Upper Congaree (IIA); MC=Middle Congaree (IIA); LC=Lower Congaree (IIA) 
Tritium Activities

Well BGO 36D

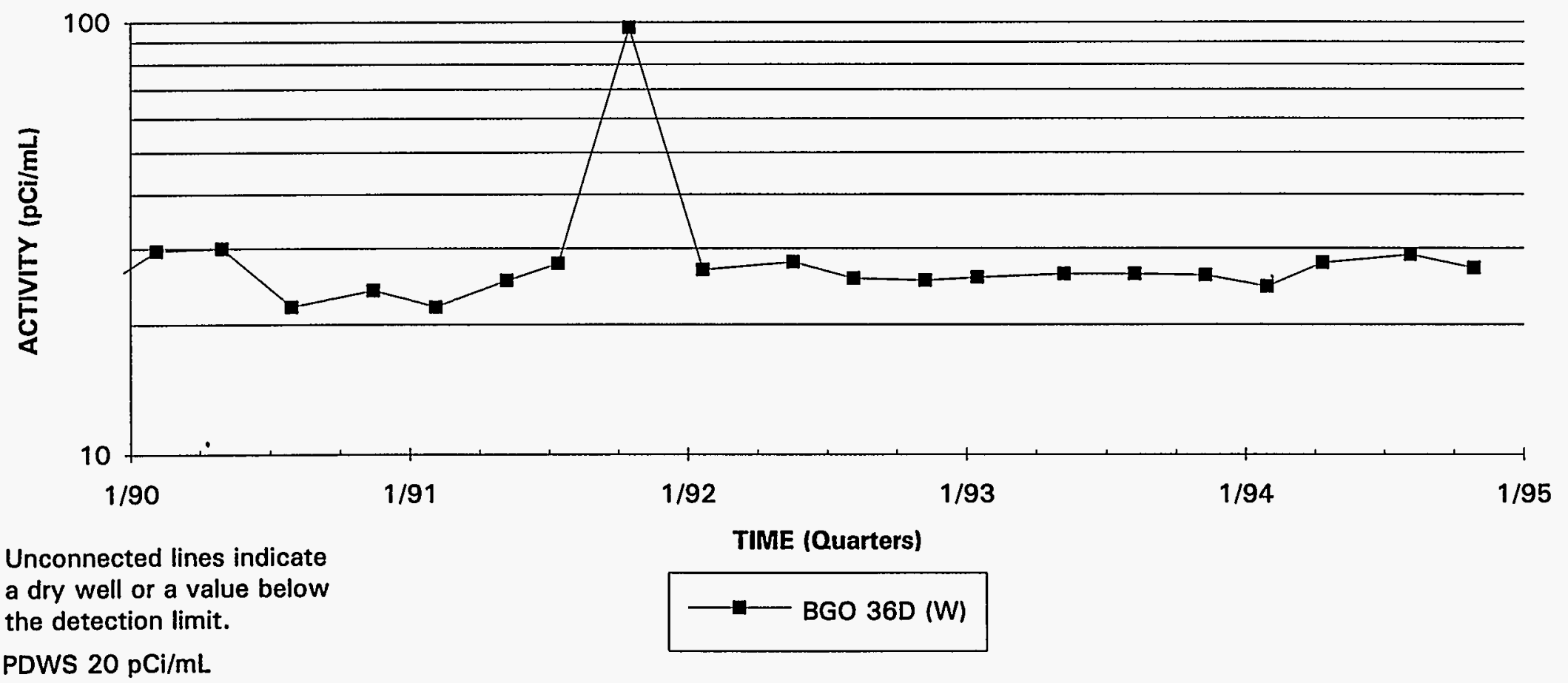

Note: W=Water Table (IIB2); B=Barnwell (IIB1); M=McBean (IIB1); UC=Upper Congaree (IIA); MC=Middle Congaree (IIA); LC=Lower Congaree (IIA) 


\section{Tritium Activities} Well Cluster BGO 37

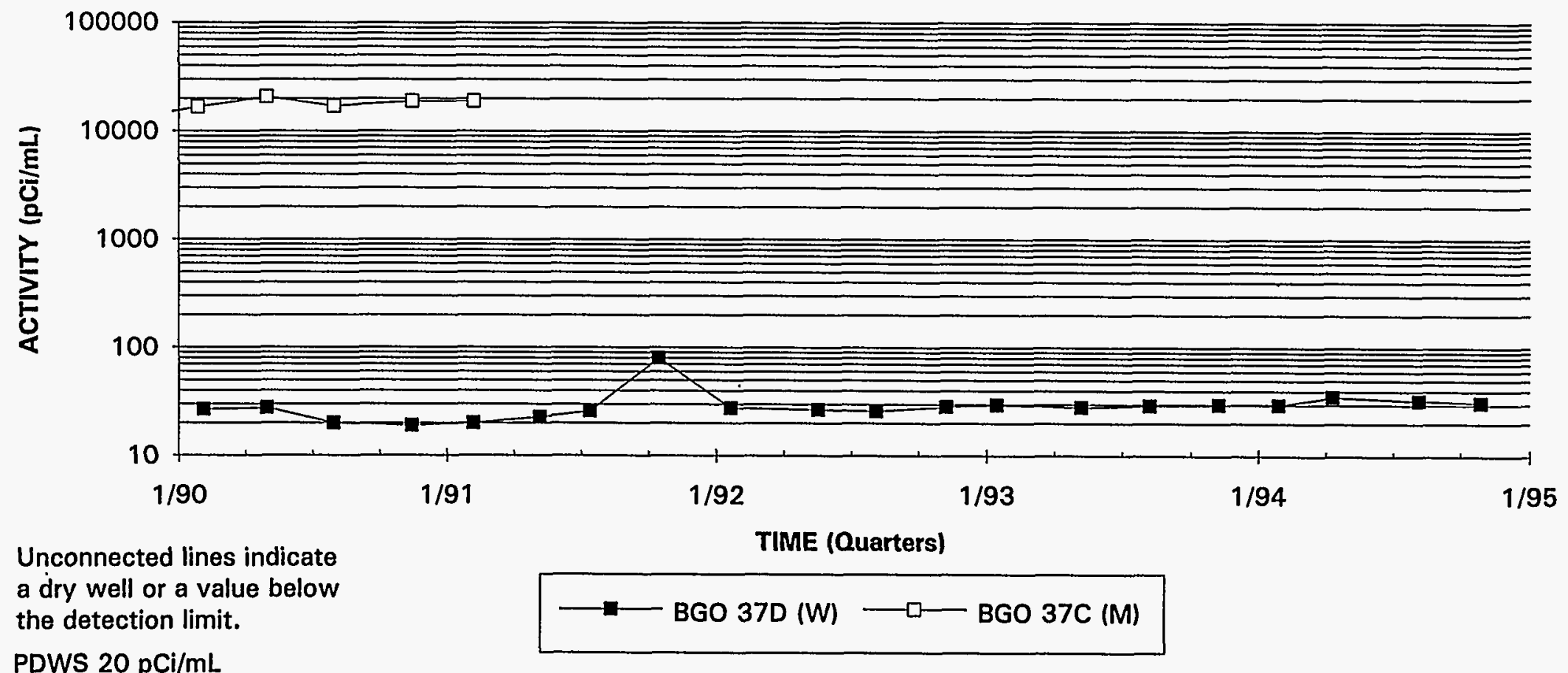

PDWS $20 \mathrm{pCi} / \mathrm{mL}$

Note: $W=$ Water Table (IIB2); B=Barnwell (IIB1); $M=$ McBean (IIB1); UC=Upper Congaree (IIA); MC=Middle Congaree (IIA); LC=Lower Congaree (IIA) 


\section{Tritium Activities \\ Well BGO 38D}

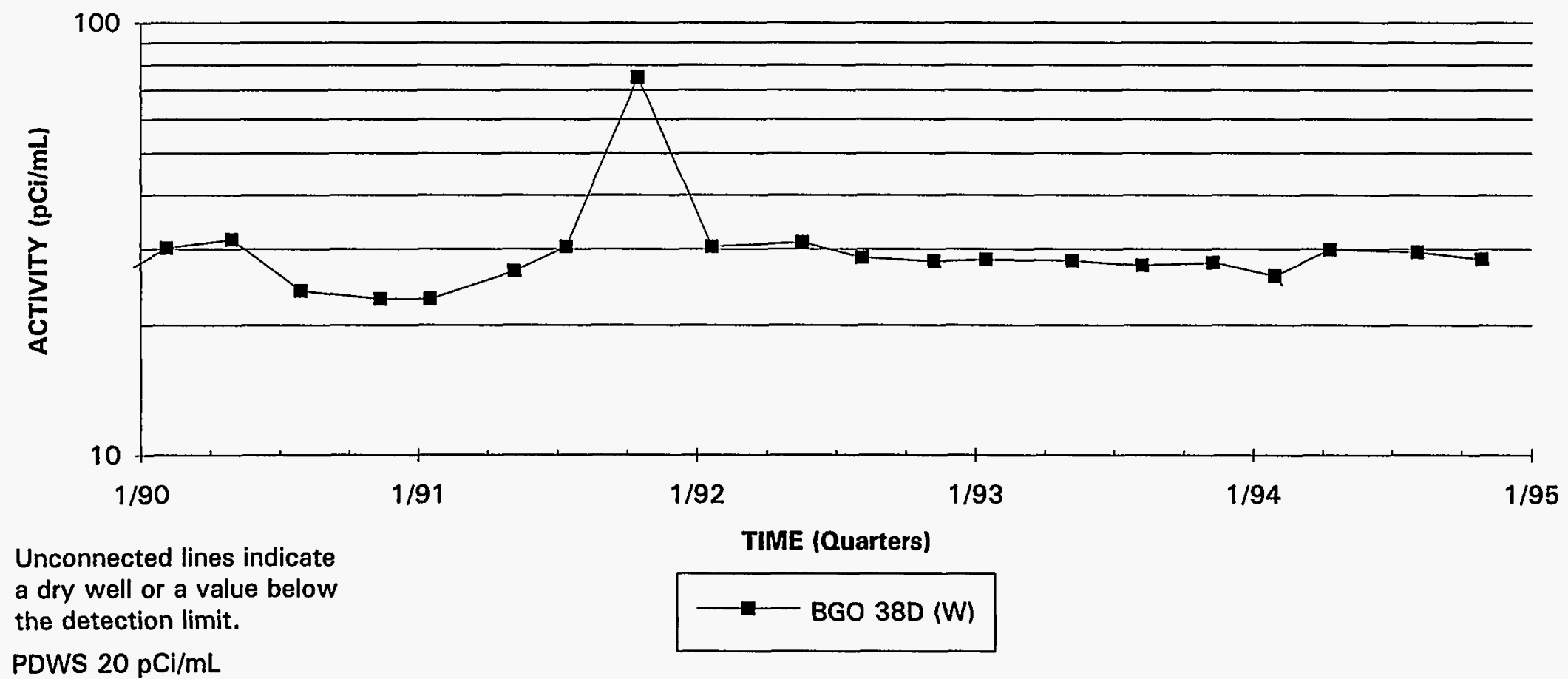

Note: W=Water Table (IIB2); B=Barnwell (IIB1); M=McBean (IIB1); UC=Upper Congaree (IIA); MC=Middle Congaree (IIA); LC=Lower Congaree (IIA) 


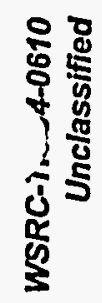
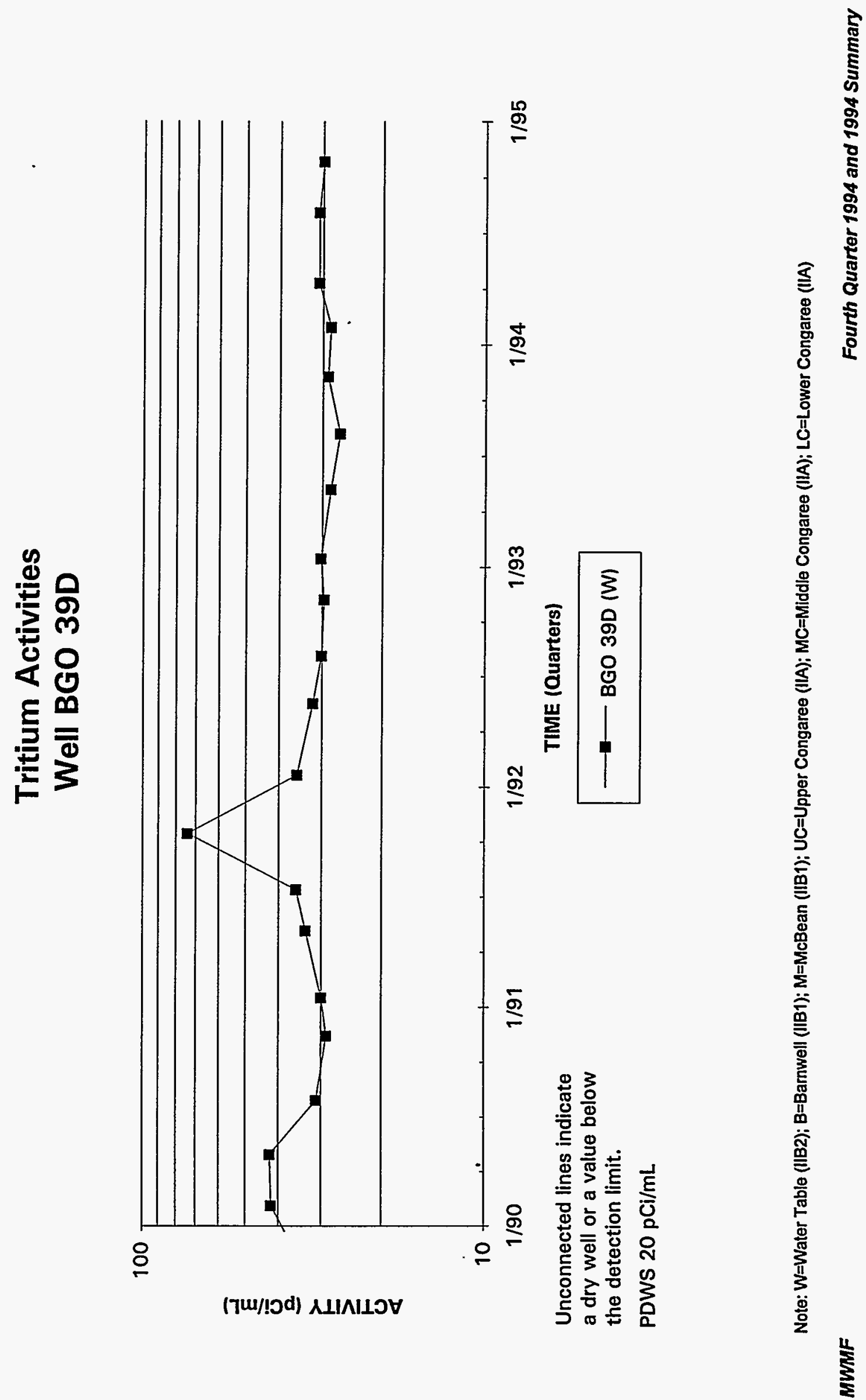

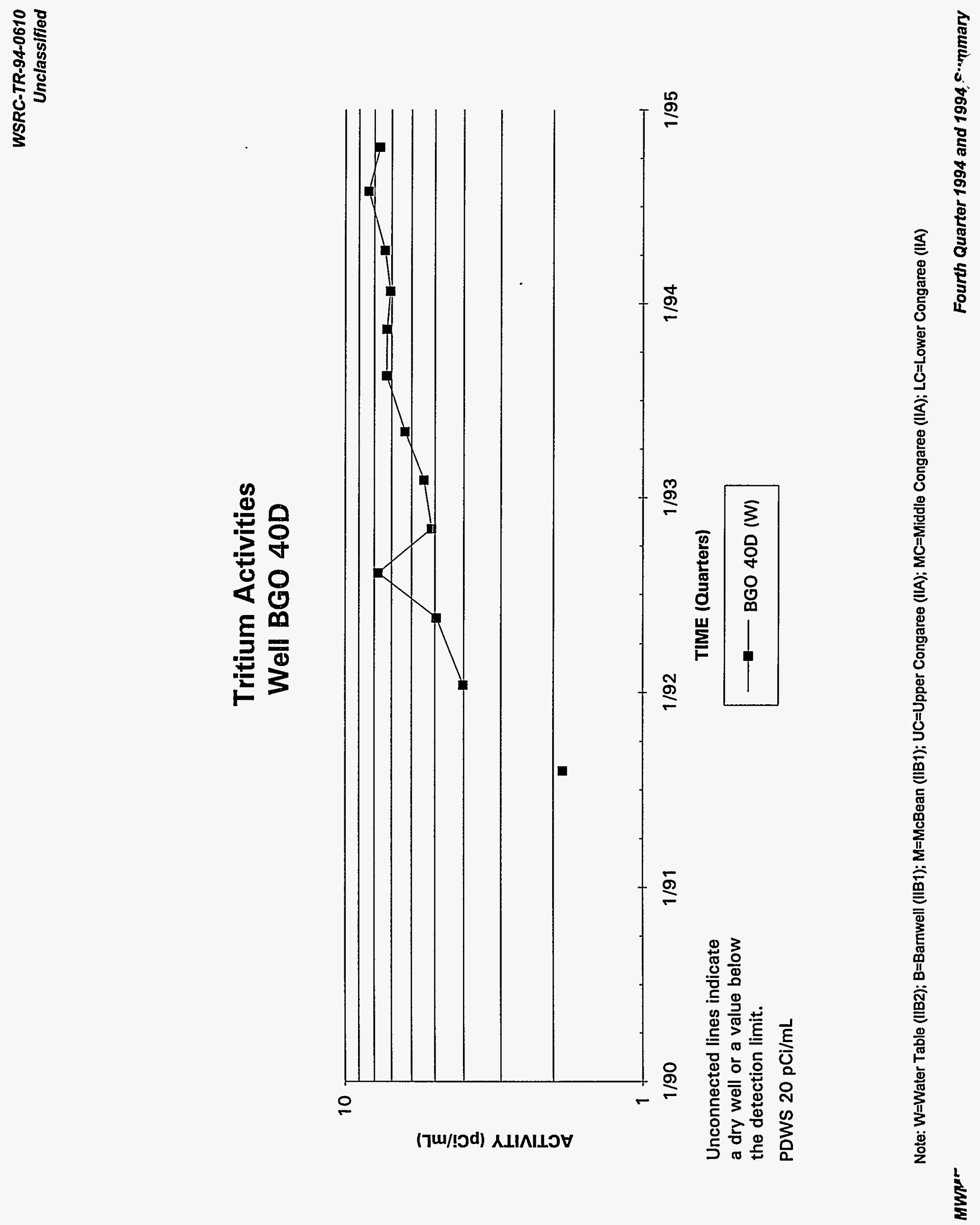


\section{Tritium Activities}

Well BGO 41A

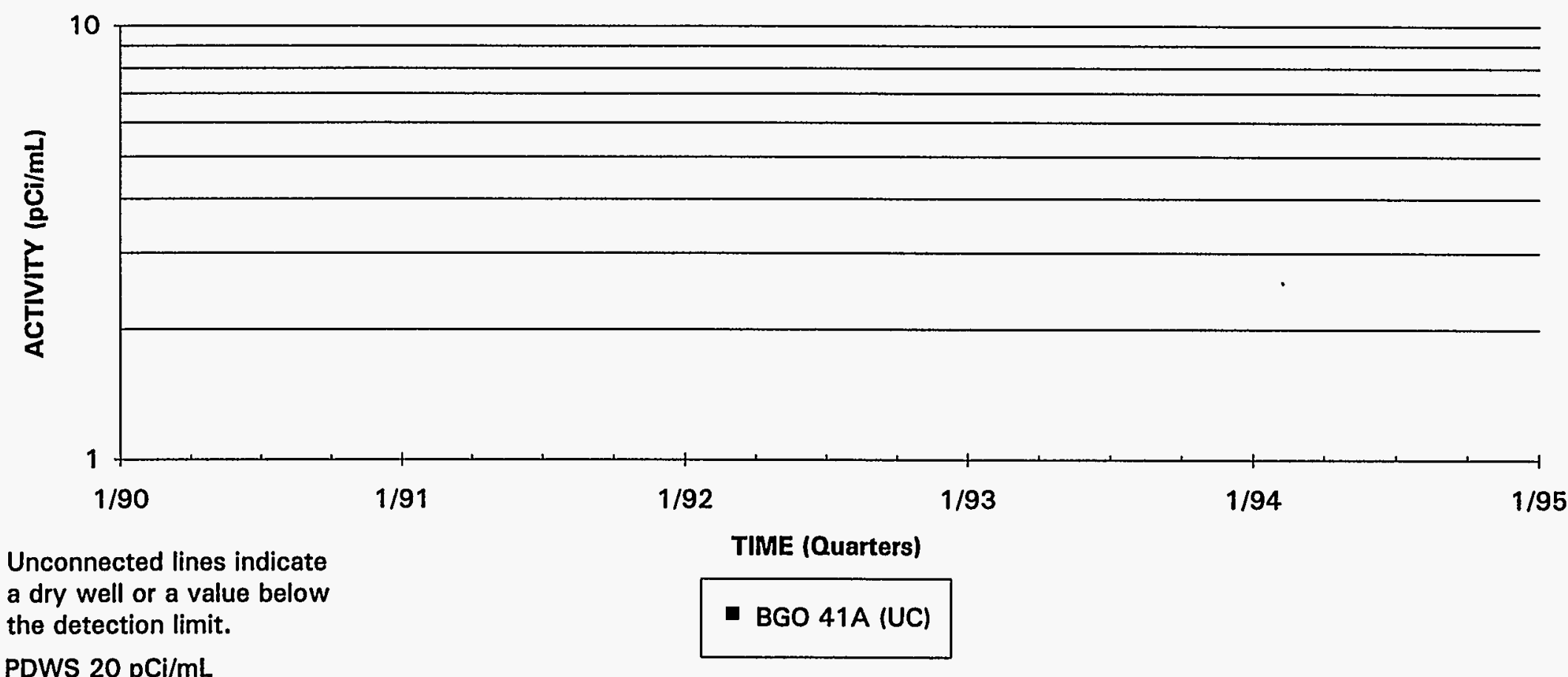

PDWS $20 \mathrm{pCi} / \mathrm{mL}$ 


\section{Tritium Activities \\ Well BGO 42C}

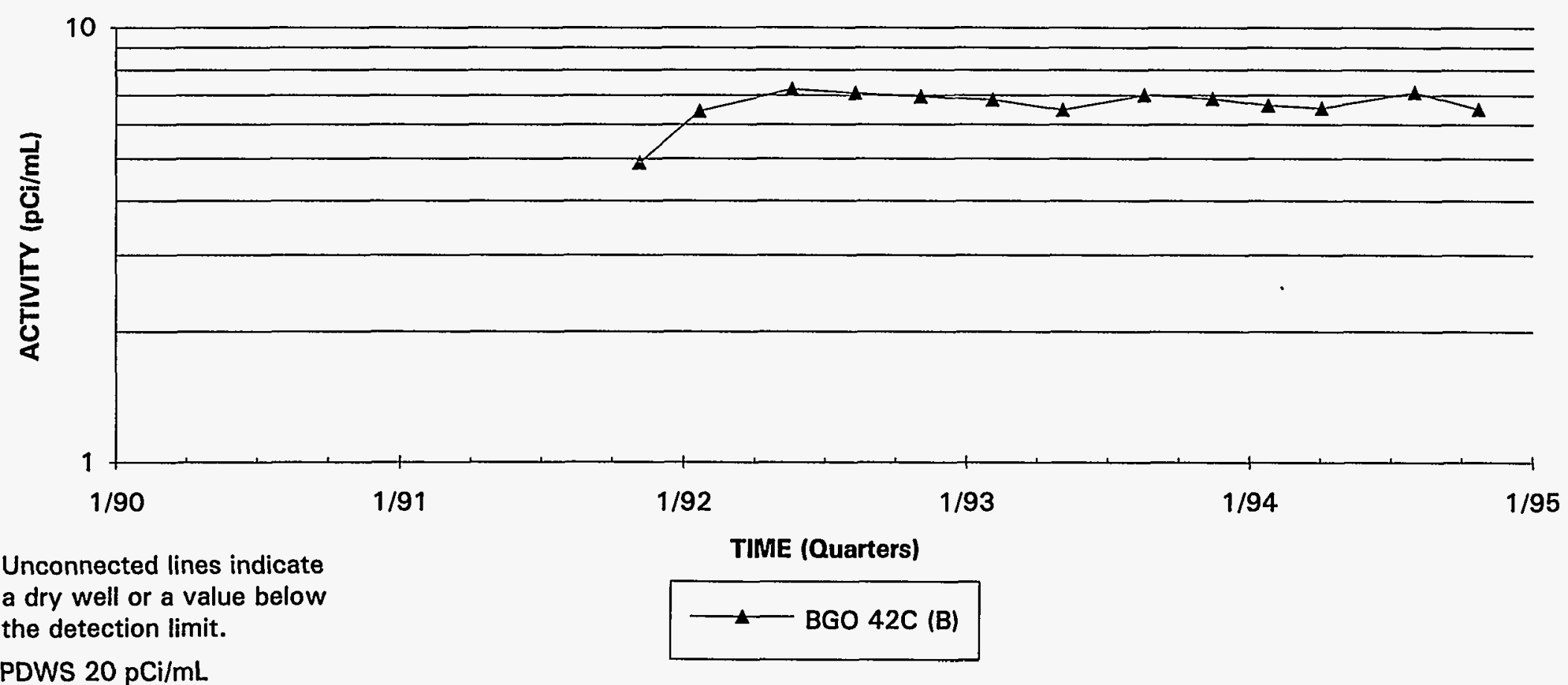

Note: W=Water Table (IIB2); B=Bamwell (IIB1); M=MCBean (IIB1); UC=Upper Congaree (IIA); MC=Middle Congaree (IIA); LC=Lower Congaree (IIA) 


\section{Tritium Activities}

\section{Well Cluster BGO 43}

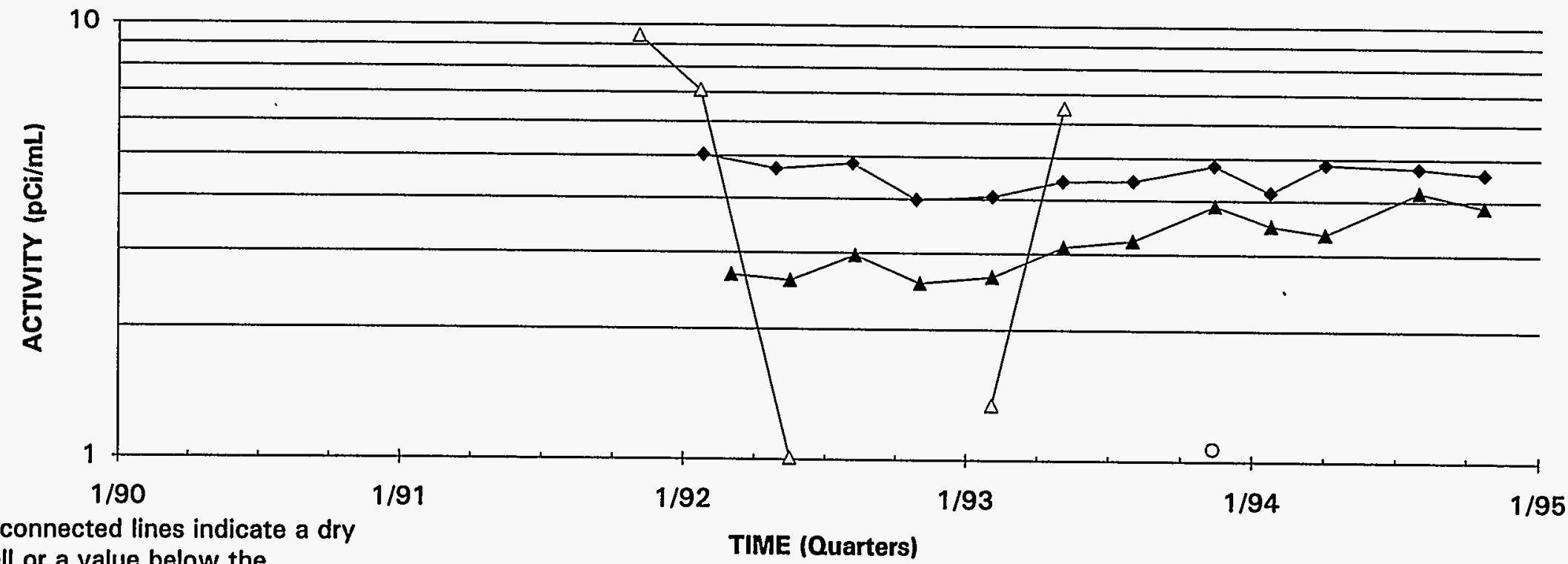

well or a value below the

detection limit.

PDWS $20 \mathrm{pCi} / \mathrm{mL}$

$\longrightarrow$ BGO 43CR (B)

$\longrightarrow$ BGO 43D (B)

$\triangle$ BGO 43A (UC)

BGO 43AA (LC)

Note: W=Water Table (IIB2); B=Barnwell (IIB1); M=McBean (IIB1); UC=Upper Congaree (IIA); MC=Middle Congaree (IIA); LC=Lower Congaree (IIA) 


\section{Tritium Activities Well Cluster BGO 44}

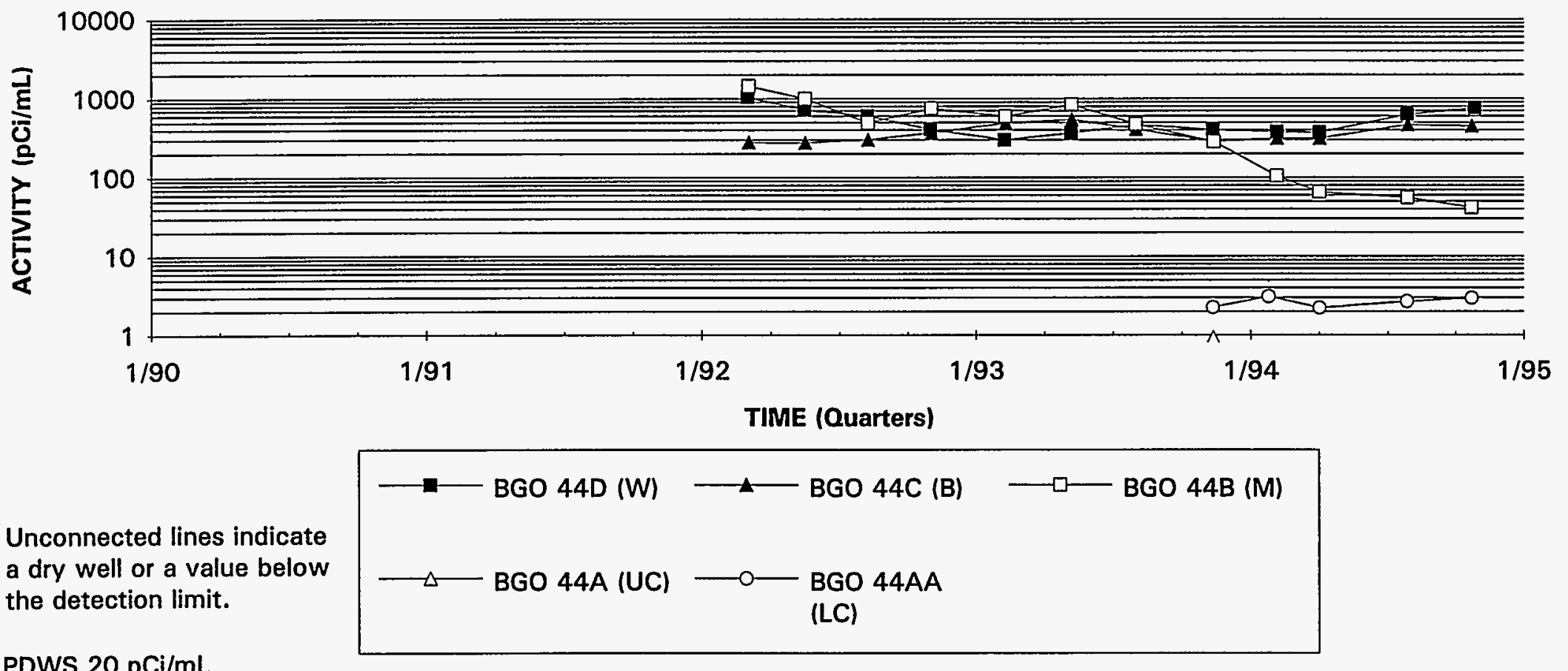

Note: $W=$ Water Table (IIB2); B=Barnwell (IIB1); $M=$ McBean (IIB1); UC=Upper Congaree (IIA); MC=Middle Congaree (IIA); LC=Lower Congaree (IIA) 


\section{Tritium Activities \\ Well Cluster BGO 45}

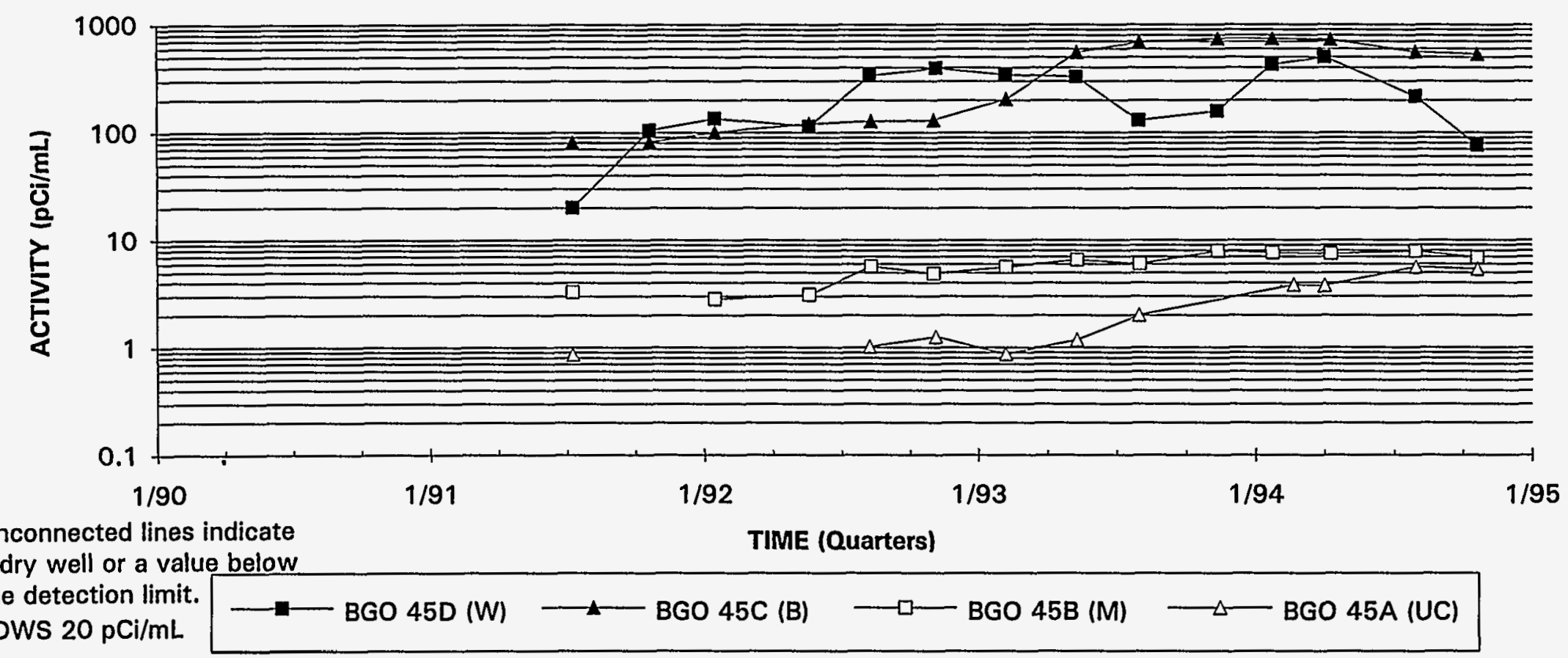

Note: W=Water Table (IIB2); B=Barnwell (IIB1); M=McBean (IIB1); UC=Upper Congaree (IIA); MC=Middle Congaree (IIA); LC=Lower Congaree (IIA) 


\section{Tritium Activities}

\section{Well Cluster BGO 46}

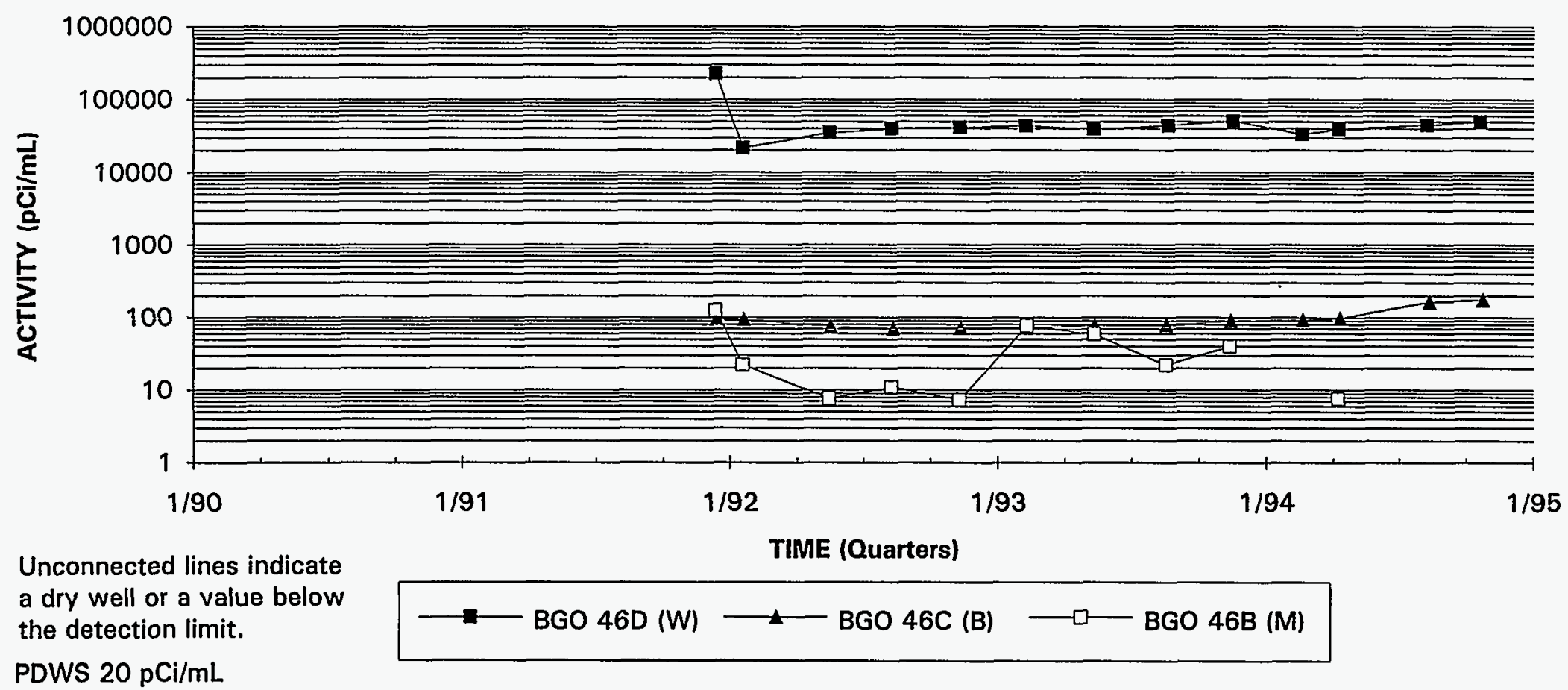

Note: W=Water Table (IIB2); B=Barnwell (IIB1); M=McBean (IIB1); UC=Upper Congaree (IIA); MC=Middle Congaree (IIA); LC=Lower Congaree (IIA) 


\section{Tritium Activities}

\section{Well Cluster BGO 47}

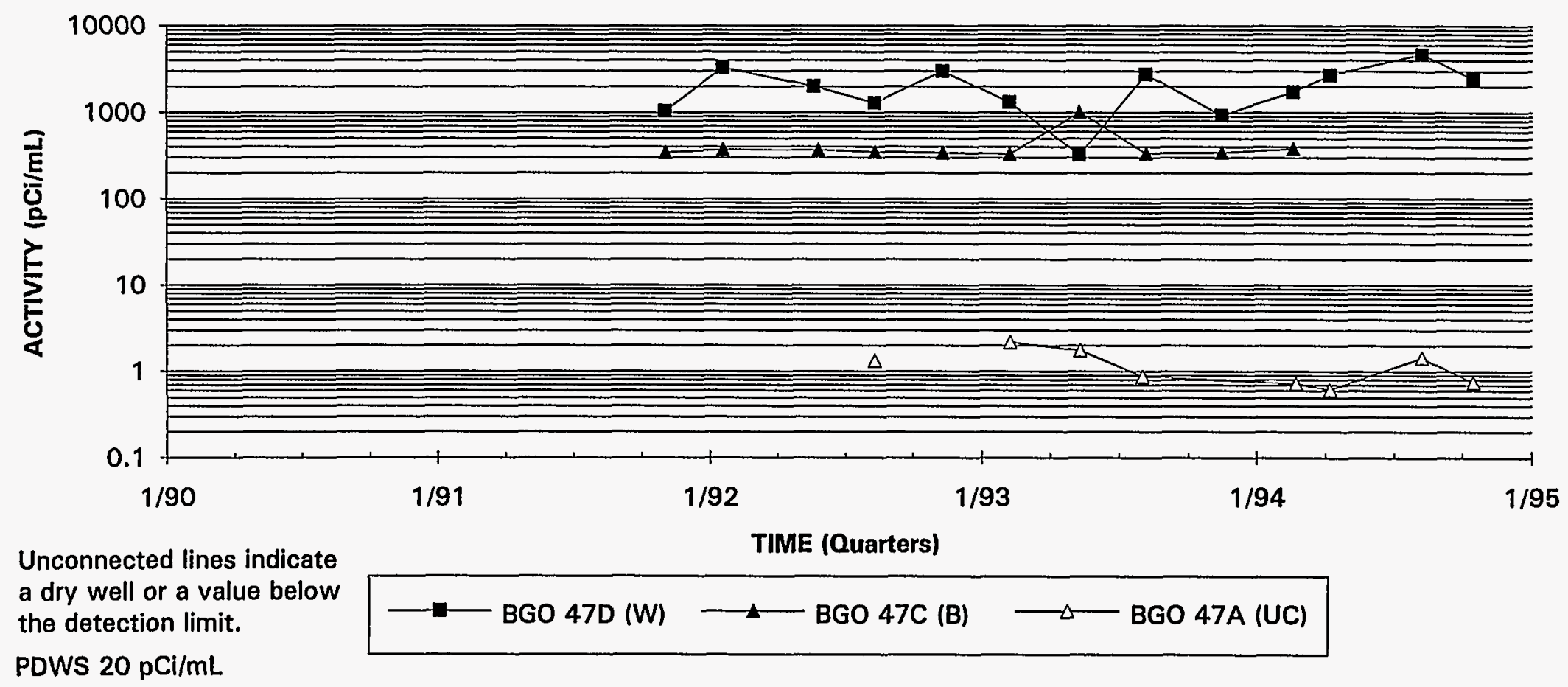

Note: $W=W a t e r$ Table (IIB2); B=Barnwell (IIB1); $M=$ McBean (IIB1); UC=Upper Congaree (IIA); MC=Middle Congaree (IIA); LC=Lower Congaree (IIA) 


\section{Tritium Activities Well Cluster BGO 48}

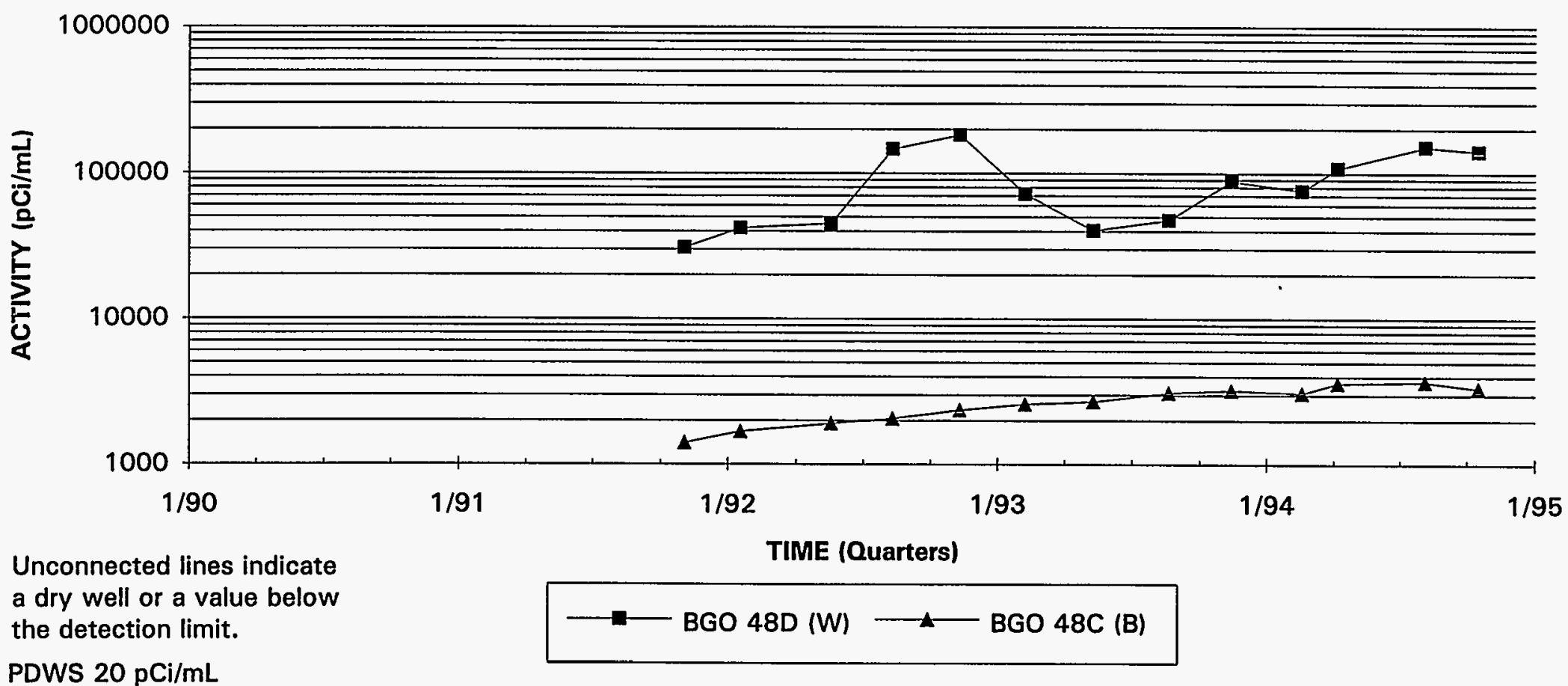

Note: W=Water Table (IIB2); B=Barnwell (IIB1); M=McBean (IIB1); UC=Upper Congaree (IIA); MC=Middle Congaree (IIA); LC=Lower Congaree (IIA) 


\section{Tritium Activities}

\section{Well Cluster BGO 49}

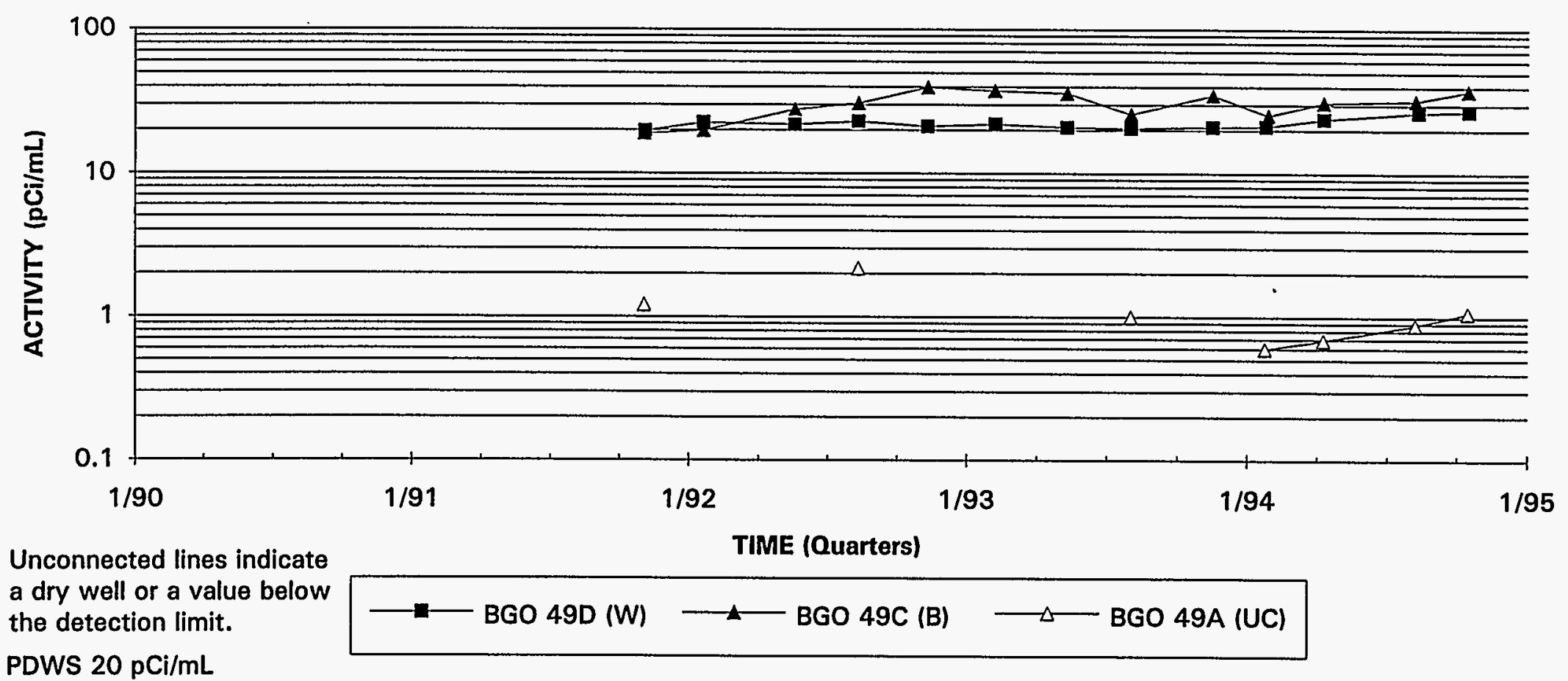

Note: W=Water Table (IIB2); B=Barnwell (IIB1); M=McBean (IIB1); UC=Upper Congaree (IIA); MC=Middle Congaree (IIA); LC=Lower Congaree (IIA) 


\section{Tritium Activities}

\section{Well Cluster BGO 50}

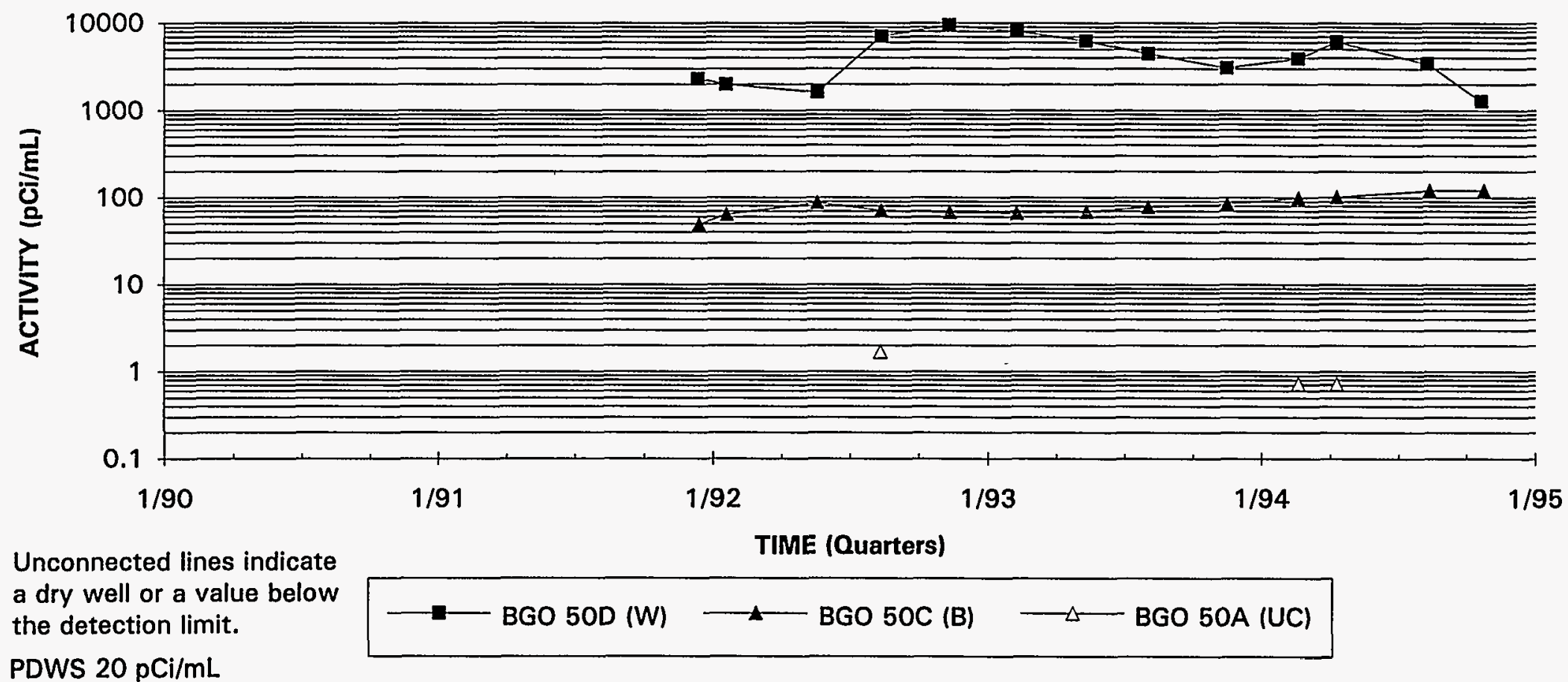

PDWS $20 \mathrm{pCi} / \mathrm{mL}$ 
Tritium Activities

\section{Well Cluster BGX 1}

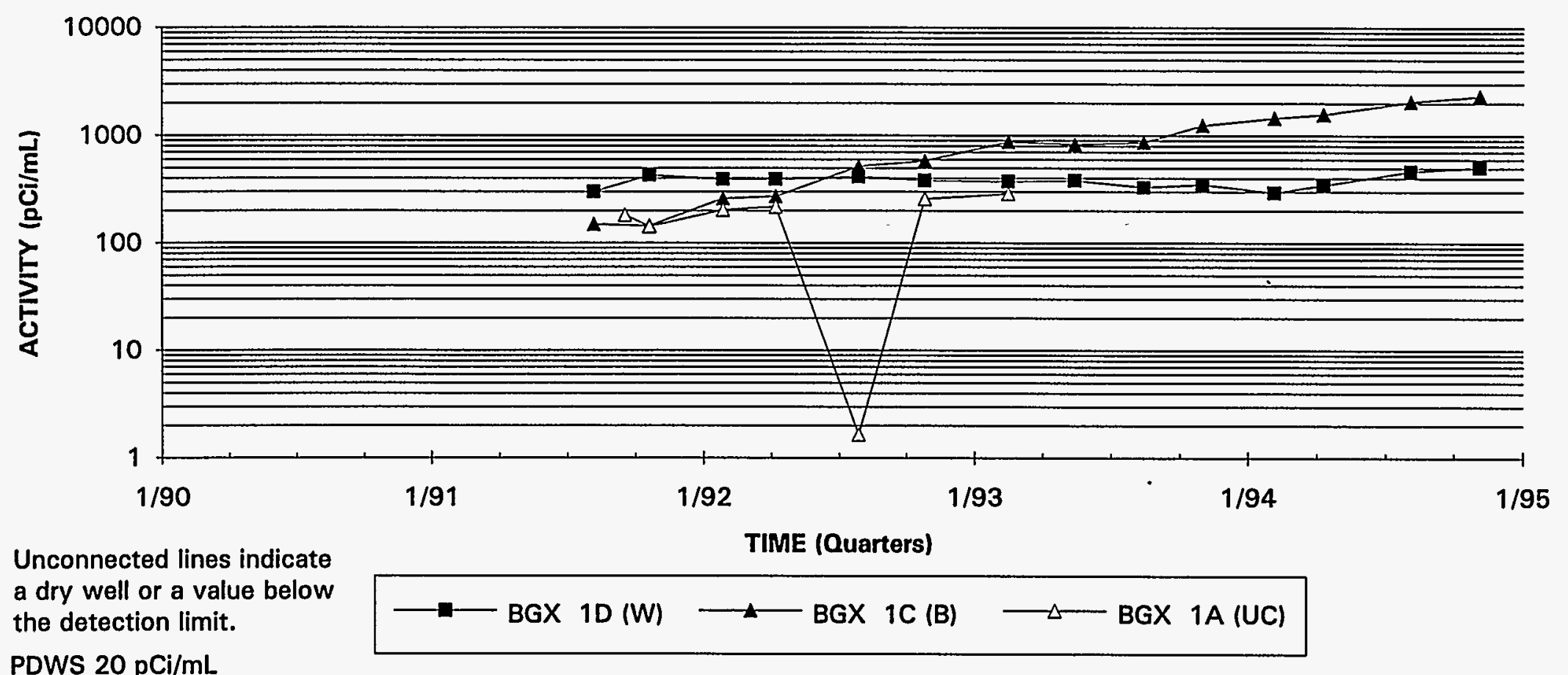

Note: W=Water Table (IIB2); B=Barnwell (IIB1); M=McBean (IIB1); UC=Upper Congaree (IIA); MC=Middle Congaree (IIA); LC=Lower Congaree (IIA) 


\section{Tritium Activities} Well Cluster BGX 2

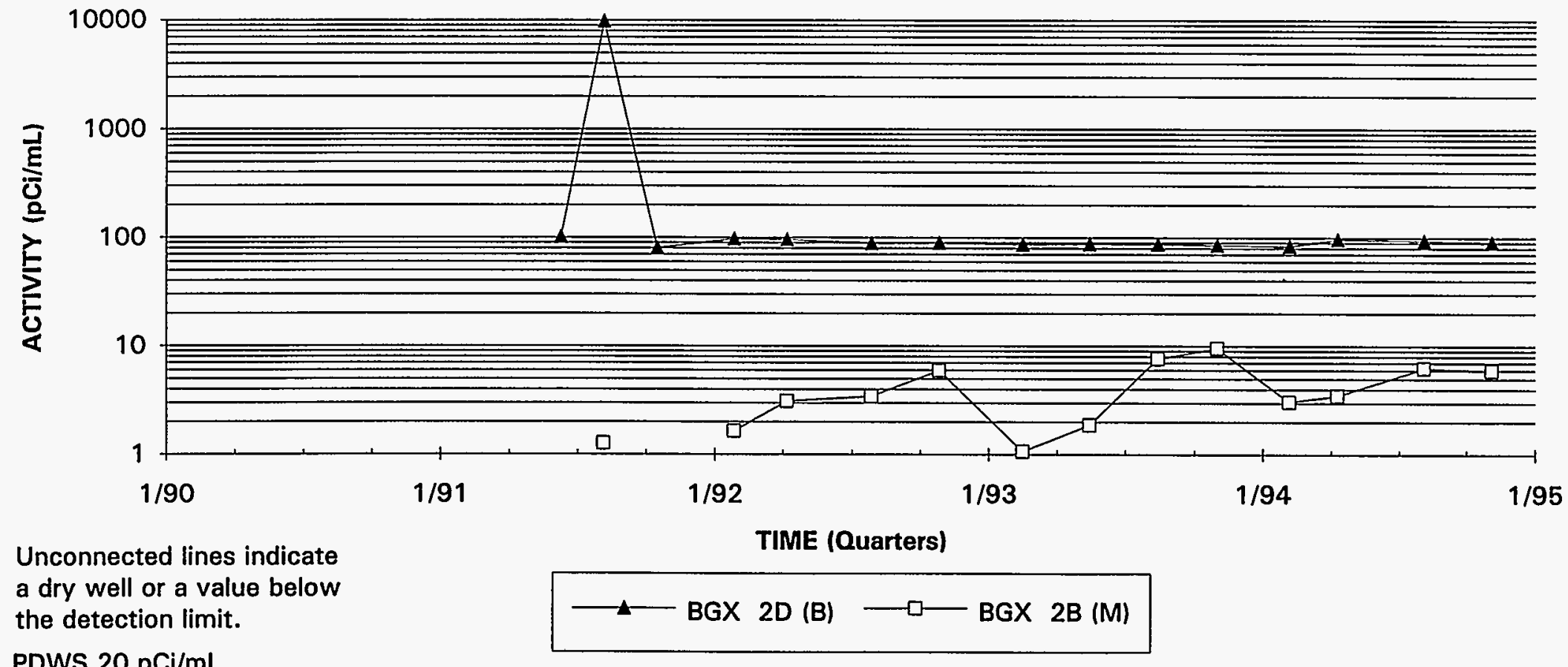

Note: W=Water Table (IIB2); B=Barnwell (IIB1); $M=$ McBean (IIB1); UC=Upper Congaree (IIA); MC=Middle Congaree (IIA); LC=Lower Congaree (IIA) 


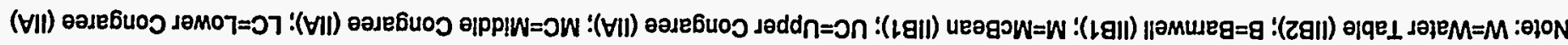

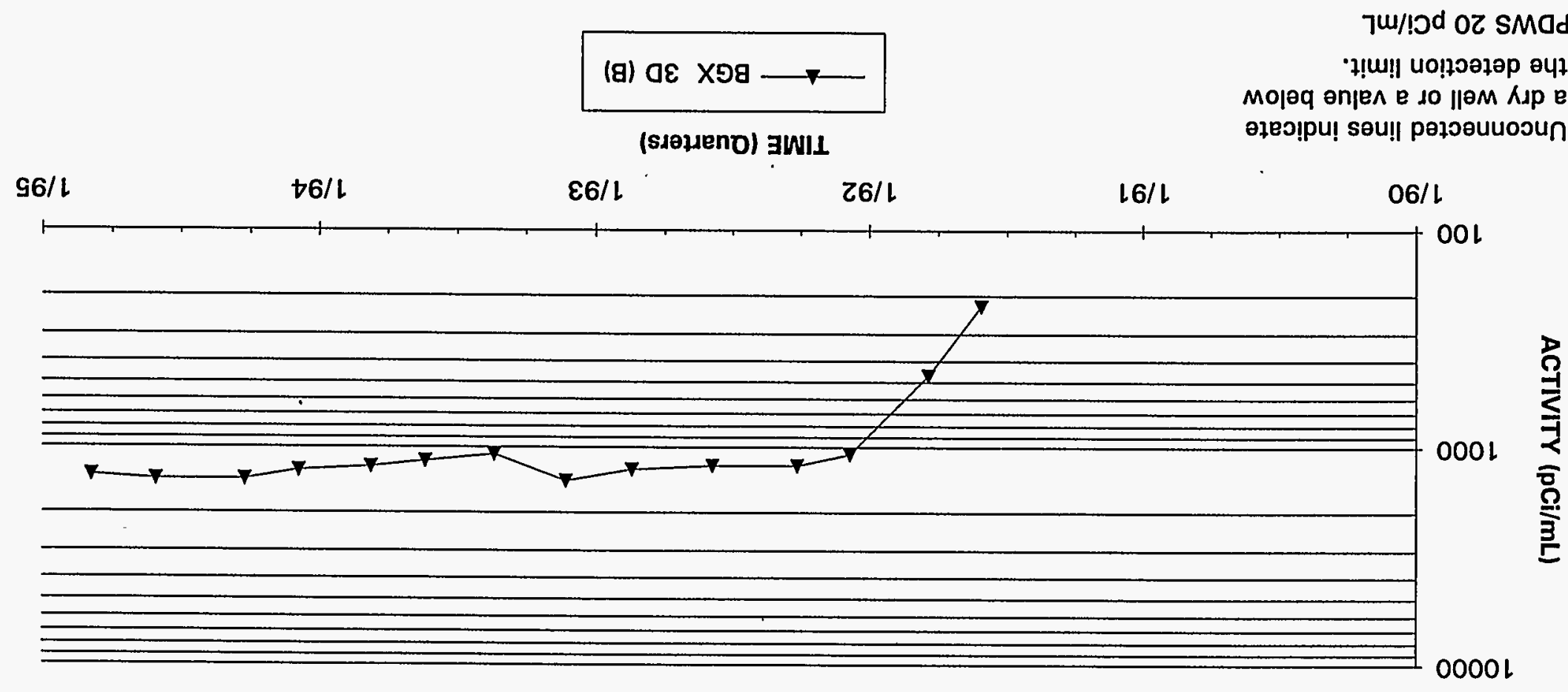

\section{वह Xפg $\| ә M$

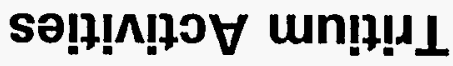




\section{Tritium Activities}

\section{Well Cluster BGX 4}

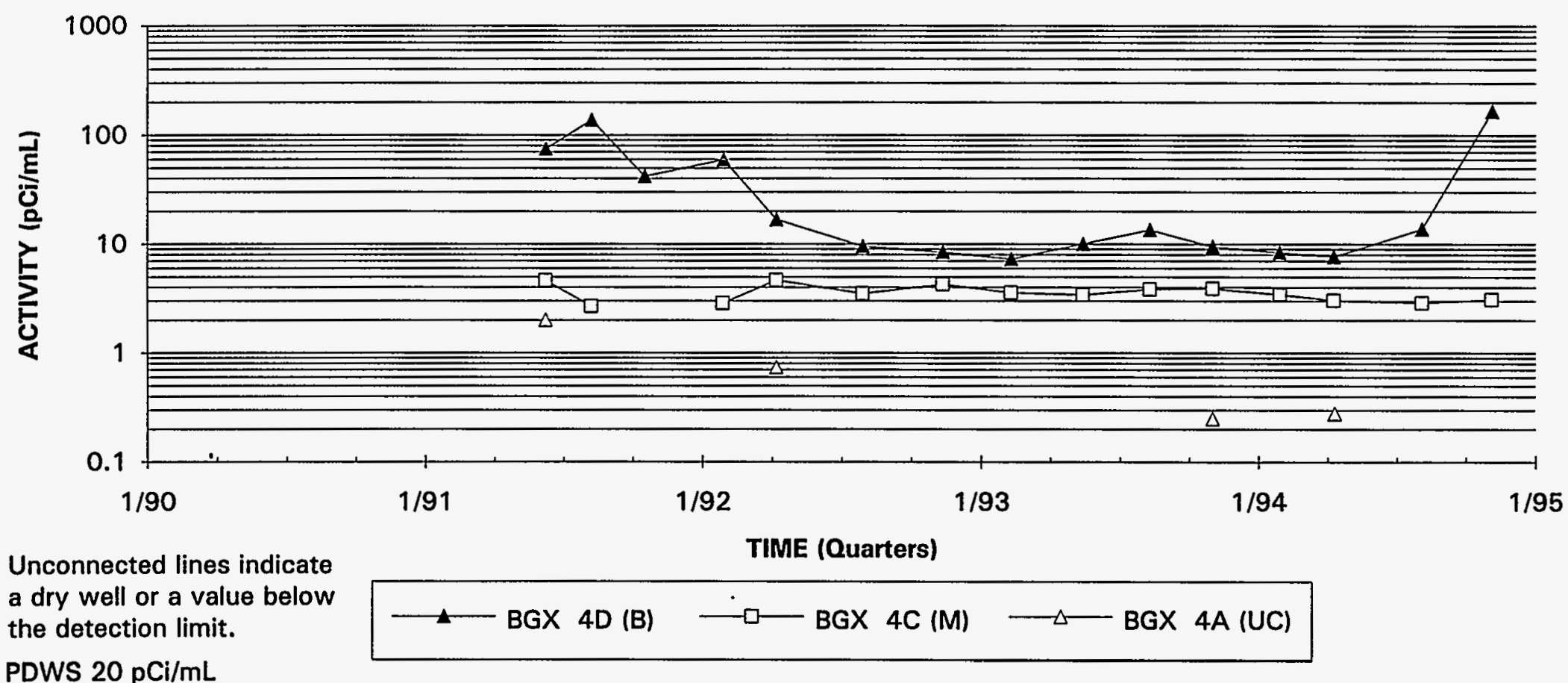

Note: W=Water Table (IIB2); B=Barnwell (IIB1); M=McBean (IIB1); UC=Upper Congaree (IIA); MC=Middle Congaree (IIA); LC=Lower Congaree (IIA) 


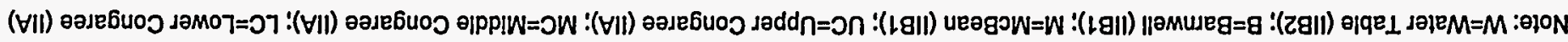

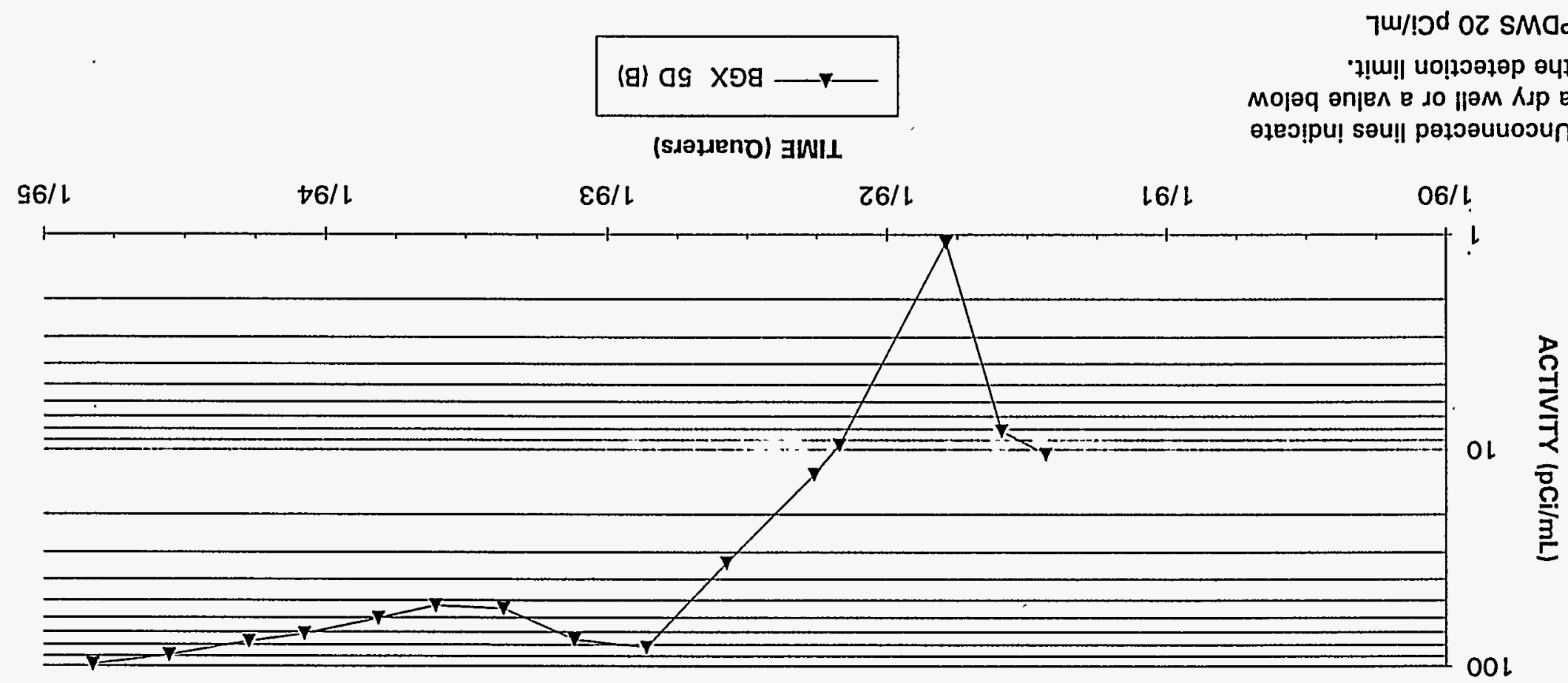

Q9 Xפg $\| \nabla M$

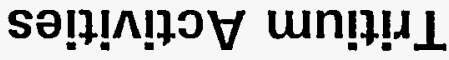




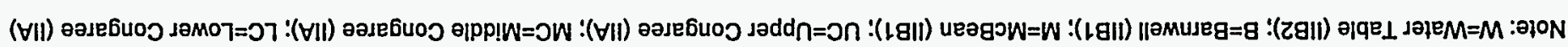

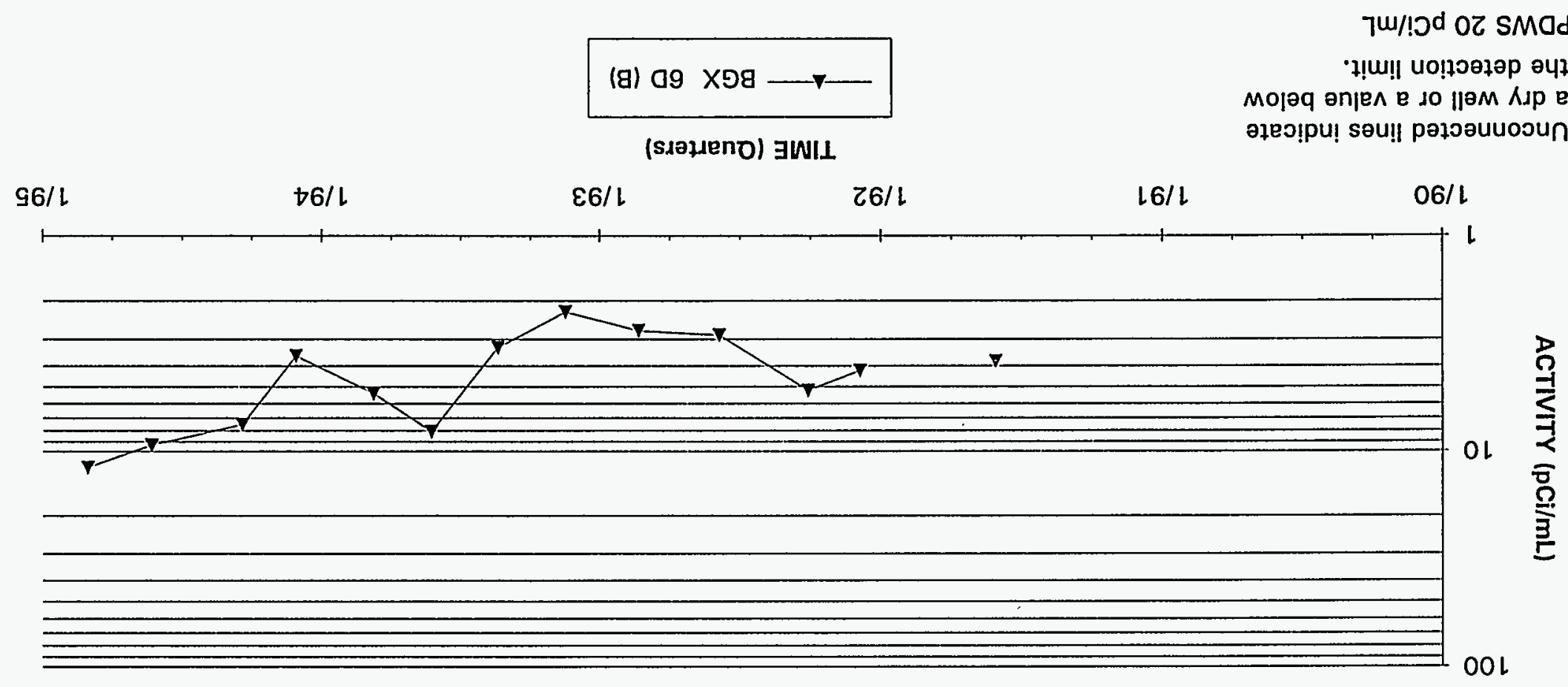

\section{Xפ9 ॥əM}

sə!ฺ!ก!ฺ० 


\section{Tritium Activities}

Well BGX 7D

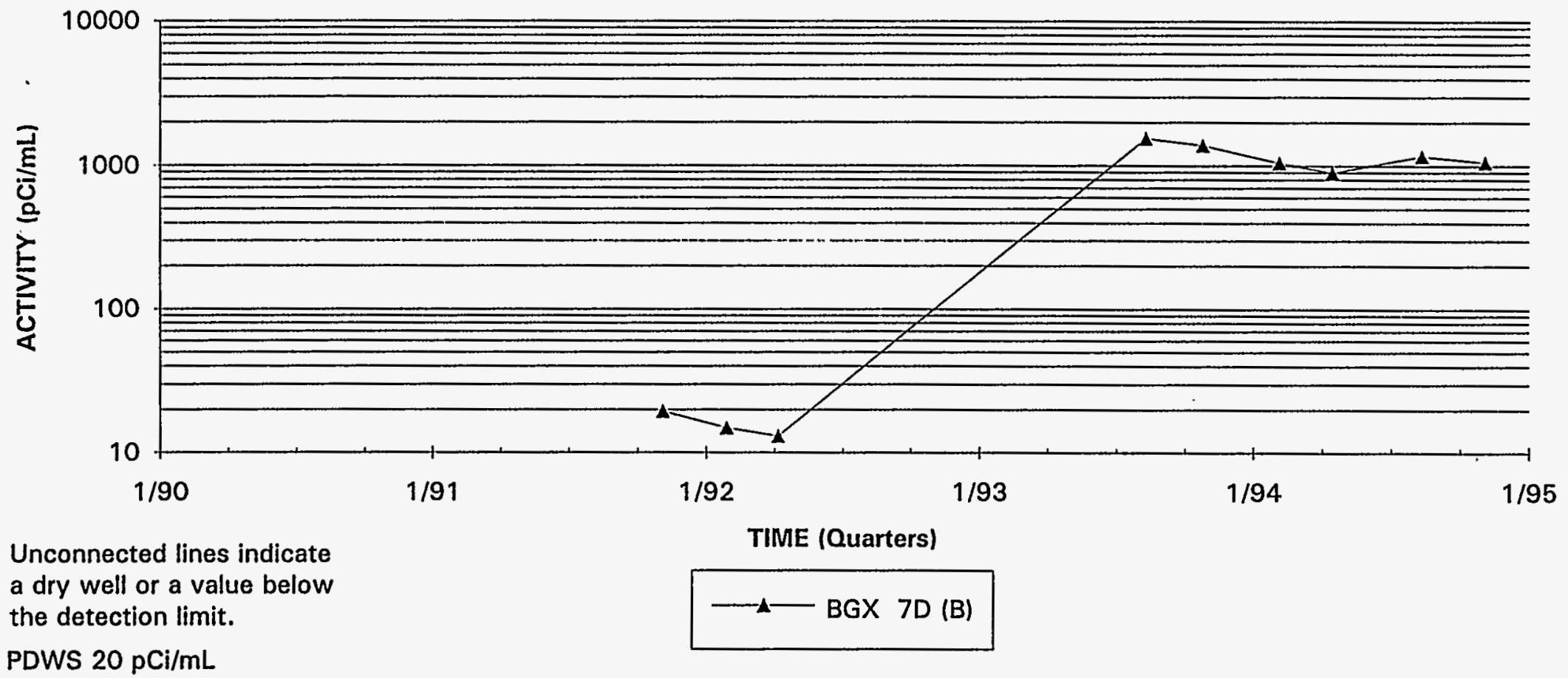

Note: $W=$ Water Table (IIB2); B=Barnwell (IIB1); $M=$ McBean (IIB1); UC=Upper Congaree (IIA); MC=Middle Congaree (IIA); LC=Lower Congaree (IIA) 


\section{Tritium Activities \\ Well Cluster BGX 8}

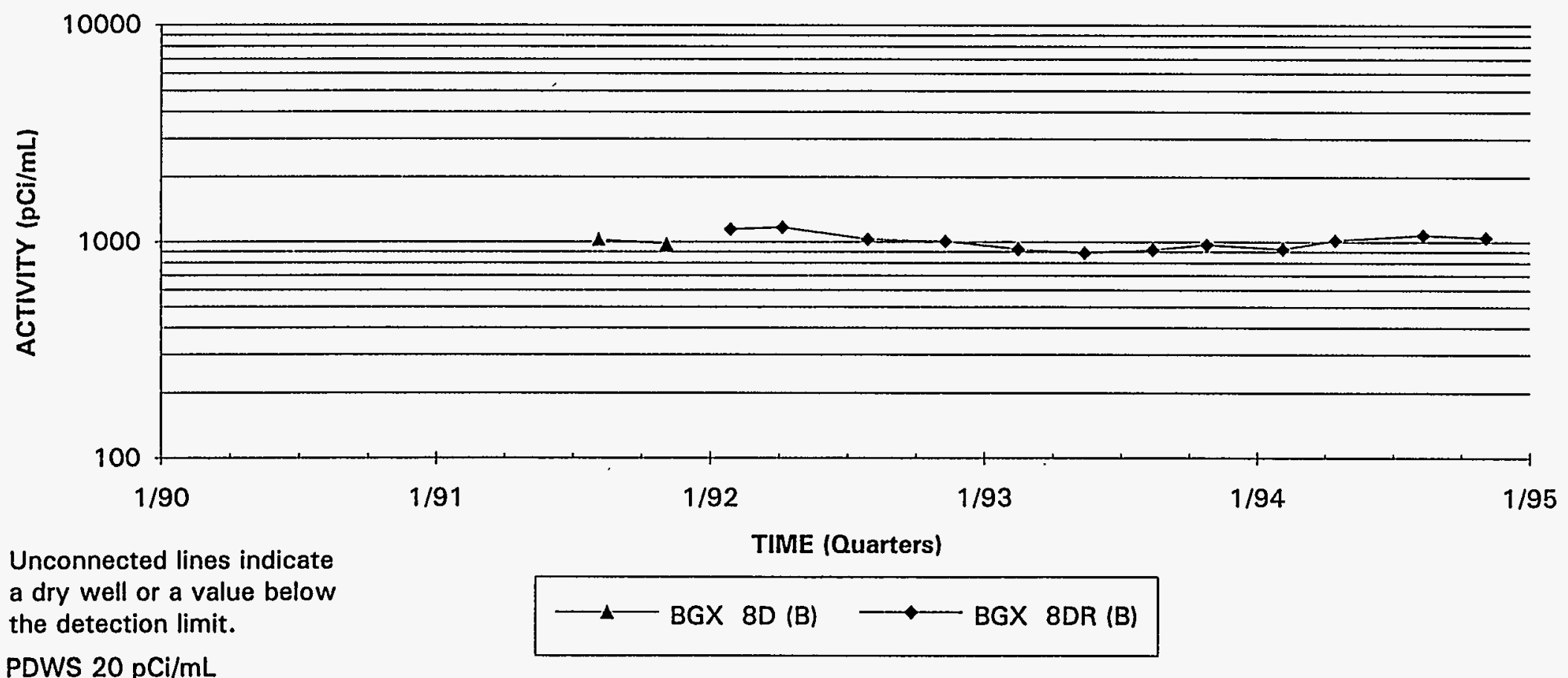

Note: W=Water Table (IIB2); B=Barnwell (IIB1); M=MCBean (IIB1); UC=Upper Congaree (IIA); MC=Middle Congaree (IIA); LC=Lower Congaree (IIA) 


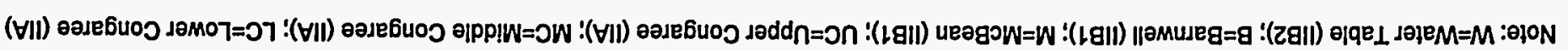

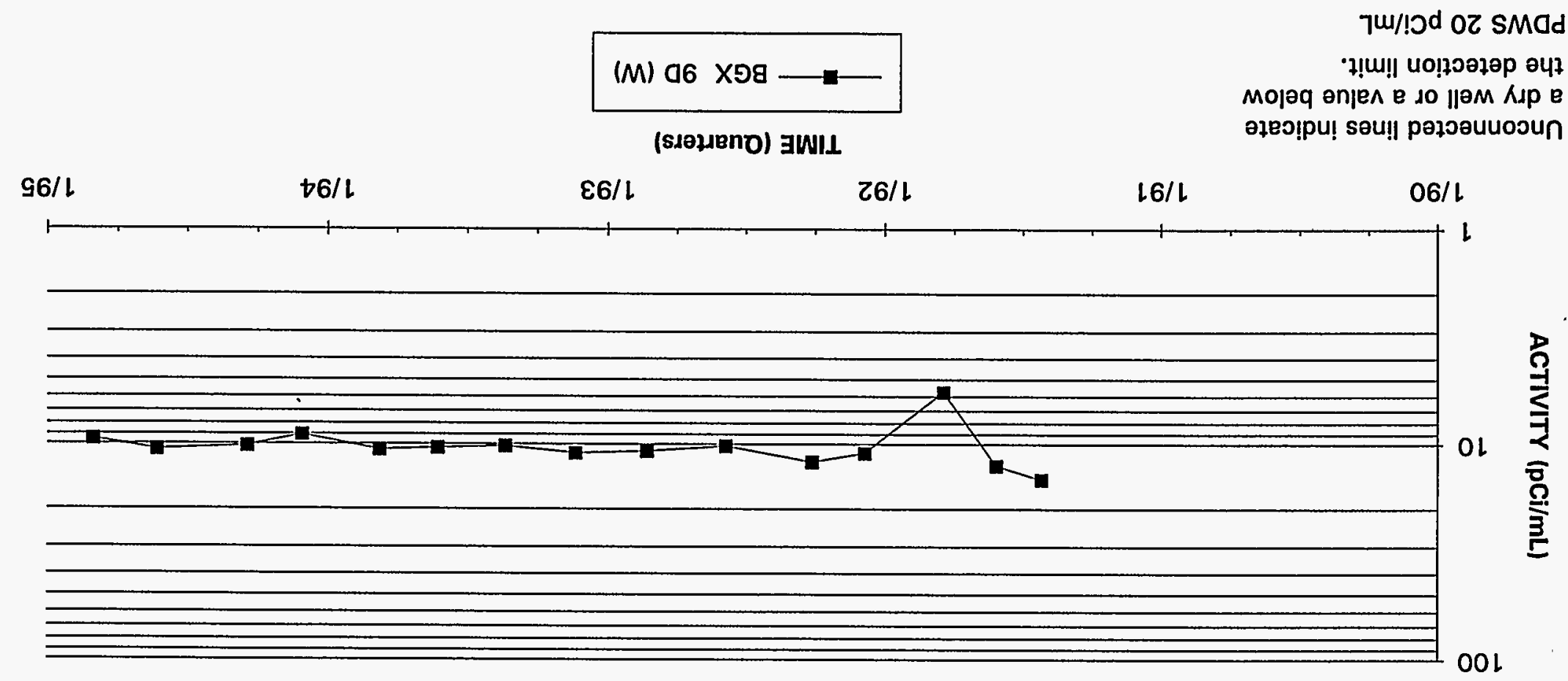

06 X99 $\| ә M$ sə!!!ก!ฺ० 


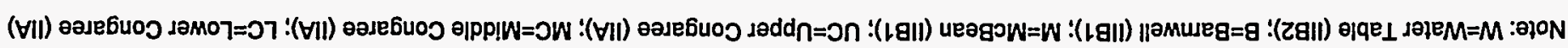

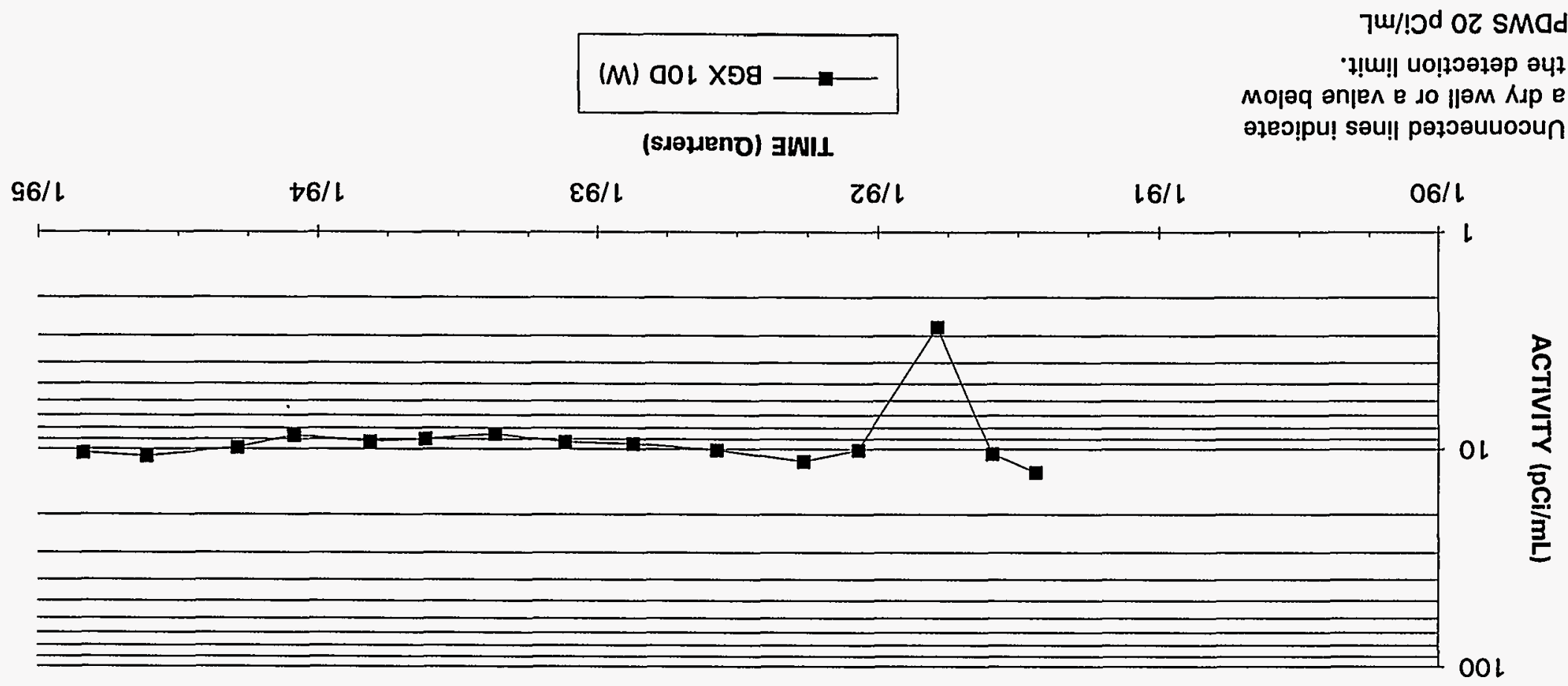

\section{GOL XOg \|әM

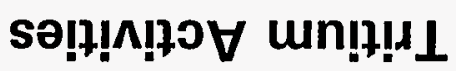




\section{Tritium Activities \\ Well BGX 11D}

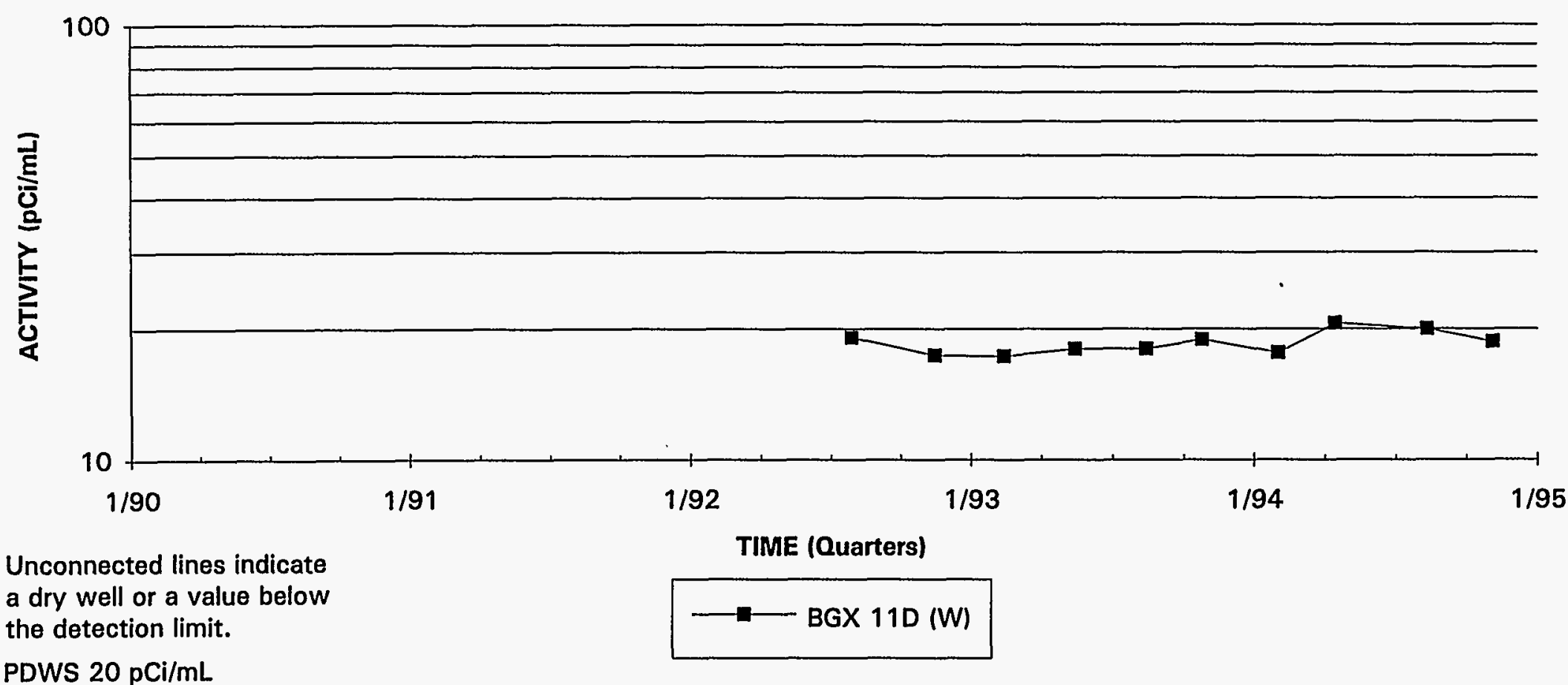

PDWS $20 \mathrm{pCi} / \mathrm{mL}$ 


\section{Tritium Activities}

\section{Well Cluster BGX 12}

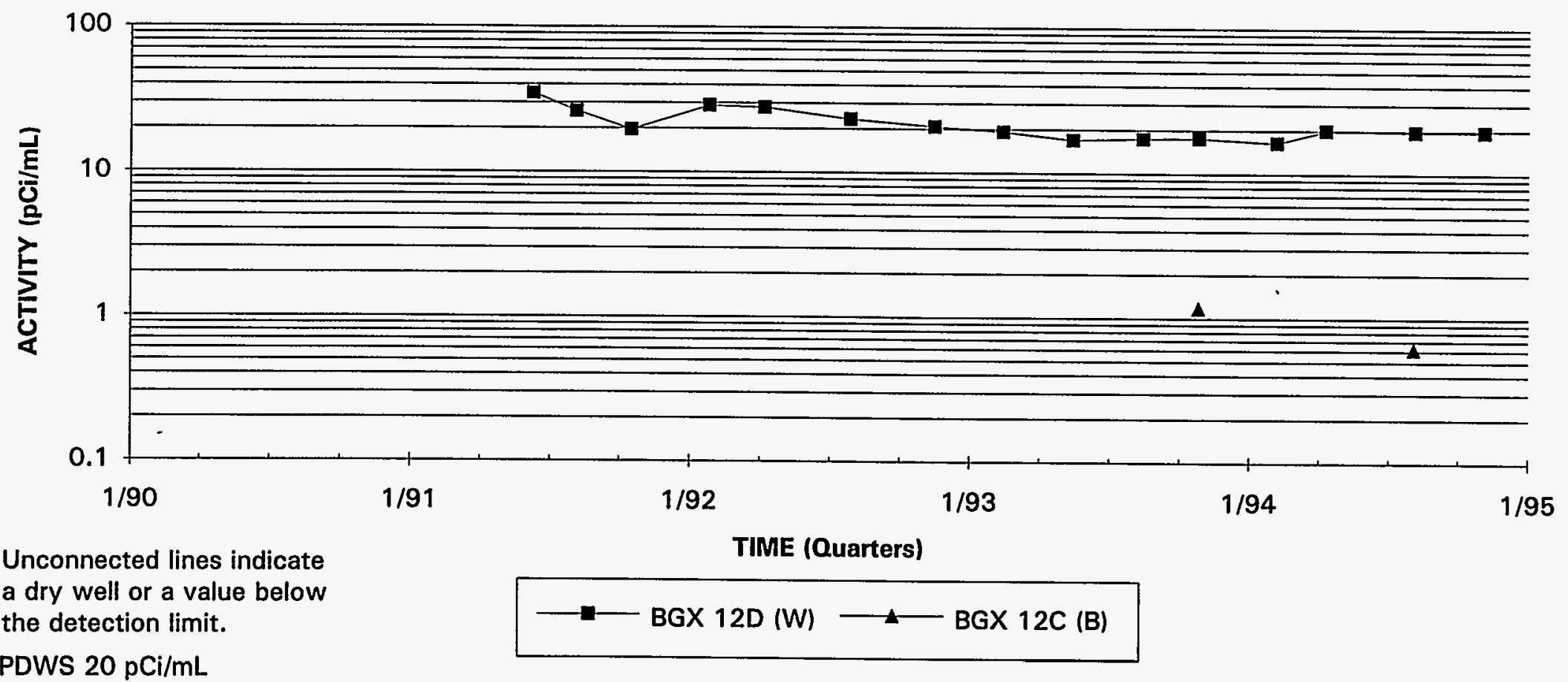

Note: W=Water Table (IIB2); B=Barnwell (IIB1); M=McBean (IIB1); UC=Upper Congaree (IIA); MC=Middle Congaree (IIA); LC=Lower Congaree (IIA) 


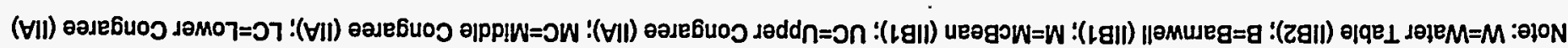

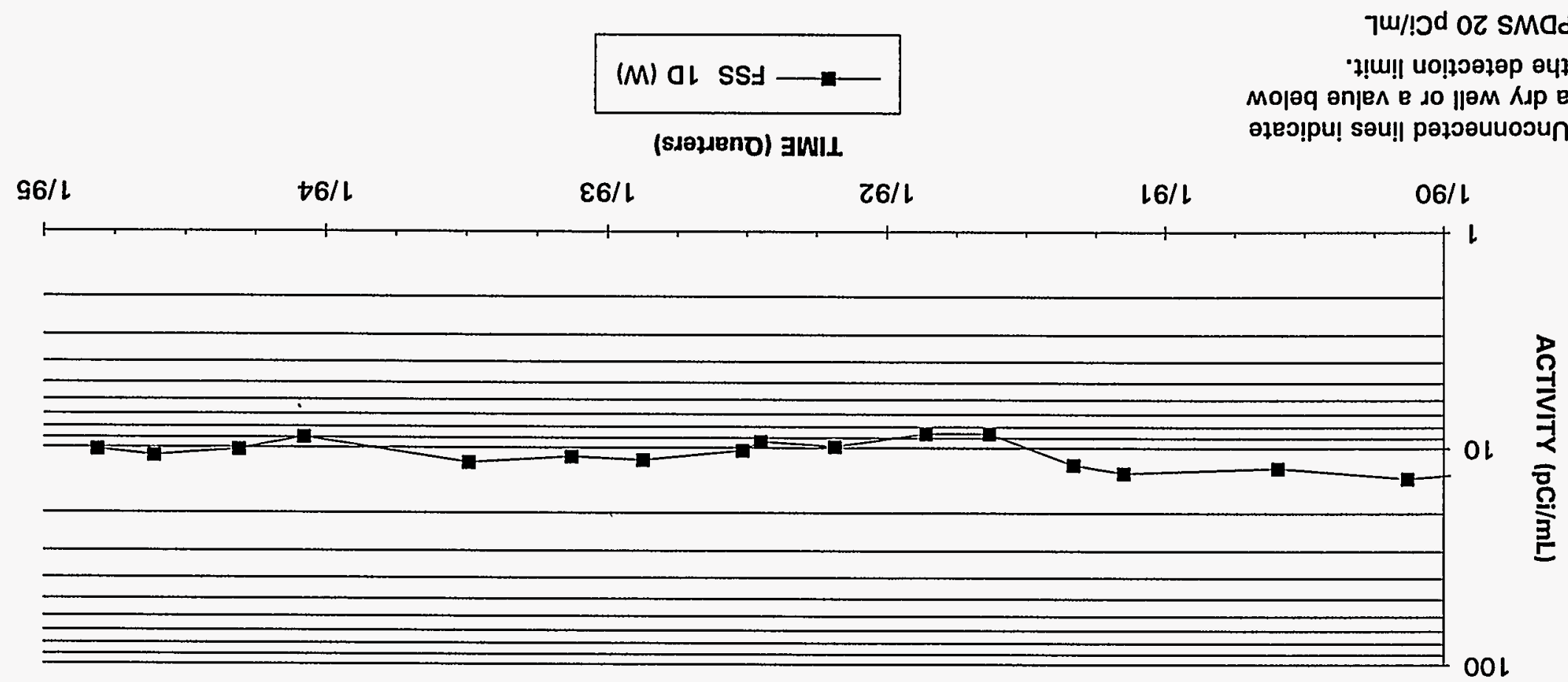

QL SS」 IIPM Sอ!!!ก!ฺ० 


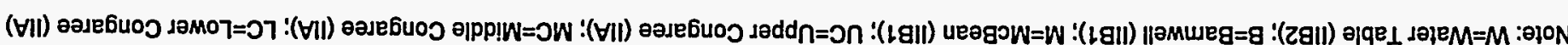

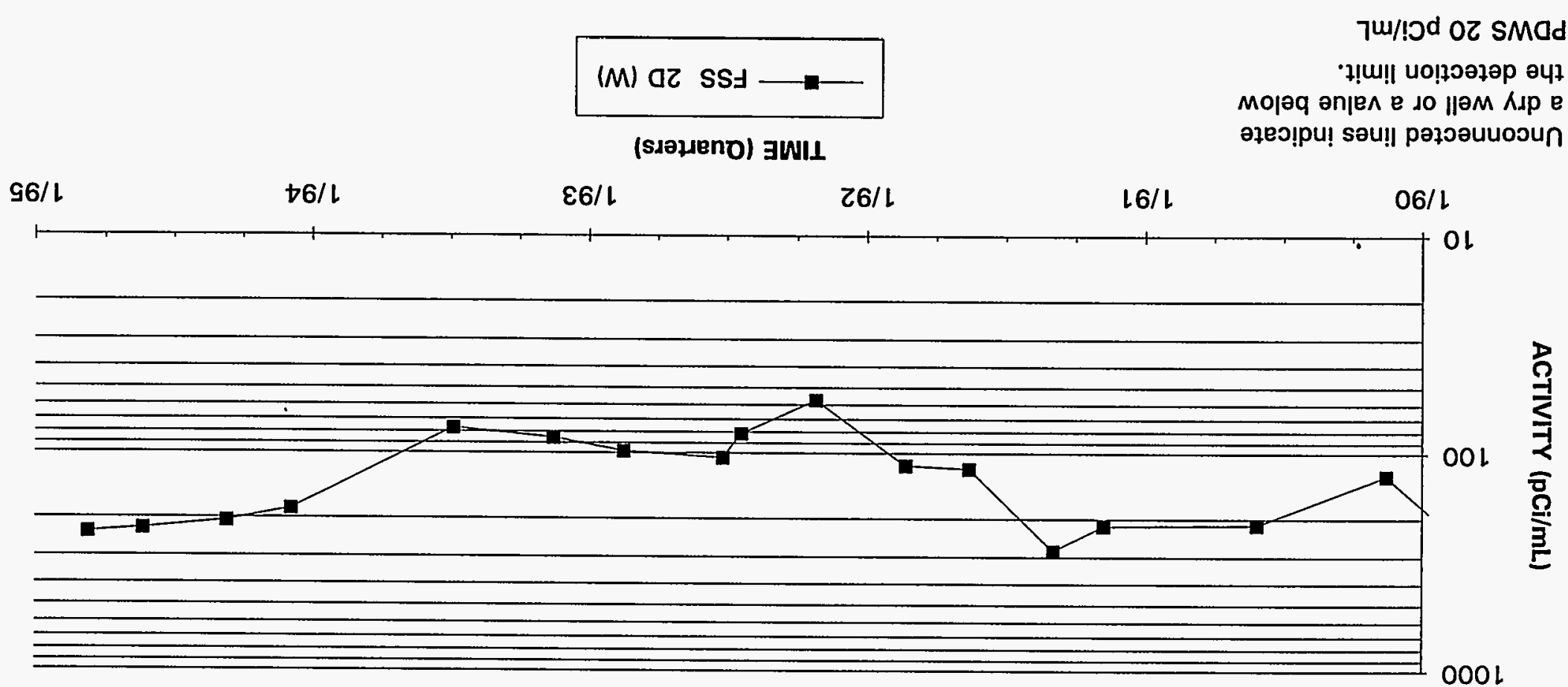

\section{aZ SS」 IIOM}

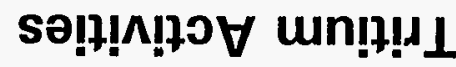




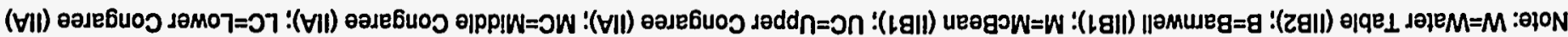

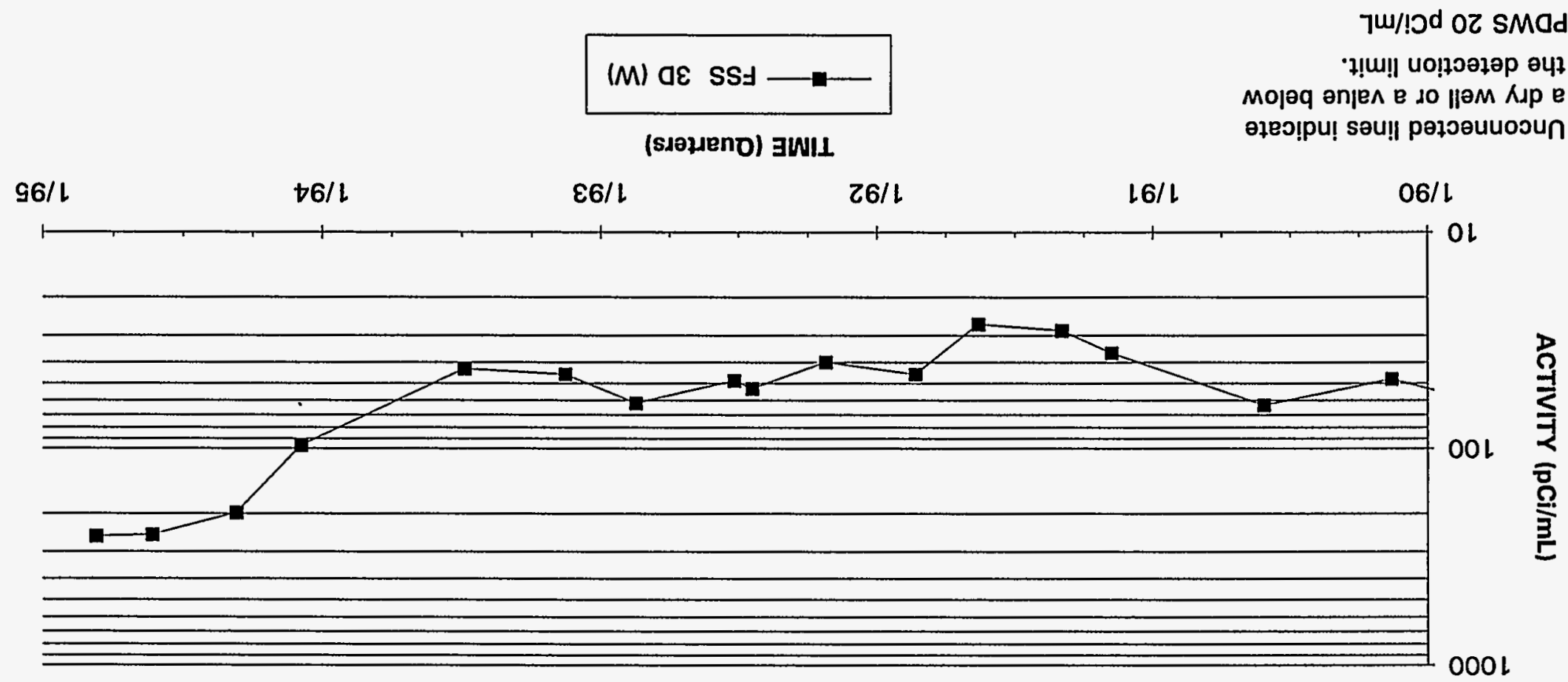

\section{वE SS $₫ \| \partial M$}

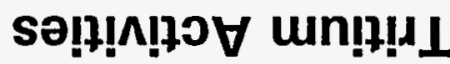




\section{Tritium Activities}

Well FSS 4D

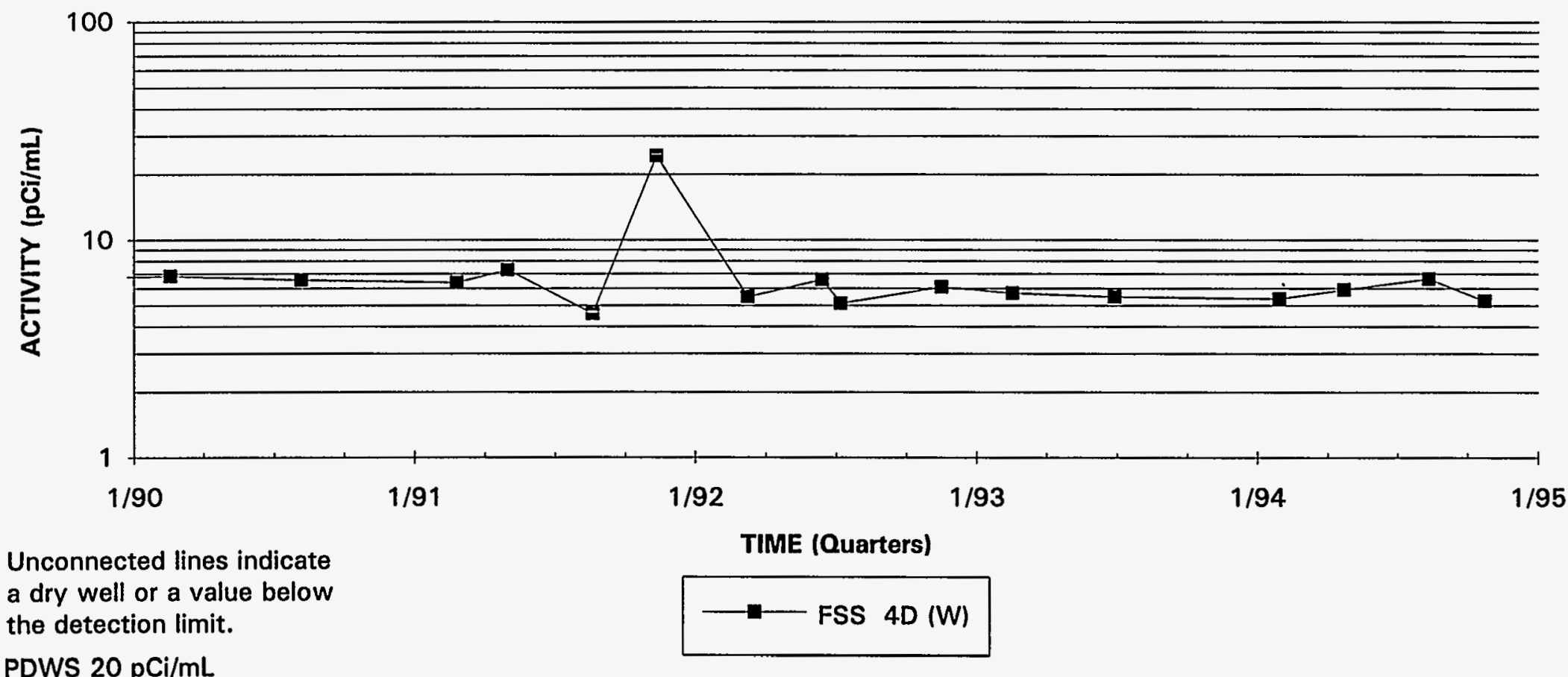

Note: W=Water Table (IIB2); B=Barnwell (IIB1); M=McBean (IIB1); UC=Upper Congaree (IIA); MC=Middle Congaree (IIA); LC=Lower Congaree (IIA) 


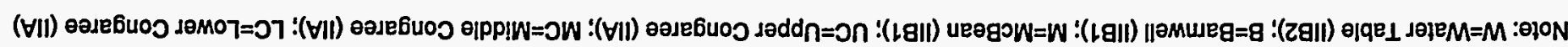

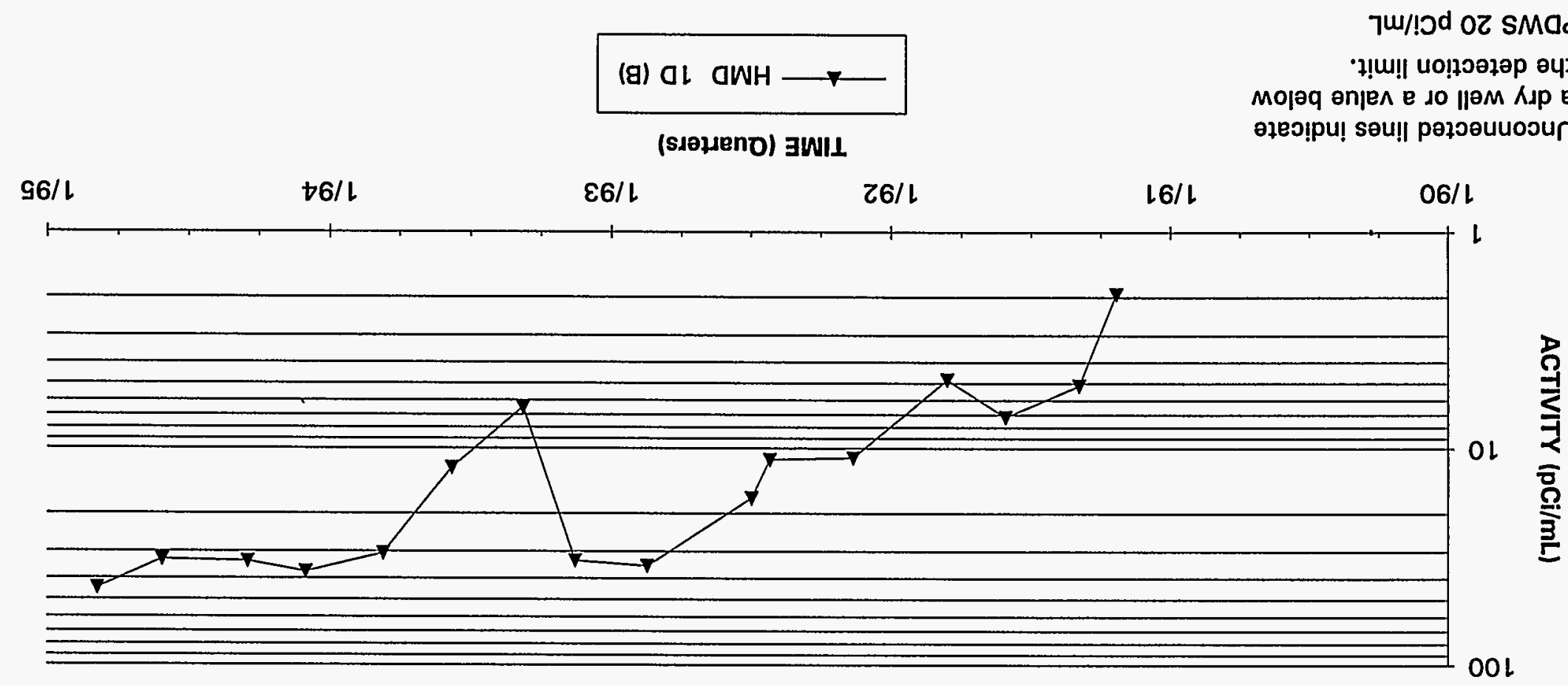

वL OWH IIәM

sə!!!ก!ฺ० 


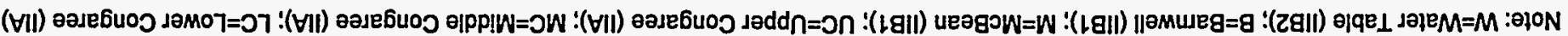

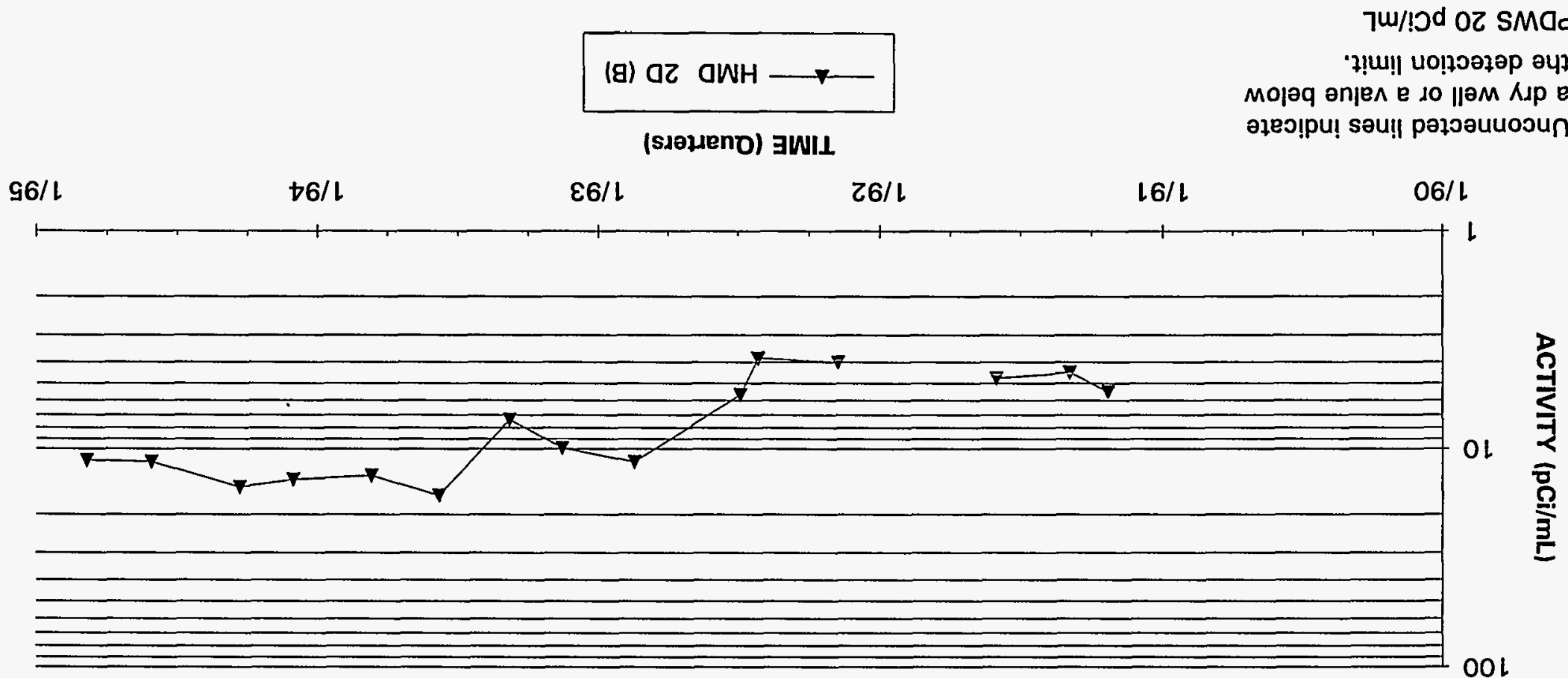

\section{वZ वWH $\|{ }^{\circ} \mathrm{M}$}

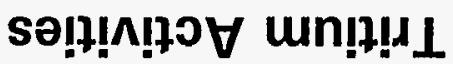




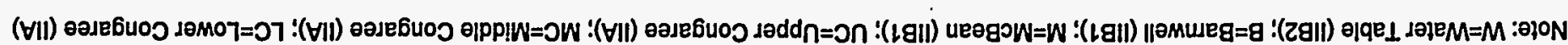

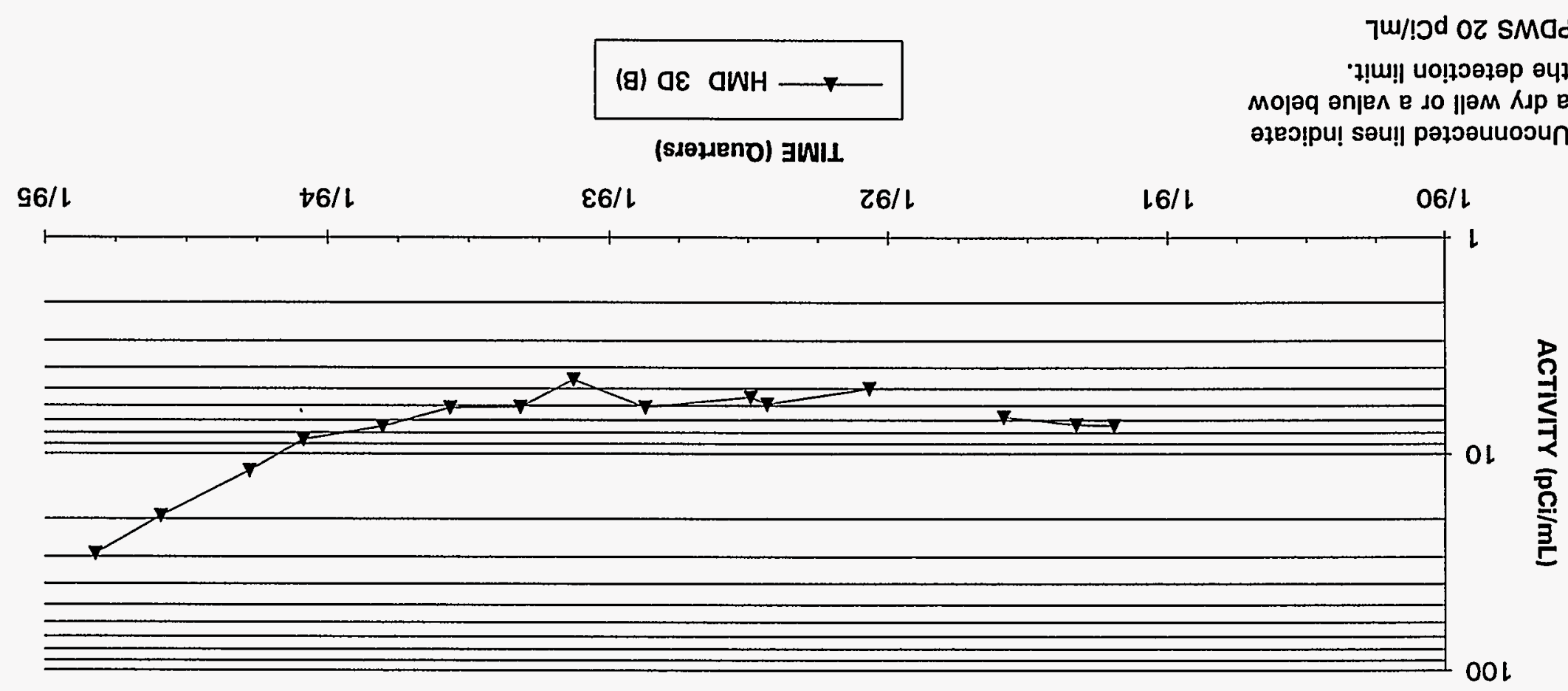

\section{वह GINH ॥әM}

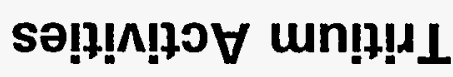




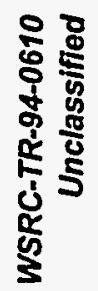
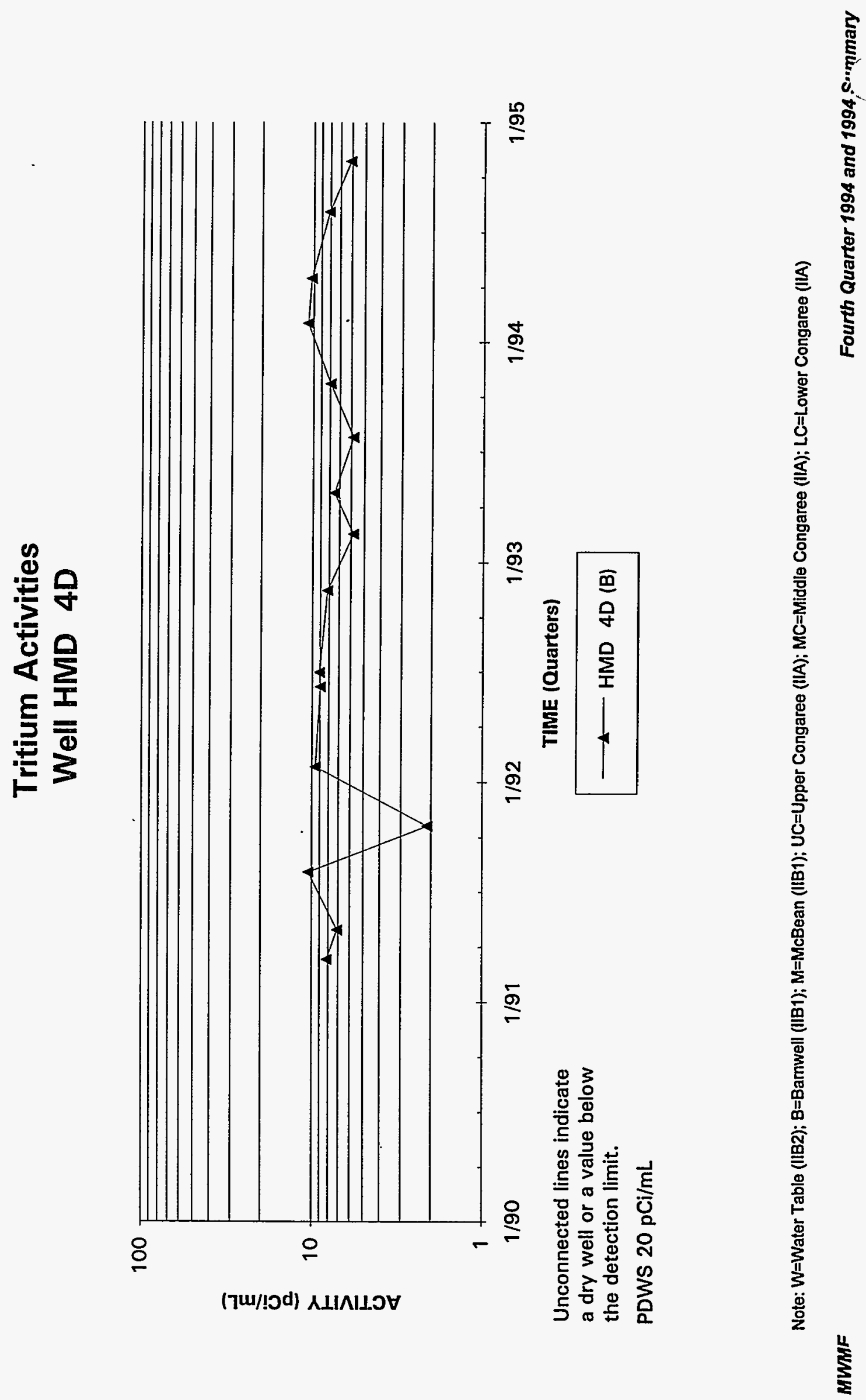


\section{Tritium Activities Well Cluster HSB 85}

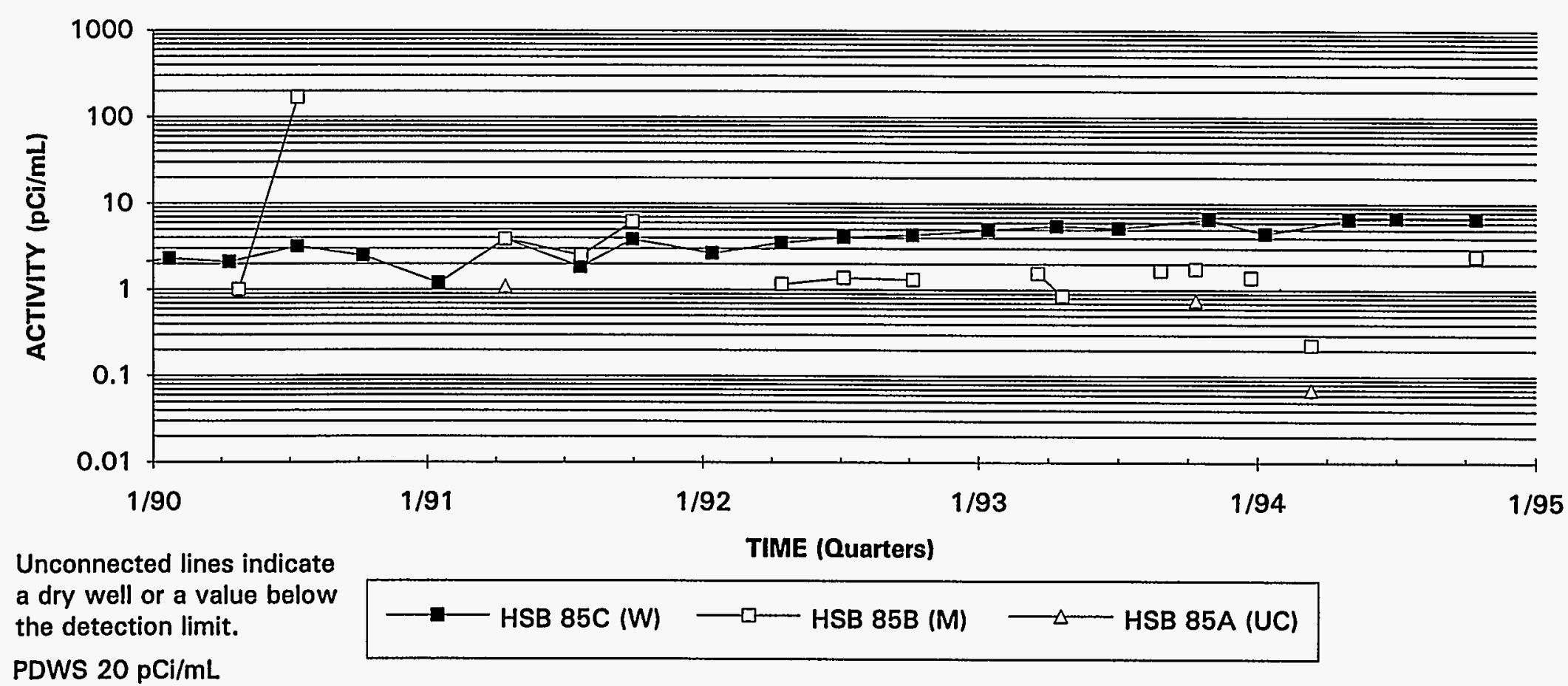

Note: W=Water Table (IIB2); B=Barnwell (IIB1); M=McBean (IIB1); UC=Upper Congaree (IIA); MC=Middle Congaree (IIA); LC=Lower Congaree (IIA) 


\section{Appendix G}

\section{Hydrographs}


WSRC-TR-94-0610

Unclassified

THIS PAGE LEFT BLANK INTENTIONALLY. 

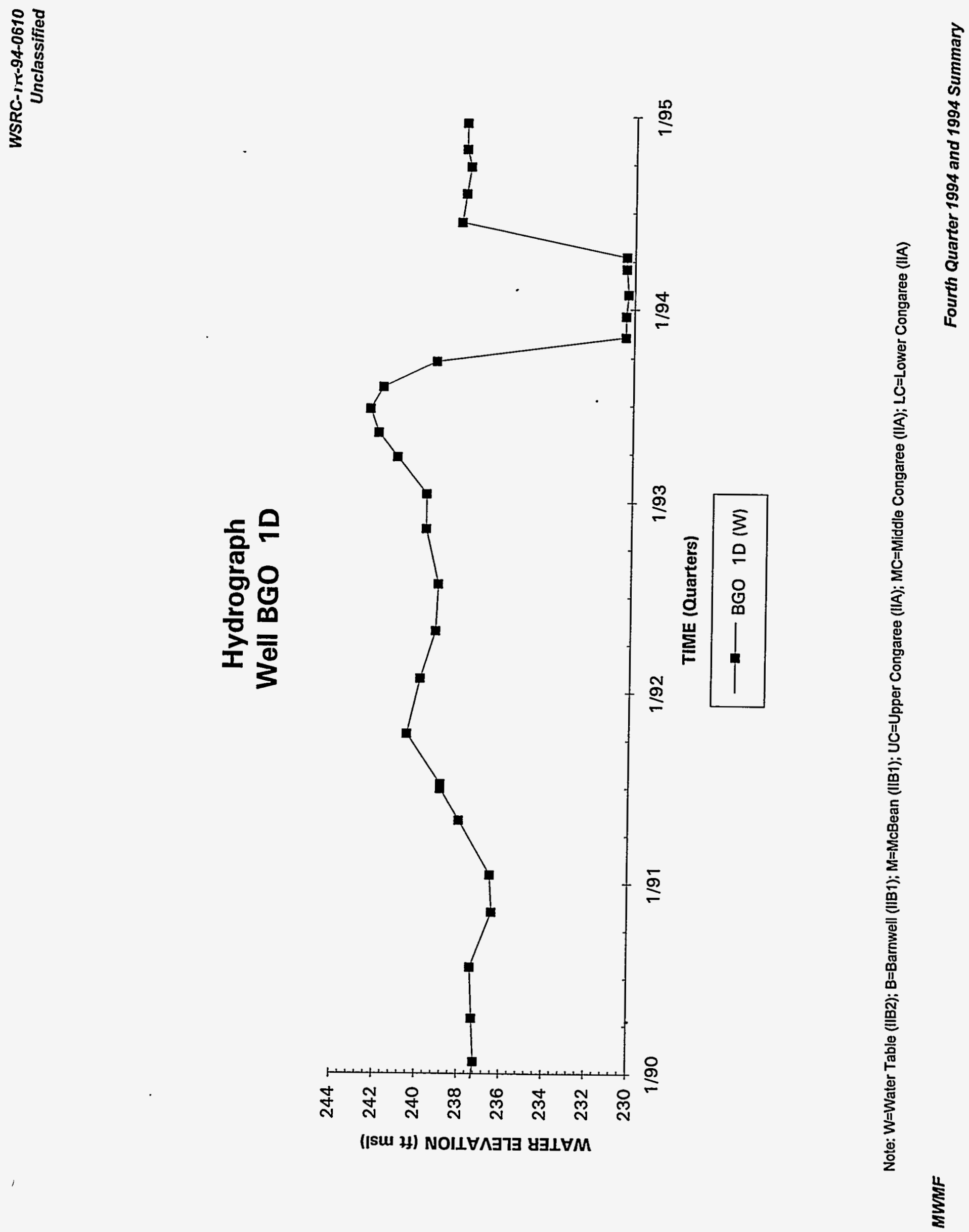


\section{Hydrograph}

Well BGO 2D

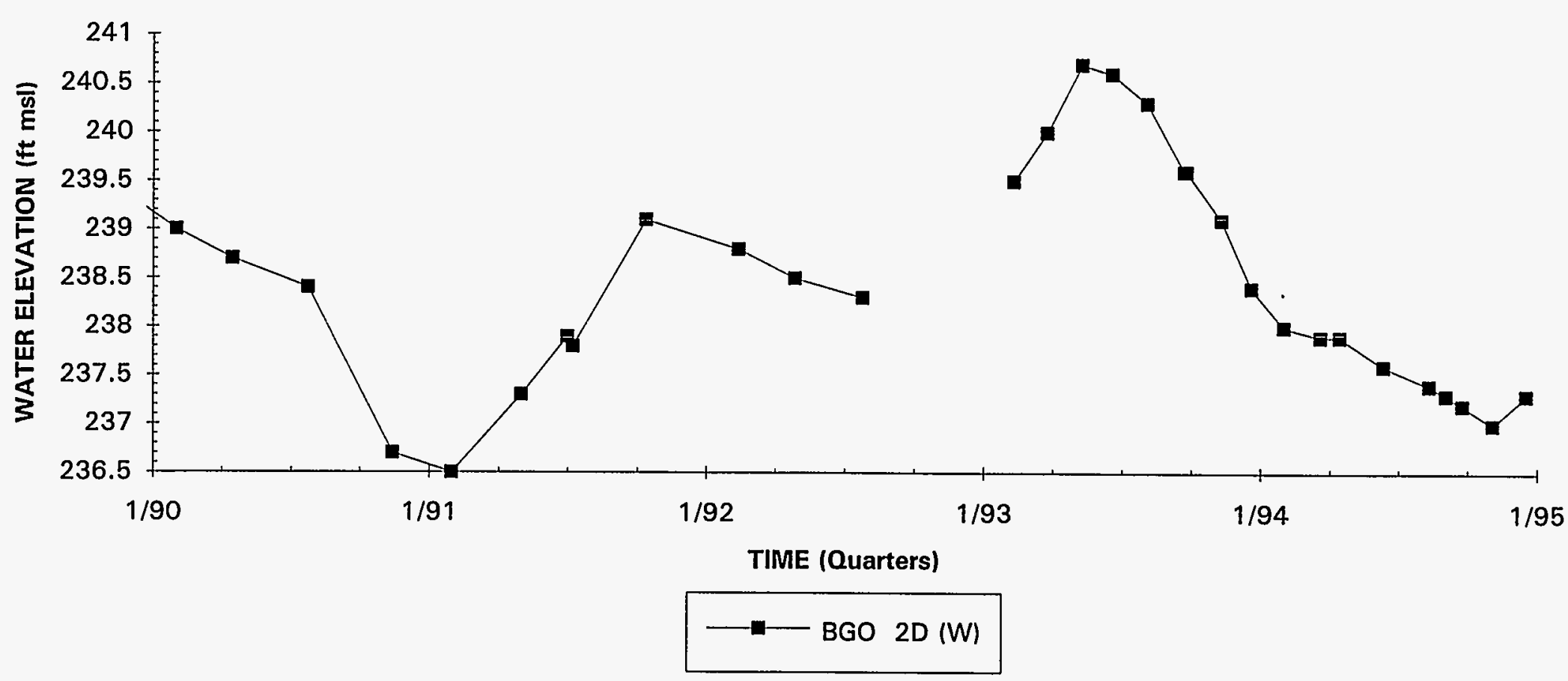

Note: W=Water Table (IIB2); B=Barnwell (IIB1); M=McBean (IIB1); UC=Upper Congaree (IIA); MC=Middle Congaree (IIA); LC=Lower Congaree (IIA) 


\section{Hydrograph}

\section{Well BGO 3D}

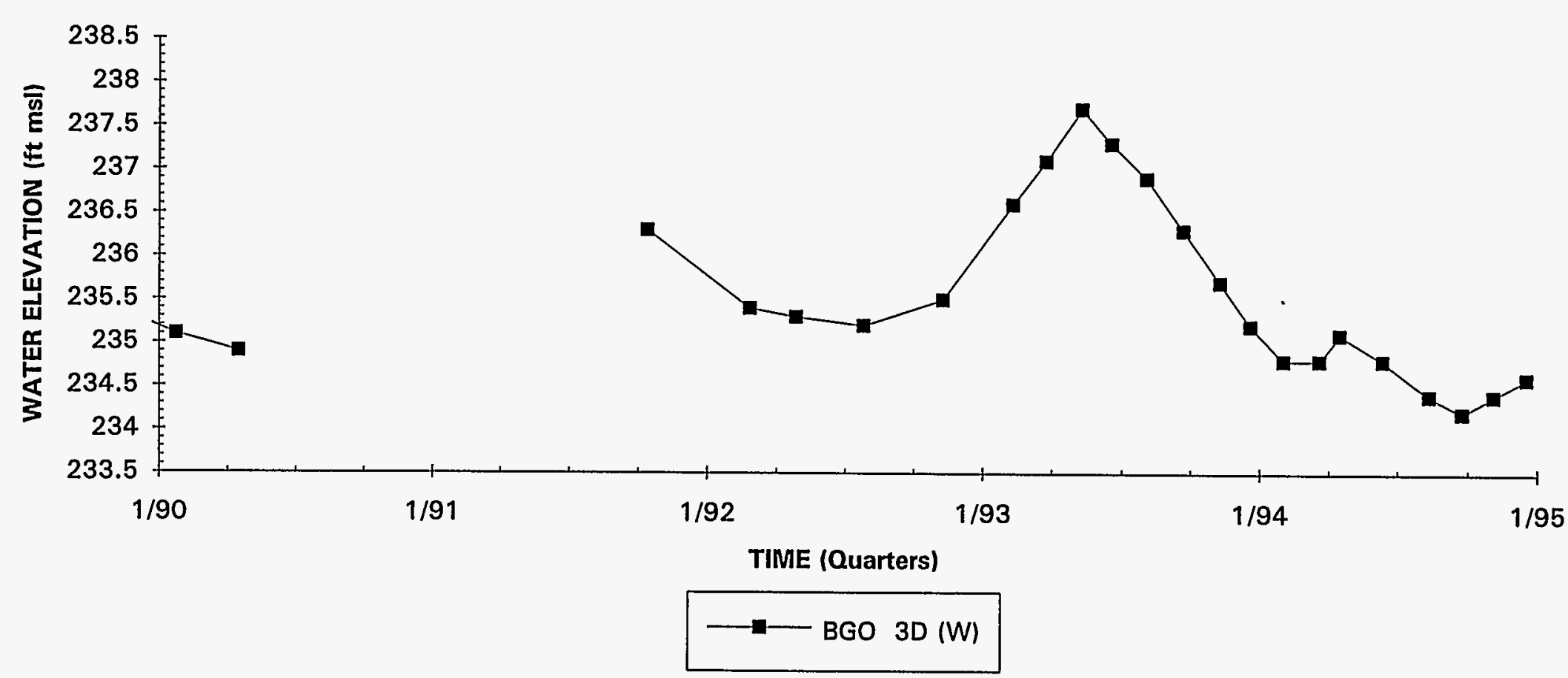

Note: W=Water Table (IIB2); B=Barnwell (IIB1); M=McBean (IIB1); UC=Upper Congaree (IIA); MC=Middle Congaree (IIA); LC=Lower Congaree (IIA) 

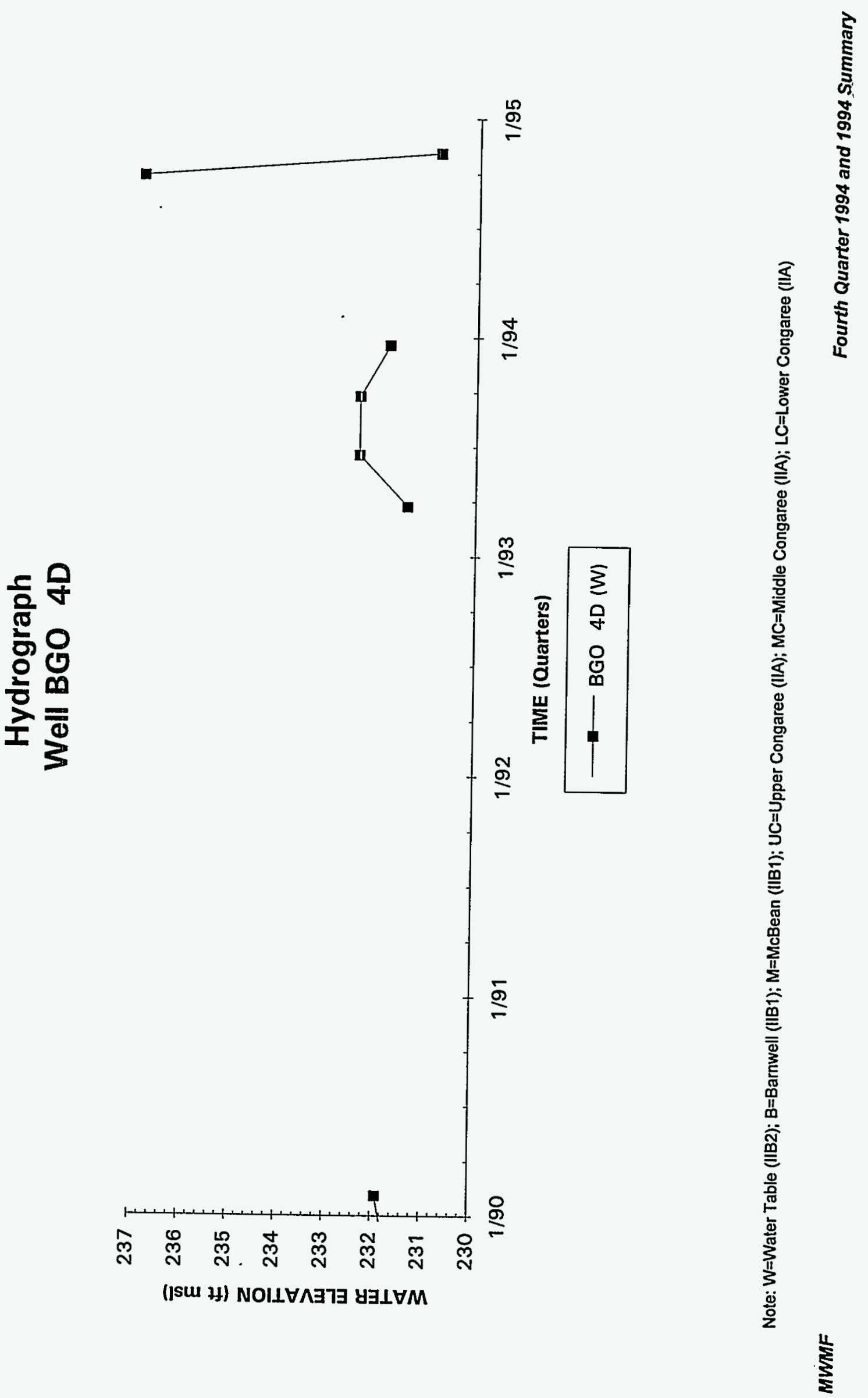

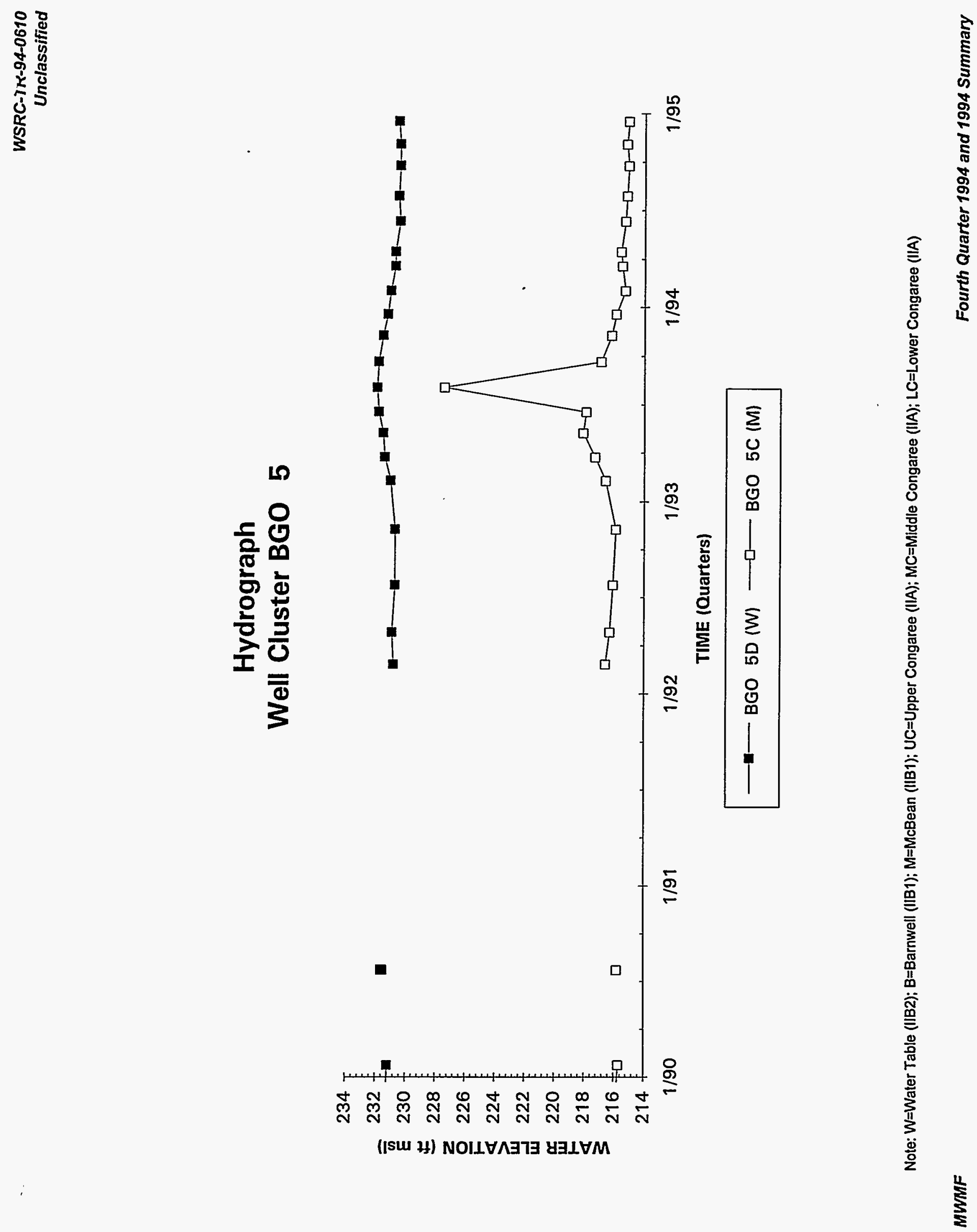

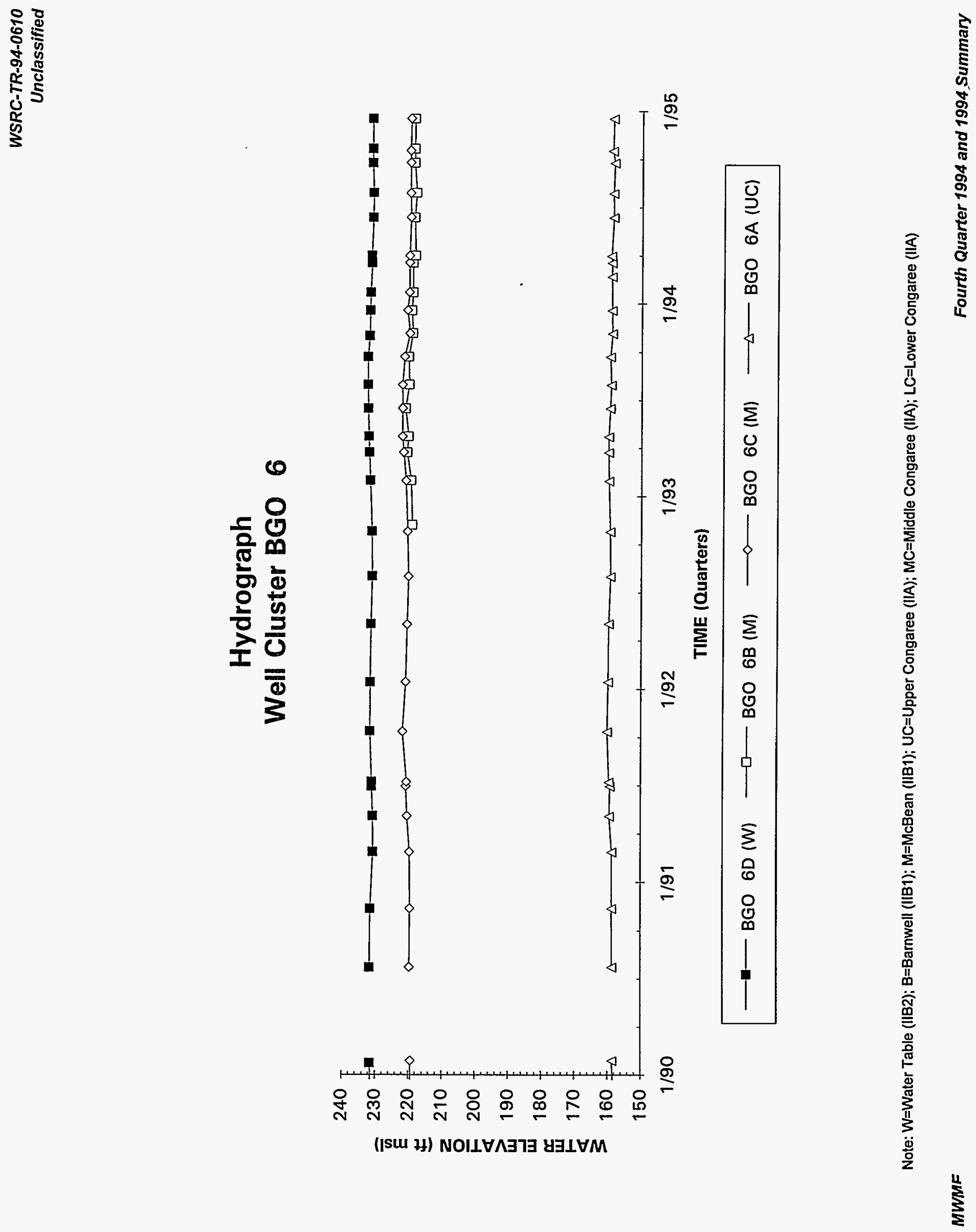


\section{Hydrograph \\ Well BGO 7D}

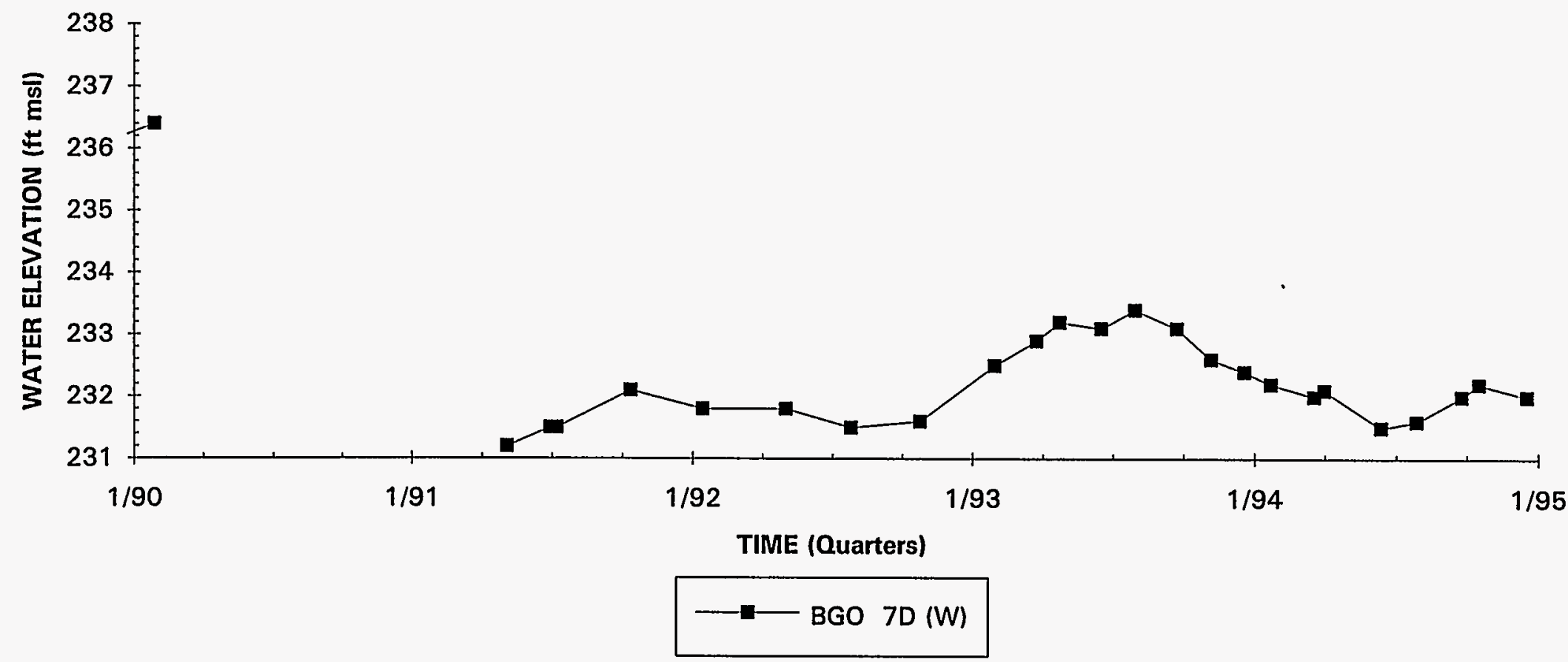

Note: $W=$ Water Table (IIB2); B=Barnwell (IIB1); M=McBean (IIB1); UC=Upper Congaree (IIA); MC=Middle Congaree (IIA); LC=Lower Congaree (IIA) 

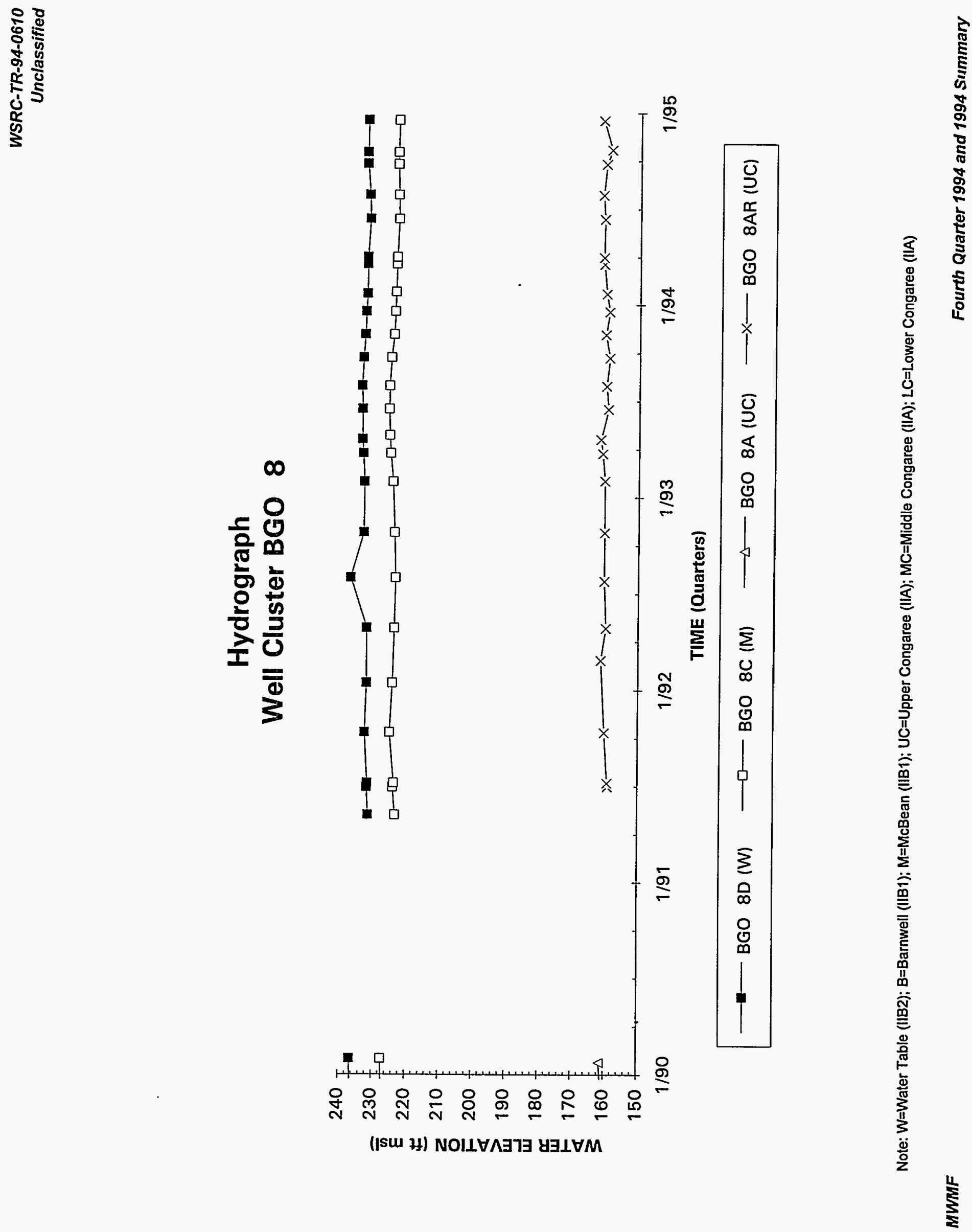

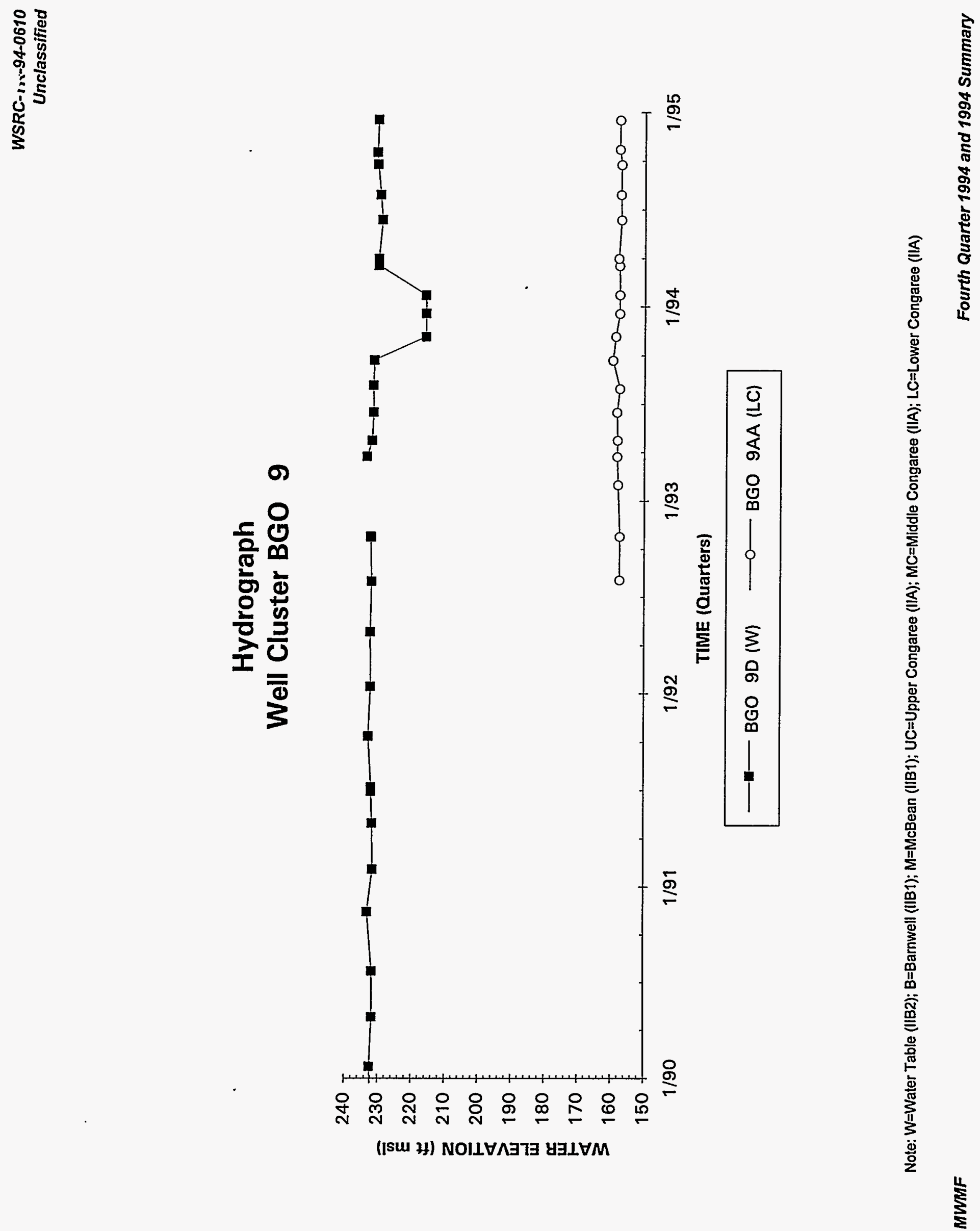


\section{Hydrograph \\ Well Cluster BGO 10}
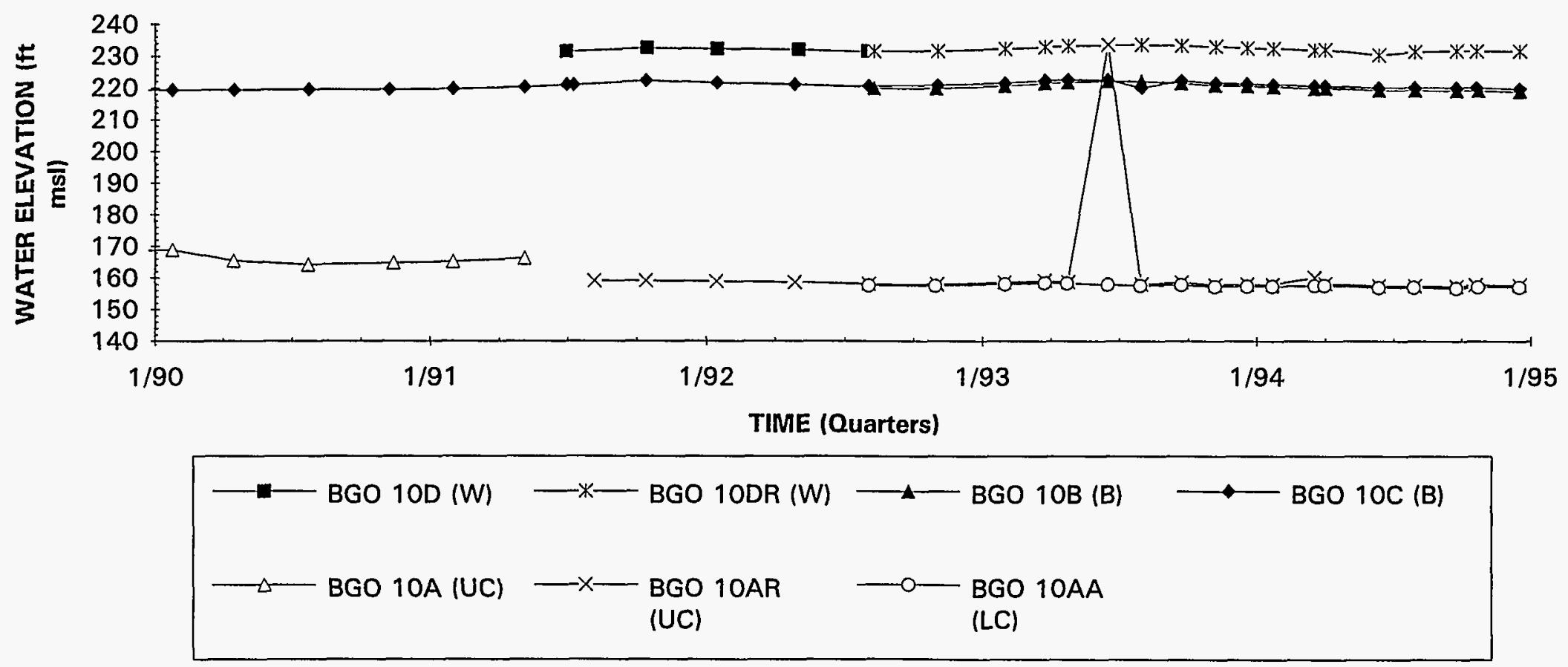

Note: $W=$ Water Table (IIB2); B=Barnwell (IIB1); $M=M c B e a n$ (IIB1); UC=Upper Congaree (IIA); MC=Middle Congaree (IIA); LC=Lower Congaree (IIA) 


\section{Hydrograph \\ Well BGO 11D}

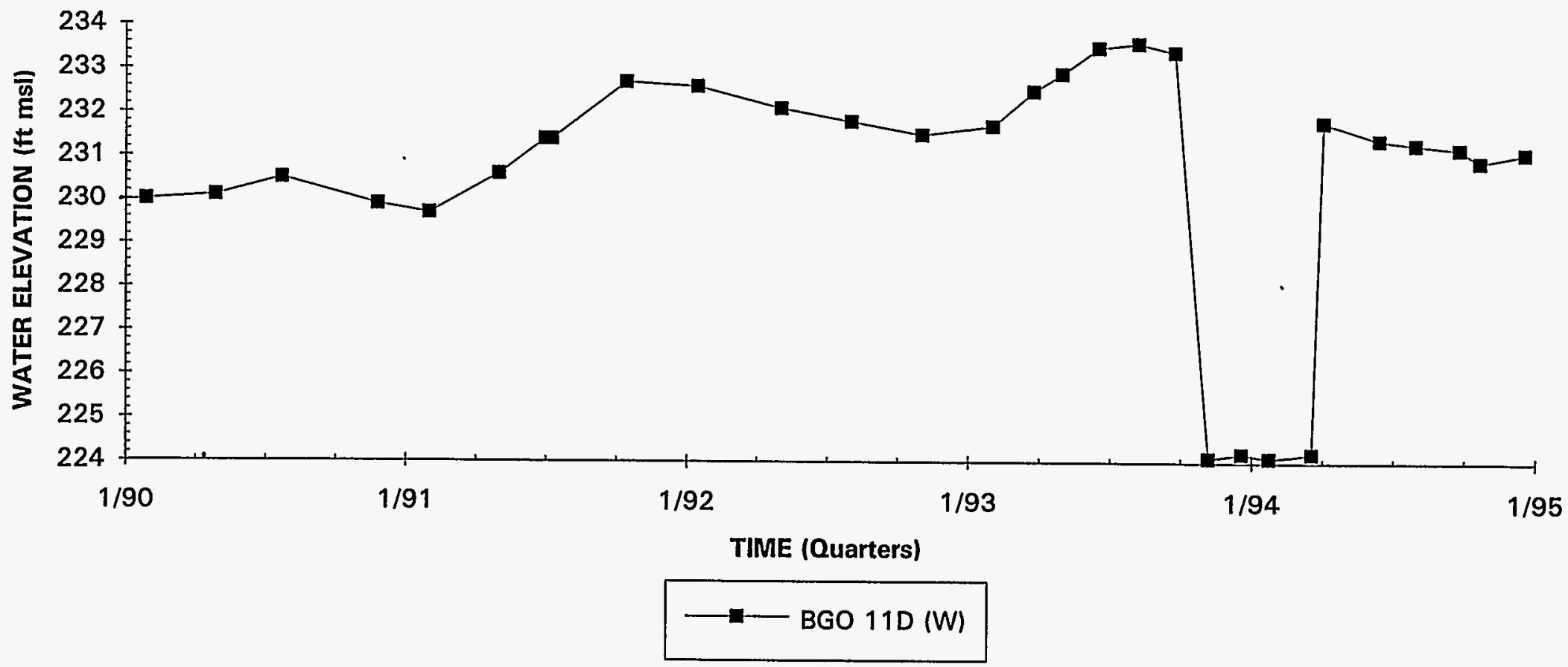

Note: W=Water Table (IIB2); B=Barnwell (IIB1); M=McBean (IIB1); UC=Upper Congaree (IIA); MC=Middle Congaree (IIA); LC=Lower Congaree (IIA) 

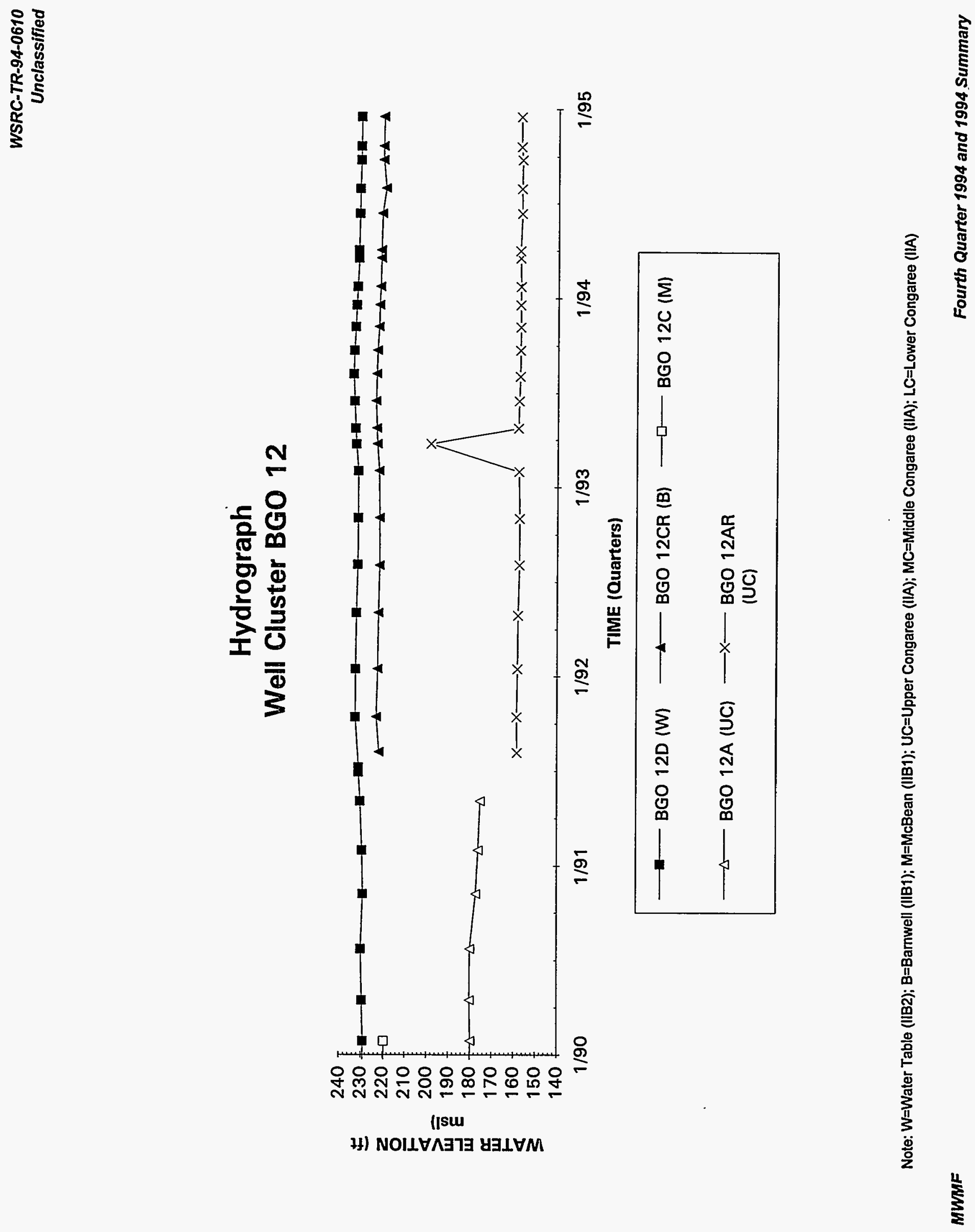


\section{Hydrograph \\ Well Cluster BGO 13}

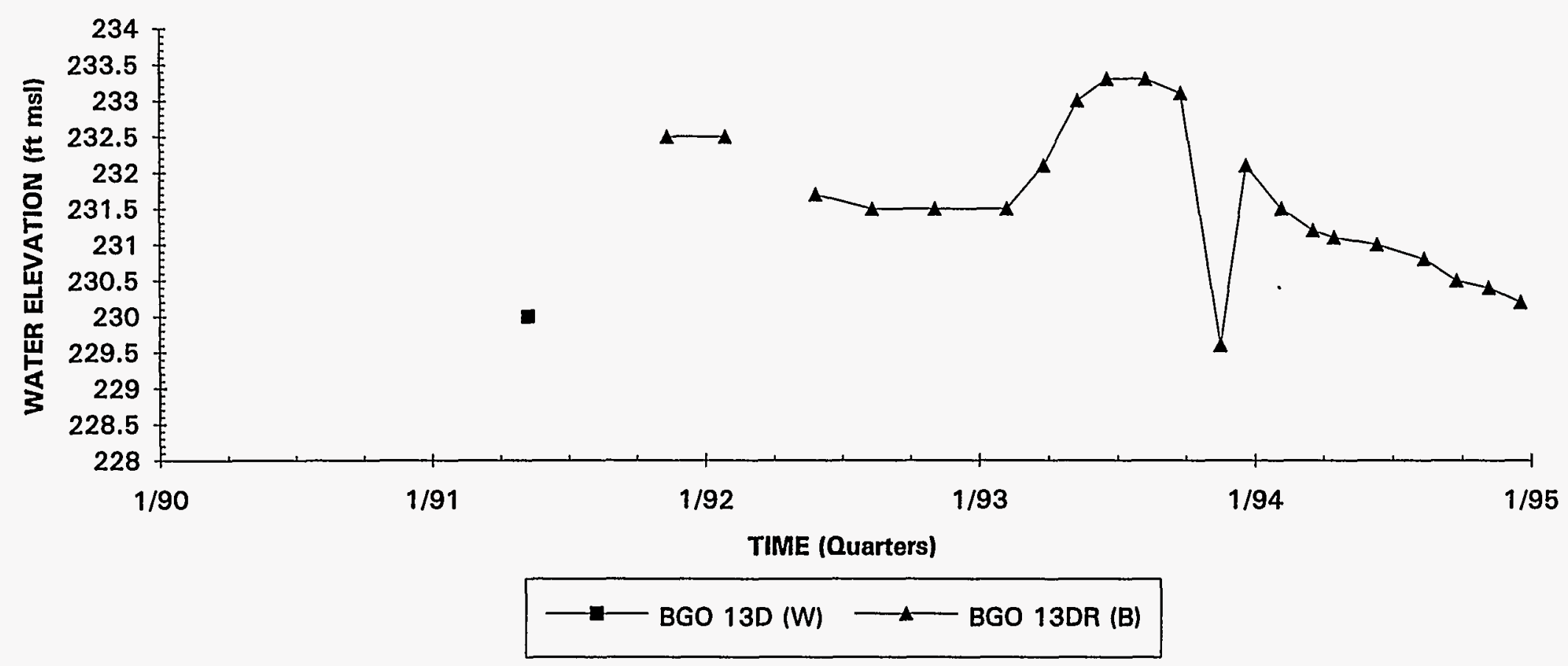

Note: W=Water Table (IIB2); B=Barnwell (IIB1); M=McBean (IIB1); UC=Upper Congaree (IIA); MC=Middle Congaree (IIA); LC=Lower Congaree (IIA) 


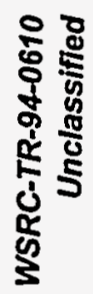
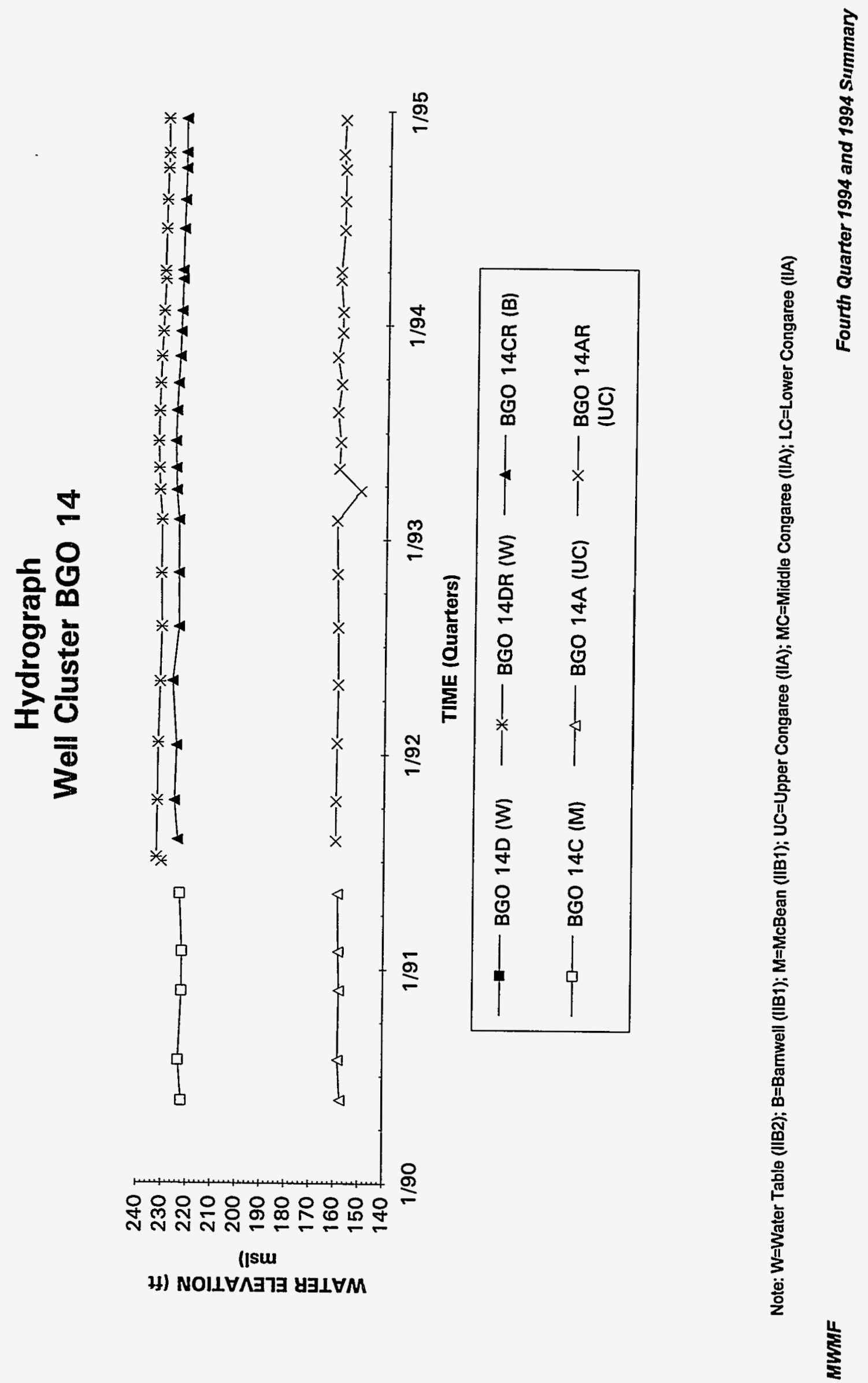


\section{Hydrograph}

\section{Well BGO 15D}

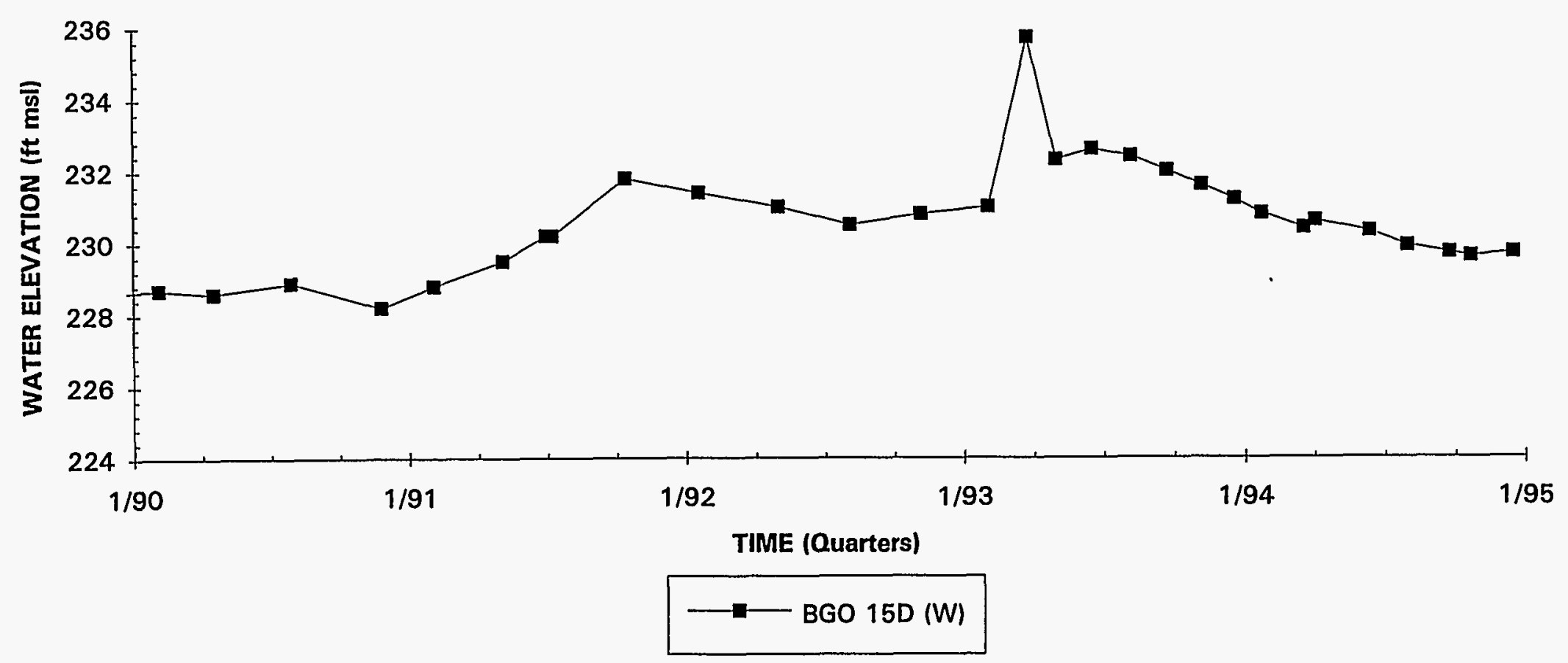

Note: W=Water Table (IIB2); B=Barnwell (IIB1); M=McBean (IIB1); UC=Upper Congaree (IIA); MC=Middle Congaree (IIA); LC=Lower Congaree (IIA) 

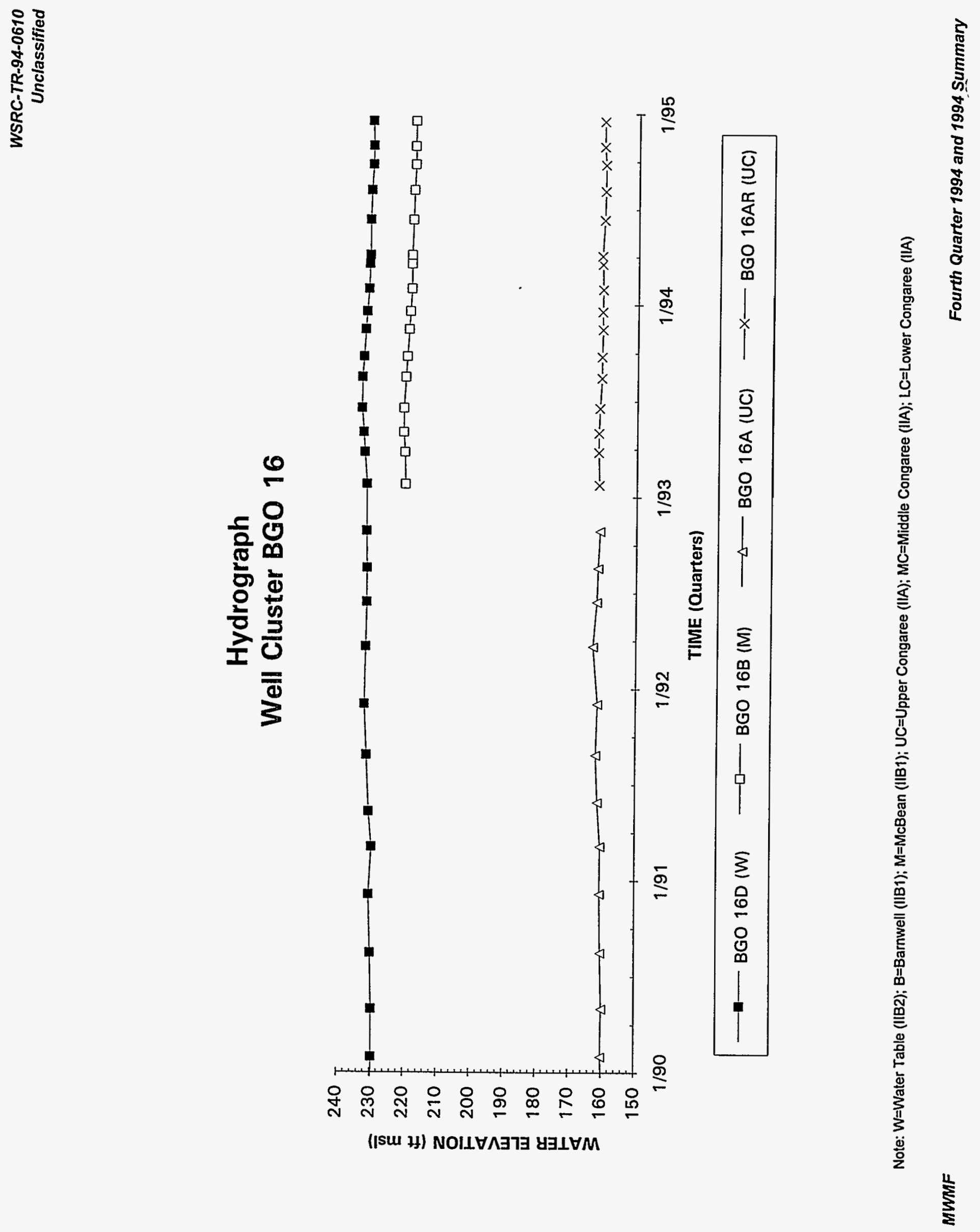

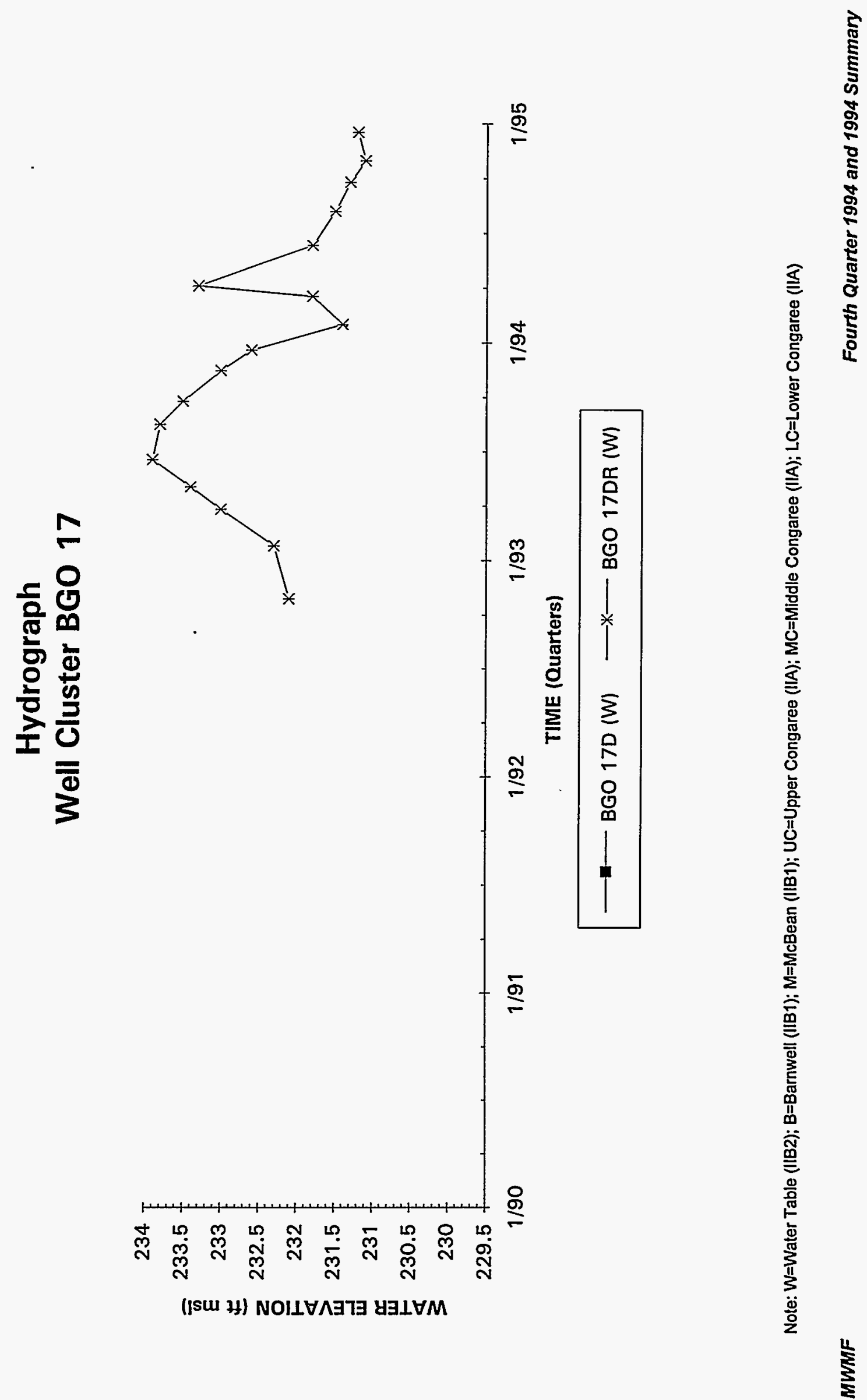

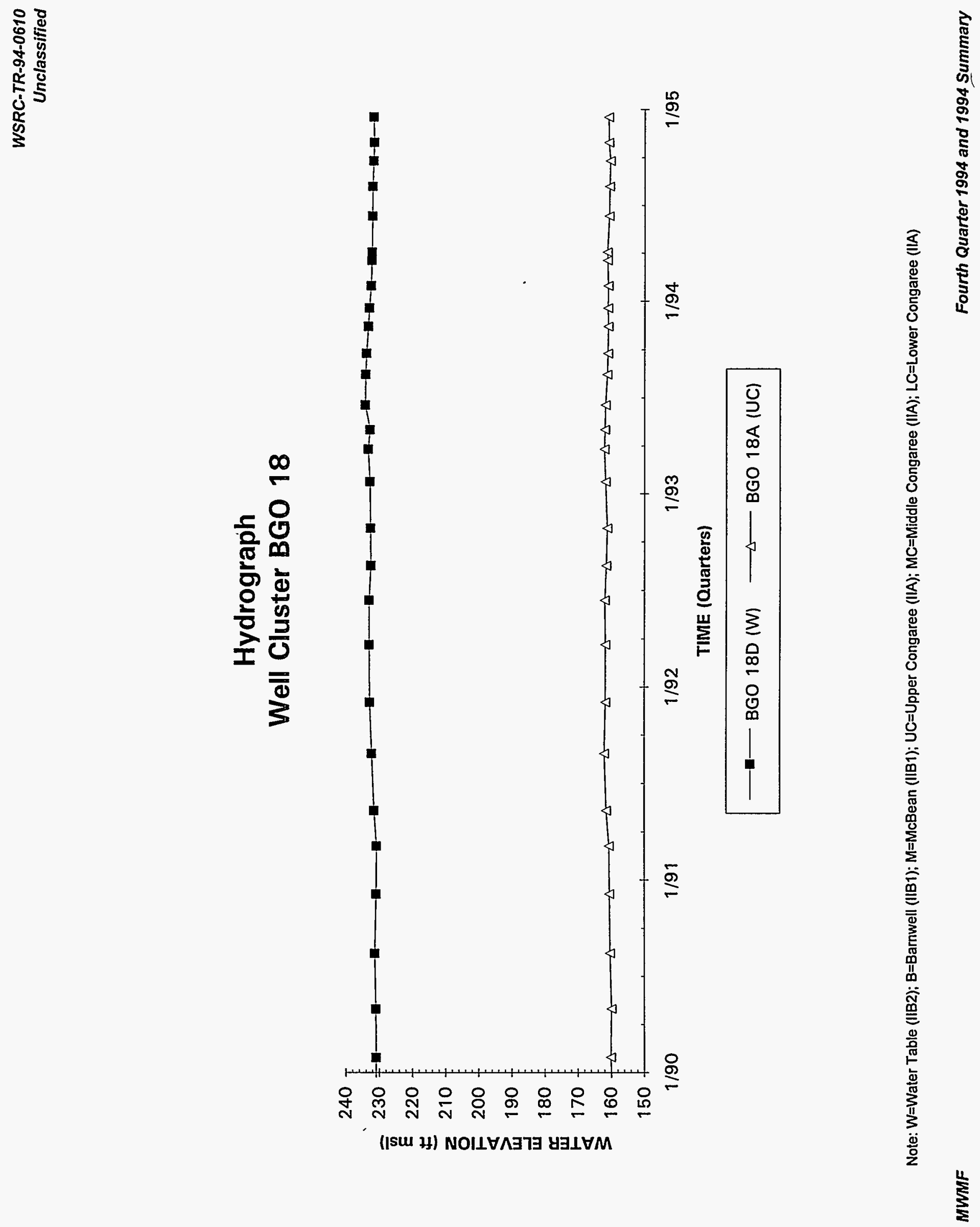


\section{Hydrograph \\ Well BGO 19D}

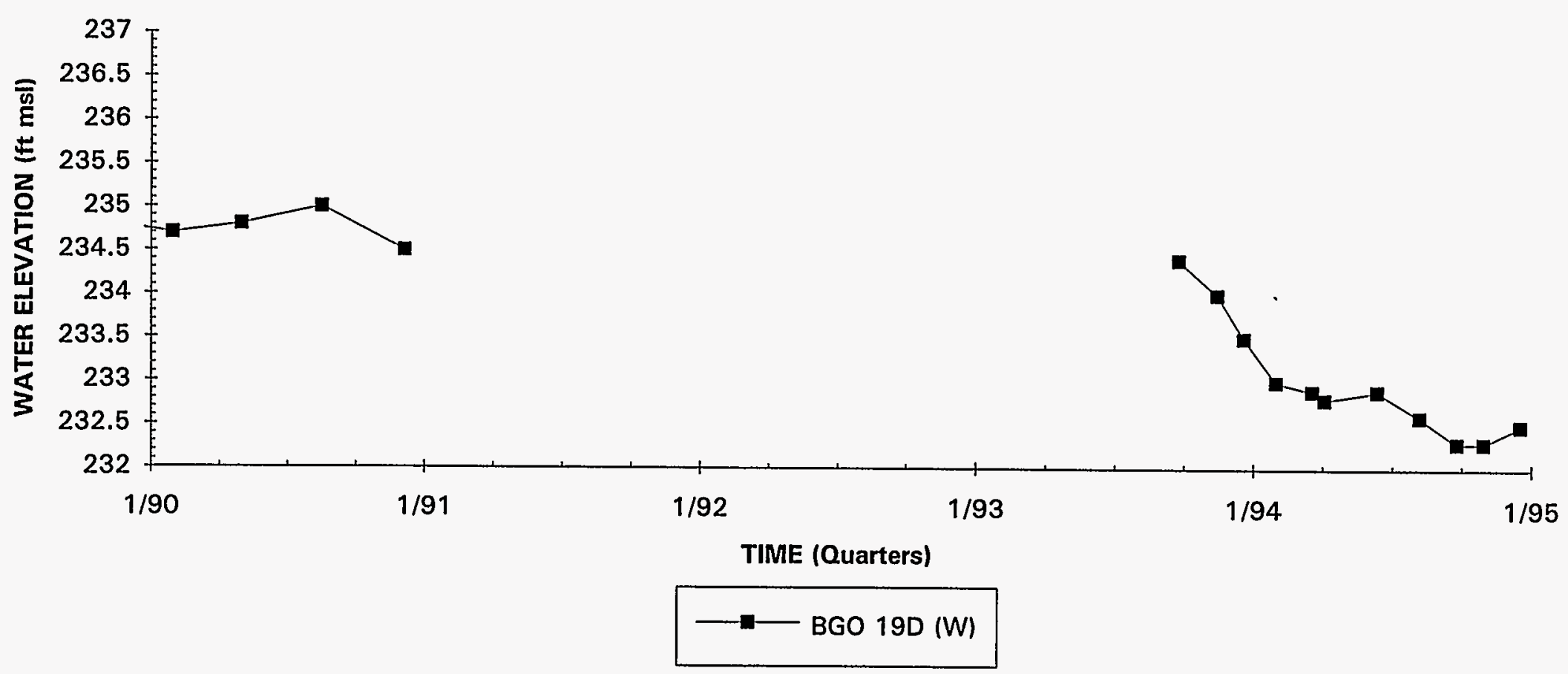

Note: W=Water Table (IIB2); B=Barnwell (IIB1); M=McBean (IIB1); UC=Upper Congaree (IIA); MC=Middle Congaree (IIA); LC=Lower Congaree (IIA) 

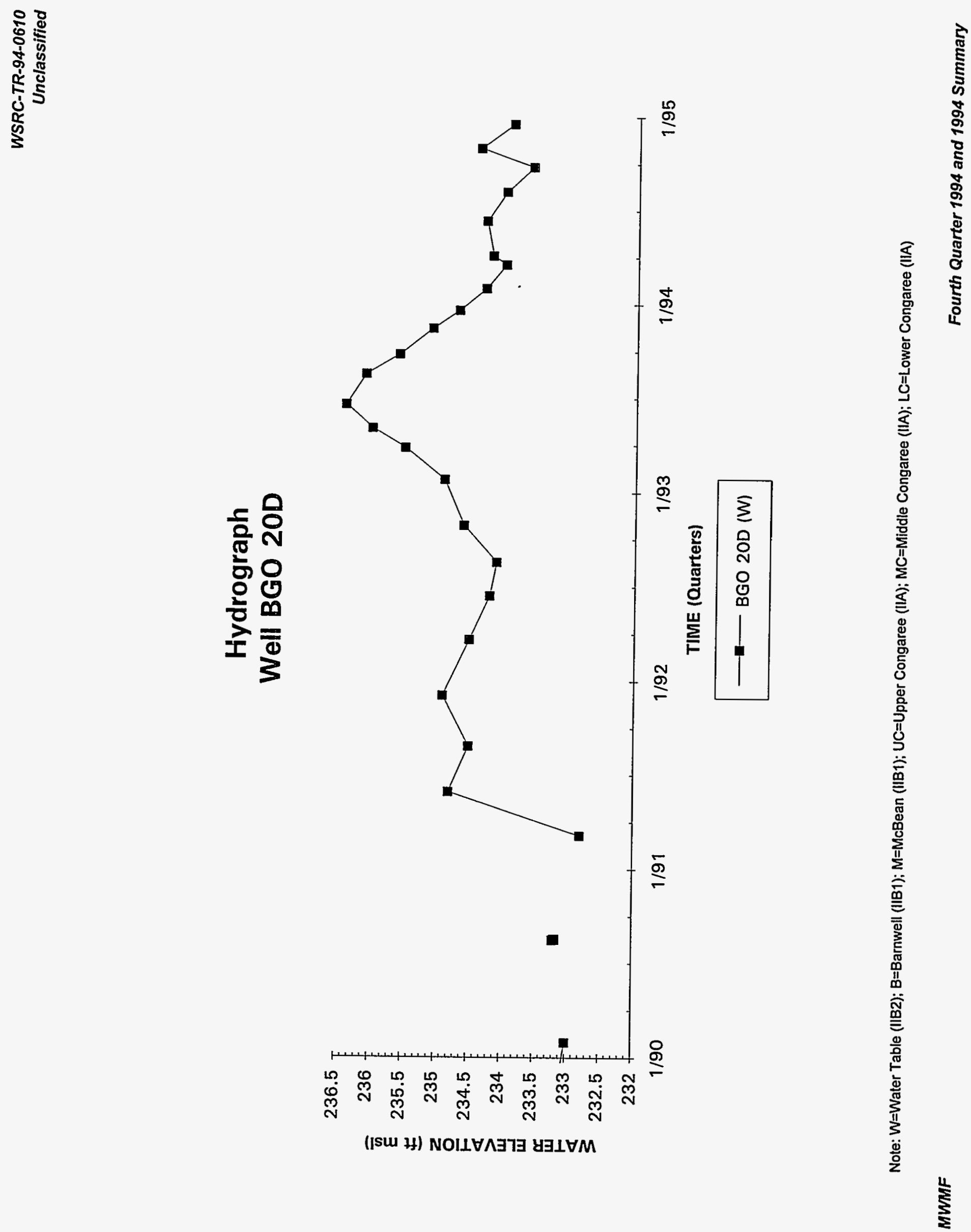

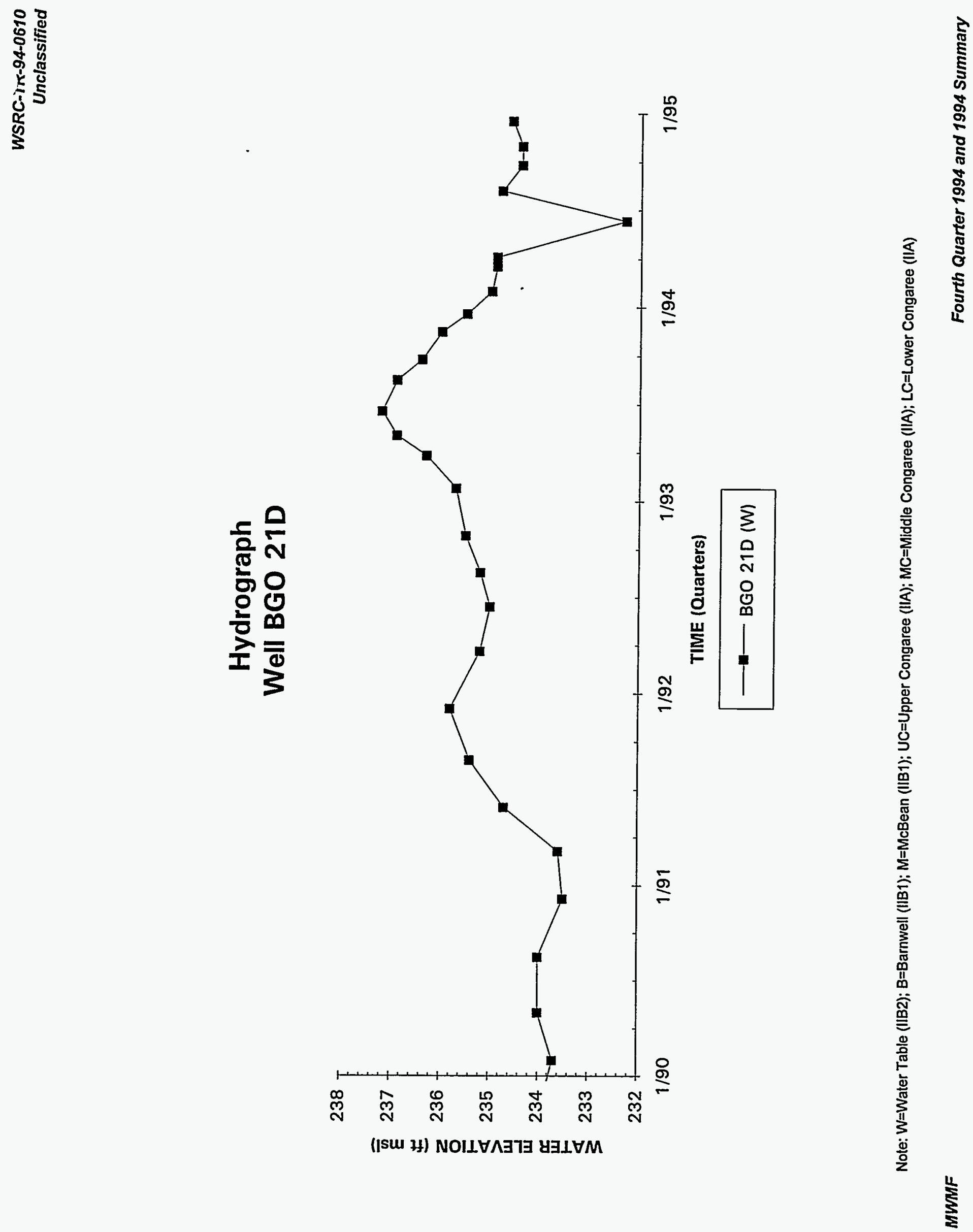

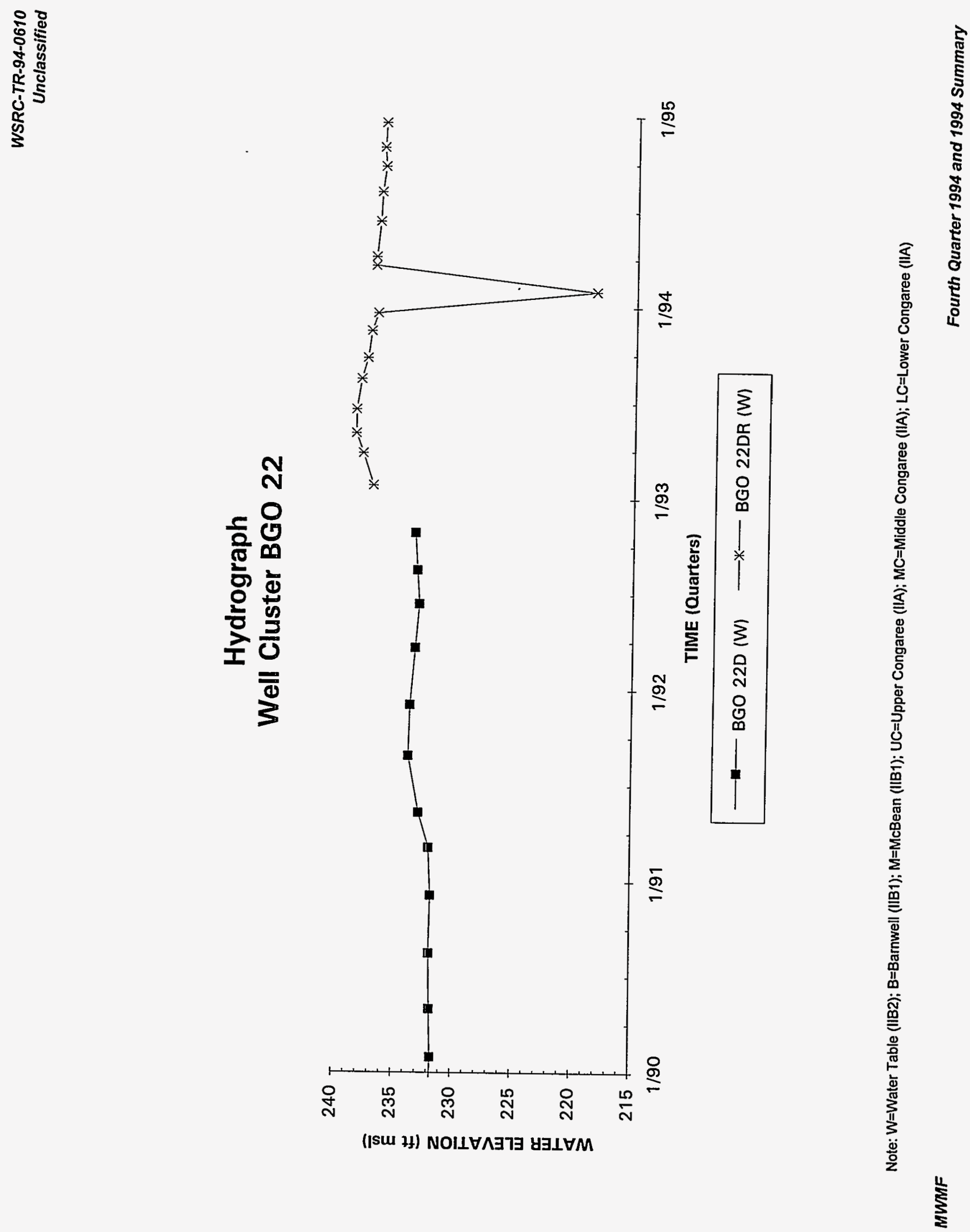

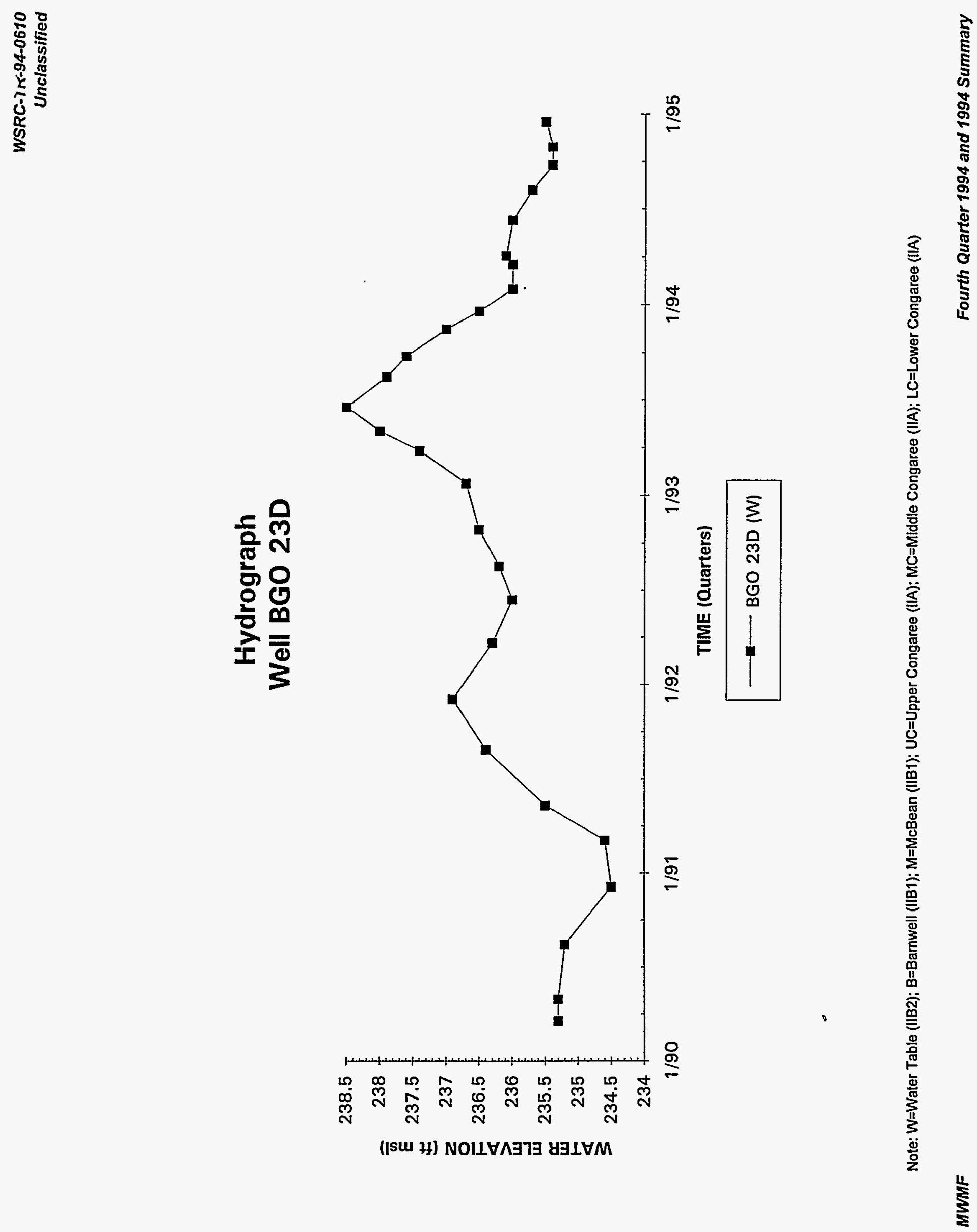

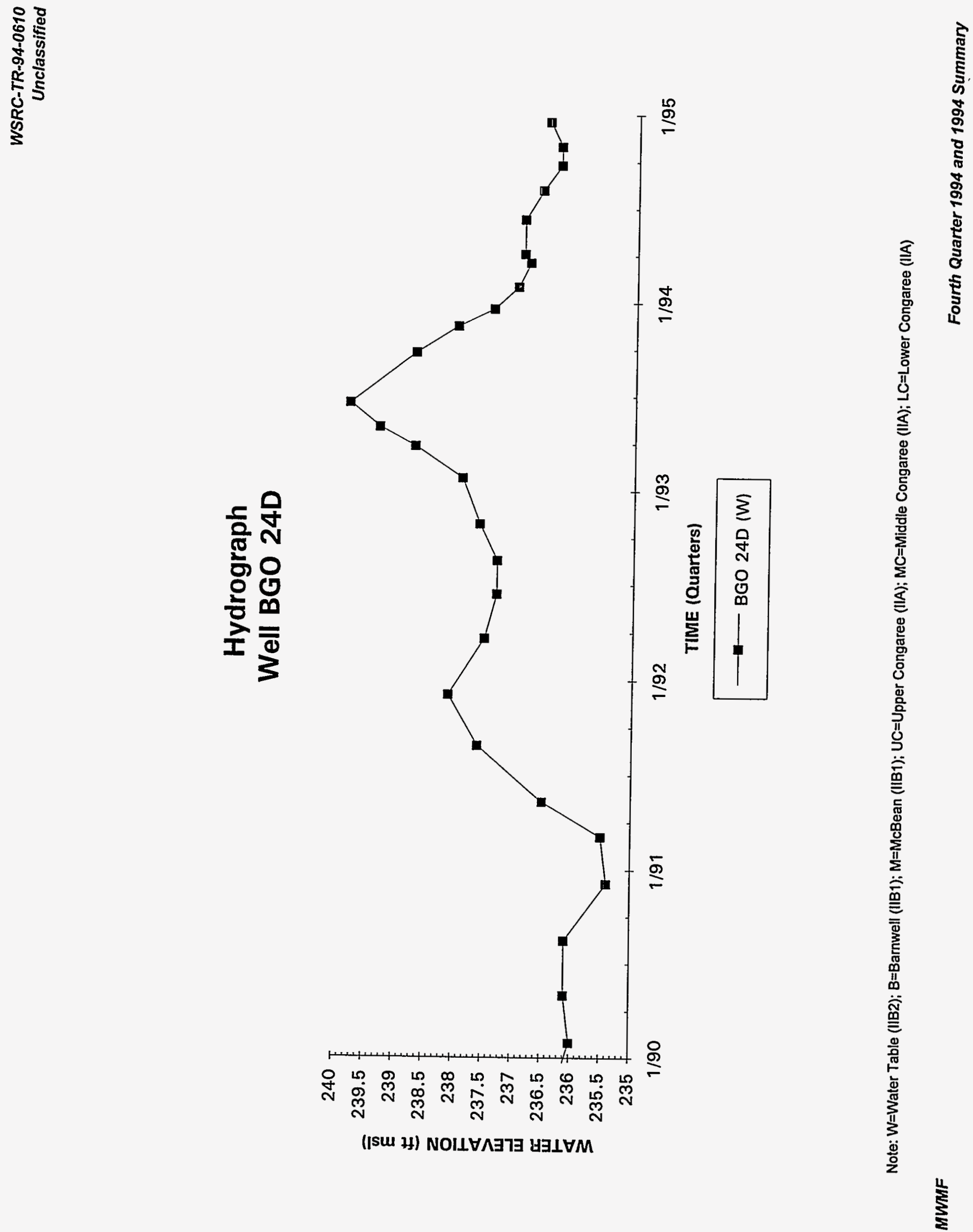


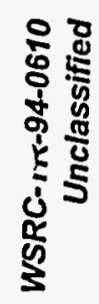
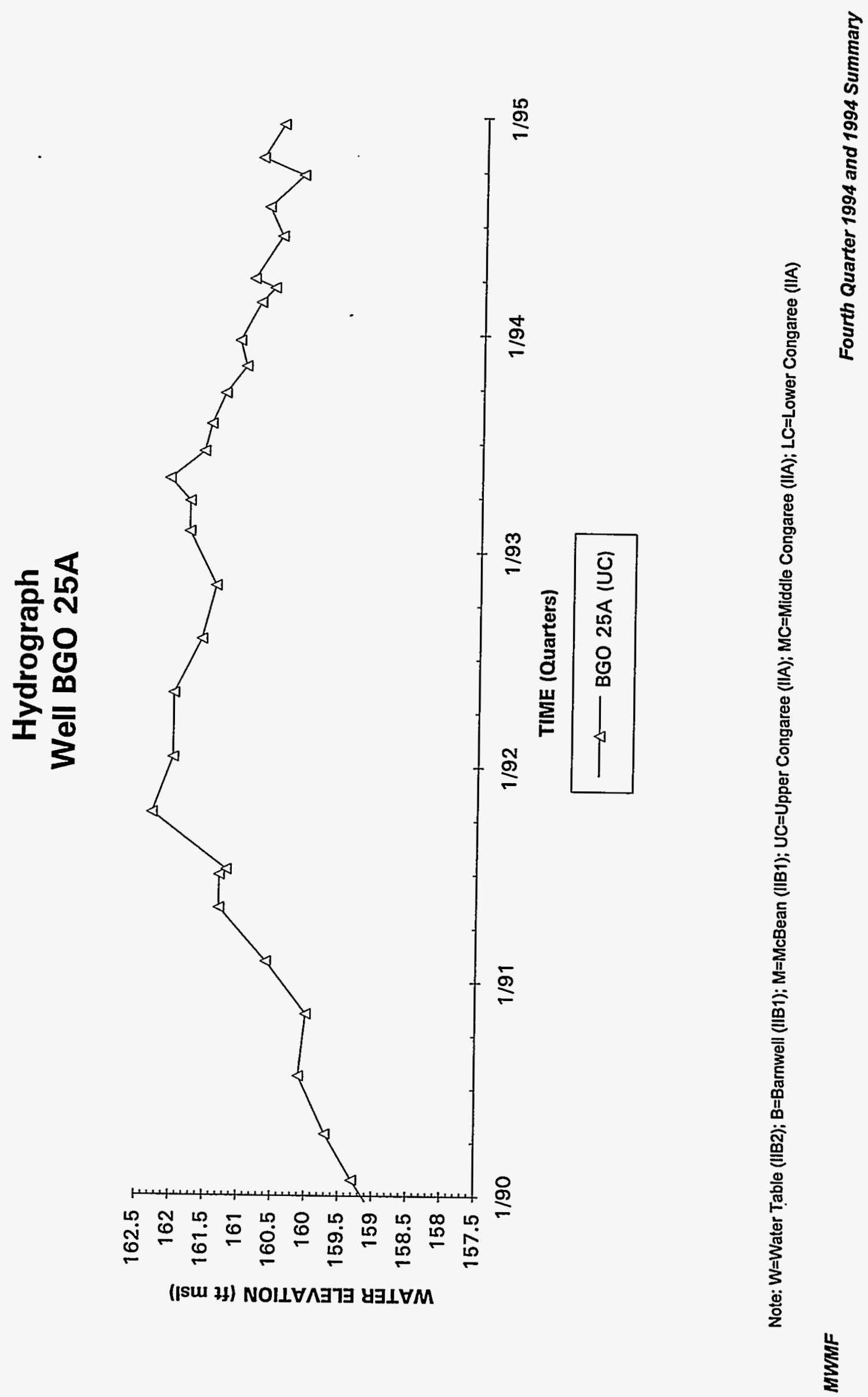

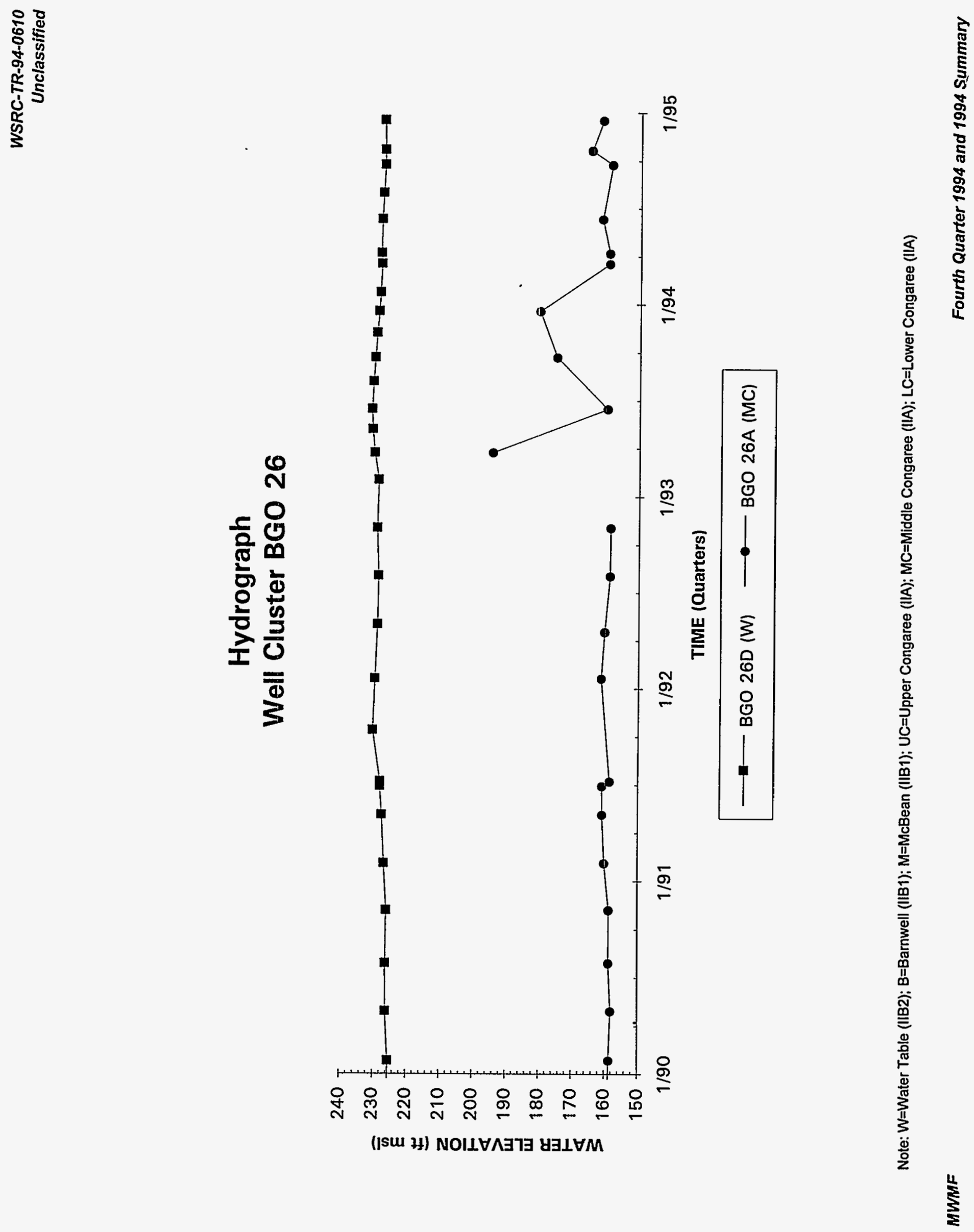


\section{Hydrograph \\ Well Cluster BGO 27}

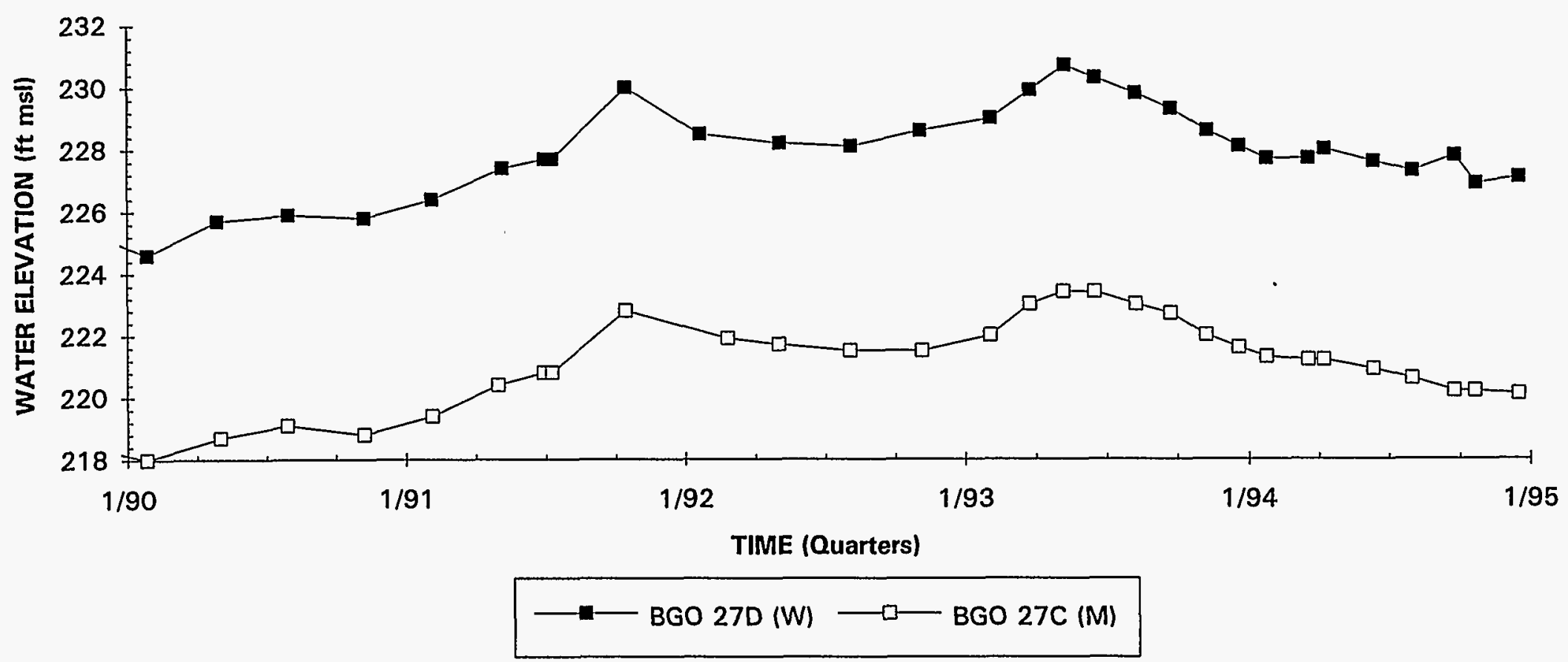

Note: W=Water Table (IIB2); B=Barnwell (IIB1); M=McBean (IIB1); UC=Upper Congaree (IIA); MC=Middle Congaree (IIA); LC=Lower Congaree (IIA) 

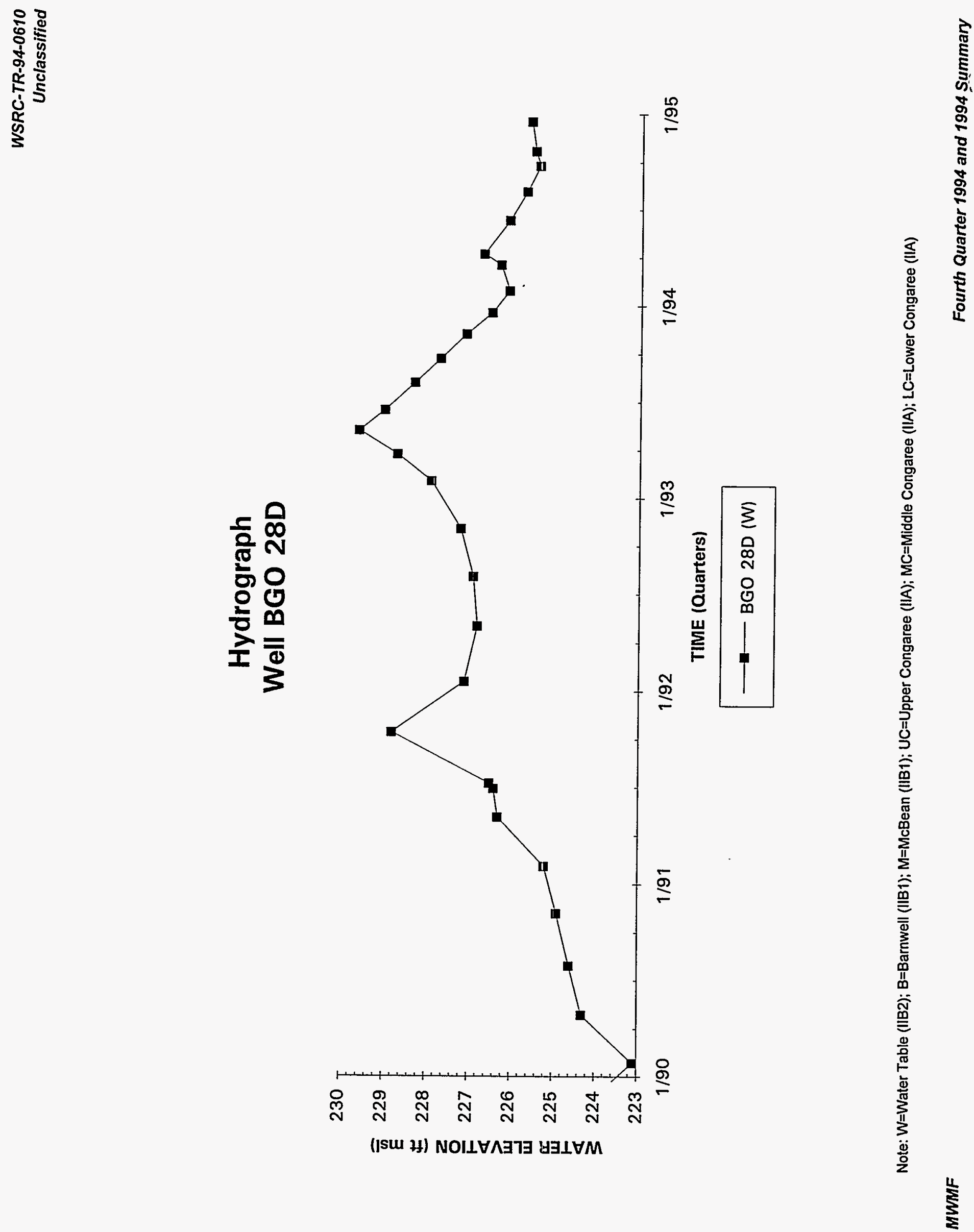


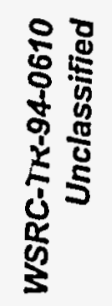
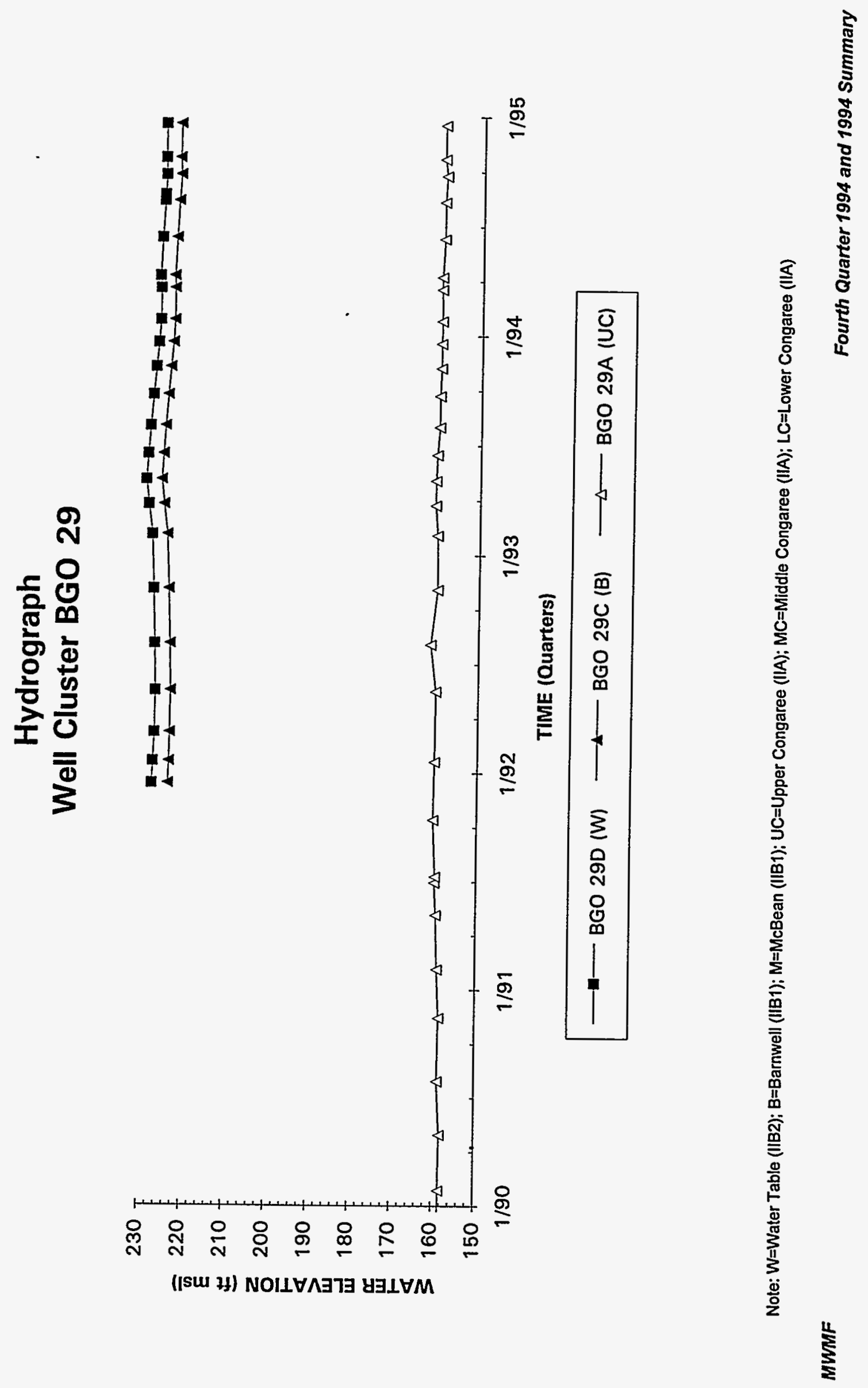

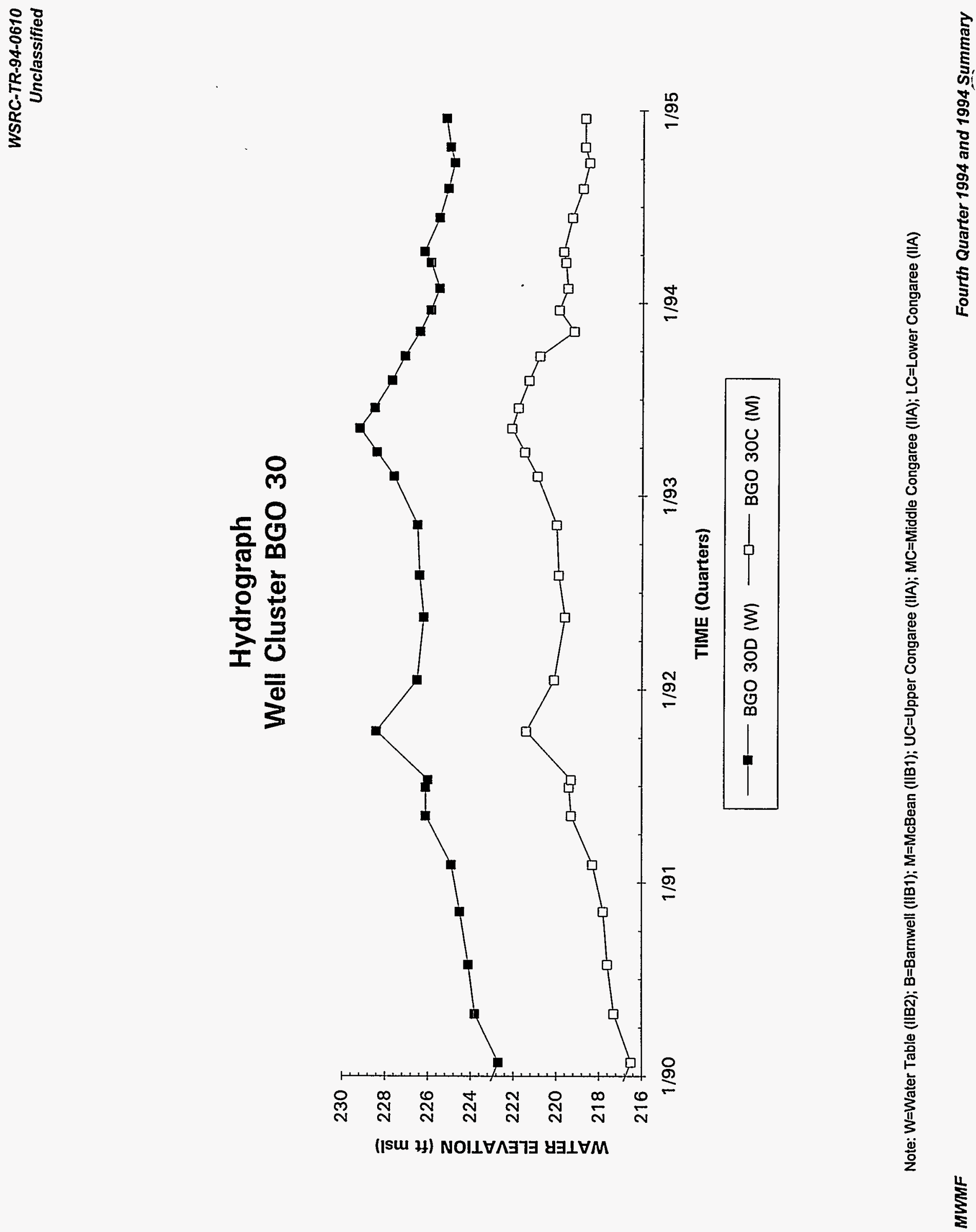

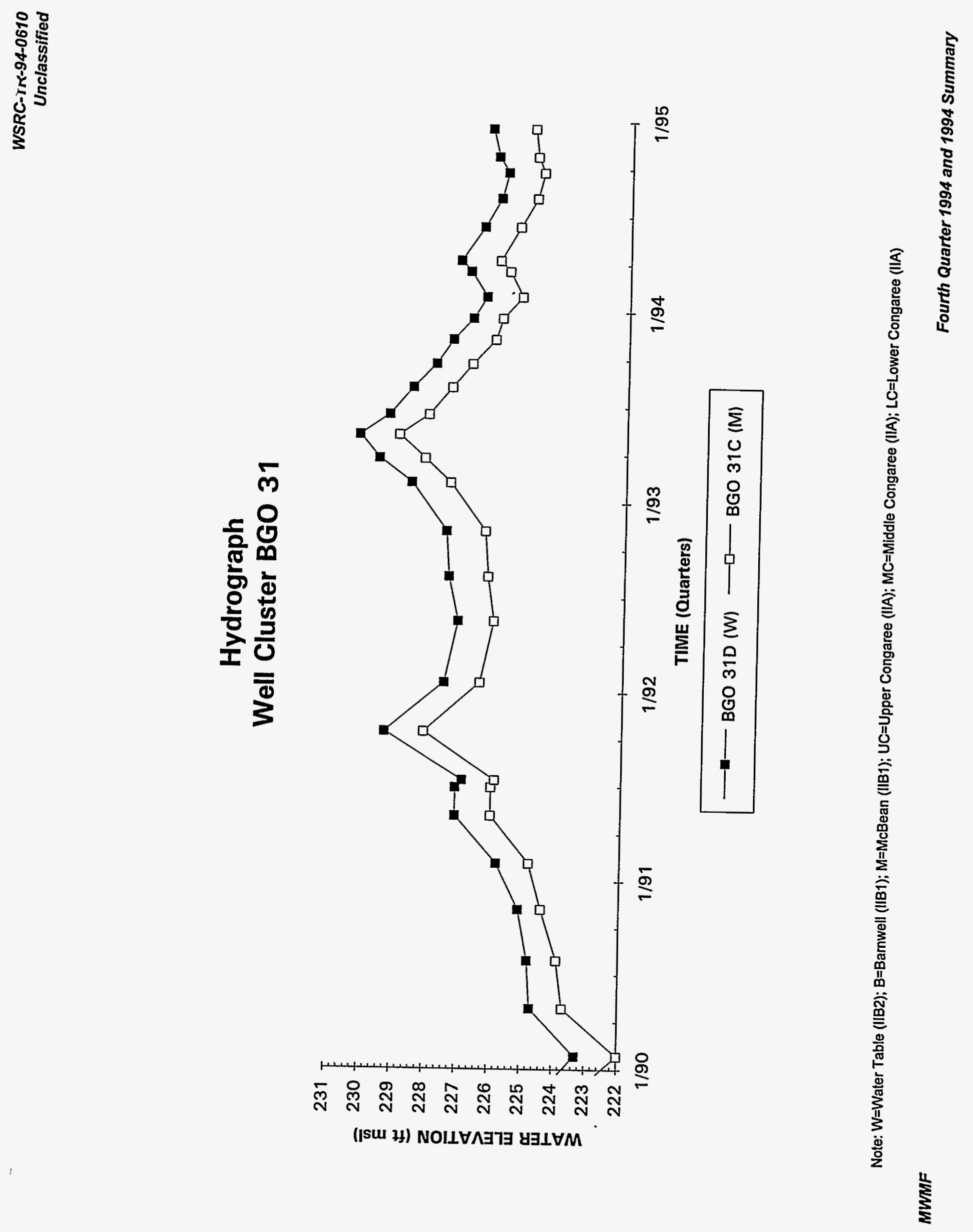

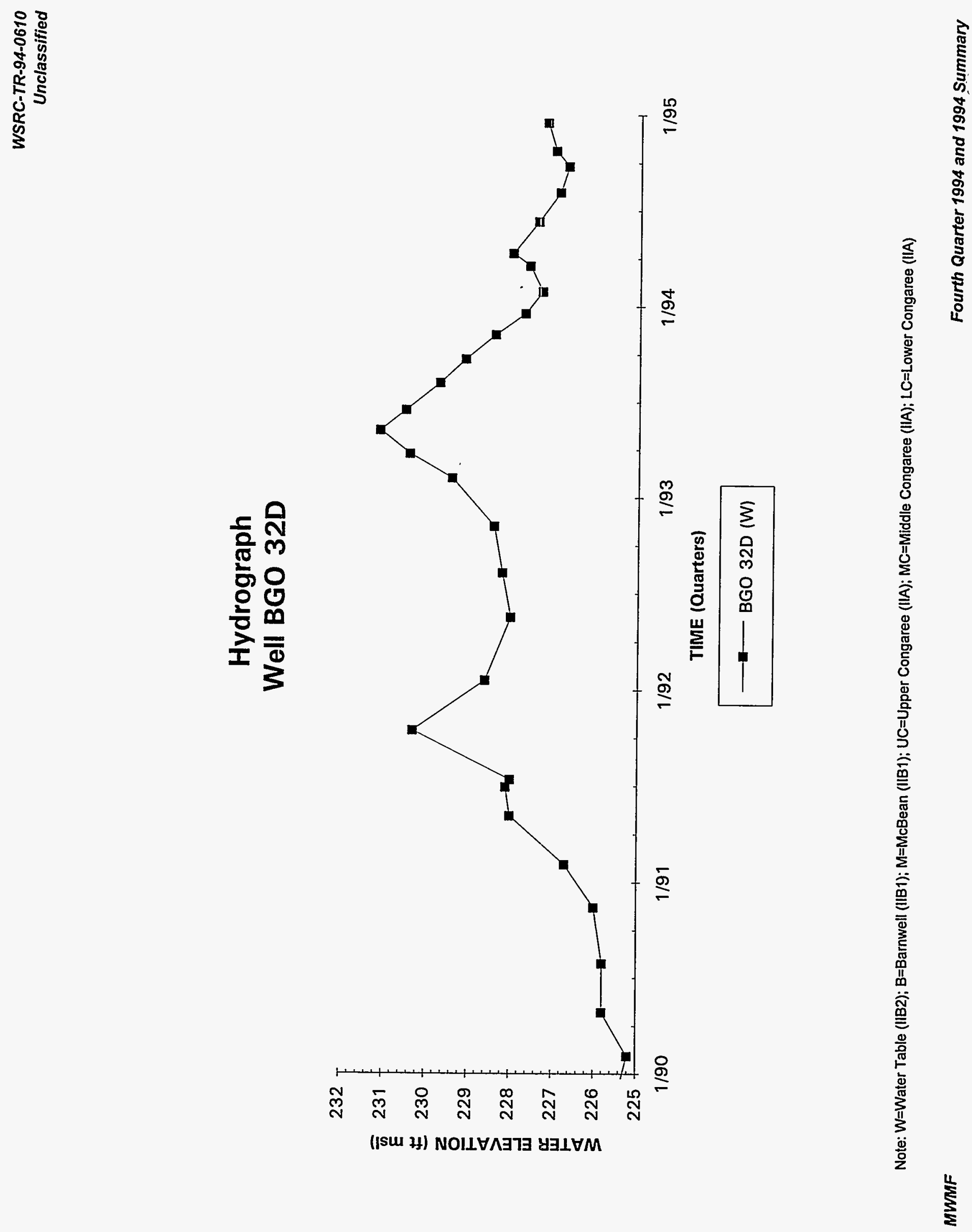

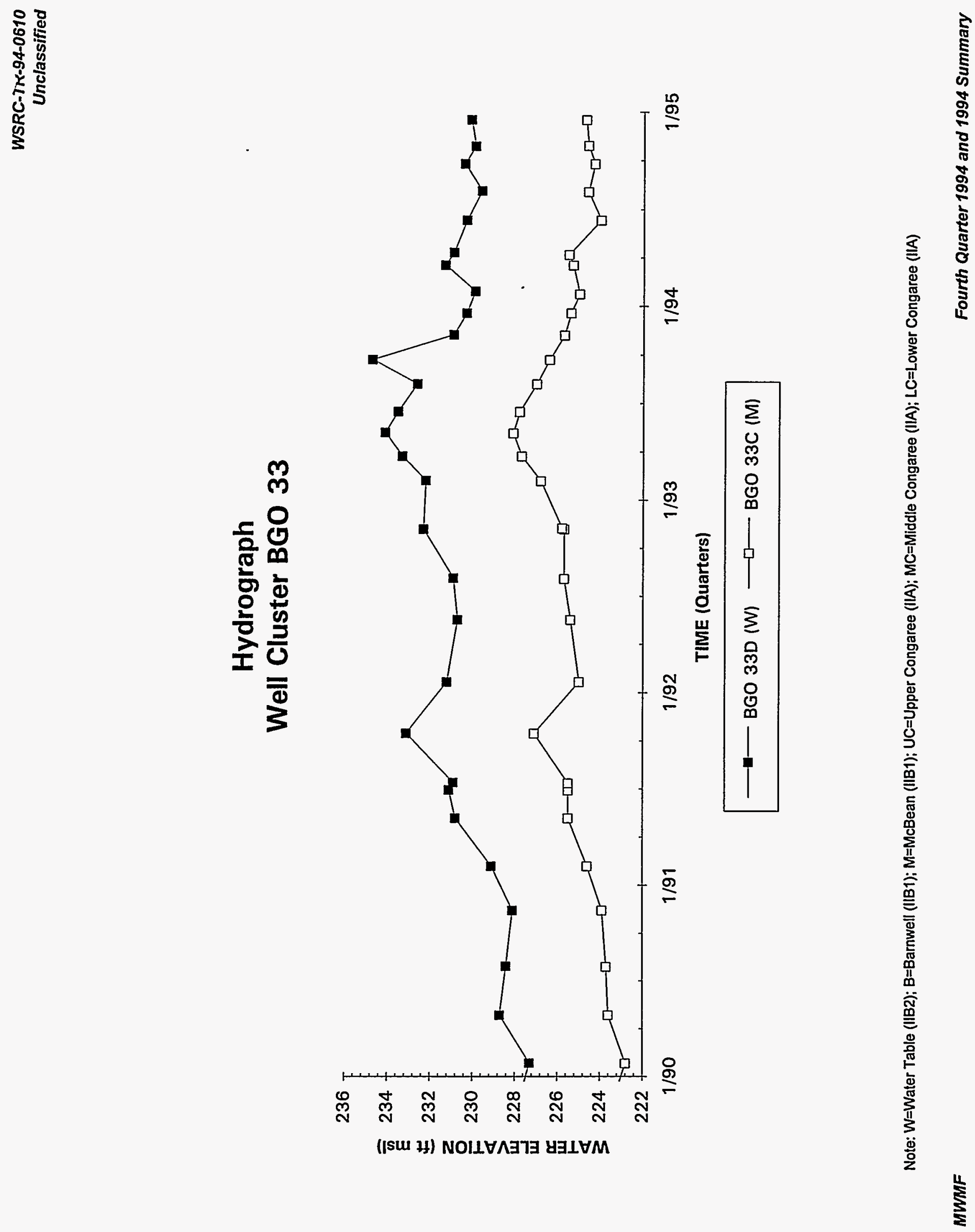


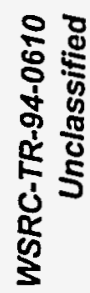
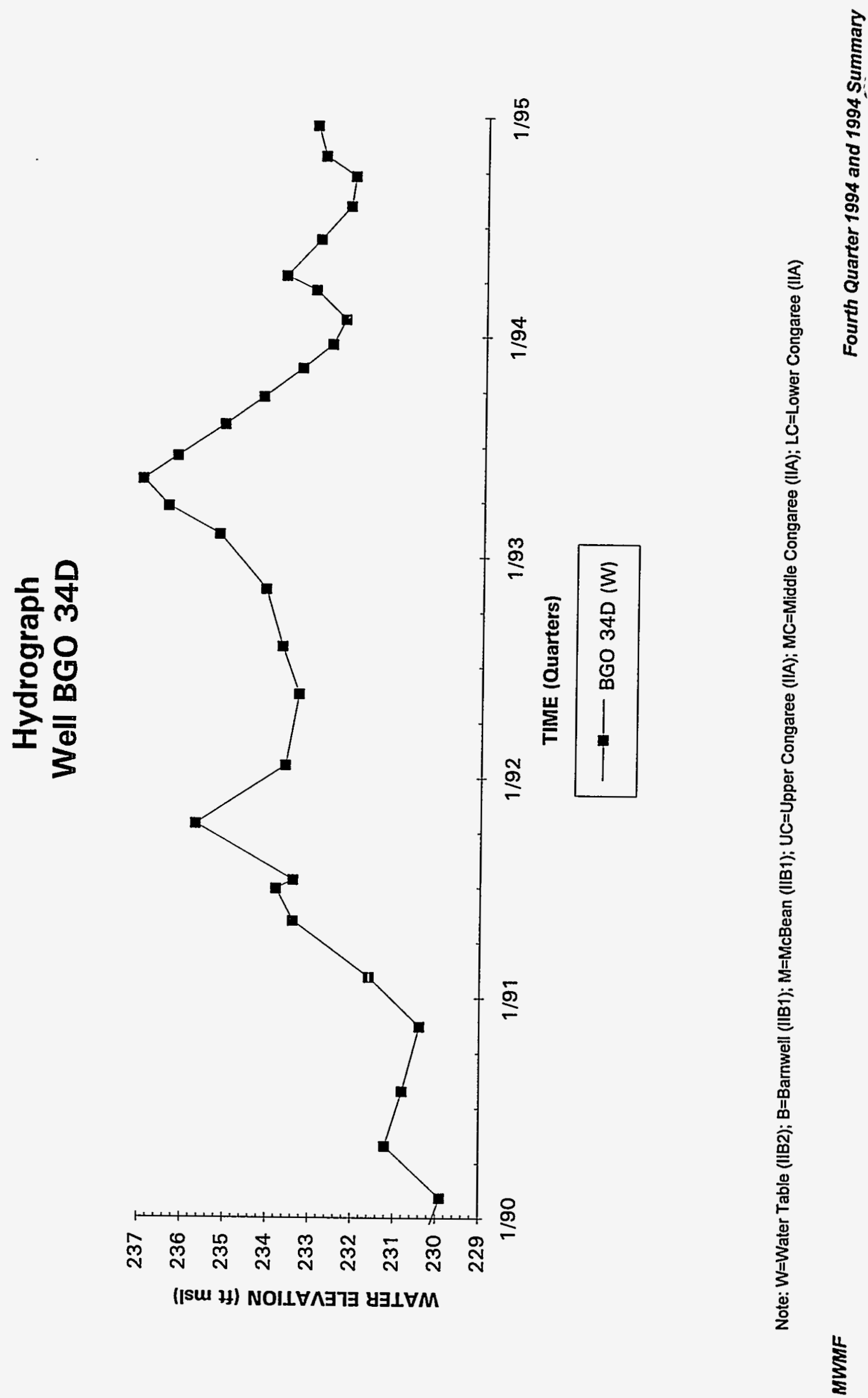


\section{Hydrograph \\ Well Cluster BGO 35}

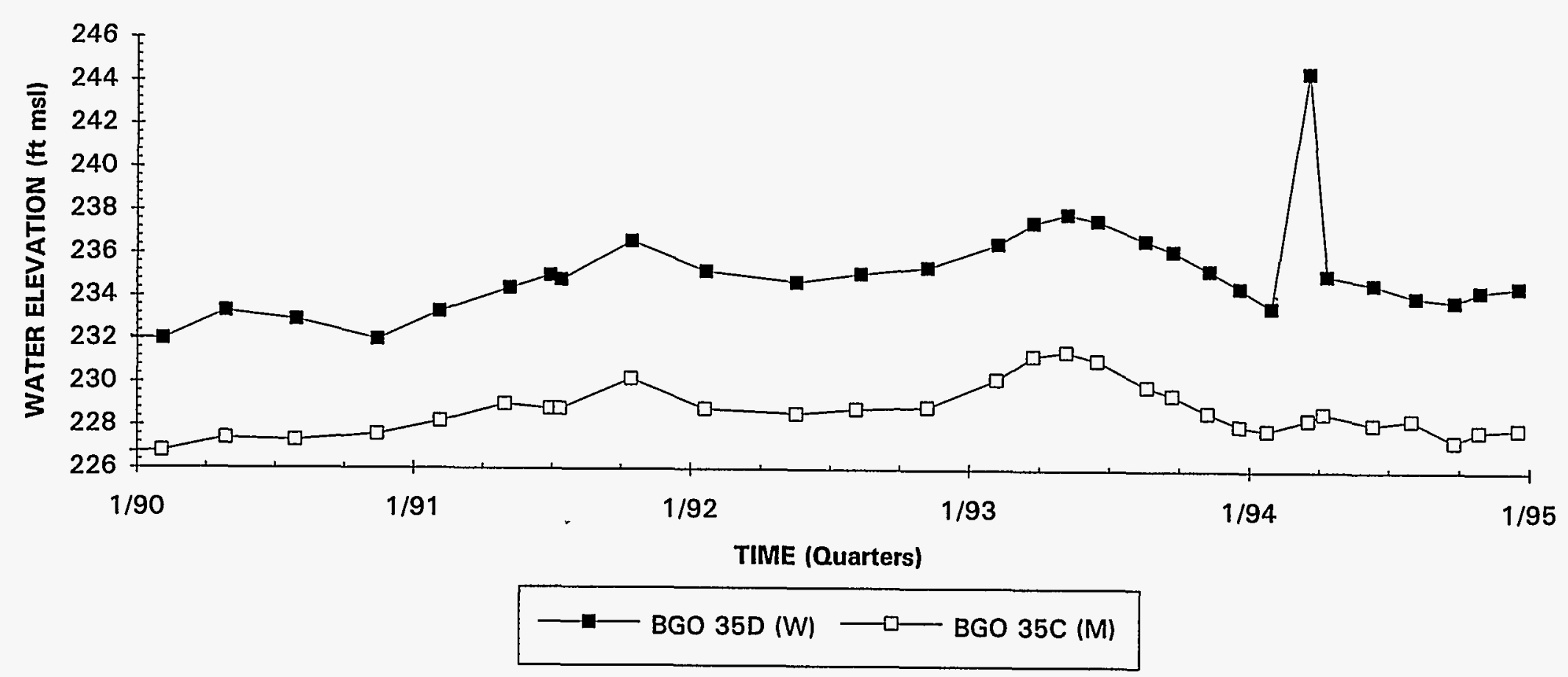

Note: W=Water Table (IIB2); B=Barnwell (IIB1); M=McBean (IIB1); UC=Upper Congaree (IIA); MC=Middle Congaree (IIA); LC=Lower Congaree (IIA) 

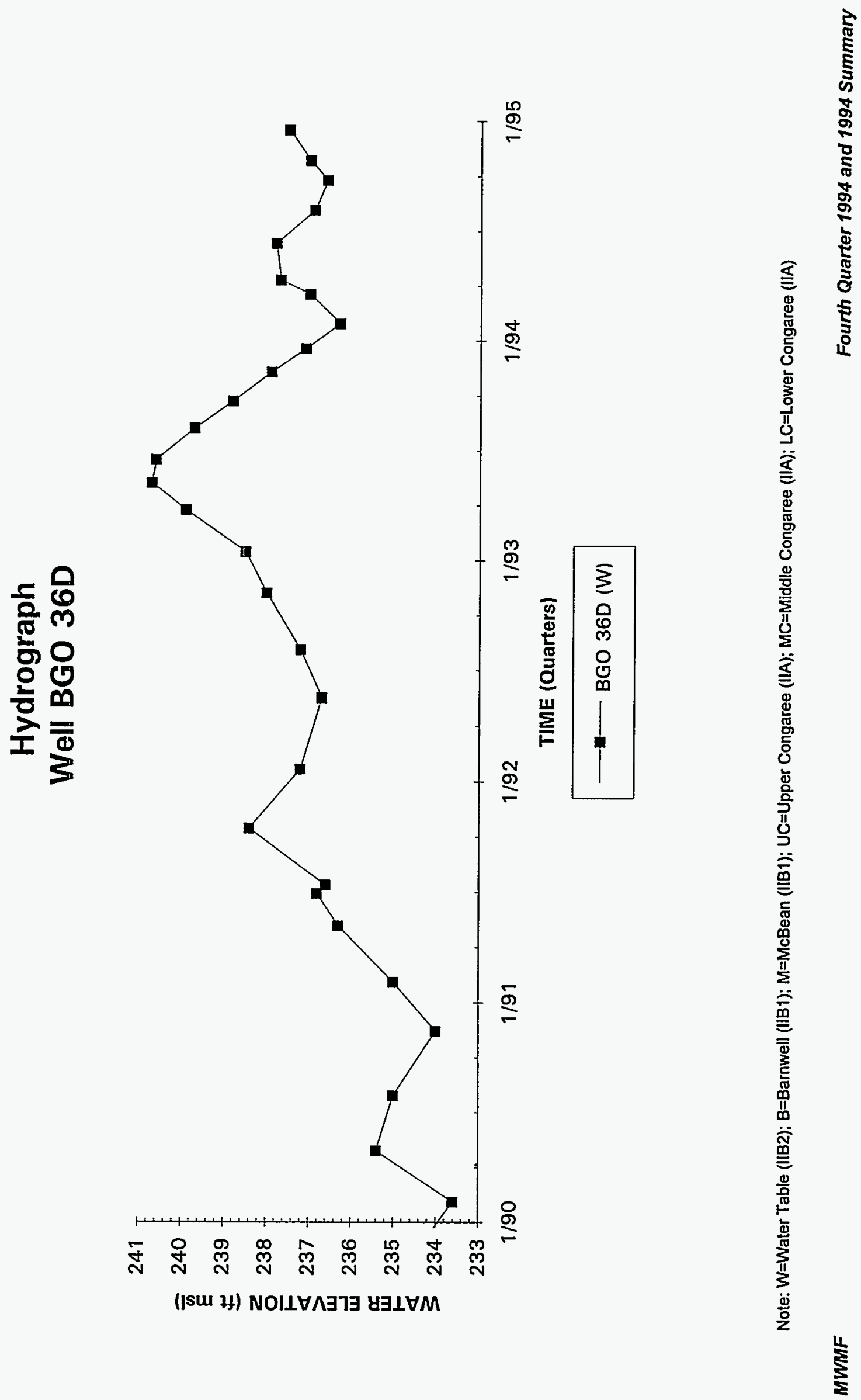


\section{Hydrograph \\ Well Cluster BGO 37}

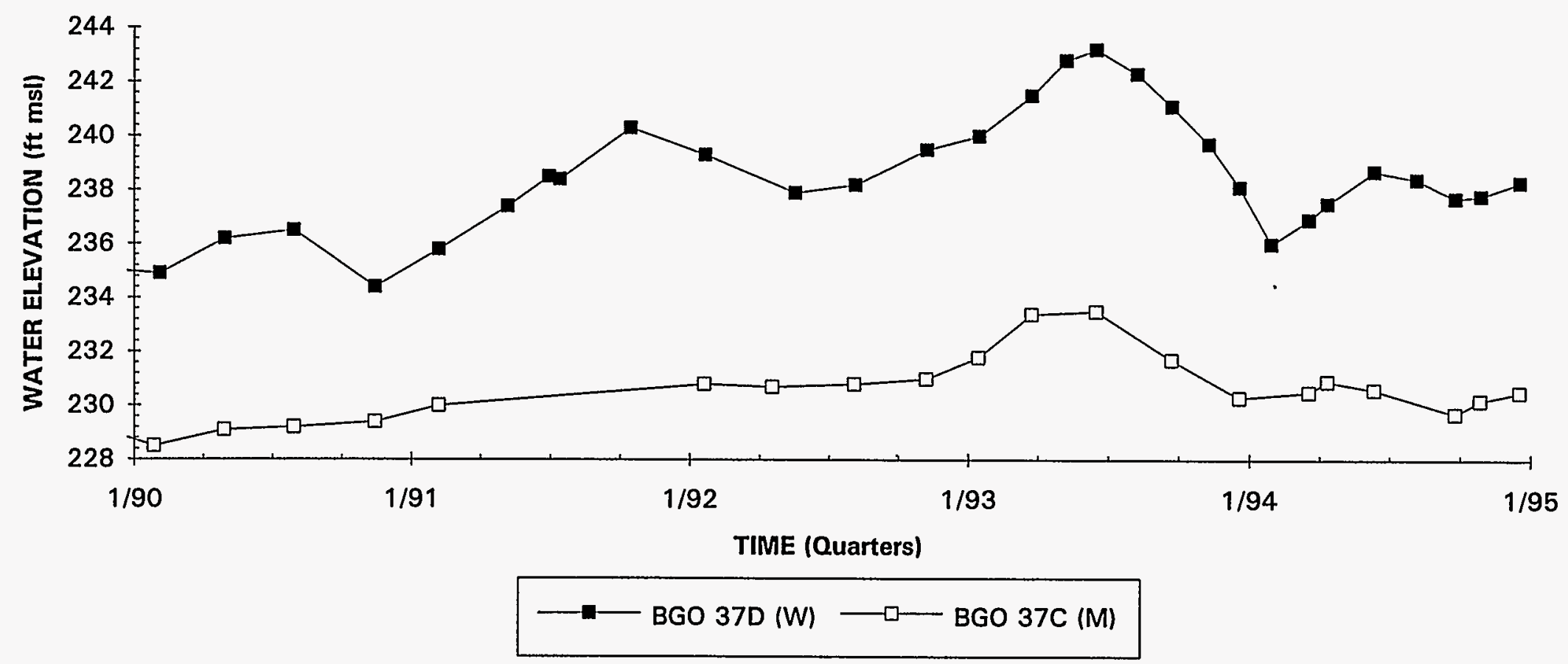

Note: W=Water Table (IIB2); B=Barnwell (IIB1); $M=$ MCBean (IIB1); UC=Upper Congaree (IIA); MC=Middle Congaree (IIA); LC=Lower Congaree (IIA) 

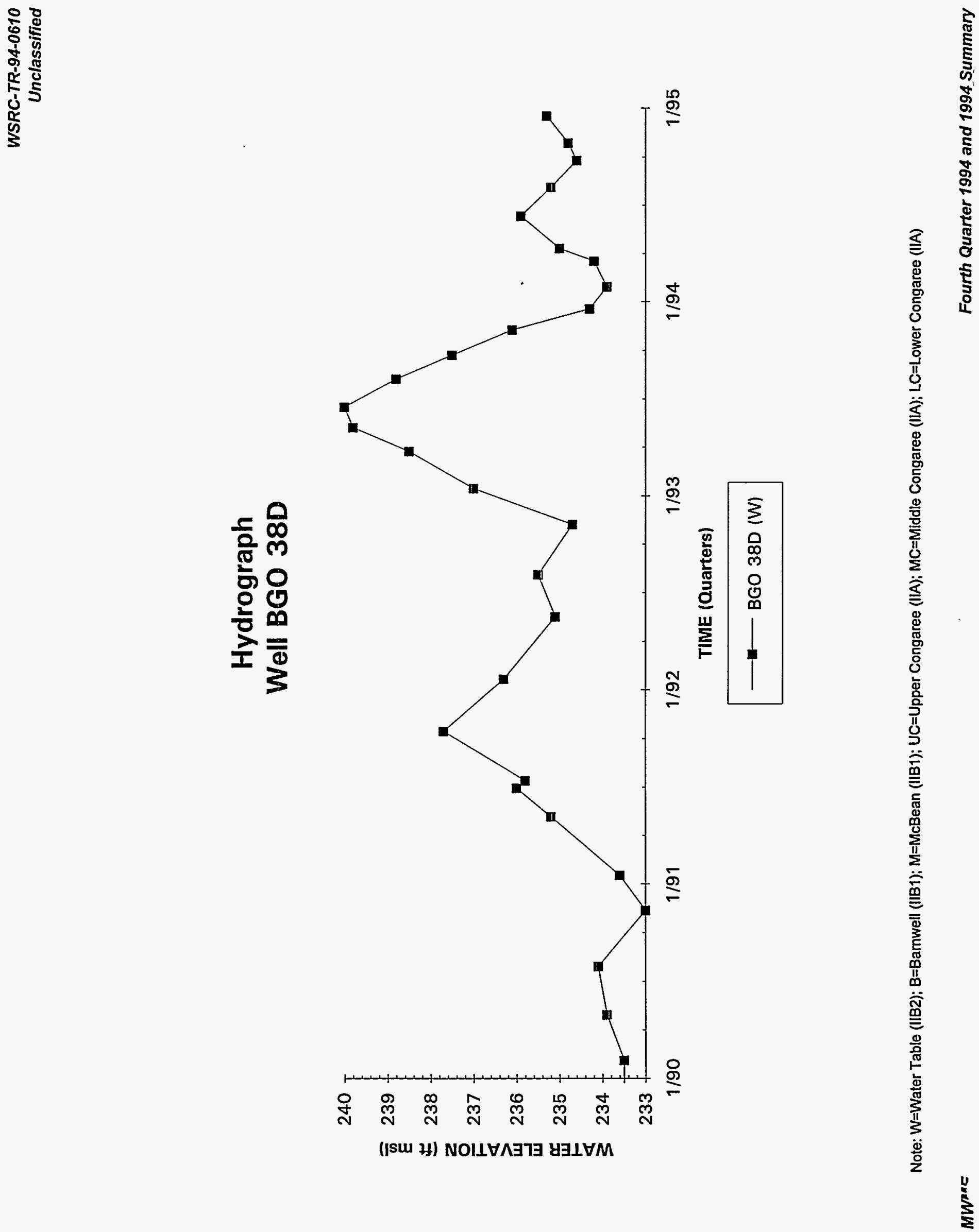

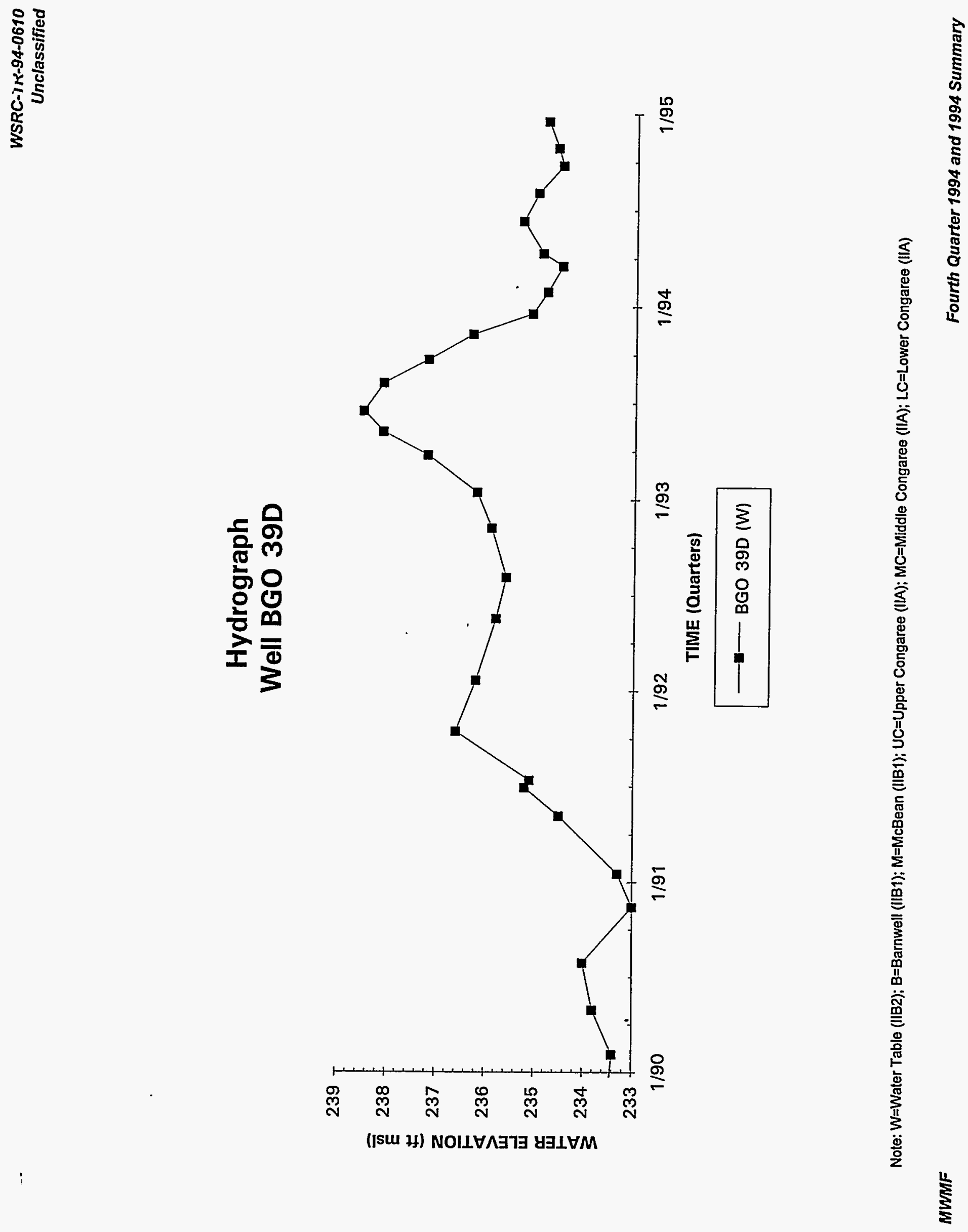


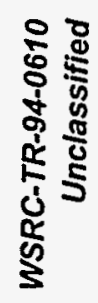
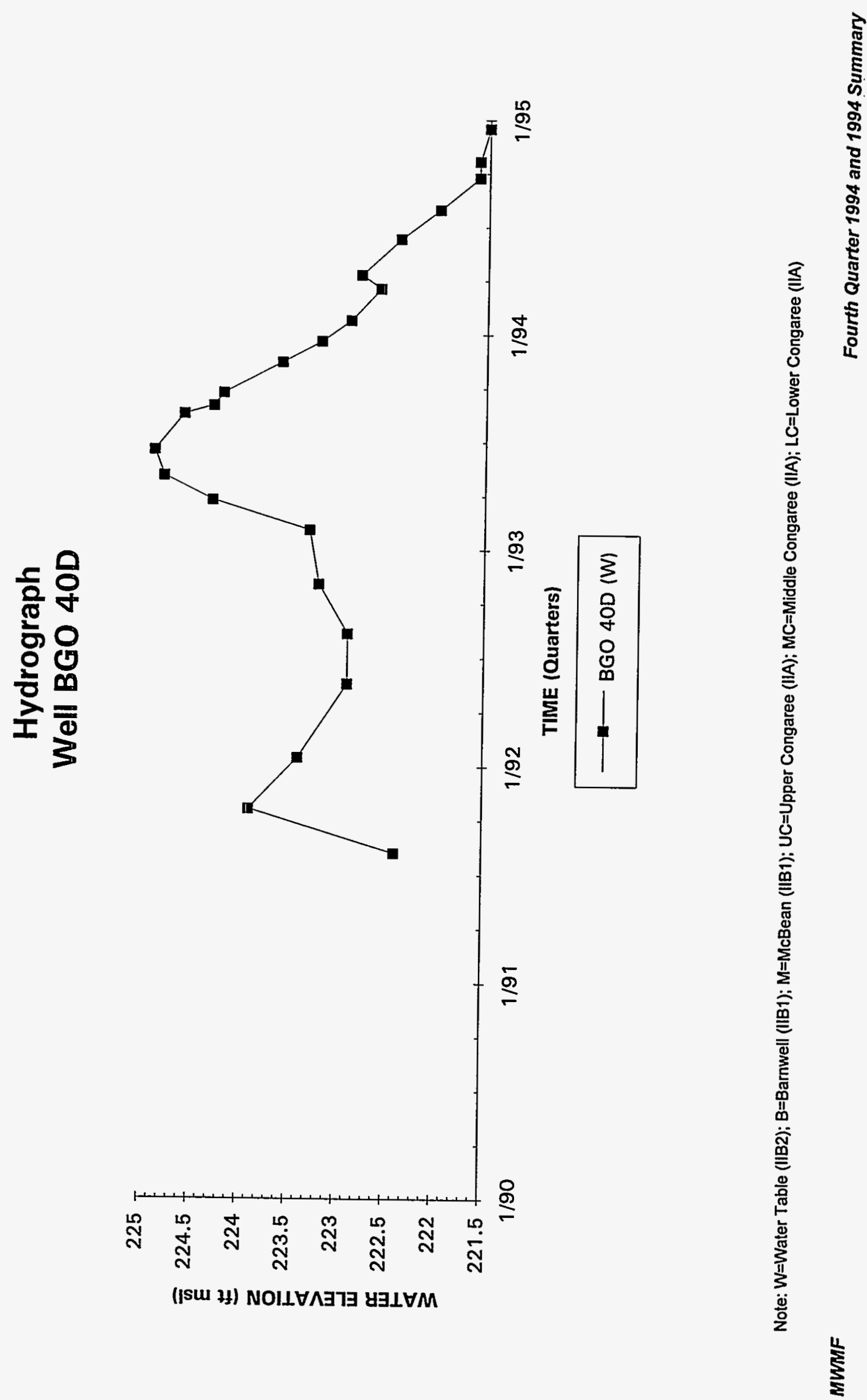


\section{Hydrograph \\ Well BGO 41A}

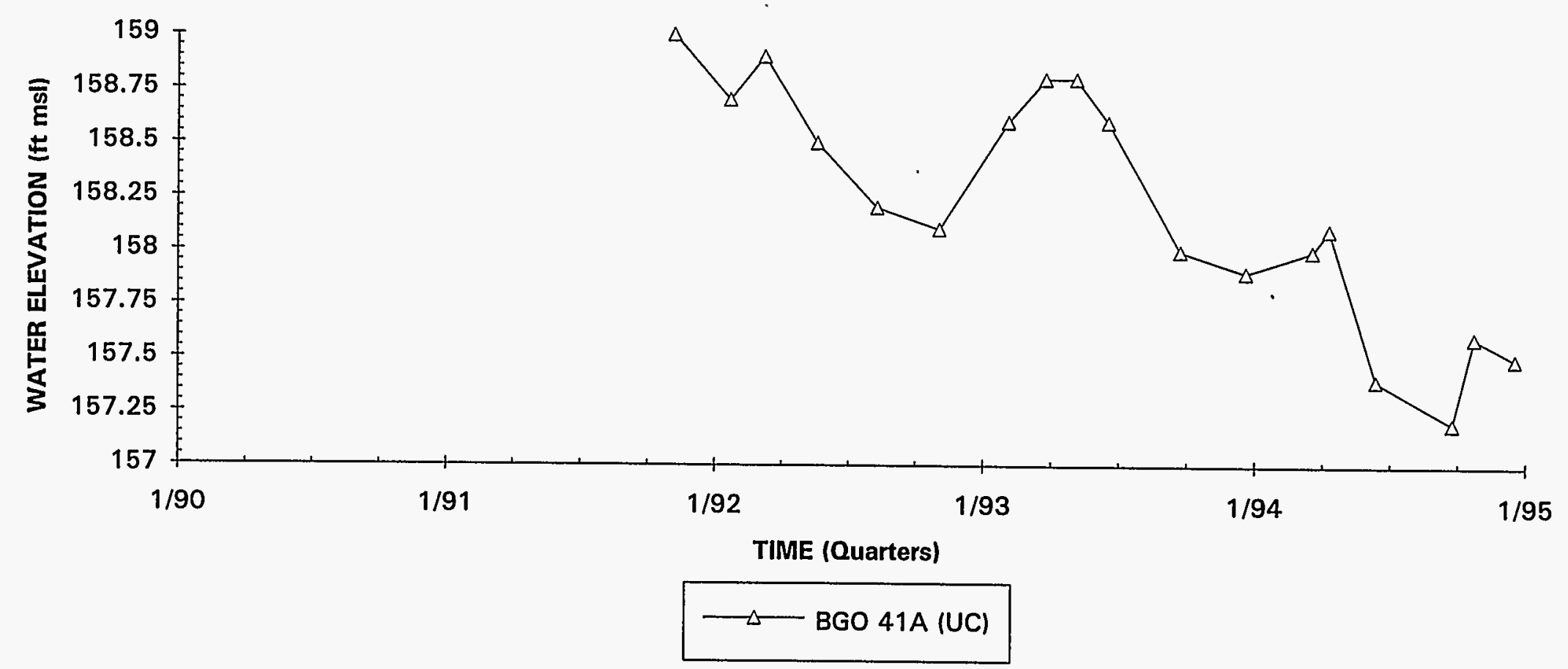

Note: W=Water Table (IIB2); B=Barnwell (IIB1); M=McBean (IIB1); UC=Upper Congaree (IIA); MC=Middle Congaree (IIA); LC=Lower Congaree (IIA) 

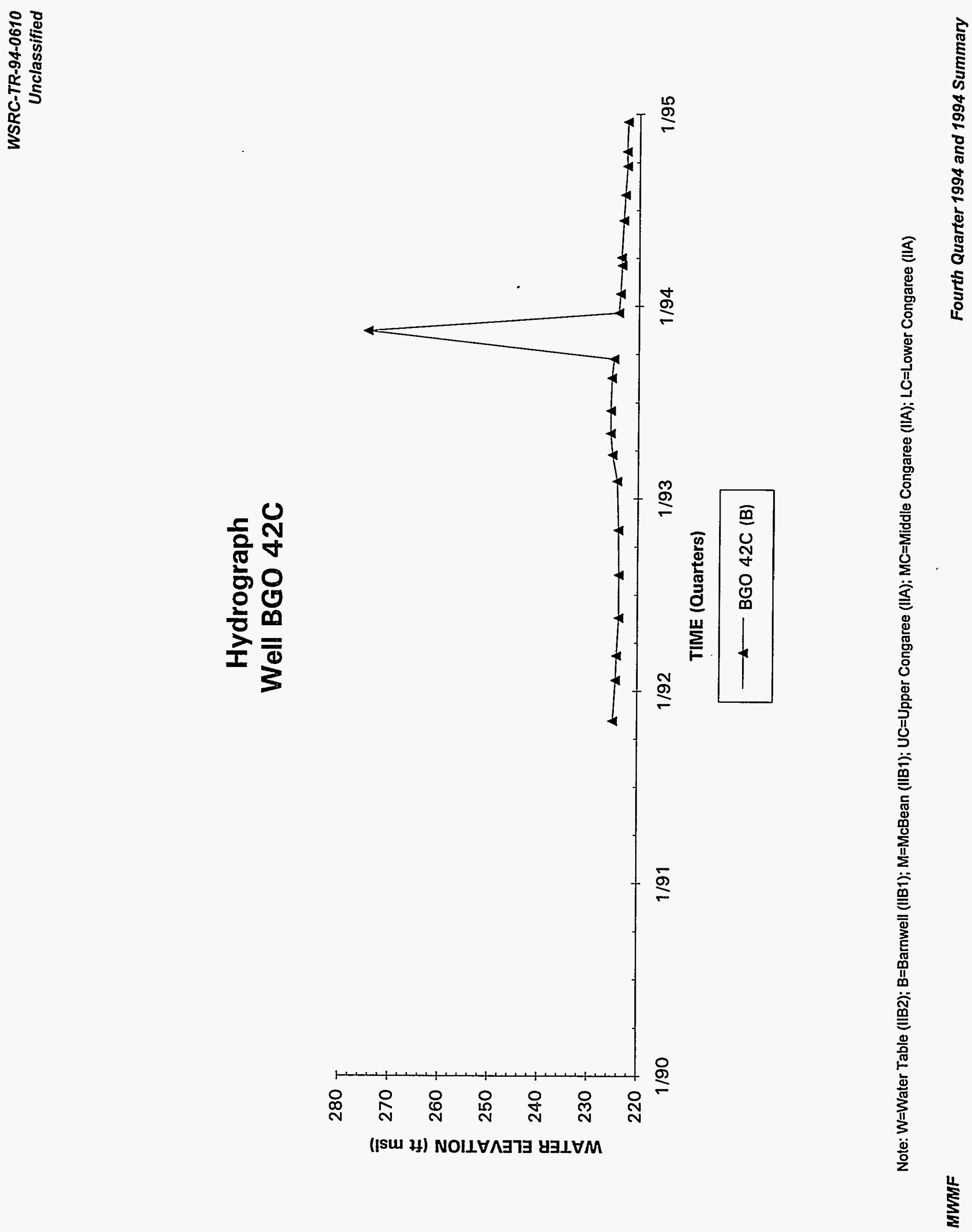

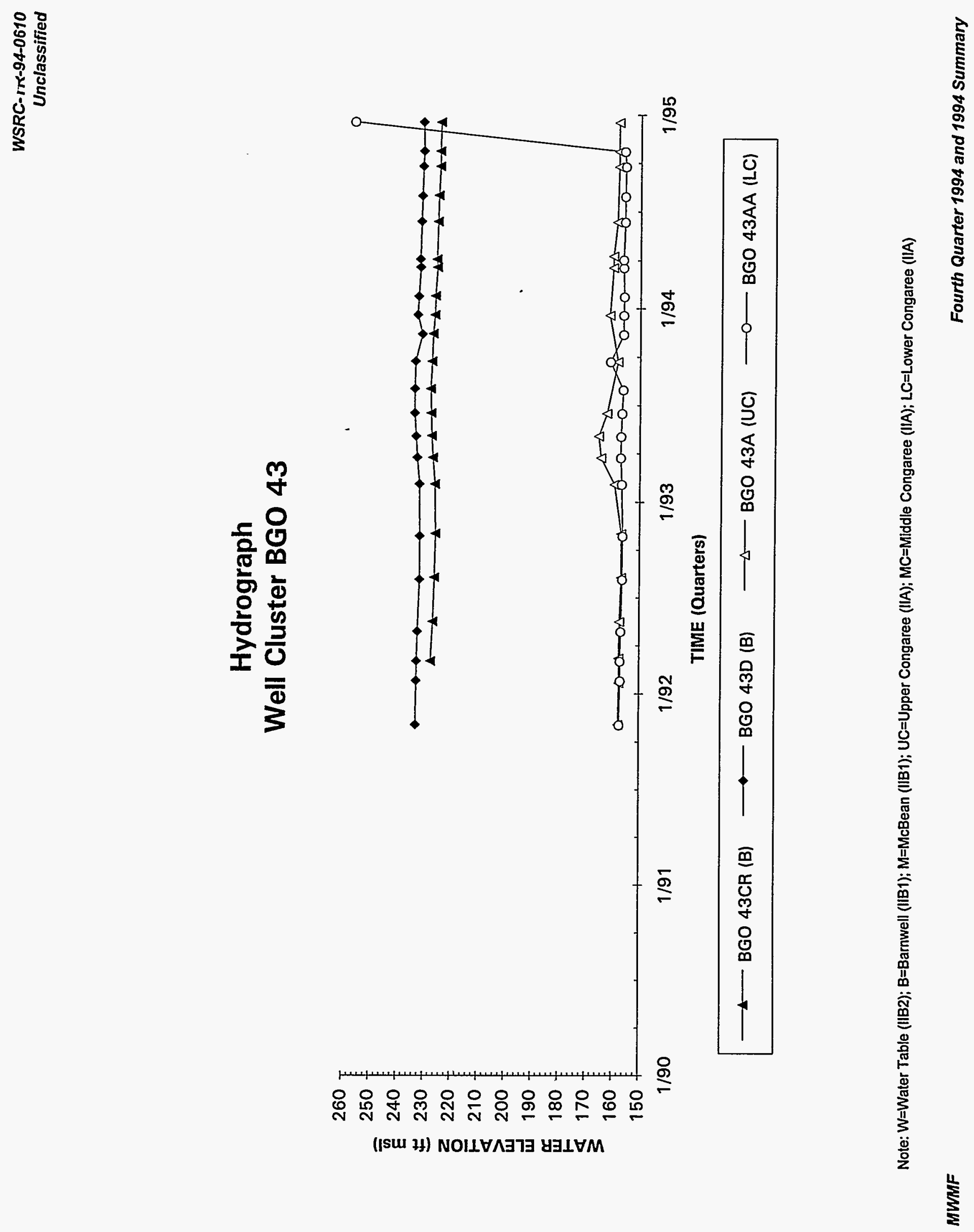

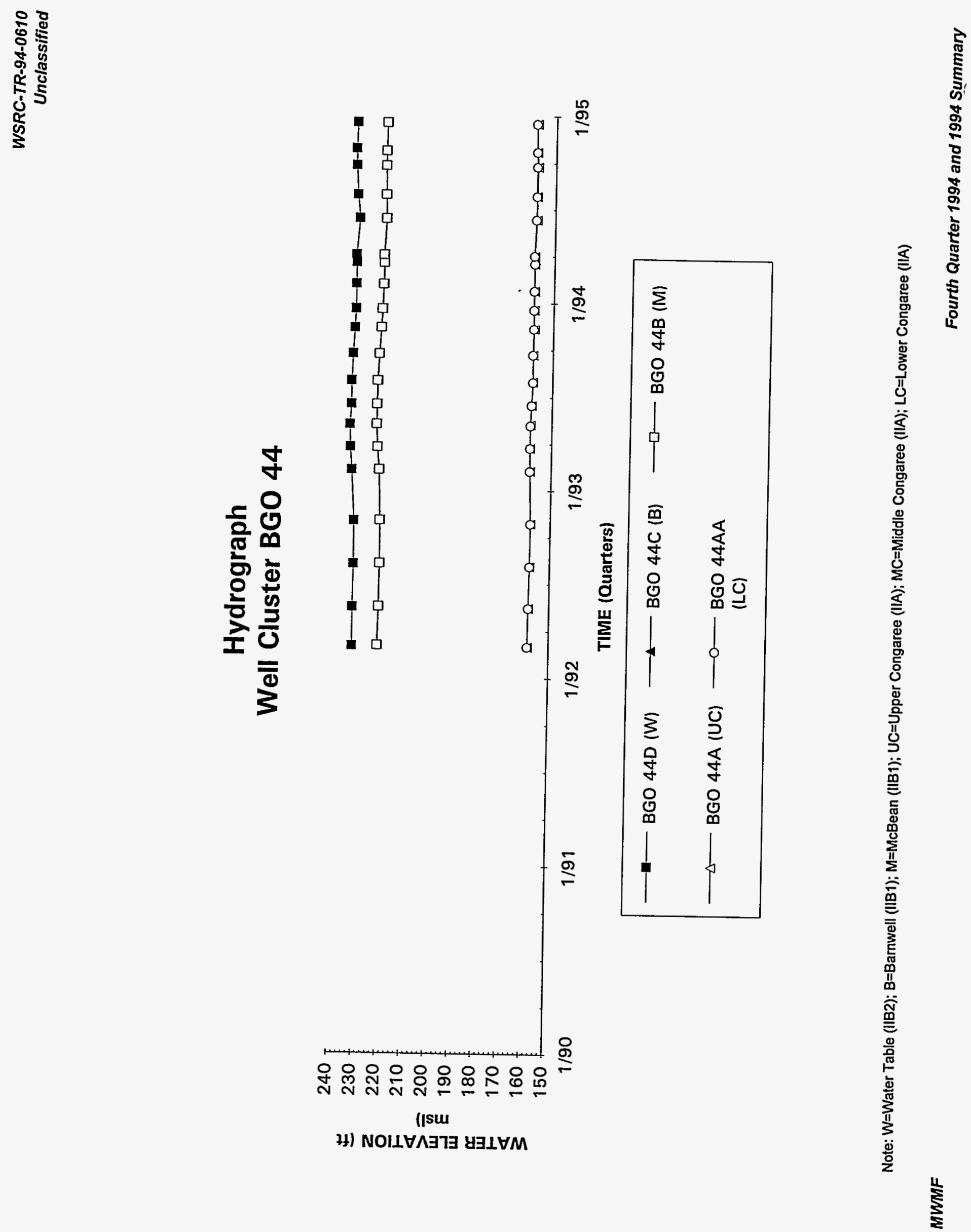


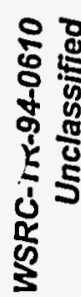
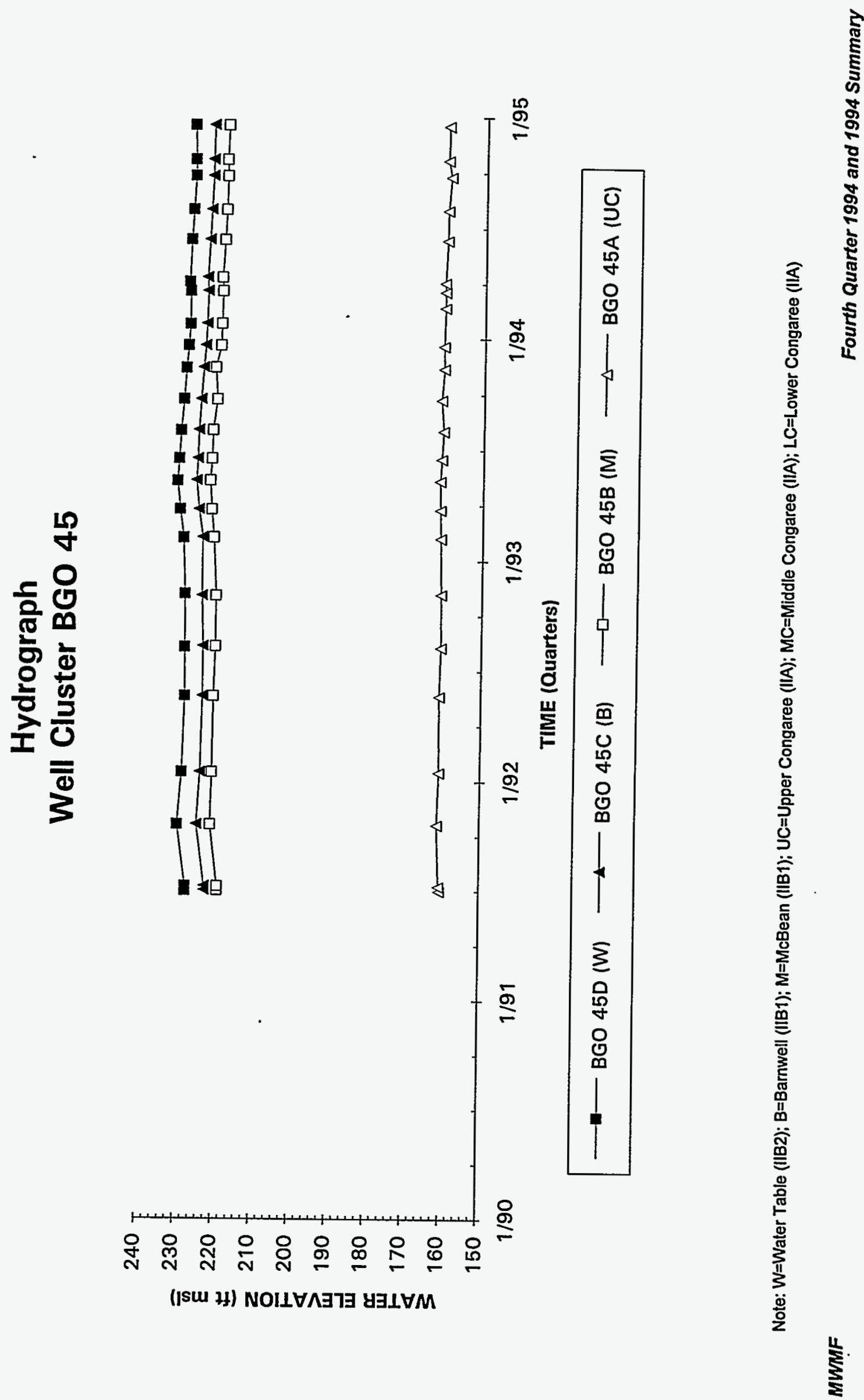


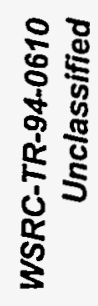
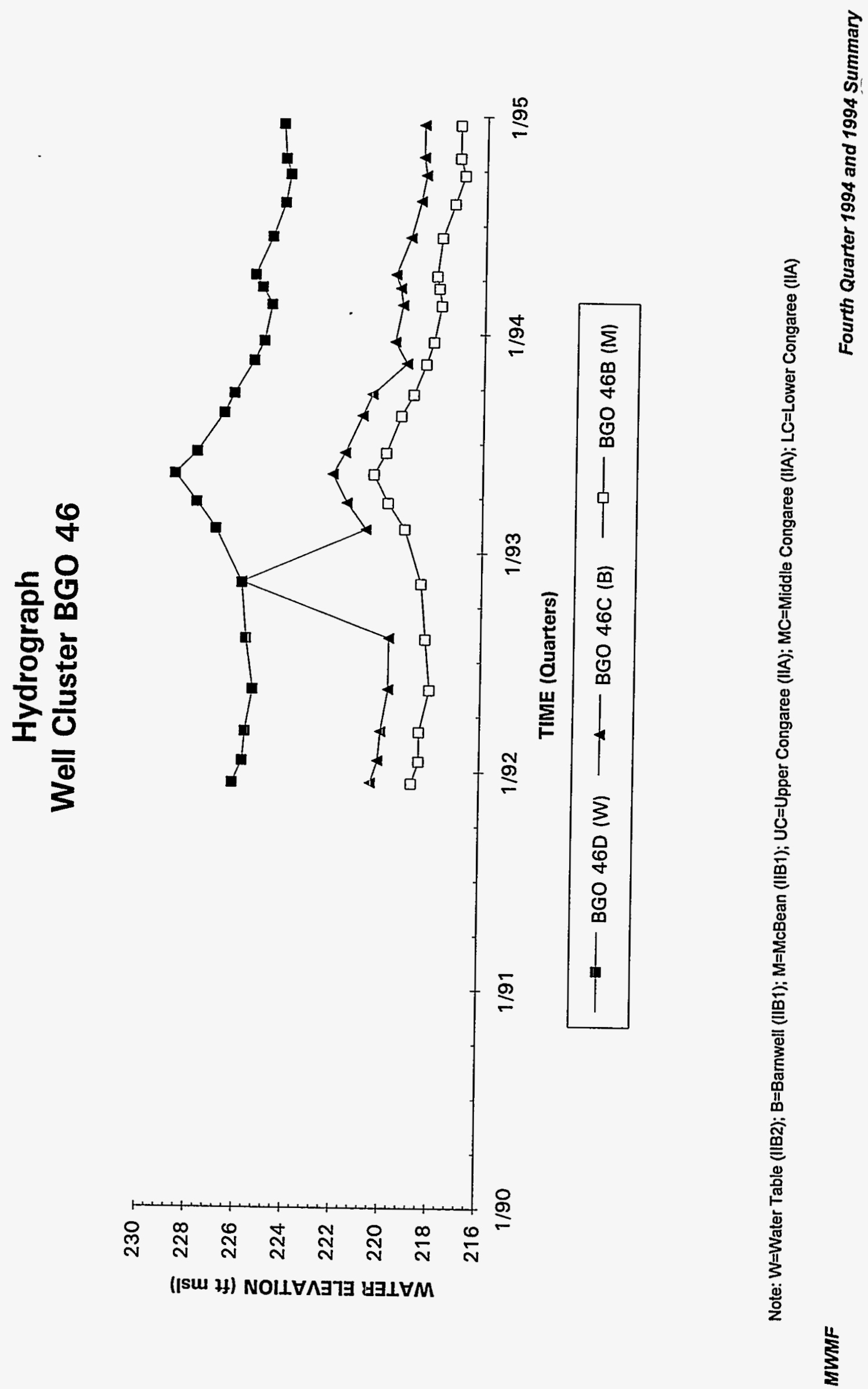

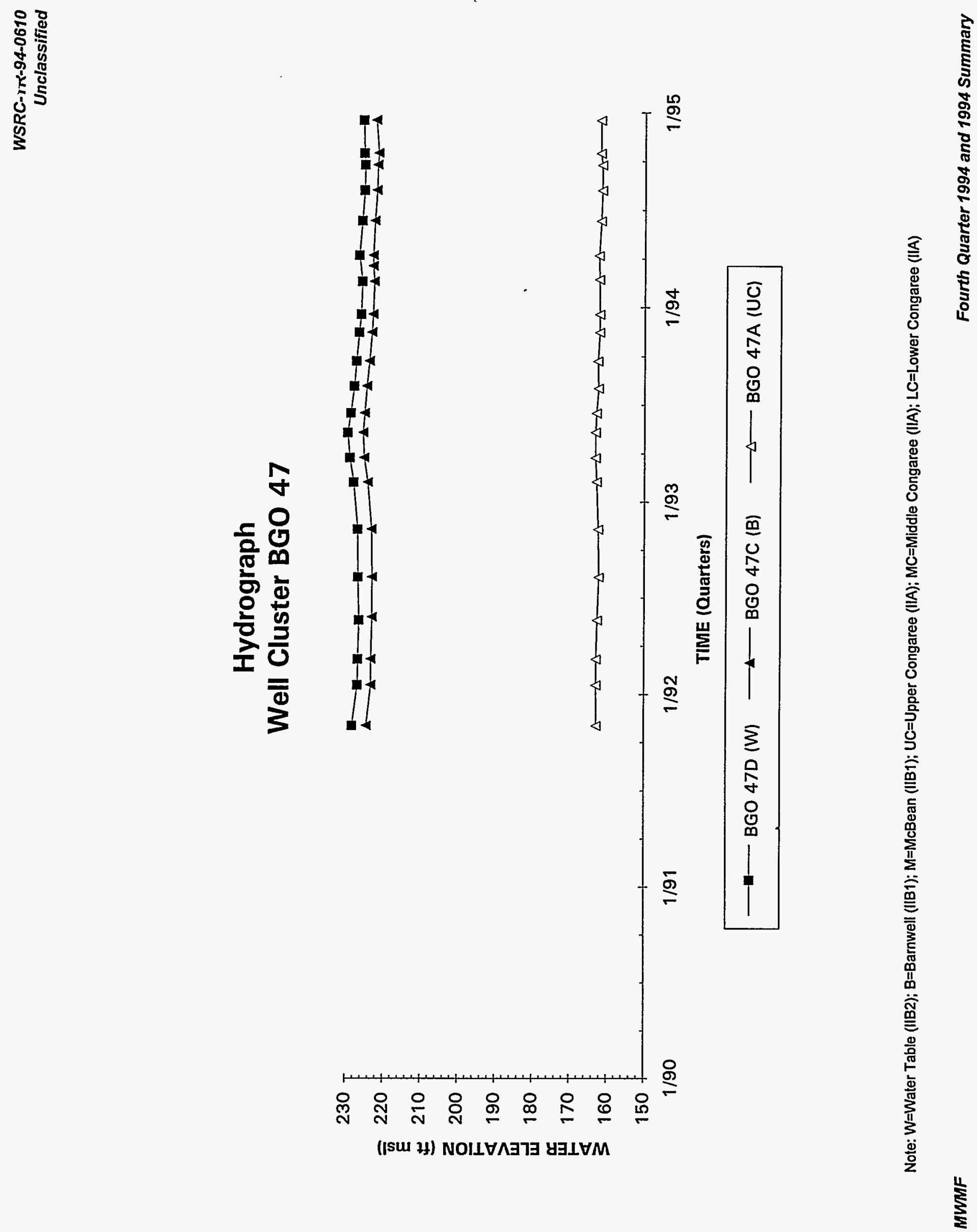


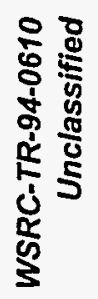
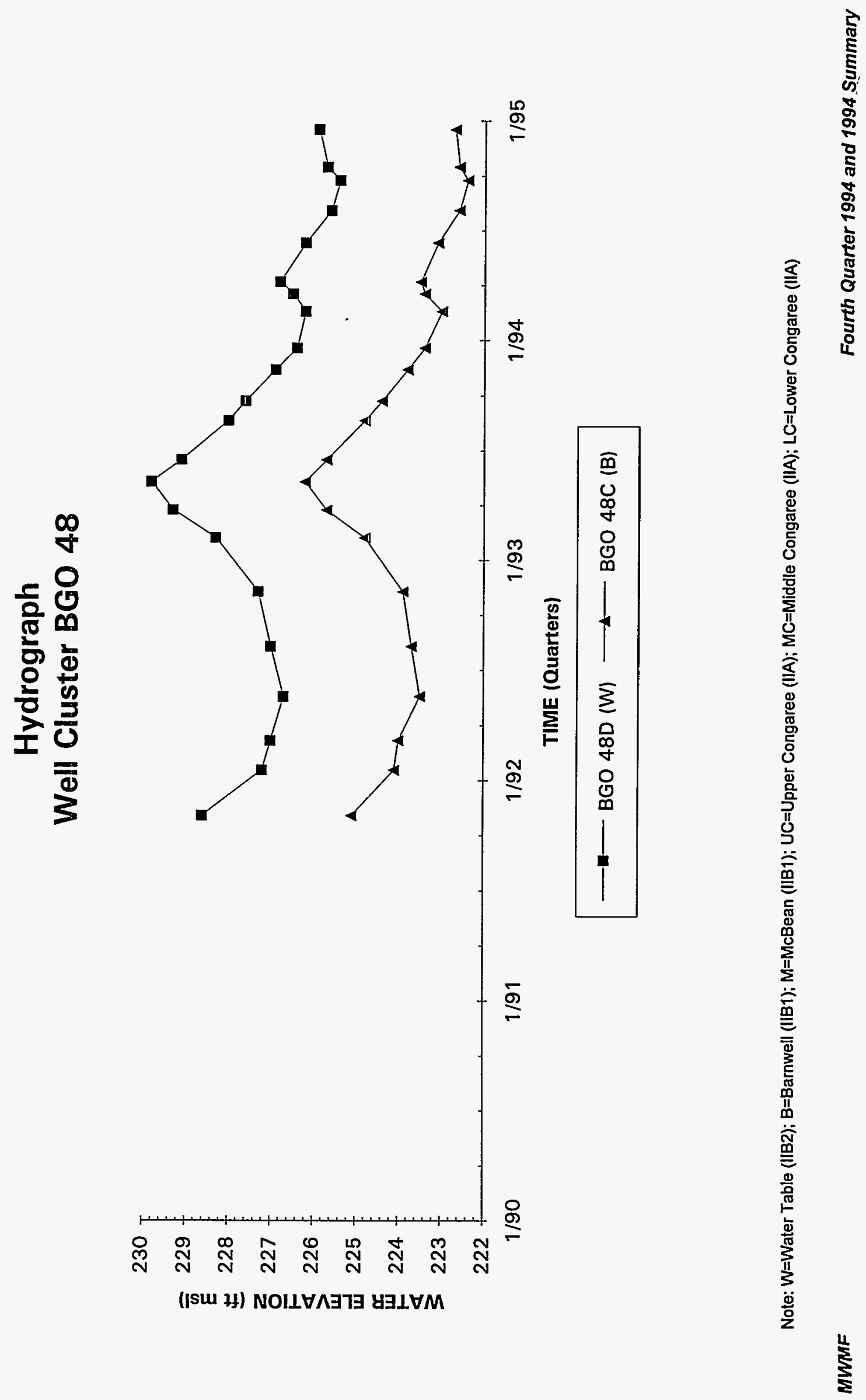


\section{Hydrograph \\ Well Cluster BGO 49}

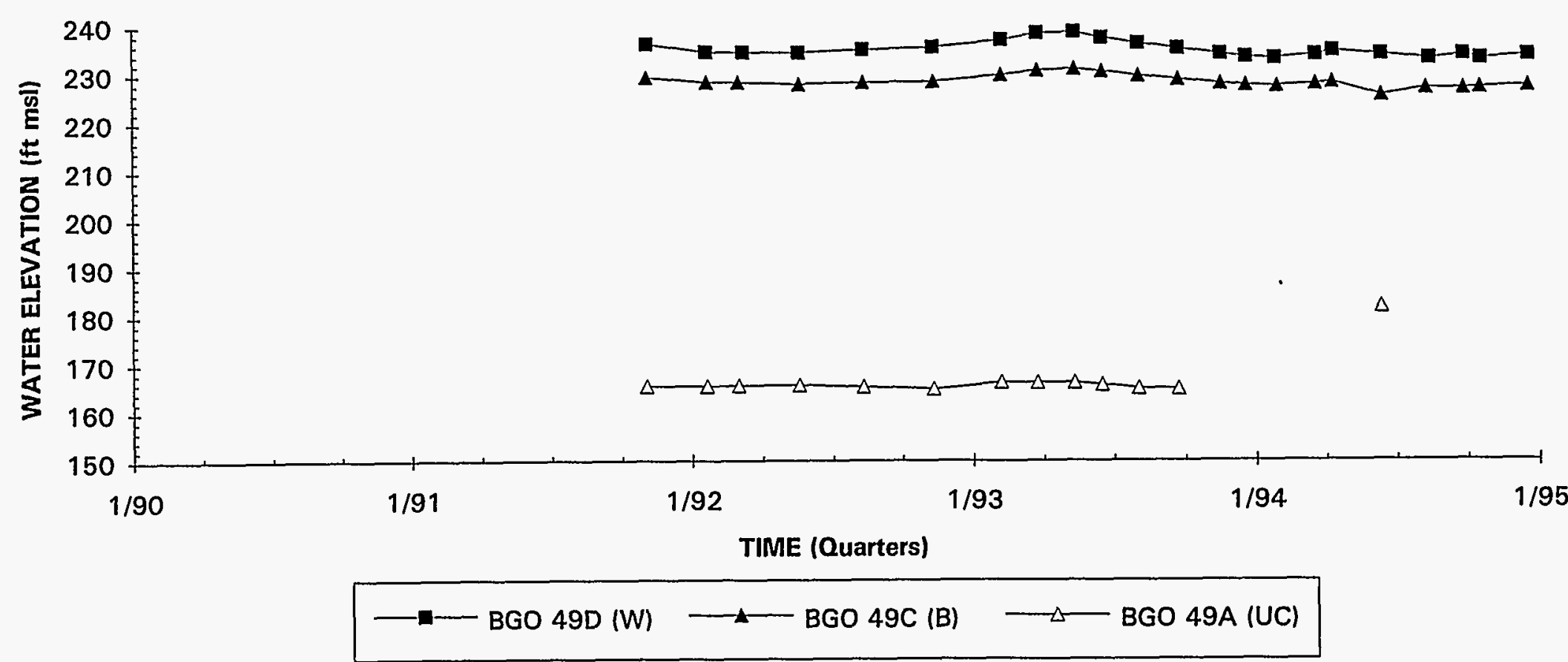

Note: W=Water Table (IIB2); B=Barnwell (IIB1); M=McBean (IIB1); UC=Upper Congaree (IIA); MC=Middle Congaree (IIA); LC=Lower Congaree (IIA) 


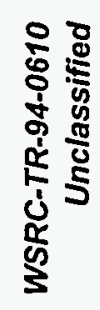
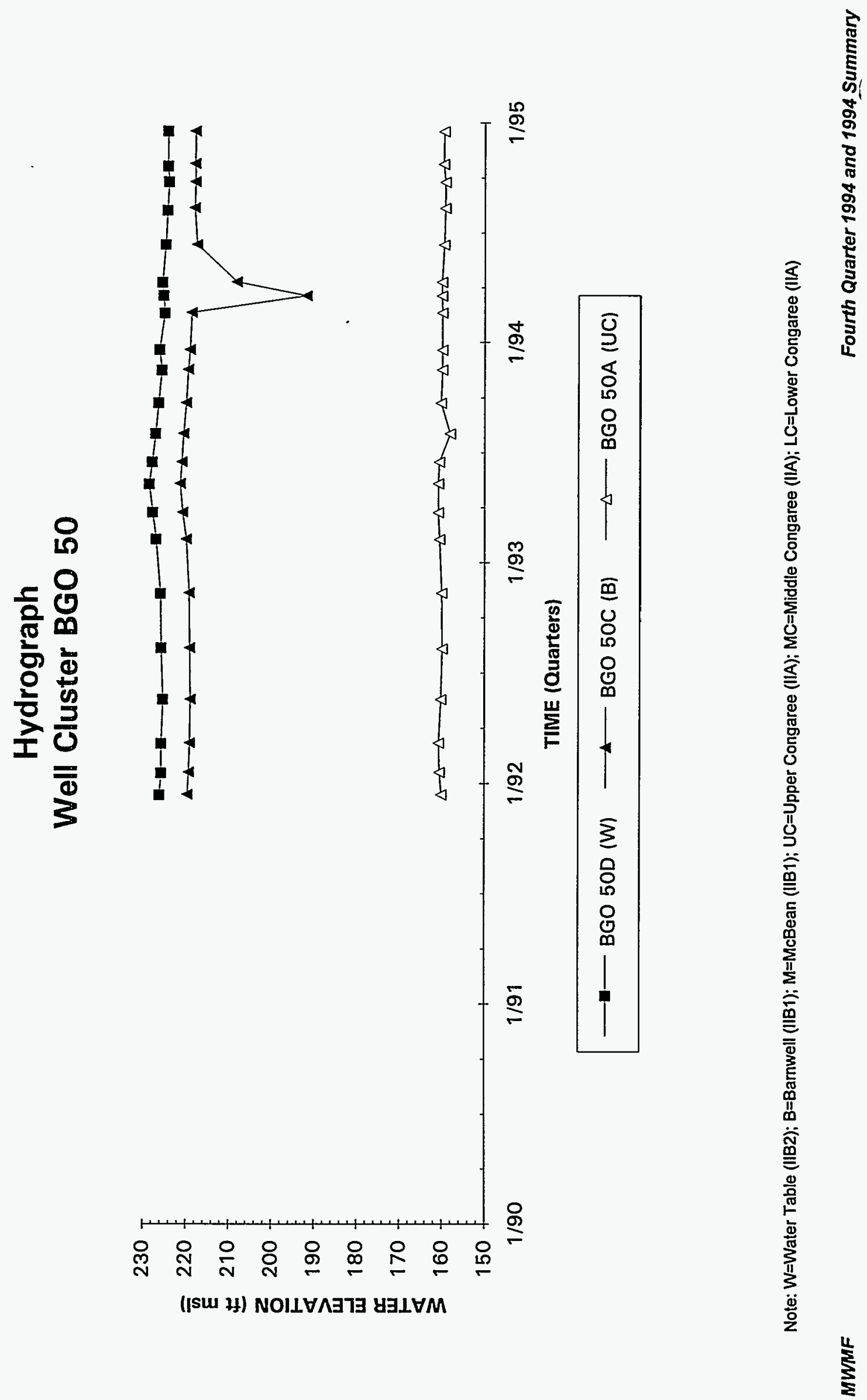


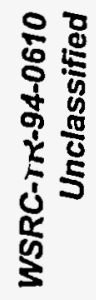
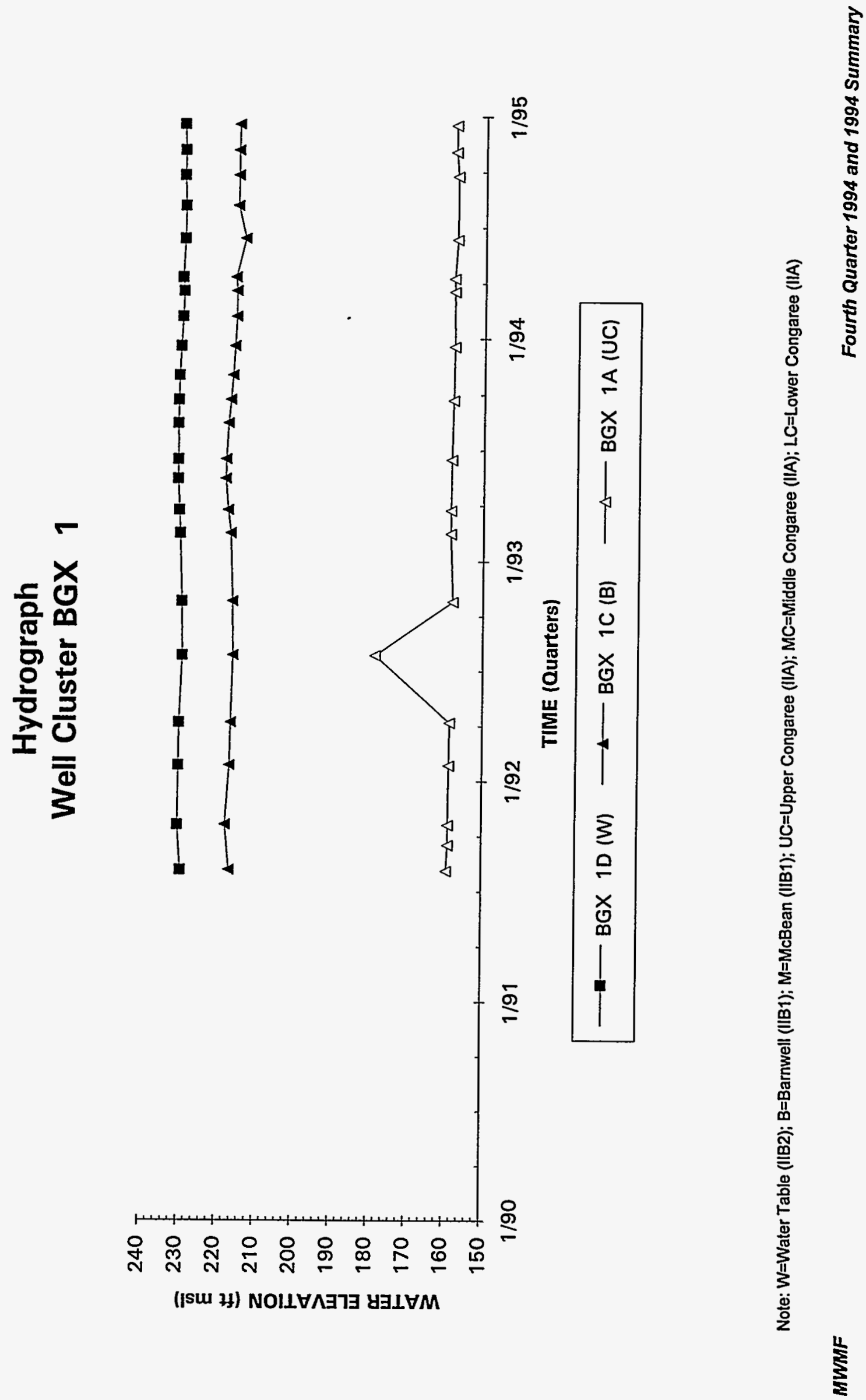


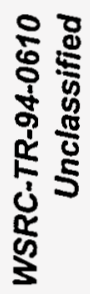
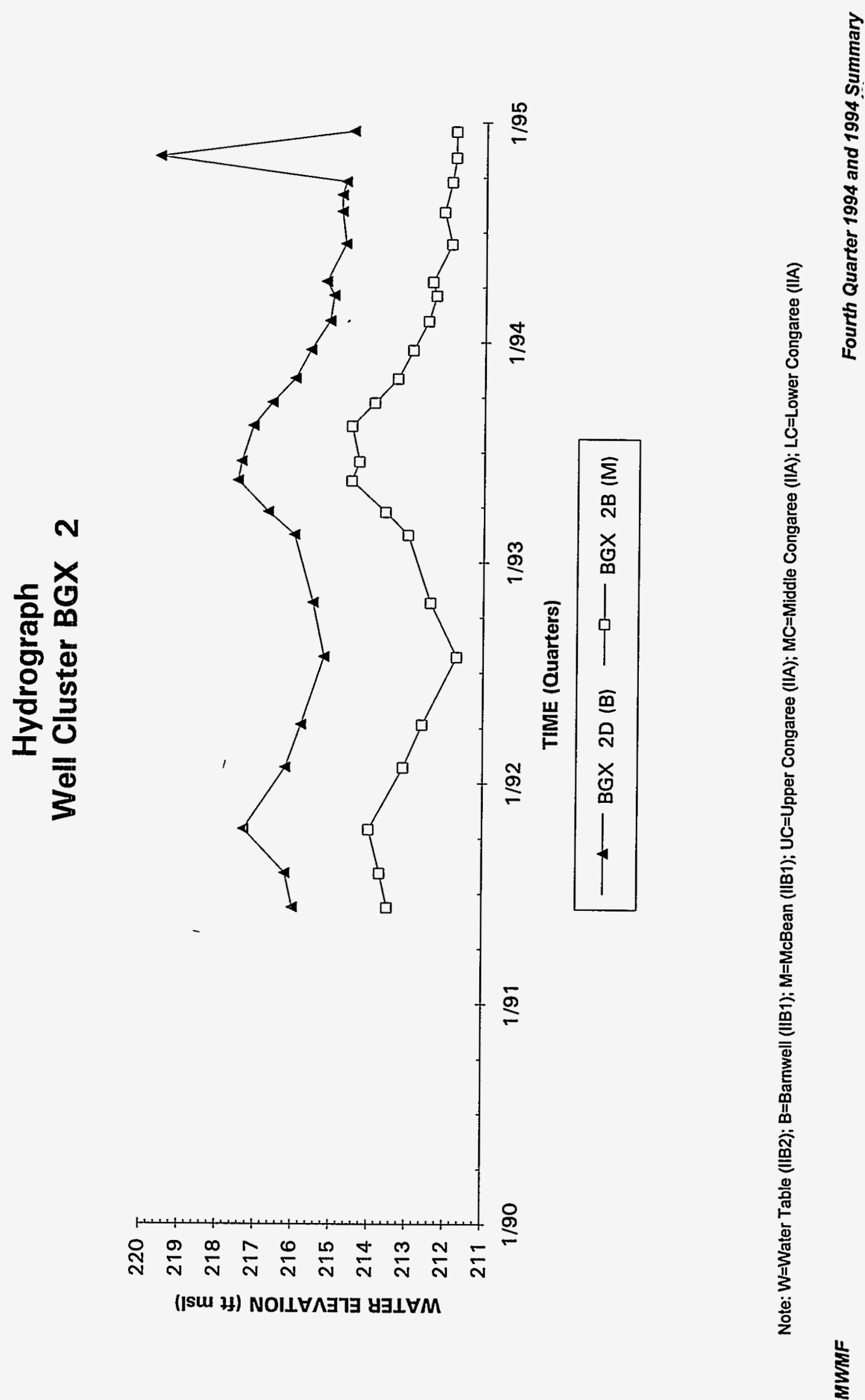


\section{Hydrograph}

Well BGX 3D

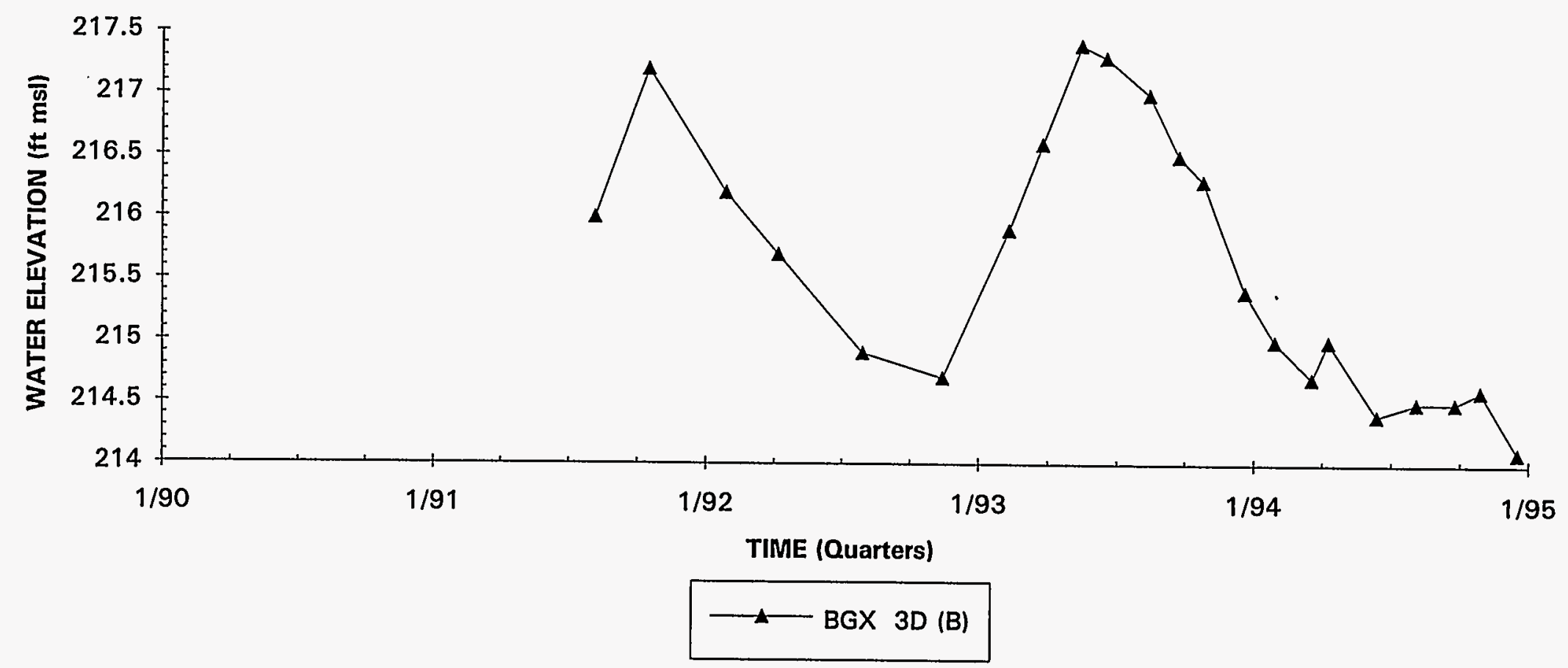

Note: W=Water Table (IIB2); B=Barnwell (IIB1); M=McBean (IIB1); UC=Upper Congaree (IIA); MC=Middle Congaree (IIA); LC=Lower Congaree (IIA) 

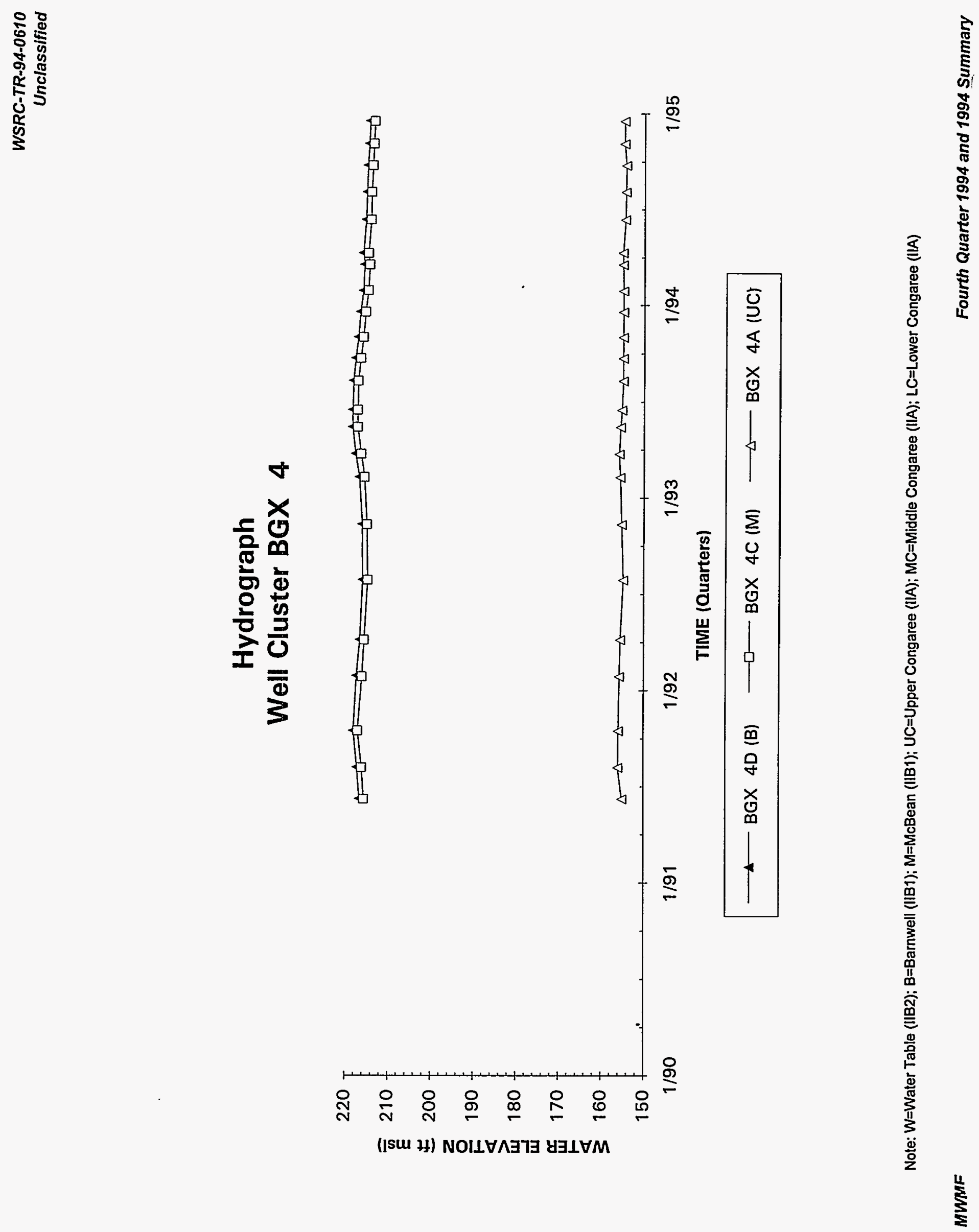

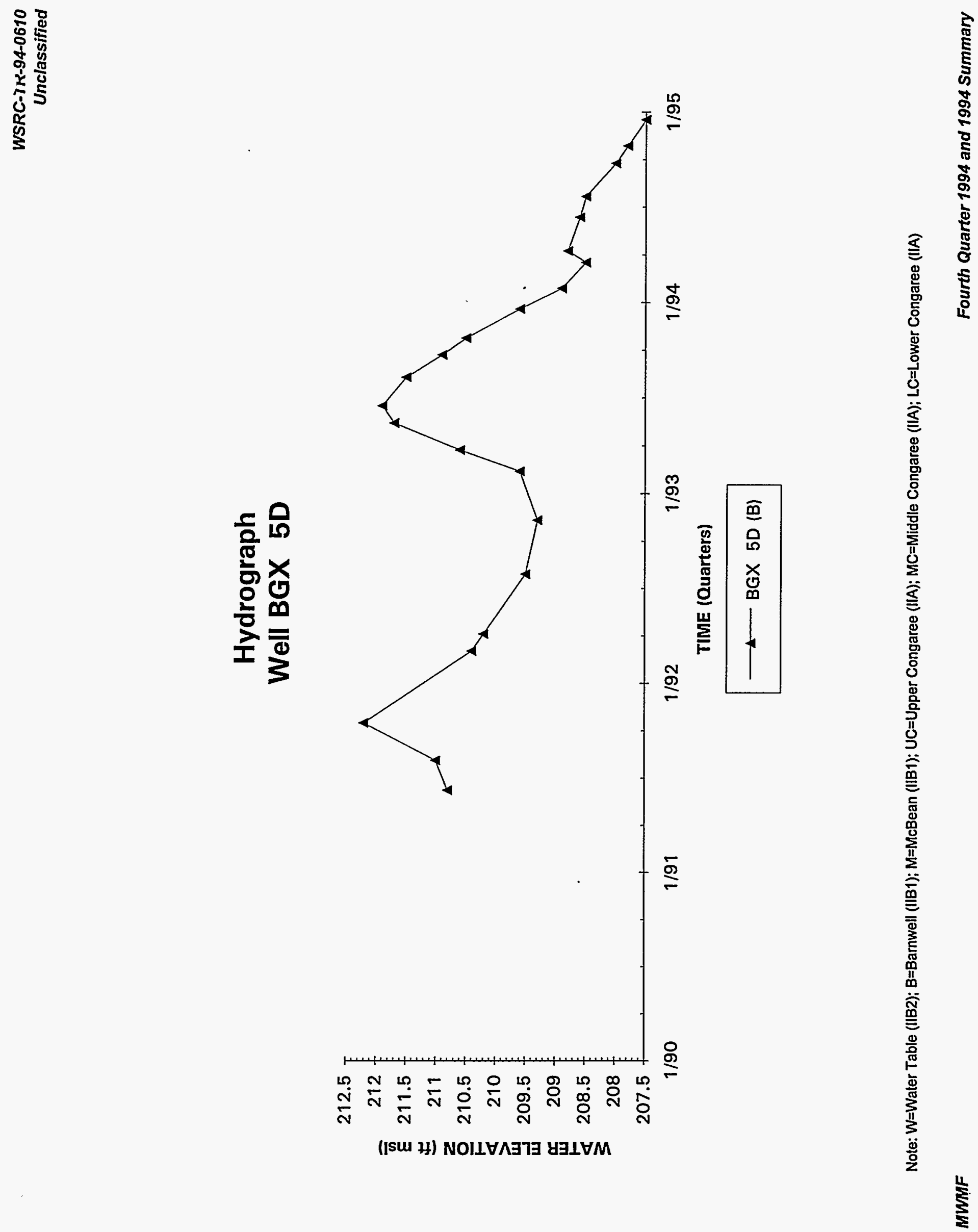

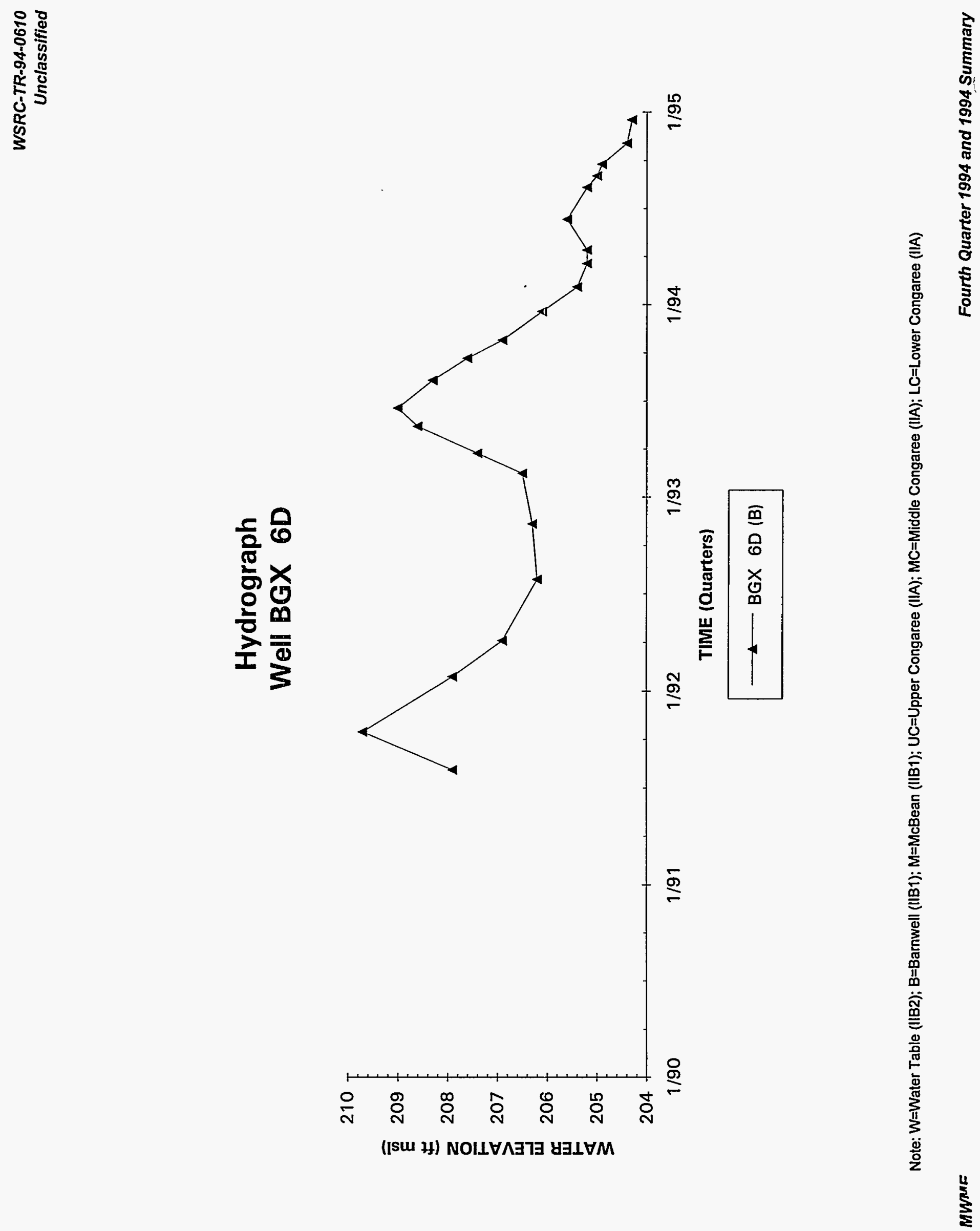

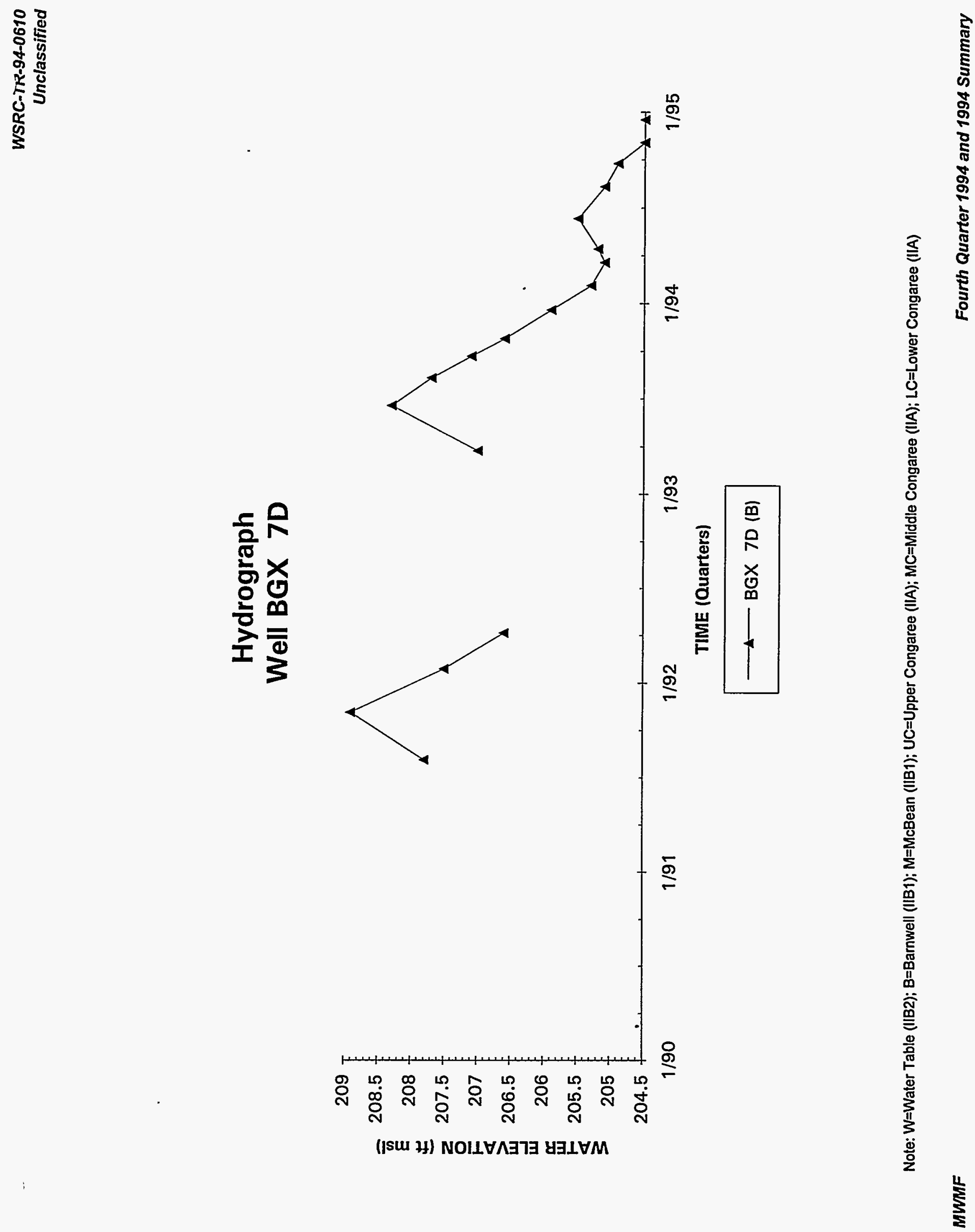

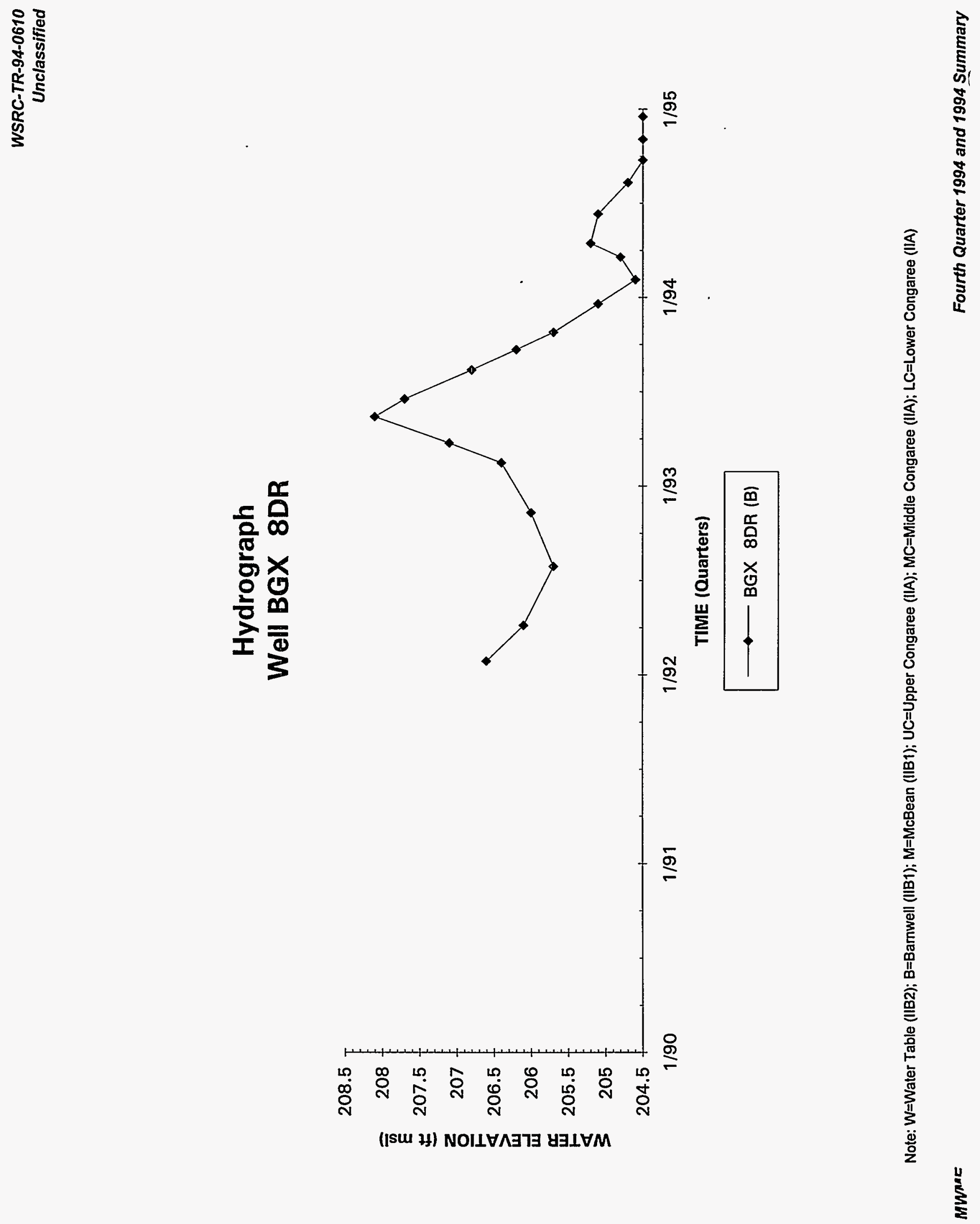


\section{Hydrograph \\ Well BGX 9D}

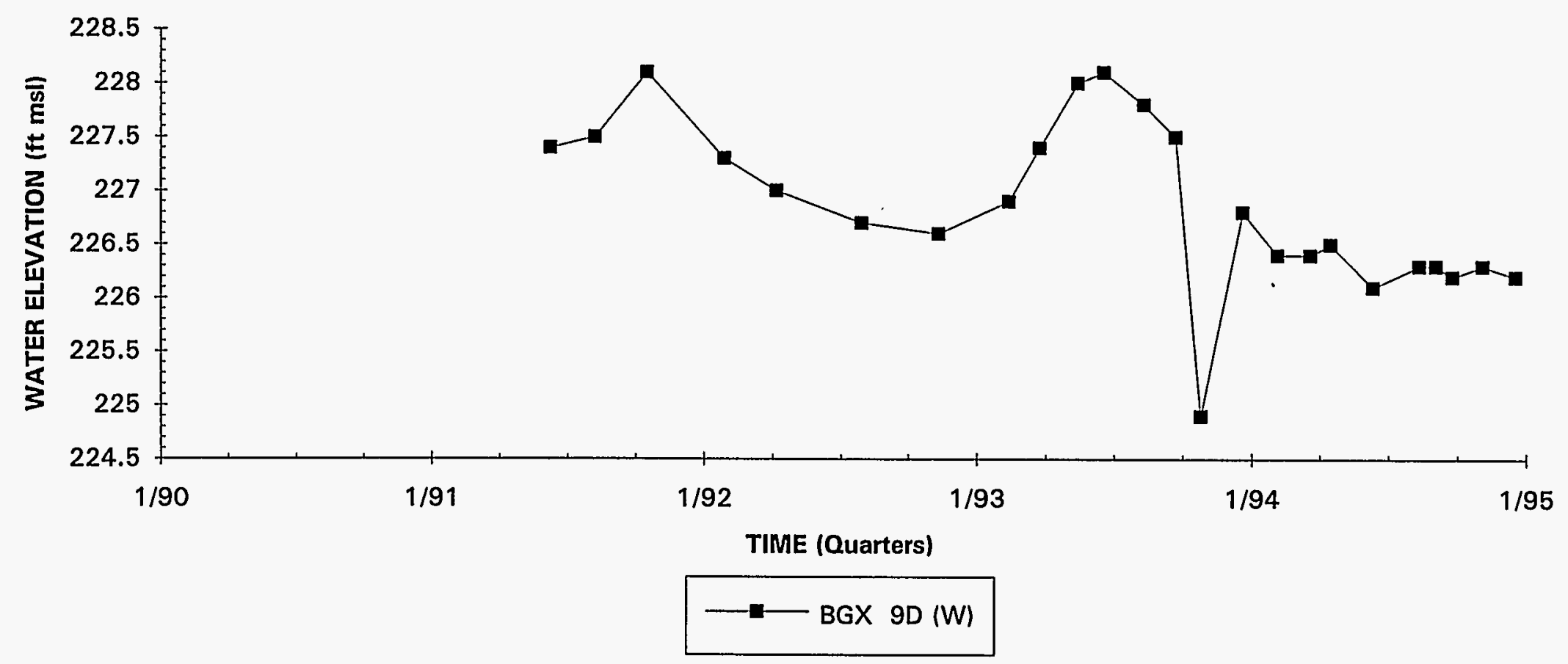

Note: W=Water Table (IIB2); B=Barnwell (IIB1); M=McBean (IIB1); UC=Upper Congaree (IIA); MC=Middle Congaree (IIA); LC=Lower Congaree (IIA) 

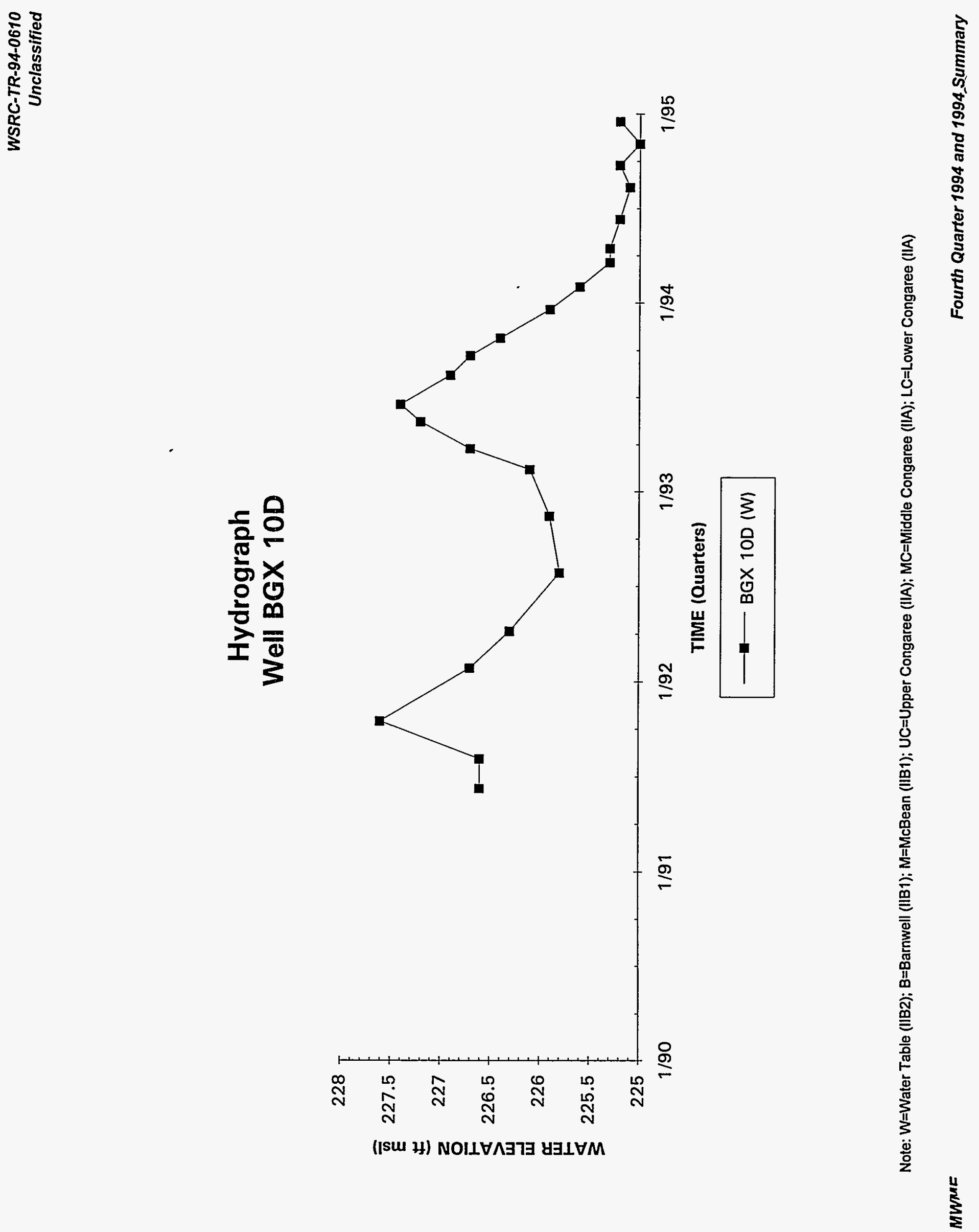

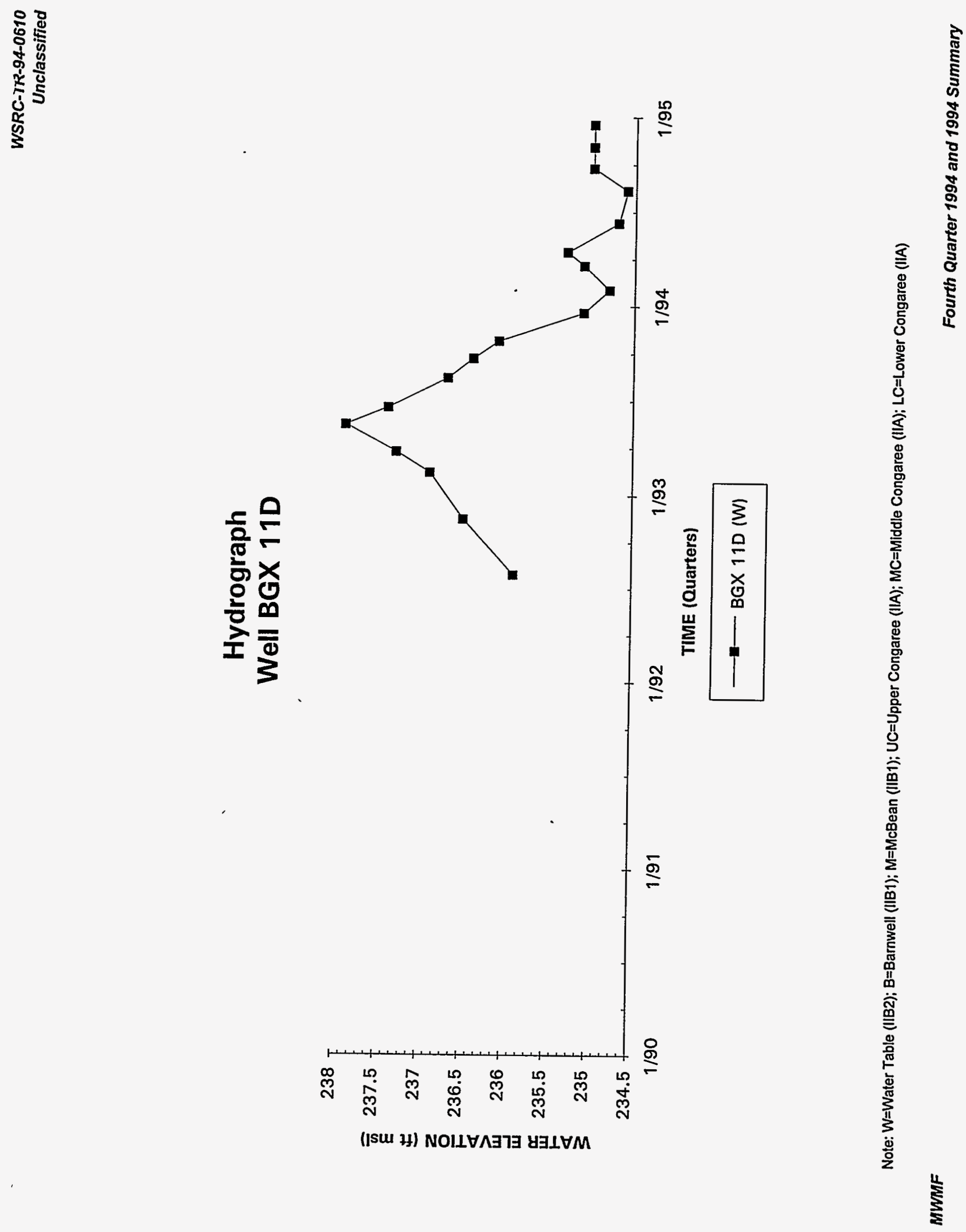


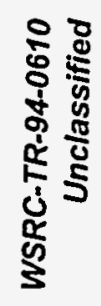
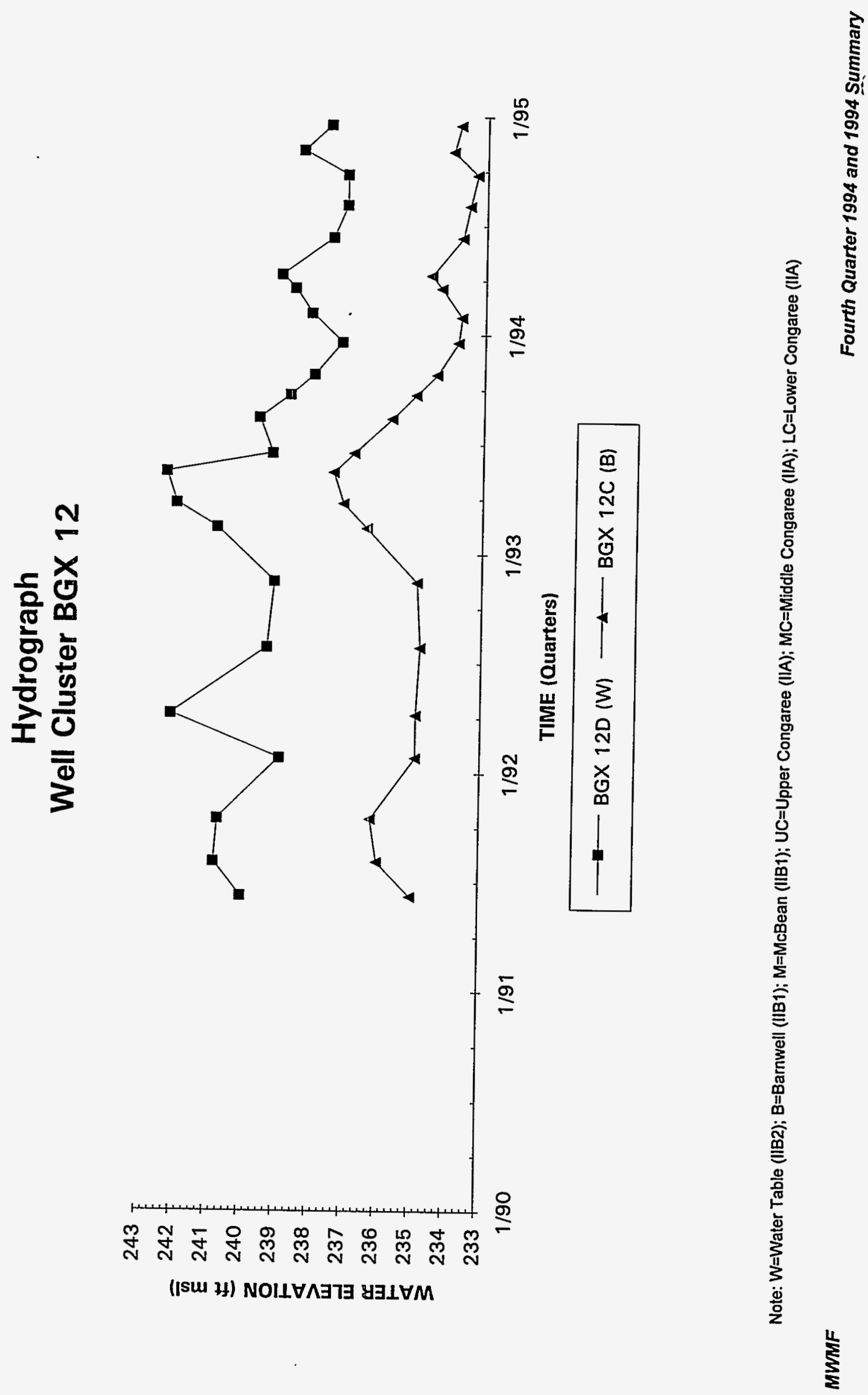

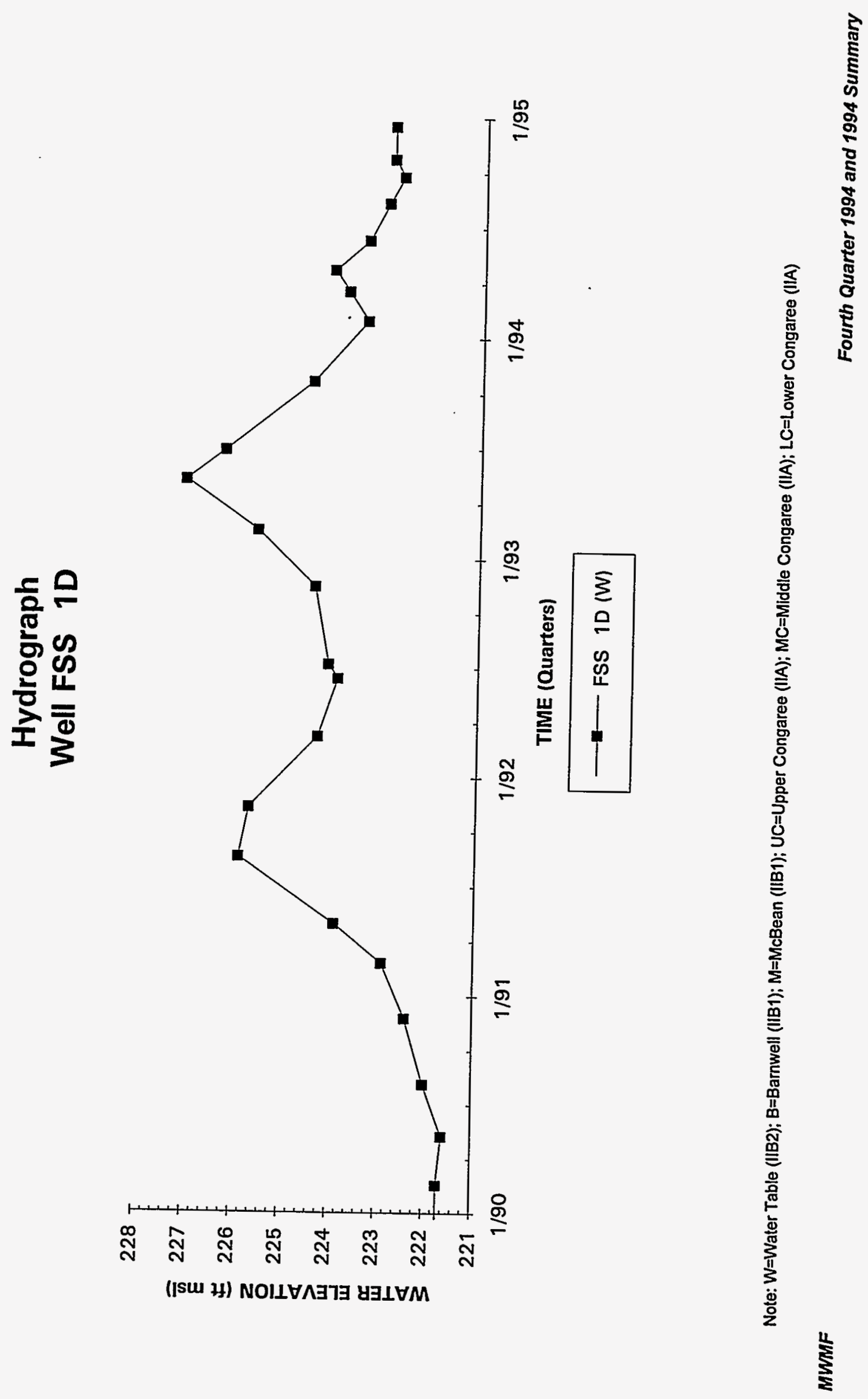

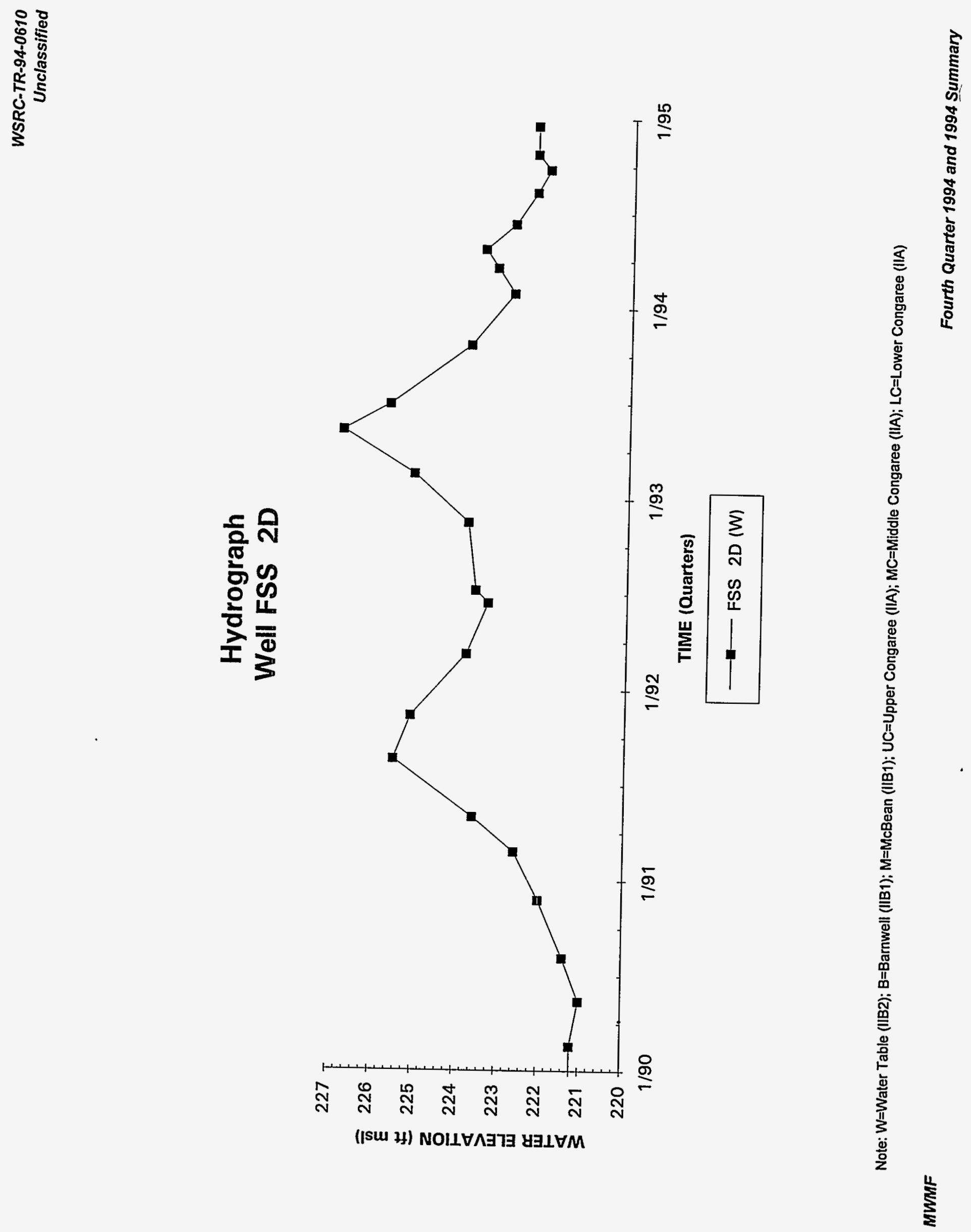

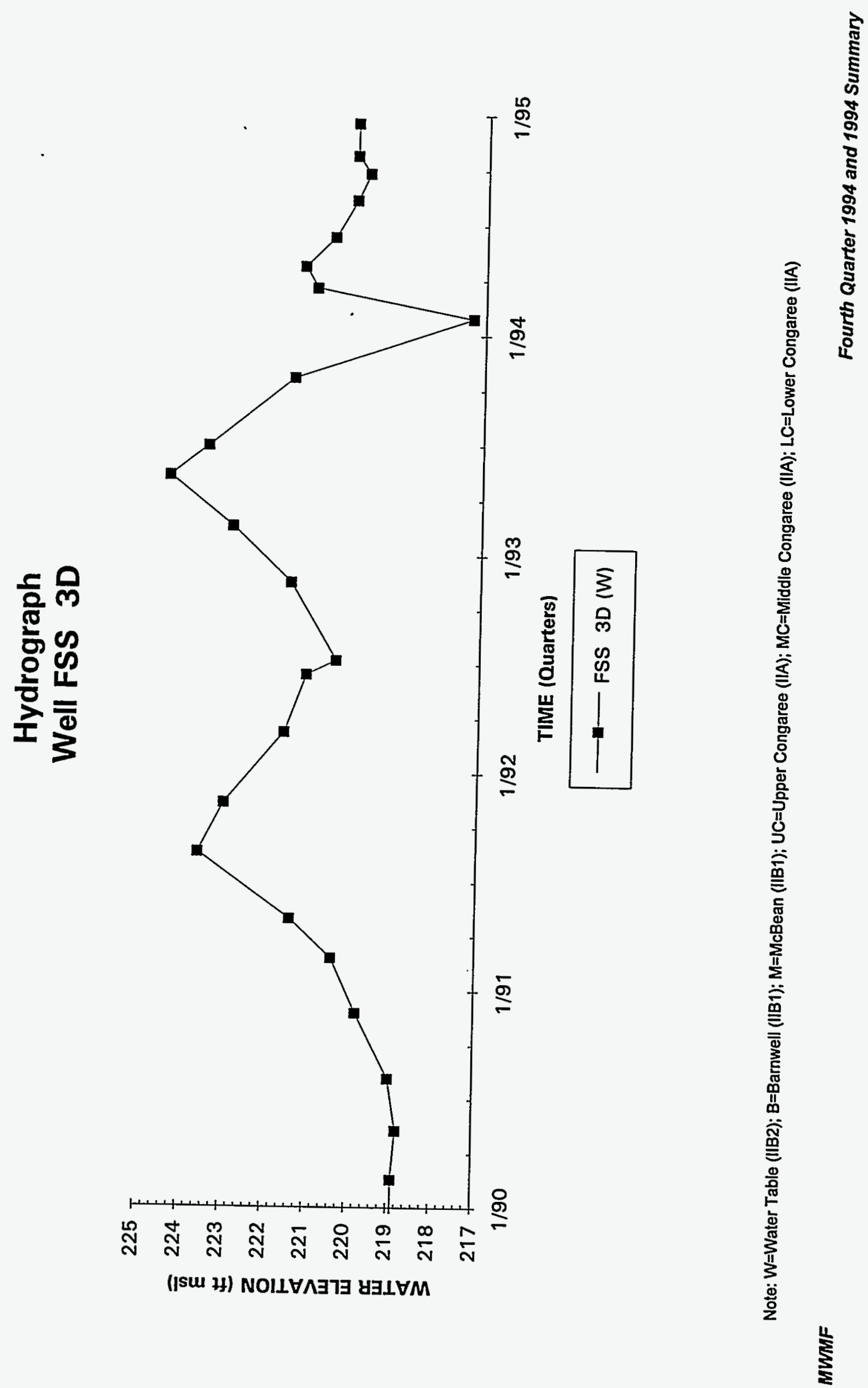

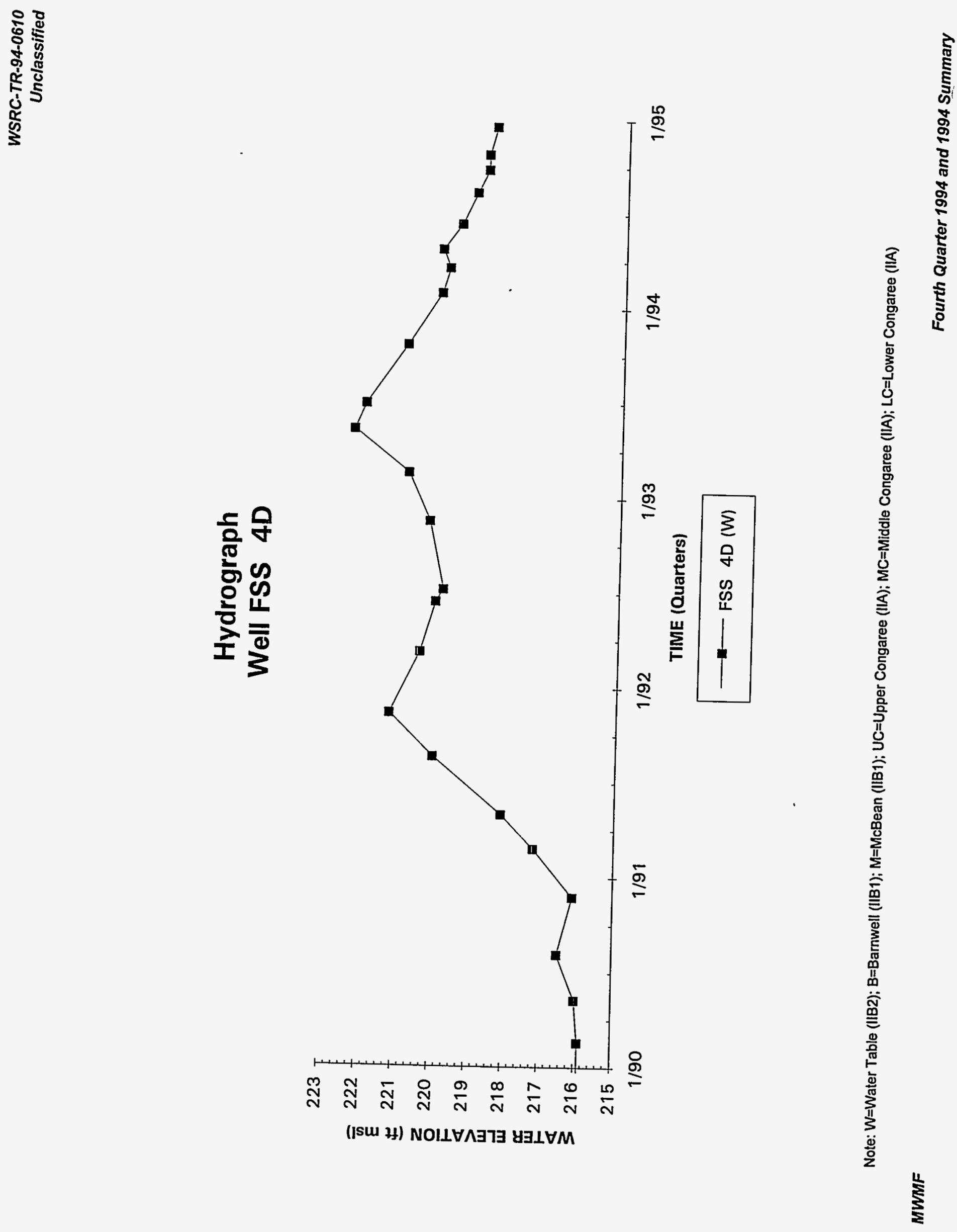


\section{Hydrograph \\ Well HMD 1D}

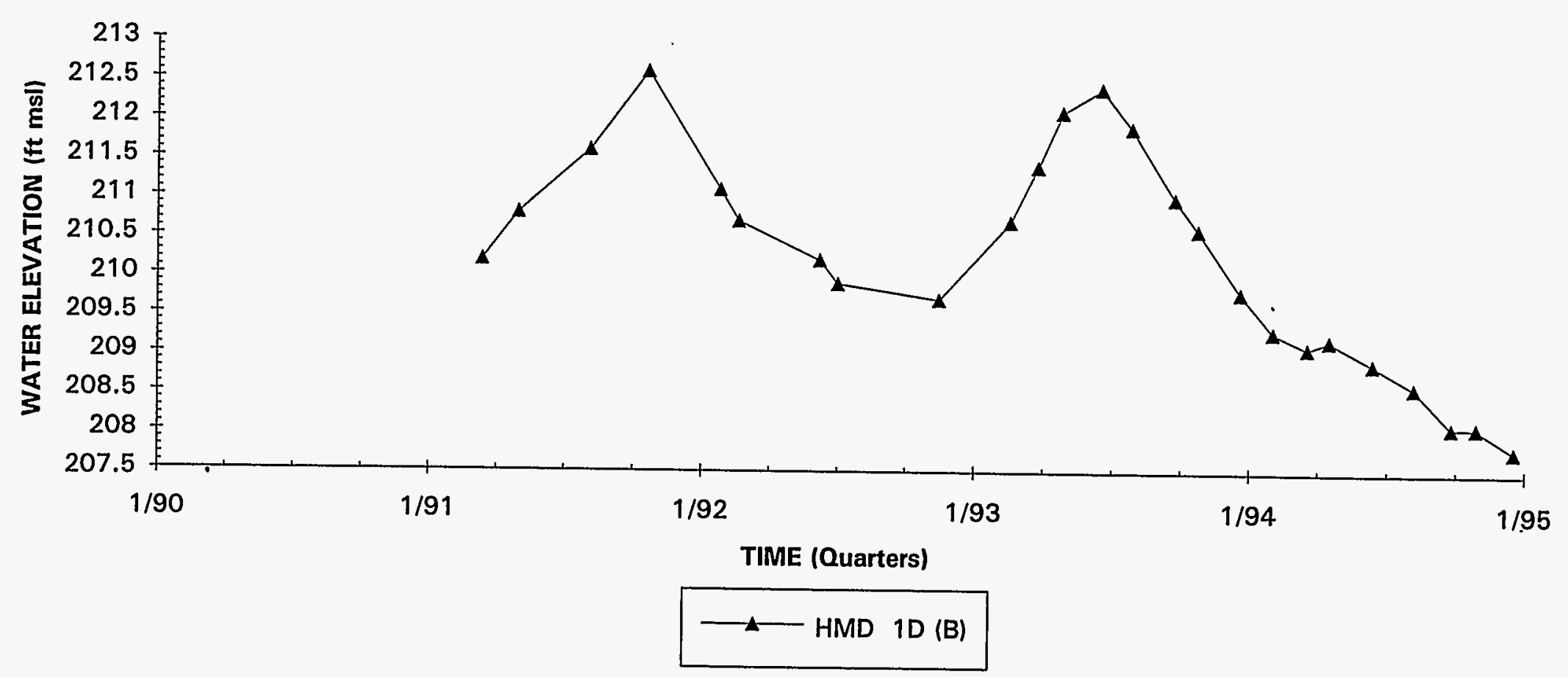

Note: W=Water Table (IIB2); B=Barnwell (IIB1); M=McBean (IIB1); UC=Upper Congaree (IIA); MC=Middle Congaree (IIA); LC=Lower Congaree (IIA) 


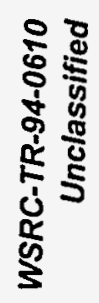
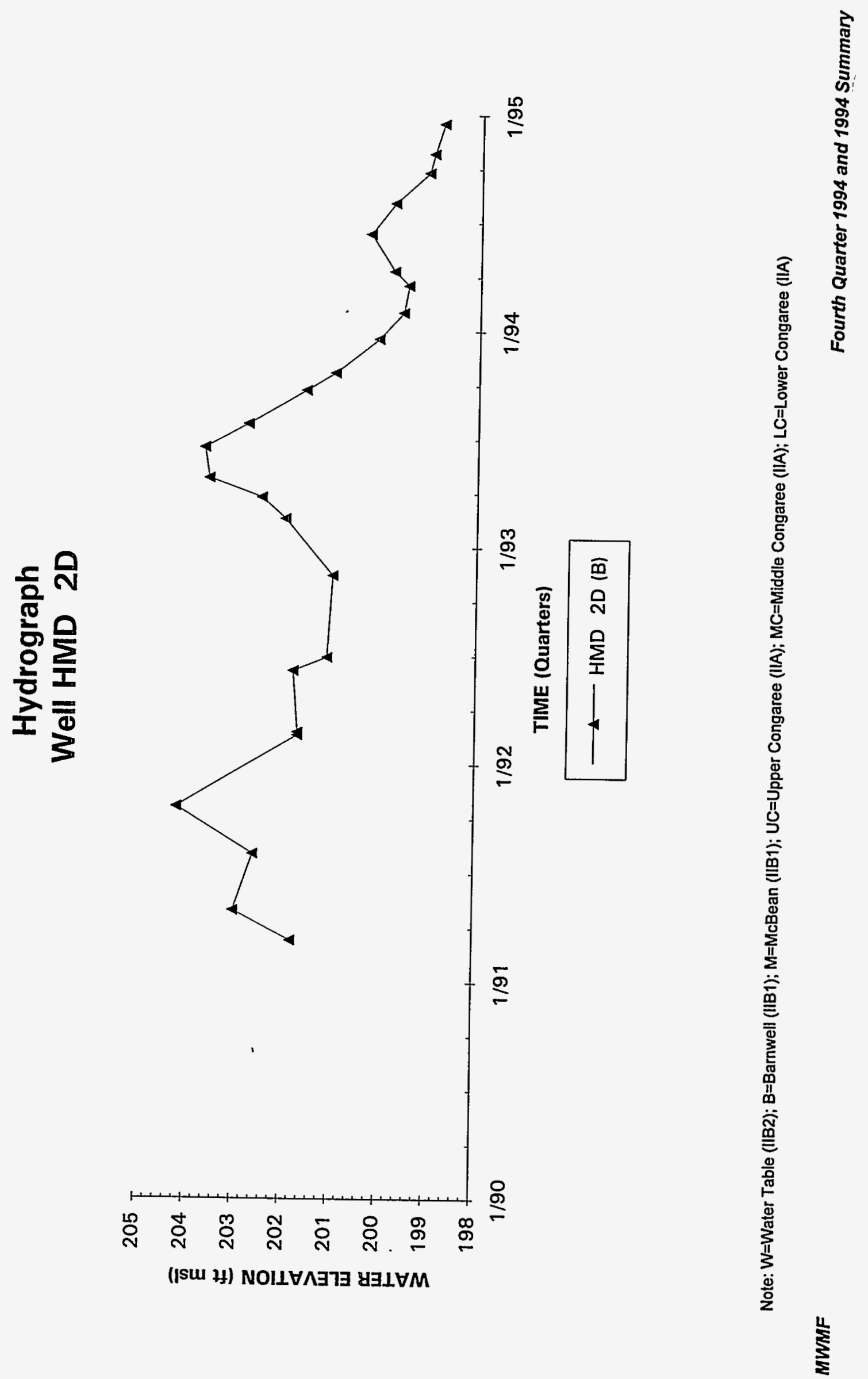

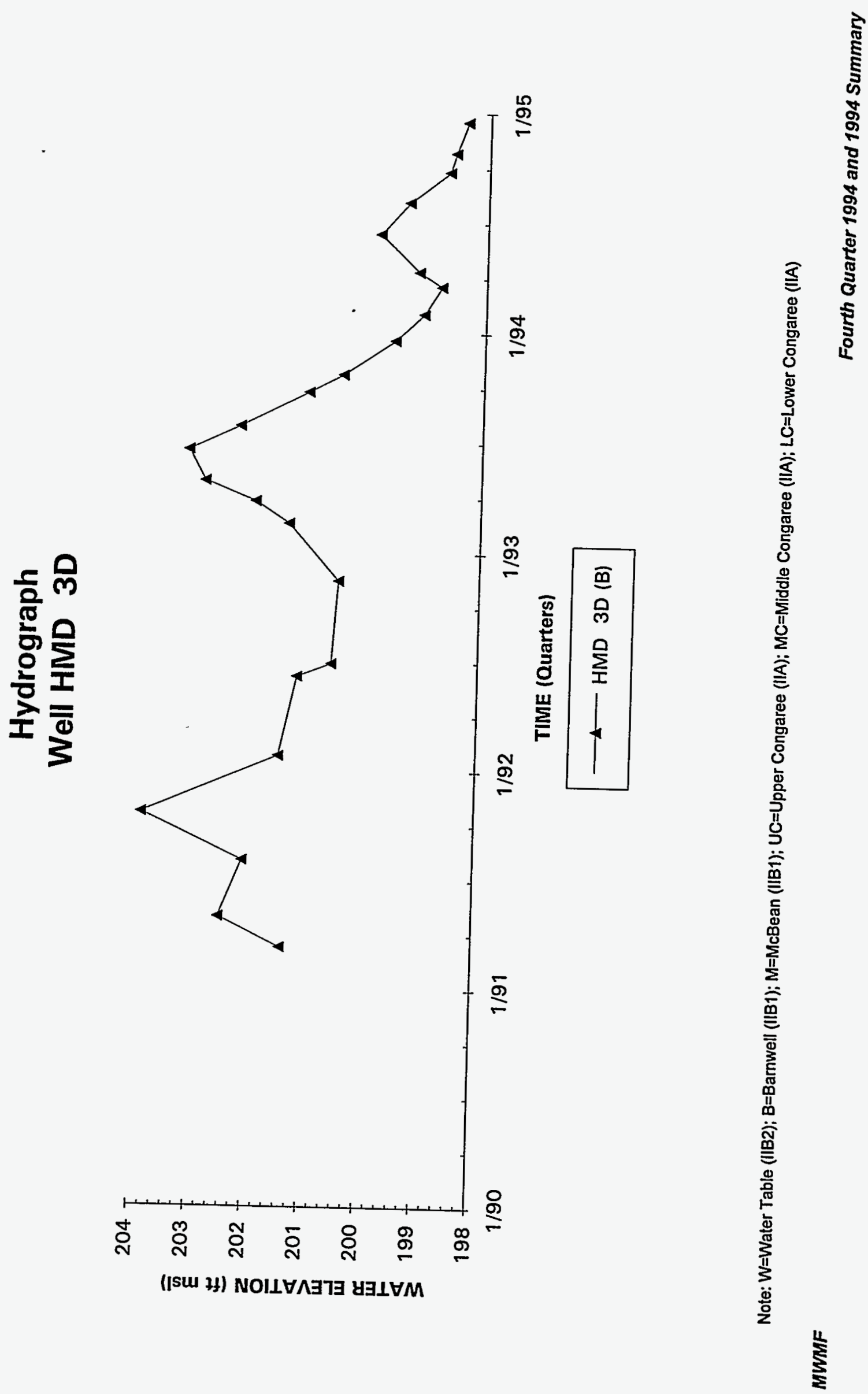

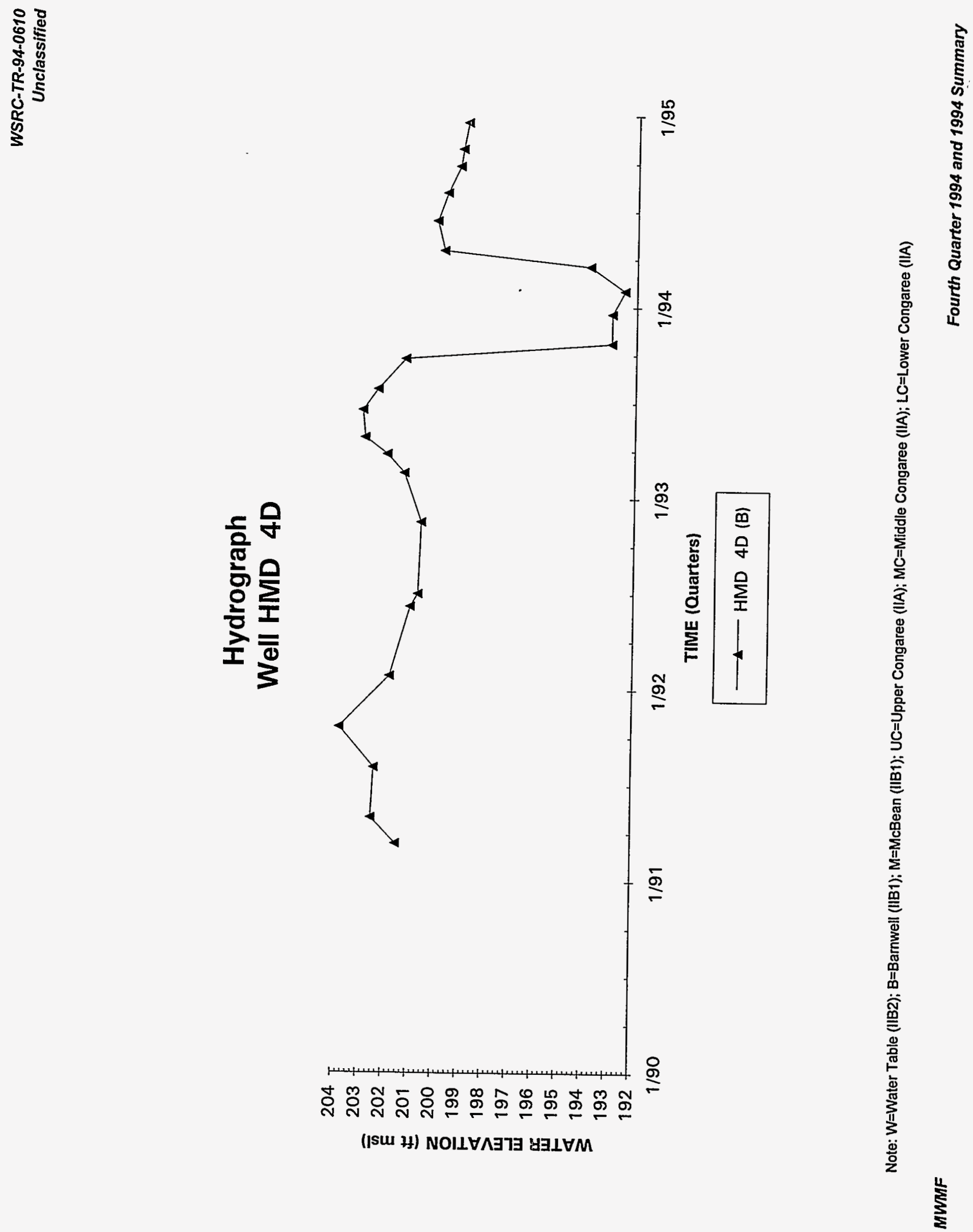


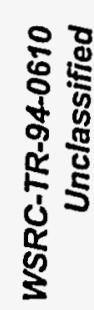
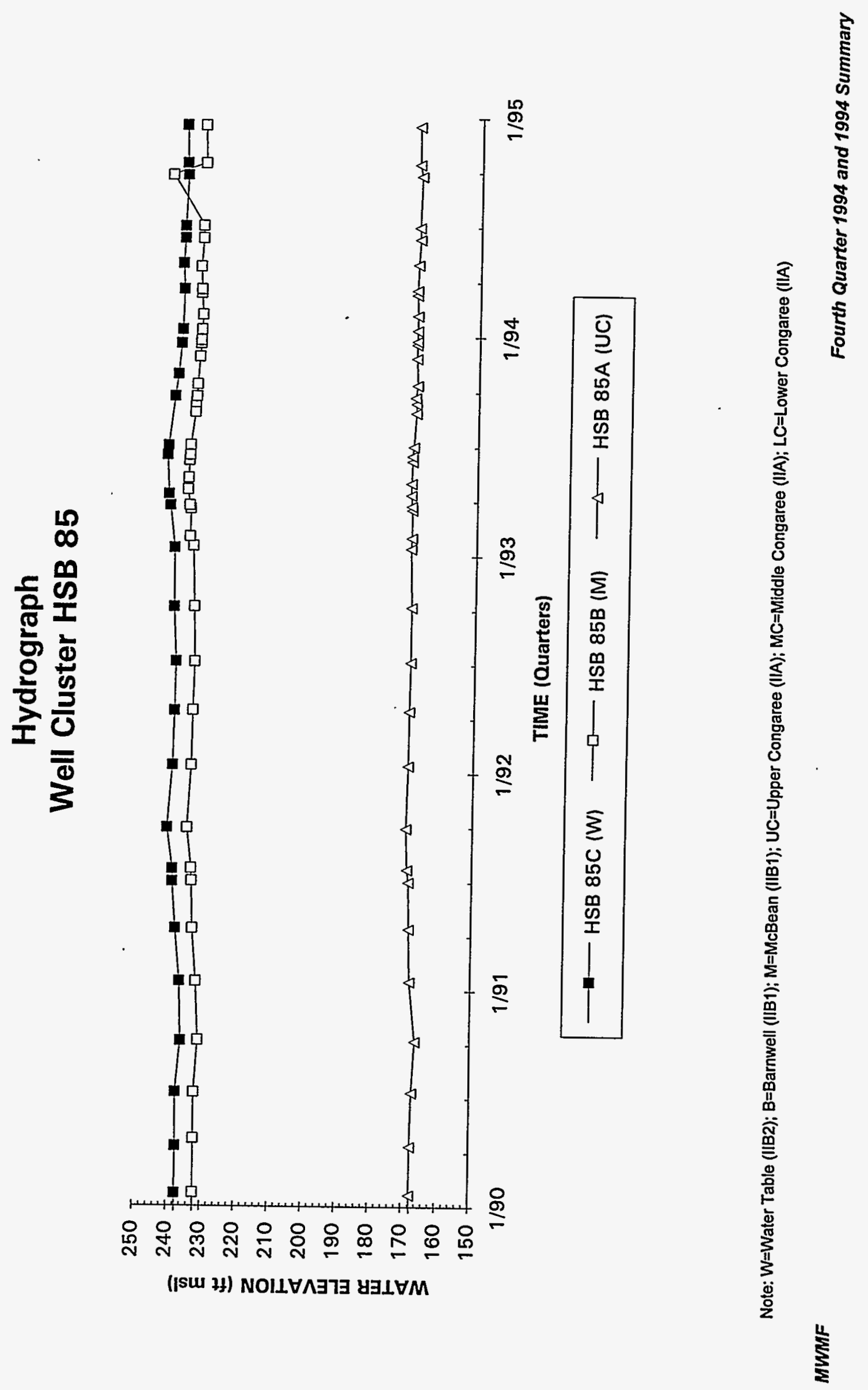